\title{
TWRS Privatization Support Project Waste Characterization Database Development
}

\author{
-. Work Order BE0216
}

Prepared for

Pacific Northwest National Laboratory

November 1995

For the U.S. Department of Energy

Contract DE-AC06-93RL12359

Prepared By

ICF Kaiser Hanford Company

Richland, Washington

VOLUME I

\section{ICF Kaiser Hanford Company}

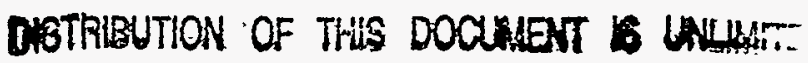


Type of Release:

[X] Technical Report

[] Speech or Presentation

[ ] Journal Article

[] Book or Book Chapter

[ ] Thesis or Dissertation

[ ] Software Documentation
[ ] Brochure/Fl ier
[ ] Trade Show Exhibit
[ ] Internet
[ ] Other

Document Number (Information Release will assign):

PNNL-10971

\begin{tabular}{c|c|c}
\hline Other Number (e.9., NUREG/CR): & Client: \\
PRV-95-64, ReV 0 & DOE-RL
\end{tabular}

Title:

TWRS Privatization Support Waste Characterization Database Development

Author(s):

K D Hiemers, report prepared by ED Johnson (ICF Kaiser Hanford)

\begin{tabular}{l|l|l|}
\hline $\begin{array}{l}\text { Lead Author's Org. Code: } \\
\text { D9T32 }\end{array}$ & $\begin{array}{l}\text { Project No.: } \\
\text { BE0216 }\end{array}$ & $\begin{array}{l}\text { B\&R COde: } \\
\text { EW3120075 }\end{array}$ \\
\hline
\end{tabular}

[ ] The distribution of this document is limited because of: (remember to mark the document with the appropriate limited distribution notice)

[ ] This information product contains intellectual property and I have discussed it with PNL's .Intellectual Property Services.

[X] This information product does Nor contain intellectual property.

[X] This subject falls under a Designated Unclassified Subject Area and does not require review by an Authorized Derivative Classifier.

[ ] This information product has been reviewed by an Authorized Derivative Classifier.

For a Presentation or Journal Article, complete the following:

Title of Conference:

Dates:

City/State:

Title of Journal:

NOTES:

If you have questions or need assistance, please contact Information Release (375-2583/K1-06).

Unless the subject work is covered under a Designated Unclassified Subject Area, the document must be reviewed by an Authorized Derivative Classifier.

If the subject work is 1830 and falls under the oversight of a DOE-RL office OTHER THAN Laboratory Management Division, the document must be reviewed by the author's DOE-RL contact person.

If the information product is not scientific/technical in content and audience but is of general content intended for a lay public audience (such as a program brochure), contact the Information Release office to see if it needs to be reviewed by DOE-RL's office of External Affairs.

For technical reports, please remember to send seven copies to the Information Release office upon completion, for transmittal to the Hanford Technical Library and DOE's Office of Scientific and Technical Information. 


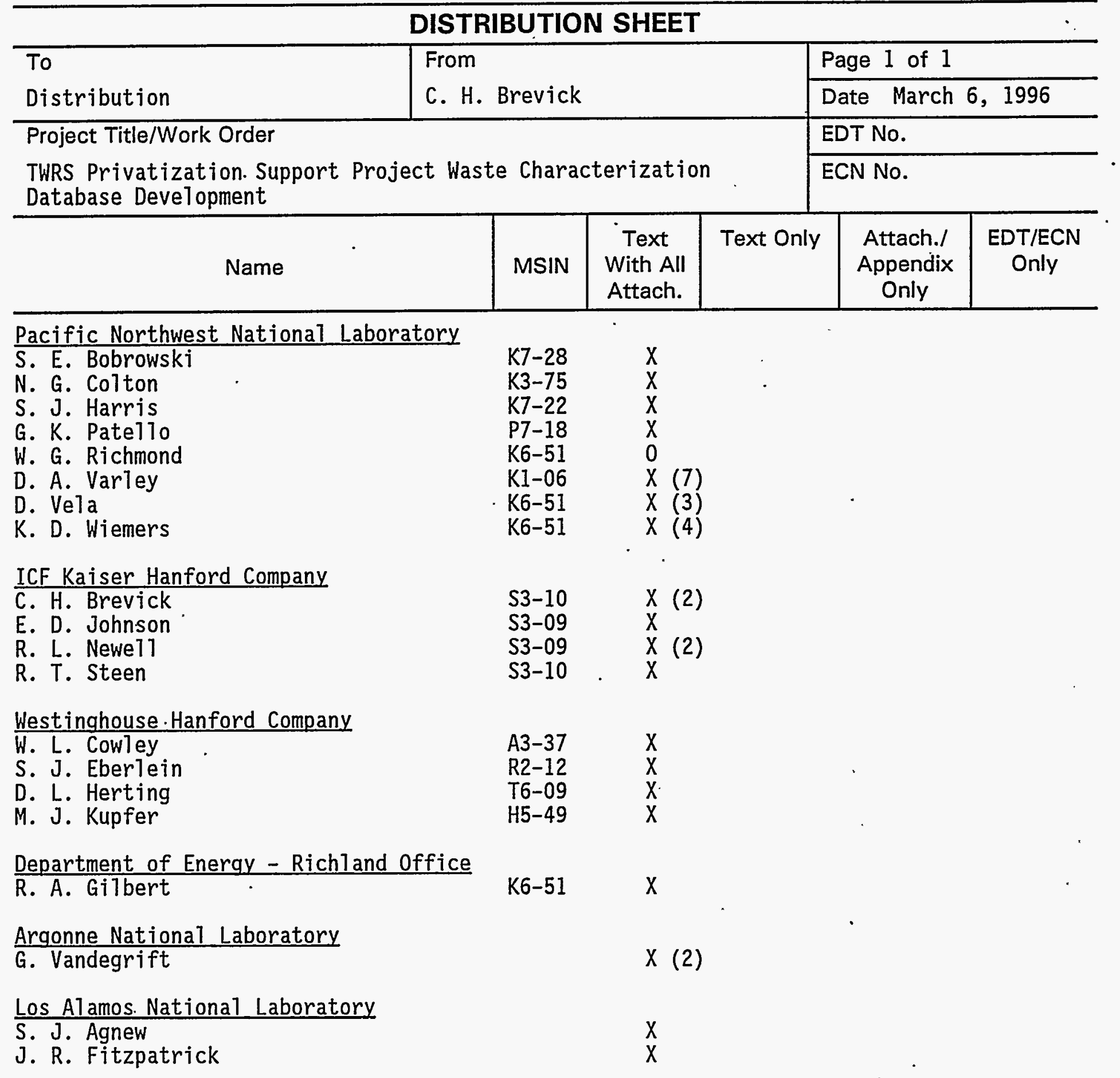




\title{
TWRS PRIVATIZATION SUPPORT PROJECT WASTE CHARACTERIZATION DATABASE DEVELOPMENT
}

\author{
Work Order BE0216 \\ Prepared for \\ Pacific Northwest National Laboratory
}

November 1995

For the U.S. Department of Energy

Contract DE-AC06-93RL12359

Prepared by

ICF Kaiser Hanford Company

Richland, Washington 
WORK ORDER BE0216

LETTER REPORT

\section{TWRS PRIVATIZATION SUPPORT PROJECT WASTE CHARACTERIZATION DATABASE DEVELOPMENT}

ICF Kaiser Hanford Company
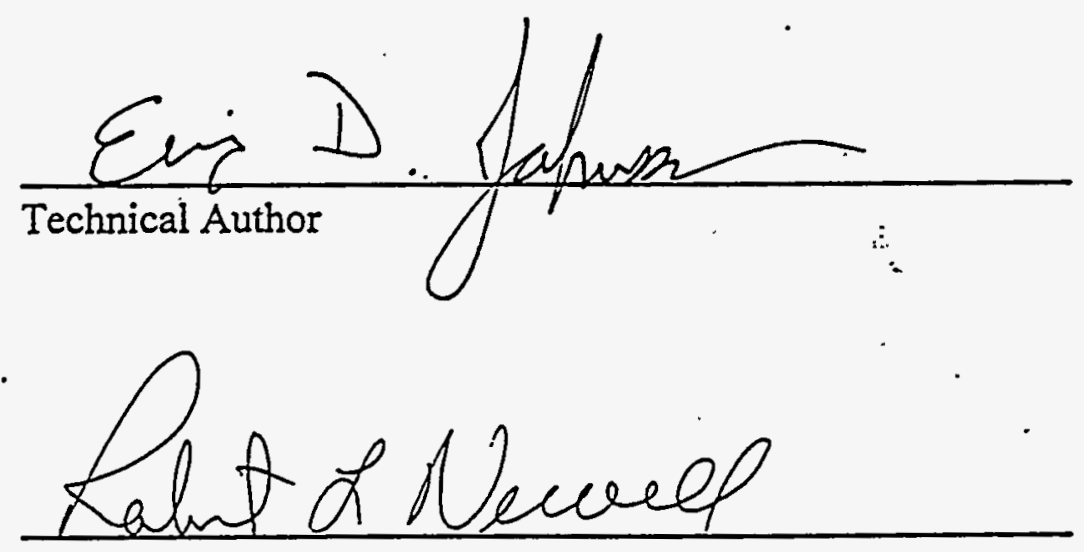

Principle Lead Engineer $\frac{11 / 17 / 95}{\text { Date }}$

$\frac{11 / 17 / 95}{\text { Date }}$

Pacific-Northwest National Laboratory
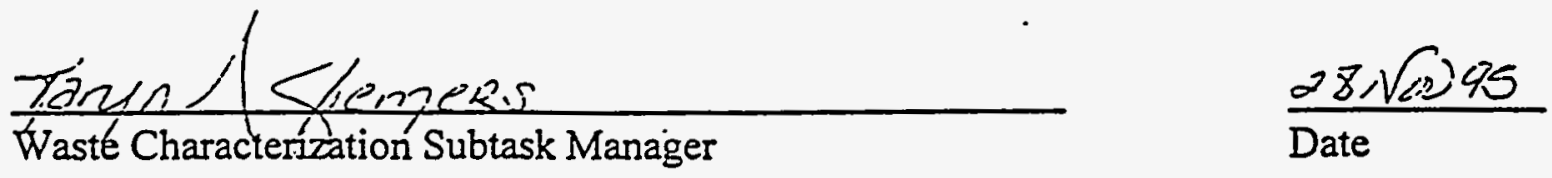


\section{ACKNOWLEDGEMENTS}

The author would like to acknowledge the contributions of the lead technical review members: L. Chen (ANL), C. J. Connor (ANL), J. R. Fitzpatrick (LANL), D. L. Herting (WHC), C. J. Mertz (ANL), G. K. Patello (PNNL), M. C. Regalguto (ANL), and G. F. Vandegrift (ANL), and the technical review panel consisting of staff from PNNL, WHC, ANL, LANL, DOE-RL, and the Technical Advisory Panel (TAP). 


\title{
DISCLAIMER
}

This report was prepared as an account of work sponsored by an agency of the United States Government. Neither the United States Government nor any agency thereof, nor Battelle Memorial Institute, nor any of their employees, makes any warranty, express or implied, or assumes any legal liability or responsibility for the accuracy, completeness, or usefulness of any information, apparatus, product, or process disclosed, or represents that its use would not infringe privately owned rights. Reference herein to any specific commercial product, process, or service by trade name, trademark, manufacturer, or otherwise does not necessarily constitute or imply its endorsement, recommendation, or favoring by the United States Government or any agency thereof, or Battelle Memorial Institute. The views and opinions expressed herein do not necessarily state or reflect those of the United States Government or any agency thereof.

\author{
PACIFIC NORTHWEST NATIONAL LABORATORY \\ operated by \\ BATTELLE \\ for the \\ UNITED STATES DEPARTMENT OF ENERGY \\ under Contract DE-AC06-76RLO 1830
}




\section{ABSTRACT}

Pacific Northwest National Laboratory requested support from ICF Kaiser Hanford Company in assembling radionuclide and chemical analyte sample data and inventory estimates for fourteen Hanford underground storage tanks: 241-AN-102, -104, -105, -106, and -107; 241-AP-102, -104, and-105; 241-AW-101, -103, and -105; 241$A Z-101$ and -102; and 241-C-109. Sample data were assembled for sixteen radionuclides and thirty-five chemical analytes. The characterization data were provided to Pacific Northwest National Laboratory in support of the Tank Waste Remediation Services Privatization Support Project.

The purpose of this report is to present the results and document the methodology used in preparing the waste characterization information data set to support the Tank Waste Remediation Services Privatization Support Project. This report describes the methodology used in assembling the waste characterization information and how that information was validated by a panel of independent technical reviewers. Also, contained in this report are the various data sets created: the master data set, a subset, and an unreviewed data set. The master data set contains waste composition information for Tanks 241-AN-102 and -107; 241-AP-102 and -105; 241-AW-101; and 241-AZ-101 and -102. The subset contains only the validated analytical sample data from the master data set. The unreviewed data set contains all collected but unreviewed sample data for Tanks 241-AN-104, -105, and 106; 241-AP-104; 241-AW-103 and -105; and 241-C-109. The methodology used to review the waste characterization information was found to be an accurate, useful way to separate the invalid or questionable data from the more reliable data. In the future, this methodology should be considered when validating waste characterization information. 
Introduction $\ldots \ldots \ldots \ldots \ldots \ldots \ldots \ldots \ldots \ldots \ldots \ldots \ldots \ldots \ldots \ldots \ldots \ldots \ldots \ldots$

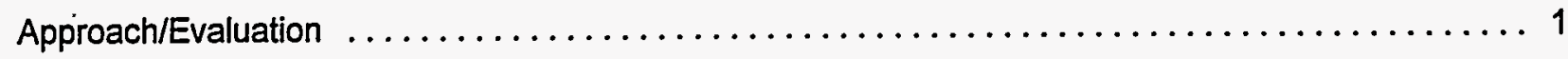

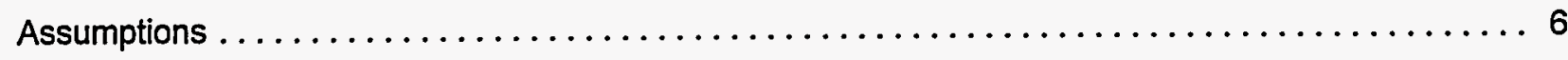

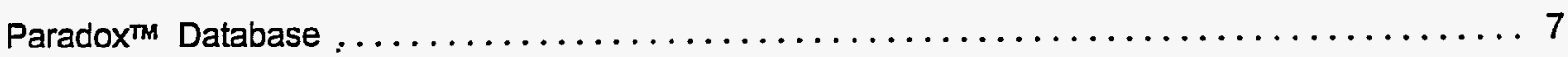

Summary and Conclusions $\ldots \ldots \ldots \ldots \ldots \ldots \ldots \ldots \ldots \ldots \ldots \ldots \ldots \ldots \ldots \ldots$

References $\ldots \ldots \ldots \ldots \ldots \ldots \ldots \ldots \ldots \ldots \ldots \ldots \ldots \ldots \ldots \ldots \ldots \ldots \ldots . \ldots \ldots$

\section{TABLES}

1. List of Radionuclides in the Data Set . . . . . . . . . . . . . . . . . . .

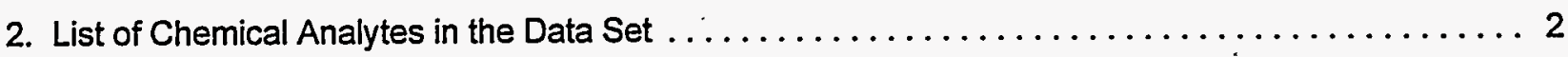

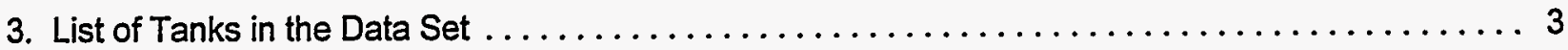

FIGURES

TWRS PSP Waste Characterization Database Development Methodology Roadmap ......... 5

\section{APPENDICES}

Volume I

A. Radionuclide and Chemical Analyte Data Tables for the Master Data Set

Volume II

B. Radionuclide and Chemical Analyte Subset Data Tables

C. Unreviewed Radionuclide and Chemical Analyte Data Tables

D. References for the Sample Data Contained in Appendices A, B, and C 


\section{LIST OF TERMS}

ANL

Braun

HTCE Inv.

ICF KH

Waste Characterization Information

Inventory Estimates

LANL

Master Data Set

PNNL

PSP

Sample Data

STIV

Subset

TCD

TRAC

TWRS

Unreviewed Data Set

Wastren

WHC
Argonne National Laboratory

Refers to the data set developed by Mr. D. J. Braun

Historical Tank Content Estimate Inventory, estimated inventory of individual tank wastes created by S. F. Agnew and published by ICF KH for WHC in the HTCE reports WHC-SD-WM-ER-349 through 352

ICF Kaiser Hanford Company

This is the combination of sample data and inventory estimates

Estimates of tank contents based on computer models, process history, process flow sheets, and waste transfer data

Los Alamos National Laboratory

This is the data set that contains all collected waste characterization information for the seven tanks selected to review

Pacific Northwest National Laboratory

Privatization Support Project

Analytical results from an actual tank sampling event

Source Term Inventory Validation data set, data from WHC-SD-WM-ER-400, Tank Waste Source Term Inventory Validation

This is a subset of the master data set. It contains only the valid waste characterization information.

Tank Characterization Database. It contains current characterization information and is operated by PNNL.

Track Radioactive Components data set. A computer estimate of the tank inventories

Tank Waste Remediation Services

This data set contains all collected waste characterization information for the seven tanks that were not selected for review. This data has not gone through a review process.

Refers to the Wastren/Van Vleet data set

Westinghouse Hanford Company

vii 


\section{TWRS PRIVATIZATION SUPPORT PROJECT WASTE CHARACTERIZATION DATABASE DEVELOPMENT}

\section{INTRODUCTION}

Pacific Northwest National Laboratory (PNNL) requested support from ICF Kaiser Hanford Company (ICF KH) in the sample data assembly, tabulation, and plotting of liquid and solid radionuclide and chemical analyte sample data and inventory estimates for up to 14 Hanford underground waste storage tanks. Sample data were to be assembled for 17 radionuclides and 35 chemical analytes that might be present in the underground waste storage tanks. The characterization data were to be provided to PNNL in support of the Tank Waste Remediation Services (TWRS) Privatization Support Project.

The PNNL requested ICF KH to perform this work because of our experience in assembling characterization data sets. In 1994 and 1995, ICF KH published Historical Tank Content Estimate (HTCE) reports for all four tank farm quadrants (northeast, northwest, southeast, and southwest), WHC-SD-WM-ER-349 through 352, Rev. 0 . The Supporting Documents, WHC-SD-WM-ER-308 through 325, Rev. 0 (by tank farm), were also published for WHC. These documents contain sample information in tables, with copies of the original reports and memoranda on all tanks until December 1989. In April 1995, ICF KH published the Tank Waste Source Term Inventory Validation (STIV) report (WHC-SD-WM-ER-400, Rev. 0) for the WHC Safety Analysis and Nuclear Engineering group. This group was preparing an accident analysis for the Hanford tank farms. The accident analysis required the calculation of unit doses, dose consequences, and source terms for radionuclides and toxic chemicals. They needed concentrations of toxic chemicals and radionuclides present in all 177 tanks. The list of constituents was limited to 24 toxic chemicals and 11 radionuclides. This initial data set (STIV data set) consisted of the Braun, Wastren, and Kaiser ("raw") data sets, the HTCE and Track Radioactive Components (TRAC) inventory estimates, and Tank Characterization Database (TCD) sample results.

The initial scope of the work consisted of assembling liquid and solid characterization data, both inventory and analytical, for up to fourteen tanks (K. D. Wiemers, PNNL, to R. T. Steen, ICF KH, "Letter of Instruction from TWRS Privatization Support Project, Waste Characterization Task, dated November 9,1995$)$. The data were to be placed in a tabular format and scatter plots prepared representing the individual sample data points in an identifiable manner. In addition, an electronic file (or electronic application) of the sample data for the selected tanks was to be made available in an electronic Site-supported database. As the project progressed, some changes to the initial work scope occurred. The scatter plots were deemed not useful for this project and were eliminated from the scope. Due to the acceleration of the schedule, time did not permit the review of the solid radionuclide and analyte data - only liquid waste characterization information was reviewed and validated. The liquid waste characterization information was needed to support the waste facility low-activity feed envelope development. Antimony-129 was deleted from the data set due to its short half-life and tin-127 was changed to tin126 (cc:Mail from K. D. Wiemers to C. H. Brevick, dated June 8, 1995). Dysprosium, which was originally in the STIV data set, was excluded from this data set because dysprosium has never been quantifiably detected in any tank at Hanford (it occasionally shows up on an inductively coupled plasma analysis because heavy-element corrections have not been made). The final data set includes 16 radionuclides and 35 chemical analytes.

\section{APPROACH/EVALUATION}

Additions to the STIV data set increased the radionuclides to 16 and chemical analytes to 35 , but narrowed the number of tanks from 177 to 14. A list of the radionuclides and chemical analytes are provided in Tables 1 and 2, respectively. The 14 tanks that waste composition information was compiled for are listed in Table 3. 
TABLE 1. List of Radionuclides in the Data Set

\begin{tabular}{|c|c|}
\hline Americium-241 $\left({ }^{241} \mathrm{Am}\right)$ & Plutonium-238 $\left({ }^{238} \mathrm{Pu}\right)$ \\
\hline *Carbon-14 $\left({ }^{14} \mathrm{C}\right)$ & Plutonium-239/240 $\left({ }^{239240} \mathrm{Pu}\right)$ \\
\hline Curium-244 $\left({ }^{244} \mathrm{Cm}\right)$ & Plutonium-241 $\left({ }^{241} \mathrm{Pu}\right)$ \\
\hline Cobalt-60 $\left({ }^{60} \mathrm{Co}\right)$ & Selenium-79 $\left({ }^{79} \mathrm{Se}\right)$ \\
\hline Cesium-137 $\left({ }^{197} \mathrm{Cs}\right)$ & ${ }^{*}$ Tin-126 $\left({ }^{126} \mathrm{Sn}\right)$ \\
\hline Europium-154 $\left({ }^{154} \mathrm{Eu}\right)$ & Strontium-90 $\left({ }^{90} \mathrm{Sr}\right)$ \\
\hline *lodine-129 $\left({ }^{129} \mathrm{l}\right)$ & ${ }^{*}$ Technetium-99 $\left({ }^{99} \mathrm{Tc}\right)$ \\
\hline Neptunium-237 $\left({ }^{237} \mathrm{~Np}\right)$ & Yttrium-90 $\left({ }^{90} \mathrm{Y}\right)$ \\
\hline
\end{tabular}

*Denotes radionuclides added for TWRS Privatization Support Project

TABLE 2. List of Chemical Analytes in the Data Set

\begin{tabular}{|c|c|c|}
\hline${ }^{\star A}$ Aluminum (Al) & Chromium (Cr) & Hydroxide $(\mathrm{OH})$ \\
\hline Arsenic (As) & ${ }^{*}$ Fluorine $(F I)$ & *Lead $(\mathrm{Pb})$ \\
\hline Barium (Ba) & *Iron (Fe) & *Phosphate $\left(\mathrm{PO}_{4}\right)$ \\
\hline Beryllium (Be) & Mercury $(\mathrm{Hg})$ & Antimony (Sb) \\
\hline Calcium (Ca) & ^Potassium (K) & Selenium (Se) \\
\hline Cadmium (Cd) & Lanthanum (La) & *Sulphate $\left(\mathrm{SO}_{4}\right)$ \\
\hline Cerium (CE) & Sodium (Na) & Strontium (Sr) \\
\hline${ }^{*}$ Chlorine (Cl) & Neodymium (Nd) & Tellerium (Te) \\
\hline Cyanide (CN) & Ammonia $\left(\mathrm{NH}_{3}\right)$ & Thallium (TI) \\
\hline Cobalt (Co) & ${ }^{*}$ Nickel (Ni) & *Total Organic Carbon (TOC) \\
\hline Chromium $^{+3}\left(\mathrm{Cr}^{+3}\right)$ & *Nitrite $\left(\mathrm{NO}_{2}\right)$ & Uranium (U) \\
\hline Chromium ${ }^{+6}\left(\mathrm{Cr}^{+6}\right)$ & ${ }^{*}$ Nitrate $\left(\mathrm{NO}_{3}\right)$ & Vanadium $(V)$ \\
\hline
\end{tabular}

*Denotes chemical analytes added for TWRS Privatization Support Project 
TABLE 3. List of Tanks in the Data Set

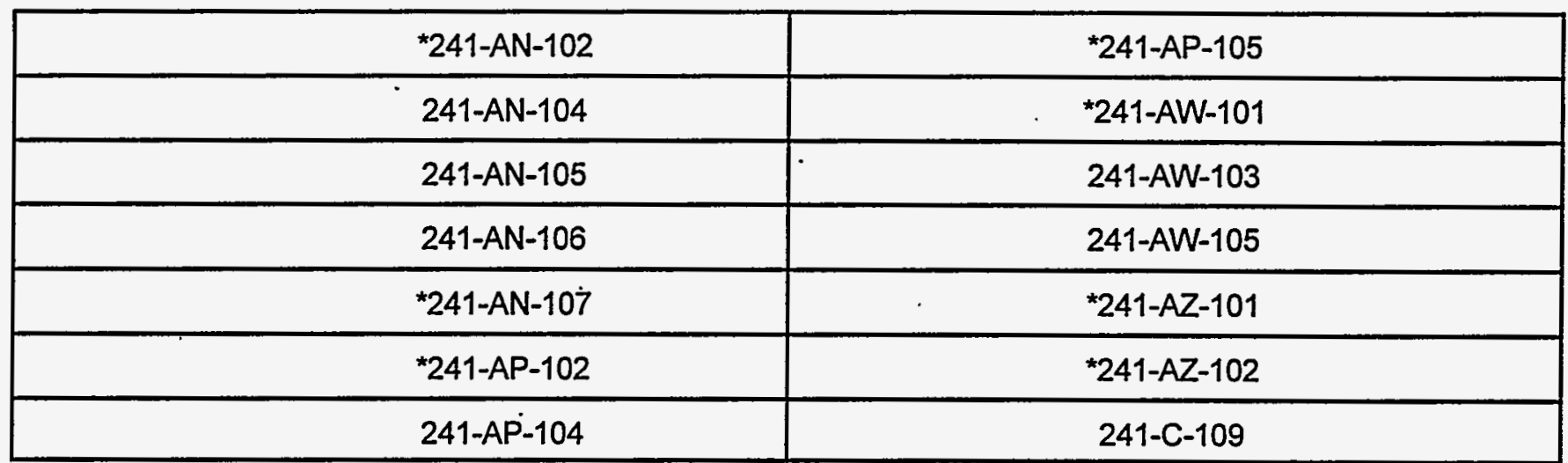

*Denotes tanks contained in the master data set and its subset

The following is a list and brief description of the six different sources of tank characterization and inventory estimate data used in compiling the data set.

Kaiser Data Set ("raw"):

Sample information from reports, memoranda, and letters found in the Tank Characterization

Resource Center (TCRC). This information was entered into an electronic database (Paradox ${ }^{\mathrm{TM}}$ ).

Braun Data Set:

This data set was developed by Mr. D. J. Braun for safety analysis. The data set contains analytical data from the TCRC selected based on highest concentrations for fissile material and lowest for neutron poisons.

Wastren Data Set:

The Wastren data set (also known as the Van Vleet data set) is a compilation of sample information before 1990 prepared by Wastren, Inc., that attempted to select the highest, most recent sample information from the TCRC to calculate a tank waste inventory. The inventories for the tanks are documented in two reports: WHC-SD-WM-TI-543, Radionuclide and Chemical Inventories for the Double-Shell Tanks, and WHC-SD-WM-TI-565, Radionuclide and Chemical Inventories for the SingleShell Tanks.

Tank Characterization Database:

Operated by PNNL, the TCD contains analytical results from Tank Characterization Reports (TCRs). This information is either received directly from the analytical laboratories or entered from the TCRs.

Historical Tank Content Estimate Inventory Data Set:

The HTCE documents provide historical evaluations of the mixed wastes stored in the Hanford Site 200 Areas underground single- and double-shell tanks. These estimates were derived by reviewing the process histories, process flow sheets, waste transfer data, and any available physical and 
chemical characterization data from the various Department of Energy and Department of Defense contractors. This work is a compilation of efforts done in 1994 and 1995 by ICF KH, Los Alamos National Laboratory, and WHC.

- Track Radioactive Components Data Set:

The TRAC system estimates the amounts of selected radionuclides and stable chemicals in the total waste management system and how they are distributed among the underground tanks. Input data are based upon historical records. Models of reactor, chemical process, and waste management were created. All computer codes except isotope generation and depletion were written within the scope of the project.

A series of four steps (described below) was completed to place the waste characterization information in a form suitable for review by the independent technical reviewers. Figure 1 is a graphical representation of the process and methodology used to produce the master data set, subset, and unreviewed data set. The remaining portion of the figure represents the iterative review process used to attain the subsets, how the data sets were derived, and where they are.

STEP 1. Extract Information

- The STIV data set is organized by analyte and the TWRS Privatization Support Project required the data to be organized by tank. Therefore, the STIV data set needed to be reorganized to allow for the extraction of , the necessary information. This was accomplished by taking the individual analyte and radionuclide files and converting from ExcelTM format to dBASE $4^{\mathrm{TM}}$ format. Once in dBASE 4TM format, all files were added to form one large database table. This table was converted to Paradox ${ }^{\top m}$ 5.0, queried by tank and radionuclide or analyte for each of the 14 tanks, and exported to Excelrm. The result was an Excelm spreadsheet that contained either all of the analytes or all of the radionuclides contained in the STIV data set for each of the fourteen tanks (e.g., an AN102 analyte file and an $\mathrm{AN}-102$ radionuclide file).

\section{STEP 2. Gather and Enter New Information}

Since the analytes and radionuclides included in the STIV data set did not include all of the radionuclides and analytes required for the TWRS Privatization Support Project, the new ones needed to be gathered, entered, and checked. For the Braun, Wastren, TCD, TRAC, and HTCE Inventory data, the original electronic sources were revisited and the new radionuclide and analytes extracted and placed into Excelrm files. Also, for the TCD data, all information entered since October 1994 (date of our last data download) was downloaded and the necessary radionuclides and analytes for the specific fourteen tanks were removed and placed in Excel'm spreadsheets. For the Kaiser ("raw") data, the original documents were revisited and the additional analytes and radionuclides were entered by hand into Excel ${ }^{\mathrm{TM}}$ and checked for accuracy.

\section{STEP 3. Integrate Information}

The information gathered in Steps 1 and 2 was integrated into 28 Exceltm spreadsheet files (i.e., two files for each tank, one containing the chemical analytes and the other the radionuclides). These files were used to create the data tables.

\section{STEP 4. Create Data Tables}

Tables were created using the spreadsheets created in Step 3. Two tables were created for each tank, one containing the chemical analytes and the other containing the radionuclides. The tables were sorted by analyte (or radionuclide) with a secondary sort by value in descending order. 


\section{PNNL-10971}

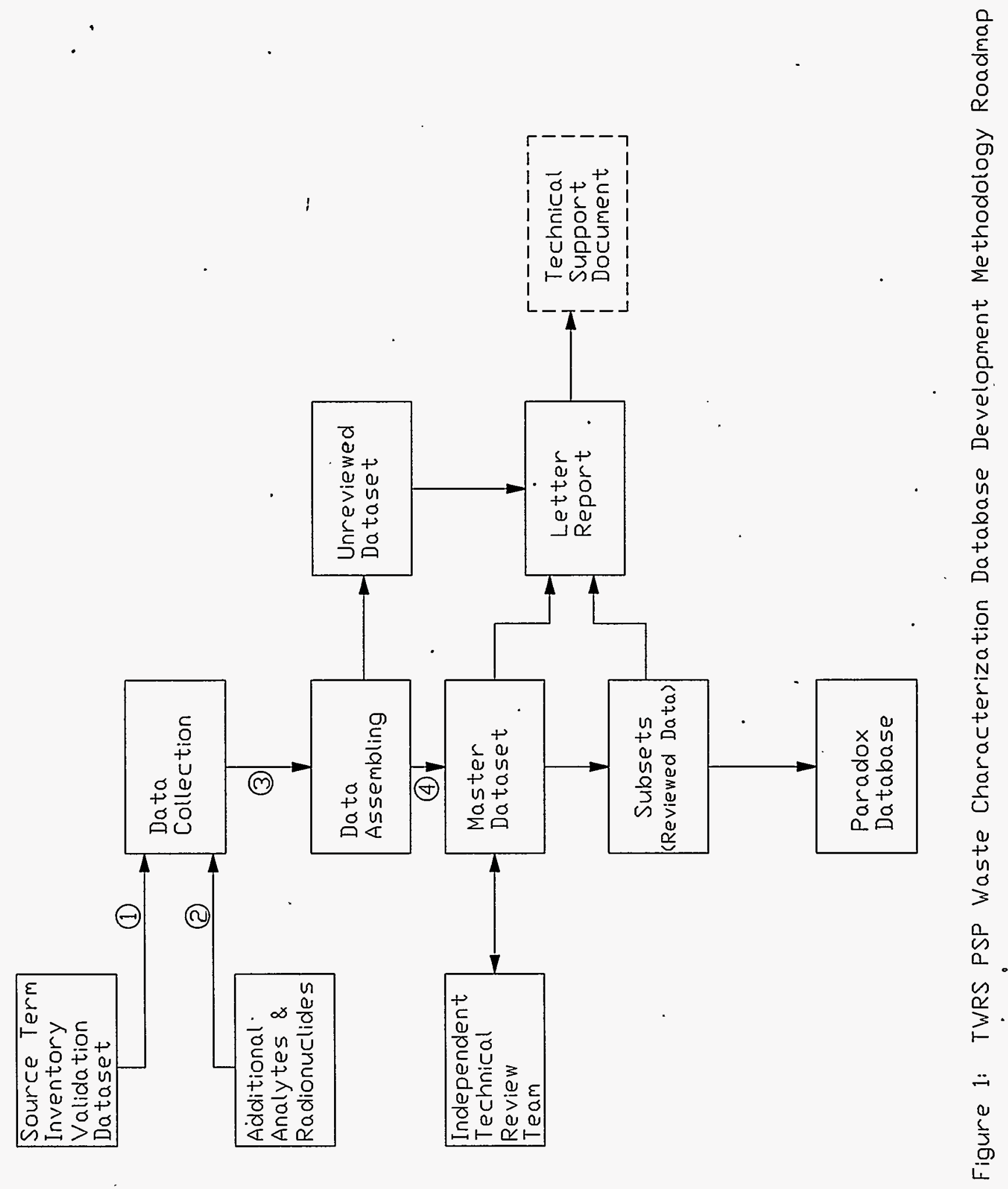


Upon completion of Step 4, the tables and plots were delivered to PNNL in draft form for review. The PNNL assembled a review team consisting of senior scientists from WHC, PNNL, Argonne National Laboratory, and LANL. From this review session, the number of tanks was narrowed from fourteen to seven. These tanks are denoted by the asterisk in Table 3.

Each tank was assigned to an independent technical review team member for review. The review process consisted of evaluating each data point for the major components and determining if it was valid waste characterization information. The major components were aluminum, chlorine, chromium, iron, potassium, sodium, nitrite, nitrate, hydroxide, phosphate, sulphate, total organic carbon, americium-241, cesium-137, neptunium-237, plutonium $239 / 240$, strontium- 90 , and technetium-99. The rest of the analytes and radionuclides in the data set are considered nonmajor components. The reviewers would consult the original memorandum or report from which the data was extracted to make their decisions. All points considered not valid (see Assumptions for validity rules) were given a justification. At the completion of this task, all data points had a qualifier (only the initials are actually entered into the database):

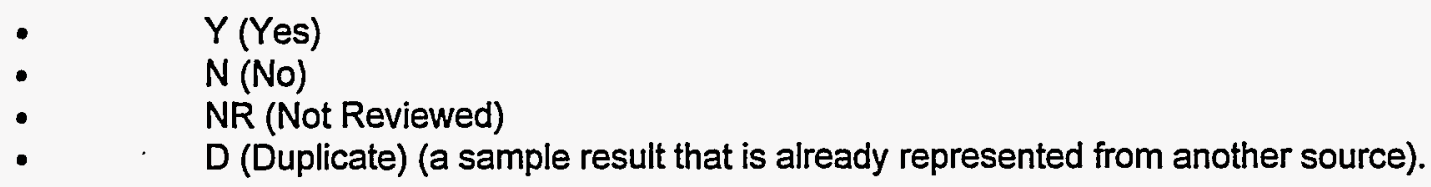

The nonmajor components were reviewed for the highest value only; i.e., the highest value for that constituent was reviewed and, if it was deemed a valid point, no other points for that constituent were reviewed, if it was deemed invalid, the next highest point would be reviewed. This process would continue until a valid data point was found and the remaining points would receive an NR. Duplicate points were not included for statistical reasons.

Once the data tables (the master data set) had been completely reviewed, a subset was created. The subset consisted of only the data points labeled Y. The master set is contained in Appendix A and the subset in Appendix B. Minimum, maximum and best-estimate tables were created from the subsets. See the Technical Support Document for more information on the creation of these tables.

\section{ASSUMPTIONS}

The following rules were used to disqualify data during the review process.

- The value is a less-than (<) value. These values would be considered valid if no other data existed for that analyte or radionuclide.

- The value was a duplicate point.

- The value was a TRAC value. TRAC was not used for this project.

- The value was an HTCE Inventory value. HTCE Inventory data were used for comparison only.

- Braun data. The Braun database was not used.

- Wastren data. The Wastren data set was not used.

- The waste is no longer in the tank; the sample was taken before a large waste transfer.

- The laboratory reported matrix interference for that particular analyte. 
- The value could not be traced back to a report or memorandum; reference unavailable.

- The value is from an evaporator feed sample.

- Miscellaneous problems with the sampling or analytical methods, based on chemical knowledge.

The following assumptions and/or rules were used in determining if a sample was solid or liquid.

Solids: $\quad$ Saltcake; sludge; composite; slurry; crust; potassium hydroxide (KOH) fusion; acid-washed, filtered, or centrifuged solids; and samples equal to or greater than 50 percent solids by volume.

Liquids: $\quad$ Supernate, filtrate, liquid/liquor, centrate, centrifuged decant, and samples with less than 50 percent solids by volume.

\section{PARADOXTM DATABASE}

The sample data subset information will be made available to the public through the Internet (http://twrs.pnl.gov:8001/twrs_rfp/homepage.htm). A Paradox ${ }^{\top M}$ runtime application database will contain all of the subset radionuclide and analyte information from the seven selected tanks, along with all of the references used. The application will allow the user to either view the information on-screen or export a query. The user can export either the radionuclide or analyte information for a specific tank, or for all tanks, while doing queries on the major column (field) headings. The on-screen view has the option of a record or spreadsheet view. The record view shows only one record (or entry) at a time, while the spreadsheet view shows multiple record entries at once. The subset data, in Excel ${ }^{\mathrm{TM}}$ format, is also available on the Internet. For more information, please contact Eric $D$. Johnson by cc:Mail, telephone [(509) 373-9542], or by e-mail (eric_d_johnson@rl.gov).

\section{SUMMARY AND CONCLUSIONS}

Of the original fourteen tanks, seven were selected for review: Tanks 241-AN-102 and -107; 241-AP-102 and 107; 241-AW-101; and 241-AZ-101 and -102. The waste characterization information gathered for these tanks was reviewed by an independent technical review team, and the valid data were extracted from the master data set. A subset of the master data set, comprising the valid data, can be found in Appendix $B$ and in the TWRS Privatization Waste Characterization Database. From this subset, the technical reviewers selected minimum and maximum values and calculated or chose a best estimate for the major components (see Approach and Evaluation section). For the nonmajor components, only a maximum value was selected. The minimum, maximum, and best-estimate values were used in the development of concentration bands that will be a part of the draft request for proposal. The master set of data for the seven tanks specified in the Approach and Evaluation section can be found in Appendix A. The unreviewed data for the remaining seven tanks can be found in Appendix C. Reference tables for sample data contained in the master data set, a subset, and unreviewed data set can be found in Appendix D. These references apply only to data from the Kaiser ( ${ }^{u}$ raw") source. Some of these references were rejected by the independent technical reviewers because they did not contain valid waste characterization data.

When entering historical information from reports, letters, or memoranda, it is recommended that all available information be entered. To validate waste characterization information accurately, all available information about that sample should be gathered (e.g., sample location, sample depth, type of sample, percent water, percent solids, viscosity, pH). A consistent vocabulary and set of rules (or assumptions) need to be established early in the process. Time should be allowed throughout the project for the vocabulary and rules to be regularly updated. This will ensure that all reviewers are using the same process to validate data. In assembling the review team, it 
is important to use experienced scientists and engineers that are familiar with the history of the Hanford processes, waste tanks, and the analytical procedures used in the laboratories. In developing an electronic application to control the sample data, knowing the limitations of the software is important. The amount of sample information available is quite large and some applications have limitations in the number of.rows (e.g., Exce|rm has a row limitation of 16,384$)$. 


\section{REFERENCES •}

Braun, D. J., 1994, Tank Sample Analysis Data File, Westinghouse Hanford Company, Richland, Washington.

Brevick, C. H.; L. A. Gaddis; and W. W. Pickett; 1994, Historical.Tank Content Estimate for the Northeast Quadrant of the Hanford 200 East Area, WHC-SD-WM-ER-349, Rev. 0, ICF Kaiser Hanford Company, Richland, Washington.

Brevick, C. H.; L. A. Gaddis; and W. W. Pickett; 1995, Historical Tank Content Estimate for the Southeast Quadrant of the Hanford 200 Areas, WHC-SD-WM-ER-350, Rev. 0, ICF Kaiser Hanford Company, Richland, Washington.

Brevick, C. H.; L. A. Gaddis; and W. W. Pickett; 1995, Historical Tank Content Estimate for the Northwest Quadrant of the Hanford 200 West Area, WHC-SD-WM-ER-351, Rev. 0, ICF Kaiser Hanford Company, Richland, Washington.

Brevick, C. H.; L. A. Gaddis; and W. W. Pickett; 1994, Historical Tank Content Estimate Report for the Southwest Quadrant of the Hanford 200 West Area, WHC-SD-WM-ER-352, Rev. 0, ICF Kaiser Hanford Company, Richland, Washington.

Brevick, C. H.; L. A. Gaddis; and E. D. Johnson; 1995, Tank Waste Source Term Inventory Validation, WHC-SD-WM-ER-400, Rev. 0, ICF Kaiser Hanford Company, Richland, Washington.

Van Vieet, R. J., 1993, Radionuclide and Chemical Inventories for the Double-Shell Tanks, WHC-SD-WM-TI-543, Rev. 1, Westinghouse Hanford Company, Richland, Washington.

Van Vleet, R. J., 1993, Radionuclide and Chemical Inventories for the Single-Shell Tanks, WHC-SD-WM-TI-565, Rev. 1, Westinghouse Hanford Company, Richland, Washington.

Wiemers, K. D., 1995, Letter of Instruction from TWRS Privatization Support Project, Waste Characterization Task,

PRV-95-96, Pacific Northwest National Laboratory, Richland, Washington.

Wiemers, K. D., et al., 1996, draft Technical Support Document, PRV-95-067, Pacific Northwest National Laboratory, Richland, Washington. 


\section{APPENDIX A}

This appendix contains the radionuclide and chemical analyte data tables for the master data set. The master data set contains all waste characterization information collected for Tanks 241-AN-102, -AN-107, -AP-102, -AP105, -AW-101, -AZ-101, and -AZ-102.

Table A-1 gives a brief description of the different fields in the data tables. Table A-2 lists the abbreviations used in the code field.

The abbreviations NA and NS will appear throughout the tables in any of the fields. They are defined as follows.

NA: Not Available. The information for that field was not given in the original report, memorandum, or letter.

NS: Not Searched For. The original report, memorandum, or letter was not searched for this information. This only applies for invalid or unreviewed data. 
Table A-1: Descriptions of Data Table Fields (Columns)

\begin{tabular}{|l|l|}
\hline COLUMN & DEFINITION \\
\hline $\begin{array}{l}\text { Reported } \\
\text { Chemical/Radionuclide }\end{array}$ & $\begin{array}{l}\text { The radionuclide or Chemical as it was reported in the } \\
\text { original report }\end{array}$ \\
\hline Memo Date & Date of the memorandum or report that value was taken from \\
\hline Start Analysis Date & Start date for the sample analysis \\
\hline Start Sample Date & Start date of the sampling event \\
\hline Sample Number & Generic sample number \\
\hline Tank Farm Number & Tank Farm assigned sample number \\
\hline Lab Sample Number & Lab assigned sample number \\
\hline Density (g/mL) & $\begin{array}{l}\text { Density as reported, if no density assumed } 1.6 \text { for solids and } \\
1.2 \text { for liquids }\end{array}$ \\
\hline Reported Value & The concentration as reported in the report \\
\hline Reported Unit & The unit the concentration was reported in \\
\hline Code & A code letter representing the type of sample \\
\hline Source & $\begin{array}{l}\text { Were the sample value originates from (i.e. Braun, Wastren, } \\
\text { Raw, etc.) }\end{array}$ \\
\hline Radionuclide/Analyte & The element or compound used \\
\hline Conc. (ug/L or Bq/L) & The standard units values were converted to \\
\hline $\begin{array}{l}\text { Conc. (mol/L) or }(\mathrm{Bq} / \mathrm{L} \\
\text { Decayed) }\end{array}$ & $\begin{array}{l}\text { Molarity concentration, chemicals only; Decayed } \\
\text { radionuclide value, decayed to } 12 / 31 / 94, \text { except }\end{array}$ \\
\hline 239/240 $\mathrm{Nu}$, and ${ }^{90} \mathrm{Y}$ \\
\hline Reference Number $(\mathrm{Ci} / \mathrm{L}$ Decayed) & Decayed radionuclide value, see Conc. (Bq/L Decayed) \\
\hline Validated (Y/N) & $\begin{array}{l}\text { For Raw data points, a reference number that refers to the } \\
\text { report or memorandum containing the data }\end{array}$ \\
\hline Notes & Is the value valid yes, no, not reviewed, or duplicate \\
\hline Reason for not validating the value, misc. Information \\
\hline
\end{tabular}


Table A-2: Definitions of Code Column Found in the Data Tables

\begin{tabular}{|c|c|c|}
\hline CODE & DESCRIPTION & PHASE \\
\hline A & Salts & $\mathbf{S}$ \\
\hline B & Solids that have been centrifuged to remove water & S . \\
\hline $\mathrm{C}$ & Centrate; Liquid removed from solids by centrifugation & $\mathrm{L}$ \\
\hline $\mathrm{D}$ & Suspended Solids & $\mathbf{S}$ \\
\hline $\mathrm{E}$ & Solids that have been washed with water & $\mathbf{S}$ \\
\hline $\mathrm{F}$ & Filtrate; solution used to wash solids & L \\
\hline $\mathrm{G}$ & Sludge & $\mathbf{S}$ \\
\hline$I^{*}$ & Composite; sample composed from more than one source & $\mathrm{S}^{\cdot}$ \\
\hline $\mathrm{K}$ & Saltcake & $\mathbf{S}$ \\
\hline $\mathrm{L}^{* *}$ & Denotes samples are to be considered as liquids & $\mathrm{L}$ \\
\hline $\mathrm{O}$ & Other (must be accompanied by a \% solid) & S or $L$ \\
\hline $\mathrm{P}$ & Untreated supernatant samples & $\mathrm{L}$ \\
\hline $\mathrm{R}$ & Slurry & $\mathbf{S}$ \\
\hline $\mathrm{S}^{* *}$ & Denotes samples are to be considered as solids & $\mathrm{S}$ \\
\hline $\mathrm{T}$ & Crust & S \\
\hline $\mathrm{U}$ & Unknown & $\mathrm{L}$ \\
\hline $\mathrm{Y}$ & Untreated sludge samples (e.g. cores) & $S$ \\
\hline$-1 * * *$ & Primary analysis result & $\mathrm{S}$ or $\mathrm{L}$ \\
\hline$-2 * * *$ & Duplicate analysis result & $\mathrm{S}$ or $\mathrm{L}$ \\
\hline$-3 * * *$ & Averaged value of primary and duplicate results & $\mathrm{S}$ or $\mathrm{L}$ \\
\hline
\end{tabular}

Used in conjunction with other codes (e.g. BI denotes that a composite solid has been centrifuged to remove water).

** Used in a separate column (L/S) to denote whether samples are considered liquid or Solid. Not used in the code column.

*** Used in conjunction with other codes (e.g. P-3 denotes a supernatant sample that is an average of the primary and duplicate results). 


\begin{tabular}{|c|c|c|c|c|c|c|c|c|c|c|c|}
\hline \multicolumn{12}{|c|}{ Tank Waste Characterization Sample Information } \\
\hline $\begin{array}{c}\text { Reported } \\
\text { Radionuclide }\end{array}$ & $\begin{array}{c}\text { Memo } \\
\text { Dato }\end{array}$ & $\begin{array}{c}\text { Start Analysis } \\
\text { Date }\end{array}$ & $\begin{array}{c}\text { Start } \\
\text { Sample } \\
\text { Date }\end{array}$ & $\begin{array}{l}\text { Sample } \\
\text { Number }\end{array}$ & $\begin{array}{c}\text { Tank Farm } \\
\text { Number }\end{array}$ & $\begin{array}{c}\text { Lab Sampie } \\
\text { Number }\end{array}$ & $\begin{array}{l}\text { Density } \\
(\mathrm{g} / \mathrm{mL})\end{array}$ & Reported Value & Reported Unit & Code & Sour \\
\hline Am241 & $2 / 28 / 85$ & NA & NA & R3640 & NA & NA & 1.39 & 268 & uCi/ & $\mathbf{L}$ & RAV \\
\hline Am241 & $2 / 2 8 \longdiv { 8 5 }$ & NA & NA & R3639 & NA & NA & 1.39 & 147.0 & $\mathrm{uCi} / \mathrm{L}$ & $\bar{P}$ & $\overline{\operatorname{Ran}}$ \\
\hline Am241 & $12 / 16 / 91$ & NA & $8 / 1 / 91$ & NA & NA & NA & 1.35 & 89.2 & $\mathrm{nCi} / \mathrm{mL}$ & $\mathbf{P}$ & Rav \\
\hline Am241 & $8 / 1 / 91$ & NS & NS & NS & NS & NS & NS & NS & NS & $\mathbf{P}$ & Waste \\
\hline $\mathrm{C} 14$ & $8 / 1 / 91$ & NS & NS & NS & NS & NS & NS & 55500 & BqR & $P$ & Wastr \\
\hline C-14 & $12 / 16 / 91$ & NA & $8 / 1 / 91$ & $\mathrm{NA}$ & NA. & NA & 1.35 & 0.00066 & $\mathrm{uCi} / \mathrm{mL}$ & $P$ & $\operatorname{Rav}$ \\
\hline $\mathrm{Cm} 243 / 244$ & $12 / 16 / 91$ & NA & $8 / 1 / 91$ & $\overline{\mathrm{NA}}$ & NA & $\overline{M A}$ & 1.35 & 6.76 & $n \mathrm{ni} / \mathrm{mL}$ & $\bar{P}$ & $\overrightarrow{\operatorname{Rav}}$ \\
\hline $\mathrm{Co60}$ & $2 / 28 / 85$ & NA & NA & R3640 & NA & NA & 1.39 & 635 & UCin & $L$ & AAV \\
\hline Co60 & $2 / 28 / 85$ & NA & NA & R3639 & NA & NA & 1.39 & 551.0 & uCin & $P$ & Raw \\
\hline Co60 & $12 / 16 / 91$ & NA & $8 / 1 / 91$ & $\mathrm{NA}$ & NA & NA & 1.35 & 0.135 & $\mathrm{UCi} / \mathrm{g}$ & $\mathbf{P}$ & $\overline{\mathrm{Rav}}$ \\
\hline Co60 & $8 / 1 / 91$ & NS & NS & NS & NS & NS & NS & NS & NS & $P$ & Wastri \\
\hline Cs137 & $2 / 28 / 85$ & NA & NA & R3640 & NA & NA & 1.39 & 448000 & uCin & $L$ & RAW \\
\hline Cs137 & $10 / 12 / 89$ & $9 / 22 / 89$ & $N A$ & R.5779 & NA & NA & 1.34 & 400000 & uCi/L & $\mathbf{P}$ & Raw \\
\hline${ }^{137} \mathrm{Cs}$ & $12 / 28 / 94$ & $\mathrm{NA}$ & $10 / 21 / 94$ & 102-AN-2 & NA & NA & 1.402 & 385000 & UCilL & $L$ & $\overline{\operatorname{Raw}}$ \\
\hline${ }^{107} \mathrm{Cs}$ & $12 / 28 / 94$ & NA & $10 / 21 / 94$ & 102-AN-1 & NA & NA & 1.4 & 382000 & uCi/L & $L$ & $\overline{\mathrm{Raw}}$ \\
\hline${ }^{137} \mathrm{Cs}$ & $12 / 28 / 94$ & $\mathrm{NA}$ & $10 / 21 / 94$ & 102-AN-4 & NA & NA & 1.403 & 378000 & UCin & $L$ & $\overline{\text { Raw }}$ \\
\hline Cs137 & $8 / 1 / 91$ & NS & NS & NS & NS & NS & NS & NS & NS & $\mathbf{P}$ & Wastrc. \\
\hline Cs137 & $2 / 28 / 85$ & NA & NA & R3639 & NA & $\overline{\mathrm{NA}}$ & 1.39 & 310000 & $\mathrm{uCi} / \mathrm{L}$ & $\overline{\mathbf{P}}$ & $\overline{\text { Raw }}$ \\
\hline Cs137 & $12 / 16 / 91$ & NA & $8 / 1 / 91$ & NA & NA & NA & 1.35 & 195 & $\mathrm{UCi} / \mathrm{g}$ & $\bar{P}$ & $\overline{\text { Raw }}$ \\
\hline Cs & NA & NA & NA & NA & NA & $\mathrm{NA}$ & 1.27 & 0.2011 & $\mathrm{Ci} / \mathrm{L}$ & $\bar{P}$ & HTCE II \\
\hline Eu154 & $8 / 1 / 91$ & NS & NS & NS & NS & NS & NS & NS & NS & $\mathbf{P}$ & Wastre \\
\hline Eu154 & $12 / 16 / 91$ & NA & $8 / 1 / 91$ & NA & NA & NA & 1.35 & 0.297 & uCi/g & $\overline{\mathbf{P}}$ & $\overrightarrow{\text { Raw }}$ \\
\hline Pu239/240 & $12 / 16 / 91$ & NA & $8 / 1 / 91$ & NA & NA & NA & 1.35 & 70.3 & $\mathrm{nCi} / \mathrm{mL}$ & $P$ & Raw \\
\hline Pu239/240 & $8 / 1 / 91$ & NS & NS & NS & NS & NS & NS & NS & NS & $P$ & Wastre \\
\hline Pu239/240 & $2 / 28 / 85$ & NA & NA & R3640 & NA & NA & 1.39 & 24.6 & UCi/L & $L$ & RAW \\
\hline Pu239/240 & $2 \sqrt{28 / 85}$ & NA & NA & R3639 & NA & $\mathrm{NA}$ & 1.39 & 13.8 & UCiR & $P$ & $\overline{R a w}$ \\
\hline Pu241 & $2 / 28 / 85$ & NA & NA & R3640 & NA & NA & 1.39 & 63.8 & UCi/L & $L$ & RAW \\
\hline Pu241 & $2 / 28 / 85$ & NA & NA & R3639 & NA & NA & 1.39 & 18.0 & UCiR & $P$ & Raw \\
\hline Se.79 & $12 / 16 / 91$ & NA & $8 / 1 / 91$ & NA & NA & NA & 1.35 & 0.000167 & $\mathrm{uCi} / \mathrm{mL}$ & $P$ & Raw \\
\hline Sr90 & $2 / 28 / 85$ & $\mathrm{NA}$ & NA & 83639 & NA & NA & 1.39 & 124000 & uCirL & $\mathbf{P}$ & Raw \\
\hline Sr89/90 & $8 / 1 / 91$ & NS & NS & NS & NS & NS & NS & NS & NS & $\mathbf{P}$ & Wastret \\
\hline Sr90 & $2 / 28 / 85$ & NA & NA & R3640 & NA & NA & 1.39 & 101000 & uCir & \llcorner & RAW \\
\hline${ }^{10} \mathrm{Sr}$ & $12 / 28 / 94$ & NA & $10 / 21 / 94$ & 102-AN-1 & NA & NA & 1.4 & 74300 & uCi/L & $L$ & Raw \\
\hline${ }^{10} \mathrm{Sr}$ & $12 / 28 / 94$ & NA & $10 / 21 / 94$ & 102-AN-2 & NA & $\mathrm{NA}$ & 1.402 & 74250 & UCiR. & L & Raw \\
\hline${ }^{\circ} \mathrm{Sr}$ & $12 / 28 / 94$ & $\mathrm{NA}$ & $10 / 21 / 94$ & $102-\mathrm{AN}^{-4}$ & NA & NA & 1.403 & 72500 & uCi/ & $L$ & Raw \\
\hline Sr90 & $12 / 16 / 91$ & $\mathrm{NA}$ & $8 / 1 / 91$ & NA & NA & NA & 1.35 & 45.1 & $\mathrm{uCi} / \mathrm{mL}$ & $P$ & Raw \\
\hline
\end{tabular}




\begin{tabular}{|c|c|c|c|c|c|c|}
\hline \multicolumn{5}{|c|}{ Radionucilidelnformation } & \multirow[b]{2}{*}{ Validated $(\mathbf{Y} / \mathbf{N})$} & \multirow[b]{2}{*}{ Notes } \\
\hline Radoinuclide & $\begin{array}{l}\text { Conc. } \\
(B q / 2)\end{array}$ & $\begin{array}{c}\text { Conc. }(B q \Omega \\
\text { decayed) }\end{array}$ & $\begin{array}{l}\text { Conc. (Cin } \\
\text { Decayed) }\end{array}$ & $\begin{array}{c}\text { Reference } \\
\text { Number }\end{array}$ & & \\
\hline Am241 & $9.92 E+06$ & $9.77 E+06$ & $2.64 E-04$ & AN102-1 & $\mathbf{Y}$ & $\begin{array}{l}\text { Centrate data point, moved from solids table, changed code } \\
\text { (S to } U \text { and sample state (Solids to Centrate) }\end{array}$ \\
\hline Am241 & \begin{tabular}{|l|}
$5.44 E+06$ \\
$3.30 E+06$ \\
\end{tabular} & $\begin{array}{l}5.35 E+06 \\
3.28 E+06\end{array}$ & $\frac{1.45 E-04}{8.88 E-05}$ & $\frac{\text { AN102-1 }}{\text { AN102-5 }}$ & $\bar{Y}$ & Supernate sample, new data (missed) \\
\hline Am241 & $3.30 E+06$ & $3.28 E+06$ & 8.86E-05 & NA & N & $\begin{array}{l}\text { Exclude, recomend to use the Raw data from } 2 / 28 / 85 \text { and } \\
12 / 18 / 91 \text { memo }\end{array}$ \\
\hline $\mathbf{C 1 4}$ & $5.55 E+04$ & $5.55 E+04$ & $1.50 E-06$ & NA & $\mathbf{N}$ & Exclude, Wastren Data \\
\hline C14 & $2.44 E+04$ & $2.44 E+04$ & $6.60 E-07$ & AN102.5 & $Y$ & Clianged units uCi/g to uCi/mL and date $11 / 1 / 91$ to $8 / 92$ \\
\hline $\mathrm{Cm} 243 / 244$ & $2.50 E+05$ & $2.23 E+05$ & $6.02 \mathrm{E}-06$ & AN102-5 & $\bar{Y}$ & Added, missed initially \\
\hline Co60 & $2.35 E+07$ & $6.47 E+06$ & $1.75 \mathrm{E}-04$ & AN102-1 & $\bar{Y}$ & Centrate data, changed code from $\mathrm{P}$ to $\mathrm{L}$ \\
\hline Co60 & $2.04 E+07$ & $5.61 E+06$ & $7.52 E-04$ & AN102-1 & $\bar{Y}$ & Supernate sample, new data (missed) \\
\hline Co60 & $6.74 E+06$ & $4.53 E+06$ & $1.22 \mathrm{E}-04$ & AN102.5 & $\bar{Y}$ & Added, missed initially \\
\hline Co60 & $5.85 E+05$ & $3.74 E+05$ & $1.01 \mathrm{E}-05$ & NA & $\mathbf{N}$ & Exclude, Wastren Data \\
\hline Cs137 & $1.66 \mathrm{E}+10$ & $1.32 E+10$ & 3.58E-01 & AN102-1 & $\mathbf{Y}$ & $\begin{array}{l}\text { Centrate data point, moved from solids table, changed code } \\
\text { (S to } L \text { ) and sample state (Solids to Centrate) }\end{array}$ \\
\hline Cs137 & $1.48 E+10$ & $1.31 E+10$ & $3.55 \mathrm{E}-01$ & AN102-6 & N & Added, new report found, reference to unpublished data \\
\hline Cs137 & $1.42 E+10$ & $1.42 E+10$ & $3.85 \mathrm{E}-01$ & AN102-6 & $Y$ & \\
\hline Cs137 & $1.41 E+10$ & $1.41 E+10$ & $3.82 \mathrm{E}-01$ & AN102-6 & $Y$ & \\
\hline Cs137 & $1.40 E+10$ & $1.40 E+10$ & $3.78 \mathrm{E}-01$ & AN102-6 & $Y$ & \\
\hline Cs137 & 1.35E+10 & $1.25 E+10$ & 3.38E-01 & NA & N & $\begin{array}{l}\text { Exclude, Wastren used data from } 2 / 28 / 85 \text { and } 12 / 18 / 91 \\
\text { memos. Need to use the original Raw data }\end{array}$ \\
\hline Cs137 & $1.15 E+10$ & $9.15 E+09$ & $2.47 E-01$ & AN102-9 & $Y$ & Supernate sample, new data (missed) \\
\hline Cs137 & $9.74 \mathrm{E}+09$ & $9.08 \mathrm{E}+09$ & $2.45 \mathrm{E}-01$ & AN102-5 & $\bar{Y}$ & Added, missed initially \\
\hline Cs137 & $7.44 \mathrm{E}+09$ & $7.44 \mathrm{E}+09$ & 2.01E-01 & NA & $N$ & HTCE Data \\
\hline Eu154 & $5.78 E+07$ & $4.41 \mathrm{E}+07$ & $1.19 \mathrm{E}-03$ & NA & 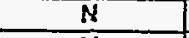 & Exclude, Wastren Data \\
\hline Eu154 & $1.48 \mathrm{E}+07$ & $1.17 E+07$ & 3.15E-04 & AN102-5 & $y$ & Added, missed initially \\
\hline Pu239/240 & $2.60 E+06$ & $2.60 E+06$ & $7.03 E-05$ & AN102-5 & $\bar{Y}$ & Added, missed initially \\
\hline Pu239/240 & $2.60 E+06$ & $2.60 E+06$ & 7.03E-05 & NA & $\boldsymbol{N}$ & $\begin{array}{l}\text { Exclude. Wastren used data from } 2 / 28 / 85 \text { and } 12 / 18 / 91 \\
\text { memos. Need to use the original Raw data }\end{array}$ \\
\hline Pu239/240 & $9.10 E+05$ & $9.10 E+05$ & 2.46E-05 & AN102-1 & $\mathbf{Y}$ & $\begin{array}{l}\text { Centrate data point, moved from solids table, changed code } \\
\text { (S to } U \text { ) and sample state (Solids to Centrate) }\end{array}$ \\
\hline Pu239/240 & $5.11 E+05$ & $5.11 E+05$ & $1.38 \mathrm{E}-05$ & ANTO2-1 & $\bar{Y}$ & Supernate sample, new data (missed) \\
\hline Pu241 & $2.36 E+06$ & $1.47 E+06$ & 3.98E-05 & AN102-1 & $Y$ & $\begin{array}{l}\text { Centrate data point, moved from solids table, changed code } \\
\text { (S to } L \text { and sample state (Solids to Centrate) }\end{array}$ \\
\hline Pu241 & $6.66 E+05$ & $4.15 E+05$ & $1.12 E-05$ & AN102-1 & $\bar{Y}$ & Supernate sample, new data (missed) \\
\hline Se79 & $6.18 E+03$ & $6.18 E+03$ & $1.67 E-07$ & AN102-5 & $Y$ & Changed units uCi/g to uCi/mL and date $11 / 1 / 91$ to $8 / 93$ \\
\hline Sr89/90 & $4.59 \mathrm{E}+09$ & $3.62 E+09$ & 9.79E-02 & ANi02-1 & $\bar{Y}$ & Supernate sample, new data (missed) \\
\hline Sr89/90 & $4.33 E+09$ & $3.99 E+09$ & $1.08 E-01$ & NA & $\mathbf{N}$ & $\begin{array}{l}\text { Exclude, Wastren used data from } 2 / 28 / 85 \text { and } 12 / 18 / 91 \\
\text { memos. Need to use the original Raw data }\end{array}$ \\
\hline Sז89/90 & $3.74 E+09$ & $2.95 E+09$ & 7.98E-02 & AN102-1 & $\mathbf{Y}$ & $\begin{array}{l}\text { Centrate data point, moved from solids table, changed code } \\
\text { (S to } L \text { ) and sample state (Solids to Centrate) }\end{array}$ \\
\hline Sr89/90 & $2.75 E+09$ & $2.75 E+09$ & $7.43 E-02$ & AN102-6 & $Y$ & \\
\hline Sr89/90 & $2.75 \mathrm{E}+09$ & $2.75 E+09$ & 7.42E-02 & AN102-6 & $\bar{Y}$ & \\
\hline Sr89/90 & $2.68 \mathrm{E}+09$ & $2.68 E+09$ & 7.25E-02 & AN102-6 & $\mathbf{Y}$ & \\
\hline Sr89/90 & $1.67 E+09$ & $1.55 E+09$ & $4.19 \mathrm{E}-02$ & AN102-5 & $Y$ & \\
\hline
\end{tabular}




\begin{tabular}{|c|c|c|c|c|c|c|c|c|c|c|c|}
\hline $\begin{array}{c}\text { Reported } \\
\text { Radionuclide }\end{array}$ & $\begin{array}{c}\text { Memo } \\
\text { Date }\end{array}$ & $\begin{array}{c}\text { Start Analysis } \\
\text { Date }\end{array}$ & $\begin{array}{c}\text { Start } \\
\text { Semple } \\
\text { Date }\end{array}$ & $\begin{array}{c}\text { Sample } \\
\text { Number }\end{array}$ & $\begin{array}{c}\text { Tank Farm } \\
\text { Number }\end{array}$ & $\begin{array}{c}\text { Lab Sample } \\
\text { Number }\end{array}$ & $\begin{array}{c}\text { Density } \\
\text { (g/mL) }\end{array}$ & Reported Value & Reported Unit & Code & Sourci \\
\hline Sr & NA & NA & NA & NA & NA & NA & 1.27 & 0.0095 & Ci/L & HTCE I \\
\hline Tc99 & $8 / 1 / 91$ & NS & NS & NS & NS & NS & NS & 11400000 & Bq/L & $P$ & Wastre \\
\hline Tc-99 & $12 / 16 / 91$ & NA & $8 / 1 / 91$ & NA & NA & NA & 1.35 & 0.0296 & uCi/mL & $P$ & Raw \\
\hline
\end{tabular}




\begin{tabular}{|c|c|c|c|c|c|c|}
\hline \multicolumn{5}{|c|}{ Radionuclidelnformation } & \multirow[b]{2}{*}{ Validated $(Y(N)$} & \multirow[b]{2}{*}{ Notes } \\
\hline Radoinuclide & $\begin{array}{l}\text { Conc. } \\
(\mathrm{Bq} / \mathrm{L})\end{array}$ & $\begin{array}{c}\text { Conc. (BqR } \\
\text { decayed) }\end{array}$ & $\begin{array}{l}\text { Conc. (CiR } \\
\text { Decayed) }\end{array}$ & $\begin{array}{c}\text { Reference } \\
\text { Number }\end{array}$ & & \\
\hline Sr89/90 & $3.52 E+08$ & $3.52 E+08$ & $9.50 \mathrm{E}-03$ & NA & $N$ & HTCE Data \\
\hline Tc99 & $1.14 E+07$ & $1.14 E+07$ & $3.08 E-04$ & NA & $\mathbf{N}$ & $\begin{array}{l}\text { Exclude, Wastren used data from } 2 / 28 / 85 \text { and } 12 / 78 / 91 \\
\text { memos. Need to use the original Raw data, this data is } \\
\text { missing }\end{array}$ \\
\hline Te99 & $1.10 E+06$ & $1.10 E+06$ & $2.96 E-05$ & AN102-5 & $\boldsymbol{\gamma}$ & Changed units uCi/g to uCi/mL and date $11 / 1 / 91$ to $8 / 91$ \\
\hline
\end{tabular}


TWRS Privatization Support Proi Radionuclide Liquic

\begin{tabular}{|c|c|c|c|c|c|c|c|c|c|c|}
\hline & & & & Tank Waste Ch & racterization & Sample Inforn & ation & & & \\
\hline $\begin{array}{c}\text { Reported } \\
\text { Radionuclide }\end{array}$ & $\begin{array}{c}\text { Memo } \\
\text { Date }\end{array}$ & $\begin{array}{c}\text { Start } \\
\text { Analysis Date } \\
\end{array}$ & $\begin{array}{c}\text { Start } \\
\text { Sample } \\
\text { Date } \\
\end{array}$ & Sample Number & $\begin{array}{c}\text { Tank Farm } \\
\text { Number }\end{array}$ & $\begin{array}{c}\text { Lab Sample } \\
\text { Number }\end{array}$ & $\begin{array}{l}\text { Density } \\
(\mathrm{g} / \mathrm{mL})\end{array}$ & Reported Value & Reported Unit & Cor \\
\hline Am-241 & $2 / 28 / 85$ & NS & NS & $R-3638$ & NS & NS & 1.35 & 875 & uCint & $L$ \\
\hline Am-241 & $2 / 28 / 85$ & NA & NA & R-3638 & NA & NA & 1.35 & 875 & uCin & $\bar{L}$ \\
\hline$A m-241$ & $3 / 6 / 85$ & NS & NS & RAT-AN107-1B & NS & NS & NS & 757 & uCi/I & $\bar{p}$ \\
\hline Am241 & $3 / 7 / 85$ & NS & NS & NS & NS & NS & NS & NS & NS & $\bar{p}$ \\
\hline Am241 & $8 / 10 / 94$ & NA & $2 / 7 / 93$ & R3155 & NA & NA & 1.38 & 0.66 & $\mathrm{uCi} / \mathrm{mL}$ & $\bar{p}$ \\
\hline Am241 & $8 / 10 / 94$ & NA & $2 / 7 / 93$ & R2686 & NA & NA & 1.41 & 0.66 & $\mathrm{uCl} / \mathrm{mL}$ & $\bar{p}$ \\
\hline Am241 & $8 / 10 / 94$ & NA & $2 / 7 / 93$ & $\mathrm{R3154}$ & NA & NA & 1.40 & 0.58 & $\mathrm{uCi} / \mathrm{mL}$ & $\vec{p}$ \\
\hline Am-241 & $2 / 28 / 85$ & NA & NA & $R-3637$ & NA & NA & 1.41 & 135 & uCin & $p$ \\
\hline Am-241 & $2 / 28 / 85$ & NA & NA & $R-3637$ & NA & NA & 1.41 & 130 & uCin & $p$ \\
\hline$A m-241$ & $2 / 28 / 85$ & NS & NS & R-3637 & NS & NS & 1.41 & 130 & $\mathrm{uCi} /$ & $\vec{p}$ \\
\hline Am-241 & $3 / 6 / 85$ & NS & NS & RAT-AN107-1A & NS & NS & NS & 111 & uCi/l & $\bar{p}$ \\
\hline Am-241 & $2 / 28 / 85$ & NA & NA & R-3637 & NA & NA & 1.41 & 111 & $\mathrm{uCi} / \mathrm{H}$ & $\bar{p}$ \\
\hline $\mathrm{C14}$ & $2 / 1 / 85$ & NS & NS & NS & NS & NS & NS & NS & NS & $\bar{p}$ \\
\hline $\mathrm{Cm}-244$ & $3 / 6 / 85$ & NS & NS & RAT-AN107-1B & NS & NS & NS & 5 & $\mathrm{uCi} / \mathrm{I}$ & $F$ \\
\hline Cm244 & $3 / 8 / 85$ & NS & NS & NS & NS & NS & NS & NS & NS & $\bar{P}$ \\
\hline Co-60 & $2 / 28 / 85$ & NS & NS & $R-3637$ & NS & NS & 1.41 & 859 & $\mathrm{uCi} / \mathrm{I}$ & $\underline{P}$ \\
\hline Co-60 & $3 / 6 / 85$ & NS & NS & RAT-AN107-1A & NS & NS & NS & 859 & $\mathrm{uCi} / \mathrm{I}$ & $\bar{P}$ \\
\hline Co60 & $3 / 1 / 85$ & NS & NS & NS & NS & NS & NS & NS & NS & $\vec{P}$ \\
\hline Co-60 & $3 / 6 / 85$ & NS & NS & RAT-AN107-1B & NS & NS & NS & 847 & $\mathrm{uCi} / \mathrm{I}$ & $\bar{P}$ \\
\hline Cs-137 & $2 / 28 / 85$ & NS & NS & $R-3637$ & NS & NS & 1.41 & 450000 & $\mathrm{uCi}$ & $F$ \\
\hline Cs-137 & $3 / 6 / 85$ & NS & NS & RAT-AN107-1A & NS & NS & NS & 450000 & $\mathrm{uCi} / \mathrm{I}$ & $\bar{P}$ \\
\hline Cs-137 & $2 / 28 / 85$ & NA & NA & $8-3637$ & NA & NA & 1.35 & 450000 & $\mathrm{uCi}$ & L \\
\hline Cs-137 & $2 / 28 / 85$ & NA & NA & $\mathrm{R}-3638$ & $\mathrm{NA}$ & NA & 1.35 & 376000 & $\mathrm{uCi} / 1$ & $\bar{P}$ \\
\hline Cs-137 & $2 / 28 / 85$ & NS & NS & R-3638 & NS & NS & 1.35 & 376000 & $\mathrm{uCi} / \mathrm{A}$ & $\bar{L}$ \\
\hline Cs & NA & NA & NA & $\mathrm{NA}$ & NA & NA & 1.43 & 358993.9911 & uCin & \\
\hline Cs137 & $3 / 3 / 85$ & NS & NS & NS & NS & NS & NS & NS & NS & $\overline{\mathbf{P}}$ \\
\hline Cs-137 & $3 / 6 / 85$ & NS & NS & RAT-AN107-1B & NS & NS & NS & 355000 & $\mathrm{uCi}$ & $\bar{P}$ \\
\hline Cs137 & $8 / 10 / 94$ & NA & $2 / 7 / 93$ & R2686 & NA & NA & 1.41 & 302 & $\mathrm{uCl} / \mathrm{mL}$ & $\bar{P}$ \\
\hline Cs137 & $8 / 10 / 94$ & NA & $2 / 7 / 93$ & $R 3154$ & NA & NA & 1.4 & 259 & $\mathrm{uCl} / \mathrm{mL}$ & $\bar{P}$ \\
\hline Cs137 & $8 / 10 / 94$ & NA & $2 / 7 / 93$ & R3155. & NA & NA & 1.384 & 197 & $\mathrm{uCi} / \mathrm{mL}$ & P \\
\hline Eu154 & $2 / 28 / 85$ & NS & NS & $R-3637$ & NS & NS & 1.41 & 2690 & uCi/l & $\mathbf{P}$ \\
\hline Eu154 & $3 / 6 / 85$ & NS & NS & RAT-AN107-1A & NS & NS & & 2690 & $\mathrm{uCi} / \mathrm{l}$ & $P$ \\
\hline Eu154 & $3 / 4 / 85$ & NS & NS & NS & NS & NS & NS & NS & NS & $\bar{P}$ \\
\hline Eu154 & $3 / 6 / 85$ & NS & NS & RAT-AN107-1B & NS & NS & NS & 2610 & $\mathrm{uCi} / 1$ & $\overline{\mathrm{P}}$ \\
\hline Np237 & $8 / 10 / 94$ & NA & $2 / 7 / 93$ & $\mathrm{R3154}$ & NA & NA & 1.40 & $<0.0005$ & $\mathrm{uCl} / \mathrm{mL}$ & $\bar{P}$ \\
\hline Np237 & $8 / 10 / 94$ & NA & $2 / 7 / 93$ & $R 2686$ & NA & NA & 1.41 & $<0.0005$ & $\mathrm{uCi} / \mathrm{mL}$ & $\bar{P}$ \\
\hline Np237 & $8 / 10 / 94$ & NA & $2 / 7 / 93$ & R3155 & NA & NA & 1.38 & 0.0009 & $\mathrm{uCi} / \mathrm{mL}$ & $\bar{P}$ \\
\hline Pu-239/240 & $2 / 28 / 85$ & NA & NA & R-3638 & NA & NA & 1.35 & 122 & uCill & $\bar{P}$ \\
\hline Pu-239/240 & $2 / 28 / 85$ & NS & NS & $R-3638$ & NS & NS & 1.35 & 122 & uCill & $L$ \\
\hline Pu.239/240 & $2 / 28 / 85$ & NS & NS & R-3637 & NS & NS & 1.41 & 108 & uCin & $\bar{P}$ \\
\hline PU-239/240 & $2 / 28 / 85$ & NA & NA & $\mathrm{R}-3637$ & NA & NA & 1.41 & 108 & $\mathrm{uCi} / \mathrm{H}$ & $\bar{P}$ \\
\hline Pu239/240 & $3 / 1 / 85$ & NS & NS & NS & NS & NS & NS & NS & NS & P. \\
\hline Pu-239/240 & $2 / 28 / 85$ & NA. & NA & R-3637 & NA & NA & 1.41 & 89 & $\mathrm{uCin}$ & $L$ \\
\hline Pu-239/240 & $2 / 28 / 85$ & NA & NA & $R-3638$ & NA & NA & 1.35 & 86 & uCi/ & $\mathbf{L}$ \\
\hline Pu239/240 & $8 / 10 / 94$ & NA & $2 / 7 / 93$ & R2686 & NA & $\mathrm{NA}$ & 1.41 & 0.035 & $\mathrm{uCi} / \mathrm{mL}$ & $P$ \\
\hline Pu239/240 & $8 / 10 / 94$ & NA & $2 / / 93$ & R3155 & NA & NA & 1.38 & 0.034 & $\mathrm{uCi} / \mathrm{mL}$ & $P$ \\
\hline Pu239/240 & $8 / 10 / 94$ & NA & $2 \sqrt{7 / 93}$ & R3154 & NA & NA & 1.40 & 0.033 & $u C \mathrm{C} / \mathrm{mL}$ & $\bar{P}$ \\
\hline Pu-241 & $2 / 28 / 85$ & NS & NS & $R-3637$ & NS & NS & 1.41 & 740 & $\mathrm{uCi}$ & $\bar{P}$ \\
\hline Pu241 & $3 / 6 / 85$ & NS & NS & NS & NS & NS & NS & NS & NS & $\bar{P}$ \\
\hline Pu-241 & $2 / 28 / 85$ & NA & NA & R-3637 & NA & NA & 1.41 & 740 & $\mathrm{uCi} / \mathrm{I}$ & $P$ \\
\hline
\end{tabular}




\begin{tabular}{|c|c|c|c|c|c|c|c|c|c|c|}
\hline \multicolumn{11}{|c|}{ Tank Waste Characterization Sample Information } \\
\hline $\begin{array}{c}\text { Reported } \\
\text { Radionuclide }\end{array}$ & $\begin{array}{l}\text { Memo } \\
\text { Date }\end{array}$ & $\begin{array}{c}\text { Start } \\
\text { Anslysis Date } \\
\end{array}$ & $\begin{array}{c}\text { Start } \\
\text { Sample } \\
\text { Date }\end{array}$ & Sample Number & $\begin{array}{c}\text { Tank Farm } \\
\text { Number }\end{array}$ & $\begin{array}{c}\text { Lab Sample } \\
\text { Number }\end{array}$ & $\begin{array}{l}\text { Density } \\
(\mathrm{g} / \mathrm{mL})\end{array}$ & Reported Value & Reported Unit & $\mathrm{Cor}$ \\
\hline Pu-241 & $2 / 28 / 85$ & NA & NA & R-3638 & NA & NA & 1.35 & 330 & $u \operatorname{cin}$ & $\mathbf{L}$ \\
\hline Pu-241 & $2 / 28 / 85$ & NS & NS & R-3638 & NS & NS & 1.35 & 330 & $\mathrm{UC \textrm {i } / \mathrm { I }}$ & L \\
\hline Sr-89/90 & $2 / 28 / 85$ & NS & NS & $\mathrm{R}-3638$ & NS & NS & 1.35 & 164000 & $u C i n$ & $\bar{L}$ \\
\hline Sr89/90 & $2 / 28 / 85$ & NA & NA & R-3638 & NA & NA & 1.35 & 164000 & $\mathrm{uCi} / 1$ & $\underline{L}$ \\
\hline $\mathrm{Sr}-89 / 90$ & $2 / 28 / 85$ & NS & NS & $R-3637$ & NS & NS & 1.41 & 148000 & $\mathrm{uCi}$ & $P$ \\
\hline Sr89/90 & $3 / 2 / 85$ & NS & NS & NS & NS & NS & NS & NS & NS & $\overline{\mathbf{P}}$ \\
\hline S189/90 & $2 / 28 / 85$ & NA & NA & R-3637 & NA & NA & 1.41 & 148000 & $\mathrm{uCi} / \mathrm{i}$ & $\bar{p}$ \\
\hline Sr9o & $8 / 10 / 94$ & NA & $2 / 7 / 93$ & 83155 & NA & NA & 1.38 & 137 & $u \mathrm{Ci} / \mathrm{L}$ & $\bar{p}$ \\
\hline Sr90 & $8 / 10 / 94$ & NA & $277 / 93$ & R3155 & NA & NA & 1.38 & 98700 & uCin & $\bar{p}$ \\
\hline Sr & NA & NA & NA & $N A$ & NA & NA & 7.43 & 11001 & $\mathrm{UC \textrm {Ci }} / \mathrm{L}$ & $i$ \\
\hline Sr90 & $8 / 10 / 94$ & NA & $2 \sqrt{7 / 93}$ & R3154 & NA & NA & 1.40 & 88.9 & $\mathrm{uCi} / \mathrm{mL}$ & $\underline{P}$ \\
\hline Sr9o & $8 / 10 / 94$ & NA & $2 \pi / 93$ & $R 2686$ & NA & NA & 1.41 & 91.5 & $\mathrm{uCi} / \mathrm{mL}$ & $\bar{P}$ \\
\hline
\end{tabular}




\begin{tabular}{|c|c|c|c|c|c|c|c|}
\hline & \multicolumn{4}{|c|}{ Radionuclide Information } & & & \\
\hline Source & Radolnuclide & Conc. (Bq/L) & $\begin{array}{c}\text { Conc. (Bq/L } \\
\text { decayed) }\end{array}$ & $\begin{array}{l}\text { Conc. (Ci/L } \\
\text { Decayed) }\end{array}$ & $\begin{array}{c}\text { Reference } \\
\text { Number }\end{array}$ & Validated $(\mathrm{Y} / \mathrm{N})$ & Notes \\
\hline Raw. & Pu241 & $1.22 E+07$ & $7.61 E+06$ & 2.06E-04 & AN-107-3 & $\mathbf{Y}$ & From Analytical Process Development Unit \\
\hline Braun & Pu241 & $1.22 E+07$ & $7.61 E+06$ & $2.06 E-04$ & NA & $\mathbf{N}$ & Duplicate of Raw R3638 \\
\hline Braun & Sr89/90 & $6.07 E+09$ & $4.79 E+09$ & $1.29 E-01$ & NA & $\mathbf{N}$ & Duplicate of Raw R3638 \\
\hline Raw & Sr89/90 & $6.07 E+09$ & $4.79 E+09$ & $1.29 E-01$ & AN-107-3 & $\bar{Y}$ & - \\
\hline Braun & Sr89/90 & $5.48 E+09$ & $4.32 E+09$ & 9.17E-01 & NA & $\mathbf{N}$ & Duplicate of Raw R3637 \\
\hline Wastren & Sr89/90 & $5.48 E+09$ & $4.33 E+09$ & $1.17 \mathrm{E}-01$ & NA & $N$ & Duplicate of Raw R3637 \\
\hline Raw & Sr89/90 & $5.48 E+09$ & $4.32 E+09$ & $1.17 E-01$ & AN-107-3 & $\mathbf{Y}$ & \\
\hline RAW & Sr89/90 & $5.07 E+09$ & $5.07 E+09$ & 1.37E-01 & AN107-8 & $\mathbf{N}$ & Units changed to UCin / Can't find \\
\hline RAW & Sr89/90 & $3.66 \mathrm{E}+09$ & $3.50 E+09$ & $9.46 E-02$ & AN107-8 & $\mathbf{Y}$ & Units changed to uCin \\
\hline HTCE InV. & Sr89/90 & 4.07E+08 & $4.07 E+08$ & $1.10 \mathrm{E}-02$ & NA & $\mathbf{N}$ & HTCE Data; Units changed to UCi/L \\
\hline RAW & Sr89/91 & $3.29 E+09$ & $3.26 E+09$ & $8.81 E-02$ & AN107-8 & $\bar{Y}$ & Added, missed initially \\
\hline RAW & Sr89/92 & $3.39 E+09$ & $3.35 E+09$ & $9.06 \mathrm{E}-02$ & AN107-8 & $\mathbf{Y}$ & Added, missed initially \\
\hline
\end{tabular}




\begin{tabular}{|c|c|c|c|c|c|c|c|c|c|c|c|}
\hline \multicolumn{12}{|c|}{ Tank Waste Characterization Sample Information } \\
\hline $\begin{array}{c}\text { Reported } \\
\text { Radlonuclide } \\
\end{array}$ & $\begin{array}{c}\text { Memo } \\
\text { Date }\end{array}$ & $\begin{array}{c}\text { Start } \\
\text { Analysis } \\
\text { Date } \\
\end{array}$ & $\begin{array}{c}\text { Start } \\
\text { Sample } \\
\text { Date } \\
\end{array}$ & $\begin{array}{l}\text { Sample } \\
\text { Number }\end{array}$ & $\begin{array}{c}\begin{array}{c}\text { Tank Farm } \\
\text { Number }\end{array} \\
\end{array}$ & $\begin{array}{c}\text { Lob Sample } \\
\text { Number }\end{array}$ & $\begin{array}{l}\text { Density } \\
\text { (g/mL) }\end{array}$ & $\begin{array}{c}\text { Reported } \\
\text { Value }\end{array}$ & Reported Unit & Code & Source \\
\hline Am241 & $6 / 1 / 90$ & $4 / 30 / 89$ & $4 / 27 / 89$ & $\mathrm{~B} 082 \mathrm{M} 2$ & G309 & G358 & 1.20 & $9.83 E-04$ & $\therefore \mathrm{uCl} / \mathrm{mL}$ & $\mathbf{P}$ & Raw \\
\hline Am241 & $6 / 1 / 90$ & $4 / 30 / 89$ & $4 / 27 / 89$ & BOB2M3 & G311 & G359 & 1.20 & $8.59 E-04$ & $\mathrm{uCi} / \mathrm{mL}$ & $\mathbf{P}$ & Raw \\
\hline Am241 & $6 / 1 / 90$ & $4 / 30 / 89$ & $4 / 27 / 89$ & $\mathrm{~B} 082 \mathrm{M} 2$ & G309 & G358 & 1.20 & $7.14 E-04$ & $\mathrm{uCi} / \mathrm{mL}$ & $\mathbf{P}$ & Raw \\
\hline Am241 & $6 / 1 / 90$ & $4 / 30 / 89$ & $4 / 27 / 89$ & BOB2N4 & G323 & G341 & 1.20 & $6.69 E-04$ & $\mathrm{uCi} / \mathrm{mL}$ & $\mathbf{P}$ & Raw \\
\hline Am241 & $6 / 1 / 90$ & $4 / 30 / 89$ & $4 / 27 / 89$ & В082M3 & G311 & G359 & 1.20 & 5.79E-O4 & $\mathrm{uCi} / \mathrm{mL}$ & $P$ & Raw \\
\hline Am241 & $6 / 1 / 90$ & $4 / 30 / 89$ & $4 / 27 / 89$ & BOB2L9 & G305 & G349 & 1.20 & $5.27 E-04$ & $\mathrm{uCi} / \mathrm{mL}$ & $\mathbf{P}$ & Raw \\
\hline Am241 & $6 / 1 / 90$ & $4 / 30 / 89$ & $4 / 27 / 89$ & В082M1 & G308 & G353 & 1.20 & 5.01E-04 & $\mathrm{uCi} / \mathrm{mL}$ & $\mathbf{P}$ & Raw \\
\hline Am241 & $6 / 1 / 90$ & $4 / 30 / 89$ & $4 / 27 / 89$ & Вов2Т9 & G327 & G470* & 1.19 & $4.88 \mathrm{E}-04$ & $\mathrm{uCi} / \mathrm{mL}$ & $\mathbf{P}$ & Raw \\
\hline Am241 & $6 / 1 / 90$ & $4 / 30 / 89$ & $4 / 27 / 89$ & ВOВ2MO & G306 & G352 & 1.20 & $4.84 E-04$ & $\mathrm{uCi} / \mathrm{mL}$ & $P$ & Raw \\
\hline Am241 & $6 / 1 / 90$ & $4 / 30 / 89$ & $4 / 27 / 89$ & BOB2M1 & G308 & G353 & 1.20 & $4.76 E-04$ & $\mathrm{uCi} / \mathrm{mL}$ & $\mathbf{P}$ & Raw \\
\hline Am241 & $6 / 1 / 90$ & $4 / 30 / 89$ & $4 / 27 / 89$ & ВОВ2M6 & G318 & G459 & 1.20 & 4.50E-0.4 & $\mathrm{uCi} / \mathrm{mL}$ & $\mathbf{P}$ & Raw \\
\hline Am241 & $6 / 1 / 90$ & $4 / 30 / 89$ & $4 / 27 / 89$ & BOB2N4 & G323 & G341 & 1.20 & 4.49E-0.4 & $\mathrm{uCi} / \mathrm{mL}$ & $\mathbf{P}$ & Raw \\
\hline Am 241 & $6 / 1 / 90$ & $4 / 30 / 89$ & $4 / 27 / 89$ & Вов2Т9 & G327 & G443 & 1.20 & 4.39E-04 & $\mathrm{uCi} / \mathrm{mL}$ & $\mathbf{P}$ & Raw \\
\hline Am241 & $6 / 1 / 90$ & $4 / 30 / 89$ & $4 / 27 / 89$ & ВОВ2МO & G306 & G352 & 1.20 & $4.32 E-04$ & $\mathrm{uCi} / \mathrm{mL}$ & $\mathbf{P}$ & Raw \\
\hline Am241 & $6 / 1 / 90$ & $4 / 30 / 89$ & $4 / 27 / 89$ & BOB2M6 & G318 & G459 & 1.20 & $4.26 \mathrm{E}-04$ & $\mathrm{uCi} / \mathrm{mL}$ & $P$ & Raw \\
\hline Am241 & $6 / 1 / 90$ & $4 / 30 / 89$ & $4 / 27 / 89$ & BOB2LA & G305 & G349 & 1.20 & $4.25 E-04$ & $\mathrm{uCi} / \mathrm{mL}$ & $\mathbf{P}$ & Raw \\
\hline Am241 & $6 / 1 / 90$ & $4 / 30 / 89$ & $4 / 27 / 89$ & Вов2М9 & G302 & G477* & 1.20 & $4.12 E-04$ & $\mathrm{uCi} / \mathrm{mL}$ & $\mathbf{P}$ & Raw \\
\hline Am241 & $6 / 1 / 90$ & $4 / 30 / 89$ & $4 / 27 / 89$ & ВOB2M7 & G319 & G460 & 1.20 & $4.08 E-04$ & $\mathrm{uCI} / \mathrm{mL}$ & $\mathbf{P}$ & Raw \\
\hline Am241 & $6 / 1 / 90$ & $4 / 30 / 89$ & $4 / 27 / 89$ & BOB2N4 & G323 & G341 & 1.20 & $3.89 E-04$ & $\mathrm{uCi} / \mathrm{mL}$ & $\mathbf{P}$ & Raw \\
\hline Am241 & $6 / 1 / 90$ & $4 / 30 / 89$ & $4 / 27 / 89$ & ВОВ2T9 & G327 & G443 & 1.20 & 3.79E-04 & uCi/mL & $\mathbf{P}$ & Raw \\
\hline
\end{tabular}




\begin{tabular}{|c|c|c|c|c|c|c|}
\hline \multicolumn{4}{|c|}{ Radionuclide Information } & & \multirow[b]{2}{*}{ Validated $(Y / N)$} & \multirow[b]{2}{*}{ Notes } \\
\hline Radoinuclide & Conc. $(\mathrm{Bq} / \mathrm{L})$ & $\begin{array}{c}\text { Conc. (Bq/L } \\
\text { decayed) }\end{array}$ & $\begin{array}{l}\text { Conc. (Ci/L } \\
\text { Decayed) }\end{array}$ & $\begin{array}{c}\text { Reference } \\
\text { Number }\end{array}$ & & \\
\hline Am241 & $3.64 E+0.4$ & $3.63 E+04$ & $\cdots 9.80 E-07$ & AP102-3 & 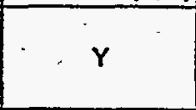 & $\begin{array}{l}\text { Density reported for } \mathrm{G} 358 \text { collected at } 334 \text { in from } \\
\text { bottom of tank at a } 150^{\circ} \text { angle. Sample was } \\
\text { unheated, unheated samples precipitated }\end{array}$ \\
\hline Am241 & $3.18 E+04$ & 3.17E+04 & 8.57E-07 & AP102-3 & $\mathbf{Y}$ & $\begin{array}{l}\text { Density reported for G359 collected at } 162 \text { in from } \\
\text { bottom of tank at a } 150^{\circ} \text { angle. Sample was } \\
\text { unheated, unheated samples precipitated }\end{array}$ \\
\hline Am241 & $2.64 E+04$ & $2.63 E+04$ & $7.12 E-07$ & AP102-3 & $\boldsymbol{\gamma}$ & $\begin{array}{l}\text { Density reported for } G 358 \text { collected at } 334 \text { in from } \\
\text { bottom of tank at a } 150^{\circ} \text { angle. Sample was } \\
\text { unheated, unheated samples precipitated }\end{array}$ \\
\hline Am241 & $2.48 E+04$ & $2.47 E+04$ & $6.67 E-07$ & AP102-3 & $Y$ & $\begin{array}{l}\text { Density reported for } \mathrm{G} 341 \text { collected at } 99 \text { in from } \\
\text { bottom of tank at a } 270^{\circ} \text { angle. }\end{array}$ \\
\hline Am241 & $2.14 E+04$ & $2.14 E+04$ & 5.77E-07 & AP102-3 & $\gamma$ & $\begin{array}{l}\text { Density reported for } G 359 \text { collected at } 162 \text { in from } \\
\text { bottom of tank at a } 150^{\circ} \text { angle. Sample was } \\
\text { unheated, unheated samples precipitated }\end{array}$ \\
\hline Am241 & $1.95 E+04$ & $1.94 E+04$ & $5.26 \mathrm{E}-07$ & AP102-3 & $\mathbf{Y}$ & $\begin{array}{l}\text { Density reported for G349 collected at } 34 \text { in from } \\
\text { bottom of tank at a } 30^{\circ} \text { angle. Sample was } \\
\text { unheated, unheated samples precipitated }\end{array}$ \\
\hline Am241 & $1.85 E+04$ & $1.85 E+04$ & $5.00 E-07$ & AP102-3 & $Y$ & $\begin{array}{l}\text { Density reported for G353 collected at } 334 \text { in from } \\
\text { bottom of tank at a } 150^{\circ} \text { angle. Sample was } \\
\text { unheated, unheated samples precipitated }\end{array}$ \\
\hline Am241 & $1.81 E+04$ & $1.80 E+04$ & 4.87E-07 & AP102-3 & $Y$ & $\begin{array}{l}\text { Density reported for } \mathrm{G} 470 \text { represents a composite } \\
\text { sample. Sample was kept heated to } 40^{\circ} \mathrm{C} \text {. }\end{array}$ \\
\hline Am241 & $1.79 E+04$ & $1.79 E+04$ & 4.83E-07 & AP102-3 & $\gamma$ & $\begin{array}{l}\text { Density reported for } \mathbf{G 3 5 2} \text { collected at } 34 \text { in from } \\
\text { bottom of tank at a } 30^{\circ} \text { angle. Sample was } \\
\text { unheated, unheated samples precipitated }\end{array}$ \\
\hline - Am241 & $1.76 E+0.4$ & $1.76 E+04$ & 4.75E-07 & AP102-3 & $Y$ & $\begin{array}{l}\text { Density reported for G353 collected at } 334 \text { in from } \\
\text { bottom of tank at a } 150^{\circ} \text { angle. Sample was } \\
\text { unheated, unheated samples precipitated }\end{array}$ \\
\hline Am241 & $1.67 E+04$ & $1.66 E+04$ & 4.49E-07 & AP102-3 & $\mathbf{Y}$ & $\begin{array}{l}\text { Density reported for G459 collected at } 226 \text { in from } \\
\text { bottom of tank at a } 270^{\circ} \text { angle. Sample was } \\
\text { unheated, unheated samples precipitated }\end{array}$ \\
\hline Am241 & $1.66 E+04$ & $1.66 E+04$ & 4.48E-07 & AP102-3 & $Y$ & $\begin{array}{l}\text { Density reported for } \mathrm{G} 341 \text { collected at } 99 \text { in from } \\
\text { bottom of tank at a } 270^{\circ} \text { angle. }\end{array}$ \\
\hline Am241 & $1.62 E+04$ & $1.62 E+04$ & $4.38 E-07$ & AP102-3 & $Y$ & $\begin{array}{l}\text { Density reported for G443 represents a composite } \\
\text { sample }\end{array}$ \\
\hline Am241 & $1.60 E+04$ & $1.59 E+04$ & $4.31 E-07$ & AP102-3 & $Y$ & $\begin{array}{l}\text { Density reported for } \mathrm{G} 352 \text { collected at } 34 \text { in from } \\
\text { bottom of tank at a } 30^{\circ} \text { angle. Sample was } \\
\text { unheated, unheated samples precipitated }\end{array}$ \\
\hline Am241 & $1.58 E+04$ & $1.57 E+04$ & $4.25 E-07$ & AP102-3 & $\gamma$ & $\begin{array}{l}\text { Density reported for G459 collected at } 226 \text { in from } \\
\text { bottom of tank at a } 270^{\circ} \text { angle. Sample was } \\
\text { unheated, unheated samples precipitated }\end{array}$ \\
\hline Am241 & $1.57 \mathrm{E}+04$ & $1.57 E+0.4$ & $4.24 E-07$ & AP102-3 & $Y$ & $\begin{array}{l}\text { Density reported for } \mathrm{G} 349 \text { collected at } 34 \text { in from } \\
\text { bottom of tank at a } 30^{\circ} \text { angle. Sample was } \\
\text { unheated, unheated samples precipitated }\end{array}$ \\
\hline Am241 & $1.52 E+04$ & $1.52 E+04$ & 4.11E-07 & AP102-3 & $Y$ & $\begin{array}{l}\text { Density reported for } 6477 \text { collected at } 182 \text { in from } \\
\text { bottom of tank at a } 30^{\circ} \text { angle and was keep heated } \\
\text { to } 40^{\circ} \mathrm{C} \text {. }\end{array}$ \\
\hline Am241 & $1.51 E+04$ & $1.51 E+04$ & 4.07E-07 & AP102-3 & $Y$ & $\begin{array}{l}\text { Density reported for G460 collected at } 226 \text { in from } \\
\text { bottom of tank at a } 270^{\circ} \text { angle. Sample was } \\
\text { unheated, unheated samples precipitated }\end{array}$ \\
\hline Am241 & $1.44 E+04$ & $1.44 E+04$ & $3.88 \mathrm{E}-07$ & AP102-3 & $Y$ & $\begin{array}{l}\text { Density reported for } \mathrm{G341} \text { collected at } 99 \text { in from } \\
\text { bottom of tank at a } 270^{\circ} \text { angle. }\end{array}$ \\
\hline Am241 & $1.40 E+04$ & $1.40 E+04$ & 3.78E-07 & AP102-3 & $Y$ & $\begin{array}{l}\text { Density reported for } \mathbf{G 4 4 3} \text { represents a composite } \\
\text { sample }\end{array}$ \\
\hline
\end{tabular}




\begin{tabular}{|c|c|c|c|c|c|c|c|c|c|c|c|}
\hline \multicolumn{12}{|c|}{ Tank Waste Characterkation Sample Information } \\
\hline $\begin{array}{c}\text { Reported } \\
\text { Radlonuclide } \\
\end{array}$ & $\begin{array}{c}\text { Memo } \\
\text { Date }\end{array}$ & $\begin{array}{c}\text { Start } \\
\text { Analysis } \\
\text { Date }\end{array}$ & $\begin{array}{c}\text { Start } \\
\text { Sample } \\
\text { Date }\end{array}$ & $\begin{array}{l}\text { Sample } \\
\text { Number }\end{array}$ & $\begin{array}{c}\text { Tank Farm } \\
\text { Number }\end{array}$ & $\begin{array}{c}\text { Lab Sample } \\
\text { Number }\end{array}$ & $\begin{array}{l}\text { Density } \\
\text { (g/mL) }\end{array}$ & $\begin{array}{c}\text { Reported } \\
\text { Value } \\
\end{array}$ & Reported Unit & Code & Source \\
\hline Am241 & $6 / 1 / 90$ & $4 / 30 / 89$ & $4 / 27 / 89$ & BOB2N1 & G310 & G471 * & 1.20 & $3.73 E-04$ & $\mathrm{uCi} / \mathrm{mL}$ & $P$ & Raw \\
\hline Am241 & $6 / 1 / 90$ & $4 / 30 / 89$ & $4 / 27 / 89$ & BOB2T9 & G327 & G470* & 1.19 & $3.72 E-04$ & $\mathrm{uCi} / \mathrm{mL}$ & $\mathbf{P}$ & Raw \\
\hline Am241 & $6 / 1 / 90$ & $4 / 30 / 89$ & $4 / 27 / 89$ & BOB2T9 & G327 & G443 & 1.20 & 3.69E-04 & $\mathrm{uCi} / \mathrm{mL}$ & $\mathbf{P}$ & Raw \\
\hline Am241 & $6 / 1 / 90$ & $4 / 30 / 89$ & $4 / 27 / 89$ & BOB2N4 & G323 & G476" & 1.21 & $3.64 E-04$ & $\mathrm{uCi} / \mathrm{mL}$ & $\mathbf{P}$ & Raw \\
\hline Am241 & $6 / 1 / 90$ & $4 / 30 / 89$ & $4 / 27 / 89$ & BOB2N4 & G323 & G341 & 1.20 & $3.51 E-04$ & $u \mathrm{Ci} / \mathrm{mL}$ & $\mathbf{P}$ & Raw \\
\hline Am241 & $6 / 1 / 90$ & $4 / 30 / 89$ & $4 / 27 / 89$ & BOB2M7 & G319 & G460 & 1.20 & $3.40 \mathrm{E}-04$ & $u \mathrm{Ci} / \mathrm{mL}$ & $P$ & Raw \\
\hline Am241 & $6 / 1 / 90$ & $4 / 30 / 89$ & $4 / 27 / 89$ & ВОВ2T9 & G327 & G443 & 1.20 & $3.35 E-04$ & $\mathrm{uCi} / \mathrm{mL}$ & $P$ & Raw \\
\hline Am241 & $6 / 1 / 90$ & $4 / 30 / 89$ & $4 / 27 / 89$ & BOB2L7 & G299 & G342 & 1.20 & $3.28 E-04$ & $u C i / m L$ & $\mathbf{P}$ & Raw \\
\hline Am241 & $6 / 1 / 90$ & $4 / 30 / 89$ & $4 / 27 / 89$ & BOB2N4 & G323 & G476* & 1.21 & $3.28 E-04$ & $\mathrm{uCi} / \mathrm{mL}$ & $\mathbf{P}$ & Raw \\
\hline Am241 & $6 / 1 / 90$ & $4 / 30 / 89$ & $4 / 27 / 89$ & BOB2L7 & G299 & G342 & 1.20 & $3.23 E-04$ & $\mathrm{uCi} / \mathrm{mL}$ & $\mathbf{P}$ & Raw \\
\hline Am241 & $6 / 1 / 90$ & $4 / 30 / 89$ & $4 / 27 / 89$ & BOB2N1 & G310 & G471* & 1.20 & $3.18 E-04$ & $\mathrm{uCi} / \mathrm{mL}$ & $\mathbf{P}$ & Raw \\
\hline Am241 & $6 / 1 / 90$ & $4 / 30 / 89$ & $4 / 27 / 89$ & B082M9 & G302 & G477* & 1.20 & $3.06 \mathrm{E}-04$ & $u C i / m L$ & $\mathbf{P}$ & Raw \\
\hline Am241 & $6 / 1 / 90$ & $4 / 30 / 89$ & $4 / 27 / 89$ & Вов2М9 & G302 & G477* & 1.20 & $2.91 E-04$ & $u C i / m L$ & $P$ & Raw \\
\hline Am241 & $6 / 1 / 90$ & $4 / 30 / 89$ & $4 / 27 / 89$ & вов2M9 & G302 & G477* & 1.20 & $2.86 E-04$ & uCi/mL & $\mathbf{P}$ & Raw \\
\hline Am241 & $6 / 1 / 90$ & $4 / 30 / 89$ & $4 / 27 / 89$ & BOB2N4 & G323 & G476* & 1.21 & $2.86 \mathrm{E}-04$ & $\mathrm{uCi} / \mathrm{mL}$ & $P$ & Raw \\
\hline Am241 & $6 / 1 / 90$ & $4 / 30 / 89$ & $4 / 27 / 89$ & BOB2N4 & G323 & G476" & 1.21 & 2.71E-04 & $\mathrm{uCi} / \mathrm{mL}$ & $\mathbf{P}$ & Raw \\
\hline Am241 & $6 / 1 / 90$ & $4 / 30 / 89$ & $4 / 27 / 89$ & BOB2M4 & G313 & G363 & 1.20 & 2.59E-04 & $\mathrm{uCi} / \mathrm{mL}$ & $\mathbf{P}$ & Raw \\
\hline Am241 & $6 / 1 / 90$ & $4 / 30 / 89$ & $4 / 27 / 89$ & BOB2M5 & G315 & G364 & 1.19 & 2.57E-04 & $\mathrm{uCl} / \mathrm{mL}$ & $\mathbf{P}$ & Raw \\
\hline Am241 & $6 / 1 / 90$ & 4/30/89 & $4 / 27 / 89$ & BOB2L8 & G303 & G348 & 1.21 & 2.49E-04 & $\mathrm{uCi} / \mathrm{mL}$ & $P$ & Raw \\
\hline Am241 & $6 / 1 / 90$ & $4 / 30 / 89$ & $4 / 27 / 89$ & BOB2M5 & G315 & G364 & 1.19 & 2.33E-04 & $\mathrm{uCi} / \mathrm{mL}$ & $P$ & Raw \\
\hline
\end{tabular}




\begin{tabular}{|c|c|c|c|c|c|c|}
\hline \multicolumn{4}{|c|}{ Radionuclide Information } & \multirow[b]{2}{*}{$\begin{array}{l}\text { Reterence } \\
\text { Number }\end{array}$} & \multirow[b]{2}{*}{ Validated $(Y / N)$} & \multirow[b]{2}{*}{ Notes } \\
\hline Radoinuclide & Conc. (Bq/L) & $\begin{array}{c}\text { Conc. (Bq/L } \\
\text { decayed) }\end{array}$ & $\begin{array}{l}\text { Conc. (Cilh } \\
\text { Decayed) }\end{array}$ & & & \\
\hline Am241 & $1.38 \mathrm{E}+0.4$ & $1.38 E+04$ & $-3.72 E-07$. & AP102-3 & $-Y$ & $\begin{array}{l}\text { Density reported for } \mathrm{G} 471 \text { collected at } 208 \text { in from } \\
\text { bottom of tank at a } 150^{\circ} \text { angle and was keep heated } \\
\text { to } 40^{\circ} \mathrm{C} \text {. }\end{array}$ \\
\hline Am241 & $1.38 E+04$ & $1.37 E+0.4$ & 3.71E-07 & AP102-3 & $Y$ & $\begin{array}{l}\text { Density reported for } \mathrm{G} 470 \text { represents a composite } \\
\text { sample. Sample was kept heated to } 40^{\circ} \mathrm{C} \text {. }\end{array}$ \\
\hline Am241 & $1.37 E+04$ & $1.36 E+04$ & $3.68 E-07$ & AP102-3 & $\gamma$ & $\begin{array}{l}\text { Density reported for G443 represents a composite } \\
\text { sample }\end{array}$ \\
\hline Am241 & $1.35 E+04$ & $1.34 E+04$ & $3.63 E-07$ & AP102-3 & $\mathbf{Y}$ & $\begin{array}{l}\text { Density reported for } 6476 \text { collected at } 99 \text { in from } \\
\text { bottom of tank at a } 270^{\circ} \text { angle and was keep heated } \\
\text { to } 40^{\circ} \mathrm{C} \text {. }\end{array}$ \\
\hline Am241 & $1.30 E+04$ & $1.30 E+0.4$ & $3.50 E-07$ & AP102-3 & $\gamma$ & $\begin{array}{l}\text { Density reported for G341 collected at } 99 \text { in from } \\
\text { bottom of tank at a } 270^{\circ} \text { angle. }\end{array}$ \\
\hline Am241 & $1.26 E+04$ & $1.25 E+04$ & 3.39E-07 & AP102-3 & $\gamma$ & $\begin{array}{l}\text { Density reported for } \mathrm{G} 460 \text { collected at } 226 \text { in from } \\
\text { bottom of tank at a } 270^{\circ} \text { angle. Sample was } \\
\text { unheated, unheated samples precipitated }\end{array}$ \\
\hline Am241 & $1.24 E+04$ & $1.24 E+04$ & 3.34E-07 & AP102-3 & $\gamma$ & $\begin{array}{l}\text { Density reported for G443 represents a composite } \\
\text { sample }\end{array}$ \\
\hline Am241 & $1.21 E+04$ & $1.21 E+04$ & $3.27 E-07$ & AP102-3 & $Y$ & $\begin{array}{l}\text { Density reported for } G 342 \text { collected at } 306 \text { in from } \\
\text { bottom of tank at a } 30^{\circ} \text { angle. Sample was } \\
\text { unheated, unheated samples precipitated }\end{array}$ \\
\hline Am241 & $1.21 E+04$ & $1.21 E+04$ & $3.27 E-07$ & AP102-3 & $\gamma$ & $\begin{array}{l}\text { Density reported for } \mathrm{G} 476 \text { collected at } 99 \text { in from } \\
\text { bottom of tank at a } 270^{\circ} \text { angle and was keep heated } \\
\text { to } 40^{\circ} \mathrm{C} \text {. }\end{array}$ \\
\hline Am241 & $1.20 E+04$ & $1.19 E+04$ & $3.22 \mathrm{E}-07$ & AP102-3 & $\mathrm{Y}$ & $\begin{array}{l}\text { Density reported for } \mathrm{G} 342 \text { collected at } 306 \text { in from } \\
\text { bottom of tank at a } 30^{\circ} \text { angle. Sample was } \\
\text { unheated, unheated samples precipitated }\end{array}$ \\
\hline Am241 & $1.18 E+04$ & $1.17 E+04$ & $3.17 E-07$ & AP102-3 & $\mathbf{Y}$ & $\begin{array}{l}\text { Density reported for } \mathrm{G} 471 \text { collected at } 208 \text { in from } \\
\text { bottom of tank at a } 150^{\circ} \text { angle and was keep heated } \\
\text { to } 40^{\circ} \mathrm{C} \text {. }\end{array}$ \\
\hline Am241 & $1.13 E+04$ & $1.13 E+04$ & $3.05 E-07$ & AP102-3 & $\mathbf{Y}$ & $\begin{array}{l}\text { Density reported for } \mathrm{G} 477 \text { collected at } 182 \text { in from } \\
\text { bottom of tank at a } 30^{\circ} \text { angle and was keep heated } \\
\text { to } 40^{\circ} \mathrm{C} \text {. }\end{array}$ \\
\hline Am241 & $1.08 E+04$ & $1.07 E+04$ & 2.90E-07 & AP102-3 & $\mathbf{Y}$ & $\begin{array}{l}\text { Density reported for } 6477 \text { collected at } 182 \text { in from } \\
\text { bottom of tank at a } 30^{\circ} \text { angle and was keep heated } \\
\text { to } 40^{\circ} \mathrm{C} \text {. }\end{array}$ \\
\hline Am 241 & $1.06 E+04$ & $1.06 E+04$ & $2.85 E-07$ & AP102-3 & $\boldsymbol{\gamma}$ & $\begin{array}{l}\text { Density reported for } \mathrm{G} 477 \text { collected at } 182 \text { in from } \\
\text { bottom of tank at a } 30^{\circ} \text { angle and was keep heated } \\
\text { to } 40^{\circ} \mathrm{C} \text {. }\end{array}$ \\
\hline Am241 & $1.06 E+04$ & $1.06 E+04$ & 2.85E-07 & AP102-3 & $Y$ & $\begin{array}{l}\text { Density reported for } \mathrm{G} 476 \text { collected at } 99 \text { in from } \\
\text { bottom of tank at a } 270^{\circ} \text { angle and was keep heated } \\
\text { to } 40^{\circ} \mathrm{C} \text {. }\end{array}$ \\
\hline Am241 & $1.00 E+04$ & $1.00 E+04$ & $2.70 E-07$ & AP102-3 & $Y$ & $\begin{array}{l}\text { Density reported for } \mathrm{G} 476 \text { collected at } 99 \text { in from } \\
\text { bottom of tank at a } 270^{\circ} \text { angle and was keep heated } \\
\text { to } 40^{\circ} \mathrm{C} \text {. }\end{array}$ \\
\hline Am241 & $9.58 E+03$ & $9.56 E+03$ & 2.58E-07 & AP102-3 & $\gamma$ & $\begin{array}{l}\text { Density reported for } G 363 \text { collected at } 18 \text { in from } \\
\text { bottom of tank at a } 150^{\circ} \text { angle. Sample was } \\
\text { unheated, unheated samples precipitated }\end{array}$ \\
\hline Am241 & $9.51 E+03$ & $9.48 E+03$ & $2.56 E-07$ & AP102-3 & $\mathbf{Y}$ & $\begin{array}{l}\text { Density reported for } \mathrm{G} 364 \text { collected at } 372 \text { in from } \\
\text { bottom of tank at a } 270^{\circ} \text { angle. Sample was } \\
\text { unheated, unheated samples precipitated }\end{array}$ \\
\hline Am241 & $9.21 E+03$ & $9.19 E+03$ & $2.48 E-07$ & AP102-3 & $\mathbf{Y}$ & $\begin{array}{l}\text { Density reported for } G 348 \text { collected at } 125 \text { in from } \\
\text { bottom of tank at a } 30^{\circ} \text { angle. Sample was } \\
\text { unheated, unheated samples precipitated }\end{array}$ \\
\hline Am241 & $8.62 E+03$ & $8.60 E+03$ & 2.32E-07 & AP102-3 & $\boldsymbol{\gamma}$ & $\begin{array}{l}\text { Density reported for G364 collected at } 372 \text { in from } \\
\text { bottom of tank at a } 270^{\circ} \text { angle. Sample was } \\
\text { unheated, unheated samples precipitated }\end{array}$ \\
\hline
\end{tabular}


TWRS Privatization Support Pro Radionuclide Liquil

\begin{tabular}{|c|c|c|c|c|c|c|c|c|c|c|c|}
\hline & & & & Tank Wast & Chasactesiza & ion Sample In & mation & & & & \\
\hline $\begin{array}{c}\text { Reported } \\
\text { Radionuclide }\end{array}$ & $\begin{array}{c}\text { Memo } \\
\text { Date }\end{array}$ & $\begin{array}{c}\text { Start } \\
\text { Analysis } \\
\text { Date }\end{array}$ & $\begin{array}{c}\text { Start } \\
\text { Sample } \\
\text { Date } \\
\end{array}$ & $\begin{array}{l}\text { Sample } \\
\text { Number }\end{array}$ & $\begin{array}{c}\text { Tank Farm } \\
\text { Number }\end{array}$ & $\begin{array}{c}\text { Lab Sample } \\
\text { Number }\end{array}$ & $\begin{array}{l}\text { Density } \\
(\mathrm{g} / \mathrm{mL})\end{array}$ & $\begin{array}{c}\text { Reported } \\
\text { Value } \\
\end{array}$ & Reported Unit & Code & Sourca \\
\hline Am241 & $6 / 1 / 90$ & $4 / 30 / 89$ & $4 / 27 / 89$ & BOB2L8 & G303 - & G348 & 1.21 & $2.26 \mathrm{E}-04$ & $\mathrm{uCi} / \mathrm{mL}$ & $\mathbf{P}$ & Raw \\
\hline Am241 & $6 / 1 / 90$ & $4 / 30 / 89$ & $4 / 27 / 89$ & ВОВ2M4 & G313 & G363 & 1.20 & 2.11E-04 & $\mathrm{uCi} / \mathrm{mL}$ & $P$ & Raw \\
\hline Am241 & $4 / 30 / 84$ & NS & NS & NS & NS & NS & NS & NS & NS & $P$ & Wastrer \\
\hline Am241 & $5 / 17 / 84$ & NS & NS & R8473 & NS & NS & NS & $7.44 E-03$ & uCI/L & $\mathbf{P}$ & $\overline{R A W}$ \\
\hline Am241 & $5 / 17 / 84$ & NS & NS & R8471 & NS & NS & NS & $4.14 E-03$ & uCin & $\bar{P}$ & $\overline{\text { RAW }}$ \\
\hline Am241 & $5 / 17 / 84$ & NS & NS & R8469 & NS & NS & NS & $1.03 E-03$ & uCi/L & $P$ & $\overline{R A W}$ \\
\hline Am241 & $5 / 77 / 84$ & NS & NS & R8472 & NS & NS & NS & $9.12 E-04$ & $\mathrm{UC \textrm {CI } / \mathrm { L }}$ & $\mathbf{P}$ & $\overline{R A W}$ \\
\hline Am241 & $5 / 17 / 84$ & NS & NS & W1549 & NS & NS & NS & $7.84 E-04$ & $u C I / L$ & $L$ & $\overline{\mathrm{RAW}}$ \\
\hline Am241 & $5 / 17 / 84$ & NS & NS & R8469 & NS & NS & WS & $1.03 E-04$ & $4 \mathrm{CI} / \mathrm{L}$ & $\bar{L}$ & $\overline{R A W}$ \\
\hline C14 & $4 / 30 / 84$ & NS & NS & NS & NS & NS & NS & $2.35 E+05$ & $8 q \pi$ & $\bar{P}$ & Wastren \\
\hline Carbon-14 & $6 / 1 / 90$ & $4 / 30 / 89$ & $4 / 27 / 89$ & BOB2MO & G306 & G352 & 1.20 & $7.38 E-04$ & $\mathrm{uCi} / \mathrm{mL}$ & $P$ & Raw \\
\hline Carbon-14 & $6 / 1 / 90$ & $4 / 30 / 89$ & $4 / 27 / 89$ & ВОВ2M6 & G318 & G459 & 1.20 & 7.17E-04 & $\mathrm{uCi} / \mathrm{mL}$ & $P$ & Raw \\
\hline Carbon-14 & $6 / 1 / 90$ & $4 / 30 / 89$ & $4 / 27 / 89$ & Вов2M6 & G318 & G459 & 1.20 & $5.61 E-04$ & $\mathrm{uCi} / \mathrm{mL}$ & $P$ & Raw \\
\hline Carbon-14 & $6 / 1 / 90$ & $4 / 30 / 89$ & $4 / 27 / 89$ & BOB2N4 & G323 & G341 & 1.20 & $5.61 E-04$ & $\mathrm{uCi} / \mathrm{mL}$ & $P$ & Raw \\
\hline Catbon-14 & 6/1/90 & $4 / 30 / 89$ & $4 / 27 / 89$ & ВОВ2M2 & G309 & G358 & 1.20 & $5.55 E-04$ & $\mathrm{uCi} / \mathrm{mL}$ & $P$ & Raw \\
\hline Carbon-14 & $6 / 1 / 90$ & $4 / 30 / 89$ & $4 / 27 / 89$ & BOB2M5 & G315 & G364 & 1.19 & $5.44 E-04$ & $\mathrm{uCi} / \mathrm{mL}$ & $P$ & Raw \\
\hline Carbon-14 & $6 / 1 / 90$ & $4 / 30 / 89$ & $4 / 27 / 89$ & BOB2M1 & G308 & G353 & 1.20 & 5.39E-04 & $\mathrm{uCi} / \mathrm{mL}$ & $P$ & Raw \\
\hline Carbon-14 & $6 / 1 / 90$ & $4 / 30 / 89$ & $4 / 27 / 89$ & $\mathrm{BOB} 2 \mathrm{~N} 4$ & G323 & G476* & 1.21 & 5.39E-0.4 & $u \mathrm{Ci} / \mathrm{mL}$ & $\mathbf{P}$ & Raw \\
\hline Carbon-14 & $6 / 1 / 90$ & $4 / 30 / 89$ & $4 / 27 / 89$ & BOB2N4 & G323 & G476* & 1.21 & 5.36E-04 & $\mathrm{uCi} / \mathrm{mL}$ & $P$ & Raw \\
\hline Carbon-14 & $6 / 1 / 90$ & $4 / 30 / 89$ & $4 / 27 / 89$ & ВОВ2M4 & G313 & G363 & 1.20 & $5.22 \mathrm{E}-04$ & $\mathrm{uCi} / \mathrm{mL}$ & $P$ & Raw \\
\hline Carbon-14 & $6 / 1 / 90$ & $4 / 30 / 89$ & $4 / 27 / 89$ & ВОВ2MO & G306 & G352 & 1.20 & $5.21 E-04$ & $\mathrm{uCl} / \mathrm{mL}$ & $P$ & Raw \\
\hline Carbon-14 & $6 / 1 / 90$ & $4 / 30 / 89$ & $4 / 27 / 89$ & BOB2N1 & G310 & G471 * & 1.20 & $5.21 E-04$ & $\mathrm{uCi} / \mathrm{mL}$ & $P$ & Raw \\
\hline Carbon-14 & $6 / 1 / 90$ & $4 / 30 / 89$ & $4 / 27 / 89$ & BOB2M3 & G311 & G359 & 1.20 & $5.20 E-04$ & $\mathrm{uCi} / \mathrm{mL}$ & $P$ & Raw \\
\hline Carbon-14 & $6 / 1 / 90$ & $4 / 30 / 89$ & $4 / 27 / 89$ & Вов2М3 & G311 & G359 & 1.20 & $5.20 E-04$ & $\mathrm{uCl} / \mathrm{mL}$ & $P$ & Raw \\
\hline
\end{tabular}




\begin{tabular}{|c|c|c|c|c|c|c|}
\hline \multicolumn{4}{|c|}{ Radionuctide Information } & \multirow[b]{2}{*}{$\begin{array}{l}\text { Reference } \\
\text { Number }\end{array}$} & \multirow[b]{2}{*}{ Validated (Y/N) } & \multirow[b]{2}{*}{ Notes } \\
\hline Radoinuclide & Conc. (Bq/L) & $\begin{array}{c}\text { Conc. }(\mathrm{Bq} / \mathrm{L} \\
\text { decayed) }\end{array}$ & $\begin{array}{l}\text { Conc. (Ci/L } \\
\text { Decayed) }\end{array}$ & & & \\
\hline Am241 & $8.36 E+03$ & $8.34 E+03$ & $2.25 E-07$ & AP102-3 & $\boldsymbol{\gamma}$ & $\begin{array}{l}\text { Density reported for G348 collected at } 125 \text { in from } \\
\text { bottom of tank at a } 30^{\circ} \text { angle. Sample was } \\
\text { unheated, unheated samples precipitated }\end{array}$ \\
\hline Am241 & $7.81 E+03$ & $7.79 E+03$ & 2.10E-07 & AP102-3 & $\mathbf{Y}$ & $\begin{array}{l}\text { Density reported for } \mathrm{G} 363 \text { collected at } 18 \text { in from } \\
\text { bottom of tank at a } 150^{\circ} \text { angle. Sample was } \\
\text { unheated, unheated samples precipitated }\end{array}$ \\
\hline Am241 & $2.75 E+02$ & $2.72 E+02$ & 7.35E-09 & $\overline{N A}$ & N & No Reference \\
\hline Am241 & $2.75 E+02$ & $2.72 E+02$ & 7.35E-09 & AP102-1 & $\mathbf{N}$ & Outdated Information \\
\hline Am241 & $1.53 \mathrm{E}+02$ & $1.52 \mathrm{E}+02$ & 4.11E-09 & AP102-1 & $\mathbf{N}$ & Outdated Information \\
\hline Am241 & $3.81 E+01$ & $3.77 E+01$ & $1.02 \mathrm{E}-09$ & AP102-1 & $\mathbf{N}$ & Outdated Information \\
\hline Am247 & $3.37 E+01$ & $3.34 E+01$ & $9.03 E-10$ & AP102-1 & $\bar{N}$ & Outdated Information \\
\hline Am241 & $2.90 E+01$ & $2.87 E+01$ & $7.76 \mathrm{E}-10$ & AP102-1 & $N$ & Outdated Information \\
\hline Am241 & $3.81 E+00$ & $3.77 \varepsilon+\infty$ & $7.02 E-10$ & AP102-1 & $\mathbf{N}$ & Outdated Information \\
\hline C14 & $2.35 \mathrm{E}+05$ & $2.35 \mathrm{E}+05$ & $6.34 E-06$ & $\overline{N A}$ & $\bar{N}$ & No Reference \\
\hline $\mathrm{C} 74$ & $2.73 E+04$ & $2.73 E+04$ & $7.38 E-07$ & AP102-3 & $\mathbf{Y}$ & $\begin{array}{l}\text { Density reported for } \mathrm{G} 352 \text { collected at } 34 \text { in from } \\
\text { bottom of tank at a } 30^{\circ} \text { angle. Sample was } \\
\text { unheated, unheated samples precipitated }\end{array}$ \\
\hline C14 & $2.65 E+04$ & $2.65 E+04$ & 7.17E-07 & AP102-3 & $\mathbf{Y}$ & $\begin{array}{l}\text { Density reported for } 6453 \text { collected at } 226 \text { in from } \\
\text { bottom of tank at a } 270^{\circ} \text { angle. Sample was } \\
\text { unheated, unheated samples precipitated }\end{array}$ \\
\hline $\mathrm{C} 14$ & $2.08 E+04$ & $2.08 E+04$ & $5.61 E-07$ & AP102-3 & $\mathbf{Y}$ & $\begin{array}{l}\text { Density reported for } \mathrm{G} 459 \text { collected at } 226 \text { in from } \\
\text { bottom of tank at a } 270^{\circ} \text { angle. Sample was } \\
\text { unheated, unheated samples precipitated }\end{array}$ \\
\hline $\mathrm{Cl4}$ & $2.08 E+04$ & $2.08 E+04$ & 5.61E-07 & AP102-3 & $\boldsymbol{\gamma}$ & $\begin{array}{l}\text { Density reported for } \mathrm{G341} \text { collected at } 99 \text { in from } \\
\text { bottom of tank at a } 270^{\circ} \text { angle. }\end{array}$ \\
\hline $\mathrm{C} 14$ & $2.05 E+04$ & $2.05 E+04$ & 5.55E-07 & AP102-3 & $\mathbf{Y}$ & $\begin{array}{l}\text { Density reported for } 6358 \text { collected at } 334 \text { in from } \\
\text { bottom of tank at a } 150^{\circ} \text { angle. Sample was } \\
\text { unheated, unheated samples precipitated }\end{array}$ \\
\hline C14 & $2.01 E+04$ & $2.01 E+04$ & 5.44E-07 & AP102-3 & $\mathbf{Y}$ & $\begin{array}{l}\text { Density reported for G364 collected at } 372 \text { in from } \\
\text { bottom of tank at a } 270^{\circ} \text { angle. Sample was } \\
\text { unheated, unheated samples precipitated }\end{array}$ \\
\hline $\mathrm{C} 14$ & $1.99 E+04$ & $1.99 E+04$ & $5.39 E-07$ & AP102-3 & $\mathbf{Y}$ & $\begin{array}{l}\text { Density reported for } 6353 \text { collected at } 334 \text { in from } \\
\text { bottom of tank at a } 150^{\circ} \text { angle. Sample was } \\
\text { unheated, unheated samples precipitated }\end{array}$ \\
\hline $\mathrm{C} 14$ & $1.99 E+04$ & $1.99 E+04$ & $5.39 E-07$ & AP102-3 & $\mathbf{Y}$ & $\begin{array}{l}\text { Density reported for } 6476 \text { collected at } 99 \text { in from } \\
\text { bottom of tank at a } 270^{\circ} \text { angle and was keep heated } \\
\text { to } 40^{\circ} \mathrm{C} \text {. }\end{array}$ \\
\hline C14 & $1.98 E+04$ & $1.98 E+04$ & $5.36 \mathrm{E}-07$ & AP102-3 & $\mathbf{Y}$ & $\begin{array}{l}\text { Density reported for } 6476 \text { collected at } 99 \text { in from } \\
\text { bottom of tank at a } 270^{\circ} \text { angle and was keep heated } \\
\text { to } 40^{\circ} \mathrm{C} \text {. }\end{array}$ \\
\hline C14 & $1.93 E+04$ & $1.93 E+04$ & $5.22 E-07$ & AP102-3 & $\mathbf{Y}$ & $\begin{array}{l}\text { Density reported for } \mathrm{G} 363 \text { collected at } 18 \text { in from } \\
\text { bottom of tank at a } 150^{\circ} \text { angle. Sample was } \\
\text { unheated, unheated samples precipitated }\end{array}$ \\
\hline C14 & $1.93 E+04$ & $1.93 E+04$ & $5.21 E-07$ & AP102-3 & $\mathbf{\gamma}$ & $\begin{array}{l}\text { Density reported for } G 352 \text { collected at } 34 \text { in from } \\
\text { bottom of tank at a } 30^{\circ} \text { angle. Sample was } \\
\text { unheated, unheated samples precipitated }\end{array}$ \\
\hline C14 & $1.93 E+04$ & $1.93 E+04$ & $5.21 E-07$ & AP102-3 & $\mathbf{Y}$ & $\begin{array}{l}\text { Density reported for } 6471 \text { collected at } 208 \text { in from } \\
\text { bottom of tank at a } 150^{\circ} \text { angle and was keep heated } \\
\text { to } 40^{\circ} \mathrm{C} \text {. }\end{array}$ \\
\hline $\mathrm{C} 14$ & $1.92 E+04$ & $1.92 E \div 04$ & $5.20 \mathrm{E}-07$ & AP102-3 & $\mathbf{Y}$ & $\begin{array}{l}\text { Density reported for } 6359 \text { collected at } 162 \text { in from } \\
\text { bottom of tank at a } 150^{\circ} \text { angle. Sample was } \\
\text { unheated, unheated samples precipitated }\end{array}$ \\
\hline C14 & $1.92 E+04$ & $1.92 E+0.4$ & $5.20 E-07$ & AP102-3 & $Y$ & $\begin{array}{l}\text { Density reported for } \mathrm{G} 359 \text { collected at } 162 \text { in from } \\
\text { bottom of tank at a } 150^{\circ} \text { angle. Sample was } \\
\text { unheated, unheated samples precipitated }\end{array}$ \\
\hline
\end{tabular}




\begin{tabular}{|c|c|c|c|c|c|c|c|c|c|c|c|}
\hline & & & & Tank Wast & Characteriza & ion Sample In & mation & & & & \\
\hline $\begin{array}{l}\text { Reported } \\
\text { Radionuclide }\end{array}$ & $\begin{array}{c}\text { Memo } \\
\text { Date }\end{array}$ & $\begin{array}{c}\text { Start } \\
\text { Analysis } \\
\text { Date }\end{array}$ & $\begin{array}{c}\text { Start } \\
\text { Sample } \\
\text { Date }\end{array}$ & $\begin{array}{l}\text { Sample } \\
\text { Number }\end{array}$ & $\begin{array}{c}\text { Tank Farm } \\
\text { Number }\end{array}$ & $\begin{array}{c}\text { Lab Sample } \\
\text { Number }\end{array}$ & $\begin{array}{c}\text { Density } \\
(\mathrm{g} / \mathrm{mL})\end{array}$ & $\begin{array}{c}\text { Reported } \\
\text { Value } \\
\end{array}$ & Reported Unit & Code & Source \\
\hline Carbon-14 & $6 / 1 / 90$ & $4 / 30 / 89$ & $4 / 27 / 89$ & BOB2L8 & G303 & G348 & 1.21 & $5.18 E-04$ & uCi $/ \mathrm{mL}$ & $\mathbf{P}$ & Raw \\
\hline Carbon-14 & $6 / 1 / 90$ & $4 / 30 / 89$ & $4 / 27 / 89$ & 80B2L8 & G303 & G348 & 1.21 & $5.18 E-04$ & $\mathrm{uCi} / \mathrm{mL}$ & $P$ & Raw \\
\hline Carbon-14 & $6 / 1 / 90$ & $4 / 30 / 89$ & $4 / 27 / 89$ & BOB2T9 & G327 & G470" . & 1.19 & $5.16 E-04$ & $\mathrm{uCi} / \mathrm{mL}$ & $\mathbf{P}$ & Raw \\
\hline Carbon-14 & $6 / 1 / 90$ & $4 / 30 / 89$ & $4 / 27 / 89$ & BOB2L7 & G299 & G342 & 1.20 & $5.14 E-04$ & $\mathrm{uCi} / \mathrm{mL}$ & $P$ & Raw \\
\hline Carbon-14 & $6 / 1 / 90$ & $4 / 30 / 89$ & $4 / 27 / 89$ & BOB2L9 & G305 & G349 & 1.20 & $5.12 E-04$ & $\mathrm{uCi} / \mathrm{mL}$ & $\mathbf{P}$ & Raw \\
\hline Carbon-14 & $6 / 1 / 90$ & $4 / 30 / 89$ & $4 / 27 / 89$ & ВОВ2MO & G306 & G352 & 1.20 & $5.07 E-04$ & uCi/mL & $\mathbf{P}$ & Raw \\
\hline Carbon-14 & $6 / 1 / 90$ & $4 / 30 / 89$ & $4 / 27 / 89$ & ВОВ2M9 & $\mathrm{G} 302$ & G477* & 1.20 & $5.05 E-04$ & $\mathrm{uCi} / \mathrm{mL}$ & $\mathbf{P}$ & Raw \\
\hline Carbon-14 & $6 / 1 / 90$ & $4 / 30 / 89$ & $4 / 27 / 89$ & ВОВ2M2 & G309 & G358 & 1.20 & $5.04 E-04$ & $\mathrm{uCi} / \mathrm{mL}$ & $\mathbf{P}$ & Raw \\
\hline Carbon-14 & $6 / 1 / 90$ & $4 / 30 / 89$ & $4 / 27 / 89$ & BOB2M7 & G319 & G460 & 1.20 & $5.03 E-04$ & $\mathrm{uCt} / \mathrm{mL}$ & $P$ & Raw \\
\hline Carbon-14 & $6 / 1 / 90$ & $4 / 30 / 89$ & $4 / 27 / 89$ & B082M5 & G315 & G364 & 1.19 & $5.01 E-04$ & $\mathrm{uCi} / \mathrm{mL}$ & $\mathbf{P}$ & Raw \\
\hline Carbon-14 & $6 / 1 / 90$ & $4 / 30 / 89$ & $4 / 27 / 89$ & BOB2L9 & G305 & G349 & 1.20 & $4.99 E-04$ & $\mathrm{uCi} / \mathrm{mL}$ & $P$ & Raw \\
\hline Carbon-14 & $6 / 1 / 90$ & $4 / 30 / 89$ & $4 / 27 / 89$ & BOB2T9 & G327 & G470* & 1.19 & $4.98 E-04$ & $\mathrm{uCi} / \mathrm{mL}$ & $P$ & Raw \\
\hline Carbon-14 & $6 / 1 / 90$ & $4 / 30 / 89$ & $4 / 27 / 89$ & BOB2N1 & G310 & G471 • & 1.20 & 4.91E-04 & $\mathrm{uCi} / \mathrm{mL}$ & $p$ & Raw \\
\hline - Carbon-14 & $6 / 1 / 90$ & $4 / 30 / 89$ & $4 / 27 / 89$ & В082M4 & G313 & G363 & 1.20 & $4.91 E-04$ & uCi/mL & $\mathbf{P}$ & Raw \\
\hline Carbon-14 & $6 / 1 / 90$ & $4 / 30 / 89$ & $4 / 27 / 89$ & BOB2N1 & G310 & G471 & 1.20 & $4.88 \mathrm{E}-04$ & $\mathrm{uCi} / \mathrm{mL}$ & $\mathbf{P}$ & Raw \\
\hline Carbon-14 & $6 / 1 / 90$ & $4 / 30 / 89$ & $4 / 27 / 89$ & ВОВ2МО & G306 & G352 & 1.20 & 4.83E-04 & $\mathrm{uCi} / \mathrm{mL}$ & $P$ & Raw \\
\hline Carbon-14 & $6 / 1 / 90$ & $4 / 30 / 89$ & $4 / 27 / 89$ & Вов2M4 & G313 & G363 & 1.20 & $4.81 E-04$ & $\mathrm{uCi} / \mathrm{mL}$ & $\mathbf{P}$ & Raw \\
\hline Carbon-14 & $6 / 1 / 90$ & $4 / 30 / 89$ & $4 / 27 / 89$ & BOB2MO & G306 & G352 & 1.20 & $4.80 E-04$ & $\mathrm{uCi} / \mathrm{mL}$ & $\mathbf{P}$ & Raw \\
\hline Carbon-14 & $6 / 1 / 90$ & $4 / 30 / 89$ & $4 / 27 / 89$ & BOB2M2 & G309 & G358 & 1.20 & 4.77E-04 & $\mathrm{uCl} / \mathrm{mL}$ & $\mathbf{p}$ & Raw \\
\hline
\end{tabular}




\begin{tabular}{|c|c|c|c|c|c|c|}
\hline \multicolumn{4}{|c|}{ Radionuclide Information } & & \multirow[b]{2}{*}{ Validated (Y/N) } & $\dot{-}$ \\
\hline Radoinucilde & Conc. (Bq/L) & $\begin{array}{c}\text { Conc. }(B q / L \\
\text { decayed) }\end{array}$ & $\begin{array}{c}\text { Conc. (Cin } \\
\text { Decayed) }\end{array}$ & $\begin{array}{c}\text { Reference } \\
\text { Number }\end{array}$ & & $\begin{array}{llll}- & - & - \\
\end{array}$ \\
\hline C14 & $1.92 E+04$ & $1.92 E+04$ & 5.18E-07 & AP102-3 & $\mathbf{Y}$ & $\begin{array}{l}\text { Density reported for G348 collected at } 125 \text { in from } \\
\text { bottom of tank at a } 30^{\circ} \text { angle. Sample was } \\
\text { unheated, unheated samples precipitated }\end{array}$ \\
\hline $\mathrm{C} 14$ & $1.92 E \div 04$ & $1.92 E+04$ & 5.18E-07 & AP102-3 & $\mathbf{Y}$ & $\begin{array}{l}\text { Density reported for G348 collected at } 125 \text { in from } \\
\text { bottom of tank at a } 30^{\circ} \text { angle. Sample was } \\
\text { unheated, unheated samples precipitated }\end{array}$ \\
\hline C14. & $1.91 E+04$ & $1.91 E+04$ & $5.16 E-07$ & AP102-3 & $\mathbf{Y}$ & $\begin{array}{l}\text { Density reported for } \mathrm{G} 470 \text { represents a composite } \\
\text { sample. Sample was kept heated to } 40^{\circ} \mathrm{C} \text {. }\end{array}$ \\
\hline $\mathrm{C} 14$ & $1.90 E \div 04$ & $1.90 E+04$ & 5.14E-07 & AP102-3 & $\mathbf{Y}$ & $\begin{array}{l}\text { Density reported for } \mathrm{G} 342 \text { collected at } 306 \text { in from } \\
\text { bottom of tank at a } 30^{\circ} \text { angle. Sample was } \\
\text { unheated, unheated samples precipitated }\end{array}$ \\
\hline $\mathrm{C14}$ & $1.89 E+04$ & $1.89 E+04$ & 5.12E-07 & AP.102-3 & $\mathbf{Y}$ & $\begin{array}{l}\text { Density reported for } G 349 \text { collected at } 34 \text { in from } \\
\text { bottom of tank at a } 30^{\circ} \text { angle. Sample was } \\
\text { unheated, unheated samples precipitated }\end{array}$ \\
\hline C14 & $1.88 E+04$ & $1.88 E+04$ & 5.07E-07 & AP102-3 & $\mathbf{Y}$ & $\begin{array}{l}\text { Density reported for } \mathrm{G} 352 \text { collected at } 34 \text { in from } \\
\text { bottom of tank at a } 30^{\circ} \text { angle. Sample was } \\
\text { unheated, unheated samples precipitated }\end{array}$ \\
\hline $\mathrm{C} 14$ & $1.87 E+04$ & $1.87 E+04$ & 5.05E-07 & AP102-3 & $\gamma$ & $\begin{array}{l}\text { Density reported for } \mathrm{G} 477 \text { collected at } 182 \text { in from } \\
\text { bottom of tank at a } 30^{\circ} \text { angle and was keep heated } \\
\text { to } 40^{\circ} \mathrm{C} \text {. }\end{array}$ \\
\hline $\mathrm{C} 14$ & $1.86 E+04$ & $1.86 E+04$ & 5.04E-07 & AP102-3 & $\mathbf{Y}$ & $\begin{array}{l}\text { Density reported for } \mathrm{G} 358 \text { collected at } 334 \text { in from } \\
\text { bottom of tank at a } 150^{\circ} \text { angle. Sample was } \\
\text { unheated, unheated samples precipitated }\end{array}$ \\
\hline $\mathrm{C} 14$ & $1.86 E+04$ & $1.86 E+04$ & $5.03 E-07$ & AP102-3 & $\mathbf{Y}$ & $\begin{array}{l}\text { Density reported for } 6460 \text { collected at } 226 \text { in from } \\
\text { bottom of tank at a } 270^{\circ} \text { angle. Sample was } \\
\text { unheated, unheated samples precipitated }\end{array}$ \\
\hline C14 & $1.85 E+04$ & $1.85 E+04$ & $5.01 E-07$ & AP102-3 & $\mathbf{Y}$ & $\begin{array}{l}\text { Density reported for } \mathrm{G} 364 \text { collected at } 372 \text { in from } \\
\text { bottom of tank at a } 270^{\circ} \text { angle. Sample was } \\
\text { unheated, unheated samples precipitated }\end{array}$ \\
\hline C14 & $1.85 E+04$ & $1.85 E+04$ & 4.99E-07 & AP102-3 & $\mathbf{Y}$ & $\begin{array}{l}\text { Density reported for } \mathrm{G} 349 \text { collected at } 34 \text { in from } \\
\text { bottom of tank at a } 30^{\circ} \text { angle. Sample was } \\
\text { unheated, unheated samples precipitated }\end{array}$ \\
\hline $\mathrm{C14}$ & $1.84 E+04$ & $1.84 E+04$ & $4.98 E-07$ & AP102-3 & $Y$ & $\begin{array}{l}\text { Density reported for } \mathrm{G} 470 \text { represents a composite } \\
\text { sample. Sample was kept heated to } 40^{\circ} \mathrm{C} \text {. }\end{array}$ \\
\hline C14 & $1.82 E+04$ & $1.82 E+04$ & 4.91E-07 & AP102-3 & $Y$ & $\begin{array}{l}\text { Density reported for } 6471 \text { collected at } 208 \text { in from } \\
\text { bottom of tank at a } 150^{\circ} \text { angle and was keep heated } \\
\text { to } 40^{\circ} \mathrm{C} \text {. }\end{array}$ \\
\hline $\mathrm{C} 14$ & $1.82 E+04$ & $1.82 E+04$ & 4.91E-07 & AP102-3 & $\mathbf{Y}$ & $\begin{array}{l}\text { Density reported for } \mathrm{G} 363 \text { collected at } 18 \text { in from } \\
\text { bottom of tank at a } 150^{\circ} \text { angle. Sample was } \\
\text { unheated, unheated samples precipitated }\end{array}$ \\
\hline C14 & $1.81 E+04$ & $1.81 E+04$ & 4.88E-07 & AP102-3 & $\mathbf{Y}$ & $\begin{array}{l}\text { Density reported for } \mathrm{G} 471 \text { collected at } 208 \text { in from } \\
\text { bottom of tank at a } 150^{\circ} \text { angle and was keep heated } \\
\text { to } 40^{\circ} \mathrm{C} \text {. }\end{array}$ \\
\hline C14 & $1.79 E+04$ & $1.79 E+04$ & $4.83 E-07$ & AP102-3 & $\gamma$ & $\begin{array}{l}\text { Density reported for } \mathrm{G} 352 \text { collected at } 34 \text { in from } \\
\text { bottom of tank at a } 30^{\circ} \text { angle. Sample was } \\
\text { unheated, unheated samples precipitated }\end{array}$ \\
\hline $\mathrm{Cl} 4$ & $1.78 E+04$ & $1.78 E+04$ & 4.81E-07 & AP102-3 & $\mathbf{Y}$ & $\begin{array}{l}\text { Density reported for G363 collected at } 18 \text { in from } \\
\text { bottom of tank at a } 150^{\circ} \text { angle. Sample was } \\
\text { unheated, unheated samples precipitated }\end{array}$ \\
\hline C14 & $1.78 E+04$ & $1.78 E+0.4$ & $4.80 E-07$ & AP102-3 & $Y$ & $\begin{array}{l}\text { Density reported for } \mathrm{G} 352 \text { collected at } 34 \text { in from } \\
\text { bottom of tank at a } 30^{\circ} \text { angle. Sample was } \\
\text { unheated, unheated samples precipitated }\end{array}$ \\
\hline C14 & $1.76 E+04$ & $1.76 E+04$ & 4.77E-07 & AP102-3 & $\mathbf{Y}$ & $\begin{array}{l}\text { Density reported for } \mathrm{G} 358 \text { collected at } 334 \text { in from } \\
\text { bottom of tank at a } 150^{\circ} \text { angle. Sample was } \\
\text { unheated, unheated samples precipitated }\end{array}$ \\
\hline
\end{tabular}




\begin{tabular}{|c|c|c|c|c|c|c|c|c|c|c|c|}
\hline \multicolumn{12}{|c|}{ Tank Waste Characterization Sample Information } \\
\hline $\begin{array}{c}\text { Reported } \\
\text { Radionucilde }\end{array}$ & $\begin{array}{c}\text { Memo } \\
\text { Date }\end{array}$ & $\begin{array}{c}\text { Start } \\
\text { Analysis } \\
\text { Date }\end{array}$ & $\begin{array}{c}\text { Start } \\
\text { Sample } \\
\text { Date } \\
\end{array}$ & $\begin{array}{l}\text { Sample } \\
\text { Number }\end{array}$ & $\begin{array}{c}\text { Tank Farm } \\
\text { Number }\end{array}$ & $\begin{array}{c}\text { Lab Sample } \\
\text { Number }\end{array}$ & $\begin{array}{l}\text { Density } \\
(\mathrm{g} / \mathrm{mL})\end{array}$ & $\begin{array}{c}\text { Reported } \\
\text { Value }\end{array}$ & Reported Unit & Code & Source \\
\hline Carbon-14 & $6 / 1 / 90$ & $4 / 30 / 89$ & $4 / 27 / 89$ & BOB2T9 & G327 & G470* & 1.19 & 4.75E-04 & $\mathrm{uCi} / \mathrm{mL}$ & $\mathbf{P}$ & Raw \\
\hline Carbon-14 & $6 / 1 / 90$ & $4 / 30 / 89$ & $4 / 27 / 89$ & Во82M1 & G308 & G353 & 1.20 & $4.74 E-04$ & $\mathrm{uCi} / \mathrm{mL}$ & $\mathbf{P}$ & Raw \\
\hline Carbon-14 & $6 / 1 / 90$ & $4 / 30 / 89$ & $4 / 27 / 89$ & BOB2MG & G318 & G459 & 1.20 & $4.72 E-04$ & $\mathrm{uCi} / \mathrm{mL}$ & $\mathbf{P}$ & Raw \\
\hline Carbon-14 & $6 / 1 / 90$ & $4 / 30 / 89$ & $4 / 27 / 89$ & BOB2M9 & G302 & G477" & 1.20 & 4.71E-04 & $u \mathrm{Ci} / \mathrm{mL}$ & $\mathbf{P}$ & Raw \\
\hline Carbon-14 & $6 / 1 / 90$ & $4 / 30 / 89$ & $4 / 27 / 89$ & BOB2N4 & G323 & G341 & 1.20 & 4.71E-04 & $\mathrm{uCi} / \mathrm{mL}$ & $\mathbf{P}$ & Raw \\
\hline Carbon-14 & $6 / 1 / 90$ & $4 / 30 / 89$ & $4 / 27 / 89$ & BOB2M1 & G308 & G353 & 1.20 & 4.59E-04 & $\mathrm{uCi} / \mathrm{mL}$ & $\mathbf{P}$ & Raw \\
\hline Carbon-14 & $6 / 1 / 90$ & $4 / 30 / 89$ & $4 / 27 / 89$ & Вов2M7 & G319 & G460 & 1.20 & 4.59E-04 & uCi/mL & $P$ & Raw \\
\hline Carbon-14 & $6 / 1 / 90$ & $4 / 30 / 89$ & $4 / 27 / 89$ & Вов2M9 & G302 & G477* & 1.20 & 4.44E-04 & $\mathrm{uCi} / \mathrm{mL}$ & $P$ & Raw \\
\hline Carbon-14 & $6 / 1 / 90$ & $4 / 30 / 89$ & $4 / 27 / 89$ & ВОВ2MI & G308 & G353 & 1.20 & 4.37E-04 & $u \mathrm{Ci} / \mathrm{mL}$ & $P$ & Raw \\
\hline Carbon-14 & $6 / 1 / 90$ & $4 / 30 / 89$ & $4 / 27 / 89$ & BOB2L7 & G299 & G342 & 1.20 & 4.31E-04 & $\mathrm{u} \mathrm{Ci} / \mathrm{mL}$ & $P$ & Raw \\
\hline Carbon-14 & $6 / 1 / 90$ & $4 / 30 / 89$ & $4 / 27 / 89$ & BOB2M2 & G309 & G358 & 1.20 & $4.28 E-04$ & $\mathrm{uCi} / \mathrm{mL}$ & $P$ & Raw \\
\hline Carbon-14 & $6 / 1 / 90$ & $4 / 30 / 89$ & . 4/27/89 & BOB2M4 & G313 & G363 & 1.20 & 4.17E-04 & $\mathrm{uCi} / \mathrm{mL}$ & $P$ & Raw \\
\hline Carbon-14 & $6 / 1 / 90$ & $4 / 30 / 89$ & $4 / 27 / 89$ & BOB2N1 & G310 & G471 • & 1.20 & 4.16E-04 & $\mathrm{uCi} / \mathrm{mL}$ & $P$ & Raw \\
\hline Carbon-14 & $6 / 1 / 90$ & $4 / 30 / 89$ & $4 / 27 / 89$ & ВОВ2M9 & G302 & G477* & 1.20 & $4.08 \mathrm{E}-04$ & $u \mathrm{Ci} / \mathrm{mL}$ & $P$ & Raw \\
\hline Carbon-14 & $6 / 1 / 90$ & $4 / 30 / 89$ & $4 / 27 / 89$ & BOB2M6 & G318 & G459 & 1.20 & $4.02 E-04$ & $\mathrm{uCi} / \mathrm{mL}$ & $\mathbf{P}$ & Raw \\
\hline Carbon-14 & $6 / 1 / 90$ & $4 / 30 / 89$ & $4 / 27 / 89$ & BOB2T9 & G327 & G470* & 1.19 & $3.93 E-04$ & $\mathrm{uCi} / \mathrm{mL}$ & $P$ & Raw \\
\hline Carbon-14 & $6 / 1 / 90$ & $4 / 30 / 89$ & $4 / 27 / 89$ & ВOB2MO & G306 & G352 & 1.20 & $3.90 E-04$ & $\mathrm{uCi} / \mathrm{mL}$ & $P$ & Raw \\
\hline Carbon-14 & $6 / 1 / 90$ & $4 / 30 / 89$ & $4 / 27 / 89$ & В082M4 & G313 & G363 & 1.20 & 3.83E-04 & $\mathrm{uCi} / \mathrm{mL}$ & P & Raw \\
\hline Carbon-14 & $6 / 1 / 90$ & $4 / 30 / 89$ & $4 / 27 / 89$ & BOB2M4 & G313 & G363 & 1.20 & $3.14 \mathrm{E}-04$ & $\mathrm{uCi} / \mathrm{mL}$ & $P$ & Raw \\
\hline $\mathrm{Cm} 244$ & $4 / 30 / 84$ & NS & NS & NS & NS & NS & NS & NS & NS & $P$ & Wastren \\
\hline
\end{tabular}




\begin{tabular}{|c|c|c|c|c|c|c|}
\hline \multicolumn{4}{|c|}{ Radionuclide Information } & \multirow[b]{2}{*}{$\begin{array}{l}\text { Reference } \\
\text { Number }\end{array}$} & \multirow[b]{2}{*}{ Validated (Y/N) } & \multirow[b]{2}{*}{ Notes } \\
\hline Radoinuclide & Conc. (Bq/L) & $\begin{array}{c}\text { Conc. }(B q / L \\
\text { decayed) }\end{array}$ & $\begin{array}{l}\text { Conc. (Cill } \\
\text { Decayed) }\end{array}$ & & & \\
\hline C14 & $1.76 E+04$ & $1.76 E+04$ & $4.75 E-07$ & AP102-3 & $\mathbf{Y}$ & $\begin{array}{l}\text { Density reported for } \mathrm{G} 470 \text { represents a composite } \\
\text { sample. Sample was kept heated to } 40^{\circ} \mathrm{C} \text {. }\end{array}$ \\
\hline $\mathrm{C14}$ & $1.75 E+04$ & $1.75 E+04$ & $4.74 E-07$ & AP102-3 & $\mathbf{Y}$ & $\begin{array}{l}\text { Density reported for } \mathrm{G} 353 \text { collected at } 334 \text { in from } \\
\text { bottom of tank at a } 150^{\circ} \text { angle. Sample was } \\
\text { unheated, unheated samples precipitated }\end{array}$ \\
\hline C14 & $1.75 E+04$ & $1.75 E \div 04$ & $4.72 E-07$ & AP102-3 & $\gamma$ & $\begin{array}{l}\text { Density reported for } 6459 \text { collected at } 226 \text { in from } \\
\text { bottom of tank at a } 270^{\circ} \text { angle. Sample was } \\
\text { unheated, unheated samples precipitated }\end{array}$ \\
\hline $\mathrm{Cl}_{4}$ & $1.74 E+04$ & $1.74 E+04$ & 4.71E-07 & AP102-3 & $Y$ & $\begin{array}{l}\text { Density reported for } \mathrm{G} 477 \text { collected at } 182 \text { in from } \\
\text { bottom of tank at a } 30^{\circ} \text { angle and was keep heated } \\
\text { to } 40^{\circ} \mathrm{C} \text {. }\end{array}$ \\
\hline $\mathrm{C} 14$ & $1.74 E+04$ & $1.74 E+04$ & 4.71E-07 & AP102-3 & $\mathbf{Y}$ & $\begin{array}{l}\text { Density reported for } \mathrm{G} 341 \text { collected at } 99 \text { in from } \\
\text { bottom of tank at a } 270^{\circ} \text { angle. }\end{array}$ \\
\hline $\mathrm{C} 14$ & $1.70 E+04$ & $1.70 E+04$ & 4.59E-07 & AP102-3 & $\mathbf{Y}$ & $\begin{array}{l}\text { Density reported for } \mathrm{G} 353 \text { collected at } 334 \text { in from } \\
\text { bottom of tank at a } 150^{\circ} \text { angle. Sample was } \\
\text { unheated, unheated samples precipitated }\end{array}$ \\
\hline $\mathrm{C} 14$ & $1.70 E+04$ & $1.70 E+04$ & $4.59 E-07$ & AP102-3 & $\mathbf{Y}$ & $\begin{array}{l}\text { Density reported for } \mathrm{G} 460 \text { collected at } 226 \text { in from } \\
\text { bottom of tank at a } 270^{\circ} \text { angle. Sample was } \\
\text { unheated, unheated samples precipitated }\end{array}$ \\
\hline C14 & $1.64 E+04$ & $1.64 E+04$ & 4.44E-07 & AP102-3 & $Y$ & $\begin{array}{l}\text { Density reported for } \mathrm{G} 477 \text { collected at } 182 \text { in from } \\
\text { bottom of tank at a } 30^{\circ} \text { angle and was keep heated } \\
\text { to } 40^{\circ} \mathrm{C} \text {. }\end{array}$ \\
\hline $\mathrm{C} 14$ & $1.62 E+04$ & $1.62 E+04$ & 4.37E-07 & AP102-3 & $\boldsymbol{Y}$ & $\begin{array}{l}\text { Density reported for } \mathrm{G} 353 \text { collected at } 334 \text { in from } \\
\text { bottom of tank at a } 150^{\circ} \text { angle. Sample was } \\
\text { unheated, unheated samples precipitated }\end{array}$ \\
\hline C14 & $1.59 E+04$ & $1.59 E+04$ & $4.31 E-07$ & AP102-3 & $\boldsymbol{\gamma}$ & $\begin{array}{l}\text { Density reported for } G 342 \text { collected at } 306 \text { in from } \\
\text { bottom of tank at a } 30^{\circ} \text { angle. Sample was } \\
\text { unheated, unheated samples precipitated }\end{array}$ \\
\hline C14 & $1.58 E+04$ & $1.58 E+04$ & $4.28 E-07$ & AP102-3 & $\mathbf{Y}$ & $\begin{array}{l}\text { Density reported for } G 358 \text { collected at } 334 \text { in from } \\
\text { bottom of tank at a } 150^{\circ} \text { angle. Sample was } \\
\text { unheated, unheated samples precipitated }\end{array}$ \\
\hline C14 & $1.54 E+04$ & $1.54 E+04$ & 4.17E-OD & AP102-3 & $\mathbf{Y}$ & $\begin{array}{l}\text { Density reported for } G 363 \text { collected at } 18 \text { in from } \\
\text { bottom of tank at a } 150^{\circ} \text { angle. Sample was } \\
\text { unheated, unheated samples precipitated }\end{array}$ \\
\hline C14 & $1.54 E+04$ & $1.54 E+04$ & 4.16E-07 & AP102-3 & $\mathbf{Y}$ & $\begin{array}{l}\text { Density reported for } \mathrm{G} 471 \text { collected at } 208 \text { in from } \\
\text { bottom of tank at a } 150^{\circ} \text { angle and was keep heated } \\
\text { to } 40^{\circ} \mathrm{C} \text {. }\end{array}$ \\
\hline C14 & $1.51 E+04$ & $1.51 E+04$ & $4.08 E-07$ & AP102-3 & $\boldsymbol{\gamma}$ & $\begin{array}{l}\text { Density reported for } \mathrm{G} 477 \text { collected at } 182 \text { in from } \\
\text { bottom of tank at a } 30^{\circ} \text { angle and was keep heated } \\
\text { to } 40^{\circ} \mathrm{C} \text {. }\end{array}$ \\
\hline $\mathrm{C14}$ & $1.49 E+04$ & $1.49 E+04$ & $4.02 E-07$ & AP102-3 & $Y$ & $\begin{array}{l}\text { Density reported for } 6459 \text { collected at } 226 \text { in from } \\
\text { bottom of tank at a } 270^{\circ} \text { angle. Sample was } \\
\text { unheated, unheated samples precipitated }\end{array}$ \\
\hline C14 & $1.45 E+04$ & $1.45 E+04$ & 3.93E-07 & AP102-3 & $\mathbf{Y}$ & $\begin{array}{l}\text { Density reported for G470 represents a composite } \\
\text { sample. Sample was kept heated to } 40^{\circ} \mathrm{C} \text {. }\end{array}$ \\
\hline C14 & $1.44 E+04$ & $1.44 E+04$ & $3.90 E-07$ & AP102-3 & $Y$ & $\begin{array}{l}\text { Density reported for G352 collected at } 34 \text { in from } \\
\text { bottom of tank at a } 30^{\circ} \text { angle. Sample was } \\
\text { unheated, unheated samples precipitated }\end{array}$ \\
\hline $\mathrm{C} 14$ & $1.42 E+04$ & $1.42 E+04$ & $3.83 E-07$ & AP102-3 & $\gamma$ & $\begin{array}{l}\text { Density reported for } \mathrm{G} 363 \text { collected at } 18 \text { in from } \\
\text { bottom of tank at a } 150^{\circ} \text { angle. Sample was } \\
\text { unheated, unheated samples precipitated }\end{array}$ \\
\hline C14 & $1.16 \mathrm{E}+04$ & $1.16 E+04$ & $3.14 E-07$ & AP102-3 & $\mathrm{Y}$ & $\begin{array}{l}\text { Density reported for } G 363 \text { collected at } 18 \text { in from } \\
\text { bottom of tank at a } 150^{\circ} \text { angle. Sample was } \\
\text { unheated, unheated samples precipitated }\end{array}$ \\
\hline $\mathrm{Cm} 243 / 244$ & $1.35 \mathrm{E}+03$ & $1.05 E+03$ & $2.84 E-08$ & NA & $\bar{N}$ & No Reference \\
\hline
\end{tabular}




\begin{tabular}{|c|c|c|c|c|c|c|c|c|c|c|c|}
\hline & 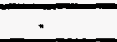 & & & Tank Was & Characteriza & on Sample Inf & mation & & & & \\
\hline $\begin{array}{c}\text { Reported } \\
\text { Radionuclide }\end{array}$ & $\begin{array}{c}\text { Memo } \\
\text { Date }\end{array}$ & $\begin{array}{c}\text { Start } \\
\text { Analysts } \\
\text { Date } \\
\end{array}$ & $\begin{array}{c}\text { Start } \\
\text { Sample } \\
\text { Date } \\
\end{array}$ & $\begin{array}{l}\text { Sample } \\
\text { Number }\end{array}$ & $\begin{array}{c}\text { Tank Farm } \\
\text { Number }\end{array}$ & $\begin{array}{c}\text { Lab Sample } \\
\text { Number }\end{array}$ & $\begin{array}{l}\text { Density } \\
\text { (g/mL) }\end{array}$ & $\begin{array}{c}\text { Reported } \\
\text { Value }\end{array}$ & Reported Unit & Code & Sourca \\
\hline $\mathrm{Cm} 243 / 244$ & $5 / 17 / 84$ & NS & NS & $R 8471$ & NS & NS & NS & $3.66 E-02$ & uCin & $P$ & RAW \\
\hline $\mathrm{Cm} 243 / 244$ & $5 / 17 / 84$ & NS & NS & R8472 & NS & NS & NS & $7.53 E-03$ & uCin & $\overline{\mathbf{P}}$ & $\overline{\text { RAW }}$ \\
\hline $\mathrm{Cm} 243 / 244$ & $5 / 17 / 84$ & NS & NS & R8473 & NS & NS & NS & $4.14 E-03$ & $\mathrm{uCi} / \mathrm{L}$ & $P$ & $\overline{\text { RAW }}$ \\
\hline $\mathrm{Cm} 243 / 244$ & $5 / 17 / 84$ & NS & NS & $\overline{R 8469}$ & NS & NS & NS & $3.27 E-03$ & uCi $/ \mathrm{h}$ & $P$ & RAW \\
\hline $\mathrm{Cm} 243 / 244$ & $5 / 17 / 84$ & NS & NS & R8469 & NS & NS & NS & $3.27 E-03$ & uCin & L & RAW \\
\hline Cm243/244 & $5 / 17 / 84$ & NS & NS & WI549 & NS & NS & NS & $1.10 E-03$ & $\mathrm{UCin}$ & $L$ & RAW \\
\hline $\mathrm{Cm} 244$ & $6 / 1 / 90$ & $4 / 30 / 89$ & $4 / 27 / 89$ & BOB2L7 & G299 & G342 & 1.20 & $<6.37 E-05$ & $u C i / m L$ & $\mathbf{P}$ & Raw \\
\hline $\mathrm{Cm} 244$ & $6 / 1 / 90$ & $4 / 30 / 89$ & $4 / 27 / 89$ & BOB2L7 & G299 & G342 & 1.20 & $<6.37 E-05$ & $\mathrm{uCi} / \mathrm{mL}$ & $\mathbf{P}$ & Raw \\
\hline $\mathrm{Cm} 244$ & $6 / 1 / 90$ & $4 / 30 / 89$ & $4 / 27 / 89$ & BOB2L8 & G303 & G348 & 1.21 & $<6.37 E-05$ & $\mathrm{uCi} / \mathrm{mL}$ & $\mathbf{P}$ & Raw \\
\hline $\mathrm{Cm} 244$ & $6 / 1 / 90$ & $4 / 30 / 89$ & $4 / 27 / 89$ & BOB2L.8 & G303 & G348 & 1.21 & $<6.37 E-05$ & $\mathrm{uCi} / \mathrm{mL}$ & $\mathbf{P}$ & Raw \\
\hline $\mathrm{Cm} 244$ & $6 / 1 / 90$ & $4 / 30 / 89$ & $4 / 27 / 89$ & BOB2M2 & G309 & G358 & 1.20 & $<6.37 E-05$ & $\mathrm{uCi} / \mathrm{mL}$ & $\mathbf{P}$ & Raw \\
\hline Cm244 & $6 / 1 / 90$ & $4 / 30 / 89$ & $4 / 27 / 89$ & ВОВ2M2 & G309 & G358 & 1.20 & $<6.37 E-05$ & $\mathrm{uCi} / \mathrm{mL}$ & $P$ & Raw \\
\hline $\mathrm{Cm} 244$ & $6 / 1 / 90$ & $4 / 30 / 89$ & $4 / 27 / 89$ & BOB2M1 & G308 & G353 & 1.20 & $<6.37 E-05$ & $u C i / m L$ & $P$ & Raw \\
\hline $\mathrm{Cm} 244$ & $6 / 1 / 90$ & $4 / 30 / 89$ & $4 / 27 / 89$ & BOB2M1 & G308 & G353 & 1.20 & $<6.37 E-05$ & $\mathrm{uCi} / \mathrm{mL}$ & $\mathbf{P}$ & Raw \\
\hline $\mathrm{Cm} 244$ & $6 / 1 / 90$ & $4 / 30 / 89$ & $4 / 27 / 89$ & ВОВ2M3 & G311 & G359 & 1.20 & $<6.37 E-05$ & $\mathrm{uCi} / \mathrm{mL}$ & $P$ & Raw \\
\hline $\mathrm{Cm} 244$ & $6 / 1 / 90$ & $4 / 30 / 89$ & $4 / 27 / 89$ & BOB2M3 & G311 & G359 & 1.20 & $<6.37 E-05$ & $u C i / m L$ & $P$ & Raw \\
\hline $\mathrm{Cm} 244$ & $6 / 1 / 90$ & $4 / 30 / 89$ & $4 / 27 / 89$ & Вов2M4 & G313 & G363 & 1.20 & $<6.37 E-05$ & $\mathrm{uCi} / \mathrm{mL}$ & $P$ & Raw \\
\hline $\mathrm{Cm} 244$ & $6 / 1 / 90$ & $4 / 30 / 89$ & $4 / 27 / 89$ & ВОВ2М'4 & G313 & G363 & 1.20 & $<6.37 E-05$ & $\mathrm{uCi} / \mathrm{mL}$ & $P$ & Raw \\
\hline Cm244 & $6 / 1 / 90$ & $4 / 30 / 89$ & $4 / 27 / 89$ & BOB2M5 & G315 & G364 & 1.19 & $<6.37 E-05$ & $\mathrm{uCI} / \mathrm{mL}$ & $P$ & Raw \\
\hline Cm244 & $6 / 1 / 90$ & $4 / 30 / 89$ & $4 / 27 / 89$ & ВOB2M5 & G315 & G364 & 1.19 & $<6.37 E-05$ & $\mathrm{uCi} / \mathrm{mL}$ & $\mathbf{P}$ & Raw \\
\hline $\mathrm{Cm} 244$ & $6 / 1 / 90$ & $4 / 30 / 89$ & $4 / 27 / 89$ & BOB2N4 & G323 & G341 & 1.20 & $<6.37 E-05$ & $\mathrm{uCi} / \mathrm{mL}$ & $\mathbf{P}$ & Raw \\
\hline Cm244 & $6 / 1 / 90$ & $4 / 30 / 89$ & $4 / 27 / 89$ & BOB2N4 & G323 & G341 & 1.20 & $<6.37 E-05$ & $\mathrm{uCi} / \mathrm{mL}$ & $\mathbf{P}$ & Raw \\
\hline Cm244 & $6 / 1 / 90$ & $4 / 30 / 89$ & $4 / 27 / 89$ & BOB2T9 & G327 & G443 & 1.20 & $<2.55 E-05$ & $\mathrm{uCi} / \mathrm{mL}$ & $P$ & Raw \\
\hline $\mathrm{Cm} 244$ & $6 / 1 / 90$ & $4 / 30 / 89$ & $4 / 27 / 89$ & ВОВ2T9 & G327 & G443 & 1.20 & $<2.55 E-05$ & uCi/mL & $\mathbf{P}$ & Raw \\
\hline
\end{tabular}




\begin{tabular}{|c|c|c|c|c|c|c|}
\hline \multicolumn{4}{|c|}{ Radionuclide Information } & \multirow[b]{2}{*}{$\begin{array}{l}\text { Reference } \\
\text { Number }\end{array}$} & \multirow[b]{2}{*}{ Validated $(\mathbf{Y} / \mathrm{N})$} & \multirow[b]{2}{*}{ Notes } \\
\hline Radoinuclide & Conc. $(\mathrm{Bq} / \mathrm{M})$ & $\begin{array}{l}\text { Conc. (Bq/L } \\
\text { decayed) }\end{array}$ & $\begin{array}{l}\text { Conc. (Cill } \\
\text { Decayed) }\end{array}$ & & & \\
\hline $\mathrm{Cm} 243 / 244$ & $1.35 \mathrm{E}+03$ & $1.05 E+03$ & $2.84 E-08$ & AP102-1 & $N$ & Outdated Information \\
\hline $\mathrm{Cm} 243 / 244$ & $2.79 E+02:$ & $2.17 E+02$ & $5.86 \mathrm{E}-09$ & AP102-1 & $\mathbf{N}$ & Outdated Information \\
\hline $\mathrm{Cm} 243 / 244$ & $1.53 E+02$ & $1.19 E+02$ & $3.22 \mathrm{E}-09$ & AP102-1 & $\mathbf{N}$ & Outdated Information \\
\hline $\mathrm{Cm} 243 / 244$ & $1.21 E+02$ & $9.41 E+01$ & $2.54 E-09$ & AP102-1 & $\bar{N}$ & Outdated Information \\
\hline $\mathrm{Cm} 243 / 244$ & $1.21 E+02$ & $9.41 E+01$ & 2.54E-09 & AP102-1 & $\mathbf{N}$ & Outdated Information \\
\hline $\mathrm{Cm} 243 / 244$ & $4.07 \mathrm{E}+01$ & $3.16 \mathrm{E}+01$ & $8.54 E-10$ & AP102-1 & $\bar{N}$ & Outdated Information \\
\hline $\mathrm{Cm} 244$ & NALUEI & FALUE! & \#ALUEI & AP102-3 & $\mathbf{Y}$ & $\begin{array}{l}\text { Density reported for } \mathrm{G} 342 \text { collected at } 306 \text { in from } \\
\text { bottom of tank at a } 30^{\circ} \text { angle. Sample was } \\
\text { unheated, unheated samples precipitated }\end{array}$ \\
\hline $\mathrm{Cm} 244$ & \#ALUE! & \#VALUE! & *VALUE! & AP102-3 & $\mathbf{Y}$ & $\begin{array}{l}\text { Density reported for } 6342 \text { collected at } 306 \text { in from } \\
\text { bottom of tank at a } 30^{\circ} \text { angle. Sample was } \\
\text { unheated, unheated samples precipitated }\end{array}$ \\
\hline $\mathrm{Cm} 244$ & *VALUE! & NALUE! & \#ALUE! & AP102-3 & $\mathbf{Y}$ & $\begin{array}{l}\text { Density reported for } \mathrm{G} 348 \text { collected at } 125 \text { in from } \\
\text { bottom of tank at a } 30^{\circ} \text { angle. Sample was } \\
\text { unheated, unheated samples precipitated }\end{array}$ \\
\hline $\mathrm{Cm} 244$ & \#VALUEI & \#VALUE! & \#VALUE! & AP102-3 & $\mathbf{Y}$ & $\begin{array}{l}\text { Density reported for } 6348 \text { collected at } 125 \text { in from } \\
\text { bottom of tank at a } 30^{\circ} \text { angle. Sample was } \\
\text { unheated, unheated samples precipitated }\end{array}$ \\
\hline $\mathrm{Cm} 244$ & \#VALUE! & \#VALUE! & *VALUE! & AP102-3 & $\mathbf{Y}$ & $\begin{array}{l}\text { Density reported for } G 358 \text { collected at } 334 \text { in from } \\
\text { bottom of tank at a } 150^{\circ} \text { angle. Sample was } \\
\text { unheated, unheated samples precipitated }\end{array}$ \\
\hline $\mathrm{Cm} 244$ & \#VALUE! & \#VALUE! & *aLue! & AP102-3 & $\mathbf{Y}$ & $\begin{array}{l}\text { Density reported for } \mathrm{G} 358 \text { collected at } 334 \text { in from } \\
\text { bottom of tank at a } 150^{\circ} \text { angle. Sample was } \\
\text { unheated, unheated samples precipitated }\end{array}$ \\
\hline $\mathrm{Cm} 244$ & \#VALUE! & \#VALUE! & \#VALUEI & AP102-3 & Y & $\begin{array}{l}\text { Density reported for } \mathrm{G} 353 \text { collected at } 334 \text { in from } \\
\text { bottom of tank at a } 150^{\circ} \text { angle. Sample was } \\
\text { unheated, unheated samples precipitated }\end{array}$ \\
\hline $\mathrm{Cm} 244$ & \#VALUE! & *VALUE! & *VALUE! & AP102-3 & $Y$ & $\begin{array}{l}\text { Density reported for } \mathrm{G} 353 \text { collected at } 334 \text { in from } \\
\text { bottom of tank at a } 150^{\circ} \text { angle. Sample was } \\
\text { unheated, unheated samples precipitated }\end{array}$ \\
\hline $\mathrm{Cm} 244$ & \#ALUE! & *VALUE! & NALUEl & AP102-3 & $\mathbf{Y}$ & $\begin{array}{l}\text { Density reported for } \mathrm{G} 359 \text { collected at } 162 \text { in from } \\
\text { bottom of tank at a } 150^{\circ} \text { angle. Sample was } \\
\text { unheated, unheated samples precipitated }\end{array}$ \\
\hline $\mathrm{Cm} 244$ & \#ALUE! & \#VALUE! & *VALUE! & AP102-3 & $\mathbf{Y}$ & $\begin{array}{l}\text { Density reported for } \mathrm{G} 359 \text { collected at } 162 \text { in from } \\
\text { bottom of tank at a } 150^{\circ} \text { angle. Sample was } \\
\text { unheated, unheated samples precipitated }\end{array}$ \\
\hline $\mathrm{Cm} 244$ & *VALUE! & FALUEI & \#VALUE! & AP102-3 & $Y$ & $\begin{array}{l}\text { Density reported for } \mathrm{G} 363 \text { collected at } 18 \text { in from } \\
\text { bottom of tank at a } 150^{\circ} \text { angle. Sample was } \\
\text { unheated, unheated samples precipitated }\end{array}$ \\
\hline $\mathrm{Cm} 244$ & \#VALUE! & \#VALUEI & * VALUE! & AP102-3 & $Y$ & $\begin{array}{l}\text { Density reported for G363 collected at } 18 \text { in from } \\
\text { bottom of tank at a } 150^{\circ} \text { angle. Sample was } \\
\text { unheated, unheated samples precipitated }\end{array}$ \\
\hline $\mathrm{Cm} 244$ & FALUEI & \#ALUEI & *VALUE! & AP102-3 & $Y$ & $\begin{array}{l}\text { Density reported for } \mathrm{G} 364 \text { collected at } 372 \text { in from } \\
\text { bottom of tank at a } 270^{\circ} \text { angle. Sample was } \\
\text { unheated, unheated samples precipitated }\end{array}$ \\
\hline $\mathrm{Cm} 244$ & NALUE! & \#ALUEI & *VALUe! & AP102-3 & $\gamma$ & $\begin{array}{l}\text { Density reported for G364 collected at } 372 \text { in from } \\
\text { bottom of tank at a } 270^{\circ} \text { angle. Sample was } \\
\text { unheated, unheated samples precipitated }\end{array}$ \\
\hline $\mathrm{Cm} 244$ & FALUE! & \#ALUE! & NALUEI & AP102-3 & $\mathbf{Y}$ & $\begin{array}{l}\text { Density reported for G341 collected at } 99 \text { in from } \\
\text { bottom of tank at a } 270^{\circ} \text { angle. }\end{array}$ \\
\hline $\mathrm{Cm} 244$ & \#VALUEI & NaLUEI & * VAlueI & AP102-3 & $\mathbf{Y}$ & $\begin{array}{l}\text { Density reported for } 6341 \text { collected at } 99 \text { in from } \\
\text { bottom of tank at a } 270^{\circ} \text { angle. }\end{array}$ \\
\hline $\mathrm{Cm} 244$ & \#ALUE! & \#ALUE! & *VALUE! & AP102-3 & $\mathbf{Y}$ & $\begin{array}{l}\text { Density reported for G443 represents a composite } \\
\text { sample }\end{array}$ \\
\hline $\mathrm{Cm} 244$ & NALUE! & *VALUE! & \#VALUEI & AP102.3 & $\mathbf{Y}$ & $\begin{array}{l}\text { Density reported for G443 represents a composite } \\
\text { sample }\end{array}$ \\
\hline
\end{tabular}




\begin{tabular}{|c|c|c|c|c|c|c|c|c|c|c|c|}
\hline \multicolumn{12}{|c|}{ Tank Waste Characterization Sample Information } \\
\hline $\begin{array}{c}\text { Reported } \\
\text { Radionuclide } \\
\end{array}$ & $\begin{array}{c}\text { Memo } \\
\text { Date }\end{array}$ & $\begin{array}{c}\text { Start } \\
\text { Analysis } \\
\text { Date }\end{array}$ & $\begin{array}{c}\text { Start } \\
\text { Sample } \\
\text { Date }\end{array}$ & $\begin{array}{c}\text { Sample } \\
\text { Numbet }\end{array}$ & $\begin{array}{c}\text { Tank Farm } \\
\text { Number }\end{array}$ & $\begin{array}{c}\text { Lab Sample } \\
\text { Number }\end{array}$ & $\begin{array}{l}\text { Density } \\
(\mathrm{g} / \mathrm{mL})\end{array}$ & $\begin{array}{c}\text { Reported } \\
\text { Value }\end{array}$ & Reported Unit & Code & Source \\
\hline $\mathrm{Cm} 244$ & $6 / 1 / 90$ & $4 / 30 / 89$ & $4 / 27 / 89$ & ВОВ2MO & G306 & G352 & 1.20 & $6.37 E-0.5$ & uCi/mL & $\mathbf{P}$ & Raw \\
\hline $\mathrm{Cm} 244$ & $6 / 1 / 90$ & $4 / 30 / 89$ & $4 / 27 / 89$ & BOB2MO & G306 & G352 & 1.20 & $6.37 E-05$ & $\mathrm{uCi} / \mathrm{mL}$ & $\mathbf{P}$ & Raw \\
\hline $\mathrm{Cm} 244$ & $6 / 1 / 90$ & $4 / 30 / 89$ & $4 / 27 / 89$ & BOB2L9 & G305 & G349 & 1.20 & $6.37 E-05$ & uCi/mL & $\mathbf{P}$ & Raw \\
\hline $\mathrm{Cm} 244$ & $6 / 1 / 90$ & $4 / 30 / 89$ & $4 / 27 / 89$ & BOB2L9 & G305 & G349 & 1.20 & 6.37E-05 & $\mathrm{uCi} / \mathrm{mL}$ & $\mathbf{P}$ & Raw \\
\hline $\mathrm{Cm} 244$ & $6 / 1 / 90$ & $4 / 30 / 89$ & $4 / 27 / 89$ & BOB2N1 & G310 & G471* & 1.20 & 4.67E-05 & $\mathrm{uCi} / \mathrm{mL}$ & $P$ & Raw \\
\hline $\mathrm{Cm} 244$ & $6 / 1 / 90$ & $4 / 30 / 89$ & $4 / 27 / 89$ & В082T9 & G327 & G470* & 1.19 & $4.20 E-05$ & $\mathrm{uCi} / \mathrm{mL}$ & $P$ & Raw \\
\hline Cm244 & $6 / 1 / 90$ & $4 / 30 / 89$ & $4 / 27 / 89$ & BOB2N1 & G310 & G471 & 1.20 & 2.96E-05 & $\mathrm{uCi} / \mathrm{mL}$ & $\mathbf{P}$ & Raw \\
\hline Cm244 & $6 / 1 / 90$ & $4 / 30 / 89$ & $4 / 27 / 89$ & BOB2M6 & G318 & G459 & 1.20 & $2.95 E-0.5$ & $\mathrm{uCi} / \mathrm{mL}$ & $P$ & Raw \\
\hline Cm244 & $6 / 1 / 90$ & $4 / 30 / 89$ & $4 / 27 / 89$ & ВОВ $2 \mathrm{M7}$ & G319 & G460 & 1.20 & $2.89 E-05$ & $\mathrm{uCi} / \mathrm{mL}$ & $\mathbf{P}$ & Raw \\
\hline Cm244 & $6 / 1 / 90$ & $4 / 30 / 89$ & $4 / 27 / 89$ & BOB2M6 & G318 & G459 & 1.20 & 2.79E-05 & $\mathrm{uCi} / \mathrm{mL}$ & $\mathbf{P}$ & Raw \\
\hline $\mathrm{Cm} 244$ & $6 / 1 / 90$ & $4 / 30 / 89$ & $4 / 27 / 89$ & ВОВ2Т9 & G327 & G470" & 1.19 & $2.70 E-05$ & uCi/mL & $P$ & Raw \\
\hline $\mathrm{Cm} 244$ & $6 / 1 / 90$ & $4 / 30 / 89$ & $4 / 27 / 89$ & BOB2M7 & G319 & G460 & 1.20 & $2.66 E-05$ & $\mathrm{uCi} / \mathrm{mL}$ & $\mathbf{P}$ & Raw \\
\hline Cm244 & $6 / 1 / 90$ & $4 / 30 / 89$ & $4 / 27 / 89$ & ВОВ2M9 & G302 & G477• & 1.20 & $2.43 E-05$ & $\mathrm{uCi} / \mathrm{mL}$ & $\mathbf{P}$ & Raw \\
\hline $\mathrm{Cm} 244$ & $6 / 1 / 90$ & $4 / 30 / 89$ & $4 / 27 / 89$ & BOB2N4 & G323 & G476* & 1.21 & $2.02 E-05$ & $\mathrm{uCi} / \mathrm{mL}$ & $\mathbf{P}$ & Raw \\
\hline $\mathrm{Cm} 244$ & $6 / 1 / 90$ & $4 / 30 / 89$ & $4 / 27 / 89$ & В082M9 & G302 & G477" & 1.20 & $1.75 E-05$ & $\mathrm{uCi} / \mathrm{mL}$ & $\mathbf{P}$ & Raw \\
\hline Cm244 & $6 / 1 / 90$ & $4 / 30 / 89$ & $4 / 27 / 89$ & BOB2N4 & G323 & G476* & 1.21 & $1.75 E-05$ & $\mathrm{uCl} / \mathrm{mL}$ & $\mathbf{P}$ & Raw \\
\hline Co60 & $6 / 1 / 90$ & $4 / 30 / 89$ & $4 / 27 / 89$ & BO82M9 & G302 & G477* & 1.20 & $<5.31 E-02$ & $\mathrm{uCl} / \mathrm{mL}$ & $P$ & Raw \\
\hline Co60 & $6 / 1 / 90$ & $4 / 30 / 89$ & $4 / 27 / 89$ & BOB2N4 & G323 & G476* & 1.21 & $<4.60 E-02$ & $\mathrm{uCi} / \mathrm{mL}$ & $P$ & Raw \\
\hline Co60 & $6 / 1 / 90$ & $4 / 30 / 89$ & $4 / 27 / 89$ & BOB2M9 & G302 & G477* & 1.20 & $<4.47 E-02$ & $\mathrm{uCi} / \mathrm{mL}$ & $P$ & Raw \\
\hline
\end{tabular}




\begin{tabular}{|c|c|c|c|c|c|c|}
\hline \multicolumn{4}{|c|}{ Radionuclide Information } & \multirow[b]{2}{*}{$\begin{array}{l}\text { Reference } \\
\text { Number }\end{array}$} & \multirow[b]{2}{*}{ Validated $(Y / N)$} & \multirow[b]{2}{*}{ Notes } \\
\hline Radoinuclide & Conc. $(\mathrm{Bq} / \mathrm{L})$ & $\begin{array}{c}\text { Conc. }(\mathrm{Bq} / \mathrm{L} \\
\text { decayed) }\end{array}$ & $\begin{array}{l}\text { Conc. (Ci/l. } \\
\text { Decayed) }\end{array}$ & & & \\
\hline $\mathrm{Cm} 244$ & $2.36 E+03$ & $2.21 E+03$ & $5.97 E-08$ & AP102-3 & $\mathbf{Y}$ & $\begin{array}{l}\text { Density reported for } \mathrm{G} 352 \text { collected at } 34 \text { in from } \\
\text { bottom of tank at a } 30^{\circ} \text { angle. Sample was } \\
\text { unheated, unheated samples precipitated }\end{array}$ \\
\hline $\mathrm{Cm} 244$ & $2.36 E+03$ & $2.21 E+03$ & $5.97 E-08$ & AP102-3 & $\mathbf{Y}$ & $\begin{array}{l}\text { Density reported for } \mathrm{G} 352 \text { collected at } 34 \text { in from } \\
\text { bottom of tank at a } 30^{\circ} \text { angle. Sample was } \\
\text { unheated, unheated samples precipitated }\end{array}$ \\
\hline $\mathrm{Cm} 244$ & $2.36 E+03$ & $2.21 E+03$ & 5.97E-08 & AP102-3 & $\mathbf{Y}$ & $\begin{array}{l}\text { Density reported for } 6349 \text { collected at } 34 \text { in from } \\
\text { bottom of tank at a } 30^{\circ} \text { angle. Sample was } \\
\text { unheated, unheated samples precipitated }\end{array}$ \\
\hline $\mathrm{Cm} 244$ & $2.36 E+03$ & $2.21 E+03$ & $5.97 E-08$ & AP102-3 & $\mathbf{Y}$ & $\begin{array}{l}\text { Density reported for } 6349 \text { collected at } 34 \text { in from } \\
\text { bottom of tank at a } 30^{\circ} \text { angle. Sample was } \\
\text { unheated, unheated samples precipitated }\end{array}$ \\
\hline Cm244 & $1.73 E+03$ & $1.62 E+03$ & $4.38 E-08$ & AP102-3 & $\mathbf{Y}$ & $\begin{array}{l}\text { Density reported for } \mathrm{G} 471 \text { collected at } 208 \text { in from } \\
\text { bottom of tank at a } 150^{\circ} \text { angle and was keep heated } \\
\text { to } 40^{\circ} \mathrm{C} \text {. }\end{array}$ \\
\hline $\mathrm{Cm} 244$ & $1.55 E+03$ & $1.46 \mathrm{E}+03$ & $3.94 E-08$ & AP102-3 & $Y$ & $\begin{array}{l}\text { Density reported for } \mathrm{G} 470 \text { represents a composite } \\
\text { sample. Sample was kept heated to } 40^{\circ} \mathrm{C} \text {. }\end{array}$ \\
\hline Cm244 & $1.10 E+03$ & $1.03 E+03$ & $2.78 E-08$ & AP102-3 & $\mathbf{Y}$ & $\begin{array}{l}\text { Density reported for } \mathrm{G} 471 \text { collected at } 208 \text { in from } \\
\text { bottom of tank at a } 150^{\circ} \text { angle and was keep heated } \\
\text { to } 40^{\circ} \mathrm{C} \text {. }\end{array}$ \\
\hline $\mathrm{Cm} 244$ & $1.09 E+03$ & $1.02 E+03$ & $2.77 E-08$ & AP102-3 & $Y$ & $\begin{array}{l}\text { Density reported for } \mathrm{G} 459 \text { collected at } 226 \text { in from } \\
\text { bottom of tank at a } 270^{\circ} \text { angle. Sample was } \\
\text { unheated, unheated samples precipitated }\end{array}$ \\
\hline $\mathrm{Cm} 244$ & $1.07 E+03$ & $1.00 E+03$ & 2.71E-08 & AP102-3 & $\mathbf{Y}$ & $\begin{array}{l}\text { Density reported for } \mathrm{G} 460 \text { collected at } 226 \text { in from } \\
\text { bottom of tank at a } 270^{\circ} \text { angle. Sample was } \\
\text { unheated, unheated samples precipitated }\end{array}$ \\
\hline $\mathrm{Cm} 244$ & $1.03 E+03$ & $9.68 E+02$ & 2.62E-08 & AP102-3 & $\gamma$ & $\begin{array}{l}\text { Density reported for } \mathrm{G} 459 \text { collected at } 226 \text { in from } \\
\text { bottom of tank at a } 270^{\circ} \text { angle. Sample was } \\
\text { unheated, unheated samples precipitated }\end{array}$ \\
\hline Cm244 & $9.99 E+02$ & $9.37 E+02$ & $2.53 E-08$ & AP102-3 & $\mathbf{Y}$ & $\begin{array}{l}\text { Density reported for G470 represents a composite } \\
\text { sample. Sample was kept heated to } 40^{\circ} \mathrm{C} \text {. }\end{array}$ \\
\hline $\mathrm{Cm} 244$ & $9.84 E+02$ & $9.23 E+02$ & $2.49 E-08$ & AP102-3 & $\mathbf{Y}$ & $\begin{array}{l}\text { Density reported for } 6460 \text { collected at } 226 \text { in from } \\
\text { bottom of tank at a } 270^{\circ} \text { angle. Sample was } \\
\text { unheated, unheated samples precipitated }\end{array}$ \\
\hline $\mathrm{Cm} 244$ & $8.99 E+02$ & $8.43 E+02$ & $2.28 E-08$ & AP102-3 & $\gamma$ & $\begin{array}{l}\text { Density reported for } G 477 \text { collected at } 182 \text { in from } \\
\text { bottom of tank at a } 30^{\circ} \text { angle and was keep heated } \\
\text { to } 40^{\circ} \mathrm{C} \text {. }\end{array}$ \\
\hline Cm244 & $7.47 E+02$ & $7.01 E+02$ & $1.89 E-08$ & AP102-3 & $Y$ & $\begin{array}{l}\text { Density reported for } \mathrm{G} 476 \text { collected at } 99 \text { in from } \\
\text { bottom of tank at a } 270^{\circ} \text { angle and was keep heated } \\
\text { to } 40^{\circ} \mathrm{C} \text {. }\end{array}$ \\
\hline $\mathrm{Cm} 244$ & $6.48 \mathrm{E}+02$ & $6.07 E+02$ & $1.64 E-08$ & AP102-3 & $\gamma$. & $\begin{array}{l}\text { Density reported for } \mathrm{G} 477 \text { collected at } 182 \text { in from } \\
\text { bottom of tank at a } 30^{\circ} \text { angle and was keep heated } \\
\text { to } 40^{\circ} \mathrm{C} \text {. }\end{array}$ \\
\hline $\mathrm{Cm} 244$ & $6.48 E+02$ & $6.07 E+02$ & $1.64 E-08$ & AP102-3 & $Y$ & $\begin{array}{l}\text { Density reported for } \mathrm{G} 476 \text { collected at } 99 \text { in from } \\
\text { bottom of tank at a } 270^{\circ} \text { angle and was keep heated } \\
\text { to } 40^{\circ} \mathrm{C} \text {. }\end{array}$ \\
\hline Co60 & NVALUE! & FALUE! & NALUE! & AP102-3 & $\mathbf{Y}$ & $\begin{array}{l}\text { Density reported for } 6477 \text { collected at } 182 \text { in from } \\
\text { bottom of tank at a } 30^{\circ} \text { angle and was keep heated } \\
\text { to } 40^{\circ} \mathrm{C} \text {. }\end{array}$ \\
\hline Co6o & *VALUE! & WALUE! & NALUEI & AP102-3 & $Y$ & $\begin{array}{l}\text { Density reported for } 6476 \text { collected at } 99 \text { in from } \\
\text { bottom of tank at a } 270^{\circ} \text { angle and was keep heated } \\
\text { to } 40^{\circ} \mathrm{C} \text {. }\end{array}$ \\
\hline Co60 & *ALUE! & Nalue! & NALUE! & AP102-3 & $\mathbf{Y}$ & $\begin{array}{l}\text { Density reported for } \mathrm{G} 477 \text { collected at } 182 \text { in from } \\
\text { bottom of tank at a } 30^{\circ} \text { angle and was keep heated } \\
\text { to } 40^{\circ} \mathrm{C} \text {. }\end{array}$ \\
\hline
\end{tabular}




\begin{tabular}{|c|c|c|c|c|c|c|c|c|c|c|c|}
\hline \multicolumn{12}{|c|}{ Tank Waste Characterization Sample Information } \\
\hline $\begin{array}{c}\text { Reported } \\
\text { Radionuclide } \\
\end{array}$ & $\begin{array}{c}\text { Memo } \\
\text { Date }\end{array}$ & $\begin{array}{c}\text { Start } \\
\text { Analysis } \\
\text { Date }\end{array}$ & $\begin{array}{c}\text { Start } \\
\text { Sample } \\
\text { Date } \\
\end{array}$ & $\begin{array}{l}\text { Sample } \\
\text { Number }\end{array}$ & $\begin{array}{c}\text { Tank Farm } \\
\text { Number }\end{array}$ & $\begin{array}{c}\text { Lab Sample } \\
\text { Number }\end{array}$ & $\begin{array}{l}\text { Density } \\
(\mathrm{g} / \mathrm{mL})\end{array}$ & $\begin{array}{c}\text { Reported } \\
\text { Value }\end{array}$ & Reportad Unit & Code & Source \\
\hline Co60 & $6 / 1 / 90$ & $4 / 30 / 89$ & $4 / 27 / 89$ & BOB2N4 & G323 & Ġ47* & 1.21 & $<4.19 E-02$ & uCi/mL & $\mathbf{P}$ & Raw \\
\hline Co60 & $6 / 1 / 90$ & $4 / 30 / 89$ & $4 / 27 / 89$ & BOB2T9 & G327 & G443 & 1.20 & $<2.07 E-02$ & $\mathrm{uCi} / \mathrm{mL}$ & $\mathbf{P}$ & Raw \\
\hline Co60 & $6 / 1 / 90$ & $4 / 30 / 89$ & $4 / 27 / 89$ & В082M4 & G313 & G363 & 1.20 & $<1.97 E-02$ & $\mathrm{uCi} / \mathrm{mL}$ & $\mathbf{P}$ & Raw \\
\hline Co60 & $6 / 1 / 90$ & $4 / 30 / 89$ & $4 / 27 / 89$ & ВОВ2M8 & G301 & G449 & NA & $<1.81 E-02$ & $u C i / m L$ & $\mathbf{P}$ & Raw \\
\hline Co60 & $6 / 1 / 90$ & $4 / 30 / 89$ & $4 / 27 / 89$ & 80B2M5 & G315 & G364 & 1.19 & $<1.81 E-02$ & $\mathrm{uCi} / \mathrm{mL}$ & $\mathbf{P}$ & Raw \\
\hline Co60 & $6 / 1 / 90$ & $4 / 30 / 89$ & $4 / 27 / 89$ & BOB2M4 & G313 & G363 & 1.20 & $<1.75 E-02$ & $\mathrm{uCi} / \mathrm{mL}$ & $\mathbf{P}$ & Raw \\
\hline Co6o & $6 / 1 / 90$ & $4 / 30 / 89$ & $4 / 27 / 89$ & $\mathrm{BOB2L7}$ & 6299 & G342 & 1.20 & $<1.69 \mathrm{E}-02$ & $\mathrm{uCi} / \mathrm{mL}$ & $\mathbf{P}$ & Raw \\
\hline Co60 & $6 / 1 / 90$ & $4 / 30 / 89$ & $4 / 27 / 89$ & BOB2L9 & G305 & G349 & 1.20 & $<1.69 E-02$ & $\mathrm{uCi} / \mathrm{mL}$ & $\mathbf{P}$ & Raw \\
\hline Co60 & $6 / 1 / 90$ & $4 / 30 / 89$ & $4 / 27 / 89$ & BOB2LS & G305 & G349 & 1.20 & $<1.69 E-02$ & $u C i / m L$ & $P$ & Raw \\
\hline Co6o & $6 / 1 / 90$ & $4 / 30 / 89$ & $4 / 27 / 89$ & ВOB2M2 & G309 & G358 & 1.20 & $<1.69 E-02$ & $u C i / m L$ & $\mathbf{P}$ & Raw \\
\hline Co60 & $6 / 1 / 90$ & $4 / 30 / 89$ & $4 / 27 / 89$ & BOB2N4 & G323 & G341 & 1.20 & $<1.69 E-02$ & $\mathrm{uCi} / \mathrm{mL}$ & $P$ & Raw \\
\hline Co6o & $6 / 1 / 90$ & $4 / 30 / 89$ & $4 / 27 / 89$ & BOB2M8 & G301 & G449 & NA & $<1.63 E-02$ & $\mathrm{uCi} / \mathrm{mL}$ & $\mathbf{P}$ & Raw \\
\hline Co6o & $6 / 1 / 90$ & $4 / 30 / 89$ & $4 / 27 / 89$ & BOB2L8 & G303 & G348 & 1.21 & $<1.63 E-02$ & $\mathrm{uCi} / \mathrm{mL}$ & $\mathbf{P}$ & Raw \\
\hline Co60 & $6 / 1 / 90$ & $4 / 30 / 89$ & $4 / 27 / 89$ & BOB2T9 & G327 & G443 & 1.20 & $<1.63 E-02$ & $\mathrm{uCi} / \mathrm{mL}$ & $\mathbf{P}$ & Raw \\
\hline Co60 & $6 / 1 / 90$ & $4 / 30 / 89$ & $4 / 27 / 89$ & BOB2M9 & G302 & G450 & 1.20 & $<1.57 E-02$ & $\mathrm{uCi} / \mathrm{mL}$ & $\mathbf{P}$ & Raw \\
\hline Co60 & $6 / 1 / 90$ & $4 / 30 / 89$ & $4 / 27 / 89$ & вов2T9 & G327 & G443 & 1.20 & $<1.57 E-02$ & $\mathrm{uCi} / \mathrm{mL}$ & $P$ & Raw \\
\hline Co6o & $6 / 1 / 90$ & $4 / 30 / 89$ & $4 / 27 / 89^{\circ}$ & вов2М5 & G315 & G364 & 1.19 & $<1.50 E-02$ & $\mathrm{uCl} / \mathrm{mL}$ & $\mathbf{P}$ & Raw \\
\hline $\mathrm{Co60}$ & $6 / 1 / 90$ & $4 / 30 / 89$ & $4 / 27 / 89$ & BOB2N1 & G310 & G454 & 1.20 & $<1.38 E-02$ & $\mathrm{uCl} / \mathrm{mL}$ & $P$ & Raw \\
\hline Co60 & $6 / 1 / 90$ & $4 / 30 / 89$ & $4 / 27 / 89$ & 8ОВ2M7 & G319 & G460 & 1.20 & $<1.36 E-02$ & $\mathrm{uCi} / \mathrm{mL}$ & $\mathbf{P}$ & Raw \\
\hline Co6o & $6 / 1 / 90$ & 4/30/89 & 4/27/89 & BOB2L8 & G303 & G348 & 1.21 & $<1.35 E-02$ & uCi/mL & $\mathbf{P}$ & Raw \\
\hline
\end{tabular}




\begin{tabular}{|c|c|c|c|c|c|c|}
\hline \multicolumn{4}{|c|}{ Radionuclide Information } & \multirow[b]{2}{*}{$\begin{array}{l}\text { Roference } \\
\text { Number }\end{array}$} & \multirow[b]{2}{*}{ Validated $(Y / N)$} & \multirow[b]{2}{*}{ Notes } \\
\hline Radoinuclide & Conc. $(\mathrm{Bq} / \mathrm{L})$ & $\begin{array}{l}\text { Conc. (Bq/L } \\
\text { decayed) }\end{array}$ & $\begin{array}{l}\text { Conc. (Cill } \\
\text { Decayed) }\end{array}$ & & & \\
\hline Co60 & \#VALUE! : & FALUE! & \#VALUE! & AP102-3 & $\mathbf{Y}$ & $\begin{array}{l}\text { Density reported for } \mathrm{G} 476 \text { collected at } 99 \text { in from } \\
\text { bottom of tank at a } 270^{\circ} \text { angle and was keep heated } \\
\text { to } 40^{\circ} \mathrm{C} \text {. }\end{array}$ \\
\hline Co60 & \#VALUE! & \#VALUE! & \#VALUE! & AP102-3 & $\mathbf{Y}$ & $\begin{array}{l}\text { Density reported for } \mathrm{G} 443 \text { represents a composite } \\
\text { sample }\end{array}$ \\
\hline Co60 & VALUEI & NALUEl & \#ALUE! & AP102-3 & $\mathbf{Y}$ & $\begin{array}{l}\text { Density reported for } \mathrm{G} 363 \text { collected at } 18 \text { in from } \\
\text { bottom of tank at a } 150^{\circ} \text { angle. Sample was } \\
\text { unheated, unheated samples precipitated }\end{array}$ \\
\hline Co6o & \#ALUEI & \# VALUEI & *VALUE! & AP102-3 & $\mathbf{Y}$ & $\begin{array}{l}\text { Density not reported for G449. No assumptions could } \\
\text { be made }\end{array}$ \\
\hline Co60 & VALUE! & $\#$ VALUEI & \#ALUE! & AP102-3 & $\mathbf{Y}$ & $\begin{array}{l}\text { Density reported for G364 collected at } 372 \text { in from } \\
\text { bottom of tank at a } 270^{\circ} \text { angle. Sample was } \\
\text { unheated, unheated samples precipitated }\end{array}$ \\
\hline Co60 & \#VALUE! & \#ALUEI & :VALUE! & AP102-3 & $\mathbf{Y}$ & $\begin{array}{l}\text { Density reported for G363 collected at } 18 \text { in from } \\
\text { bottom of tank at a } 150^{\circ} \text { angle. Sample was } \\
\text { unheated, unheated samples precipitated }\end{array}$ \\
\hline Co60 & \#VALUE! & \#VALUE! & \#VALUE! & AP102-3 & $\mathbf{Y}$ & $\begin{array}{l}\text { Density reported for } 6342 \text { collected at } 306 \text { in from } \\
\text { bottom of tank at a } 30^{\circ} \text { angle. Sample was } \\
\text { unheated, unheated samples precipitated }\end{array}$ \\
\hline Co60 & \#VALUE! & \#VALUEl & \#VALUe! & AP102-3 & $\mathbf{Y}$ & $\begin{array}{l}\text { Density reported for } 6349 \text { collected at } 34 \text { in from } \\
\text { bottom of tank at a } 30^{\circ} \text { angle. Sample was } \\
\text { unheated, unheated samples precipitated }\end{array}$ \\
\hline Co60 & *VALUE! & \#ALUE! & *VALUE! & AP102-3 & $\mathbf{Y}$ & $\begin{array}{l}\text { Density reported for G349 collected at } 34 \text { in from } \\
\text { bottom of tank at a } 30^{\circ} \text { angle. Sample was } \\
\text { unheated, unheated samples precipitated }\end{array}$ \\
\hline Co60 & \#VALUE! & \#VALUE! & \#VALUE! & AP102-3 & $\mathbf{Y}$ & $\begin{array}{l}\text { Density reported for G358 collected at } 334 \text { in from } \\
\text { bottom of tank at a } 150^{\circ} \text { angle. Sample was } \\
\text { unheated, unheated samples precipitated }\end{array}$ \\
\hline Co60 & *VALUE! & \#VALUEI & *aLUE! & AP102-3 & $\mathbf{Y}$ & $\begin{array}{l}\text { Density reported for } \mathrm{G} 341 \text { collected at } 99 \text { in from } \\
\text { bottom of tank at a } 270^{\circ} \text { angle. }\end{array}$ \\
\hline Co60 & \#VALUE! & \#VALUE! & \#VALUE! & AP102-3 & $\gamma$ & $\begin{array}{l}\text { Density not reported for G449. No assumptions could } \\
\text { be made }\end{array}$ \\
\hline Co6o & $\#$ \#ALUE! & *VALUE! & \#ALUE! & AP102-3 & $\mathbf{Y}$ & $\begin{array}{l}\text { Density reported for } \mathrm{G} 348 \text { collected at } 125 \text { in from } \\
\text { bottom of tank at a } 30^{\circ} \text { angle. Sample was } \\
\text { unheated, unheated samples precipitated }\end{array}$ \\
\hline Co60 & *alueI & *VAlueI & *VALUEI & AP102-3 & $\mathbf{Y}$ & $\begin{array}{l}\text { Density reported for } \mathrm{G} 443 \text { represents a composite } \\
\text { sample }\end{array}$ \\
\hline $\mathrm{Co} 60$ & \# VAlUEI & *VALUE! & \#ALUE! & AP102-3 & $\mathbf{Y}$ & $\begin{array}{l}\text { Density not reported for G450. Density assumed } \\
\text { same as } G 477 \text { which was collected at } 182 \text { in from } \\
\text { bottom of tank at a } 30^{\circ} \text { angle and was keep heated } \\
\text { to } 40^{\circ} \mathrm{C} \text {. }\end{array}$ \\
\hline Co60 & \#VALUE! & *VALUE! & \#VALUEI & AP102-3 & $\mathbf{Y}$ & $\begin{array}{l}\text { Density reported for } \mathrm{G} 443 \text { represents a composite } \\
\text { sample }\end{array}$ \\
\hline Co60 & VALUEI & *VALUEI & *VALUE! & AP102-3 & $\gamma$ & $\begin{array}{l}\text { Density reported for G364 collected at } 372 \text { in from } \\
\text { bottom of tank at a } 270^{\circ} \text { angle. Sample was } \\
\text { unheated, unheated samples precipitated }\end{array}$ \\
\hline Co60 & \#VALUEI & NALUE! & NaLUE! & AP102-3 & $\boldsymbol{\gamma}$ & $\begin{array}{l}\text { Density not reported for G454. Density assumed } \\
\text { same as G47i which was collected at } 208 \text { in from } \\
\text { bottom of tank at a } 150^{\circ} \text { angle and was keep heated } \\
\text { to } 40^{\circ} \mathrm{C} \text {. }\end{array}$ \\
\hline Co60 & \#VALUE! & FALUEI & \#ALUEI & AP102-3 & $\boldsymbol{Y}$ & $\begin{array}{l}\text { Density reported for } G 460 \text { collected at } 226 \text { in from } \\
\text { bottom of tank at a } 270^{\circ} \text { angle. Sample was } \\
\text { unheated, unheated samples precipitated }\end{array}$ \\
\hline Co60 & NALUE! & NaLUE! & FALUEI & AP102-3 & $\mathbf{Y}$ & $\begin{array}{l}\text { Density reported for G348 collected at } 125 \text { in from } \\
\text { bottom of tank at a } 30^{\circ} \text { angle. Sample was } \\
\text { unheated, unheated samples precipitated }\end{array}$ \\
\hline
\end{tabular}




\begin{tabular}{|c|c|c|c|c|c|c|c|c|c|c|c|}
\hline \multicolumn{12}{|c|}{ Tank Wasto Characterization Sample Information } \\
\hline $\begin{array}{c}\text { Reported } \\
\text { Radionuclide }\end{array}$ & $\begin{array}{c}\text { Memo } \\
\text { Date } \\
\end{array}$ & $\begin{array}{c}\text { Start } \\
\text { Analysis } \\
\text { Date }\end{array}$ & $\begin{array}{c}\text { Start } \\
\text { Sample } \\
\text { Date }\end{array}$ & $\begin{array}{l}\text { Sample } \\
\text { Number }\end{array}$ & $\begin{array}{c}\text { Tank Farm } \\
\text { Number }\end{array}$ & $\begin{array}{c}\text { Lab Sample } \\
\text { Number }\end{array}$ & $\begin{array}{l}\text { Density } \\
(\mathrm{g} / \mathrm{mL})\end{array}$ & $\begin{array}{c}\text { Reported } \\
\text { Value } \\
\end{array}$ & Reported Unit & Code & Sourc \\
\hline Co60 & $6 / 1 / 90$ & $4 / 30 / 89$ & $4 / 27 / 89$ & BOB2N4 & G323 & G341 & 1.20 & $<1.35 E-02$ & $\mathrm{uCi} / \mathrm{mL}$ & $P$ & Raw \\
\hline Co60 & $6 / 1 / 90$ & $4 / 30 / 89$ & $4 / 27 / 89$ & ВОВ2T9 & G327 & G443 & 1.20 & $<1.35 \mathrm{E}-02$ & $\mathrm{uCi} / \mathrm{mL}$ & $\mathbf{P}$ & Raw \\
\hline Co60 & $6 / 1 / 90$ & $4 / 30 / 89$ & $4 / 27 / 89$ & ВОВ2T9 & G327 & G470* & 1.19 & $<1.31 E-02$ & uCi/mL. & $\mathbf{p}$ & Raw \\
\hline Co60 & $6 / 1 / 90$ & $4 / 30 / 89$ & $4 / 27 / 89$ & BOB2N1 & G310 & G471* & 1.20 & $<1.30 E-02$ & $\mathrm{uCi} / \mathrm{mL}$ & $\mathbf{P}$ & Raw \\
\hline Co60 & $6 / 1 / 90$ & $4 / 30 / 89$ & $4 / 27 / 89$ & ВОВ2M6 & G318 & G459 & 1.20 & $<1.30 E-02$ & $\mathrm{uCi} / \mathrm{mL}$ & $\mathbf{P}$ & Raw \\
\hline Co6o & $6 / 1 / 90$ & $4 / 30 / 89$ & $4 / 27 / 89$ & ВOB2M7 & G319 & G460 & 1.20 & $<1.28 \mathrm{E}-02$ & $\mathrm{uCi} / \mathrm{mL}$ & $P$ & Raw \\
\hline Co60 & $6 / 1 / 90$ & $4 / 30 / 89$ & $4 / 27 / 89$ & BOB2MO & G306 & G352 & 1.20 & $<1.23 \mathrm{E}-02$ & uCi/mL & $P$ & Raw \\
\hline Co6o & $6 / 1 / 90$ & $4 / 30 / 89$ & $4 / 27 / 89$ & BOB2M1 & G308 & G353 & 1.20 & $<1.23 E-02$ & $\mathrm{uCi} / \mathrm{mL}$ & $\mathbf{P}$ & Raw \\
\hline Co60 & $6 / 1 / 90$ & $4 / 30 / 89$ & $4 / 27 / 89$ & BOB2N1 & G310 & G471* & 1.20 & $<1.23 E-02$ & $\mathrm{uCi} / \mathrm{mL}$ & $\mathbf{P}$ & Raw \\
\hline Co60 & $6 / 1 / 90$ & $4 / 30 / 89$ & $4 / 27 / 89$ & BOB2N3 & G322 & G338 & NA & $<1.23 \mathrm{E}-02$ & $\mathrm{uCi} / \mathrm{mL}$ & $\mathbf{P}$ & Raw \\
\hline Co60 & $6 / 7 / 90$ & $4 / 30 / 89$ & $4 / 27 / 89$ & 8OB2NO & G307 & G453 & NA & $<1.17 E-02$ & $\mathrm{uCi} / \mathrm{mL}$ & $\mathbf{P}$ & Raw \\
\hline Co60 & $6 / 1 / 90$ & $4 / 30 / 89$ & $4 / 27 / 89$ & BOB2NO & G307 & G453 & NA & $<1.17 E-02$ & $\mathrm{uCi} / \mathrm{mL}$ & $P$ & Raw \\
\hline Co6o & $6 / 1 / 90$ & $4 / 30 / 89$ & $4 / 27 / 89$ & BOB2N1 & G310 & G454 & 1.20 & $<1.17 E-02$ & $\mathrm{uCi} / \mathrm{mL}$ & $\mathbf{P}$ & Raw \\
\hline Co60 & $6 / 1 / 90$ & $4 / 30 / 89$ & $4 / 27 / 89$ & ВОВ2MO & G306 & G352 & 1.20 & $<1.15 \mathrm{E}-02$ & $\mathrm{uCi} / \mathrm{mL}$ & $P$ & Raw \\
\hline $\mathrm{Co60}$ & $6 / 1 / 90$ & $4 / 30 / 89$ & $4 / 27 / 89$ & $\mathrm{BOB} 2 \mathrm{~N} 3$ & G322 & G338 & NA & $<1.15 E-02$ & uCi/mL & $P$ & Raw \\
\hline Co60 & $6 / 1 / 90$ & $4 / 30 / 89$ & $4 / 27 / 89$ & BOB2M2 & G309 & G358 & 1.20 & $<1.12 E-02$ & $\mathrm{uCi} / \mathrm{mL}$ & $P$ & Raw \\
\hline Co6o & $6 / 1 / 90$ & $4 / 30 / 89$ & $4 / 27 / 89$ & В082M3 & G311 & G359 & 1.20 & $<1.12 E-02$ & $\mathrm{uCl} / \mathrm{mL}$ & $P$ & Raw \\
\hline Co60 & $6 / 1 / 90$ & $4 / 30 / 89$ & $4 / 27 / 89$ & BOB2N2 & G317 & G333 & NA & $<1.10 E-02$ & $\mathrm{uCi} / \mathrm{mL}$ & $P$ & Raw \\
\hline Co60 & $6 / 1 / 90$ & $4 / 30 / 89$ & $4 / 27 / 89$ & B082M6 & G318 & G459 & 1.20 & $<1.10 E-02$ & $\mathrm{uCl} / \mathrm{mL}$ & $P$ & Raw \\
\hline Co60 & $6 / 1 / 90$ & $4 / 30 / 89$ & $4 / 27 / 89$ & BOB2M9 & G302 & G450 & 1.20 & $<1.09 E-02$ & $\mathrm{uCi} / \mathrm{mL}$ & $P$ & Raw \\
\hline
\end{tabular}




\begin{tabular}{|c|c|c|c|c|c|c|}
\hline \multicolumn{4}{|c|}{ Radionuclide Information } & \multirow[b]{2}{*}{$\begin{array}{c}\text { Reference } \\
\text { Number }\end{array}$} & \multirow[b]{2}{*}{ Validated $(Y / N)$} & \multirow[b]{2}{*}{ Notes } \\
\hline Radoinuclide & Conc. $(\mathrm{Bq} / \mathrm{L})$ & $\begin{array}{c}\text { Conc. (Bq/h } \\
\text { decayed) }\end{array}$ & $\begin{array}{c}\text { Conc. (Ci/L } \\
\text { Decayed) }\end{array}$ & & & \\
\hline Co60 & NALUE! & \#VALUE! & NALUE! & AP102-3 & $\mathbf{Y}$ & $\begin{array}{l}\text { Density reported for } \mathrm{G} 341 \text { collected at } 99 \text { in from } \\
\text { bottom of tank at a } 270^{\circ} \text { angle. }\end{array}$ \\
\hline Co60 & \#VALUE! & \#VALUE! & \#VALUE! & AP102-3 & $\mathbf{Y}$ & $\begin{array}{l}\text { Density reported for G443 represents a composite } \\
\text { sample }\end{array}$ \\
\hline Co60 & NALUE! & \#VALUE! & *VALUE! & AP102-3 & $\mathbf{Y}$ & $\begin{array}{l}\text { Density reported for } \mathrm{G} 470 \text { represents a composite } \\
\text { sample. Sample was kept heated to } 40^{\circ} \mathrm{C} \text {. }\end{array}$ \\
\hline Co60 & FALUE! & NALUE! & \#VALUEI & AP102-3 & $\mathbf{Y}$ & $\begin{array}{l}\text { Density reported for G471 collected at } 208 \text { in from } \\
\text { bottom of tank at a } 150^{\circ} \text { angle and was keep heated } \\
\text { to } 40^{\circ} \mathrm{C} \text {. }\end{array}$ \\
\hline Co60 & *VAlUE! & NALUE! & NALUE! & AP102-3 & $\mathbf{Y}$ & $\begin{array}{l}\text { Density reported for } \mathrm{G} 459 \text { collected at } 226 \text { in from } \\
\text { bottom of tank at a } 270^{\circ} \text { angle. Sample was } \\
\text { unheated, unheated samples precipitated }\end{array}$ \\
\hline Co6o & \#VALUE! & \#VALUE! & \#VALUEI & AP102-3 & $\mathbf{Y}$ & $\begin{array}{l}\text { Density reported for } G 460 \text { collected at } 226 \text { in from } \\
\text { bottom of tank at a } 270^{\circ} \text { angle. Sample was } \\
\text { unheated, unheated samples precipitated }\end{array}$ \\
\hline Co60 & \#VALUE! & \#VALUE! & \#VALUE! & AP102-3 & $\mathbf{Y}$ & $\begin{array}{l}\text { Density reported for } \mathrm{G} 352 \text { collected at } 34 \text { in from } \\
\text { bottom of tank at a } 30^{\circ} \text { angle. Sample was } \\
\text { unheated, unheated samples precipitated }\end{array}$ \\
\hline Co6o & * VALUE! & \#VALUE! & \#ALUE! & AP102-3 & $\mathbf{Y}$ & $\begin{array}{l}\text { Density reported for } \mathrm{G} 353 \text { collected at } 334 \text { in from } \\
\text { bottom of tank at a } 150^{\circ} \text { angle. Sample was } \\
\text { unheated, unheated samples precipitated }\end{array}$ \\
\hline Co60 & *VALUE! & \#VALUE! & \#ALUE! & AP102-3 & Y & $\begin{array}{l}\text { Density reported for } \mathrm{G} 471 \text { collected at } 208 \text { in from } \\
\text { bottom of tank at a } 150^{\circ} \text { angle and was keep heated } \\
\text { to } 40^{\circ} \mathrm{C} \text {. }\end{array}$ \\
\hline Co60 & \#VALUEI & \#VALUEI & \#ALUE! & AP102-3 & $Y$ & $\begin{array}{l}\text { Density not reported for G338. No assumptions could } \\
\text { be made }\end{array}$ \\
\hline Co6o & IVALUEI. & \#VALUEI & \#VALUE! & AP102-3 & $\mathbf{Y}$ & $\begin{array}{l}\text { Density not reported for G453. No assumptions could } \\
\text { be made }\end{array}$ \\
\hline Co60 & *VALUE! & \#VALUE! & \#VALUEI & AP102-3 & $\mathbf{Y}$ & $\begin{array}{l}\text { Density not reported for G453. No assumptions could } \\
\text { be made }\end{array}$ \\
\hline Co6o & *VALUE! & \#VALUE! & \#VALUE! & AP102-3 & $\mathbf{Y}$ & $\begin{array}{l}\text { Density not reported for G454. Density assumed } \\
\text { same as } 6471 \text { which was collected at } 208 \text { in from } \\
\text { bottom of tank at a } 150^{\circ} \text { angle and was keep heated } \\
\text { to } 40^{\circ} \mathrm{C} \text {. }\end{array}$ \\
\hline Co60 & *VALUE! & \#VALUE! & *VALUEI & AP102-3 & $Y$ & $\begin{array}{l}\text { Density reported for } \mathrm{G} 352 \text { collected at } 34 \text { in from } \\
\text { bottom of tank at a } 30^{\circ} \text { angle. Sample was } \\
\text { unheated, unheated samples precipitated }\end{array}$ \\
\hline Co60 & \#VALUE! & \#VALUE! & \#VALUE! & AP102-3 & $\mathbf{Y}$ & $\begin{array}{l}\text { Density not reported for G338. No assumptions could } \\
\text { be made }\end{array}$ \\
\hline Co60 & $\#$ \#ALUE! & \#VALUE! & *VALUE! & AP102-3 & $Y$ & $\begin{array}{l}\text { Density reported for } G 358 \text { collected at } 334 \text { in from } \\
\text { bottom of tank at a } 150^{\circ} \text { angle. Sample was } \\
\text { unheated, unheated samples precipitated }\end{array}$ \\
\hline Co6o & FALUEI & \#ALUEI & NALUE! & AP102-3 & $\mathbf{Y}$ & $\begin{array}{l}\text { Density reported for } 6359 \text { collected at } 162 \text { in from } \\
\text { bottom of tank at a } 150^{\circ} \text { angle. Sample was } \\
\text { unheated, unheated samples precipitated }\end{array}$ \\
\hline Co60 & NALUEI & *VALUEI & *VALUEI & AP102-3 & $Y$ & $\begin{array}{l}\text { Density not reported for } 6333 \text {. No assumptions could } \\
\text { be made }\end{array}$ \\
\hline $\mathrm{Co60}$ & VALUEI & *VALUEI & FALUE! & AP102-3 & $Y$ & $\begin{array}{l}\text { Density reported for G459 collected at } 226 \text { in from } \\
\text { bottom of tank at a } 270^{\circ} \text { angle. Sample was } \\
\text { unheated, unheated samples precipitated }\end{array}$ \\
\hline Co60 & NALUEI & \#VALUE! & NVALUE! & AP102-3 & $Y$ & $\begin{array}{l}\text { Density not reported for G450. Density assumed } \\
\text { same as } \mathrm{G} 477 \text { which was collected at } 182 \text { in from } \\
\text { bottom of tank at a } 30^{\circ} \text { angle and was keep heated } \\
\text { to } 40^{\circ} \mathrm{C} \text {. }\end{array}$ \\
\hline
\end{tabular}


TWRS Privatization Support Pro Radionuclide Liqui

\begin{tabular}{|c|c|c|c|c|c|c|c|c|c|c|c|}
\hline & & & & Tank Wast & Characteriza & ion Sample Inf & mation & & & & \\
\hline $\begin{array}{c}\text { Reported } \\
\text { Radionuclide } \\
\end{array}$ & $\begin{array}{c}\text { Memo } \\
\text { Date }\end{array}$ & $\begin{array}{c}\text { Start } \\
\text { Analysis } \\
\text { Date } \\
\end{array}$ & $\begin{array}{c}\text { Start } \\
\text { Sample } \\
\text { Date } \\
\end{array}$ & $\begin{array}{l}\text { Sample } \\
\text { Number }\end{array}$ & $\begin{array}{c}\text { Tank Farm } \\
\text { Number }\end{array}$ & $\begin{array}{c}\text { Lab Sample } \\
\text { Number }\end{array}$ & $\begin{array}{l}\text { Density } \\
\text { (g/mL) }\end{array}$ & $\begin{array}{c}\text { Reported } \\
\text { Value }\end{array}$ & [Reported Unit] & Code & Source \\
\hline Co60 & $6 / 1 / 90$ & $4 / 30 / 89$ & $4 / 27 / 89$ & ВОВ2M2 & G309 & G358 & 1.20 & $<1.08 E-02$ & $\mathrm{uCi} / \mathrm{mL}$ & $P$ & Raw \\
\hline Co60 & $6 / 1 / 90$ & $4 / 30 / 89$ & $4 / 27 / 89$ & В082M1 & G308 & G353 & 1.20 & $<1.08 E-02$ & $\mathrm{uCi} / \mathrm{mL}$ & $P$ & Raw \\
\hline Co60 & $6 / 1 / 90$ & $4 / 30 / 89$ & $4 / 27 / 89$ & $80 B 2 N 2$ & G317 & G333 & NA & $<1.06 \mathrm{E}-02$ & $\mathrm{uCi} / \mathrm{mL}$ & $\mathbf{P}$ & Plaw \\
\hline Co60 & $6 / 1 / 90$ & $4 / 30 / 89$ & $4 / 27 / 89$ & вов2Т9 & G327 & G470*, & 1.19 & $<1.01 E-02$ & $\mathrm{uCi} / \mathrm{mL}$ & $\mathbf{P}$ & Raw \\
\hline Co60 & $6 / 1 / 90$ & $4 / 30 / 89$ & $4 / 27 / 89$ & вов2М3 & G311 & G359 & 1.20 & $<8.18 E-03$ & $\mathrm{uCi} / \mathrm{mL}$ & $\mathbf{P}$ & Raw \\
\hline Co60 & $6 / 1 / 90$ & $4 / 30 / 89$ & $4 / 27 / 89$ & Вов2М2 & G309 & G358 & 1.20 & $8.09 E-02$ & $\mathrm{uCi} / \mathrm{mL}$ & $\mathbf{P}$ & Raw \\
\hline Co60 & $6 / 1 / 90$ & $4 / 30 / 89$ & $4 / 27 / 89$ & BOB2M1 & G308 & G353 & 1.20 & $7.49 \mathrm{E}-02$ & $\mathrm{uCi} / \mathrm{mL}$ & $\mathbf{P}$ & Raw \\
\hline Co60 & $6 / 1 / 90$ & $4 / 30 / 89$ & $4 / 27 / 89$ & BOB2M1 & G308 & G353 & 1.20 & $6.84 E-02$ & $\mathrm{uCi} / \mathrm{mL}$ & $\mathbf{P}$ & Raw \\
\hline Co60 & $6 / 1 / 90$ & $4 / 30 / 89$ & $4 / 27 / 89$ & BOB2L7 & G299 & G342 & 1.20 & $6.29 E-02$ & $\mathrm{uCi} / \mathrm{mL}$ & $\mathbf{P}$ & Raw \\
\hline Co60 & $5 / 17 / 84$ & NS & NS & R8473 & NS & NS & NS & $6.35 E+00$ & uCi/L & $\mathbf{P}$ & RAW \\
\hline Co60 & $5 / 17 / 84$ & NS & NS & R8472 & NS & NS & NS & $6.13 E+00$ & uCi/L & $\mathbf{P}$ & RAW \\
\hline Co60 & $5 / 17 / 84$ & NS & NS & R8469 & NS & NS & NS & $5.89 E+\infty$ & uCi/L & $\mathbf{P}$ & RAW \\
\hline Co60 & $5 / 17 / 84$ & NS & NS & $\mathbf{R 8 4 7 1}$ & NS & NS & NS & $5.81 E+\infty$ & uCin & $\mathbf{P}$ & RAW \\
\hline Co60 & $4 / 30 / 84$ & NS & NS & NS & NS & NS & NS & NS & NS & $\mathbf{P}$ & Wastren \\
\hline Cs137 & $6 / 1 / 90$ & $4 / 30 / 89$ & $4 / 27 / 89$ & ВОВ2T9 & G327 & G443 & 1.20 & $2.42 E+02$ & $\mathrm{uCi} / \mathrm{mL}$ & $\mathbf{P}$ & Raw \\
\hline Cs137 & $6 / 1 / 90$ & $4 / 30 / 89$ & $4 / 27 / 89$ & BOB2T9 & G327 & G443 & 1.20 & $2.41 E+02$ & $\mathrm{uCi} / \mathrm{mL}$ & $P$ & Raw \\
\hline Cs137 & 6/1/90 & $4 / 30 / 89$ & $4 / 27 / 89$ & 80B2L9 & G305 & G349 & 1.20 & $2.38 E+02$ & $\mathrm{uCi} / \mathrm{mL}$ & $P$ & Raw \\
\hline Cs137 & $6 / 1 / 90$ & $4 / 30 / 89$ & $4 / 27 / 89$ & BOB2M4 & G313 & G363 & 1.20 & $2.37 E+02$ & $\mathrm{uCi} / \mathrm{mL}$ & $P$ & Raw \\
\hline Cs137 & $6 / 1 / 90$ & $4 / 30 / 89$ & $4 / 27 / 89$ & BOB2L.9 & G305 & G349 & 1.20 & $2.36 E+02$ & $\mathrm{uCi} / \mathrm{mL}$ & $\mathbf{P}$ & Raw \\
\hline Cs137 & $6 / 1 / 90$ & $4 / 30 / 89$ & $4 / 27 / 89$ & BOB2N4 & G323 & G341 & 1.20 & $2.36 E+02$ & $\mathrm{uCi} / \mathrm{mL}$ & $P$ & Raw \\
\hline Cs137 & $6 / 1 / 90$ & $4 / 30 / 89$ & $4 / 27 / 89$ & BOB2L7 & G299 & G342 & 1.20 & $2.35 \mathrm{E}+02$ & $\mathrm{uCi} / \mathrm{mL}$ & $P$ & Raw \\
\hline Cs137 & $6 / 1 / 90$ & $4 / 30 / 89$ & $4 / 27 / 89$ & ВOB2M9 & G302 & G450 & 1.20 & $2.35 E+02$ & $\mathrm{uCi} / \mathrm{mL}$ & $P$ & Raw \\
\hline Cs137 & $6 / 1 / 90$ & $4 / 30 / 89$ & $4 / 27 / 89$ & ВОВ2M4 & G313 & G363 & 1.20 & $2.35 E+02$ & $\mathrm{uCi} / \mathrm{mL}$ & $\mathbf{P}$ & Raw \\
\hline
\end{tabular}




\begin{tabular}{|c|c|c|c|c|c|c|}
\hline \multicolumn{4}{|c|}{ Radionuclide Information } & \multirow[b]{2}{*}{$\begin{array}{c}\text { Reference } \\
\text { Number }\end{array}$} & \multirow[b]{2}{*}{ Validated (Y/N) } & \multirow{3}{*}{$\begin{array}{l}\text { Notes - } \\
\text { Density reported for } \mathrm{G} 358 \text { collected at } 334 \text { in from } \\
\text { bottom of tank at a } 150^{\circ} \text { angle. Sample was } \\
\text { unheated, unheated samples precipitated }\end{array}$} \\
\hline Radoinuclide & Conc. $(\mathrm{Bq} / \mathrm{L})$ & $\begin{array}{l}\text { Conc. }(B q / L \\
\text { decayed) }\end{array}$ & $\begin{array}{l}\text { Conc. (Ci/l } \\
\text { Decayed) }\end{array}$ & & & \\
\hline Co60 & \#VALUEI & \#VALUEI & \#VALUE! & AP102-3 & $\mathbf{Y}$ & \\
\hline Co60 & \#ALUEI & \#VALUE! & *NALUE! & AP102-3 & $\mathbf{Y}$ & $\begin{array}{l}\text { Density reported for } \mathrm{G} 353 \text { collected at } 334 \text { in from } \\
\text { bottom of tank at a } 150^{\circ} \text { angle. Sample was } \\
\text { unheated, unheated samples precipitated }\end{array}$ \\
\hline Co60 & \#AALUEI & \#VALUEl & VALUE! & AP102-3 & $\mathbf{Y}$ & $\begin{array}{l}\text { Density not reported for G333. No assumptions could } \\
\text { be made }\end{array}$ \\
\hline Co60 & NALUEI & *VALUEI & *VALUE! & AP102-3 & $Y$ & $\begin{array}{l}\text { Density reported for } \mathrm{G} 470 \text { represents a composite } \\
\text { sample. Sample was kept heated to } 40^{\circ} \mathrm{C} \text {. }\end{array}$ \\
\hline Co60 & FALUEI & *VALUEi & *VALUE! & AP102-3 & $\mathbf{Y}$ & $\begin{array}{l}\text { Density reported for } \mathrm{G} 359 \text { collected at } 162 \text { in from } \\
\text { bottom of tank at a } 150^{\circ} \text { angle. Sample was } \\
\text { unheated, unheated samples precipitated }\end{array}$ \\
\hline Co60 & $2.99 E+06$ & $2.40 E+06$ & $6.49 E-05$ & AP102-3 & $\mathbf{Y}$ & $\begin{array}{l}\text { Density reported for } 6358 \text { collected at } 334 \text { in from } \\
\text { bottom of tank at a } 150^{\circ} \text { angle. Sample was } \\
\text { unheated, unheated samples precipitated }\end{array}$ \\
\hline Co6o & $2.77 E+06$ & $2.22 E+06$ & 6.01E-05 & AP102-3 & $Y$ & $\begin{array}{l}\text { Density reported for } \mathrm{G} 353 \text { collected at } 334 \text { in from } \\
\text { bottom of tank at a } 150^{\circ} \text { angle. Sample was } \\
\text { unheated, unheated samples precipitated }\end{array}$ \\
\hline Co60 & $2.53 E+06$ & $2.03 E+06$ & $5.49 E-05$ & AP102-3 & $\mathrm{Y}$ & $\begin{array}{l}\text { Density reported for } \mathrm{G} 353 \text { collected at } 334 \text { in from } \\
\text { bottom of tank at a } 150^{\circ} \text { angle. Sample was } \\
\text { unheated, unheated samples precipitated }\end{array}$ \\
\hline Co6o & $2.33 E+06$ & $1.87 E+06$ & $5.05 E-05$ & AP102-3 & $\mathbf{Y}$ & $\begin{array}{l}\text { Density reported for } \mathrm{G} 342 \text { collected at } 306 \text { in from } \\
\text { bottom of tank at a } 30^{\circ} \text { angle. Sample was } \\
\text { unheated, unheated samples precipitated }\end{array}$ \\
\hline Co60 & $2.35 E+05$ & $9.86 E+04$ & $2.66 \mathrm{E}-06$ & AP102-1 & $\mathbf{N}$ & Outdated Information \\
\hline $\mathbf{C o 6 0}$ & $2.27 E+05$ & $9.52 E+04$ & $2.57 E-06$ & AP102-1 & $\mathbf{N}$ & Outdated Information \\
\hline Co60 & $2.18 E+05$ & $9.15 E+04$ & 2.47E-06 & AP102-1 & $N$ & Outdated information \\
\hline Co60 & $2.15 E+05$ & $9.03 E+04$ & 2.44E-06 & AP102-1 & $\mathbf{N}$ & Outdated Information \\
\hline Co60 & $1.26 E+03$ & $5.26 \mathrm{E}+02$ & $1.42 \mathrm{E}-08$ & NA & $\mathbf{N}$ & No Reference \\
\hline Cs137 & $8.95 E+09$ & $8.62 E+09$ & 2.33E-01 & AP102-3 & $\mathbf{Y}$ & $\begin{array}{l}\text { Density reported for } \mathrm{G} 443 \text { represents a composite } \\
\text { sample }\end{array}$ \\
\hline Cs137 & $8.92 E+09$ & $8.58 E+09$ & 2.32E-01 & AP102-3 & $\mathbf{Y}$ & $\begin{array}{l}\text { Density reported for G443 represents a composite } \\
\text { sample }\end{array}$ \\
\hline Csi37 & $8.81 E+09$ & $8.47 E+09$ & 2.29E-01 & AP102-3 & $Y$ & $\begin{array}{l}\text { Density reported for } 6349 \text { collected at } 34 \text { in from } \\
\text { bottom of tank at a } 30^{\circ} \text { angle. Sample was } \\
\text { unheated, unheated samples precipitated }\end{array}$ \\
\hline Cs137 & $8.77 E+09$ & $8.44 E+09$ & $2.28 \mathrm{E}-01$ & AP102-3 & $Y$ & $\begin{array}{l}\text { Density reported for } G 363 \text { collected at } 18 \text { in from } \\
\text { bottom of tank at a } 150^{\circ} \text { angle. Sample was } \\
\text { unheated, unheated samples precipitated }\end{array}$ \\
\hline $\operatorname{Cs137}$ & $8.73 E+09$ & $8.40 E+09$ & 2.27E-01 & AP102-3 & $Y$ & $\begin{array}{l}\text { Density reported for } 6349 \text { collected at } 34 \text { in from } \\
\text { bottom of tank at a } 30^{\circ} \text { angle. Sample was } \\
\text { unheated, unheated samples precipitated }\end{array}$ \\
\hline Cs137 & $8.73 E+09$ & $8.40 E+09$ & 2.27E-01 & AP102-3 & $Y$ & $\begin{array}{l}\text { Density reported for } 6341 \text { collected at } 99 \text { in from } \\
\text { bottom of tank at a } 270^{\circ} \text { angle. }\end{array}$ \\
\hline Cs137 & $8.70 E+09$ & $8.37 E+09$ & $2.26 E-01$ & AP102-3 & $Y$ & $\begin{array}{l}\text { Density reported for } \mathrm{G} 342 \text { collected at } 306 \text { in from } \\
\text { bottom of tank at a } 30^{\circ} \text { angle. Sample was } \\
\text { unheated, unheated samples precipitated }\end{array}$ \\
\hline Cs137 & $8.70 E+09$ & $8.37 E+09$ & $2.26 E-01$ & AP102-3 & $Y$ & $\begin{array}{l}\text { Density not reported for G } 450 \text {. Density assumed } \\
\text { same as G477 which was collected at } 182 \text { in from } \\
\text { bottom of tank at a } 30^{\circ} \text { angle and was keep heated } \\
\text { to } 40^{\circ} \mathrm{C} \text {. }\end{array}$ \\
\hline Csi37 & $8.70 E+09$ & $8.37 E+09$ & $2.26 E-01$ & AP102-3 & $Y$ & $\begin{array}{l}\text { Density reported for } \mathrm{G} 363 \text { collected at } 18 \text { in from } \\
\text { bottom of tank at a } 150^{\circ} \text { angle. Sample was } \\
\text { unheated, unheated samples precipitated }\end{array}$ \\
\hline
\end{tabular}




\begin{tabular}{|c|c|c|c|c|c|c|c|c|c|c|c|}
\hline \multicolumn{12}{|c|}{ Tank Waste Characterization Sample Information } \\
\hline $\begin{array}{c}\text { Reported } \\
\text { Radionuclide }\end{array}$ & $\begin{array}{c}\text { Memo } \\
\text { Date }\end{array}$ & $\begin{array}{c}\text { Start } \\
\text { Analysis } \\
\text { Date }\end{array}$ & $\begin{array}{c}\text { Start } \\
\text { Sample } \\
\text { Date }\end{array}$ & $\begin{array}{l}\text { Sample } \\
\text { Number }\end{array}$ & $\begin{array}{c}\text { Tank Farm } \\
\text { Number }\end{array}$ & $\begin{array}{c}\text { Lab Sample } \\
\text { Number }\end{array}$ & $\begin{array}{c}\text { Density } \\
(\mathrm{g} / \mathrm{mL})\end{array}$ & $\begin{array}{c}\text { Reported } \\
\text { Value } \\
\end{array}$ & Reported Untt & Code & Source \\
\hline Cs137 & $6 / 1 / 90$ & $4 / 30 / 89$ & $4 / 27 / 89$ & BOB2N4 & G323 & G341 & 1.20 & $2.34 E+02$ & $\mathrm{uCl} / \mathrm{mL}$ & $\mathbf{P}$ & Raw \\
\hline Cs137 & $6 / 1 / 90$ & $4 / 30 / 89$ & $4 / 27 / 89$ & BOB2NO & G307 & G453 & NA & $2.33 E+02$ & $\mathrm{uCi} / \mathrm{mL}$ & $\mathbf{P}$ & Raw \\
\hline Cs137 & $6 / 1 / 90$ & $4 / 30 / 89$ & $4 / 27 / 89$ & BOB2NO & G307 & G453 & NA & $2.33 E+02$ & $\mathrm{uCi} / \mathrm{mL}$ & $\mathbf{P}$ & Raw \\
\hline Cs137 & $6 / 1 / 90$ & $4 / 30 / 89$ & $4 / 27 / 89$ & BOB2L7 & G299 & G342 & 1.20 & $2.32 E+02$ & $\mathrm{uCi} / \mathrm{mL}$ & $\mathbf{P}$ & Raw \\
\hline Cs137 & $6 / 1 / 90$ & $4 / 30 / 89$ & $4 / 27 / 89$ & BOB2L8 & G303 & G348 & 1.21 & $2.32 E+02$ & $\mathrm{uCl} / \mathrm{mL}$ & $p$ & Raw \\
\hline Cs137 & $6 / 1 / 90$ & $4 / 30 / 89$ & $4 / 27 / 89$ & BOB2M8 & G301 & G449 & NA & $2.30 E+02$ & $\mathrm{uCi} / \mathrm{mL}$ & $\mathbf{P}$ & Raw \\
\hline Cs137 & $6 / 1 / 90$ & $4 / 30 / 89$ & $4 / 27 / 89$ & В0В2M8 & G301 & G449 & NA & $2.30 E+02$ & $u \mathrm{Ci} / \mathrm{mL}$ & $\mathbf{P}$ & Raw \\
\hline Cs137 & $6 / 1 / 90$ & $4 / 30 / 89$ & $4 / 27 / 89$ & Вов2M9 & G302 & G450 & 1.20 & $2.29 E+02$ & $\mathrm{uCi} / \mathrm{mL}$ & $\mathbf{P}$ & Raw \\
\hline Cs137 & $6 / 1 / 90$ & $4 / 30 / 89$ & $4 / 27 / 89$ & BOB2N1 & G310 & G454 & 1.20 & $2.29 E+02$ & $\mathrm{uCi} / \mathrm{mL}$ & $P$ & Raw \\
\hline $\operatorname{Cs137}$ & $6 / 1 / 90$ & $4 / 30 / 89$ & $4 / 27 / 89$ & BOB2M1 & G308 & G353 & 1.20 & $2.28 E+02$ & $\mathrm{uCi} / \mathrm{mL}$ & $P$ & Raw \\
\hline Cs137 & $6 / 1 / 90$ & $4 / 30 / 89$ & $4 / 27 / 89$ & ВOB2M1 & G308 & G353 & 1.20 & $2.28 E+02$ & uCi/mL - & $\mathbf{P}$ & Raw \\
\hline Cs137 & $6 / 1 / 90$ & $4 / 30 / 89$ & $4 / 27 / 89$ & BOB2N1 & G310 & G454 & 1.20 & $2.28 E+02$ & $\mathrm{uCi} / \mathrm{mL}$ & $P$ & Raw \\
\hline Cs137 & $6 / 1 / 90$ & $4 / 30 / 89$ & $4 / 27 / 89$ & BOB2N3 & G322 & G338 & NA & $2.27 E+02$ & $u \mathrm{Ci} / \mathrm{mL}$ & $\mathbf{P}$ & Raw \\
\hline Cs137 & $6 / 1 / 90$ & $4 / 30 / 89$ & $4 / 27 / 89$ & BOB2N3 & G322 & G338 & NA & $2.26 E+02$ & $\mathrm{uCi} / \mathrm{mL}$ & $\mathbf{P}$ & Raw \\
\hline Cs137 & $6 / 1 / 90$ & $4 / 30 / 89$ & $4 / 27 / 89$ & ВO82MO & G306 & G352 & 1.20 & $2.25 E+02$ & $\mathrm{uCi} / \mathrm{mL}$ & $\mathbf{P}$ & Raw \\
\hline Cs137 & $6 / 1 / 90$ & $4 / 30 / 89$ & $4 / 27 / 89$ & ВOВ2MO & G306 & G352 & 1.20 & $2.25 E+02$ & $\mathrm{uCi} / \mathrm{mL}$ & $\mathbf{P}$ & Raw \\
\hline Cs137 & $6 / 1 / 90$ & $4 / 30 / 89$ & $4 / 27 / 89$ & ВОВ2М3 & G311 & G359 & 1.20 & $2.25 E+02$ & $\mathrm{uCi} / \mathrm{mL}$ & $P$ & Raw \\
\hline Cs137 & $6 / 1 / 90$ & $4 / 30 / 89$ & $4 / 27 / 89$ & $8082 M 3$ & G311 & G359 & 1.20 & $2.25 E+02$ & $\mathrm{uCl} / \mathrm{mL}$ & $\mathbf{P}$ & Raw \\
\hline Cs137 & $6 / 1 / 90$ & 4/30/89 & $4 / 27 / 89$ & BOB2M5 & G315 & G364 & 1.19 & $2.25 E+02$ & $\mathrm{uCI} / \mathrm{mL}$ & $P$ & Raw \\
\hline Cs137 & $6 / 1 / 90$ & $4 / 30 / 89$ & $4 / 27 / 89$ & BOB2N2 & G317 & G333 & NA & $2.25 E+02$ & $\mathrm{uCi} / \mathrm{mL}$ & $\mathbf{P}$ & Raw \\
\hline
\end{tabular}




\begin{tabular}{|c|c|c|c|c|c|c|}
\hline \multicolumn{4}{|c|}{ Radionuclide Information } & \multirow[b]{2}{*}{$\begin{array}{l}\text { Reference } \\
\text { Number }\end{array}$} & \multirow[b]{2}{*}{ Validated $(Y / N)$} & \multirow[b]{2}{*}{ Notes } \\
\hline Radoinuclide & Conc. $(\mathrm{Bq} \Omega \mathrm{L})$ & $\begin{array}{l}\text { Conc. }(\mathrm{Bq} / \mathrm{h} \\
\text { decayed })\end{array}$ & $\begin{array}{l}\text { Conc. (Ci/L } \\
\text { Decayed) }\end{array}$ & & & \\
\hline Cs137 & $8.66 E+09$ & $8.33 E+09$ & $2.25 \mathrm{E}-01$ & AP102-3 & $\mathbf{Y}$ & $\begin{array}{l}\text { Density reported for } G 341 \text { collected at } 99 \text { in from } \\
\text { bottom of tank at a } 270^{\circ} \text { angle. }\end{array}$ \\
\hline Cs137 & $8.62 E+09$ & $8.30 E+09$ & $2.24 E-01$ & AP102-3 & $\mathbf{Y}$ & $\begin{array}{l}\text { Density not reported for G453. No assumptions could } \\
\text { be made }\end{array}$ \\
\hline Cs137 & $8.62 E+09$ & $8.30 E+09$ & $2.24 \mathrm{E}-01$ & AP102-3 & $\mathbf{Y}$ & $\begin{array}{l}\text { Density not reported for G453. No assumptions could } \\
\text { be made }\end{array}$ \\
\hline Cs137 & $8.58 E+09$ & $8.26 E+09$ & $2.23 E-01$ & AP102-3 & $\mathbf{Y}$ & $\begin{array}{l}\text { Density reported for } \mathrm{G} 342 \text { collected at } 306 \text { in from } \\
\text { bottom of tank at a } 30^{\circ} \text { angle. Sample was } \\
\text { unheated, unheated samples precipitated }\end{array}$ \\
\hline Cs137 & $8.58 E+09$ & $\begin{array}{c}8.26 E+09 \\
\end{array}$ & 2.23E-01 & AP102-3 & $\mathbf{Y}$ & $\begin{array}{l}\text { Density reported for } G 348 \text { collected at } 125 \text { in from } \\
\text { bottom of tank at a } 30^{\circ} \text { angle. Sample was } \\
\text { unheated, unheated samples precipitated }\end{array}$ \\
\hline Cs137 & $8.51 E+09$ & $8.19 E+09$ & 2.21E-01 & AP102-3 & $\mathbf{Y}$ & $\begin{array}{l}\text { Density not reported for G449. No assumptions could } \\
\text { be made }\end{array}$ \\
\hline Cs137 & $8.51 E+09$ & $8.19 E+09$ & 2.21E-01 & AP102-3 & $\mathbf{Y}$ & $\begin{array}{l}\text { Density not reported for G449. No assumptions could } \\
\text { be made }\end{array}$ \\
\hline Cs137 & $8.47 E+09$ & $8.15 E+09$ & $2.20 E-01$ & AP102-3 & $\mathbf{Y}$ & $\begin{array}{l}\text { Density not reported for G450. Density assumed } \\
\text { same as } G 477 \text { which was collected at } 182 \text { in from } \\
\text { bottom of tank at a } 30^{\circ} \text { angle and was keep heated } \\
\text { to } 40^{\circ} \mathrm{C} \text {. }\end{array}$ \\
\hline Cs137 & $8.47 E+09$ & $8.15 E+09$ & $2.20 E-01$ & AP102-3 & $\mathbf{Y}$ & $\begin{array}{l}\text { Density not reported for G454. Density assumed } \\
\text { same as G471 which was collected at } 208 \text { in from } \\
\text { bottom of tank at a } 150^{\circ} \text { angle and was keep heated } \\
\text { to } 40^{\circ} \mathrm{C} \text {. }\end{array}$ \\
\hline Cs137 & $8.44 E+09$ & $8.12 E+09$ & 2.19E-01 & AP102-3 & $\mathbf{Y}$ & $\begin{array}{l}\text { Density reported for } 6353 \text { collected at } 334 \text { in from } \\
\text { bottom of tank at a } 150^{\circ} \text { angle. Sample was } \\
\text { unheated, unheated samples precipitated }\end{array}$ \\
\hline Cs137 & $8.44 E+09$ & $8.12 E+09$ & 2.19E-01 & AP102-3 & $\boldsymbol{Y}$ & $\begin{array}{l}\text { Density reported for } \mathrm{G} 353 \text { collected at } 334 \text { in from } \\
\text { bottom of tank at a } 150^{\circ} \text { angle. Sample was } \\
\text { unheated, unheated samples precipitated }\end{array}$ \\
\hline Cs137 & $8.44 E+09$ & $8.12 E+09$ & 2.19E-01 & AP102-3 & $\mathbf{Y}$ & $\begin{array}{l}\text { Density not reported for G454. Density assumed } \\
\text { same as G } 471 \text { which was collected at } 208 \text { in from } \\
\text { bottom of tank at a } 150^{\circ} \text { angle and was keep heated } \\
\text { to } 40^{\circ} \mathrm{C} \text {. }\end{array}$ \\
\hline Cs137 & $8.40 E+09$ & $8.08 E+09$ & $2.18 E-01$ & AP102-3 & $\mathbf{Y}$ & $\begin{array}{l}\text { Density not reported for G338. No assumptions could } \\
\text { be made }\end{array}$ \\
\hline Cs137 & $8.36 E+09$ & $8.05 E+09$ & 2.17E-01 & AP102-3 & $\mathrm{Y}$ & $\begin{array}{l}\text { Density not reported for G338. No assumptions could } \\
\text { be made }\end{array}$ \\
\hline Cs137 & $8.33 E+09$ & $8.01 E+09$ & 2.17E-01 & AP102-3 & $\mathbf{Y}$ & $\begin{array}{l}\text { Density reported for G352 collected at } 34 \text { in from } \\
\text { bottom of tank at a } 30^{\circ} \text { angle. Sample was } \\
\text { unheated, unheated samples precipitated }\end{array}$ \\
\hline Cs137 & $8.33 E+09$ & $8.01 E+09$ & 2.17E-01 & AP102-3 & $\mathbf{Y}$ & $\begin{array}{l}\text { Density reported for G352 collected at } 34 \text { in from } \\
\text { bottom of tank at a } 30^{\circ} \text { angle. Sample was } \\
\text { unheated, unheated samples precipitated }\end{array}$ \\
\hline Cs137 & 8.33E+09 & $8.01 E+09$ & 2.17E-01 & AP102-3 & $\boldsymbol{\gamma}$ & $\begin{array}{l}\text { Density reported for } \mathrm{G} 359 \text { collected at } 162 \text { in from } \\
\text { bottom of tank at a } 150^{\circ} \text { angle. Sample was } \\
\text { unheated, unheated samples precipitated }\end{array}$ \\
\hline Cs137 & $8.33 E+09$ & $8.01 E+09$ & 2.17E-01 & AP102-3 & $\mathbf{Y}$ & $\begin{array}{l}\text { Density reported for } \mathrm{G} 359 \text { collected at } 162 \text { in from } \\
\text { bottom of tank at a } 150^{\circ} \text { angle. Sample was } \\
\text { unheated, unheated samples precipitated }\end{array}$ \\
\hline Cs137 & $8.33 E+09$ & $8.01 E+09$ & 2.17E-01 & AP102-3 & $\mathbf{Y}$ & $\begin{array}{l}\text { Density reported for G364 collected at } 372 \text { in from } \\
\text { bottom of tank at a } 270^{\circ} \text { angle. Sample was } \\
\text { unheated, unheated samples precipitated }\end{array}$ \\
\hline Cs137 & $8.33 E+09$ & $8.01 E+09$ & $2.17 E-01$ & AP102-3 & $\mathbf{Y}$ & $\begin{array}{l}\text { Density not reported for G333. No assumptions could } \\
\text { be made }\end{array}$ \\
\hline
\end{tabular}




\begin{tabular}{|c|c|c|c|c|c|c|c|c|c|c|c|}
\hline \multicolumn{12}{|c|}{ Tank Waste Characterization Sample Information } \\
\hline $\begin{array}{c}\text { Reported } \\
\text { Radionuclide }\end{array}$ & $\begin{array}{c}\text { Memo } \\
\text { Date }\end{array}$ & $\begin{array}{c}\text { Start } \\
\text { Analysis } \\
\text { Date }\end{array}$ & $\begin{array}{c}\text { Start } \\
\text { Sample } \\
\text { Date } \\
\end{array}$ & $\begin{array}{l}\text { Sample } \\
\text { Number }\end{array}$ & $\begin{array}{c}\text { Tank Farm } \\
\text { Number }\end{array}$ & $\begin{array}{c}\text { Lab Sample } \\
\text { Number }\end{array}$ & $\begin{array}{l}\text { Density } \\
(\mathrm{g} / \mathrm{mL})\end{array}$ & $\begin{array}{c}\text { Reported } \\
\text { Value } \\
\end{array}$ & Reported Unit & Codo & Sourc \\
\hline Cs137 & $6 / 1 / 90$ & $4 / 30 / 89$ & $4 / 27 / 89$ & BOB2N4 & G323 & G476* & 1.21 & $2.25 E+02$ & $\mathrm{uCi} / \mathrm{mL}$ & $\mathbf{P}$ & Raw \\
\hline Cs137 & $6 / 1 / 90$ & $4 / 30 / 89$ & $4 / 27 / 89$ & BOB2M1 & G308 & G353 & 1.20 & $2.24 \mathrm{E}+02$ & $\mathrm{uCi} / \mathrm{mL}$ & $\mathbf{P}$ & Raw \\
\hline Cs137 & $6 / 1 / 90$ & $4 / 30 / 89$ & $4 / 27 / 89$ & BOB2N2 & G317 & G333. & NA & $2.24 E+02$ & $-u C i / m L$ & $\mathbf{P}$ & Raw \\
\hline Cs137 & $6 / 1 / 90$ & $4 / 30 / 89$ & $4 / 27 / 89$ & $80 B 2 N 4$ & G323 & G476* & 1.21 & $2.24 E+02$ & $\mathrm{uCi} / \mathrm{mL}$ & $\mathbf{P}$ & Raw \\
\hline Cs137 & $6 / 1 / 90$ & $4 / 30 / 89$ & $4 / 27 / 89$ & ВОВ2M2 & G309 & G358 & 1.20 & $2.23 E+02$ & $\mathrm{uCi} / \mathrm{mL}$ & $P$ & Raw \\
\hline $\operatorname{Cs} 137$ & $6 / 1 / 90$ & $4 / 30 / 89$ & $4 / 27 / 89$ & ВОВ2M9 & G302 & G477* & 1.20 & $2.22 E+02$ & $\mathrm{uCi} / \mathrm{mL}$ & $\mathbf{P}$ & Raw \\
\hline $\operatorname{Cs137}$ & $6 / 1 / 90$ & $4 / 30 / 89$ & $4 / 27 / 89$ & ВОВ2M1 & G308 & G353 & 1.20 & $2.22 E+02$ & $\mathrm{uCi} / \mathrm{mL}$ & $\mathbf{P}$ & Raw \\
\hline Cs137 & $6 / 1 / 90$ & 4/30/89 & $4 / 27 / 89$ & BOB2M5 & G315 & G364 & 1.19 & $2.22 E+02$ & $\mathrm{uCi} / \mathrm{mL}$ & $\mathbf{P}$ & Raw \\
\hline Cs137 & $6 / 1 / 90$ & $4 / 30 / 89$ & $4 / 27 / 89$ & Вов2М9 & G302 & G477* & 1.20 & $2.20 E+02$ & uCi/mL & $\mathbf{P}$ & Raw \\
\hline Cs137 & $6 / 1 / 90$ & $4 / 30 / 89$ & $4 / 27 / 89$ & BOB2M6 & G318 & G459 & 1.20 & $2.19 E+02$ & $\mathrm{uCi} / \mathrm{mL}$ & $P$ & Raw \\
\hline Cs137 & $6 / 1 / 90$ & $4 / 30 / 89$ & $4 / 27 / 89$ & BOB2L8 & G303 & G348 & 1.21 & $2.17 E+02$ & uCi/mL & $P$ & Raw \\
\hline Cs137 & $6 / 1 / 90$ & $4 / 30 / 89$ & $4 / 27 / 89$ & ВОВ2M2 & G309 & G358 & 1.20 & $2.17 E+02$ & $\mathrm{uCi} / \mathrm{mL}$ & $P$ & Raw \\
\hline Cs 137 & $6 / 1 / 90$ & $4 / 30 / 89$ & $4 / 27 / 89$ & ВОВ2T9 & G327 & G470* & 1.19 & $2.17 E+02$ & $\mathrm{uCi} / \mathrm{mL}$ & $\mathbf{P}$ & Raw \\
\hline Cs137 & $6 / 1 / 90$ & $4 / 30 / 89$ & $4 / 27 / 89$ & ВOB2M2 & G309 & G358 & 1.20 & $2.16 E+02$ & $\mathrm{uCi} / \mathrm{mL}$ & $\mathbf{P}$ & Raw \\
\hline Cs137 & $6 / 1 / 90$ & $4 / 30 / 89$ & $4 / 27 / 89$ & ВOB2M7 & G319 & G460 & 1.20 & $2.16 \mathrm{E}+02$ & $\mathrm{uCi} / \mathrm{mL}$ & $\mathbf{P}$ & Raw \\
\hline Cs137 & $6 / 1 / 90$ & $4 / 30 / 89$ & $4 / 27 / 89$ & BOB2M2 & G309 & G358 & 1.20 & $2.15 E+02$ & $\mathrm{uCi} / \mathrm{mL}$ & $\mathbf{P}$ & Raw \\
\hline Cs137 & $6 / 1 / 90$ & $4 / 30 / 89$ & $4 / 27 / 89$ & BOB2N1 & G310 & G471* & 1.20 & $2.15 E+02$ & $\mathrm{uCi} / \mathrm{mL}$ & $P$ & Raw \\
\hline Cs137 & $6 / 1 / 90$ & $4 / 30 / 89$ & $4 / 27 / 89$ & BOB2M6 & G318 & G459 & 1.20 & $2.15 E+02$ & $\mathrm{uCi} / \mathrm{mL}$ & $P$ & Raw \\
\hline Cs137 & $6 / 1 / 90$ & $4 / 30 / 89$ & $4 / 27 / 89$ & вов2T9 & G327 & G470* & 1.19 & $2.15 E+02$ & $\mathrm{uCi} / \mathrm{mL}$ & $\mathbf{P}$ & Raw \\
\hline
\end{tabular}




\begin{tabular}{|c|c|c|c|c|c|c|}
\hline \multicolumn{4}{|c|}{ Radionuclide Information } & \multirow[b]{2}{*}{$\begin{array}{c}\text { Reference } \\
\text { Number }\end{array}$} & \multirow[b]{2}{*}{ Validated $(Y / N)$} & \multirow[b]{2}{*}{ Notes } \\
\hline Radoinuclide & Conc. (Bq/L) & $\begin{array}{c}\text { Conc. }(B q / L- \\
\text { decayed) }\end{array}$ & $\begin{array}{c}\text { Conc. (Ci/L } \\
\text { Decayed) }\end{array}$ & & & \\
\hline Cs137 & $8.33 E+09$ & $8.01 E+09$ & 2.17E-01 & AP102-3 & $\mathbf{Y}$ & $\begin{array}{l}\text { Density reported for } \mathrm{G} 476 \text { collected at } 99 \text { in from } \\
\text { bottom of tank at a } 270^{\circ} \text { angle and was keep heated } \\
\text { to } 40^{\circ} \mathrm{C} \text {. }\end{array}$ \\
\hline Cs137 & $8.29 E+09$ & $7.98 E+09$ & $2.16 E-01$ & AP102-3 & $\mathbf{Y}$ & $\begin{array}{l}\text { Density reported for } \mathrm{G} 353 \text { collected at } 334 \text { in from } \\
\text { bottom of tank at a } 150^{\circ} \text { angle. Sample was } \\
\text { unheated, unheated samples precipitated }\end{array}$ \\
\hline Cs137 & $8.29 E+09$ & $7.98 E+09$ & $2.16 \mathrm{E}-01$ & AP102-3 & $\mathbf{Y}$ & $\begin{array}{l}\text { Density not reported for G333. No assumptions could } \\
\text { be made }\end{array}$ \\
\hline Cs137 & $8.29 E+09$ & $7.98 E+09$ & 2.16E-01 & AP102-3 & $\mathbf{Y}$ & $\begin{array}{l}\text { Density reported for } \mathrm{G} 476 \text { collected at } 99 \text { in from } \\
\text { bottom of tank at a } 270^{\circ} \text { angle and was keep heated } \\
\text { to } 40^{\circ} \mathrm{C} \text {. }\end{array}$ \\
\hline Cs137 & $8.25 E+09$ & $7.94 E+09$ & 2.15E-01 & AP102-3 & $\gamma$ & $\begin{array}{l}\text { Density reported for } \mathrm{G} 358 \text { collected at } 334 \text { in from } \\
\text { bottom of tank at a } 150^{\circ} \text { angle. Sample was } \\
\text { unheated, unheated samples precipitated }\end{array}$ \\
\hline Cs137 & $8.21 E+09$ & $7.90 E+09$ & $2.74 E-01$ & AP102-3 & $\mathbf{Y}$ & $\begin{array}{l}\text { Density reported for } \mathrm{G} 477 \text { collected at } 182 \text { in from } \\
\text { bottom of tank at a } 30^{\circ} \text { angle and was keep heated } \\
\text { to } 40^{\circ} \mathrm{C} \text {. }\end{array}$ \\
\hline Cs137 & $8.21 E+09$ & $7.90 E+09$ & $2.14 \mathrm{E}-01$ & AP102-3 & $\mathbf{Y}$ & $\begin{array}{l}\text { Density reported for } \mathrm{G} 353 \text { collected at } 334 \text { in from } \\
\text { bottom of tank at a } 150^{\circ} \text { angle. Sample was } \\
\text { unheated, unheated samples precipitated }\end{array}$ \\
\hline Cs137 & $8.21 E+09$ & $7.90 E+09$ & 2.14E-01 & AP102-3 & $\mathbf{Y}$ & $\begin{array}{l}\text { Density reported for G364 collected at } 372 \text { in from } \\
\text { bottom of tank at a } 270^{\circ} \text { angle. Sample was } \\
\text { unheated, unheated samples precipitated }\end{array}$ \\
\hline Cs137 & $8.14 E+09$ & $7.83 E+09$ & $2.12 E-01$ & AP102-3 & $Y$ & $\begin{array}{l}\text { Density reported for } \mathrm{G} 477 \text { collected at } 182 \text { in from } \\
\text { bottom of tank at a } 30^{\circ} \text { angle and was keep heated } \\
\text { to } 40^{\circ} \mathrm{C} \text {. }\end{array}$ \\
\hline Cs137. & $8.10 E+09$ & $7.80 E+09$ & 2.11E-01 & AP102-3 & $\mathbf{Y}$ & $\begin{array}{l}\text { Density seported for G459 collected at } 226 \text { in from } \\
\text { bottom of tank at a } 270^{\circ} \text { angle. Sample was } \\
\text { unheated, unheated samples precipitated }\end{array}$ \\
\hline Cs137 & $8.03 E+09$ & $7.73 E+09$ & 2.09E-01 & AP102-3 & $\mathbf{Y}$ & $\begin{array}{l}\text { Density reported for } \mathrm{G} 348 \text { collected at } 125 \text { in from } \\
\text { bottom of tank at a } 30^{\circ} \text { angle. Sample was } \\
\text { unheated, unheated samples precipitated }\end{array}$ \\
\hline Cs137 & $8.03 E+09$ & $7.73 E+09$ & 2.09E-01 & AP102-3 & $\mathbf{Y}$ & $\begin{array}{l}\text { Density reported for } \mathrm{G} 358 \text { collected at } 334 \text { in from } \\
\text { bottom of tank at a } 150^{\circ} \text { angle. Sample was } \\
\text { unheated, unheated samples precipitated }\end{array}$ \\
\hline Cs137 & $8.03 E+09$ & $7.73 E+09$ & 2.09E-01 & AP102-3 & $\mathbf{Y}$ & $\begin{array}{l}\text { Density reported for G470 represents a composite } \\
\text { sample. Sample was kept heated to } 40^{\circ} \mathrm{C} \text {. }\end{array}$ \\
\hline Cs137 & $7.99 E+09$ & $7.69 E+09$ & $2.08 E-01$ & AP102-3 & $\mathbf{Y}$ & $\begin{array}{l}\text { Density reported for } \mathrm{G} 358 \text { collected at } 334 \text { in from } \\
\text { bottom of tank at a } 150^{\circ} \text { angle. Sample was } \\
\text { unheated, unheated samples precipitated }\end{array}$ \\
\hline Cs137 & $7.99 E+09$ & $7.69 E+09$ & 2.08E-01 & AP102-3 & $Y$ & $\begin{array}{l}\text { Density reported for } \mathrm{G} 460 \text { collected at } 226 \text { in from } \\
\text { bottom of tank at a } 270^{\circ} \text { angle. Sample was } \\
\text { unheated, unheated samples precipitated }\end{array}$ \\
\hline Cs137 & $7.96 \mathrm{E}+09$ & $7.66 E+09$ & 2.07E-01 & AP102-3 & $Y$ & $\begin{array}{l}\text { Density reported for } \mathrm{G} 358 \text { collected at } 334 \text { in from } \\
\text { bottom of tank at a } 150^{\circ} \text { angle. Sample was } \\
\text { unheated, unheated samples precipitated }\end{array}$ \\
\hline Cs137 & $7.96 E+09$ & $7.66 \mathrm{E}+09$ & 2.07E-01 & AP102-3 & $\mathbf{Y}$ & $\begin{array}{l}\text { Density reported for } \mathrm{G} 471 \text { collected at } 208 \text { in from } \\
\text { bottom of tank at a } 150^{\circ} \text { angle and was keep heated } \\
\text { to } 40^{\circ} \mathrm{C} \text {. }\end{array}$ \\
\hline Cs137 & $7.96 E+09$ & $7.66 E+09$ & 2.07E-01 & AP102-3 & $Y$ & $\begin{array}{l}\text { Density reported for } \mathrm{G} 459 \text { collected at } 226 \text { in from } \\
\text { bottom of tank at a } 270^{\circ} \text { angle. Sample was } \\
\text { unheated, unheated samples precipitated }\end{array}$ \\
\hline Cs137 & $7.96 E+09$ & $7.66 E+09$ & 2.07E-01 & AP102-3 & $\mathbf{Y}$ & $\begin{array}{l}\text { Density reported for G470 represents a composite } \\
\text { sample. Sample was kept heated to } 40^{\circ} \mathrm{C} \text {. }\end{array}$ \\
\hline
\end{tabular}




\begin{tabular}{|c|c|c|c|c|c|c|c|c|c|c|c|}
\hline \multicolumn{12}{|c|}{ Tank Waste Characterization Sample Information } \\
\hline $\begin{array}{c}\text { Reported } \\
\text { Radionuclide }\end{array}$ & $\begin{array}{c}\text { Memo } \\
\text { Date }\end{array}$ & $\begin{array}{c}\text { Start } \\
\text { Analysis } \\
\text { Date }\end{array}$ & $\begin{array}{c}\text { Start } \\
\text { Sample } \\
\text { Date } \\
\end{array}$ & $\begin{array}{l}\text { Sample } \\
\text { Number }\end{array}$ & $\begin{array}{c}\text { Tank Farm } \\
\text { Number }\end{array}$ & $\begin{array}{c}\text { Lab Sample } \\
\text { Number }\end{array}$ & $\begin{array}{l}\text { Density } \\
(\mathrm{g} / \mathrm{mL})\end{array}$ & $\begin{array}{c}\text { Reported } \\
\text { Value }\end{array}$ & Reported Unit & Code & Source \\
\hline Cs 137 & $6 / 1 / 90$ & $4 / 30 / 89$ & $4 / 27 / 89$ & В0В2M7 & G319 & G460 & 1.20 & $2.12 E+02$ & $\mathrm{uCi} / \mathrm{mL}$ & $P$ & Raw \\
\hline Cs137 & $6 / 1 / 90$ & $4 / 30 / 89$ & $4 / 27 / 89$ & BOB2N1 & G310 & G471* & 1.20 & $2.10 E+02$ & $\mathrm{uCi} / \mathrm{mL}$ & $\mathbf{P}$ & Raw \\
\hline Cs137 & $6 / 1 / 90$ & $4 / 30 / 89$ & $4 / 27 / 89$ & ВОВ2T9 & G327 & G443 & 1.20 & $2.08 E \div 02$ & $\mathrm{uCi} / \mathrm{mL}$ & $P$ & Raw \\
\hline Cs137 & $6 / 1 / 90$ & $4 / 30 / 89$ & $4 / 27 / 89$ & 8OB2T9 & G327 & G443 & 1.20 & $2.03 E+02$ & $\mathrm{uCi} / \mathrm{mL}$ & $P$ & Raw \\
\hline Cs & & $4 / 89$ & $4 / 27 / 89$ & NS & NS & NS & 1.24 & $1.77 \mathrm{E}-01$ & $\mathrm{Cin}$ & $L$ & HTCE Inv. \\
\hline Cs137 & $4 / 30 / 84$ & NS & NS & NS & NS & NS & NS & NS & NS & $P$ & Wastren \\
\hline Cs137 & $5 / 17 / 84$ & NS & NS & R8472 & NS & NS & NS & $4.89 \mathrm{E}-01$ & uCi/L & $P$ & RAW \\
\hline Cs137 & $5 / 17 / 84$ & NS & NS & R8473 & MS & NS & NS & $4.83 \mathrm{E}-01$ & uCin & $P$ & RAW \\
\hline Cs137 & $5 / 17 / 84$ & NS & NS & R8469 & NS & NS & NS & $4.65 \mathrm{E}-01$ & $\mathrm{uCi} / \mathrm{L}$ & $P$ & RAW \\
\hline Cs137 & $5 / 17 / 84$ & NS & NS & R8471 & NS & NS & NS & $4.43 E-01$ & $\mathrm{uCi} / \mathrm{L}$ & $P$ & RAW \\
\hline lodine-129 & '́n/1/90 & $4 / 30 / 89$ & $4 / 27 / 89$ & BOB2N4 & G323 & G476* & 1.21 & $<5.33 E-05$ & $\mathrm{uCi} / \mathrm{mL}$ & $P$ & Raw \\
\hline lodine-129 & $6 / 1 / 90$ & $4 / 30 / 89$ & $4 / 27 / 89$ & ВOB2M9 & G302 & G477* & 1.20 & $<5.05 \mathrm{E}-05$ & $\mathrm{uCi} / \mathrm{mL}$ & $\mathbf{P}$ & Raw \\
\hline lodine- 129 & $6 / 1 / 90$ & $4 / 30 / 89$ & $4 / 27 / 89$ & BOB2T9 & G327 & G470 & 1.19 & $<4.91 E-05$ & $\mathrm{uCi} / \mathrm{mL}$ & $P$ & Raw \\
\hline lodine-129 & $6 / 1 / 90$ & $4 / 30 / 89$ & $4 / 27 / 89$ & ВOB2M9 & G302 & G477* & 1.20 & $<4.83 E-05$ & $\mathrm{uCi} / \mathrm{mL}$ & $P$ & Raw \\
\hline lodine-129 & $6 / 1 / 90$ & $4 / 30 / 89$ & $4 / 27 / 89$ & ВОВ2T9 & G327 & G470* & 1.19 & $<4.78 \mathrm{E}-05$ & $\mathrm{uCi} / \mathrm{mL}$ & $P$ & Raw \\
\hline lodine-129 & $6 / 1 / 90$ & $4 / 30 / 89$ & $4 / 27 / 89$ & BOB2N1 & G310 & G471• & 1.20 & $<4.73 E-05$ & $\mathrm{uCi} / \mathrm{mL}$ & $\mathbf{P}$ & Raw \\
\hline lodine-129 & $6 / 1 / 90$ & $4 / 30 / 89$ & $4 / 27 / 89$ & BOB2N1 & G310 & $\mathrm{G} 471^{\circ}$ & 1.20 & $<4.73 E-05$ & $\mathrm{uCi} / \mathrm{mL}$ & $P$ & Raw \\
\hline lodine-129 & $6 / 1 / 90$ & 4/30/89 & $4 / 27 / 89$ & BOB2N1 & G310 & G471* & 1.20 & $<4.70 E-05$ & $\mathrm{uCi} / \mathrm{mL}$ & $P$ & Raw \\
\hline lodine-129 & $6 / 1 / 90$ & $4 / 30 / 89$ & $4 / 27 / 89$ & BOB2N4 & G323 & G476* & 1.21 & $<4.38 E-05$ & $\mathrm{uCi} / \mathrm{mL}$ & $P$ & Raw \\
\hline lodine-129 & $6 / 1 / 90$ & $4 / 30 / 89$ & $4 / 27 / 89$ & ВОВ2T9 & G327 & G443 & 1.20 & $<3.71 E-05$ & $\mathrm{uCi} / \mathrm{mL}$ & $P$ & Raw \\
\hline lodine-129 & $6 / 1 / 90$ & $4 / 30 / 89$ & $4 / 27 / 89$ & BOB2T9 & G327 & G443 & 1.20 & $<3.33 E-05$ & $\mathrm{uCl} / \mathrm{mL}$ & $P$ & Raw \\
\hline lodine-129 & $6 / 1 / 90$ & $4 / 30 / 89$ & $4 / 27 / 89$ & BOB2N1 & G310 & G471* & 1.20 & $<6.44 E-06$ & $\mathrm{uCi} / \mathrm{mL}$ & $P$ & Raw \\
\hline lodine-129 & $3 / 31 / 89$ & $4 / 89$ & $4 / 27 / 89$ & BOB2M9 & G302 & G477 & 1.20 & $5.49 E-05$ & $\mathrm{uCi} / \mathrm{mL}$ & $P$ & TCD \\
\hline lodine-129 & $3 / 31 / 89$ & $4 / 89$ & $4 / 27 / 89$ & 8082T9 & G327 & NS & NS & $4.91 E-05$ & $\mathrm{uCi} / \mathrm{mL}$ & $\mathbf{P}$ & TCD \\
\hline lodine-129 & $3 / 31 / 89$ & $4 / 89$ & $4 / 27 / 89$ & BOB2T9 & G327 & NS & NS & $4.78 \mathrm{E}-05$ & $\mathrm{uCl} / \mathrm{mL}$ & $P$ & TCD \\
\hline Np237 & $6 / 1 / 90$ & $4 / 30 / 89$ & $4 / 27 / 89$ & вов2M1 & G308 & G353 & 1.20 & $<4.65 E-04$ & $\mathrm{uCl} / \mathrm{mL}$ & $\mathbf{P}$ & Raw \\
\hline
\end{tabular}




\begin{tabular}{|c|c|c|c|c|c|c|}
\hline \multicolumn{4}{|c|}{ Radionuclide Information } & \multirow[b]{2}{*}{$\begin{array}{c}\text { Reference } \\
\text { Number }\end{array}$} & \multirow[b]{2}{*}{ Validated $(Y / N)$} & \multirow[b]{2}{*}{ Notes } \\
\hline Radoinucide & Conc. $(\mathrm{Bq} / \mathrm{L})$ & $\begin{array}{c}\text { Conc. (Bq/L } \\
\text { decayed) }\end{array}$ & $\begin{array}{l}\text { Conc. (Ci/L } \\
\text { Decayed) }\end{array}$ & & & \\
\hline Cs137 .. & $7.84 E+09$ & $7.55 \mathrm{E}+09$ & 2.04E-01 & AP102-3 & $-x$ & $\begin{array}{l}\text { Density reported for } \mathrm{G} 460 \text { collected at } 226 \text { in from } \\
\text { bottom of tank at a } 270^{\circ} \text { angle. Sample was } \\
\text { unheated, unheated samples precipitated }\end{array}$ \\
\hline Cs137 & $7.77 E+09$ & $7.48 \mathrm{E}+09$ & 2.02E-01 & AP102-3 & Y & $\begin{array}{l}\text { Density reported for } \mathrm{G} 471 \text { collected at } 208 \text { in from } \\
\text { bottom of tank at a } 150^{\circ} \text { angle and was keep heated } \\
\text { to } 40^{\circ} \mathrm{C} \text {. }\end{array}$ \\
\hline Cs137 & $7.70 E+09$. & $7.41 E+09$ & 2.00E-01 & AP102-3 & $\mathbf{Y}$ & $\begin{array}{l}\text { Density reported for G443 represents a composite } \\
\text { sample }\end{array}$ \\
\hline Cs137 & $7.51 E+09$ & $7.23 E+09$ & $1.95 \mathrm{E}-01$ & AP102-3 & $\mathbf{Y}$ & $\begin{array}{l}\text { Density reported for G443 represents a composite } \\
\text { sample }\end{array}$ \\
\hline Cs137 & $6.53 E+09$ & $6.53 E+09$ & 1.77E-01 & NA & $\mathbf{N}$ & No Reference \\
\hline Cs137 & $1.81 E+04$ & $1.55 E+04$ & $4.19 \mathrm{E}-07$ & NA & $\mathbf{N}$ & No Reference \\
\hline Cs137 & $1.81 E+04$ & $1.55 E+04$ & 4.19E-07 & AP102-1 & $\mathbf{N}$ & Outdated Information \\
\hline Cs137 & $1.79 E+04$ & $1.53 E+04$ & 4.14E-07 & AP102-1 & $\mathbf{N}$ & Outdated Information \\
\hline Cs137 & $1.72 E+04$ & $1.48 \mathrm{E}+04$ & 4.00E-07 & AP102-1 & $\mathbf{N}$ & Outdated Information \\
\hline Cs137 & $1.64 E+04$ & $1.41 E+04$ & 3.81E-07 & AP102-1 & $N$ & Outdated Information \\
\hline 1129 & \#VALUE! & \#VALUE! & \#VALUE! & AP102-3 & $Y$ & $\begin{array}{l}\text { Density reported for } \mathrm{G} 476 \text { collected at } 99 \text { in from } \\
\text { bottom of tank at a } 270^{\circ} \text { angle and was keep heated } \\
\text { to } 40^{\circ} \mathrm{C} \text {. }\end{array}$ \\
\hline 1129 & \#VALUEI & \#VALUE! & \#VALUE] & AP102-3 & $\mathbf{Y}$ & $\begin{array}{l}\text { Density reported for } \mathrm{G} 477 \text { collected at } 182 \text { in from } \\
\text { bottom of tank at a } 30^{\circ} \text { angle and was keep heated } \\
\text { to } 40^{\circ} \mathrm{C} \text {. }\end{array}$ \\
\hline 1129 & \#ALUE! & \#VALUE! & \#VALUE! & AP102-3 & $Y$ & $\begin{array}{l}\text { Density reported for G470 represents a composite } \\
\text { sample. Sample was kept heated to } 40^{\circ} \mathrm{C} \text {. }\end{array}$ \\
\hline 1129 & \#ALUEl & \#VALUE! & WALUE! & AP102-3 & $\mathbf{Y}$ & $\begin{array}{l}\text { Density reported for } \mathrm{G} 477 \text { collected at } 182 \text { in from } \\
\text { bottom of tank at a } 30^{\circ} \text { angle and was keep heated } \\
\text { to } 40^{\circ} \mathrm{C} \text {. }\end{array}$ \\
\hline 1129 & \#VALUEI & \#VALUEI & NALUE! & AP102-3 & $\mathbf{Y}$ & $\begin{array}{l}\text { Density reported for G470 represents a composite } \\
\text { sample. Sample was kept heated to } 40^{\circ} \mathrm{C} \text {. }\end{array}$ \\
\hline 1129 & \#VALUE! & \#ALUE! & \#ALUE! & AP102-3 & $\mathbf{Y}$ & $\begin{array}{l}\text { Density reported for } \mathrm{G} 471 \text { collected at } 208 \text { in from } \\
\text { bottom of tank at a } 150^{\circ} \text { angle and was keep heated } \\
\text { to } 40^{\circ} \mathrm{C} \text {. }\end{array}$ \\
\hline 1129 & \#VALUE! & \#VALUE! & \#VALUE! & AP102-3 & $\mathbf{Y}$ & $\begin{array}{l}\text { Density reported for } 6471 \text { collected at } 208 \text { in from } \\
\text { bottom of tank at a } 150^{\circ} \text { angle and was keep heated } \\
\text { to } 40^{\circ} \mathrm{C} \text {. }\end{array}$ \\
\hline 1129 & \#VALUE! & \#ALUE! & \#ALUEI & AP102-3 & $Y$ & $\begin{array}{l}\text { Density reported for } \mathrm{G} 471 \text { collected at } 208 \text { in from } \\
\text { bottom of tank at a } 150^{\circ} \text { angle and was keep heated } \\
\text { to } 40^{\circ} \mathrm{C} \text {. }\end{array}$ \\
\hline 1129 & \#ALUE! & \#ALUE! & \#VALUE! & AP102-3 & $\mathbf{Y}$ & $\begin{array}{l}\text { Density reported for } \mathrm{G} 476 \text { collected at } 99 \text { in from } \\
\text { bottom of tank at a } 270^{\circ} \text { angle and was keep heated } \\
\text { to } 40^{\circ} \mathrm{C} \text {. }\end{array}$ \\
\hline 1129 & FVALUEI & \#VALUEl & \#VALUE! & AP102-3 & $Y$ & $\begin{array}{l}\text { Density reported for G443 represents a composite } \\
\text { sample }\end{array}$ \\
\hline 1129 & *VALUEI & NALUE! & \#ALUE! & AP102-3 & $\mathbf{Y}$ & $\begin{array}{l}\text { Density reported for G443 represents a composite } \\
\text { sample }\end{array}$ \\
\hline 1129 & WALUEI & FALUEI & FVALUE! & AP102-3 & $\mathbf{Y}$ & $\begin{array}{l}\text { Density reported for } \mathrm{G} 471 \text { collected at } 208 \text { in from } \\
\text { bottom of tank at a } 150^{\circ} \text { angle and was keep heated } \\
\text { to } 40^{\circ} \mathrm{C} \text {. }\end{array}$ \\
\hline 1129 & $2.03 E+03$ & $2.03 E+03$ & $5.49 E-08$ & NA & N & THIS POINT NOT NEEDED FOR DATA 8 ASE \\
\hline 1129 & $1.82 E+03$ & $1.82 E+03$ & 4.91E-08 & NA & $\mathbf{N}$ & THIS POINT NOT NEEDED FOR DATA BASE \\
\hline 1129 & $1.77 E+03$ & 1.77E +03 & $4.78 \mathrm{E}-08$ & NA & $\mathbf{N}$ & THIS POINT NOT NEEDED FOR DATA BASE \\
\hline Np237 & $\#$ VALUE! & \#VALUE! & NALUEI & AP102-3 & Y & $\begin{array}{l}\text { Density reported for G353 collected at } 334 \text { in from } \\
\text { bottom of tank at a } 150^{\circ} \text { angle. Sample was } \\
\text { unheated, unheated samples precipitated }\end{array}$ \\
\hline
\end{tabular}




\begin{tabular}{|c|c|c|c|c|c|c|c|c|c|c|c|}
\hline$\cdots$ & & & & Tank Wast & Characteriz: & Ion Sample In & mation & & & & \\
\hline $\begin{array}{c}\text { Reported } \\
\text { Radionuclide }\end{array}$ & $\begin{array}{c}\text { Memo } \\
\text { Date }\end{array}$ & $\begin{array}{c}\text { Start } \\
\text { Analysis } \\
\text { Date } \\
\end{array}$ & $\begin{array}{c}\text { Start } \\
\text { Sample } \\
\text { Date } \\
\end{array}$ & $\begin{array}{l}\text { Sample } \\
\text { Number }\end{array}$ & $\begin{array}{c}\text { Tank Farm } \\
\text { Number }\end{array}$ & $\begin{array}{c}\text { Lab Sample } \\
\text { Number }\end{array}$ & $\begin{array}{l}\text { Density } \\
\text { (g/mL) }\end{array}$ & $\begin{array}{c}\text { Reported } \\
\text { Value }\end{array}$ & Reported Untt & Code & Sourca \\
\hline Np237 & $6 / 1 / 90$ & $4 / 30 / 89$ & $4 / 27 / 89$ & BOB2M2 & G309 & G358 & 1.20 & $<4.65 E-04$ & $\mathrm{uCi} / \mathrm{mL}$ & $\mathbf{P}$ & Raw \\
\hline Np237 & $6 / 1 / 90$ & $4 / 30 / 89$ & $4 / 27 / 89$ & ВОВ2M9 & G3O2 & G477* & 1.20 & $<4.64 E-04$ & $\mathrm{uCi} / \mathrm{mL}$ & $\mathbf{P}$ & Raw \\
\hline Np237 & $6 / 1 / 90$ & $4 / 30 / 89$ & $4 / 27 / 89$ & BOB2N1 & G310 & G471* & 1.20 & $<4.64 E-04$ & $\mathrm{UCi} / \mathrm{mL}$ & $\mathbf{P}$ & Raw \\
\hline Np237 & $6 / 1 / 90$ & $4 / 30 / 89$ & $4 / 27 / 89$ & BOB2N4 & G323 & G476* & 1.21 & $<4.64 \mathrm{E}-04$ & $u C i / m L$ & $\mathbf{P}$ & Raw \\
\hline$x^{\mathrm{Np237}}$ & $6 / 1 / 90$ & $4 / 30 / 89$ & $4 / 27 / 89$ & BOB2M9 & G302 & G477* & 1.20 & $<4.32 E-04$ & $\mathrm{uCi} / \mathrm{mL}$ & $P$ & Raw \\
\hline Np237 & $6 / 1 / 90$ & $4 / 30 / 89$ & $4 / 27 / 89$ & BOB2M4 & G313 & G363 & 1.20 & $<4.32 \mathrm{E}-04$ & $\mathrm{uCi} / \mathrm{mL}$ & $P$ & Raw \\
\hline Np237 & $6 / 1 / 90$ & $4 / 30 / 89$ & $4 / 27 / 89$ & В082M6 & G318 & G459 & 1.20 & $<4.32 E-04$ & $\mathrm{uCi} / \mathrm{mL}$ & $P$ & Raw \\
\hline Np237 & $6 / 1 / 90$ & $4 / 30 / 89$ & $4 / 27 / 89$ & BOB2M7 & G319 & G460 & 1.20 & $<4.32 E-04$ & $\mathrm{uCl} / \mathrm{mL}$ & $P$ & Raw \\
\hline $\mathrm{Np237}$ & $6 / 1 / 90$ & $4 / 30 / 89$ & $4 / 27 / 89$ & BOB2N4 & G323 & G341 & 1.20 & $<4.32 E-04$ & $\mathrm{uCi} / \mathrm{mL}$ & $P$ & Raw \\
\hline Np237 & $6 / 7 / 90$ & $4 / 30 / 89$ & $4 / 27 / 89$ & в082T9 & G327 & G470* & 1.19 & $<4.32 E-04$ & $\mathrm{uCi} / \mathrm{mL}$ & $\mathbf{P}$ & Raw \\
\hline Np237 & $6 / 1 / 90$ & $4 / 30 / 89$ & $4 / 27 / 89$ & BOB2L7 & G299 & G342 & 1.20 & $<3.98 E-04$ & $\mathrm{uCi} / \mathrm{mL}$ & $P$ & Raw \\
\hline Np237 & $6 / 1 / 90$ & $4 / 30 / 89$ & $4 / 27 / 89$ & BOB2L8 & G303 & G348 & 1.21 & $<3.89 \mathrm{E}-04$ & $u C i / m L$ & $\mathbf{P}$ & Raw \\
\hline Np237 & $6 / 1 / 90$ & $4 / 30 / 89$ & $4 / 27 / 89$ & BOB2L8 & G303 & G348 & 1.21 & $<3.89 E-04$ & $\mathrm{uCi} / \mathrm{mL}$ & $\mathbf{P}$ & Raw \\
\hline Np237 & $6 / 1 / 90$ & $4 / 30 / 89$ & $4 / 27 / 89$ & BOB2L9 & G305 & G349 & 1.20 & $<3.89 E-04$ & $\mathrm{uCi} / \mathrm{mL}$ & $\mathbf{P}$ & Raw \\
\hline Np237 & $6 / 1 / 90$ & $4 / 30 / 89$ & $4 / 27 / 89$ & BOB2L9 & G305 & G349 & 1.20 & $<3.89 \mathrm{E}-04$ & $\mathrm{uCi} / \mathrm{mL}$ & $P$ & Raw \\
\hline Np237 & $6 / 1 / 90$ & $4 / 30 / 89$ & $4 / 27 / 89$ & ВОВ2MO & G306 & G352 & 1.20 & $<3.89 E-04$ & $u \mathrm{Ci} / \mathrm{mL}$ & $\mathbf{P}$ & Raw \\
\hline Np237 & $6 / 1 / 90$ & $4 / 30 / 89$ & $4 / 27 / 89$ & ВОВ2MO & G306 & G352 & 1.20 & $<3.89 E-04$ & $\mathrm{uCi} / \mathrm{mL}$ & $\mathbf{P}$ & Raw \\
\hline Np237 & $6 / 1 / 90$ & $4 / 30 / 89$ & $4 / 27 / 89$ & BOB2M1 & G308 & G353 & 1.20 & $<3.89 E-04$ & $\mathrm{uCl} / \mathrm{mL}$ & $\mathbf{P}$ & Raw \\
\hline Np237 & $6 / 1 / 90$ & 4/30/89 & $4 / 27 / 89$ & 8OB2M3 & G311 & G359 & 1.20 & $<3.89 E-0.4$ & $\mathrm{uCi} / \mathrm{mL}$ & $P$ & Raw \\
\hline
\end{tabular}




\begin{tabular}{|c|c|c|c|c|c|c|}
\hline \multicolumn{4}{|c|}{ Radionuclide Information } & \multirow[b]{2}{*}{$\begin{array}{l}\text { Reference } \\
\text { Number }\end{array}$} & \multirow[b]{2}{*}{ Validated $(Y / N)$} & \multirow[b]{2}{*}{ Notes } \\
\hline Radoinuclide & Conc. $(\mathrm{Bq} / \mathrm{L})$ & $\begin{array}{l}\text { Cone. (8q/L } \\
\text { decayed) }\end{array}$ & $\begin{array}{l}\text { Conc. (Cin } \\
\text { Decayed) }\end{array}$ & & & \\
\hline Np237 & FALUE! & \#VALUE! & NALUE! & AP102-3 & $\mathbf{Y}$ & $\begin{array}{l}\text { Density reported for } 6358 \text { collected at } 334 \text { in from } \\
\text { bottom of tank at a } 150^{\circ} \text { angle. Sample was } \\
\text { unheated, unheated samples precipitated }\end{array}$ \\
\hline Np237 & \#VALUE! & \#VALUEI & \#VALUEI & AP102-3 & $\mathbf{Y}$ & $\begin{array}{l}\text { Density reported for } \mathrm{G} 477 \text { collected at } 182 \text { in from } \\
\text { bottom of tank at a } 30^{\circ} \text { angle and was keep heated } \\
\text { to } 40^{\circ} \mathrm{C} \text {. }\end{array}$ \\
\hline Np237 & VALUE! & \#VALUEI & FVALUE! & AP102-3 & $\mathbf{Y}$ & $\begin{array}{l}\text { Density reported for } \mathrm{G} 471 \text { collected at } 208 \text { in from } \\
\text { bottom of tank at a } 150^{\circ} \text { angle and was keep heated } \\
\text { to } 40^{\circ} \mathrm{C} \text {. }\end{array}$ \\
\hline Np237 & \#ALUE! & \#ALUE! & *VALUE! & AP102-3 & $\mathbf{Y}$ & $\begin{array}{l}\text { Density reported for } 6476 \text { collected at } 99 \text { in from } \\
\text { bottom of tank at a } 270^{\circ} \text { angle and was keep heated } \\
\text { to } 40^{\circ} \mathrm{C} \text {. }\end{array}$ \\
\hline Np237 & *Value! & Walue! & NALUE! & AP102-3 & $\mathbf{Y}$ & $\begin{array}{l}\text { Density reported for G477 collected at } 182 \text { in from } \\
\text { bottom of tank at a } 30^{\circ} \text { angle and was keep heated } \\
\text { to } 40^{\circ} \mathrm{C} \text {. }\end{array}$ \\
\hline Np237 & \#VALUE! & \#VALUE! & FVALUE! & AP102-3 & $\mathbf{Y}$ & $\begin{array}{l}\text { Density reported for } 6363 \text { collected at } 18 \text { in from } \\
\text { bottom of tank at a } 150^{\circ} \text { angle. Sample was } \\
\text { unheated, unheated samples precipitated }\end{array}$ \\
\hline Np237 & \#VALUE! & IVALUE! & \#VALUE! & AP102-3 & $\mathbf{Y}$ & $\begin{array}{l}\text { Density reported for } 6459 \text { collected at } 226 \text { in from } \\
\text { bottom of tank at a } 270^{\circ} \text { angle. Sample was } \\
\text { unheated, unheated samples precipitated }\end{array}$ \\
\hline Np237 & \#VALUE! & \#VALUE! & \#VALUE! & AP102-3 & $\mathbf{Y}$ & $\begin{array}{l}\text { Density reported for } G 460 \text { collected at } 226 \text { in from } \\
\text { bottom of tank at a } 270^{\circ} \text { angle. Sample was } \\
\text { unheated, unheated samples precipitated }\end{array}$ \\
\hline Np237 & \#VALUE! & \#alue! & \#VALUE! & AP102-3 & $Y$ & $\begin{array}{l}\text { Density reported for } 6341 \text { collected at } 99 \text { in from } \\
\text { bottom of tank at a } 270^{\circ} \text { angle. }\end{array}$ \\
\hline Np237 & \#VALUE! & *alue! & *VALUEI & AP102-3 & $\mathbf{Y}$ & $\begin{array}{l}\text { Density reported for } \mathrm{G} 470 \text { represents a composite } \\
\text { sample. Sample was kept heated to } 40^{\circ} \mathrm{C} \text {. }\end{array}$ \\
\hline Np237 & \#VALUE! & \#ALUE! & NaLUE! & AP102-3 & $\mathbf{Y}$ & $\begin{array}{l}\text { Density reported for } \mathrm{G} 342 \text { collected at } 306 \text { in from } \\
\text { bottom of tank at a } 30^{\circ} \text { angle. Sample was } \\
\text { unheated, unheated samples precipitated }\end{array}$ \\
\hline Np237 & *VALUEI & *VALUE! & * VALUE! & AP102-3 & $\mathbf{Y}$ & $\begin{array}{l}\text { Density reported for G348 collected at } 125 \text { in from } \\
\text { bottom of tank at a } 30^{\circ} \text { angle. Sample was } \\
\text { unheated, unheated samples precipitated }\end{array}$ \\
\hline Np237 & \#ALUEI & NALUE! & NALUEI & AP102-3 & $\boldsymbol{\gamma}$ & $\begin{array}{l}\text { Density reported for } \mathrm{G} 348 \text { collected at } 125 \text { in from } \\
\text { bottom of tank at a } 30^{\circ} \text { angle. Sample was } \\
\text { unheated, unheated samples precipitated }\end{array}$ \\
\hline Np237 & *VALUEI & * VALUEI & * VALUEI & AP102-3 & $Y$ & $\begin{array}{l}\text { Density reported for } \mathrm{G} 349 \text { collected at } 34 \text { in from } \\
\text { bottom of tank at a } 30^{\circ} \text { angle. Sample was } \\
\text { unheated, unheated samples precipitated }\end{array}$ \\
\hline Np237 & *ValueI & NaLUEI & *VALUEl & AP102-3 & $\mathbf{Y}$ & $\begin{array}{l}\text { Density reported for G349 collected at } 34 \text { in from } \\
\text { bottom of tarik at a } 30^{\circ} \text { angle. Sample was } \\
\text { unheated, unheated samples precipitated }\end{array}$ \\
\hline Np237 & *Value! & NaLUE! & NALUEI & AP102-3 & $\mathbf{Y}$ & $\begin{array}{l}\text { Density reported for } \mathrm{G352} \text { collected at } 34 \text { in from } \\
\text { bottom of tank at a } 30^{\circ} \text { angle. Sample was } \\
\text { unheated, unheated samples precipitated }\end{array}$ \\
\hline Np237 & *VALUEI & *VALUEI & VALUEI & AP102-3 & $\mathbf{Y}$ & $\begin{array}{l}\text { Density reported for } \mathrm{G} 352 \text { collected at } 34 \text { in from } \\
\text { bottom of tank at a } 30^{\circ} \text { angle. Sample was } \\
\text { unheated. unheated samples precipitated }\end{array}$ \\
\hline Np237 & FALUE! & NALUEI & ॠVALUEI & AP102-3 & $\mathbf{Y}$ & $\begin{array}{l}\text { Density reported for } \mathrm{G} 353 \text { collected at } 334 \text { in from } \\
\text { bottom of tank at a } 150^{\circ} \text { angle. Sample was } \\
\text { unheated, unheated samples precipitated }\end{array}$ \\
\hline Np237 & FALUEI & VALUEI & NALUEI & AP102-3 & $\mathbf{Y}$ & $\begin{array}{l}\text { Density reported for G359 collected at } 162 \text { in from } \\
\text { bottom of tank at a } 150^{\circ} \text { angle. Sample was } \\
\text { unheated, unheated samples precipitated }\end{array}$ \\
\hline
\end{tabular}




\begin{tabular}{|c|c|c|c|c|c|c|c|c|c|c|c|}
\hline \multicolumn{12}{|c|}{ Tank Waste Characterization Sample Information } \\
\hline $\begin{array}{c}\text { Reported } \\
\text { Radionuclide }\end{array}$ & $\begin{array}{c}\text { Memo } \\
\text { Date }\end{array}$ & $\begin{array}{c}\text { Start } \\
\text { Analysis } \\
\text { Date }\end{array}$ & $\begin{array}{c}\text { Start } \\
\text { Sample } \\
\text { Date } \\
\end{array}$ & $\begin{array}{l}\text { Sample } \\
\text { Number }\end{array}$ & $\begin{array}{c}\text { Tank Farm } \\
\text { Number }\end{array}$ & $\begin{array}{c}\text { Lab Sample } \\
\text { Number }\end{array}$ & $\begin{array}{l}\text { Density } \\
(\mathrm{g} / \mathrm{mL})\end{array}$ & $\begin{array}{c}\text { Reported } \\
\text { Value }\end{array}$ & Reported Unit & Code & Source \\
\hline Np237 & $6 / 1 / 90$ & $4 / 30 / 89$ & $4 / 27 / 89$ & BOB2N4 & G323 & G341 & .1 .20 & $<3.89 E-04$ & $\mathrm{uCi} / \mathrm{mL}$ & $\mathbf{P}$ & Raw \\
\hline Np237 & $6 / 1 / 90$ & $4 / 30 / 89$ & $4 / 27 / 89$ & BOB2N4 & G323 & G476* & 1.21 & $<3.89 E-04$ & $\mathrm{uCl} / \mathrm{mL}$ & $P$ & Raw \\
\hline Np237 & $6 / 1 / 90$ & $4 / 30 / 89$ & $4 / 27 / 89$ & 8OB2M6 & G318 & G459 & 1.20 & $<3.46 E-04$ & $\mathrm{uCi} / \mathrm{mL}$ & $\mathbf{P}$ & Raw \\
\hline Np237 & $6 / 1 / 90$ & $4 / 30 / 89$ & $4 / 27 / 89$ & ВОВ2M7 & G319 & G460 & 1.20 & $<3.46 \mathrm{E}-04$ & $\mathrm{uCi} / \mathrm{mL}$ & $P$ & Raw \\
\hline Np237 & $6 / 1 / 90$ & $4 / 30 / 89$ & $4 / 27 / 89$ & ВОВ2M1 & G308 & G353 & 1.20 & $<2.81 E-04$ & $\mathrm{uCi} / \mathrm{mL}$ & $P$ & Raw \\
\hline Np237 & $6 / 1 / 90$ & $4 / 30 / 89$ & $4 / 27 / 89$ & ВОВ2M1 & G308 & G353 & 1.20 & $<2.81 E-04$ & $\mathrm{uCi} / \mathrm{mL}$ & $P$ & Raw \\
\hline Np237 & $6 / 1 / 90$ & $4 / 30 / 89$ & $4 / 27 / 89$ & BOB2N1 & G310 & G471* & 1.20 & $<2.81 E-04$ & $\mathrm{uCi} / \mathrm{mL}$ & $P$ & Raw \\
\hline Np237 & $6 / 1 / 90$ & $4 / 30 / 89$ & $4 / 27 / 89$ & В0В2М3 & G311 & G359 & 1.20 & $<2.81 E-04$ & $\mathrm{uCi} / \mathrm{mL}$ & $P$ & Raw \\
\hline Np237 & $6 / 1 / 90$ & 4/30/89 & $4 / 27 / 89$ & Вов2M4 & G313 & G363 & 1.20 & $<2.81 E-0.4$ & $\mathrm{uCi} / \mathrm{mL}$ & $P$ & Raw \\
\hline Np237 & $6 / 1 / 90$ & $4 / 30 / 89$ & $4 / 27 / 89$ & В0В2M5 & G315 & G364 & 1.19 & $<2.81 E-04$ & $\mathrm{uCi} / \mathrm{mL}$ & $P$ & Raw \\
\hline Np237 & $6 / 1 / 90$ & $4 / 30 / 89$ & $4 / 27 / 89$ & BOB2M5 & G315。 & G364 & 1.19 & $<2.81 E-04$ & $\mathrm{uCi} / \mathrm{mL}$ & $P$ & Raw \\
\hline Np237 & $6 / 1 / 90$ & $4 / 30 / 89$ & $4 / 27 / 89$ & ВОВ2T9 & G327 & G470 & 1.19 & $<2.81 E-04$ & $\mathrm{uCi} / \mathrm{mL}$ & $P$ & Raw \\
\hline Np237 & $6 / 1 / 90$ & $4 / 30 / 89$ & $4 / 27 / 89$ & BOB2M2 & G309 & G358 & 1.20 & $<2.18 \mathrm{E}-04$ & uCi/mL & $P$ & Raw \\
\hline Np237 & $6 / 1 / 90$ & $4 / 30 / 89$ & $4 / 27 / 89$ & BOB2L7 & G299 & G342 & 1.20 & $1.00 E-03$ & $\mathrm{uCi} / \mathrm{mL}$ & $P$ & Raw \\
\hline Pu238 & $6 / 1 / 90$ & $4 / 30 / 89$ & $4 / 27 / 89$ & BOB2N1 & G310 & G471 & 1.20 & $<2.17 E-04$ & $\mathrm{uCi} / \mathrm{mL}$ & $\mathbf{P}$ & Raw \\
\hline Pu238 & $6 / 1 / 90$ & $4 / 30 / 89$ & 4/27/89 & ВОВ2M2 & G309 & G358 & 1.20 & $<1.63 E-04$ & $\mathrm{uCi} / \mathrm{mL}$ & $P$ & Raw \\
\hline Pu238 & $6 / 1 / 90$ & $4 / 30 / 89$ & $4 / 27 / 89$ & ВОВ2M2 & G309 & G358 & 1.20 & $<1.56 \mathrm{E}-04$ & uCi/mL & $\mathbf{P}$ & Raw \\
\hline Pu238 & $6 / 1 / 90$ & $4 / 30 / 89$ & $4 / 27 / 89$ & ВОВ2M3 & G311 & G359 & 1.20 & $<1.50 E-04$ & $\mathrm{uCi} / \mathrm{mL}$ & $P$ & Raw \\
\hline Pu238 & $6 / 1 / 90$ & $4 / 30 / 89$ & $4 / 27 / 89$ & BOB2N1 & G310 & G471 • & 1.20 & $<1.42 E-04$ & $u C i / m L$ & $P$ & Raw \\
\hline
\end{tabular}


aste Characterization Database Tank 241-AP-102

\begin{tabular}{|c|c|c|c|c|c|c|}
\hline & Radionuclide & rmation & & & & \\
\hline Radoinuclide" & Conc. (Bq $/ \mathrm{L})$ & $\begin{array}{l}\text { Conc. }(B q / L \\
\text { decayed }\end{array}$ & $\begin{array}{l}\text { Conc. (Ci/L } \\
\text { Decayed) }\end{array}$ & $\begin{array}{c}\text { Reference } \\
\text { Number }\end{array}$ & Validated $(\mathbf{Y} / \mathbf{N})$ & Notes \\
\hline $\mathrm{Np} 237$ & \#ALUE! & NALUE! & \#ALUEI & AP102-3 & $-1,-\pi-1,0$ & $\begin{array}{l}\text { Density reported for } \mathrm{G} 341 \text { collected at } 99 \text { in from - } \\
\text { bottom of tank at a } 270^{\circ} \text { angle. }\end{array}$ \\
\hline Np237 & \#VALUE! & *VALUE! & \#VALUE! & AP102-3 & $\mathbf{Y}$ & $\begin{array}{l}\text { Density reported for } \mathrm{G} 476 \text { collected at } 99 \text { in from } \\
\text { bottom of tank at a } 270^{\circ} \text { angle and was keep heated } \\
\text { to } 40^{\circ} \mathrm{C} \text {. }\end{array}$ \\
\hline Np237 & FALUEI & *VALUE! & "VALUE! & AP102-3 & $\mathbf{Y}$ & $\begin{array}{l}\text { Density reported for } 6459 \text { collected at } 226 \text { in from } \\
\text { bottom of tank at a } 270^{\circ} \text { angle. Sample was } \\
\text { unheated, unheated samples precipitated }\end{array}$ \\
\hline Np237 & FVALUE! & FALUE! & \#ALUE! & AP102-3 & $\mathbf{Y}$ & $\begin{array}{l}\text { Density reported for G460 collected at } 226 \text { in from } \\
\text { bottom of tank at a } 270^{\circ} \text { angle. Sample was } \\
\text { unheated, unheated samples precipitated }\end{array}$ \\
\hline Np237 & * VALUEI & * VALUEI & \#VALUE! & AP102-3 & $\mathbf{Y}$ & $\begin{array}{l}\text { Density reported for } \mathrm{G} 353 \text { collected at } 334 \text { in from } \\
\text { bottom of tank at a } 150^{\circ} \text { angle. Sample was } \\
\text { unheated, unheated samples precipitated }\end{array}$ \\
\hline Np237 & \#VALUE! & \#ALUE! & \#VALUE! & AP102-3 & $\mathbf{Y}$ & $\begin{array}{l}\text { Density reported for } \mathrm{G} 353 \text { collected at } 334 \text { in from } \\
\text { bottom of tank at a } 150^{\circ} \text { angle. Sample was } \\
\text { unheated, unheated samples precipitated }\end{array}$ \\
\hline Np237 & \#VALUE! & NALUE! & \#VALUE! & AP102-3 & $\mathbf{Y}$ & $\begin{array}{l}\text { Density reported for } \mathbf{G 4 7 1} \text { collected at } 208 \text { in from } \\
\text { bottom of tank at a } 150^{\circ} \text { angle and was keep heated } \\
\text { to } 40^{\circ} \mathrm{C} \text {. }\end{array}$ \\
\hline Np237 & \#VALUE! & *VALUE! & \#VALUE! & AP102-3 & $\mathbf{Y}$ & $\begin{array}{l}\text { Density reported for } \mathbf{G 3 5 9} \text { collected at } 162 \text { in from } \\
\text { bottom of tank at a } 150^{\circ} \text { angle. Sample was } \\
\text { unheated, unheated samples precipitated }\end{array}$ \\
\hline Np237 & \#VALUEI & \#VALUE! & \#VALUE! & AP102-3 & $\mathbf{Y}$ & $\begin{array}{l}\text { Density reported for } \mathrm{G} 363 \text { collected at } 18 \text { in from } \\
\text { bottom of tank at a } 150^{\circ} \text { angle. Sample was } \\
\text { unheated, unheated samples precipitated }\end{array}$ \\
\hline Np237 & \#VALUE! & * VALUE! & FVALUE! & AP102-3 & $\mathbf{Y}$ & $\begin{array}{l}\text { Density reported for } \mathrm{G} 364 \text { collected at } 372 \text { in from } \\
\text { bottom of tank at a }-270^{\circ} \text { angle. Sample was } \\
\text { unheated, unheated samples precipitated }\end{array}$ \\
\hline Np237 & \#VALUE! & FVALUE! & \#VALUE! & AP102-3 & $Y$ & $\begin{array}{l}\text { Density reported for } \mathrm{G} 364 \text { collected at } 372 \text { in from } \\
\text { bottom of tank at a } 270^{\circ} \text { angle. Sample was } \\
\text { unheated, unheated samples precipitated }\end{array}$ \\
\hline Np237 & *VALUE! & \#ALUE! & \#VALUEI & AP102-3 & $\mathbf{Y}$ & $\begin{array}{l}\text { Density reported for } \mathrm{G} 470 \text { represents a composite } \\
\text { sample. Sample was kept heated to } 40^{\circ} \mathrm{C} \text {. }\end{array}$ \\
\hline No237 & \#VALUE! & \#ALLUE! & NALUEI & AP102-3 & $Y$ & $\begin{array}{l}\text { Density reported for } 6358 \text { collected at } 334 \text { in from } \\
\text { bottom of tank at a } 150^{\circ} \text { angle. Sample was } \\
\text { unheated, unheated samples precipitated }\end{array}$ \\
\hline Np237 & $3.70 E+0.4$ & $3.70 E+04$ & $1.00 E-06$ & AP102-3 & $Y$ & $\begin{array}{l}\text { Density reported for } \mathrm{G} 342 \text { collected at } 306 \text { in from } \\
\text { bottom of tank at a } 30^{\circ} \text { angle. Sample was } \\
\text { unheated, unheated samples precipitated }\end{array}$ \\
\hline Pu238 & FALUEI & NALUE! & \#VALUE! & AP102-3 & $\mathbf{Y}$ & $\begin{array}{l}\text { Density reported for } \mathrm{G} 471 \text { collected at } 208 \text { in from } \\
\text { bottom of tank at a } 150^{\circ} \text { angle and was keep heated } \\
\text { to } 40^{\circ} \mathrm{C} \text {. }\end{array}$ \\
\hline Pu238 & * VALUE! & FALUE! & *VALUE! & AP102-3 & $\mathbf{Y}$ & $\begin{array}{l}\text { Density reported for } \mathrm{G} 358 \text { collected at } 334 \text { in from } \\
\text { bottom of tank at a } 150^{\circ} \text { angle. Sample was } \\
\text { unheated, unheated samples precipitated }\end{array}$ \\
\hline Pu238 & N VALUEI & FVALUE! & FVALUEI & AP102-3 & $Y$ & $\begin{array}{l}\text { Density reported for } \mathbf{G 3 5 8} \text { collected at } 334 \text { in from } \\
\text { bottom of tank at a } 150^{\circ} \text { angle. Sample was } \\
\text { unheated, unheated samples precipitated }\end{array}$ \\
\hline Pu238 & NALUEI & FVALUEI & \#VALUE! & AP102-3 & $Y$ & $\begin{array}{l}\text { Density reported for } 6359 \text { collected at } 162 \text { in from } \\
\text { bottom of tank at a } 150^{\circ} \text { angle. Sample was } \\
\text { unheated, unheated samples precipitated }\end{array}$ \\
\hline Pu238 & VALUE! & NALUEI & NALUEI & AP102-3 & $\mathbf{Y}$ & $\begin{array}{l}\text { Density reported for } \mathrm{G} 471 \text { collected at } 208 \text { in from } \\
\text { bottom of tank at a } 150^{\circ} \text { angle and was keep heated } \\
\text { to } 40^{\circ} \mathrm{C} \text {. }\end{array}$ \\
\hline
\end{tabular}




\begin{tabular}{|c|c|c|c|c|c|c|c|c|c|c|c|}
\hline \multicolumn{12}{|c|}{ Tank Waste Characterization Sample Information } \\
\hline $\begin{array}{c}\text { Reported } \\
\text { Radionuclide }\end{array}$ & $\begin{array}{c}\text { Memo } \\
\text { Date }\end{array}$ & $\begin{array}{c}\text { Start } \\
\text { Analysis } \\
\text { Date }\end{array}$ & $\begin{array}{c}\text { Start } \\
\text { Sample } \\
\text { Date }\end{array}$ & $\begin{array}{l}\text { Sample } \\
\text { Number }\end{array}$ & $\begin{array}{c}\text { Tank Farm } \\
\text { Number }\end{array}$ & $\begin{array}{c}\text { Lab Sample } \\
\text { Number }\end{array}$ & $\begin{array}{l}\text { Density } \\
(\mathrm{g} / \mathrm{mL})\end{array}$ & $\begin{array}{c}\text { Reported } \\
\text { Value }\end{array}$ & Reported Unit & Code & Source \\
\hline Pu238 & $6 / 1 / 90$ & $4 / 30 / 89$ & $4 / 27 / 89$ & B082T9 & G327 & G470* & 1.19 & $<1.25 E-04$ & $\mathrm{uCi} / \mathrm{mL}$ & $\mathbf{P}$ & Raw \\
\hline Pu238 & $6 / 1 / 90$ & $4 / 30 / 89$ & $4 / 27 / 89$ & ВOB2M1 & G308 & G353 & 1.20 & $<1.23 E-04$ & uCi/mL & $\mathbf{P}$ & Raw \\
\hline Pu238 & $6 / 1 / 90$ & $4 / 30 / 89$ & $4 / 27 / 89$ & BOB2M5 & G315 & G364 & 1.19 & $<1.22 \mathrm{E}-04$ & uCi/mL & $\mathbf{P}$ & gaw \\
\hline Pu238 & $6 / 1 / 90$ & $4 / 30 / 89$ & $4 / 27 / 89$ & ВOB2M1 & G308 & G353 & 1.20 & $<1.14 E-0.4$ & $\mathrm{uCi} / \mathrm{mL}$ & $P$ & Raw \\
\hline Pu238 & $6 / 1 / 90$ & $4 / 30 / 89$ & $4 / 27 / 89$ & BOB2MO & G306 & G352 & 1.20 & $<1.11 E-04$ & $\mathrm{uCi} / \mathrm{mL}$ & $P$ & Raw \\
\hline Pu238 & $6 / 1 / 90$ & $4 / 30 / 89$ & $4 / 27 / 89$ & BOB2N4 & G323 & G341 & 1.20 & $<1.08 E-04$ & $\mathrm{uCi} / \mathrm{mL}$ & $P$ & Raw \\
\hline Pu238 & $6 / 1 / 90$ & $4 / 30 / 89$ & $4 / 27 / 89$ & вов2M7 & G319 & G460 & 1.20 & $<1.02 E-04$ & uCi/mL & $P$ & Raw \\
\hline Pu238 & $6 / 1 / 90$ & $4 / 30 / 89$ & $4 / 27 / 89$ & ВОВ2MO & G306 & G352 & 1.20 & $<1.01 E-04$ & $\mathrm{uCi} / \mathrm{mL}$ & $P$ & Raw \\
\hline Pu238 & $6 / 1 / 90$ & $4 / 30 / 89$ & $4 / 27 / 89$ & BOB2M6 & G318 & G459 & 1.20 & $<1.01 E-04$ & $\mathrm{uCi} / \mathrm{mL}$ & $P$ & Raw \\
\hline Pu238 & $6 / 1 / 90$ & $4 / 30 / 89$ & $4 / 27 / 89$ & B082N4 & G323 & G476" & 1.21 & $<1.01 E-04$ & $\mathrm{uCi} / \mathrm{mL}$ & 8 & Raw \\
\hline Pu238 & $6 / 1 / 90$ & $4 / 30 / 89$ & $4 / 27 / 89$ & BOB2L] & G299 & G342 & 1.20 & $<1.00 \mathrm{E}-04$ & $\mathrm{uCi} / \mathrm{mL}$ & $P$ & Raw \\
\hline Pu23B & $6 / 1 / 90$ & $4 / 30 / 89$ & $4 / 27 / 89$ & BOB2M4 & G313 & G363 & 1.20 & $<9.88 E-05$ & $\mathrm{uCi} / \mathrm{mL}$ & $P$ & Raw \\
\hline Pu238 & $6 / 1 / 90$ & $4 / 30 / 89$ & $4 / 27 / 89$ & BOB2L.9 & G305 & G349 & 1.20 & $<9.87 E-05$ & $\mathrm{uCi} / \mathrm{mL}$ & $P$ & Raw \\
\hline Pu238 & $6 / 1 / 90$ & $4 / 30 / 89$ & $4 / 27 / 89$ & BOB2L9 & G305 & G349 & 1.20 & $<9.82 E-05$ & $\mathrm{uCi} / \mathrm{mL}$ & $P$ & Raw \\
\hline Pu238 & $6 / 1 / 90$ & $4 / 30 / 89$ & $4 / 27 / 89$ & BOB2M7 & G319 & G460 & $1.20^{\circ}$ & $<9.75 E-05$ & $\mathrm{uCi} / \mathrm{mL}$ & $P$ & Raw \\
\hline Pu238 & $6 / 1 / 90$ & $4 / 30 / 89$ & $4 / 27 / 89$ & BOB2L8 & G303 & G348 & 1.21 & $<9.64 E-05$ & uCi/mL & $\mathbf{P}$ & Raw \\
\hline Pu238 & $6 / 1 / 90$ & $4 / 30 / 89$ & $4 / 27 / 89$ & B082M9 & G302 & G477" & 1.20 & $<9.50 E-05$ & $\mathrm{uCi} / \mathrm{mL}$ & $P$ & Raw \\
\hline Pu238 & $6 / 1 / 90$ & $4 / 30 / 89$ & $4 / 27 / 89$ & BOB2L8 & G303 & G348 & 1.21 & $<9.48 E-05$ & $\mathrm{uCi} / \mathrm{mL}$ & $P$ & Raw \\
\hline Pu238 & $6 / 1 / 90$ & 4/30/89 & $4 / 27 / 89$ & BOB2M4 & G313 & G363 & 1.20 & $<9.40 E-05$ & $\mathrm{uCi} / \mathrm{mL}$ & $P$ & Raw \\
\hline
\end{tabular}




\begin{tabular}{|c|c|c|c|c|c|c|}
\hline \multicolumn{4}{|c|}{ Radionuclide Information } & \multirow[b]{2}{*}{$\begin{array}{l}\text { Referance } \\
\text { Number }\end{array}$} & \multirow[b]{2}{*}{ Validated $(Y / N)$} & \multirow[b]{2}{*}{ Notes } \\
\hline Radoinuclide & Conc. (Bq/L) & $\begin{array}{c}\text { Conc. }(\mathrm{Bq} / \mathrm{L} \\
\text { decayed) }\end{array}$ & $\begin{array}{c}\text { Conc. (Ci/L } \\
\text { Decayed) }\end{array}$ & & & \\
\hline Pu238 & \#VALUE! & \#VALUE! & \#VALUE! & AP102-3 & $\mathbf{Y}$ & $\begin{array}{l}\text { Density reported for } \mathrm{G} 470 \text { represents a composite } \\
\text { sample. Sample was kept heated to } 40^{\circ} \mathrm{C} \text {. }\end{array}$ \\
\hline Pu238 & \#VALUE! & \#ALUE! & \#ALUE! & AP102-3 & $\mathbf{Y}$ & $\begin{array}{l}\text { Density reported for G353 collected at } 334 \text { in from } \\
\text { bottom of tank at a } 150^{\circ} \text { angle. Sample was } \\
\text { unheated, unheated samples precipitated }\end{array}$ \\
\hline Pu238 & \#ALUE! & \#VALUE! & \#ALUE! & AP102-3 & $\mathbf{Y}$ & $\begin{array}{l}\text { Density reported for G364 collected at } 372 \text { in from } \\
\text { bottom of tank at a } 270^{\circ} \text { angle. Sample was } \\
\text { unheated, unheated samples precipitated }\end{array}$ \\
\hline Pu238 & \#VALUE! & \#VALUE! & \#VALUE! & AP102-3 & $Y$ & $\begin{array}{l}\text { Density reported for } \mathrm{G} 353 \text { collected at } 334 \text { in from } \\
\text { bottom of tank at a } 150^{\circ} \text { angle. Sample was } \\
\text { unheated, unheated samples precipitated }\end{array}$ \\
\hline Pu238 & \#VALUE! & \#VALUE! & \#VALUEI & AP102-3 & $Y$ & $\begin{array}{l}\text { Density reported for G352 collected at } 34 \text { in from } \\
\text { bottom of tank at a } 30^{\circ} \text { angle. Sample was } \\
\text { unheated, unheated samples precipitated }\end{array}$ \\
\hline Pu238 & \#VALUE! & \#VALUE! & WALUE! & AP102-3 & $Y$ & $\begin{array}{l}\text { Density reported for } \mathrm{G} 341 \text { collected at } 99 \text { in from } \\
\text { bottom of tank at a } 270^{\circ} \text { angle. }\end{array}$ \\
\hline Pu238 & \#VALUE! & \#VALUE! & \#VALUE! & AP102-3 & $Y$ & $\begin{array}{l}\text { Density reported for } \mathrm{G} 460 \text { collected at } 226 \text { in from } \\
\text { bottom of tank at a } 270^{\circ} \text { angle. Sample was } \\
\text { unheated, unheated samples precipitated }\end{array}$ \\
\hline Pu238 & \#ALUE! & \#VALUE! & \#ALUE! & AP102-3 & $\mathbf{Y}$ & $\begin{array}{l}\text { Density reported for } \mathrm{G} 352 \text { collected at } 34 \text { in from } \\
\text { bottom of tank at a } 30^{\circ} \text { angle. Sample was } \\
\text { unheated, unheated samples precipitated }\end{array}$ \\
\hline Pu238 & \#VALUE! & \#VALUE! & \#VALUE! & AP102-3 & $\mathbf{Y}$ & $\begin{array}{l}\text { Density reported for } \mathrm{G} 459 \text { collected at } 226 \text { in from } \\
\text { bottom of tank at a } 270^{\circ} \text { angle. Sample was } \\
\text { unheated, unheated samples precipitated }\end{array}$ \\
\hline Pu238 & \#VALUE! & \#VALUE! & \#VALUE! & AP102-3 & $\gamma$ & $\begin{array}{l}\text { Density reported for } \mathrm{G} 476 \text { collected at } 99 \text { in from } \\
\text { bottom of tank at a } 270^{\circ} \text { angle and was keep heated } \\
\text { to } 40^{\circ} \mathrm{C} \text {. }\end{array}$ \\
\hline Pu238 & \#VALUE! & \#VALUE! & \#VALUE! & AP102-3 & $\mathbf{Y}$ & $\begin{array}{l}\text { Density reported for G342 collected at } 306 \text { in from } \\
\text { bottom of tank at a } 30^{\circ} \text { angle. Sample was } \\
\text { unheated, unheated samples precipitated }\end{array}$ \\
\hline Pu238 & NALUEI & \#VALUEI & \#VALUE! & AP102-3 & $\mathbf{Y}$ & $\begin{array}{l}\text { Density reported for } \mathrm{G} 363 \text { collected at } 18 \text { in from } \\
\text { bottom of tank at a } 150^{\circ} \text { angle. Sample was } \\
\text { unheated, unheated samples precipitated }\end{array}$ \\
\hline Pu238 & \#VALUE! & FALUE! & \#VALUE! & AP102-3 & $Y$ & $\begin{array}{l}\text { Density reported for } \mathrm{G} 349 \text { collected at } 34 \text { in from } \\
\text { bottom of tank at a } 30^{\circ} \text { angle. Sample was } \\
\text { unheated, unheated samples precipitated }\end{array}$ \\
\hline Pu238 & \#VALUEI & \#VALUE! & \#VALUE! & AP102-3 & $Y$ & $\begin{array}{l}\text { Density reported for } G 349 \text { collected at } 34 \text { in from } \\
\text { bottom of tank at a } 30^{\circ} \text { angle. Sample was } \\
\text { unheated, unheated samples precipitated }\end{array}$ \\
\hline Pu238 & *aLue! & \#VALUEI & \#ALUE! & AP102-3 & $Y$ & $\begin{array}{l}\text { Density reported for } \mathrm{G} 460 \text { collected at } 226 \text { in from } \\
\text { bottom of tank at a } 270^{\circ} \text { angle. Sample was } \\
\text { unheated, unheated samples precipitated }\end{array}$ \\
\hline Pu238 & * VALUE! & \#VALUE! & \#VALUE! & AP102-3 & $Y$ & $\begin{array}{l}\text { Density reported for G348 collected at } 125 \text { in from } \\
\text { bottom of tank at a } 30^{\circ} \text { angle. Sample was } \\
\text { unheated, unheated samples precipitated }\end{array}$ \\
\hline Pu238 & \#ALUE! & \#VALUE! & \#VALUEI & AP102-3 & $\gamma$ & $\begin{array}{l}\text { Density reported for } \mathrm{G} 477 \text { collected at } 182 \text { in from } \\
\text { bottom of tank at a } 30^{\circ} \text { angle and was keep heated } \\
\text { to } 40^{\circ} \mathrm{C} \text {. }\end{array}$ \\
\hline $\begin{array}{c}\text { Pu238 } \\
.\end{array}$ & \#ALUE! & \#VALUE! & \#ALUEI & AP102-3 & $Y$ & $\begin{array}{l}\text { Density reported for } \mathrm{G} 348 \text { collected at } 125 \text { in from } \\
\text { bottom of tank at a } 30^{\circ} \text { angle. Sample was } \\
\text { unheated, unheated samples precipitated }\end{array}$ \\
\hline Pu238 & *VALUE! & "VALUE! & \#VALUE! & AP102-3 & $\gamma$ & $\begin{array}{l}\text { Density reported for } G 363 \text { collected at } 18 \text { in from } \\
\text { bottom of tank at a } 150^{\circ} \text { angle. Sample was } \\
\text { unheated, unheated samples precipitated }\end{array}$ \\
\hline
\end{tabular}




\begin{tabular}{|c|c|c|c|c|c|c|c|c|c|c|c|}
\hline \multicolumn{12}{|c|}{ Tank Waste Characterization Sample Information } \\
\hline $\begin{array}{c}\text { Reported } \\
\text { Radionuclide }\end{array}$ & $\begin{array}{c}\text { Memo } \\
\text { Date }\end{array}$ & $\begin{array}{c}\text { Start } \\
\text { Analysis } \\
\text { Date }\end{array}$ & $\begin{array}{c}\text { Start } \\
\text { Sample } \\
\text { Date } \\
\end{array}$ & $\begin{array}{l}\text { Sample } \\
\text { Number }\end{array}$ & $\begin{array}{c}\text { Tank Farm } \\
\text { Number }\end{array}$ & $\begin{array}{l}\text { Lab Sample } \\
\text { Number }\end{array}$ & $\begin{array}{c}\text { Density } \\
(\mathrm{g} / \mathrm{mL})\end{array}$ & $\begin{array}{c}\text { Reported } \\
\text { Value } \\
\end{array}$ & Reported Unit & Code & Source \\
\hline Pu238 & $6 / 1 / 90$ & $4 / 30 / 89$ & $4 / 27 / 89$ & BOB2M9 & G302 & G477* & 1.20 & $<9.34 E-05$ & uCi/mL & $P$ & Raw \\
\hline Pu238 & $6 / 1 / 90$ & $4 / 30 / 89$ & $4 / 27 / 89$ & Вов2M5 & G315 & G364 & 1.19 & $<9.34 E-05$ & $\mathrm{uCi} / \mathrm{mL}$ & $\mathbf{P}$ & Raw \\
\hline Pu238 & $6 / 1 / 90$ & $4 / 30 / 89$ & $4 / 27 / 89$ & ВOB2T9 & G327 & G470* & 1.19 & $<9.11 E-05$ & $\mathrm{uCi} / \mathrm{mL}$ & $\mathbf{P}$ & Raw \\
\hline Pu238 & $6 / 1 / 90$ & $4 / 30 / 89$ & $4 / 27 / 89$ & BOB2L7 & G299 & G342 & 1.20 & $<9.08 E-05$ & $\mathrm{uCi} / \mathrm{mL}$ & $P$ & Raw \\
\hline Pu238 & $6 / 1 / 90$ & $4 / 30 / 89$ & $4 / 27 / 89$ & ВOB2M3 & G311 & 6359 & 1.20 & $<9.06 E-05$ & $\mathrm{uCi} / \mathrm{mL}$ & $\mathbf{p}$ & Raw \\
\hline Pu238 & $6 / 1 / 90$ & $4 / 30 / 89$ & $4 / 27 / 89$ & ВОВ2М6 & G318 & G459 & 1.20 & $<9.03 E-05$ & $\mathrm{uCi} / \mathrm{mL}$ & $P$ & Raw \\
\hline Pu238 & $6 / 1 / 90$ & $4 / 30 / 89$ & $4 / 27 / 89$ & BOB2N4 & G323 & G341 & 1.20 & $<8.99 E-05$ & $\mathrm{uCi} / \mathrm{mL}$ & $P$ & Raw \\
\hline Pu238 & $6 / 1 / 90$ & $4 / 30 / 89$ & $4 / 27 / 89$ & BOB2N4 & G323 & G476* & 1.21 & $<8.80 E-05$ & $\mathrm{uCi} / \mathrm{mL}$ & $P$ & Raw \\
\hline Pu239/240 & $6 / 1 / 90$ & $4 / 30 / 89$ & $4 / 27 / 89$ & BOB2N4 & G323 & G341 & 1.20 & $<7.48 \mathrm{E}-05$ & uCi/mL & $\mathbf{P}$ & Raw \\
\hline Pu239/240 & $6 / 1 / 90$ & $4 / 30 / 89$ & $4 / 27 / 89$ & BOB2N4 & G323 & G341 & 1.20 & $<7.44$ E-05 & $\mathrm{uCi} / \mathrm{mL}$ & $P$ & Raw \\
\hline Pu239/240 & $6 / 1 / 90$ & $4 / 30 / 89$ & $4 / 27 / 89$ & BOB2M5 & G315 & G364 & 1.19 & $<6.99 E-05$ & $\mathrm{uCi} / \mathrm{mL}$ & $P$ & Paw \\
\hline Pu239/240 & $6 / 1 / 90$ & $4 / 30 / 89$ & $4 / 27 / 89$ & BOB2L8 & G303 & G348 & 1.21 & $<6.96 E-05$ & $\mathrm{uCl} / \mathrm{mL}$ & $P$ & Raw \\
\hline Pu239/240 & $6 / 1 / 90$ & $4 / 30 / 89$ & $4 / 27 / 89$ & BOB2L9 & G305 & G349 & 1.20 & $<6.96 E-05$ & $\mathrm{uCi} / \mathrm{mL}$ & $P$ & Raw \\
\hline Pu239/240 & $6 / 1 / 90$ & $4 / 30 / 89$ & $4 / 27 / 89$ & ВОВ2M9 & G302 & G477* & 1.20 & $<6.90 E-05$ & $\mathrm{uCi} / \mathrm{mL}$ & $P$ & Raw \\
\hline Pu239/240 & $6 / 1 / 90$ & $4 / 30 / 89$ & $4 / 27 / 89$ & BOB2M6 & G318 & G459 & 1.20 & $<6.90 \mathrm{E}-05$ & $\mathrm{uCi} / \mathrm{mL}$ & $P$ & Raw \\
\hline Pu239/240 & $6 / 1 / 90$ & $4 / 30 / 89$ & $4 / 27 / 89$ & B082M5 & G315 & G364 & 1.19 & $<6.89 E-05$ & $\mathrm{uCi} / \mathrm{mL}$ & 8 & Raw \\
\hline Pu239/240 & $6 / 1 / 90$ & $4 / 30 / 89$ & $4 / 27 / 89$ & вов2M6 & G318 & G459 & 1.20 & $<6.88 E-05$ & $\mathrm{uCi} / \mathrm{mL}$ & $P$ & Raw \\
\hline Pu239/240 & $6 / 1 / 90$ & $4 / 30 / 89$ & $4 / 27 / 89$ & BOB2N4 & G323 & G476* & 1.21 & $<6.88 \mathrm{E}-05$ & $\mathrm{uCi} / \mathrm{mL}$ & $\mathbf{P}$ & Raw \\
\hline Pu239/240 & $6 / 1 / 90$ & $4 / 30 / 89$ & $4 / 27 / 89$ & BOB2L8 & G303 & G348 & 1.21 & $<6.87 E-05$ & $\mathrm{uCi} / \mathrm{mL}$ & $P$ & Raw \\
\hline Pu239/240 & $6 / 1 / 90$ & $4 / 30 / 89$ & $4 / 27 / 89$ & BOB2L9 & G305 & G349 & 1.20 & $<6.86 E-05$ & $\mathrm{uCi} / \mathrm{mL}$ & $\mathbf{P}$ & Raw \\
\hline
\end{tabular}




\begin{tabular}{|c|c|c|c|c|c|c|}
\hline \multicolumn{4}{|c|}{ Radionuclide Information } & \multirow[b]{2}{*}{$\begin{array}{l}\text { Reference } \\
\text { Number }\end{array}$} & \multirow[b]{2}{*}{ Validated (Y/N) } & \multirow[b]{2}{*}{ Notes } \\
\hline Radoinuclide & Conc. (Bq/L) & $\begin{array}{l}\text { Conc. }(B q / L \\
\text { decayed) }\end{array}$ & $\begin{array}{l}\text { Conc. (Ci/l } \\
\text { Decayed) }\end{array}$ & & & \\
\hline Pu238 & \#VALUE! & \#VALUE! & \#VALUE! & AP102-3 & $\log ^{-1}$ & $\begin{array}{l}\text { Density reported for } \mathrm{G} 477 \text { collected at } 182 \text { in from } \\
\text { bottom of tank at a } 30^{\circ} \text { angle and was keep heated } \\
\text { to } 40^{\circ} \mathrm{C} \text {. }\end{array}$ \\
\hline Pu238 & \#VALUE! & \#VALUE! & *VALUE! & AP102-3 & $\gamma$ & $\begin{array}{l}\text { Density reported for } \mathrm{G} 364 \text { collected at } 372 \text { in from } \\
\text { bottom of tank at a } 270^{\circ} \text { angle. Sample was } \\
\text { unheated, unheated samples precipitated }\end{array}$ \\
\hline Pu238 & Falue! & \#VALUE! & NALUEI & AP102-3 & $\mathbf{Y}$ & $\begin{array}{l}\text { Density reported for } \mathrm{G} 470 \text { represents a composite } \\
\text { sample. Sample was kept heated to } 40^{\circ} \mathrm{C} \text {. }\end{array}$ \\
\hline Pu238 & \#VALUE! & \#VALUE! & * VAlUE! & AP102-3 & $Y$ & $\begin{array}{l}\text { Density reported for } \mathrm{G} 342 \text { collected at } 306 \text { in from } \\
\text { bottom of tank at a } 30^{\circ} \text { angle. Sample was } \\
\text { unheated, unheated samples precipitated }\end{array}$ \\
\hline Pu238 & \#VALUE! & \#VALUE! & "VALUEI & AP102-3 & $Y$ & $\begin{array}{l}\text { Density reported for } \mathrm{G} 359 \text { collected at } 162 \text { in from } \\
\text { bottom of tank at a } 150^{\circ} \text { angle. Sample was } \\
\text { unheated, unheated samples precipitated }\end{array}$ \\
\hline Pu238 & \#ALUE! & \#VALUE! & \#VALUE! & AP102-3 & $\mathbf{Y}$ & $\begin{array}{l}\text { Density reported for } 6459 \text { collected at } 226 \text { in from } \\
\text { bottom of tank at a } 270^{\circ} \text { angle. Sample was } \\
\text { unheated, unheated samples precipitated }\end{array}$ \\
\hline Pu238 & \#ALUE! & \#VALUE! & *VALUE! & AP102-3 & $Y$ & $\begin{array}{l}\text { Density reported for } \mathrm{G} 341 \text { collected at } 99 \text { in from } \\
\text { bottom of tank at a } 270^{\circ} \text { angle. }\end{array}$ \\
\hline Pu238 & \#VALUE! & \#VALUE! & VALUE! & AP102-3 & $Y$ & $\begin{array}{l}\text { Density reported for G476 collected at } 99 \text { in from } \\
\text { bottom of tank at a } 270^{\circ} \text { angle and was keep heated } \\
\text { to } 40^{\circ} \mathrm{C} \text {. }\end{array}$ \\
\hline Pu239/240 & \#ALUE! & \#VALUE! & \#VALUE! & AP102-3 & Y & $\begin{array}{l}\text { Density reported for } \mathrm{G} 341 \text { collected at } 99 \text { in from } \\
\text { bottom of tank at a } 270^{\circ} \text { angle. }\end{array}$ \\
\hline Pu239/240 & \#VALUE! & \#VALUE! & \#VALUE! & AP102-3 & $\mathbf{Y}$ & $\begin{array}{l}\text { Density reported for G341 collected at } 99 \text { in from } \\
\text { bottom of tank at a } 270^{\circ} \text { angle. }\end{array}$ \\
\hline Pu239/240 & \#VALUEI & VALUEI & \#ALUE! & AP102-3 & $\mathbf{Y}$ & $\begin{array}{l}\text { Density reported for } \mathrm{G} 364 \text { collected at } 372 \text { in from } \\
\text { bottom of tank at a } 270^{\circ} \text { angle. Sample was } \\
\text { unheated, unheated samples precipitated }\end{array}$ \\
\hline Pu239/240 & FALUE! & *VALUE! & *alue! & AP102-3 & $\mathbf{Y}$ & $\begin{array}{l}\text { Density reported for } \mathrm{G} 348 \text { collected at } 125 \text { in from } \\
\text { bottom of tank at a } 30^{\circ} \text { angle. Sample was } \\
\text { unheated, unheated samples precipitated }\end{array}$ \\
\hline Pu239/240 & \#VALUE! & \#VALUE! & \#VALUE! & AP102-3 & $\mathbf{Y}$ & $\begin{array}{l}\text { Density reported for } \mathrm{G} 349 \text { collected at } 34 \text { in from } \\
\text { bottom of tank at a } 30^{\circ} \text { angle. Sample was } \\
\text { unheated, unheated samples precipitated }\end{array}$ \\
\hline Pu239/240 & \#ALUE! & \#ALUE! & \#VALUE! & AP102-3 & $\gamma$ & $\begin{array}{l}\text { Density reported for } \mathrm{G} 477 \text { collected at } 182 \text { in from } \\
\text { bottom of tank at a } 30^{\circ} \text { angle and was keep heated } \\
\text { to } 40^{\circ} \mathrm{C} \text {. }\end{array}$ \\
\hline Pu239/240 & \#VALUE! & \#VALUE! & \#VALUE! & AP102-3 & $Y$ & $\begin{array}{l}\text { Density reported for } 6459 \text { collected at } 226 \text { in from } \\
\text { bottom of tank at a } 270^{\circ} \text { angle. Sample was } \\
\text { unheated, unheated samples precipitated }\end{array}$ \\
\hline Pu239/240 & \#ALUE! & \#ALUE! & *VALUEI & AP102-3 & $\gamma$ & $\begin{array}{l}\text { Density reported for G364 collected at } 372 \text { in from } \\
\text { bottom of tank at a } 270^{\circ} \text { angle. Sample was } \\
\text { unheated, unheated samples precipitated }\end{array}$ \\
\hline Pu239/240 & NALUE! & \#VALUEI & *VALUEI & AP102-3 & $\mathbf{Y}$ & $\begin{array}{l}\text { Density reported for G } 459 \text { collected at } 226 \text { in from } \\
\text { bottom of tank at a } 270^{\circ} \text { angle. Sample was } \\
\text { unheated, unheated samples precipitated }\end{array}$ \\
\hline Pu239/240 & *VALUE! & *VALUEI & *VALUE! & AP102-3 & $Y$ & $\begin{array}{l}\text { Density reported for } \mathrm{G} 476 \text { collected at } 99 \text { in from } \\
\text { bottom of tank at a } 270^{\circ} \text { angle and was keep heated } \\
\text { to } 40^{\circ} \mathrm{C} \text {. }\end{array}$ \\
\hline Pu239/240 & \#ALUE! & \#VALUEI & VALUEI & AP102-3 & $\gamma$ & $\begin{array}{l}\text { Density reported for } G 348 \text { collected at } 125 \text { in from } \\
\text { bottom of tank at a } 30^{\circ} \text { angle. Sample was } \\
\text { unheated, unheated samples precipitated }\end{array}$ \\
\hline Pu239/240 & WALUE! & *VALUE! & NALUE! & AP102-3 & $\gamma$ & $\begin{array}{l}\text { Density reported for G349 collected at } 34 \text { in from } \\
\text { bottom of tank at a } 30^{\circ} \text { angle. Sample was } \\
\text { unheated, unheated samples precipitated }\end{array}$ \\
\hline
\end{tabular}




\begin{tabular}{|c|c|c|c|c|c|c|c|c|c|c|c|}
\hline \multicolumn{12}{|c|}{ Tank Waste Characterization Sample Information } \\
\hline $\begin{array}{c}\text { Reported } \\
\text { Radionuclide }\end{array}$ & $\begin{array}{c}\text { Memo } \\
\text { Date }\end{array}$ & $\begin{array}{c}\text { Start } \\
\text { Analysis } \\
\text { Date }\end{array}$ & $\begin{array}{c}\text { Start } \\
\text { Sample } \\
\text { Date }\end{array}$ & $\begin{array}{l}\text { Sample } \\
\text { Number }\end{array}$ & $\begin{array}{c}\text { Tank Farm } \\
\text { Numbar }\end{array}$ & $\begin{array}{c}\text { Lab Sample } \\
\text { Number }\end{array}$ & $\begin{array}{l}\text { Density } \\
(\mathrm{g} / \mathrm{mL})\end{array}$ & $\begin{array}{c}\text { Reported } \\
\text { Yalue }\end{array}$ & Reported Unit & Code & Source \\
\hline Pu239/240 & $6 / 1 / 90$ & $4 / 30 / 89$ & $4 / 27 / 89$ & В082M4 & G313 & G363 & 1.20 & $<6.86 E-05$ & uCi/mL & $\mathbf{P}$ & Raw \\
\hline Pu239/240 & $6 / 1 / 90$ & $4 / 30 / 89$ & $4 / 27 / 89$ & BOB2M7 & G319 & G460 & 1.20 & $<6.86 \mathrm{E}-05$ & $\mathrm{uCi} / \mathrm{mL}$ & $\mathbf{P}$ & Raw \\
\hline Pu239/240 & $6 / 1 / 90$ & $4 / 30 / 89$ & $4 / 27 / 89$ & BOB2M2 & G309 & G358 & 1.20 & $<6.82 E-05$ & uCi/mL & $\mathbf{P}$ & Raw \\
\hline Pu239/240 & $6 / 1 / 90$ & $4 / 30 / 89$ & $4 / 27 / 89$ & ВОВ2M3 & G311 & G359 & 1.20 & $<6.81 E-05$ & uCi/mL & $\mathbf{P}$ & Raw \\
\hline Pu239/240 & $6 / 1 / 90$ & $4 / 30 / 89$ & $4 / 27 / 89$ & BOB2M4 & G313 & G363 & 1.20 & $<6.80 E-05$ & $\mathrm{uCi} / \mathrm{mL}$ & $\mathbf{P}$ & Raw \\
\hline Pu239/240 & $6 / 1 / 90$ & $4 / 30 / 89$ & $4 / 27 / 89$ & BOB2N4 & 6323 & G476* & 1.21 & $<6.79 E-05$ & $\mathrm{uCi} / \mathrm{mL}$ & $P$ & Raw \\
\hline Pu239/240 & $6 / 1 / 90$ & $4 / 30 / 89$ & $4 / 27 / 89$ & ВОВ2M9 & G302 & G477* & 1.20 & $<6.78 E-05$ & uCi/mL & $P$ & Raw \\
\hline Pu239/240 & $6 / 1 / 90$ & $4 / 30 / 89$ & $4 / 27 / 89$ & BOB2N1 & G310 & $\mathrm{G} 471^{\circ}$ & 1.20 & $<6.77 E-05$ & $\mathrm{uCi} / \mathrm{mL}$ & $\mathbf{P}$ & Raw \\
\hline Pu239/240 & $6 / 1 / 90$ & $4 / 30 / 89$ & 4/27/89 & вов2M3 & G311 & G359 & 1.20 & $<6.77 E-05$ & $u C i / m L$ & $\mathbf{P}$ & Raw \\
\hline Pu239/240 & $6 / 1 / 90$ & $4 / 30 / 89$ & $4 / 27 / 89$ & BOB2M1 & G308 & G353 & 1.20 & $<6.74 \mathrm{E}-05^{\circ}$ & $\mathrm{uCi} / \mathrm{mL}$ & $\mathbf{P}$ & Raw \\
\hline Pu239/240 & $6 / 1 / 90$ & $4 / 30 / 89$ & $4 / 27 / 89$ & BOB2L7 & G299 & G342 & 1.20 & $<6.72 E-05$ & $\mathrm{uCi} / \mathrm{mL}$ & $P$ & Raw \\
\hline Pu239/240 & $6 / 1 / 90$ & $4 / 30 / 89$ & $4 / 27 / 89$ & вов2М2 & G309 & G358 & 1.20 & $<6.72 E-05$ & $\mathrm{uCi} / \mathrm{mL}$ & $\mathbf{P}$ & Raw \\
\hline Pu239/240 & $6 / 1 / 90$ & $4 / 30 / 89$ & $4 / 27 / 89$ & BOB2L7 & G299 & G342 & 1.20 & $<6.71 E-05$ & $. u C i / m L$ & $P$ & Raw \\
\hline Pu239/240 & $6 / 1 / 90$ & $4 / 30 / 89$ & $4 / 27 / 89$ & BOB2N1 & G310 & G471" & -1.20 & $<6.70 E-05$ & $\mathrm{uCi} / \mathrm{mL}$ & $\mathbf{P}$ & Raw \\
\hline Pu239/240 & $6 / 1 / 90$ & $4 / 30 / 89$ & $4 / 27 / 89$ & BOB2M1 & G308 & G353 & 1.20 & $<6.69 E-05$ & uCi/mL & $P$ & Raw \\
\hline Pu239/240 & $6 / 1 / 90$ & $4 / 30 / 89$ & $4 / 27 / 89$ & ВОВ2Т9 & G327 & G470* & 1.19 & $<6.69 E-05$ & uCi/mL & $P$ & Raw \\
\hline Pu239/240 & $6 / 1 / 90$ & $4 / 30 / 89$ & $4 / 27 / 89$ & B082MO & G306 & G352 & 1.20 & $<6.64 E-05$ & $\mathrm{uCl} / \mathrm{mL}$ & $\mathbf{P}$ & Raw \\
\hline Pu239/240 & $6 / 1 / 90$ & $4 / 30 / 89$ & $4 / 27 / 89$ & BOB2MO & G306 & G352 & 1.20 & $<6.64 E-05$ & $\mathrm{uCl} / \mathrm{mL}$ & $P$ & Raw \\
\hline Pu239/240 & $6 / 1 / 90$ & $4 / 30 / 89$ & $4 / 27 / 89$ & BOB2M7 & G319 & G460 & 1.20 & $<6.62 E-05$ & $\mathrm{uCi} / \mathrm{mL}$ & $p$ & Raw \\
\hline
\end{tabular}


aste Characterization Database

Tank 241-AP-102

\begin{tabular}{|c|c|c|c|c|c|c|}
\hline \multicolumn{4}{|c|}{ Radionuclide Information } & \multirow[b]{2}{*}{$\begin{array}{l}\text { Reference } \\
\text { Number }\end{array}$} & \multirow[b]{2}{*}{ Validated $(Y / N)$} & \multirow[b]{2}{*}{ Notes } \\
\hline Radoinuclide & Conc. (Bq/L) & $\begin{array}{c}\text { Conc. (Bq/L } \\
\text { decayed) }\end{array}$ & $\begin{array}{l}\text { Conc. (Ci/L } \\
\text { Decayed) }\end{array}$ & & & \\
\hline Pu239/240 & \#aLue! & \#VALUE! & \#VALUe! & AP102-3 & $\gamma$ & $\begin{array}{l}\text { Density reported for } \mathrm{G} 363 \text { collected at } 18 \text { in from } \\
\text { bottom of tank at a } 150^{\circ} \text { angle. Sample was } \\
\text { unheated, unheated samples precipitated }\end{array}$ \\
\hline Pu239/240 & $¥$ VALUE! & *VALUE! & \#VALUE! & AP102-3 & $\mathbf{Y}$ & $\begin{array}{l}\text { Density reported for } G 460 \text { collected at } 226 \text { in from } \\
\text { bottom of tank at a } 270^{\circ} \text { angle. Sample was } \\
\text { unheated, unheated samples precipitated }\end{array}$ \\
\hline Pu239/240 & \#ALUE! & \#VALUE! & NALUE! & AP102-3 & $\mathbf{Y}$ & $\begin{array}{l}\text { Density reported for } \mathrm{G} 358 \text { collected at } 334 \text { in from } \\
\text { bottom of tank at a } 150^{\circ} \text { angle. Sample was } \\
\text { unheated, unheated samples precipitated }\end{array}$ \\
\hline Pu239/240 & \#VALUE! & *VALUEI. & FALUE! & AP102-3 & $\mathbf{Y}$ & $\begin{array}{l}\text { Density reported for G359 collected at } 162 \text { in from } \\
\text { bottom of tank at a } 150^{\circ} \text { angle. Sample was } \\
\text { unheated, unheated samples precipitated }\end{array}$ \\
\hline Pu239/240 & *VALUE! & \#VALUE! & FALUE! & AP102-3 & $\mathbf{Y}$ & $\begin{array}{l}\text { Density reported for G363 collected at } 18 \text { in from } \\
\text { bottom of tank at a } 150^{\circ} \text { angle. Sample was } \\
\text { unheated, unheated samples precipitated }\end{array}$ \\
\hline Pu239/240 & \#VALUE! & \#VALUE! & \#VALUE! & AP102-3 & $Y$ & $\begin{array}{l}\text { Density reported for } 6476 \text { collected at } 99 \text { in from } \\
\text { bottom of tank at a } 270^{\circ} \text { angle and was keep heated } \\
\text { to } 40^{\circ} \mathrm{C} \text {. }\end{array}$ \\
\hline Pu239/240 & \#VALUE! & \#VALUE! & \#VALUE! & AP102-3 & $Y$ & $\begin{array}{l}\text { Density reported for } \mathrm{G} 477 \text { collected at } 182 \text { in from } \\
\text { bottom of tank at a } 30^{\circ} \text { angle and was keep heated } \\
\text { to } 40^{\circ} \mathrm{C} \text {. }\end{array}$ \\
\hline Pu239/240 & \#VALUE! & \#VALUE! & \#VALUE! & AP102-3 & $Y$ & $\begin{array}{l}\text { Density reported for } \mathrm{G} 471 \text { collected at } 208 \text { in from } \\
\text { bottom of tank at a } 150^{\circ} \text { angle and was keep heated } \\
\text { to } 40^{\circ} \mathrm{C} \text {. }\end{array}$ \\
\hline Pu239/240 & *VALUE! & \#VALUE! & \#VALUE! & AP102-3 & $Y$ & $\begin{array}{l}\text { Density reported for } G 359 \text { collected at } 162 \text { in from } \\
\text { bottom of tank at a } 150^{\circ} \text { angle. Sample was } \\
\text { unheated, unheated samples precipitated }\end{array}$ \\
\hline Pu239/240 & NALUE! & \#Value! & \#VALUE! & AP102-3 & $\mathbf{Y}$ & $\begin{array}{l}\text { Density reported for } 6353 \text { collected at } 334 \text { in from } \\
\text { bottom of tank at a } 150^{\circ} \text { angle. Sample was } \\
\text { unheated, unheated samples precipitated }\end{array}$ \\
\hline Pu239/240 & \#VALUE! & \#VALUE! & Nalue! & AP102-3 & $\mathbf{Y}$ & $\begin{array}{l}\text { Density reported for } \mathrm{G} 342 \text { collected at } 306 \text { in from } \\
\text { bottom of tank at a } 30^{\circ} \text { angle. Sample was } \\
\text { unheated, unheated samples precipitated }\end{array}$ \\
\hline Pu239/240 & \#VALUE! & \#VALUEI & \#ALUE! & AP102-3 & $Y$ & $\begin{array}{l}\text { Density reported for } G 358 \text { collected at } 334 \text { in from } \\
\text { bottom of tank at a } 150^{\circ} \text { angle. Sample was } \\
\text { unheated, unheated samples precipitated }\end{array}$ \\
\hline Pu239/240 & \#VALUE! & \#VALUE! & \#ALUE! & AP102-3 & $\mathbf{Y}$ & $\begin{array}{l}\text { Density reported for } \mathrm{G} 342 \text { collected at } 306 \text { in from } \\
\text { bottom of tank at a } 30^{\circ} \text { angle. Sample was } \\
\text { unheated, unheated samples precipitated }\end{array}$ \\
\hline Pu239/240 & *VALUEI & \#VALUE! & FVALUE! & AP102-3 & $Y$ & $\begin{array}{l}\text { Density reported for } \mathrm{G} 47 \mathrm{y} \text { collected at } 208 \text { in from } \\
\text { bottom of tank at a } 150^{\circ} \text { angle and was keep heated } \\
\text { to } 40^{\circ} \mathrm{C} \text {. }\end{array}$ \\
\hline Pu239/240 & \#VALUE! & *VALUEI & NALUE! & AP102-3 & $Y$ & $\begin{array}{l}\text { Density reported for } \mathrm{G} 353 \text { collected at } 334 \text { in from } \\
\text { bottom of tank at a } 150^{\circ} \text { angle. Sample was } \\
\text { unheated, unheated samples precipitated }\end{array}$ \\
\hline Pu239/240 & NALUE! & * VALUEI & \#VALUE! & AP102-3 & $Y$ & $\begin{array}{l}\text { Density reported for } \mathrm{G} 470 \text { represents a composite } \\
\text { sample. Sample was kept heated to } 40^{\circ} \mathrm{C} \text {. }\end{array}$ \\
\hline Pu239/240 & \#VALUEI & \#VALUEI & ÆVALUE! & AP102-3 & $\mathbf{Y}$ & $\begin{array}{l}\text { Density reported for G352 collected at } 34 \text { in from } \\
\text { bottom of tank at a } 30^{\circ} \text { angle. Sample was } \\
\text { unheated, unheated samples precipitated }\end{array}$ \\
\hline Pu239/240 & \#VALUE! & \#ALUEI & NALUE! & AP102-3 & $Y$ & $\begin{array}{l}\text { Density reported for } \mathrm{G} 352 \text { collected at } 34 \text { in from } \\
\text { bottom of tank at a } 30^{\circ} \text { angle. Sample was } \\
\text { unheated, unheated samples precipitated }\end{array}$ \\
\hline Pu239/240 & \#VALUE! & \#VALUE! & \#ALUE! & AP102-3 & $\mathbf{Y}$ & $\begin{array}{l}\text { Density reported for } \mathrm{G} 460 \text { collected at } 226 \text { in from } \\
\text { bottom of tank at a } 270^{\circ} \text { angle. Sample was } \\
\text { unheated, unheated samples precipitated }\end{array}$ \\
\hline
\end{tabular}




\begin{tabular}{|c|c|c|c|c|c|c|c|c|c|c|c|}
\hline & & & & Tank Wast & Characterize & ion Sample In & imation & & & & \\
\hline $\begin{array}{c}\text { Reported } \\
\text { Radionuclide }\end{array}$ & $\begin{array}{c}\text { Memo } \\
\text { Date }\end{array}$ & $\begin{array}{c}\text { Start } \\
\text { Analysis } \\
\text { Date }\end{array}$ & $\begin{array}{c}\text { Start } \\
\text { Sample } \\
\text { Date }\end{array}$ & $\begin{array}{l}\text { Sample } \\
\text { Number }\end{array}$ & $\begin{array}{c}\text { Tank Farm } \\
\text { Number }\end{array}$ & $\begin{array}{c}\text { Lab Sample } \\
\text { Number }\end{array}$ & $\begin{array}{l}\text { Density } \\
(\mathrm{g} / \mathrm{mL})\end{array}$ & $\begin{array}{c}\text { Reported } \\
\text { Value }\end{array}$ & Reported Unit & Code & Source \\
\hline Pu239/240 & $6 / 1 / 90$ & $4 / 30 / 89$ & $4 / 27 / 89$ & BOB2T9 & G327 & G470* & 1.19 & $<6.60 E-05$ & uCi/mL & $P$ & Raw \\
\hline $\mathrm{Sr}$ & & $4 / 89$ & $4 / 27 / 89$ & NS & NS & NS & 1.24 & $5.16 \mathrm{E}-03$ & $\mathrm{Ci} / \mathrm{L}$ & $\mathrm{L}$ & HTCE In \\
\hline Sr90 & $6 / 1 / 90$ & $4 / 30 / 89$ & $4 / 27 / 89$ & BOB2L8 & G303 & G348 & 1.21 & $1.75 E+\infty 0$ & $\mathrm{uCi} / \mathrm{mL}$ & $P$ & Raw \\
\hline Sr9o & $6 / 1 / 90$ & $4 / 30 / 89$ & $-4 / 27 / 89$ & BOB2L7 & G299 & G342 & 1.20 & $1.64 \mathrm{E}+00$ & $\mathrm{uCi} / \mathrm{mL}$ & $P$ & Raw \\
\hline Sr9o & $6 / 1 / 90$ & $4 / 30 / 89$ & $4 / 27 / 89$ & BOB2M5 & G315 & G364 & 1.19 & $1.61 E+\infty$ & $\mathrm{uCi} / \mathrm{mL}$ & $P$ & Raw \\
\hline Sr9o & $6 / 1 / 90$ & $4 / 30 / 89$ & $4 / 27 / 89$ & $\mathrm{BOB} 2 \mathrm{L7}$ & G299 & G342 & 1.20 & $1.59 E+00$ & $\mathrm{uCi} / \mathrm{mL}$ & $P$ & Raw \\
\hline Sr9o & $6 / 1 / 90$ & $4 / 30 / 89$ & $4 / 27 / 89$ & BOB2MO & G306 & G352 & 1.20 & $1.58 E+\infty 0$ & $\mathrm{uCi} / \mathrm{mL}$ & $P$ & Raw \\
\hline Sr9o & $6 / 1 / 90$ & $4 / 30 / 89$ & $4 / 27 / 89$ & $80 B 2 M 3$ & G311 & G359 & 1.20 & $1.53 E+00$ & $\mathrm{uCi} / \mathrm{mL}$ & $\mathbf{P}$ & Raw \\
\hline St90 & $6 / 1 / 90$ & $4 / 30 / 89$ & $4 / 27 / 89$ & BOB2T9 & G327 & G443 & 1.20 & $1.52 E+\infty 0$ & $\mathrm{uCi} / \mathrm{mL}$ & $\mathbf{P}$ & Raw \\
\hline Sr9o & $6 / 1 / 90$ & $4 / 30 / 89$ & $4 / 27 / 89$ & 8082L9 & G305 & G349 & 1.20 & $1.49 E+\infty$ & $\mathrm{uCi} / \mathrm{mL}$ & $P$ & Raw \\
\hline Sr9o & $6 / 1 / 90$ & $4 / 30 / 89$ & $4 / 27 / 89$ & BOB2M3 & G311 & G359 & 1.20 & $1.48 E+00$ & $\mathrm{uCi} / \mathrm{mL}$ & $P$ & Raw \\
\hline Sr9o & $6 / 1 / 90$ & $4 / 30 / 89$ & $4 / 27 / 89$ & BOB2M2 & G309 & G358 & 1.20 & $1.47 E+\infty$ & $\mathrm{uCi} / \mathrm{mL}$ & $P$ & Raw. \\
\hline Sr9o & $6 / 1 / 90$ & $4 / 30 / 89$ & $4 / 27 / 89$ & 8082T9 & G327 & G443 & 1.20 & $1.47 E+\infty$ & $\mathrm{uCi} / \mathrm{mL}$ & $\mathbf{P}$ & Raw \\
\hline Sr9o & $6 / 1 / 90$ & $4 / 30 / 89$ & $4 / 27 / 89$ & BOB2L7 & G299 & G342 & 1.20 & $1.46 E+\infty$ & $\mathrm{uCi} / \mathrm{mL}$ & $\mathbf{P}$ & Raw \\
\hline Sr9o & $6 / 1 / 90$ & $4 / 30 / 89$ & $4 / 27 / 89$ & BOB2L9 & G305 & G349 & 1.20 & $1.46 E+\infty$ & $\mathrm{uCi} / \mathrm{mL}$ & $P$ & Raw \\
\hline Sr9o & $6 / 1 / 90$ & $4 / 30 / 89$ & $4 / 27 / 89$ & ВOB2M3 & G311 & G359 & 1.20 & $1.45 E+\infty$ & $\mathrm{uCi} / \mathrm{mL}$ & $P$ & Raw \\
\hline Sr9o & $6 / 1 / 90$ & $4 / 30 / 89$ & $4 / 27 / 89$ & Bo82M5 & G315 & G364 & 1.19 & $1.45 E+\infty$ & uCi/mL & $\mathbf{P}$ & Raw \\
\hline Sr9o & $6 / 1 / 90$ & $4 / 30 / 89$ & $4 / 27 / 89$ & BOB2M6 & G318 & G459 & 1.20 & $1.44 E+\infty$ & $\mathrm{uCi} / \mathrm{mL}$ & $\mathbf{P}$ & Raw \\
\hline St90 & $6 / 1 / 90$ & $4 / 30 / 89$ & $4 / 27 / 89$ & $\mathrm{BOB} 2 \mathrm{~L} 7$ & G299 & G342 & 1.20 & $1.43 E+\infty$ & $\mathrm{uCi} / \mathrm{mL}$ & $\mathbf{P}$ & Raw \\
\hline Sr9o & $6 / 1 / 90$ & $4 / 30 / 89$ & $4 / 27 / 89$ & B082M5 & G315 & G364 & 1.19 & $1.43 E+\infty$ & $\mathrm{uCi} / \mathrm{mL}$ & $\mathbf{P}$ & Raw \\
\hline
\end{tabular}




\begin{tabular}{|c|c|c|c|c|c|c|}
\hline \multicolumn{4}{|c|}{ Radionuclide Information } & \multirow[b]{2}{*}{$\begin{array}{l}\text { Reference } \\
\text { Number }\end{array}$} & \multirow[b]{2}{*}{ Validated $(Y / N)$} & \multirow[b]{2}{*}{ Notes } \\
\hline Radoinuclide & Conc. (Bq/L) & $\begin{array}{c}\text { Conc. (Bq/L } \\
\text { decayed) }\end{array}$ & $\begin{array}{c}\text { Conc. (Ci/L } \\
\text { Decayed) }\end{array}$ & & & \\
\hline Pu239/240 & \#VALUE! & \#VALUE! & \#ALUE! & AP102-3 & $\mathbf{Y}$ & $\begin{array}{l}\text { Density reported for } \mathrm{G} 470 \text { represents a composite } \\
\text { sample. Sample was kept heated to } 40^{\circ} \mathrm{C} \text {. }\end{array}$ \\
\hline Sr89/90 & $1.91 E+08$ & $1.91 E+08$ & $5.16 \mathrm{E}-03$ & NA & $\mathrm{N}$ & No Reference \\
\hline Sr89/90 & $6.48 E+07$ & $6.22 E+07$ & $1.68 E-03$ & AP102-3 & $\mathbf{Y}$ & $\begin{array}{l}\text { Density reported for } \mathrm{G} 348 \text { collected at } 125 \text { in from } \\
\text { bottom of tank at a } 30^{\circ} \text { angle. Sample was } \\
\text { unheated, unheated samples precipitated }\end{array}$ \\
\hline Sr89/90 & $6.07 E+07$ & $5.83 E+07$ & $1.58 \mathrm{E}-03$ & AP102-3 & $\mathbf{\gamma}$ & $\begin{array}{l}\text { Density reported for } \mathrm{G} 342 \text { collected at } 306 \text { in from } \\
\text { bottom of tank at a } 30^{\circ} \text { angle. Sample was } \\
\text { unheated, unheated samples precipitated }\end{array}$ \\
\hline Sr89/90 & $5.96 E+07$ & $5.72 E+07$ & $1.55 E-03$ & AP102-3 & $Y$ & $\begin{array}{l}\text { Density reported for G364 collected at } 372 \text { in from } \\
\text { bottom of tank at a } 270^{\circ} \text { angle. Sample was } \\
\text { unheated, unheated samples precipitated }\end{array}$ \\
\hline Sr89/90 & $5.88 E+07$ & $5.65 E+07$ & $1.53 \mathrm{E}-03$ & AP102-3 & $\mathbf{Y}$ & $\begin{array}{l}\text { Density reported for } \mathrm{G} 342 \text { collected at } 306 \text { in from } \\
\text { bottom of tank at a } 30^{\circ} \text { angle. Sample was } \\
\text { unheated, unheated samples precipitated }\end{array}$ \\
\hline Sr89/90 & $5.85 E+07$ & $5.62 E+07$ & 1.52E-03 & AP102-3 & $\mathbf{Y}$ & $\begin{array}{l}\text { Density reported for } \mathrm{G} 352 \text { collected at } 34 \text { in from } \\
\text { bottom of tank at a } 30^{\circ} \text { angle. Sample was } \\
\text { unheated, unheated samples precipitated }\end{array}$ \\
\hline Sr89/90 & $5.66 E+07$ & $5.44 E+07$ & $1.47 E-03$ & AP102-3 & $\mathbf{Y}$ & $\begin{array}{l}\text { Density reported for } \mathrm{G} 359 \text { collected at } 162 \text { in from } \\
\text { bottom of tank at a } 150^{\circ} \text { angle. Sample was } \\
\text { unheated, unheated samples precipitated }\end{array}$ \\
\hline Sr89/90 & $5.62 \mathrm{E}+07$ & $5.40 E+07$ & $1.46 E-03$ & AP102-3 & $\mathbf{Y}$ & $\begin{array}{l}\text { Density reported for G443 represents a composite } \\
\text { sample }\end{array}$ \\
\hline Sr89/90 & $5.51 E+07$ & $5.30 E+07$ & $1.43 E-03$ & AP102-3 & $\mathbf{Y}$ & $\begin{array}{l}\text { Density reported for } 6349 \text { collected at } 34 \text { in from } \\
\text { bottom of tank at a } 30^{\circ} \text { angle. Sample was } \\
\text { unheated, unheated samples precipitated }\end{array}$ \\
\hline Sr89/90 & $5.48 E+07$ & $5.26 \varepsilon+07$ & $1.42 \mathrm{E}-03$ & AP102-3 & $\mathbf{Y}$ & $\begin{array}{l}\text { Density reported for } \mathrm{G} 359 \text { collected at } 162 \text { in from } \\
\text { bottom of tank at a } 150^{\circ} \text { angle. Sample was } \\
\text { unheated, unheated samples precipitated }\end{array}$ \\
\hline Sr89/90 & $5.44 E+07$ & $5.22 E+07$ & $1.41 E-03$ & AP102-3 & $\mathbf{Y}$ & $\begin{array}{l}\text { Density reported for } \mathrm{G} 358 \text { collected at } 334 \text { in from } \\
\text { bottom of tank at a } 150^{\circ} \text { angle. Sample was } \\
\text { unheated, unheated samples precipitated }\end{array}$ \\
\hline Sr89/90 & $5.44 E+07$ & $5.22 E+07$ & $1.41 E-03$ & AP102-3 & $\mathbf{Y}$ & $\begin{array}{l}\text { Density reported for G443 represents a composite } \\
\text { sample }\end{array}$ \\
\hline Sr89/90 & $5.40 E+07$ & $5.19 E+07$ & $1.40 E-03$ & AP102-3 & $\mathbf{Y}$ & $\begin{array}{l}\text { Density reported for } \mathrm{G} 342 \text { collected at } 306 \text { in from } \\
\text { bottom of tank at a } 30^{\circ} \text { angle. Sample was } \\
\text { unheated, unheated samples precipitated }\end{array}$ \\
\hline Sr89/90 & $5.40 E+07$ & $5.19 E+07$ & $1.40 \mathrm{E}-03$ & AP102-3. & $\mathbf{Y}$ & $\begin{array}{l}\text { Density reported for } \mathrm{G} 349 \text { collected at } 34 \text { in from } \\
\text { bottom of tank at a } 30^{\circ} \text { angle. Sample was } \\
\text { unheated, unheated samples precipitated }\end{array}$ \\
\hline Sr89/90 & $5.37 E \div 07$ & $5.15 E+07$ & 1.39E-03 & AP102-3 & $\mathbf{Y}$ & $\begin{array}{l}\text { Density reported for } \mathrm{G} 359 \text { collected at } 162 \text { in from } \\
\text { bottom of tank at a } 150^{\circ} \text { angle. Sample was } \\
\text { unheated, unheated samples precipitated }\end{array}$ \\
\hline Sr89/90 & $5.37 E+07$ & $5.15 E \div 07$ & 1.39E-03 & AP102-3 & $\mathbf{Y}$ & $\begin{array}{l}\text { Density reported for } \mathrm{G} 364 \text { collected at } 372 \text { in from } \\
\text { bottom of tank at a } 270^{\circ} \text { angle. Sample was } \\
\text { unheated, unheated samples precipitated }\end{array}$ \\
\hline Sr89/90 & $5.33 E \div 07$ & $5.12 E+07$ & $1.38 E-03$ & AP102-3 & $\mathbf{Y}$ & $\begin{array}{l}\text { Density reported for } \mathrm{G} 459 \text { collected at } 226 \text { in from } \\
\text { bottom of tank at a } 270^{\circ} \text { angle. Sample was } \\
\text { unheated, unheated samples precipitated }\end{array}$ \\
\hline Sr89/90 & $5.29 E+07$ & $5.08 E+07$ & $1.37 E-03$ & AP102-3 & $\mathbf{Y}$ & $\begin{array}{l}\text { Density reported for } \mathrm{G} 342 \text { collected at } 306 \text { in from } \\
\text { bottom of tank at a } 30^{\circ} \text { angle. Sample was } \\
\text { unheated, unheated samples precipitated }\end{array}$ \\
\hline Sr89/90 & $5.29 E+07$ & $5.08 E+07$ & 1.37E-03 & AP102-3 & $\mathbf{Y}$ & $\begin{array}{l}\text { Density reported for G364 collected at } 372 \text { in from } \\
\text { bottom of tank at a } 270^{\circ} \text { angle. Sample was } \\
\text { unheated, unheated samples precipitated }\end{array}$ \\
\hline
\end{tabular}




\begin{tabular}{|c|c|c|c|c|c|c|c|c|c|c|c|}
\hline \multicolumn{12}{|c|}{ Tank Waste Characterization Sample Information } \\
\hline $\begin{array}{c}\text { Reported } \\
\text { Radionuclide }\end{array}$ & $\begin{array}{c}\text { Memo } \\
\text { Date }\end{array}$ & $\begin{array}{c}\text { Start } \\
\text { Analysis } \\
\text { Date }\end{array}$ & $\begin{array}{c}\text { Start } \\
\text { Sample } \\
\text { Date } \\
\end{array}$ & $\begin{array}{l}\text { Sample } \\
\text { Number }\end{array}$ & $\begin{array}{c}\text { Tank Farm } \\
\text { Number }\end{array}$ & $\begin{array}{c}\text { Lab Sample } \\
\text { Number }\end{array}$ & $\begin{array}{l}\text { Density } \\
(\mathrm{g} / \mathrm{mL})\end{array}$ & $\begin{array}{c}\text { Reported } \\
\text { Value }\end{array}$ & Reported Unit & Code & Source \\
\hline Sr9o & 6/1/90 & $4 / 30 / 89$ & $4 / 27 / 89$ & ВОВ2М7 & G319 & G460 & 1.20 & $1.42 E+\infty 0$ & $\mathrm{uCi} / \mathrm{mL}$ & $\mathbf{P}$ & Raw \\
\hline Sr9o & $6 / 1 / 90$ & $4 / 30 / 89$ & $4 / 27 / 89$ & ВОВ2МО & G306 & G352 & 1.20 & $1.41 E+\infty$ & $\mathrm{uCi} / \mathrm{mL}$ & $\mathbf{P}$ & Raw \\
\hline Sr9o & $6 / 1 / 90$ & $4 / 30 / 89$ & $4 / 27 / 89$ & ВОВ2М3 & G311 & G359 & 1.20 & $1.41 E+\infty 0$ & $\mathrm{uCi} / \mathrm{mL}$ & $\mathbf{P}$ & Raw \\
\hline Sr9o & $6 / 1 / 90$ & $4 / 30 / 89$ & $4 / 27 / 89$ & ВОВ2M5 & G315 & G364 & 1.19 & $1.41 E+\infty 0$ & $\mathrm{uCi} / \mathrm{mL}$ & $P$ & Raw \\
\hline Sr9o & $6 / 1 / 90$ & $4 / 30 / 89$ & $4 / 27 / 89$ & BOB2L8 & G303 & G348 & 1.21 & $1.40 E+\infty 0$ & $\mathrm{uCi} / \mathrm{mL}$ & $P$ & Raw \\
\hline Sr9o & $6 / 1 / 90$ & $4 / 30 / 89$ & $4 / 27 / 89$ & 80B2M1 & G308 & G353 & 1.20 & $1.40 E+\infty$ & uCi/mL & $P$ & Raw \\
\hline Sr9o & $6 / 1 / 90$ & $4 / 30 / 89$ & $4 / 27 / 89$ & 80B2N4 & G323 & G341 & 1.20 & $1.40 E+\infty 0$ & uCi/mL & $P$ & Raw \\
\hline Sr90 & $6 / 1 / 90$ & $4 / 30 / 89$ & $4 / 27 / 89$ & BOB2M4 & G313 & G363 & 1.20 & $1.39 E+00$ & $\mathrm{uCi} / \mathrm{mL}$ & $P$ & Raw \\
\hline Sr9o & $6 / 1 / 90$ & $4 / 30 / 89$ & $4 / 27 / 89$ & ВОВ2M4 & G313 & G363 & 1.20 & $1.39 E+\infty 0$ & $\mathrm{uCi} / \mathrm{mL}$ & $P$ & Raw \\
\hline Sr9o & $6 / 1 / 90$ & 4/30/89 & $4 / 27 / 89$ & 8OB2M6 & G318 & G459 & 1.20 & $1.39 E+\infty 0$ & $\mathrm{uCi} / \mathrm{mL}$ & $P$ & Raw \\
\hline Sr9o & $6 / 1 / 90$ & $4 / 30 / 89$ & $4 / 27 / 89$ & ВОВ2M1 & G308 & G353 & 1.20 & $1.38 E+\infty$ & $\mathrm{uCi} / \mathrm{mL}$ & $P$ & Raw \\
\hline Sr9o & $6 / 1 / 90$ & $4 / 30 / 89$ & $4 / 27 / 89$ & BOB2N4 & G323 & G341 & 1.20 & $1.38 E+\infty 0$ & $\mathrm{uCi} / \mathrm{mL}$ & $\mathbf{P}$ & Raw \\
\hline Sr90 & $6 / 1 / 90$ & $4 / 30 / 89$ & $4 / 27 / 89$ & BOB2MO & G306 & G352 & 1.20 & $1.37 E+\infty$ & $\mathrm{uCi} / \mathrm{mL}$ & $P$ & Raw \\
\hline Si90 & $6 / 1 / 90$ & $4 / 30 / 89$ & $4 / 27 / 89$ & BOB2M7 & G319 & G460 & 1.20 & $1.37 E+\infty$ & $\mathrm{uCi} / \mathrm{mL}$ & $P$ & Raw \\
\hline Srgo & $6 / 1 / 90$ & $4 / 30 / 89$ & $4 / 27 / 89$ & BOB2MO & G306 & G352 & 1.20 & $1.36 E+\infty 0$ & $\mathrm{uCi} / \mathrm{mL}$ & $P$ & Raw \\
\hline Sr9o & $6 / 1 / 90$ & $4 / 30 / 89$ & $4 / 27 / 89$ & ВОВ2M2 & G309 & G358 & 1.20 & $1.36 E+\infty 0$ & $\mathrm{uCi} / \mathrm{mL}$ & $P$ & Raw \\
\hline Srgo & $6 / 1 / 90$ & $4 / 30 / 89$ & $4 / 27 / 89$ & BOB2L8 & G303 & G348 & 1.21 & $1.25 E+\infty$ & $\mathrm{uCi} / \mathrm{mL}$ & $P$ & Raw \\
\hline St9o & $6 / 1 / 90$ & $4 / 30 / 89$ & $4 / 27 / 89$ & BOB2L8 & G303 & G348 & 1.21 & $1.23 E+\infty 0$ & $\mathrm{uCi} / \mathrm{mL}$ & $P$ & Raw \\
\hline Sr90 & $6 / 1 / 90$ & $4 / 30 / 89$ & $4 / 27 / 89$ & BOB2N4 & G323 & G476: & 1.21 & $1.10 E+\infty$ & $\mathrm{uCi} / \mathrm{mL}$ & $\mathbf{P}$ & Raw \\
\hline
\end{tabular}




\begin{tabular}{|c|c|c|c|c|c|c|}
\hline \multicolumn{4}{|c|}{ Radionuclide Information } & \multirow[b]{2}{*}{$\begin{array}{c}\text { Reference } \\
\text { Number }\end{array}$} & \multirow[b]{2}{*}{ Validated $(\mathrm{Y} / \mathrm{N})$} & \multirow[b]{2}{*}{ Notes } \\
\hline Radoinuclide & Conc. (Bq/L) & $\begin{array}{c}\text { Conc. }(B q / L \\
\text { decayed) }\end{array}$ & $\begin{array}{c}\text { Conc. (Ci/L } \\
\text { Decayed) }\end{array}$ & & & \\
\hline Sr89/90 & $5.25 E+07$ & $5.05 E+07$ & $1.36 \mathrm{E}-03$ & AP102-3 & 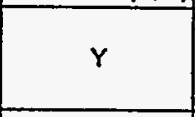 & $\begin{array}{l}\text { Density reported for } \mathrm{G} 460 \text { collected at } 226 \text { in from } \\
\text { bottom of tank at a } 270^{\circ} \text { angle. Sample was } \\
\text { unheated, unheated samples precipitated }\end{array}$ \\
\hline Sr89/90 & $5.22 E+07$ & $5.01 E+07$ & $1.35 E-03$ & AP102-3 & $\mathbf{Y}$ & $\begin{array}{l}\text { Density reported for } \mathrm{G} 352 \text { collected at } 34 \text { in from } \\
\text { bottom of tank at a } 30^{\circ} \text { angle. Sample was } \\
\text { unheated, unheated samples precipitated }\end{array}$ \\
\hline Sr89/90 & $5.22 \mathrm{E}+07$ & $5.01 E+07$ & $1.35 E-03$ & AP102-3 & $\mathbf{Y}$ & $\begin{array}{l}\text { Density reported for } \mathrm{G} 359 \text { collected at } 162 \text { in from } \\
\text { bottom of tank at a } 150^{\circ} \text { angle. Sample was } \\
\text { unheated, unheated samples precipitated }\end{array}$ \\
\hline Sr89/90 & $5.22 E+07$ & $5.01 E+07$ & $1.35 E-03$ & AP102-3 & $\mathrm{Y}$ & $\begin{array}{l}\text { Density reported for } \mathrm{G} 364 \text { collected at } 372 \text { in from } \\
\text { bottom of tank at a } 270^{\circ} \text { angle. Sample was } \\
\text { unheated, unheated samples precipitated }\end{array}$ \\
\hline Sr89/90 & $5.18 E+07$ & $4.98 E+07$ & $1.34 \mathrm{E}-03$ & AP102-3 & Y & $\begin{array}{l}\text { Density reported for G348 collected at } 125 \text { in from } \\
\text { bottom of tank at a } 30^{\circ} \text { angle. Sample was } \\
\text { unheated, unheated samples precipitated }\end{array}$ \\
\hline Sr89/90 & $5.18 E+07$ & $4.98 E+07$ & $1.34 \mathrm{E}-03$ & AP102-3 & $Y$ & $\begin{array}{l}\text { Density reported for } \mathrm{G} 353 \text { collected at } 334 \text { in from } \\
\text { bottom of tank at a } 150^{\circ} \text { angle. Sample was } \\
\text { unheated, unheated samples precipitated }\end{array}$ \\
\hline Sr89/90 & $5.18 E+07$ & $4.98 E+07$ & $1.34 E-03$ & AP102-3 & $\gamma$ & $\begin{array}{l}\text { Density reported for } \mathrm{G} 341 \text { collected at } 99 \text { in from } \\
\text { bottom of tank at a } 270^{\circ} \text { angle. }\end{array}$ \\
\hline Sr89/90 & $5.14 E+07$ & $4.94 E+07$ & $1.34 E-03$ & AP102-3 & $\mathbf{Y}$ & $\begin{array}{l}\text { Density reported for } G 363 \text { collected at } 18 \text { in from } \\
\text { bottom of tank at a } 150^{\circ} \text { angle. Sample was } \\
\text { unheated, unheated samples precipitated }\end{array}$ \\
\hline Sr89/90 & $5.14 E+07$ & $4.94 E+07$ & $1.34 E-03$ & AP102-3 & $Y$ & $\begin{array}{l}\text { Density reported for G363 collected at } 18 \text { in from } \\
\text { bottom of tank at a } 150^{\circ} \text { angle. Sample was } \\
\text { unheated, unheated samples precipitated }\end{array}$ \\
\hline Sr89/90 & $5.14 E+07$ & $4.94 E+07$ & $1.34 E-03$ & AP102-3 & $\mathbf{Y}$ & $\begin{array}{l}\text { Density reported for G459 collected at } 226 \text { in from } \\
\text { bottom of tank at a } 270^{\circ} \text { angle. Sample was } \\
\text { unheated, unheated samples precipitated }\end{array}$ \\
\hline Sr89/90 & $5.11 E+07$ & $4.90 E \div 07$ & $1.33 E-03$ & AP102-3 & $\mathrm{Y}$ & $\begin{array}{l}\text { Density reported for } \mathrm{G} 353 \text { collected at } 334 \text { in from } \\
\text { bottom of tank at a } 150^{\circ} \text { angle. Sample was } \\
\text { unheated, unheated samples precipitated }\end{array}$ \\
\hline Sr89/90 & $5.11 E+07$ & $4.90 E+07$ & $1.33 E-03$ & AP102-3 & $Y$ & $\begin{array}{l}\text { Density reported for } \mathrm{G} 341 \text { collected at } 99 \text { in from } \\
\text { bottom of tank at a } 270^{\circ} \text { angle. }\end{array}$ \\
\hline Sr89/90 & $5.07 E+07$ & $4.87 E+07$ & $1.32 \mathrm{E}-03$ & AP102-3 & $Y$ & $\begin{array}{l}\text { Density reported for } G 352 \text { collected at } 34 \text { in from } \\
\text { bottom of tank at a } 30^{\circ} \text { angle. Sample was } \\
\text { unheated, unheated samples precipitated }\end{array}$ \\
\hline Sr89/90 & $5.07 E+07$ & $4.87 E+07$ & $1.32 E-03$ & AP102-3 & $Y$ & $\begin{array}{l}\text { Density reported for } \mathrm{G} 460 \text { collected at } 226 \text { in from } \\
\text { bottom of tank at a } 270^{\circ} \text { angle. Sample was } \\
\text { unheated, unheated samples precipitated }\end{array}$ \\
\hline Sr89/90 & $5.03 E+07$ & $4.83 E+07$ & $1.31 \mathrm{E}-03$ & AP102-3 & $Y$ & $\begin{array}{l}\text { Density reported for } \mathrm{G} 352 \text { collected at } 34 \text { in from } \\
\text { bottom of tank at a } 30^{\circ} \text { angle. Sample was } \\
\text { unheated, unheated samples precipitated }\end{array}$ \\
\hline Sr89/90 & $5.03 E+07$ & $4.83 E+07$ & $1.31 E-03$ & AP102-3 & $Y$ & $\begin{array}{l}\text { Density reported for } \mathrm{G} 358 \text { collected at } 334 \text { in from } \\
\text { bottom of tank at a } 150^{\circ} \text { angle. Sample was } \\
\text { unheated, unheated samples precipitated }\end{array}$ \\
\hline Sr89/90 & $4.63 E+07$ & $4.44 E+07$ & $1.20 \mathrm{E}-03$ & AP102-3 & $Y$ & $\begin{array}{l}\text { Density reported for G348 collected at } 125 \text { in from } \\
\text { bottom of tank at a } 30^{\circ} \text { angle. Sample was } \\
\text { unheated, unheated samples precipitated }\end{array}$ \\
\hline Sr89/90 & $4.55 E+07$ & $4.37 E+07$ & $1.18 \mathrm{E}-03$ & AP102-3 & $Y$ & $\begin{array}{l}\text { Density reported for } \mathrm{G} 348 \text { collected at } 125 \text { in from } \\
\text { bottom of tank at a } 30^{\circ} \text { angle. Sample was } \\
\text { unheated, unheated samples precipitated }\end{array}$ \\
\hline Sr89/90 & $4.07 E+07$ & $3.91 E+07$ & $1.06 E-03$ & AP102-3 & $\mathbf{Y}$ & $\begin{array}{l}\text { Density reported for } \mathrm{G} 476 \text { collected at } 99 \text { in from } \\
\text { bottom of tank at a } 270^{\circ} \text { angle and was keep heated } \\
\text { to } 40^{\circ} \mathrm{C} \text {. }\end{array}$ \\
\hline
\end{tabular}


TWRS Privatization Support PSC Radionuclide Liqui

\begin{tabular}{|c|c|c|c|c|c|c|c|c|c|c|c|}
\hline \multicolumn{12}{|c|}{ Tank Waste Characterization Sample Information } \\
\hline $\begin{array}{c}\text { Reported } \\
\text { Radionuclide } \\
\end{array}$ & $\begin{array}{c}\text { Memo } \\
\text { Date }\end{array}$ & $\begin{array}{c}\text { Start } \\
\text { Analysis } \\
\text { Date }\end{array}$ & $\begin{array}{c}\text { Start } \\
\text { Sample } \\
\text { Date }\end{array}$ & $\begin{array}{l}\text { Sample } \\
\text { Number }\end{array}$ & $\begin{array}{c}\text { Tank Farm } \\
\text { Number }\end{array}$ & $\begin{array}{c}\text { Lab Sample } \\
\text { Number }\end{array}$ & $\begin{array}{l}\text { Density } \\
(\mathrm{g} / \mathrm{mL})\end{array}$ & $\begin{array}{c}\text { Reported } \\
\text { Value }\end{array}$ & Reported Unit & Code & Sourc: \\
\hline Sr9o & $6 / 1 / 90$ & $4 / 30 / 89$ & $4 / 27 / 89$ & вов2М9 & G302 & G477* & 1.20 & 8.34E-01 & uCi/mL & $\mathbf{P}$ & Raw \\
\hline Sr9o & $6 / 1 / 90$ & $4 / 30 / 89$ & $4 / 27 / 89$ & BOB2N4 & G323 & G476* & 1.21 & 7.79E-01 & $\mathrm{uCi} / \mathrm{mL}$ & $P$ & Raw \\
\hline Sr9o & $6 / 1 / 90$ & $4 / 30 / 89$ & $4 / 27 / 89$ & BOB2N1 & G310 & G471* & 1.20 & 7.78E-01 & uCi/mL & $\mathbf{P}$ & Raw \\
\hline Sr9o & $6 / 1 / 90$ & $4 / 30 / 89$ & $4 / 27 / 89$ & Во82M9 & G302 & G477" & 1.20 & $7.59 E-01$ & $\mathrm{uCi} / \mathrm{mL}$ & $\mathbf{P}$ & Raw \\
\hline Sr9o & $6 / 1 / 90$ & $4 / 30 / 89$ & $4 / 27 / 89$ & BOB2N1 & G310 & G471• & 1.20 & 7.54E-01 & $\mathrm{uCi} / \mathrm{mL}$ & $P$ & Raw \\
\hline Sr9o & $6 / 1 / 90$ & $4 / 30 / 89$ & $4 / 27 / 89$ & BOB2T9 & G327 & G470 & 1.19 & 7.19E-01 & $\mathrm{uCi} / \mathrm{mL}$ & $p$ & Raw \\
\hline Sr9o & $6 / 1 / 90$ & $4 / 30 / 89$ & $4 / 27 / 89$ & 8082T9 & G327 & G470 & 1.19 & $7.00 E-01$ & $u \mathrm{Ci} / \mathrm{mL}$ & $\mathbf{P}$ & Raw \\
\hline Sr9o & $6 / 1 / 90$ & $4 / 30 / 89$ & $4 / 27 / 89$ & $8082 N 4$ & G323 & G476* & 1.21 & 5.89E-01 & $\mathrm{uCi} / \mathrm{mL}$ & $\mathbf{P}$ & Raw \\
\hline Sr9o & $6 / 1 / 90$ & $4 / 30 / 89$ & $4 / 27 / 89$ & BOB2N4 & G323 & G476* & 1.21 & 5.77E-01 & $\mathrm{uCi} / \mathrm{mL}$ & $\mathbf{P}$ & Raw \\
\hline Sr90 & $5 / 17 / 84$ & NS & NS & R8469 & NS & NS & NS & $3.41 E-02$ & uCi/L & $P$ & RAW \\
\hline Sr90 & $5 / 17 / 84$ & NS & NS & $\mathbf{R 8 4 7 2}$ & NS & NS & NS & $3.32 \mathrm{E}-02$ & $\mathrm{uCi} / \mathrm{L}$ & $P$ & RAW \\
\hline Sr90 & $5 / 17 / 84$ & NS & NS & $\mathrm{R} 8473$ & NS & NS & NS & $3.22 \mathrm{E}-02$ & uCin & $P$ & RAW \\
\hline Sr90 & $5 / 17 / 84$ & NS & NS & R8471 & NS & NS & NS & $3.20 \mathrm{E}-02$ & uCi/L. & $P$ & RAW \\
\hline Technetium-99 & 6/1/90 & $4 / 30 / 89$ & $4 / 27 / 89$ & ВОВ2T9 & G327 & G443 & 1.20 & $1.02 E-01$ & $\mathrm{uCi} / \mathrm{mL}$ & $\mathbf{P}$ & Raw \\
\hline Technetium-99 & $6 / 1 / 90$ & $4 / 30 / 89$ & $4 / 27 / 89$ & BOB2T9 & G327 & G443 & 1.20 & $9.49 E-02$ & $u C \mathrm{i} / \mathrm{mL}$ & $P$ & Raw \\
\hline Technetium-99 & $6 / 1 / 90$ & $4 / 30 / 89$ & $4 / 27 / 89$ & BOB2T9 & G327 & G443 & 1.20 & $9.42 E-02$ & $\mathrm{uCi} / \mathrm{mL}$ & $\mathbf{P}$ & Raw \\
\hline Technetium-99 & $6 / 1 / 90$ & $4 / 30 / 89$ & $4 / 27 / 89$ & вов2M5 & G315 & G364 & 1.19 & $9.36 E-02$ & $u C i / m L$ & $P$ & Raw \\
\hline Technetium-99 & $6 / 1 / 90$ & $4 / 30 / 89$ & $4 / 27 / 89$ & $80 B 2 L 7$ & G299 & G342 & 1.20 & $9.25 E-02$ & $\mathrm{uCi} / \mathrm{mL}$ & $\mathbf{P}$ & Raw \\
\hline Technetium-99 & $6 / 1 / 90$ & $4 / 30 / 89$ & $4 / 27 / 89$ & ВОВ2M1 & G308 & G353 & 1.20 & $9.24 E-02$ & $\mathrm{uCi} / \mathrm{mL}$ & $\mathbf{P}$ & Raw \\
\hline Technetium-99 & $6 / 1 / 90$ & $4 / 30 / 89$ & $4 / 27 / 89$ & $\mathrm{BOB2N4}$ & G323 & G341 & 1.20 & $9.08 E-02$ & $\mathrm{uCi} / \mathrm{mL}$ & $\mathbf{P}$ & Raw \\
\hline Technetium-99 & $6 / 1 / 90$ & $4 / 30 / 89$ & 4/27/89 & BOB2M4 & G313 & G363 & 1.20 & $9.04 E-02$ & $\mathrm{uCi} / \mathrm{mL}$ & $\mathbf{P}$ & Raw \\
\hline Technetium-99 & $6 / 1 / 90$ & $4 / 30 / 89$ & $4 / 27 / 89$ & B082M9 & G302 & G477* & 1.20 & $9.03 E-02$ & $\mathrm{uCi} / \mathrm{mL}$ & $P$ & Raw \\
\hline Technetium-99 & $6 / 1 / 90$ & $4 / 30 / 89$ & $4 / 27 / 89$ & BOB2L9 & G305 & G349 & 1.20 & 9.02E-02 & $\mathrm{uCi} / \mathrm{mL}$ & $\mathbf{P}$ & Raw \\
\hline
\end{tabular}




\begin{tabular}{|c|c|c|c|c|c|c|}
\hline \multicolumn{4}{|c|}{ Radionuclide Information } & \multirow[b]{2}{*}{$\begin{array}{l}\text { References } \\
\text { Number }\end{array}$} & \multirow[b]{2}{*}{ Validated $(Y / N)$} & \multirow[b]{2}{*}{ Notes } \\
\hline Radoinuclide & Conc. $(\mathrm{Bq} / \mathrm{L})$ & $\begin{array}{c}\text { Conc. (Bq/L } \\
\text { decayed) }\end{array}$ & $\begin{array}{l}\text { Conc. (Cill } \\
\text { Decayed) }\end{array}$ & & & \\
\hline Sr89/90 & $3.09 E+07$ & $2.96 E+07$ & 8.01E-04 & AP102-3 & $\mathbf{Y}$ & $\begin{array}{l}\text { Density reported for } \mathrm{G} 477 \text { collected at } 182 \text { in from } \\
\text { bottom of tank at a } 30^{\circ} \text { angle and was keep heated } \\
\text { to } 40^{\circ} \mathrm{C} \text {. }\end{array}$ \\
\hline Sr89/90 & $2.88 E+07$ & $2.77 E+07$ & $7.48 E-04$ & AP102-3 & $Y$ & $\begin{array}{l}\text { Density reported for } \mathrm{G} 476 \text { collected at } 99 \text { in from } \\
\text { bottom of tank at a } 270^{\circ} \text { angle and was keep heated } \\
\text { to } 40^{\circ} \mathrm{C} \text {. }\end{array}$ \\
\hline Sr89/90 & $2.88 E+07$ & $2.77 E+07$ & 7.47E-0.4 & AP102-3 & $\gamma$ & $\begin{array}{l}\text { Density reported for } 6471 \text { collected at } 208 \text { in from } \\
\text { bottom of tank at a } 150^{\circ} \text { angle and was keep heated } \\
\text { to } 40^{\circ} \mathrm{C} \text {. }\end{array}$ \\
\hline Sr89/90 & $2.81 E+07$ & $2.70 E+07$ & $7.29 E-0.4$ & AP102-3 & $Y$ & $\begin{array}{l}\text { Density reported for } 6477 \text { collected at } 182 \text { in from } \\
\text { bottom of tank at a } 30^{\circ} \text { angle and was keep heated } \\
\text { to } 40^{\circ} \mathrm{C} \text {. }\end{array}$ \\
\hline SrB9/90 & $2.79 E+07$ & $2.68 E+07$ & $7.24 E-0.4$ & AP102-3 & $\mathbf{Y}$ & $\begin{array}{l}\text { Density reported for } 6471 \text { collected at } 208 \text { in from } \\
\text { bottom of tank at a } 150^{\circ} \text { angle and was keep heated } \\
\text { to } 40^{\circ} \mathrm{C} \text {. }\end{array}$ \\
\hline Sr89/90 & $2.66 E+07$ & $2.56 E+07$ & $6.91 E-0.4$ & AP102-3 & $Y$ & $\begin{array}{l}\text { Density reported for G470 represents a composite } \\
\text { sample. Sample was kept heated to } 40^{\circ} \mathrm{C} \text {. }\end{array}$ \\
\hline Sr89/90 & $2.59 E+07$ & $2.49 E+07$ & $6.72 \mathrm{E}-04$ & AP102-3 & $Y$ & $\begin{array}{l}\text { Density reported for } \mathrm{G} 470 \text { represents a composite } \\
\text { sample. Sample was kept heated to } 40^{\circ} \mathrm{C} \text {. }\end{array}$ \\
\hline Sr89/90 & $2.18 E+07$ & $2.09 E+07$ & $5.66 \mathrm{E}-0.4$ & AP102-3 & $\mathbf{Y}$ & $\begin{array}{l}\text { Density reported for } 6476 \text { collected at } 99 \text { in from } \\
\text { bottom of tank at a } 270^{\circ} \text { angle and was keep heated } \\
\text { to } 40^{\circ} \mathrm{C} \text {. }\end{array}$ \\
\hline Sr89/90 & $2.13 E+07$ & $2.05 E+07$ & $5.54 E-0.4$ & AP102-3 & $\mathbf{Y}$ & $\begin{array}{l}\text { Density reported for } \mathrm{G} 476 \text { collected at } 99 \text { in from } \\
\text { bottom of tank at a } 270^{\circ} \text { angle and was keep heated } \\
\text { to } 40^{\circ} \mathrm{C} \text {. }\end{array}$ \\
\hline Sr89/90 & $1.26 \mathrm{E}+03$ & $1.08 \mathrm{E}+03$ & $2.92 E-08$ & AP102-1 & $\mathbf{N}$ & Outdated Information \\
\hline Sr89/90 & $1.23 E+03$ & $1.05 \mathrm{E}+03$ & $2.84 E-08$ & AP102-1 & $\mathbf{N}$ & Outdated Information \\
\hline Sr89/90 & $1.19 E+03$ & $1.02 E+03$ & $2.76 \mathrm{E}-08$ & AP102-1 & $N$ & Outdated information \\
\hline Sr89/90 & $1.18 \mathrm{E}+03$ & $1.01 E+03$ & $2.73 E-08$ & AP102-1 & $\mathbf{N}$ & Outdated Information \\
\hline Tc99 & $3.77 E+06$ & $3.77 E+06$ & $1.02 \mathrm{E}-04$ & AP102-3 & $\mathbf{Y}$ & $\begin{array}{l}\text { Density reported for G443 represents a composite } \\
\text { sample }\end{array}$ \\
\hline Tc99 & $3.51 E+06$ & $3.51 E+06$ & $9.49 E-05$ & AP102-3 & $Y$ & $\begin{array}{l}\text { Density reported for G443 represents a composite } \\
\text { sample }\end{array}$ \\
\hline Tc99 & $3.49 E+06$ & $3.49 E+06$ & 9.42E-05 & AP102-3 & $Y$ & $\begin{array}{l}\text { Density reported for } \mathrm{G} 443 \text { represents a composite } \\
\text { sample }\end{array}$ \\
\hline Tc99 & $3.46 E+06$ & $3.46 E+06$ & $9.36 E-05$ & AP102-3 & $Y$ & $\begin{array}{l}\text { Density reported for } \mathrm{G} 364 \text { collected at } 372 \text { in from } \\
\text { bottom of tank at a } 270^{\circ} \text { angle. Sample was } \\
\text { unheated, unheated samples precipitated }\end{array}$ \\
\hline Tc99 & $3.42 E+06$ & $3.42 E+06$ & $9.25 E-05$ & AP102-3 & $Y$ & $\begin{array}{l}\text { Density reported for } G 342 \text { collected at } 306 \text { in from } \\
\text { bottom of tank at a } 30^{\circ} \text { angle. Sample was } \\
\text { unheated, unheated samples precipitated }\end{array}$ \\
\hline Tc99 & $3.42 E+06$ & $3.42 E+06$ & $9.24 E-05$ & AP102-3 & $\boldsymbol{Y}$ & $\begin{array}{l}\text { Density reported for } \mathrm{G} 353 \text { collected at } 334 \text { in from } \\
\text { bottom of tank at a } 150^{\circ} \text { angle. Sample was } \\
\text { unheated, unheated samples precipitated }\end{array}$ \\
\hline Tc99 & $3.36 E+06$ & $3.36 E+06$ & $9.08 E-05$ & AP102-3 & $Y$ & $\begin{array}{l}\text { Density reported for } \mathrm{G} 341 \text { collected at } 99 \text { in from } \\
\text { bottom of tank at a } 270^{\circ} \text { angle. }\end{array}$ \\
\hline Tc99 & $3.34 E+06$ & $3.34 E+06$ & $9.04 E-05$ & AP102-3 & $\mathbf{Y}$ & $\begin{array}{l}\text { Density reported for } G 363 \text { collected at } 18 \text { in from } \\
\text { bottom of tank at a } 150^{\circ} \text { angle. Sample was } \\
\text { unheated, unheated samples precipitated }\end{array}$ \\
\hline Tc99 & $3.34 E+06$ & $3.34 E+06$ & 9.03E-05 & AP102-3 & $Y$ & $\begin{array}{l}\text { Density reported for G477 collected at } 182 \text { in from } \\
\text { bottom of tank at } 230^{\circ} \text { angle and was keep heated } \\
\text { to } 40^{\circ} \mathrm{C} \text {. }\end{array}$ \\
\hline Tc99 & $3.34 E+06$ & $3.34 E+06$ & $9.02 E-05$ & AP102-3 & $Y$ & $\begin{array}{l}\text { Density reported for G349 collected at } 34 \text { in from } \\
\text { bottom of tank at a } 30^{\circ} \text { angle. Sample was } \\
\text { unheated, unheated samples precipitated }\end{array}$ \\
\hline
\end{tabular}




\begin{tabular}{|c|c|c|c|c|c|c|c|c|c|c|c|}
\hline \multicolumn{12}{|c|}{ Tank Waste Characterization Sample Information } \\
\hline $\begin{array}{c}\text { Reported } \\
\text { Radionuclide }\end{array}$ & $\begin{array}{c}\text { Memo } \\
\text { Date }\end{array}$ & $\begin{array}{c}\text { Start } \\
\text { Analysis } \\
\text { Dato }\end{array}$ & $\begin{array}{c}\text { Start } \\
\text { Sample } \\
\text { Date } \\
\end{array}$ & $\begin{array}{l}\text { Sample } \\
\text { Number }\end{array}$ & $\begin{array}{c}\text { Tank Farm } \\
\text { Number }\end{array}$ & $\begin{array}{c}\text { Lab Sample } \\
\text { Number }\end{array}$ & $\begin{array}{l}\text { Density } \\
(\mathrm{g} / \mathrm{mL})\end{array}$ & $\begin{array}{c}\text { Reported } \\
\text { Value } \\
\end{array}$ & Reported Unit & Code & Soure: \\
\hline Technetium-99 & $6 / 1 / 90$ & $4 / 30 / 89$ & $4 / 27 / 89$ & вОВ2T9 & G327 & G443 & 1.20 & $9.02 E-02$ & $\mathrm{uCl} / \mathrm{mL}$ & $\mathbf{P}$ & Raw \\
\hline Technetium-99 & $6 / 1 / 90$ & $4 / 30 / 89$ & $4 / 27 / 89$ & BOB2N1 & G310 & G471* & 1.20 & 8.98E-02 & $\mathrm{uCi} / \mathrm{mL}$ & P & Raw \\
\hline Technetium-99 & $6 / 1 / 90$ & $4 / 30 / 89$ & $4 / 27 / 89$ & BOB2L8 & G303 & G348 & 1.21 & 8.97E-02 & uCi/mL & $\mathbf{P}$ & Raw \\
\hline Technetium-99 & 6/1/90 & $4 / 30 / 89$ & $4 / 27 / 89$ & Вов2МО & G306 & G352 & 1.20 & $8.95 E-02$ & $\mathrm{uCi} / \mathrm{mL}$ & $\mathbf{P}$ & Raw \\
\hline Technetium-99 & $6 / 1 / 90$ & $4 / 30 / 89$ & $4 / 27 / 89$ & BOB2M1 & G308 & G353 & 1.20 & $8.92 E-02$ & $\mathrm{uCi} / \mathrm{mL}$ & $\mathbf{P}$ & Raw \\
\hline Technetium-99 & $6 / 1 / 90$ & $4 / 30 / 89$ & $4 / 27 / 89$ & BOB2N4 & G323 & G341 & 1.20 & $8.89 \mathrm{E}-02$ & uCi/mL & $P$ & Raw \\
\hline Technetium-99 & $6 / 1 / 90$ & $4 / 30 / 89$ & $4 / 27 / 89$ & BOB2T9 & G32? & G470* & 1.19 & $8.84 E-02$ & $\mathrm{uCi} / \mathrm{mL}$ & $P$ & Raw \\
\hline Technetium-99 & $6 / 1 / 90$ & $4 / 30 / 89$ & $4 / 27 / 89$ & BOB2L9 & G305 & G349 & 1.20 & 8.77E-02 & $u C i / m L$ & $\mathbf{P}$ & Raw \\
\hline Technetium-99 & $6 / 1 / 90$ & $4 / 30 / 89$ & $4 / 27 / 89$ & ВОВ2М3 & G311 & G359 & 1.20 & $8.76 E-02$ & $\mathrm{uCi} / \mathrm{mL}$ & $\mathbf{P}$ & Raw \\
\hline Technetium-99 & $6 / 1 / 90$ & $4 / 30 / 89$ & $4 / 27 / 89$ & BOB2N4 & G323 & G476* & 1.21 & $8.75 \mathrm{E}-02$ & $u C i / m L$ & $\mathbf{P}$ & Raw \\
\hline Technetium-99 & $6 / 1 / 90$ & $4 / 30 / 89$ & $4 / 27 / 89$ & BOB2L7 & G299 & G342 & 1.20 & $8.72 E-02$ & $\mathrm{uCi} / \mathrm{mL}$ & $\mathbf{P}$ & Raw \\
\hline Technetium-99 & $6 / 1 / 90$ & $4 / 30 / 89$ & $4 / 27 / 89$ & BOB2L8 & G303 & G348 & 1.21 & $8.71 E-02$ & $\mathrm{uCi} / \mathrm{mL}$ & $\mathbf{P}$ & Raw \\
\hline Technetium-99 & $6 / 1 / 90$ & $4 / 30 / 89$ & $4 / 27 / 89$ & ВОВ2M4 & G313 & G363 & 1.20 & $8.71 E-02$ & uCi/mL & $\mathbf{P}$ & Raw \\
\hline Technetium-99 & $6 / 1 / 90$ & $4 / 30 / 89$ & $4 / 27 / 89$ & ВОВ2M5 & G315 & G364 & 1.19 & 8.59E-02 & $\mathrm{uCi} / \mathrm{mL}$ & $P$ & Raw \\
\hline Technetium-99 & $6 / 1 / 90$ & $4 / 30 / 89$ & $4 / 27 / 89$ & 8OB2M5 & G315 & G364 & 1.19 & $8.57 E-02$ & $\mathrm{uCi} / \mathrm{mL}$ & $\mathbf{P}$ & Raw \\
\hline Technetium-99 & $6 / 1 / 90$ & $4 / 30 / 89$ & $4 / 27 / 89$ & вов2T9 & G327 & G470* & 1.19 & $8.55 E-02$ & $u C i / m L$ & $P$ & Raw \\
\hline Technetium-99 & $3 / 31 / 89$ & $4 / 89$ & $4 / 27 / 89$ & BOB2T9 & G327 & NS & NS & $8.55 E-02$ & $\mathrm{uCl} / \mathrm{mL}$ & $\mathbf{P}$ & TCD \\
\hline Technetium-99 & $6 / 1 / 90$ & $4 / 30 / 89$ & $4 / 27 / 89$ & ВOB2M4 & G313 & G363 & 1.20 & 8.54E-02 & $\mathrm{uCi} / \mathrm{mL}$ & $P$ & Raw \\
\hline Technetium-99 & $6 / 1 / 90$ & $4 / 30 / 89$ & $4 / 27 / 89$ & BOB2M2 & G309 & G358 & 1.20 & $8.44 E-02$ & $\mathrm{uCi} / \mathrm{mL}$ & $P$ & Raw \\
\hline Technetium-99 & $6 / 1 / 90$ & $4 / 30 / 89$ & $4 / 27 / 89$ & ВОВ2T9 & G327 & G470* & 1.19 & 8.38E-02 & $\mathrm{uCi} / \mathrm{mL}$ & $P$ & Raw \\
\hline Technetium-99 & $3 / 31 / 89$ & $4 / 89$ & $4 / 27 / 89$ & BOB2T9 & G327 & NS & NS & $8.38 E-02$ & $\mathrm{uCi} / \mathrm{mL}$ & $P$ & $T C D$ \\
\hline
\end{tabular}


Vaste Characterization Database

Tank 241-AP-102

\begin{tabular}{|c|c|c|c|c|c|c|}
\hline \multicolumn{4}{|c|}{ Radionuclide Information } & \multirow[b]{2}{*}{$\begin{array}{l}\text { Reference } \\
\text { Number }\end{array}$} & \multirow[b]{2}{*}{ Validated $[Y / N]$} & \multirow[b]{2}{*}{ Notes } \\
\hline Radoinuclide & Conc. $(\mathrm{Bq} / \mathrm{L})$ & $\begin{array}{c}\text { Conc. }(B q / L \\
\text { decayed) }\end{array}$ & $\begin{array}{l}\text { Conc. (Ci/L } \\
\text { Decayed) }\end{array}$ & & & \\
\hline Tc99 & $3.34 E+06$ & $3.34 E+06$ & $9.02 E-05$ & AP102-3 & $\mathrm{Y}$ & $\begin{array}{l}\text { Density reported for G443 represents a composite } \\
\text { sample }\end{array}$ \\
\hline $\operatorname{Tc} 99$ & $3.32 E+06$ & $3.32 \mathrm{E}+06$ & $8.98 E-05$ & AP102-3 & $\mathbf{Y}$ & $\begin{array}{l}\text { Density reported for G471 collected at } 208 \text { in from } \\
\text { bottom of tank at a } 150^{\circ} \text { angle and was keep heated } \\
\text { to } 40^{\circ} \mathrm{C} \text {. }\end{array}$ \\
\hline Te99 & $3.32 E+06$ & $3.32 \mathrm{E}+06$ & 8.97E-05 & AP102-3 & $Y$ & $\begin{array}{l}\text { Density reported for G348 collected at } 125 \text { in from } \\
\text { bottom of tank at a } 30^{\circ} \text { angle. Sample was } \\
\text { unheated, unheated samples precipitated }\end{array}$ \\
\hline Tc99 & $3.31 E+06$ & $3.31 E+06$ & $8.95 E-05$ & AP102-3 & $\mathbf{Y}$ & $\begin{array}{l}\text { Density reported for } \mathrm{G} 352 \text { collected at } 34 \text { in from } \\
\text { bottom of tank at a } 30^{\circ} \text { angle. Sample was } \\
\text { unheated, unheated samples preclpitated }\end{array}$ \\
\hline Tc99 & $3.30 E+06$ & $3.30 E+06$ & $8.92 E-05$ & AP102-3 & $Y$ & $\begin{array}{l}\text { Density reported for } G 353 \text { collected at } 334 \text { in from } \\
\text { bottom of tank at a } 150^{\circ} \text { angle. Sample was } \\
\text { unheated, unheated samples precipitated }\end{array}$ \\
\hline Tc99 & $3.29 E+06$ & $3.29 E+06$ & 8.89E-05 & AP102-3 & $\mathbf{Y}$ & $\begin{array}{l}\text { Density reported for } \mathrm{G} 341 \text { collected at } 99 \text { in from } \\
\text { bottom of tank at a } 270^{\circ} \text { angle. }\end{array}$ \\
\hline Tc99 & $3.27 E+06$ & $3.27 E \div 06$ & 8.84E-05 & AP102-3 & $Y$ & $\begin{array}{l}\text { Density reported for G470 represents a composite } \\
\text { sample. Sample was kept heated to } 40^{\circ} \mathrm{C} \text {. }\end{array}$ \\
\hline Tc99 & $3.24 E+06$ & $3.24 E+06$ & 8.77E-05 & AP102-3 & $\mathbf{Y}$ & $\begin{array}{l}\text { Density reported for } \mathbf{G 3 4 9} \text { collected at } 34 \text { in from } \\
\text { bottom of tank at a } 30^{\circ} \text { angle. Sample was } \\
\text { Unheated, unheated samples precipitated }\end{array}$ \\
\hline Tc99 & $3.24 E+06$ & $3.24 E+06$ & $8.76 E-05$ & AP102-3 & $Y$ & $\begin{array}{l}\text { Density reported for G359 collected at } 162 \text { in from } \\
\text { bottom of tank at a } 150^{\circ} \text { angle. Sample was } \\
\text { unheated, unheated samples precipitated }\end{array}$ \\
\hline Tc99 & $3.24 E+06$ & $3.24 E+06$ & $8.75 E-05$ & AP102-3 & $Y$ & $\begin{array}{l}\text { Density reported for } \mathrm{G} 476 \text { collected at } 99 \text { in from } \\
\text { bottom of tank at a } 270^{\circ} \text { angle and was keep heated } \\
\text { to } 40^{\circ} \mathrm{C} \text {. }\end{array}$ \\
\hline Tc99 & $3.23 E+06$ & $3.23 E+06$ & $8.72 E-05$ & AP102-3 & $\mathbf{Y}$ & $\begin{array}{l}\text { Density reported for } \mathrm{G} 342 \text { collected at } 306 \text { in from } \\
\text { bottom of tank at a } 30^{\circ} \text { angle. Sample was } \\
\text { unheated, unheated samples precipitated }\end{array}$ \\
\hline Tc99 & $3.22 E+06$ & $3.22 E+06$ & 8.71E-05 & AP102-3 & $\mathbf{Y}$ & $\begin{array}{l}\text { Density reported for } G 348 \text { collected at } 125 \text { in from } \\
\text { bottom of tank at a } 30^{\circ} \text { angle. Sample was } \\
\text { unheated, unheated samples precipitated }\end{array}$ \\
\hline Tc99 & $3.22 E+06$ & $3.22 E+06$ & 8.71E-05 & AP102-3 & $\gamma$ & $\begin{array}{l}\text { Density reported for } \mathrm{G} 363 \text { collected at } 18 \text { in from } \\
\text { bottom of tank at a } 150^{\circ} \text { angle. Sample was } \\
\text { unheated, unheated samples precipitated }\end{array}$ \\
\hline Tc99 & $3.18 E+06$ & $3.18 E+06$ & $8.59 E-05$ & AP102-3 & $Y$ & $\begin{array}{l}\text { Density reported for G364 collected at } 372 \text { in from } \\
\text { bottom of tank at a } 270^{\circ} \text { angle. Sample was } \\
\text { unheated, unheated samples precipitated }\end{array}$ \\
\hline Tc99 & $3.17 E \div 06$ & $3.17 E+06$ & 8.57E-05 & AP102-3 & $Y$ & $\begin{array}{l}\text { Density reported for } \mathrm{G} 364 \text { collected at } 372 \text { in from } \\
\text { bottom of tank at a } 270^{\circ} \text { angle. Sample was } \\
\text { unheated, unheated samples precipitated }\end{array}$ \\
\hline Tc99 & $3.16 E+06$ & $3.16 E+06$ & 8.55E-05 & AP102-3 & $\mathbf{Y}$ & $\begin{array}{l}\text { Density reported for } \mathrm{G} 470 \text { represents a composite } \\
\text { sample. Sample was kept heated to } 40^{\circ} \mathrm{C} \text {. }\end{array}$ \\
\hline Tc99 & $3.16 E+06$ & $3.16 E+06$ & 8.55E-05 & NA & $N$ & THIS POINT NOT NEEDED FOR DATA BASE \\
\hline Tc99 & $3.16 E+06$ & $3.16 E+06$ & $8.54 E-05$ & AP102-3 & $\mathbf{Y}$ & $\begin{array}{l}\text { Density reported for } G 363 \text { collected at } 18 \text { in from } \\
\text { bottom of tank at a } 150^{\circ} \text { angle. Sample was } \\
\text { unheated, unheated samples precipitated }\end{array}$ \\
\hline $\operatorname{Tc99}$ & $3.12 E+06$ & $3.12 E+06$ & 8.44E-05 & AP102-3 & $\mathbf{Y}$ & $\begin{array}{l}\text { Density reported for } \mathrm{G} 358 \text { collected at } 334 \text { in from } \\
\text { bottom of tank at a } 150^{\circ} \text { angle. Sample was } \\
\text { unheated, unheated samples precipitated }\end{array}$ \\
\hline Tc99 & $3.10 E+06$ & $3.10 E+06$ & $8.38 E-05$ & AP102-3 & $\mathbf{Y}$ & $\begin{array}{l}\text { Density reported for } \mathrm{G} 470 \text { represents a composite } \\
\text { sample. Sample was kept heated to } 40^{\circ} \mathrm{C} \text {. }\end{array}$ \\
\hline Tc99 & $3.10 E+06$ & $3.10 E+06$ & $8.38 E-05$ & NA & $\mathbf{N}$ & THIS POINT NOT NEEDED FOR DATA BASE \\
\hline
\end{tabular}




\begin{tabular}{|c|c|c|c|c|c|c|c|c|c|c|c|}
\hline \multicolumn{12}{|c|}{ Tank Waste Characterization Sample Information } \\
\hline $\begin{array}{c}\text { Reported } \\
\text { Radionuclide }\end{array}$ & $\begin{array}{c}\text { Memo } \\
\text { Date }\end{array}$ & $\begin{array}{c}\text { Start } \\
\text { Analysis } \\
\text { Date }\end{array}$ & $\begin{array}{c}\text { Start } \\
\text { Sample } \\
\text { Date }\end{array}$ & $\begin{array}{l}\text { Sample } \\
\text { Number }\end{array}$ & $\begin{array}{c}\text { Tank Farm } \\
\text { Number }\end{array}$ & $\begin{array}{c}\text { Lab Sample } \\
\text { Number }\end{array}$ & $\begin{array}{l}\text { Density } \\
(\mathrm{g} / \mathrm{mL})\end{array}$ & $\begin{array}{c}\text { Reported } \\
\text { Value } \\
\end{array}$ & Reported Unit & Code & Source \\
\hline Technetium-99 & $6 / 1 / 90$ & $4 / 30 / 89$ & $4 / 27 / 89$ & Вов2М6 & G318 & G459 & 1.20 & 8.34E-02 & $\mathrm{uCi} / \mathrm{mL}$ & $\mathbf{P}$ & Raw \\
\hline Technetium-99 & $6 / 1 / 90$ & $4 / 30 / 89$ & $4 / 27 / 89$ & ВОВ2M6 & G318 & G459 & 1.20 & 8.14E-02 & $\mathrm{uCi} / \mathrm{mL}$ & $\mathbf{P}$ & Raw \\
\hline Technetium-99 & $6 / 1 / 90$ & $4 / 30 / 89$ & $4 / 27 / 89$ & ВOB2M3 & G311 & G359 & 1.20 & $8.11 E-02$ & $\mathrm{uCi} / \mathrm{mL}$ & $\mathbf{P}$ & Raw \\
\hline Technetium-99 & $6 / 1 / 90$ & $4 / 30 / 89$ & $4 / 27 / 89$ & BOB2M5 & G315 & G364 & 1.19 & 7.83E-02 & $u C i / m L$ & $\mathbf{P}$ & Raw \\
\hline Technetium-99 & $6 / 1 / 90$ & $4 / 30 / 89$ & $4 / 27 / 89$ & BOB2MO & G306 & G352 & 1.20 & 7.82E-02 & $\mathrm{uCi} / \mathrm{mL}$ & $\mathbf{P}$ & Raw \\
\hline Technetium-99 & $6 / 1 / 90$ & $4 / 30 / 89$ & $4 / 27 / 89$ & BOB2M4 & G313 & G363 & 1.20 & 7.81E-02 & $\mathrm{uCi} / \mathrm{mL}$ & $\mathbf{P}$ & Raw \\
\hline Technetlum-99 & $6 / 1 / 90$ & $4 / 30 / 89$ & $4 / 27 / 89$ & ВOB2M2 & G309 & G358 & 1.20 & 7.69E-02 & $\mathrm{uCi} / \mathrm{mL}$ & $\mathbf{P}$ & Raw \\
\hline Technetium-99 & $6 / 1 / 90$ & 4/30/89 & $4 / 27 / 89$ & $\mathrm{BOB} 2 \mathrm{N1}$ & G310 & $\mathrm{G} 471^{\circ}$ & 1.20 & $7.66 \mathrm{E}-02$ & $\mathrm{uCi} / \mathrm{mL}$ & $\mathbf{P}$ & Raw \\
\hline Technetium-99 & $6 / 1 / 90$ & $4 / 30 / 89$ & $4 / 27 / 89$ & BOB2M9 & G302 & G477* & 1.20 & $7.59 E-02$ & $\mathrm{uCi} / \mathrm{mL}$ & $P$ & Raw \\
\hline Technetium-99 & $6 / 1 / 90$ & $4 / 30 / 89$ & $4 / 27 / 89$ & BOB2N4 & G323 & G476* & 1.21 & $6.99 E-02$ & $\mathrm{uCi} / \mathrm{mL}$ & $\mathbf{P}$ & Raw \\
\hline Technetium-9g & $6 / 1 / 90$ & $4 / 30 / 89$ & $4 / 27 / 89$ & ВОВ2M7 & G319 & G460 & 1.20 & $6.97 E-02$ & uCi $/ \mathrm{mL}$ & $P$ & Raw \\
\hline Technetium-99 & $6 / 1 / 90$ & $4 / 30 / 89$ & $4 / 27 / 89$ & $8082 M 7$ & G319 & G460 & 1.20 & $6.84 E-02$ & $u C i / m L$ & $P$ & Raw \\
\hline Technetium-99 & $6 / 1 / 90$ & $4 / 30 / 89$ & $4 / 27 / 89$ & Вов2Т9 & G327 & G470* & 1.19 & $6.39 E-02$ & $\mathrm{uCi} / \mathrm{mL}$ & $P$ & Raw \\
\hline Technetium-99 & $6 / 1 / 90$ & $4 / 30 / 89$ & $4 / 27 / 89$ & ВОВ2M3 & G311 & G359 & 1.20 & 2.39E-O2 & $\mathrm{uCi} / \mathrm{mL}$ & $P$ & Raw \\
\hline Technetium-99 & $6 / 1 / 90$ & $4 / 30 / 89$ & $4 / 27 / 89$ & ВОВ2M3 & G311 & G359 & 1.20 & 2.19E-02 & uCi/mL & $P$ & Raw \\
\hline Technetium-99 & $6 / 1 / 90$ & $4 / 30 / 89$ & $4 / 27 / 89$ & Вов2M2 & G309 & G358 & 1.20 & $1.72 E-02$ & $\mathrm{uCi} / \mathrm{mL}$ & $P$ & Raw \\
\hline Technetium-99 & $6 / 1 / 90$ & $4 / 30 / 89$ & $4 / 27 / 89$ & во82M2 & G309 & G358 & 1.20 & $1.70 E-02$ & $\mathrm{uCi} / \mathrm{mL}$ & $\mathbf{P}$ & Raw \\
\hline Technetium-99 & $6 / 1 / 90$ & $4 / 30 / 89$ & $4 / 27 / 89$ & BOB2M1 & G308 & G353 & 1.20 & $1.40 \mathrm{E}-02$ & $\mathrm{uCi} / \mathrm{mL}$ & $P$ & Raw \\
\hline Technetium-99 & $6 / 1 / 90$ & $4 / 30 / 89$ & $4 / 27 / 89$ & BOB2M1 & G308 & G353 & 1.20 & $1.27 \mathrm{E}-02$ & $\mathrm{uCi} / \mathrm{mL}$ & $p$ & Raw \\
\hline
\end{tabular}




\begin{tabular}{|c|c|c|c|c|c|c|}
\hline \multicolumn{4}{|c|}{ Radionuclide information } & \multirow[b]{2}{*}{$\begin{array}{c}\text { Reference } \\
\text { Number }\end{array}$} & \multirow[b]{2}{*}{ Validated (Y/N) } & \multirow[b]{2}{*}{ Notes } \\
\hline Radoinuclide & Conc. $(\mathrm{Bq} / \mathrm{L})$ & $\begin{array}{c}\text { Conc. (Bq/L } \\
\text { decayed) }\end{array}$ & $\begin{array}{c}\text { Conc. (Ci/h } \\
\text { Decayed) }\end{array}$ & & & \\
\hline Tc99 & $3.09 E+06$ & $3.09 E+06$ & 8.34E-05 & AP102-3 & $Y$ & $\begin{array}{l}\text { Density reported for } \mathrm{G} 459 \text { collected at } 226 \text { in from } \\
\text { bottom of tank at a } 270^{\circ} \text { angle. Sample was } \\
\text { unheated, unheated samples precipitated }\end{array}$ \\
\hline Tc99 & $3.01 E+06$ & $3.01 E+06$ & $8.14 E-05$ & AP102-3 & $\mathbf{Y}$ & $\begin{array}{l}\text { Density reported for } 6459 \text { collected at } 226 \text { in from } \\
\text { bottom of tank at a } 270^{\circ} \text { angle. Sample was } \\
\text { unheated, unheated samples precipitated }\end{array}$ \\
\hline Tc99 & $3.00 E+06$ & $3.00 E+06$ & $8.11 E-05$ & AP102-3 & $Y$ & $\begin{array}{l}\text { Density reported for } G 359 \text { collected at } 162 \text { in from } \\
\text { bottom of tank at a } 150^{\circ} \text { angle. Sample was } \\
\text { unheated, unheated samples precipitated }\end{array}$ \\
\hline Te99 & $2.90 E+06$ & $2.90 E+06$ & $7.83 E-05$ & AP102-3 & $\mathbf{Y}$ & $\begin{array}{l}\text { Density reported for } G 364 \text { collected at } 372 \text { in from } \\
\text { bottom of tank at a } 270^{\circ} \text { angle. Sample was } \\
\text { unheated, unheated samples precipitated }\end{array}$ \\
\hline Tc99 & $2.89 E+06$ & $2.89 E+06$ & 7.82E-05 & AP102-3 & $Y$ & $\begin{array}{l}\text { Density reported for } \mathrm{G} 352 \text { collected at } 34 \text { in from } \\
\text { bottom of tank at a } 30^{\circ} \text { angle. Sample was } \\
\text { unheated, unheated samples precipitated }\end{array}$ \\
\hline Tc99 & $2.89 E+06$ & $2.89 E+06$ & 7.81E-05 & AP102-3 & $Y$ & $\begin{array}{l}\text { Density reported for } \mathrm{G} 363 \text { collected at } 18 \text { in from } \\
\text { bottom of tank at a } 150^{\circ} \text { angle. Sample was } \\
\text { unheated, unheated samples precipitated }\end{array}$ \\
\hline Tc99 & $2.85 E+06$ & $2.85 E+06$ & 7.69E-05 & AP102-3 & $Y$ & $\begin{array}{l}\text { Density reported for } G 358 \text { collected at } 334 \text { in from } \\
\text { bottom of tank at a } 150^{\circ} \text { angle. Sample was } \\
\text { unheated, unheated samples precipitated }\end{array}$ \\
\hline Tc99 & $2.83 E+06$ & $2.83 E+06$ & $7.66 \mathrm{E}-05$ & AP102-3 & $\mathbf{Y}$ & $\begin{array}{l}\text { Density reported for } \mathrm{G} 471 \text { collected at } 208 \text { in from } \\
\text { bottom of tank at a } 150^{\circ} \text { angle and was keep heated } \\
\text { to } 40^{\circ} \mathrm{C} \text {. }\end{array}$ \\
\hline Tc99 & $2.81 E+06$ & $2.81 E+06$ & 7.59E-05 & AP102-3 & $\mathrm{Y}$ & $\begin{array}{l}\text { Density reported for } \mathrm{G} 477 \text { collected at } 182 \text { in from } \\
\text { bottom of tank at a } 30^{\circ} \text { angle and was keep heated } \\
\text { to } 40^{\circ} \mathrm{C} \text {. }\end{array}$ \\
\hline Tc9s & $2.59 E+06$ & $2.59 E+06$ & 6.99E-05 & AP102-3 & $\mathbf{Y}$ & $\begin{array}{l}\text { Density reported for } \mathrm{G} 476 \text { collected at } 99 \text { in from } \\
\text { bottom of tank at a } 270^{\circ} \text { angle and was keep heated } \\
\text { to } 40^{\circ} \mathrm{C} \text {. }\end{array}$ \\
\hline Tc99 & $2.58 E+06$ & $2.58 E+06$ & 6.97E-05 & AP102-3 & $Y$ & $\begin{array}{l}\text { Density reported for } \mathrm{G} 460 \text { collected at } 226 \text { in from } \\
\text { bottom of tank at a } 270^{\circ} \text { angle. Sample was } \\
\text { unheated, unheated samples precipitated }\end{array}$ \\
\hline Tc99 & $2.53 E+06$ & $2.53 E+06$ & $6.84 \mathrm{E}-05$ & AP102-3 & $Y$ & $\begin{array}{l}\text { Density reported for } \mathrm{G} 460 \text { collected at } 226 \text { in from } \\
\text { bottom of tank at a } 270^{\circ} \text { angle. Sample was } \\
\text { unheated, unheated samples precipitated }\end{array}$ \\
\hline Tc99 & $2.36 E+06$ & $2.36 \varepsilon+06$ & $6.39 E-05$ & AP102-3 & $Y$ & $\begin{array}{l}\text { Density reported for G470 represents a composite } \\
\text { sample. Sample was kept heated to } 40^{\circ} \mathrm{C} \text {. }\end{array}$ \\
\hline Tc99 & $8.84 E+05$ & $8.84 E+05$ & 2.39E-05 & AP102-3 & $Y$ & $\begin{array}{l}\text { Density reported for } \mathrm{G359} \text { collected at } 162 \text { in from } \\
\text { bottom of tank at a } 150^{\circ} \text { angle. Sample was } \\
\text { unheated, unheated samples precipitated }\end{array}$ \\
\hline Tc99 & $8.10 E+05$ & $8.10 E+05$ & 2.19E-05 & AP102-3 & $\mathbf{Y}$ & $\begin{array}{l}\text { Density reported for G359 collected at } 162 \text { in from } \\
\text { bottom of tank at a } 150^{\circ} \text { angle. Sample was } \\
\text { unheated, unheated samples precipitated }\end{array}$ \\
\hline $\operatorname{Ta99}$ & $6.36 E+05$ & $6.36 E+05$ & $1.72 E-05$ & AP102-3 & $\mathbf{Y}$ & $\begin{array}{l}\text { Density reported for } G 358 \text { collected at } 334 \text { in from } \\
\text { bottom of tank at a } 150^{\circ} \text { angle. Sample was } \\
\text { unheated, unheated samples precipitated }\end{array}$ \\
\hline Te99 & $6.29 E+05$ & $6.29 E+05$ & $1.70 E-05$ & AP102-3 & $\mathbf{Y}$ & $\begin{array}{l}\text { Density reported for G358 collected at } 334 \text { in from } \\
\text { bottom of tank at a } 150^{\circ} \text { angle. Sample was } \\
\text { unheated, unheated samples precipitated }\end{array}$ \\
\hline Tc99 & $5.18 E+05$ & $5.18 E+05$ & $1.40 E-05$ & APIO2-3 & $\mathbf{Y}$ & $\begin{array}{l}\text { Density reported for } \mathrm{G} 353 \text { collected at } 334 \text { in from } \\
\text { bottom of tank at a } 150^{\circ} \text { angle. Sample was } \\
\text { unheated, unheated samples precipitated }\end{array}$ \\
\hline Tc99 & $4.70 E+05$ & $4.70 E \div 05$ & 1.27E-05 & AP102-3 & $Y$ & $\begin{array}{l}\text { Density reported for } \mathrm{G} 353 \text { collected at } 334 \text { in from } \\
\text { bottom of tank at a } 150^{\circ} \text { angle. Sample was } \\
\text { unheated. unheated samples precipitated }\end{array}$ \\
\hline
\end{tabular}




\begin{tabular}{|c|c|c|c|c|c|c|c|c|c|c|c|}
\hline & & & & Tank Wast & haracterizatic & Sample Infor: & ation & & & & \\
\hline $\begin{array}{l}\text { Reported } \\
\text { Radionuclide }\end{array}$ & Memo Date & $\begin{array}{c}\text { Start Analysis } \\
\text { Date }\end{array}$ & $\begin{array}{c}\text { Start } \\
\text { Sample } \\
\text { Date }\end{array}$ & $\begin{array}{l}\text { Sample } \\
\text { Number }\end{array}$ & $\begin{array}{c}\text { Tank Farm } \\
\text { Number }\end{array}$ & $\begin{array}{c}\text { Lab Sample } \\
\text { Number }\end{array}$ & $\begin{array}{l}\text { Density } \\
\text { (g/mL) }\end{array}$ & Reported Value & Reported Unit & Cade & \\
\hline Am241 & $5 / 10 / 94$ & NA & $3 / 10 / 93$ & $808 V \times 4$ & G238 & G401 & 1.1 & 0.000602 & $\mathrm{uCi} / \mathrm{mL}$ & $L$ & \\
\hline Am241 & $5 / 10 / 94$ & NA & $3 / 10 / 93$ & $\mathrm{~B} 08 \mathrm{~V} \times 4$ & G238 & G401 & 1.1 & 0.000512 & uCi/mL & $L$ & \\
\hline Am241 & $5 / 10 / 94$ & NA & $3 / 10 / 93$ & $\mathrm{BOB} 3 \mathrm{C9}$ & G249 & G418 & 1.3 & 0.000446 & $\mathrm{uCi} / \mathrm{mL}$ & $L$ & \\
\hline Am241 & $5 / 10 / 94$ & NA & $3 / 10 / 93$ & BOBVX1 & G253 & G392 & 1.34 & 0.000429 & uCi/mL & $L$ & \\
\hline Am241 & $5 / 10 / 94$ & NA & $3 / 10 / 93$ & B08VX7 & G243 & G409 & 1.298 & 0.000424 & $\mathrm{uCi} / \mathrm{mL}$ & $L$ & \\
\hline Am241 & $5 / 10 / 94$ & NA & $3 / 10 / 93$ & B08302 & NA & G379 & 1.329 & 0.000415 & $\mathrm{uCi} / \mathrm{mL}$ & $L$ & \\
\hline Am241 & $5 / 10 / 94$ & NA & $3 / 10 / 93$ & $\mathrm{BOB} 3 \mathrm{CB}$ & G248 & G417 & 1.327 & 0.000413 & $\mathrm{uCi} / \mathrm{mL}$ & $L$ & \\
\hline Am241 & $5 / 10 / 94$ & NA & NA & $\mathrm{NA}$ & $\mathrm{NA}$ & NA & 1.336 & 0.000408 & $\mathrm{uCi} / \mathrm{mL}$ & $P$ & \\
\hline Am241 & $5 / 10 / 94$ & NA & $3 / 10 / 93$ & B08VX8 & G244 & $\mathrm{G} 412$ & 1.377 & 0.000405 & uCilmL & $L$ & \\
\hline Am241 & $5 / 10 / 94$ & $\mathrm{NA}$ & $3 / 10 / 93$ & $808 \vee \times 6$ & G241 & G407 & 1.3375 & 0.000394 & $\mathrm{uCi} / \mathrm{mL}$ & $L$ & \\
\hline Am241 & $5 / 10 / 94$ & $\mathrm{NA}$ & $3 / 10 / 93$ & $808 V \times 5$ & G240 & G402 & 1.3375 & 0.000385 & $\mathrm{uCi} / \mathrm{mL}$ & $L$ & \\
\hline Am241 & $5 / 10 / 94$ & NA & $3 / 10 / 93$ & $808 \vee \times 0$ & G251 & G391 & 1.345 & 0.000384 & $\mathrm{uCi} / \mathrm{mL}$ & L & \\
\hline Am241 & $5 / 10 / 94$ & NA & $3 / 10 / 93$ & B08VX3 & G271 & G397 & 1.3 & 0.000376 & $\mathrm{uCi} / \mathrm{mL}$ & $L$ & \\
\hline Am241 & $5 / 10 / 94$ & $\mathrm{NA}$ & $3 / 10 / 93$ & $808 V \times 2$ & G254 & G396 & 1.2975 & 0.000365 & $\mathrm{uCi} / \mathrm{mL}$ & $\underline{L}$ & \\
\hline Am241 & $5 / 10 / 94$ & $\overline{N A}$ & $3 / 10 / 93$ & B08VX9 & G245 & G413 & 1.3335 & 0.00035 & $\mathrm{uCi} / \mathrm{mL}$ & $L$ & \\
\hline Am241 & $5 / 10 / 94$ & NA & $3 / 10 / 93$ & $\mathrm{BOB} 301$ & NA & G378 & 1.334 & 0.000348 & $\mathrm{uCi} / \mathrm{mL}$ & $L$ & \\
\hline Am241 & $5 / 10 / 94$ & NA & $3 / 10 / 93$ & NA & NA & G422 & 1.334 & 0.000302 & $\mathrm{uCi} / \mathrm{mL}$ & $L$ & \\
\hline $\operatorname{Am} 241$ & $5 / 70 / 94$ & NA & $3 / 10 / 93$ & BOB3D1 & NA & G378 & 1.334 & 0.000264 & $\mathrm{uCi} / \mathrm{mL}$ & $L$ & \\
\hline Carbon-14 & $5 / 10 / 94$ & NS & $3 / 10 / 93$ & $808 \mathrm{~V} \times 5$ & $\mathrm{G} 240$ & G402 & 1.339 & 0.000389 & $\mathrm{uCi} / \mathrm{mL}$ & $L$ & \\
\hline Carbon-14 & $5 / 10 / 94$ & NS & $3 / 10 / 93$ & B08VX4 & G238 & G401 & 1.39 & 0.0002405 & $\mathrm{uCi} / \mathrm{mL}$ & $L$ & \\
\hline Carbon-14 & $5 / 10 / 94$ & NS & $3 / 10 / 93$ & BOBVX6 & G241 & G407 & 1.339 & 0.000234 & $\mathrm{uCi} / \mathrm{mL}$ & L & \\
\hline Carbon-14 & $5 / 10 / 94$ & NS & $3 / 10 / 93$ & B08V $\times 8$ & G244 & G412 & 1.379 & 0.000223 & $\mathrm{uCi} / \mathrm{mL}$ & $L$ & \\
\hline Carbon-14 & $5 / 10 / 94$ & NS & $3 / 10 / 93$ & $808 \mathrm{~V} \times 2$ & G254 & G396 & 1.297 & 0.000219 & $\mathrm{uCi} / \mathrm{mL}$ & $L$ & \\
\hline Carbon-14 & $5 / 10 / 94$ & NS & $3 / 10 / 93$ & $80 \overline{B 3 C 9}$ & G249 & G418 & 1.301 & 0.000219 & uCi/mL & $L$ & \\
\hline $\mathrm{C} 14$ & $5 / 10 / 94$ & NS & $3 / 10 / 93$ & NS & NS & NS & 1.336 & 0.000217 & $\mathrm{uCi} / \mathrm{mL}$ & $\mathbf{P}$ & \\
\hline Carbon-14 & $5 / 10 / 94$ & NS & $3 / 10 / 93$ & $808 \mathrm{~V} \times 5$ & G240 & G402 & & 0.0002165 & $\mathrm{uCi} / \mathrm{mL}$ & $L$ & \\
\hline Carbon-14 & $5 / 10 / 94$ & NS & $3 / 10 / 93$ & B08VX1 & G253 & G392 & & 0.000216 & $\mathrm{uCi} / \mathrm{mL}$ & $L$ & \\
\hline Carbon-14 & $5 / 10 / 94$ & NS & $3 / 10 / 93$ & $808 \mathrm{~V} \times 3$ & G271 & G397 & & 0.000214 & $\mathrm{uCi} / \mathrm{mL}$ & $L$ & \\
\hline Carbon-14 & $5 / 10 / 94$ & NS & $3 / 10 / 93$ & $808 \vee \times 9$ & G245 & G413 & 1.335 & 0.0002135 & $\mathrm{uCi} / \mathrm{mL}$ & $L$ & \\
\hline Carbon-14 & $5 / 10 / 94$ & NS & $3 / 10 / 93$ & B08VX3 & G271 & G397 & 1.299 & 0.0002115 & $\mathrm{uCi} / \mathrm{mL}$ & $\bar{L}$ & \\
\hline Carbon-14 & $5 / 10 / 94$ & NS & $3 / 10 / 93$ & B08VX7 & G243 & G409 & & 0.0002115 & $\mathrm{uCi} / \mathrm{mL}$ & $\mathbf{L}$ & \\
\hline Carbon-14 & $5 / 10 / 94$ & NS & $3 / 10 / 93$ & B08VX8 & G244 & G412 & & 0.000211 & $\mathrm{uClimL}$ & L & \\
\hline Carbon-14 & $5 / 70 / 94$ & NS & $3 / 10 / 93$ & $\mathrm{BOB} 3 \mathrm{CB}$ & G248 & G417 & 1.328 & 0.0002095 & $\mathrm{uCi} / \mathrm{mL}$ & $L$ & 7 \\
\hline Carbon-14 & $5 / 10 / 94$ & NS & $3 / 10 / 93$ & BOBVXO & G251 & G391 & 1.35 & 0.3002085 & $\mathrm{uCi} / \mathrm{mL}$ & $\bar{L}$ & \\
\hline Carbon-14 & $5 / 10 / 94$ & NS & $3 / 10 / 93$ & BOBVX1 & G253 & G392 & & 0.0002065 & $\mathrm{uCi} / \mathrm{mL}$ & $L$ & 7 \\
\hline Carbon-14 & $5 / 10 / 94$ & NS & $3 / 10 / 93$ & $808 V \times 8$ & G244 & G412 & 1.375 & 0.000204 & $\mathrm{uCi} / \mathrm{mL}$ & $L$ & T \\
\hline Carbon-14 & $5 / 10 / 94$ & NS & $3 / 10 / 93$ & B08VX7 & G243 & 6409 & 1.293 & 0.000201 & $\mathrm{uCi} / \mathrm{mL}$ & $L$ & 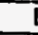 \\
\hline Carbon-14 & $5 / 10 / 94$ & NS & $3 / 10 / 93$ & $808 \vee \times 2$ & G254 & G396 & & 0.0001935 & $\mathrm{uCi} / \mathrm{mL}$ & $L$ & 7 \\
\hline Carbon-14 & $5 / 10 / 94$ & NS & $3 / 10 / 93$ & $808 V \times 0$ & G251 & G391 & & 0.000176 & $\mathrm{uCi} / \mathrm{mL}$ & $I$ & - \\
\hline Carbon-14 & $5 / 10 / 94$ & NS & $3 / 10 / 93$ & B08VX1 & $625 \overline{3}$ & G392 & 1.34 & 0.000165 & $\mathrm{uCi} / \mathrm{mL}$ & $L$ & T \\
\hline Carbon-14 & $5 / 10 / 94$ & NS & $3 / 10 / 93$ & $808 V \times 2$ & G254 & 6396 & 1.298 & 0.00014 & $\mathrm{uCi} / \mathrm{mL}$ & L & 1 \\
\hline Co60 & $5 / 10 / 94$ & NS & $3 / 10 / 93$ & $808 \mathrm{~V} \times 0$ & G251 & G391 & 1.345 & $<1.18 \mathrm{E}-02$ & $\mathrm{uCi} / \mathrm{mL}$ & $L$ & 4 \\
\hline Co60 & $5 / 10 / 94$ & NS & $3 / 10 / 93$ & B08VXI & G253 & G392 & 1.34 & $<1.34 \mathrm{E}-02$ & $\mathrm{uCi} / \mathrm{mL}$ & $L$ & 7 \\
\hline Co60 & $5 / 10 / 94$ & NS & $3 / 10 / 93$ & $808 V \times 3$ & G271 & G397 & 1.3 & $<3.96 \mathrm{E}-02$ & $\mathrm{uCi} / \mathrm{mL}$ & $\mathrm{L}$ & 7 \\
\hline Co60 & $5 / 10 / 94$ & NS & $3 / 10 / 93$ & $808 \mathrm{~V} \times 4$ & G238 & G401 & 1.1 & $<1.16 \mathrm{E}-02$ & $\mathrm{uCi} / \mathrm{mL}$ & $L$ & 7 \\
\hline Co60 & $5 / 10 / 94$ & NS & $3 / 10 / 93$ & B08VX5 & G240 & G402 & 1.3375 & $<1.06 E-02$ & $\mathrm{uCi} / \mathrm{mL}$ & $L$ & 7 \\
\hline C060 & $5 / 10 / 94$ & NS & $3 / 10 / 93$ & B08VX7 & G243 & G409 & 1.298 & $<1.84 \mathrm{E}-02$ & $\mathrm{uCi} / \mathrm{mL}$ & $L$ & 1 \\
\hline Co60 & $5 / 10 / 94$ & NS & $3 / 10 / 93$ & $\mathrm{BOB3C8}$ & G248 & G417 & 1.327 & $<1.25 \mathrm{E}-02$ & $\mathrm{uCi} / \mathrm{mL}$ & $L$ & I \\
\hline Cs137 & $5 / 10 / 94$ & NA & $3 / 10 / 93$ & BOBVX8 & G244 & G412 & 1.377 & 266 & $\mathrm{uCi} / \mathrm{mL}$ & $L$ & $T$ \\
\hline Csi37 & $5 / 10 / 94$ & NA & $3 / 10 / 93$ & B08VX4 & G238 & G401 & 1.1 & 255 & $\mathrm{uCi} / \mathrm{mL}$ & $L$ & 1 \\
\hline Cs 137 & $5 / 10 / 94$ & NA & $3 / 10 / 93$ & NA & NA & G422 & 1.377 & 236 & $\mathrm{uCi} / \mathrm{mL}$ & $L$ & 1 \\
\hline Cs137 & $5 / 10 / 94$ & NA & $3 / 10 / 93$ & $808 \mathrm{~V} \times 9$ & G245 & G413 & 1.3335 & 235 & $\mathrm{uCi} / \mathrm{mL}$ & $L$ & I \\
\hline Cs137 & $5 / 10 / 94$ & NA & $3 / 10 / 93$ & B08VX1 & G253 & G392 & 1.34 & 233 & $u \mathrm{Ci} / \mathrm{mL}$ & $L$ & I \\
\hline Cs137 & $5 / 10 / 94$ & NA & $3 / 10 / 93$ & B08VX0 & G251 & G391 & 1.345 & 232 & $\mathrm{uCi} / \mathrm{mL}$ & $L$ & $T$ \\
\hline Cs137 & $5 / 10 / 94$ & NA & $3 / 10 / 93$ & B08VX5 & G240 & G402 & 1.3375 & 232 & $\mathrm{uCi} / \mathrm{mL}$ & $L$ & $T$ \\
\hline Cs137 & $5 / 10 / 94$ & NA & $3 / 10 / 93$ & $808 V \times 6$ & G241 & G407 & 1.3375 & 232 & $\mathrm{uCi} / \mathrm{mL}$ & $L$ & \\
\hline Cs137 & $5 / 10 / 94$ & NA & $3 / 10 / 93$ & NA & $\mathrm{NA}$ & $\mathrm{NA}$ & 1.336 & 227 & $\mathrm{uCi} / \mathrm{mL}$ & $P$ & $\bar{R}$ \\
\hline
\end{tabular}




\begin{tabular}{|c|c|c|c|c|c|c|c|}
\hline & \multicolumn{4}{|c|}{ Radionuclidelnformation } & \multirow[b]{2}{*}{$\begin{array}{c}\text { Reference } \\
\text { Number }\end{array}$} & \multirow[b]{2}{*}{ Validated $(\mathbf{Y} / \mathbf{N})$} & \multirow[b]{2}{*}{ Notes } \\
\hline rce & Radoinuclide & Conc. $(B q / L)$ & $\begin{array}{c}\text { Conc. (Bq/2 } \\
\text { decayed) }\end{array}$ & $\begin{array}{c}\text { Conc. (Ci/L } \\
\text { Decayed) }\end{array}$ & & & \\
\hline$w$ & Am241 & $2.23 E+04$ & $2.23 \mathrm{E}+04$ & 6.01E-07 & AP105-2 & $\mathrm{Y}$ & Average of two results \\
\hline$\underline{w}$ & Am241 & $1.89 \mathrm{E}+04$ & $1.89 E+04$ & 5.11E-07 & AP105-2 & $\bar{Y}$ & Average of two results \\
\hline$w$ & Am241 & $1.65 E+04$ & $1.65 E+04$ & $4.46 \mathrm{E}-07$ & AP105-2 & $Y$ & Average of two results \\
\hline$\underline{w}$ & Am 241 & $1.59 E+04$ & $1.59 \mathrm{E}+04$ & $4.29 \mathrm{E}-07$ & AP105-2 & $Y$ & Average of two results \\
\hline$\underline{w}$ & Am241 & $1.57 E+04$ & $1.57 E+04$ & $4.24 \mathrm{E}-07$ & AP105.2 & $Y$ & Average of two results \\
\hline$\underline{N}$ & Am241 & $1.54 E+04$ & $1.53 E+04$ & 4.15E-07 & AP105.2 & $Y$ & Average of two results \\
\hline$N$ & Am241 & $1.53 E+04$ & $1.53 E+04$ & 4.13E-07 & AP105-2 & $\mathbf{Y}$ & Average of two results \\
\hline$N$ & Am241 & $1.51 E+04$ & $1.50 E+04$ & 4.05E-07 & AP105-2 & $Y$ & Average of 13 samples \\
\hline$N$ & Am241 & $1.50 E+04$ & $1.50 E+04$ & $4.05 E-07$ & AP105-2 & $Y$ & Average of two results \\
\hline$\underline{N}$ & Am241 & $1.46 E+04$ & $1.46 \mathrm{E}+04$ & $3.94 E-07$ & AP105-2 & $\bar{Y}$ & Average of two results \\
\hline$v$ & Am241 & $1.42 \mathrm{E}+04$ & $1.42 E+04$ & $3.85 E-07$ & AP105-2 & $\bar{Y}$ & Average of two results \\
\hline$\underline{v}$ & Am241 & $1.42 E+0.4$ & $1.42 E+04$ & $3.84 E-07$ & AP105-2 & $\bar{Y}$ & Average of two results \\
\hline$\underline{v}$ & Am241 & $1.39 E+04$ & $1.39 \mathrm{E}+\overline{04}$ & $3.76 \mathrm{E}-07$ & AP105-2 & $Y$ & Average of two results \\
\hline$\underline{v}$ & Am241 & $1.35 \mathrm{E}+04$ & $1.35 E+04$ & $3.65 \mathrm{E}-07$ & AP105-2 & $\bar{Y}$ & Average of two results \\
\hline$\checkmark$ & Am241 & $1.30 E+04$ & $1.29 \mathrm{E}+04$ & $3.50 \mathrm{E}-07$ & AP105-2 & $Y$ & Average of two results \\
\hline 8 & Am241 & $1.29 E+04$ & $1.29 E+04$ & $3.48 \mathrm{E}-07$ & AP105-2 & $\bar{Y}$ & Average of two results \\
\hline 1 & Am241 & $1.12 E+04$ & $1.12 E+04$ & $3.02 E-07$ & AP105-2 & $\mathbf{Y}$ & Average of two results \\
\hline 1 & Am241 & $9.77 E+03$ & $9.76 E+03$ & $2.64 E-07$ & AP105-2 & $Y$ & Average of two results \\
\hline 1 & $\mathrm{C14}$ & $1.44 E+04$ & $1.44 E+04$ & $3.89 \mathrm{E}-07$ & AP105-2 & NR & Average of two results \\
\hline 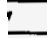 & $\mathrm{C} 14$ & $8.90 \mathrm{E}+03$ & $8.90 \mathrm{E}+03$ & $2.40 \mathrm{E}-07$ & AP105-2 & NR & Average of two results \\
\hline 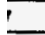 & $\mathrm{C14}$ & $8.66 \mathrm{E}+03$ & $8.66 E+03$ & $2.34 \mathrm{E}-07$ & AP105.2 & NR & Average of two results \\
\hline 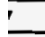 & C14 & $8.25 E+03$ & $8.25 E+03$ & $2.23 \mathrm{E}-07$ & AP105-2 & NR & Cannot locate value \\
\hline 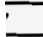 & $\mathrm{CI4}$ & $8.10 E+03$ & $8.10 E+03$ & $2.19 E-07$ & AP105-2 & NR & Cannot locate value \\
\hline 7 & C14 & $8.10 E+0 \overline{3}$ & $8.10 E+03$ & $2.19 \mathrm{E}-07$ & AP105-2 & NR & Average of two results \\
\hline & C14 & $8.03 E+03$ & $8.03 E+03$ & 2.17E-07 & AP105-2 & NR & \\
\hline & $\mathrm{C} 14$ & $8.01 E+03$ & $8.01 E+03$ & $2.16 \mathrm{E}-07$ & AP105-2 & NR & Average of two results \\
\hline & $\mathrm{C14}$ & $7.99 E+03$ & $7 . \overline{99 E}+03$ & $2.16 \mathrm{E}-07$ & AP105-2 & NR & Average of two results \\
\hline & $\mathrm{Cl4}$ & $7.92 E+03$ & $7 . \overline{92 E+03}$ & $2.14 \mathrm{E}-07$ & AP105-2 & NR & Average of two results \\
\hline & $\mathrm{Ci4}$ & $7.90 E+03$ & $7.90 E+03$ & $2.13 \bar{E}-07$ & AP105-2 & NR & Average of two results \\
\hline & C14 & $7.83 E+03$ & $7.82 \mathrm{E}+03$ & $2.11 E-07$ & AP105-2 & NR & Average of two results \\
\hline & C14 & $7.83 E+03$ & $7.82 E+03$ & 2.11E-07 & AP105-2 & NR & Average of two results \\
\hline & $\mathrm{C} 14$ & 7.81E +03 & $7.81 E+03$ & 2.11E-07 & AP105-2 & $\mathrm{NR}$ & Average of two results \\
\hline & $\mathrm{C14}$ & $7.75 E+03$ & $7.75 E+03$ & $2.09 E-07$ & AP105-2 & NR & Average of two results \\
\hline & C14 & $7.71 E+03$ & $7.71 E+03$ & $2.08 E-07$ & AP105-2 & NR & Average of two results \\
\hline & $\mathrm{C14}$ & $7.64 E+03$ & $7.64 E+03$ & $2.06 \mathrm{E}-07$ & AP105-2 & NR & Average of two results \\
\hline & $\mathrm{C14}$ & $7.55 E+03$ & $7.55 \mathrm{E}+0 \overline{3}$ & $2.04 E-07$ & AP105-2 & NR & Cannot locate value \\
\hline & $\overline{C 14}$ & $7.44 E+03$ & $7.44 E+03$ & $2.01 E-07$ & AP105-2 & NR & Average of two results \\
\hline & C14 & $7.16 E+03$ & $7.16 E+03$ & 1.93E-07 & AP105-2 & NR & Average of two results \\
\hline & $\mathrm{C14}$ & $6.51 E+03$ & $6.51 E+03$ & $1.76 \mathrm{E}-07$ & AP105-2 & NR & Average of two results \\
\hline & $\mathrm{C14}$ & $6.11 E+03$ & $6.10 \mathrm{E}+03$ & $1.65 \mathrm{E}-07$ & AP105-2 & NR & Average of two results \\
\hline & $\overline{C 14}$ & $5.18 E+03$ & $5.18 E+03$ & $1.40 \mathrm{E}-07$ & AP105-2 & NR & Average of two results \\
\hline & Co60 & \#VALUE! & "VALUE! & WALUE! & AP105-2 & NR & Average of two results \\
\hline & $\mathrm{Co60}$ & IVALUE! & \#VALUE! & \#VALUE! & AP105-2 & NR & Average of two results \\
\hline & Co60 & \#VALUE! & \#VALUEI & "VALUE! & AP105-2 & NR & Average of two results \\
\hline & Co60 & "VALUE! & \#VALUE! & \#VALUE! & AP105-2 & $\mathrm{NR}$ & Average of two results \\
\hline & Co60 & \#VALUE! & HVALUE! & \#VALUE! & AP105-2 & NR & Average of two results \\
\hline & Co60 & "VALUE! & \#VALUE! & VALUE! & AP105-2 & NR & Average of two results \\
\hline & $\mathrm{Co60}$ & \#VALUE! & \#VALUE! & VALUEI & AP105-2 & NR & Average of two results \\
\hline & Csi37 & $9.84 E+09$ & $9.70 \mathrm{E}+09$ & 2.62E-01 & AP105-2 & $\mathrm{Y}$ & Average of two results \\
\hline & Cs137 & $9.44 E+09$ & $9.30 E+09$ & $2.51 E-01$ & AP105-2 & $Y$ & Average of two results \\
\hline & Cs137 & $8.73 E+09$ & $8.60 \mathrm{E}+09$ & $2.33 \mathrm{E}-01$ & AP105-2 & $Y$ & Average of two resuits \\
\hline & Cs137 & $8.70 E+09$ & $8.57 E+09$ & $2.32 E-01$ & AP105.2 & $\bar{Y}$ & Average of two resuits \\
\hline & Cs137 & $8.62 E+09$ & $8.49 E+09$ & $2.30 \mathrm{E}-01$ & AP105-2 & $Y$ & Average of two results \\
\hline & Cs137 & $8.58 E+09$ & $8.46 E+09$ & 2.29E-01 & AP105-2 & $\mathbf{Y}$ & Average of two results \\
\hline & Cs137 & $8.58 E+09$ & $8.46 \mathrm{E}+09$ & $2.29 E-01$ & AP105-2 & $\mathbf{Y}$ & Average of two results \\
\hline & Cs137 & $8.58 E+09$ & $8.46 E+09$ & $2.29 \mathrm{E}-01$ & AP105-2 & $\bar{Y}$ & Average of two resuits \\
\hline & Cs137 & $8.40 E+09$ & $8.28 E+09$ & $2.24 E-01$ & AP105-2 & $\bar{Y}$ & Average of 13 samples \\
\hline
\end{tabular}




\begin{tabular}{|c|c|c|c|c|c|c|c|c|c|c|c|}
\hline \multicolumn{12}{|c|}{ Tank Waste Characterization Sample Information } \\
\hline $\begin{array}{c}\text { Reported } \\
\text { Radionuclide }\end{array}$ & Memo Date & $\begin{array}{c}\text { Start Analysis } \\
\text { Date }\end{array}$ & $\begin{array}{c}\text { Start } \\
\text { Sample } \\
\text { Date } \\
\end{array}$ & $\begin{array}{l}\text { Sample } \\
\text { Number }\end{array}$ & $\begin{array}{c}\text { Tank Farm } \\
\text { Number }\end{array}$ & $\begin{array}{c}\text { Lab Sample } \\
\text { Number }\end{array}$ & $\begin{array}{c}\text { Density } \\
\text { (g/mL) }\end{array}$ & Reported Value & Reported Unit & Code & si \\
\hline Cs137 & $5 / 10 / 94$ & NA & $3 / 10 / 93$ & BOB3D2 & NA & G379 & 1.329 & 226 & $\mathrm{uCi} / \mathrm{mL}$ & $\mathrm{L}$ & 7 \\
\hline Cs137 & $5 / 10 / 94$ & NA & $3 / 10 / 93$ & BOB3D1 & NA & G378 & 1.334 & 225 & $\mathrm{uCi} / \mathrm{mL}$ & L & $T$ \\
\hline CS137 & $5 / 10 / 94$ & NA & $3 / 10 / 93$ & $\mathrm{BOB} 3 \mathrm{C8}$ & G248 & $\mathrm{G} 417$ & 1.327 & 222 & $\mathrm{uCi} / \mathrm{mL}$ & $\mathrm{L}$ & 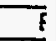 \\
\hline Cs137 & $5 / 10 / 94$ & NA & $3 / 10 / 93$ & $808 \mathrm{~V} \times 2$ & G254 & G396 & 1.2975 & 195 & $\mathrm{uCi} / \mathrm{mL}$ & L & 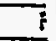 \\
\hline Cs137 & $5 / 10 / 94$ & NA & $3 / 10 / 93$ & 808VX3 & $\mathbf{G 2 7 1}$ & G397 & 1.3 & 195 & $\mathrm{uCi} / \mathrm{mL}$ & $L$ & 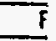 \\
\hline Cs137 & $5 / 10 / 94$ & NA & $3 / 10 / 93$ & B08VX7 & G243 & G409 & 1.298 & 194 & $\mathrm{uCi} / \mathrm{mL}$ & $\mathrm{L}$ & $\bar{F}$ \\
\hline Cs137 & $5 / 10 / 94$ & NA & $3 / 10 / 93$ & $8083 \mathrm{Cg}$ & G249 & G418 & 1.3 & 194 & $\mathrm{uCi} / \mathrm{mL}$ & $L$ & $\bar{F}$ \\
\hline Cs & $5 / 10 / 94$ & NS & NS & NS & NS & NS & 1.332491 & 0.158840102 & $\mathrm{Ci} / \mathrm{L}$ & $L$ & $\overline{H T C}$ \\
\hline 1.129 & $5 / 10 / 94$ & NS & $3 / 10 / 93$ & NS & NS & NS & 1.336 & $<2.12 E-04$ & $\mathrm{uCi} / \mathrm{mL}$ & $\bar{P}$ & $\bar{F}$ \\
\hline lodine-129 & $5 / 10 / 94$ & NS & $3 / 10 / 93$ & B08VX6 & G241 & G407 & 1.339 & 0.000196 & $\mathrm{uCi} / \mathrm{mL}$ & $\mathrm{L}$ & $\vec{F}$ \\
\hline lodine-129 & $5 / 10 / 94$ & NS & $3 / 10 / 93$ & B08VX0 & G251 & G391 & 1.34 & 0.00017595 & $\mathrm{uCi} / \mathrm{mL}$ & $L$ & $\bar{F}$ \\
\hline lodine-129 & $5 / 10 / 94$ & NS & $3 / 10 / 93$ & B08VX4 & G238 & G401 & 1.39 & 0.000156 & $\mathrm{uCi} / \mathrm{mL}$ & $L$ & $\bar{R}$ \\
\hline lodine-129 & $5 / 10 / 94$ & NS & $3 / 10 / 93$ & $B 08 v \times 3$ & G271 & G397 & 1.299 & 0.0001376 & $\mathrm{uCi} / \mathrm{mL}$ & $L$ & $\bar{R}$ \\
\hline lodine-129 & $5 / 10 / 94$ & NS & $3 / 10 / 93$ & $\mathrm{BOB3C8}$ & G248 & G417 & 1.328 & 0.0001315 & $\mathrm{uCi} / \mathrm{mL}$ & $L$ & $\bar{A}$ \\
\hline Todine-129 & $5 / 10 / 94$ & NS & $3 / 10 / 93$ & B08VX5 & G240 & G402 & 1.339 & 0.00013115 & $\mathrm{uCi} / \mathrm{mL}$ & $L$ & $\bar{A}$ \\
\hline lodine-129 & $5 / 10 / 94$ & NS & $3 / 10 / 93$ & BOB3C9 & G249 & G418 & 1.301 & 0.00012175 & $\mathrm{uCi} / \mathrm{mL}$ & $\bar{L}$ & $\vec{R}$ \\
\hline lodine-129 & $5 / 10 / 94$ & NS & $3 / 10 / 93$ & $\mathrm{BOB} 3 \mathrm{D} 2$ & NA & G379 & 1.328 & 0.0001188 & $\mathrm{uCi} / \mathrm{mL}$ & $L$ & $\bar{A}$ \\
\hline lodine -129 & $5 / 10 / 94$ & NS & $3 / 10 / 93$ & B0B3D1 & NA & G378 & & 0.00011525 & $\mathrm{uCi} / \mathrm{mL}$ & $L$ & $\bar{R}$ \\
\hline lodine-129 & $5 / 10 / 94$ & NS & $3 / 10 / 93$ & BOBVX8 & G244 & G412 & 1.375 & 0.00011225 & $\mathrm{uCi} / \mathrm{mL}$ & $L$ & $\bar{R}$ \\
\hline lodine-129 & $5 / 10 / 94$ & NS & $3 / 10 / 93$ & B083D1 & NA & G378 & 1.348 & 0.0001003 & $\mathrm{uCi} / \mathrm{mL}$ & L & $\bar{A}$ \\
\hline lodine-129 & $5 / 10 / 94$ & NS & $3 / 10 / 93$ & B08VX5 & G240 & G402 & & 0.00009315 & $\mathrm{uCi} / \mathrm{mL}$ & $L$ & $\bar{R}$ \\
\hline lodine-129 & $5 / 10 / 94$ & NS & $3 / 10 / 93$ & $\mathrm{BOB3D2}$ & $\overline{N A}$ & G379 & & 0.00008935 & $\mathrm{uCi} / \mathrm{mL}$ & $L$ & $\bar{R}$ \\
\hline lodine-129 & $5 / 10 / 94$ & NS & $3 / 10 / 93$ & $808 V \times 3$ & G271 & G397 & & 0.00007575 & $\mathrm{uCi} / \mathrm{mL}$ & $L$ & $\bar{R}$ \\
\hline lodine-129 & $5 / 10 / 94$ & NS & $3 / 10 / 93$ & B08VX7 & G243 & G409 & 1.293 & 0.00007295 & $\mathrm{uCi} / \mathrm{mL}$ & $L$ & $\overline{\mathbf{R}}$ \\
\hline lodine-129 & $5 / 10 / 94$ & NS & $3 / 10 / 93$ & BOB3C9 & G249 & G418 & & 0.00005755 & $\mathrm{uCi} / \mathrm{mL}$ & $L$ & $\bar{R}$ \\
\hline lodine-129 & $5 / 10 / 94$ & NS & $3 / 10 / 93$ & $808 \vee \times 7$ & G243 & G409 & & 0.00005295 & $\mathrm{uCi} / \mathrm{mL}$ & $L$ & $\bar{R}$ \\
\hline lodine-129 & $5 / 10 / 94$ & NS & $3 / 10 / 93$ & B08VX9 & G245 & G413 & 1.332 & 0.0000519 & $\mathrm{uCi} / \mathrm{mL}$ & $L$ & $\bar{R}$ \\
\hline lodine-129 & $5 / 10 / 94$ & NS & $3 / 10 / 93$ & B08VX2 & G254 & G396 & 1.297 & 0.00004525 & $\mathrm{uCi} / \mathrm{mL}$ & $\mathrm{L}$ & $\overline{R_{i}}$ \\
\hline lodine-129 & $5 / 10 / 94$ & NS & $3 / 10 / 93$ & $808 \mathrm{~V} \times 1$ & G253 & G392 & 1.34 & 0.0000374 & $\mathrm{uCi} / \mathrm{mL}$ & $\mathrm{L}$ & $\overline{n_{1}}$ \\
\hline Np237 & $5 / 10 / 94$ & NS & $3 / 10 / 93$ & $808 \mathrm{~V} \times 9$ & G245 & G413 & 1.3335 & $<3.57 \mathrm{E}-04$ & $\mathrm{uCi} / \mathrm{mL}$ & $L$ & $\overline{R i}$ \\
\hline Np237 & $5 / 10 / 94$ & NA & $3 / 10 / 93$ & BosvXo & G251 & G391 & 1.345 & $<4.32 E-04$ & $\mathrm{uCi} / \mathrm{mL}$ & $L$ & $\mathrm{Ri}$ \\
\hline No237 & $5 / 10 / 94$ & NA & $3 / 10 / 93$ & B08VXI & G253 & G392 & 1.34 & $<4.32 \mathrm{E}-04$ & $\mathrm{uCi} / \mathrm{mL}$ & L & Ri \\
\hline Np237 & $5 / 10 / 94$ & NA & $3 / 10 / 93$ & B08VX8 & G244 & G412 & 1.377 & $<4.32 \mathrm{E}-04$ & $\mathrm{uCi} / \mathrm{mL}$ & L & Rq \\
\hline Pu238 & $5 / 10 / 94$ & $\overline{N S}$ & $3 / 10 / 93$ & $808 \vee \times 0$ & G251 & 6391 & 1.345 & $<1.05 \mathrm{E}-04$ & $\mathrm{uCi} / \mathrm{mL}$ & $L$ & $\overrightarrow{\boldsymbol{R} z}$ \\
\hline Pu238 & $5 / 10 / 94$ & NS & $3 / 10 / 93$ & $808 \mathrm{VX1}$ & G253 & G392 & 1.34 & $<9.62 \mathrm{E}-05$ & $\mathrm{uCi} / \mathrm{mL}$ & $L$ & $\bar{R}$ \\
\hline Pu238 & $5 / 10 / 94$ & NS & $3 / 10 / 93$ & $808 V \times 2$ & G254 & G396 & 1.2975 & $<2.43 E-04$ & $\mathrm{uCi} / \mathrm{mL}$ & $I$ & $\overline{R a}$ \\
\hline Pu238 & $5 / 10 / 94$ & NS & $3 / 10 / 93$ & B08VX3 & G271 & G397 & 1.3 & $<3.31 \mathrm{E}-04$ & $\mathrm{uCi} / \mathrm{mL}$ & $L$ & $\overline{A d}$ \\
\hline Pu238 & $5 / 10 / 94$ & NS & $3 / 10 / 93$ & B08VX4 & G238 & G401 & 1.1 & $<4.11 \mathrm{E}-04$ & $\mathrm{uCi} / \mathrm{mL}$ & $L$ & $\overrightarrow{\mathrm{Aa}}$ \\
\hline Pu238 & $5 / 10 / 94$ & NS & $3 / 10 / 93$ & B08VX6 & G241 & 6407 & 1.3375 & $<9.72 \mathrm{E}-05$ & $\mathrm{uCi} / \mathrm{mL}$ & $L$ & $\overrightarrow{\mathrm{Aa}_{3}}$ \\
\hline Pu238 & $5 / 10 / 94$ & NS & $3 / 10 / 93$ & $B 08 V \times 7$ & G243 & G409 & 1.298 & $<9.77 \mathrm{E}-05$ & $\mathrm{uCi} / \mathrm{mL}$ & $L$ & $\mathrm{Ra}$ \\
\hline Pu238 & $5 / 10 / 94$ & NS & $3 / 10 / 93$ & B08VX8 & G244 & G412 & 1.377 & $<4.08 \mathrm{E}-04$ & $\mathrm{uCi} / \mathrm{mL}$ & $L$ & $\overrightarrow{\mathrm{Aa}}$ \\
\hline Pu238 & $5 / 10 / 94$ & NS & $3 / 10 / 93$ & BOBVXB & G244 & G412 & 1.377 & $<9.80 E-05$ & $\mathrm{uCi} / \mathrm{mL}$ & $L$ & $\overline{\mathrm{Ra}}$ \\
\hline Pu238 & $5 / 10 / 94$ & NS & $3 / 10 / 93$ & B08VX9 & G245 & G413 & 1.3335 & $<3.27 E-04$ & $\mathrm{uCi} / \mathrm{mL}$ & $L$ & $\mathrm{Ra}$ \\
\hline Pu238 & $5 / 10 / 94$ & NS & $3 / 10 / 93$ & B08VX9 & G245 & G413 & 1.3335 & $<9.38 \mathrm{E}-05$ & $\mathrm{uCi} / \mathrm{mL}$ & $L$ & $\bar{R}$ \\
\hline Pu238 & $5 / 10 / 94$ & NS & $3 / 10 / 93$ & $\mathrm{BOB3C8}$ & G248 & G417 & 1.327 & $<2.00 E-04$ & $\mathrm{uCi} / \mathrm{mL}$ & $L$ & $\overline{\mathrm{Ra}}$ \\
\hline Pu238 & $5 / 10 / 94$ & NS & $3 / 10 / 93$ & BOB3C9 & G249 & G418 & 1.3 & $<7.34 \mathrm{E}-05$ & $\mathrm{uCi} / \mathrm{mL}$ & $L$ & $\mathrm{Ra}$ \\
\hline Pu238 & $5 / 10 / 94$ & NA & $3 / 10 / 93$ & B08VX5 & G240 & G402 & 1.3375 & $<4.82 E-04$ & $\mathrm{uCi} / \mathrm{mL}$ & L & $\mathbf{R a}$ \\
\hline Pu239/240 & $5 / 10 / 94$ & NS & $3 / 10 / 93$ & BO8VX4 & G238 & G401 & 1.1 & $<1.08 E-04$ & $\mathrm{uCi} / \mathrm{mL}$ & $L$ & $\overline{\mathrm{Ra}}$ \\
\hline Pu239/240 & $5 / 10 / 94$ & NS & $3 / 10 / 93$ & B08VX5 & G240 & G402 & 1.3375 & $<1.48 E-04$ & $\mathrm{uCi} / \mathrm{mL}$ & $\bar{L}$ & $\overline{\text { Ra }}$ \\
\hline Pu239/240 & $5 / 10 / 94$ & NS & $3 / 10 / 93$ & B08VX8 & G244 & G412 & 1.377 & $<1.33 E-04$ & $\mathrm{uCi} / \mathrm{mL}$ & $\mathbf{L}$ & $\mathrm{Ra}$ \\
\hline
\end{tabular}




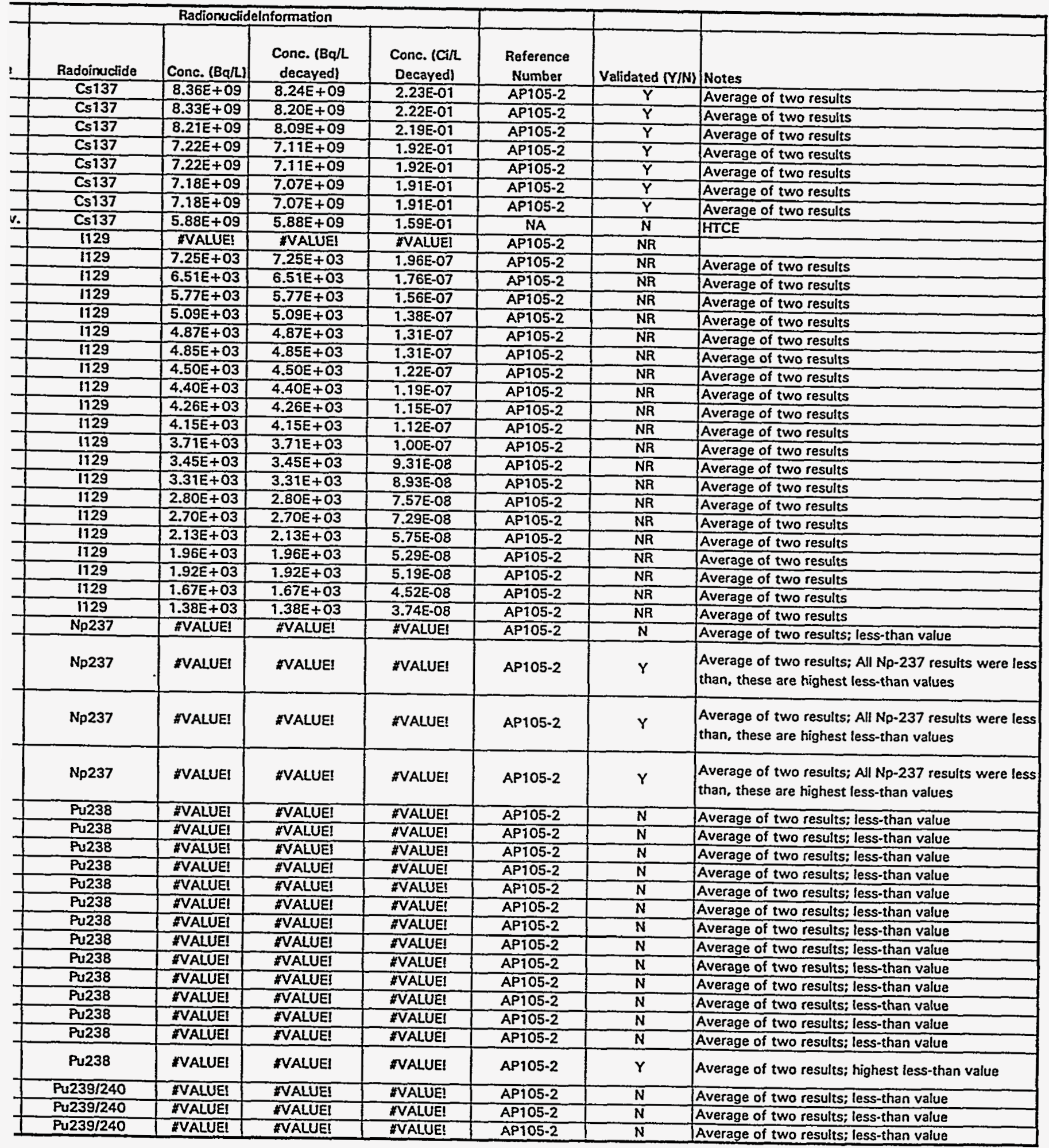




\begin{tabular}{|c|c|c|c|c|c|c|c|c|c|c|c|}
\hline & & & & Tank Wast & haracterizatio & Sample Infor & ation & & & & \\
\hline $\begin{array}{c}\text { Reported } \\
\text { Radionuclide }\end{array}$ & Memo Date & $\begin{array}{c}\text { Start Analysis } \\
\text { Date }\end{array}$ & $\begin{array}{c}\text { Start } \\
\text { Sample } \\
\text { Date }\end{array}$ & $\begin{array}{l}\text { Sample } \\
\text { Number }\end{array}$ & $\begin{array}{c}\text { Tank Farm } \\
\text { Number }\end{array}$ & $\begin{array}{c}\text { Lab Sample } \\
\text { Number }\end{array}$ & $\begin{array}{l}\text { Density } \\
(\mathrm{g} / \mathrm{m} L)\end{array}$ & Reported Value & Reported Unit & Code & \\
\hline Pu239/240 & $5 / 10 / 94$ & NS & $3 / 10 / 93$ & B0B3C.9 & $\mathbf{G 2 4 9}$ & G418 & 1.3 & $<1.88 \mathrm{E}-04$ & $\mathrm{uCi} / \mathrm{mL}$ & $L$ & \\
\hline Pu239/240 & $5 / 10 / 94$ & NA & $3 / 10 / 93$ & $\mathrm{BOB3C8}$ & G248 & G417 & 1.327 & 0.000181 & uCi/mL & $L$ & \\
\hline Pu239/240 & $5 / 10 / 94$ & NA & $3 / 10 / 93$ & Bo8VX7 & G243 & 6409 & 1.298 & 0.00018 & $\mathrm{uCi} / \mathrm{mL}$ & $L$ & \\
\hline Pu239/240 & $5 / 10 / 94$ & NA & $3 / 10 / 93$ & BOB3C9 & G249 & G418 & 1.3 & 0.000179 & $\mathrm{tC} \mathrm{i} / \mathrm{mL}$ & $L$ & \\
\hline Pu239/240 & $5 / 10 / 94$ & NA & $3 / 10 / 93$ & $808 \mathrm{~V} \times 3$ & $\mathbf{G 2 7 1}$ & G397 & 1.3 & 0.000169 & $\mathrm{uCi} / \mathrm{mL}$ & $\mathbf{L}$ & \\
\hline Pu239/240 & $5 / 10 / 94$ & NA & $3 / 10 / 93$ & $808 \mathrm{~V} \times 9$ & G245 & G413 & 1.3335 & 0.000161 & $\mathrm{uCi} / \mathrm{mL}$ & $L$ & \\
\hline Pu239/240 & $5 / 10 / 94$ & NA & $3 / 10 / 93$ & NA & NA & G422 & 1.3 & 0.00016 & $\mathrm{uCi} / \mathrm{mL}$ & $L$ & \\
\hline Pu239/240 & $5 / 10 / 94$ & NA & $3 / 10 / 93$ & B08VX1 & G253 & G392 & 1.34 & 0.000159 & $\mathrm{uCi} / \mathrm{mL}$ & $L$ & \\
\hline Pu2391240 & $5 / 10 / 94$ & NA & $3 / 10 / 93$ & B08VX2 & G254 & G396 & 1.2975 & 0.000159 & $\mathrm{uCi} / \mathrm{mL}$ & $L$ & \\
\hline Pu239/240 & $5 / 10 / 94$ & NA & NA & NA & NA & NA & 1.336 & 0.000158 & $\mathrm{uCi} / \mathrm{mL}$ & $\mathbf{P}$ & \\
\hline Pu239/240 & $5 / 10 / 94$ & NA & $3 / 10 / 93$ & $808 \mathrm{~V} \times 9$ & G245 & G413 & 1.3335 & 0.000152 & $\mathrm{uCi} / \mathrm{mL}$ & $L$ & \\
\hline Pu239/240 & $5 / 10 / 94$ & NA & $3 / 10 / 93$ & B08VXO & G251 & G391 & 1.345 & 0.000148 & $\mathrm{uCi} / \mathrm{mL}$ & $\mathrm{L}$ & \\
\hline Pu239/240 & $5 / 10 / 94$ & NA & $3 / 10 / 93$ & $808 \vee \times 8$ & G244 & G412 & 1.377 & 0.00013 & $\mathrm{uCi} / \mathrm{mL}$ & $\mathrm{L}$ & \\
\hline Pu239/240 & $5 / 10 / 94$ & $\mathrm{NA}$ & $3 / 10 / 93$ & B08VX6 & G241 & G407 & 1.3375 & 0.00012 & $\mathrm{uCi} / \mathrm{mL}$ & $L$ & \\
\hline Sr & $5 / 10 / 94$ & NA & NA & NA & NA & NA & 1.332491 & 0.021470845 & $\mathrm{Cin}$ & $L$ & $\overline{\mathrm{Hit}}$ \\
\hline Sr90 & $5 / 10 / 94$ & NA & $3 / 10 / 93$ & $808 \vee \times 8$ & G244 & G412 & 1.377 & 0.253 & $\mathrm{uCi} / \mathrm{mL}$ & $L$ & \\
\hline Sr90 & $5 / 10 / 94$ & NA & $3 / 10 / 93$ & BOB3C9 & G249 & $\mathrm{G} 418$ & 1.3 & 0.243 & $\mathrm{uCi} / \mathrm{mL}$ & $L$ & \\
\hline 5,90 & $5 / 10 / 94$ & NA & $3 / 10 / 93$ & B08VX4 & G238 & G401 & 1.1 & 0.242 & $\mathrm{uCi} / \mathrm{mL}$ & $\underline{L}$ & \\
\hline Sr90 & $5 \longdiv { 1 0 / 9 4 }$ & NA & $3 / 70 / 93$ & $808 V \times 3$ & G271 & G397 & 1.3 & 0.24 & $\mathrm{uCi} / \mathrm{mL}$ & $L$ & \\
\hline Sr90 & $5 / 10 / 94$ & NA & $3 / 10 / 93$ & B08VX7 & G243 & G409 & 1.298 & 0.234 & $\mathrm{uCi} / \mathrm{mL}$ & $L$ & \\
\hline Sr90 & $5 / 10 / 94$ & NA & $3 / 10 / 93$ & $\mathrm{BOB} 3 \mathrm{C9}$ & G249 & G418 & 1.3 & 0.23 & $\mathrm{uCi} / \mathrm{mL}$ & $\underline{L}$ & \\
\hline Sr90 & $5 / 10 / 94$ & NA & $3 / 10 / 93$ & $808 V \times 2$ & 6254 & G396 & 1.2975 & 0.219 & $\mathrm{uCi} / \mathrm{ml}$ & $L$ & \\
\hline Srgo & $5 / 10 / 94$ & NA & NA & NA & NA & NA & 1.336 & 0.208 & $\mathrm{uCi} / \mathrm{mL}$ & $P$ & 1 \\
\hline Sr90 & $5 / 10 / 94$ & NA & $3 / 10 / 93$ & BOB3D1 & NA & G378 & 1.334 & 0.208 & $\mathrm{uCi} / \mathrm{mL}$ & $L$ & \\
\hline Sr90 & $5 / 10 / 94$ & NA & $3 / 10 / 93$ & $\mathrm{BOB} 3 \mathrm{CB}$ & G248 & G417 & 1.327 & 0.207 & $\mathrm{uCi} / \mathrm{mL}$ & $L$ & \\
\hline Sr90 & $5 / 10 / 94$ & NA & $3 / 10 / 93$ & $80 B 302$ & NA & 6379 & 1.329 & 0.201 & $\mathrm{uCi} / \mathrm{mL}$ & $L$ & \\
\hline Si90 & $5 / 10 / 94$ & NA & $3 / 10 / 93$ & $808 V \times 9$ & G245 & G413 & 1.3335 & 0.189 & $u \mathrm{Ci} / \mathrm{mL}$ & $L$ & -1 \\
\hline Sr90 & $5 / 10 / 94$ & NA & $3 / 10 / 93$ & NA & NA & $\mathrm{G} 422$ & 1.377 & 0.188 & $\mathrm{uCi} / \mathrm{mL}$ & $L$ & 1 \\
\hline Sr90 & $5 / 10 / 94$ & NA & $3 / 10 / 93$ & $\mathrm{~B} 08 \mathrm{~V} \times 0$ & G251 & G391 & 1.345 & 0.182 & $\mathrm{uCi} / \mathrm{mL}$ & $L$ & 7 \\
\hline Sr90 & $5 / 10 / 94$ & NA & $3 / 10 / 93$ & B08VX6 & G241 & G407 & 1.3375 & 0.182 & $\mathrm{uCi} / \mathrm{mL}$ & $L$ & 1 \\
\hline Sr90 & $5 / 10 / 94$ & NA & $3 / 10 / 93$ & B08VX5 & 6240 & $\mathbf{G 4 0 2}$ & 1.3375 & 0.18 & $u \mathrm{Ci} / \mathrm{mL}$ & $L$ & 1 \\
\hline $5+90$ & $5 / 10 / 94$ & NA & $3 / 10 / 93$ & $808 \mathrm{~V} \times 1$ & G253 & G392 & 1.34 & 0.176 & $\mathrm{uCi} / \mathrm{ml}$ & $L$ & 1 \\
\hline Technetium-99 & $5 / 10 / 94$ & NA & $3 / 10 / 93$ & B08VX4 & G238 & G401 & 1.388 & 0.086 & $\mathrm{uCi} / \mathrm{mL}$ & $L$ & 1 \\
\hline Technetium-99 & $5 / 10 / 94$ & $\mathrm{NA}$ & $3 / 10 / 93$ & $\mathrm{~B} 08 \mathrm{~V} \times 8$ & G244 & $\mathrm{G} 412$ & 1.375 & 0.0794 & $\mathrm{uCi} / \mathrm{mL}$ & $L$ & 7 \\
\hline Technetium-99 & $5 / 10 / 94$ & NA & $3 / 10 / 93$ & BOB3DI & NA & G378 & 1.32 & 0.0772 & $\mathrm{uCi} / \mathrm{mL}$ & $L$ & $\underline{I}$ \\
\hline Technetium-99 & $5 / 10 / 94$ & NA & $3 / 10 / 93$ & $808 V \times 5$ & 6240 & G402 & 1.336 & 0.0746 & $\mathrm{uCi} / \mathrm{mL}$ & L & 7 \\
\hline Technetium-99 & $5 / 10 / 94$ & NA & $3 / 10 / 93$ & BOEVXO & 6251 & G391 & 1.34 & 0.0746 & $\mathrm{vCi} / \mathrm{mL}$ & $L$ & $\underline{F}$ \\
\hline Technetium-\$9 & $5 \longdiv { 1 0 / 9 4 }$ & NA & $3 / 10 / 93$ & B08VX6 & G241 & G407 & 1.336 & 0.0739 & $\mathrm{LCi} / \mathrm{mL}$ & $L$ & $\bar{F}$ \\
\hline Technetium-99 & $5 / 10 / 94$ & NA & $3 / 10 / 93$ & $\mathrm{BO8VX1}$ & G253 & G392 & 1.34 & 0.0703 & $\omega \mathrm{Ci} / \mathrm{mL}$ & $L$ & $\underline{F}$ \\
\hline Tc-99 & $5 / 10 / 94$ & NA & NA & NA & NA & NA & 1.336 & 0.0698 & $\mathrm{uCi} / \mathrm{mL}$ & $P$ & F \\
\hline Technetium-99 & $5 / 10 / 94$ & NA & $3 / 10 / 93$ & 808302 & NA & G379 & 1.328 & 0.0652 & $\mathrm{uCi} / \mathrm{mL}$ & $L$ & $\widetilde{F}$ \\
\hline Technetium-99 & $5 / 10 / 94$ & NA & $3 / 10 / 93$ & $\mathrm{BOB3C8}$ & G248 & G417 & 1.328 & 0.0647 & $\mathrm{uCi} / \mathrm{mL}$ & $L$ & F \\
\hline Technetium-99 & $5 / 10 / 94$ & NA & $3 / 10 / 93$ & $808 V \times 9$ & G245 & G413 & 1.335 & 0.0625 & $\mathrm{uCi} / \mathrm{mL}$ & $L$ & $\bar{A}$ \\
\hline Technetium-99 & $5 / 10 / 94$ & NA & $3 / 10 / 93$ & B08VX3 & E271 & G397 & & 0.0611 & $\mathrm{uCi} / \mathrm{mL}$ & $\mathrm{L}$ & $\bar{n}$ \\
\hline Technetium-99 & $5 / 10 / 94$ & $\overline{N A}$ & $3 / 10 / 93$ & $808 \mathrm{~V} \times 3$ & G271 & G397 & 1.301 & 0.0611 & $\mathrm{uCi} / \mathrm{mL}$ & L & $\vec{R}$ \\
\hline Technetium-99 & $5 / 10 / 94$ & NA & $3 / 10 / 93$ & $808 \mathrm{~V} \times 7$ & G243 & G409 & 1.303 & 0.0597 & $\mathrm{uCi} / \mathrm{mL}$ & L & $\pi$ \\
\hline Technetium-99 & $5 / 10 / 94$ & NA & $3 / 10193$ & $\mathrm{BOB3C9}$ & G249 & G418 & 1.299 & 0.0587 & $\mathrm{uCi} / \mathrm{mL}$ & $L$ & $\underline{R}$ \\
\hline Technetium-99 & $5 / 10 / 94$ & NA & $3 / 10 / 93$ & B08VX2 & $\mathrm{G} 254$ & G396 & & 0.0567 & $\mathrm{uCi} / \mathrm{mL}$ & $L$ & $\bar{R}$ \\
\hline Technetium-99 & $5 / 10 / 94$ & NA & $3 / 10 / 93$ & $808 V \times 2$ & G254 & G396 & 1.297 & 0.05605 & $\mathrm{uCi} / \mathrm{mL}$ & $\mathbf{L}$ & $\bar{R}$ \\
\hline
\end{tabular}




\begin{tabular}{|c|c|c|c|c|c|c|c|}
\hline & & Radionuclid & information & & & & \\
\hline 30 & Radoinuclide & Conc. $(\mathrm{Bq} / \mathrm{L})$ & $\begin{array}{c}\text { Conc. }(\mathrm{Bq} / \mathrm{L} \\
\text { decayed) }\end{array}$ & $\begin{array}{c}\text { Conc. (Cil } \\
\text { Decayed) }\end{array}$ & $\begin{array}{c}\text { Reference } \\
\text { Number } \\
\end{array}$ & Validated (Y/N) & Notes \\
\hline & Pu239/240 & \#VALUE! & NALLUE! & VALUE! & AP105-2 & $\mathbf{N}$ & Average of two results; less-than value \\
\hline & Pu239/240 & $6.70 E+03$ & $6.70 E+03$ & $1.81 \mathrm{E}-07$ & AP105-2 & $Y$ & Average of two results \\
\hline & Pu239/240 & $6.66 E+03$ & $6.66 E+03$ & $1.80 \mathrm{E}-07$ & AP105-2 & $\bar{Y}$ & Average of two results \\
\hline & Pu239/240 & $6.33 E+03$ & $6.33 E+03$ & $1.71 E-07$ & AP105-2 & $\bar{Y}$ & Average of two results \\
\hline & Pu239/240 & $6.25 E+03$ & $6.25 E+03$ & $1.69 E-07$ & AP105-2 & $\mathbf{Y}$ & Average of two results \\
\hline & Pu239/240 & $5.96 \mathrm{E}+03$ & $5.96 E+03$ & $1.61 E-07$ & AP105-2 & $Y$ & Average of two results \\
\hline & Pu239/240 & $5.92 E+03$ & $5.92 E+03$ & 1.60E-07 & AP105-2 & $Y$ & Average of two results \\
\hline & Pu239/240 & $5.88 E+03$ & $5.88 \mathrm{E}+03$ & 1.59E-07 & AP105-2 & $Y$ & Average of two results \\
\hline & Pu239/240 & $5.88 E+03$ & $5.88 E+03$ & $1.59 \mathrm{E}-07$ & AP105-2 & $\bar{Y}$ & Average of two results \\
\hline & Pu239/240 & $5.85 \mathrm{E}+03$ & $5.85 E+03$ & $1.58 \mathrm{E}-07$ & AP105-2 & $\mathbf{Y}$ & Average of all valid points \\
\hline & Pu239/240 & $5.62 E+03$ & $5.62 E+03$ & $1.52 \mathrm{E}-07$ & AP105-2 & $\bar{Y}$ & Average of two results \\
\hline & $P u 239 / 240$ & $5.48 \mathrm{E}+03$ & $5.48 E+03$ & $1.48 E-07$ & AP105-2 & $\bar{Y}$ & Average of two results \\
\hline & Pu239/240 & $4.81 E+03$ & $4.81 E+03$ & $1.30 E-07$ & AP105-2 & $\bar{Y}$ & Average of two results \\
\hline & Pu239/240 & $4.44 E+03$ & $4.44 E+03$ & $1.20 E-07$ & AP105-2 & $Y$ & Average of two results \\
\hline 3v. & St89/90 & $7.94 E+08$ & $7.94 E+08$ & 2.15E-02 & NA & $N$ & HTCE \\
\hline & Sr89/90 & $9.36 E+06$ & $9.22 E+06$ & 2.49E-04 & AP105-2 & $\bar{Y}$ & Average of two results \\
\hline & Sr89/90 & $8.99 \mathrm{E}+06$ & $8.85 E+06$ & $2.39 E-04$ & AP105-2 & $\mathbf{Y}$ & Average of two results \\
\hline & Sr89/90 & $8.95 E+06$ & $8.82 E+06$ & $2.38 \mathrm{E}-04$ & AP105-2 & $Y$ & Average of two results \\
\hline & Sr89/90 & $8.88 E+06$ & $8.74 \mathrm{E}+06$ & $2.36 E-04$ & AP105-2 & $Y$ & Average of two results \\
\hline & Sr89/90 & $8.66 E+06$ & $8.53 E+06$ & $2.30 E-04$ & AP105-2 & $\bar{Y}$ & Average of two results \\
\hline & Sr89/90 & $8.51 E+06$ & $8.38 E+06$ & $2.26 \mathrm{E}-04$ & AP105-2 & $\mathbf{Y}$ & Average of two results \\
\hline & Sr89/90 & $8.10 E+06$ & $7.98 \mathrm{E}+06$ & $2.16 E-04$ & AP105-2 & $\bar{Y}$ & Average of two results \\
\hline & Sr89/90 & $7.70 E+06$ & $7.37 E+06$ & $1.99 E-04$ & AP105-2 & $\mathbf{Y}$ & $\begin{array}{l}\text { This is a statistical average of } 13 \text { individual tank } \\
\text { samples and } 2 \text { composite samples, and represents } \\
\text { the "nominal value" for this analyte; average for all } \\
\text { valid points }\end{array}$ \\
\hline & Sr89/90 & $7.70 E+06$ & $7.58 E+06$ & $2.05 E-04$ & AP $105-2$ & $\bar{Y}$ & Average of two results \\
\hline & Sr89/90 & $7.66 \mathrm{E}+06$ & $7.54 \mathrm{E}+06$ & $2.04 \mathrm{E}-04$ & AP105-2 & $\mathbf{Y}$ & Average of two results \\
\hline & Sr89/90 & $7.44 \mathrm{E}+06$ & $7.32 E+06$ & $1.98 \mathrm{E}-04$ & AP105-2 & $\mathbf{Y}$ & Average of two results \\
\hline & Sr89/90 & $6.99 \mathrm{E}+06$ & $6.89 E+06$ & $1.86 \mathrm{E}-04$ & AP105-2 & $\bar{Y}$ & Average of two results \\
\hline & Sr89/90 & $6.96 \mathrm{E}+06$ & $6.85 E+06$ & $1.85 E-04$ & AP105-2 & $\bar{Y}$ & Average of two results \\
\hline & St89/90 & $6.73 E+06$ & $6.63 E+06$ & $1.79 \mathrm{E}-04$ & AP105-2 & $\bar{Y}$ & Average of two results \\
\hline & Sr89/90 & $6.73 E+06$ & $6.63 \bar{E}+06$ & $1.79 E-04$ & AP105-2 & $\bar{Y}$ & Average of two results \\
\hline & Sr89/90 & $6.66 \mathrm{E}+06$ & $6.56 \mathrm{E}+06$ & 1.77E-04 & AP105-2 & $\bar{Y}$ & Average of two results \\
\hline & Sr89/90 & $6.51 E+06$ & $6.41 E+06$ & $1.73 \mathrm{E}-04$ & AP105-2 & $Y$ & Average of two results \\
\hline & Tc99 & $3.18 \mathrm{E}+06$ & $3.18 \mathrm{E}+06$ & $8.60 E-05$ & AP105-2 & $Y$ & Average of two results \\
\hline & Tc99 & $2.94 E+06$ & $2.94 E+06$ & 7.94E-05 & AP105-2 & $Y$ & Average of two results \\
\hline & To99 & $2.86 E+06$ & $2.86 E+06$ & $7.72 \mathrm{E}-05$ & AP105-2 & $\bar{Y}$ & Average of two results \\
\hline & Tc99 & $2.76 E+06$ & $2.76 \varepsilon+06$ & $7.46 \mathrm{E}-05$ & AP105-2 & $\bar{Y}$ & Average of two results \\
\hline & Tc99 & $2.76 \mathrm{E}+06$ & $2.76 E+06$ & $7.46 \mathrm{E}-05$ & AP105-2 & $Y$ & Average of two results \\
\hline & Tc99 & $2.73 E+06$ & $2.73 E+06$ & $7.39 E-05$ & AP105-2 & $\bar{Y}$ & Average of two results \\
\hline & Tc99 & $2.60 E+06$ & $2.60 \mathrm{E}+06$ & 7.03E-05 & AP105-2 & $Y$ & Average of two results \\
\hline & Tc99 & $2.58 E+06$ & $2.58 E+06$ & 6.98E-05 & AP105-2 & $Y$ & $\begin{array}{l}\text { This is a statistical average of } 13 \text { individual tank } \\
\text { samples and } 2 \text { composite samples, and represents } \\
\text { the "nominal value" for this analyte }\end{array}$ \\
\hline & Tc99 & $2.41 E+06$ & $2.41 E+06$ & $6.52 E-05$ & AP105-2 & $\mathbf{Y}$ & Average of two results \\
\hline & Tc99 & $2.39 E+06$ & $2.39 E+06$ & 6.47E-05 & AP105-2 & $\bar{Y}$ & Average of two results \\
\hline & Tc99 & $2.31 E+06$ & $2.31 E+06$ & 6.25E-05 & AP105-2 & $Y$ & Average of two results \\
\hline & Tc99 & $2.26 E+06$ & $2.26 E+06$ & 6.11E-05 & AP105-2 & $\bar{Y}$ & Average of two results \\
\hline & Tc99 & $2.26 \mathrm{E}+06$ & $2.26 E+06$ & $6.11 E-05$ & AP105-2 & $\mathbf{Y}$ & Average of two results \\
\hline & Tc99 & $2.21 E+06$ & $2.21 E+06$ & 5.97E-05 & AP105-2 & $\mathbf{Y}$ & Average of two results \\
\hline & Tc99 & 2.17E+06 & $2.17 \mathrm{E}+06$ & 5.87E-05 & AP105-2 & $Y$ & Average of two results \\
\hline & Tc99 & $2.10 E+06$ & $2.10 E+06$ & 5.67E-05 & AP105-2 & $Y$ & Average of two results \\
\hline & Tc99 & $2.07 E+06$ & $2.07 E+06$ & 5.60E-05 & AP105-2 & $\bar{Y}$ & Average of two results \\
\hline
\end{tabular}




\begin{tabular}{|c|c|c|c|c|c|c|c|c|c|c|}
\hline \multicolumn{11}{|c|}{ Tank Waste Characterization Sample Information } \\
\hline $\begin{array}{c}\text { Reported } \\
\text { Radionuclide }\end{array}$ & $\begin{array}{c}\text { Memo } \\
\text { Date }\end{array}$ & $\begin{array}{c}\text { Start Analysis } \\
\text { Date }\end{array}$ & $\begin{array}{c}\text { Start } \\
\text { Sample } \\
\text { Date } \\
\end{array}$ & $\begin{array}{l}\text { Sample } \\
\text { Number }\end{array}$ & $\begin{array}{c}\text { Tank Farm } \\
\text { Number }\end{array}$ & $\begin{array}{c}\text { Lab Sample } \\
\text { Number }\end{array}$ & $\begin{array}{l}\text { Density } \\
(\mathrm{g} / \mathrm{mL})\end{array}$ & Reported Valua & Reported Unit & Coda \\
\hline Am-241 & $12 / 1 / 91$ & $7 \longdiv { 1 7 / 9 0 }$ & $6 / 28 / 90$ & NA & NA & R8804/R8805 & 1.540 & 1.47 & $\mathrm{uCi} / \mathrm{L}$ & $\mathbf{P}$ \\
\hline Am241 & $6 / 1 / 90$ & $7 / 17 / 90$ & $6 / 28 / 90$ & NA & NA & R8804/R8805 & 1.540 & 1.204 & uCi/L & $\mathbf{P}$ \\
\hline Am-241 & $12 / 1 / 91$ & $7 \longdiv { 1 7 7 / 9 0 }$ & $6 / 28 / 90$ & NA & NA & $88804 / 28805$ & 1.540 & 0.938 & $\mathrm{uCi} / \mathrm{L}$ & $\mathbf{P}$ \\
\hline Am241 & $7 / 21 / 87$ & NS & NS & NS & NS & NS & NS & 2.2 & nCirh & $\mathbf{p}$ \\
\hline $\mathrm{C}-14$ & $6 \longdiv { 2 6 / 8 6 }$ & NS & NS & NS & NS & NS & 1.490 & $<4.55 E-01$ & uCi/L & $F$ \\
\hline$C 14$ & $7 / 2 / 86$ & NS & NS & NS & NS & NS & 1.500 & $<4.75 E-01$ & uCill & $F$ \\
\hline$C-14$ & $7 / 6 / 86$ & NS & NS & R9897 & NS & NS & 1.460 & $<4.75 E-01$ & $u \mathrm{Ci} / \mathrm{L}$ & $\bar{F}$ \\
\hline$C-14$ & $6 / 30 / 86$ & NS & NS & NS & NS & NS & 1.54 & 1.55 & uCin & $\bar{F}$ \\
\hline C.14 & $6 / 30 / 86$ & NA & NA & NA & NA & NA & 1.540 & 1.55 & $u \mathrm{Ci} / \Omega$ & $\bar{F}$ \\
\hline$C-14$ & $7 / 2 / 86$ & NS & NS & NS & NS & NS & 1.5 & 0.475 & uCi/L & $\bar{F}$ \\
\hline C-14 & $6 / 23 / 86$ & NS & NS & NS & NS & NS & 1.370 & 0.475 & uCin & $F$ \\
\hline$C-14$ & $6 / 23 / 86$ & $\mathrm{NA}$ & $\mathrm{NA}$ & $\mathrm{NA}$ & NA & NA & 1.37 & 0.475 & uCi/L & $\bar{F}$ \\
\hline$C-14$ & $6 / 26 / 86$ & NS & NS & NS & NS & NS & 9.49 & 0.455 & $u \mathrm{Cin}$ & $F$ \\
\hline Carbon-14 & $12 / 1 / 91$ & $7 / 17 / 90$ & $6 / 28 / 90$ & NA & $\overline{\mathrm{NA}}$ & R8804/R8805 & 1.560 & 0.37 & uCin & $\bar{P}$ \\
\hline C.14 & $6 / 26 / 86$ & NS & NS & T686 & NS & NS & 1.310 & 0.329 & $\mathrm{uCi} / 2$ & $\mathbf{P}$ \\
\hline$C-14$ & $6 / 26 / 86$ & NS & NS & T686 & NS & NS & 1.31 & 0.329 & $\mathrm{uCi} / \mathrm{L}$ & $\bar{L}$ \\
\hline $\mathrm{Cm}-243 / 244$ & $12 / 1 / 91$ & $7 / 17 / 90$ & $6 / 28 / 90$ & NA & $\overline{N A}$ & R8804/R8805 & 1.560 & $5.33 E-02$ & $\mathrm{uCi} / \mathrm{L}$ & $\mathbf{P}$ \\
\hline Cobalt-60 & $12 / 1 / 91$ & $7 / 17 / 90$ & $6 / 28 / 90$ & NA & R4792 & R7912/R7929 & 1.530 & $<18.7$ & $u \mathrm{Ci}_{\mathrm{i} / \mathrm{L}}$ & $\overline{\mathbf{P}}$ \\
\hline Cobalt-60 & $12 / 1 / 91$ & $7 / 17 / 90$ & $6 / 28 / 90$ & NA & R4793 & R7913/R7930 & 1.535 & $<16.6$ & uCin & $\bar{P}$ \\
\hline Cobalt-60 & $12 / 1 / 91$ & $7 / 17 / 90$ & $6 / 28 / 90$ & NA & R4794 & R7914/R7933 & 1.570 & $<22.15$ & $\mathrm{uCi} / \mathrm{L}$ & $\bar{P}$ \\
\hline Cobalt -60 & $12 / 1 / 91$ & $7 / 17 / 90$ & $6 / 28 / 90$ & NA & R4795 & R7915/R7934 & 1.540 & $<16.25$ & $\mathrm{uCi} / \mathrm{L}$ & $\overline{\mathbf{P}}$ \\
\hline Cobalt -60 & $12 / 1 / 91$ & $7 / 17 / 90$ & $6 / 28 / 90$ & NA & R4796 & R7916/R7935 & 1.625 & $<30.4$ & UCin & $\bar{P}$ \\
\hline Cobalt-60 & $12 / 1 / 91$ & $7 / 17 / 90$ & $6 / 28 / 90$ & NA & R4797 & R7919/R7936 & 1.560 & $<15.45$ & uCin & $\bar{P}$ \\
\hline Cobalt-60 & $12 / 1 / 91$ & $7 / 17 / 90$ & $6 / 28 / 90$ & NA & R4798 & R7920/R7937 & 1.560 & $<18.6$ & $u C i n$ & $\mathbf{P}$ \\
\hline Cobalt.60 & $12 / 1 / 91$ & $7 / 17 / 90$ & $6 / 28 / 90$ & NA & R4799 & R7921/R7940 & 1.555 & $<21.9$ & uCin & $\bar{P}$ \\
\hline Cobalt-60 & $12 / 1 / 91$ & $7 / 17 / 90$ & $6 / 28 / 90$ & NA & R4800 & R7922/R7941 & 1.560 & $<27.5$ & $\mathrm{uCi} / \mathrm{L}$ & $\mathrm{P}$ \\
\hline Cobalt-60 & $12 / 1 / 91$ & $7 / 17 / 90$ & $6 / 28 / 90$ & NA & R4801 & R7923/R7942 & 1.560 & $<19.7$ & uCin & $\mathbf{P}$ \\
\hline Cobalt-60 & $12 / 1 / 91$ & $7 / 17 / 90$ & $6 / 28 / 90$ & NA & $\mathrm{R} 4802$ & R7926/R7943 & 1.550 & $<31.45$ & UCi/L & $\cdot P$ \\
\hline Cobalt- 60 & $12 / 1 / 91$ & $7 / 17 / 90$ & $6 / 28 / 90$ & NA & R4803 & R7927/R7944 & 1.575 & $<117$ & $\mathrm{UCi} / \mathrm{L}$ & $\mathbf{P}$ \\
\hline Cobalt-60 & $12 / 1 / 91$ & $7 / 17 / 90$ & $6 / 28 / 90$ & NA & NA & $R 8804 / R 8805$ & 1.560 & $<2.964 \mathrm{E}+01$ & uCi/L & $P$ \\
\hline Cs137 & $7 / 2 / 86$ & NA & NA & NA & NA & NA & 1.5 & 563000 & uCin & $\mathbf{F}$ \\
\hline Cesium-137 & $12 / 1 / 91$ & $7 / 17 / 30$ & $6 / 28 / 90$ & NA & $\mathrm{R} 4796$ & R7916/R7935 & 1.625 & $5.520 E+05$ & ucin & $P$ \\
\hline Cs137 & $6 / 23 / 86$ & NS & NS & T621 & NS & NS & 1.44 & 550000 & UCiR & $\mathrm{U}$ \\
\hline Cesium-137 & $12 / 1 / 91$ & $7 / 17 / 90$ & $6 / 28 / 90$ & NA & R4803 & R7927/R7944 & 1.575 & $5.410 E+05$ & uCiR & $\mathbf{p}$ \\
\hline Cesium-137 & $12 / 1 / 91$ & $7 / 17 / 90$ & $6 / 28 / 90$ & NA & R4799 & R792V/R7940 & 1.555 & $5.385 E+05$ & uCin & $\mathbf{P}$ \\
\hline Cs137 & $6 / 30 / 86$ & NA & NA & NA & NA & NA & 1.54 & 530000 & uCin & $\mathbf{F}$ \\
\hline Cesium-137 & $12 / 1 / 91$ & $7 / 17 / 90$ & $6 / 28 / 90$ & NA & R4794 & R7914/R7933 & 1.570 & $5.220 E+05$ & uCin & $\mathbf{P}$ \\
\hline Cesium-137 & $12 / 1 / 91$ & $7 / 17 / 90$ & $6 / 28 / 90$ & NA & $\mathrm{R} 4802$ & R7926/R7943 & 1.550 & $5.215 E+05$ & ucin & $\mathbf{P}$ \\
\hline Cesium-137 & $12 / 1 / 91$ & $7 / 17 / 90$ & $6 / 28 / 90$ & NA & NA & R8804/R8805 & 1.560 & $5.20 \varepsilon+05$ & uCin & $\mathbf{P}$ \\
\hline Cesium-137 & $12 / 1 / 91$ & $7 / 17 / 90$ & $6 / 28 / 90$ & NA & R4801 & R7923/R7942 & 1.560 & $5.190 E+05$ & uCiR & $P$ \\
\hline Cesium.137 & $12 / 1 / 91$ & $7 / 17 / 90$ & $6 / 28 / 90$ & NA & R4792 & R7912/R7929 & 1.530 & $5.180 E+05$ & uCin & $\mathbf{P}$ \\
\hline Cesium-137 & $12 / 1 / 91$ & $7 / 17 / 90$ & $6 / 28 / 90$ & NA & R4793 & R7913/R7930 & 1.535 & $5.175 E+05$ & uCin & $\mathbf{P}$ \\
\hline Cs137 & $6 / 26 / 86$ & NA & NA & NA & NA & NA & 1.49 & 515000 & uCiR & $\mathbf{F}$ \\
\hline
\end{tabular}




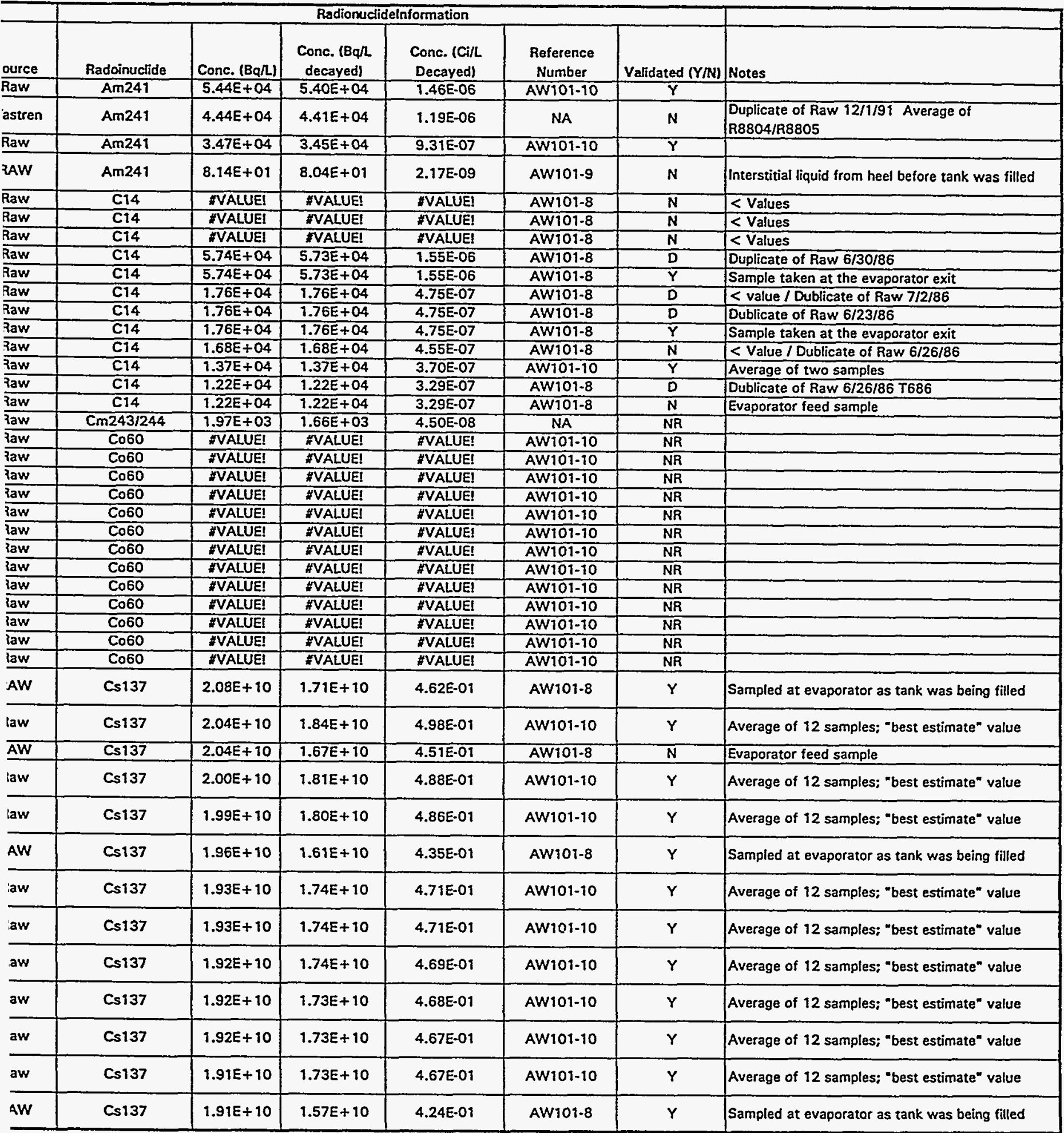


TWRS Privatization Support Pro

\begin{tabular}{|c|c|c|c|c|c|c|c|c|c|c|}
\hline \multicolumn{11}{|c|}{ Tank Waste Characterization Sample Information } \\
\hline $\begin{array}{c}\text { Reported } \\
\text { Radionuclide } \\
\end{array}$ & $\begin{array}{c}\text { Memo } \\
\text { Date }\end{array}$ & $\begin{array}{c}\text { Start Anzlysis } \\
\text { Date }\end{array}$ & $\begin{array}{c}\text { Start } \\
\text { Sample } \\
\text { Date }\end{array}$ & $\begin{array}{c}\text { Sample } \\
\text { Number } \\
\end{array}$ & $\begin{array}{c}\text { Tank Farm } \\
\text { Number }\end{array}$ & $\begin{array}{c}\text { Lab Sample } \\
\text { Number }\end{array}$ & $\begin{array}{l}\text { Density } \\
\text { (g/mL) }\end{array}$ & Reported Value & Reported Unit & Code \\
\hline Cesium-137 & $12 / 1 / 91$ & $7 / 17 / 90$ & $6 / 28 / 90$ & NA & R4800 & R7922/R7941 & 1.560 & $5.125 E+05$ & uCill & $\mathbf{P}$ \\
\hline Cesium-137 & $12 / 1 / 91$ & $7 / 17 / 90$ & $6 / 28 / 90$ & NA & R4798 & R7920/R7937 & 1.560 & $5.010 E+05$ & uCi/L & $\mathbf{P}$ \\
\hline Cesium-137 & $12 / 1 / 91$ & $7 / 17 / 90$ & $6 / 28 / 90$ & NA & R4797 & R7919/R7936 & 1.560 & $4.970 E+05$ & uCill & $\mathbf{P}$ \\
\hline Cesium-137 & $12 / 1 / 91$ & $7 / 17 / 90$ & $6 / 28 / 90$ & NA & R4795 & R7915/R7934 & 1.540 & $4.965 E+05$ & uCin & $\mathbf{P}$ \\
\hline $\operatorname{Cs} 137$ & $6 / 1 / 90$ & NA & NA & NA & NA & NA & NA & NA & NA & $P$ \\
\hline Cs137 & $12 / 30 / 81$ & NS & NS & $T 1049$ & NS & NS & 1.329 & 455000 & uCi/L & $L$ \\
\hline Cs137 & $12 / 30 / 81$ & NS & NS & $T 1048$ & NS & NS & 1.324 & 439000 & uCi $/ / 2$ & $L$ \\
\hline Cs137 & $6 / 23 / 86$ & NA & NA & NA & NA & NA & 1.37 & 368000 & uCin & $\mathbf{F}$ \\
\hline Cs137 & $7 / 2 / 86$ & NS & NS & $T 882$ & NS & NS & 1.32 & 348000 & UCin & 0 \\
\hline Cs137 & $6 / 26 / 86$ & NS & NS & T686 & NS & NS & 1.31 & 319000 & UCiR & $\mathbf{U}$ \\
\hline Cs & NS & NS & NS & NS & NS & NS & 1.393161 & 0.253805421 & $\mathrm{Ci} / \mathrm{L}$ & \\
\hline Cs137 & $7 / 6 / 86$ & NS & NS & T959 & NS & NS & 1.23 & 245000 & uCi $/ \mathrm{L}$ & $\bar{U}$ \\
\hline Cs137 & $6 / 30 / 86$ & NS & NS & $T 812$ & NS & NS & 1.13 & 134000 & uCi/L & $\bar{U}$ \\
\hline Cs137 & $11 / 20 / 84$ & NS & NS & $R 2644$ & NS & NS & NS & 54400 & uCin & $\mathbf{P}$ \\
\hline Cs137 & $11 / 20 / 84$ & NS & NS & R2645 & NS & NS & NS & 27600 & uCi/L & 0 \\
\hline Cs-137 & $11 / 9 / 84$ & NS & NS & R.3185 & NS & NS & 1.024 & 21800 & uCin & $\bar{P}$ \\
\hline Cs137 & $11 / 9 / 84$ & NS & NS & NS & NS & NS & 1.024 & 21800 & uCi/L & $\mathbf{P}$ \\
\hline Cs137 & $11 / 9 / 84$ & NS & NS & R3185 & NS & NS & 1.024 & 21800 & uCin & $I$ \\
\hline Cs137 & $4 / 21 / 85$ & NS & NS & T5351 & $\mathrm{NS}$ & NS & 1.0411 & 15200 & $u \mathrm{Ci} / \mathrm{L}$ & 0 \\
\hline Cs137 & $4 / 21 / 85$ & NS & NS & $T 5350$ & NS & NS & 1.0279 & 9640 & uCi/L & $\mathrm{L}$ \\
\hline Cs137 & $3 / 30 / 86$ & NS & NS & T7698 & NS & NS & 0.993 & 5480 & uCi/L & $L$ \\
\hline Cs137 & $3 / 30 / 86$ & NS & NS & r7697 & NS & NS & 0.997 & 5470 & uCin & $L$ \\
\hline Cs137 & $11 / 14 / 85$ & NS & NS & T2641 & NS & NS & 1.01 & 4470 & $u \mathrm{Ci} / \mathrm{L}$ & $L$ \\
\hline Cs137 & $7 / 21 / 87$ & NS & NS & NS & NS & NS & NS & 548000 & nCill & $\mathbf{P}$ \\
\hline lodine-129 & $12 / 7 / 91$ & $7 / 17 / 90$ & $6 / 28 / 90$ & NA & $\overline{N A}$ & $\mathrm{R8804/R8805}$ & 1.560 & 0.303 & uCin & $\mathbf{P}$ \\
\hline $\mathrm{Np}-237$ & $12 / 1 / 91$ & $7 / 17 / 90$ & $6 / 28 / 90$ & NA & NA & R8804/R8805 & 1.560 & $<3.060 \mathrm{E}-03$ & $\mathrm{uCi} / \mathrm{L}$ & $\mathbf{P}$ \\
\hline Pu239/240 & $6 / 26 / 86$ & NS & NS & T686 & NS & NS & 1.31 & 0.00229 & $g / L$ & U \\
\hline Pu239/240 & $6 / 23 / 86$ & NS & NS & $T 621$ & NS & NS & 1.44 & 0.000169 & $g / 2$ & $\underline{U}$ \\
\hline Pu239/240 & $6 / 26 / 86$ & NA & NA & NA & NA & NA & 1.49 & 0.0000204 & $g /$ & $\mathbf{F}$ \\
\hline Pu-239/240 & $12 / 1 / 91$ & $7 / 17 / 90$ & $6 / 28 / 90$ & NA & NA & R8804/R8805 & 1.560 & 1.25 & UCi/L & $P$ \\
\hline$P \mathrm{P}-239 / 240$ & $12 / 1 / 91$ & $7 / 17 / 90$ & $6 / 28 / 90$ & NA & NA & $R 8804 / R 8805$ & 1.560 & 1.04 & $\mathrm{uCi} / \mathrm{L}$ & $\mathbf{P}$ \\
\hline Pu239/240 & $7 / 2 / 86$ & NS & NS & $T 882$ & NS & NS & 1.32 & 0.0000144 & $g / h$ & U \\
\hline Pu239/240 & $6 / 30 / 86$ & NA & NA & NA & NA & NA & 1.54 & 0.0000126 & $g / L$ & $F$ \\
\hline Pu239/240 & $7 / 2 / 86$ & NA & NA & NA & NA & NA & 1.5 & 0.0000107 & $g n$ & $F$ \\
\hline Pu239/240 & $7 / 6 / 86$ & NS & NS & T959 & NS & NS & 1.23 & 0.00000896 & $g / L$ & 0 \\
\hline Pu239/240 & $6 / 23 / 86$ & NA & NA & NA & NA & NA & 1.36 & 0.00000625 & $g h$ & $F$ \\
\hline Pu239/240 & \begin{tabular}{|l|}
$6 / 30 / 86$ \\
\end{tabular} & NS & NS & T812 & NS & NS & 1.13 & 0.00000562 & $g h$ & U \\
\hline Pu239/240 & $7 / 21 / 87$ & NS & NS & NS & NS & NS & NS & 0.82 & $\mathrm{nCi} /$ & $P$ \\
\hline Pu239/240 & $11 / 14 / 85$ & NS & NS & T2641 & NS & NS & 1.01 & 0.0000304 & $u \mathrm{Ci} / \mathrm{L}$ & $\mathrm{L}$ \\
\hline Pu239/240 & \begin{tabular}{|l|}
$3 / 30 / 86$ \\
\end{tabular} & NS & NS & T7698 & NS & NS & 0.993 & 0.00000103 & uCi $i \Omega$ & L \\
\hline Pu239/240 & $3 / 30186$ & NS & NS & T7697 & NS & NS & 0.997 & $1.34 \mathrm{E}-08$ & uCi/l. & $\mathrm{L}$ \\
\hline Selenium-79 & \begin{tabular}{|l|}
$12 / 1 / 91$ \\
\end{tabular} & $7 / 17 / 90$ & $6 / 28 / 90$ & NA & $\overline{\mathrm{NA}}$ & R8804/R8805 & 1.560 & 0.48 & $\mathrm{uCi} / \mathrm{L}$ & $\bar{P}$ \\
\hline Sr & NS & NS & NS & NS & NS & NS & 1.393161 & 0.017804195 & $\mathrm{Ci} / \mathrm{L}$ & \\
\hline Sr90 & \begin{tabular}{|l|}
$12 / 30 / 81$ \\
\end{tabular} & NS & NS & T1049 & NS & NS & 1.329 & 14600 & uCin & $L$ \\
\hline Sr90 & $12 / 30 / 81$ & NS & NS & $T 1048$ & NS & NS & 1.324 & 13600 & $u \mathrm{Ci} / \mathrm{L}$ & $L$ \\
\hline
\end{tabular}




\begin{tabular}{|c|c|c|c|c|c|c|c|c|c|c|}
\hline \multicolumn{11}{|c|}{ Tank Waste Characterization Sample Information } \\
\hline $\begin{array}{c}\text { Reported } \\
\text { Radionuclido }\end{array}$ & $\begin{array}{c}\text { Memo } \\
\text { Date } \\
\end{array}$ & $\begin{array}{c}\text { Start Analysis } \\
\text { Date }\end{array}$ & $\begin{array}{c}\text { Start } \\
\text { Sample } \\
\text { Date }\end{array}$ & $\begin{array}{l}\text { Sample } \\
\text { Number }\end{array}$ & $\begin{array}{c}\text { Tank Farm } \\
\text { Number }\end{array}$ & $\begin{array}{c}\text { Lab Sample } \\
\text { Number }\end{array}$ & $\begin{array}{l}\text { Density } \\
\text { (g/mL) }\end{array}$ & Reported Value & Reported Unit & Code \\
\hline Sr90 & $6 / 26 / 86$ & \begin{tabular}{|c|} 
NS \\
\end{tabular} & NS & $T 686$ & NS & NS & 1.31 & 13200 & uCi/L & $\mathrm{U}$ \\
\hline Sr90 & $6 / 23 / 86$ & NA & NA & NA & NA & $\mathrm{NA}$ & 1.36 & 9400 & uCin & $F$ \\
\hline Sr90 & $6 / 26 / 86$ & NA & NA & NA & $\overline{N A}$ & NA & 1.49 & 8780 & uCint & $F$ \\
\hline Sr90 & $6 / 23 / 86$ & NS & NS & $T 621$ & NS & NS & 1.44 & 8240 & UCi/L & $\bar{U}$ \\
\hline Sr90 & $7 / 2 / 86$ & NS & NS & $T 882$ & NS & NS & 1.32 & 6340 & uCin & $\mathrm{U}$ \\
\hline Sr90 & $6 / 30 / 86$ & $\mathrm{NA}$ & NA & NA & NA & NA & 1.54 & 6000 & uCill. & $F$ \\
\hline Sr90 & $7 / 6 / 86$ & NS & NS & $T 959$ & NS & NS & 1.23 & 4010 & $\mathrm{uCi} / \mathrm{L}$ & $\bar{U}$ \\
\hline Sr90 & $11 / 20 / 84$ & NS & NS & $\mathbf{R 2 6 4 4}$ & NS & NS & NS & 2700 & uCin & $\mathbf{P}$ \\
\hline Sr90 & $6 / 30 / 86$ & NS & NS & T812 & NS & NS & 1.13 & 2200 & $\mathrm{uCi} / \mathrm{L}$ & $\mathbf{U}$ \\
\hline Sत90 & $7 / 2 / 86$ & NA & NA & NA & NA & NA & 1.5 & 1830 & uCi $/$ L & $F$ \\
\hline Si-89/90 & $12 / 1 / 91$ & $7 / 17 / 90$ & $6 / 28 / 90$ & NA & NA & R8804/R8805 & 1.560 & $1.14 E+03$ & UCIR & $P$ \\
\hline $5 r 89 / 90$ & $6 / 1 / 90$ & NS & NS & NS & NS & NS & NS & NS & & $L$ \\
\hline $5 r-89 / 90$ & $12 / 1 / 91$ & $7 / 17 / 90$ & $6 / 28 / 90$ & NA & NA & R8804/R8805 & 1.560 & $1.03 E+03$ & uCin & $\mathbf{P}$ \\
\hline Sr90 & $11 / 9 / 84$ & NS & NS & NS & NS & NS & 1.024 & 428 & $\mathrm{uCi} / \mathrm{L}$ & $\underline{P}$ \\
\hline Sr-90 & $11 / 9 / 84$ & NS & NS & $R-3185$ & NS & NS & 1.024 & 425 & uCin & $P$ \\
\hline Sr90 & $11 / 9 / 84$ & NS & NS & $\mathbf{R 3 1 8 5}$ & NS & NS & 1.024 & 425 & $\mathrm{uCi} / \mathrm{L}$ & $L$ \\
\hline Sr90 & $11 / 20 / 84$ & NS & NS & R2645 & NS & NS & NS & 340 & $u C i / L$ & 0 \\
\hline Sr90 & $3 / 30 / 86$ & NS & NS & T7697 & NS & NS & 0.997 & 45.9 & uCi/L & $L$ \\
\hline Sr90 & $3 / 30 / 86$ & NS & NS & T7698 & NS & NS & 0.993 & 32.8 & $\mathrm{uCi} / \mathrm{L}$ & $\mathrm{L}$ \\
\hline Tc99 & $11 / 20 / 84$ & NS & NS & R2644 & NS & NS & NS & 7350 & uCi/L & $P$ \\
\hline Tc99 & $7 / 6 / 86$ & NA & NA & R9897 & NA & NA & 1.460 & 440 & $\mathrm{UCi} / \mathrm{L}$ & $F$ \\
\hline Tc99 & $7 / 2 / 86$ & NS & NS & NS & NS & NS & 1.500 & 248 & $u \mathrm{Ci} / \mathrm{L}$ & $\mathbf{F}$ \\
\hline Tc-99 & $7 / 2 / 86$ & NA & NA & NA & NA & NA & 1.5 & 248 & $\mathrm{uCI} / \mathrm{L}$ & $F$ \\
\hline Tc99 & $6 / 30 / 86$ & NS & NS & NS & NS & NS & 1.540 & 215 & $\mathrm{UC \textrm {Ci } / \mathrm { L }}$ & $F$ \\
\hline Tc-99 & $6 / 30 / 86$ & NA & NA & NA & NA & $\overline{N A}$ & 1.54 & 215 & UCi/L & $F$ \\
\hline Tc99 & $6 / 23 / 86$ & NS & NS & NS & NS & NS & 1.370 & 205 & uCiR & $F$ \\
\hline Tc-99 & $6 / 23 / 86$ & NA & NA & NA & NA & NA & 1.37 & 205 & $\mathrm{uCi} / \mathrm{L}$ & $F$ \\
\hline Tc99 & $6 / 26 / 86$ & NS & NS & NS & NS & NS & 1.490 & 200 & UCi/L & $F$ \\
\hline TC-99 & $6 / 26 / 86$ & NA & NA & NA & NA & NA & 1.49 & 200 & uCin & $F$ \\
\hline Tc-99 & $12 / 1 / 91$ & $7 / 17 / 90$ & $6 / 28 / 90$ & $\mathrm{NA}$ & NA & R8804/R8805 & 1.560 & 176 & uCin & P \\
\hline Tc99 & $6 / 26 / 86$ & NS & NS & T686 & NS & NS & 1.310 & 148 & $\mathrm{uCi} / \mathrm{L}$ & $P$ \\
\hline Tc-99 & $6 / 26 / 86$ & NS & NS & $T 686$ & NS & NS & 1.31 & 148 & $\mathrm{uCi} / \mathrm{L}$ & $L$ \\
\hline Tc-99 & $12 / 1 / 91$ & $7 / 17 / 90$ & $6 / 28 / 90$ & NA & NA & $R 8804 / \mathrm{R} 8805$ & 1.560 & 128 & uCi/L. & $\bar{p}$ \\
\hline Tc-99 & $11 / 20 / 84$ & NS & NS & R2644 & NS & NS & $\mathrm{NS}$ & 118 & $u \mathrm{Ci} / \mathrm{L}$ & $P$ \\
\hline Tc99 & $11 / 20 / 84$ & NS & NS & $\mathrm{R} 2644$ & NS & NS & NS & 118 & uCi/L & $P$ \\
\hline Te9g & $6 / 23 / 86$ & NS & NS & T621 & NS & NS & 1.440 & 105 & uCir & $\mathbf{P}$ \\
\hline Tc-99 & $6 / 23 / 86$ & NS & NS & T621 & NS & NS & 1.44 & 105 & uCill & $L$ \\
\hline Tc-99 & $3 / 30 / 86$ & NS & NS & T7697 & NS & NS & 0.997 & 22 & $\mathrm{UC \textrm {Ci } / \mathrm { L }}$ & $\mathbf{P}$ \\
\hline Tc99 & $4 / 29 / 86$ & NS & NS & 7697 & NS & NS & 0.993 & 22 & $\mathrm{UCi} / \mathrm{L}$ & $P$ \\
\hline Tc-99 & $3 / 30 / 86$ & NS & NS & T7698 & NS & NS & 0.993 & 18.3 & UCilL & $P$ \\
\hline Tc99 & $4 / 29 / 86$ & NS & NS & T7698 & NS & NS & 0.993 & 18.3 & $u \mathrm{Ci} / \mathrm{L}$ & $P$ \\
\hline
\end{tabular}




\begin{tabular}{|c|c|c|c|c|c|c|c|c|c|c|}
\hline & & & & Tank Waste Char & cterizations & Sample Inform & lation & & & \\
\hline Reported Radionuclide & $\begin{array}{c}\text { Memo } \\
\text { Date }\end{array}$ & $\begin{array}{c}\text { Start } \\
\text { Analysis Date }\end{array}$ & $\begin{array}{c}\text { Start } \\
\text { Sample } \\
\text { Date } \\
\end{array}$ & Sample Number & $\begin{array}{c}\text { Tank Farm } \\
\text { Number }\end{array}$ & $\begin{array}{c}\text { Lab Sample } \\
\text { Number }\end{array}$ & Density $(\mathrm{g} / \mathrm{mL})$ & Reported Value & Reported Unit & Codt \\
\hline $\operatorname{Am} 241$ & $9 / 22 / 86$ & NA & $\mathrm{NA}$ & NA & NA & NA & NA & $<1.45 \mathrm{E}-04$ & $\mathrm{uCi} / \mathrm{mL}$ & $P$ \\
\hline Am241 & $2 / 8 / 89$ & $3 / 11 / 88$ & $10 / 1 / 87$ & $T-3493$ & NA & NA & NA & $<10$ & $\mathrm{uCi} / \Omega$ & $\mathbf{P}$ \\
\hline Americium-241 & $7 / 26 / 95$ & NA & $3 / 3 / 95$ & 8OD9P9 & NA & NA & 1.20 & $<0.0469$ & $\mathrm{uCi} / \mathrm{mL}$ & $\mathbf{L}$ \\
\hline Americium-241 & $7 / 26 / 95$ & NA & $3 / 3 / 95$ & 80D9P6 & NA & NA & 1.19 & $<0.0219$ & $\mathrm{uCi} / \mathrm{mL}$ & $L$ \\
\hline Americium-241 & $7 / 26 / 95$ & NA & $3 / 3 / 95$ & 8009P7 & NA & NA & 1.18 & $<0.0216$ & $\mathrm{uCi} / \mathrm{mL}$ & $\mathbf{L}$ \\
\hline Am241 & NS & NS & NS & NS & NS & NS & NS & NS & $\because \mathrm{NS}$ & $\bar{L}$ \\
\hline Am241 & $9 / 1 / 89$ & NS & NS & RAT-AZ101-4A & NS & NS & 1.2 & 0.011 & uCi/gm & L \\
\hline Am241 & $4 / 1 / 89$ & NS & NS & NS & NS & NS & NS & NS & NS & $\bar{P}$ \\
\hline Am241 & $7 / 30 / 92$ & NA & $4 / 1 / 89$ & Core 1 & NA & NA & NA & 13.2 & $U \mathrm{Ci} / \mathrm{L}$ & $\bar{P}$ \\
\hline Am-241 & $9 / 1 / 93$ & NA & $4 / 1 / 89$ & Core 2 & NA & NA & 1.22 & 0.013 & $u \mathrm{Ci} / \mathrm{g}$ & $\bar{P}$ \\
\hline Am-241 & $9 / 1 / 93$ & NA & $4 / 1 / 89$ & Core 2 & NA & NA & 1.14 & 0.0139 & uCi/g & $\bar{P}$ \\
\hline $\mathrm{C14}$ & $1 / 1 / 81$ & NS & NS & NS & NS & NS & NS & 2.016561292 & ug $/ \mathrm{L}$ & $\mathrm{L}$ \\
\hline$C-14$ & $9 / 1 / 93$ & NA & $4 / 1 / 89$ & Core 2 & NA & NA & NA & 0.0013 & $\mathrm{uCi} / \mathrm{g}$ & $\bar{P}$ \\
\hline $\mathrm{C} 14$ & $4 / 1 / 89$ & NS & NS & NS & NS & NS & NS & 53300 & $B q / L$ & $\overrightarrow{\mathbf{P}}$ \\
\hline$C-14$ & $9 / 1 / 89$ & NS & NS & RAT-AZ101-4A & NS & NS & 1.2 & 0.0012 & uCi/gm & $P$ \\
\hline C. 14 & $7 / 30 / 92$ & NA & $4 / 1 / 89$ & Core 1 & NA & NA & NA & 1.44 & $\mathrm{uCi} / \mathrm{L}$ & $\bar{P}$ \\
\hline C. 14 & $9 / 13 / 85$ & NS & NS & RAT-AZ101-3A & NS & NS & 1.17 & 1.06 & $\mathrm{uCin}$ & $\bar{P}$ \\
\hline $\mathrm{C}-14$ & $9 / 13 / 85$ & NA & $\overline{\mathrm{NA}}$ & NA & NA & NA & 1.17 & 1.06 & uCi/L & $\bar{P}$ \\
\hline$C-14$ & $9 / 1 / 93$ & $\overline{N A}$ & $4 / 1 / 89$ & Core 2 & NA & NA & 1.22 & 0.0013 & $\mathrm{uCi} / \mathrm{g}$ & $P$ \\
\hline $\mathrm{C}-14$ & $9 / 1 / 93$ & NA & $4 / 1 / 89$ & Core 2 & NA & $\overline{N A}$ & 1.14 & 0.000694 & $\mathrm{uCi} / \mathrm{g}$ & $P$ \\
\hline $\mathrm{Cm} 243,4$ & $9 / 1 / 93$ & NA & $4 / 1 / 89$ & Core 2 & NA & NA & 1.14 & $<0.00058$ & $\mathrm{uCi} / \mathrm{g}$ & $\bar{p}$ \\
\hline Cm243,4 & $9 / 1 / 93$ & NA & $4 / 1 / 89$ & Core 2 & NA & NA & 1.22 & $<0.00068$ & $u \mathrm{Ci} / \mathrm{g}$ & $P$ \\
\hline $\mathrm{Cm} 244$ & NS & NS & NS & NS & NS & NS & NS & NS & NS & $L$ \\
\hline $\mathrm{Cm} 244$ & $4 / 1 / 89$ & NS & NS & NS & NS & NS & NS & NS & NS & $\vec{P}$ \\
\hline Cm243/244 & $7 / 30 / 92$ & NA & $4 / 1 / 89$ & Core 1 & $\mathrm{NA}$ & NA & NA & 0.144 & UCi/L & $\bar{P}$ \\
\hline $\mathrm{Cm} 243 / 244$ & $9 / 1 / 89$ & NS & NS & RAT-AZ101-4A & NS & NS & 7.2 & 0.00012 & uCin & $\mathrm{L}$ \\
\hline Co-60 & $9 / 1 / 93$ & NA & $4 / 1 / 89$ & Core 2 & NA & NA & 1.14 & $<0 . \overline{31}$ & $\mathrm{uCi} / \mathrm{g}$ & $\bar{P}$ \\
\hline Co.60 & $9 / 1 / 93$ & NA & $4 / 1 / 89$ & Core 2 & NA & NA & 1.22 & $<2.0$ & uCi/g & $\bar{P}$ \\
\hline Co60 & $9 / 29 / 89$ & 1989 & 1989 & Core 1 & NA & NA & 1.2 & $<7.5 \mathrm{E}-04$ & $\mathrm{mCi} / \mathrm{g}$ & $\bar{P}$ \\
\hline Co60 & $4 / 26 / 78$ & NS & NS & NS & NS & NS & NS & 1 & UCi/L & $L$ \\
\hline Cs & NA & $\overline{N A}$ & NA & NA & NA & NA & 1.12432389 & 2.521827668 & CinL & $\bar{L}$ \\
\hline Cesium-137 & $7 / 26 / 95$ & NA & $3 / 3 / 95$ & B0D9P7 & NA & NA & 1.18 & 1590 & $\mathrm{uCi} / \mathrm{mL}$ & $\bar{L}$ \\
\hline Cesium-137 & $7 / 26 / 95$ & NA & $3 / 3 / 95$ & BOD9P6 & NA & NA & 1.19 & 1585 & $\mathrm{uCi} / \mathrm{mL}$ & $L$ \\
\hline Cesium-137 & $7 / 26 / 95$ & NA & $3 / 3 / 95$ & BOD9P9 & NA & NA & 1.20 & 1585 & $\mathrm{uCl} / \mathrm{mL}$ & $L$ \\
\hline Cs137 & NS & NS & NS & NS & NS & NS & NS & NS & NS & $\mathrm{L}$ \\
\hline Cs137 & $9 / 1 / 93$ & NA & $4 / 1 / 89$ & Core 2 & NA & NA & $\overline{\mathrm{NA}}$ & 2540 & uCi/g & $\bar{P}$ \\
\hline Cs137 & $10 / 1 / 87$ & NS & NS & RAT-AZ101-6A & NS & NS & NS & 2270000 & $\mathrm{uCin}$ & $\mathrm{L}$ \\
\hline Cs137 & $10 / 30 / 80$ & $\mathrm{NA}$ & $5 / 2 / 90$ & R1103B8 & NA & NA & NA & 2270000 & $\mathrm{uCi} / \mathrm{L}$ & $P$ \\
\hline Cs137 & $2 / 8 / 89$ & NA & $10 / 7 / 87$ & T3493 & NA & $\overline{N A}$ & NA & 2270000 & uCi/L & $P$ \\
\hline Cs137 & $4 / 8 / 86$ & NA & $11 / 1 / 85$ & NA & NA & NA & 1.17 & 2200000 & $\mathrm{uCi} / \mathrm{L}$ & $L$ \\
\hline Cs137 & $8 / 7 / 86$ & NS & NS & R9964/R0247 & NS & NS & 1.2 & 2110000 & $\mathrm{uCin}$ & $\mathrm{L}$ \\
\hline Cs137 & $10 / 29 / 90$ & NA & $5 / 2 / 90$ & R280187 & NA & NA & $\mathrm{NA}$ & 2110000 & UCill & $P$ \\
\hline Cs137 & $1 / 28 / 87$ & NA & NA & $\overline{N A}$ & $\overline{N A}$ & $\overline{\mathrm{NA}}$ & NA & 2110000 & $\mathrm{UCi}$ & $P$ \\
\hline Cs137 & $9 / 1 / 89$ & NS & NS & RAT-AZ101-4A & NS & NS & 1.2 & 1700 & uCi/gm & $L$ \\
\hline Cs137 & $4 / 1 / 89$ & NS & NS & NS & NS & NS & NS & NS & NS & $\mathbf{P}$ \\
\hline $\operatorname{cs137}$ & $7 / 30 / 92$ & NA & $4 / 1 / 89$ & Core 1 & NA & NA & $\mathrm{NA}$ & 2040000 & $\mathrm{uCi} / \mathrm{L}$ & $P$ \\
\hline Cs137 & $9 / 13 / 85$ & NA & NA & RAT-AZ101-3A & NA & $\overline{N A}$ & 1.17 & 1330000 & uCin & $L$ \\
\hline Cs137 & $9 / 13 / 85$ & NA & NA & NA & NA & NA & 1.17 & 1330000 & UCi/L & $L$ \\
\hline Cs137 & $9 / 13 / 85$ & NA & NA & B & NA & NA & NA & 1290000 & uCi/L & $P$ \\
\hline Cs137 & $9 / 13 / 85$ & NA & NA & A & NA & NA & NA & 1240000 & uCill & $\mathbf{P}$ \\
\hline Cs137 & $11 / 18 / 85$ & NS & NS & RAT-AZ101-I & NS & NS & 1.17 & 1090000 & uCin & $\bar{L}$ \\
\hline Cs137 & $10 / 29 / 90$ & NA & $5 / 2 / 90$ & R101283 & $\mathrm{NA}$ & NA & $\mathrm{NA}$ & 1090000 & UCin & $\bar{P}$ \\
\hline
\end{tabular}




\begin{tabular}{|c|c|c|c|c|c|c|c|}
\hline & \multicolumn{4}{|c|}{ Radionuclide Information } & \multirow[b]{2}{*}{$\begin{array}{c}\text { Reference } \\
\text { Number }\end{array}$} & \multirow[b]{2}{*}{$\begin{array}{c}\text { Validated } \\
\text { (Y/N) }\end{array}$} & \multirow[b]{2}{*}{ Notes } \\
\hline Source & Radionuclide & $\begin{array}{l}\text { Conc. } \\
(\mathrm{Bq} / \mathrm{L})\end{array}$ & $\begin{array}{c}\text { Conc. }(B q / L \\
\text { decayed) }\end{array}$ & $\begin{array}{l}\text { Conc. (Ci/L } \\
\text { Decayed) }\end{array}$ & & & \\
\hline Raw & Am241 & \#VALUE! & \#VALUE! & \#VALUE! & AZ101-10 & $Y$ & \\
\hline Raw & Am241 & \#VALUE! & \#VALUE! & \#VALUE! & $A 7101-16$ & $\mathbf{Y}$ & \\
\hline TCD & Am241 & \#VALUE! & \#VALUE! & \#VALUE! & NA & $\mathbf{Y}$ & $\begin{array}{l}<\text { Value, } \$ 1 \text { - Replace } \# 1 \& \$ 2 \text { with Avg } \\
\text { value }=<0.0469\end{array}$ \\
\hline TCD & Am241 & \#VALUE! & \#VALUE! & \#VALUE! & NA & $\mathbf{Y}$ & $\begin{array}{l}<\text { Value, } \# 3-\text { Replace } \# 3 \& \text { \& with Avg } \\
\text { value }=<0.0219\end{array}$ \\
\hline TCD & Am241 & \#VALUE! & \#VALUE! & \#VALUE! & NA & $\mathbf{Y}$ & $\begin{array}{l}<\text { Value, } \# 5 \text { - Replace } \# 5 \text { \& } \# 6 \text { with Avg } \\
\text { value }=<0.0216\end{array}$ \\
\hline TRAC & Am241 & NS & $2.75 E+06$ & 7.43E-05 & NA & $\mathbf{N}$ & Unknown date, samplë̈, TRAC data, not used \\
\hline Braun & Am241 & $4.88 \mathrm{E}+05$ & $4.84 E+05$ & $1.31 \mathrm{E}-05$ & NA & $N$ & Unknown sample date, Braun data, not used \\
\hline Vastren & Am241 & $4.88 E+05$ & $4.84 E+05$ & $1.31 E-05$ & NA & $\mathbf{N}$ & Wastren data, not used \\
\hline RAW & Am241 & $4.88 E+05$ & $4.87 E+05$ & $1.32 \mathrm{E}-05$ & AZ101-25 & $\mathbf{Y}$ & Data from AZ101-22 \\
\hline Raw & Am241 & $5.87 E+02$ & $5.86 E+02$ & $1.58 \mathrm{E}-08$ & AZ101-19 & $\mathbf{Y}$ & \\
\hline Raw & Am241 & $5.86 \mathrm{E}+02$ & $5.85 \mathrm{E}+02$ & $1.58 \mathrm{E}-08$ & AZ101-19 & $N$ & Combined supernate from the washed solids \\
\hline TRAC & $\mathrm{C} 14$ & $3.33 E+05$ & $3.32 E+05$ & 8.98E-06 & NA & $\bar{N}$ & TRAC data, not used \\
\hline Raw & $\mathbf{C 1 4}$ & $5.77 E+04$ & $5.77 E+04$ & $1.56 \mathrm{E}-06$ & AZ101-19 & $Y$ & \\
\hline Vastren & $\mathrm{C} 14$ & $5.33 E+04$ & $5.33 E+04$ & $1.44 \mathrm{E}-06$ & NA & $N$ & Wastren data, not used \\
\hline Braun & $\mathrm{Cl4}$ & $5.33 E+04$ & $5.32 \bar{E}+04$ & 1.44E-06 & NA & $N$ & Braun data, not used \\
\hline Raw & C14 & $5.33 E+04$ & $5.33 E+04$ & $1.44 E-06$ & AZ101-25 & $\mathrm{Y}$ & Data from AZ101-22 \\
\hline Braun & $\mathrm{C14}$ & $3.92 E+04$ & $3.92 E+04$ & $1.06 \mathrm{E}-06$ & NA & $\mathbf{N}$ & Braun data, not used \\
\hline Raw & $\mathrm{Cl4}$ & $3.92 E+04$ & $3.92 E+04$ & $1.06 \mathrm{E}-06$ & AZ101-7 & $\mathbf{Y}$ & \\
\hline Raw & $\mathrm{C14}$ & $5 . \overline{87}+01$ & $5.87 \mathrm{E}+01$ & 1.59E-09 & AZ101-19 & $\bar{N}$ & Duplicate \\
\hline Raw & $\mathrm{C14}$ & $2.93 \mathrm{E}+01$ & $2.93 E+01$ & $7.91 \mathrm{E}-10$ & AZ101-19 & $N$ & Combined supernate from the washed solids \\
\hline Raw & $\mathrm{Cm} 243 / 244$ & \#VALUE! & \#VALUE! & \#VALUE! & A2101-19 & $N$ & Combined supernate from the washed solids \\
\hline Raw & $\mathrm{Cm} 243 / 244$ & \#VALUE! & \#VALUE! & \#VALUE! & AZ101-19 & $\bar{Y}$ & \\
\hline TRAC & $\mathrm{Cm} 243 / 244$ & NS & $5.44 \mathrm{E}+03$ & 1.47E-07 & NA & $\mathrm{N}$ & TRAC data, not used \\
\hline lastren & $\mathrm{Cm} 243 / 244$ & $5.33 \mathrm{E}+03$ & $4.28 E+03$ & $1.16 \mathrm{E}-07$ & NA & $\mathbf{N}$ & Wastren data, not used \\
\hline RAW & $\mathrm{Cm} 243 / 244$ & $5.33 \mathrm{E}+03$ & $4.86 \mathrm{E}+03$ & 1.31E-07 & AZ101-25 & $\bar{Y}$ & Data from AZ101-22 \\
\hline Braun & $\mathrm{Cm} 243 / 244$ & $4.44 E+00$ & $3.63 \mathrm{E}+00$ & $9.81 E-11$ & NA & $\mathbf{N}$ & Braun data, not used \\
\hline Raw & $\mathrm{Co60}$ & \#VALUE! & \#VALUE! & \#VALUE! & AZ101-19 & $\mathbf{N}$ & Combined supernate from the washed solids \\
\hline Raw & Co60 & \#VALUE! & \#VALUE! & FVALUE! & A2101-19 & $\mathbf{Y}$ & $<$ Value \\
\hline Raw & Co60 & \#VALUE! & \#VALUE! & \#VALUE! & AZ101-24 & $\bar{Y}$ & \\
\hline RAW & Co60 & $3.70 \mathrm{E}+04$ & $4.15 E+03$ & $1.12 \mathrm{E}-07$ & AZ101-1 & $N$ & Data from pre-03-1984 \\
\hline CE Inv. & Cs-137 & $9.33 E+10$ & $9.33 \mathrm{E}+10$ & $2.52 \mathrm{E}+00$ & NA & $\mathbf{N}$ & HTCE Data \\
\hline TCD & Cs-137 & $5.88 \mathrm{E}+10$ & $5.88 E+10$ & $1.59 E+00$ & NA & $\bar{Y}$ & $\# 1,-$ Avg. Value of $\# 1 \& \# 6=1590$ \\
\hline TCD & Cs-137 & $5.86 \mathrm{E}+10$ & $5.86 E+10$ & $1.59 \mathrm{E}+00$ & NA & $\bar{Y}$ & $\$ 2,-$ Avg. Value of $\$ 2 \& \$ 5=1585$ \\
\hline TCD & Cs-137 & $5.86 E+10$ & $5.86 E+10$ & $1.59 \mathrm{E}+00$ & NA & $\bar{Y}$ & $\# 3,-$ Avg. Value of $\# 3 \& \& 4=1585$ \\
\hline TRAC & Cs137 & NS & $2.20 E+08$ & 5.95E-03 & NA & $N$ & TRAC data, not used \\
\hline RAW & Cs137 & $1.03 E+11$ & $9.07 E+10$ & $2.45 \mathrm{E}+00$ & AZ101-19 & $\bar{Y}$ & \\
\hline Braun & Cs137 & $8.40 \bar{E}+10$ & $7.11 E+10$ & $1.92 \mathrm{E}+00$ & NA & $\mathrm{N}$ & Braun data, not used \\
\hline RAW & Cs137 & $8.40 E+10$ & $7.54 \mathrm{E}+10$ & $2.04 \mathrm{E}+00$ & $A Z 101-22$ & $\bar{Y}$ & \\
\hline RAW & Cs137 & $8.40 E+10$ & $7.18 \mathrm{E}+10$ & $1.94 E+00$ & $A Z 101-16$ & $\bar{Y}$ & \\
\hline RAW & Cs137 & $8.14 E+10$ & $6.66 \mathrm{E}+10$ & $1.80 \mathrm{E}+00$ & AZ101-9 & $N$ & Duplicate \\
\hline Braun & Cs137 & $7.81 E+10$ & $6.43 E+10$ & $1.74 \mathrm{E}+00$ & NA & $\mathbf{N}$ & Braun data, not used \\
\hline RAW & Cs137 & $7.81 E+10$ & $7.01 E+10$ & $1.89 E+00$ & A2101-21 & $Y$ & \\
\hline RAW & Csi37 & $7.81 E+10$ & $6.51 E+10$ & $1.76 E+00$ & A2101-12 & $Y$ & \\
\hline 3raun & $\operatorname{Cs} 137$ & $7.55 E+10$ & $6.68 \mathrm{E}+10$ & $1.81 E+00$ & NA & $N$ & Braun data, not used \\
\hline 'astren & Cs137 & $7.55 E+10$ & $6.61 E+10$ & $1.79 E+00$ & NA & $\mathbf{N}$ & $\begin{array}{l}\text { from? sample date is different, Wastren data, not } \\
\text { used }\end{array}$ \\
\hline RAW & Csi37 & $7.55 E+10$ & $7.14 E+10$ & $1.93 E+00$ & $A Z 101-25$ & $\bar{Y}$ & Data from AZ101-22 \\
\hline 3raun & Cs137 & $4.92 E+10$ & $3.97 E+10$ & $1.07 E+00$ & NA & $\mathbf{N}$ & Braun data, not used \\
\hline ZAW & Cs137 & $4.92 \mathrm{E}+10$ & $3.97 E+10$ & $1.07 \mathrm{E}+00$ & AZ101-7 & $Y$ & \\
\hline RAW & Cs 137 & $4.77 E+10$ & $3.85 E+10$ & $1.04 E+00$ & AZ101-7 & $\mathbf{N}$ & Aliquots diluted - are A, B corrected for this dilution? \\
\hline ZAW & Cs137 & $4.59 E+10$ & $3.70 E+10$ & $1.00 E+00$ & AZ101-7 & $\mathbf{N}$ & Aliquots diluted - are A, B corrected for this dilution? \\
\hline iraun & Cs137 & $4.03 E+10$ & 3.27E+10 & $8.84 E-01$ & NA & $\mathbf{N}$ & Braun data, not used \\
\hline ZAW & Cs137 & $4.03 E+10$ & $3.62 E+10$ & 9.78E-01 & AZ101-21 & $\mathbf{Y}$ & \\
\hline
\end{tabular}


TWRS Privatization Support Pre Radionuclide Liqu

\begin{tabular}{|c|c|c|c|c|c|c|c|c|c|c|}
\hline \multicolumn{11}{|c|}{ Tank Waste Characterization Sample Information } \\
\hline Reported Radionuclide & $\begin{array}{c}\text { Memo } \\
\text { Date }\end{array}$ & $\begin{array}{c}\text { Start } \\
\text { Analysis Date }\end{array}$ & $\begin{array}{c}\text { Start } \\
\text { Sample } \\
\text { Date }\end{array}$ & Sample Number & $\begin{array}{c}\text { Tank Farm } \\
\text { Number }\end{array}$ & $\begin{array}{c}\text { Lab Sample } \\
\text { Number }\end{array}$ & Density $(\mathrm{g} / \mathrm{mL})$ & Reported Value & Reported Unit & Co. \\
\hline Cs137 & $1 / 10 / 86$ & NA & $11 / 1 / 85$ & NA & NA & NA & NA & 1090000 & $\mu \mathrm{Ci} / \mathrm{L}$ & 4 \\
\hline Cs137 & $11 / 18 / 85$ & NS & NS & RAT-AZ101-1A & NS & NS & NS & 595000 & uCi/ & $\mathbf{L}$ \\
\hline Cs137 & $2 / 5 / 90$ & NA & $6 / 1 / 84$ & $R 100984$ & $\overline{N A}$ & NA & NA & 360000 & uCi/L & $\bar{p}$ \\
\hline Cs137 & $6 / 1 / 84$ & NS & NS & RAT-AZ101-2A & NS & NS & NS & 360000 & $u \mathrm{Cin}$ & $i$ \\
\hline Cs137 & $6 / 11 / 84$ & NS & NS & RAT-AZ101-2B & NS & NS & NS & 350000 & uCin & $\bar{L}$ \\
\hline Cs137 & $9 / 10 / 84$ & NA & $6 / 1 / 84$ & NA & $\overline{\mathrm{NA}}$ & $\overline{N A}$ & NA & 350000 & $\mathrm{UCi} / \mathrm{L}$ & $\bar{p}$ \\
\hline Cs 137 & $12 / 28 / 83$ & NS & NS & R281283 & NS & NS & NS & 234000 & $u \mathrm{Ci} / \mathrm{L}$ & $P$ \\
\hline Cs137 & $8 / 27 / 81$ & NS & NS & NS & NS & NS & 1.143 & 129000 & uCin & $F$ \\
\hline Cs137 & $1 / 10 / 86$ & NA & $11 / 1 / 85$ & NA & $\mathrm{NA}$ & NA & NA & 41000 & $\mathrm{uCi}$ & L \\
\hline Cs137 & $9 / 10 / 84$ & NA & $6 / 1 / 84$ & NA & NA & NA & NA & 360000 & $\mathrm{uCi} / \mathrm{L}$ & $\underline{P}$ \\
\hline Cs137 & $1 / 10 / 86$ & NA & $11 / 1 / 85$ & NA & NA & NA & NA & 14800 & uCi/L & $\mathrm{L}$ \\
\hline Cs-137 & $9 / 1 / 93$ & NA & $4 / 1 / 89$ & Core 2 & NA & NA & 1.22 & 2540 & $\mathrm{uCi} / \mathrm{g}$ & $\bar{p}$ \\
\hline Cs-137 & $9 / 1 / 93$ & NA & $4 / 1 / 89$ & Core 2 & NA & NA & $(1.2)$ & 1930 & $\mathrm{uCi} / \mathrm{g}$ & $p$ \\
\hline Cs-137 & $9 / 1 / 93$ & NA & $4 / 1 / 89$ & Core 2 & NA & NA & $(1.2)$ & 1930 & uCi/g & $\bar{P}$ \\
\hline Cs-137 & $9 / 1 / 93$ & $\mathrm{NA}$ & $4 / 7 / 89$ & Core 2 & NA & NA & $(1.2)$ & 996 & $\mathrm{uCi} / \mathrm{g}$ & $\vec{p}$ \\
\hline Cs-137 & $9 / 1 / 93$ & NA & $4 / 1189$ & Core 2 & NA & NA & $(1.2)$ & 899 & $\mathrm{uCi} / \mathrm{g}$ & $\bar{p}$ \\
\hline Cs-137 & $9 / 1 / 93$ & NA & $4 / 1 / 89$ & Core 2 & NA & NA & 1.14 & 363 & $\mathrm{uCi} / \mathrm{g}$ & $\bar{p}$ \\
\hline Cs137 & $4 / 26 / 78$ & NS & NS & NS & NS & NS & NS & 119 & UCi/L & $\tau$ \\
\hline Eu154 & $7 / 30 / 92$ & NA & $4 / 1 / 89$ & Core 1 & NA & NA & $\overline{N A}$ & 6600 & uCirl & $\overline{\mathrm{p}}$ \\
\hline Eu154 & $4 / 26 / 78$ & NS & NS & NS & NS & NS & NS & 3.5 & $\mathrm{uCi} / \mathrm{L}$ & L \\
\hline $1-129$ & 9/1/93 & NA & $4 / 1 / 89$ & Core 2 & NA & NA & 1.14 & $<0.00000040$ & $\mathrm{uCi} / \mathrm{g}$ & $P$ \\
\hline $1-129$ & $9 / 7 / 93$ & NA & $4 / 1 / 89$ & Core 2 & NA & NA & 1.22 & $<0.00000063$ & uCi/g & $\bar{p}$ \\
\hline 1.129 & $9 / 1 / 89$ & NS & NS & RAT-AZ101-4A & NS & NS & 1.2 & 0.0006 & $\mathrm{uCi} / \mathrm{gm}$ & P \\
\hline 1.129 & $7 / 30 / 92$ & NA & $4 / 1 / 89$ & Core 1 & NA & NA & NA & 0.72 & uCi/L & $\bar{F}$ \\
\hline 1129 & $1 / 1 / 81$ & NS & NS & NS & NS & NS & NS & 50.52023697 & $u g / L$ & $\underline{L}$ \\
\hline $\mathrm{Np}-237$ & $9 / 1 / 93$ & NA & $4 / 1 / 89$ & Core 2 & NA & NA & 1.14 & $<0.00000041$ & $\mathrm{uCi} / \mathrm{g}$ & $p$ \\
\hline $\mathrm{Np}-237$ & $9 / 1 / 93$ & NA & $4 / 1 / 89$ & Core 2 & NA & NA & 1.22 & $<0.00000069$ & $\mathrm{uCi} / 9$ & $\vec{P}$ \\
\hline Np-237 & $7 / 30 / 92$ & NA & $4 / 1 / 89$ & Core 1 & NA & NA & NA & $<0.0708$ & UCi/L & $\bar{P}$ \\
\hline $\mathrm{Np}-237$ & $1 / 10 / 86$ & NA & $11 / 1 / 85$ & NA & NA & NA & NA & 26800 & uCi/L & L \\
\hline Putonium-238 & $7 / 26 / 95$ & NA & $3 / 3 / 95$ & BOD9P6 & NA & NA & 1.19 & $<2.94 E-05$ & $\mathrm{uCi} / \mathrm{mL}$ & $\bar{L}$ \\
\hline Plutonium-238 & $7 / 26 / 95$ & NA & $3 / 3 / 95$ & BOD9P7 & NA & NA & 9.18 & $<3.54 \mathrm{E}-05$ & $\mathrm{uCi} / \mathrm{mL}$ & $\bar{L}$ \\
\hline Plutonium-238 & $7 / 26 / 95$ & NA & $3 / 3 / 95$ & BODSP9 & NA & NA & 1.20 & $<6.11 \mathrm{E}-05$ & $\mathrm{uCi} / \mathrm{mL}$ & $I$ \\
\hline Pu238 & $7 / 30 / 92$ & NA & $4 / 1 / 89$ & Core 1 & NA & NA & NA & $<0.0792$ & uCi/L & $\mathbf{P}$ \\
\hline Pu238 & NS & NS & NS & NS & NS & NS & NS & NS & NS & L \\
\hline Pu238 & $9 / 1 / 89$ & NS & NS & RAT-AZ101-4A & NS & NS & 1.2 & 0.000066 & $\mathrm{uCi} / \mathrm{gm}$ & $\overline{\mathbf{L}}$ \\
\hline Pu238 & $9 / 1 / 89$ & NS & NS & RAT-AZ101-4A & NS & NS & 1.2 & 0.000066 & uCi/gm & $\bar{P}$ \\
\hline Pu238 & $4 / 1 / 89$ & NS & NS & NS & NS & NS & NS & NS & NS & $P$ \\
\hline Plutonium-239/240 & $7 / 26 / 95$ & NA & $3 / 3 / 95$ & BOD9P6 & NA & NA & 1.19 & $<2.94 E-05$ & $\mathrm{uCi} / \mathrm{mL}$ & $L$ \\
\hline Plutonium-239/240 & $7 / 26 / 95$ & NA & $3 / 3 / 95$ & $8009 P 7$ & NA & $\overline{N A}$ & 1.18 & $<3.54 \mathrm{E}-05$ & $\mathrm{uCi} / \mathrm{mL}$ & $\bar{L}$ \\
\hline Plutonium-239/240 & $7 / 26 / 95$ & NA & $3 / 3 / 95$ & B0D9P9 & NA & NA & 1.20 & $<6.11 E-05$ & $\mathrm{uCi} / \mathrm{mL}$ & $\bar{L}$ \\
\hline Pu & $9 / 10 / 84$ & NA & $6 / 1 / 84$ & NA & NA & NA & NA & 9.44 & $\mathrm{uCi} / \mathrm{L}$ & $\bar{p}$ \\
\hline Pu & $6 / 1 / 84$ & NS & NS & RAT-AZ101-2A & NS & NS & NS & 9.44 & uCin & $\mathbf{L}$ \\
\hline PU & $9 / 10 / 84$ & NA & $6 / 1 / 84$ & NA & $\mathrm{NA}$ & NA & NA & 8.28 & $\mathrm{uCi} / \mathrm{L}$ & $\bar{P}$ \\
\hline Pu & $6 / 1 / 84$ & NS & NS & RAT-AZ101-2B & NS & NS & NS & 8.28 & uCin & $\mathbf{L}$ \\
\hline Pu-239/240 & $9 / 1 / 89$ & NS & NS & RAT-AZ101-4A & NS & NS & 1.2 & 0.00043 & uCilgm & $L$ \\
\hline Pu-239 & $9 / 1 / 89$ & NS & NS & RAT-AZ101-4A & NS & NS & 1.2 & 0.00034 & uCi/gm & L \\
\hline Py-239 & $7 / 30 / 92$ & NA & $4 / 1 / 89$ & NA & NA & NA & NA & 0.408 & uCi/L & $\bar{P}$ \\
\hline Pu-239/240 & $7 / 30 / 92$ & NA & $4 / 7 / 89$ & Core 1 & NA & NA & NA & 0.113 & uCi/L & $\bar{P}$ \\
\hline Pu239/240 & $12 / 4 / 86$ & $3 / 20 / 86$ & $11 / 1 / 85$ & $\mathrm{LT}$ & NA & NA & 9.24 & $1.00 \mathrm{E}-05$ & $\mathrm{uCi} / \mathrm{mL}$ & $\bar{P}$ \\
\hline PU-239/240 & $9 / 29 / 89$ & NA & $4 / 1 / 89$ & Core 1 & NA & NA & 1.2 & 0.00043 & $u \mathrm{Ci} / \mathrm{g}$ & $\bar{p}$ \\
\hline Pu241 & NS & N5 & NS & NS & NS & NS & NS & NS & NS & 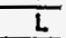 \\
\hline Pu241 & $9 / 1 / 89$ & NS & NS & RAT-AZ101-4A & NS & NS & 1.2 & 0.0036 & $\mathrm{uCi} / \mathrm{gm}$ & $\bar{L}$ \\
\hline
\end{tabular}




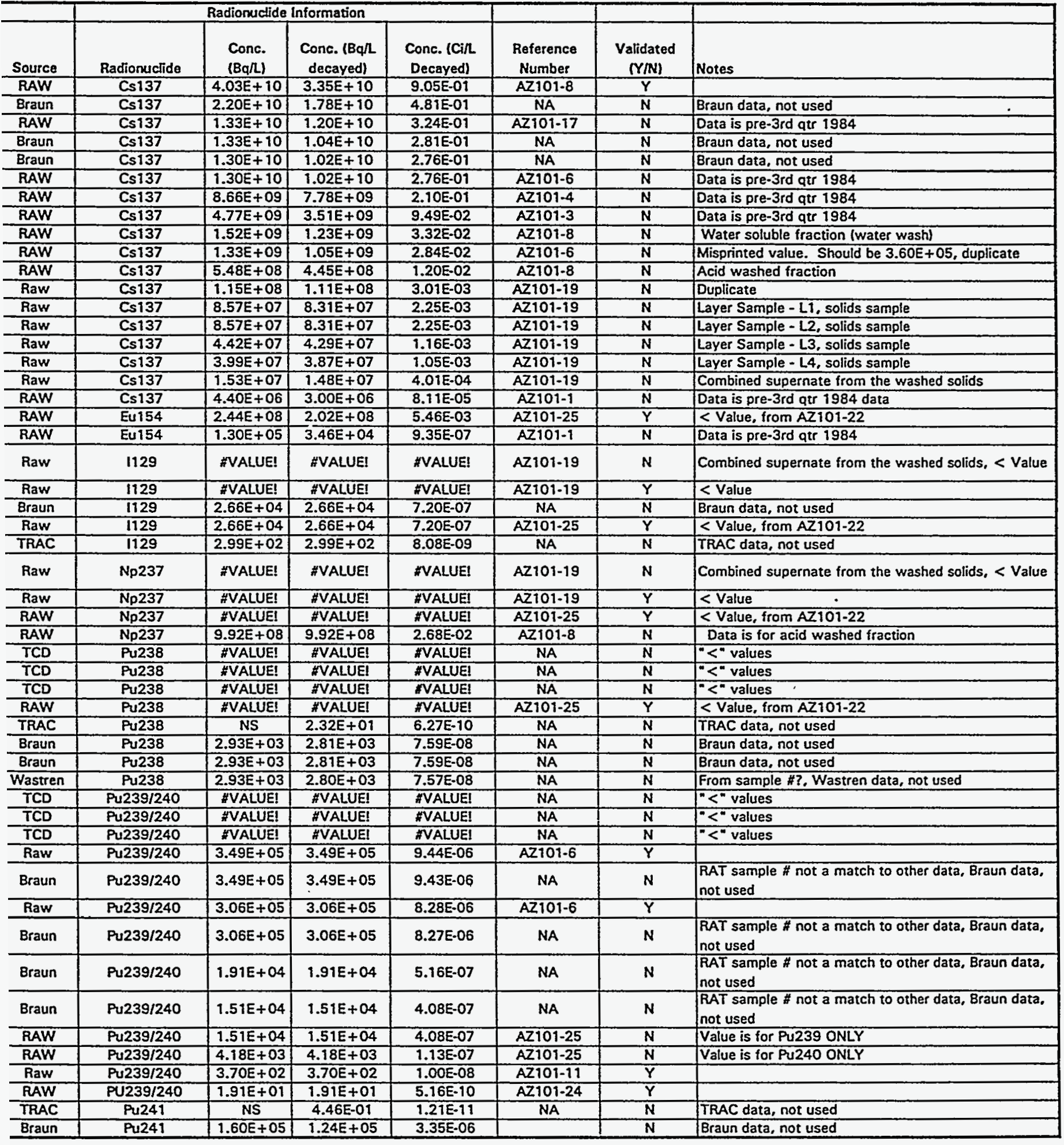


TWRS Privatization Support Prc Radionuclide Liqui

\begin{tabular}{|c|c|c|c|c|c|c|c|c|c|c|}
\hline & & & & Tank Waste Char & cterization S & Sample Inform & ation & & & \\
\hline Reported Radionuclide & $\begin{array}{c}\text { Merno } \\
\text { Date }\end{array}$ & $\begin{array}{c}\text { Start } \\
\text { Analysis Date }\end{array}$ & $\begin{array}{c}\text { Start } \\
\text { Sample } \\
\text { Date }\end{array}$ & Sample Number & $\begin{array}{c}\text { Tank Farm } \\
\text { Number }\end{array}$ & $\begin{array}{c}\text { Lab Sample } \\
\text { Number }\end{array}$ & Density $(\mathrm{g} / \mathrm{mL})$ & Reported Value & Reported Unit & Cor \\
\hline Pu241 & $4 / 1 / 89$ & NS & NS & NS & NS & NS & NS & NS & NS & $p$ \\
\hline Pu241 & $9 / 29 / 89$ & $\overline{N A}$ & $4 / 1 / 89$ & Core 1 & NA & NA & NA & 0.0036 & $u C i / L$ & $\bar{p}$ \\
\hline $\mathrm{Sb}-125$ & $4 / 8 / 86$ & $\overline{\mathrm{NA}}$ & $11 / 1 / 85$ & NA & NA & NA & 1.17 & 120000 & uCi/l & L \\
\hline$S b-125$ & $1 / 10 / 86$ & NA & $11 / 1 / 85$ & NA & NA & NA & NA & 28800 & uCi/L & $\bar{s}$ \\
\hline$S b-125$ & $7 / 30 / 92$ & NA & $4 / 1 / 89$ & Core 1 & NA & NA & NA & 25200 & $\mathrm{uCi} / \mathrm{L}$ & $P$ \\
\hline $5 \mathrm{~b}-125$ & $4 / 26 \pi 8$ & NS & NS & NS & NS & NS & NS & 2.02 & $u C i / L$ & $\mathrm{~L}$ \\
\hline Se79 & $1 / 1 / 81$ & NS & NS & NS & NS & NS & NS & 2.325650089 & $u g / L$ & $\bar{L}$ \\
\hline Se.79 & $9 / 1 / 93$ & NA & $4 / 1 / 89$ & Core 2 & NA & NA & NA & 0.00215 & uCi/g & $P$ \\
\hline Se-79 & $9 / 1 / 89$ & NS & NS & RAT-AZ101-4A & NS & NS & 1.2 & 0.00021 & $u \mathrm{Ci} / \mathrm{gm}$ & $\bar{P}$ \\
\hline Se-79 & $7 / 30 / 92$ & NA & $4 / 1 / 89$ & Core 1 & NA & NA & NA & 0.252 & $\mathrm{uCi} / \mathrm{L}$ & $\bar{p}$ \\
\hline Se-79 & 9/1/93 & NA & $4 / 1 / 89$ & Core 2 & NA & NA & 1.22 & 0.00215 & $\mathrm{uCi} / \mathrm{g}$ & $\bar{P}$ \\
\hline Se-79 & $9 / 1 / 93$ & NA & $4 / 1 / 89$ & Core 2 & NA & NA & 1.14 & 0.000332 & $u C i / g$ & $\bar{p}$ \\
\hline Sn126 & $1 / 1 / 81$ & NS & NS & NS & NS & NS & NS & 82.79982078 & ug/L & $\bar{L}$ \\
\hline Sr90 & NS & NS & NS & NS & NS & NS & NS & NS & NS & $t$ \\
\hline $\mathrm{Sr}$ & $\overline{N A}$ & NA & $\overline{N A}$ & $\mathrm{NA}$ & NA & NA & 1.12432389 & 0.006582357 & $\mathrm{Cin}$ & $\bar{L}$ \\
\hline Sr90 & $911 / 89$ & NS & NS & RAT-AZ101-4A & NS & NS & 1.2 & 4.8 & $\mathrm{uCi} / \mathrm{gm}$ & $\bar{L}$ \\
\hline Sr89/90 & $4 / 1 / 89$ & NS & NS & NS & NS & NS & NS & NS & NS & $\mathbf{P}$ \\
\hline Sr90 & $7 / 30 / 92$ & NA & $4 / 1 / 89$ & Core 1 & NA & NA & NA & 5760 & $u \mathrm{Ci} / \mathrm{L}$ & $P$ \\
\hline Sr90 & $9 / 13 / 85$ & NA & NA & R130985 & NA & NA & NA & 2110 & $\mathrm{UC \textrm {C } / \mathrm { L }}$ & $\mathbf{P}$ \\
\hline Sr90 & $9 / 13 / 85$ & NS & NS & RAT-AZ101-3A & NS & NS & 1.17 & 2110 & $\mathrm{uCin}$ & $\bar{L}$ \\
\hline Sr90 & $9 / 13 / 85$ & $\overline{\mathrm{NA}}$ & NA & NA & NA & $\overline{N A}$ & 1.17 & 2110 & $\mathrm{uCi} / \mathrm{L}$ & $P$ \\
\hline Sr90 & $11 / 18 / 85$ & NS & NS & RAT-AZ107-1A & NS & NS & NS & 2000 & uCin & $\bar{L}$ \\
\hline Sr90 & $3 / 11 / 88$ & NA & NA & T3494 & NA & NA & NA & 1660 & UCi/L & P \\
\hline Sr89/90 & $10 / 1 / 87$ & NS & NS & RAT-AZ101-6A & NS & NS & NS & 1660 & $u \mathrm{Cin}$ & 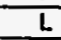 \\
\hline Sr90 & $10 / 30 / 90$ & NA & $5 / 2 / 90$ & 8110388 & NA & NA & NA & 1660 & $\mathrm{UCi} / \mathrm{L}$ & $P$ \\
\hline Sr9o & $2 / 8 / 89$ & NA & $10 / 1 / 87$ & $T 3493$ & NA & NA & NA & 1660 & uCin & $\bar{p}$ \\
\hline Strontium-90 & $7 / 26 / 95$ & $\overline{N A}$ & $3 / 3 / 95$ & BOD9P6 & NA & NA & 1.19 & 1.23 & $\mathrm{uCi} / \mathrm{mL}$ & $L$ \\
\hline Strontium-90 & $7 / 26 / 95$ & NA & $3 / 3 / 95$ & BOD9P7 & NA & NA & 1.18 & 1.18 & $\mathrm{uCi} / \mathrm{mL}$ & $\mathrm{L}$ \\
\hline Strontium-90 & $7 / 26 / 95$ & NA & $3 / 3 / 95$ & BOD9P9 & $\overline{N A}$ & $\overline{\text { NA }}$ & 1.20 & 1.18 & $\mathrm{uCi} / \mathrm{mL}$ & $\mathrm{L}$ \\
\hline Sr90 & $1 / 10 / 86$ & $\mathrm{NA}$ & $11 / 1 / 85$ & $N A$ & $\overline{N A}$ & NA & NA & 1160 & $\mathrm{uCi} / \mathrm{L}$ & $\mathbf{L}$ \\
\hline Sr90 & $10 / 30 / 90$ & $\overline{N A}$ & $5 / 2 / 90$ & $\mathrm{Ri01283}$ & NA & NA & NA & 206 & uCi/L & $\bar{P}$ \\
\hline Sr90 & $1 / 10 / 86$ & NA & $11 / 1 / 85$ & NA & NA & NA & NA & 203 & uCi/L & $\mathrm{L}$ \\
\hline Sr90 & $1 / 10 / 86$ & NA & $11 / 1 / 85$ & NA & NA & NA & NA & 203 & UCi/L & $P$ \\
\hline Sr90 & $4 / 8 / 86$ & $\mathrm{NA}$ & $11 / 1 / 85$ & NA & NA & NA & 1.17 & 200 & $\mathrm{UCi} / \mathrm{L}$ & $L$ \\
\hline Sr9o & $10 / 29 / 90$ & NA & $5 / 2 / 90$ & R100984 & NA & NA & NA & 178 & uCi/L & $P$ \\
\hline Sr90 & $9 / 10 / 84$ & NA & $6 / 1 / 84$ & NA & NA & NA & NA & 178 & $\mathrm{UCi} / \mathrm{L}$ & $P$ \\
\hline Si90 & $6 / 1 / 84^{\circ}$ & NS & NS & RAT-AZ101-2A & NS & NS & NS & 178 & $\mathrm{uCi} /$ & $\mathrm{L}$ \\
\hline Sr90 & $9 / 10 / 84$ & NA & $6 / 1 / 84$ & NA & $\mathrm{NA}$ & NA & NA & 169 & $\mathrm{uCi} / \mathrm{L}$ & $P$ \\
\hline Sr90 & $6 / 1 / 84$ & NS & NS & RAT-AZ101-2B & NS & NS & NS & 169 & $\mathrm{uCi} / 1$ & $L$ \\
\hline Sr90 & $8 / 27 / 81$ & NS & NS & NS & NS & NS & 1.143 & 3.57 & uCill & $F$ \\
\hline Sr90 & $12 / 28 / 83$ & NS & NS & $\mathrm{R} 281283$ & NS & NS & NA & 1.11 & $\mathrm{uCi} / \mathrm{L}$ & $p$ \\
\hline Sr.90 & $9 / 1 / 93$ & NA & $4 / 1 / 89$ & Core 2 & NA & NA & 1.22 & 3.47 & $\mathrm{uCi} / \mathrm{g}$ & $\bar{P}$ \\
\hline Sr-90 & $9 / 1 / 93$ & NA & $4 / 1 / 89$ & Core 2 & NA & NA & 1.14 & 1.58 & uCi/g & $P$ \\
\hline TC-99 & $9 / 10 / 87$ & NA & $\overline{\mathrm{NA}}$ & 1 & $\overline{N A}$ & NA & NA & 12000 & $\mathrm{mCi} / \mathrm{L}$ & $\mathrm{P}$ \\
\hline TC-99 & $9 / 10 / 87$ & NA & NA & 2 & NA & NA & NA & 6380 & $\mathrm{mCi} / \mathrm{L}$ & $\mathrm{P}$ \\
\hline TC-99 & $9 / 10 / 87$ & NA & NA & 3 & $\overline{N A}$ & NA & NA & 6380 & $\mathrm{mCi} / \mathrm{L}$ & $\bar{P}$ \\
\hline Tc-99 & $4 / 8 / 86$ & NA & $11 / 1 / 85$ & NA & NA & NA & 1.17 & 12000 & UCi/L & $L$ \\
\hline Tc-99 & $1 / 10 / 86$ & NA & $11 / 1 / 85$ & NA & NA & $\mathrm{NA}$ & NA & 6380 & $\mathrm{UCi} / \mathrm{L}$ & $L$ \\
\hline Tc-99 & $3 / 11 / 88$ & NA & NA & $T 3494$ & NA & NA & NA & 5300 & $\mathrm{UCi} / \mathrm{L}$ & $\mathrm{L}$ \\
\hline Tc-99 & $9 / 13 / 85$ & NS & NS & RAT-AZ101-3A & NS & NS & 1.17 & 3680 & uCin & $\bar{P}$ \\
\hline TC-99 & $9 / 13 / 85$ & $\overline{N A}$ & $\mathrm{NA}$ & $\overline{N A}$ & NA & $\overline{N A}$ & 1.17 & 3680 & $u C i / L$ & $\bar{P}$ \\
\hline$T C-99$ & $4 / 8 / 86$ & $\overline{N A}$ & $911 / 1 / 85$ & $\overline{N A}$ & NA & $\overline{N A}$ & 1.17 & 2000 & $\mathrm{UCi}$ & $P$ \\
\hline Tc.99 & $11 / 18 / 85$ & NS & NS & RAT-AZ101-1A & NS & NS & NS & 1950 & $4 \mathrm{Cin}$ & $P$ \\
\hline$T c-99$ & $1 / 10 / 86$ & $\overline{\mathrm{NA}}$ & $11 / 1 / 85$ & $\overline{\mathrm{NA}}$ & $\overline{\mathrm{NA}}$ & NA & $\overline{\text { NA }}$ & 1950 & UCi/L & $P$ \\
\hline TC-99 & $3 / 11 / 88$ & NA & NA & T3494 & $\overline{\mathrm{NA}}$ & NA & NA & 402 & UCi/L & $L$ \\
\hline Tc-99 & $10 / 1 / 87$ & NS & NS & RAT-AZ101-6A & NS & NS & NS & 402 & $4 \mathrm{Cin}$ & $\bar{P}$ \\
\hline Tc-99 & $2 / 8 / 89$ & NA & $10 / 1 / 87$ & $T 3493$ & NA & $\overline{N A}$ & NA & 402 & $\mathrm{uCi} / \mathrm{L}$ & L \\
\hline
\end{tabular}




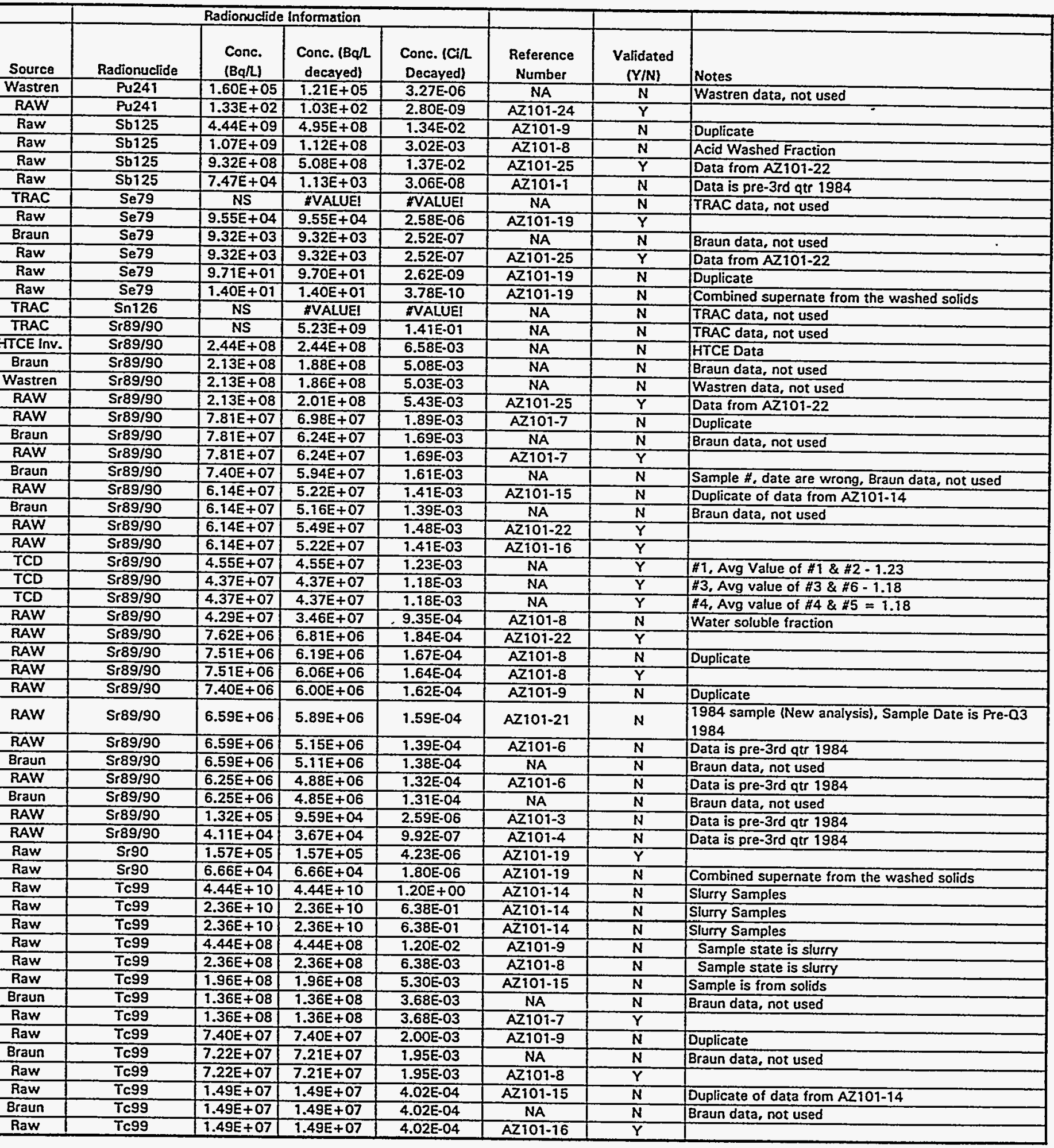




\begin{tabular}{|c|c|c|c|c|c|c|c|c|c|c|}
\hline & & & & Tank Waste Chat & cterization S & ample Inform & tation & & & \\
\hline Reported Radionuclide & $\begin{array}{l}\text { Memo } \\
\text { Date } \\
\end{array}$ & $\begin{array}{c}\text { Start } \\
\text { Analysis Date }\end{array}$ & $\begin{array}{c}\text { Start } \\
\text { Sample } \\
\text { Date }\end{array}$ & Sample Number & $\begin{array}{c}\text { Tank Farm } \\
\text { Number }\end{array}$ & $\begin{array}{c}\text { Lab Sample } \\
\text { Number }\end{array}$ & Density $(\mathrm{g} / \mathrm{mL})$ & Reported Value & Reported Unit & Cod \\
\hline TC-99 & $9 / 1 / 89$ & NS & NS & RAT-AZ101-4A & NS & NS & 1.2 & 0.32 & uCi/gm & $\mathrm{P}$ \\
\hline Tc-99 & $7 / 30 / 92$ & NA & $4 / 1 / 89$ & Core 1 & $\mathrm{NA}$ & NA & NA & 384 & UCi/L & $\mathbf{P}$ \\
\hline Tc99 & $4 / 1 / 89$ & NS & NS & NS & NS & NS & NS & 14200000 & $B q / L$ & $P$ \\
\hline Tc-99 & $9 / 1 / 93$ & NA & $4 / 1 / 89$ & Core 2 & NA & $\overline{N A}$ & $\overline{N A}$ & 0.271 & $\mathrm{uCi} / \mathrm{g}$ & $P$ \\
\hline Tc-99 & $3 / 11 / 88$ & NA & $\overline{N A}$ & $T 3494$ & NA & NA & $\overline{\mathrm{NA}}$ & 32 & uCi/L & $\underline{L}$ \\
\hline Te99 & $1 / 1 / 81$ & NS & NS & NS & NS & NS & NS & 335.1421317 & $u g / L$ & L \\
\hline Tc-99 & $9 / 1 / 93$ & NA & $4 / 1 / 89$ & Core 2 & NA & $\overline{N A}$ & 1.22 & 0.271 & $\mathrm{uCi} / \mathrm{g}$ & $P$ \\
\hline Tc-99 & $9 / 1 / 93$ & NA & $4 / 1 / 89$ & Core 2 & NA & NA & 1.14 & 0.0464 & uCi/g & $P$ \\
\hline Y90 & NS & NS & NS & NS & NS & NS & NS & NS & NS & $t$ \\
\hline
\end{tabular}




\begin{tabular}{|c|c|c|c|c|c|c|c|}
\hline \multirow[b]{2}{*}{ Source } & \multicolumn{4}{|c|}{ Radionuclide Information } & \multirow[b]{2}{*}{$\begin{array}{c}\text { Reference } \\
\text { Number }\end{array}$} & \multirow[b]{2}{*}{$\begin{array}{l}\text { Validated } \\
\text { (Y/N) }\end{array}$} & \multirow[b]{2}{*}{ Notes } \\
\hline & Radionuclide & $\begin{array}{l}\text { Conc. } \\
\text { (Bq/L) }\end{array}$ & $\begin{array}{c}\text { Conc. (Bq/L } \\
\text { decayed) }\end{array}$ & $\begin{array}{l}\text { Conc. (Ci/L. } \\
\text { Decayed) }\end{array}$ & & & \\
\hline Braun & Tc99 & $1.42 E+07$ & $1.42 E+07$ & $3.84 \mathrm{E}-04$ & NA & $\mathbf{N}$ & Braun data, not used \\
\hline Raw & Tc99 & $1.42 E+07$ & $1.42 E+07$ & $3.84 \mathrm{E} \cdot 04$ & AZ101-25 & $\bar{Y}$ & Data from AZ101-22 \\
\hline Wastren & Te99 & $1.42 E+07$ & $1.42 E+07$ & $3.84 E-04$ & NA & $\mathbf{N}$ & $\begin{array}{l}\text { No date, no sample \# (Duplicate of Raw data??). } \\
\text { Wastren data, not used }\end{array}$ \\
\hline Raw & Tc99 & $1.20 \varepsilon+07$ & $1.20 E+07$ & $3.25 E-04$ & AZ101-19 & $\bar{Y}$ & \\
\hline Raw & Tc99 & $1.18 E+06$ & $1.18 E+06$ & $3.20 E-05$ & AZ101-15 & $\mathbf{N}$ & Acid Wash sample \\
\hline TRAC & Tc99 & $2.11 E+05$ & $2.11 E+05$ & $5.70 E-06$ & NA & $\mathbf{N}$ & TRAC data, not used \\
\hline Raw & Tc99 & $1.22 \mathrm{E}+04$ & $1.22 E+04$ & 3.31E-07 & AZ101-19 & $\mathbf{N}$ & Duplicate \\
\hline Raw & Tc99 & $1.96 \mathrm{E}+03$ & $1.96 \mathrm{E}+03$ & $5.29 E-08$ & AZ101-19 & $\mathbf{N}$ & Combined supernate from the washed solids \\
\hline TRAC & Y90 & NS & $\overline{7.40 E+09}$ & 2.00E-01 & $\overline{N A}$ & $\mathbf{N}$ & TRAC data, not used \\
\hline
\end{tabular}




\begin{tabular}{|c|c|c|c|c|c|c|c|c|c|c|}
\hline \multicolumn{11}{|c|}{ Tank Waste Characterization Sample Information } \\
\hline $\begin{array}{c}\text { Reported } \\
\text { Radionuclide } \\
\end{array}$ & $\begin{array}{c}\text { Memo } \\
\text { Date }\end{array}$ & $\begin{array}{c}\text { Start } \\
\text { Analysis } \\
\text { Date } \\
\end{array}$ & $\begin{array}{c}\text { Start } \\
\text { Sample } \\
\text { Date } \\
\end{array}$ & Sample Number & $\begin{array}{c}\text { Tank Farm } \\
\text { Number }\end{array}$ & $\begin{array}{c}\text { Lab Sampie } \\
\text { Number }\end{array}$ & $\begin{array}{l}\text { Density } \\
\text { (g/mL) }\end{array}$ & $\begin{array}{l}\text { Reported } \\
\text { Value }\end{array}$ & $\begin{array}{l}\text { Reported } \\
\text { Unit }\end{array}$ & Codu \\
\hline Americlum-241 & $3 / 2 / 91$ & N/A & $3 / 2 / 91$ & BOD9R3 & $A Z-102-1$ & S95T000263 & 1.1 & $<4.29 E-03$ & $\mathrm{uCi} / \mathrm{mL}$ & $P-1$ \\
\hline Americium-241 & $3 / 2 / 91$ & N/A & $3 / 2 / 91$ & BOD9R4 & $A Z-102-2$ & S95T000264 & 1.1 & $<4.29 \mathrm{E}-03$ & $\mathrm{uCi} / \mathrm{mL}$ & $\overline{P-1}$ \\
\hline Americium-241 & $3 / 2 / 91$ & N/A & $3 / 2 / 91$ & BOD9R3 & $A Z-102-1$ & S95T000263 & 1.1 & $<4.29 \mathrm{E}-0 \overline{3}$ & $\mathrm{uCi} / \mathrm{mL}$ & $\overline{\mathbf{P}-2}$ \\
\hline Americium-241 & $3 / 2 / 91$ & N/A & $3 / 2 / 91$ & BOD9R4 & AZ-102-2 & $595 T 000264$ & 1.1 & $<4.39 \mathrm{E}-03$ & $\mathrm{uCi} / \mathrm{mL}$ & $\overline{P-2}$ \\
\hline Americium-241 & $3 / 2 / 91$ & N/A & $3 / 2 / 91$ & BOD9R5 & $A Z-102-3$ & 5957000265 & 1.1 & $<4.53 E-03$ & $\mathrm{uCi} / \mathrm{mL}$ & $\overline{P-2}$ \\
\hline Americium-241 & $3 / 2 / 91$ & N/A & $3 / 2 / 91$ & B0D9R5 & $A Z-102-3$ & S95T000265 & 1.1 & $<4.62 \mathrm{E}-03$ & $\mathrm{uCi} / \mathrm{mL}$ & $P=1$ \\
\hline Am241 & NS & NS & NS & NS & NS & NS & NS & NS & NS & $L$ \\
\hline Am241 & $8 / 1 / 85$ & NS & NS & NS & NS & NS & NS & NS & NS & $\mathbf{s}$ \\
\hline Am241 & $8 / 31 / 89$ & NS & $6 / 30 / 85$ & Composite Core & NS & NS & 1.11 & $2.50 E+01$ & uCi/g & EI \\
\hline Am241 & $7 / 6 / 84$ & NS & NS & T3496A & NS & NS & NS & $8.18 E+03$ & uCi/L & $S$ \\
\hline Am241 & $7 / 6 / 84$ & NS & NS & T3496B & NS & NS & NS & $6.71 E+03$ & uCi/L & $\mathbf{S}$ \\
\hline Am241 & $11 / 5 / 81$ & NS & NS & RAT-AZ102-2B & NS & NS & 1.4 & $1.79 E-03$ & $\mathrm{gm} / \mathrm{l}$ & $\mathbf{s}$ \\
\hline Am241 & $5 / 6 / 82$ & NS & NS & NS & NS & NS & NS & $1.79 \mathrm{E}-03$ & $g / L$ & S \\
\hline Am241 & $11 / 5 / 81$ & NS & NS & RAT-AZ102-2C & NS & NS & 1.26 & 1.89E-05 & $\mathrm{gm} / \mathrm{l}$ & s \\
\hline Am241 & $11 / 5 / 81$ & NS & NS & RAT-AZ102-2A & NS & NS & 1.09 & $6.70 E-07$ & $\mathrm{gm} / \mathrm{I}$ & $\mathbf{L}$ \\
\hline Am241 & $5 / 6 / 82$ & NS & NS & NS & NS & NS & 1.09 & $6.70 \mathrm{E}-07$ & $g / L$ & $P$ \\
\hline Am241 & $8 / 1 / 85$ & NS & NS & NS & NS & NS & NS & NS & NS & $\mathbf{P}$ \\
\hline Am241 & $8 / 31 / 89$ & N/A & $6 / 30 / 85$ & Composite Core & NA & $\overline{N A}$ & 7.14 & $1.30 \mathrm{E}-03$ & $\mathrm{uCi} / \mathrm{g}$ & $\mathrm{Cl}$ \\
\hline Am241 & $8 / 31 / 89$ & NS & $6 / 30 / 85$ & Composite Core & NS & NS & $(1.0)$ & $<1.20 \mathrm{E}-03$ & uCi/g & FI \\
\hline Am241 & $5 / 6 / 82$ & NS & NS & NS & NS & NS & 1.26 & $1.89 \mathrm{E}-05$ & $\mathrm{UCi} / \mathrm{L}$ & G \\
\hline C-14 & $8 / 31 / 89$ & N/A & $6 / 30 / 85$ & Composite Core & NA & NA & 1.14 & $1.06 \mathrm{E}-03$ & $\mathrm{uCi} / \mathrm{g}$ & $\mathrm{Cl}$ \\
\hline$C-14$ & $9 / 1 / 89$ & NS & NS & NS & NS & NS & 1.36 & $1.50 \mathrm{E}-01$ & $u \mathrm{Ci} / \mathrm{g}$ & $S$ \\
\hline$C-14$ & $9 / 1 / 89$ & NS & NS & NS & NS & NS & 1.11 & $6.00 \mathrm{E}-02$ & $\mathrm{uCi} / 9$ & $\mathrm{~s}$ \\
\hline$C 14$ & $1 / 1 / 77$ & NS & NS & NS & NS & NS & NS & $3.34 E+00$ & $\mathrm{ug} / \mathrm{L}$ & L \\
\hline $\mathrm{C74}$ & $8 / 1 / 85$ & NS & NS & NS & NS & NS & NS & $1.01 E+05$ & $B q / L$ & s \\
\hline$c-14$ & $9 / 1 / 89$ & NS & NS & NS & NS & NS & 1.49 & $1.83 \mathrm{E}-03$ & uCi/g & s \\
\hline C-14 & $9 / 1 / 89$ & NS & NS & NS & NS & NS & 1.49 & $1.06 \mathrm{E}-03$ & uCi/g & P \\
\hline $\mathrm{C}_{14}$ & $8 / 1 / 85$ & NS & NS & NS & NS & NS & NS & $4.63 E+04$ & $B q \Omega$ & $P$ \\
\hline $\mathrm{Cm} 244$ & NS & NS & NS & NS & NS & NS & NS & NS & NS & $L$ \\
\hline $\mathrm{Cm} 243 / 244$ & $9 / 1 / 89$ & NS & NS & NS & NS & NS & 1.49 & 3.77E-01 & $\mathrm{uCi} / \mathrm{g}$ & $S$ \\
\hline $\mathrm{Cm} 244$ & $8 / 1 / 85$ & NS & NS & NS & NS & NS & NS & NS & NS & $\frac{2}{2}$ \\
\hline Co-60 & $8 / 31 / 89$ & N/A & $6 / 30 / 85$ & Composite Core & NA & NA & 1.14 & $<1.70 \mathrm{e}-01$ & UCi/g & $\mathrm{Cl}$ \\
\hline Co60 & $5 / 3 / 86$ & NS & NS & $L 1$ & NS & NS & NS & $1.90 \mathrm{E}+01$ & uCi/g & 1 \\
\hline Co60 & $5 / 3 / 86$ & NS & NS & $L 4$ & NS & NS & NS & $1.69 E+01$ & $u C i / g$ & 1 \\
\hline Co60 & $5 / 3 / 86$ & NS & - NS & $L 3$ & NS & NS & NS & $1.00 E+00$ & uCi/g & $i$ \\
\hline $\mathrm{Co60}$ & $4 / 26 / 74$ & NS & NS & NS & NS & NS & 1.299 & $2.59 E+02$ & uCi/L & $\bar{L}$ \\
\hline C5137 & NS & NS & NS & NS & NS & NS & NS & NS & NS & $L$ \\
\hline Cs & NS & NS & NS & NS & NS & NS & 1.3927616 & $2.12 E+\infty$ & $\mathrm{Ci} / \mathrm{L}$ & $s$ \\
\hline Cs137 & $5 / 3 / 86$ & NS & NS & L1 & NS & NS & NS & $9.50 E+02$ & uCi/g & $T$ \\
\hline Cs137 & $5 / 3 / 86$ & NS & NS & 14 & NS & NS & NS & $9.26 E+02$ & $\mathrm{uCl} / \mathrm{g}$ & $t$ \\
\hline Cs & NS & NS & NS & NS & NS & NS & 1.03625374 & $1.34 E+00$ & cin & L \\
\hline Cs137 & $8 / 1 / 85$ & NS & NS & NS & NS & NS & NS & NS & NS & s \\
\hline Cs137 & $5 / 3 / 86$ & NS & NS & 23 & NS & NS & NS & $7.49 E+02$ & $\mathrm{uCi} / \mathrm{g}$ & $T$ \\
\hline Cesium-137 & $3 / 2 / 91$ & N/A & $3 / 2 / 91$ & BOD9R5 & $A Z-102-3$ & 595T000265 & 1.1 & $1.06 \mathrm{E}+03$ & $\mathrm{uCi} / \mathrm{mL}$ & $P-1$ \\
\hline Cesium-137 & $3 / 2 / 91$ & N/A & $3 / 2 / 91$ & BOD9R4 & $A Z-102-2$ & S95T000264 & 1.1 & $1.05 E+03$ & $\mathrm{uCi} / \mathrm{mL}$ & $P=1$ \\
\hline Cesium-137 & $3 / 2 / 91$ & N/A & $3 / 2 / 91$ & BOD9R3 & AZ-102-1 & S95T000263 & 7.1 & $1.05 E+03$ & $\mathrm{uCi} / \mathrm{mL}$ & $P-2$ \\
\hline Cesium-137 & $3 / 2 / 91$ & N/A & $3 / 2 / 91$ & BOD9R3 & $A Z-102-1$ & S95T000263 & 1.1 & $1.04 E+03$ & $\mathrm{uCi} / \mathrm{mL}$ & $P-1$ \\
\hline Cesium-137 & $3 / 2 / 91$ & N/A & $3 / 2 / 91$ & BOD9R5 & $A Z-102-3$ & S95T000265 & 1.1 & $1.03 E+03$ & $\mathrm{uCl} / \mathrm{mL}$ & $P-2$ \\
\hline Cesium-137 & $3 / 2 / 91$ & N/A & $3 / 2 / 91$ & BOD9R4 & $A Z-102-2$ & S95T000264 & 1.1 & $1.03 E+03$ & $\mathrm{uCi} / \mathrm{mL}$ & $P-2$ \\
\hline
\end{tabular}




\begin{tabular}{|c|c|c|c|c|c|c|c|}
\hline & \multicolumn{4}{|c|}{ Radionuclide Information } & \multirow[b]{2}{*}{$\begin{array}{l}\text { Reference } \\
\text { Number }\end{array}$} & \multirow[b]{2}{*}{$\begin{array}{l}\text { Validated } \\
(\mathrm{Y} / \mathrm{N})\end{array}$} & \multirow[b]{2}{*}{ Notes } \\
\hline Source & Radionuclide & Conc. $(\mathrm{Bq} / \mathrm{L})$ & $\begin{array}{c}\text { Conc. (Bq/L } \\
\text { decayed) }\end{array}$ & $\begin{array}{l}\text { Conc. (Ci/L } \\
\text { Decayed) }\end{array}$ & & & \\
\hline Raw & $\operatorname{Am} 241$ & IVALUE! & \#VALUEI & \#VALUE! & AZ102-24 & $\mathrm{Y}$ & Updated per memo $8 / 17 / 95$ \\
\hline Raw & Am241 & VALUE! & \#VALUE! & \#VALUE! & AZ102-24 & $Y$ & Updated per memo 8/17/95 \\
\hline Raw & Am241 & \#VALUE! & $\sharp$ VALUE! & \#VALUE! & AZ102-24 & $\bar{Y}$ & Updated per memo $8 / 17 / 95$ \\
\hline Raw & Am241 & \#VALUE! & \#VALUE! & \#VALUE! & AZ102-24 & $Y$ & Updated per memo $8 / 17 / 95$ \\
\hline Raw & Am241 & \#VALUE! & FVALUE! & \#VALUE! & AZ102-24 & $\bar{Y}$ & Updated per memo 8/17/95 \\
\hline Raw & Am241 & \#VALUE! & \#VALUE! & $\#$ \#ALUE! & AZ102-24 & $\bar{Y}$ & Updated per memo $8 / 17 / 95$ \\
\hline IRAC & Am241 & NS & NS & NS & NA & $\mathbf{N}$ & Privitization is not using TRAC data \\
\hline Vastren & Am241 & $5.57 E+09$ & $5.52 E+09$ & $1.49 E-01$ & NA & $\mathbf{N}$ & $\begin{array}{l}\text { Privitization is not using Braun, Wastren or HTCE Inv. } \\
\text { data }\end{array}$ \\
\hline Raw & Am241 & $1.02 E+09$ & $1.01 E+09$ & $2.73 E-02$ & AZ102-23 & N & Solid sample after has been washed with fresh water \\
\hline Raw & Am241 & $3.03 E+08$ & $3.00 E+08$ & 8.11E-03 & A2102-16 & N & Analysis prior to $7 / 89$ \\
\hline Raw & Am241 & $2.48 E+08$ & $2.46 E+08$ & $6.65 \mathrm{E}-03$ & AZ102-16 & $\mathbf{N}$ & Analysis prior to $7 / 89$ \\
\hline Braun & Am241 & $2.15 E+08$ & $2.11 E+08$ & $5.70 E-03$ & NA & $N$ & $\begin{array}{l}\text { Privitization is not using Braun, Wastren or HTCE Inv. } \\
\text { data }\end{array}$ \\
\hline Raw & Am241 & $2.15 E+08$ & $2.12 E+08$ & $5.73 E-03$ & AZ102-11 & $\mathrm{N}$ & Analysis prior to $7 / 89$ \\
\hline Braun & Am241 & $2.27 E+06$ & $2.23 E+06$ & $6.03 E-05$ & NA & N & $\begin{array}{l}\text { Privitization is not using Braun, Wastren or HTCE Inv. } \\
\text { data }\end{array}$ \\
\hline Braun & Am241 & $8.03 E+04$ & $7.92 E+04$ & 2.14E-06 & NA & $\mathbf{N}$ & $\begin{array}{l}\text { Privitization is not using Braun, Wastren or HTCE Inv. } \\
\text { data }\end{array}$ \\
\hline Raw & Am241 & $8.03 E+04$ & $7.92 E+04$ & $2.14 E-06$ & AZ102-11 & $\mathrm{N}$ & Analysis prior to $7 / 89$ \\
\hline Vastren & Am241 & $5.68 E+04$ & $5.63 E+04$ & $1.52 E-06$ & NA & $\mathrm{N}$ & $\begin{array}{l}\text { Privitization is not using Braun, Wastren or HTCE Inv. } \\
\text { data }\end{array}$ \\
\hline Raw & Am241 & $5.48 E+04$ & $5.44 \mathrm{E}+04$ & 1.47E-06 & $A 2102-23$ & $\bar{Y}$ & \\
\hline Raw & Am241 & $<4.40 E+04$ & $<4.36 E+04$ & $<1.18 E-06$ & AZ102-23 & $N$ & Water wash of solid sample not supernatent \\
\hline Raw & Am241 & 6.99E-01 & $6.90 \mathrm{E}-01$ & $1.86 \mathrm{E}-11$ & $A 2102-11$ & $\mathbf{N}$ & Analysis prior to $7 / 89$ \\
\hline Raw & $\mathrm{C}-14$ & $4.43 E+04$ & $4.43 E+04$ & $1.20 \mathrm{E}-06$ & A2102-23 & $\bar{Y}$ & \\
\hline Raw & $\mathrm{C14}$ & $7.55 E+06$ & $7.55 E+06$ & $2.04 E-04$ & AZ102-23 & $N$ & Not currently of interest to privitization \\
\hline Raw & $\mathrm{C14}$ & $2.46 \mathrm{E}+06$ & $2.46 E+06$ & 6.66E-05 & AZ102-23 & $N$ & Not currently of interest to privitization \\
\hline TRAC & C14 & $5.51 E+05$ & $5.50 E+05$ & $1.49 \mathrm{E}-05$ & NA & $N$ & Not currently of interest to privitization \\
\hline Vastren & C14 & $1.01 E+05$ & $1.01 E+05$ & $2.73 E-06$ & NA & $N$ & Not currently of interest to privitization \\
\hline Raw & C14 & $1.01 E+05$ & $1.01 E+05$ & 2.73E-06 & AZ102-23 & $\mathbf{N}$ & Not currently of interest to privitization \\
\hline Raw & $\mathrm{C14}$ & $5.84 E+04$ & $5.84 E \div 04$ & $1.58 \mathrm{E}-06$ & AZ102-23 & $\mathbf{N}$ & Not currently of interest to privitization \\
\hline Vastren & C14 & $4.63 E+04$ & $4.63 E+04$ & $1.25 \mathrm{E}-06$ & NA & $\mathbf{N}$ & Not currently of interest to privitization \\
\hline TRAC & $\mathrm{Cm} 243 / 244$ & NS & NS & NS & NA & $N$ & Not currently of interest to privitization \\
\hline Raw & $\mathrm{Cm} 243 / 244$ & $2.08 \mathrm{E}+07$ & $1.98 E+07$ & $5.35 \mathrm{E}-04$ & AZ102-23 & $N$ & Not currently of interest to privitization \\
\hline Vastren & $\mathrm{Cm} 243 / 244$ & $2.08 \mathrm{E}+07$ & $1.69 \mathrm{E}+07$ & 4.57E-04 & NA & $\mathbf{N}$ & Not currently of interest to privitization \\
\hline Raw & Co-60 & \#VALUE! & \#VALUE! & \#VALUE! & AZ102-23 & $\bar{Y}$ & \\
\hline Raw & Co6o & $1.12 E+09$ & $6.10 E+08$ & $1.65 E-02$ & AZ102-17 & $\mathbf{N}$ & Repeated data from report AZ-102-17 \\
\hline Raw & $\mathrm{Co60}$ & $1.00 E+09$ & $5.43 E+08$ & 1.47E-02 & AZ102-17 & $\mathbf{N}$ & Repeated data from report AZ-102-17 \\
\hline Raw & Co60 & $5.92 E+07$ & $3.21 E+07$ & $8.68 \mathrm{E}-04$ & $A Z 102-17$ & $\bar{N}$ & Repeated data from report AZ-102-17 \\
\hline Raw & $\mathrm{Co60}$ & $9.58 E+06$ & $1.08 E+06$ & 2.92E-05 & AZ102-1 & $\mathbf{N}$ & Analysis prior to $7 / 89$ \\
\hline TRAC & Cs137 & NS & $3.15 E+07$ & $8.51 E-04$ & NA & $\mathbf{N}$ & Privitization is not using TRAC data \\
\hline TCE lnv. & Cs137 & $7.84 E+10$ & $7.84 E+10$ & $2.12 E+\infty$ & NA & $\mathbf{N}$ & $\begin{array}{l}\text { Privitization is not using Braun, Wastren or HTCE Inv. } \\
\text { data }\end{array}$ \\
\hline Raw & Cs137 & $5.62 \mathrm{E}+10$ & $5.05 E+10$ & $1.36 \mathrm{E}+00$ & AZ102-17 & $\mathbf{N}$ & Repeated data from report AZ-102-17 \\
\hline Raw & Cs137 & $5.48 \mathrm{E}+10$ & $4.92 E+10$ & $1.33 E+00$ & AZ102-17 & $\mathbf{N}$ & Repeated data from report AZ-102-17 \\
\hline "CE Inv. & Cs137 & $4.96 E+10$ & $4.96 E+10$ & $1.34 E+\infty 0$ & NA & $\mathrm{N}$ & $\begin{array}{l}\text { Privitization is not using Braun, Wastren or HTCE Inv. } \\
\text { data }\end{array}$ \\
\hline fastren & Cs137 & $4.85 E+10$ & $4.28 E+10$ & $1.16 \mathrm{E}+00$ & NA & $\mathbf{N}$ & $\begin{array}{l}\text { Privitization is not using Braun, Wastren or HTCE Inv. } \\
\text { data }\end{array}$ \\
\hline Raw & Cs137 & $4.43 \mathrm{E}+10$ & $3.98 E+10$ & $1.08 E+00$ & AZ102-17 & $\bar{N}$ & Repeated data from report AZ-102-17 \\
\hline Raw & Cs137 & $3.92 \mathrm{E}+10$ & $3.94 E+10$ & $1.06 E+00$ & AZ102-24 & $\bar{Y}$ & Updated per memo 8/17/95 \\
\hline Raw & Cs137 & $3.89 E+10$ & $3.90 E+10$ & $1.05 E+00$ & AZ102-24 & $\mathbf{Y}$ & Updated per memo $8 / 17 / 95$ \\
\hline Raw & Cs137 & $3.89 E+10$ & $3.90 E+10$ & $1.05 E+00$ & AZ102-24 & $Y$ & Updated per memo 8/17/95 \\
\hline Raw & Cs137 & $3.85 E+10$ & $3.86 E+10$ & $1.04 E+00$ & AZ102-24 & $\bar{Y}$ & Updated per memo $8 / 17 / 95$ \\
\hline Raw & Cs137 & $3.81 E+10$ & $3.83 E+10$ & $1.03 E+00$ & A2102-24 & $Y$ & Updated per memo $8 / 17 / 95$ \\
\hline Raw & Cs137 & $3.81 E+10$ & $3.83 E+10$ & $1.03 E+\infty 0$ & AZ102-24 & $\bar{Y}$ & Updated per memo $8 / 17 / 95$ \\
\hline
\end{tabular}


TWRS Privatization Support Pro] Radionuclide Llquid

\begin{tabular}{|c|c|c|c|c|c|c|c|c|c|c|}
\hline \multicolumn{11}{|c|}{ Tank Waste Characterization Sample Information } \\
\hline $\begin{array}{c}\text { Reported } \\
\text { Radionuclide }\end{array}$ & $\begin{array}{c}\text { Memo } \\
\text { Date }\end{array}$ & $\begin{array}{c}\text { Start } \\
\text { Analysis } \\
\text { Date }\end{array}$ & $\begin{array}{c}\text { Start } \\
\text { Sample } \\
\text { Date } \\
\end{array}$ & Sample Number & $\begin{array}{c}\text { Tank Farm } \\
\text { Number }\end{array}$ & $\begin{array}{c}\text { Lab Sample } \\
\text { Number }\end{array}$ & $\begin{array}{l}\text { Density } \\
(\mathrm{g} / \mathrm{mL})\end{array}$ & $\begin{array}{c}\text { Reported } \\
\text { Value }\end{array}$ & $\begin{array}{c}\begin{array}{c}\text { Reported } \\
\text { Unit }\end{array} \\
\end{array}$ & Code \\
\hline Cs137 & $10 / 1 / 83$ & NS & NS & RAT-AZ102-3 & NS & NS & NS & $9.36 \mathrm{E}+05$ & uCin & $\mathbf{L}$ \\
\hline Cs137 & $7 / 6 / 84$ & NS & NS & T3495 & NS & NS & NS & $9.36 E+05$ & $\overline{\mathrm{UCi} / \mathrm{L}}$ & $P$ \\
\hline Cs137 & $3 / 11 / 84$ & NS & NS & NS & NS & NS & NS & $9.36 E+05$ & uCill & $P$ \\
\hline Cs137 & $10 / 31 / 86$ & NS & NS & R880311 & NS & NS & NS & $9.36 E+05$ & $u \mathrm{Ci} / \mathrm{L}$ & $P$ \\
\hline Cs 137 & $8 / 1 / 85$ & NS & NS & NS & NS & NS & NS & NS & NS & $P$ \\
\hline Cs137 & $8 / 31 / 89$ & N/A & $6 / 30 / 85$ & Composite Core & NA & NA & 1.14 & $7.47 E+02$ & $u C i / g$ & $\mathrm{Cl}$ \\
\hline Cs137 & $7 / 13 / 84$ & NS & NS & T1520 & NS & NS & NS & $7.35 E+05$ & uCi/L & $P$ \\
\hline Cs137 & $12 / 8 / 83$ & NS & NS & NS & NS & NS & NS & $7.35 E+05$ & $\mathrm{uCi} / \mathrm{L}$ & $\bar{P}$ \\
\hline Cs137 & $10 / 31 / 86$ & NS & NS & R081287 & NS & NS & NS & $7.35 E+05$ & uCiR & $P$ \\
\hline Cs 137 & $8 / 27 / 77$ & NS & NS & RAT-AZ102-4B & NS & NS & 1.39 & $3.75 \mathrm{E}-01$ & $\mathrm{Cin}$ & L \\
\hline Cs137 & $6 / 26 / 75$ & NS & NS & RAT-AZ102-4a & NS & NS & 1.39 & 3.31E-01 & $\mathrm{Ci} / \mathrm{I}$ & $L$ \\
\hline Cs137 & $6 / 26 / 75$ & NS & NS & NS & NS & NS & 1.392 & $3.31 \mathrm{E}-01$ & $\overline{\mathrm{Ci} / \mathrm{L}}$ & $S$ \\
\hline Cs137 & $7 / 28 / 79$ & NS & NS & RAT-AZ102-4D & NS & NS & 1.41 & $3.14 \mathrm{E}-01$ & $\mathrm{Ci} / 1$ & $\mathbf{L}$ \\
\hline Cs137 & $10 / 30 / 86$ & NS & NS & R280783 & NS & NS & NS & $3.14 E+05$ & uCi/L & $\bar{P}$ \\
\hline Cs137 & $7 / 28 / 79$ & NS & NS & NS & NS & NS & 1.41 & $3.14 E+05$ & uCi/L & $P$ \\
\hline Cs137 & $7 / 28 / 79$ & NS & NS & RAT-AZ102-6 & NS & NS & 1.41 & $3.14 E+05$ & $\mathrm{uCi} \lambda$ & s \\
\hline Cs137 & $12 / 3 / 75$ & NS & NS & 1458 & NS & NS & 1.391 & $3.07 E+05$ & uCi/L & $\mathrm{s}$ \\
\hline Cs137 & $12 / 3 / 75$ & NS & NS & 1457 & NS & NS & 1.398 & $3.01 E+05$ & $\mathrm{uCi} / \mathrm{L}$ & s \\
\hline Cs137 & $12 / 3 / 75$ & NS & NS & 1456 & NS & NS & 1.393 & $2.96 E+05$ & uCi/L. & s \\
\hline Cs137 & $9 / 1 / 79$ & NS & NS & NS & NS & NS & NS & $2.54 E+05$ & uCi/L & $\mathbf{G}$ \\
\hline Cs 137 & $9 / 20 / 78$ & NS & NS & RAT-AZ102-4C & NS & NS & 1.39 & $2.20 \mathrm{E}-01$ & $\mathrm{Ci} / \mathrm{I}$ & $L$ \\
\hline Cs137 & $9 / 20 / 78$ & NS & NS & NS & NS & NS & 1.39 & $2.20 E-01$ & $\mathrm{Ci} / \mathrm{L}$ & $\mathrm{s}$ \\
\hline Cs 137 & $8 / 31 / 89$ & NS & $6 / 30 / 85$ & Composite Core & NS & NS & 1.11 & $1.46 \mathrm{E}+02$ & uCi/g & EI \\
\hline Cs137 & $8 / 31 / 89$ & NS & $6 / 30 / 85$ & Composite Core & NS & NS & $(1.0)$ & $1.10 E+02$ & $u \mathrm{Ci} / \mathrm{g}$ & $F I$ \\
\hline Cs 137 & $2 / 4 / 81$ & NS & NS & $R-3699$ & NS & NS & 1.11 & $8.00 E+04$ & $\mathrm{uCi} / \mathrm{I}$ & $L$ \\
\hline Cs 137 & $2 / 4 / 81$ & NS & NS & R-3700 & NS & NS & 1.11 & $7.89 E+04$ & uCin & $L$ \\
\hline Cs137 & $7 / 6 / 84$ & NS & NS & T3496A & NS & NS & NS & $7.54 E+04$ & $\mathrm{uCi} / \mathrm{L}$ & $\mathbf{S}$ \\
\hline Cs137 & $3 / 11 / 84$ & NS & NS & $A$ & NS & NS & 11.9 & $7.54 E+04$ & uCi/L & 1 \\
\hline Cs137 & $7 / 6 / 84$ & NS & NS & T3496B & NS & NS & NS & $6.95 E+04$ & uCi/L & $\mathbf{S}$ \\
\hline Cs137 & $3 / 11 / 84$ & NS & NS & 8 & NS & NS & 12 & $6.95 E+04$ & $\mathrm{uCi} / \mathrm{L}$ & 1 \\
\hline Cs137 & $11 / 5 / 81$ & NS & NS & RAT-AZ102-2B & NS & NS & 1.4 & $5.79 E+04$ & uCin & $\mathbf{s}$ \\
\hline Cs137 & $5 / 6 / 82$ & NS & NS & NS & NS & NS & NS & $5.79 E+04$ & uCin & 5 \\
\hline Cs137 & $11 / 5 / 81$ & NS & NS & RAT-AZ102-2A & NS & NS & 1.09 & $4.87 E+04$ & $\mathrm{uCi} / \Lambda$ & $\mathbf{L}$ \\
\hline Cs137 & $5 / 6 / 82$ & NS & NS & NS & NS & NS & NS & $4.87 E+04$ & $\mathrm{uCi} / \mathrm{L}$ & $P$ \\
\hline Cs137 & $5 / 6 / 82$ & NS & NS & NS & NS & NS & 1.09 & $4.87 E+04$ & $\mathrm{uCi} / \mathrm{L}$ & $\dot{P}$ \\
\hline Cs137 & $10 / 30 / 86$ & NS & NS & R060386 & NS & NS & NS & $4.8 \overline{7 E}+04$ & uCi/L & $\bar{P}$ \\
\hline Cs137 & $7 / 6 / 84$ & NS & NS & T34968 & NS & NS & NS & $2.12 E+04$ & $\mathrm{UCi} / \mathrm{L}$ & $\mathrm{s}$ \\
\hline Cs137 & $4 / 26 / 74$ & NS & NS & NS & NS & NS & 1.299 & $1.97 E+04$ & $\mathrm{uCi} / \mathrm{L}$ & $\mathrm{L}$ \\
\hline Cs137 & $7 / 6 / 84$ & NS & NS & T3496A & NS & NS & NS & $1.76 \mathrm{E}+04$ & $\mathrm{uCi} / \mathrm{L}$ & $\bar{S}$ \\
\hline Cs137 & $12 / 2 / 81$ & NS & NS & T3251 & NS & NS & 1.03 & $1.46 E+04$ & UCi/L & $L$ \\
\hline Cs137 & $11 / 5 / 81$ & NS & NS & RAT-AZ102-2C & NS & NS & 1.26 & $4.59 E+03$ & uCi/ & $\mathbf{s}$ \\
\hline Cs137 & $5 / 6 / 82$ & NS & NS & NS & NS & NS & 1.26 & $4.59 E+03$ & uCi/L & $\mathbf{G}$ \\
\hline Cs137 & $1 / 30 / 82$ & NS & NS & $T-5387$ & NS & NS & 1.008 & $9.59 E+02$ & $\mathrm{uCi} / n$ & $L$ \\
\hline
\end{tabular}




\begin{tabular}{|c|c|c|c|c|c|c|c|}
\hline & \multicolumn{4}{|c|}{ Radionuclide Information } & \multirow[b]{2}{*}{$\begin{array}{l}\text { Reference } \\
\text { Number }\end{array}$} & \multirow[b]{2}{*}{$\begin{array}{l}\text { Validated } \\
\text { (Y/N) }\end{array}$} & \multirow[b]{2}{*}{ Notes } \\
\hline iource & Radionuclide & Conc. $(\mathrm{Bq} / \mathrm{L})$ & $\begin{array}{c}\text { Conc. }(\mathrm{Bq} / \mathrm{L} \\
\text { decayed) }\end{array}$ & $\begin{array}{l}\text { Conc. (Ci/L } \\
\text { Decayed) }\end{array}$ & & & \\
\hline Braun & Cs137 & $3.46 E+10$ & $2.93 E+10$ & 7.92E-01 & NA & $\mathbf{N}$ & $\begin{array}{l}\text { Privitization is not using Braun, Wastren or HTCE Inv. } \\
\text { data }\end{array}$ \\
\hline$\overline{\text { Raw }}$ & Cs137. & $3.46 E+10$ & $2.98 E+10$ & $8.05 \mathrm{E}-01$ & AZ102-16 & $\mathrm{N}$ & Analysis prior to $7 / 89$ \\
\hline Raw & Cs137 & $3.46 E+10$ & $2.96 E+10$ & $8.00 E-01$ & AZ102-16 & $\mathbf{N}$ & Analysis prior to $7 / 89$ \\
\hline Raw & Cs137 & $3.46 E+10$ & $3.15 E+10$ & $8.51 E-01$ & AZ102-19 & N & Not a traceable reference \\
\hline lastren & Cs137 & $3.26 E+10$ & $2.88 E+10$ & 7.78E-01 & NA & $\mathbf{N}$ & $\begin{array}{l}\text { Privitization is not using Braun, Wastren or HTCE Inv. } \\
\text { data }\end{array}$ \\
\hline Raw & Cs137 & $3.15 E+10$ & $2.78 E+10$ & $7.50 E-01$ & AZ102-23 & $\mathbf{Y}$ & $\begin{array}{l}\text { Cs isotopic(wt\%): }-133(47.8 \%),-134(0.06 \%),-135 \\
(13.6 \%),-137(38.6 \%)\end{array}$ \\
\hline Raw & Cs137 & $2.72 \mathrm{E}+10$ & $2.34 E+10$ & $6.32 \mathrm{E}-01$ & AZ102-14 & $\mathrm{N}$ & Analysis prior to $7 / 89$ \\
\hline Raw & Cs137 & $2.72 E+10$ & $2.31 E+10$ & $6.24 E-01$ & AZ102-14 & $\bar{N}$ & Analysis prior to $7 / 89$ \\
\hline Raw & Cs137 & $2.72 \mathrm{E}+10$ & $2.47 E+10$ & $6.68 \mathrm{E}-01$ & AZ102-22 & $N$ & Not a traceable reference \\
\hline 3raun & Cs137 & $1.39 E+10$ & $1.02 E+10$ & 2.76E-01 & NA & $\mathbf{N}$ & $\begin{array}{l}\text { Privitization is not using Braun, Wastren or HTCE lnv. } \\
\text { data }\end{array}$ \\
\hline 3raun & Cs137 & $1.22 E+10$ & $8.57 E+09$ & $2.32 \mathrm{E}-01$ & NA & $\mathbf{N}$ & $\begin{array}{l}\text { Privitization is not using Braun, Wastren or HTCE Inv. } \\
\text { data }\end{array}$ \\
\hline Raw & Cs137 & $1.22 E+10$ & $8.57 E+09$ & $2.32 \mathrm{E}-01$ & AZ102-6 & $\bar{N}$ & Analysis prior to $7 / 89$ \\
\hline Braun & Cs137 & $1.16 E+10$ & $8.93 E+09$ & 2.41E-01 & NA & $\mathbf{N}$ & $\begin{array}{l}\text { Privitization is not using Braun, Wastren or HTCE Inv. } \\
\text { data }\end{array}$ \\
\hline Raw & Cs137 & $1.16 E+10$ & $1.06 E+10$ & $2.86 \mathrm{E}-01$ & AZ102-20 & $\mathbf{N}$ & Not a traceable reference \\
\hline Raw & Cs137 & $1.16 \mathrm{E}+10$ & $8.93 E+09$ & $2.41 \mathrm{E}-01$ & AZ102-5 & $\mathbf{N}$ & Analysis prior to $7 / 89$ \\
\hline Braun & Cs137 & $1.16 E+10$ & $8.93 E+09$ & $2.41 \mathrm{E}-01$ & NA & N & $\begin{array}{l}\text { Privitization is not using Braun, Wastren or HTCE Inv. } \\
\text { data }\end{array}$ \\
\hline Raw & Cs137 & $1.14 E+10$ & $8.03 E+09$ & 2.17E-01 & $A Z 102-3$ & $\bar{N}$ & Analysis prior to $7 / 89$ \\
\hline Raw & Cs137 & $1.11 E+10$ & $7.87 E+09$ & $2.13 \mathrm{E}-01$ & AZ102-3 & $\bar{N}$ & Analysis prior to $7 / 89$ \\
\hline Raw & Cs137 & $1.10 E+10$ & $7.74 E+09$ & $2.09 \mathrm{E}-01$ & AZ102-3 & $\mathbf{N}$ & Analysis prior to $7 / 89$ \\
\hline Raw & Cs137 & $9.40 E+09$ & $7.24 E+09$ & $1.96 \mathrm{E}-01$ & $A Z 102-7$ & $\bar{N}$ & Analysis prior to $7 / 89$ \\
\hline 3raun & Cs137 & $8.14 E+09$ & $6.14 E+09$ & $1.66 \mathrm{E}-01$ & NA & $\mathrm{N}$ & $\begin{array}{l}\text { Privitization is not using Braun, Wastren or HTCE Inv. } \\
\text { data }\end{array}$ \\
\hline Raw & Cs137 & $8.14 E+09$ & $6.14 E+09$ & $1.66 \mathrm{E}-01$ & AZ102-6 & $N$ & Analysis prior to $7 / 89$ \\
\hline Raw. & Cs137 & $5.94 E+09$ & $5.24 \mathrm{E}+09$ & $1.42 E-01$ & $A Z 102-23$ & N & Solid sample after has been washed with fresh water \\
\hline Raw & Cs137 & $4.03 E+09$ & $3.55 \mathrm{E}+09$ & $9.61 \mathrm{E}-02$ & AZ102-23 & $N$ & Water wash of solid sample not supernatent \\
\hline 3raun & Cs137 & $2.96 \mathrm{E}+09$ & $2.36 \mathrm{E}+09$ & $6.38 \mathrm{E}-02$ & NA & $\mathbf{N}$ & $\begin{array}{l}\text { Privitization is not using Braun, Wastren or HTCE Inv. } \\
\text { data }\end{array}$ \\
\hline 3raun & Cs137 & $2.92 E+09$ & $2.32 E+09$ & $6.27 E-02$ & NA & $\mathbf{N}$ & $\begin{array}{l}\text { Privitization is not using Braun, Wastren or HTCE Inv. } \\
\text { data }\end{array}$ \\
\hline Raw & Cs137 & $2.79 E+09$ & $2.40 \mathrm{E}+09$ & $6.49 \mathrm{E}-02$ & $A Z 102-16$ & $N$ & Analysis prior to $7 / 89$ \\
\hline Raw & Cs137 & $2.79 E+09$ & $2.39 E+09$ & $6.46 \mathrm{E}-02$ & A2102-16 & $\bar{N}$ & Analysis prior to $7 / 89$ \\
\hline Raw & Cs137 & $2.57 E+09$ & $2.21 E+09$ & 5.97E-02 & AZ102-16 & $\bar{N}$ & Analysis prior to $7 / 89$ \\
\hline Raw & Cs137 & $2.57 E+09$ & $2.20 E+09$ & 5.95E-02 & AZ102-16 & $\bar{N}$ & Analysis prior to $7 / 89$ \\
\hline 3raun & Cs137 & $2.14 E+09$ & $1.74 E+09$ & 4.70E-02 & NA & $\mathbf{N}$ & $\begin{array}{l}\text { Privitization is not using Braun, Wastren or HTCE Inv. } \\
\text { data }\end{array}$ \\
\hline Raw & Cs137 & $2.14 E+09$ & $1.76 \mathrm{E}+09$ & $4.76 \mathrm{E}-02$ & AZ102-11 & $\mathbf{N}$ & Analysis prior to $7 / 89$ \\
\hline 3raun & Cs137 & $1.80 E+09$ & $1.46 \mathrm{E}+09$ & $3.95 \mathrm{E}-02$ & NA & $\mathbf{N}$ & $\begin{array}{l}\text { Privitization is not using Braun, Wastren or HTCE Inv. } \\
\text { data }\end{array}$ \\
\hline Raw & Cs137 & $1.80 E+09$ & $1.48 E+09$ & $4.00 \mathrm{E}-02$ & AZ102-11 & $\mathbf{N}$ & Analysis prior to $7 / 89$ \\
\hline Raw & Cs137 & $1.80 E+09$ & $1.48 \mathrm{E}+09$ & $4.00 \mathrm{E}-02$ & AZ102-11 & $N$ & Analysis prior to $7 / 89$ \\
\hline Raw & Cs137 & $1.80 E+09$ & $1.64 E+09$ & 4.43E-02 & AZ102-21 & $\mathbf{N}$ & Not a traceable reference \\
\hline Raw & Cs137 & $7.84 E+08$ & $6.76 \mathrm{E}+08$ & $1.83 \mathrm{E}-02$ & AZ102-16 & $\bar{N}$ & Analysis prior to 7/89 \\
\hline Raw & Cs137 & $7.29 E+08$ & $4.97 E+08$ & $1.34 \mathrm{E}-02$ & $A Z 102-1$ & N & Analysis prior to $7 / 89$ \\
\hline Raw & Cs137 & $6.51 E+08$ & $5.61 E+08$ & 1.52E-02 & AZ102-16 & $\bar{N}$ & Analysis prior to $7 / 89$ \\
\hline Raw & Cs137 & $5.40 E+08$ & $4.38 E+08$ & $1.18 \mathrm{E}-02$ & $A Z 102-10$ & $\bar{N}$ & Analysis prior to $7 / 89$ \\
\hline 3raun & $\operatorname{Cs137}$ & $1.70 E+08$ & $1.38 \mathrm{E}+08$ & $3.73 E-03$ & NA & $\mathbf{N}$ & $\begin{array}{l}\text { Privitization is not using Braun, Wastren or HTCE Inv. } \\
\text { data }\end{array}$ \\
\hline Raw & Cs137 & $1.70 E+08$ & $1.39 E+08$ & $3.76 \mathrm{E}-03$ & AZ102-11 & $\bar{N}$ & Analysis prior to $7 / 89$ \\
\hline Braun & Cs137 & $3.55 E+07$ & $2.89 E+07$ & 7.81E-0.4 & NA & $N$ & $\begin{array}{l}\text { Privitization is not using Braun, Wastren or HTCE Inv. } \\
\text { data }\end{array}$ \\
\hline
\end{tabular}




\begin{tabular}{|c|c|c|c|c|c|c|c|c|c|c|}
\hline & & & & Tank Waste & racterization & ample Informati & & & & \\
\hline $\begin{array}{c}\text { Reported } \\
\text { Radionuclide }\end{array}$ & $\begin{array}{c}\text { Memo } \\
\text { Date }\end{array}$ & $\begin{array}{c}\text { Start } \\
\text { Analysis } \\
\text { Date } \\
\end{array}$ & $\begin{array}{c}\text { Start } \\
\text { Sample } \\
\text { Date } \\
\end{array}$ & Sample Number & $\begin{array}{c}\text { Tank Farm } \\
\text { Number }\end{array}$ & $\begin{array}{c}\text { Lab Sample } \\
\text { Number }\end{array}$ & $\begin{array}{l}\text { Density } \\
(\mathrm{g} / \mathrm{mL})\end{array}$ & $\begin{array}{c}\text { Reported } \\
\text { Value }\end{array}$ & $\begin{array}{l}\text { Reported } \\
\text { Unit }\end{array}$ & Code \\
\hline Cs137 & $2 / 19 / 82$ & NS & NS & $T 5387$ & NS & NS & 1.008 & $9.59 E+02$ & $\mathrm{uCi} / \mathrm{L}$ & $\mathrm{L}$ \\
\hline Cs137 & $8 / 27 / 77$ & NS & NS & NS & NS & NS & 1.391 & $3.75 \mathrm{E}+00$ & $u C i / L$ & $\mathbf{L}$ \\
\hline Cs137 & $1 2 / 3 \longdiv { 7 5 }$ & NS & NS & 1459 & NS & - NS & 1.386 & $2.93 E+00$ & $\mathrm{uCi} / \mathrm{L}$ & $\bar{s}$ \\
\hline Eu-154 & $8 / 31 / 89$ & N/A & $6 / 30 / 85$ & Composite Core & NA & NA & 1.14 & $<4.80 \mathrm{e}-01$ & uCi/g & $\mathrm{Cl}$ \\
\hline Eu154 & $5 / 3 / 86$ & NS & NS & $L 1$ & NS & NS & NS & $9.75 E+01$ & uCi/g & 1 \\
\hline Eu154 & $5 / 3 / 86$ & NS & NS & $L 4$ & NS & NS & NS & $6.54 E+01$ & $\mathrm{uCi} / \mathrm{g}$ & 1 \\
\hline Eu154 & $5 / 3 / 86$ & NS & NS & L3 & NS & NS & NS & $1.85 E+01$ & $\mathrm{uCi} / \mathrm{g}$ & 1 \\
\hline Eu154 & $8 / 27 / 77$ & NS & NS & NS & NS & NS & 1.391 & $5.85 E+03$ & UCi & $\mathrm{L}$ \\
\hline Eu154 & $4 / 26 / 74$ & NS & NS & NS & NS & NS & 1.299 & $6.65 E+02$ & UCin & $\mathbf{L}$ \\
\hline 1.129 & $8 / 31 / 89$ & N/A & $6 / 30 / 85$ & Composite Core & NA & NA & 1.14 & $<1.00$ E-06 & uCi $/ \mathrm{g}$ & $\mathrm{Cl}$ \\
\hline $1-129$ & $8 / 31 / 89$ & NS & $6 / 30 / 85$ & Composite Core & NS & NS & 1.11 & $<3.00 E-05$ & $\mathrm{uCi} / \mathrm{g}$ & EI \\
\hline 1129 & $1 / 1 / 77$ & NS & NS & NS & NS & NS & NS & $3.68 \mathrm{E}+01$ & ug $/$ & L \\
\hline $1-129$ & $8 / 31 / 89$ & NS & $6 / 30 / 85$ & Composite Core & NS & NS & $(1.0)$ & $<4.00 \mathrm{E}-07$ & $\mathrm{uCi} / \mathrm{g}$ & $F$ \\
\hline $\mathrm{Np}-237$ & $8 / 31 / 89$ & NS & $6 / 30 / 85$ & Composite Core & NS & NS & 1.11 & 5.16E-03 & uCi/g & EI \\
\hline Np-237 & $8 / 31 / 89$ & N/A & $6 / 30 / 85$ & Composite Core & NA & NA & 1.14 & $1.67 \mathrm{E}-03$ & $\mathrm{uCi} / \mathrm{g}$ & $\mathrm{Cl}$ \\
\hline $\mathrm{Np}-237$ & $8 / 31 / 89$ & NS & $6 / 30 / 85$ & Composite Core & NS & NS & $(1.0)$ & $7.40 \mathrm{E} \cdot 05$ & $u \mathrm{Ci} / \mathrm{g}$ & FI \\
\hline Total Pu & $8 / 31 / 89$ & N/A & $6 / 30 / 85$ & Composite Core & NA & NA & 1.14 & $4.50 E-01$ & $u g / g$ & $\mathrm{Cl}$ \\
\hline Total Pu & $8 / 31 / 89$ & N/A & $6 / 30 / 85$ & Composite Core & NA & NA & 1.14 & 4.50E-01 & $u g / g$ & $\mathrm{Cl}$ \\
\hline Plutonium-238 & $3 / 2 / 91$ & N/A & $3 / 2 / 91$ & BOD9R4 & $A Z-102-2$ & S95T000264 & 1.1 & $<1.01 E-03$ & $\mathrm{uCi} / \mathrm{mL}$ & $P-1$ \\
\hline Plutonium-238 & $3 / 2 / 91$ & N/A & $3 / 2 / 91$ & BOD9R4 & $A Z-102-2$ & S95T000264 & 1.1 & $<1.19 \mathrm{E}-03$ & $\mathrm{uCi} / \mathrm{mL}$ & $P-2$ \\
\hline Plutonium-238 & $3 / 2 / 91$ & N/A & $3 / 2 / 91$ & 80D9R5 & $A Z-102-3$ & S95T000265 & 1.1 & $<2.85 E-03$ & $\mathrm{uCi} / \mathrm{mL}$ & $P-2$ \\
\hline Plutonium-238 & $3 / 2 / 91$ & N/A & $3 / 2 / 91$ & BOD9R5 & $A Z-102-3$ & S95T000265 & 1.1 & $<2.96 \mathrm{E}-03$ & $\mathrm{uCi} / \mathrm{mL}$ & $P-1$ \\
\hline Plutonium-238 & $3 / 2 / 91$ & N/A & $3 / 2 / 91$ & BOD9R3 & $A Z-102-1$ & S95T000263 & 1.1 & $<4.21 \mathrm{E}-03$ & $\mathrm{uCi} / \mathrm{mL}$ & $P-1$ \\
\hline Plutonium-238 & $3 / 2 / 91$ & N/A & $3 / 2 / 91$ & BOD9R3 & $A Z-102-1$ & S95T000263 & 1.1 & $<8.63 \mathrm{E}-03$ & $\mathrm{uCi} / \mathrm{mL}$ & $\mathrm{P}-2$ \\
\hline Pu238 & NS & NS & NS & NS & NS & NS & NS & NS & NS & $\mathrm{L}$ \\
\hline Total Pu & $8 / 31 / 89$ & NS & $6 / 30 / 85$ & Composite Core & NS & NS & 1.11 & $1.84 E+01$ & $u g / g$ & EI \\
\hline Total Pu & $8 / 31 / 89$ & N/A & $6 / 30 / 85$ & Composite Core & NA & NA & 1.14 & 4.50E-01 & ug/g & $\mathrm{Cl}$ \\
\hline Total Pu & $8 / 31 / 89$ & NS & $6 / 30 / 85$ & Composite Core & NS & NS & 1.11 & $1.84 E+01$ & ug/g & El \\
\hline Total Pu & $8 / 31 / 89$ & N/A & $6 / 30 / 85$ & Composite Core & NA & NA & 1.14 & $4.50 E-01$ & $u g / g$ & $\mathrm{Cl}$ \\
\hline Plutonium-239/40 & $3 / 2 / 91$ & N/A & $3 / 2 / 91$ & BOD9R4 & $A Z-102-2$ & S95T000264 & 1.1 & $<1.01 E-03$ & $\mathrm{uCi} / \mathrm{mL}$ & $P-1$ \\
\hline Plutonium-239/40 & $3 / 2 / 91$ & N/A & $3 / 2 / 91$ & 80D9R4 & $A Z-102-2$ & S95T000264 & 1.1 & $<1.19 E-03$ & $\mathrm{uCi} / \mathrm{mL}$ & $P-2$ \\
\hline Plutonium-239/40 & $3 / 2 / 91$ & N/A & $3 / 2 / 91$ & BOD9R5 & $A Z-102-3$ & S95T000265 & 1.1 & $<2.85 E-03$ & $u \mathrm{Ci} / \mathrm{mL}$ & P-2 \\
\hline Plutonium-239/40 & $3 / 2 / 91$ & N/A & $3 / 2 / 91$ & BOD9R5 & $A Z-102-3$ & S95T000265 & 1.1 & $<2.96 E-03$ & $\mathrm{uCi} / \mathrm{mL}$ & $P-1$ \\
\hline Plutonium-239/40 & $3 / 2 / 91$ & N/A & $3 / 2 / 91$ & BOD9R3 & $A Z-102-1$ & S95T000263 & 1.1 & $<4.21 E-03$ & $\mathrm{uCi} / \mathrm{mL}$ & $P-1$ \\
\hline Plutonium-239/40 & $3 / 2 / 91$ & N/A & $3 / 2 / 91$ & BOD9R3 & $A Z-102-1$ & S95T000263 & 1.1 & $<8.63 E-03$ & $\mathrm{uCi} / \mathrm{mL}$ & $P-2$ \\
\hline
\end{tabular}




\begin{tabular}{|c|c|c|c|c|c|c|c|}
\hline & \multicolumn{4}{|c|}{ Radionuclide Information } & \multirow[b]{2}{*}{$\begin{array}{l}\text { Reference } \\
\text { Number }\end{array}$} & \multirow[b]{2}{*}{$\begin{array}{l}\text { Validated } \\
\text { (Y/N) }\end{array}$} & \multirow[b]{2}{*}{ Notes } \\
\hline jource & Radionuclide & Conc. $(B q / L)$ & $\begin{array}{l}\text { Conc. }(\mathrm{Bq} / \mathrm{L} \\
\text { decayed) }\end{array}$ & $\begin{array}{l}\text { Conc. }(\mathrm{Ci} / \mathrm{L} \\
\text { Decayed) }\end{array}$ & & & \\
\hline Raw & Cs137 & $3.55 E+07$ & $2.89 E+07$ & 7.81E-04 & $A Z 102-9$ & $\mathbf{N}$ & Not a traceable reference \\
\hline$\overline{\text { Raw }}$ & Cs137 & $1.39 E+05$ & $1.02 E+05$ & $2.76 \mathrm{E}-06$ & $A Z 102-4$ & $\mathbf{N}$ & Analysis prior to $7 / 89$ \\
\hline Raw & Cs137 & $1.08 E+05$ & $7.66 \mathrm{E}+04$ & 2.07E-06 & A2102-3 & $\mathbf{N}$ & Analysis prior to $7 / 89$ \\
\hline Raw & Eu-154 & \#VALUE! & \#VALUE! & \#VALUEI & $A Z 102-23$ & $\bar{Y}$ & \\
\hline Raw & Eu154 & $5.77 E+09$ & $3.99 E+09$ & $1.08 E-01$ & AZ102-17 & $\mathbf{N}$ & Repeated data from report AZ-102-17 \\
\hline Raw & Eu154 & $3.87 E+09$ & $2.68 E+09$ & $7.24 \mathrm{E}-02$ & $\overline{A Z 102.17}$ & $N$ & Repeated data from report AZ-102-17 \\
\hline Raw & Eu154 & $1.10 E+09$ & $7.58 \mathrm{E}+08$ & $2.05 E-02$ & AZ102-17 & $N$ & Repeated data from report AZ-102-17 \\
\hline Raw & Eu154 & $2.16 E+08$ & $7.54 \mathrm{E}+07$ & $2.04 E-03$ & AZ102-4 & $\mathbf{N}$ & Analysis prior to $7 / 89$ \\
\hline Raw & Eu154 & $2.46 E+07$ & $6.58 E+06$ & $1.78 \mathrm{E}-04$ & AZ102-1 & $\mathbf{N}$ & Analysis prior to $7 / 89$ \\
\hline Raw & 1129 & \#ALUE! & \#VALUE! & \#VALUE! & AZ102-23 & $Y$ & \\
\hline Raw & 1129 & $<1.22 \mathrm{E}+03$ & $<1.22 E+03$ & $<3.30 E-08$ & $A Z 102-23$ & $\mathbf{N}$ & Solid sample after has been washed with tresh water \\
\hline TRAC & 1729 & $2.18 E+02$ & $2.18 E+02$ & 5.89E-09 & NA & $\mathbf{N}$ & Not currently of interest to privitization \\
\hline Raw & 1129 & $<1.47 E+01$ & $<1.47 E+01$ & $<3.96 \mathrm{E}-10$ & $A Z 102-23$ & $\mathbf{N}$ & Water wash of solid sample not supernatent \\
\hline Raw & Np237 & $2.10 E+05$ & $2.10 E+05$ & $5.68 \mathrm{E}-06$ & AZ102-23 & N & Solid sample after has been washed with fresh water \\
\hline Raw & Np237 & $7.04 E+04$ & $7.04 E+04$ & $1.90 \mathrm{E}-06$ & $A Z 102-23$ & $\mathbf{Y}$ & \\
\hline Raw & Np237 & $2.71 E+03$ & $2.71 E+03$ & 7.33E-08 & $A 2102-23$ & $\mathrm{~N}$ & Water wash of solid sample not supernatent \\
\hline Raw & Pu-241 & $1.23 E+07$ & $9.45 E+06$ & 2.56E-04 & AZ102-23 & $\mathrm{Y}$ & $\begin{array}{l}\text { Values reported as total Pu, isotopic(wt\%): }-238 \\
(0.0471 \%),-239(92.26 \%),-240(7.00 \%),-241 \\
(0.624 \%),-242(0.0761), \text { Bq/L value multiplied by } \\
0.00624\end{array}$ \\
\hline Raw & Pu-242 & $5.73 E+01$ & $5.73 E+01$ & $1.55 \mathrm{E}-09$ & AZ102-23 & $\mathrm{Y}$ & $\begin{array}{l}\text { Values reported as total Pu, isotopic(wt\%): }-238 \\
(0.0471 \%),-239(92.26 \%),-240(7.00 \%),-241 \\
(0.624 \%),-242(0.0761), \text { Bq/L value multiplied by } \\
0.000761\end{array}$ \\
\hline Raw & Pu238 & \#VALUE! & \#VALUE! & \#VALUE! & AZ102-24 & $Y$ & Updated per memo $8 / 17 / 95$ \\
\hline Raw & Pu238 & \#VALUE! & \#VALUE! & \#VALUE! & AZ102-24 & $\bar{Y}$ & Updated per memo $8 / 17 / 95$ \\
\hline Raw & Pu238 & \#VALUE! & \#VALUE! & \#VALUE! & $A Z 102-24$ & $\bar{Y}$ & Updated per memo 8/17/95 \\
\hline Raw & Pu238 & \#VALUE! & \#VALUE! & \#VALUE! & $A Z 102-24$ & $\bar{Y}$ & Updated per memo 8/17/95 \\
\hline Raw & Pu238 & \#VALUE! & \#VALUE! & \#VALUE! & AZ102-24 & $\mathbf{Y}$ & Updated per memo 8/17/95 \\
\hline Raw & Pu238 & \#VALUE! & \#VALUE! & \#VALUE! & AZ102-24 & $\bar{Y}$ & Updated per memo $8 / 17 / 95$ \\
\hline IRAC & Pu238 & NS & NS & NS & NA & N & Not currently of interest to privitization \\
\hline Raw & Pu238 & $6.10 E+06$ & $5.84 E+06$ & $1.58 \mathrm{E}-04$ & $A Z 102-23$ & $\mathbf{N}$ & Solid sample after has been washed with fresh water \\
\hline Raw & Pu238 & $1.55 E+05$ & $1.48 \mathrm{E}+05$ & $4.00 E-06$ & AZ102-23 & $\mathbf{Y}$ & $\begin{array}{l}\text { Values reported as total Pu, isotopic(wt } \%):-238 \\
(0.0471 \%),-239(92.26 \%),-240(7.00 \%),-241 \\
(0.624 \%),-242(0.0761), 8 q / L \text { value multiplied by } \\
0.000471\end{array}$ \\
\hline Raw & Pu239 & $4.33 E+07$ & $4.33 E+07$ & $1.17 E-03$ & $A Z 102-23$ & $\mathbf{N}$ & Solid sample after has been washed with fresh water \\
\hline Raw & Pu239 & $1.10 E+06$ & $1.10 E+06$ & 2.97E-05 & AZ102-23 & $\mathrm{Y}$ & $\begin{array}{l}\text { Values reported as total Pu, isotopic(wt \%): }-238 \\
(0.0471 \%),-239(92.26 \%),-240(7.00 \%),-241 \\
(0.624 \%),-242(0.0761), \text { Bq/L value multiplied by } \\
0.9226\end{array}$ \\
\hline Raw & Pu239/240 & \#VALUE! & \#VALUE! & \#VALUE! & $A Z 102-24$ & $\mathbf{Y}$ & $\begin{array}{l}\text { Assumed half-life and atomic weight of Pu-239. } \\
\text { Updated per memo } 8 / 17 / 95\end{array}$ \\
\hline Raw & Pu239/240 & NaLUE! & $\#$ \#ALUE! & \#VALUE! & $A Z 102-24$ & Y & $\begin{array}{l}\text { Assumed half-life and atomic weight of Pu-239. } \\
\text { Updated per memo } 8 / 17 / 95\end{array}$ \\
\hline Raw & Pu239/240 & *VALUE! & \#VALUE! & \#VALUE! & AZ102-24 & $\boldsymbol{\gamma}$ & $\begin{array}{l}\text { Assumed half-life and atomic weight of Pu-239. } \\
\text { Updated per memo } 8 / 17 / 95\end{array}$ \\
\hline Raw & Pu239/240 & \#VALUE! & \#VALUE! & \#VALUE! & AZ102-24 & Y & $\begin{array}{l}\text { Assumed half-life and atomic weight of Pu-239. } \\
\text { Updated per memo } 8 / 17 / 95\end{array}$ \\
\hline Raw & Pu239/240 & *VALUE! & FALUE! & *VAluel & AZ102-24 & Y & $\begin{array}{l}\text { Assumed half-life and atomic weight of Pu-239. } \\
\text { Updated per memo } 8 / 17 / 95\end{array}$ \\
\hline Raw & Pu239/240 & *alue! & "VALUE! & \#VALUE! & AZ102-24 & $Y$ & $\begin{array}{l}\text { Assumed half-life and atomic weight of Pu-239. } \\
\text { Updated per memo 8/17/95 }\end{array}$ \\
\hline
\end{tabular}




\begin{tabular}{|c|c|c|c|c|c|c|c|c|c|c|}
\hline \multicolumn{11}{|c|}{ Tank Waste Characterization Sample Information } \\
\hline $\begin{array}{c}\begin{array}{c}\text { Reported } \\
\text { Radionuclide }\end{array} \\
\end{array}$ & $\begin{array}{c}\text { Memo } \\
\text { Date }\end{array}$ & $\begin{array}{c}\text { Start } \\
\text { Analysis } \\
\text { Date } \\
\end{array}$ & $\begin{array}{c}\text { Start } \\
\text { Sample } \\
\text { Date } \\
\end{array}$ & Sample Number & $\begin{array}{c}\text { Tank Farm } \\
\text { Number }\end{array}$ & $\begin{array}{c}\text { Lab Sample } \\
\text { Number }\end{array}$ & $\begin{array}{l}\text { Density } \\
(\mathrm{g} / \mathrm{mL})\end{array}$ & $\begin{array}{c}\text { Reported } \\
\text { Value }\end{array}$ & $\begin{array}{c}\text { Reported } \\
\text { Unit }\end{array}$ & Codt \\
\hline Pu-239/240 & $11 / 5 / 81$ & NS & NS & RAT-AZ102-2B & NS & NS & 1.4 & 2.41E-02 & $\mathrm{gm} / \mathrm{l}$ & $\mathbf{s}$ \\
\hline Pu-239/240 & $5 / 6 / 82$ & NS & NS & NS & NS & NS & NS & $2.41 E-02$ & $g / L$ & $\bar{S}$ \\
\hline Pu-239/240 & $9 / 20 / 78$ & NS & NS & RAT-AZ102-4C & NS & NS & 1.39 & $3.60 E-03$ & $\mathrm{gm} n$ & $L$ \\
\hline Pu-239/240 & $9 / 2 0 \longdiv { 7 8 }$ & NS & NS & NS & NS & NS & 1.39 & $3.60 \mathrm{E}-03$ & 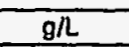 & $\bar{s}$ \\
\hline$P u-239 / 240$ & $6 / 26 / 75$ & NS & NS & RAT-AZ102-4a & NS & NS & 1.39 & $2.40 E-03$ & $\mathrm{gm} / \mathrm{l}$ & $L$ \\
\hline $\mathrm{Pu}-239 / 240$ & $6 / 26 / 75$ & NS & NS & NS & NS & NS & 1.392 & $2.40 \mathrm{E}-03$ & $g \Omega$ & $s$ \\
\hline$P u-239 / 240$ & $8 / 27 / 77$ & NS & NS & RAT-AZ102-4B & NS. & NS & 1.39 & $1.06 E-03$ & $g \mathrm{~m} n$ & $L$ \\
\hline Pu-239/240 & $11 / 5 / 81$ & NS & NS & RAT-AZ102-2C & NS & NS & 1.26 & $9.69 E-05$ & $\operatorname{gmn}$ & $\mathbf{s}$ \\
\hline$P u-239 / 240$ & $11 / 5 / 81$ & NS & NS & RAT-AZ102-2A & NS & NS & 1.09 & $7.22 E-05$ & $\mathrm{gm} / \mathrm{I}$ & $L$ \\
\hline Pu-239/240 & $5 / 6 / 82$ & NS & NS & NS & NS & NS & 1.09 & 7.22E-05 & $g / L$ & $P$ \\
\hline Pu & $2 / 4 / 81$ & NS & NS & R-3699 & NS & NS & 1.11 & $2.11 E+\infty$ & uCin & $l$ \\
\hline Pu & $2 / 4 / 81$ & NS & NS & $R-3700$ & NS & NS & 1.11 & $1.30 E+\infty$ & $u C i n$ & $L$ \\
\hline Pu & $1 / 30 / 82$ & NS & NS & $T-5387$ & NS & NS & 1.008 & $3.58 E-07$ & $\mathrm{gm} n$ & $L$ \\
\hline Pu-239/240 & $5 / 6 / 82$ & NS & NS & NS & NS & NS & 1.26 & $9.69 E-05$ & uCin & $\bar{G}$ \\
\hline Total Pu & $8 / 31 / 89$ & NS & $6 / 30 / 85$ & Composite Core & NS & NS & 1.11 & $1.84 \varepsilon+01$ & ugig & EI \\
\hline Total Pu & $8 / 31 / 89$ & N/A & $6 / 30 / 85$ & Composite Core & NA & NA & 1.14 & $4.50 E-01$ & ug/g & Cl \\
\hline Pu241 & NS & NS & NS & NS & NS & NS & NS & NS & NS & $L$ \\
\hline$S b-125$ & $9 / 1 / 89$ & NS & NS & NS & NS & NS & 1.36 & $3.43 E+04$ & uCi/g & S \\
\hline Sb-125 & $9 / 1 / 89$ & NS & NS & NS & NS & NS & 1.11 & $1.62 E+04$ & uCi/g & s \\
\hline $\mathrm{Sb}-125$ & $9 / 1 / 89$ & NS & NS & NS & NS & NS & 1.49 & $5.76 E+02$ & $\mathrm{uCi} / \mathrm{g}$ & $\mathrm{S}$ \\
\hline $\mathrm{sb}-125$ & $9 / 7 / 89$ & NS & NS & L1 & NS & NS & 1.49 & $5.15 E+02$ & uCi/g & S \\
\hline $\mathrm{Sb}-125$ & $9 / 1 / 89$ & NS & NS & $\mathrm{L} 2$ & NS & NS & 1.49 & $5.01 E+02$ & $\mathrm{uCi} / \mathrm{g}$ & $S$ \\
\hline $\mathrm{Sb}-125$ & $9 / 1 / 89$ & NS & NS & $L 4$ & NS & NS & 1.49 & $2.26 \mathrm{E}+02$ & $\mathrm{uCi} / \mathrm{g}$ & S \\
\hline $\mathrm{Sb}-125$ & $9 / 1 / 89$ & NS & NS & L3 & NS & NS & 1.49 & $3.10 E+01$ & uCi/g & 5 \\
\hline $\mathrm{Sb}-125$ & $4 / 2 6 \longdiv { 7 4 }$ & NS & NS & NS & NS & NS & 1.299 & $2.37 E+02$ & UCi/L & $L$ \\
\hline Se-79 & $8 / 31 / 89$ & N/A & $6 / 30 / 85$ & Composite Core & NA & NA & 1.14 & $3.66 \mathrm{E}-04$ & $\mathrm{uCi} / \mathrm{g}$ & CI \\
\hline Se-79 & $9 / 1 / 89$ & NS & NS & NS & NS & NS & 1.1 & $3.66 \mathrm{E}-04$ & $u \mathrm{Ci} / \mathrm{g}$ & $P$ \\
\hline Se79 & $1 / 1 / 77$ & NS & NS & NS & NS & NS & NS & $1.70 E+\infty$ & ug $/ \mathrm{L}$ & $L$ \\
\hline Sn126 & $1 / 1 / 77$ & NS & NS & NS & NS & NS & NS & $2.52 \mathrm{E}-12$ & $\mathrm{ug} / \mathrm{L}$ & $L$ \\
\hline Sr90 & NS & NS & NS & NS & NS & NS & NS & NS & NS & $L$ \\
\hline sr & NS & NS & NS & NS & NS & NS & 1.3927616 & $3.50 E+01$ & Ci/L & $s$ \\
\hline Sr89/90 & $8 / 1 / 85$ & NS & NS & NS & NS & NS & NS & NS & NS & $\mathbf{s}$ \\
\hline Sr90 & $8 / 31 / 89$ & NS & $6 / 30 / 85$ & Composite Core & NS & NS & 1.11 & $5.53 E+03$ & uCi/g & EI \\
\hline Sr89/90 & $11 / 5 / 81$ & NS & NS & RAT-AZ102-2B & NS & NS & 1.4 & $5.21 E+06$ & uCin & s \\
\hline Sr90 & $5 / 6 / 82$ & NS & NS & NS & NS & NS & NS & $5.21 E+06$ & uCi/L & $\bar{s}$ \\
\hline S?90 & $7 / 6 / 84$ & NS & NS & T3496A & NS & NS & NS & $6.21 E+05$ & $\mathrm{UCi} / \mathrm{L}$ & $S$ \\
\hline Sr90 & $7 / 6 / 84$ & NS & NS & T3496B & NS & NS & NS & $6.10 E+05$ & UCi $/ \mathrm{L}$ & $s$ \\
\hline St90 & $8 / 27 / 77$ & NS & NS & RAT-AZ102-48 & NS & NS & 1.39 & 2.92E-01 & $\mathrm{Ci} / 1$ & $L$ \\
\hline Sr90 & $8 / 27 / 77$ & NS & NS & NS & NS & NS & 1.391 & $2.92 E+05$ & uCill & $L$ \\
\hline
\end{tabular}




\begin{tabular}{|c|c|c|c|c|c|c|c|}
\hline \multirow[b]{2}{*}{ Source } & \multicolumn{4}{|c|}{ Radionuclide Information } & \multirow[b]{2}{*}{$\begin{array}{l}\text { Reference } \\
\text { Number }\end{array}$} & \multirow[b]{2}{*}{$\begin{array}{l}\text { Validated } \\
\text { (Y/N) }\end{array}$} & \multirow[b]{2}{*}{ Notes } \\
\hline & Radionuclide & Conc. $(\mathrm{Bq} / \mathrm{L})$ & $\begin{array}{l}\text { Conc. (BqIL } \\
\text { decayed) }\end{array}$ & $\begin{array}{l}\text { Conc. (Cill } \\
\text { Decayed) }\end{array}$ & & & \\
\hline Braun & Pu239/240 & $6.36 E+07$ & $6.36 \varepsilon+07$ & $1.72 \mathrm{E}-03$ & NA & $\mathbf{N}$ & $\begin{array}{l}\text { Privitization is not using Braun, Wastren or HTCE Inv. } \\
\text { data }\end{array}$ \\
\hline Raw & Pu239/240 & $6.36 E+07$ & $6.36 E+07$ & $1.72 \mathrm{E}-03$ & AZ102-11 & $\mathbf{N}$ & Analysis prior to $7 / 89$ \\
\hline Braun & Pu239/240 & $9.50 E+06$ & $9.50 E+06$ & 2.57E-04 & NA & $\mathbf{N}$ & $\begin{array}{l}\text { Privitization is not using Braun, Wastren or HTCE Inv. } \\
\text { data }\end{array}$ \\
\hline Raw & Pu239/240 & $9.50 \mathrm{E}+06$ & $9.50 E+06$ & $2.57 E-04$ & $A Z 102-6$ & $\bar{N}$ & Analysis prior to 7/89 \\
\hline Braun & Pu239/240 & $6.33 E+06$ & $6.33 E+06$ & $1.71 E-04$ & NA & $\mathbf{N}$ & $\begin{array}{l}\text { Privitization is not using Braun, Wastren or HTCE Inv. } \\
\text { data }\end{array}$ \\
\hline Raw & $84239 / 240$ & $6.33 E+06$ & $6.33 \mathrm{E}+06$ & 1.71E-04 & $A Z 102-6$ & $\mathbf{N}$ & Analysis prior to $7 / 89$ \\
\hline Braun & Pu239/240 & $2.80 E \div 06$ & $2.80 \varepsilon+06$ & 7.57E-05 & NA & N & $\begin{array}{l}\text { Privitization is not using Braun, Wastren or HTCE Inv. } \\
\text { data }\end{array}$ \\
\hline Braun & Pu239/240 & $2.56 E+05$ & $2.56 E+05$ & $6.92 \mathrm{E}-06$ & NA & $\mathbf{N}$ & $\begin{array}{l}\text { Privitization is not using Braun, Wastren or HTCE Inv. } \\
\text { data }\end{array}$ \\
\hline Braun & Pu239/240 & $1.90 E+05$ & $1.90 E+05$ & $5.14 E-06$ & NA & $\mathbf{N}$ & $\begin{array}{l}\text { Privitization is not using Braun, Wastren or HTCE Inv. } \\
\text { data }\end{array}$ \\
\hline Raw & Pu239/240 & $1.90 \mathrm{E} \div 05$ & $1.90 E+05$ & $5.14 E-06$ & $A \overline{Z 102-11}$ & $\bar{N}$ & Analysis prior to $7 / 89$ \\
\hline Braun & Pu239/240 & $7.81 E+04$ & $7.81 E+04$ & 2.11E-06 & NA & $\mathbf{N}$ & $\begin{array}{l}\text { Privitization is not using Braun, Wastren or HTCE Inv. } \\
\text { data }\end{array}$ \\
\hline Braun & Pu239/240 & $4.81 E \div 04$ & $4.81 E+04$ & $1.30 E-06$ & NA & $\mathbf{N}$ & $\begin{array}{l}\text { Privitization is not using Braun, Wastren or HTCE Inv. } \\
\text { data }\end{array}$ \\
\hline Braun & Pu239/240 & $9.44 E+02$ & $9.44 E+02$ & $2.55 \mathrm{E}-08$ & NA & $\mathbf{N}$ & $\begin{array}{l}\text { Privitization is not using Braun, Wastren or HTCE Inv. } \\
\text { data }\end{array}$ \\
\hline Raw & Pu239/240 & $3.59 E+00$ & $3.59 \mathrm{E}+\infty$ & $9.70 \mathrm{E}-11$ & $A 2102-11$ & $N$ & Analysis prior to $7 / 89$ \\
\hline Raw & Pu240 & $1.21 E+07$ & $1.21 E+07$ & $3.26 E-04$ & $A Z 102-23$ & N & Solid sample after has been washed with fresh water \\
\hline $\begin{array}{r}\text { Raw } \\
. \\
\end{array}$ & Pu240 & $3.06 E+05$ & $3.06 E+05$ & $8.26 E-06$ & $A Z 102-23$ & Y & $\begin{array}{l}\text { Values reported as total Pu, isotopic(wt\%): }-238 \\
(0.0471 \%),-239(92.26 \%),-240(7.00 \%),-241 \\
(0.624 \%),-242(0.0761), \text { Bq/L value multiplied by } \\
0.0700\end{array}$ \\
\hline IRAC & Pu241 & NS & NS & NS & NA & $\bar{N}$ & Not currently of interest to privitization \\
\hline Raw & Sb125 & $1.73 E+12$ & $1.24 E+12$ & $3.34 E+01$ & $A Z 102-23$ & $\bar{N}$ & Not currently of interest to privitization \\
\hline Raw & Sb125 & $6.65 E+11$ & $4.77 E+11$ & $1.29 E+01$ & AZ102-23 & $\bar{N}$ & Not currently of interest to privitization \\
\hline Raw & Sb125 & $3.18 E+10$ & $2.27 E+10$ & 6.15E-01 & AZ102-23 & $\mathrm{N}$ & Not currently of interest to privitization \\
\hline Raw & Sb125 & $2.84 E+10$ & $2.03 E+10$ & 5.50E-01 & AZ102-23 & $\mathrm{N}$ & Not currently of interest to privitization \\
\hline Raw & Sb125 & $2.76 E+10$ & $1.98 E+10$ & $5.35 E-01$ & $A Z 102-23$ & $\mathrm{~N}$ & Not currently of interest to privitization \\
\hline Raw & Sb125 & $1.25 E+10$ & $8.93 E+09$ & 2.41E-01 & AZ102-23 & $N$ & Not currently of interest to privitization \\
\hline Raw & Sb125 & $1.71 E+09$ & $1.22 E+09$ & $3.31 E-02$ & $A Z 102-23$ & $N$ & Not currently of interest to privitization \\
\hline Raw & $5 \mathrm{bb} 125$ & $8.77 E+06$ & $1.33 E+05$ & $3.59 \mathrm{E}-06$ & AZ102-1 & $\bar{N}$ & Analysis prior to $7 / 89$ \\
\hline Raw & Se-79 & $1.53 E+04$ & $1.53 E+04$ & 4.13E-07 & AZ102-23 & $\bar{Y}$ & \\
\hline$\overline{R a w}$ & Se79 & $1.49 E+04$ & $1.49 E+04$ & $4.03 E-07$ & $A Z 102-23$ & $\mathbf{N}$ & Not cursently of interest to privitization \\
\hline$\overline{\mathrm{RAC}}$ & Se79 & $4.38 E+03$ & $4.38 E+03$ & $1.18 \mathrm{E}-07$ & NA & $\mathbf{N}$ & Not currently of interest to privitization \\
\hline$\overline{R A C}$ & Sn126 & $2.64 \mathrm{E}-09$ & $2.64 \mathrm{E}-09$ & $7.14 \mathrm{E}-20$ & NA & $\bar{N}$ & Not currently of interest to privitization \\
\hline RAC & Sr89/90 & NS & NS & NS & $\overline{N A}$ & $\mathrm{~N}$ & Privitization is not using TRAC data \\
\hline CE Inv. & Sr89/90 & $1.29 E+12$ & $1.29 E+12$ & $3.50 E+01$ & NA & $\mathbf{N}$ & $\begin{array}{l}\text { Privitization is not using Braun, Wastren or HTCE InV. } \\
\text { data }\end{array}$ \\
\hline astren & $5 r 89 / 90$ & $1.21 E+12$ & $1.06 E+12$ & $2.86 E+01$ & NA & $\mathbf{N}$ & $\begin{array}{l}\text { Privitization is not using Braun, Wastren or HTCE inv. } \\
\text { data }\end{array}$ \\
\hline Zaw & Sr89/90 & $2.25 E+11$ & $1.97 E+11$ & $5.33 E+\infty$ & $A Z 102-23$ & $\mathbf{N}$ & Solid sample after has been washed with fresh water \\
\hline raun & Sr89/90 & $1.93 E+11$ & $1.55 E+11$ & 4.19E $+\infty 0$ & NA & $\mathbf{N}$ & $\begin{array}{l}\text { Privitization is not using Braun, Wastren or HTCE Inv. } \\
\text { data }\end{array}$ \\
\hline Zaw & Sr89/90 & $1.93 E+11$ & $1.57 E+11$ & $4.24 E+00$ & A2102-11 & $\mathbf{N}$ & Analysis prior to $7 / 89$ \\
\hline Raw & Sr89/90 & $2.30 E+10$ & $1.97 E+10$ & 5.32E-01 & AZ102-16 & $\bar{N}$ & Analysis prior to $7 / 89$ \\
\hline law & Sr89/90 & $2.26 E+10$ & $1.93 E+10$ & $5.22 E-01$ & AZ102-16 & $\mathbf{N}$ & Analysis prior to $7 / 89$ \\
\hline raun & Sr89/90 & $1.08 E+10$ & $7.84 E+09$ & 2.12E-01 & NA & $\mathbf{N}$ & $\begin{array}{l}\text { Privitization is not using Braun, Wastren or HTCE Inv. } \\
\text { data }\end{array}$ \\
\hline law & Sr89/90 & $1.08 E+10$ & $7.84 E+09$ & 2.12E-01 & AZ102-4 & $N$ & Analysis prior to $7 / 89$ \\
\hline
\end{tabular}


TWRS Privatization Support Proj: Radionuclide Llquid

\begin{tabular}{|c|c|c|c|c|c|c|c|c|c|c|}
\hline \multicolumn{11}{|c|}{ Tank Waste Characterization Sample Information } \\
\hline $\begin{array}{c}\text { Reported } \\
\text { Radionucllde } \\
\end{array}$ & $\begin{array}{c}\text { Memo } \\
\text { Date }\end{array}$ & $\begin{array}{c}\text { Start } \\
\text { Analysis } \\
\text { Date } \\
\end{array}$ & $\begin{array}{c}\text { Start } \\
\text { Sample } \\
\text { Date } \\
\end{array}$ & Sample Number & $\begin{array}{c}\text { Tank Farm } \\
\text { Number }\end{array}$ & $\begin{array}{c}\text { Lab Sample } \\
\text { Number }\end{array}$ & $\begin{array}{l}\text { Density } \\
(\mathrm{g} / \mathrm{mL})\end{array}$ & $\begin{array}{c}\text { Reported } \\
\text { Value }\end{array}$ & $\begin{array}{c}\begin{array}{c}\text { Reported } \\
\text { Unit }\end{array} \\
\end{array}$ & Code \\
\hline Sr90 & $7 / 28 / 79$ & NS & NS & RAT-AZ102-4D & NS & NS & 1.41 & $2.65 E-01$ & $\mathrm{Ci} / \mathrm{I}$ & $L$ \\
\hline Sr90 & $10 / 30 / 86$ & NS & NS & R280783 & NS & NS & NS & $2.65 E+05$ & $\mathrm{uCi} / \mathrm{L}$ & $P$ \\
\hline Sr90 & $7 / 28 / 79$ & NS & NS & NS & NS & NS & NS & $2.65 \mathrm{E}+05$ & $\mathrm{uCi} / \mathrm{L}$ & $P$ \\
\hline Sr90 & $7 / 28799$ & NS & NS & RAT-AZ102-6 & NS & NS & 1.41 & $2.65 E+05$ & $\mathrm{uCi} /$ & $\mathbf{s}$ \\
\hline Sr90 & $12 / 3 / 75$ & NS & NS & 1457 & NS & NS & 1.398 & $2.60 E+05$ & $\mathrm{UCi} / \mathrm{L}$ & $\mathbf{S}$ \\
\hline Sr90 & $12 / 3 / 75$ & NS & NS & 1458 & NS & NS & 1.391 & $2.59 E+05$ & $\mathrm{UCi} / \mathrm{L}$ & $\mathbf{S}$ \\
\hline Sr90 & $12 / 3 / 75$ & NS & NS & 1456 & NS & NS & 1.393 & $2.50 \mathrm{E}+05$ & UCi/L & $\mathbf{S}$ \\
\hline Sr90 & $12 / 3 / 75$ & NS & NS & 1459 & NS & NS & 1.386 & $2.49 E+05$ & $\mathrm{UCI} / \mathrm{L}$ & $\mathbf{S}$ \\
\hline Sr9o & $6 / 26 / 75$ & NS & NS & RAT-AZ102-4a & NS & NS & 1.39 & 2.44E-01 & $\mathrm{Ci} / \mathrm{I}$ & $L$ \\
\hline Sr90 & $6 / 26 / 75$ & NS & NS & NS & NS & NS & 1.392 & $2.44 E-01$ & $\mathrm{Ci} / \mathrm{L}$ & $\mathbf{S}$ \\
\hline Sr90 & $9 / 1 / 79$ & NS & NS & NS & NS & NS & NS & $2.19 E+05$ & $\mathrm{UCi} / \mathrm{L}$ & G \\
\hline Sr90 & $4 / 26 / 74$ & NS & NS & NS & NS & NS & 1.299 & $1.95 \mathrm{E}+05$ & $\mathrm{uCi} / \mathrm{L}$ & $L$ \\
\hline Sr9o & $9 / 20 / 78$ & NS & NS & RAT-AZ102-4C & NS & NS & 1.39 & $7.20 \mathrm{E}-02$ & $\mathrm{Ci} / \mathrm{I}$ & $L$ \\
\hline Sr90 & $9 / 20 / 78$ & NS & NS & NS & NS & NS & 1.39 & $7.20 \mathrm{E}-02$ & $\mathrm{Ci} / \mathrm{L}$ & S \\
\hline Sr89/90 & $11 / 5 / 81$ & NS & NS & RAT-AZ102-2C & NS & NS & 1.26 & $1.29 E+04$ & uCi/l & s \\
\hline Sr89/90 & $5 / 6 / 82$ & NS & NS & NS & NS & NS & 1.26 & $1.29 E+04$ & uCi/L & $\mathbf{G}$ \\
\hline Sr89/90 & $11 / 5 / 81$ & NS & NS & RAT-AZ102-2A & NS & NS & 1.09 & $7.45 E+03$ & $\mathrm{uCin}$ & $L$ \\
\hline Sr90 & $5 / 6 / 82$ & NS & NS & NS & NS & NS & 1.09 & $7.45 \mathrm{E}+03$ & uCi/L & $P$ \\
\hline Sr90 & $10 / 30 / 86$ & NS & NS & R060386 & NS & NS & NS & $7.45 E+03$ & uCi/L & $\mathbf{P}$ \\
\hline Sr89/90 & $8 / 1 / 85$ & NS & NS & NS & NS & NS & NS & NS & NS & $P$ \\
\hline Si90 & $8 / 31 / 89$ & N/A & $6 / 30 / 85$ & Composite Core & NA & NA & 1.14 & $5.96 \mathrm{E}+00$ & $\mathrm{uCi} / \mathrm{g}$ & $\mathrm{Cl}$ \\
\hline $\mathrm{Sr}$ & NS & NS & NS & NS & NS & NS & 1.03625374 & $2.43 E-03$ & $\mathrm{Ci} / \mathrm{L}$ & $L$ \\
\hline Strontium-90 & $3 / 2 / 91$ & N/A & $3 / 2 / 91$ & BOD9R5 & $A Z-102-3$ & S95T000265 & 1.1 & $2.40 E+00$ & $\mathrm{uCi} / \mathrm{mL}$ & $P-1$ \\
\hline Sr89/90 & $2 / 4 / 81$ & NS & NS & R-3699 & NS & NS & 1.71 & $2.14 E+03$ & uCi/l & $L$ \\
\hline Strontium-90 & $3 / 2 / 91$ & N/A & $3 / 2 / 91$ & BOD9R5 & $A Z-102-3$ & S95T000265 & 1.1 & $2.12 E+00$ & $\mathrm{uCi} / \mathrm{mL}$ & $P-2$ \\
\hline Strontium-90 & $3 / 2 / 91$ & N/A & $3 / 2 / 91$ & BOD9R3 & $A Z-102-1$ & S95T000263 & 1.1 & $1.64 E+00$ & $\mathrm{uCi} / \mathrm{mL}$ & P-1 \\
\hline Sr90 & $8 / 31 / 89$ & NS & $6 / 30 / 85$ & Composite Ccre & NS & NS & $(1.0)$ & $1.65 E+00$ & uCi/g & $\mathrm{FI}$ \\
\hline Strontium-90 & $3 / 2 / 91$ & N/A & $3 / 2 / 91$ & BOD9R4 & $A Z-102-2$ & $595 T 000264$ & 1.1 & $1.60 \mathrm{E}+00$ & uCi/mL & P-2 \\
\hline Strontium-90 & $3 / 2 / 91$ & N/A & $3 / 2 / 91$ & BOD9R3 & $A Z-102-1$ & S95T000263 & 1.1 & $1.53 E+00$ & $\mathrm{uCi} / \mathrm{mL}$ & P-2 \\
\hline Strontium-90 & $3 / 2 / 91$ & N/A & $3 / 2 / 91$ & BOD9R4 & $A Z-102-2$ & S95T000264 & 1.1 & $1.46 \mathrm{E}+00$ & $\mathrm{uCi} / \mathrm{mL}$ & P.1 \\
\hline Sr89/90 & $10 / 1 / 83$ & NS & NS & RAT-AZ102-3 & NS & NS & NS & $1.46 E+03$ & uСiл & $L$ \\
\hline Sr90 & $7 / 6 / 84$ & NS & NS & T3495 & NS & NS & NS & $1.46 \mathrm{E}+03$ & uCi/L & $P$ \\
\hline Sr90 & $3 / 11 / 84$ & NS & NS & NS & NS & NS & NS & $1.46 \mathrm{E}+03$ & $\mathrm{uCi} / \mathrm{L}$ & $P$ \\
\hline Sr90 & $10 / 31 / 86$ & NS & NS & R880311 & NS & NS & NS & $1.46 E+03$ & $\mathrm{uCi} / \mathrm{L}$ & $P$ \\
\hline Sr89/90 & $2 / 4 / 81$ & NS & NS & $R-3700$ & NS & NS & 1.11 & $1.06 E+03$ & $\mathrm{uCi} / \mathrm{I}$ & $L$ \\
\hline Sr90 & $7 / 6 / 84$ & NS & NS & T3496B & NS & NS & NS & $1.67 E+02$ & $\mathrm{uC \textrm {i } / \mathrm { L }}$ & S \\
\hline Sr90 & $3 / 11 / 84$ & NS & NS & $\mathrm{B}$ & NS & NS & 12 & $1.67 E+02$ & $\mathrm{uCi} / \mathrm{L}$ & $T$ \\
\hline Sr90 & $7 / 6 / 84$ & NS & NS & T3496A & NS & NS & NS & $6.20 \mathrm{E}+01$ & $\mathrm{UC \textrm {C } / \mathrm { L }}$ & $\mathbf{S}$ \\
\hline Sr90 & $3 / 11 / 84$ & NS & NS & $A$ & NS & NS & 11.9 & $6.20 E+01$ & uCill & $T$ \\
\hline Sr90 & $7 / 13 / 84$ & NS & NS & $T 1520$ & NS & NS & NS & $1.65 E+\infty$ & $\mathrm{UCi} / \mathrm{L}$ & $P$ \\
\hline Sr90 & $12 / 8 / 83$ & NS & NS & NS & NS & NS & NS & $1.65 \mathrm{E}+\infty$ & UCin & $P$ \\
\hline Sr90 & $10 / 31 / 86$ & NS & NS & $R 081287$ & NS & NS & NS & $1.65 E+00$ & $\mathrm{uCi} / \mathrm{L}$ & $P$ \\
\hline Tc-99 & $10 / 1 / 83$ & NS & NS & RAT-AZ102-3 & NS & NS & NS & $4.03 E+04$ & $\mathrm{uCi} / \mathrm{I}$ & $\mathbf{P}$ \\
\hline Tc-99 & $7 / 6 / 84$ & NS & NS & T3495 & NS & NS & NS & $4.03 E+04$ & uCi/L & $P$ \\
\hline TC-99 & $7 / 6 / 84$ & NS & NS & T3496A & NS & NS & NS & $4.03 E+04$ & $\mathrm{UCi} / \mathrm{L}$ & $\mathbf{P}$ \\
\hline Tc-99 & $7 / 13 / 84$ & NS & NS & $T 1520$ & NS & NS & NS & $3.72 E+04$ & uCin & $P$ \\
\hline
\end{tabular}




\begin{tabular}{|c|c|c|c|c|c|c|c|}
\hline & \multicolumn{4}{|c|}{ Radionuclide information } & \multirow[b]{2}{*}{$\begin{array}{c}\text { Reference } \\
\text { Number }\end{array}$} & \multirow[b]{2}{*}{$\begin{array}{l}\text { Validated } \\
\text { (Y/N) }\end{array}$} & \multirow[b]{2}{*}{ Notes } \\
\hline iource & Radionuclide & Conc. $(\mathrm{Bq} / \mathrm{L})$ & $\begin{array}{c}\text { Conc. }(\mathrm{Bq} / \mathrm{L} \\
\text { decayed) }\end{array}$ & $\begin{array}{c}\text { Conc. (Ci/L } \\
\text { Decayed) }\end{array}$ & & & \\
\hline Braun & Sr89/90 & $9.81 E+09$ & $7.45 E+09$ & 2.01E-01 & NA & $\mathbf{N}$ & $\begin{array}{l}\text { Privitization is not using Braun, Wastren or HTCE Inv. } \\
\text { data }\end{array}$ \\
\hline Raw & Sr89/90 & $9.81 E+09$ & $8.87 E+09$ & $2.40 \mathrm{E}-01$ & $A Z 102-20$ & $\mathbf{N}$ & Not a traceable reference \\
\hline Raw & Sr89/90 & $9.81 E+09$ & $7.45 E+09$ & $2.01 \mathrm{E}-01$ & AZ102-5 & $\mathrm{N}$ & Analysis prior to $7 / 89$ \\
\hline Braun & Sr89/90 & $9.81 E+09$ & $7.45 E+09$ & 2.01E-01 & NA & N & $\begin{array}{l}\text { Privitization is not using Braun, Wastren or HTCE Inv. } \\
\text { data }\end{array}$ \\
\hline Raw & Sr89/90 & $9.62 E+09$ & $6.70 \mathrm{E}+09$ & $1.81 \mathrm{E}-01$ & AZ102-3 & $\mathbf{N}$ & Analysis prior to $7 / 89$ \\
\hline Raw & Sr89/90 & $9.58 E+09$ & $6.67 E+09$ & $1.80 \mathrm{E}-01$ & $A Z 102-3$ & $\mathbf{N}$ & Analysis prior to $7 / 89$ \\
\hline Raw & Sr89/90 & $9.25 \mathrm{E}+09$ & $6.44 E+09$ & $1.74 \mathrm{E}-01$ & AZ102-3 & $\bar{N}$ & Analysis prior to $7 / 89$ \\
\hline Raw & Sr89/90 & $9.21 E+09$ & $6.41 E+09$ & $1.73 \mathrm{E}-01$ & $A \overline{Z 102-3}$ & $\bar{N}$ & Analysis prior to $7 / 89$ \\
\hline Braun & Sr89/90 & $9.03 E+09$ & $6.22 E+09$ & $1.68 E-01$ & NA & $\mathbf{N}$ & $\begin{array}{l}\text { Privitization is not using Braun, Wastren or HTCE Inv. } \\
\text { data }\end{array}$ \\
\hline Raw & Sr89/90 & $9.03 E+09$ & $6.22 \mathrm{E}+09$ & $1.68 \mathrm{E}-01$ & AZ102.6 & $N$ & Analysis prior to $7 / 89$ \\
\hline Raw & Sr89/90 & $8.10 E+09$ & $6.17 E+09$ & 1.67E-01 & $A \overline{Z 102-7}$ & $\bar{N}$ & Analysis prior to $7 / 89$ \\
\hline Raw & Sr89/90 & $7.22 E+09$ & $4.83 E+09$ & $1.31 \mathrm{E}-01$ & AZ102-1 & $N$ & Analysis prior to $7 / 89$ \\
\hline Braun & Sr89/90 & $2.66 E+09$ & $1.98 E+09$ & 5.35E-02 & NA & $\mathbf{N}$ & $\begin{array}{l}\text { Privitization is not using Braun, Wastren or HTCE Inv. } \\
\text { data }\end{array}$ \\
\hline Raw & Sr89/90 & $2.66 E+09$ & $1.98 \mathrm{E}+09$ & 5.35E-02 & AZ102.6 & $\mathbf{N}$ & Analysis prior to $7 / 89$ \\
\hline Braun & Sr89/90 & $4.77 E+08$ & $3.83 E+08$ & $1.04 \mathrm{E}-02$ & NA & $\mathrm{N}$ & $\begin{array}{l}\text { Privitization is not using Braun, Wastren or HTCE Inv. } \\
\text { data }\end{array}$ \\
\hline Raw & Sr89/90 & $4.77 E+08$ & $3.88 E+08$ & $1.05 \mathrm{E}-02$ & AZ102-11 & $\bar{N}$ & Analysis prior to $7 / 89$ \\
\hline Braun & Sr89/90 & $2.76 E+08$ & $2.21 E+08$ & $5.97 \mathrm{E}-03$ & NA & $\mathbf{N}$ & $\begin{array}{l}\text { Privitization is not using Braun, Wastren or HTCE Inv. } \\
\text { data }\end{array}$ \\
\hline Raw & Sr89/90 & $2.76 E+08$ & $2.24 E+08$ & $6.05 \mathrm{E}-03$ & AZ102-11 & $\mathbf{N}$ & Analysis prior to $7 / 89$ \\
\hline Raw & Sr89/90 & $2.76 E+08$ & $2.49 E+08$ & $6.73 \mathrm{E}-03$ & $A Z 102-21$ & $\mathbf{N}$ & Not a traceable reference \\
\hline 'astren & Sr89/90 & $2.60 E+08$ & $2.28 E+08$ & $6.16 \mathrm{E}-03$ & NA & $\mathbf{N}$ & $\begin{array}{l}\text { Privitization is not using Braun, Wastren or HTCE Inv. } \\
\text { data }\end{array}$ \\
\hline Raw & Sr89/90 & $2.51 E+08$ & $2.20 E+08$ & $5.96 \mathrm{E}-03$ & AZ102-23 & $\bar{Y}$ & \\
\hline CE Inv. & Sr89/90 & $8.99 E \div 07$ & $8.99 E+07$ & $2.43 E-03$ & NA & $\mathbf{N}$ & $\begin{array}{l}\text { Privitization is not using Braun, Wastren or HTCE Inv. } \\
\text { data }\end{array}$ \\
\hline Raw & Sr89/90 & $8.88 E+07$ & $8.92 \mathrm{E}+07$ & $2.41 \mathrm{E}-03$ & AZ102-24 & $\bar{Y}$ & Updated per memo $8 / 17 / 95$ \\
\hline 3raun & Sr89/90 & $7.92 E+07$ & $6.24 E+07$ & 1.69E-03 & NA & $\mathbf{N}$ & $\begin{array}{l}\text { Privitization is not using Braun, Wastren or HTCE Inv. } \\
\text { data }\end{array}$ \\
\hline Raw & Sr89/90 & $7.84 E+07$ & $7.88 E+07$ & $2.13 E-03$ & AZ102-24 & $\bar{Y}$ & Updated per memo $8 / 17 / 95$ \\
\hline$\overline{\text { Raw }}$ & Sr89/90 & $6.07 E+07$ & $6.09 E+07$ & $1.65 \mathrm{E}-03$ & AZ102-24 & $\bar{Y}$ & Updated per memo $8 / 17 / 95$ \\
\hline Raw & Sr89/90 & $6.05 E+07$ & $5.30 \mathrm{E}+07$ & $1.43 E-03$ & AZ102-23 & $\mathbf{N}$ & Water wash of solid sample not supernatent \\
\hline Raw & Sr89/90 & $5.92 E+07$ & $5.94 E+07$ & $1.61 \mathrm{E}-03$ & AZ102-24 & $\bar{Y}$ & Updated per memo 8/17/95 \\
\hline Raw & Sr89/90 & $5.66 \mathrm{E}+07$ & $5.68 \mathrm{E}+07$ & 1.54E-03 & AZ102-24 & $\bar{Y}$ & Updated per memo $8 / 17 / 95$ \\
\hline Raw & Sr89/90 & $5.40 E+07$ & $5.42 E+07$ & 1.47E-03 & $A Z 102-24$ & $\bar{Y}$ & Updated per memo 8/17/95 \\
\hline 3raun & Sr89/90 & $5.40 E+07$ & $4.54 E+07$ & $1.23 \mathrm{E}-03$ & NA & $\mathbf{N}$ & $\begin{array}{l}\text { Privitization is not using Braun, Wastren or HTCE InV. } \\
\text { data }\end{array}$ \\
\hline Raw & Sr89/90 & $5.40 E+07$ & $4.62 E+07$ & $1.25 \mathrm{E}-03$ & $A Z 102-16$ & $\mathbf{N}$ & Analysis prior to $7 / 89$ \\
\hline Raw & Sr89/90 & $5.40 E+07$ & $4.59 E+07$ & $1.24 \mathrm{E}-03$ & $A \overline{Z 102.16}$ & N & Analysis prior to $7 / 89$ \\
\hline Raw & Sr89/90 & $5.40 \mathrm{E}+07$ & $4.89 \mathrm{E}+07$ & $1.32 \mathrm{E}-03$ & AZ102-19 & $\mathbf{N}$ & Not a traceable reference \\
\hline 3raun & Sr89/90 & $3.92 E+07$ & $3.09 E+07$ & $8.35 \mathrm{E}-04$ & NA & $\mathbf{N}$ & $\begin{array}{l}\text { Privitization is not using Braun, Wastren or HTCE Inv. } \\
\text { data }\end{array}$ \\
\hline Raw & Sr89/90 & $6.18 E+06$ & $5.29 E+06$ & $1.43 E-04$ & A2102-16 & $\bar{N}$ & Analysis prior to $7 / 89$ \\
\hline Raw & Sr89/90 & $6.18 E+06$ & $5.25 E+06$ & $1.42 \mathrm{E}-04$ & $A Z 102-16$ & $\mathbf{N}$ & Analysis prior to $7 / 89$ \\
\hline Raw & Sr89/90 & $2.29 E+06$ & $1.96 E+06$ & $5.30 E-05$ & AZ102-16 & $\bar{N}$ & Analysis prior to $7 / 89$ \\
\hline Raw & Sr89/90 & $2.29 E+06$ & $1.95 \mathrm{E}+06$ & 5.27E-05 & AZ102-16 & $\mathbf{N}$ & Analysis prior to $7 / 89$ \\
\hline Raw & Sr89/90 & $6.11 E+04$ & $5.23 E+04$ & $1.41 E-06$ & AZ102-14 & $\mathrm{N}$ & Analysis prior to $7 / 89$ \\
\hline Raw & Sr89/90 & $6.11 E+04$ & $5.15 E+04$ & $1.39 \mathrm{E}-06$ & AZ102-14 & $\mathbf{N}$ & Analysis prior to $7 / 89$ \\
\hline Raw & Sr89/90 & $6.11 E+04$ & $5.52 E+04$ & $1.49 E-06$ & AZ102.22 & $\mathbf{N}$ & Not a traceable reference \\
\hline 3raun & Tc99 & $1.49 E+09$ & $1.49 E+09$ & 4.03E-02 & NA & $\mathbf{N}$ & $\begin{array}{l}\text { Privitization is not using Braun, Wastren or HTCE Inv. } \\
\text { data }\end{array}$ \\
\hline Raw & Tc99 & $1.49 E+09$ & $1.49 E+09$ & $4.03 E-02$ & $A Z 102-16$ & $\mathbf{N}$ & Analysis prior to $7 / 89$ \\
\hline Raw & Tc99 & $1.49 E+09$ & $1.49 E+09$ & $4.03 E-02$ & $\mathrm{AZ102-16}$ & $N$ & Analysis prior to $7 / 89$ \\
\hline Raw & Tc99 & $1.38 \mathrm{E}+09$ & $1.38 E+09$ & $3.72 \mathrm{E}-02$ & AZ102-14 & $\bar{N}$ & Analysis prior to $7 / 89$ \\
\hline
\end{tabular}




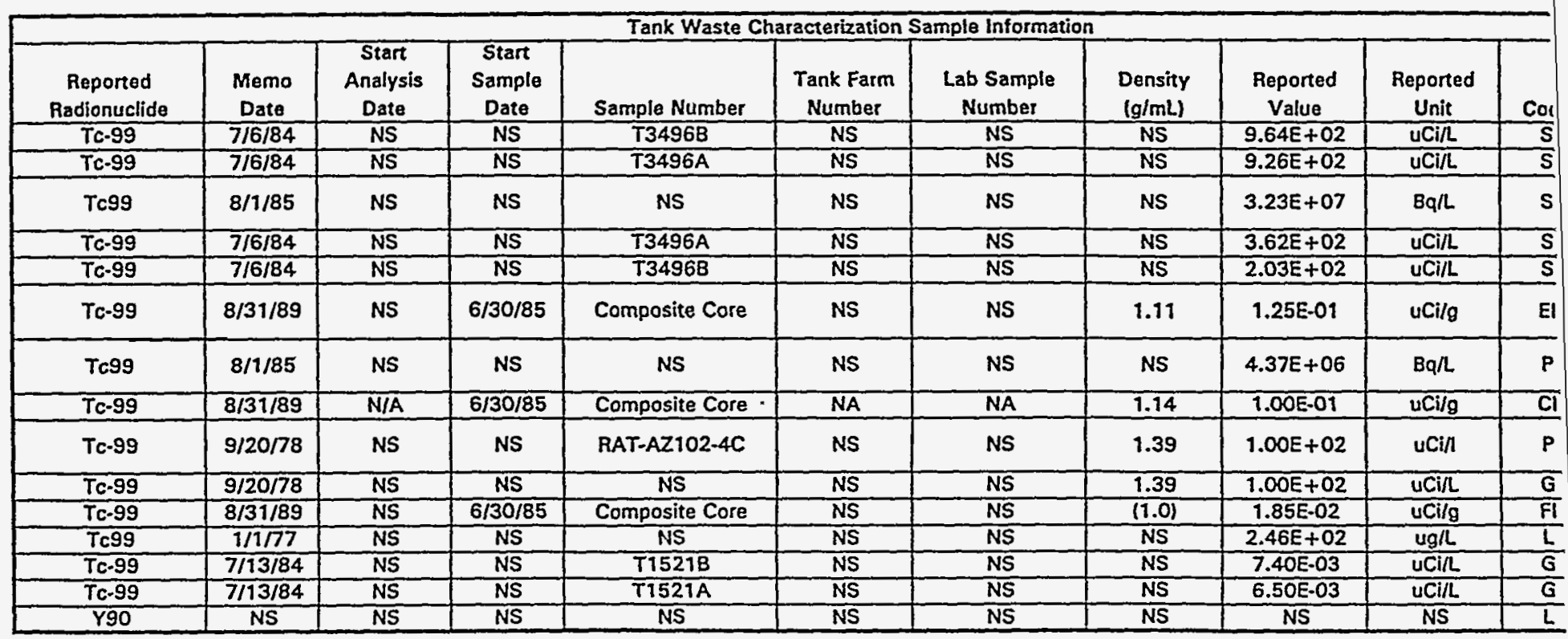




\begin{tabular}{|c|c|c|c|c|c|c|c|}
\hline & \multicolumn{4}{|c|}{ Radionuclide Information } & \multirow[b]{2}{*}{$\begin{array}{c}\text { Reference } \\
\text { Number }\end{array}$} & \multirow[b]{2}{*}{$\begin{array}{c}\text { Validated } \\
\text { (Y/N) }\end{array}$} & \multirow[b]{2}{*}{ Notes } \\
\hline Source & Radionuclide & Conc. $(\mathrm{Bq} / \mathrm{L})$ & $\begin{array}{c}\text { Conc. (Bq/L } \\
\text { decayed) }\end{array}$ & $\begin{array}{l}\text { Conc. (Ci/h. } \\
\text { Decayed). }\end{array}$ & & & \\
\hline Raw & Tc99 & $3.57 E+07$ & $3.57 E+07$ & $9.64 \mathrm{E}-04$ & A2102-16 & $\mathbf{N}$ & Analysis prior to $7 / 89$ \\
\hline Raw & Tc99 & $3.43 E+07$ & $3.43 E+07$ & $9.26 \mathrm{E} \cdot 04$ & $A Z 102-16$ & $\mathbf{N}$ & Analysis prior to $7 / 89$ \\
\hline Wastren & Tc99 & $3.23 E+07$ & $3.23 E \div 07$ & 8.73E-04 & NA & $\mathbf{N}$ & $\begin{array}{l}\text { Privitization is not using Braun, Wastren or HTCE Inv. } \\
\text { data }\end{array}$ \\
\hline Raw & Tc99 & $1.34 E+07$ & $1.34 E+07$ & 3.62E-04 & AZ102-16 & $\mathbf{N}$ & Analysis prior to $7 / 89$ \\
\hline Raw & Tc99 & $7.51 E+06$ & $7.51 \mathrm{E}+06$ & $2.03 \mathrm{E}-04$ & AZ102-16 & $\mathbf{N}$ & Analysis prior to $7 / 89$ \\
\hline Raw & Tc99 & $5.09 E+06$ & $5.09 E+06$ & $1.37 E-04$ & $A Z 102-23$ & N & Solid șample after has been washed with fresh water \\
\hline Wastren & Tc99 & $4.37 E+06$ & $4.37 E+06$ & $1.18 E-04$ & NA & $\mathbf{N}$ & $\begin{array}{l}\text { Privitization is not using Braun, Wastren or HTCE Inv. } \\
\text { data }\end{array}$ \\
\hline Raw & Tc99 & $4.22 \mathrm{E}+06$ & $4.22 E+06$ & $1.14 \mathrm{E}-04$ & AZ102-23 & $\bar{Y}$ & \\
\hline Braun & Tc99 & $3.70 E+06$ & $3.70 E+06$ & $1.00 E-04$ & NA & $\mathbf{N}$ & $\begin{array}{l}\text { Privitization is not using Braun, Wastren or HTCE lnv. } \\
\text { data }\end{array}$ \\
\hline Raw & Tc99 & $3.70 E+06$ & $3.70 E+06$ & $1.00 \mathrm{E}-04$ & AZ102-6 & $\mathbf{N}$ & Analysis prior to $7 / 89$ \\
\hline Raw & Tc99 & $6.78 E+05$ & $6.78 \mathrm{E}+05$ & $1.83 E-05$ & AZ102-23 & $\bar{N}$ & Water wash of solid sample not supernatent \\
\hline TRAC & Tc99 & $1.54 \mathrm{E}+05$ & $1.54 \mathrm{E}+05$ & $4.18 \mathrm{E}-06$ & $\overline{N A}$ & $\mathbf{N}$ & Privitization is not using TRAC data \\
\hline Raw & Tc99 & $2.74 E+02$ & $2.74 \mathrm{E}+02$ & $7.40 \mathrm{E}-09$ & AZ102-14 & $\bar{N}$ & Analysis prior to $7 / 89$ \\
\hline Raw & Tc99 & $2.41 E+02$ & $2.40 \mathrm{E}+02$ & $6.50 E-09$ & $A Z 102-14$ & $\bar{N}$ & Analysis prior to $7 / 89$ \\
\hline TRAC & Y90 & NS & NS & NS & $\overline{N A}$ & $\mathbf{N}$ & Not currently of interest to privitization \\
\hline
\end{tabular}




\begin{tabular}{|c|c|c|c|c|c|c|c|c|c|c|}
\hline \multicolumn{11}{|c|}{ Tank Waste Characterization Sample Information } \\
\hline Reported Chemical & Memo Date & $\begin{array}{c}\text { Start } \\
\text { Analysis } \\
\text { Date } \\
\end{array}$ & $\begin{array}{c}\text { Start } \\
\text { Sample } \\
\text { Date }\end{array}$ & $\begin{array}{l}\text { Sample } \\
\text { Number }\end{array}$ & $\begin{array}{c}\text { Tank Farm } \\
\text { Number }\end{array}$ & $\begin{array}{c}\text { Lab Sample } \\
\text { Number }\end{array}$ & $\begin{array}{l}\text { Density } \\
(\mathrm{g} / \mathrm{mL})\end{array}$ & $\begin{array}{l}\text { Reported } \\
\text { Value }\end{array}$ & Reported Unit & Code \\
\hline $\mathrm{Al}+$ & NA & NA & NA & NA & NA & NA & 1.27 & $7.28 \mathrm{E}-01$ & Mole/L & L \\
\hline Al & $12 / 28 / 94$ & NA & $10 / 21 / 94$ & 102-AN-4 & NA & NA & 1.403 & 0.56 & $M$ & L \\
\hline Al & $12 / 28 / 94$ & NA & $10 / 21 / 94$ & 102-AN-1 & NA & NA & 1.4 & 0.55 & $\bar{M}$ & $\bar{L}$ \\
\hline $\mathrm{Al}$ & $12 / 28 / 94$ & NA & $10 / 21 / 94$ & 102-AN-2 & NA & NA & 1.402 & 0.55 & $M$ & L \\
\hline Al & $2 / 28 / 85$ & NA & NA & R3640 & NA & NA & 1.39 & $5.38 E-01$ & $M$ & $\mathrm{~L}$ \\
\hline A) & $2 / 28 / 85$ & NS & NS & NS & NS & NS & NS & $1.45 E+01$ & $g / L$ & $P$ \\
\hline $\mathrm{Al}$ & $2 / 28 / 85$ & NA & NA & R3639 & $\mathrm{NA}$ & NA & 1.39 & 5.37E-01 & $M$ & L \\
\hline A & $2 / 28 / 85$ & NA & NA & R-3639 & NA & NA & 1.39 & 0.537 & moles $A$ & P \\
\hline $\mathrm{Al}$ & $12 / 16 / 91$ & NA & NA & NA & NA & NA & 1.35 & 12894.1 & $\mathrm{ug} / \mathrm{mL}$ & $P$ \\
\hline AI & $10 / 12 / 89$ & $9 / 22 / 89$ & NA & R-5779 & NA & NA & 1.34 & $4.60 \mathrm{E}-01$ & $M$ & $\bar{P}$ \\
\hline $\mathbf{B a}$ & $2 / 28 / 85$ & $\mathrm{NA}$ & NA & R3639 & NA & NA & 1.39 & $<1.25 \mathrm{E}-04$ & $M$ & $L$ \\
\hline $\mathrm{Ba}$ & $5 / 16 / 90$ & NA & NA & NA & NA & NA & (1.2) & $<1.3 \mathrm{E}-4$ & moles/L & $P$ \\
\hline $\mathrm{Ba}$ & $2 / 28 / 85$ & NA & NA & $\mathrm{R} 3640$ & NA & NA & 1.39 & 0.000133 & $M$ & L \\
\hline $\mathrm{Ba}$ & $2 / 28 / 85$ & NS & NS & NS & NS & NS & NS & 0.0172 & $g / L$ & $P$ \\
\hline $\mathrm{Ca}$ & $2 / 28 / 85$ & NA & NA & $\mathrm{R} 3640$ & NA & NA & 1.39 & 0.0203 & $M$ & L \\
\hline $\mathrm{Ca}$ & $2 / 28 / 85$ & NS & NS & NS & NS & NS & NS & 0.493 & $g / L$ & $\mathbf{P}$ \\
\hline $\mathrm{Ca}$ & $2 / 28 / 85$ & NA & NA & R-3639 & $\mathrm{NA}$ & NA & 1.39 & 0.0123 & moles $n$ & $P$ \\
\hline $\mathrm{Ca}$ & $2 / 28 / 85$ & NA & NA & R3639 & NA & NA & 1.39 & 0.0123 & $M$ & $P$ \\
\hline $\mathrm{Ca}$ & $12 / 16 / 91$ & NA & NA & NA & NA & $\mathrm{NA}$ & 1.35 & 450 & $\mathrm{ug} / \mathrm{mL}$ & P \\
\hline $\mathrm{Ca}$ & $12 / 28 / 94$ & NA & $10 / 21 / 94$ & 102-AN-2 & NA & NA & 1.402 & 0.011 & $\mathrm{M}$ & $L$ \\
\hline $\mathrm{Ca}$ & $12 / 28 / 94$ & NA & $10 / 21 / 94$ & $102-\mathrm{AN}-4$ & NA & NA & 1.403 & 0.011 & $M$ & L \\
\hline $\mathrm{Ca}$ & $12 / 28 / 94$ & NA & $10 / 22 / 94$ & 102-AN-1 & NA & NA & 1.4 & 0.011 & $M$ & $L$ \\
\hline $\mathrm{Ca}$ & $10 / 12 / 89$ & $9 / 22 / 89$ & $\mathrm{NA}$ & R-5779 & NA & NA & 1.34 & $1.00 \mathrm{E}-02$ & $M$ & $P$ \\
\hline Cd & $2 / 28 / 85$ & NA & NA & 83639 & NA & NA & 1.39 & 0.000503 & $M$ & $P$ \\
\hline Cd & $2 / 28 / 85$ & NS & NS & NS & NS & NS & NS & 0.0565 & $g / L$ & $P$ \\
\hline Cd & $2 / 28 / 85$ & NA & NA & R-3639 & NA & NA & 1.39 & 0.000503 & moles $n$ & $P$ \\
\hline $\mathrm{Cd}$ & $5 / 16 / 90$ & NA & NA & NA & NA & NA & $(1.2)$ & $5.00 \mathrm{E}-04$ & moles $/ \mathrm{L}$ & $\bar{P}$ \\
\hline$\overline{C d}$ & $2 / 28 / 85$ & NA & NA & $\mathrm{R} 3640$ & NA & $\mathrm{NA}$ & 1.39 & 0.000448 & $M$ & $L$ \\
\hline $\mathrm{Cl}$ & $12 / 28 / 94$ & NA & $10 / 21 / 94$ & 102-AN-4 & NA & NA & 1.403 & 0.12 & $M$ & $L$ \\
\hline $\mathrm{Cr}^{2}$ & $12 / 28 / 94$ & NA & $10 / 21 / 94$ & 102-AN-2 & NA & NA & 1.402 & 0.11 & $M$ & $L$ \\
\hline $\mathrm{Cl}^{\circ}$ & $12 / 28 / 94$ & NA & $10 / 21 / 94$ & $102-\mathrm{AN}-1$ & NA & NA & 1.4 & 0.1 & $M$ & L \\
\hline $\mathrm{Cl}-$ & NA & NA & NA & NA & NA & NA & 1.27 & $9.98 \mathrm{E}-02$ & Mole $/ \mathrm{L}$ & $L$ \\
\hline $\mathrm{Cl}$ & $2 / 28 / 85$ & NA & NA & 83639 & $\mathrm{NA}$ & NA & 1.39 & $9.49 E-02$ & $M$ & $L$ \\
\hline $\mathrm{Cl}$ & $2 / 28 / 85$ & NA & NA & $R-3639$ & NA & $\mathrm{NA}$ & 1.39 & 0.0949 & moles n & $p$ \\
\hline $\mathrm{Cl}-$ & $2 / 28 / 85$ & NS & NS & NS & NS & NS & NS & $3.36 E+00$ & $g / L$ & $P$ \\
\hline $\mathrm{Cl}$ & $2 / 28 / 85$ & NA & NA & $\mathrm{R} 3640$ & NA & $\mathrm{NA}$ & 1.39 & 9.03E-02 & $M$ & $L$ \\
\hline $\mathrm{Cl}-$ & $12 / 16 / 91$ & NA & NA & NA & NA & NA & 1.35 & 1500 & $\mathrm{ug} / \mathrm{mL}$ & $P$ \\
\hline $\mathrm{Cr}$ & $2 / 28 / 85$ & NA & NA & R3640 & NA & NA & 1.39 & 0.0101 & $M$ & $L$ \\
\hline $\mathrm{Cr}$ & $5 / 16 / 90$ & NA & NA & NA & NA & $N A$ & (1.2) & $8.00 \mathrm{E}-03$ & moles/L & $P$ \\
\hline $\mathrm{Cr}$ & $2 / 28 / 85$ & NA & NA & R3639 & NA & NA & 1.39 & 0.00796 & $M$ & $P$ \\
\hline $\mathrm{Cr}$ & $2 / 28 / 85$ & NS & NS & NS & NS & NS & NS & 0.414 & $g / L$ & $\overline{\mathbf{P}}$ \\
\hline $\mathrm{Cr}$ & $2 / 28 / 85$ & NA & $\overline{\text { NA }}$ & $R-3639$ & NA & NA & 1.39 & 0.00796 & moles & $\bar{P}$ \\
\hline $\mathrm{Cr}$ & $12 / 16 / 91$ & NA & NA & NA & NA & $\mathrm{NA}$ & 1.35 & 399 & $\mathrm{ug} / \mathrm{mL}$ & $P$ \\
\hline $\mathrm{Cr}$ & $12 / 28 / 94$ & NA & $10 / 21 / 94$ & $102-A N-1$ & NA & NA & 1.4 & 0.006 & $M$ & L \\
\hline $\mathrm{Cr}$ & $12 / 28 / 94$ & NA & $10 / 21 / 94$ & 102-AN-2 & NA & NA & 1.402 & 0.006 & $M$ & $\mathbf{L}$ \\
\hline $\mathrm{Cr}$ & $12 / 28 / 94$ & NA & $10 / 21 / 94$ & 102-AN-4 & NA & NA & 1.403 & 0.006 & $M$ & $L$ \\
\hline$F$ & $2 / 28 / 85$ & NA & NA & $\mathrm{R} 3640$ & NA & NA & 1.39 & $<1.32 \mathrm{E}-01$ & $M$ & $L$ \\
\hline$F$ & $2 / 28 / 85$ & NA & NA & R3639 & NA & NA & 1.39 & $<0.118$ & $M$ & L \\
\hline$F_{0}$ & $12 / 16 / 91$ & NA & NA & $\mathrm{NA}$ & $N A$ & NA & 1.35 & 2500 & $\mathrm{ug} / \mathrm{mL}$ & $P$ \\
\hline$F(2)$ & $12 / 28 / 94$ & NA & $10 / 21 / 94$ & 102-AN-4 & NA & NA & 1.403 & 0.12 & $M$ & $L$ \\
\hline
\end{tabular}




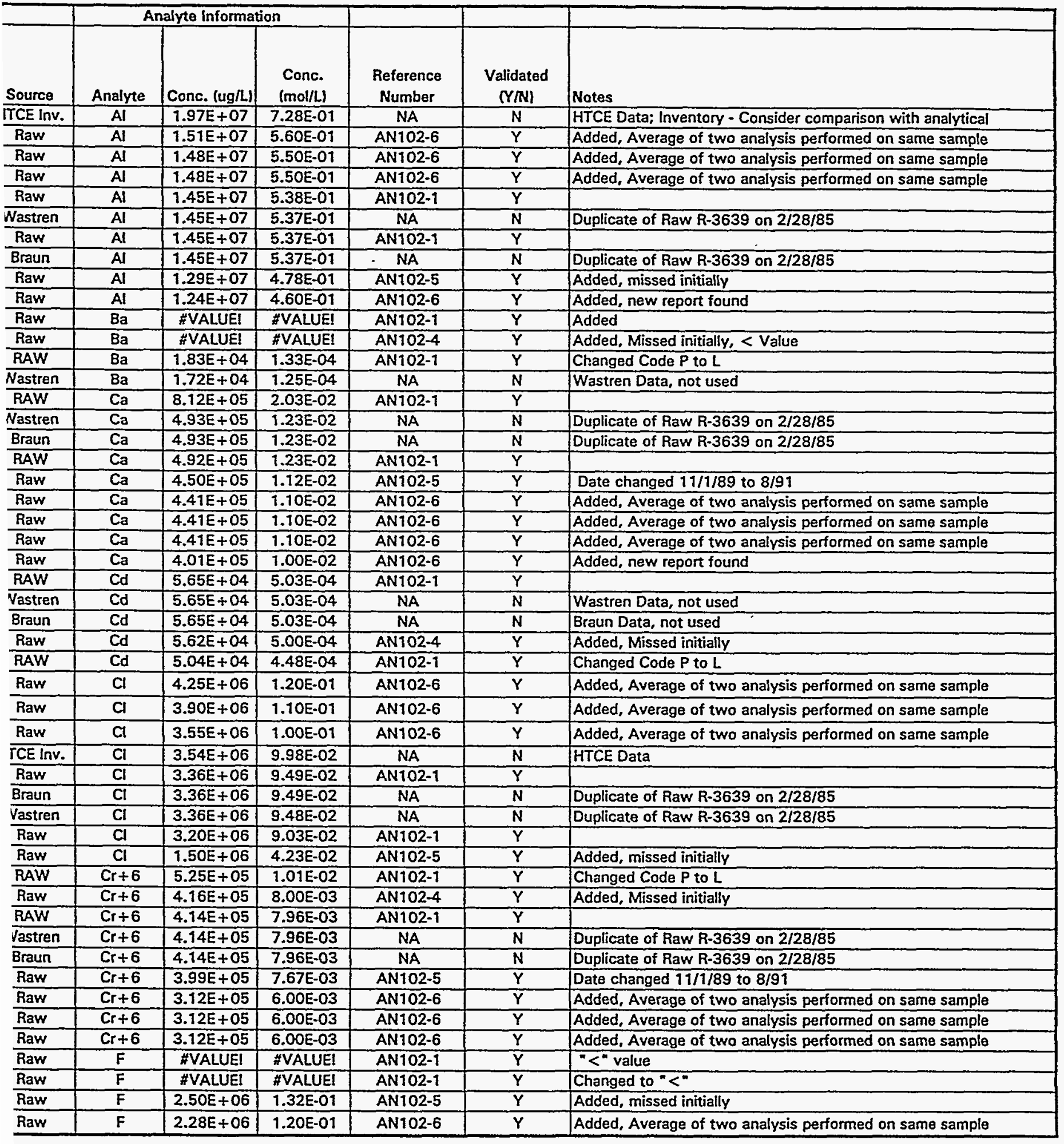


TWRS Privatization Support Pre Chemical Liquid

\begin{tabular}{|c|c|c|c|c|c|c|c|c|c|c|}
\hline \multicolumn{11}{|c|}{ Tank Waste Characterization Sample Information } \\
\hline Reported Chemical & Memo Date & $\begin{array}{c}\text { Start } \\
\text { Analysis } \\
\text { Date }\end{array}$ & $\begin{array}{c}\text { Start } \\
\text { Sample } \\
\text { Date }\end{array}$ & $\begin{array}{l}\text { Sample } \\
\text { Number }\end{array}$ & $\begin{array}{c}\text { Tank Farm } \\
\text { Number }\end{array}$ & $\begin{array}{c}\text { Lab Sample } \\
\text { Number }\end{array}$ & $\begin{array}{l}\text { Density } \\
\text { (g/mL) }\end{array}$ & $\begin{array}{c}\text { Reported } \\
\text { Value }\end{array}$ & Reported Unit & Cods \\
\hline$F$ & $2 / 28 / 85$ & NA & NA & $8-3639$ & NA & NA & 1.39 & 0.118 & moles $n$ & $P$ \\
\hline$F$ & $2 / 28 / 85$ & NS & NS & NS & NS & NS & NS & $2.24 E+00$ & $g / L$ & $P$ \\
\hline$F(2)$ & $12 / 28 / 94$ & NA & $10 / 21 / 94$ & 102-AN-1 & NA & NA & 1.4 & 0.1 & $M$ & $\bar{L}$ \\
\hline$F(2)$ & $12 / 28 / 94$ & NA & $10 / 21 / 94$ & 102-AN-2 & NA & NA & 1.402 & 0.1 & $M$ & $\bar{L}$ \\
\hline F- & NA & NA & NA & NA & NA & NA & 1.27 & $5.06 E-02$ & Mole/L & $L$ \\
\hline Fe & $12 / 16 / 91$ & NA & NA & NA & NA & NA & 1.35 & 298.6 & $\mathrm{ug} / \mathrm{mL}$ & $\mathbf{P}$ \\
\hline $\mathrm{Fe}$ & $2 / 28 / 85$ & $\mathrm{NA}$ & NA & R3640 & NA & NA & 1.39 & $4.68 E-03$ & $M$ & $L$ \\
\hline $\mathrm{Fe} 3+$ (total Fe) & NA & NA & NA & NA & NA & NA & 1.27 & $3.41 E-03$ & Mole/L & $L$ \\
\hline $\mathrm{Fe}$ & $2 / 28 / 85$ & NS & NS & NS & NS & NS & NS & $1.01 E-01$ & $g / L$ & $P$ \\
\hline $\mathrm{Fe}$ & $2 / 28 / 85$ & NA & NA & R3639 & NA & NA & 1.39 & $1.80 \mathrm{E}-03$ & $M$ & $L$ \\
\hline $\mathrm{Fe}$ & $2 / 28 / 85$ & NA & $\mathrm{NA}$ & $R-3639$ & NA & NA & 1.39 & 0.0018 & moles $n$ & $P$ \\
\hline$K$ & $12 / 28 / 94$ & NA & $10 / 21 / 94$ & $102-\mathrm{AN}-4$ & NA & NA & 1.403 & 0.112 & $M$ & $\mathrm{~L}$ \\
\hline$K$ & $12 / 28 / 94$ & NA & $10 / 21 / 94$ & $102-A N-2$ & NA & NA & 1.402 & 0.098 & $M$ & $L$ \\
\hline$K$ & $12 / 28 / 94$ & NA & $10 / 21 / 94$ & 102-AN-1 & NA & NA & 1.4 & 0.089 & $M$ & $L$ \\
\hline$k$ & $2 / 28 / 85$ & NS & NS & NS & NS & NS & NS & $2.08 E+00$ & $g / L$ & $P$ \\
\hline$K$ & $2 / 28 / 85$ & NA & $\mathrm{NA}$ & 83639 & NA & NA & 1.39 & $5.31 E-02$ & $M$ & $L$ \\
\hline$K$ & $2 / 28 / 85$ & $\mathrm{NA}$ & NA & R-3639 & NA & $\mathrm{NA}$ & 1.39 & 0.0531 & moles $A$ & $P$ \\
\hline$K$ & $12 / 16 / 91$ & NA & NA & NA & NA & NA & 1.35 & 1903.7 & $\mathrm{ug} / \mathrm{mL}$ & $P$ \\
\hline$K$ & $2 / 28 / 85$ & NA. & NA & R3640 & NA & NA & 1.39 & $4.68 \mathrm{E}-02$ & $M$ & $\mathrm{~L}$ \\
\hline$K$ & $10 / 12 / 89$ & $9 / 22 / 89$ & NA & R-5779 & NA & $\mathrm{NA}$ & 1.34 & $4.10 \mathrm{E}-02$ & $M$ & $P$ \\
\hline$x+$ & $\mathrm{NA}$ & NA & NA & NA & NA & NA & 1.27 & $2.06 E-02$ & Mole/L & $\bar{L}$ \\
\hline La & $2 / 28 / 85$ & NS & NS & NS & NS & NS & NS & 0.158 & $g / L$ & $P$ \\
\hline $\mathrm{Na}$ & $2 / 28 / 85$ & NA & NA & $R 3640$ & NA & NA & 1.39 & 40.6 & $M$ & $\mathrm{~L}$ \\
\hline $\mathrm{Na}$ & $12 / 28 / 94$ & NA & $10 / 21 / 94$ & $102-\mathrm{AN}-1$ & NA & NA & 1.4 & 11.77 & $M$ & $\mathrm{~L}$ \\
\hline $\mathrm{Na}$ & $12 / 28 / 94$ & NA & $10 / 21 / 94$ & $102-A N-4$ & $\mathrm{NA}$ & NA & 1.403 & 11.09 & $M$ & $\mathrm{~L}$ \\
\hline $\mathrm{Na}$ & $12 / 28 / 94$ & NA & $10 / 21 / 94$ & 102-AN-2 & NA & NA & 1.402 & 10.74 & $M$ & $\bar{L}$ \\
\hline $\mathrm{Na}$ & $2 / 28 / 85$ & NA & NA & R3639 & NA & NA & 1.39 & 10.4 & $M$ & $p$ \\
\hline $\mathrm{Na}$ & $2 / 28 / 85$ & $\mathrm{NA}$ & $\mathrm{NA}$ & $R-3639$ & NA & NA & 1.39 & 10.4 & moles $A$ & $P$ \\
\hline $\mathrm{Na}$ & $10 / 12 / 89$ & $9 / 22 / 89$ & NA & R.5779 & NA & NA & 1.34 & $7.65 \mathrm{E}+00$ & $M$ & $P$ \\
\hline $\mathrm{Na}$ & $12 / 16 / 91$ & NA & NA & NA & NA & NA & 1.35 & 174621 & $\mathrm{ug} / \mathrm{mL}$ & $\mathbf{P}$ \\
\hline $\mathrm{Ni}$ & $12 / 28 / 94$ & $\mathrm{NA}$ & $10 / 21 / 94$ & 102-AN-2 & NA & $\mathrm{NA}$ & 1.402 & 0.007 & $M$ & L \\
\hline $\mathrm{Ni}$ & $2 / 28 / 85$ & NA & NA & R3640 & NA & NA & 1.39 & $6.55 E-03$ & $M$ & $L$ \\
\hline $\mathrm{Ni}$ & $2 / 28 / 85$ & $N A$ & $\mathrm{NA}$ & $\mathbf{R 3 6 3 9}$ & $\mathrm{NA}$ & NA & 1.39 & $6.43 E-03$ & $M$ & L \\
\hline $\mathrm{Ni}$ & $2 / 28 / 85$ & NA & NA & $R-3639$ & NA & NA & 1.39 & 0.00643 & moles $n$ & $\mathbf{P}$ \\
\hline $\mathrm{Ni}$ & $2 / 28 / 85$ & NS & NS & NS & NS & NS & NS & 3.77E-01 & $g / L$ & $P$ \\
\hline $\mathrm{Ni}$ & $10 / 12 / 89$ & $9 / 22 / 89$ & NA & $8-5779$ & NA & NA & 1.34 & $6.00 \mathrm{E}-03$ & $M$ & $P$ \\
\hline $\mathrm{Ni}$ & $12 / 28 / 94$ & NA & $10 / 21 / 94$ & $102-\mathrm{AN}-1$ & NA & NA & 1.4 & 0.006 & $M$ & $L$ \\
\hline $\mathrm{Ni}$ & $12 / 28 / 94$ & NA & $10 / 21 / 94$ & $102-A N-4$ & NA & NA & 1.403 & 0.006 & $M$ & $L$ \\
\hline $\mathrm{Ni2}+$ & NA & $\mathrm{NA}$ & $\mathrm{NA}$ & $\mathrm{NA}$ & NA & NA & 1.27 & $2.97 \mathrm{E}-03$ & Mole/L & $L$ \\
\hline $\mathrm{NO}_{2}^{-}$ & $12 / 28 / 94$ & NA & $10 / 21 / 94$ & 102-AN-4 & NA & $\mathrm{NA}$ & 1.403 & 1.84 & $M$ & $L$ \\
\hline $\mathrm{NO}_{2}^{-}$ & $12 / 28 / 94$ & NA & $10 / 21 / 94$ & 102-AN-2 & NA & NA & 1.402 & 1.81 & $M$ & $L$ \\
\hline $\mathrm{NO}_{2}^{-}$ & $12 / 28 / 94$ & NA & $10 / 21 / 94$ & 102-AN-1 & $\mathrm{NA}$ & NA & 1.4 & 1.79 & $M$ & L \\
\hline NO2- & $10 / 12 / 89$ & $9 / 22 / 89$ & NA & $R-5779$ & $\mathrm{NA}$ & $\mathrm{NA}$ & 1.34 & $1.36 \mathrm{E}+00$ & $M$ & $P$ \\
\hline NO2 & $2 / 28 / 85$ & NA & NA & R3639 & NA & NA & 1.39 & $1.32 \mathrm{E}+00$ & $M$ & L \\
\hline $\mathrm{NO2}$ & $2 / 28 / 85$ & NA & NA & $\mathrm{R}-3639$ & NA & NA & 1.39 & 1.32 & moles $\Lambda$ & $P$ \\
\hline NO2- & $2 / 28 / 85$ & NS & NS & NS & NS & NS & NS & $6.07 E+01$ & $g / L$ & $P$ \\
\hline $\mathrm{No2}$ & $2 / 28 / 85$ & $\overrightarrow{N A}$ & NA & R3640 & $\mathrm{NA}$ & NA & 1.39 & $1.28 \mathrm{E}+00$ & $M$ & $L$ \\
\hline NO2- & NA & NA & $\mathrm{NA}$ & $\mathrm{NA}$ & NA & NA & 1.27 & $1.24 \mathrm{E}+00$ & Mole/L & $\mathrm{L}$ \\
\hline NO2 & $12 / 16 / 91$ & NA & NA & $\mathrm{NA}$ & NA & NA & 1.35 & 14000 & $\mathrm{ug} / \mathrm{mL}$ & $P$ \\
\hline
\end{tabular}




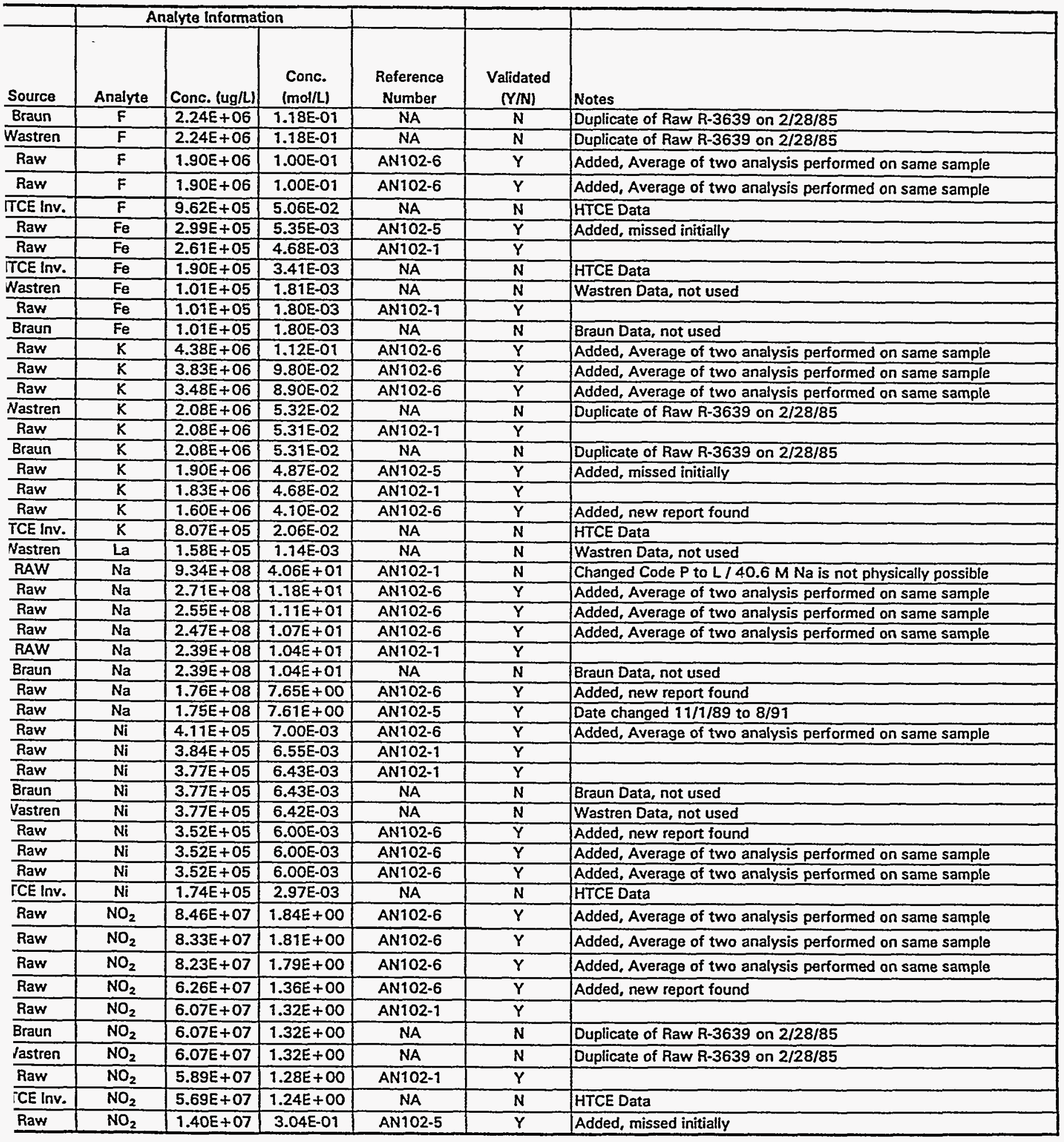




\begin{tabular}{|c|c|c|c|c|c|c|c|c|c|c|}
\hline & & & & k Waste Ch & racterization & Sample Inform & tion & & & \\
\hline Reported Chemical & Memo Date & $\begin{array}{c}\text { Start } \\
\text { Analysis } \\
\text { Date } \\
\end{array}$ & $\begin{array}{c}\text { Start } \\
\text { Sample } \\
\text { Date }\end{array}$ & $\begin{array}{l}\text { Sample } \\
\text { Number }\end{array}$ & $\begin{array}{c}\text { Tank Farm } \\
\text { Number }\end{array}$ & $\begin{array}{c}\text { Lab Sample } \\
\text { Number }\end{array}$ & $\begin{array}{l}\text { Density } \\
(\mathrm{g} / \mathrm{mL})\end{array}$ & $\begin{array}{c}\text { Reported } \\
\text { Value }\end{array}$ & Reported Unit & Cod \\
\hline NO3. & $2 / 28 / 85$ & NS & NS & NS & NS & NS & NS & $2.24 E+02$ & $g / L$ & $\mathbf{P}$ \\
\hline NO3 & $2 / 28 / 85$ & NA & NA & R3639 & NA & $\mathrm{NA}$ & 1.39 & $3.61 E+00$ & $M$ & $L$ \\
\hline $\mathrm{NO3}$ & $2 / 28 / 85$ & NA & NA & 8.3639 & NA & NA & 1.39 & 3.61 & moles I & $P$ \\
\hline $\mathrm{NO}_{3}{ }^{-}$ & $12 / 28 / 94$ & NA & $10 / 21 / 94$ & 102-AN-4 & NA & NA & 1.403 & 3.6 & $M$ & $L$ \\
\hline $\mathrm{NO}_{3}^{-}$ & $12 / 28 / 94$ & NA & $10 / 21 / 94$ & 102-AN-2 & NA & NA & 1.402 & 3.57 & $M$ & $L$ \\
\hline $\mathrm{NO}_{3}^{-}$ & $12 / 28 / 94$ & NA & $10 / 21 / 94$ & 102-AN-1 & NA & $\mathrm{NA}$ & 1.4 & 3.55 & $M$ & $\mathbf{L}$ \\
\hline NO3. & $10 / 12 / 89$ & $9 / 22 / 89$ & NA & R-5779 & NA & NA & 1.34 & $3.54 E+00$ & $M$ & $\mathbf{P}$ \\
\hline $\mathrm{NO3}$ & $2 / 28 / 85$ & NA & NA & 83640 & NA & $\mathrm{NA}$ & 1.39 & $3.38 E+00$ & $M$ & $L$ \\
\hline N03- & NA & NA & NA & NA & NA & NA & 1.27 & $2.07 E+00$ & Mole/L & L \\
\hline NO3 & $12 / 16 / 91$ & NA & NA & $\mathrm{NA}$ & NA & $\mathrm{NA}$ & 1.35 & 41000 & $\mathrm{ug} / \mathrm{mL}$ & $P$ \\
\hline $\mathrm{OH}$ & $2 / 28 / 85$ & NA & NA & R3640 & NA & NA & 1.39 & 0.648 & $M$ & $\mathbf{L}$ \\
\hline $\mathrm{OH}-$ & $10 / 12 / 89$ & $9 / 22 / 89$ & NA & R-5779 & $\mathrm{NA}$ & NA & 1.34 & $4.50 E-01$ & $M$ & $P$ \\
\hline $\mathrm{OH}^{\circ}$ & $12 / 28 / 94$ & $\mathrm{NA}$ & $10 / 21 / 94$ & 102-AN-1 & NA & NA & 1.4 & 0.24 & $M$ & $L$ \\
\hline $\mathrm{OH}^{-}$ & $12 / 28 / 94$ & NA & $10 / 21 / 94$ & 102-AN-2 & NA & NA & 1.402 & 0.24 & $M$ & $L$ \\
\hline $\mathrm{OH}^{\circ}$ & $12 / 28 / 94$ & NA & $10 / 21 / 94$ & 102-AN-4 & NA & NA & 1.403 & 0.24 & $M$ & $L$ \\
\hline $\mathrm{OH}-$ & $2 / 28 / 85$ & NS & NS & NS & NS & NS & NS & 3.42 & $g / L$ & $P$ \\
\hline $\mathrm{OH}$ & $2 / 28 / 85$ & $\mathrm{NA}$ & NA & R3639 & NA & NA & 1.39 & 0.201 & $M$ & $P$ \\
\hline $\mathrm{OH}$ & $2 / 28 / 85$ & NA & $\overline{N A}$ & R-3639 & NA & NA & 1.39 & 0.201 & moles 1 & $P$ \\
\hline $\mathrm{Pb}$ & $2 / 28 / 85$ & $\mathrm{NA}$ & NA & R3639 & NA & NA & 1.39 & $<0.00114$ & $M$ & $L$ \\
\hline $\mathrm{Pb}$ & $5 / 16 / 90$ & NA & NA & NA & NA & NA & (11.2) & $<1.1 \mathrm{e}-3$ & moles/L & $P$ \\
\hline $\mathrm{Pb}$ & $5 / 16 / 90$ & NA & NA & NA & NA & NA & (1.2) & $1.30 \mathrm{E}-03$ & moles $/ L$ & $P$ \\
\hline $\mathrm{Pb}$ & $2 / 28 / 85$ & $\mathrm{NA}$ & NA & R-3639 & NA & NA & 1.39 & 0.00114 & moles $\Lambda$ & $P$ \\
\hline $\mathrm{Pb}$ & $2 / 28 / 85$ & NS & NS & NS & NS & NS & NS & $2.36 \mathrm{E}-01$ & $g / L$ & $P$ \\
\hline $\mathrm{Pb}$ & $2 / 28 / 85$ & NA & NA & R3640 & $\mathrm{NA}$ & NA & 1.39 & 0.00105 & $M$ & L \\
\hline $\mathrm{Pb} 2+$ & NA & NA & $\mathrm{NA}$ & NA & $N A$ & NA & 1.27 & $6.48 E-05$ & Mole/L & L \\
\hline P043- & NA & NA & NA & NA & NA & NA & 1.27 & $1.08 \mathrm{E}-01$ & Mole/L & $L$ \\
\hline $\mathrm{PO}_{4}{ }^{3 \cdot}$ & $12 / 28 / 94$ & NA & $10 / 21 / 94$ & 102-AN-4 & NA & NA & 1.403 & 0.055 & $M$ & L \\
\hline $\mathrm{PO}_{4}{ }^{3-}$ & $12 / 28 / 94$ & NA & $10 / 21 / 94$ & $102-A N-2$ & NA & NA & 1.402 & 0.051 & $M$ & $L$ \\
\hline $\mathrm{PO}_{4}{ }^{3}$ & $12 / 28 / 94$ & $N A$ & $10 / 21 / 94$ & 102-AN-1 & $\mathrm{NA}$ & NA & 1.4 & 0.050 & $M$ & $L$ \\
\hline PO4 & $2 / 28 / 85$ & NA & NA & 83640 & NA & NA & 1.39 & $4.80 E-02$ & $M$ & L \\
\hline $\mathrm{PO4}$ & $2 / 28 / 85$ & NA & $\mathrm{NA}$ & R3639 & NA & NA & 1.39 & $4.73 E-02$ & $M$ & $L$ \\
\hline $\mathrm{PO4}$ & $2 / 28 / 85$ & NA & NA & $\mathrm{R}-3639$ & $\mathrm{NA}$ & NA & 1.39 & 0.0473 & moles $n$ & $P$ \\
\hline PO4- & $2 / 28 / 85$ & NS & NS & NS & NS & NS & NS & $4.49 E+00$ & $g / L$ & $P$ \\
\hline PO4 & $12 / 16 / 91$ & NA & $\mathrm{NA}$ & $N A$ & NA & NA & 1.35 & 3200 & $\mathrm{ug} / \mathrm{mL}$ & $P$ \\
\hline s042. & NA & NA & NA & NA & $\mathrm{NA}$ & $\mathrm{NA}$ & 1.27 & $1.62 E-01$ & Mole/L & $L$ \\
\hline $\mathrm{SO}_{4}^{2 \cdot}$ & $12 / 28 / 94$ & $\mathrm{NA}$ & $10 / 21 / 94$ & 102-AN-4 & NA & $\mathrm{NA}$ & 1.403 & 0.16 & $M$ & $L$ \\
\hline $\mathrm{SO}_{4}^{2-}$ & $12 / 28 / 94$ & NA & $10 / 21 / 94$ & 102-AN-1 & NA & NA & 1.4 & 0.14 & $M$ & $\bar{L}$ \\
\hline $\mathrm{SO}_{4}{ }^{2-}$ & $12 / 28 / 94$ & NA & $10 / 21 / 94$ & 102-AN-2 & NA & NA & 1.402 & 0.14 & $M$ & 4 \\
\hline SO4 & $2 / 28 / 85$ & NS & NS & NS & NS & NS & NS & $1.10 \mathrm{E}+01$ & $g / L$ & $P$ \\
\hline 504 & $2 / 28 / 85$ & NA & NA & R3639 & $\mathrm{NA}$ & NA & 1.39 & $1.14 \mathrm{E}-01$ & $M$ & $L$ \\
\hline S04 & $2 / 28 / 85$ & NA & NA & R-3639 & NA & NA & 1.39 & 0.114 & moles $n$ & $P$ \\
\hline S04 & $12 / 16 / 91$ & $\mathrm{NA}$ & NA & NA & NA & NA & 1.35 & 4300 & $\mathrm{ug} / \mathrm{mL}$ & $\mathbf{P}$ \\
\hline 504 & $2 / 28 / 85$ & NA & NA & R3640 & NA & NA & 1.39 & 1.96E-02 & $M$ & $\mathrm{~L}$ \\
\hline TOC & $12 / 28 / 94$ & NA & $10 / 21 / 94$ & $102-\mathrm{AN}-1$ & $\mathrm{NA}$ & NA & 1.4 & 27.1 & $M$ & L \\
\hline TOC & $12 / 28 / 94$ & NA & $10 / 21 / 94$ & $102-\mathrm{AN}-4$ & NA & NA & 1.403 & 26.2 & $\bar{M}$ & $L$ \\
\hline TOC & $12 / 28 / 94$ & NA & $10 / 21 / 94$ & $102-A N-2$ & NA & NA & 1.402 & 25 & $M$ & $L$ \\
\hline
\end{tabular}




\begin{tabular}{|c|c|c|c|c|c|c|}
\hline \multirow[b]{2}{*}{ Source } & \multicolumn{3}{|c|}{ Analyte Information } & \multirow[b]{2}{*}{$\begin{array}{c}\text { Reference } \\
\text { Number }\end{array}$} & \multirow[b]{2}{*}{$\begin{array}{c}\text { Validated } \\
\text { (YIN) }\end{array}$} & \multirow[b]{2}{*}{ Notes } \\
\hline & Analyte & Conc. (ug/L) & $\begin{array}{l}\text { Conc. } \\
\text { (mol/L) }\end{array}$ & & & \\
\hline Wastren & $\mathrm{NO}_{3}$ & $2.24 E+08$ & $3.61 E+00$ & NA & $\mathbf{N}$ & Duplicate of Raw R-3639 on 2/28/85 \\
\hline Raw & $\mathrm{NO}_{3}$ & $2.24 E+08$ & $3.61 E+00$ & AN102-1 & $Y$ & \\
\hline Braun & $\mathrm{NO}_{3}$ & $2.24 E+08$ & $3.61 \mathrm{E}+00$ & NA & $\mathbf{N}$ & Duplicate of Raw R-3639 on $2 / 28 / 85$ \\
\hline Raw & $\mathrm{NO}_{3}$ & $2.23 E+08$ & $3.60 E+00$ & AN $102-6$ & $\mathbf{Y}$ & Added, Average of two analysis performed on same sample \\
\hline Raw & $\mathrm{NO}_{3}$ & $2.21 E+08$ & $3.57 E+00$ & AN102-6 & $Y$ & Added, Average of two analysis performed on same sample \\
\hline Raw & $\mathrm{NO}_{3}$ & $2.20 E+08$ & $3.55 E+00$ & AN102-6 & $\mathbf{Y}$ & Added, Average of two analysis performed on same sample \\
\hline Raw & $\mathrm{NO}_{3}$ & $2.19 E+08$ & $3.54 E+00$ & AN102-6 & $\mathbf{Y}$ & Added, new report found \\
\hline Raw & $\mathrm{NO}_{3}$ & $2.10 E+08$ & $3.38 E+00$ & AN102-1 & $Y$ & \\
\hline ITCE InV. & $\mathrm{NO}_{3}$ & $1.29 E+08$ & 2.07E+00 & NA & $\mathbf{N}$ & HTCE Data \\
\hline Raw & $\mathrm{NO}_{3}$ & $4.10 E+07$ & 6.61E-01 & AN102-5 & $\mathbf{Y}$ & Added, missed initially \\
\hline RAW & $\mathrm{OH}$ & $2.59 E+07$ & $1.52 E+00$ & AN102-1 & $\bar{Y}$ & Changed Code $P$ to $L$ \\
\hline Raw & $\mathrm{OH}$ & $1.80 E+07$ & $1.06 E+00$ & AN102-6 & $\mathbf{Y}$ & Added, new report found \\
\hline Raw & $\mathrm{OH}$ & $9.60 E+06$ & $5.65 \mathrm{E}-01$ & AN102-6 & $\mathbf{Y}$ & Added, Average of two analysis performed on same sample \\
\hline Raw & $\mathrm{OH}$ & $9.60 E+06$ & 5.65E-01 & AN102-6 & $\mathbf{Y}$ & Added, Average of two analysis performed on same sample \\
\hline Raw & $\mathrm{OH}$ & $9.60 E+06$ & $5.65 \mathrm{E}-01$ & AN102-6 & $\mathbf{Y}$ & Added, Average of two analysis performed on same sample \\
\hline Wastren & $\mathrm{OH}$ & $8.05 E+06$ & 4.74E-01 & NA & $\mathbf{N}$ & Wastren Data, not used \\
\hline RAW & $\mathrm{OH}$ & $8.04 E+06$ & $4.73 \mathrm{E}-01$ & AN102-1 & $\mathbf{Y}$ & \\
\hline Braun & $\mathrm{OH}$ & $8.04 E+06$ & $4.73 E-01$ & NA & $\mathbf{N}$ & Braun Data, not used \\
\hline Raw & $\mathrm{Pb}$ & \#VALUE! & \#VALUE! & AN102-1 & $Y$ & $<$ Value \\
\hline Raw & $\mathrm{Pb}$ & \#VALUE! & \#VALUE! & AN102-4 & $\mathbf{Y}$ & Added, Missed initially \\
\hline Raw & $\mathrm{Pb}$ & 2.c9E+05 & $1.30 E-03$ & AN102-4 & $\mathbf{Y}$ & Added, Missed initially \\
\hline Braun & $\mathrm{Pb}$ & $2.36 E+05$ & $1.14 \mathrm{E}-03$ & NA & $\mathbf{N}$ & Braun Data, not used \\
\hline Nastren & $\mathrm{Pb}$ & $2.36 E+05$ & $1.14 \mathrm{E}-03$ & NA & $N$ & Wastren Data, not used \\
\hline Raw & $\mathrm{Pb}$ & $2.18 E+05$ & $1.05 \mathrm{E}-03$ & AN102-1 & $Y$ & NOT $<$ value! \\
\hline TCE Inv. & $\mathrm{Pb}$ & $1.34 \mathrm{E}+04$ & $6.48 E-05$ & NA & $N$ & HTCE Data \\
\hline TCE Inv. & $\mathrm{PO}_{4}$ & $1.03 E+07$ & $1.08 \mathrm{E}-01$ & NA & $\mathbf{N}$ & HTCE Data \\
\hline Raw & $\mathrm{PO}_{4}$ & $5.22 E+06$ & $5.50 E-02$ & AN102-6 & $\mathbf{Y}$ & Added, Average of two analysis performed on same sample \\
\hline Raw & $\mathrm{PO}_{4}$ & $4.84 E+06$ & $5.10 E-02$ & AN102-6 & $Y$ & Added, Average of two analysis performed on same sample \\
\hline Raw & $\mathrm{PO}_{4}$ & $4.75 E+06$ & $5.00 \mathrm{E}-02$ & AN102-6 & $\mathbf{Y}$ & Added, Average of two analysis performed on same sample \\
\hline Raw & $\mathrm{PO}_{4}$ & $4.56 \mathrm{E}+06$ & 4.80E-02 & AN102-1 & $\mathbf{Y}$ & \\
\hline Raw & $\mathrm{PO}_{4}$ & $4.49 E+06$ & $4.73 \mathrm{E}-02$ & AN102-1 & $Y$ & \\
\hline Braun & $\mathrm{PO}_{4}$ & $4.49 E+06$ & $4.73 E-02$ & NA & $\mathbf{N}$ & Duplicate of Raw R-3639 on 2/28/85 \\
\hline Nastren & $\mathrm{PO}_{4}$ & $4.49 E+06$ & 4.73E-02 & NA & $N$ & Duplicate of Raw R-3639 on 2/28/85 \\
\hline Raw & $\mathrm{PO}_{4}$ & $3.20 E+06$ & 3.37E-02 & AN102-5 & $\mathbf{Y}$ & Added, missed initially \\
\hline TCE Inv. & $\mathrm{SO}_{4}$ & $1.56 \mathrm{E}+07$ & $1.63 \mathrm{E}-01$ & NA & $N$ & HTCE Data \\
\hline Raw & $\mathrm{SO}_{4}$ & $1.54 E+07$ & $1.60 \mathrm{E}-01$ & AN102.6 & $Y$ & Added, Average of two analysis performed on same sample \\
\hline Raw & $\mathrm{SO}_{4}$ & $1.34 E+07$ & $1.40 E-01$ & AN102-6 & $\mathbf{Y}$ & Added, Average of two analysis performed on same sample \\
\hline Raw & $\mathrm{SO}_{4}$ & $1.34 E+07$ & $1.40 E-01$ & AN102-6 & 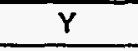 & Added, Average of two analysis performed on same sample \\
\hline Vastren & $\mathrm{SO}_{4}$ & $1.10 E+07$ & $1.15 \mathrm{E}-01$ & NA & $N$ & Duplicate of Raw R-3639 on $2 / 28 / 85$ \\
\hline Raw & $\mathrm{SO}_{4}$ & $1.10 E+07$ & $1.14 \mathrm{E}-01$ & AN102-1 & $\mathbf{Y}$ & \\
\hline Braun & $\mathrm{SO}_{4}$ & $1.10 \mathrm{E}+07$ & $1.74 \mathrm{E}-01$ & NA & $\mathbf{N}$ & Duplicate of Raw R-3639 on $2 / 28 / 85$ \\
\hline Raw & $\mathrm{SO}_{4}$ & $4.30 E+06$ & $4.48 \mathrm{E}-02$ & AN102-5 & $\mathbf{Y}$ & Added, missed initially \\
\hline Raw & $\mathrm{SO}_{4}$ & $1.88 \mathrm{E}+06$ & $1.96 \mathrm{E}-02$ & AN102-1 & $\mathbf{Y}$ & \\
\hline Raw & TOC & $3.25 E+08$ & $2.71 E+01$ & AN102-6 & $\underline{Y}$ & Added, Average of two analysis performed on same sample \\
\hline Raw & TOC & $3.15 E+08$ & $2.62 E+01$ & AN102-6 & $\mathbf{Y}$ & Added, Average of two analysis performed on same sample \\
\hline Raw & TOC & $3.00 E+08$ & $2.50 E+01$ & AN102-6 & $\bar{Y}$ & Added, Average of two analysis performed on same sample \\
\hline
\end{tabular}




\begin{tabular}{|c|c|c|c|c|c|c|c|c|c|c|}
\hline \multicolumn{11}{|c|}{ Tank Waste Characterization Sample Information } \\
\hline Reported Chemical & Memo Date & $\begin{array}{c}\text { Start } \\
\text { Analysis } \\
\text { Date }\end{array}$ & $\begin{array}{c}\text { Start } \\
\text { Sample } \\
\text { Date } \\
\end{array}$ & $\begin{array}{l}\text { Sample } \\
\text { Number }\end{array}$ & $\begin{array}{c}\text { Tank Farm } \\
\text { Number }\end{array}$ & $\begin{array}{c}\text { Lab Sampla } \\
\text { Number }\end{array}$ & $\begin{array}{c}\text { Density } \\
\text { (g/mL) }\end{array}$ & $\begin{array}{c}\text { Reported } \\
\text { Value }\end{array}$ & Reported Unit & Cod \\
\hline TOC & $2 / 28 / 85$ & NA & NA & R3639 & NA & NA & 1.39 & $3.37 E+01$ & $\mathrm{gC} n$ & L \\
\hline TOC & $2 / 28 / 85$ & NA & NA & R-3639 & NA & NA & 1.39 & 33.7 & $\mathrm{gmn}$ & P \\
\hline TOC & $2 / 28 / 85$ & NS & NS & NS & NS & NS & NS & $3.37 E+01$ & $g / L$ & $\dot{\mathrm{P}}$ \\
\hline TOC & $2 / 28 / 85$ & NA & NA & R3640 & NA & NA & 1.39 & $2.98 \mathrm{E}+01$ & $\mathrm{gCn}$ & L \\
\hline TOC & $10 / 12 / 89$ & $9 / 22 / 89$ & NA & R-5779 & NA & NA & 1.34 & $2.73 E+01$ & $g / L$ & $\overline{\mathrm{P}}$ \\
\hline TOC wt\% C (wet) & NA & NA & NA & $\mathrm{NA}$ & NA & NA & 1.27 & $7.36 \mathrm{E}-01$ & $w+\%$ & L \\
\hline$U$ & $2 / 28 / 85$ & NA & NA & R3640 & $\mathrm{NA}$ & NA & 1.39 & $<0.107$ & $g / L$ & $\bar{L}$ \\
\hline U & $2 / 28 / 85$ & NA & NA & 83639 & NA & $\overline{N A}$ & 1.39 & 0.0247 & $g n$ & 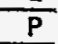 \\
\hline
\end{tabular}




\begin{tabular}{|c|c|c|c|c|c|c|c|c|c|}
\hline & & & & Tank Waste Char & erization Sar & ple Informatic & & & \\
\hline Reported Chemical & Memo Date & $\begin{array}{c}\text { Start Analysis } \\
\text { Date }\end{array}$ & $\begin{array}{c}\text { Start Sample } \\
\text { Date }\end{array}$ & Sample Number & $\begin{array}{c}\text { Tank Farm } \\
\text { Number }\end{array}$ & $\begin{array}{c}\text { Lab Sample } \\
\text { Number }\end{array}$ & $\begin{array}{l}\text { Density } \\
\text { (g/mL) }\end{array}$ & $\begin{array}{l}\text { Reported } \\
\text { Value }\end{array}$ & Reported \\
\hline $\mathrm{Al3+}$ & NA & $\mathrm{NA}$ & NA & NA & NA & NA & 1.43 & $1.20 E+00$ & Molell \\
\hline Al & $3 / 6 / 85$ & NS & NS & RAT-AN107-1B & NS & NS & NS & 0.23 & moles. \\
\hline $\mathrm{Al}$ & $2 / 28 / 85$ & NA & NA & R3638 & NA & NA & 1.35 & 0.211 & $M$ \\
\hline $\mathrm{Al}$ & $2 / 28 / 85$ & NS & NS & R-3638 & NS & NS & 1.35 & 0.211 & moles! \\
\hline Al & $2 / 28 / 85$ & $\mathrm{NA}$ & NA & R3637 & $\overline{N A}$ & NA & 1.41 & 0.198 & $\bar{M}$ \\
\hline Al & $2 / 28 / 85$ & NS & NS & R-3637 & NS & NS & 1.41 & 0.198 & molesi \\
\hline A! & $3 / 6 / 85$ & NS & NS & RAT-AN107-1A & NS & NS & NS & 0.198 & moles! \\
\hline Al & $12 / 4 / 86$ & NS & NS & NS & NS & NS & NS & $3.78 E+\infty 0$ & $g / L$ \\
\hline$\overline{A l}$ & $12 / 4 / 86$ & NA & $\overline{N A}$ & 8650 & NA & NA & NA & 0.14 & $M$ \\
\hline $\mathrm{Al}$ & $12 / 4 / 86$ & NS & NS & R-8650 & NS & NS & NS & 0.14 & moles I \\
\hline$\overline{A l}$ & $6 / 29 / 90$ & NA & NA & R-5887/CC- $44 \mathrm{H}$ & NA & NA & NA & 0.0762 & $M$ \\
\hline $\mathrm{Al}$ & $8 / 10 / 94$ & $\overline{N A}$ & $2 \pi / 93$ & $R 3155$ & NA & NA & 1.384 & 0.089 & WT $\%$ \\
\hline $\mathrm{Al}$ & $8 / 10 / 94$ & NA & $2 / 7 / 93$ & ก3154 & NA & NA & 1.392 & 0.085 & WT \% \\
\hline Al & $8 / 10 / 94$ & $\overline{N A}$ & $2 / 7 / 93$ & R2686 & NA & NA & 1.047 & 0.084 & WT $\%$ \\
\hline$\overline{A l}$ & $8 / 10 / 94$ & NA & $5 / 13 / 94$ & R4047 & $\mathrm{NA}$ & $\overline{\mathrm{NA}}$ & 1.345 & 489 & $\mathrm{ug} / \mathrm{mL}$ \\
\hline $\mathrm{Al}$ & $8 / 10 / 94$ & NA & $5 / 13 / 94$ & $\mathrm{R} 4047$ & NA & NA & 1.363 & 413 & $\mathrm{ug} / \mathrm{ml}$ \\
\hline Al & $8 / 10 / 94$ & NA & $5 / 73 / 94$ & R4048 & NA & NA & 1.349 & 409 & $u g / m L$ \\
\hline Al & $8 / 10 / 94$ & NA & $5 / 13 / 94$ & R4048 & NA & NA & 1.352 & 386 & $\mathrm{ug} / \mathrm{mL}$ \\
\hline Al & $8 / 10 / 94$ & NA & $5 / 13 / 94$ & $\mathrm{R} 4047$ & NA & NA & NA & 0.0154 & $g / 100 \mathrm{~g}$ \\
\hline Al & $8 / 10 / 94$ & NA & $5 / 13 / 94$ & R4047 & NA & NA & 1.15 & 171 & $\overline{u g} / \mathrm{mL}$ \\
\hline A! & $8 / 10 / 94$ & NA & $5 / 13 / 94$ & $\mathrm{R} 4048$ & NA & NA & 1.157 & 169 & $\mathrm{ug} / \mathrm{mL}$ \\
\hline Al & $8 / 10 / 94$ & NA & $5 / 13 / 94$ & R4048 & NA & NA & & 0.0136 & $g / 100 g$ \\
\hline $\mathrm{Al}$ & $8 / 10 / 94$ & NA & $5 / 13 / 94$ & 84047 & NA & NA & 1.141 & 122 & $\mathrm{ug} / \mathrm{mL}$ \\
\hline Al & $8 / 10 / 94$ & $\mathrm{NA}$ & $5 / 13 / 94$ & R4048 & NA & NA & 1.139 & 78 & $\mathrm{ug} / \mathrm{mL}$ \\
\hline Al & $8 / 10 / 94$ & NA & $5 / 13 / 94$ & R4047 & NA & NA & NA & 0.006 & $g / 100 \mathrm{~g}$ \\
\hline Al & $8 / 10 / 94$ & $\mathrm{NA}$ & $5 / 13 / 94$ & R4048 & NA & NA & NA & 0.0054 & $g / 100 \mathrm{~g}$ \\
\hline $\mathrm{Ba}$ & $5 / 16 / 90$ & NA & NA & NA & NA & NA & NA & $5.00 \mathrm{E}-03$ & $M$ \\
\hline $\mathrm{Ba}$ & $3 / 6 / 85$ & NS & NS & RAT-AN107-1B & NS & NS & NS & 0.0049 & moles $n$ \\
\hline $\mathrm{Ba}$ & $2 / 28 / 85$ & NA & $\mathrm{NA}$ & R3638 & NA & NA & 1.35 & 0.000242 & $M$ \\
\hline $\mathrm{Ba}$ & $2 / 28 / 85$ & NA & NA & $R-3638$ & NA & NA & 1.35 & 0.000242 & moles $\Lambda$ \\
\hline $\mathrm{Ba}$ & $2 / 28 / 85$ & NA & NA & 83637 & NA & NA & 1.41 & 0.0000503 & $M$ \\
\hline $\mathrm{Ba}$ & $2 / 28 / 85$ & NS & NS & 8-3637 & NS & NS & 1.41 & 0.0000503 & moles $\hat{n}$ \\
\hline $\mathrm{Ba}$ & $3 / 6 / 85$ & NS & NS & RAT-AN107-1A & NS & NS & NS & 0.0000503 & moles $n$ \\
\hline $\mathrm{Ca}$ & $3 / 6 / 85$ & NS & NS & RAT-AN107-1B & NS & NS & NS & 0.032 & moles $n$ \\
\hline $\mathrm{Ca}$ & $2 / 28 / 85$ & NS & NS & R-3637 & NS & NS & 1.41 & 0.0173 & moles $/$ \\
\hline $\mathrm{Ca}$ & $3 / 6 / 85$ & NS & NS & RAT-AN107-1A & NS & NS & NS & 0.0173 & moles $n$ \\
\hline $\mathrm{Ca}$ & $2 / 28 / 85$ & NA & $N A$ & 83637 & NA & NA & 1.41 & 0.0173 & $M$ \\
\hline $\mathrm{Ca}$ & $2 / 28 / 85$ & NS & NS & R-3638 & NS & NS & 1.35 & 0.0159 & moles $A$ \\
\hline $\mathrm{Ca}$ & $2 / 28 / 85$ & NA & NA & R3638 & NA & NA & 1.35 & 0.0159 & $M$ \\
\hline $\mathrm{Ca}$ & $8 / 10 / 94$ & NA & $5 / 13 / 94$ & $R 4048$ & NA & NA & 1.349 & 589 & $\mathrm{ug} / \mathrm{mL}$ \\
\hline $\mathrm{Ca}$ & $8 / 10 / 94$ & NA & $5 / 13 / 94$ & R4047 & NA & NA & 1.345 & 585 & $\mathrm{ug} / \mathrm{mL}$ \\
\hline $\mathrm{Ca}$ & $8 / 10 / 94$ & $\mathrm{NA}$ & $2 / 7 / 93$ & $\mathrm{R} 3155$ & NA & NA & 1.384 & 0.041 & $W t \%$ \\
\hline $\mathrm{Ca}$ & $8 / 10 / 94$ & NA & $5 / 13 / 94$ & R4048 & NA & NA & 1.352 & 549 & $\mathrm{ug} / \mathrm{mL}$ \\
\hline $\mathrm{Ca}$ & $8 / 10 / 94$ & NA & $5 / 13 / 94$ & R4047 & NA & NA & 1.363 & 545 & $\mathrm{ug} / \mathrm{mL}$ \\
\hline $\mathrm{Ca}$ & $6 / 29 / 90$ & NA & NA & R5887 & NA & NA & NA & 0.0154 & $M$ \\
\hline $\mathrm{Ca}$ & $8 / 10 / 94$ & NA & $2 / 7 / 93$ & R3154 & NA & NA & 1.392 & 0.038 & $W t \%$ \\
\hline $\mathrm{Ca}$ & $8 / 10 / 94$ & NA & $2 n 7193$ & R2686 & NA & NA & 1.047 & 0.038 & $W_{t} \%$ \\
\hline $\mathrm{Ca}$ & $8 / 10 / 94$ & NA & $5 / 13 / 94$ & R4047 & NA & NA & 1.157 & 256 & $\mathrm{ug} / \mathrm{mL}$ \\
\hline
\end{tabular}




\begin{tabular}{|c|c|c|c|c|c|c|c|}
\hline \multirow[b]{2}{*}{ Code } & \multirow[b]{2}{*}{ Source } & \multicolumn{3}{|c|}{ Analyte Information } & \multirow[b]{2}{*}{$\begin{array}{c}\text { Reference } \\
\text { Number } \\
\end{array}$} & \multirow[b]{2}{*}{ Validated (Y/N) } & \multirow[b]{2}{*}{ Notes } \\
\hline & & Analyte & Conc. (ug/L) & $\begin{array}{l}\text { Conc. } \\
\text { (mollt) }\end{array}$ & & & \\
\hline$L$ & HTCE InV. & $\mathrm{Al}$ & $3.23 E+07$ & $1.20 E+00$ & NA & $\mathbf{N}$ & HTCE Data \\
\hline$P$ & Braun & $\mathrm{Al}$ & $6.21 E+06$ & 2.30E-01 & $\mathrm{NA}$ & $N$ & No reference \\
\hline$L$ & Raw & Al & $5.69 E+06$ & $2.11 E-01$ & AN107.3 & $\bar{Y}$ & \\
\hline$L$ & Braun & $\mathrm{Al}$ & $5.69 \mathrm{E}+06$ & 2.11E-01 & NA & $D$ & Duplicate of Raw R3638 \\
\hline$P$ & Raw & $\overline{A l}$ & $5.34 E+06$ & $1.98 \mathrm{E}-01$ & AN107-3 & $\bar{Y}$ & \\
\hline$P$ & Braun & $\overrightarrow{\mathrm{Al}}$ & $5.34 E+06$ & $1.98 \mathrm{E}-01$ & NA & $D$ & Duplicate of Raw R3637 \\
\hline$P$ & Braun & Al & $5.34 \mathrm{E}+06$ & $1.98 \mathrm{E}-01$ & NA & $\mathrm{D}$ & Duplicate of Raw R3637 \\
\hline$P$ & Wastren & Al & $3.78 E+06$ & $1.40 \mathrm{E}-01$ & NA & $D$ & Duplicate of Raw 8650 \\
\hline$P$ & Raw & $\mathrm{Al}$ & $3.78 \mathrm{E}+06$ & $1.40 \mathrm{E}-01$ & AN107-4 & $\mathrm{Y}$ & \\
\hline $\mathbf{P}$ & Braun & Al & $3.78 \mathrm{E}+06$ & $1.40 E-01$ & NA & $D$ & Duplicate of Raw 8650 \\
\hline$P$ & Raw & $\mathrm{Al}$ & $2.06 E+06$ & $7.62 \mathrm{E}-02$ & AN107-7 & $Y$ & \\
\hline$P$ & Raw & $\mathrm{Al}$ & $1.23 E+06$ & $4.57 \mathrm{E}-02$ & AN107-8 & $N$ & Average of 3 duplicate samples \\
\hline $\mathrm{P}$ & Raw & $\mathrm{Al}$ & $1.18 \mathrm{E}+06$ & $4.39 E-02$ & AN107.8 & $N$ & Average of 3 duplicate samples \\
\hline$P$ & Raw & Al & $8.79 E+05$ & $3.26 E-02$ & AN107-8 & $N$ & Average of 3 duplicate samples \\
\hline$P$ & Raw & $\mathrm{Al}$ & $4.89 E+05$ & $1.81 E .02$ & AN107.8 & $\bar{Y}$ & Table 4 \\
\hline$P$ & Raw & Al & $4.13 E+05$ & $1.53 E-02$ & AN107-8 & $Y$ & Table 4 \\
\hline$P$ & Raw & $\mathrm{Al}$ & $4.09 E+05$ & $1.52 \mathrm{E}-02$ & AN107-8 & $\bar{Y}$ & Table 4 \\
\hline$P$ & Raw & $\mathrm{Al}$ & $3.86 E+05$ & $1.43 E-02$ & AN107-8 & $\bar{Y}$ & Table 4 \\
\hline$L$ & Raw & Al & $1.85 E+05$ & $6.85 E-03$ & AN107-8 & $Y$ & $\begin{array}{l}\text { Value is for Centrate / Changed sample state } \\
\text { Supernatant to Centrate and Code P to L }\end{array}$ \\
\hline$P$ & Raw & Al & $1.71 E+05$ & $6.34 E-03$ & AN107.8 & $N$ & Water washed value, not true centrate \\
\hline$P$ & Raw & Al & $1.69 E+05$ & $6.26 \mathrm{E}-03$ & AN107-8 & $N$ & Water washed value, not true centrate \\
\hline L & Raw & Al & $1.63 E+05$ & $6.05 E-03$ & AN107-8 & $Y$ & $\begin{array}{l}\text { Value is for Centrate / Changed sample state } \\
\text { Supernatant to Centrate and Code } \mathrm{P} \text { to } 1 .\end{array}$ \\
\hline$P$ & Raw & $\overline{A l}$ & $7.22 E+05$ & $4.52 \mathrm{E}-03$ & AN107-8 & $N$ & Water washed value, not true centrate \\
\hline$P$ & Raw & $\mathrm{Al}$ & $7.80 E+04$ & $2.89 E-03$ & AN107-8 & $\mathbf{N}$ & Water washed value, not true centrate \\
\hline$P$ & Raw & Al & $7.20 \mathrm{E}+04$ & 2.67E-03 & AN107-8 & $N$ & Water washed value, not true centrate \\
\hline$P$ & Raw & Al & $6.48 E+04$ & $2.40 E-03$ & AN107-8 & $N$ & Water washed value, not true centrate \\
\hline$P$ & Raw & $\mathrm{Ba}$ & $6.87 E+05$ & $5.00 \mathrm{E}-03$ & AN107-6 & $\bar{Y}$ & \\
\hline $\mathbf{P}$ & Braun & $\mathrm{Ba}$ & $6.73 E+05$ & $4.90 \mathrm{E}-03$ & NA & $\mathbf{N}$ & No refference \\
\hline $\mathbf{P}$ & RAW & $\mathrm{Ba}$ & $3.32 E+04$ & $2.42 \mathrm{E}-04$ & AN107-3 & $\bar{Y}$ & Added Sample State Supernate \\
\hline$L$ & Braun & $\mathrm{Ba}$ & $3.32 E+04$ & $2.42 E-04$ & NA & $D$ & Duplicate of Raw R3638 \\
\hline$P$ & RAW & $\overline{B a}$ & $6.91 E+03$ & $5.03 E-05$ & AN107-3 & $\bar{Y}$ & Added Sample State Supernate \\
\hline $\mathbf{P}$ & Braun & $\overline{\mathrm{Ba}}$ & $6.91 E+03$ & $5.03 E-05$ & NA & $\mathrm{D}$ & Duplicate of Raw R3637 \\
\hline$P$ & Braun & $\mathrm{Ba}$ & $6.91 E+03$ & 5.03E-05 & NA & $D$ & Duplicate of Raw R3637 \\
\hline $\mathbf{P}$ & Braun & $\mathrm{Ca}$ & $1.28 E+06$ & $3.19 E-02$ & NA & $N$ & No reference \\
\hline$P$ & Braun & $\mathrm{Ca}$ & $6.93 E+05$ & $1.73 \mathrm{E}-02$ & NA & D & Duplicate of Raw R3637 \\
\hline$P$ & Braun & $\mathrm{Ca}$ & $6.93 E+05$ & $1.73 E-02$ & NA & $D$ & Duplicate of Raw $\mathrm{R3} 637$ \\
\hline $\mathbf{P}$ & RAW & $\mathrm{Ca}$ & $6.92 E+05$ & $1.73 E-02$ & AN107-3 & $\bar{Y}$ & \\
\hline$L$ & Braun & $\mathrm{Ca}$ & $6.37 E+05$ & $1.59 \mathrm{E}-02$ & NA & D & Duplicate of Raw R3638 \\
\hline $\mathbf{P}$ & RAW & $\mathrm{Ca}$ & $6.36 E+05$ & $1.59 \mathrm{E}-02$ & AN107.3 & $\bar{Y}$ & Table 4 \\
\hline$P$ & RAW & $\mathrm{Ca}$ & $5.89 E+05$ & $1.47 E-02$ & AN707-8 & $\bar{Y}$ & Table 4 \\
\hline$P$ & RAW & $\mathrm{Ca}$ & $5.85 E+05$ & $1.46 E-02$ & AN107-8 & $Y$ & Table 4 \\
\hline$P$ & RAW & $\mathbf{C a}$ & $5.67 E+05$ & $1.41 \mathrm{E}-02$ & AN107-8 & $N$ & Average of 3 duplicate samples \\
\hline $\mathbf{P}$ & RAW & $\mathbf{C a}$ & $5.49 E+05$ & $1.37 \mathrm{E}-02$ & AN107-8 & $\bar{Y}$ & Table 4 \\
\hline$P$ & RAW & $\mathrm{Ca}$ & $5.45 E+05$ & $1.36 E-02$ & AN107-8 & $\bar{Y}$ & Table 4 \\
\hline $\mathbf{P}$ & RAW & $\mathrm{Ca}$ & $5.32 E+05$ & 1.33E-02 & AN107-7 & $\bar{Y}$ & Changed value 0.0133 to 0.0154 \\
\hline$P$ & RAW & $\mathrm{Ca}$ & $5.29 E+05$ & $1.32 \mathrm{E}-02$ & AN107-8 & $N$ & Average of 3 duplicate samples \\
\hline$P$ & RAW & $\mathbf{C a}$ & $3.98 E+05$ & $9.93 E-03$ & AN107-8 & $N$ & Average of 3 duplicate samples \\
\hline$P$ & RAW & $\mathrm{Ca}$ & $2.56 E+05$ & $6.39 \mathrm{E}-03$ & AN107-8 & $\mathbf{N}$ & Water washed value, not true centrate \\
\hline
\end{tabular}




\begin{tabular}{|c|c|c|c|c|c|c|c|c|c|}
\hline Reported Chemical & Memo Date & $\begin{array}{c}\text { Start Analysis } \\
\text { Date }\end{array}$ & $\begin{array}{c}\text { Start Sample } \\
\text { Date }\end{array}$ & Sample Number & $\begin{array}{l}\text { Tank Farm } \\
\text { Number }\end{array}$ & $\begin{array}{c}\text { Lab Sample } \\
\text { Number }\end{array}$ & $\begin{array}{l}\text { Density } \\
(\mathrm{g} / \mathrm{mL})\end{array}$ & $\begin{array}{l}\text { Reported } \\
\text { Value }\end{array}$ & Reported U \\
\hline $\mathrm{Ca}$ & $8 / 10 / 94$ & NA & $5 / 13 / 94$ & R4047 & NA & NA & 1.15 & 242 & $\mathrm{ug} / \mathrm{mL}$ \\
\hline $\mathrm{Ca}$ & $8 / 10 / 94$ & NA & $5 / 13 / 94$ & R4048 & $\overline{N A}$ & NA & 1.141 & 175 & $\mathrm{ug} / \mathrm{mL}$ \\
\hline $\mathrm{Ca}$ & $8 / 10 / 94$ & NA & $5 / 13 / 94$ & R4047 & NA & NA & 1.349 & 160 & $\mathrm{ug} / \mathrm{mL}$ \\
\hline Cd & $8 / 10 / 94$ & $\mathrm{NA}$ & $5 / 13 / 94$ & $\mathrm{R} 4048$ & $\mathrm{NA}$ & NA & 1.349 & 70 & $\mathrm{ug} / \mathrm{mL}$ \\
\hline Cd & $8 / 10 / 94$ & $\mathrm{NA}$ & $5 / 13 / 94$ & R4047 & NA & NA & 1.345 & 69 & $\mathrm{ug} / \mathrm{mL}$ \\
\hline Cd & $8 / 10 / 94$ & NA & $5 / 13 / 94$ & R4047 & NA & NA & 1.363 & 66 & $\mathrm{ug} / \mathrm{mL}$ \\
\hline Cd & $8 / 10 / 94$ & NA & $5 / 13 / 94$ & R4048 & NA & NA & 1.352 & 66 & $\mathrm{ug} / \mathrm{mL}$ \\
\hline Cd & $5 / 16 / 90$ & NA & NA & NA & NA & NA & NA & $5.50 \mathrm{E}-04$ & $M$ \\
\hline Cd & $2 / 28 / 85$ & $\overline{N A}$ & NA & R3637 & NA & NA & 1.41 & 0.000545 & $\bar{M}$ \\
\hline Cd & $2 / 28 / 85$ & NS & NS & $\mathrm{R}-3637$ & NS & NS & 1.41 & 0.000545 & moles $n$ \\
\hline Cd & $3 / 6 / 85$ & NS & NS & RAT-AN107-1A & NS & NS & NS & 0.000545 & moles $n$ \\
\hline $\overrightarrow{C d}$ & $8 / 10 / 94$ & NA & $5 / 13 / 94$ & 84047 & NA & NA & 1.157 & 30 & $\mathrm{ug} / \mathrm{mL}$ \\
\hline$\overline{C d}$ & $8 / 10 / 94$ & NA & $5 / 13 / 94$ & R4047 & NA & NA & 1.15 & 28 & $\mathrm{ug} / \mathrm{mL}$ \\
\hline Cd & $8 / 10 / 94$ & NA & $5 / 13 / 94$ & R4048 & NA & NA & 1.141 & 20 & $\mathrm{ug} / \mathrm{mL}$ \\
\hline $\mathrm{Cd}$ & $8 / 10 / 94$ & NA & $5 / 13 / 94^{\circ}$ & R4047 & NA & $\overline{N A}$ & 1.349 & 16 & $\mathrm{ug} / \mathrm{mL}$ \\
\hline $\mathrm{Ce}$ & $8 / 10 / 94$ & NA & $5 / 13 / 94$ & $R 4048$ & NA & NA & 1.352 & 58 & $\mathrm{ug} / \mathrm{mL}$ \\
\hline $\mathrm{Ce}$ & $8 / 10 / 94$ & NA & $5 / 13 / 94$ & R4048 & NA & NA & 1.349 & 56 & $u g / m L$ \\
\hline $\mathrm{Ce}$ & $8 / 10 / 94$ & NA & $5 / 13 / 94$ & R4047 & NA & NA & 1.345 & 54 & $\mathrm{ug} / \mathrm{mL}$ \\
\hline $\mathrm{Ce}$ & $8 / 10 / 94$ & NA & $5 / 13 / 94$ & 84047 & NA & NA & 1.363 & 50 & $\mathrm{ug} / \mathrm{mL}$ \\
\hline $\mathrm{Cr}$ & $2 / 28 / 85$ & NA & NA & 83638 & NA & NA & 1.35 & $<5.93 E-02$ & $M$ \\
\hline $\mathrm{Cl}$ & NA & NA & NA & NA & NA & NA & 1.43 & $1.60 \mathrm{E}-01$ & Mole/L \\
\hline $\mathrm{Cl}^{2}$ & $8 / 10 / 94$ & $\mathrm{NA}$ & $2 \pi / 93$ & R3154 & NA & NA & 1.392 & 0.23 & $W T \%$ \\
\hline $\mathrm{Cl}^{\circ}$ & $8 / 10 / 94$ & NA & $2 / 7 / 93$ & R3155 & NA & NA & 1.384 & 0.19 & $W T \%$ \\
\hline $\mathrm{Cl}^{\circ}$ & $8 / 10 / 94$ & NA & $2 / 7 / 93$ & 82686 & NA & NA & 1.047 & 0.25 & WT \% \\
\hline $\mathrm{Cl}$ & $8 / 10 / 94$ & NA & $5 / 13 / 94$ & $\mathrm{R} 4048$ & NA & NA & 1.352 & 2130 & $\mathrm{ug} / \mathrm{mL}$ \\
\hline $\mathrm{Cl}$ & $2 / 28 / 85$ & NS & NS & R-3638 & NS & NS & 1.35 & 0.0593 & moles $n$ \\
\hline $\mathrm{Cr}$ & $8 / 10 / 94$ & NA & $5 / 13 / 94$ & R4047 & NA & NA & 1.345 & 2030 & $\mathrm{ug} / \mathrm{mL}$ \\
\hline $\mathrm{Cl}^{\circ}$ & $8 / 10 / 94$ & $N A$ & $5 / 13 / 94$ & R4048 & NA & NA & 1.349 & 2000 & $u g / \mathrm{mL}$ \\
\hline $\mathrm{Cl}^{-}$ & $8 / 10 / 94$ & NA & $5 / 13 / 94$ & $\mathrm{R} 4047$ & NA & NA & 1.363 & 1870 & ug/mL \\
\hline $\mathrm{Cl}^{2}$ & $2 / 28 / 85$ & NA & NA & R3637 & NA & NA & 1.41 & 0.041 & $M$ \\
\hline $\mathrm{Cl}$ & $2 / 28 / 85$ & NS & NS & $\mathrm{R}-3637$ & NS & NS & 1.41 & 0.041 & moles $\Lambda$ \\
\hline $\mathrm{Cr}^{\circ}$ & $8 / 10 / 94$ & NA & $5 / 13 / 94$ & R4048 & $\mathrm{NA}$ & NA & 1.157 & 1060 & $\mathrm{ug} / \mathrm{mL}$ \\
\hline $\mathrm{Cr}$ & $8 / 10 / 94$ & $\mathrm{NA}$ & $5 / 13 / 94$ & 84047 & NA & NA & 1.15 & 994 & $\mathrm{ug} / \mathrm{mL}$ \\
\hline $\mathrm{Cr}$ & $8 / 10 / 94$ & NA & $5 / 13 / 94$ & R4048 & $N A$ & NA & NA & 0.08 & $\mathrm{~g} / 100 \mathrm{~g}$ \\
\hline Cr & $8 / 10 / 94$ & NA & $5 / 13 / 94$ & R4047 & NA & NA & NA & 0.07 & $\mathrm{~g} / 100 \mathrm{~g}$ \\
\hline $\mathrm{Cl}^{-}$ & $8 / 10 / 94$ & NA & $5 / 13 / 94$ & 84047 & $\mathrm{NA}$ & NA & 1.141 & 593 & $\mathrm{ug} / \mathrm{mL}$ \\
\hline $\mathrm{Cl}$ & $8 / 10 / 94$ & NA & $5 / 13 / 94$ & $\mathrm{R} 4048$ & NA & NA & 1.139 & 574 & $\mathrm{ug} / \mathrm{mL}$ \\
\hline$a^{\prime}$ & $8 / 10 / 94$ & NA & $5 / 13 / 94$ & 84047 & NA & NA & NA & 0.03 & $g / 100 g$ \\
\hline $\mathrm{Cr}$ & $8 / 10 / 94$ & NA & $5 / 13 / 94$ & R4048 & NA & NA & NA & 0.03 & $g / 100 \mathrm{~g}$ \\
\hline $\mathrm{Cr}_{\mathrm{r}}$ & $5 / 16 / 90$ & NA & NA & $\mathrm{NA}$ & NA & NA & NA & $4.40 \mathrm{E}-03$ & $M$ \\
\hline $\mathrm{Cr}$ & $6 / 29 / 90$ & NA & NA & R-5887/CC- $44 \mathrm{H}$ & NA & NA & NA & 0.00428 & $M$ \\
\hline $\mathrm{Cr}$ & $2 / 28 / 85$ & NA & NA & R3638 & NA & NA & 1.35 & 0.00514 & $M$ \\
\hline $\mathrm{Cr}$ & $2 / 28 / 85$ & NS & NS & $R-3638$ & NS & NS & 1.35 & 0.00514 & moles $n$ \\
\hline $\mathrm{Cr}$ & $3 / 6 / 85$ & NS & NS & RAT-AN107-1B & NS & NS & NS & 0.0046 & moles $\Lambda$ \\
\hline $\mathrm{Cr}$ & $2 / 28 / 85$ & NA & NA & R3637 & NA & NA & 1.41 & 0.00442 & $M$ \\
\hline $\mathrm{Cl}$ & $2 / 28 / 85$ & NS & NS & R-3637 & NS & NS & 1.41 & 0.00442 & moles $n$ \\
\hline
\end{tabular}




\begin{tabular}{|c|c|c|c|c|c|c|c|}
\hline \multirow[b]{2}{*}{ Code } & \multirow[b]{2}{*}{ Source } & \multicolumn{3}{|c|}{ Analyte Information } & \multirow[b]{2}{*}{$\begin{array}{l}\text { Reference } \\
\text { Number }\end{array}$} & \multirow[b]{2}{*}{ Vafidated $(Y / N)$} & \multirow[b]{2}{*}{ Notes } \\
\hline & & Analyte & Conc. (ug/L) & $\begin{array}{l}\text { Conc. } \\
\text { (mol/L) }\end{array}$ & & & \\
\hline $\mathbf{P}$ & RAW & $\mathrm{Ca}$ & $2.42 E+05$ & $6.04 E-03$ & AN107-8 & $N$ & Water washed value, not true centrate \\
\hline $\mathbf{P}$ & RAW & $\mathrm{Ca}$ & $1.75 \mathrm{E}+05$ & 4.37E-03 & AN107-8 & $\bar{N}$ & Water washed value, not true centrate \\
\hline $\mathbf{P}$ & RAW & $\mathrm{Ca}$ & $1.60 E+05$ & $3.99 \mathrm{E}-03$ & AN107-8 & $\mathbf{N}$ & Water washed value, not true centrate \\
\hline $\mathbf{P}$ & RAW & $\mathrm{Cd}$ & $7.00 E+04$ & $6.23 E-0.4$ & AN107-8 & $Y$ & Table 4 \\
\hline $\mathbf{P}$ & RAW & Cd & $6.90 \mathrm{E}+04$ & $6.14 E-04$ & AN107-8 & $\bar{Y}$ & Table 4 \\
\hline $\mathbf{P}$ & RAW & $\mathrm{Cd}$ & $6.60 \mathrm{E}+04$ & $5.87 E-04$ & AN107-8 & $\bar{Y}$ & Table 4 \\
\hline $\mathbf{P}$ & RAW & $\mathrm{Cd}$ & $6.60 \mathrm{E}+04$ & 5.87E-04 & AN107-8 & $Y$ & Table 4 \\
\hline $\mathbf{P}$ & Raw & $\mathrm{Cd}$ & $6.18 E+04$ & $5.50 E-04$ & AN107-6 & $Y$ & \\
\hline $\mathbf{P}$ & RAW & $\mathrm{Cd}$ & $6.13 E+04$ & $5.45 \mathrm{E}-04$ & AN107-3 & $Y$ & \\
\hline $\mathbf{P}$ & Braun & $\mathrm{Cd}$ & $6.13 E+04$ & 5.45E-04 & NA & $N$ & Braun Data, not used \\
\hline $\mathrm{P}$ & Braun & Cd & $6.13 E+04$ & 5.45E-04 & NA & $\mathbf{N}$ & Braun Data, not used \\
\hline $\mathbf{P}$ & RAW & $\mathrm{Cd}$ & $3 . \mathrm{COE}+04$ & 2.67E-04 & AN107-8 & $N$ & Water Washed Sample \\
\hline $\mathbf{P}$ & RAW & $\mathrm{Cd}$ & $2.80 E+04$ & $2.49 E-04$ & AN107-8 & $N$ & Water Washed Sample \\
\hline $\mathbf{P}$ & RAW & $\mathrm{Cd}$ & $2.00 E+04$ & $1.78 \mathrm{E}-04$ & AN107-8 & $N$ & Water Washed Sample \\
\hline $\mathbf{P}$ & RAW & $\mathrm{Cd}$ & $1.60 E+04$ & 1.42E-04 & AN107-8 & $\mathrm{N}$ & Water Washed Sample \\
\hline$P$ & RAW & $\mathrm{Ce}$ & $5.80 E+04$ & $4.14 E-04$ & AN107-8 & $\bar{Y}$ & Table 4 \\
\hline $\mathbf{P}$ & RAW & $\mathrm{Ce}$ & $5.60 E+04$ & $4.00 \mathrm{E}-04$ & AN107-8 & $\bar{Y}$ & Table 4 \\
\hline$P$ & RAW & $\mathrm{Ce}$ & $5.40 E+04$ & $3.85 \mathrm{E}-04$ & AN107-8 & $\bar{Y}$ & Table 4 \\
\hline$P$ & RAW & $\mathrm{Ce}$ & $5.00 E+04$ & $3.57 \mathrm{E}-04$ & AN107-8 & $Y$ & Table 4 \\
\hline $\mathbf{L}$ & Raw & $\mathrm{Cl}$ & \#VALUE! & \#VALUE! & AN107-3 & $\bar{Y}$ & $<$ Value \\
\hline L & HTCE Inv. & $\mathrm{Cl}$ & $5.66 \mathrm{E}+06$ & $1.60 \mathrm{E}-01$ & NA & $N$ & HTCE Data \\
\hline$P$ & Raw & $\mathrm{Cl}$ & $3.20 E+06$ & $9.03 \mathrm{E}-02$ & AN107-8 & $N$ & Average of 3 duplicate samples \\
\hline$P$ & Raw & $\mathrm{Cl}$ & $2.63 E+06$ & 7.42E-02 & AN107-8 & $N$ & Average of 2 duplicate samples \\
\hline$P$ & Raw & $\mathrm{Cl}$ & $2.62 E+06$ & $7.38 \mathrm{E}-02$ & AN107.8 & $N$ & Average of 3 duplicate samples \\
\hline $\mathbf{P}$ & Raw & $\mathrm{Cl}$ & $2.13 E+06$ & 6.01E-02 & AN107-8 & $\bar{Y}$ & Table 4 \\
\hline$L$ & Braun & $\mathrm{Cl}$ & $2.10 E+06$ & $5.93 E-02$ & NA & $\mathrm{N}$ & $<$ Value, Braun dated, not used \\
\hline$P$ & Raw & $\mathrm{Cl}$ & $2.03 E+06$ & $5.73 \mathrm{E}-02$ & AN107-8 & $Y$ & Table 4 \\
\hline$P$ & Raw & $\mathrm{Cl}$ & $2.00 E+06$ & $5.64 E-02$ & AN107-8 & $Y$ & Table 4 \\
\hline $\mathbf{P}$ & Raw & $\mathrm{Cl}$ & $1.87 E+06$ & $5.27 \mathrm{E}-02$ & AN107-8 & $\bar{Y}$ & Table 4 \\
\hline$P$ & Raw & $\mathrm{Cl}$ & $1.45 \mathrm{E}+06$ & $4.10 E-02$ & AN107-3 & $\bar{Y}$ & \\
\hline$P$ & Braun & $\mathrm{Cl}$ & $1.45 E+06$ & $4.10 \mathrm{E}-02$ & NA & $D$ & \begin{tabular}{|l|} 
Duplicate of Raw R3637 \\
\end{tabular} \\
\hline$P$ & Raw & $\mathrm{Cl}$ & $1.06 E+06$ & 2.99E-02 & AN107-8 & $N$ & Water washed value, not true centrate \\
\hline$P$ & Raw & $\mathrm{Cl}$ & $9.94 \mathrm{E}+05$ & $2.80 \mathrm{E}-02$ & AN107-8 & $N$ & Water washed value, not true centrate \\
\hline$\underline{P}$ & Raw & a & $9.60 E+05$ & $2.71 E-02$ & AN107-8 & $Y$ & Table 11B \\
\hline$\underline{P}$ & Raw & $\mathrm{Cl}$ & $8.40 E+05$ & $2.37 \mathrm{E}-02$ & AN107-8 & $Y$ & Table 11A \\
\hline $\mathbf{P}$ & Raw & C1 & $5.93 E+05$ & $1.67 E-02$ & AN107-8 & $N$ & Water washed value, not true centrate \\
\hline$P$ & Raw & $\mathrm{Cl}$ & $5.74 E+05$ & $1.62 \mathrm{E}-02$ & AN107.8 & $N$ & Water washed value, not true centrate \\
\hline$\underline{P}$ & Raw & $\mathrm{Cl}$ & $3.60 E+05$ & $1.02 \mathrm{E}-02$ & AN107.8 & $\mathbf{N}$ & Water washed value, not true centrate \\
\hline$\underline{P}$ & Raw & $\mathrm{Cl}$ & $3.60 E \div 05$ & $1.02 \mathrm{E}-02$ & AN107-8 & $\mathrm{N}$ & Water washed value, not true centrate \\
\hline$\underline{P}$ & Raw & $\mathrm{Cr}$ & $2.29 E+05$ & $4.40 \mathrm{E}-03$ & AN107-6 & $\bar{Y}$ & \\
\hline P & Raw & $\mathrm{Cr}$ & $2.23 E+05$ & $4.28 \mathrm{E}-03$ & AN107-7 & $\bar{Y}$ & \\
\hline$\underline{P}$ & RAW & $\mathrm{Cr}+6$ & $2.67 E+05$ & $5.14 E-03$ & AN107-3 & $\bar{Y}$ & \\
\hline$\underline{L}$ & Braun & $\mathrm{Cr}+6$ & $2.67 E+05$ & $5.14 \mathrm{E}-03$ & NA & $D$ & Duplicate of Raw R3638 \\
\hline$\underline{\underline{P}}$ & Braun & $\mathrm{Cr}+6$ & $2.39 E+05$ & $4.60 \mathrm{E}-03$ & NA & $N$ & No reference \\
\hline $\bar{P}$ & RAW & $\mathrm{Cr}+6$ & $2.30 E+05$ & $4.42 \mathrm{E}-03$ & AN107-3 & $Y$ & \\
\hline$\underline{P}$ & Braun & $\mathrm{Cr}+6$ & $2.30 E+05$ & $4.42 E-03$ & NA & $\bar{D}$ & Duplicate of Raw R3637 \\
\hline
\end{tabular}




\begin{tabular}{|c|c|c|c|c|c|c|c|c|c|}
\hline & & & & Tank Waste Charc & erization Sam & ple Informatio & & & \\
\hline Reported Chemical & Memo Date & $\begin{array}{c}\text { Start Analysis } \\
\text { Date }\end{array}$ & $\begin{array}{c}\text { Start Sample } \\
\text { Date }\end{array}$ & Sample Number & $\begin{array}{c}\text { Tank Farm } \\
\text { Number }\end{array}$ & $\begin{array}{c}\text { Lab Sample } \\
\text { Number }\end{array}$ & $\begin{array}{l}\text { Density } \\
(\mathrm{g} / \mathrm{mL})\end{array}$ & $\begin{array}{c}\text { Reported } \\
\text { Value }\end{array}$ & Reported 1 \\
\hline $\mathrm{Cr}$ & $3 / 6 / 85$ & NS & NS & RAT-AN107-1A & NS & NS & NS & 0.00442 & molesf \\
\hline $\mathrm{Cr}$ & $8 / 10 / 94$ & NA & $2 / 7 / 93$ & 83155 & $\mathrm{NA}$ & NA & 1.384 & 0.013 & $\mathrm{Wt} \%$ \\
\hline $\mathrm{Cr}$ & $8 / 10 / 94$ & NA & $2 / 7 / 93$ & R3154 & NA & NA & 1.392 & 0.012 & $W t \%$ \\
\hline $\mathrm{Cr}$ & $8 / 10 / 94$ & NA & $2 / 7 / 93$ & $R 2686$ & NA & $\mathrm{NA}$ & 1.047 & 0.012 & Wt\% \\
\hline$F$ & $2 / 28 / 85$ & NA & NA & 83637 & NA & NA & 1.41 & $<2.22 \mathrm{E}-01$ & $M$ \\
\hline$F$ & $2 / 28 / 85$ & NA & NA & $\mathrm{R} 3638$ & $\mathrm{NA}$ & NA & 1.35 & $<1.86 E-01$ & $M$ \\
\hline$F$ & $2 / 28 / 85$ & NS & NS & R-3637 & NS & NS & 1.41 & 0.222 & moles $n$ \\
\hline$F$ & $2 / 28 / 85$ & NS & NS & $R \cdot 3638$ & NS & NS & 1.35 & 0.186 & moles $\Lambda$ \\
\hline$F$ & $8 / 10 / 94$ & $\mathrm{NA}$ & $5 / 13 / 94$ & R4047 & NA & NA & 1.15 & 1870 & $\mathrm{ug} / \mathrm{mL}$ \\
\hline $\mathbf{F}$ & $8 / 10 / 94$ & NA & $5 / 13 / 94$ & R4048 & NA & NA & 1.157 & 1750 & $u \mathrm{~g} / \mathrm{mL}$ \\
\hline $\mathrm{F}-$ & $\mathrm{NA}$ & NA & NA & NA & $\mathrm{NA}$ & NA & 1.43 & $8.54 E-02$ & Mole/L \\
\hline$F$ & $8 / 10 / 94$ & NA & $5 / 13 / 94$ & R4047 & NA & NA & 1.141 & 934 & $\mathrm{ug} / \mathrm{mL}$ \\
\hline$F$ & $8 / 10 / 94$ & NA & $5 / 13 / 94$ & R4048 & NA & $\mathrm{NA}$ & 1.139 & 843 & $\mathrm{ug} / \mathrm{mL}$ \\
\hline$F$ & $8 / 10 / 94$ & NA & $5 / 13 / 94$ & $\mathrm{R} 4047$ & NA & NA & NA & 0.07 & $\mathrm{~g} / 100_{\mathrm{g}}$ \\
\hline$F$ & $8 / 10 / 94$ & $\mathrm{NA}$ & $5 / 13 / 94$ & $\mathrm{R} 4048$ & NA & NA & NA & 0.06 & $\mathrm{~g} / 100 \mathrm{~g}$ \\
\hline $\mathrm{Fe}$ & $2 / 28 / 85$ & NA & NA & R3638 & $\mathrm{NA}$ & NA & 1.35 & 0.0526 & $M$ \\
\hline $\mathrm{Fe}$ & $2 / 28 / 85$ & NS & NS & R-3638 & NS & NS & 1.35 & 0.0526 & moles $\Pi$ \\
\hline $\mathrm{Fe}$ & $3 / 6 / 85$ & NS & NS & RAT-AN107-1B & NS & NS & NS & 0.043 & moles $n$ \\
\hline $\mathrm{Fe}$ & $8 / 10 / 94$ & NA & $5 / 13 / 94$ & $\mathrm{R} 4048$ & NA & NA & 1.349 & 1980 & $\mathrm{ug} / \mathrm{mL}$ \\
\hline $\mathrm{Fe}$ & $8 / 10 / 94$ & NA & $5 / 13 / 94$ & $\mathrm{R} 4048$ & NA & $\overline{N A}$ & 1.352 & 1850 & $\mathrm{ug} / \mathrm{mL}$ \\
\hline $\mathrm{Fe}$ & $2 / 28 / 85$ & NA & NA & R3637 & NA & NA & 1.41 & 0.0331 & $\bar{M}$ \\
\hline $\mathrm{Fe}$ & $2 / 28 / 85$ & NS & NS & R-3637 & NS & NS & 1.41 & 0.0331 & moles $n$ \\
\hline $\mathrm{Fe}$ & $3 / 6 / 85$ & NS & NS & RAT-AN107-1A & NS & NS & NS & 0.0331 & moles $\Lambda$ \\
\hline $\mathrm{Fe}$ & $12 / 4 / 86$ & NA & NA & 8650 & NA & NA & NA & 0.033 & $M$ \\
\hline $\mathrm{Fe}$ & $12 / 4 / 86$ & NS & NS & R-8650 & NS & NS & NS & 0.033 & moles $n$ \\
\hline $\mathrm{Fe}$ & $12 / 4 / 86$ & NS & NS & NS & NS & NS & NS & $1.84 E+00$ & $g / L$ \\
\hline $\mathrm{Fe}$ & $6 / 29 / 90$ & NA & NA & $\mathrm{R}-5887 / \mathrm{CC}-44 \mathrm{H}$ & NA & NA & $\mathrm{NA}$ & 0.0309 & $M$ \\
\hline $\mathrm{Fe}$ & $8 / 10 / 94$ & $\mathrm{NA}$ & $5 / 13 / 94$ & R4047 & $\overline{\mathrm{NA}}$ & NA & 1.345 & 1640 & $\mathrm{ug} / \mathrm{mL}$ \\
\hline $\mathrm{Fe}$ & $8 / 10 / 94$ & NA & $2 \sqrt{7 / 93}$ & R3155 & NA & NA & 1.384 & 0.11 & WT \% \\
\hline $\mathrm{Fe}$ & $8 / 10 / 94$ & NA & $5 / 13 / 94$ & $\mathrm{R} 4047$ & NA & NA & 1.363 & 1520 & $\mathrm{ug} / \mathrm{mL}$ \\
\hline $\mathrm{Fe}$ & $8 / 10 / 94$ & NA & $2 \pi / 93$ & 83154 & NA & NA & 1.392 & 0.107 & WT \% \\
\hline $\mathrm{Fe}$ & $8 / 10 / 94$ & NA & $2 / 7 / 93$ & $R 2686$ & $\mathrm{NA}$ & $\mathrm{NA}$ & 1.047 & 0.105 & WT $\%$ \\
\hline $\mathrm{Fe}$ & $8 / 10 / 94$ & NA & $5 / 13 / 94$ & R4048 & NA & NA & NA & 0.0654 & $\mathrm{~g} / 100 \mathrm{~g}$ \\
\hline $\mathrm{Fe}$ & $8 / 10 / 94$ & NA & $5 / 13 / 94$ & R4048 & NA & $\mathrm{NA}$ & 1.157 & 709 & $\mathrm{ug} / \mathrm{mL}$ \\
\hline $\mathrm{Fe}$ & $8 / 10 / 94$ & NA & $5 / 13 / 94$ & $\mathrm{R} 4047$ & NA & $\mathrm{NA}$ & NA & 0.0568 & $\mathrm{~g} / 100 \mathrm{~g}$ \\
\hline $\mathrm{Fe}$ & $8 / 10 / 94$ & NA & $5 / 13 / 94$ & R4047 & NA & NA & 1.15 & 423 & $\mathrm{ug} / \mathrm{mL}$ \\
\hline $\mathrm{Fe}$ & $8 / 10 / 94$ & NA & $5 / 13 / 94$ & $R 4048$ & NA & $\mathrm{NA}$ & NA & 0.0227 & $g / 100 g$ \\
\hline $\mathrm{Fe} 3+($ total $\mathrm{Fe})$ & NA & NA & NA & NA & $\mathrm{NA}$ & NA & 1.43 & $3.95 \mathrm{E}-03$ & Mole/L \\
\hline $\mathrm{Fe}$ & $8 / 10 / 94$ & NA & $5 / 13 / 94$ & 84047 & NA & NA & NA & 0.0148 & $g / 100 \mathrm{~g}$ \\
\hline $\mathrm{Fe}$ & $8 / 10 / 94$ & NA & $5 / 13 / 94$ & $\mathrm{R} 4047$ & NA & NA & 1.141 & 156 & $u \mathrm{~g} / \mathrm{mL}$ \\
\hline $\mathrm{Fe}$ & $8 / 10 / 94$ & NA & $5 / 13 / 94$ & $\mathrm{R} 4048$ & NA & NA & 1.139 & 102 & $\mathrm{ug} / \mathrm{mL}$ \\
\hline$K$ & $8 / 10 / 94$ & NA & $2 / 7193$ & 83155 & $\overline{N A}$ & NA & 1.384 & 0.188 & WT\% \\
\hline $\bar{K}$ & $8 / 10 / 94$ & NA & $2 / 7 / 93$ & R3154 & NA & NA & 1.392 & 0.146 & WT \% \\
\hline$K$ & $3 / 6 / 85$ & NS & NS & RAT-AN107-18 & NS & NS & NS & 0.048 & molesn \\
\hline$K$ & $8 / 10 / 94$ & $\mathrm{NA}$ & $5 / 13 / 94$ & 84048 & NA & NA & 1.349 & 1800 & $\mathrm{ug} / \mathrm{mL}$ \\
\hline$K$ & $8 / 10 / 94$ & NA & $5 / 13 / 94$ & R4047 & NA & $\mathrm{NA}$ & 1.345 & 1770 & ug/mL \\
\hline
\end{tabular}




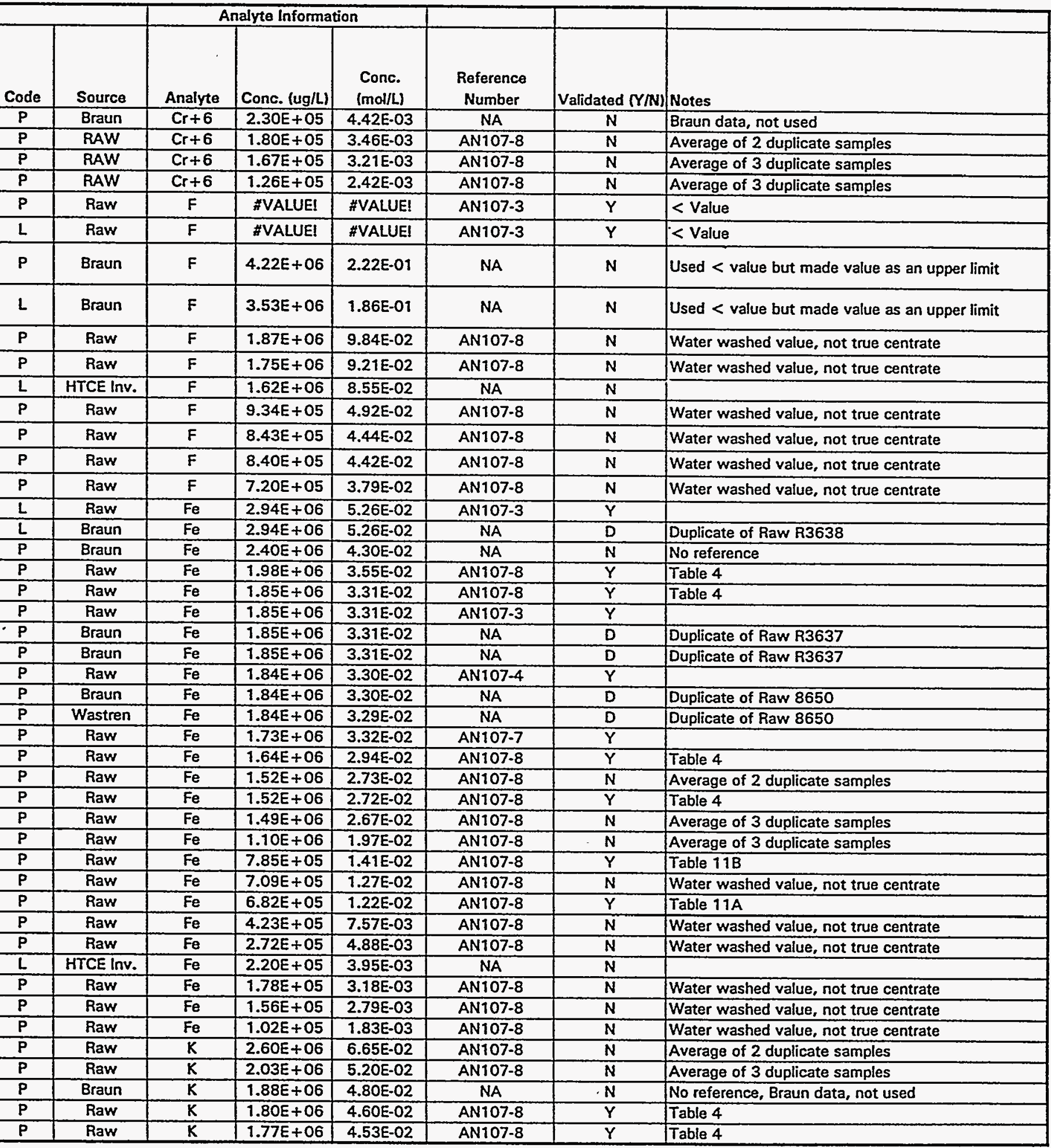


TWRS Privatization Support Pro Chemical Liquids

\begin{tabular}{|c|c|c|c|c|c|c|c|c|c|}
\hline & & & & Tank Waste Char & terization San & ple Informatio & & & \\
\hline Reported Chemical & Memo Date & $\begin{array}{c}\text { Start Analysis } \\
\text { Date }\end{array}$ & $\begin{array}{c}\text { Start Sample } \\
\text { Date }\end{array}$ & Sample Number & $\begin{array}{c}\text { Tank Farm } \\
\text { Number }\end{array}$ & $\begin{array}{c}\text { Lab Sample } \\
\text { Number }\end{array}$ & $\begin{array}{c}\text { Density } \\
(\mathrm{g} / \mathrm{mL})\end{array}$ & $\begin{array}{c}\text { Reported } \\
\text { Value }\end{array}$ & Reported \\
\hline$K$ & $8 / 10 / 94$ & \begin{tabular}{|l|}
$\mathrm{NA}$ \\
\end{tabular} & $5 / 13 / 94$ & $\mathrm{R} 4048$ & NA & NA & 1.352 & 1730 & $\mathrm{ug} / \mathrm{mL}$ \\
\hline$K$ & $2 / 28 / 85$ & NA & $\mathrm{NA}$ & R3637 & NA & NA & 1.41 & $4.39 \mathrm{E} \cdot 02$ & $M$ \\
\hline$K$ & $2 / 28 / 85$ & NS & NS & R-3637 & NS & NS & 1.41 & 0.0439 & moles I \\
\hline$K$ & $3 / 6 / 85$ & NS & NS & RAT-AN107-1A & NS & NS & NS & 0.0439 & moles $\lambda$ \\
\hline$K$ & $8 / 10 / 94$ & NA & $5 / 13 / 94$ & R4047 & NA & NA & 1.363 & 1690 & $\mathrm{ug} / \mathrm{mL}$ \\
\hline$K$ & $2 / 28 / 85$ & NA & $\mathrm{NA}$ & R3638 & NA & NA & 1.35 & 0.0425 & $M$ \\
\hline$K$ & $2 / 28 / 85$ & NS & NS & R-3638 & NS & NS & 1.35 & 0.0425 & moles $n$ \\
\hline$K$ & $8 / 10 / 94$ & NA & $2 \pi / 93$ & $\mathrm{R} 2686$ & NA & NA & 1.047 & 0.123 & WT \% \\
\hline$K+$ & NA & NA & NA & NA & NA & NA & 1.43 & $2.84 E-02$ & Mole $/ 2$ \\
\hline$K$ & $8 / 10 / 94$ & NA & $5 / 13 / 94$ & $\mathrm{R} 4047$ & NA & NA & NA & 0.0632 & $g / 100 g$ \\
\hline$K$ & $8 / 10 / 94$ & NA & $5 / 13 / 94$ & $\mathrm{R} 4048$ & NA & NA & 1.157 & 738 & $\mathrm{ug} / \mathrm{mL}$ \\
\hline$K$ & $8 / 10 / 94$ & NA & $5 / 13 / 94$ & 84048 & NA & NA & NA & 0.0611 & $g / 100 \mathrm{~g}$ \\
\hline$K$ & $8 / 10 / 94$ & NA & $5 / 13 / 94$ & R4047 & NA & NA & 1.15 & 728 & $\mathrm{ug} / \mathrm{mL}$ \\
\hline$K$ & $8 / 10 / 94$ & NA & $5 / 13 / 94$ & R4047 & NA & NA & 1.141 & 495 & $\mathrm{ug} / \mathrm{mL}$ \\
\hline$K$ & $8 / 10 / 94$ & NA & $5 / 13 / 94$ & R4048 & NA & $\mathrm{NA}$ & 1.139 & 427 & $\mathrm{ug} / \mathrm{mL}$ \\
\hline $\mathrm{K}$ & $8 / 10 / 94$ & NA & $5 / 13 / 94$ & R4047 & $\mathrm{NA}$ & NA & NA & 0.0255 & $g / 100 \mathrm{~g}$ \\
\hline$K$ & $8 / 10 / 94$ & NA & $5 / 13 / 94$ & $\mathrm{R} 4048$ & NA & NA & NA & 0.0236 & $g / 100 \mathrm{~g}$ \\
\hline $\mathrm{La}$ & $2 / 28 / 85$ & NS & NS & $\mathrm{R}-3638$ & NS & NS & 1.35 & 0.00101 & moles $\hat{n}$ \\
\hline $\mathrm{La}$ & $2 / 28 / 85$ & NS & NS & R-3637 & NS & NS & 1.41 & 0.000277 & moles $n$ \\
\hline La & $3 / 6 / 85$ & NS & NS & RAT-AN107-1A & NS & NS & NS & 0.000277 & moles $\Lambda$ \\
\hline $\mathrm{Na}$ & $6 / 29 / 90$ & NA & NA & $\mathrm{R}-5887 / \mathrm{CC}-44 \mathrm{H}$ & NA & NA & NA & 8.22 & $M$ \\
\hline $\mathrm{Na}$ & $2 / 28 / 85$ & NA & NA & 83637 & $\mathrm{NA}$ & NA & 1.41 & 10.1 & $M$ \\
\hline $\mathrm{Na}$ & $2 / 28 / 85$ & NS & NS & $R-3637$ & NS & NS & 1.41 & 10.1 & moles $\Lambda$ \\
\hline $\mathrm{Na}$ & $3 / 6 / 85$ & NS & NS & RAT-AN107-1A & NS & NS & NS & 10.1 & moles \\
\hline $\mathrm{Na}$ & $2 / 28 / 85$ & NA & NA & R3638 & NA & NA & 1.35 & 9.8 & $M$ \\
\hline $\mathrm{Na}$ & $2 / 28 / 85$ & NS & NS & R-3638 & NS & NS & 1.35 & 9.8 & moles $n$ \\
\hline $\mathrm{Na}$ & $12 / 4 / 86$ & NA & NA & $R 8650$ & NA & NA & NA & 9.15 & $\mathrm{moln}$ \\
\hline $\mathrm{Na}$ & $12 / 4 / 86$ & NS & NS & R-8650 & NS & NS & NS & 9.15 & moles $n$ \\
\hline $\mathrm{Na}$ & $12 / 4 / 86$ & NS & NS & NS & NS & NS & NS & 210 & $g / L$ \\
\hline $\mathrm{Na}$ & $8 / 10 / 94$ & NA & $5 / 13 / 94$ & $\mathrm{R} 4048$ & $\mathrm{NA}$ & NA & 1.349 & 207000 & $\mathrm{ug} / \mathrm{mL}$ \\
\hline $\mathrm{Na}$ & $8 / 10 / 94$ & $\mathrm{NA}$ & $5 / 13 / 94$ & $\mathrm{R} 4047$ & NA & NA & 1.345 & 201000 & $\mathrm{ug} / \mathrm{mL}$ \\
\hline $\mathrm{Na}$ & $8 / 10 / 94$ & NA & $2 \pi / 93$ & $\mathbf{R 3 1 5 5}$ & NA & NA & 1.384 & 14.275 & Wt\% \\
\hline $\mathrm{Na}$ & $3 / 6 / 85$ & NS & NS & RAT-AN107-1B & NS & NS & NS & 8.5 & moles $n$ \\
\hline $\mathrm{Na}$ & $8 / 10 / 94$ & NA & $2 / 7 / 93$ & R3154 & NA & NA & 1.392 & 14.108 & Wt\% \\
\hline $\mathrm{Na}$ & $8 / 10 / 94$ & NA & $5 / 13 / 94$ & $\mathrm{R} 4047$ & NA & $\mathrm{NA}$ & 1.363 & 196000 & $\mathrm{ug} / \mathrm{mL}$ \\
\hline $\mathrm{Na}$ & $8 / 10 / 94$ & NA & $5 / 13 / 94$ & R4048 & NA & NA & 1.352 & 196000 & $\mathrm{ug} / \mathrm{mL}$ \\
\hline $\mathrm{Na}$ & $6 / 29 / 90$ & NA & NA & R5887 & NA & NA & NA & 8.22 & $M$ \\
\hline $\mathrm{Na}$ & $8 / 10 / 94$ & NA & $2 / 7 / 93$ & R2686 & NA & NA & 1.047 & 14.431 & Wt\% \\
\hline $\mathrm{Na}$ & $8 / 10 / 94$ & NA & $5 / 13 / 94$ & 84047 & NA & $\mathrm{NA}$ & 1.157 & 87500 & $\mathrm{ug} / \mathrm{mL}$ \\
\hline $\mathrm{Na}$ & $8 / 10 / 94$ & NA & $5 / 13 / 94$ & R4047 & NA & NA & 1.15 & 84100 & ug $/ \mathrm{mL}$ \\
\hline $\mathrm{Na}$ & $8 / 10 / 94$ & NA & $5 / 13 / 94$ & R4048 & NA & NA & 1.141 & 61000 & $\mathrm{ug} / \mathrm{mL}$ \\
\hline $\mathrm{Na}$ & $8 / 10 / 94$ & NA & $5 / 13 / 94$ & R4047 & NA & NA & 1.349 & 50800 & $\mathrm{ug} / \mathrm{mL}$ \\
\hline Nd & $3 / 6 / 85$ & NS & NS & RAT-AN107-18 & NS & NS & NS & 0.00098 & moles $n$ \\
\hline Nd & $8 / 10 / 94$ & NA & $5 / 13 / 94$ & $R 4048$ & $\mathrm{NA}$ & $\mathrm{NA}$ & 1.349 & 113 & $\mathrm{ug} / \mathrm{mL}$ \\
\hline Nd & $8 / 10 / 94$ & NA & $5 / 13 / 94$ & R4048 & NA & NA & 1.352 & 109 & $\mathrm{ug} / \mathrm{mL}$ \\
\hline $\mathrm{Nd}$ & $8 / 10 / 94$ & NA & $5 / 13 / 94$ & R4047 & NA & NA & 1.345 & 103 & Ug $/ \mathrm{mL}$ \\
\hline $\mathrm{Nd}$ & $8 / 10 / 94$ & $\mathrm{NA}$ & $5 / 13 / 94$ & 84047 & NA & NA & 1.363 & 99 & $\mathrm{ug} / \mathrm{mL}$ \\
\hline $\mathrm{Nd}$ & $8 / 10 / 94$ & NA & $5 / 13 / 94$ & 84047 & NA & NA & 1.157 & 41 & $\mathrm{ug} / \mathrm{mL}$ \\
\hline $\mathrm{Nd}$ & $8 / 10 / 94$ & NA & $5 / 13 / 94$ & R4047 & $\mathrm{NA}$ & NA & 1.15 & 36 & $\mathrm{ug} / \mathrm{mL}$ \\
\hline Nd & $8 / 10 / 94$ & $\mathrm{NA}$ & $5 / 13 / 94$ & $\mathrm{R} 4048$ & NA & NA & 1.141 & 22 & $\mathrm{ug} / \mathrm{mL}$ \\
\hline
\end{tabular}




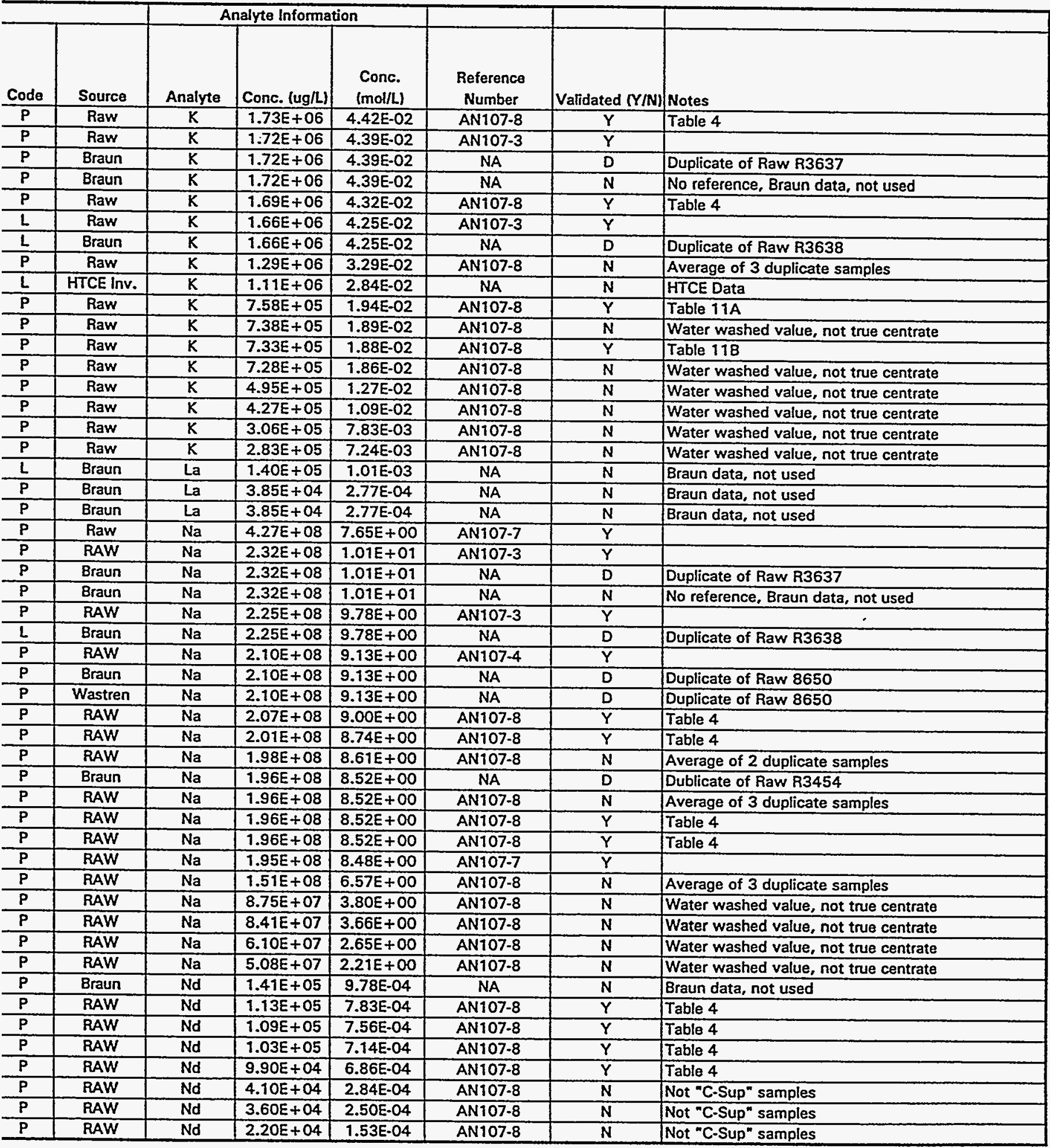


TWRS Privatization Support Prc Chemical Liquids

\begin{tabular}{|c|c|c|c|c|c|c|c|c|c|}
\hline & & & & Tank Waste Char & erization San & ple Informatio & & & \\
\hline Reported Chemical & Memo Date & $\begin{array}{c}\text { Start Analysis } \\
\text { Date }\end{array}$ & $\begin{array}{c}\text { Start Sample } \\
\text { Date }\end{array}$ & Sample Number & $\begin{array}{c}\text { Tank Farm } \\
\text { Number }\end{array}$ & $\begin{array}{c}\text { Lab Sample } \\
\text { Number }\end{array}$ & $\begin{array}{c}\text { Density } \\
(\mathrm{g} / \mathrm{mL})\end{array}$ & $\begin{array}{c}\text { Reported } \\
\text { Value } \\
\end{array}$ & Reported \\
\hline Nd & $8 / 10 / 94$ & NA & $5 / 13 / 94$ & R4047 & NA & NA & 1.349 & 16 & $\mathrm{ug} / \mathrm{mL}$ \\
\hline $\mathrm{Ni}$ & $8 / 10 / 94$ & NA & $5 / 13 / 94$ & R3155 & NA & NA & 1.384 & 0.33 & WT $\%$ \\
\hline $\mathrm{Ni}$ & $6 / 29 / 90$ & $\mathrm{NA}$ & NA & $\mathrm{R}-5887 / \mathrm{CC}-44 \mathrm{H}$ & NA & NA & NA & 0.01 & $\bar{M}$ \\
\hline $\mathrm{Ni}$ & $8 / 10 / 94$ & NA & $5 / 13 / 94$ & $R 4047$ & $\mathrm{NA}$ & NA & 1.345 & 573 & $\mathrm{ug} / \mathrm{mL}$ \\
\hline $\mathrm{Ni}$ & $8 / 10 / 94$ & NA & $5 / 13 / 94$ & R4048 & NA & NA & 1.349 & 573 & $u g / m L$ \\
\hline $\mathrm{Ni}$ & $8 / 10 / 94$ & NA & $5 / 13 / 94$ & R4047 & NA & NA & 1.363 & 541 & $\mathrm{ug} / \mathrm{mL}$ \\
\hline $\mathrm{Ni}$ & $8 / 10 / 94$ & NA & $5 / 13 / 94$ & $R 4048$ & NA & NA & 1.352 & .537 & $\mathrm{ug} / \mathrm{mL}$ \\
\hline$\cdot \mathrm{Ni}$ & $2 / 28 / 85$ & NA & NA & R3637 & NA & NA & 1.41 & 0.0088 & $M$ \\
\hline $\mathrm{Ni}$ & $2 / 28 / 85$ & NS & NS & R-3637 & NS & NS & 1.41 & 0.0088 & moles $\lambda$ \\
\hline $\mathrm{Ni}$ & $3 / 6 / 85$ & NS & NS & RAT-AN107-1A & NS & NS & NS & 0.0088 & moles $\hat{n}$ \\
\hline $\mathrm{Ni}$ & $2 / 28 / 85$ & NA & NA & R3638 & NA & $\mathrm{NA}$ & 1.35 & 0.00816 & $\bar{M}$ \\
\hline $\mathrm{Ni}$ & $2 / 28 / 85$ & NS & NS & $R-3638$ & NS & NS & 1.35 & 0.00816 & moles $\hat{n}$ \\
\hline $\mathrm{Ni}$ & $8 / 10 / 94$ & NA & $5 / 13 / 94$ & R3154 & NA & NA & 1.392 & 0.032 & WT \% \\
\hline $\mathrm{Ni}$ & $3 / 6 / 85$ & NS & NS & RAT-AN107-1B & NS & NS & NS & 0.0071 & moles $\hat{n}$ \\
\hline $\mathrm{Ni}$ & $8 / 10 / 94$ & NA & $5 / 13 / 94$ & $R 2686$ & NA & NA & 1.047 & 0.032 & WT \% \\
\hline $\mathrm{Ni}$ & $8 / 10 / 94$ & NA & $5 / 13 / 94$ & R4048 & $\mathrm{NA}$ & NA & 1.157 & 245 & $u g / m L$ \\
\hline $\mathrm{Ni}$ & $8 / 10 / 94$ & NA & $5 / 13 / 94$ & $\mathrm{R} 4047$ & NA & NA & NA & 0.0202 & $g / 100 \mathrm{~g}$ \\
\hline $\mathrm{Ni}$ & $8 / 10 / 94$ & NA & $5 / 13 / 94$ & R4047 & NA & NA & 1.15 & 232 & $\mathrm{ug} / \mathrm{mL}$ \\
\hline $\mathrm{Ni}$ & $8 / 10 / 94$ & NA & $5 / 13 / 94$ & R4048 & NA & NA & NA & 0.019 & $\mathrm{~g} / 100 \mathrm{~g}$ \\
\hline $\mathrm{Ni2}+$ & NA & NA & NA & $\mathrm{NA}$ & NA & NA & 1.43 & 3.33E-03 & Mole/L \\
\hline $\mathrm{Ni}$ & $8 / 10 / 94$ & NA & $5 / 13 / 94$ & R4047 & NA & NA & 1.141 & 160 & $\mathrm{ug} / \mathrm{mL}$ \\
\hline $\mathrm{Ni}$ & $8 / 10 / 94$ & NA & $5 / 13 / 94$ & R4048 & NA & NA & 1.139 & 132 & $\mathrm{ug} / \mathrm{mL}$ \\
\hline $\mathrm{Ni}$ & $8 / 10 / 94$ & NA & $5 / 13 / 94$ & $\mathrm{R} 4047$ & NA & NA & NA & 0.0081 & $\mathrm{~g} / 100 \mathrm{~g}$ \\
\hline $\mathrm{Ni}$ & $8 / 10 / 94$ & NA & $5 / 13 / 94$ & R4048 & NA & NA & NA & 0.0078 & $\mathrm{~g} / 100 \mathrm{~g}$ \\
\hline NO2- & NA & NA & $\mathrm{NA}$ & NA & NA & NA & 1.43 & $2.17 E+00$ & Mole/L \\
\hline $\mathrm{NO}_{2}^{-}$ & $8 / 10 / 94$ & NA & $5 / 13 / 94$ & R4048 & NA & NA & 1.352 & 63800 & ug $/ \mathrm{mL}$ \\
\hline $\mathrm{NO}_{2}^{-}$ & $8 / 10 / 94$ & NA & $5 / 13 / 94$ & R4047 & NA & NA & 1.345 & 61100 & $u g / m L$ \\
\hline $\mathrm{NO}_{2}^{-}$ & $8 / 10 / 94$ & NA & $2 \pi / 93$ & R3155 & NA & NA & 1.384 & 4.32 & WT \% \\
\hline $\mathrm{NO}_{2}^{-}$ & $8 / 10 / 94$ & NA & $5 / 13 / 94$ & R4048 & NA & $\mathrm{NA}$ & 1.349 & 59700 & $\mathrm{ug} / \mathrm{mL}$ \\
\hline $\mathrm{NO}_{2}^{-}$ & $8 / 10 / 94$ & $N A$ & $5 / 13 / 94$ & $\mathrm{R} 4047$ & NA & NA & 1.363 & 52500 & $\mathrm{ug} / \mathrm{mL}$ \\
\hline NO2 & $6 / 29 / 90$ & NA & NA & R-5887CC- 428 & NA & NA & $\mathrm{NA}$ & 1.09 & $M$ \\
\hline $\mathrm{NO}_{2}^{\circ}$ & $8 / 10 / 94$ & NA & $2 / 7 / 93$ & R3154 & NA & NA & 1.392 & 3.35 & WT $\%$ \\
\hline NO2 & $6 / 29 / 90$ & NA & NA & R-5887/CC-44H & NA & NA & NA & 1.01 & $M$ \\
\hline $\mathrm{NO}_{2}$ & $4 / 23 / 84$ & NS & NS & $\mathrm{R} 8843$ & NS & NS & NS & 0.84 & Moles/L \\
\hline NO2- & $12 / 4 / 86$ & NS & NS & NS & NS & NS & NS & $3.82 \mathrm{E}+01$ & $g / L$ \\
\hline $\mathrm{NO}_{2}$ & $12 / 4 / 86$ & NA & NA & 8650 & NA & NA & NA & 0.83 & $M$ \\
\hline $\mathrm{NO2}$ & $12 / 4 / 86$ & NS & NS & $R-8650$ & NS & NS & NS & 0.83 & moles $n$ \\
\hline $\mathrm{NO}_{2}^{-}$ & $2 / 28 / 85$ & NA & NA & 83637 & NA & NA & 1.41 & 0.829 & $M$ \\
\hline $\mathrm{NO2}$ & $2 / 28 / 85$ & NS & NS & R-3637 & NS & NS & 1.41 & 0.829 & molesn \\
\hline $\mathrm{NO}_{2}^{-}$ & $8 / 10 / 94$ & NA & $5 / 13 / 94$ & R4048 & NA & $N A$ & 1.157 & 37500 & $\mathrm{ug} / \mathrm{mL}$ \\
\hline $\mathrm{NO}_{2}$ & $4 / 23 / 84$ & NS & NS & R8844 & NS & NS & NS & 0.81 & Moles/L \\
\hline $\mathrm{NO}_{2}^{-}$ & $2 / 28 / 85$ & NA & NA & R3638 & NA & NA & 1.35 & 0.776 & $M$ \\
\hline NO2 & $2 / 28 / 85$ & NS & NS & $R-3638$ & NS & NS & 1.35 & 0.776 & moles $\lambda$ \\
\hline $\mathrm{NO}_{2}^{-}$ & $8 / 10 / 94$ & NA & $2 \pi / 93$ & R2686 & NA & $N A$ & 1.047 & 2.95 & WT \% \\
\hline $\mathrm{NO}_{2}^{-}$ & $8 / 10 / 94$ & NA & $5 / 13 / 94$ & 84047 & NA & NA & 1.15 & 29400 & $\mathrm{ug} / \mathrm{mL}$ \\
\hline $\mathrm{NO}_{2}^{-}$ & $8 / 10 / 94$ & NA & $5 / 13 / 94$ & $\mathrm{R} 4048$ & NA & NA & NA & 2.25 & $g / 100 g$ \\
\hline
\end{tabular}




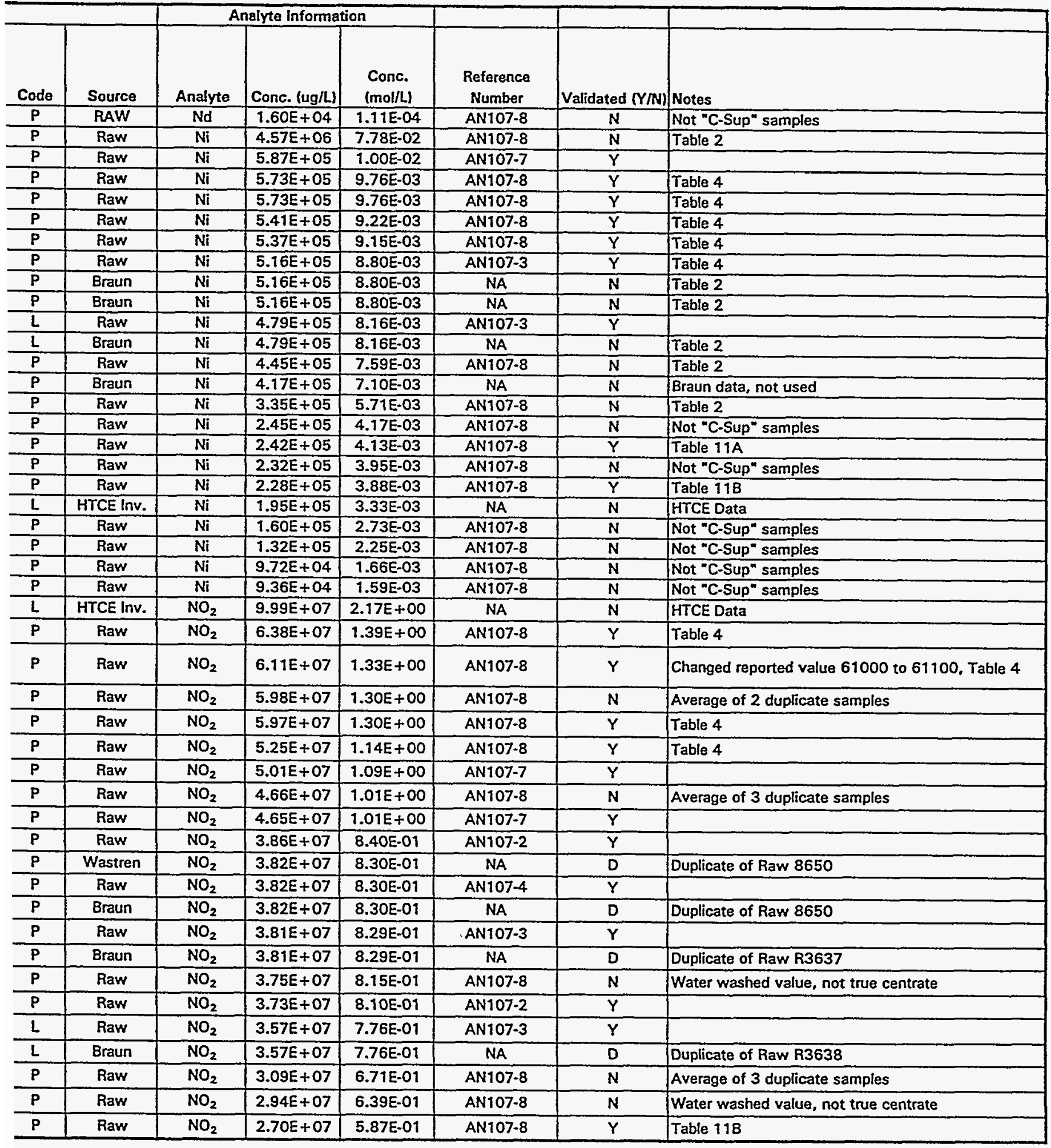


TWRS Privatization Support Pro Chemical Liquids

\begin{tabular}{|c|c|c|c|c|c|c|c|c|c|}
\hline & & & & Tank Waste Char & erization Sam & ple Informatio & & & \\
\hline Reported Chemical & Memo Date & $\begin{array}{c}\text { Start Analysis } \\
\text { Date }\end{array}$ & $\begin{array}{c}\text { Start Sample } \\
\text { Date }\end{array}$ & Sample Number & $\begin{array}{c}\text { Tank Farm } \\
\text { Number }\end{array}$ & $\begin{array}{c}\text { Lab Sample } \\
\text { Number }\end{array}$ & $\begin{array}{c}\text { Density } \\
\text { (g/mL) }\end{array}$ & $\begin{array}{c}\text { Reported } \\
\text { Value } \\
\end{array}$ & Reported I \\
\hline $\mathrm{NO}_{2}^{-}$ & $8 / 10 / 94$ & NA & $5 / 13 / 94$ & 84047 & NA & $\mathrm{NA}$ & NA & 1.96 & $g / 100$ \\
\hline $\mathrm{NO}_{2}^{-}$ & $8 / 10 / 94$ & NA & $5 / 13 / 94$ & R4047 & NA & NA & 1.141 & 21200 & $\mathrm{ug} / \mathrm{mL}$. \\
\hline $\mathrm{NO}_{2}^{-}$ & $8 / 10 / 94$ & NA & $5 / 13 / 94$ & R4048 & NA & NA & 1.139 & 16800 & $\mathrm{ug} / \mathrm{mL}$ \\
\hline $\mathrm{NO}_{2}^{-}$ & $8 / 10 / 94$ & NA & $5 / 13 / 94$ & 84048 & NA & NA & NA & 1.2 & $g / 100 \mathrm{~g}$ \\
\hline $\mathrm{NO}_{2}^{-}$ & $8 / 10 / 94$ & NA & $5 / 13 / 94$ & $\mathrm{R} 4047$ & NA & NA & NA & 1.03 & $\mathrm{~g} / 100 \mathrm{~g}$ \\
\hline $\mathrm{NO2}$ & $10 / 10 / 80$ & NA & NA & 5439 & NA & NA & NA & 0.258 & $M$ \\
\hline $\mathrm{NO2}$ & $10 / 10 / 80$ & NA & NA & 5440 & NA & NA & NA & 0.258 & $M$ \\
\hline $\mathrm{NO}_{3}$ & $4 / 23 / 84$ & NS & NS & R8843 & NS & NS & NS & 4.08 & Moles/L \\
\hline $\mathrm{NO}_{3}$ & $4 / 23 / 84$ & NS & NS & R8844 & NS & NS & NS & 3.98 & Moles/L \\
\hline $\mathrm{NO}_{3}^{-}$ & $8 / 10 / 94$ & NA & $2 / 7 / 93$ & R3154 & NA & NA & 1.392 & 16.67 & WT \% \\
\hline $\mathrm{NO}_{3}{ }^{-}$ & $8 / 10 / 94$ & NA & $5 / 13 / 94$ & R4048 & NA & NA & 1.352 & 229000 & $u g / \mathrm{mL}$ \\
\hline $\mathrm{NO}_{3}^{\circ}$ & $8 / 10 / 94$ & $\mathrm{NA}$ & $2 \pi / 93$ & R3155 & NA & NA & 1.384 & 16.41 & WT \% \\
\hline $\mathrm{NO}_{3}{ }^{\circ}$ & $2 / 28 / 85$ & NA & NA & R3637 & NA & NA & 1.41 & 3.54 & $M$ \\
\hline NO3 & $2 / 28 / 85$ & NS & NS & R-3637 & NS & NS & 1.41 & 3.54 & moles $\hat{n}$ \\
\hline $\mathrm{NO}_{3}{ }^{\circ}$ & $8 / 10 / 94$ & NA & $5 / 13 / 94$ & 84047 & $\mathrm{NA}$ & NA & 1.345 & 218000 & $\mathrm{ug} / \mathrm{mL}$ \\
\hline $\mathrm{NO}_{3}^{-}$ & $2 / 28 / 85$ & NA & $\mathrm{NA}$ & R3638 & NA & $\mathrm{NA}$ & 1.35 & 3.51 & $M$ \\
\hline $\mathrm{NO3}$ & $2 / 28 / 85$ & NS & NS & $\mathrm{R}-3638$ & NS & NS & 1.35 & 3.51 & moles $n$ \\
\hline $\mathrm{NO}_{3}^{-}$ & $8 / 10 / 94$ & NA & $5 / 13 / 94$ & 84048 & NA & NA & 1.349 & 216000 & $\mathrm{ug} / \mathrm{mL}$ \\
\hline NO3- & NA & NA & NA & NA & NA & NA & 1.43 & $3.06 \mathrm{E}+00$ & Mole/L \\
\hline $\mathrm{NO}_{3}^{-}$ & $8 / 10 / 94$ & NA & $5 / 13 / 94$ & R4047 & NA & NA & 1.363 & 189000 & $\mathrm{ug} / \mathrm{mL}$ \\
\hline $\mathrm{NO}_{3}^{-}$ & $8 / 10 / 94$ & NA & $2 \pi / 93$ & R2686 & NA & NA & 1.047 & 17.88 & WT \% \\
\hline $\mathrm{NO}_{3}$ & $12 / 4 / 86$ & NA & NA & 8650 & NA & $\mathrm{NA}$ & NA & 2.7 & $M$ \\
\hline $\mathrm{NO3}$ & $12 / 4 / 86$ & NS & NS & R-8650 & NS & NS & NS & 2.7 & moles $n$ \\
\hline NO3. & $12 / 4 / 86$ & NS & NS & NS & NS & NS & NS & $1.67 E+02$ & $g / L$ \\
\hline $\mathrm{NO}_{3}^{-}$ & $8 / 10 / 94$ & NA & $5 / 13 / 94$ & R4048 & NA & $\mathrm{NA}$ & 1.157 & 128000 & $\mathrm{ug} / \mathrm{mL}$ \\
\hline $\mathrm{NO}_{3}^{-}$ & $8 / 10 / 94$ & NA & $5 / 13 / 94$ & $\mathrm{R} 4047$ & $\mathrm{NA}$ & NA & 1.15 & 98100 & $\mathrm{ug} / \mathrm{mL}$ \\
\hline $\mathrm{NO}_{3}{ }^{-}$ & $8 / 10 / 94$ & NA & $5 / 13 / 94$ & R4048 & $\mathrm{NA}$ & NA & NA & 8.09 & $g / 100 g$ \\
\hline $\mathrm{NO}_{3}{ }^{\circ}$ & $8 / 10 / 94$ & NA & $5 / 13 / 94$ & $R 4047$ & NA & NA & NA & 7.07 & $g / 100 \mathrm{~g}$ \\
\hline $\mathrm{NO}_{3}^{-}$ & $8 / 10 / 94$ & NA & $5 / 13 / 94$ & R4047 & $\mathrm{NA}$ & NA & 1.141 & 67800 & $\mathrm{ug} / \mathrm{mL}$ \\
\hline $\mathrm{NO}_{3}^{-}$ & $8 / 10 / 94$ & NA & $5 / 13 / 94$ & R4048 & NA & NA & 1.139 & 56100 & $\mathrm{ug} / \mathrm{mL}$ \\
\hline $\mathrm{NO}_{3}^{\circ}$ & $8 / 10 / 94$ & NA & $5 / 13 / 94$ & 84048 & NA & NA & NA & 4.1 & $\mathrm{~g} / 100 \mathrm{~g}$ \\
\hline $\mathrm{NO}_{3}^{-}$ & $8 / 10 / 94$ & NA & $5 / 13 / 94$ & $\mathrm{R} 4047$ & $\mathrm{NA}$ & NA & NA & 3.44 & $\mathrm{~g} / 100 \mathrm{~g}$ \\
\hline $\mathrm{OH}$ & $12 / 4 / 86$ & $12 / 4 / 86$ & NA & $R-8650$ & $\mathrm{NA}$ & NA & NA & $<0.05$ & $M$ \\
\hline $\mathrm{OH}$ & $6 / 29 / 90$ & NA & NA & R5887 & NA & $\mathrm{NA}$ & NA & 0.646 & $M$ \\
\hline $\mathrm{OH}-$ & $6 / 29 / 90$ & NA & NA & R5887 & NA & NA & NA & 0.571 & $M$ \\
\hline $\mathrm{OH}-$ & $6 / 29 / 90$ & NA & NA & R5887 & NA & NA & NA & 0.476 & $M$ \\
\hline $\mathrm{OH}-$ & $10 / 10 / 80$ & NS & NS & 5440 & NS & NS & NS & 0.243 & $M$ \\
\hline $\mathrm{OH}$ & $10 / 10 / 80$ & NS & NS & 5439 & NS & NS & NS & 0.229 & $M$ \\
\hline $\mathrm{OH}-$ & $12 / 4 / 86$ & NS & NS & NS & NS & NS & NS & 0.85 & $g / L$ \\
\hline $\mathrm{OH}$ & $6 / 29 / 90$ & NA & NA & R-5887/CC-44H & NA & NA & NA & 0.0603 & $M$ \\
\hline $\mathrm{OH}$ & $6 / 29 / 90$ & NA & NA & $\mathrm{R}-5887 \mathrm{CC}-42 \mathrm{~B}$ & NA & NA & $\mathrm{NA}$ & 0.0525 & $M$ \\
\hline $\mathrm{Pb}$ & $2 / 28 / 85$ & NA & NA & R3638 & NA & $\overline{\mathrm{NA}}$ & 1.35 & $<2.60 \mathrm{E}-03$ & $M$ \\
\hline $\mathrm{Pb}$ & $2 / 28 / 85$ & NS & NS & $\mathrm{R}-3638$ & NS & NS & 1.35 & 0.0026 & moles $n$ \\
\hline $\mathrm{Pb}$ & $8 / 10 / 94$ & NA & $5 / 13 / 94$ & R4048 & NA & NA & 1.349 & 461 & $\mathrm{ug} / \mathrm{mL}$ \\
\hline
\end{tabular}


TWRS Privatization Support Pro Chemical Liquids

\begin{tabular}{|c|c|c|c|c|c|c|c|c|c|}
\hline & & & & Tank Waste Char & erization San & ple Informatio & & & \\
\hline Reported Chemical & Memo Date & $\begin{array}{c}\text { Start Analysis } \\
\text { Date }\end{array}$ & $\begin{array}{c}\text { Start Sample } \\
\text { Date }\end{array}$ & Sample Number & $\begin{array}{l}\text { Tank Farm } \\
\text { Number }\end{array}$ & $\begin{array}{c}\text { Lab Sample } \\
\text { Number }\end{array}$ & $\begin{array}{l}\text { Density } \\
(\mathrm{g} / \mathrm{mL})\end{array}$ & $\begin{array}{l}\text { Reported } \\
\text { Value }\end{array}$ & Reported I \\
\hline $\mathrm{Pb}$ & $8 / 10 / 94$ & NA & $5 / 13 / 94$ & $\mathrm{R} 4047$ & NA & NA & 1.345 & 417 & $\mathrm{ug} / \mathrm{mL}$ \\
\hline $\mathrm{Pb}$ & $8 / 10 / 94$ & NA & $5 / 13 / 94$ & R4048 & NA & NA & 1.352 & 413 & $u g / m L$ \\
\hline $\mathrm{Pb}$ & $8 / 10 / 94$ & NA & $5 / 13 / 94$ & R4047 & NA & NA & 1.363 & 409 & $\mathrm{ug} / \mathrm{mL}$ \\
\hline $\mathrm{Pb}$ & $2 / 28 / 85$ & NA & NA & $R-3637$ & NA & NA & 1.41 & 0.00182 & moles \\
\hline $\mathrm{Pb}$ & $3 / 6 / 85$ & NS & NS & RAT-AN107-1A & NS & NS & NS & 0.00182 & moles $A$ \\
\hline $\mathrm{Pb}$ & $5 / 16 / 90$ & NA & NA & NA & NA & NA & NA & 0.0018 & Moles \\
\hline $\mathrm{Pb}$ & $5 / 16 / 90$ & NA & NA & NA & NA & NA & NA & $1.80 \mathrm{E}-03$ & $M$ \\
\hline $\mathrm{Pb}$ & $8 / 10 / 94$ & NA & $5 / 13 / 94$ & R3154 & NA & NA & 1.392 & 0.024 & WT \% \\
\hline $\mathrm{Pb}$ & $8 / 10 / 94$ & NA & $5 / 13 / 94$ & R3155 & NA & NA & 1.384 & 0.024 & WT \% \\
\hline $\mathrm{Pb}$ & $8 / 10 / 94$ & NA & $5 / 13 / 94$ & R2686 & NA & NA & 1.047 & 0.023 & WT \% \\
\hline $\mathrm{Pb}$ & $8 / 10 / 94$ & NA & $5 / 13 / 94$ & R4048 & NA & NA & 1.157 & 195 & $\mathrm{ug} / \mathrm{mL}$ \\
\hline $\mathrm{Pb}$ & $8 / 10 / 94$ & NA & $5 / 13 / 94$ & R4047 & NA & NA & NA & 0.0153 & $\mathrm{~g} / 100 \mathrm{~g}$ \\
\hline$\overline{\mathrm{Pb}}$ & $8 / 10 / 94$ & $\overline{N A}$ & $5 / 13 / 94$ & 84048 & NA & NA & NA & 0.0146 & $g / 100 g$ \\
\hline$\overline{\mathrm{Pb}}$ & $8 / 10 / 94$ & NA & $5 / 13 / 94$ & R4047 & NA & NA & 1.15 & 162 & $\mathrm{ug} / \mathrm{mL}$ \\
\hline $\mathrm{Pb}$ & $8 / 10 / 94$ & NA & $5 / 13 / 94$ & R4048 & NA & NA & 1.139 & 130 & $\mathrm{ug} / \mathrm{mL}$ \\
\hline $\mathrm{Pb}$ & $8 / 10 / 94$ & NA & $5 / 13 / 94$ & R4047 & NA & NA & 9.141 & 110 & $\mathrm{ug} / \mathrm{mL}$ \\
\hline $\mathrm{Pb}$ & $8 / 10 / 94$ & NA & $5 / 13 / 94$ & R4048 & NA & NA & NA & 0.0062 & $g / 100 \mathrm{~g}$ \\
\hline $\mathrm{Pb}$ & $8 / 10 / 94$ & NA & $5 / 13 / 94$ & R4047 & NA & NA & NA & 0.0057 & $g / 100 g$ \\
\hline $\mathrm{Pb} 2+$ & NA & $N A$ & NA & NA & NA & NA & 1.43 & $1.07 E-04$ & Molell \\
\hline P043- & NA & NA & NA & NA & NA & NA & 1.43 & $1.25 \mathrm{E}-01$ & Mole/L \\
\hline $\mathrm{PO}_{4}{ }^{3-}$ & $8 / 10 / 94$ & NA & $5 / 13 / 94$ & R4047 & NA & NA & 1.345 & 6350 & $\mathrm{ug} / \mathrm{mL}$ \\
\hline $\mathrm{PO}_{4}^{3 \cdot}$ & $8 / 10 / 94$ & NA & $5 / 13 / 94$ & R4048 & NA & NA & 1.352 & 6140 & $\mathrm{ug} / \mathrm{mL}$ \\
\hline $\mathrm{PO}_{4}^{3-}$ & $8 / 10 / 94$ & NA & $5 / 13 / 94$ & R4048 & NA & NA & 1.349 & 6100 & $\mathrm{ug} / \mathrm{mL}$ \\
\hline $\mathrm{PO}_{4}{ }^{3-}$ & $8 / 10 / 94$ & NA & $5 / 13 / 94$ & R4047 & NA & $\overline{N A}$ & 1.363 & 6000 & $\mathrm{ug} / \mathrm{mL}$ \\
\hline $\mathrm{PO}_{4}^{3 \cdot}$ & $8 / 10 / 94$ & NA & $5 / 13 / 94$ & R4048 & NA & NA & 1.157 & 2950 & $u g / \mathrm{mL}$ \\
\hline $\mathrm{PO}_{4}{ }^{3-}$ & $8 / 10 / 94$ & NA & $5 / 13 / 94$ & R4047 & NA & NA & 1.15 & 2910 & $\mathrm{ug} / \mathrm{mL}$ \\
\hline PO4- & $12 / 4 / 86$ & NS & NS & NS & NS & NS & NS & $2.85 E+00$ & $g / L$ \\
\hline $\mathrm{PO}_{4}$ & $12 / 4 / 86$ & NA & NA & 8650 & NA & $\overline{N A}$ & NA & 0.03 & $M$ \\
\hline PO4 & $12 / 4 / 86$ & NS & NS & R-8650 & NS & NS & NS & 0.03 & moles $\Lambda$ \\
\hline $\mathrm{PO}_{4}{ }^{3-}$ & $8 / 10 / 94$ & NA & $5 / 13 / 94$ & R4047 & NA & $\overline{N A}$ & NA & 0.22 & $g / 100 \mathrm{~g}$ \\
\hline $\mathrm{PO}_{4}{ }^{3-}$ & $8 / 10 / 94$ & NA & $5 / 13 / 94$ & R4048 & NA & NA & NA & 0.22 & $g / 100 g$ \\
\hline $\mathrm{PO}_{4}^{-3}$ & $2 / 28 / 85$ & $\mathrm{NA}$ & NA & $R 3637$ & $\overline{N A}$ & NA & 1.41 & 0.0233 & $M$ \\
\hline $\mathrm{PO4}$ & $2 / 28 / 85$ & NS & NS & R-3637 & NS & NS & 1.41 & 0.0233 & moles $n$ \\
\hline $\mathrm{PO}_{4}^{-3}$ & $2 / 28 / 85$ & NA & $\overline{N A}$ & R3638 & NA & NA & 1.35 & 0.0207 & $M$ \\
\hline $\mathrm{PO4}$ & $2 / 28 / 85$ & NS & NS & R-3638 & NS & NS & 1.35 & 0.0207 & moles $n$ \\
\hline $\mathrm{PO}_{4}{ }^{3-}$ & $8 / 10 / 94$ & NA & $5 / 13 / 94$ & 84047 & NA & NA & 1.141 & 1510 & $\mathrm{ug} / \mathrm{mL}$ \\
\hline $\mathrm{PO}_{4}^{3-}$ & $8 / 10 / 94$ & NA & $5 / 13 / 94$ & R4048 & NA & NA & 1.139 & 1490 & $\mathrm{ug} / \mathrm{mL}$ \\
\hline $\mathrm{PO}_{4}{ }^{3-}$ & $8 / 10 / 94$ & NA & $5 / 13 / 94$ & R4047 & NA & NA & NA & 0.1 & $g / 100 g$ \\
\hline $\mathrm{PO}_{4}{ }^{3-}$ & $8 / 10 / 94$ & NA & $5 / 13 / 94$ & R4048 & NA & $\mathrm{NA}$ & NA & 0.09 & $g / 100 g$ \\
\hline $\mathrm{PO}_{4}{ }^{3+}$ & $8 / 10 / 94$ & NA & $2 / 7 / 93$ & 83154 & NA & NA & 1.392 & 0.04 & WT $\%$ \\
\hline $\mathrm{PO}_{4}{ }^{3-}$ & $8 / 10 / 94$ & NA & $2 \pi / 93$ & 83155 & NA & NA & 1.384 & 0.04 & WT \% \\
\hline $\mathrm{PO}_{4}^{3-}$ & $8 / 10 / 94$ & $N A$ & $2 / 7 / 93$ & R2686 & NA & NA & 1.047 & 0.03 & WT \% \\
\hline $\mathrm{SO}_{4}^{-2}$ & $2 / 28 / 85$ & NA & NA & R3638 & NA & NA & 1.35 & $<1.81 E-01$ & $M$ \\
\hline s042- & NA & $\overline{N A}$ & $\overline{N A}$ & NA & NA & NA & 1.43 & $2.72 \mathrm{E}-01$ & Mole/L \\
\hline SO4 & $2 / 28 / 85$ & NS & NS & R-3638 & NS & NS & 1.35 & 0.181 & moles $n$ \\
\hline
\end{tabular}


TWRS Privatization Support Pro] Chemical Liquids

\begin{tabular}{|c|c|c|c|c|c|c|c|c|c|}
\hline & & & & Tank Waste Char & erization San & ple Informatio & & & \\
\hline Reported Chemical & Memo Date & $\begin{array}{c}\text { Start Analysis } \\
\text { Date }\end{array}$ & $\begin{array}{c}\text { Start Sample } \\
\text { Date } \\
\end{array}$ & Sample Number & $\begin{array}{c}\text { Tank Farm } \\
\text { Number }\end{array}$ & $\begin{array}{c}\text { Lab Sample } \\
\text { Number }\end{array}$ & $\begin{array}{c}\text { Density } \\
\text { (g/mL) }\end{array}$ & $\begin{array}{c}\text { Reported } \\
\text { Value } \\
\end{array}$ & Reported U \\
\hline $\mathrm{SO}_{4}^{2-}$ & $8 / 10 / 94$ & NA & $2 \pi / 93$ & R3154 & NA & NA & 1.392 & 1.16 & WT \% \\
\hline $\mathrm{SO}_{4}^{2-}$ & $8 / 10 / 94$ & NA & $2 / 7 / 93$ & $\mathrm{R} 3155$ & $\overline{N A}$ & NA & 1.384 & 1.00 & WT \% \\
\hline $\mathrm{SO}_{4}^{2-}$ & $8 / 10 / 94$ & NA & $5 / 13 / 94$ & R4047 & NA & NA & 1.345 & 12500 & $\mathrm{ug} / \mathrm{mL}$ \\
\hline $\mathrm{SO}_{4}{ }^{2-}$ & $8 / 10 / 94$ & NA & $5 / 13 / 94$ & R4048 & NA & NA & 1.352 & 12500 & $\mathrm{ug} / \mathrm{mL}$ \\
\hline $\mathrm{SO}_{4}{ }^{2-}$ & $8 / 10 / 94$ & NA & $5 / 13 / 94$ & $\mathrm{R} 4048$ & NA & $\mathrm{NA}$ & 1.349 & 12100 & $\mathrm{ug} / \mathrm{mL}$ \\
\hline $\mathrm{SO}_{4}^{2-}$ & $8 / 10 / 94$ & NA & $5 / 13 / 94$ & 84047 & NA & NA & 1.363 & 11300 & $\mathrm{ug} / \mathrm{mL}$ \\
\hline $\mathrm{SO}_{4}{ }^{2-}$ & $8 / 10 / 94$ & NA & $2 / 7 / 93$ & R2686 & NA & NA & 1.047 & 0.79 & WT $\%$ \\
\hline $\mathrm{SO}_{4}{ }^{20}$ & $8 / 10 / 94$ & NA & $5 / 13 / 94$ & $\mathrm{R} 4048$ & $\mathrm{NA}$ & NA & 1.157 & 6640 & $\mathrm{ug} / \mathrm{mL}$ \\
\hline $\mathrm{SO}_{4}{ }^{20}$ & $8 / 10 / 94$ & NA & $5 / 13 / 94$ & R4047 & NA & NA & 1.15 & 6380 & $\mathrm{ug} / \mathrm{mL}$ \\
\hline $\mathrm{SO}_{4}^{-2}$ & $2 / 28 / 85$ & NA & $\mathrm{NA}$ & R3637 & NA & NA & 1.41 & 0.0633 & $M$ \\
\hline 504 & $2 / 28 / 85$ & NS & NS & $\mathrm{R}-3637$ & NS & NS & 1.41 & 0.0633 & moles I \\
\hline $\mathrm{SO}_{4}$ & $12 / 4 / 86$ & NA & NA & 8650 & NA & NA & $\overline{N A}$ & 0.06 & $M$ \\
\hline SO4 & $12 / 4 / 86$ & NS & NS & R-8650 & NS & NS & NS & 0.06 & moles n \\
\hline SO4- & $12 / 4 / 86$ & NS & NS & NS & NS & NS & NS & $5.76 \mathrm{E}+00$ & $g / L$ \\
\hline $\mathrm{SO}_{4}{ }^{2-}$ & $8 / 10 / 94$ & NA & $5 / 13 / 94$ & R4048 & NA & NA & NA & 0.44 & $\mathrm{~g} / 100 \mathrm{~g}$ \\
\hline $\mathrm{SO}_{4}{ }^{2-}$ & $8 / 10 / 94$ & NA & $5 / 13 / 94$ & R4047 & NA & NA & $\mathrm{NA}$ & 0.42 & $g / 100 \mathrm{~g}$ \\
\hline $\mathrm{SO}_{4}{ }^{2-}$ & $8 / 10 / 94$ & NA & $5 / 13 / 94$ & 84047 & NA & NA & 1.141 & 3490 & $\mathrm{ug} / \mathrm{mL}$ \\
\hline $\mathrm{SO}_{4}{ }^{2-}$ & $8 / 10 / 94$ & NA & $5 / 13 / 94$ & $\mathrm{R} 4048$ & NA & NA & 1.139 & 3350 & $\mathrm{ug} / \mathrm{mL}$ \\
\hline $\mathrm{SO}_{4}{ }^{2-}$ & $8 / 10 / 94$ & NA & $5 / 13 / 94$ & R4047 & NA & NA & NA & 0.22 & $g / 100 \mathrm{~g}$ \\
\hline $\mathrm{SO}_{4}{ }^{2-}$ & $8 / 10 / 94$ & NA & $5 / 13 / 94$ & R4048 & NA & NA & $N A$ & 0.21 & $\mathrm{~g} / 100 \mathrm{~g}$ \\
\hline $\mathrm{Sr}$ & $3 / 6 / 85$ & NS & NS & RAT-AN107 & NS & NS & NS & 0.000074 & moles $A$ \\
\hline TOC & $6 / 29 / 90$ & NA & NA & $\mathrm{R}-5887 \mathrm{CC}-42 \mathrm{~B}$ & NA & $\mathrm{NA}$ & NA & 46.7 & $g / L$ \\
\hline TOC & $4 / 23 / 84$ & NS & NS & $\mathrm{R} 8843$ & NA & $\mathrm{NA}$ & NA & 46 & $g / L$ \\
\hline TOC & $8 / 10 / 94$ & $\mathrm{NA}$ & $2 / 7 / 93$ & R3155 & NA & NA & 1.384 & 3.24 & WT \% \\
\hline TOC & $4 / 23 / 84$ & NS & NS & R8844 & NS & NS & NA & 44.4 & $g / L$ \\
\hline TOC & $2 / 28 / 85$ & NA & NA & R3637 & $\mathrm{NA}$ & NA & 1.41 & 44.3 & $\mathrm{gC/L}$ \\
\hline TOC & $2 / 28 / 85$ & NS & NS & R-3637 & NS & NS & 1.41 & 44.3 & $\mathrm{gm} / \mathrm{I}$ \\
\hline TOC & $6 / 29 / 90$ & NA & NA & R-5887/CC-44H & NA & NA & NA & 42.8 & $g / L$ \\
\hline TOC & $8 / 10 / 94$ & NA & $5 / 13 / 94$ & $\mathrm{R} 4048$ & NA & NA & 1.352 & 42200 & $\mathrm{ug} / \mathrm{mL}$ \\
\hline TOC & $8 / 10 / 94$ & NA & 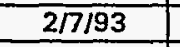 & 83154 & NA & $\mathrm{NA}$ & 1.392 & 2.99 & WT \% \\
\hline TOC & $12 / 4 / 86$ & NA & NA & 8650 & NA & NA & NA & 39.1 & $g / L$ \\
\hline TOC & $12 / 4 / 86$ & NS & NS & R-8650 & NS & NS & NS & 39.1 & $\mathrm{gm} / 1$ \\
\hline TOC & $12 / 4 / 86$ & NS & NS & NS & NS & NS & NS & $3.91 E+01$ & $g / L$ \\
\hline TOC & $2 / 28 / 85$ & NA & NA & R3638 & $\mathrm{NA}$ & NA & 1.35 & 38.8 & $\mathrm{gC/L}$ \\
\hline TOC & $2 / 28 / 85$ & NS & NS & $R-3638$ & NS & NS & 1.35 & 38.8 & $\mathrm{gm} / \mathrm{I}$ \\
\hline TOC & $8 / 10 / 94$ & NA & $5 / 13 / 94$ & R4047 & NA & NA & 1.345 & 38000 & $\mathrm{ug} / \mathrm{mL}$ \\
\hline TOC & $8 / 10 / 94$ & NA & $5 / 13 / 94$ & R4048 & NA & NA & 1.349 & 37900 & $\mathrm{ug} / \mathrm{mL}$ \\
\hline TOC & $8 / 10 / 94$ & NA & $5 / 13 / 94$ & R4047 & NA & NA & 1.363 & 37300 & $\mathrm{ug} / \mathrm{mL}$ \\
\hline TOC & $8 / 10 / 94$ & NA & $2 / 7 / 93$ & R2686 & NA & NA & 1.047 & 2.99 & WT \% \\
\hline TOC & $8 / 10 / 94$ & NA & $5 / 13 / 94$ & R4048 & NA & NA & 1.157 & 20900 & $\mathrm{ug} / \mathrm{mL}$ \\
\hline TOC wt\% C (wet) & NA & NA & NA & NA & NA & NA & 1.43 & $1.46 \mathrm{E}+00$ & $w t \%$ \\
\hline TOC & $8 / 10 / 94$ & NA & $5 / 13 / 94$ & R4047 & NA & NA & 1.15 & 19100 & $\mathrm{ug} / \mathrm{mL}$ \\
\hline TOC & $8 / 10 / 94$ & $\mathrm{NA}$ & $5 / 13 / 94$ & R4048 & NA & NA & NA & 1.49 & $\mathrm{~g} / 100 \mathrm{~g}$ \\
\hline TOC & $8 / 10 / 94$ & $\mathrm{NA}$ & $5 / 13 / 94$ & R4047 & NA & NA & NA & 1.39 & $\mathrm{~g} / 100 \mathrm{~g}$ \\
\hline TOC & $8 / 10 / 94$ & NA & $5 / 13 / 94$ & R4047 & NA & NA & 1.141 & 14400 & $\mathrm{ug} / \mathrm{mL}$ \\
\hline TOC & $8 / 10 / 94$ & NA & $5 / 13 / 94$ & R4048 & NA & NA & 1.139 & 10800 & $\mathrm{ug} / \mathrm{mL}$ \\
\hline
\end{tabular}




\begin{tabular}{|c|c|c|c|c|c|c|c|}
\hline \multirow[b]{2}{*}{ Code } & \multirow[b]{2}{*}{ Source } & \multicolumn{3}{|c|}{ Analyte Information } & \multirow[b]{2}{*}{$\begin{array}{c}\text { Reference } \\
\text { Number }\end{array}$} & \multirow[b]{2}{*}{ Validated $\mathbf{Y} / \mathbf{N})$} & \multirow[b]{2}{*}{ Notes } \\
\hline & & Analyte & Conc. (ug/L) & $\begin{array}{l}\text { Conc. } \\
\text { (mol/L) }\end{array}$ & & & \\
\hline$P$ & Raw & $\mathrm{SO}_{4}$ & $1.61 \mathrm{E}+07$ & $1.68 \mathrm{E}-01$ & AN107-8 & $N$ & Average of 3 duplicate samples \\
\hline $\mathrm{P}$ & Raw & $\mathrm{SO}_{4}$ & $1.38 E+07$ & $1.44 \mathrm{E}-01$ & AN107-8 & $\bar{N}$ & Average of 2 duplicate samples \\
\hline$P$ & Raw & $\mathrm{SO}_{4}$ & $1.25 E+07$ & $1.30 \mathrm{E}-01$ & AN107-8 & $Y$ & Table 4 \\
\hline$P$ & Raw & $\mathrm{SO}_{4}$ & $1.21 E+07$ & $1.26 \mathrm{E}-01$ & AN107-8 & $Y$ & Table 4 \\
\hline $\mathbf{P}$ & Raw & $\mathrm{SO}_{4}$ & $1.13 E+07$ & $1.18 E-01$ & AN107-8 & $\bar{Y}$ & Table 4 \\
\hline $\mathbf{P}$ & Raw & $\mathrm{SO}_{4}$ & $8.27 E+06$ & $8.62 \mathrm{E}-02$ & AN107-8 & $N$ & Average of 3 duplicate samples \\
\hline$P$ & Raw & $\mathrm{SO}_{4}$ & $6.64 E+06$ & $6.92 \mathrm{E}-02$ & AN107-8 & $N$ & Water washed value, not true centrate \\
\hline $\mathbf{P}$ & Raw & $\mathrm{SO}_{4}$ & $6.38 \mathrm{E}+06$ & $6.65 E-02$ & AN107-8 & $\mathrm{N}$ & Water washed value, not true centrate \\
\hline$P$ & Wastren & $\mathrm{SO}_{4}$ & $5.76 E+06$ & $6.00 \mathrm{E}-02$ & NA & D & Duplicate of Raw 8650 \\
\hline $\mathbf{P}$ & Raw & $\mathrm{SO}_{4}$ & $5.28 E+06$ & 5.50E-02 & AN107-8 & $\mathrm{Y}$ & Table 11B \\
\hline $\mathbf{P}$ & Raw & $\mathrm{SO}_{4}$ & $5.04 E+06$ & $5.25 E-02$ & AN107-8 & $\bar{Y}$ & Table 11A \\
\hline $\mathbf{P}$ & Raw & $\mathrm{SO}_{4}$ & $3.49 E+06$ & $3.64 \mathrm{E}-02$ & AN107-8 & $\mathbf{N}$ & Water washed value, not true centrate \\
\hline$P$ & Raw & $\mathrm{SO}_{4}$ & $3.35 E+06$ & $3.49 E-02$ & AN107-8 & $N$ & Water washed value, not true centrate \\
\hline $\mathbf{P}$ & Raw & $\mathrm{SO}_{4}$ & $2.64 E+06$ & $2.75 E-02$ & AN107-8 & $\mathbf{N}$ & Water washed value, not true centrate \\
\hline $\mathbf{P}$ & Raw & $\mathrm{SO}_{4}$ & $2.52 E+06$ & $2.63 E-02$ & AN107-8 & $\mathbf{N}$ & Water washed value, not true centrate \\
\hline $\mathrm{P}$ & Braun & $\mathrm{Sr}$ & $6.48 E+03$ & $7.40 E-05$ & NA & $N$ & Braun data, not used \\
\hline $\mathbf{P}$ & Raw & TOC & $4.67 E+07$ & $3.89 E+00$ & AN107-7 & $Y$ & \\
\hline$P$ & Raw & TOC & $4.60 E+07$ & $3.83 E+00$ & AN107-2 & $\bar{Y}$ & \\
\hline$P$ & Raw & TOC & $4.48 E+07$ & $3.74 E+00$ & AN107-8 & N & Average of 2 duplicate samples \\
\hline$P$ & Wastren & TOC & $3.91 \mathrm{E}+07$ & $3.26 \varepsilon+00$ & NA & D & Duplicate of Raw 8650 \\
\hline L & Raw & TOC & $3.88 E+07$ & $3.23 E+00$ & AN107-3 & $\mathbf{Y}$ & \\
\hline $\mathbf{L}$ & Braun & TOC & $3.88 E+07$ & $3.23 E+00$ & NA & D & Duplicate of Raw R3638 \\
\hline $\mathbf{P}$ & Raw & TOC & $3.80 E+07$ & $3.17 E+00$ & AN107-8 & $Y$ & Table 4 \\
\hline$P$ & Raw & TOC & $3.79 E+07$ & $3.16 E+00$ & AN107-8 & $\mathbf{Y}$ & Table 4 \\
\hline$P$ & Raw & TOC & $3.73 E+07$ & $3.11 E+00$ & AN107-8 & $\bar{Y}$ & Table 4 \\
\hline $\mathbf{P}$ & Raw & TOC & $3.13 E+07$ & $2.61 E+00$ & AN107-8 & $\mathbf{N}$ & Average of 3 duplicate samples \\
\hline $\mathbf{P}$ & Raw & TOC & $2.09 E+07$ & $1.74 E+00$ & AN107-8 & $\mathrm{N}$ & Water washed value, not true centrate \\
\hline $\mathbf{L}$ & HTCE Inv. & TOC & $2.09 E+07$ & $1.74 E+00$ & NA & $N$ & HTCE Data \\
\hline $\mathbf{P}$ & Raw & TOC & $1.91 E+07$ & $1.59 \varepsilon+00$ & AN107-8 & $\mathbf{N}$ & Water washed value, not true centrate \\
\hline $\mathbf{P}$ & Raw & TOC & $1.79 E+07$ & $1.49 E+00$ & AN107-8 & $\mathbf{Y}$ & Table 11B \\
\hline $\mathbf{P}$ & Raw & TOC & $1.67 E+07$ & $1.39 E+00$ & AN107-8 & $\mathbf{Y}$ & Table 11A \\
\hline $\mathbf{P}$ & Raw & TOC & $1.44 \mathrm{E}+07$ & $1.20 E+00$ & AN107-8 & $\mathbf{N}$ & Water washed value, not true centrate \\
\hline $\mathbf{P}$ & $\overline{\text { Raw }}$ & TOC & $1.08 \mathrm{E}+07$ & $9.00 \mathrm{E}-01$ & AN107-8 & $N$ & Water washed value, not true centrate \\
\hline
\end{tabular}


TWRS Privatization Support Proje Chemical Liquids $\mathbf{l}$

\begin{tabular}{|c|c|c|c|c|c|c|c|c|c|}
\hline & & & & Tank Waste Char & erization San & de lnformatio & & & \\
\hline Reported Chemical & Memo Date & $\begin{array}{c}\text { Start Analysis } \\
\text { Date }\end{array}$ & $\begin{array}{c}\text { Start Sample } \\
\text { Date }\end{array}$ & Sample Number & $\begin{array}{l}\text { Tank Farm } \\
\text { Number }\end{array}$ & $\begin{array}{c}\text { Lab Sample } \\
\text { Number }\end{array}$ & $\begin{array}{l}\text { Density } \\
\text { (g/mL) }\end{array}$ & $\begin{array}{l}\text { Reported } \\
\text { Value }\end{array}$ & Reported U \\
\hline TOC & $8 / 10 / 94$ & NA & \begin{tabular}{|l|}
$5 / 13 / 94$ \\
\end{tabular} & $\mathrm{R} 4047$ & NA & NA & NA & 0.67 & $\mathrm{~g} / 100 \mathrm{~g}$ \\
\hline TOC & $8 / 10 / 94$ & NA & $5 / 13 / 94$ & R4048 & NA & NA & NA & 0.67 & $g / 100 g$ \\
\hline$U$ & $2 / 28 / 85$ & NA & NA & R3638 & NA & NA & 1.35 & 0.172 & $g / 1$ \\
\hline $\bar{U}$ & $2 / 28 / 85$ & NS & NS & $R-3638$ & NS & NS & 1.35 & 0.172 & $g m /$ \\
\hline$U$ & $2 / 28 / 85$ & NA & NA & R3637 & NA & NA & 1.41 & 0.107 & $g n$ \\
\hline$U$ & $2 / 28 / 85$ & NS & NS & $\mathrm{R}-3637$ & NS & NS & 1.41 & 0.107 & $g m h$ \\
\hline
\end{tabular}

A- 


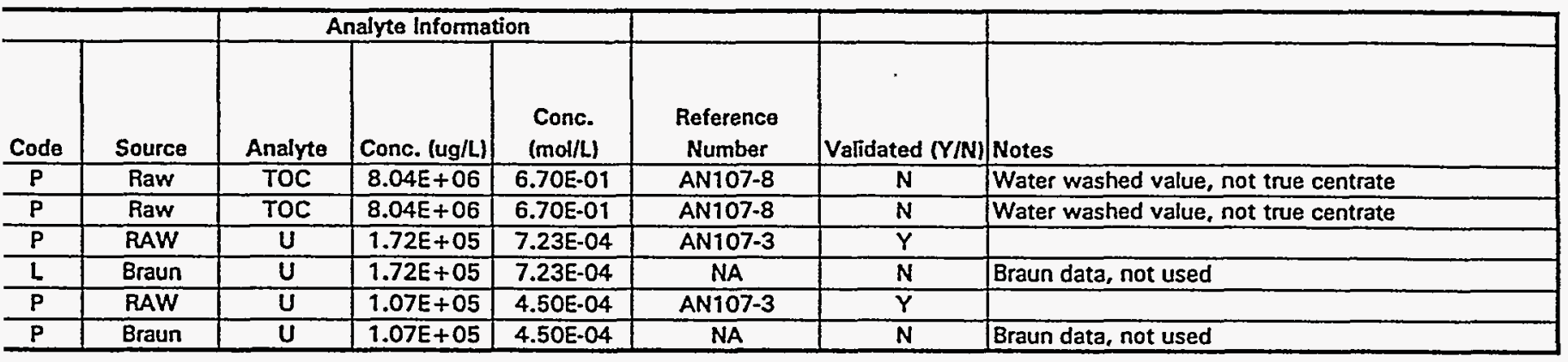




\begin{tabular}{|c|c|c|c|c|c|c|c|c|c|c|c|}
\hline & & & & & ank Waste & haracteriz: & ion Sample & ormation & & & \\
\hline Reported Chemical & $\begin{array}{c}\text { Memo } \\
\text { Date }\end{array}$ & $\begin{array}{c}\text { Start } \\
\text { Analysis } \\
\text { Date } \\
\end{array}$ & $\begin{array}{c}\text { Finish } \\
\text { Analysis Date } \\
\end{array}$ & $\begin{array}{c}\text { Start } \\
\text { Sample } \\
\text { Date } \\
\end{array}$ & $\begin{array}{c}\text { Finish } \\
\text { Sample } \\
\text { Date }\end{array}$ & $\begin{array}{l}\text { Sample } \\
\text { Number }\end{array}$ & $\begin{array}{c}\text { Tank Farm } \\
\text { Number }\end{array}$ & $\begin{array}{c}\text { Lab Sample } \\
\text { Number }\end{array}$ & $\begin{array}{l}\text { Density } \\
(\mathrm{g} / \mathrm{mL})\end{array}$ & $\begin{array}{c}\text { Reported } \\
\text { Value } \\
\end{array}$ & $\begin{array}{r}\text { Repont } \\
\text { Unit } \\
\end{array}$ \\
\hline $\mathrm{Al}+\mathrm{H}$ & NS & NS & NS & NS & NS & NS & NS & NS & 1.24 & 6.65E-01 & Mole \\
\hline Aluminum & $6 / 1 / 90$ & $4 / 30 / 89$ & $12 / 30 / 89$ & $4 / 27 / 89$ & $4 / 29 / 89$ & BOB2L9 & G305 & G349 & 1.20 & $1.21 E+07$ & $u g / \mathrm{L}$ \\
\hline Aluminum & $6 / 1 / 90$ & $4 / 30 / 89$ & $12 / 30 / 89$ & $4 / 27 / 89$ & $4 / 29 / 89$ & BOB2NO & G307 & G453 & NA & $1.19 E+07$ & $u g h$. \\
\hline Aluminum & $6 / 1 / 90$ & $4 / 30 / 89$ & $12 / 30 / 89$ & $4 / 27 / 89$ & $4 / 29 / 89$ & BOB2T9 & G327 & G470* & 1.19 & $1.19 E+07$ & $\operatorname{ug} \Omega$ \\
\hline Aluminum & $6 / 1 / 90$ & $4 / 30 / 89$ & $12 / 30 / 89$ & $4 / 27 / 89$ & $4 / 29 / 89$ & Вов2М9 & G302 & G450 & $(1.20)$ & $1.18 E+07$ & $u g / \Omega$ \\
\hline Aluminum & $6 / 1 / 90$ & $4 / 30 / 89$ & $12 / 30 / 89$ & $4 / 27 / 89$ & $4 / 29 / 89$ & ВОВ2T9 & G327 & G470 & 1.19 & $1.18 E+07$ & $u g / L$ \\
\hline Aluminum & $6 / 1 / 90$ & $4 / 30 / 89$ & $12 / 30 / 89$ & $4 / 27 / 89$ & $4 / 29 / 89$ & BOB2L8 & G303 & G348 & 1.21 & $1.18 E+07$ & ug/L \\
\hline Aluminum & $6 / 1 / 90$ & $4 / 30 / 89$ & $12 / 30 / 89$ & $4 / 27 / 89$ & $4 / 29 / 89$ & BOB2N3 & G322 & G338 & NA & $1.17 E+07$ & $u g / L$ \\
\hline Aluminum & $6 / 1 / 90$ & $4 / 30 / 89$ & $12 / 30 / 89$ & $4 / 27 / 89$ & $4 / 29 / 89$ & BOB2LO & G305 & G349 & 1.20 & $1.17 E+07$ & $u g / L$ \\
\hline Aluminum & $6 / 1 / 90$ & $4 / 30 / 89$ & $12 / 30 / 89$ & $4 / 27 / 89$ & $4 / 29 / 89$ & BOB2N1 & G310 & G454 & $(1.19)$ & $1.17 E+07$ & $u g / 2$ \\
\hline Aluminum & $6 / 1 / 90$ & $4 / 30 / 89$ & $12 / 30 / 89$ & $4 / 27 / 89$ & $4 / 29 / 89$ & ВОВ2M6 & G318 & G459 & 1.20 & $1.17 E+07$ & ugh \\
\hline Aluminum & $6 / 1 / 90$ & $4 / 30 / 89$ & $12 / 30 / 89$ & $4 / 27 / 89$ & $4 / 29 / 89$ & BOB2N3 & G322 & G338 & NA & $1.17 E+07$ & ug $/$ \\
\hline Aluminum & $6 / 1 / 90$ & 4/30/89 & $12 / 30 / 89$ & $4 / 27 / 89$ & $4 / 29 / 89$ & ВОВ2М9 & G302 & G450 & $(1.20)$ & $1.17 E+07$ & $u g / L$ \\
\hline Aluminum & $6 / 1 / 90$ & $4 / 30 / 89$ & $12 / 30 / 89$ & $4 / 27 / 89$ & $4 / 29 / 89$ & $B O B 2 N 1$ & G310 & G454 & $(1.19)$ & $1.17 E+07$ & $u g / h$ \\
\hline Aluminum & $6 / 1 / 90$ & $4 / 30 / 89$ & $12 / 30 / 89$ & $4 / 27 / 89$ & $4 / 29 / 89$ & BOB2M6 & G318 & G459 & 1.20 & $1.17 E+07$ & ug $/ \mathrm{L}$ \\
\hline Aluminum & $6 / 1 / 90$ & $4 / 30 / 89$ & $12 / 30 / 89$ & $4 / 27 / 89$ & $4 / 29 / 89$ & BOB2L8 & G303 & 6348 & 1.21 & $1.16 E+07$ & $u g / h$ \\
\hline Aluminum & $6 / 1 / 90$ & $4 / 30 / 89$ & $12 / 30 / 89$ & $4 / 27 / 89$ & $4 / 29 / 89$ & ВOB2T9 & G327 & G443 & 1.20 & $1.16 E+07$ & $u g / \Omega$ \\
\hline Aluminum & $6 / 1 / 90$ & $4 / 30 / 89$ & $12 / 30 / 89$ & $4 / 27 / 89$ & $4 / 29 / 89$ & BOB2N4 & G323 & G341 & 1.20 & $1.16 E+07$ & $u g / L$ \\
\hline Aluminum & $6 / 1 / 90$ & $4 / 30 / 89$ & $12 / 30 / 89$ & $4 / 27 / 89$ & $4 / 29 / 89$ & Вов2М2 & G309 & G358 & 1.20 & $1.16 E+07$ & $u g / L$ \\
\hline Aluminum & $6 / 1 / 90$ & $4 / 30 / 89$ & $12 / 30 / 89$ & $4 / 27 / 89$ & $4 / 29 / 89$ & BOB2M5 & G315 & G364 & 1.19 & $1.16 E+07$ & $u g / L$ \\
\hline Aluminum & $6 / 1 / 90$ & $4 / 30 / 89$ & $12 / 30 / 89$ & $4 / 27 / 89$ & $4 / 29 / 89$ & ВОВ2T9 & G327 & G443 & 1.20 & $1.16 E+07$ & ugh \\
\hline Aluminum & $6 / 1 / 90$ & $4 / 30 / 89$ & $12 / 30 / 89$ & $4 / 27 / 89$ & $4 / 29 / 89$ & BOB2M8 & G301 & G449 & NA & $1.16 \mathrm{E}+07$ & $u g R$ \\
\hline Aluminum & $6 / 1 / 90$ & $4 / 30 / 89$ & $12 / 30 / 89$ & $4 / 27 / 89$ & $4 / 29 / 89$ & BO82M7 & G319 & G460 & 1.20 & $1.16 E+07$ & ug $/$ L \\
\hline
\end{tabular}




\begin{tabular}{|c|c|c|c|c|c|c|c|}
\hline & & & nalyte Inforn & ation & & & \\
\hline Code & Source & Analyte & $\begin{array}{l}\text { Conc. } \\
\text { (ug/L) }\end{array}$ & Conc. (mol/L) & $\begin{array}{l}\text { Reference } \\
\text { Number }\end{array}$ & $\begin{array}{l}\text { Validated } \\
\text { (Y/N) }\end{array}$ & Notes \\
\hline$L$ & HTCE Inv. & $\mathrm{Al}$ & $1.80 E+13$ & $6.65 E+05$ & NA & $N$ & No Reference \\
\hline $\mathbf{P}$ & Raw & Al & $1.21 E+07$ & 4.48E-01 & AP102-3 & $Y$ & $\begin{array}{l}\text { Density reported for G349 collected at } 34 \text { in from bottom of } \\
\text { tank at a } 30^{\circ} \text { angle. Sample was unheated, unheated } \\
\text { samples precipitated }\end{array}$ \\
\hline $\mathbf{P}$ & Raw & Al & $1.19 E \div 07$ & 4.41E-01 & AP102-3 & $\mathbf{Y}$ & $\begin{array}{l}\text { Density not reported for G453. No assumptions could be } \\
\text { made }\end{array}$ \\
\hline$P$ & Raw & Al & $1.19 E \div 07$ & 4.41E-01 & AP102-3 & $\mathbf{Y}$ & $\begin{array}{l}\text { Density reported for } \mathrm{G} 470 \text { represents a composite sample. } \\
\text { Sample was kept heated to } 40^{\circ} \mathrm{C} \text {. }\end{array}$ \\
\hline$P$ & Raw & Al & $1.18 E+07$ & 4.37E-01 & AP102-3 & $Y$ & $\begin{array}{l}\text { Density not reported for G450. Density assumed same as } \\
\text { G477 which was collected at } 182 \text { in from bottom of tank at } \\
\text { a } 30^{\circ} \text { angle and was keep heated to } 40^{\circ} \mathrm{C} \text {. }\end{array}$ \\
\hline$P$ & Raw & $\mathrm{Al}$ & $1.18 E+07$ & 4.37E-01 & AP102-3 & $\mathbf{Y}$ & $\begin{array}{l}\text { Density reported for G470 represents a composite sample. } \\
\text { Sample was kept heated to } 40^{\circ} \mathrm{C} \text {. }\end{array}$ \\
\hline $\mathbf{P}$ & Raw & Al & $1.18 E+07$ & 4.37E-01 & AP102-3 & Y & $\begin{array}{l}\text { Density reported for G348 collected at } 125 \text { in from bottom } \\
\text { of tank at a } 30^{\circ} \text { angle. Sample was unheated, unheated } \\
\text { samples precipitated }\end{array}$ \\
\hline $\mathrm{P}$ & Raw & Al & $1.17 E+07$ & 4.34E-01 & AP102-3 & $Y$ & $\begin{array}{l}\text { Density not reported for G338. No assumptions could be } \\
\text { made }\end{array}$ \\
\hline $\mathbf{P}$ & Raw & Al & $1.17 E+07$ & 4.34E-01 & AP102-3 & $Y$ & $\begin{array}{l}\text { Density reported for } 6349 \text { collected at } 34 \text { in from bottom of } \\
\text { tank at a } 30^{\circ} \text { angle. Sample was unheated, unheated } \\
\text { samples precipitated }\end{array}$ \\
\hline $\mathbf{P}$ & Raw & Al & $1.17 E+07$ & $4.34 \mathrm{E}-01$ & AP102-3 & $\gamma$ & $\begin{array}{l}\text { Density not reported for G454. Density assumed same as } \\
\text { G471 which was collected at } 208 \text { in from bottom of tank at } \\
\text { a } 150^{\circ} \text { angle and was keep heated to } 40^{\circ} \mathrm{C} \text {. }\end{array}$ \\
\hline $\mathbf{P}$ & Raw & Al & $1.17 E+07$ & 4.34E-01 & AP102-3 & $\mathrm{Y}$ & $\begin{array}{l}\text { Density reported for G459 collected at } 226 \text { in from bottom } \\
\text { of tank at a } 270^{\circ} \text { angle. Sample was unheated, unheated } \\
\text { samples precipitated }\end{array}$ \\
\hline $\mathbf{P}$ & Raw & Al & $1.17 \mathrm{E}+07$ & 4.34E-01 & AP102-3 & $\mathbf{Y}$ & $\begin{array}{l}\text { Density not reported for G338. No assumptions could be } \\
\text { made }\end{array}$ \\
\hline $\mathbf{P}$ & Raw & Al & $1.17 E+07$ & 4.34E-01 & AP102-3 & $\gamma$ & $\begin{array}{l}\text { Density not reported for G450. Density assumed same as } \\
\text { G477 which was collected at } 182 \text { in from bottom of tank at } \\
\text { a } 30^{\circ} \text { angle and was keep heated to } 40^{\circ} \mathrm{C} \text {. }\end{array}$ \\
\hline $\mathbf{P}$ & Raw & Al & $1.17 E+07$ & 4.34E-01 & AP102-3 & $Y$ & $\begin{array}{l}\text { Density not reported for G454. Density assumed same as } \\
6471 \text { which was collected at } 208 \text { in from bottom of tank at } \\
\text { a } 150^{\circ} \text { angle and was keep heated to } 40^{\circ} \mathrm{C} \text {. }\end{array}$ \\
\hline $\mathbf{P}$ & Raw & Al & $1.17 E+07$ & 4.34E-01 & AP102-3 & $\mathbf{Y}$ & $\begin{array}{l}\text { Density reported for } 6459 \text { collected at } 226 \text { in from bottom } \\
\text { of tank at a } 270^{\circ} \text { angle. Sample was unheated, unheated } \\
\text { samples precipitated }\end{array}$ \\
\hline$P$ & Raw & Al & $1.16 E+07$ & 4.30E-01 & AP102-3 & $Y$ & $\begin{array}{l}\text { Density reported for } G 348 \text { collected at } 125 \text { in from bottom } \\
\text { of tank at a } 30^{\circ} \text { angle. Sample was unheated, unheated } \\
\text { samples precipitated }\end{array}$ \\
\hline$P$ & Raw & Al & $1.16 E+07$ & 4.30E-01 & AP102-3 & $Y$ & Density reported for $\mathrm{G} 443$ represents a composite sample \\
\hline$P$ & Raw & Al & $1.16 E+07$ & 4.30E-01 & AP102-3 & $Y$ & $\begin{array}{l}\text { Density reported for } \mathrm{G} 341 \text { collected at } 99 \text { in from bottom of } \\
\text { tank at a } 270^{\circ} \text { angle. }\end{array}$ \\
\hline$P$ & Raw & Al & $1.16 E+07$ & $4.30 E-01$ & AP102-3 & $Y$ & $\begin{array}{l}\text { Density reported for } \mathrm{G} 358 \text { collected at } 334 \text { in from bottom } \\
\text { of tank at a } 150^{\circ} \text { angle. Sample was unheated, unheated } \\
\text { samples precipitated }\end{array}$ \\
\hline $\mathbf{P}$ & Raw & Al & $1.16 E+07$ & 4.30E-01 & AP102-3 & $Y$ & $\begin{array}{l}\text { Density reported for G364 collected at } 372 \text { in from bottom } \\
\text { of tank at a } 270^{\circ} \text { angle. Sample was unheated, unheated } \\
\text { samples precipitated }\end{array}$ \\
\hline $\mathbf{P}$ & Raw & Al & $1.16 E+07$ & 4.30E-01 & AP102-3 & $\mathbf{Y}$ & Density reported for G443 represents a composite sample \\
\hline $\mathbf{P}$ & Raw & Al & $1.16 E+07$ & 4.30E-01 & AP102-3 & $\boldsymbol{Y}$ & $\begin{array}{l}\text { Density not reported for G449. No assumptions could be } \\
\text { made }\end{array}$ \\
\hline $\mathbf{P}$ & Raw & Al & $1.16 \mathrm{E}+07$ & 4.30E-01 & AP102-3 & $\mathbf{Y}$ & $\begin{array}{l}\text { Density reported for G460 collected at } 226 \text { in from bottom } \\
\text { of tank at a } 270^{\circ} \text { angle. Sample was unheated, unheated } \\
\text { samples precipitated }\end{array}$ \\
\hline
\end{tabular}




\begin{tabular}{|c|c|c|c|c|c|c|c|c|c|c|c|}
\hline & & & & & nk Waste & haracteriza & Ion Sample I & rmation & & & \\
\hline Reported Chemical & $\begin{array}{c}\text { Memo } \\
\text { Date }\end{array}$ & $\begin{array}{c}\text { Start } \\
\text { Analysis } \\
\text { Date }\end{array}$ & $\begin{array}{c}\text { Finish } \\
\text { Analysis Date } \\
\end{array}$ & $\begin{array}{c}\text { Start } \\
\text { Sample } \\
\text { Date } \\
\end{array}$ & $\begin{array}{c}\text { Finish } \\
\text { Sample } \\
\text { Date } \\
\end{array}$ & $\begin{array}{l}\text { Sample } \\
\text { Number }\end{array}$ & $\begin{array}{c}\text { Tank Farm } \\
\text { Number }\end{array}$ & $\begin{array}{c}\text { Lab Sample } \\
\text { Number }\end{array}$ & $\begin{array}{l}\text { Density } \\
\text { (g/mL) }\end{array}$ & $\begin{array}{c}\text { Reported } \\
\text { Value } \\
\end{array}$ & $\begin{array}{r}\text { Repc } \\
\mathbf{U}_{\mathrm{r}} \\
\end{array}$ \\
\hline Aluminum & $6 / 1 / 90$ & $4 / 30 / 89$ & $12 / 30 / 89$ & $4 / 27 / 89$ & $4 / 29 / 89$ & BOB2N4 & G323 & G341 & 1.20 & $1.15 E+07$ & ug \\
\hline Aluminum & $6 / 1 / 90$ & $4 / 30 / 89$ & $12 / 30 / 89$ & $4 / 27 / 89$ & $4 / 29 / 89$ & BOB2MO & G306 & G352 & 1.20 & $1.15 E+07$ & ug \\
\hline Aluminum & $6 / 1 / 90$ & $4 / 30 / 89$ & $12 / 30 / 89$ & $4 / 27 / 89$ & $4 / 29 / 89$ & ВОВ2M8 & G301 & G449 & NA & $1.15 E+07$ & ug \\
\hline Aluminum: & $6 / 1 / 90$ & $4 / 30 / 89$ & $12 / 30 / 89$ & $4 / 27 / 89$ & $4 / 29 / 89$ & $8082 L 7$ & G299 & G342 & 1.20 & $1.15 \mathrm{E}+07$ & ug. \\
\hline Aluminum & $6 / 1 / 90$ & $4 / 30 / 89$ & $12 / 30 / 89$ & $4 / 27 / 89$ & $4 / 29 / 89$ & BOB2MO & G306 & G352 & 1.20 & $1.15 E+07$ & $u g_{t}$ \\
\hline Aluminum & $6 / 1 / 90$ & $4 / 30 / 89$ & $12 / 30 / 89$ & $4 / 27 / 89$ & $4 / 29 / 89$ & BOB2NO & G307 & G453 & NA & $1.15 E+07$ & ug \\
\hline Aluminum & $6 / 1 / 90$ & $4 / 30 / 89$ & $12 / 30 / 89$ & $4 / 27 / 89$ & $4 / 29 / 89$ & $\mathrm{BOB} 2 \mathrm{~N} 2$ & G317 & G333 & NA & $1.14 E+07$ & ugi \\
\hline Aluminum & $6 / 1 / 90$ & $4 / 30 / 89$ & $12 / 30 / 89$ & $4 / 27 / 89$ & $4 / 29 / 89$ & BOB2L7 & G299 & G342 & 1.20 & $1.14 E+07$ & ugi \\
\hline Aluminum & $6 / 1 / 90$ & $4 / 30 / 89$ & $12 / 30 / 89$ & $4 / 27 / 89$ & $4 / 29 / 89$ & $\mathrm{BOB2M1}$ & G308 & G353 & 1.20 & $1.14 E+07$ & ugl \\
\hline Aluminum & $6 / 1 / 90$ & $4 / 30 / 89$ & $12 / 30 / 89$ & $4 / 27 / 89$ & $4 / 29 / 89$ & BOB2M5 & G315 & G364 & 1.19 & $1.14 E+07$ & ugt \\
\hline Aluminum & $6 / 1 / 90$ & $4 / 30 / 89$ & $12 / 30 / 89$ & $4 / 27 / 89$ & $4 / 29 / 89$ & BOB2M7 & G319 & G460 & 1.20 & $1.14 E+07$ & ugf \\
\hline Aluminum & $6 / 1 / 90$ & $4 / 30 / 89$ & $12 / 30 / 89$ & $4 / 27 / 89$ & $4 / 29 / 89$ & BOB2N2 & G317 & G333 & NA & $1.14 E+07$ & ug/. \\
\hline Aluminum & $6 / 1 / 90$ & $4 / 30 / 89$ & $12 / 30 / 89$ & $4 / 27 / 89$ & $4 / 29 / 89$ & BOB2M4 & G313 & G363 & 1.20 & $1.14 E+07$ & ug/l \\
\hline Aluminum & $6 / 1 / 90$ & $4 / 30 / 89$ & $12 / 30 / 89$ & $4 / 27 / 89$ & $4 / 29 / 89$ & BOB2M4 & G313 & G363 & 1.20 & $1.13 E+07$ & $\mathrm{ug} / \mathrm{I}$ \\
\hline Aluminum & $6 / 1 / 90$ & $4 / 30 / 89$ & $12 / 30 / 89$ & $4 / 27 / 89$ & $4 / 29 / 89$ & $8082 M 1$ & G308 & G353 & 1.20 & $1.13 E+07$ & $u g / l$ \\
\hline Aluminum & $6 / 1 / 90$ & $4 / 30 / 89$ & $12 / 30 / 89$ & $4 / 27 / 89$ & $4 / 29 / 89$ & ВОВ2M3 & G311 & G359 & 1.20 & $1.12 E+07$ & ugh $h$ \\
\hline Aluminum & $6 / 1 / 90$ & $4 / 30 / 89$ & $12 / 30 / 89$ & $4 / 27 / 89$ & $4 / 29 / 89$ & ВОВ2M2 & G309 & G358 & 1.20 & $1.11 E+07$ & $u g h$ \\
\hline Aluminum & 6/1/90 & $4 / 30 / 89$ & $12 / 30 / 89$ & $4 / 27 / 89$ & $4 / 29 / 89$ & ВOB2M3 & G311 & G359 & 1.20 & $1.11 E+07$ & ug $\Omega$ \\
\hline Arsenic & $6 / 1 / 90$ & $4 / 30 / 89$ & $12 / 30 / 89$ & $4 / 27 / 89$ & $4 / 29 / 89$ & $8082 T 9$ & G327 & G470 & 1.19 & $<2.50 E+02$ & $\operatorname{ug} \Omega$ \\
\hline Arsenic & $6 / 1 / 90$ & $4 / 30 / 89$ & $12 / 30 / 89$ & $4 / 27 / 89$ & $4 / 29 / 89$ & BOB2T9 & G327 & G470 & 1.19 & $<2.50 E+02$ & $u g /$ \\
\hline Arsenic & $6 / 1 / 90$ & $4 / 30 / 89$ & $12 / 30 / 89$ & $4 / 27 / 89$ & $4 / 29 / 89$ & ВОВ2M9 & G302 & G477* & 1.20 & $<2.50 E+02$ & $u g h$ \\
\hline
\end{tabular}




\begin{tabular}{|c|c|c|c|c|c|c|c|}
\hline \multirow[b]{2}{*}{ Code } & \multirow[b]{2}{*}{ Source } & \multicolumn{3}{|c|}{ Analyte Information } & \multirow[b]{2}{*}{$\begin{array}{c}\text { Reference } \\
\text { Number }\end{array}$} & \multirow[b]{2}{*}{$\begin{array}{l}\text { Validated } \\
\text { (Y/N) }\end{array}$} & \multirow[b]{2}{*}{ Notes } \\
\hline & & Analyte & $\begin{array}{l}\text { Cone. } \\
\text { (ug/L) }\end{array}$ & Conc. (mol/L) & & & \\
\hline $\mathbf{P}$ & Raw & Al & $1.15 E+07$ & $4.26 \mathrm{E}-01$ & AP102-3 & $Y$ & $\begin{array}{l}\text { Density reported for } \mathrm{G} 341 \text { collected at } 99 \text { in from bottom of } \\
\text { tank at a } 270^{\circ} \text { angle. }\end{array}$ \\
\hline $\mathbf{P}$ & Raw & Al & $1.15 \mathrm{E}+07$ & $4.26 \mathrm{E}-01$ & AP102-3 & $Y$ & $\begin{array}{l}\text { Density reported for } \mathrm{G} 352 \text { collected at } 34 \text { in from bottom of } \\
\text { tank at a } 30^{\circ} \text { angle. Sample was unheated, unheated } \\
\text { samples precipitated }\end{array}$ \\
\hline $\mathbf{P}$ & Raw & Al & $1.15 E+07$ & $4.26 E-01$ & AP102-3 & $Y$ & $\begin{array}{l}\text { Density not reported for G449. No assumptions could be } \\
\text { made }\end{array}$ \\
\hline $\mathbf{P}$ & Raw & Al & $1.15 E+07$ & $4.26 E-01$ & AP102-3 & $Y$ & $\begin{array}{l}\text { Density reported for G342 collected at } 306 \text { in from bottom } \\
\text { of tank at a } 30^{\circ} \text { angle. Sample was unheated, unheated } \\
\text { samples precipitated }\end{array}$ \\
\hline $\mathbf{P}$ & Raw & $\mathrm{Al}$ & $1.15 E+07$ & $4.26 E-01$ & AP102-3 & $\mathbf{Y}$ & $\begin{array}{l}\text { Density reported for } \mathrm{G} 352 \text { collected at } 34 \text { in from bortom of } \\
\text { tank at a } 30^{\circ} \text { angle. Sample was unheated, unheated } \\
\text { samples precipitated }\end{array}$ \\
\hline $\mathbf{P}$ & Raw & Al & $1.15 E+07$ & $4.26 E-01$ & AP102-3 & $Y$ & $\begin{array}{l}\text { Density not reported for G453. No assumptions could be } \\
\text { made }\end{array}$ \\
\hline$P$ & Raw & Al & $1.14 E+07$ & $4.23 E-01$ & AP102-3 & Y & $\begin{array}{l}\text { Density not reported for G333. No assumptions could be } \\
\text { made }\end{array}$ \\
\hline$P$ & Raw & Al & $1.14 E+07$ & 4.23E-01 & AP102-3 & Y & $\begin{array}{l}\text { Density reported for } \mathrm{G} 342 \text { collected at } 306 \text { in from bottom } \\
\text { of tank at a } 30^{\circ} \text { angle. Sample was unheated, unheated } \\
\text { samples precipitated }\end{array}$ \\
\hline $\mathbf{P}$ & Raw & Al & $1.14 E+07$ & 4.23E-01 & AP102-3 & $\mathbf{Y}$ & $\begin{array}{l}\text { Density reported for G353 collected at } 334 \text { in from bottom } \\
\text { of tank at a } 150^{\circ} \text { angle. Sample was unheated, unheated } \\
\text { samples precipitated }\end{array}$ \\
\hline$P$ & Raw & Al & $1.14 E+07$ & $4.23 E-01$ & AP $102-3$ & $\mathbf{Y}$ & $\begin{array}{l}\text { Density reported for G364 collected at } 372 \text { in from bottom } \\
\text { of tank at a } 270^{\circ} \text { angle. Sample was unheated, unheated } \\
\text { samples precipitated }\end{array}$ \\
\hline $\mathbf{P}$ & Raw & Al & $1.14 E+07$ & $4.23 E-01$ & AP102-3 & $Y$ & $\begin{array}{l}\text { Density reported for G } 460 \text { collected at } 226 \text { in from bottom } \\
\text { of tank at a } 270^{\circ} \text { angle. Sample was unheated, unheated } \\
\text { samples precipitated }\end{array}$ \\
\hline$P$ & Raw & Al & $1.14 E+07$ & 4.23E-01 & AP102-3 & 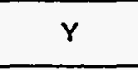 & $\begin{array}{l}\text { Density not reported for G333. No assumptions could be } \\
\text { made }\end{array}$ \\
\hline$P$ & Raw & Al & $1.14 E+07$ & $4.23 E-01$ & AP102-3 & $Y$ & $\begin{array}{l}\text { Density reported for } \mathrm{G} 363 \text { collected at } 18 \text { in from bottom of } \\
\text { tank at a } 150^{\circ} \text { angle. Sample was unheated, unheated } \\
\text { samples precipitated }\end{array}$ \\
\hline $\mathbf{P}$ & Raw & Al & $1.13 E+07$ & 4.19E-01 & AP102-3 & $\mathbf{Y}$ & $\begin{array}{l}\text { Density reported for G363 collected at } 18 \text { in from bottom of } \\
\text { tank at a } 150^{\circ} \text { angle. Sample was unheated, unheated } \\
\text { samples precipitated }\end{array}$ \\
\hline$P$ & Raw & Al & $1.13 E+07$ & 4.19E-01 & AP102-3 & $\mathrm{Y}$ & $\begin{array}{l}\text { Density reported for G353 collected at } 334 \text { in from bottom } \\
\text { of tank at a } 150^{\circ} \text { angle. Sample was unheated, unheated } \\
\text { samples precipitated }\end{array}$ \\
\hline $\mathbf{P}$ & Raw & Al & $1.12 E+07$ & 4.15E-01 & AP102-3 & $Y$ & $\begin{array}{l}\text { Density reported for G359 collected at } 162 \text { in from bottom } \\
\text { of tank at a } 150^{\circ} \text { angle. Sample was unheated, unheated } \\
\text { samples precipitated }\end{array}$ \\
\hline $\mathbf{P}$ & Raw & Al & $1.11 E+07$ & $4.11 E-01$ & AP102-3 & $Y$ & $\begin{array}{l}\text { Density reported for G358 collected at } 334 \text { in from bottom } \\
\text { of tank at a } 150^{\circ} \text { angle. Sample was unheated, unheated } \\
\text { samples precipitated }\end{array}$ \\
\hline$P$ & Raw & $\mathrm{Al}$ & $1.11 E+07$ & 4.11E-01 & AP102-3 & $\mathbf{Y}$ & $\begin{array}{l}\text { Density reported for G359 collected at } 162 \text { in from bottom } \\
\text { of tank at a } 150^{\circ} \text { angle. Sample was unheated, unheated } \\
\text { samples precipitated }\end{array}$ \\
\hline $\mathbf{P}$ & Raw & As & \#VALUEI & \#ALUE! & AP102-3 & $Y$ & $\begin{array}{l}\text { Density reported for G470 represents a composite sample. } \\
\text { Sample was kept heated to } 40^{\circ} \mathrm{C} \text {. }\end{array}$ \\
\hline$P$ & Raw & As & *VALUE! & \#VALUE! & AP102-3 & $Y$ & $\begin{array}{l}\text { Density reported for G470 represents a composite sample. } \\
\text { Sample was kept heated to } 40^{\circ} \mathrm{C} \text {. }\end{array}$ \\
\hline$P$ & Raw & As & \#VALUEI & \#VALUE! & AP102-3 & $Y$ & $\begin{array}{l}\text { Density reported for G477 collected at } 182 \text { in from bottom } \\
\text { of tank at a } 30^{\circ} \text { angle and was keep heated to } 40^{\circ} \mathrm{C} \text {. }\end{array}$ \\
\hline
\end{tabular}




\begin{tabular}{|c|c|c|c|c|c|c|c|c|c|c|c|}
\hline & & & & & ank Waste & haracteriza & ion Sample I & ormation & & & \\
\hline Reported Chemical & $\begin{array}{c}\text { Memo } \\
\text { Date }\end{array}$ & $\begin{array}{c}\text { Start } \\
\text { Analysis } \\
\text { Date } \\
\end{array}$ & $\begin{array}{c}\text { Finish } \\
\text { Analysis Date } \\
\end{array}$ & $\begin{array}{c}\text { Start } \\
\text { Sample } \\
\text { Date }\end{array}$ & $\begin{array}{c}\text { Finish } \\
\text { Sample } \\
\text { Date }\end{array}$ & $\begin{array}{l}\text { Sample } \\
\text { Number }\end{array}$ & $\begin{array}{c}\text { Tank Farm } \\
\text { Number }\end{array}$ & $\begin{array}{c}\text { Lab Sample } \\
\text { Number }\end{array}$ & $\begin{array}{l}\text { Density } \\
\text { (g/mL) }\end{array}$ & $\begin{array}{c}\text { Reported } \\
\text { Value } \\
\end{array}$ & $\begin{array}{r}\text { Repo } \\
\mathbf{U}_{\mathrm{n}} \\
\end{array}$ \\
\hline Arsenic & $6 / 1 / 90$ & $4 / 30 / 89$ & $12 / 30 / 89$ & $4 / 27 / 89$ & $4 / 29 / 89$ & ВOB2M9 & G302 & G477* & 1.20 & $<2.50 E+02$ & ug/ \\
\hline Arsenic & $6 / 1 / 90$ & $4 / 30 / 89$ & $12 / 30 / 89$ & $4 / 27 / 89$ & $4 / 29 / 89$ & BOB2N1 & G310 & G471* & 1.19 & $<2.50 E+02$ & $u g f$ \\
\hline Arsenic & $6 / 1 / 90$ & $4 / 30 / 89$ & $12 / 30 / 89$ & $4 / 27 / 89$ & $4 / 29 / 89$ & BOB2N1 & G310 & G471• & 1.19 & $<2.50 E+02$ & ugl \\
\hline Arsenic & $6 / 1 / 90$ & $4 / 30 / 89$ & $12 / 30 / 89$ & $4 / 27 / 89$ & $4 / 29 / 89$ & BOB2N4 & G323 & G476* & 1.21 & $<2.50 E+02$ & ugn \\
\hline Arsenic & $6 / 1 / 90$ & $4 / 30 / 89$ & $12 / 30 / 89$ & $4 / 27 / 89$ & $4 / 29 / 89$ & $\mathrm{BOB2N4}$ & G323 & G476* & 1.21 & $<2.50 E+02$ & $\operatorname{ug} n$ \\
\hline AS & $2 / 10 / 89$ & NS & NS & NS & NS & NS & NS & NS & 0.956764 & 4.74E-02 & $\overline{p p n}$ \\
\hline Arsenic & $6 / 1 / 90$ & $4 / 30 / 89$ & $12 / 30 / 89$ & $4 / 27 / 89$ & $4 / 29 / 89$ & вов2Т9 & G327 & G443 & 1.20 & $1.02 E+02$ & ug/l \\
\hline Arsenic & $6 / 1 / 90$ & $4 / 30 / 89$ & $12 / 30 / 89$ & $4 / 27 / 89$ & $4 / 29 / 89$ & вов2Т9 & G327 & G443 & 1.20 & $7.59 E+01$ & $u g n$ \\
\hline Barium & $6 / 1 / 90$ & $4 / 30 / 89$ & $12 / 30 / 89$ & $4 / 27 / 89$ & $4 / 29 / 89$ & ВОВ2T9 & G327 & G470* & 1.19 & $<3.00 E+03$ & $u g / h$ \\
\hline Barium & $6 / 1 / 90$ & $4 / 30 / 89$ & $12 / 30 / 89$ & $4 / 27 / 89$ & $4 / 29 / 89$ & ВOB2T9 & G327 & G470 & 1.19 & $<3.00 E+03$ & $u g / h$ \\
\hline $\mathrm{Ba}$ & $2 / 10 / 89$ & NS & NS & NS & NS & NS & NS & NS & 0.956764 & $4.74 \mathrm{E}-01$ & $\overline{p p r}$ \\
\hline Barium & $6 / 1 / 90$ & $4 / 30 / 89$ & $12 / 30 / 89$ & $4 / 27 / 89$ & $4 / 29 / 89$ & BOB2N3 & G322 & G338 & NA & $4.19 E+02$ & ugh \\
\hline Barium & $6 / 1 / 90$ & $4 / 30 / 89$ & $12 / 30 / 89$ & $4 / 27 / 89$ & $4 / 29 / 89$ & BO82N3 & G322 & G338 & NA & $3.65 E+02$ & ug/h \\
\hline Barium & $6 / 1 / 90$ & $4 / 30 / 89$ & $12 / 30 / 89$ & $4 / 27 / 89$ & $4 / 29 / 89$ & ВОВ2M9 & G302 & G450 & $(1.20)$ & $3.59 E+02$ & ugh \\
\hline Barium & $6 / 1 / 90$ & $4 / 30 / 89$ & $12 / 30 / 89$ & $4 / 27 / 89$ & $4 / 29 / 89$ & ВОВ2M9 & G302 & G450 & $(1.20)$ & $3.54 E+02$ & ught. \\
\hline Barium & $6 / 1 / 90$ & $4 / 30 / 89$ & $12 / 30 / 89$ & $4 / 27 / 89$ & $4 / 29 / 89$ & BOB2N2 & 6317 & G333 & NA & $3.45 E+02$ & ug/h. \\
\hline Barium & $6 / 1 / 90$ & $4 / 30 / 89$ & $12 / 30 / 89$ & $4 / 27 / 89$ & $4 / 29 / 89$ & В0В2M8 & G301 & G449 & NA & $3.43 E+02$ & $u g / L$ \\
\hline Barium & $6 / 1 / 90$ & $4 / 30 / 89$ & $12 / 30 / 89$ & $4 / 27 / 89$ & $4 / 29 / 89$ & BOB2L9 & G305 & G349 & 1.20 & $3.42 E+02$ & $u g / L$ \\
\hline Barium & $6 / 1 / 90$ & $4 / 30 / 89$ & $12 / 30 / 89$ & $4 / 27 / 89$ & $4 / 29 / 89$ & ВОВ2М2 & G309 & G358 & 1.20 & $3.42 E+02$ & ug $/ \mathrm{L}$ \\
\hline Barium & $6 / 1 / 90$ & $4 / 30 / 89$ & $12 / 30 / 89$ & $4 / 27 / 89$ & $4 / 29 / 89$ & BOB2N1 & G310 & G454 & $(1.19)$ & $3.40 E+02$ & $u g / \Omega$ \\
\hline Barium & $6 / 1 / 90$ & $4 / 30 / 89$ & $12 / 30 / 89$ & $4 / 27 / 89$ & $4 / 29 / 89$ & ВОВ2M8 & G301 & G449 & NA & $3.38 E+02$ & ug $\Omega$ \\
\hline Barium & $6 / 1 / 90$ & $4 / 30 / 89$ & $12 / 30 / 89$ & $4 / 27 / 89$ & $4 / 29 / 89$ & BOB2NO & G307 & G453 & NA & $3.35 E+02$ & $u g / h$ \\
\hline Barium & $6 / 1 / 90$ & $4 / 30 / 89$ & $12 / 30 / 89$ & $4 / 27 / 89$ & $4 / 29 / 89$ & BOB2N1 & G310 & G454 & $(1.19)$ & $3.27 E+02$ & ug $n$ \\
\hline Barium & $6 / 1 / 90$ & $4 / 30 / 89$ & $12 / 30 / 89$ & $4 / 27 / 89$ & $4 / 29 / 89$ & BOB2L9 & G305 & G349 & 1.20 & $3.22 E+02$ & $u g / L$ \\
\hline
\end{tabular}




\begin{tabular}{|c|c|c|c|c|c|c|c|}
\hline \multirow[b]{2}{*}{ Code } & \multirow[b]{2}{*}{ Source } & \multicolumn{3}{|c|}{ Analyte information } & \multirow[b]{2}{*}{$\begin{array}{l}\text { Reference } \\
\text { Number }\end{array}$} & \multirow[b]{2}{*}{$\begin{array}{l}\text { Validated } \\
(Y / N)\end{array}$} & \multirow[b]{2}{*}{ Notes } \\
\hline & & Analyte & $\begin{array}{l}\text { Conc. } \\
\text { (ug/L) }\end{array}$ & Conc. (mol/L) & & & \\
\hline$P$ & Raw & As & *VALUE! & \#VALUE! & AP102-3 & $Y$ & $\begin{array}{l}\text { Density reported for } \mathrm{G} 477 \text { collected at } 182 \text { in from bottom } \\
\text { of tank at a } 30^{\circ} \text { angle and was keep heated to } 40^{\circ} \mathrm{C} \text {. }\end{array}$ \\
\hline $\mathbf{P}$ & Raw & As & *VALUEI & \#VALUE! & AP102-3 & $\gamma$ & $\begin{array}{l}\text { Density reported for } \mathrm{G} 471 \text { collected at } 208 \text { in from bottom } \\
\text { of tank at a } 150^{\circ} \text { angle and was keep heated to } 40^{\circ} \mathrm{C} \text {. }\end{array}$ \\
\hline $\mathbf{P}$ & Raw & As & Falue! & FVALUEI & AP102-3 & $Y$ & $\begin{array}{l}\text { Density reported for G471 collected at } 208 \text { in from bottom } \\
\text { of tank at a } 150^{\circ} \text { angle and was keep heated to } 40^{\circ} \mathrm{C} \text {. }\end{array}$ \\
\hline$P$ & Raw & As & \#VALUE! & \#VALUE! & AP102-3 & $Y$ & $\begin{array}{l}\text { Density reported for } \mathrm{G} 476 \text { collected at } 99 \text { in from bottom of } \\
\text { tank at a } 270^{\circ} \text { angle and was keep heated to } 40^{\circ} \mathrm{C} \text {. }\end{array}$ \\
\hline$P$ & Raw & As & FALUEI & \#VALUEl & AP102-3 & $\begin{array}{l}Y \\
.\end{array}$ & $\begin{array}{l}\text { Density reported for } 6476 \text { collected at } 99 \text { in from bottom of } \\
\text { tank at a } 270^{\circ} \text { angle and was keep heated to } 40^{\circ} \mathrm{C} \text {. }\end{array}$ \\
\hline$\underline{U}$ & RAW & As & $4.53 E+07$ & 6.05E-01 & AP102-2 & $\mathbf{N}$ & Projected value, not a real data point \\
\hline$P$ & Raw & As & $1.02 E+02$ & $1.36 \mathrm{E}-06$ & AP102-3 & $\mathbf{Y}$ & Density reported for $\mathrm{G} 443$ represents a composite sample \\
\hline$P$ & Raw & As & $7.59 E+01$ & $1.01 E-06$ & AP102-3 & Y & Density reported for $\mathrm{G} 443$ represents a composite sample \\
\hline$P$ & Raw & $\mathrm{Ba}$ & \#VALUE! & \#VALUE! & AP102-3 & $Y$ & $\begin{array}{l}\text { Density reported for } \mathrm{G} 470 \text { represents a composite sample. } \\
\text { Sample was kept heated to } 40^{\circ} \mathrm{C} \text {. }\end{array}$ \\
\hline$P$ & Raw & $8 a$ & \#VALUE! & \#VALUE! & AP102-3 & $Y$ & $\begin{array}{l}\text { Density reported for G470 represents a composite sample. } \\
\text { Sample was kept heated to } 40^{\circ} \mathrm{C} \text {. }\end{array}$ \\
\hline$\underline{U}$ & RAW & $\mathrm{Ba}$ & $4.53 \mathrm{E}+08$ & $3.30 E+00$ & AP102-2 & N & Projected value, not a real data point \\
\hline$P$ & Raw & $\mathrm{Ba}$ & $4.19 E+02$ & $3.05 E-06$ & AP102-3 & $Y$ & $\begin{array}{l}\text { Density not reported for } \mathbf{G} 338 \text {. No assumptions could be } \\
\text { made }\end{array}$ \\
\hline $\mathbf{P}$ & Raw & $\mathrm{Ba}$ & $3.65 E+02$ & $2.66 \mathrm{E}-06$ & AP102-3. & $\mathbf{Y}$ & $\begin{array}{l}\text { Density not reported for G338. No assumptions could be } \\
\text { made }\end{array}$ \\
\hline $\mathbf{P}$ & Raw & $\mathrm{Ba}$ & $3.59 E+02$ & 2.61E-06 & AP102-3 & $Y$ & $\begin{array}{l}\text { Density not reported for G450. Density assumed same as } \\
\mathrm{G} 477 \text { which was collected at } 182 \text { in from bottom of tank at } \\
\text { a } 30^{\circ} \text { angle and was keep heated to } 40^{\circ} \mathrm{C} \text {. }\end{array}$ \\
\hline$P$ & Raw & $\mathrm{Ba}$ & $3.54 E+02$ & $2.58 \mathrm{E}-06$ & AP102-3 & $Y$ & $\begin{array}{l}\text { Density not reported for G450. Density assumed same as } \\
\text { G477 which was collected at } 182 \text { in from bottom of tank at } \\
\text { a } 30^{\circ} \text { angle and was keep heated to } 40^{\circ} \mathrm{C} \text {. }\end{array}$ \\
\hline$P$ & Raw & $\mathrm{Ba}$ & $3.45 E+02$ & $2.51 E-06$ & AP102-3 & $Y$ & $\begin{array}{l}\text { Density not reported for G333. No assumptions could be } \\
\text { made }\end{array}$ \\
\hline $\mathbf{P}$ & Raw & $\mathrm{Ba}$ & $3.43 E+02$ & $2.50 E-06$ & AP102-3 & $Y$ & $\begin{array}{l}\text { Density not reported for G449. No assumptions could be } \\
\text { made }\end{array}$ \\
\hline$P$ & Raw & $\mathrm{Ba}$ & $3.42 E+02$ & $2.49 E-06$ & AP102-3 & $Y$ & $\begin{array}{l}\text { Density reported for } \mathrm{G} 349 \text { collected at } 34 \text { in from bottom of } \\
\text { tank at a } 30^{\circ} \text { angle. Sample was unheated, unheated } \\
\text { samples precipitated }\end{array}$ \\
\hline $\mathbf{P}$ & Raw & $\mathrm{Ba}$ & $3.42 E+02$ & 2.49E-06 & AP102-3 & $Y$ & $\begin{array}{l}\text { Density reported for } \mathrm{G} 358 \text { collected at } 334 \text { in from bottom } \\
\text { of tank at a } 150^{\circ} \text { angle. Sample was unheated, unheated } \\
\text { samples precipitated }\end{array}$ \\
\hline$P$ & Raw & $\mathrm{Ba}$ & $3.40 E+02$ & $2.48 E-06$ & AP102-3 & $\mathbf{Y}$ & $\begin{array}{l}\text { Density not reported for G454. Density assumed same as } \\
\text { G471 which was collected at } 208 \text { in from bottom of tank at } \\
\text { a } 150^{\circ} \text { angle and was keep heated to } 40^{\circ} \mathrm{C} \text {. }\end{array}$ \\
\hline$P$ & Raw & $\mathrm{Ba}$ & $3.38 \mathrm{E}+02$ & $2.46 E-06$ & AP102-3 & $Y$ & $\begin{array}{l}\text { Density not reported for } \mathrm{G} 449 \text {. No assumptions could be } \\
\text { made }\end{array}$ \\
\hline$P$ & Raw & $\mathrm{Ba}$ & $3.35 E+02$ & 2.44E-06 & AP102-3 & $Y$ & $\begin{array}{l}\text { Density not reported for G453. No assumptions could be } \\
\text { made }\end{array}$ \\
\hline$P$ & Raw & $8 a$ & $3.27 E+02$ & 2.38E-06 & AP102-3 & $Y$ & $\begin{array}{l}\text { Density not reported for G454. Density assumed same as } \\
\text { G471 which was collected at } 208 \text { in from bottom of tank at } \\
\text { a } 150^{\circ} \text { angle and was keep heated to } 40^{\circ} \mathrm{C} \text {. }\end{array}$ \\
\hline$P$ & Raw & $\mathrm{Ba}$ & $3.22 E+02$ & 2.34E-06 & AP102-3 & $\mathbf{Y}$ & $\begin{array}{l}\text { Density reported for G349 collected at } 34 \text { in from bottom of } \\
\text { tank at a } 30^{\circ} \text { angle. Sample was unheated, unheated } \\
\text { samples precipitated }\end{array}$ \\
\hline
\end{tabular}




\begin{tabular}{|c|c|c|c|c|c|c|c|c|c|c|c|}
\hline Reported Chemical & $\begin{array}{c}\text { Memo } \\
\text { Date }\end{array}$ & $\begin{array}{c}\text { Start } \\
\text { Analysis } \\
\text { Date }\end{array}$ & $\begin{array}{c}\text { Finish } \\
\text { Analysis Date } \\
\end{array}$ & $\begin{array}{c}\text { Start } \\
\text { Sample } \\
\text { Date }\end{array}$ & $\begin{array}{c}\text { Finish } \\
\text { Sample } \\
\text { Date } \\
\end{array}$ & $\begin{array}{l}\text { Sample } \\
\text { Number }\end{array}$ & $\begin{array}{c}\text { Tank Farm } \\
\text { Number }\end{array}$ & $\begin{array}{c}\text { Lab Samplo } \\
\text { Number } \\
\end{array}$ & $\begin{array}{l}\text { Density } \\
(\mathrm{g} / \mathrm{mL})\end{array}$ & $\begin{array}{c}\text { Reported } \\
\text { Value }\end{array}$ & $\begin{array}{r}\text { Repo: } \\
\text { U } \\
\end{array}$ \\
\hline Barium & $6 / 1 / 90$ & $4 / 30 / 89$ & $12 / 30 / 89$ & $4 / 27 / 89$ & $4 / 29 / 89$ & вов2мо & G306 & G352 & 1.20 & $3.14 E+02$ & ug \\
\hline Barium & $6 / 1 / 90$ & $4 / 30 / 89$ & $12 / 30 / 89$ & $4 / 27 / 89$ & $4 / 29 / 89$ & ВОВ2M3 & G311 & G359 & 1.20 & $3.09 E+02$ & ug. \\
\hline Barium & $6 / 1 / 90$ & $4 / 30 / 89$ & $12 / 30 / 89$ & $4 / 27 / 89$ & $4 / 29 / 89$ & ВОВ2M3 & G311 & G359 & 1.20 & $3.00 E+02$ & ugi \\
\hline Barium & $6 / 1 / 90$ & $4 / 30 / 89$ & $12 / 30 / 89$ & $4 / 27 / 89$ & $4 / 29 / 89$ & воB2M4 & G313 & G363 & 1.20 & $2.89 E+02$ & ugl \\
\hline Barium & $6 / 1 / 90$ & $4 / 30 / 89$ & $12 / 30 / 89$ & $4 / 27 / 89$ & $4 / 29 / 89$ & BOB2N2 & G317 & G333 & NA & $2.88 E+02$ & ug/ \\
\hline Barium & $6 / 1 / 90$ & $4 / 30 / 89$ & $12 / 30 / 89$ & $4 / 27 / 89$ & $4 / 29 / 89$ & BOB2M4 & G313 & G363 & 1.20 & $2.86 E+02$ & ugi \\
\hline Barium & $6 / 1 / 90$ & $4 / 30 / 89$ & $12 / 30 / 89$ & $4 / 27 / 89$ & $4 / 29 / 89$ & $\mathrm{BOB} 2 \mathrm{M2}$ & 6309 & G358 & 1.20 & $2.84 E+02$ & ug/l \\
\hline Barium & $6 / 1 / 90$ & $4 / 30 / 89$ & $12 / 30 / 89$ & $4 / 27 / 89$ & $4 / 29 / 89$ & BOB2N4 & G323 & G341 & 1.20 & $2.83 E+02$ & ug/l \\
\hline Barium & $6 / 1 / 90$ & $4 / 30 / 89$ & $12 / 30 / 89$ & $4 / 27 / 89$ & $4 / 29 / 89$ & BOB2MG & G318 & G459 & 1.20 & $2.73 E+02$ & $u g / h$ \\
\hline Barium & $6 / 1 / 90$ & $4 / 30 / 89$ & $12 / 30 / 89$ & $4 / 27 / 89$ & $4 / 29 / 89$ & BOB2L8 & G303 & G348 & 1.21 & $2.68 E+02$ & ug/h \\
\hline Barium & $6 / 1 / 90$ & $4 / 30 / 89$ & $12 / 30 / 89$ & $4 / 27 / 89$ & $4 / 29 / 89$ & BOB2L8 & G303 & G348 & 1.21 & $2.60 E+02$ & ugh $n$ \\
\hline Barium & $6 / 1 / 90$ & $4 / 30 / 89$ & $12 / 30 / 89$ & $4 / 27 / 89$ & $4 / 29 / 89$ & BOB2L7 & G299 & G342 & 1.20 & $2.58 E+02$ & ug $\Omega$ \\
\hline Barium & $6 / 1 / 90$ & $4 / 30 / 89$ & $12 / 30 / 89$ & $4 / 27 / 89$ & $4 / 29 / 89$ & BOB2M6 & G318 & G459 & 1.20 & $2.52 E+02$ & ug/h. \\
\hline Barium & $6 / 1 / 90$ & $4 / 30 / 89$ & $12 / 30 / 89$ & $4 / 27 / 89$ & $4 / 29 / 89$ & BOB2M7 & G319 & G460 & 1.20 & $2.52 E+02$ & ug/h \\
\hline Barium & $6 / 1 / 90$ & $4 / 30 / 89$ & $12 / 30 / 89$ & $4 / 27 / 89$ & $4 / 29 / 89$ & ВOВ2MO & G306 & G352 & 1.20 & $2.48 E+02$ & $u g / L$ \\
\hline Barium & $6 / 1 / 90$ & $4 / 30 / 89$ & $12 / 30 / 89$ & $4 / 27 / 89$ & $4 / 29 / 89$ & BOB2M1 & G308 & G353 & 1.20 & $2.48 E+02$ & ug $/ \mathrm{L}$ \\
\hline Barium & $6 / 1 / 90$ & $4 / 30 / 89$ & $12 / 30 / 89$ & $4 / 27 / 89$ & $4 / 29 / 89$ & Вов2T9 & G327 & G443 & 1.20 & $2.48 E+02$ & ugh \\
\hline Barium & $6 / 1 / 90$ & $4 / 30 / 89$ & $12 / 30 / 89$ & $4 / 27 / 89$ & $4 / 29 / 89$ & Вов2M1 & G308 & G353 & 1.20 & $2.37 E+02$ & $\mathrm{ug} / \mathrm{L}$ \\
\hline Barium & $6 / 1 / 90$ & $4 / 30 / 89$ & $12 / 30 / 89$ & $4 / 27 / 89$ & $4 / 29 / 89$ & BOB2NO & G307 & G453 & NA & $2.11 E+02$ & $\operatorname{ug} h$ \\
\hline Barium & $6 / 1 / 90$ & $4 / 30 / 89$ & $12 / 30 / 89$ & $4 / 27 / 89$ & $4 / 29 / 89$ & BOB2M7 & G319 & G460 & 1.20 & $2.07 E+02$ & ug/L. \\
\hline Barium & $6 / 1 / 90$ & $4 / 30 / 89$ & $12 / 30 / 89$ & $4 / 27 / 89$ & $4 / 29 / 89$ & BOB2M5 & G315 & G364 & 1.19 & $1.70 E+02$ & $u g h$ \\
\hline
\end{tabular}




\begin{tabular}{|c|c|c|c|c|c|c|c|}
\hline \multirow[b]{2}{*}{ Code } & \multirow[b]{2}{*}{ Source } & \multicolumn{3}{|c|}{ Analyte Information } & \multirow[b]{2}{*}{$\begin{array}{l}\text { Referenco } \\
\text { Number }\end{array}$} & \multirow[b]{2}{*}{$\begin{array}{l}\text { Validated } \\
\text { (Y/N) }\end{array}$} & \multirow[b]{2}{*}{ Notes } \\
\hline & & Analyte & $\begin{array}{l}\text { Conc. } \\
\text { (ug/L) }\end{array}$ & Conc. (mol/L) & & & \\
\hline $\mathbf{P}$ & Raw & Ba & $3.14 E+02$ & 2.29E-06 & AP102-3 & $Y$ & $\begin{array}{l}\text { Density reported for } G 352 \text { collected at } 34 \text { in from bottom of } \\
\text { tank at a } 30^{\circ} \text { angle. Sample was unheated, unheated } \\
\text { samples precipitated }\end{array}$ \\
\hline $\mathbf{P}$ & Raw & Ba & $3.09 E+02$ & $2.25 E-06$ & AP102-3 & $Y$ & $\begin{array}{l}\text { Density reported for } 6359 \text { collected at } 162 \text { in from bottom } \\
\text { of tank at a } 150^{\circ} \text { angle. Sample was unheated, unheated } \\
\text { samples precipitated }\end{array}$ \\
\hline $\mathbf{P}$ & Raw & $\mathrm{Ba}$ & $3.00 E+02$ & $2.18 \mathrm{E}-06$ & AP102-3 & $Y$ & $\begin{array}{l}\text { Density reported for } 6359 \text { collected at } 162 \text { in from bottom } \\
\text { of tank at a } 150^{\circ} \text { angle. Sample was unheated, unheated } \\
\text { samples precipitated }\end{array}$ \\
\hline $\mathbf{P}$ & Raw & $\mathrm{Ba}$ & $2.89 E+02$ & 2.10E-06 & AP102-3 & $Y$ & $\begin{array}{l}\text { Density reported for } \mathrm{G} 363 \text { collected at } 18 \text { in from bottom of } \\
\text { tank at a } 150^{\circ} \text { angle. Sample was unheated, unheated } \\
\text { samples precipitated }\end{array}$ \\
\hline $\mathbf{P}$ & Raw & $\mathrm{Ba}$ & $2.88 E+02$ & $2.10 E-06$ & AP102-3 & $Y$ & $\begin{array}{l}\text { Density not reported for G333. No assumptions could be } \\
\text { made }\end{array}$ \\
\hline $\mathbf{P}$ & Raw & $8 a$ & $2.86 E+02$ & 2.08E-06 & AP102-3 & $Y$ & $\begin{array}{l}\text { Density reported for } G 363 \text { collected at } 18 \text { in from bottom of } \\
\text { tank at a } 150^{\circ} \text { angle. Sample was unheated, unheated } \\
\text { samples precipitated }\end{array}$ \\
\hline $\mathbf{P}$ & Raw & $\mathrm{Ba}$ & $2.84 E+02$ & 2.07E-06 & AP102-3 & $Y$ & $\begin{array}{l}\text { Density reported for } \mathrm{G} 358 \text { collected at } 334 \text { in from bottom } \\
\text { of tank at a } 150^{\circ} \text { angle. Sample was unheated, unheated } \\
\text { samples precipitated }\end{array}$ \\
\hline $\mathbf{P}$ & Raw & $\mathrm{Ba}$ & $2.83 E+02$ & 2.06E-06 & AP102-3 & $Y$ & $\begin{array}{l}\text { Density reported for } 6341 \text { collected at } 99 \text { in from bottom of } \\
\text { tank at a } 270^{\circ} \text { angle. }\end{array}$ \\
\hline $\mathbf{P}$ & Raw & Ba & $2.73 E+02$ & 1.99E-06 & AP102-3 & Y & $\begin{array}{l}\text { Density reported for G459 collected at } 226 \text { in from bottom } \\
\text { of tank at a } 270^{\circ} \text { angle. Sample was unheated, unheated } \\
\text { samples precipitated }\end{array}$ \\
\hline $\mathbf{P}$ & Raw & $\mathrm{Ba}$ & $2.68 E+02$ & $1.95 E-06$ & AP102-3 & $Y$ & $\begin{array}{l}\text { Density reported for } \mathrm{G} 348 \text { collected at } 125 \text { in from bottom } \\
\text { of tank at a } 30^{\circ} \text { angle. Sample was unheated, unheated } \\
\text { samples precipitated }\end{array}$ \\
\hline $\mathbf{P}$ & Raw & $\mathrm{Ba}$ & $2.60 E+02$ & $1.89 E-06$ & AP102-3 & Y & $\begin{array}{l}\text { Density reported for G348 collected at } 125 \text { in from bottom } \\
\text { of tank at a } 30^{\circ} \text { angle. Sample was unheated, unheated } \\
\text { samples precipitated }\end{array}$ \\
\hline$P$ & Raw & $\mathrm{Ba}$ & $2.58 E+02$ & $1.88 \mathrm{E}-06$ & AP102-3 & $Y$ & $\begin{array}{l}\text { Density reported for G342 collected at } 306 \text { in from bottom } \\
\text { of tank at a } 30^{\circ} \text { angle. Sample was unheated, unheated } \\
\text { samples precipitated }\end{array}$ \\
\hline$P$ & Raw & $\mathbf{B a}$ & $2.52 E+02$ & $1.84 \mathrm{E}-06$ & AP102-3 & $Y$ & $\begin{array}{l}\text { Density reported for G459 collected at } 226 \text { in from bottom } \\
\text { of tank at a } 270^{\circ} \text { angle. Sample was unheated, unheated } \\
\text { samples precipitated }\end{array}$ \\
\hline$P$ & Raw & $\mathrm{Ba}$ & $2.52 E+02$ & $1.84 E-06$ & AP102-3 & Y & $\begin{array}{l}\text { Density reported for } G 460 \text { collected at } 226 \text { in from bottom } \\
\text { of tank at a } 270^{\circ} \text { angle. Sample was unheated, unheated } \\
\text { samples precipitated }\end{array}$ \\
\hline$P$ & Raw & $\mathbf{B a}$ & $2.48 E+02$ & $1.81 E-06$ & AP102-3 & $Y$ & $\begin{array}{l}\text { Density reported for G352 collected at } 34 \text { in from bottom of } \\
\text { tank at a } 30^{\circ} \text { angle. Sample was unheated, unheated } \\
\text { samples precipitated }\end{array}$ \\
\hline$P$ & Raw & Ba & $2.48 E+02$ & 1.81E-06 & AP102-3 & $\mathbf{Y}$ & $\begin{array}{l}\text { Density reported for } \mathrm{G} 353 \text { collected at } 334 \text { in from bottom } \\
\text { of tank at a } 150^{\circ} \text { angle. Sample was unheated, unheated } \\
\text { samples precipitated }\end{array}$ \\
\hline $\mathbf{P}$ & Raw & $\mathrm{Ba}$ & $2.48 E+02$ & $1.81 E-06$ & AP102-3 & $\mathbf{Y}$ & Density reported for $\mathrm{G} 443$ represents a composite sample \\
\hline $\mathbf{P}$ & Raw & $\mathrm{Ba}$ & $2.37 E+02$ & $1.73 E-06$ & AP102-3 & $\mathbf{Y}$ & $\begin{array}{l}\text { Density reported for G353 collected at } 334 \text { in from bottom } \\
\text { of tank at a } 150^{\circ} \text { angle. Sample was unheated, unheated } \\
\text { samples precipitated }\end{array}$ \\
\hline $\mathbf{P}$ & Raw & $\mathrm{Ba}$ & $2.11 E+02$ & $1.54 E-06$ & AP102-3 & $Y$ & $\begin{array}{l}\text { Density not reported for G453. No assumptions could be } \\
\text { made }\end{array}$ \\
\hline$P$ & Raw & $\mathrm{Ba}$ & $2.07 E+02$ & $1.51 E-06$ & AP102-3 & $Y$ & $\begin{array}{l}\text { Density reported for G460 collected at } 226 \text { in from botton } \\
\text { of tank at a } 270^{\circ} \text { angle. Sample was unheated, unheated } \\
\text { samples precipitated }\end{array}$ \\
\hline $\mathbf{P}$ & Raw & $8 a$ & $1.70 E+02$ & $1.24 E-06$ & AP102-3 & $Y$ & $\begin{array}{l}\text { Density reported for G364 collected at } 372 \text { in from bottom } \\
\text { of tank at a } 270^{\circ} \text { angle. Sample was unheated, unheated } \\
\text { samples precipitated }\end{array}$ \\
\hline
\end{tabular}




\begin{tabular}{|c|c|c|c|c|c|c|c|c|c|c|c|}
\hline & & & & & ank Waste & haracteriza & ion Sample & ormation & & & \\
\hline Reported Chemical & $\begin{array}{c}\text { Memo } \\
\text { Date }\end{array}$ & $\begin{array}{c}\text { Start } \\
\text { Analysis } \\
\text { Date }\end{array}$ & $\begin{array}{c}\text { Finish } \\
\text { Analysis Date }\end{array}$ & $\begin{array}{c}\text { Start } \\
\text { Sample } \\
\text { Date }\end{array}$ & $\begin{array}{c}\text { Finish } \\
\text { Sample } \\
\text { Date }\end{array}$ & $\begin{array}{l}\text { Sample } \\
\text { Number }\end{array}$ & $\begin{array}{c}\text { Tank Farm } \\
\text { Number }\end{array}$ & $\begin{array}{c}\text { Lab Sample } \\
\text { Number }\end{array}$ & $\begin{array}{l}\text { Density } \\
(\mathrm{g} / \mathrm{mL})\end{array}$ & $\begin{array}{c}\text { Reported } \\
\text { Value } \\
\end{array}$ & $\begin{array}{r}\text { Repor } \\
\text { Uni }\end{array}$ \\
\hline Barium & $6 / 1 / 90$ & $4 / 30 / 89$ & $12 / 30 / 89$ & $4 / 27 / 89$ & $4 / 29 / 89$ & BOB2L7 & G299 & G342 & 1.20 & $1.70 E+02$ & ugf \\
\hline Barium & $6 / 1 / 90$ & $4 / 30 / 89$ & $12 / 30 / 89$ & $4 / 27 / 89$ & $4 / 29 / 89$ & вов2T9 & G327 & G443 & 1.20 & $1.40 E+02$ & ug $/$ i \\
\hline Barium & $6 / 1 / 90$ & $4 / 30 / 89$ & $12 / 30 / 89$ & $4 / 27 / 89$ & $4 / 29 / 89$ & BOB2N4 & G323 & G341 & 1.20 & $1.39 E+02$ & ug $/$ \\
\hline Barium & $6 / 1 / 90$ & $4 / 30 / 89$ & $12 / 30 / 89$ & $4 / 27 / 89$ & $4 / 29 / 89$ & BOB2M5 & G315 & G364 & 1.19 & $9.02 E+01$ & $u g /$ \\
\hline Beryllium & $6 / 1 / 90$ & $4 / 30 / 89$ & $12 / 30 / 89$ & $4 / 27 / 89$ & $4 / 29 / 89$ & вОВ2T9 & G327 & G470* & 1.19 & $<3.00 E+03$ & ug/l \\
\hline Beryllium & $6 / 1 / 90$ & $4 / 30 / 89$ & $12 / 30 / 89$ & $4 / 27 / 89$ & $4 / 29 / 89$ & ВOB2T9 & G327 & G470* & 1.19 & $<3.00 E+03$ & $\operatorname{ug} n$ \\
\hline $\mathrm{Be}$ & $2 / 10 / 89$ & NS & NS & NS & NS & NS & NS & NS & 0.956764 & $1.89 \mathrm{E}-02$ & $\overline{\mathrm{ppr}}$ \\
\hline Beryilium & $6 / 1 / 90$ & $4 / 30 / 89$ & $12 / 30 / 89$ & $4 / 27 / 89$ & $4 / 29 / 89$ & BOB2N1 & G310 & G454 & $(1.19)$ & $1.68 E+02$ & ug/h \\
\hline Beryllium & $6 / 1 / 90$ & $4 / 30 / 89$ & $12 / 30 / 89$ & $4 / 27 / 89$ & $4 / 29 / 89$ & BOB2N3 & G322 & G338 & NA & $1.63 E+02$ & $u g / h$ \\
\hline Beryllium & $6 / 1 / 90$ & $4 / 30 / 89$ & $12 / 30 / 89$ & $4 / 27 / 89$ & $4 / 29 / 89$ & BOB2M6 & G318 & G459 & 1.20 & $1.63 E+02$ & $\mathrm{ug} / \mathrm{L}$ \\
\hline Beryllium & $6 / 1 / 90$ & $4 / 30 / 89$ & $12 / 30 / 89$ & $4 / 27 / 89$ & $4 / 29 / 89$ & BOB2M5 & G315 & G364 & 1.19 & $1.61 E+02$ & $u g / h$ \\
\hline Beryllium & $6 / 1 / 90$ & $4 / 30 / 89$ & $12 / 30 / 89$ & $4 / 27 / 89$ & $4 / 29 / 89$ & BOB2NO & G307 & G453 & NA & $1.59 E+02$ & ug/h. \\
\hline Beryllium & $6 / 1 / 90$ & $4 / 30 / 89$ & $12 / 30 / 89$ & $4 / 27 / 89$ & $4 / 29 / 89$ & BOB2N2 & G317 & G333 & NA & $1.58 E+02$ & ugh. \\
\hline Beryllium & $6 / 1 / 90$ & $4 / 30 / 89$ & $12 / 30 / 89$ & $4 / 27 / 89$ & $4 / 29 / 89$ & ВОВ2M7 & G319 & G460 & 1.20 & $1.58 E+02$ & ug $h$ \\
\hline Beryllium & $6 / 1 / 90$ & $4 / 30 / 89$ & $12 / 30 / 89$ & $4 / 27 / 89$ & $4 / 29 / 89$ & BOB2N2 & G317 & G333 & NA & $1.58 E+02$ & ug/h. \\
\hline Beryllium & $6 / 1 / 90$ & $4 / 30 / 89$ & $12 / 30 / 89$ & $4 / 27 / 89$ & $4 / 29 / 89$ & BOB2N4 & G323 & G341 & 1.20 & $1.54 E+02$ & $u g h$. \\
\hline Beryllium & $6 / 1 / 90$ & $4 / 30 / 89$ & $12 / 30 / 89$ & $4 / 27 / 89$ & $4 / 29 / 89$ & BOB2N3 & G322 & G338 & NA & $1.54 E+02$ & $u g R$ \\
\hline Beryllium & $6 / 1 / 90$ & $4 / 30 / 89$ & $12 / 30 / 89$ & $4 / 27 / 89$ & $4 / 29 / 89$ & ВОВ2T9 & G327 & G443 & 1.20 & $1.52 E+02$ & $u g / L$ \\
\hline Beryllium & $6 / 1 / 90$ & $4 / 30 / 89$ & $12 / 30 / 89$ & $4 / 27 / 89$ & $4 / 29 / 89$ & BOB2N1 & G310 & G454 & (1.19) & $1.52 E+02$ & $u g / L$ \\
\hline Beryilium & $6 / 1 / 90$ & $4 / 30 / 89$ & $12 / 30 / 89$ & $4 / 27 / 89$ & $4 / 29 / 89$ & BOB2NO & G307 & G453 & NA & $1.51 E+02$ & ug $/$. \\
\hline Beryllium & $6 / 1 / 90$ & $4 / 30 / 89$ & $12 / 30 / 89$ & $4 / 27 / 89$ & $4 / 29 / 89$ & BOB2L8 & G303 & G348 & 1.21 & $1.50 E+02$ & ug/L \\
\hline Beryllium & $6 / 1 / 90$ & $4 / 30 / 89$ & $12 / 30 / 89$ & $4 / 27 / 89$ & $4 / 29 / 89$ & BOB2M1 & G308 & G353 & 1.20 & $1.49 E+02$ & ug $n$. \\
\hline Beryllium & $6 / 1 / 90$ & $4 / 30 / 89$ & $12 / 30 / 89$ & $4 / 27 / 89$ & $4 / 29 / 89$ & BOB2M7 & G319 & $\mathrm{G} 460$ & 1.20 & $1.49 E+02$ & $u g / L$ \\
\hline Beryllium & $6 / 1 / 90$ & $4 / 30 / 89$ & $12 / 30 / 89$ & $4 / 27 / 89$ & $4 / 29 / 89$ & BOB2M4 & G313 & G363 & 1.20 & $1.47 E+02$ & ug $/ 2$ \\
\hline
\end{tabular}




\begin{tabular}{|c|c|c|c|c|c|c|c|}
\hline \multirow[b]{2}{*}{ Code } & \multirow[b]{2}{*}{ Source } & \multicolumn{3}{|c|}{ Analyte Information } & \multirow[b]{2}{*}{$\begin{array}{c}\text { Reference } \\
\text { Number }\end{array}$} & \multirow[b]{2}{*}{$\begin{array}{c}\text { Validated } \\
\text { (Y/N) }\end{array}$} & \multirow[b]{2}{*}{ Notes } \\
\hline & & Analyte & $\begin{array}{l}\text { Conc. } \\
\text { (ug/L) }\end{array}$ & Conc. (mol/L) & & & \\
\hline$P$ & Raw & $\mathrm{Ba}$ & $1.70 E+02$ & $1.24 E-06$ & AP102-3 & $\gamma$ & $\begin{array}{l}\text { Density reported for G342 collected at } 306 \text { in from bottom } \\
\text { of tank at a } 30^{\circ} \text { angle. Sample was unheated, unheated } \\
\text { samples precipitated }\end{array}$ \\
\hline$P$ & Raw & Ba & $1.40 E+02$ & $1.02 \mathrm{E}-06$ & AP102-3 & Y & Density reported for 6443 represents a composite sample \\
\hline$P$ & Raw & $\mathrm{Ba}$ & $1.39 E+02$ & $1.01 E-06$ & AP102-3 & $Y$ & $\begin{array}{l}\text { Density reported for G341 collected at } 99 \text { in from bottom of } \\
\text { tank at a } 270^{\circ} \text { angle. }\end{array}$ \\
\hline$P$ & Raw & $\mathrm{Ba}$ & $9.02 E+01$ & 6.57E-07 & AP102-3 & Y & $\begin{array}{l}\text { Density reported for } \mathrm{G} 364 \text { collected at } 372 \text { in from bottom } \\
\text { of tank at a } 270^{\circ} \text { angle. Sample was unheated, unheared } \\
\text { samples precipitated }\end{array}$ \\
\hline$P$ & Raw & Be & NALUE! & \# VALUEI & AP102-3 & $Y$ & $\begin{array}{l}\text { Density reported for G470 represents a composite sample. } \\
\text { Sample was kept heated to } 40^{\circ} \mathrm{C} \text {. }\end{array}$ \\
\hline $\mathbf{P}$ & Raw & $\mathrm{Be}$ & \#VALUE! & \#VALUE! & AP102-3 & $Y$ & $\begin{array}{l}\text { Density reported for G470 represents a composite sample. } \\
\text { Sample was kept heated to } 40^{\circ} \mathrm{C} \text {. }\end{array}$ \\
\hline $\mathbf{U}$ & RAW & $\mathrm{Be}$ & $1.81 E+07$ & $2.01 E+\infty 0$ & AP102-2 & $\mathrm{N}$ & Projected value, not a real data point \\
\hline $\mathbf{P}$ & Raw & $\mathrm{Be}$ & $1.68 E+02$ & $1.86 \mathrm{E}-05$ & AP102-3 & $\mathrm{Y}$ & $\begin{array}{l}\text { Density not reported for G454. Density assumed same as } \\
\text { G471 which was collected at } 208 \text { in from bottom of tank at } \\
\text { a } 150^{\circ} \text { angle and was keep heated to } 40^{\circ} \mathrm{C} \text {. }\end{array}$ \\
\hline $\mathbf{P}$ & Raw & $\mathrm{Be}$ & $1.63 E+02$ & $1.81 E-05$ & AP102-3 & $Y$ & $\begin{array}{l}\text { Density not reported for G338. No assumptions could be } \\
\text { made }\end{array}$ \\
\hline $\mathbf{P}$ & Raw & Be & $1.63 E+02$ & 1.81E-05 & AP102-3 & $Y$ & $\begin{array}{l}\text { Density reported for G459 collected at } 226 \text { in from bottom } \\
\text { of tank at a } 270^{\circ} \text { angle. Sample was unheated, unheated } \\
\text { samples precipitated }\end{array}$ \\
\hline$P$ & Raw & $\mathrm{Be}$ & $1.61 E+02$ & 1.79E-05 & AP102-3 & Y & $\begin{array}{l}\text { Density reported for G364 collected at } 372 \text { in from bottom } \\
\text { of tank at a } 270^{\circ} \text { angle. Sample was unheated, unheated } \\
\text { samples precipitated }\end{array}$ \\
\hline $\mathbf{P}$ & Raw & $\mathrm{Be}$ & $1.59 E+02$ & $1.76 \mathrm{E}-05$ & AP102-3 & $Y$ & $\begin{array}{l}\text { Density not reported for G453. No assumptions could be } \\
\text { made }\end{array}$ \\
\hline. $\mathbf{P}$ & Raw & $\mathrm{Be}$ & $1.58 E+02$ & $1.75 E-05$ & AP102-3 & $Y$ & $\begin{array}{l}\text { Density not reported for G333. No assumptions could be } \\
\text { made }\end{array}$ \\
\hline $\mathbf{P}$ & Raw & Be & $1.58 E+02$ & $1.75 E-05$ & AP102-3 & $Y$ & $\begin{array}{l}\text { Density reported for } \mathrm{G} 460 \text { collected at } 226 \text { in from bottom } \\
\text { of tank at a } 270^{\circ} \text { angle. Sample was unheated, unheated } \\
\text { samples precipitated }\end{array}$ \\
\hline $\mathbf{P}$ & Raw & $\mathrm{Be}$ & $1.58 E+02$ & $1.75 \mathrm{E}-05$ & AP102-3 & $\mathbf{Y}$ & $\begin{array}{l}\text { Density not reported for G333. No assumptions could be } \\
\text { made }\end{array}$ \\
\hline$P$ & Raw & Be & $1.54 E+02$ & 1.71E-05 & AP102-3 & $Y$ & $\begin{array}{l}\text { Density reported for } 6341 \text { collected at } 99 \text { in from bottom of } \\
\text { tank at a } 270^{\circ} \text { angle. }\end{array}$ \\
\hline$P$ & Raw & $8 e$ & $1.54 E+02$ & $1.71 E-05$ & AP $102-3$ & $Y$ & $\begin{array}{l}\text { Density not reported for G338. No assumptions could be } \\
\text { made }\end{array}$ \\
\hline$P$ & Raw & $\mathrm{Be}$ & $1.52 E+02$ & $1.69 E-05$ & AP102-3 & $Y$ & Density reported for $\mathrm{G} 443$ represents a composite sample \\
\hline $\mathbf{P}$ & Raw & $\mathrm{Be}$ & $1.52 E+02$ & $1.69 \mathrm{E}-05$ & AP102-3 & Y & $\begin{array}{l}\text { Density not reported for G454. Density assumed same as } \\
\text { G471 which was collected at } 208 \text { in from bottom of tank at } \\
\text { a } 150^{\circ} \text { angle and was keep heated to } 40^{\circ} \mathrm{C} \text {. }\end{array}$ \\
\hline$P$ & Raw & Be & $1.51 E+02$ & $1.68 E-05$ & AP102-3 & $\mathbf{Y}$ & $\begin{array}{l}\text { Density not reported for G453. No assumptions could be } \\
\text { made }\end{array}$ \\
\hline $\mathbf{P}$ & Raw & Be & $1.50 E+02$ & $1.66 E-05$ & AP102-3 & Y & $\begin{array}{l}\text { Density reported for G348 collected at } 125 \text { in from bottom } \\
\text { of tank at a } 30^{\circ} \text { angle. Sample was unheated, unheated } \\
\text { samples precipitated }\end{array}$ \\
\hline $\mathbf{P}$ & Raw & $\mathrm{Be}$ & $1.49 E+02$ & $1.65 \mathrm{E}-05$ & AP102-3 & $\mathbf{Y}$ & $\begin{array}{l}\text { Density reported for G353 collected at } 334 \text { in from bottom } \\
\text { of tank at a } 150^{\circ} \text { angle. Sample was unheated, unheated } \\
\text { samples precipitated }\end{array}$ \\
\hline $\mathbf{P}$ & Raw & $\mathrm{Be}$ & $1.49 E+02$ & $1.65 E-05$ & AP102-3 & $\boldsymbol{Y}$ & $\begin{array}{l}\text { Density reported for G460 collected at } 226 \text { in from bottom } \\
\text { of tank at a } 270^{\circ} \text { angle. Sample was unheated, unheated } \\
\text { samples precipitated }\end{array}$ \\
\hline $\mathbf{P}$ & Raw & $\mathrm{Be}$ & $1.47 E+02$ & $1.63 E-05$ & AP102-3 & $\mathbf{Y}$ & $\begin{array}{l}\text { Density reported for G363 collected at } 18 \text { in from bottom of } \\
\text { tank at a } 150^{\circ} \text { angle. Sample was unheated, unheated } \\
\text { samples precipitated }\end{array}$ \\
\hline
\end{tabular}




\begin{tabular}{|c|c|c|c|c|c|c|c|c|c|c|c|}
\hline & & & & & ank Waste & Sharacteriza & ion Sample 1 & ormation & & & \\
\hline Reported Chemical & $\begin{array}{c}\text { Memo } \\
\text { Date }\end{array}$ & $\begin{array}{c}\text { Start } \\
\text { Analysis } \\
\text { Date }\end{array}$ & $\begin{array}{c}\text { Finish } \\
\text { Analysis Date } \\
\end{array}$ & $\begin{array}{c}\text { Start } \\
\text { Sample } \\
\text { Date } \\
\end{array}$ & $\begin{array}{c}\text { Finish } \\
\text { Sample } \\
\text { Date } \\
\end{array}$ & $\begin{array}{l}\text { Sample } \\
\text { Number }\end{array}$ & $\begin{array}{c}\text { Tank Farm } \\
\text { Number }\end{array}$ & $\begin{array}{c}\text { Lab Sample } \\
\text { Number }\end{array}$ & $\begin{array}{l}\text { Density } \\
(\mathrm{g} / \mathrm{mL})\end{array}$ & $\begin{array}{c}\text { Reported } \\
\text { Value }\end{array}$ & $\begin{array}{r}\text { Repo: } \\
\text { Un: } \\
\end{array}$ \\
\hline Beryllium & 6/1/9̣0 & $4 / 30 / 89$ & $12 / 30 / 89$ & $4 / 27 / 89$ & $4 / 29 / 89$ & BOB2MO & G306 & G352 & 1.20 & $1.47 E+02$ & ugl \\
\hline Beryllium & $6 / 1 / 90$ & $4 / 30 / 89$ & $12 / 30 / 89$ & $4 / 27 / 89$ & $4 / 29 / 89$ & ВOB2M2 & G309 & G358 & 1.20 & $1.47 E+02$ & ugi \\
\hline Beryllium & $6 / 1 / 90$ & $4 / 30 / 89$ & $12 / 30 / 89$ & $4 / 27 / 89$ & $4 / 29 / 89$ & BOB2L7 & G299 & G342 & 1.20 & $1.46 E+02$ & ugh \\
\hline Beryllium & $6 / 1 / 90$ & $4 / 30 / 89$ & $12 / 30 / 89$ & $4 / 27 / 89$ & $4 / 29 / 89$ & BOB2N4 & G323 & G341 & 1.20 & $1.46 E+02$ & $\operatorname{ug} /$ \\
\hline Berylium & $6 / 1 / 90$ & $4 / 30 / 89$ & $12 / 30 / 89$ & $4 / 27 / 89$ & $4 / 29 / 89$ & BOB2L7 & G299 & G342 & 1.20 & $1.46 E+02$ & ug/l \\
\hline Beryllium & $6 / 1 / 90$ & $4 / 30 / 89$ & $12 / 30 / 89$ & $4 / 27 / 89$ & $4 / 29 / 89$ & BOB2L8 & G303 & G348 & 1.21 & $1.45 E+02$ & ug/l \\
\hline Beryllium & $6 / 1 / 90$ & $4 / 30 / 89$ & $12 / 30 / 89$ & $4 / 27 / 89$ & $4 / 29 / 89$ & BOB2L9 & G305 & G349 & 1.20 & $1.45 E+02$ & $u g / 1$ \\
\hline Beryllium & $6 / 1 / 90$ & $4 / 30 / 89$ & $12 / 30 / 89$ & $4 / 27 / 89$ & $4 / 29 / 89$ & BOB2M8 & G301 & G449 & NA & $1.45 E+02$ & $u g / 1$ \\
\hline Beryllium & $6 / 1 / 90$ & $4 / 30 / 89$ & $12 / 30 / 89$ & $4 / 27 / 89$ & $4 / 29 / 89$ & BOB2L9 & G305 & G349 & 1.20 & $1.43 E+02$ & $u g / h$ \\
\hline Beryllium & $6 / 1 / 90$ & $4 / 30 / 89$ & $12 / 30 / 89$ & $4 / 27 / 89$ & $4 / 29 / 89$ & ВОВ2MO & G306 & G352 & 1.20 & $1.43 E+02$ & $u g / h$ \\
\hline Beryilium & $6 / 1 / 90$ & $4 / 30 / 89$ & $12 / 30 / 89$ & $4 / 27 / 89$ & $4 / 29 / 89$ & BOB2M2 & G309 & G358 & 1.20 & $1.43 E+02$ & ugh \\
\hline Beryllium & $6 / 1 / 90$ & $4 / 30 / 89$ & $12 / 30 / 89$ & $4 / 27 / 89$ & $4 / 29 / 89$ & В082T9 & G327 & G443 & 1.20 & $1.40 E+02$ & ugh. \\
\hline Beryllium & $6 / 1 / 90$ & $4 / 30 / 89$ & $12 / 30 / 89$ & $4 / 27 / 89$ & $4 / 29 / 89$ & Вов2M6 & G318 & G459 & 1.20 & $1.40 E+02$ & ug/h. \\
\hline Beryllium & $6 / 1 / 90$ & 4/30/89 & $12 / 30 / 89$ & $4 / 27 / 89$ & $4 / 29 / 89$ & воB2M3 & G311 & G359 & 1.20 & $1.39 E+02$ & ug/h \\
\hline Beryllium & $6 / 1 / 90$ & $4 / 30 / 89$ & $12 / 30 / 89$ & $4 / 27 / 89$ & $4 / 29 / 89$ & BOB2M4 & G313 & G363 & 1.20 & $1.36 E+02$ & ug/L \\
\hline Beryllium & $6 / 1 / 90$ & $4 / 30 / 89$ & $12 / 30 / 89$ & $4 / 27 / 89$ & $4 / 29 / 89$ & BOB2M5 & G315 & G364 & 1.19 & $1.35 E+02$ & ug/h \\
\hline Beryllium & $6 / 1 / 90$ & $4 / 30 / 89$ & $12 / 30 / 89$ & $4 / 27 / 89$ & $4 / 29 / 89$ & вов2М3 & G311 & G359 & 1.20 & $1.32 E+02$ & ugh \\
\hline Beryllium & $6 / 1 / 90$ & $4 / 30 / 89$ & $12 / 30 / 89$ & $4 / 27 / 89$ & $4 / 29 / 89$ & BOB2M9 & G302 & G450 & $(1.20)$ & $1.29 E+02$ & $\operatorname{ug} / \mathrm{L}$ \\
\hline Beryllium & $6 / 1 / 90$ & $4 / 30 / 89$ & $12 / 30 / 89$ & $4 / 27 / 89$ & $4 / 29 / 89$ & BOB2M1 & 6308 & $\mathbf{6 3 5 3}$ & 1.20 & $1.27 E+02$ & $u g / L$ \\
\hline Berylitum & $6 / 1 / 90$ & $4 / 30 / 89$ & $12 / 30 / 89$ & $4 / 27 / 89$ & $4 / 29 / 89$ & Вов2M9 & G302 & G450 & $\{1.20\}$ & $1.27 E+02$ & ugh \\
\hline Beryilium & $6 / 1 / 90$ & $4 / 30 / 89$ & $12 / 30 / 89$ & $4 / 27 / 89$ & $4 / 29 / 89$ & 8082M8 & G301 & 6449 & NA & $1.09 E+02$ & $u g / L$ \\
\hline
\end{tabular}




\begin{tabular}{|c|c|c|c|c|c|c|c|}
\hline \multirow[b]{2}{*}{ Code } & \multirow[b]{2}{*}{ Source } & \multicolumn{3}{|c|}{ Analyte Information } & \multirow[b]{2}{*}{$\begin{array}{c}\text { Reference } \\
\text { Number }\end{array}$} & \multirow[b]{2}{*}{$\begin{array}{l}\text { Validated } \\
(Y / N) \\
\end{array}$} & \multirow[b]{2}{*}{ Notes } \\
\hline & & Analyte & $\begin{array}{l}\text { Conc. } \\
\text { (ug/L) }\end{array}$ & Conc. (mol/L) & & & \\
\hline$P$ & Raw & $\mathrm{Be}$ & $1.47 E+02$ & $1.63 E-05$ & AP102-3 & $Y$ & $\begin{array}{l}\text { Density reported for } 6352 \text { collected at } 34 \text { in from bottom of } \\
\text { tank at a } 30^{\circ} \text { angle. Sample was unheated, unheated } \\
\text { samples precipitated }\end{array}$ \\
\hline$P$ & Raw & $\mathrm{Be}$ & $1.47 \mathrm{E}+02$ & $1.63 E-05$ & AP102-3 & $Y$ & $\begin{array}{l}\text { Density reported for G358 collected at } 334 \text { in from bottom } \\
\text { of tank at a } 150^{\circ} \text { angle. Sample was unheated, unheated } \\
\text { samples precipitated }\end{array}$ \\
\hline $\mathbf{P}$ & Raw & $\mathrm{Be}$ & $1.46 E+02$ & $1.62 E-05$ & AP102-3 & $Y$ & $\begin{array}{l}\text { Density reported for G342 collected at } 306 \text { in from bottom } \\
\text { of tank at a } 30^{\circ} \text { angle. Sample was unheated, unheated } \\
\text { samples precipitated }\end{array}$ \\
\hline $\mathbf{P}$ & Raw & $\mathrm{Be}$ & $1.46 E+02$ & $1.62 E-05$ & AP102-3 & $Y$ & $\begin{array}{l}\text { Density reported for } \mathrm{G} 341 \text { collected at } 99 \text { in from bottom of } \\
\text { tank at a } 270^{\circ} \text { angle. }\end{array}$ \\
\hline $\mathbf{P}$ & Raw & $\mathrm{Be}$ & $1.46 E+02$ & 1.62E-05 & AP102-3 & $\mathbf{Y}$ & $\begin{array}{l}\text { Density reported for } \mathrm{G} 342 \text { collected at } 306 \text { in from bottom } \\
\text { of tank at a } 30^{\circ} \text { angle. Sample was unheated, unheated } \\
\text { samples precipitated }\end{array}$ \\
\hline $\mathbf{P}$ & Raw & $\mathrm{Be}$ & $1.45 E+02$ & 1.61E-05 & AP102-3 & $\mathrm{Y}$ & $\begin{array}{l}\text { Density reported for G348 collected at } 125 \text { in from bottom } \\
\text { of tank at a } 30^{\circ} \text { angle. Sample was unheated, unheated } \\
\text { samples precipitated }\end{array}$ \\
\hline$P$ & Raw & $\mathrm{Be}$ & $1.45 E+02$ & 1.61E-05 & AP102-3 & $\mathbf{Y}$ & $\begin{array}{l}\text { Density reported for } G 349 \text { collected at } 34 \text { in from bottom of } \\
\text { tank at a } 30^{\circ} \text { angle. Sample was unheated, unheated } \\
\text { samples precipitated }\end{array}$ \\
\hline $\mathbf{P}$ & Raw & $\mathrm{Be}$ & $1.45 E+02$ & $1.61 \mathrm{E}-05$ & AP102-3 & $Y$ & $\begin{array}{l}\text { Density not reported for G449. No assumptions could be } \\
\text { made }\end{array}$ \\
\hline$P$ & Raw & $\mathrm{Be}$ & $1.43 E+02$ & 1.59E-05 & AP102-3 & $\mathrm{Y}$ & $\begin{array}{l}\text { Density reported for } \mathrm{G} 349 \text { collected at } 34 \text { in from bottom of } \\
\text { tank at a } 30^{\circ} \text { angle. Sample was unheated, unheated } \\
\text { samples precipitated }\end{array}$ \\
\hline $\mathbf{P}$ & Raw & $\mathrm{Be}$ & $1.43 E+02$ & $1.59 \mathrm{E}-05$ & AP102-3 & $\gamma$ & $\begin{array}{l}\text { Density reported for G352 collected at } 34 \text { in from bottom of } \\
\text { tank at a } 30^{\circ} \text { angle. Sample was unheated, unheated } \\
\text { samples precipitated }\end{array}$ \\
\hline $\mathbf{P}$ & Raw & $\mathrm{Be}$ & $1.43 E+02$ & $1.59 E-05$ & AP102-3 & $\mathbf{Y}$ & $\begin{array}{l}\text { Density reported for } G 358 \text { collected at } 334 \text { in from bottom } \\
\text { of tank at a } 150^{\circ} \text { angle. Sample was unheated, unheated } \\
\text { samples precipitated }\end{array}$ \\
\hline$P$ & Raw & $\mathrm{Be}$ & $1.40 E+02$ & 1.55E-05 & AP102-3 & $Y$ & Density reported for $\mathrm{G} 443$ represents a composite sample \\
\hline $\mathbf{P}$ & Raw & $\mathrm{Be}$ & $1.40 E+02$ & $1.55 E-05$ & AP102-3 & Y & $\begin{array}{l}\text { Density reported for G459 collected at } 226 \text { in from bottom } \\
\text { of tank at a } 270^{\circ} \text { angle. Sample was unheated, unheated } \\
\text { samples precipitated }\end{array}$ \\
\hline$P$ & Raw & Be & $1.39 E+02$ & $1.54 E-05$ & AP102-3 & Y & $\begin{array}{l}\text { Density reported for G359 collected at } 162 \text { in from bottom } \\
\text { of tank at a } 150^{\circ} \text { angle. Sample was unheated, unheated } \\
\text { samples precipitated }\end{array}$ \\
\hline$P$ & Raw & $\mathrm{Be}$ & $1.36 E+02$ & $1.51 E-05$ & AP102-3 & $Y$ & $\begin{array}{l}\text { Density reported for G363 collected at } 18 \text { in from bottom of } \\
\text { tank at a } 150^{\circ} \text { angle. Sample was unheated, unheated } \\
\text { samples precipitated }\end{array}$ \\
\hline $\mathbf{P}$ & Raw & Be & $1.35 E+02$ & $1.50 E-05$ & AP102-3 & $\mathbf{Y}$ & $\begin{array}{l}\text { Density reported for } \mathrm{G} 364 \text { collected at } 372 \text { in from bottom } \\
\text { of tank at a } 270^{\circ} \text { angle. Sample was unheated, unheated } \\
\text { samples precipitated }\end{array}$ \\
\hline $\mathbf{P}$ & Raw & $\mathrm{Be}$ & $1.32 E+02$ & $1.46 \mathrm{E}-05$ & AP102-3 & $\mathbf{Y}$ & $\begin{array}{l}\text { Density reported for G359 collected at } 162 \text { in from bottom } \\
\text { of tank at a } 150^{\circ} \text { angle. Sample was unheated, unheated } \\
\text { samples precipitated }\end{array}$ \\
\hline $\mathbf{P}$ & Raw & $\mathrm{Be}$ & $1.29 E+02$ & $1.43 E-05$ & AP102-3 & $\mathbf{Y}$ & $\begin{array}{l}\text { Density not reported for G450. Density assumed same as } \\
\text { G477 which was collected at } 182 \text { in from bottom of tank at } \\
\text { a } 30^{\circ} \text { angle and was keep heated to } 40^{\circ} \mathrm{C} \text {. }\end{array}$ \\
\hline $\mathbf{P}$ & Raw & Be & $1.27 E+02$ & 1.41E-05 & AP102-3 & $\mathrm{Y}$ & $\begin{array}{l}\text { Density reported for G353 collected at } 334 \text { in from bottom } \\
\text { of tank at a } 150^{\circ} \text { angle. Sample was unheated, unheated } \\
\text { samples precipitated }\end{array}$ \\
\hline $\mathbf{P}$ & Raw & $\mathrm{Be}$ & $1.27 E+02$ & $1.41 E-05$ & AP102-3 & $\mathbf{Y}$ & $\begin{array}{l}\text { Density not reported for G450. Density assumed same as } \\
\text { G477 which was collected at } 182 \text { in from bottom of tank at } \\
\text { a } 30^{\circ} \text { angle and was keep heated to } 40^{\circ} \mathrm{C} \text {. }\end{array}$ \\
\hline$P$ & Raw & $8 e$ & $1.09 E+02$ & $1.21 E-05$ & AP102-3 & $Y$ & $\begin{array}{l}\text { Density not reported for } 6449 \text {. No assumptions could be } \\
\text { made }\end{array}$ \\
\hline
\end{tabular}




\begin{tabular}{|c|c|c|c|c|c|c|c|c|c|c|c|}
\hline Reported Chemical & $\begin{array}{c}\text { Memo } \\
\text { Date }\end{array}$ & $\begin{array}{c}\text { Start } \\
\text { Analysis } \\
\text { Date } \\
\end{array}$ & $\begin{array}{c}\text { Finish } \\
\text { Analysis Date }\end{array}$ & $\begin{array}{c}\text { Start } \\
\text { Sample } \\
\text { Date } \\
\end{array}$ & $\begin{array}{c}\text { Finish } \\
\text { Sample } \\
\text { Date }\end{array}$ & $\begin{array}{l}\text { Sample } \\
\text { Number }\end{array}$ & $\begin{array}{c}\text { Tank Farm } \\
\text { Number }\end{array}$ & $\begin{array}{l}\text { Lab Sample } \\
\text { Number }\end{array}$ & $\begin{array}{l}\text { Densiny } \\
(\mathrm{g} / \mathrm{mL})\end{array}$ & $\begin{array}{l}\text { Reported } \\
\text { Value }\end{array}$ & $\begin{array}{r}\text { Repa } \\
\text { Un }\end{array}$ \\
\hline $\mathrm{Ca}$ & $4 / 28 / 84$ & NS & NS & NS & NS & R8469 & NS & NS & NS & $5.30 E+\infty 0$ & UGI \\
\hline $\mathrm{Ca}$ & $4 / 28 / 84$ & NS & NS & NS & NS & NS & NS & NS & NS & $5.30 \mathrm{E}-03$ & $g l$ \\
\hline $\mathrm{Ca}$ & $4 / 28 / 84$ & NS & NS & NS & NS & R-8469 & NS & NS & NS & $5.30 E+\infty 0$ & ugm \\
\hline $\mathrm{Ca}_{3}$ & $2 / 10 / 89$ & NS & NS & NS & NS & NS & NS & NS & 1.01 & $9.00 \mathrm{E}-01$ & $\mathrm{ppl}$ \\
\hline Ca & $2 / 10 / 89$ & NS & NS & NS & NS & NS & NS & NS & 0.956764 & $8.53 \mathrm{E}-01$ & $p p !$ \\
\hline Cadmium & $6 / 1 / 90$ & $4 / 30 / 89$ & $12 / 30 / 89$ & $4 / 27 / 89$ & $4 / 29 / 89$ & BOB2T9 & G327 & G470* & 1.19 & $<6.00 E+03$ & ugl \\
\hline Cadmium & $6 / 1 / 90$ & 4/30/89 & $12 / 30 / 89$ & $4 / 27 / 89$ & $4 / 29 / 89$ & BOB2T9 & G327 & G470* & 1.19 & $<6.00 E+03$ & ugl \\
\hline Cd & $2 / 10 / 89$ & NS & NS & NS & NS & NS & NS & NS & 0.956764 & 1.89E-01 & $p p t$ \\
\hline Cadmium & $6 / 1 / 90$ & $4 / 30 / 89$ & $12 / 30 / 89$ & $4 / 27 / 89$ & $4 / 29 / 89$ & BOB2NO & G307 & G453 & NA & $1.79 E+03$ & ug/ \\
\hline Cadmium & $6 / 1 / 90$ & 4/30/89 & $12 / 30 / 89$ & $4 / 27 / 89$ & $4 / 29 / 89$ & BOB2N1 & G310 & G454 & (1.19) & $1.78 E+03$ & $u g f$ \\
\hline Cadmium & $6 / 1 / 90$ & $4 / 30 / 89$ & $12 / 30 / 89$ & $4 / 27 / 89$ & $4 / 29 / 89$ & В0В2M5 & G315 & G364 & 1.19 & $1.65 E+03$ & $u g r_{i}$ \\
\hline Cadmium & $6 / 1 / 90$ & $4 / 30 / 89$ & $12 / 30 / 89$ & $4 / 27 / 89$ & $4 / 29 / 89$ & BOB2L9 & G305 & G349 & 1.20 & $1.54 E+03$ & ug $\Lambda$ \\
\hline Cadmium & $6 / 1 / 90$ & $4 / 30 / 89$ & $12 / 30 / 89$ & $4 / 27 / 89$ & $4 / 29 / 89$ & BOB2L9 & G305 & G349 & 1.20 & $1.54 E+03$ & ug $n$ \\
\hline Cadmium & $6 / 1 / 90$ & $4 / 30 / 89$ & $12 / 30 / 89$ & $4 / 27 / 89$ & $4 / 29 / 89$ & ВОВ2M9 & G302 & G450 & $(1.20)$ & $1.53 E+03$ & ug $/ 2$ \\
\hline Cadmium & $6 / 7 / 90$ & $4 / 30 / 89$ & $12 / 30 / 89$ & $4 / 27 / 89$ & $4 / 29 / 89$ & вов2M4 & G313 & G363 & 1.20 & $1.52 E+03$ & ug $\Omega$ \\
\hline Cadmium & $6 / 1 / 90$ & $4 / 30 / 89$ & $12 / 30 / 89$ & $4 / 27 / 89$ & $4 / 29 / 89$ & ВОВ2M6 & G318 & G459 & 1.20 & $1.52 E+03$ & ug/t. \\
\hline Cadmium & $6 / 1 / 90$ & $4 / 30 / 89$ & $12 / 30 / 89$ & $4 / 27 / 89$ & $4 / 29 / 89$ & ВОВ2Т9 & G327 & G443 & 1.20 & $1.52 E+03$ & $u g h$ \\
\hline Cadmium & $6 / 1 / 90$ & $4 / 30 / 89$ & $12 / 30 / 89$ & $4 / 27 / 89$ & $4 / 29 / 89$ & BOB2NO & G307 & G453 & NA & $1.51 E+03$ & Ug/L \\
\hline Cadrnium & $6 / 1 / 90$ & $4 / 30 / 89$ & $12 / 30 / 89$ & $4 / 27 / 89$ & $4 / 29 / 89$ & $80 B 2 N 2$ & G317 & G333 & NA & $1.50 E+03$ & ug/h \\
\hline Cadmium & $6 / 1 / 90$ & 4/30/89 & $12 / 30 / 89$ & $4 / 27 / 89$ & $4 / 29 / 89$ & BOB2N3 & G322 & G338 & NA & $1.50 E+03$ & $\lg / \mathrm{h}$ \\
\hline Cadmium & $6 / 1 / 90$ & $4 / 30 / 89$ & $12 / 30 / 89$ & $4 / 27 / 89$ & $4 / 29 / 89$ & B082M7 & 6319 & G460 & 1.20 & $1.50 E+03$ & $\operatorname{ug} / \mathrm{h}$ \\
\hline Cadmium & $6 / 1 / 90$ & $4 / 30 / 89$ & $12 / 30 / 89$ & $4 / 27 / 89$ & $4 / 29 / 89$ & BOB2N3 & G322 & G338 & NA & $1.50 E+03$ & ug/L \\
\hline Cadmium & $6 / 1 / 90$ & $4 / 30 / 89$ & $12 / 30 / 89$ & $4 / 27 / 89$ & $4 / 29 / 89$ & BOB2M5 & G315 & G364 & 1.19 & $1.48 \mathrm{E}+03$ & ug $\Omega$ \\
\hline Cadmium & $6 / 1 / 90$ & 4/30/89 & $12 / 30 / 89$ & $4 / 27 / 89$ & $4 / 29 / 89$ & BOB2M2 & G309 & G358 & 1.20 & $1.48 E+03$ & $u g h$ \\
\hline Cadmium & $6 / 1 / 90$ & 4/30/89 & $12 / 30 / 89$ & $4 / 27 / 89$ & $4 / 29 / 89$ & $\mathrm{BOB} 2 \mathrm{~N} 4$ & G323 & G341 & 1.20 & $1.47 E+03$ & ugh \\
\hline Cadmium & $6 / 1 / 90$ & $4 / 30 / 89$ & $12 / 30 / 89$ & $4 / 27 / 89$ & $4 / 29 / 89$ & BOB2T9 & G327 & G443 & 1.20 & $1.47 E+03$ & $\operatorname{ug} \Omega$ \\
\hline Cadmium & $6 / 1 / 90$ & $4 / 30 / 89$ & $12 / 30 / 89$ & $4 / 27 / 89$ & 4/29/89 & $\mathrm{BOB} 2 \mathrm{~N} 4$ & G323 & G341 & 1.20 & $1.47 E+03$ & $\operatorname{ug} / \mathrm{L}$ \\
\hline
\end{tabular}




\begin{tabular}{|c|c|c|c|c|c|c|c|}
\hline \multirow[b]{2}{*}{ Code } & \multirow[b]{2}{*}{ Source } & \multicolumn{3}{|c|}{ Analyte Information } & \multirow[b]{2}{*}{$\begin{array}{l}\text { Feference } \\
\text { Number }\end{array}$} & \multirow[b]{2}{*}{$\begin{array}{l}\text { Validated } \\
\text { (Y/N) }\end{array}$} & \multirow[b]{2}{*}{ Notes } \\
\hline & & Analyte & $\begin{array}{l}\text { Conc. } \\
\text { (ug/L) }\end{array}$ & Conc. (mol/L) & & & \\
\hline $\mathbf{P}$ & RAW & $\mathrm{Ca}$ & $5.30 E+09$ & $1.32 \mathrm{E}+02$ & AP102-1 & $\mathbf{N}$ & Outdated Information \\
\hline $\bar{P}$ & Wastren & $\mathrm{Ca}$ & $5.30 E+09$ & $1.32 E+02$ & NS & $\mathbf{N}$ & No Reference \\
\hline $\bar{P}$ & Braun & $\mathrm{Ca}$ & $5.30 \mathrm{E}+09$ & $1.32 \mathrm{E}+02$ & NS & $\mathbf{N}$ & No Reference \\
\hline $\mathbf{U}$ & RAW & $\mathrm{Ca}$ & $9.09 E+08$ & $2.27 E+01$ & AP102-2 & $\mathbf{N}$ & Projected value, not a real data point \\
\hline $\mathbf{u}$ & RAW & $\mathrm{Ca}$ & $8.16 \mathrm{E}+08$ & $2.04 E+01$ & AP102-2 & $\mathbf{N}$ & Projected value, not a real data point \\
\hline $\mathbf{P}$ & Raw & $\mathrm{Cd}$ & \#VALUE! & \#ALUE! & AP102-3 & $\mathbf{Y}$ & $\begin{array}{l}\text { Density reported for } \mathrm{G} 470 \text { represents a composite sample. } \\
\text { Sample was kept heated to } 40^{\circ} \mathrm{C} \text {. }\end{array}$ \\
\hline $\mathbf{P}$ & Raw & Cd & \#VALUEI & NALUE! & AP102-3 & $\mathbf{Y}$ & $\begin{array}{l}\text { Density reported for } 6470 \text { represents a composite sample. } \\
\text { Sample was kept heated to } 40^{\circ} \mathrm{C} \text {. }\end{array}$ \\
\hline U & RAW & Cd & $1.81 E+08$ & $1.61 E+\infty$ & AP102-2 & $\mathbf{N}$ & Projected value, not a real data point \\
\hline $\mathbf{P}$ & Raw & Cd & $1.79 E+03$ & $1.59 \mathrm{E}-05$ & AP102-3 & $Y$ & $\begin{array}{l}\text { Density not reported for G453. No assumptions could be } \\
\text { made }\end{array}$ \\
\hline $\mathbf{P}$ & Raw & Cod & $1.78 E+03$ & $1.58 E-05$ & AP102-3 & $Y$ & $\begin{array}{l}\text { Density not reported for } \mathrm{G} 454 \text {. Density assumed same as } \\
6471 \text { which was collected at } 208 \text { in from bottom of tank at } \\
\text { a } 150^{\circ} \text { angle and was keep heated to } 40^{\circ} \mathrm{C} \text {. }\end{array}$ \\
\hline $\mathbf{P}$ & Raw & Cd & $1.65 E+03$ & 1.47E-05 & AP102-3 & $Y$ & $\begin{array}{l}\text { Density reported for G364 collected at } 372 \text { in from bottom } \\
\text { of tank at a } 270^{\circ} \text { angle. Sample was unheated, unheated } \\
\text { samples precipitated }\end{array}$ \\
\hline $\mathbf{P}$ & Raw & Cd & $1.54 E+03$ & $1.37 E-05$ & AP102-3 & Y & $\begin{array}{l}\text { Density reported for } \mathrm{G} 349 \text { collected at } 34 \text { in from bottom of } \\
\text { tank at a } 30^{\circ} \text { angle. Sample was unheated, unheated } \\
\text { samples precipitated }\end{array}$ \\
\hline $\mathbf{P}$ & Raw & Cd & $1.54 E+03$ & $1.37 E-05$ & AP102-3 & $\mathbf{Y}$ & $\begin{array}{l}\text { Density reported for } \mathrm{G} 349 \text { collected at } 34 \text { in from bottom of } \\
\text { tank at a } 30^{\circ} \text { angle. Sample was unheated, unheated } \\
\text { samples precipitated }\end{array}$ \\
\hline $\mathbf{P}$ & $\begin{array}{r}\text { Raw } \\
.\end{array}$ & Cd & $1.53 E+03$ & $1.36 E-05$ & AP102-3 & $\mathbf{Y}$ & $\begin{array}{l}\text { Density not reported for } 6450 \text {. Density assumed same as } \\
\text { G477 which was collected at } 182 \text { in from bottom of tank at } \\
\text { a } 30^{\circ} \text { angle and was keep heated to } 40^{\circ} \mathrm{C} \text {. }\end{array}$ \\
\hline $\mathbf{P}$ & - Raw & Cd & $1.52 E+03$ & $1.35 E-05$ & AP102-3 & $Y$ & $\begin{array}{l}\text { Density reported for } \mathrm{G} 363 \text { collected at } 18 \text { in from bottom of } \\
\text { tank at a } 150^{\circ} \text { angle. Sample was unheated, unheated } \\
\text { samples precipitated }\end{array}$ \\
\hline $\mathbf{P}$ & Raw & $\mathrm{Cd}$ & $1.52 E+03$ & $1.35 \mathrm{E}-05$ & AP102-3 & $Y$ & $\begin{array}{l}\text { Density reported for G459 collected at } 226 \text { in from bottom } \\
\text { of tank at a } 270^{\circ} \text { angle. Sample was unheated, unheated } \\
\text { samples precipitated }\end{array}$ \\
\hline$P$ & Raw & $\mathrm{Cd}$ & $1.52 E+03$ & $1.35 E-05$ & AP102-3 & Y & Density reported for G443 represents a composite sample \\
\hline$P$ & Raw & $\mathrm{Cd}$ & $1.51 E+03$ & $1.34 E-05$ & AP102-3 & $Y$ & $\begin{array}{l}\text { Density not reported for G453. No assumptions could be } \\
\text { made }\end{array}$ \\
\hline $\mathbf{P}$ & Raw & $\mathrm{Cd}$ & $1.50 E+03$ & $1.33 E-05$ & AP102-3 & $Y$ & $\begin{array}{l}\text { Density not reported for G333. No assumptions could be } \\
\text { made }\end{array}$ \\
\hline $\mathbf{P}$ & Raw & $\mathrm{Cd}$ & $1.50 E+03$ & 1.33E-05 & AP102-3 & $Y$ & $\begin{array}{l}\text { Density not reported for G338. No assumptions could be } \\
\text { made }\end{array}$ \\
\hline $\mathbf{P}$ & Raw & $\mathrm{Cd}$ & $1.50 E+03$ & 1.33E-05 & AP102-3 & $Y$ & $\begin{array}{l}\text { Density reported for } G 460 \text { collected at } 226 \text { in from bottom } \\
\text { of tank at a } 270^{\circ} \text { angle. Sample was unheated, unheated } \\
\text { samples precipitated }\end{array}$ \\
\hline $\mathbf{P}$ & Raw & Cd & $1.50 E+03$ & $1.33 E-05$ & AP102-3 & $Y$ & $\begin{array}{l}\text { Density not reported for G338. No assumptions could be } \\
\text { made }\end{array}$ \\
\hline $\mathbf{P}$ & Raw & $\mathrm{Cd}$ & $1.48 E+03$ & 1.32E-05 & AP102-3 & $Y$ & $\begin{array}{l}\text { Density reported for } 6364 \text { collected at } 372 \text { in from bottom } \\
\text { of tank at a } 270^{\circ} \text { angle. Sample was unheated, unheated } \\
\text { samples precipitated }\end{array}$ \\
\hline $\mathbf{P}$ & Raw & Cd & $1.48 E+03$ & $1.32 E-05$ & AP102-3 & $\gamma$ & $\begin{array}{l}\text { Density reported for } G 358 \text { collected at } 334 \text { in from bottom } \\
\text { of tank at a } 150^{\circ} \text { angle. Sample was unheated, unheated } \\
\text { samples precipitated }\end{array}$ \\
\hline $\mathbf{P}$ & Raw & $\mathrm{Cd}$ & $1.47 E+03$ & 1.31E-05 & AP102-3 & $Y$ & $\begin{array}{l}\text { Density reported for } 6341 \text { collected at } 99 \text { in from bottom of } \\
\text { tank at a } 270^{\circ} \text { angle. }\end{array}$ \\
\hline $\mathbf{P}$ & Raw & Cd & $1.47 E+03$ & $1.31 E-05$ & AP102-3 & $\gamma$ & Density reported for G443 represents a composite sample \\
\hline $\mathbf{P}$ & Raw & Cd & $1.47 E+03$ & 1.31E-05 & AP102-3 & $\mathbf{Y}$ & $\begin{array}{l}\text { Density reported for } \mathrm{G} 341 \text { collected at } 99 \text { in from bottom of } \\
\text { tank at a } 270^{\circ} \text { angle. }\end{array}$ \\
\hline
\end{tabular}




\begin{tabular}{|c|c|c|c|c|c|c|c|c|c|c|c|}
\hline Reported Chemical & $\begin{array}{c}\text { Memo } \\
\text { Date }\end{array}$ & $\begin{array}{c}\text { Start } \\
\text { Analysis } \\
\text { Date }\end{array}$ & $\begin{array}{c}\begin{array}{c}\text { Finish } \\
\text { Analysis Date }\end{array} \\
\end{array}$ & $\begin{array}{c}\text { Start } \\
\text { Sample } \\
\text { Date } \\
\end{array}$ & $\begin{array}{c}\text { Finish } \\
\text { Sample } \\
\text { Date } \\
\end{array}$ & $\begin{array}{l}\text { Sample } \\
\text { Number }\end{array}$ & $\begin{array}{c}\text { Tank Farm } \\
\text { Number }\end{array}$ & $\begin{array}{c}\text { Lab Sample } \\
\text { Number }\end{array}$ & $\begin{array}{l}\text { Density } \\
(\mathrm{g} / \mathrm{mL})\end{array}$ & $\begin{array}{c}\text { Reported } \\
\text { Value }\end{array}$ & $\begin{array}{r}\text { Repa } \\
U_{n} \\
\end{array}$ \\
\hline Cadmium & $6 / 1 / 90$ & $4 / 30 / 89$ & $12 / 30 / 89$ & $4 / 27 / 89$ & $4 / 29 / 89$ & ВОВ2MO & G306 & G352 & 1.20 & $1.47 E+03$ & ug! \\
\hline Cadmium & $6 / 1 / 90$ & $4 / 30 / 89$ & $12 / 30 / 89$ & $4 / 27 / 89$ & $4 / 29 / 89$ & Вов2M1 & G308 & G353 & 1.20 & $1.47 E+03$ & ugi! \\
\hline Cadmium & $6 / 1 / 90$ & $4 / 30 / 89$ & $12 / 30 / 89$ & $4 / 27 / 89$ & $4 / 29 / 89$ & BOB2M1 & G308 & G353 & 1.20 & $1.46 \varepsilon+03$ & $u g i$ \\
\hline Cadmium & $6 / 1 / 90$ & $4 / 30 / 89$ & $12 / 30 / 89$ & $4 / 27 / 89$ & $4 / 29 / 89$ & BOB2L7 & G299 & G342 & 1.20 & $1.46 E+03$ & ug $\ell$ \\
\hline Cadmium & $6 / 1 / 90$ & $4 / 30 / 89$ & $12 / 30 / 89$ & $4 / 27 / 89$ & $4 / 29 / 89$ & $80 B 2 M 4$ & G313 & G363 & 1.20 & $1.45 E+03$ & ug $/ l$ \\
\hline Cadmium & $6 / 1 / 90$ & $4 / 30 / 89$ & $12 / 30 / 89$ & $4 / 27 / 89$ & $4 / 29 / 89$ & BOB2M7 & G319 & G460 & 1.20 & $1.45 E+03$ & ug $/ 1$ \\
\hline Cadmium & $6 / 1 / 90$ & $4 / 30 / 89$ & $12 / 30 / 89$ & $4 / 27 / 89$ & $4 / 29 / 89$ & $\mathrm{BOB} 2 \mathrm{LT}$ & G299 & G342 & 1.20 & $1.44 E+03$ & $u g / L$ \\
\hline Cadmium & $6 / 1 / 90$ & $4 / 30 / 89$ & $12 / 30 / 89$ & $4 / 27 / 89$ & $4 / 29 / 89$ & BOB2N2 & G317 & G333 & NA & $1.43 E \div 03$ & $u g h$ \\
\hline Cadmium & $6 / 1 / 90$ & $4 / 30 / 89$ & $12 / 30 / 89$ & $4 / 27 / 89$ & $4 / 29 / 89$ & BOB2M6 & G318 & G459 & 1.20 & $1.42 E+03$ & $u g / \Omega$ \\
\hline Cadmium & $6 / 1 / 90$ & $4 / 30 / 89$ & $12 / 30 / 89$ & $4 / 27 / 89$ & $4 / 29 / 89$ & BOB2N1 & G310 & G454 & (1.19) & $1.41 \varepsilon+03$ & $u g / L$ \\
\hline Cadmium & $6 / 1 / 90$ & $4 / 30 / 89$ & $12 / 30 / 89$ & $4 / 27 / 89$ & $4 / 29 / 89$ & BOB2M8 & G301 & G449 & NA & $1.40 E+03$ & ugh \\
\hline Cadmium & $6 / 1 / 90$ & $4 / 30 / 89$ & $12 / 30 / 89$ & $4 / 27 / 89$ & $4 / 29 / 89$ & BOB2M8 & G301 & G449 & NA & $1.39 E+03$ & ug $/$ L \\
\hline Cadmium & $6 / 1 / 90$ & $4 / 30 / 89$ & $12 / 30 / 89$ & $4 / 27 / 89$ & $4 / 29 / 89$ & BOB2L8 & G303 & G348 & 1.21 & $1.36 \mathrm{E}+03$ & ug/L \\
\hline Cadmium & $6 / 1 / 90$ & $4 / 30 / 89$ & $12 / 30 / 89$ & $4 / 27 / 89$ & $4 / 29 / 89$ & BOB2L8 & G303 & G348 & 1.21 & $1.36 \mathrm{E}+03$ & $u g / L$ \\
\hline Cadmium & $6 / 1 / 90$ & $4 / 30 / 89$ & $12 / 30 / 89$ & $4 / 27 / 89$ & $4 / 29 / 89$ & $8082 \mathrm{M} 3$ & G311 & G359 & 1.20 & $1.34 \mathrm{E}+03$ & $u g / L$ \\
\hline Cadmium & $6 / 1 / 90$ & $4 / 30 / 89$ & $12 / 30 / 89$ & $4 / 27 / 89$ & $4 / 29 / 89$ & BOB2M3 & G311 & G359 & 1.20 & $1.33 E+03$ & $u g / L$ \\
\hline Cadmium & $6 / 1 / 90$ & $4 / 30 / 89$ & $12 / 30 / 89$ & $4 / 27 / 89$ & $4 / 29 / 89$ & BO82M9 & G302 & G450 & $(1.20)$ & $1.32 E+03$ & $\mathrm{ug} / \mathrm{h}$ \\
\hline Cadmium & $6 / 1 / 90$ & $4 / 30 / 89$ & $12 / 30 / 89$ & $4 / 27 / 89$ & $4 / 29 / 89$ & $\mathrm{~B} 082 \mathrm{M} 2$ & G309 & G358 & 1.20 & $1.31 E+03$ & ug $/$. \\
\hline Cadmium & $6 / 1 / 90$ & $4 / 30 / 89$ & $12 / 30 / 89$ & $4 / 27 / 89$ & $4 / 29 / 89$ & 8OB2MO & G306 & G352 & 1.20 & $1.30 E+03$ & $u g h$ \\
\hline CI & $4 / 28 / 84$ & NS & NS & NS & NS & R8469 & NS & NS & NS & $<4.38 \mathrm{E}-04$ & $M$ \\
\hline Cl- & NS & NS & NS & NS & NS & NS & NS & NS & 1.24 & $9.13 E-02$ & Mole $/ \mathrm{L}$ \\
\hline $\mathrm{Cl}$ & $4 / 28 / 84$ & NS & NS & NS & NS & R-8469 & NS & NS & NS & $4.38 \mathrm{E}-0.4$ & moles $n$ \\
\hline Cl. & $4 / 28 / 84$ & NS & NS & NS & NS & NS & NS & NS & NS & $1.55 E-02$ & $g / L$ \\
\hline
\end{tabular}




\begin{tabular}{|c|c|c|c|c|c|c|c|}
\hline \multirow[b]{2}{*}{ Code } & \multirow[b]{2}{*}{ Source } & \multicolumn{3}{|c|}{ Analyte Information } & \multirow[b]{2}{*}{$\begin{array}{c}\text { Reference } \\
\text { Number }\end{array}$} & \multirow[b]{2}{*}{$\begin{array}{c}\text { Validated } \\
(Y / N)\end{array}$} & \multirow[b]{2}{*}{ Notes } \\
\hline & & Analyte & $\begin{array}{l}\text { Conc. } \\
\text { (ug/L) }\end{array}$ & Conc. (mol/L) & & & \\
\hline $\mathbf{P}$ & Raw & Cd & $1.47 E+03$ & 1.31E-05 & AP102-3 & 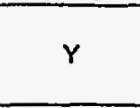 & $\begin{array}{l}\text { Density reported for G352 collected at } 34 \text { in from bottom of } \\
\text { tank at a } 30^{\circ} \text { angle. Sample was unheated, unheated } \\
\text { samples precipitated }\end{array}$ \\
\hline$P$ & Raw & Cd & $1.47 E+03$ & 1.31E-05 & AP102-3 & $\mathbf{Y}$ & $\begin{array}{l}\text { Density reported for G353 collected at } 334 \text { in from bottom } \\
\text { of tank at a } 150^{\circ} \text { angle. Sample was unheated, unheated } \\
\text { samples precipitated }\end{array}$ \\
\hline $\mathbf{P}$ & Raw & $\mathrm{Cd}$ & $1.46 E+03$ & $1.30 E-05$ & AP102-3 & $Y$ & $\begin{array}{l}\text { Density reported for } \mathrm{G} 353 \text { collected at } 334 \text { in from botrom } \\
\text { of tank at a } 150^{\circ} \text { angle. Sample was unheated, unheated } \\
\text { samples precipitated }\end{array}$ \\
\hline $\mathbf{P}$ & Raw & $\mathrm{Cd}$ & $1.46 \mathrm{E}+03$ & $1.30 \mathrm{E}-05$ & AP102-3 & $\mathbf{Y}$ & $\begin{array}{l}\text { Density reported for G342 collected at } 306 \text { in from bottom } \\
\text { of tank at a } 30^{\circ} \text { angle. Sample was unheated, unheated } \\
\text { samples precipitated }\end{array}$ \\
\hline $\mathbf{P}$ & Raw & Cd & $1.45 E+03$ & $1.29 E-05$ & AP102-3 & $Y$ & $\begin{array}{l}\text { Density reported for } \mathrm{G} 363 \text { collected at } 18 \text { in from bottom of } \\
\text { tank at a } 150^{\circ} \text { angle. Sample was unheated, unheated } \\
\text { samples precipitated }\end{array}$ \\
\hline $\mathbf{P}$ & Raw & $C d$ & $1.45 E+03$ & $1.29 E-05$ & AP102-3 & $\mathrm{Y}$ & $\begin{array}{l}\text { Density seported for } \mathrm{G} 460 \text { collected at } 226 \text { in from bottom } \\
\text { of tank at a } 270^{\circ} \text { angle. Sample was unheated, unheated } \\
\text { samples precipitated }\end{array}$ \\
\hline $\mathbf{P}$ & Raw & Cod & $1.44 E+03$ & $1.28 \mathrm{E}-05$ & AP102-3 & $Y$ & $\begin{array}{l}\text { Density reported for G342 collected at } 306 \text { in from bottom } \\
\text { of tank at a } 30^{\circ} \text { angle. Sample was unheated, unheated } \\
\text { samples precipitated }\end{array}$ \\
\hline$P$ & Raw & $\mathrm{Cd}$ & $1.43 E+03$ & $1.27 \mathrm{E}-05$ & AP102-3 & $\mathbf{Y}$ & $\begin{array}{l}\text { Density not reported for G333. No assumptions could be } \\
\text { made }\end{array}$ \\
\hline $\mathbf{P}$ & Raw & Cd & $1.42 E+03$ & $1.26 E-05$ & AP102-3 & $\mathbf{Y}$ & $\begin{array}{l}\text { Density reported for G } 459 \text { collected at } 226 \text { in from bottom } \\
\text { of tank at a } 270^{\circ} \text { angle. Sample"was unheated, unheated } \\
\text { samples precipitated }\end{array}$ \\
\hline$P$ & Raw & $\mathrm{Cd}$ & $1.41 E+03$ & $1.25 E-05$ & AP102-3 & $Y$ & $\begin{array}{l}\text { Density not reported for G454. Density assumed same as } \\
\text { G471 which was collected at } 208 \text { in from bottom of tank at } \\
\text { a } 150^{\circ} \text { angle and was keep heated to } 40^{\circ} \mathrm{C} \text {. }\end{array}$ \\
\hline$P$ & Raw & $\mathrm{Cd}$ & $1.40 E+03$ & $1.25 \mathrm{E}-05$ & AP102-3 & $Y$ & $\begin{array}{l}\text { Density not reported for } 6449 \text {. No assumptions could be } \\
\text { made }\end{array}$ \\
\hline$P$ & Raw & Cd & $1.39 E+03$ & $1.24 E-05$ & AP102-3 & $Y$ & $\begin{array}{l}\text { Density not reported for G449. No assumptions could be } \\
\text { made }\end{array}$ \\
\hline$P$ & Raw & $C d$ & $1.36 E+03$ & 1.21E-05 & AP102-3 & Y & $\begin{array}{l}\text { Density reported for G348 collected at } 125 \text { in from bottom } \\
\text { of tank at a } 30^{\circ} \text { angle. Sample was unheated, unheated } \\
\text { samples precipitated }\end{array}$ \\
\hline $\mathbf{P}$ & Raw & $\mathrm{Cd}$ & $1.36 E+03$ & $1.21 E-05$ & AP102-3 & $Y$ & $\begin{array}{l}\text { Density reported for G348 collected at } 125 \text { in from bottom } \\
\text { of tank at a } 30^{\circ} \text { angle. Sample was unheated, unheated } \\
\text { samples precipitated }\end{array}$ \\
\hline $\mathbf{P}$ & Raw & $\mathrm{Cd}$ & $1.34 E+03$ & $1.19 E-05$ & AP102-3 & $\mathbf{Y}$ & $\begin{array}{l}\text { Density reported for G359 collected at } 162 \text { in from bottom } \\
\text { of tank at a } 150^{\circ} \text { angle. Sample was unheated, unheated } \\
\text { samples precipitated }\end{array}$ \\
\hline $\mathbf{P}$ & Raw & $\mathrm{Cd}$ & $1.33 E+03$ & $1.18 \mathrm{E}-05$ & AP102-3 & $Y$ & $\begin{array}{l}\text { Density reported for G359 collected at } 162 \text { in from bottom } \\
\text { of tank at a } 150^{\circ} \text { angle. Sample was unheated, unheated } \\
\text { samples precipitated }\end{array}$ \\
\hline $\mathbf{P}$ & Raw & $\mathrm{Cd}$ & $1.32 E+03$ & $1.17 E-05$ & AP102-3 & $\mathbf{Y}$ & $\begin{array}{l}\text { Density not reported for G450. Density assumed same as } \\
\text { G477 which was collected at } 182 \text { in from bottom of tank at } \\
\text { a } 30^{\circ} \text { angle and was keep heated to } 40^{\circ} \mathrm{C} \text {. }\end{array}$ \\
\hline$P$ & Raw & Cd & $1.31 E+03$ & 1.17E-05 & AP102-3 & $Y$ & $\begin{array}{l}\text { Density reported for G358 collected at } 334 \text { in from bottom } \\
\text { of tank at a } 150^{\circ} \text { angle. Sample was unheated, unheated } \\
\text { samples precipitated }\end{array}$ \\
\hline $\mathbf{P}$ & Raw & $\mathrm{Cd}$ & $1.30 E \div 03$ & $1.16 \mathrm{E}-05$ & AP102-3 & $\gamma$ & $\begin{array}{l}\text { Density reported for } \mathrm{G} 352 \text { collected at } 34 \text { in from bottom of } \\
\text { tank at a } 30^{\circ} \text { angle. Sample was unheated, unheated } \\
\text { samples precipitated }\end{array}$ \\
\hline$L$ & Raw & $\mathrm{Cl}$ & NVALUE! & \#VALUE! & AP102-1 & $N$ & Outdated Information \\
\hline$L$ & HTCE Inv. & $\mathrm{Cl}$ & $3.24 E+12$ & $9.13 E+04$ & NA & $\mathbf{N}$ & No Reference \\
\hline $\mathbf{P}$ & Braun & $\mathrm{Cl}$ & $1.55 \mathrm{E}+10$ & $4.38 \mathrm{E}+02$ & NS & $N$ & No Reference \\
\hline $\mathbf{P}$ & Wastren & $\mathrm{Cl}$ & $1.55 E+10$ & $4.37 E+02$ & NS & $N$ & No Reference \\
\hline
\end{tabular}


TWRS Privatization Support $P$ Chemical Liqui

\begin{tabular}{|c|c|c|c|c|c|c|c|c|c|c|c|}
\hline & & & & & ank Waste & hatacteriza & On Sample I & ormation & & & \\
\hline Reported Chemical & $\begin{array}{c}\text { Memo } \\
\text { Date }\end{array}$ & $\begin{array}{c}\text { Start } \\
\text { Analysis } \\
\text { Date } \\
\end{array}$ & $\begin{array}{c}\text { Finish } \\
\text { Analysis Date } \\
\end{array}$ & $\begin{array}{c}\text { Start } \\
\text { Sample } \\
\text { Date }\end{array}$ & $\begin{array}{c}\text { Finish } \\
\text { Sample } \\
\text { Date }\end{array}$ & $\begin{array}{l}\text { Sample } \\
\text { Number }\end{array}$ & $\begin{array}{c}\text { Tank Farm } \\
\text { Number }\end{array}$ & $\begin{array}{c}\text { Lab Sample } \\
\text { Number }\end{array}$ & $\begin{array}{l}\text { Density } \\
(\mathrm{g} / \mathrm{mL})\end{array}$ & $\begin{array}{c}\text { Reported } \\
\text { Value }\end{array}$ & $\begin{array}{r}\text { Ref } \\
2 \\
\end{array}$ \\
\hline Chloride & $6 / 1 / 90$ & $4 / 30 / 89$ & $12 / 30 / 89$ & $4 / 27 / 89$ & $4 / 29 / 89$ & ВОВ2T9 & G327 & G443 & 1.20 & $4.56 E+03$ & ug \\
\hline Chloride & $6 / 1 / 90$ & $4 / 30 / 89$ & $12 / 30 / 89$ & $4 / 27 / 89$ & $4 / 29 / 89$ & ВОВ2T9 & G327 & G443 & 1.20 & $4.36 E+03$ & ug \\
\hline Chloride & $6 / 1 / 90$ & $4 / 30 / 89$ & $12 / 30 / 89$ & $4 / 27 / 89$ & $4 / 29 / 89$ & BOB2M7 & G319 & G460 & 1.2 & $3.63 E+03$ & $\mathrm{ug}$ \\
\hline Chloride & $6 / 1 / 90$ & $4 / 30 / 89$ & $12 / 30 / 89$ & $4 / 27 / 89$ & $4 / 29 / 89$ & BOB2M7 & G319 & G460 & 1.2 & $3.57 E+03$ & ug \\
\hline Chloride & $6 / 1 / 90$ & $4 / 30 / 89$ & $12 / 30 / 89$ & $4 / 27 / 89$ & $4 / 29 / 89$ & ВОВ2Т9 & G327 & G470* & 1.19 & $3.15 E+03$ & ug. \\
\hline Chloride & $6 / 1 / 90$ & $4 / 30 / 89$ & $12 / 30 / 89$ & $4 / 27 / 89$ & $4 / 29 / 89$ & BOB2T9 & G327 & G470* & 1.19 & $3.12 E+03$ & ugi \\
\hline Chloride & $6 / 1 / 90$ & $4 / 30 / 89$ & $12 / 30 / 89$ & $4 / 27 / 89$ & $4 / 29 / 89$ & Вов2M9 & G302 & G477* & 1.20 & $3.08 E+03$ & ug. \\
\hline Chloride & $6 / 1 / 90$ & $4 / 30 / 89$ & $12 / 30 / 89$ & $4 / 27 / 89$ & $4 / 29 / 89$ & BOB2M9 & G302 & G450 & $(1.20)$ & $3.05 E+03$ & ugi \\
\hline Chloride & $6 / 1 / 90$ & $4 / 30 / 89$ & $12 / 30 / 89$ & $4 / 27 / 89$ & $4 / 29 / 89$ & B082N3 & G322 & G338 & NA & $3.02 E+03$ & $u g f$ \\
\hline Chloride & $6 / 1 / 90$ & $4 / 30 / 89$ & $12 / 30 / 89$ & $4 / 27 / 89$ & $4 / 29 / 89$ & BOB2NO & G307 & G453 & NA & $3.02 E+03$ & ugl \\
\hline Chloride & $6 / 1 / 90$ & $4 / 30 / 89$ & $12 / 30 / 89$ & $4 / 27 / 89$ & $4 / 29 / 89$ & BOB2N3 & G322 & G338 & NA & $3.00 E+03$ & ug/. \\
\hline Chloride & $6 / 1 / 90$ & $4 / 30 / 89$ & $12 / 30 / 89$ & $4 / 27 / 89$ & $4 / 29 / 89$ & BOB2M8 & G301 & G449 & NA & $2.99 E+03$ & $u g / 1$ \\
\hline Chloride & $6 / 1 / 90$ & $4 / 30 / 89$ & $12 / 30 / 89$ & $4 / 27 / 89$ & $4 / 29 / 89$ & BOB2N1 & G310 & G471* & 1.19 & $2.99 E+03$ & ug/s \\
\hline Chloride & $6 / 1 / 90$ & $4 / 30 / 89$ & $12 / 30 / 89$ & $4 / 27 / 89$ & $4 / 29 / 89$ & BOB2M9 & G302 & G450 & $(1.20)$ & $2.97 E+03$ & $\mathrm{ug} / \mathrm{r}$ \\
\hline Chloride & $6 / 1 / 90$ & $4 / 30 / 89$ & $12 / 30 / 89$ & $4 / 27 / 89$ & $4 / 29 / 89$ & BOB2M8 & G301 & G449 & NA & $2.96 E+03$ & ugir \\
\hline Chloride & $6 / 1 / 90$ & $4 / 30 / 89$ & $12 / 30 / 89$ & $4 / 27 / 89$ & $4 / 29 / 89$ & BOB2N4 & G323 & G476* & 1.21 & $2.96 E+03$ & $\mathrm{ug} / \mathrm{n}$ \\
\hline Chloride & $6 / 1 / 90$ & $4 / 30 / 89$ & $12 / 30 / 89$ & $4 / 27 / 89$ & $4 / 29 / 89$ & BOB2M5 & G315 & G364 & 1.19 & $2.94 E+03$ & $u g / n$ \\
\hline Chloride & $6 / 1 / 90$ & $4 / 30 / 89$ & $12 / 30 / 89$ & $4 / 27 / 89$ & $4 / 29 / 89$ & BOB2NO & G307 & G453 & NA & $2.94 E+03$ & $u g / n$ \\
\hline Chloride & $6 / 1 / 90$ & $4 / 30 / 89$ & $12 / 30 / 89$ & $4 / 27 / 89$ & $4 / 29 / 89$ & BOB2M5 & G315 & G364 & 1.19 & $2.94 E+03$ & $u g / \alpha$ \\
\hline Chloride & $6 / 1 / 90$ & $4 / 30 / 89$ & $12 / 30 / 89$ & $4 / 27 / 89$ & $4 / 29 / 89$ & BOB2N2 & G317 & G333 & NA & $2.93 E+03$ & $u g / m$ \\
\hline Chloride & $6 / 1 / 90$ & $4 / 30 / 89$ & $12 / 30 / 89$ & $4 / 27 / 89$ & $4 / 29 / 89$ & BOB2M4 & G313 & 6363 & 1.20 & $2.92 E+03$ & $\mathrm{ug} / \mathrm{m}$ \\
\hline Chloride & $6 / 1 / 90$ & $4 / 30 / 89$ & $12 / 30 / 89$ & $4 / 27 / 89$ & $4 / 29 / 89$ & BOB2NO & G307 & G527* & NA & $2.92 E+03$ & $\mathrm{ug} / \mathrm{m}$ \\
\hline Chloride & $6 / 1 / 90$ & $4 / 30 / 89$ & $12 / 30 / 89$ & $4 / 27 / 89$ & $4 / 29 / 89$ & BOB2N1 & G310 & G454 & $(1.19)$ & $2.91 E+03$ & $\mathrm{ug} / \mathrm{m}$ \\
\hline
\end{tabular}




\begin{tabular}{|c|c|c|c|c|c|c|c|}
\hline & & \multicolumn{3}{|c|}{ Analyte Information } & \multirow[b]{2}{*}{$\begin{array}{c}\text { Reference } \\
\text { Number }\end{array}$} & \multirow[b]{2}{*}{$\begin{array}{c}\text { Validated } \\
\text { (Y/N) }\end{array}$} & \multirow[b]{2}{*}{ Notes } \\
\hline Code & Source & Analyte & $\begin{array}{l}\text { Conc. } \\
\text { (ug/L) }\end{array}$ & Conc. (mol/L) & & & \\
\hline $\mathbf{P}$ & Raw & $\mathrm{Cl}$ & $4.56 \mathrm{E}+06$ & $1.29 E-01$ & AP102-3 & $\mathbf{Y}$ & Density reported for $\mathrm{G} 443$ represents a composite sample \\
\hline $\mathbf{P}$ & Raw & $\mathrm{Cl}$ & $4.36 E+06$ & $1.23 E-01$ & AP102-3 & Y & Density reported for 6443 represents a composite sample \\
\hline $\mathbf{P}$ & Raw & $\mathrm{Cl}$ & $3.63 E+06$ & $1.02 E-01$ & AP102-3 & $Y$ & $\begin{array}{l}\text { Density reported for G460 collected at } 226 \text { in from bottom } \\
\text { of tank at a } 270^{\circ} \text { angle. Sample was unheated, unheated } \\
\text { samples precipitated }\end{array}$ \\
\hline $\mathbf{P}$ & Raw & $\mathrm{Cl}$ & $3.57 E+06$ & $1.01 E-01$ & AP102-3 & $\mathbf{Y}$ & $\begin{array}{l}\text { Density reported for } \mathrm{G} 460 \text { collected at } 226 \text { in from bottom } \\
\text { of tank at a } 270^{\circ} \text { angle. Sample was unheated, unheated } \\
\text { samples precipitated }\end{array}$ \\
\hline $\mathbf{P}$ & Raw & $\mathrm{Cl}$ & $3.15 E+06$ & $8.89 E-02$ & AP102-3 & $\mathbf{Y}$ & $\begin{array}{l}\text { Density reported for } \mathrm{G} 470 \text { represents a composite sample. } \\
\text { Sample was kept heated to } 40^{\circ} \mathrm{C} \text {. }\end{array}$ \\
\hline $\mathbf{P}$ & Raw & $\mathrm{Cl}$ & $3.12 E+06$ & $8.80 E-02$ & AP102-3 & $Y$ & $\begin{array}{l}\text { Density reported for G470 represents a composite sample. } \\
\text { Sample was kept heated to } 40^{\circ} \mathrm{C} \text {. }\end{array}$ \\
\hline $\mathbf{P}$ & Raw & $\mathrm{Cl}$ & $3.08 E+06$ & $8.69 \varepsilon-02$ & AP102-3 & $Y$ & $\begin{array}{l}\text { Density reported for } \mathrm{G} 477 \text { collected at } 182 \text { in from bottom } \\
\text { of tank at a } 30^{\circ} \text { angle and was keep heated to } 40^{\circ} \mathrm{C} \text {. }\end{array}$ \\
\hline $\mathbf{P}$ & Raw & $\mathrm{Cl}$ & $3.05 E+06$ & 8.60E-02 & AP102-3 & $Y$ & $\begin{array}{l}\text { Density not reported for G450. Density assumed same as } \\
\text { G } 477 \text { which was collected at } 182 \text { in from bottom of tank at } \\
\text { a } 30^{\circ} \text { angle and was keep heated to } 40^{\circ} \mathrm{C} \text {. }\end{array}$ \\
\hline$P$ & Raw & $\mathrm{Cl}$ & $3.02 E+06$ & 8.52E-02 & AP102-3 & $Y$ & $\begin{array}{l}\text { Density not reported for G338. No assumptions could be } \\
\text { made }\end{array}$ \\
\hline$P$ & Raw & $\mathrm{Cl}$ & $3.02 E+06$ & $8.52 E-02$ & AP102-3 & $Y$ & $\begin{array}{l}\text { Density not reported for G453. No assumptions could be } \\
\text { made }\end{array}$ \\
\hline$P$ & Raw & $\mathrm{Cl}$ & $3.00 E+06$ & $8.46 E-02$ & AP102-3 & $\gamma$ & $\begin{array}{l}\text { Density not reported for G338. No assumptions could be } \\
\text { made }\end{array}$ \\
\hline $\mathbf{P}$ & Raw & Cl & $2.99 E+06$ & $8.43 E-02$ & AP102-3 & $\gamma$ & $\begin{array}{l}\text { Density not reported for G449. No assumptions could be } \\
\text { made }\end{array}$ \\
\hline $\mathbf{P}$ & Raw & $\mathrm{Cl}$ & $2.99 E+06$ & $8.43 E-02$ & AP102-3 & $Y$ & $\begin{array}{l}\text { Density reported for } \mathrm{G} 471 \text { collected at } 208 \text { in from bottom } \\
\text { of tank at a } 150^{\circ} \text { angle and was keep heated to } 40^{\circ} \mathrm{C} \text {. }\end{array}$ \\
\hline $\mathbf{P}$ & Raw & $\mathrm{Cl}$ & $2.97 E+06$ & 8.38E-02 & AP102-3 & $\mathbf{Y}$ & $\begin{array}{l}\text { Density not reported for G450. Density assumed same as } \\
\mathrm{G} 477 \text { which was collected at } 182 \text { in from bottom of tank at } \\
\text { a } 30^{\circ} \text { angle and was keep heated to } 40^{\circ} \mathrm{C} \text {. }\end{array}$ \\
\hline $\mathbf{P}$ & Raw & $\mathrm{Cl}$ & $2.96 E+06$ & 8.35E-02 & AP102-3 & $\mathbf{Y}$ & $\begin{array}{l}\text { Density not reported for G449. No assumptions could be } \\
\text { made }\end{array}$ \\
\hline$P$ & Raw & $\mathrm{Cl}$ & $2.96 E+06$ & 8.35E-02 & AP102-3 & $\gamma$ & $\begin{array}{l}\text { Density reported for } G 476 \text { collected at } 99 \text { in from bottom of } \\
\text { tank at a } 270^{\circ} \text { angle and was keep heated to } 40^{\circ} \mathrm{C} \text {. }\end{array}$ \\
\hline $\mathbf{P}$ & Raw & $\mathrm{Cl}$ & $2.94 E+06$ & 8.29E-02 & AP102-3 & $Y$ & $\begin{array}{l}\text { Density reported for G364 collected at } 372 \text { in from bottom } \\
\text { of tank at a } 270^{\circ} \text { angle. Sample was unheated, unheated } \\
\text { samples precipitated }\end{array}$ \\
\hline$P$ & Raw & $\mathrm{Cl}$ & $2.94 E+06$ & 8.29E-02 & AP102-3 & $Y$ & $\begin{array}{l}\text { Density not reported for G453. No assumptions could be } \\
\text { made }\end{array}$ \\
\hline $\mathbf{P}$ & Raw & $\mathrm{Cl}$ & $2.94 E+06$ & $8.29 E-02$ & AP102-3 & $Y$ & $\begin{array}{l}\text { Density reported for G364 collected at } 372 \text { in from bottom } \\
\text { of tank at a } 270^{\circ} \text { angle. Sample was unheated, unheated } \\
\text { samples precipitated }\end{array}$ \\
\hline$P$ & Raw & $\mathrm{Cl}$ & $2.93 E+06$ & $8.27 E-02$ & AP102-3 & $Y$ & $\begin{array}{l}\text { Density not reported for G333. No assumptions could be } \\
\text { made }\end{array}$ \\
\hline $\mathbf{P}$ & Raw & $\mathrm{Cl}$ & $2.92 \mathrm{E}+06$ & 8.24E-02 & AP102-3 & $Y$ & $\begin{array}{l}\text { Density reported for } \mathrm{G} 363 \text { collected at } 18 \text { in from bottom of } \\
\text { tank at a } 150^{\circ} \text { angle. Sample was unheated, unheated } \\
\text { samples precipitated }\end{array}$ \\
\hline$P$ & Raw & $\mathrm{Cl}$ & $2.92 E+06$ & 8.24E-02 & AP102-3 & $Y$ & $\begin{array}{l}\text { Density not reported for G527. No assumptions could be } \\
\text { made }\end{array}$ \\
\hline$P$ & Raw & $\mathrm{Cl}$ & $2.91 E+06$ & 8.21E-02 & AP102-3 & $Y$ & $\begin{array}{l}\text { Density not reported for G454. Density assumed same as } \\
\text { G471 which was collected at } 208 \text { in from bottom of tank at } \\
\text { a } 150^{\circ} \text { angle and was keep heated to } 40^{\circ} \mathrm{C} \text {. }\end{array}$ \\
\hline
\end{tabular}




\begin{tabular}{|c|c|c|c|c|c|c|c|c|c|c|c|}
\hline & & & & & ank Waste & haracteriza & Oon Sample I & ormation & & & \\
\hline Reported Chemical & $\begin{array}{c}\text { Memo } \\
\text { Date }\end{array}$ & $\begin{array}{c}\text { Start } \\
\text { Analysis } \\
\text { Date }\end{array}$ & $\begin{array}{c}\text { Finish } \\
\text { Analysis Date } \\
\end{array}$ & $\begin{array}{c}\text { Start } \\
\text { Sample } \\
\text { Date }\end{array}$ & $\begin{array}{c}\text { Finish } \\
\text { Sample } \\
\text { Date } \\
\end{array}$ & $\begin{array}{l}\text { Sample } \\
\text { Number }\end{array}$ & $\begin{array}{c}\text { Tank Farm } \\
\text { Number }\end{array}$ & $\begin{array}{c}\text { Lab Sample } \\
\text { Number }\end{array}$ & $\begin{array}{l}\text { Density } \\
\text { (g/mL) }\end{array}$ & $\begin{array}{c}\text { Reported } \\
\text { Value } \\
\end{array}$ & $\begin{array}{r}\text { Repo } \\
U_{n} \\
\end{array}$ \\
\hline Chloride & $6 / 1 / 90$ & $4 / 30 / 89$ & $12 / 30 / 89$ & $4 / 27 / 89$ & $4 / 29 / 89$ & BOB2N1 & G310 & G454 & (1.19) & $2.90 E+03$ & ug/r \\
\hline Chloride & $6 / 1 / 90$ & $4 / 30 / 89$ & $12 / 30 / 89$ & $4 / 27 / 89$ & $4 / 29 / 89$ & BOB2N2 & G317 & G333 & NA & $2.90 E+03$ & ug/r \\
\hline Chloride & $6 / 1 / 90$ & $4 / 30 / 89$ & $12 / 30 / 89$ & $4 / 27 / 89$ & $4 / 29 / 89$ & BOB2NO & G307 & G527* & NA & $2.90 E+03$ & $u g / n$ \\
\hline Chloride & $6 / 1 / 90$ & $4 / 30 / 89$ & $12 / 30 / 89$ & $4 / 27 / 89$ & $4 / 29 / 89$ & $8082 \mathrm{M1}$ & G308 & G353 & 1.20 & $2.89 E+03$ & $u g / n$ \\
\hline Chloride & $6 / 1 / 90$ & $4 / 30 / 89$ & $12 / 30 / 89$ & $4 / 27 / 89$ & $4 / 29 / 89$ & ВОВ2MO & G306 & G352 & 1.20 & $2.88 E+03$ & $u g / n$ \\
\hline Chloride & $6 / 1 / 90$ & $4 / 30 / 89$ & $12 / 30 / 89$ & $4 / 27 / 89$ & $4 / 29 / 89$ & BOB2M6 & G318 & G459 & 1.20 & $2.87 E+03$ & $u g / \pi$ \\
\hline Chloride & $6 / 1 / 90$ & $4 / 30 / 89$ & $12 / 30 / 89$ & $4 / 27 / 89$ & $4 / 29 / 89$ & BOB2L7 & 6299 & G342 & 1.20 & $2.85 E+03$ & $\mathrm{ug} / \mathrm{n}$ \\
\hline Chloride & $6 / 1 / 90$ & $4 / 30 / 89$ & $12 / 30 / 89$ & $4 / 27 / 89$ & $4 / 29 / 89$ & вов2М1 & G308 & G522* & $(1.20)$ & $2.85 E+03$ & $\mathrm{ug} / \mathrm{m}$ \\
\hline Chloride & $6 / 1 / 90$ & $4 / 30 / 89$ & $12 / 30 / 89$ & $4 / 27 / 89$ & $4 / 29 / 89$ & BOB2L9 & G305 & G349 & 1.20 & $2.84 E+03$ & $\mathrm{ug} / \mathrm{m}$ \\
\hline Chloride & $6 / 1 / 90$ & $4 / 30 / 89$ & $12 / 30 / 89$ & $4 / 27 / 89$ & $4 / 29 / 89$ & BOB2M3 & G311 & G516* & $(1.20)$ & $2.84 E+03$ & $\mathrm{ug} / \mathrm{m}$ \\
\hline Chloride & $6 / 1 / 90$ & $4 / 30 / 89$ & $12 / 30 / 89$ & $4 / 27 / 89$ & $4 / 29 / 89$ & BOB2N4 & G323 & G341 & 1.20 & $2.83 E+03$ & $\mathrm{ug} / \mathrm{m}^{\prime}$ \\
\hline Chloride & $6 / 1 / 90$ & $4 / 30 / 89$ & $12 / 30 / 89$ & $4 / 27 / 89$ & $4 / 29 / 89$ & BOB2M3 & G311 & G359 & 1.20 & $2.83 E+03$ & $\mathrm{ug} / \mathrm{ml}$ \\
\hline Chloride & $6 / 1 / 90$ & $4 / 30 / 89$ & $12 / 30 / 89$ & $4 / 27 / 89$ & $4 / 29 / 89$ & BOB2M4 & G313 & G363 & 1.20 & $2.83 E+03$ & $\mathrm{ug} / \mathrm{ml}$ \\
\hline Chloride & $6 / 1 / 90$ & $4 / 30 / 89$ & $12 / 30 / 89$ & $4 / 27 / 89$ & $4 / 29 / 89$ & BOB2M6 & G318 & G459 & 1.20 & $2.83 E+03$ & $\mathrm{ug} / \mathrm{ml}$ \\
\hline Chloride & $6 / 1 / 90$ & $4 / 30 / 89$ & $12 / 30 / 89$ & $4 / 27 / 89$ & $4 / 29 / 89$ & B082M2 & G309 & G358 & 1.20 & $2.82 E+03$ & $\mathrm{ug} / \mathrm{ml}$ \\
\hline Chloride & $6 / 1 / 90$ & $4 / 30 / 89$ & $12 / 30 / 89$ & $4 / 27 / 89$ & $4 / 29 / 89$ & BOB2N4 & G323 & G476" & 1.21 & $2.82 E+03$ & ug/mL \\
\hline Chloride & $6 / 1 / 90$ & $4 / 30 / 89$ & $12 / 30 / 89$ & $4 / 27 / 89$ & $4 / 29 / 89$ & BOB2M6 & G318 & G517* & $(1.20)$ & $2.82 E+03$ & $u g / m L$ \\
\hline Chloride & $6 / 1 / 90$ & $4 / 30 / 89$ & $12 / 30 / 89$ & $4 / 27 / 89$ & $4 / 29 / 89$ & BOB2NO & G307 & G527* & NA & $2.82 E+03$ & $u g / \mathrm{mL}$ \\
\hline Chloride & $6 / 1 / 90$ & $4 / 30 / 89$ & $12 / 30 / 89$ & $4 / 27 / 89$ & $4 / 29 / 89$ & BOB2M2 & G309 & G515* & $(1.20)$ & $2.81 E+03$ & $\mathrm{ug} / \mathrm{mL}$ \\
\hline Chloride & $6 / 1 / 90$ & $4 / 30 / 89$ & $12 / 30 / 89$ & $4 / 27 / 89$ & $4 / 29 / 89$ & BOB2M3 & G311 & G516* & $(1.20)$ & $2.81 E \div 03$ & $\mathrm{ug} / \mathrm{mL}$ \\
\hline Chloride & $6 / 1 / 90$ & $4 / 30 / 89$ & $12 / 30 / 89$ & $4 / 27 / 89$ & $4 / 29 / 89$ & BOB2M1 & G308 & G522* & $(1.20)$ & $2.81 E+03$ & $\mathrm{ug} / \mathrm{mL}$ \\
\hline
\end{tabular}




\begin{tabular}{|c|c|c|c|c|c|c|c|}
\hline \multirow[b]{2}{*}{ Code } & \multirow[b]{2}{*}{ Source } & \multicolumn{3}{|c|}{ Analyte Information } & \multirow[b]{2}{*}{$\begin{array}{l}\text { Reference } \\
\text { Number }\end{array}$} & \multirow[b]{2}{*}{$\begin{array}{l}\text { Validated } \\
\text { (Y/N) }\end{array}$} & \multirow[b]{2}{*}{ Notes } \\
\hline & & Analyte & $\begin{array}{l}\text { Conc. } \\
\text { (ug/L) }\end{array}$ & Conc. (mol/L) & & & \\
\hline $\mathbf{P}$ & Raw & $\mathrm{Cl}$ & $2.90 \mathrm{E}+06$ & $8.18 \mathrm{E}-02$ & AP102-3 & $\mathbf{Y}$ & $\begin{array}{l}\text { Density not reported for G454. Density assumed same as } \\
\text { GS71 which was collected at } 208 \text { in from bottom of tank at } \\
\text { a } 150^{\circ} \text { angle and was keep heated to } 40^{\circ} \mathrm{C} \text {. }\end{array}$ \\
\hline $\mathbf{P}$ & Raw & $\mathrm{Cl}$ & $2.90 E+06$ & 8.18E-02 & AP102-3 & $\boldsymbol{Y}$ & $\begin{array}{l}\text { Density not reported for G333. No assumptions could be } \\
\text { made }\end{array}$ \\
\hline $\mathbf{P}$ & Raw & $\mathrm{Cl}$ & $2.90 E+06$ & 8.18E-02 & AP102-3 & $\mathbf{Y}$ & $\begin{array}{l}\text { Density not reported for G527. No assumptions could be } \\
\text { made }\end{array}$ \\
\hline $\mathbf{P}$ & Raw & $\mathrm{Cl}$ & $2.89 E+06$ & 8.15E-02 & AP102-3 & $Y$ & $\begin{array}{l}\text { Density reported for G353 collected at } 334 \text { in from bottom } \\
\text { of tank at a } 150^{\circ} \text { angle. Sample was unheated, unheated } \\
\text { samples precipitated }\end{array}$ \\
\hline $\mathbf{P}$ & Raw & $\mathrm{Cl}$ & $2.88 E+06$ & 8.12E-02 & AP102-3 & $Y$ & $\begin{array}{l}\text { Density reported for } \mathrm{G} 352 \text { collected at } 34 \text { in from bottom of } \\
\text { tank at a } 30^{\circ} \text { angle. Sample was unheated, unheated } \\
\text { samples precipitated }\end{array}$ \\
\hline $\mathbf{P}$ & Raw & $\mathrm{Cl}$ & $2.87 E+06$ & $8.10 \varepsilon \cdot 02$ & AP102-3 & $Y$ & $\begin{array}{l}\text { Density reported for G459 collected at } 226 \text { in from bottom } \\
\text { of tank at a } 270^{\circ} \text { angle. Sample was unheated, unheated } \\
\text { samples precipitated }\end{array}$ \\
\hline $\mathbf{P}$ & Raw & $\mathrm{Cl}$ & $2.85 E+06$ & 8.04E-02 & AP102-3 & $\mathbf{Y}$ & $\begin{array}{l}\text { Density reported for G342 collected at } 306 \text { in from bottom } \\
\text { of tank at a } 30^{\circ} \text { angle. Sample was unheated, unheated } \\
\text { samples precipitated }\end{array}$ \\
\hline$P$ & Raw & $\mathrm{Cl}$ & $2.85 E+06$ & 8.04E-02 & AP102-3 & $\gamma$ & $\begin{array}{l}\text { Density not reported for G522. Density assumed same as } \\
\text { G353 which was collected at } 334 \text { in from bottom of tank at } \\
\text { a } 150^{\circ} \text { angle. This sample was heated. }\end{array}$ \\
\hline$P$ & Raw & $\mathrm{Cl}$ & $2.84 E+06$ & 8.01E-02 & AP102-3 & $\gamma$ & $\begin{array}{l}\text { Density reported for G349 collected at } 34 \text { in from bottom of } \\
\text { tank at a } 30^{\circ} \text { angle. Sample was unheated, unheated } \\
\text { samples precipitated }\end{array}$ \\
\hline $\mathbf{P}$ & Raw & $\mathrm{Cl}$ & $2.84 E+06$ & 8.01E-02 & AP102-3 & $\gamma$ & $\begin{array}{l}\text { Density not reported for G516. Density assumed same as } \\
\text { G359 which was collected at } 162 \text { in from bottom of tank at } \\
\text { a } 150^{\circ} \text { angle. This sample was heated. }\end{array}$ \\
\hline $\mathbf{P}$ & Raw & $\mathrm{Cl}$ & $2.83 E+06$ & 7.98E-02 & AP102-3 & $\gamma$ & $\begin{array}{l}\text { Density reported for } \mathrm{G} 341 \text { collected at } 99 \text { in from bottom of } \\
\text { tank at a } 270^{\circ} \text { angle. }\end{array}$ \\
\hline $\mathbf{P}$ & Raw & $\mathrm{Cl}$ & $2.83 E+06$ & 7.98E-02 & AP102-3 & $Y$ & $\begin{array}{l}\text { Density reported for G359 collected at } 162 \text { in from bottom } \\
\text { of tank at a } 150^{\circ} \text { angle. Sample was unheated, unheated } \\
\text { samples precipitated }\end{array}$ \\
\hline $\mathbf{P}$ & Raw & $\mathrm{Cl}$ & $2.83 E+06$ & 7.98E-02 & AP102-3 & $Y$ & $\begin{array}{l}\text { Density reported for } \mathrm{G} 363 \text { collected at } 18 \text { in from bottom of } \\
\text { tank at a } 150^{\circ} \text { angle. Sample was unheated, unheated } \\
\text { samples precipitated }\end{array}$ \\
\hline$P$ & Raw & $\mathrm{Cl}$ & $2.83 E+06$ & 7.98E-02 & AP102-3 & $\mathbf{Y}$ & $\begin{array}{l}\text { Density reported for } 6459 \text { collected at } 226 \text { in from bottom } \\
\text { of tank at a } 270^{\circ} \text { angle. Sample was unheated, unheated } \\
\text { samples precipitated }\end{array}$ \\
\hline$P$ & Raw & $\mathrm{Cl}$ & $2.82 \mathrm{E}+06$ & 7.95E-02 & AP102-3 & $\gamma$ & $\begin{array}{l}\text { Density reported for } G 358 \text { collected at } 334 \text { in from bottom } \\
\text { of tank at a } 150^{\circ} \text { angle. Sample was unheated, unheated } \\
\text { samples precipitated }\end{array}$ \\
\hline$P$ & Raw & $\mathrm{Cl}$ & $2.82 E+06$ & 7.95E-02 & AP102-3 & $\mathbf{Y}$ & $\begin{array}{l}\text { Density reported for G476 collected at } 99 \text { in from bottom of } \\
\text { tank at a } 270^{\circ} \text { angle and was keep heated to } 40^{\circ} \mathrm{C} \text {. }\end{array}$ \\
\hline$P$ & Raw & $\mathrm{Cl}$ & $2.82 E+06$ & 7.95E-02 & AP102-3 & $\mathbf{Y}$ & $\begin{array}{l}\text { Density not reported for G517. Density assumed same as } \\
\text { G459 which was collected at } 226 \text { in from bottom of tank at } \\
\text { a } 270^{\circ} \text { angle. This sample was heated. }\end{array}$ \\
\hline $\mathbf{P}$ & Raw & $\mathrm{Cl}$ & $2.82 E+06$ & $7.95 E-02$ & AP102-3 & $\mathbf{Y}$ & $\begin{array}{l}\text { Density not reported for G527. No assumptions could be } \\
\text { made }\end{array}$ \\
\hline $\mathbf{P}$ & Raw & $\mathrm{Cl}$ & $2.81 E+06$ & 7.93E-02 & AP102-3 & $Y$ & $\begin{array}{l}\text { Density not reported for G515. Density assumed same as } \\
\text { G358 which was collected at } 334 \text { in from bottom of tank at } \\
\text { a } 150^{\circ} \text { angle. This sample was heated. }\end{array}$ \\
\hline $\mathbf{P}$ & Raw & $\mathrm{Cl}$ & $2.81 E+06$ & 7.93E-02 & AP102-3 & $\mathrm{Y}$ & $\begin{array}{l}\text { Density not reported for G516. Density assumed same as } \\
\text { G359 which was collected at } 162 \text { in from bottom of tank at } \\
\text { a } 150^{\circ} \text { angle. This sample was heated. }\end{array}$ \\
\hline $\mathbf{P}$ & Raw & $\mathrm{Cl}$ & $2.81 E+06$ & 7.93E-02 & AP102-3 & $\mathbf{Y}$ & $\begin{array}{l}\text { Density not reported for } \mathrm{G522} \text {. Density assumed same as } \\
\mathrm{G} 353 \text { which was collected at } 334 \text { in from bottom of tank at } \\
\text { a } 150^{\circ} \text { angle. This sample was heated. }\end{array}$ \\
\hline
\end{tabular}




\begin{tabular}{|c|c|c|c|c|c|c|c|c|c|c|c|}
\hline & & & & & ank Waste & haracteriz & On Sample I & ormation & & & \\
\hline Reported Chemlcal & $\begin{array}{c}\text { Memo } \\
\text { Date }\end{array}$ & $\begin{array}{c}\text { Start } \\
\text { Analysis } \\
\text { Date }\end{array}$ & $\begin{array}{c}\text { Finish } \\
\text { Analysis Date }\end{array}$ & $\begin{array}{c}\text { Start } \\
\text { Sample } \\
\text { Date } \\
\end{array}$ & $\begin{array}{c}\text { Finish } \\
\text { Sample } \\
\text { Date } \\
\end{array}$ & $\begin{array}{l}\text { Sample } \\
\text { Number }\end{array}$ & $\begin{array}{c}\text { Tank Farm } \\
\text { Number }\end{array}$ & $\begin{array}{c}\text { Lab Sample } \\
\text { Number }\end{array}$ & $\begin{array}{l}\text { Density } \\
(\mathrm{g} / \mathrm{mL})\end{array}$ & $\begin{array}{c}\text { Reported } \\
\text { Value } \\
\end{array}$ & $\begin{array}{r}\text { Repil } \\
\mathbf{U}\end{array}$ \\
\hline Chioride & $6 / 1 / 90$ & $4 / 30 / 89$ & $12 / 30 / 89$ & $4 / 27 / 89$ & $4 / 29 / 89$ & BOB2MO & G306 & G521* & $(1.20)$ & $2.81 E+03$ & ugi \\
\hline Chloride & $6 / 1 / 90$ & $4 / 30 / 89$ & $12 / 30 / 89$ & $4 / 27 / 89$ & $4 / 29 / 89$ & BOB2MO & G306 & G352 & 1.20 & $2.80 E+03$ & $u g l$ \\
\hline Chloride & $6 / 1 / 90$ & $4 / 30 / 89$ & $12 / 30 / 89$ & $4 / 27 / 89$ & $4 / 29 / 89$ & BOB2M3 & G311 & G359 & 1.20 & $2.80 E+03$ & $u_{g} g ?$ \\
\hline Chloride & $6 / 1 / 90$ & $4 / 30 / 89$ & $12 / 30 / 89$ & $4 / 27 / 89$ & $4 / 29 / 89$ & BOB2M4 & G313 & G533* & $(1.20)$ & $2.80 E+03$ & $\mathrm{ug} / \mathrm{h}$ \\
\hline Chloride & $6 / 1 / 90$ & $4 / 30 / 89$ & $12 / 30 / 89$ & $4 / 27 / 89$ & $4 / 29 / 89$ & BOB2N1 & G310 & G471* & 1.19 & $2.78 \mathrm{E}+03$ & ug/r \\
\hline Chloride & $6 / 1 / 90$ & $4 / 30 / 89$ & $12 / 30 / 89$ & $4 / 27 / 89$ & $4 / 29 / 89$ & Вов2M3 & G311 & G516* & $(1.20)$ & $2.78 E+03$ & $u g / r$ \\
\hline Chloride & $6 / 1 / 90$ & $4 / 30 / 89$ & $12 / 30 / 89$ & $4 / 27 / 89$ & $4 / 29 / 89$ & ВОВ2M6 & G318 & G517* & $(1.20)$ & $2.78 E+03$ & $u g / n$ \\
\hline Chtoride & $6 / 1 / 90$ & $4 / 30 / 89$ & $12 / 30 / 89$ & $4 / 27 / 89$ & $4 / 29 / 89$ & BOB2MO & G306 & G521* & $(1.20)$ & $2.78 E+03$ & $u g / r$ \\
\hline Chloride & $6 / 1 / 90$ & $4 / 30 / 89$ & $12 / 30 / 89$ & $4 / 27 / 89$ & $4 / 29 / 89$ & ВОВ2M2 & G309 & G358 & 1.20 & $2.78 E+03$ & $u g / r$ \\
\hline Chloride & $6 / 1 / 90$ & $4 / 30 / 89$ & $12 / 30 / 89$ & $4 / 27 / 89$ & $4 / 29 / 89$ & BOB2N4 & G323 & G341 & 1.20 & $2.76 E+03$ & $\mathrm{ug} / \mathrm{m}$ \\
\hline Chloride & $6 / 1 / 90$ & $4 / 30 / 89$ & $12 / 30 / 89$ & $4 / 27 / 89$ & $4 / 29 / 89$ & BOB2L8 & G303 & G348 & 1.21 & $2.75 E+03$ & $u g / m$ \\
\hline Chloride & $6 / 1 / 90$ & $4 / 30 / 89$ & $12 / 30 / 89$ & $4 / 27 / 89$ & $4 / 29 / 89$ & BOB2L9 & G305 & G349 & 1.20 & $2.74 E+03$ & $\mathrm{ug} / \mathrm{m}$ \\
\hline Chloride & $6 / 1 / 90$ & $4 / 30 / 89$ & $12 / 30 / 89$ & $4 / 27 / 89$ & $4 / 29 / 89$ & $\mathrm{BOB2M1}$ & G308 & G353 & 1.20 & $2.74 E+03$ & $\mathrm{ug} / \mathrm{ml}$ \\
\hline Chloride & $6 / 1 / 90$ & $4 / 30 / 89$ & $12 / 30 / 89$ & $4 / 27 / 89$ & $4 / 29 / 89$ & BOB2L9 & G305 & G511* & $(1.20)$ & $2: 74 E+03$ & $\mathrm{ug} / \mathrm{ml}$ \\
\hline Chloride & $6 / 1 / 90$ & $4 / 30 / 89$ & $12 / 30 / 89$ & $4 / 27 / 89$ & $4 / 29 / 89$ & BOB2L7 & G299 & G342 & 1.20 & $2.73 E+03$ & $\mathrm{ug} / \mathrm{ml}$ \\
\hline Chioride & $6 / 1 / 90$ & $4 / 30 / 89$ & $12 / 30 / 89$ & $4 / 27 / 89$ & $4 / 29 / 89$ & BOB2L7 7 & G299 & G509* & $(1.20)$ & $2.73 E+03$ & $u g / \mathrm{mL}$ \\
\hline Chloride & $6 / 1 / 90$ & $4 / 30 / 89$ & $12 / 30 / 89$ & $4 / 27 / 89$ & $4 / 29 / 89$ & BOB2L8 & G303 & G510* & 1.21 & $2.73 E+03$ & $\mathrm{ug} / \mathrm{mL}$ \\
\hline Chloride & $6 / 1 / 90$ & $4 / 30 / 89$ & $12 / 30 / 89$ & $4 / 27 / 89$ & $4 / 29 / 89$ & $80 B 2 M 5$ & G315 & G534* & (1.19) & $2.73 E+03$ & $\mathrm{ug} / \mathrm{mL}$ \\
\hline Chloride & $6 / 1 / 90$ & $4 / 30 / 89$ & $12 / 30 / 89$ & $4 / 27 / 89$ & $4 / 29 / 89$ & BOB2M9 & G302 & 6477* & 1.20 & $2.72 E+03$ & $\mathrm{\omega g} / \mathrm{mL}$ \\
\hline Chloride & $6 / 1 / 90$ & $4 / 30 / 89$ & $12 / 30 / 89$ & $4 / 27 / 89$ & 4/29/89 & BOB2L7 & G299 & G509" & $(1.20)$ & $2.71 E+03$ & $\mathrm{ug} / \mathrm{mL}$ \\
\hline
\end{tabular}




\begin{tabular}{|c|c|c|c|c|c|c|c|}
\hline & & \multicolumn{3}{|c|}{ Analyte information } & \multirow[b]{2}{*}{$\begin{array}{c}\text { Reference } \\
\text { Number }\end{array}$} & \multirow[b]{2}{*}{$\begin{array}{l}\text { Validated } \\
\text { (Y/N) }\end{array}$} & \multirow[b]{2}{*}{ Notes } \\
\hline Code & Source & Analyte & $\begin{array}{l}\text { Conc. } \\
\text { (ug/L) }\end{array}$ & Conc. (mol/L) & & & \\
\hline $\mathbf{P}$ & Raw & $\mathrm{Cl}$ & $2.81 E+06$ & $7.93 E-02$ & AP102-3 & $\mathbf{Y}$ & $\begin{array}{l}\text { Density not reported for G521. Density assumed same as } \\
\text { G352 which was collected at } 34 \text { in from bottom of tank at a } \\
30^{\circ} \text { angle. This sample was heated. }\end{array}$ \\
\hline $\mathbf{P}$ & Raw & $\mathrm{Cl}$ & $2.80 E+06$ & $7.90 E-02$ & AP102-3 & $Y$ & $\begin{array}{l}\text { Density reported for G352 collected at } 34 \text { in from bottom of } \\
\text { tank at a } 30^{\circ} \text { angle. Sample was unheated, unheated } \\
\text { samples precipitated }\end{array}$ \\
\hline $\mathbf{P}$ & Raw & $\mathrm{Cl}$ & $2.80 E+06$ & $7.90 \mathrm{E}-02$ & AP102-3 & $Y$ & $\begin{array}{l}\text { Density reported for G359 collected at } 162 \text { in from bottom } \\
\text { of tank at a } 150^{\circ} \text { angle. Sample was unheated, unheated } \\
\text { samples precipitated }\end{array}$ \\
\hline$P$ & Raw & $\mathrm{Cl}$ & $2.80 E+06$ & $7.90 E-02$ & AP102-3 & $Y$ & $\begin{array}{l}\text { Density not reported for G533. Density assumed same as } \\
\text { G363 which was collected at } 18 \text { in from bottom of tank at a } \\
150^{\circ} \text { angle. This sample was heated. }\end{array}$ \\
\hline $\mathbf{P}$ & Raw & $\mathrm{Cl}$ & $2.78 E+06$ & $7.84 E-02$ & AP102-3 & $Y$ & $\begin{array}{l}\text { Density reported for } 6471 \text { collected at } 208 \text { in from bottom } \\
\text { of tank at a } 150^{\circ} \text { angle and was keep heated to } 40^{\circ} \mathrm{C} \text {. }\end{array}$ \\
\hline $\mathbf{P}$ & Raw & $\mathrm{Cl}$ & $2.78 E+06$ & $7.84 E-02$ & AP102-3 & $\mathbf{Y}$ & $\begin{array}{l}\text { Density not reported for G516. Density assumed same as } \\
\text { G359 which was collected at } 162 \text { in from bottom of tank at } \\
\text { a } 150^{\circ} \text { angle. This sample was heated. }\end{array}$ \\
\hline $\mathbf{P}$ & Raw & $\mathrm{Cl}$ & $2.78 E+06$ & $7.84 E-02$ & AP102-3 & $Y$ & $\begin{array}{l}\text { Density not reported for G517. Density assumed same as } \\
\text { G459 which was collected at } 226 \text { in from bottom of tank at } \\
\text { a } 270^{\circ} \text { angle. This sample was heated. }\end{array}$ \\
\hline $\mathbf{P}$ & Raw & $\mathrm{Cl}$ & $2.78 \mathrm{E}+06$ & $7.84 E-02$ & AP102-3 & $\gamma$ & $\begin{array}{l}\text { Density not reported for G521. Density assumed same as } \\
6352 \text { which was collected at } 34 \text { in from bottom of tank at a } \\
30^{\circ} \text { angle. This sample was heated. }\end{array}$ \\
\hline$P$ & Raw & $\mathrm{CI}$ & $2.78 E+06$ & $7.84 E-02$ & AP102-3 & $Y$ & $\begin{array}{l}\text { Density reported for G358 collected at } 334 \text { in from bottom } \\
\text { of tank at a } 150^{\circ} \text { angle. Sample was unheated, unheated } \\
\text { samples precipitated }\end{array}$ \\
\hline$P$ & Raw & $\mathrm{Cl}$ & $2.76 E+06$ & 7.79E-02 & AP102-3 & $Y$ & $\begin{array}{l}\text { Density reported for G341 collected at } 99 \text { in from bottom of } \\
\text { tank at a } 270^{\circ} \text { angle. }\end{array}$ \\
\hline$P$ & Raw & $\mathrm{Cl}$ & $2.75 E+06$ & 7.76E-02 & AP102-3 & $Y$ & $\begin{array}{l}\text { Density reported for } \mathrm{G} 348 \text { collected at } 125 \text { in from bottom } \\
\text { of tank at a } 30^{\circ} \text { angle. Sample was unheated, unheated } \\
\text { samples precipitated }\end{array}$ \\
\hline$P$ & Raw & $\mathrm{Cl}$ & $2.74 E+06$ & 7.73E-02 & AP102-3 & $Y$ & $\begin{array}{l}\text { Density reported for } \mathrm{G} 349 \text { collected at } 34 \text { in from bottom of } \\
\text { tank at a } 30^{\circ} \text { angle. Sample was unheated, unheated } \\
\text { samples precipitated }\end{array}$ \\
\hline $\mathbf{P}$ & Raw & $\mathrm{Cl}$ & $2.74 E+06$ & 7.73E-02 & AP102-3 & $Y$ & $\begin{array}{l}\text { Density reported for G353 collected at } 334 \text { in from bottom } \\
\text { of tank at a } 150^{\circ} \text { angle. Sample was unheated, unheated } \\
\text { samples precipitated }\end{array}$ \\
\hline 8 & Raw & $\mathrm{Cl}$ & $2.74 E+06$ & 7.73E-02 & AP102-3 & $Y$ & $\begin{array}{l}\text { Density not reported for G511. Density assumed same as } \\
\text { G349 which was collected at } 34 \text { in from bottom of tank at a } \\
30^{\circ} \text { angle. This sample was heated. }\end{array}$ \\
\hline$P$ & Raw & $\mathrm{Cl}$ & $2.73 E+06$ & $7.70 E-02$ & AP102-3 & $Y$ & $\begin{array}{l}\text { Density reported for } \mathrm{G} 342 \text { collected at } 306 \text { in from bottom } \\
\text { of tank at a } 30^{\circ} \text { angle. Sample was unheated, unheated } \\
\text { samples precipitated }\end{array}$ \\
\hline $\mathbf{P}$ & Raw & $\mathrm{Cl}$ & $2.73 E+06$ & 7.70E-02 & AP102-3 & $Y$ & $\begin{array}{l}\text { Density not reported for G509. Density assumed same as } \\
\text { G342 which was collected at } 306 \text { in from bottom of tank at } \\
\text { a } 30^{\circ} \text { angle. This sample was heated. }\end{array}$ \\
\hline$P$ & Raw & Cl & $2.73 E+06$ & 7.70E-02 & AP102-3 & $\gamma$ & $\begin{array}{l}\text { Density not reported for } \mathrm{G} 510 \text {. Density assumed same as } \\
\mathrm{G} 348 \text { which was collected at } 125 \text { in from bottom of tank at } \\
\text { a } 30^{\circ} \text { angle. This sample was heated. }\end{array}$ \\
\hline$P$ & Raw & $\mathrm{Cl}$ & $2.73 E+06$ & 7.70E-02 & AP102-3 & $Y$ & $\begin{array}{l}\text { Density not reported for G534. Density assumed same as } \\
\text { G364 which was collected at } 372 \text { in from bottom of tank at } \\
\text { a } 270^{\circ} \text { angle. This sample was heated. }\end{array}$ \\
\hline$P$ & Raw & $\mathrm{Cl}$ & $2.72 E+06$ & $7.67 E-02$ & AP102-3 & $Y$ & $\begin{array}{l}\text { Density reported for } \mathrm{G} 477 \text { collected at } 182 \text { in from bottom } \\
\text { of tank at a } 30^{\circ} \text { angle and was keep heated to } 40^{\circ} \mathrm{C} \text {. }\end{array}$ \\
\hline $\mathbf{P}$ & Raw & $\mathrm{Cl}$ & $2.71 E+06$ & 7.64E-02 & AP102-3 & $Y$ & $\begin{array}{l}\text { Density not reported for G509. Density assumed same as } \\
6342 \text { which was collected at } 306 \text { in from bottom of tank at } \\
\text { a } 30^{\circ} \text { angle. This sample was heated. }\end{array}$ \\
\hline
\end{tabular}




\begin{tabular}{|c|c|c|c|c|c|c|c|c|c|c|c|}
\hline & & & & & ank Waste & haracteriza & on Sample Is & ormation & & & \\
\hline Reported Chemical & $\begin{array}{c}\text { Memo } \\
\text { Date }\end{array}$ & $\begin{array}{c}\text { Start } \\
\text { Analysis } \\
\text { Date } \\
\end{array}$ & $\begin{array}{c}\text { Finish } \\
\text { Anzlysis Date }\end{array}$ & $\begin{array}{c}\text { Start } \\
\text { Sample } \\
\text { Date } \\
\end{array}$ & $\begin{array}{c}\text { Finish } \\
\text { Sample } \\
\text { Date } \\
\end{array}$ & $\begin{array}{l}\text { Sample } \\
\text { Number }\end{array}$ & $\begin{array}{c}\text { Tank Farm } \\
\text { Number }\end{array}$ & $\begin{array}{c}\text { Lab Sampie } \\
\text { Number }\end{array}$ & $\begin{array}{l}\text { Density } \\
(\mathrm{g} / \mathrm{mL})\end{array}$ & $\begin{array}{c}\text { Reported } \\
\text { Value }\end{array}$ & $\begin{array}{r}\text { Repo } \\
\text { Ur } \\
\end{array}$ \\
\hline Chloride & $6 / 1 / 90$ & $4 / 30 / 89$ & $12 / 30 / 89$ & $4 / 27 / 89$ & $4 / 29 / 89$ & BOB2M4 & G313 & G533* & $(1.20)$ & $2.70 E+03$ & ug/h \\
\hline Chloride & $6 / 1 / 90$ & $4 / 30 / 89$ & $12 / 30 / 89$ & $4 / 27 / 89$ & $4 / 29 / 89$ & BOB2L9 & G305 & G511* & $(1.20)$ & $2.69 E+03$ & ug/r \\
\hline Chloride & $6 / 1 / 90$ & $4 / 30 / 89$ & $12 / 30 / 89$ & $4 / 27 / 89$ & $4 / 29 / 89$ & BOB2M7 & G319 & G536* & $(1.20)$ & $2.69 E+03$ & $u g / r$ \\
\hline Chloride & $6 / 1 / 90$ & $4 / 30 / 89$ & $12 / 30 / 89$ & $4 / 27 / 89$ & $4 / 29 / 89$ & BOB2M5 & G315 & G534* & $(1.19)$ & $2.68 E+03$ & ug/r \\
\hline Chloride & $6 / 1 / 90$ & $4 / 30 / 89$ & $12 / 30 / 89$ & $4 / 27 / 89$ & $4 / 29 / 89$ & ВОВ2M9 & G302 & G477* & 1.20 & $2.68 E+03$ & $u g / n$ \\
\hline Chloride & $6 / 1 / 90$ & $4 / 30 / 89$ & $12 / 30 / 89$ & $4 / 27 / 89$ & $4 / 29 / 89$ & BOB2L8 & G303 & G348 & 1.21 & $2.67 E+03$ & $\mathbf{u g} / \mathbf{n}$ \\
\hline Chloride & $6 / 1 / 90$ & $4 / 30 / 89$ & $12 / 30 / 89$ & $4 / 27 / 89$ & $4 / 29 / 89$ & BOB2M7 & G319 & G536* & $(1.20)$ & $2.67 E+03$ & $u g / n$ \\
\hline Chloride & $6 / 1 / 90$ & $4 / 30 / 89$ & $12 / 30 / 89$ & $4 / 27 / 89$ & $4 / 29 / 89$ & BOB2N3 & G322 & G529* & NA & $2.66 E+03$ & $u g / \pi$ \\
\hline Chloride & $6 / 1 / 90$ & $4 / 30 / 89$ & $12 / 30 / 89$ & $4 / 27 / 89$ & $4 / 29 / 89$ & ВОВ2M8 & G301 & G523* & NA & $2.66 E+03$ & $\mathrm{ug} / \mathrm{r}$ \\
\hline Chlorlde & $6 / 1 / 90$ & $4 / 30 / 89$ & $12 / 30 / 89$ & $4 / 27 / 89$ & $4 / 29 / 89$ & BOB2M2 & G309 & G515* & $(1.20)$ & $2.65 E+03$ & ug/m \\
\hline Chloride & $6 / 1 / 90$ & $4 / 30 / 89$ & $12 / 30 / 89$ & $4 / 27 / 89$ & $4 / 29 / 89$ & $8082 \mathrm{M9}$ & G302 & G477* & 1.20 & $2.63 E+03$ & $u g / m$ \\
\hline Chloride & $6 / 1 / 90$ & $4 / 30 / 89$ & $12 / 30 / 89$ & $4 / 27 / 89$ & $4 / 29 / 89$ & BOB2M8 & G301 & G523* & NA & $2.61 E+03$ & $u g / m$ \\
\hline Chloride & $6 / 1 / 90$ & $4 / 30 / 89$ & $12 / 30 / 89$ & $4 / 27 / 89$ & $4 / 29 / 89$ & BOB2N3 & G322 & G529* & NA & $2.58 E+03$ & $\mathrm{ug} / \mathrm{m}$ \\
\hline Chloride & $6 / 1 / 90$ & $4 / 30 / 89$ & $12 / 30 / 89$ & $4 / 27 / 89$ & $4 / 29 / 89$ & BOB2N2 & G317 & G528* & NA & $2.55 E+03$ & $\mathrm{ug} / \mathrm{m}$ \\
\hline Chloride & $6 / 1 / 90$ & $4 / 30 / 89$ & $12 / 30 / 89$ & $4 / 27 / 89$ & $4 / 29 / 89$ & BOB2L8 & G303 & G510* & 1.21 & $2.54 E+03$ & $\mathrm{ug} / \mathrm{ml}$ \\
\hline Chloride & $6 / 1 / 90$ & $4 / 30 / 89$ & $12 / 30 / 89$ & $4 / 27 / 89$ & $4 / 29 / 89$ & BOB2N2 & G317 & G528* & NA & $2.42 E+03$ & $\mathrm{ug} / \mathrm{ml}$ \\
\hline Chloride & $6 / 1 / 90$ & $4 / 30 / 89$ & $12 / 30 / 89$ & $4 / 27 / 89$ & $4 / 29 / 89$ & ВОВ2M3 & G311 & G516* & $(1.20)$ & $2.39 E+03$ & $\mathrm{ug} / \mathrm{ml}$ \\
\hline Chloride & $6 / 1 / 90$ & $4 / 30 / 89$ & $12 / 30 / 89$ & $4 / 27 / 89$ & $4 / 29 / 89$ & BOB2NO & G307 & G527* & NA & $2.36 E+03$ & ug $/ \mathrm{ml}$ \\
\hline Cyanide & $4 / 1 / 93$ & NS & NS & NS & NS & $80 B 2 T 9$ & G327 & NS & 1.19 & $2.46 E+01$ & $\mathrm{ug} / \mathrm{ml}$ \\
\hline Cyanide & $4 / 1 / 93$ & NS & NS & NS & NS & BOB2T9 & G327 & NS & 1.2 & $2.46 E+01$ & $\mathrm{ug} / \mathrm{ml}$ \\
\hline Cyanide & $4 / 1 / 93$ & NS & NS & NS & NS & BOB2T9 & G327 & NS & 1.2 & $2.46 E+01$ & $\mathrm{ug} / \mathrm{ml}$ \\
\hline Cyanide & $4 / 1 / 93$ & NS & NS & NS & NS & BOB2N1 & G310 & NS & 1.2 & $2.44 E+01$ & ug/mL \\
\hline Cyanide & $4 / 1 / 93$ & $4 / 30 / 89$ & $11 / 30 / 89$ & $4 / 27 / 89$ & $4 / 29 / 89$ & BOB2N4 & G323 & G341 & 1.20 & $2.41 E+01$ & $\mathrm{ug} / \mathrm{mL}$ \\
\hline Cyanide & $4 / 1 / 93$ & NS & NS & NS & NS & $\mathrm{BOB2M9}$ & G302 & NS & 1.2 & $2.32 E+01$ & $\mathrm{ug} / \mathrm{mL}$ \\
\hline Cyanide & $4 / 1 / 93$ & NS & NS & NS & NS & ВОВ2T9 & G327 & NS & 1.19 & $2.32 \mathrm{E}+01$ & $\mathrm{ug} / \mathrm{mL}$ \\
\hline Cyanide & $4 / 1 / 93$ & $4 / 30 / 89$ & $11 / 30 / 89$ & $4 / 27 / 89$ & $4 / 29 / 89$ & BOB2N4 & G323 & G341 & 1.20 & $2.32 E+01$ & $\mathrm{ug} / \mathrm{mL}$ \\
\hline Cyanide & $4 / 1 / 93$ & NS & NS & NS & NS & $\mathrm{BOB2N1}$ & G310 & NS & 1.2 & $2.30 E+01$ & $\mathrm{ug} / \mathrm{mL}$ \\
\hline Cyanide & $4 / 1 / 93$ & NS & NS & NS & NS & BOB2M9 & $\mathrm{G} 302$ & NS & 1.2 & $2.26 \mathrm{E}+01$ & $\mathrm{ug} / \mathrm{mL}$ \\
\hline $\mathrm{CN}$ & $6 / 1 / 90$ & $4 / 30 / 89$ & $12 / 30 / 89$ & $4 / 27 / 89$ & $4 / 29 / 89$ & BOB2T9 & G327 & G443 & 1.20 & $2.46 E+01$ & $\mathrm{ug} / \mathrm{mL}$ \\
\hline
\end{tabular}




\begin{tabular}{|c|c|c|c|c|c|c|c|}
\hline & & & nalyte Inform & ation & & & \\
\hline Code & Source & Analyte & $\begin{array}{l}\text { Conc. } \\
\text { (ug/L) }\end{array}$ & Conc. (molll) & $\begin{array}{c}\text { Reference } \\
\text { Number }\end{array}$ & $\begin{array}{c}\text { Validated } \\
\text { (Y/N) }\end{array}$ & Notes \\
\hline $\mathbf{P}$ & Raw & $\mathrm{Cl}$ & $2.70 \varepsilon+06$ & $7.62 E-02$ & AP102-3 & $Y$ & $\begin{array}{l}\text { Density not reported for G533. Density assumed same as } \\
\text { G363 which was collected at } 18 \text { in from bottom of tank at a } \\
150^{\circ} \text { angle. This sample was heated. }\end{array}$ \\
\hline $\mathbf{P}$ & Raw & $\mathrm{Cl}$ & $2.69 E+06$ & 7.59E-02 & AP102-3 & $\gamma$ & $\begin{array}{l}\text { Density not reported for G511. Density assumed same as } \\
\mathrm{G} 349 \text { which was collected at } 34 \text { in from bottom of tank at.a } \\
30^{\circ} \text { angle. This sample was heated. }\end{array}$ \\
\hline $\mathbf{P}$ & Raw & $\mathrm{Cl}$ & $2.69 E+06$ & 7.59E-02 & $\because A P 102-3$ & $Y$ & $\begin{array}{l}\text { Density not reported for G536. Density assumed same as } \\
\text { G460 which was collected at } 226 \text { in from bottom of tank at } \\
\text { a } 270^{\circ} \text { angle. This sample was heated. }\end{array}$ \\
\hline $\mathbf{P}$ & Raw & $\mathrm{Cl}$ & $2.68 E+06$ & $7.56 \mathrm{E}-02$ & AP102-3 & $Y$ & $\begin{array}{l}\text { Density not reported for G534. Density assumed same as } \\
\text { G364 which was collected at } 372 \text { in from bottom of tank at } \\
\text { a } 270^{\circ} \text { angle. This sample was heated. }\end{array}$ \\
\hline $\mathbf{P}$ & Raw & $\mathrm{Cl}$ & $2.68 E+06$ & $7.56 E-02$ & AP102-3 & $Y$ & $\begin{array}{l}\text { Density reported for } \mathrm{G} 477 \text { collected at } 182 \text { in from bottom } \\
\text { of tank at a } 30^{\circ} \text { angle and was keep heated to } 40^{\circ} \mathrm{C} \text {. }\end{array}$ \\
\hline $\mathbf{P}$ & Raw & $\mathrm{Cl}$ & $2.67 E+06$ & $7.53 E-02$ & AP102-3 & $Y$ & $\begin{array}{l}\text { Density reported for G348 collected at } 125 \text { in from bottom } \\
\text { of tank at a } 30^{\circ} \text { angle. Sample was unheated, unheated } \\
\text { samples precipitated }\end{array}$ \\
\hline $\mathbf{P}$ & Raw & $\mathrm{Cl}$ & $2.67 E+06$ & 7.53E-02 & AP102-3 & $\mathbf{Y}$ & $\begin{array}{l}\text { Density not reported for G536. Density assumed same as } \\
\text { G460 which was collected at } 226 \text { in from bottom of tank at } \\
\text { a } 270^{\circ} \text { angle. This sample was heated. }\end{array}$ \\
\hline $\mathbf{P}$ & Raw & $\mathrm{Cl}$ & $2.66 E+06$ & $7.50 E-02$ & AP102-3 & $Y$ & $\begin{array}{l}\text { Density not reported for } 6529 \text {. No assumptions could be } \\
\text { made }\end{array}$ \\
\hline $\mathbf{P}$ & Raw & $\mathrm{Cl}$ & $2.66 \varepsilon+06$ & 7.50E-02 & AP102-3 & $Y$ & $\begin{array}{l}\text { Density not reported for G523. No assumptions could be } \\
\text { made }\end{array}$ \\
\hline $\mathbf{P}$ & Raw & $\mathrm{Cl}$ & $2.65 E+06$ & $7.48 E-02$ & AP102-3 & $Y$ & $\begin{array}{l}\text { Density not reported for G515. Density assumed same as } \\
\text { G358 which was collected at } 334 \text { in from bottom of tank at } \\
\text { a } 150^{\circ} \text { angle. This sample was heated. }\end{array}$ \\
\hline $\mathbf{P}$ & Raw & $\mathrm{Cl}$ & $2.63 E+06$ & 7.42E-02 & AP102-3 & $Y$ & $\begin{array}{l}\text { Density reported for } \mathrm{G} 477 \text { collected at } 182 \text { in from bottom } \\
\text { of tank at a } 30^{\circ} \text { angle and was keep heated to } 40^{\circ} \mathrm{C} \text {. }\end{array}$ \\
\hline $\mathbf{P}$ & Raw & $\mathrm{Cl}$ & $2.61 E+06$ & 7.36E-02 & AP102-3 & $Y$ & $\begin{array}{l}\text { Density not reported for G523. No assumptions could be } \\
\text { made }\end{array}$ \\
\hline $\mathbf{P}$ & Raw & $\mathrm{Cl}$ & $2.58 E+06$ & $7.28 \mathrm{E}-02$ & AP102-3 & $Y$ & $\begin{array}{l}\text { Density not reported for G529. No assumptions could be } \\
\text { made }\end{array}$ \\
\hline$P$ & Raw & $\mathrm{Cl}$ & $2.55 E+06$ & 7.19E-02 & AP102-3 & $\gamma$ & $\begin{array}{l}\text { Density not reported for G528. No assumptions could be } \\
\text { made }\end{array}$ \\
\hline $\mathbf{P}$ & Raw & $\mathrm{Cl}$ & $2.54 E+06$ & 7.17E-02 & AP102-3 & $\gamma$ & $\begin{array}{l}\text { Density not reported for G510. Density assumed same as } \\
\text { G348 which was collected at } 125 \text { in from bottom of tank at } \\
\text { a } 30^{\circ} \text { angle. This sample was heated. }\end{array}$ \\
\hline$P$ & Raw & $\mathrm{Cl}$ & $2.42 E+06$ & 6.83E-02 & AP102-3 & $Y$ & $\begin{array}{l}\text { Density not reported for G528. No assumptions could be } \\
\text { made }\end{array}$ \\
\hline$P$ & Raw & $\mathrm{Cl}$ & $2.39 E+05$ & $6.74 E-02$ & AP102-3 & $Y$ & $\begin{array}{l}\text { Density not reported for G516. Density assumed same as } \\
\text { G359 which was collected at } 162 \text { in from bottom of tank at } \\
\text { a } 150^{\circ} \text { angle. This sample was heated. }\end{array}$ \\
\hline $\mathbf{P}$ & Raw & $\mathrm{Cl}$ & $2.36 E+06$ & $6.66 \mathrm{E}-02$ & AP102-3 & $Y$ & $\begin{array}{l}\text { Density not reported for G527. No assumptions could be } \\
\text { made }\end{array}$ \\
\hline$P$ & $\overline{T C D}$ & $\mathrm{CN}$ & $2.46 E+10$ & $9.46 E+02$ & NS & $N$ & THIS POINT NOT NEEDED FOR DATA BASE \\
\hline $\mathbf{P}$ & TCD & $\mathrm{CN}$ & $2.46 \mathrm{E}+10$ & $9.46 \mathrm{E}+02$ & NS & $\mathbf{N}$ & THIS POINT NOT NEEDED FOR DATA BASE \\
\hline $\mathbf{P}$ & TCD & $\overline{C N}$ & $2.46 E+10$ & $9.46 E+02$ & NS & $\mathbf{N}$ & THIS POINT NOT NEEDED FOR DATA BASE \\
\hline $\mathbf{P}$ & TCD & $\mathrm{CN}$ & $2.44 E+10$ & $9.38 E+02$ & NS & $\mathbf{N}$ & THIS POINT NOT NEEOED FOR DATA BASE \\
\hline $\mathbf{P}$ & TCD & $\mathrm{CN}$ & $2.41 E+10$ & $9.27 E+02$ & AP102-44 & $N$ & THIS POINT NOT NEEDED FOR DATA BASE \\
\hline $\mathbf{P}$ & TCD & $\mathrm{CN}$ & $2.32 E+10$ & $8.92 E+02$ & NS & $\mathbf{N}$ & THIS POINT NOT NEEDED FOR DATA BASE \\
\hline$P$ & TCD & $\mathrm{CN}$ & $2.32 E+10$ & $8.92 E+02$ & NS & $N$ & THIS POINT NOT NEEDED FOR DATA BASE \\
\hline$P$ & TCD & $\mathrm{CN}$ & $2.32 E+10$ & $8.92 E+02$ & AP102-44 & $N$ & THIS POINT NOT NEEDED FOR DATA BASE \\
\hline $\mathbf{P}$ & TCD & $\mathrm{CN}$ & $2.30 E+10$ & $8.85 E+02$ & NS & $N$ & THIS POINT NOT NEEDED FOR DATA BASE \\
\hline $\bar{P}$ & TCD & $\mathrm{CN}$ & $2.26 E+10$ & $8.69 E+02$ & NS & $N$ & TTHIS POINT NOT NEEDED FOR DATA BASE \\
\hline $\mathbf{p}$ & Raw & $\mathrm{CN}$ & $2.46 E+04$ & $9.46 E-04$ & AP102-3 & $\mathbf{Y}$ & Density reported for $\mathrm{G} 443$ represents a composite \\
\hline
\end{tabular}




\begin{tabular}{|c|c|c|c|c|c|c|c|c|c|c|c|}
\hline & & & & & ank Waste & haracteriza & ion Sample In & ormation & & & \\
\hline Reported Chemical & $\begin{array}{c}\text { Memo } \\
\text { Date }\end{array}$ & $\begin{array}{c}\text { Start } \\
\text { Analysis } \\
\text { Date }\end{array}$ & $\begin{array}{c}\text { Finish } \\
\text { Analysis Date }\end{array}$ & $\begin{array}{c}\text { Start } \\
\text { Sample } \\
\text { Date }\end{array}$ & $\begin{array}{c}\text { Finish } \\
\text { Sample } \\
\text { Date }\end{array}$ & $\begin{array}{c}\text { Sample } \\
\text { Number }\end{array}$ & $\begin{array}{c}\text { Tank Farm } \\
\text { Number }\end{array}$ & $\begin{array}{c}\text { Lab Sample } \\
\text { Number }\end{array}$ & $\begin{array}{l}\text { Density } \\
(\mathrm{g} / \mathrm{mL})\end{array}$ & $\begin{array}{c}\text { Reported } \\
\text { Value } \\
\end{array}$ & $\begin{array}{r}\text { Repe } \\
\mathrm{U}_{t} \\
\end{array}$ \\
\hline $\mathrm{CN}$ & $6 / 1 / 90$ & $4 / 30 / 89$ & $12 / 30 / 89$ & $4 / 27 / 89$ & $4 / 29 / 89$ & 8082T9 & G327 & G443 & 1.20 & $2.46 E+01$ & ugl \\
\hline $\mathrm{CN}$ & $6 / 1 / 90$ & $4 / 30 / 89$ & $12 / 30 / 89$ & $4 / 27 / 89$ & $4 / 29 / 89$ & BOB2N1 & G310 & G471* & 1.19 & $2.44 E+01$ & ug $/$ \\
\hline $\mathrm{CN}$ & $6 / 1 / 90$ & $4 / 30 / 89$ & $12 / 30 / 89$ & $4 / 27 / 89$ & $4 / 29 / 89$ & BOB2N4 & G323 & G476* & 1.21 & $2.41 E+01$ & $\mathrm{ug} h$ \\
\hline $\mathrm{CN}$ & $6 / 1 / 90$ & $4 / 30 / 89$ & $12 / 30 / 89$ & $4 / 27 / 89$ & $4 / 29 / 89$ & ВОВ2Т9 & G327 & G470* & 1.19 & $2.34 E+01$ & $u g / 1$ \\
\hline $\mathrm{CN}$ & $6 / 1 / 90$ & $4 / 30 / 89$ & $12 / 30 / 89$ & $4 / 27 / 89$ & $4 / 29 / 89$ & ВОВ2T9 & G327 & G470* & 1.19 & $2.32 E+01$ & $u g / t$ \\
\hline CN & $6 / 1 / 90$ & $4 / 30 / 89$ & $12 / 30 / 89$ & $4 / 27 / 89$ & $4 / 29 / 89$ & BOB2M9 & G302 & G477• & 1.20 & $2.32 E+01$ & $u g / r$ \\
\hline $\mathrm{CN}$ & $6 / 1 / 90$ & $4 / 30 / 89$ & $12 / 30 / 89$ & $4 / 27 / 89$ & $4 / 29 / 89$ & BOB2N4 & G323 & G476 & 1.21 & $2.32 E+01$ & $u g / \mathbf{r}$ \\
\hline CN & $6 / 1 / 90$ & $4 / 30 / 89$ & $12 / 30 / 89$ & $4 / 27 / 89$ & $4 / 29 / 89$ & BOB2N1 & G310 & G471" & 1.19 & $2.30 E+01$ & ug/n \\
\hline $\mathrm{CN}$ & $6 / 1 / 90$ & $4 / 30 / 89$ & $12 / 30 / 89$ & $4 / 27 / 89$ & $4 / 29 / 89$ & вОВ2М9 & $\mathrm{G} 302$ & G477* & 1.20 & $2.26 E+01$ & $u g / n$ \\
\hline Chromium & $6 / 1 / 90$ & $4 / 30 / 89$ & $12 / 30 / 89$ & $4 / 27 / 89$ & $4 / 29 / 89$ & ВОВ2Т9 & G327 & G470* & 1.19 & $7.16 E+05$ & ug/ $/$ \\
\hline Chromium & $6 / 1 / 90$ & $4 / 30 / 89$ & $12 / 30 / 89$ & $4 / 27 / 89$ & $4 / 29 / 89$ & вов2Т9 & G327 & G470. & 1.19 & $7.08 E+05$ & ugh \\
\hline Chromium & $6 / 1 / 90$ & $4 / 30 / 89$ & $12 / 30 / 89$ & $4 / 27 / 89$ & $4 / 29 / 89$ & BOB2L9 & G305 & G349 & 1.20 & $6.55 E+05$ & ug $/$ \\
\hline Chromium & $6 / 1 / 90$ & $4 / 30 / 89$ & $12 / 30 / 89$ & $4 / 27 / 89$ & $4 / 29 / 89$ & BOB2L.9 & G305 & G349 & 1.20 & $6.46 E+05$ & - ugh \\
\hline Chromium & $6 / 1 / 90$ & $4 / 30 / 89$ & $12 / 30 / 89$ & $4 / 27 / 89$ & $4 / 29 / 89$ & BOB2NO & G307 & G453 & NA & $6.38 E+05$ & $u g / h$ \\
\hline Chromium & $6 / 1 / 90$ & $4 / 30 / 89$ & $12 / 30 / 89$ & $4 / 27 / 89$ & $4 / 29 / 89$ & BOB2L8 & G303 & G348 & 1.21 & $6.37 E+05$ & ug/L \\
\hline Chromium & $6 / 1 / 90$ & $4 / 30 / 89$ & $12 / 30 / 89$ & $4 / 27 / 89$ & $4 / 29 / 89$ & BOB2M6 & G318 & G459 & 1.20 & $6.35 E+05$ & $u g / h$ \\
\hline Chromium & $6 / 1 / 90$ & 4/30/89 & $12 / 30 / 89$ & $4 / 27 / 89$ & $4 / 29 / 89$ & BOB2L8 & G303 & G348 & 1.21 & $6.34 E+05$ & ug/L \\
\hline Chromium & $6 / 1 / 90^{*}$ & $4 / 30 / 89^{\circ}$ & $12 / 30 / 89$ & $4 / 27 / 89$ & $4 / 29 / 89$ & BOB2N2 & G317 & G333 & NA & $6.32 E+05$ & ug $\Omega$ \\
\hline Chromium & $6 / 1 / 90$ & $4 / 30 / 89$ & $12 / 30 / 89$ & $4 / 27 / 89$ & $4 / 29 / 89$ & ВОВ2M5 & G315 & G364 & 1.19 & $6.31 E+05$ & $u g / L$ \\
\hline Chromium & $6 / 1 / 90$ & $4 / 30 / 89$ & $12 / 30 / 89$ & $4 / 27 / 89$ & $4 / 29 / 89$ & в082T9 & G327 & G443 & 1.20 & $6.31 E+05$ & ugh \\
\hline Chromium & $6 / 1 / 90$ & 4/30/89 & $12 / 30 / 89$ & $4 / 27 / 89$ & $4 / 29 / 89$ & BOB2N3 & G322 & G338 & NA & $6.29 E+05$ & ug/L \\
\hline Chromium & $6 / 1 / 90$ & $4 / 30 / 89$ & $12 / 30 / 89$ & $4 / 27 / 89$ & $4 / 29 / 89$ & BOB2M7 & G319 & G460 & 1.20 & $6.28 E+05$ & Ug/L \\
\hline
\end{tabular}




\begin{tabular}{|c|c|c|c|c|c|c|c|}
\hline \multirow[b]{2}{*}{ Code } & \multirow[b]{2}{*}{ Source } & \multirow[b]{2}{*}{ Analyte } & \multicolumn{2}{|c|}{ Analyte Information } & \multirow[b]{2}{*}{$\begin{array}{c}\text { Reference } \\
\text { Number }\end{array}$} & \multirow[b]{2}{*}{$\begin{array}{l}\text { Validated } \\
(Y / N)\end{array}$} & \multirow[b]{2}{*}{ Notes } \\
\hline & & & $\begin{array}{l}\text { Conc. } \\
(\text { ug/L) }\end{array}$ & Conc. (mol/L) & & & \\
\hline $\mathbf{P}$ & Raw & $\mathrm{CN}$ & $2.46 \mathrm{E}+04$ & $9.46 \mathrm{E}-04$ & AP102-3 & $\mathbf{Y}$ & Density reported for $\mathrm{G} 443$ represents a composite sample \\
\hline $\mathbf{P}$ & Raw & $\mathrm{CN}$ & $2.44 E+04$ & $9.38 E-04$ & AP102-3 & $\mathbf{Y}$ & $\begin{array}{l}\text { Density reported for G471 collected at } 208 \text { in from bottom } \\
\text { of tank at a } 150^{\circ} \text { angle and was keep heated to } 40^{\circ} \mathrm{C} \text {. }\end{array}$ \\
\hline$P$ & Raw & $\mathrm{CN}$ & $2.41 E+04$ & $9.27 E-04$ & AP102-3 & $\mathbf{Y}$ & $\begin{array}{l}\text { Density reported for } \mathrm{G} 476 \text { collected at } 99 \text { in from bottom of } \\
\text { tank at a } 270^{\circ} \text { angle and was keep heated to } 40^{\circ} \mathrm{C} \text {. }\end{array}$ \\
\hline$P$ & Raw & CN & $2.34 E+04$ & 9.00E-04 & AP102-3 & $\mathbf{Y}$ & $\begin{array}{l}\text { Density reported for } \mathrm{G} 470 \text { represents a composite sample. } \\
\text { Sample was kept heated to } 40^{\circ} \mathrm{C} \text {. }\end{array}$ \\
\hline$P$ & Raw & $\mathrm{CN}$ & $2.32 E+04$ & 8.92E-04 & AP102-3 & $Y$ & $\begin{array}{l}\text { Density reported for } \mathrm{G} 470 \text { represents a composite sample. } \\
\text { Sample was kept heated to } 40^{\circ} \mathrm{C} \text {. }\end{array}$ \\
\hline$P$ & Raw & $\mathrm{CN}$ & $2.32 E+04$ & 8.92E-04 & AP102-3 & $Y$ & $\begin{array}{l}\text { Density reported for G477 collected at } 182 \text { in from bottom } \\
\text { of tank at a } 30^{\circ} \text { angle and was keep heated to } 40^{\circ} \mathrm{C} \text {. }\end{array}$ \\
\hline$P$ & Raw & $\mathrm{CN}$ & $2.32 E+04$ & 8.92E-04 & AP102-3 & $Y$ & $\begin{array}{l}\text { Density reported for } G 476 \text { collected at } 99 \text { in from bottom of } \\
\text { tank at a } 270^{\circ} \text { angle and was keep heated to } 40^{\circ} \mathrm{C} \text {. }\end{array}$ \\
\hline$P$ & Raw & CN & $2.30 E+04$ & 8.85E-04 & AP102-3 & $Y$ & $\begin{array}{l}\text { Density reported for G471 collected at } 208 \text { in from bottom } \\
\text { of tank at a } 150^{\circ} \text { angle and was keep heated to } 40^{\circ} \mathrm{C} \text {. }\end{array}$ \\
\hline$P$ & Raw & CN & $2.26 E+04$ & 8.69E-04 & AP102-3 & $Y$ & $\begin{array}{l}\text { Density reported for } \mathrm{G} 477 \text { collected at } 182 \text { in from bottom } \\
\text { of tank at a } 30^{\circ} \text { angle and was keep heated to } 40^{\circ} \mathrm{C} \text {. }\end{array}$ \\
\hline$P$ & Raw & $\mathrm{Cr}$ & $7.16 E+05$ & 1.38E-02 & AP102-3 & $Y$ & $\begin{array}{l}\text { Density reported for G470 represents a composite sample. } \\
\text { Sample was kept heated to } 40^{\circ} \mathrm{C} \text {. }\end{array}$ \\
\hline$P$ & Raw & $\mathrm{Cr}$ & $7.08 E+05$ & 1.36E-02 & AP102-3 & $Y$ & $\begin{array}{l}\text { Density reported for G470 represents a composite sample. } \\
\text { Sample was kept heated to } 40^{\circ} \mathrm{C} \text {. }\end{array}$ \\
\hline$P$ & Raw & $\mathrm{Cr}$ & $6.55 E+05$ & $1.26 E-02$ & AP102-3 & $\gamma$ & $\begin{array}{l}\text { Density reported for G349 collected at } 34 \text { in from bottom of } \\
\text { tank at a } 30^{\circ} \text { angle. Sample was unheated, unheated } \\
\text { samples precipitated }\end{array}$ \\
\hline$P$ & Raw & . $\mathrm{Cr}$ & $6.46 E+05$ & $1.24 E-02$ & AP102-3 & $Y$ & $\begin{array}{l}\text { Density reported for G349 collected at } 34 \text { in from bottom of } \\
\text { tank at a } 30^{\circ} \text { angle. Sample was unheated, unheated } \\
\text { samples precipitated }\end{array}$ \\
\hline$P$ & Raw & $\mathrm{Cr}$ & $6.38 E+05$ & $1.23 E-02$ & AP102-3 & $Y$ & $\begin{array}{l}\text { Density not reported for G453. No assumptions could be } \\
\text { made }\end{array}$ \\
\hline$P$ & Raw & $\mathrm{Cr}$ & $6.37 E+05$ & $1.23 E-02$ & AP102-3 & $Y$ & $\begin{array}{l}\text { Density reported for } \mathrm{G} 348 \text { collected at } 125 \text { in from bottom } \\
\text { of tank at a } 30^{\circ} \text { angle. Sample was unheated, unheated } \\
\text { samples precipitated }\end{array}$ \\
\hline$P$ & Raw & $\mathrm{Cr}$ & $6.35 E+05$ & 1.22E-02 & AP102-3 & $Y$ & $\begin{array}{l}\text { Density reported for G459 collected at } 226 \text { in from bottom } \\
\text { of tank at a } 270^{\circ} \text { angle. Sample was unheated, unheated } \\
\text { samples precipitated }\end{array}$ \\
\hline$P$ & Raw & $\mathrm{Cr}$ & $6.34 E+05$ & $1.22 E-02$ & AP102-3 & $Y$ & $\begin{array}{l}\text { Density reported for } G 348 \text { collected at } 125 \text { in from bottom } \\
\text { of tank at a } 30^{\circ} \text { angle. Sample was unheated, unheated } \\
\text { samples precipitated }\end{array}$ \\
\hline$P$ & Raw & $\mathrm{Cr}$ & $6.32 E+05$ & $1.22 E-02$ & AP102-3 & $Y$ & $\begin{array}{l}\text { Density not reported for G333. No assumptions could be } \\
\text { made }\end{array}$ \\
\hline $\mathbf{P}$ & Raw & $\mathrm{Cr}$ & $6.31 E+05$ & $1.21 E-02$ & AP102-3 & $Y$ & $\begin{array}{l}\text { Density reported for G364 collected at } 372 \text { in from bottom } \\
\text { of tank at a } 270^{\circ} \text { angle. Sample was unheated, unheated } \\
\text { samples precipitated }\end{array}$ \\
\hline$P$ & Raw & $\mathrm{Cr}$ & $6.31 E+05$ & $1.21 E-02$ & AP102-3 & $Y$ & Density reported for 6443 represents a composite sample \\
\hline $\mathbf{P}$ & Raw & $\mathbf{C r}$ & $6.29 E+05$ & $1.21 \mathrm{E}-02$ & AP102-3 & Y & $\begin{array}{l}\text { Density not reported for G338. No assumptions could be, } \\
\text { made }\end{array}$ \\
\hline $\mathbf{P}$ & Raw & $\mathrm{Cr}$ & $6.28 E+05$ & $1.21 E-02$ & AP102-3 & Y & $\begin{array}{l}\text { Density reported for G460 collected at } 226 \text { in from bottom } \\
\text { of tank at a } 270^{\circ} \text { angle. Sample was unheated, unheated } \\
\text { samples precipitated }\end{array}$ \\
\hline
\end{tabular}




\begin{tabular}{|c|c|c|c|c|c|c|c|c|c|c|c|}
\hline & & & & & ank Waste & haracteriza & Ion Sample II & ormation & & & \\
\hline Reported Chemical & $\begin{array}{c}\text { Memo } \\
\text { Date }\end{array}$ & $\begin{array}{c}\text { Start } \\
\text { Analysis } \\
\text { Date }\end{array}$ & $\begin{array}{c}\text { Finish } \\
\text { Analysis Date }\end{array}$ & $\begin{array}{c}\text { Start } \\
\text { Sample } \\
\text { Date } \\
\end{array}$ & $\begin{array}{c}\text { Finish } \\
\text { Sample } \\
\text { Date }\end{array}$ & $\begin{array}{l}\text { Sampla } \\
\text { Number }\end{array}$ & $\begin{array}{c}\text { Tank Farm } \\
\text { Number }\end{array}$ & $\begin{array}{c}\text { Lab Sample } \\
\text { Number }\end{array}$ & $\begin{array}{l}\text { Density } \\
\text { (g/mL) }\end{array}$ & $\begin{array}{c}\text { Reported } \\
\text { Value } \\
\end{array}$ & $\begin{array}{r}\text { Repo } \\
U_{n} \\
\end{array}$ \\
\hline Chromium & $6 / 1 / 90$ & $4 / 30 / 89$ & $12 / 30 / 89$ & $4 / 27 / 89$ & $4 / 29 / 89$ & BOB2MO & G306 & G352 & 1.20 & $6.27 E+05$ & ugi \\
\hline Chromium & $6 / 1 / 90$ & $4 / 30 / 89$ & $12 / 30 / 89$ & $4 / 27 / 89$ & $4 / 29 / 89$ & BOB2N4 & G323 & G341 & 1.20 & $6.25 E+05$ & ugl \\
\hline Chromium & $6 / 1 / 90$ & $4 / 30 / 89$ & $12 / 30 / 89$ & $4 / 27 / 89$ & $4 / 29 / 89$ & ВOB2M1 & G308 & G353 & 1.20 & $6.24 E+05$ & ug! \\
\hline Chromium & $6 / 1 / 90$ & $4 / 30 / 89$ & $12 / 30 / 89$ & $4 / 27 / 89$ & $4 / 29 / 89$ & BOB2NO & G307 & G453 & NA & $6.22 E+05$ & ugh \\
\hline Chromium & $6 / 1 / 90$ & $4 / 30 / 89$ & $12 / 30 / 89$ & $4 / 27 / 89$ & $4 / 29 / 89$ & BOB2NT & G310 & G454 & $(1.19)$ & $6.22 E+05$ & ug/ \\
\hline Chromium & $6 / 1 / 90$ & $4 / 30 / 89$ & $12 / 30 / 89$ & $4 / 27 / 89$ & $4 / 29 / 89$ & BOB2L7 & G299 & G342 & 1.20 & $6.22 E+05$ & $u g / h$ \\
\hline Chromium & $6 / 1 / 90$ & $4 / 30 / 89$ & $12 / 30 / 89$ & $4 / 27 / 89$ & $4 / 29 / 89$ & B082N4 & G323 & G341 & 1.20 & $6.21 E+05$ & $u g / t$ \\
\hline Chromium & $6 / 1 / 90$ & $4 / 30 / 89$ & $12 / 30 / 89$ & $4 / 27 / 89$ & $4 / 29 / 89$ & $\mathrm{BOB2N3}$ & G322 & G338 & NA & $6.19 E+05$ & ug $/ \mathrm{h}$ \\
\hline Chromium & $6 / 1 / 90$ & $4 / 30 / 89$ & $12 / 30 / 89$ & $4 / 27 / 89$ & $4 / 29 / 89$ & $\mathrm{BOB} 2 \mathrm{M7}$ & G319 & G460 & 1.20 & $6.19 E+05$ & ugh. \\
\hline Chromium & $6 / 1 / 90$ & $4 / 30 / 89$ & $12 / 30 / 89$ & $4 / 27 / 89$ & $4 / 29 / 89$ & BOB2N2 & G317 & G333 & NA & $6.18 E+05$ & $u g h$ \\
\hline Chromium & $6 / 1 / 90$ & $4 / 30 / 89$ & $12 / 30 / 89$ & $4 / 27 / 89$ & $4 / 29 / 89$ & Во82M9 & G302 & G450 & $(1.20)$ & $6.16 E+05$ & ug/h \\
\hline Chromium & $6 / 1 / 90$ & $4 / 30 / 89$ & $12 / 30 / 89$ & $4 / 27 / 89$ & $4 / 29 / 89$ & ВOB2M2 & G309 & G358 & 1.20 & $6.16 E+05$ & $u g / L$ \\
\hline Chromium & $6 / 1 / 90$ & $4 / 30 / 89$ & $12 / 30 / 89$ & $4 / 27 / 89$ & $4 / 29 / 89$ & BOB2T9 & G327 & G443 & 1.20 & $6.15 E+05$ & $u g / L$ \\
\hline Chromium & $6 / 1 / 90$ & $4 / 30 / 89$ & $12 / 30 / 89$ & $4 / 27 / 89$ & $4 / 29 / 89$ & BOB2M1 & G308 & G353 & 1.20 & $6.14 E+05$ & $4 g / L$ \\
\hline Chromium & $6 / 1 / 90$ & $4 / 30 / 89$ & $12 / 30 / 89$ & $4 / 27 / 89$ & $4 / 29 / 89$ & Вов2М8 & G301 & G449 & NA & $6.12 E+05$ & ug/L \\
\hline Chromium & $6 / 1 / 90$ & $4 / 30 / 89$ & $12 / 30 / 89$ & $4 / 27 / 89$ & $4 / 29 / 89$ & BOB2M4 & G313 & G363 & 1.20 & $6.11 E+05$ & $u g / L$ \\
\hline Chromium & $6 / 1 / 90$ & $4 / 30 / 89$ & $12 / 30 / 89$ & $4 / 27 / 89$ & $4 / 29 / 89$ & BOB2M4 & G313 & G363 & 1.20 & $6.09 E+05$ & $u g / L$ \\
\hline Chromlum & $6 / 1 / 90$ & $4 / 30 / 89$ & $12 / 30 / 89$ & $4 / 27 / 89$ & $4 / 29 / 89$ & вов2М3 & G311 & G359 & 1.20 & $6.08 E+05$ & $u g / L$ \\
\hline Chromium & $6 / 1 / 90$ & $4 / 30 / 89$ & $12 / 30 / 89$ & $4 / 27 / 89$ & $4 / 29 / 89$ & BOB2N1 & G310 & G454 & (1.19) & $6.07 E+05$ & ugh \\
\hline Chromium & $6 / 1 / 90$ & $4 / 30 / 89$ & $12 / 30 / 89$ & $4 / 27 / 89$ & $4 / 29 / 89$ & ВОВ2MO & G306 & G352 & 1.20 & $6.05 E+05$ & ugh \\
\hline Chromium & $6 / 1 / 90$ & $4 / 30 / 89$ & $12 / 30 / 89$ & $4 / 27 / 89$ & $4 / 29 / 89$ & BOB2L7 & G299 & G342 & 1.20 & $6.04 E+05$ & ug $\Omega$ \\
\hline
\end{tabular}




\begin{tabular}{|c|c|c|c|c|c|c|c|}
\hline & & & Analvte Inforn & ation & & & \\
\hline Code & Source & Analyte & $\begin{array}{l}\text { Conc. } \\
\text { (ug/L) }\end{array}$ & Conc. (mol/L) & $\begin{array}{l}\text { Reference } \\
\text { Number }\end{array}$ & $\begin{array}{l}\text { Validated } \\
\text { (Y/N) }\end{array}$ & Notes \\
\hline $\mathbf{P}$ & Raw & $\mathrm{Cr}$ & $6.27 E+05$ & $1.21 E-02$ & AP102-3 & $Y$ & $\begin{array}{l}\text { Density reported for G352 collected at } 34 \text { in from bottom of } \\
\text { tank at a } 30^{\circ} \text { angle. Sample was unheated, unheated } \\
\text { samples precipitated }\end{array}$ \\
\hline $\mathbf{P}$ & Raw & $\mathrm{Cr}$ & $6.25 E+05$ & $1.20 \mathrm{E}-02$ & AP102-3 & $Y$ & $\begin{array}{l}\text { Density reported for } \mathrm{G} 341 \text { collected at } 99 \text { in from bottom of } \\
\text { tank at a } 270^{\circ} \text { angle. }\end{array}$ \\
\hline $\mathbf{P}$ & Raw & $\mathrm{Cr}$ & $6.24 E+05$ & $1.20 \mathrm{E}-02$ & AP102-3 & $Y$ & $\begin{array}{l}\text { Density reported for G353 collected at } 334 \text { in from bottom } \\
\text { of tank at a } 150^{\circ} \text { angle. Sample was unheated, unheated } \\
\text { samples precipitated }\end{array}$ \\
\hline $\mathbf{P}$ & Raw & $\mathrm{Cr}_{\mathrm{r}}$ & $6.22 E+05$ & $1.20 E-02$ & AP102-3 & $Y$ & $\begin{array}{l}\text { Density not reported for } \mathrm{G} 453 \text {. No assumptions could be } \\
\text { made }\end{array}$ \\
\hline$P$ & Raw & $\mathrm{Cr}$ & $6.22 E+05$ & $1.20 E-02$ & $A P 102-3$ & $\mathbf{Y}$ & $\begin{array}{l}\text { Density not reported for G454. Density assumed same as } \\
6471 \text { which was collected at } 208 \text { in from bottom of tank at } \\
\text { a } 150^{\circ} \text { angle and was keep heated to } 40^{\circ} \mathrm{C} \text {. }\end{array}$ \\
\hline $\mathbf{P}$ & Raw & $\mathrm{Cr}$ & $6.22 \mathrm{E}+05$ & 1.20E-02 & AP102-3 & $\mathbf{Y}$ & $\begin{array}{l}\text { Density reported for G342 coliected at } 306 \text { in from bottom } \\
\text { of tank at a } 30^{\circ} \text { angle. Sample was unheated, unheated } \\
\text { samples precipitated }\end{array}$ \\
\hline$P$ & Raw & $\mathrm{Cr}$ & $6.21 E+05$ & 1.19E-02 & AP102-3 & $Y$ & $\begin{array}{l}\text { Density reported for } G 341 \text { collected at } 99 \text { in from bottom of } \\
\text { tank at a } 270^{\circ} \text { angle. }\end{array}$ \\
\hline $\mathbf{P}$ & Raw & $\mathrm{Cr}$ & $6.19 E+05$ & 1.19E-02 & AP102-3 & $\mathbf{Y}$ & $\begin{array}{l}\text { Density not reported for G338. No assumptions could be } \\
\text { made }\end{array}$ \\
\hline $\mathbf{P}$ & Raw & $\mathrm{Cr}$ & $6.19 E+05$ & 1.19E-02 & AP102-3 & $\mathbf{Y}$ & $\begin{array}{l}\text { Density reported for G460 collected at } 226 \text { in from bottom } \\
\text { of tank at a } 270^{\circ} \text { angle. Sample was unheated, unheated } \\
\text { samples precipitated }\end{array}$ \\
\hline $\mathbf{P}$ & Raw & $\mathrm{Cr}$ & $6.18 E+05$ & $1.19 E-02$ & AP102-3 & $\mathbf{Y}$ & $\begin{array}{l}\text { Density not reported for } \mathrm{G} 333 \text {. No assumptions could be } \\
\text { made }\end{array}$ \\
\hline $\mathbf{P}$ & Raw & $\mathrm{Cr}$ & $6.16 E+05$ & $1.18 \mathrm{E}-02$ & AP102-3 & $\mathbf{Y}$ & $\begin{array}{l}\text { Density not reported for G450. Density assumed same as } \\
\text { G477 which was collected at } 182 \text { in from bottom of tank at } \\
\text { a } 30^{\circ} \text { angle and was keep heated to } 40^{\circ} \mathrm{C} \text {. }\end{array}$ \\
\hline$P$ & Raw & $\mathrm{Cr}$ & $6.16 \mathrm{E}+05$ & $1.18 E-02$ & AP102-3 & $Y$ & $\begin{array}{l}\text { Density reported for G358 collected at } 334 \text { in from bottom } \\
\text { of tank at a } 150^{\circ} \text { angle. Sample was unheated, unheated } \\
\text { samples precipitated }\end{array}$ \\
\hline $\mathbf{P}$ & Raw & $\mathrm{Cr}$ & $6.15 E+05$ & $1.18 E-02$ & AP102-3 & $Y$ & Density reported for $\mathrm{G} 443$ represents a composite sample \\
\hline $\mathbf{P}$ & Raw & $\mathrm{Cr}$ & $6.14 E+05$ & $1.18 E-02$ & AP102-3 & $Y$ & $\begin{array}{l}\text { Density reported for } \mathrm{G} 353 \text { collected at } 334 \text { in from bottom } \\
\text { of tank at a } 150^{\circ} \text { angle. Sample was unheated, unheated } \\
\text { samples precipitated }\end{array}$ \\
\hline$P$ & Raw & $\mathrm{Cr}$ & $6.12 E+05$ & $1.18 E-02$ & AP102-3 & $Y$ & $\begin{array}{l}\text { Density not reported for G449. No assumptions could be } \\
\text { made }\end{array}$ \\
\hline $\mathbf{P}$ & Raw & $\mathrm{Cr}$ & $6.11 E+05$ & $1.18 \mathrm{E}-02$ & AP102-3 & Y & $\begin{array}{l}\text { Density reported for G363 collected at } 18 \text { in from bottom of } \\
\text { tank at a } 150^{\circ} \text { angle. Sample was unheated, unheated } \\
\text { samples precipitated }\end{array}$ \\
\hline $\mathbf{P}$ & Raw & $\mathrm{Cr}$ & $6.09 E+05$ & $1.17 E-02$ & AP102-3 & Y & $\begin{array}{l}\text { Density reported for G363 collected at } 18 \text { in from bottom of } \\
\text { tank at a } 150^{\circ} \text { angle. Sample was unheated, unheated } \\
\text { samples precipitated }\end{array}$ \\
\hline $\mathbf{P}$ & Raw & $\mathrm{Cr}$ & $6.08 E+05$ & 1.17E-02 & AP102-3 & $Y$ & $\begin{array}{l}\text { Density reported for G359 collected at } 162 \text { in from bottom } \\
\text { of tank at a } 150^{\circ} \text { angle. Sample was unheated, unheated } \\
\text { samples precipitated }\end{array}$ \\
\hline $\mathbf{P}$ & Raw & $\mathrm{Cr}$ & $6.07 E+05$ & $1.17 E-02$ & AP102-3 & Y & $\begin{array}{l}\text { Density not reported for G454. Density assumed same as } \\
\text { G471 which was collected at } 208 \text { in from bottom of tank at } \\
\text { a } 150^{\circ} \text { angle and was keep heated to } 40^{\circ} \mathrm{C} \text {. }\end{array}$ \\
\hline$P$ & Raw & $\mathrm{Cr}$ & $6.05 \varepsilon+05$ & $1.16 E-02$ & AP102-3 & $Y$ & $\begin{array}{l}\text { Density reported for } \mathrm{G} 352 \text { collected at } 34 \text { in from bottom bf } \\
\text { tank at a } 30^{\circ} \text { angle. Sample was unheated, unheated } \\
\text { samples precipitated }\end{array}$ \\
\hline $\mathbf{P}$ & Raw & $\mathrm{Cr}$ & $6.04 E+05$ & $1.16 E-02$ & AP102-3 & $\mathbf{Y}$ & 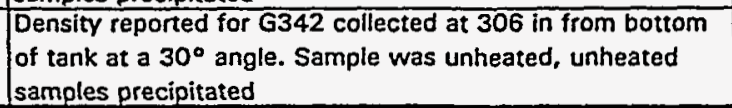 \\
\hline
\end{tabular}




\begin{tabular}{|c|c|c|c|c|c|c|c|c|c|c|c|}
\hline & & & & & ank Waste & haracteriza & On Sample 1 & ormation & & & \\
\hline Reported Chemical & $\begin{array}{c}\text { Memo } \\
\text { Date }\end{array}$ & $\begin{array}{c}\text { Start } \\
\text { Analysis } \\
\text { Date }\end{array}$ & $\begin{array}{c}\text { Finish } \\
\text { Analysis Date }\end{array}$ & $\begin{array}{c}\text { Start } \\
\text { Sample } \\
\text { Date } \\
\end{array}$ & $\begin{array}{c}\text { Finish } \\
\text { Sample } \\
\text { Date } \\
\end{array}$ & $\begin{array}{l}\text { Sample } \\
\text { Number }\end{array}$ & $\begin{array}{c}\text { Tank Farm } \\
\text { Number }\end{array}$ & $\begin{array}{c}\text { Lab Sample } \\
\text { Number }\end{array}$ & $\begin{array}{l}\text { Density } \\
\text { (g/mL) }\end{array}$ & $\begin{array}{c}\text { Reported } \\
\text { Value }\end{array}$ & $\begin{array}{r}\text { Repo } \\
U_{n}\end{array}$ \\
\hline Chromium & $6 / 1 / 90$ & $4 / 30 / 89$ & $12 / 30 / 89$ & $4 / 27 / 89$ & $4 / 29 / 89$ & ВОВ2M6 & G318 & G459 & 1.20 & $6.04 E+05$ & ugi \\
\hline Chromium & $6 / 1 / 90$ & $4 / 30 / 89$ & $12 / 30 / 89$ & $4 / 27 / 89$ & $4 / 29 / 89$ & BOB2M2 & G309 & G358 & 1.20 & $6.03 E+05$ & ugi \\
\hline Chromium & $6 / 1 / 90$ & $4 / 30 / 89$ & $12 / 30 / 89$ & $4 / 27 / 89$ & $4 / 29 / 89$ & BOB2M5 & G315 & G364 & 1.19 & $6.02 E+05$ & ugf \\
\hline Chromium & $6 / 1 / 90$ & $4 / 30 / 89$ & $12 / 30 / 89$ & $4 / 27 / 89$ & $4 / 29 / 89$ & ВOB2M8 & G301 & G449 & NA & $6.01 E+05$ & ugi \\
\hline Chromium & $6 / 1 / 90$ & $4 / 30 / 89$ & $12 / 30 / 89$ & $4 / 27 / 89$ & $4 / 29 / 89$ & $8082 \mathrm{M9}$ & G302 & G450 & $(1.20)$ & $5.97 E+05$ & ug $l$ \\
\hline Chromium & $6 / 1 / 90$ & $4 / 30 / 89$ & $12 / 30 / 89$ & $4 / 27 / 89$ & $4 / 29 / 89$ & ВОВ2M3 & G311 & G359 & 1.20 & $5.91 E+05$ & ugf \\
\hline $\mathrm{Cr}$ & $4 / 28 / 84$ & NS & NS & NS & NS & R-8469 & NS & NS & NS & $1.70 E+\infty$ & $\overline{u g m /}$ \\
\hline Chromium & $4 / 1 / 93$ & NS & NS & NS & NS & BOB2T9 & G327 & NS & 1.19 & $7.16 E+05$ & UGI \\
\hline Chromium & $4 / 1 / 93$ & NS & NS & NS & NS & BOB2T9 & G327 & NS & 1.19 & $7.08 E+05$ & $\overline{U G 1}$ \\
\hline $\mathrm{Cr}$ & $2 / 10 / 89$ & NS & NS & NS & NS & NS & NS & NS & 1.01 & $1.70 E+\infty 0$ & ppri \\
\hline $\mathrm{Cr}$ & $4 / 28 / 84$ & NS & NS & NS & NS & R8469 & NS & $\overline{N S}$ & NS & $1.70 E+00$ & $\overline{U G / M}$ \\
\hline $\mathrm{Cr}$ & $4 / 28 / 84$ & NS & NS & NS & NS & NS & NS & NS & NS & $1.70 \mathrm{E}-03$ & $g / L$ \\
\hline$\overline{C r}$ & $2 / 10 / 89$ & NS & NS & NS & NS & NS & NS & NS & 0.956764 & $1.61 E+\infty 0$ & ppn \\
\hline Fluoride & $6 / 1 / 90$ & $4 / 30 / 89$ & $12 / 30 / 89$ & $4 / 27 / 89$ & $4 / 29 / 89$ & BOB2N2 & G317 & G333 & NA & $<2.09 E+02$ & $u g / \pi$ \\
\hline Fluoride & $6 / 1 / 90$ & $4 / 30 / 89$ & $12 / 30 / 89$ & $4 / 27 / 89$ & $4 / 29 / 89$ & BOB2N2 & G317 & G333 & NA & $<2.09 E+02$ & ug/m \\
\hline Fluoride & $6 / 1 / 90$ & $4 / 30 / 89$ & $12 / 30 / 89$ & $4 / 27 / 89$ & $4 / 29 / 89$ & BOB2N3 & G322 & G338 & NA & $<1.68 E+02$ & $\omega g / m$ \\
\hline Fluoride & $6 / 1 / 90$ & $4 / 30 / 89$ & $12 / 30 / 89$ & $4 / 27 / 89$ & $4 / 29 / 89$ & BOB2N4 & G323 & G341 & 1.20 & $<1.68 E+02$ & $u g / \pi$ \\
\hline Fiuoride & $6 / 1 / 90$ & $4 / 30 / 89$ & $12 / 30 / 89$ & $4 / 27 / 89$ & $4 / 29 / 89$ & BOB2L7 & G299 & G342 & 1.20 & $<1.68 E+02$ & $\mathrm{ug} / \mathrm{m}$ \\
\hline Fluoride & $6 / 1 / 90$ & 4/30/89 & $12 / 30 / 89$ & $4 / 27 / 89$ & $4 / 29 / 89$ & BOB2L8 & G303 & G348 & 1.21 & $<1.68 E+02$ & $\mathrm{ug} / \mathrm{m}$ \\
\hline Fluoride & $6 / 1 / 90$ & $4 / 30 / 89$ & $12 / 30 / 89$ & $4 / 27 / 89$ & $4 / 29 / 89$ & BOB2L9 & G305 & G349 & 1.20 & $<1.68 E+02$ & $\mathrm{ug} / \mathrm{m}$ \\
\hline Fluoride & $6 / 1 / 90$ & $4 / 30 / 89$ & $12 / 30 / 89$ & $4 / 27 / 89$ & $4 / 29 / 89$ & BOB2MO & G306 & G352 & 1.20 & $<1.68 E+02$ & $\mathrm{ug} / \mathrm{m}$ \\
\hline Fluoride & $6 / 1 / 90$ & $4 / 30 / 89$ & $12 / 30 / 89$ & $4 / 27 / 89$ & $4 / 29 / 89$ & BOB2M1 & G308 & G353 & 1.20 & $<1.68 E+02$ & $\mathrm{ug} / \mathrm{ml}$ \\
\hline Fluoride & $6 / 1 / 90$ & $4 / 30 / 89$ & $12 / 30 / 89$ & $4 / 27 / 89$ & $4 / 29 / 89$ & BOB2M2 & G309 & G358 & 1.20 & $<1.68 E+02$ & $u g / m l$ \\
\hline Fluoride & $6 / 1 / 90$ & $4 / 30 / 89$ & $12 / 30 / 89$ & $4 / 27 / 89$ & $4 / 29 / 89$ & BOB2M3 & G311 & G359 & 1.20 & $<1.68 E+02$ & $\mathrm{ug} / \mathrm{ml}$ \\
\hline Fluoride & $6 / 1 / 90$ & $4 / 30 / 89$ & $12 / 30 / 89$ & $4 / 27 / 89$ & $4 / 29 / 89$ & BOB2M4 & G313 & G363 & 1.20 & $<1.68 E+02$ & $\mathrm{ug} / \mathrm{ml}$ \\
\hline Fluoride & $6 / 1 / 90$ & $4 / 30 / 89$ & $12 / 30 / 89$ & $4 / 27 / 89$ & 4/29/89 & BO82M5 & G315 & G364 & 1.19 & $<1.68 E+02$ & $\mathrm{ug} / \mathrm{mL}$ \\
\hline
\end{tabular}




\begin{tabular}{|c|c|c|c|c|c|c|c|}
\hline \multirow[b]{2}{*}{ Code } & \multirow[b]{2}{*}{ Source } & \multicolumn{3}{|c|}{ Analyte Information } & \multirow[b]{2}{*}{$\begin{array}{l}\text { Reference } \\
\text { Number }\end{array}$} & \multirow[b]{2}{*}{$\begin{array}{c}\text { Validated } \\
\text { (Y/N) }\end{array}$} & \multirow[b]{2}{*}{ Notes } \\
\hline & & Analyte & $\begin{array}{l}\text { Conc. } \\
\text { (ug/L) }\end{array}$ & Conc. (mol/h) & & & \\
\hline$P$ & Raw & $\mathrm{Cr}$ & $6.04 E+05$ & $1.16 E-02$ & AP102-3 & $Y$ & $\begin{array}{l}\text { Density reported for } \mathrm{G} 459 \text { collected at } 226 \text { in from bottom } \\
\text { of tank at a } 270^{\circ} \text { angle. Sample was unheated, unheated } \\
\text { samples precipitated }\end{array}$ \\
\hline$P$ & Raw & $\mathrm{Cr}$ & $6.03 E+05$ & $1.16 \mathrm{E}-02$ & AP102-3 & $Y$ & $\begin{array}{l}\text { Density reported for G358 collected at } 334 \text { in from bottom } \\
\text { of tank at a } 150^{\circ} \text { angle. Sample was unheated, unheated } \\
\text { samples precipitated }\end{array}$ \\
\hline $\mathbf{P}$ & Raw & $\mathrm{Cr}$ & $6.02 E+05$ & $1.16 E-02$ & AP102-3 & $Y$ & $\begin{array}{l}\text { Density reported for } \mathrm{G} 364 \text { collected at } 372 \text { in from bottom } \\
\text { of tank at a } 270^{\circ} \text { angle. Sample was unheated, unheated } \\
\text { samples precipitated }\end{array}$ \\
\hline $\mathbf{P}$ & Raw & $\mathrm{Cr}$ & $6.01 E+05$ & $1.16 \mathrm{E}-02$ & AP102-3 & $Y$ & $\begin{array}{l}\text { Density not reported for } 6449 \text {. No assumptions could be } \\
\text { made }\end{array}$ \\
\hline $\mathbf{P}$ & Raw & $\mathrm{Cr}$ & $5.97 E+05$ & $1.15 E-02$ & AP102-3 & $\mathbf{Y}$ & $\begin{array}{l}\text { Density not reported for } G 450 \text {. Density assumed same as } \\
\text { G477 which was collected at } 182 \text { in from bottom of tank at } \\
\text { a } 30^{\circ} \text { angle and was keep heated to } 40^{\circ} \mathrm{C} \text {. }\end{array}$ \\
\hline $\mathbf{P}$ & Raw & $\mathrm{Cr}$ & $5.91 E+05$ & $1.14 E-02$ & AP102-3 & $Y$ & $\begin{array}{l}\text { Density reported for G359 collected at } 162 \text { in from bottom } \\
\text { of tank at a } 150^{\circ} \text { angle. Sample was unheated, unheated } \\
\text { samples precipitated }\end{array}$ \\
\hline $\mathbf{P}$ & Braun & $\mathrm{Cr}+3$ & $1.70 E+09$ & $3.27 \mathrm{E}+01$ & NS & $\mathbf{N}$ & No Reference \\
\hline $\mathbf{P}$ & TCO & $\mathrm{Cr}+6$ & $7.16 \mathrm{E}+11$ & $1.38 E+04$ & NS & $\mathbf{N}$ & THIS POINT NOT GIVEN IN REPORT WHC-SD-WM-TRP-169 \\
\hline $\mathbf{P}$ & TCD & $\mathrm{Cr}+6$ & $7.08 E+11$ & $1.36 E+04$ & NS & $\bar{N}$ & THIS POINT NOT GIVEN IN REPORT WHC-SD-WM-TRP-169 \\
\hline $\mathbf{U}$ & RAW & $\mathrm{Cr}+6$ & $1.72 E+09$ & $3.31 E+01$ & AP102-2 & $N$ & Projected value, not a real data point \\
\hline $\mathbf{P}$ & RAW & $\mathrm{Cr}+6$ & $1.70 E+09$ & $3.27 E+01$ & AP102-1 & $\mathbf{N}$ & Outdated Information \\
\hline $\mathbf{P}$ & Wastren & $\mathrm{Cr}+6$ & $1.70 E+09$ & $3.27 \mathrm{E}+01$ & NS & $\mathbf{N}$ & No Reference \\
\hline $\mathbf{U}$ & RAW & $\mathrm{Cr}+6$ & $1.54 E+09$ & $2.96 E+01$ & AP102-2 & $\mathbf{N}$ & Projected value, not a real data point \\
\hline $\mathbf{P}$ & Raw & $\mathbf{F}$ & \#VALUE! & \#VALUe! & AP102-3 & $\mathbf{Y}$ & $\begin{array}{l}\text { Density not reported for G333. No assumptions could be } \\
\text { made }\end{array}$ \\
\hline $\mathbf{P}$ & Raw & $\mathbf{F}$ & VALUE! & \#VALUE! & AP102-3 & $Y$ & $\begin{array}{l}\text { Density not reported for G333. No assumptions could be } \\
\text { made }\end{array}$ \\
\hline $\mathbf{P}$ & Raw & $\mathbf{F}$ & \#VALUE! & \#VALUE! & AP102-3 & $\gamma$ & $\begin{array}{l}\text { Density not reported for } \mathrm{G338} \text {. No assumptions could be } \\
\text { made }\end{array}$ \\
\hline$P$ & Raw & $\mathbf{F}$ & \#VALUE! & \#ALUE! & AP102-3 & $\mathbf{Y}$ & $\begin{array}{l}\text { Density reported for } 6341 \text { collected at } 99 \text { in from bottom of } \\
\text { tank at a } 270^{\circ} \text { angle. }\end{array}$ \\
\hline $\mathbf{P}$ & Raw & $F$ & \#VALUE! & \#VALUE! & AP102-3 & $\mathbf{Y}$ & $\begin{array}{l}\text { Density reported for G342 collected at } 306 \text { in from bottom } \\
\text { of tank at a } 30^{\circ} \text { angle. Sample was unheated, unheated } \\
\text { samples precipitated }\end{array}$ \\
\hline$P$ & Raw & $F$ & \#VALUE! & \#VALUEI & AP102-3 & $\mathbf{Y}$ & $\begin{array}{l}\text { Density reported for G348 collected at } 125 \text { in from bottom } \\
\text { of tank at a } 30^{\circ} \text { angle. Sample was unheated, unheated } \\
\text { samples precipitated }\end{array}$ \\
\hline $\mathbf{P}$ & Raw & $F$ & \#ALUEI & \#VALUEI & AP102-3 & $Y$ & $\begin{array}{l}\text { Density reported for G349 collected at } 34 \text { in from bottom of } \\
\text { tank at a } 30^{\circ} \text { angle. Sample was unheated, unheated } \\
\text { samples precipitated }\end{array}$ \\
\hline$P$ & Raw & $F$ & NALUE! & \#VALUE! & AP102-3 & $\mathbf{Y}$ & $\begin{array}{l}\text { Density reported for G352 collected at } 34 \text { in from bottom of } \\
\text { tank at a } 30^{\circ} \text { angle. Sample was unheated, unheated } \\
\text { samples precipitated }\end{array}$ \\
\hline$P$ & Raw & $F$ & \#VALUE! & \#ALUE! & AP102-3 & $Y$ & $\begin{array}{l}\text { Density reported for } 6353 \text { collected at } 334 \text { in from bottom } \\
\text { of tank at a } 150^{\circ} \text { angle. Sample was unheated, unheated } \\
\text { samples precipitated }\end{array}$ \\
\hline$P$ & Raw & $\mathbf{F}$ & \#VALUEI & \#VALUE! & AP102-3 & $Y$ & $\begin{array}{l}\text { Density reported for G358 collected at } 334 \text { in from bottom } \\
\text { of tank at a } 150^{\circ} \text { angle. Sample was unheated, unheated } \\
\text { samples precipitated }\end{array}$ \\
\hline$P$ & Raw & $\mathbf{F}$ & \#ALUE! & \#ALUE! & AP102-3 & $\mathbf{Y}$ & $\begin{array}{l}\text { Density reported for } G 359 \text { collected at } 162 \text { in from bottom } \\
\text { of tank at a } 150^{\circ} \text { angle. Sample was unheated, unheated } \\
\text { samples precipitated }\end{array}$ \\
\hline $\mathbf{P}$ & Raw & $F$ & \#ALUE! & FALUE! & AP102-3 & $Y$ & $\begin{array}{l}\text { Density reported for G363 collected at } 18 \text { in from bottom of } \\
\text { tank at a } 150^{\circ} \text { angle. Sample was unheated, unheated } \\
\text { samples precipitated }\end{array}$ \\
\hline $\mathbf{P}$ & Raw & $F$ & \#VALUE! & \#VALUE! & AP102-3 & $Y$ & $\begin{array}{l}\text { Density reported for G364 collected at } 372 \text { in from bottom } \\
\text { of tank at a } 270^{\circ} \text { angle. Sample was unheated, unheated } \\
\text { samples precipitated }\end{array}$ \\
\hline
\end{tabular}




\begin{tabular}{|c|c|c|c|c|c|c|c|c|c|c|c|}
\hline$\eta w / 5 n$ & $20+389^{\circ} 1>$ & $07 \cdot 1$ & ع9عפ & EเEפ & tw2808 & $68 / 6 z / t$ & $68 / L Z / t$ & $68 / 0 \varepsilon / 2 !$ & $68 / 0 \varepsilon / t$ & $06 / L / 9$ & әp!yon!y \\
\hline (jw/6n & $20+389^{\circ} L>$ & $0 Z !$ & 6989 & $\lfloor\llcorner\varepsilon 5$ & Ewz808 & $68 / 6 z / 5$ & $68 / \angle Z / b$ & $68 / 0 \varepsilon / 2 L$ & $68 / 08 / t$ & $06 / 1 / 9$ & op!jonity \\
\hline fw/Bn & $20+389^{\circ} t>$ & $0 z \cdot 1$ & 8589 & 6089 & ZwZ808 & $68 / 6 z / t$ & $68 / L Z / t$ & 68/0ع/ZL & $68 / 0 \varepsilon / t$ & $06 / 1 / 9$ & opplonys \\
\hline (W/6n & $20+389 \cdot 1>$ & or'l & ع૬ع९ & $80 \varepsilon 9$ & IwZgog & $68 / 6 z / t$ & $68 / L Z / t$ & $68 / 0 \varepsilon / Z L$ & $68 / 0 \varepsilon / 5$ & $06 / L / 9$ & ap!lonys \\
\hline ן $w / 6 n$ & $20+389^{\circ} 1>$ & or: & ZSES & $90 \varepsilon 9$ & owz808 & $68 / 62 / t$ & $68 / L Z / t$ & 68/0ع/ZL & $68 / 0 \varepsilon / t$ & $06 / 1 / 9$ & op!son|s \\
\hline |w/bn & $20+389^{\circ} 1>$ & $O Z^{*} L$ & 6†ยง & soعง & 672809 & $68 / 62 / b$ & G8/LZ/t & $68 / 0 \varepsilon / 2 L$ & $68 / 08 / 7$ & $06 / L / 9$ & op!lon/s \\
\hline 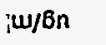 & $20+389^{\circ} 1>$ & IZ゙ & $8 t \varepsilon 9$ & ع૦६९ & 872809 & $68 / 6 z / 5$ & $68 / L Z / t$ & $68 / 0 \varepsilon / Z 1$ & $68 / 08 / t$ & $06 / 1 / 9$ & ap!sonys \\
\hline$w / 6 n$ & $20+389^{\circ} 1>$ & $0 z \cdot 1$ & 乙†६९ & 6620 & $\angle 72808$ & $68 / 6 Z / t$ & $68 / L Z / t$ & $68 / 0 \varepsilon / 2 L$ & $68 / 0 \varepsilon / t$ & $06 / L / 9$ & әp!yony \\
\hline$w / 8 n$ & $20+389^{\circ} 1>$ & $O Z^{\prime} i$ & เ†६פ & ยてะ૭ & tNZROB & $68 / 62 / b$ & $68 / L Z / b$ & $68 / 0 \varepsilon / Z L$ & $68 / 0 \varepsilon / t$ & $06 / 4 / 9$ & optronts \\
\hline$\omega / 8 n$ & $\left.20+389^{\circ} 1\right\rangle$ & $\forall N$ & $8 \varepsilon \varepsilon 9$ & 乙2ย९ & ENZ808 & $68 / 62 / 5$ & $68 / L Z / t$ & $68 / 0 \varepsilon / Z L$ & $68 / 0 \varepsilon / t$ & $06 / L / 9$ & op!yonis \\
\hline (2)/6n & $20+389^{\circ} 1>$ & or:1 &.$\angle L t 9$ & ZOED & 6WZ808 & $68 / 62 / b$ & $68 / \angle Z / t$ & $68 / 0 \varepsilon / Z L$ & $68 / 0 \varepsilon / t$ & $06 / L / 9$ & optyonys \\
\hline$w / 6 n$ & $\left.20+389^{\circ} L\right\rangle$ & 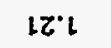 & $.9<t 5$ & $\varepsilon \succsim \varepsilon ૭$ & tNZBOB & $68 / 6 \mathrm{C} / \mathrm{t}$ & $68 / L Z / t$ & $68 / 0 \varepsilon / 2 L$ & $68 / 0 \varepsilon / 7$ & $06 / L / 9$ & әp!Jonjs \\
\hline wu/6n & $20+389 \cdot \iota>$ & $6 ! \cdot !$ & $\cdot 1<t 5$ & OLEง & INZ8OG & $68 / 62 / 4$ & $68 / \angle Z / \downarrow$ & $68 / 0 \varepsilon / Z L$ & $68 / 0 \varepsilon / t$ & $06 / L / 9$ & oppuonis \\
\hline$w / 6 n$ & $\left.20+389^{\circ} 1\right\rangle$ & $6 L^{\circ}$ & $.0<t 5$ & LZEง & 612808 & $68 / 62 / t$ & $68 / L Z / t$ & $68 / 0 \varepsilon / 21$ & $68 / 0 \varepsilon / t$ & $06 / L / 9$ & opponits \\
\hline $4 / 8 \mathrm{n}$ & $20+389^{\circ} L>$ & $2 \cdot 1$ & $09+9$ & 6เEง & LWZ8OS & $68 / 62 / t$ & $68 / L Z / t$ & $68 / 0 \varepsilon / Z 1$ & $68 / 0 \varepsilon / 5$ & $06 / 1 / 9$ & ap!jon!t \\
\hline W/En & $\left.20+389^{\circ} \iota\right\rangle$ & or*l & $6 S+9$ & 8189 & 9wzgog & $68 / 62 / t$ & $68 / L Z / t$ & $68 / 0 \varepsilon / Z 1$ & $68 / 0 \varepsilon / t$ & $06 / L / 9$ & opuonis \\
\hline د/8n & $20+389 \cdot 1\rangle$ & $\left(6 t^{\circ} L\right)$ & $\nabla S+9$ & OLED & LNZgOS & $68 / 62 / t$ & $68 / L Z / 4$ & 68/08/ZL & 68/0E/t & $06 / 2 / 9$ & eptuonis \\
\hline Lu/Bn & $\left.20+389^{\circ} L\right\rangle$ & $\forall N$ & $\varepsilon S+\vartheta$ & LOES & ONZ808 & $68 / 62 / t$ & $68 / 22 / 7$ & 68/0E/ZL & $68 / 0 \varepsilon / t$ & $06 / 1 / 9$ & op!yonjy \\
\hline $4 / 6 n$ & $\left.20+389^{\circ} L\right\rangle$ & $\left(0 Z^{\prime} L\right)$ & osts & 2089 & 6W2808 & $68 / 62 / t$ & $68 / L Z / t$ & $68 / 0 \varepsilon / 2 L$ & $68 / 0 \varepsilon / t$ & $06 / L / 9$ & op!yonis \\
\hline$y / B n$ & $20+389^{\circ} \mathrm{L}>$ & $\forall N$ & $6 \nleftarrow 9$ & LOعפ & 8WZвоg & $68 / 6 \tau / t$ & $68 / \angle Z / b$ & $68 / 0 \varepsilon / Z L$ & $68 / 0 \varepsilon / b$ & $06 / 1 / 9$ & ap!lon!y \\
\hline u/6n & $20+389^{\circ} 1>$ & or:1 & 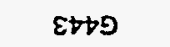 & LZEפ & 612808 & $68 / 62 / b$ & $68 / L Z / b$ & $68 / 0 \varepsilon / 2 !$ & $68 / 0 \varepsilon / t$ & $06 / 6 / 9$ & әp!nony \\
\hline iodey & $\begin{array}{c}\text { onjen } \\
\text { poyodey }\end{array}$ & $\begin{array}{l}(7 w / B) \\
\text { hỵsuəa }\end{array}$ & $\begin{array}{l}\text { JoqunN } \\
\text { ofdues qR? }\end{array}$ & \begin{tabular}{|c|} 
dequnN \\
wse yur
\end{tabular} & $\begin{array}{l}\text { JequnN } \\
\text { eldues }\end{array}$ & $\begin{array}{c}\text { olea } \\
\text { oldues } \\
\text { 4sị! }\end{array}$ & $\begin{array}{l}\text { olea } \\
\text { opdues } \\
\text { Heis }\end{array}$ & $\begin{array}{c}\text { elea s!skiput } \\
\text { ys!u!t }\end{array}$ & $\begin{array}{c}\text { ojeO } \\
\text { sishjeu } \\
\text { yEIS }\end{array}$ & $\begin{array}{l}\text { O2Ra } \\
\text { ourW }\end{array}$ & 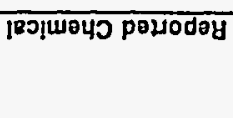 \\
\hline
\end{tabular}




\begin{tabular}{|c|c|c|c|c|c|c|c|}
\hline \multirow[b]{2}{*}{ Code } & \multirow[b]{2}{*}{ Source } & \multicolumn{3}{|c|}{ Analyte Information } & \multirow[b]{2}{*}{$\begin{array}{l}\text { Reference } \\
\text { Number }\end{array}$} & \multirow[b]{2}{*}{$\begin{array}{l}\text { Validated } \\
\text { (Y/N) }\end{array}$} & \multirow[b]{2}{*}{ Notes } \\
\hline & & Analyte & $\begin{array}{l}\text { Conc. } \\
\text { (ugg/L) }\end{array}$ & Conc. (mol/L) & & & \\
\hline $\mathbf{P}$ & Raw & $\mathbf{F}$ & \#VALUE! & \#VALUE! & AP102-3 & $\mathbf{Y}$ & Density reported for $\mathrm{G} 443$ represents a composite sample \\
\hline $\mathbf{P}$ & Raw & $\mathbf{F}$ & \#VALUEl & \#VALUE! & AP102-3 & $\mathbf{Y}$ & $\begin{array}{l}\text { Density not reported for G449. No assumptions could be } \\
\text { made }\end{array}$ \\
\hline $\mathbf{P}$ & Raw & $\mathbf{F}$ & \#VALUE! & \#VALUE! & AP102-3 & $\mathbf{Y}$ & $\begin{array}{l}\text { Density not reported for G450. Density assumed same as } \\
\text { G477 which was collected at } 182 \text { in from bottom of tank at } \\
\text { a } 30^{\circ} \text { angle and was keep heated to } 40^{\circ} \mathrm{C} \text {. }\end{array}$ \\
\hline $\mathbf{P}$ & Raw & F & NALUE! & \#VALUE! & AP102-3 & $\mathbf{Y}$ & $\begin{array}{l}\text { Density not reported for G453. No assumptions could be } \\
\text { made }\end{array}$ \\
\hline $\mathbf{P}$ & Raw & $F$ & \#VALUE! & *VALUE! & AP102-3 & $\mathbf{Y}$ & $\begin{array}{l}\text { Density not reported for G454. Density assumed same as } \\
\text { G471 which was collected at } 208 \text { in from bottom of tank at } \\
\text { a } 150^{\circ} \text { angle and was keep heated to } 40^{\circ} \mathrm{C} \text {. }\end{array}$ \\
\hline$P$ & Raw & $F$ & \#VALUE! & \#VALUE! & AP102-3 & $\mathbf{Y}$ & $\begin{array}{l}\text { Density reported for G } 459 \text { collected at } 226 \text { in from bottom } \\
\text { of tank at a } 270^{\circ} \text { angle. Sample was unheated, unheated } \\
\text { samples precipitated }\end{array}$ \\
\hline $\mathbf{P}$ & Raw & $F$ & \#VALUE! & \#VALUE! & AP102-3 & $Y$ & $\begin{array}{l}\text { Density reported for G460 collected at } 226 \text { in from bottom } \\
\text { of tank at a } 270^{\circ} \text { angle. Sample was unheated, unheated } \\
\text { samples precipitated }\end{array}$ \\
\hline$P$ & Raw & $\mathbf{F}$ & \#VALUE! & \#VALUE! & AP102-3 & $Y$ & $\begin{array}{l}\text { Density reported for } \mathrm{G} 470 \text { represents a composite sample. } \\
\text { Sample was kept heated to } 40^{\circ} \mathrm{C} \text {. }\end{array}$ \\
\hline$P$ & Raw & $F$ & \#VALUE! & "VALUE! & AP102-3 & $Y$ & $\begin{array}{l}\text { Density reported for G471 collected at } 208 \text { in from bottom } \\
\text { of tank at a } 150^{\circ} \text { angle and was keep heated to } 40^{\circ} \mathrm{C} \text {. }\end{array}$ \\
\hline$P$ & Raw & $F$ & \#VALUE! & \#VALUE! & AP102-3 & $\mathbf{Y}$ & $\begin{array}{l}\text { Density reported for G } 476 \text { collected at } 99 \text { in from bottom of } \\
\text { tank at a } 270^{\circ} \text { angle and was keep heated to } 40^{\circ} \mathrm{C} \text {. }\end{array}$ \\
\hline$P$ & Raw & $F$ & \#VALUE! & \#VALUE! & AP102-3 & $Y$ & $\begin{array}{l}\text { Density reported for } G 477 \text { collected at } 182 \text { in from bottom } \\
\text { of tank at a } 30^{\circ} \text { angle and was keep heated to } 40^{\circ} \mathrm{C} \text {. }\end{array}$ \\
\hline $\mathbf{P}$ & Raw & $F$ & \#VALUE! & \#VALUE! & AP102-3 & $\mathbf{Y}$ & $\begin{array}{l}\text { Density not reported for } \mathrm{G} 338 \text {. No assumptions could be } \\
\text { made }\end{array}$ \\
\hline$P$ & Raw & $\mathbf{F}$ & \#ALUE! & \#VALUE! & AP102-3 & $\mathbf{Y}$ & $\begin{array}{l}\text { Density reported for } \mathrm{G} 341 \text { collected at } 99 \text { in from bottom of } \\
\text { tank at a } 270^{\circ} \text { angle. }\end{array}$ \\
\hline$P$ & Raw & $\mathbf{F}$ & \#VALUE! & \#VALUE! & AP102-3 & $\mathbf{Y}$ & $\begin{array}{l}\text { Density reported for G342 collected at } 306 \text { in from bottom } \\
\text { of tank at a } 30^{\circ} \text { angle. Sample was unheated, unheated } \\
\text { samples precipitated }\end{array}$ \\
\hline$P$ & Raw & $\mathbf{F}$ & NALUE! & \#VALUE! & AP102-3 & $Y$ & $\begin{array}{l}\text { Density reported for G348 collected at } 125 \text { in from bottom } \\
\text { of tank at a } 30^{\circ} \text { angle. Sample was unheated, unheated } \\
\text { samples precipitated }\end{array}$ \\
\hline $\mathbf{P}$ & Raw & $F$ & \#VALUE! & \#VALUE! & AP102-3 & Y & $\begin{array}{l}\text { Density reported for G349 collected at } 34 \text { in from bottom of } \\
\text { tank at a } 30^{\circ} \text { angle. Sample was unheated, unheated } \\
\text { samples precipitated }\end{array}$ \\
\hline$P$ & Raw & $\mathbf{F}$ & \#VALUE! & \#VALUE! & AP102-3 & $Y$ & $\begin{array}{l}\text { Density reported for } \mathrm{G} 352 \text { collected at } 34 \text { in from bottom of } \\
\text { tank at a } 30^{\circ} \text { angle. Sample was unheated, unheated } \\
\text { samples precipitated }\end{array}$ \\
\hline$P$ & Raw & $\mathbf{F}$ & \#ALUEI & *VALUEI & AP102-3 & $\mathrm{Y}$ & $\begin{array}{l}\text { Density reported for } \mathrm{G} 353 \text { collected at } 334 \text { in from bottom } \\
\text { of tank at a } 150^{\circ} \text { angle. Sample was unheated, unheated } \\
\text { samples precipitated }\end{array}$ \\
\hline$P$ & Raw & $F$ & \#VALUEI & NALUE! & AP102-3 & $\mathbf{Y}$ & $\begin{array}{l}\text { Density reported for } G 358 \text { collected at } 334 \text { in from bottom } \\
\text { of tank at a } 150^{\circ} \text { angle. Sample was unheated, unheated } \\
\text { samples precipitated }\end{array}$ \\
\hline$P$ & Raw & $\mathbf{F}$ & \#VALUE! & \#VALUE! & AP102-3 & $\mathbf{Y}$ & $\begin{array}{l}\text { Density reported for } \mathbf{G 3 5 9} \cdot \text { collected at } 162 \text { in from bottom } \\
\text { of tank at a } 150^{\circ} \text { angle. Sample was unheated, unheated } \\
\text { samples precipitated }\end{array}$ \\
\hline $\mathbf{P}$ & Raw & $F$ & \#VALUE! & \#VALUE! & AP102-3 & $\mathbf{Y}$ & $\begin{array}{l}\text { Density reported for G363 collected at } 18 \text { in from bottom of } \\
\text { tank at a } 150^{\circ} \text { angle. Sample was unheated, unheated } \\
\text { samples precipitated }\end{array}$ \\
\hline
\end{tabular}


TWRS Privatization Support Pre Chemical Liquid;

\begin{tabular}{|c|c|c|c|c|c|c|c|c|c|c|c|}
\hline & & & & & ank Waste & haracteriza & on Sample I & ormation & & & \\
\hline Reported Chemical & $\begin{array}{c}\text { Memo } \\
\text { Date }\end{array}$ & $\begin{array}{c}\text { Start } \\
\text { Analysis } \\
\text { Date }\end{array}$ & $\begin{array}{c}\text { Finish } \\
\text { Analysis Date }\end{array}$ & $\begin{array}{c}\text { Start } \\
\text { Sample } \\
\text { Date } \\
\end{array}$ & $\begin{array}{c}\text { Finish } \\
\text { Sample } \\
\text { Date } \\
\end{array}$ & $\begin{array}{l}\text { Sample } \\
\text { Number }\end{array}$ & $\begin{array}{c}\text { Tank Farm } \\
\text { Number }\end{array}$ & $\begin{array}{c}\text { Lab Sample } \\
\text { Number }\end{array}$ & $\begin{array}{l}\text { Density } \\
(\mathrm{g} / \mathrm{mL})\end{array}$ & $\begin{array}{c}\text { Reported } \\
\text { Value }\end{array}$ & $\begin{array}{r}\text { Repo } \\
U_{n} \\
\end{array}$ \\
\hline Fluoride & $6 / 1 / 90$ & $4 / 30 / 89$ & $12 / 30 / 89$ & $4 / 27 / 89$ & $4 / 29 / 89$ & BOB2M5 & G315 & G364 & 1.19 & $<1.68 E+02$ & $u g / r$ \\
\hline Fluoride & $6 / 1 / 90$ & $4 / 30 / 89$ & $12 / 30 / 89$ & $4 / 27 / 89$ & $4 / 29 / 89$ & BOB2T9 & G327 & G443 & 1.20 & $<1.68 E+02$ & ug/r \\
\hline Fluoride & $6 / 1 / 90$ & $4 / 30 / 89$ & $12 / 30 / 89$ & $4 / 27 / 89$ & $4 / 29 / 89$ & 80B2M8 & G301 & G449 & NA & $<1.68 E+02$ & $u g / r$ \\
\hline Fluoride . & $6 / 1 / 90$ & $4 / 30 / 89$ & $12 / 30 / 89$ & $4 / 27 / 89$ & $4 / 29 / 89$ & ВОВ2M9 & G302 & G450 & $(1.20)$ & $<1.68 E+02$ & $u g / n$ \\
\hline Fluoride & $6 / 1 / 90$ & $4 / 30 / 89$ & $12 / 30 / 89$ & $4 / 27 / 89$ & $4 / 29 / 89$ & BOB2NO & G307 & G453 & NA & $<1.68 E+02$ & $u g / n$ \\
\hline Fluoride & $6 / 1 / 90$ & $4 / 30 / 89$ & $12 / 30 / 89$ & $4 / 27 / 89$ & $4 / 29 / 89$ & BOB2N1 & G310 & G454 & (1.19) & $<1.68 \varepsilon+02$ & $u g / n$ \\
\hline Fluoride & $6 / 1 / 90$ & $4 / 30 / 89$ & $12 / 30 / 89$ & $4 / 27 / 89$ & $4 / 29 / 89$ & BOB2ME & G318 & G459 & 1.20 & $<1.68 E+02$ & $\mathrm{ug} / \mathbf{\pi}$ \\
\hline Fluoride & $6 / 1 / 90$ & $4 / 30 / 89$ & $12 / 30 / 89$ & $4 / 27 / 89$ & $4 / 29 / 89$ & BOB2M7 & G319 & G460 & 1.2 & $<1.68 E+02$ & $u g / \pi$ \\
\hline Fluoride & $6 / 1 / 90$ & $4 / 30 / 89$ & $12 / 30 / 89$ & $4 / 27 / 89$ & $4 / 29 / 89$ & Bов2T9 & G327 & $\mathrm{G} 470^{*}$ & 1.19 & $<1.68 E+02$ & $\mathrm{ug} / \mathrm{m}$ \\
\hline Fluoride & $6 / 1 / 90$ & $4 / 30 / 89$ & $12 / 30 / 89$ & $4 / 27 / 89$ & $4 / 29 / 89$ & BOB2N1 & G310 & G471* & 1.19 & $<1.68 E+02$ & $\mathrm{ug} / \mathrm{m}$ \\
\hline Fluoride & $6 / 1 / 90$ & $4 / 30 / 89$ & $12 / 30 / 89$ & $4 / 27 / 89$ & $4 / 29 / 89$ & BOB2N4 & G323 & G476* & 1.21 & $<1.68 E+02$ & $\mathrm{ug} / \mathrm{m}$ \\
\hline Fluoride & $6 / 1 / 90$ & $4 / 30 / 89$ & $12 / 30 / 89$ & $4 / 27 / 89$ & $4 / 29 / 89$ & 8OB2M9 & G302 & G477* & 1.20 & $<1.68 \mathrm{E}+02$ & $u g / m$ \\
\hline Fluoride & $6 / 1 / 90$ & $4 / 30 / 89$ & $12 / 30 / 89$ & $4 / 27 / 89$ & $4 / 29 / 89$ & вов2T9 & G327 & & NA & $<1.32 E+01$ & $u g / m l$ \\
\hline$F$ & $4 / 28 / 84$ & NS & NS & NS & NS & R8469 & NS & NS & NS & $<6.64 E-04$ & $\bar{M}$ \\
\hline $\mathrm{F}$ - & NS & NS & NS & NS & NS & NS & NS & NS & 1.24 & $4.71 \mathrm{E}-02$ & Molel \\
\hline$F$ & $4 / 28 / 84$ & NS & NS & NS & NS & $R-8469$ & NS & NS & NS & $6.64 E-04$ & moles \\
\hline$F$. & $4 / 28 / 84$ & NS & NS & NS & NS & NS & NS & NS & NS & $1.26 \mathrm{E}-02$ & $g / L$ \\
\hline Iron & $6 / 1 / 90$ & $4 / 30 / 89$ & $12 / 30 / 89$ & $4 / 27 / 89$ & $4 / 29 / 89$ & ВОВ2Т9 & G327 & G470* & 1.19 & $<1.50 E+04$ & $u g / h$ \\
\hline Iron & $6 / 1 / 90$ & $4 / 30 / 89$ & $12 / 30 / 89$ & $4 / 27 / 89$ & $4 / 29 / 89$ & Вов2T9 & G327 & G470* & 1.19 & $<1.50 E+04$ & $\operatorname{ug} \boldsymbol{h}^{!}$ \\
\hline $\mathrm{Fe} 3+($ total $\mathrm{Fe})$ & NS & NS & NS & NS & NS & NS & NS & NS & 1.24 & $2.83 E-03$ & Mole/ \\
\hline $\mathrm{Fe}$ & $4 / 28 / 84$ & NS & NS & NS & NS & R8469 & NS & NS & NS & $1.90 \mathrm{E}+00$ & $\mathrm{ug} / \mathrm{mL}$ \\
\hline $\mathrm{Fe}$ & $4 / 28 / 84$ & NS & NS & NS & NS & NS & NS & NS & NS & $1.90 \mathrm{E}-03$ & $g / L$ \\
\hline $\mathrm{Fe}$ & $4 / 28 / 84$ & NS & NS & NS & NS & R-8469 & NS & NS & NS & $1.90 E+00$ & ugm/m \\
\hline Iron & $6 / 1 / 90$ & $4 / 30 / 89$ & $12 / 30 / 89$ & $4 / 27 / 89$ & $4 / 29 / 89$ & ВОВ2M2 & G309 & G358 & 1.20 & $1.05 E+04$ & $u g / L$ \\
\hline Iron & $6 / 1 / 90$ & $4 / 30 / 89$ & $12 / 30 / 89$ & $4 / 27 / 89$ & $4 / 29 / 89$ & BOB2NO & G307 & G453 & NA & $7.16 E+03$ & $u g h$ \\
\hline Iron & $6 / 1 / 90$ & $4 / 30 / 89$ & $12 / 30 / 89$ & $4 / 27 / 89$ & $4 / 29 / 89$ & 80B2T9 & G327 & G443 & 1.20 & $6.44 E+03$ & $\mathrm{ug} / \mathrm{L}$ \\
\hline Iron & 6/1/90 & $4 / 30 / 89$ & $12 / 30 / 89$ & $4 / 27 / 89$ & $4 / 29 / 89$ & BOB2L8 & G303 & G348 & 1.21 & $6.16 E+03$ & ug/L \\
\hline
\end{tabular}




\begin{tabular}{|c|c|c|c|c|c|c|c|}
\hline \multirow[b]{2}{*}{ Code } & \multirow[b]{2}{*}{ Source } & \multicolumn{3}{|c|}{ Analyte Information } & \multirow[b]{2}{*}{$\begin{array}{c}\text { Reference } \\
\text { Number }\end{array}$} & \multirow[b]{2}{*}{$\begin{array}{l}\text { Validated } \\
\text { (Y/N) }\end{array}$} & \multirow{3}{*}{$\begin{array}{l}\text { Notes } \\
\text { Density reported for G364 collected at } 372 \text { in from bottom } \\
\text { of tank at a } 270^{\circ} \text { angle. Sample was unheated, unheated } \\
\text { samples precipitated }\end{array}$} \\
\hline & & Analyte & $\begin{array}{l}\text { Conc. } \\
\text { (ug/L) }\end{array}$ & Conc. (mal/L) & & & \\
\hline $\mathbf{P}$ & Raw & $\mathbf{F}$ & \#VALUE! & \#VALUE! & AP102-3 & $Y$ & \\
\hline $\mathbf{P}$ & Raw & $\mathbf{F}$ & \#VALUE! & \#VALUE! & AP102-3 & $\mathbf{Y}$ & Density reported for $\mathbf{G} 443$ represents a composite sample \\
\hline $\mathbf{P}$ & Raw & $\mathbf{F}$ & \#ALUE! & \#VALUE! & AP102-3 & $Y$ & $\begin{array}{l}\text { Density not reported for G449. No assumptions could be } \\
\text { made }\end{array}$ \\
\hline $\mathbf{P}$ & Raw & $\mathbf{F}$ & \#NALUE! & \#VALUE! & AP102-3 & $\mathbf{Y}$ & $\begin{array}{l}\text { Density not reported for G450. Density assumed same as } \\
6477 \text { which was collected at } 182 \text { in from bottom of tank at } \\
\text { a } 30^{\circ} \text { angle and was keep heated to } 40^{\circ} \mathrm{C} \text {. }\end{array}$ \\
\hline $\mathbf{P}$ & Raw & $\mathbf{F}$ & \#VALUEI & *VALUE! & AP102-3 & Y & $\begin{array}{l}\text { Density not reported for G453. No assumptions could be } \\
\text { made }\end{array}$ \\
\hline $\mathbf{P}$ & Raw & $\mathbf{F}$ & \#VALUE! & \#VALUE! & AP102-3 & $Y$ & $\begin{array}{l}\text { Density not reported for G454. Density assumed same as } \\
G 471 \text { which was collected at } 208 \text { in from bottom of tank at } \\
\text { a } 150^{\circ} \text { angle and was keep heated to } 40^{\circ} \mathrm{C} \text {. }\end{array}$ \\
\hline$P$ & Raw & $F$ & \#VALUE! & \#VALUE! & AP102-3 & $\gamma$ & $\begin{array}{l}\text { Density reported for G459 collected at } 226 \text { in from bottom } \\
\text { of tank at a } 270^{\circ} \text { angle. Sample was unheated, unheated } \\
\text { samples precipitated }\end{array}$ \\
\hline$P$ & Raw & $\mathbf{F}$ & \#VALUE! & \#VALUE! & AP102-3 & $Y$ & $\begin{array}{l}\text { Density reported for } \mathrm{G} 460 \text { collected at } 226 \text { in from bottom } \\
\text { of tank at a } 270^{\circ} \text { angle. Sample was unheated, unheated } \\
\text { samples precipitated }\end{array}$ \\
\hline$P$ & Raw & $F$ & \#VALUE! & \#VALUE! & AP102-3 & $Y$ & $\begin{array}{l}\text { Density reported for G470 represents a composite sample. } \\
\text { Sample was kept heated to } 40^{\circ} \mathrm{C} \text {. }\end{array}$ \\
\hline$P$ & Raw & $F$ & \#VALUE! & \#VALUE! & AP102-3 & $Y$ & $\begin{array}{l}\text { Density reported for G471 collected at } 208 \text { in from bottom } \\
\text { of tank at a } 150^{\circ} \text { angle and was keep heated to } 40^{\circ} \mathrm{C} \text {. }\end{array}$ \\
\hline$P$ & Raw & $F$ & \#VALUEI & \#VALUE! & AP102-3 & $Y$ & $\begin{array}{l}\text { Density reported for G } 476 \text { collected at } 99 \text { in from bottom of } \\
\text { tank at a } 270^{\circ} \text { angle and was keep heated to } 40^{\circ} \mathrm{C} \text {. }\end{array}$ \\
\hline$P$ & Raw & $F$ & \#VALUE! & \#VALUE! & AP102-3 & $Y$ & $\begin{array}{l}\text { Density reported for } \mathrm{G} 477 \text { collected at } 182 \text { in from bottom } \\
\text { of tank at a } 30^{\circ} \text { angle and was keep heated to } 40^{\circ} \mathrm{C} \text {. }\end{array}$ \\
\hline $\mathbf{P}$ & Raw & $F$ & \#VALUE! & \#VALUEI & AP102-3 & $Y$ & $\begin{array}{l}\text { An aliquot from the composite sample was shipped to the } \\
\text { PUREX analytical laboratory for analysis of F- using the ion } \\
\text { specific electrode analytical method }\end{array}$ \\
\hline$L$ & Raw & $F$ & \#VALUE! & \#VALUEI & AP102-1 & $N$ & Outdated Information \\
\hline$L$ & HTCE Inv. & $F$ & $8.95 E+11$ & 4.71E+04 & NA & $\mathrm{N}$ & No Reference \\
\hline$P$ & Braun & $F$ & $1.26 E+10$ & $6.64 E+02$ & & $N$ & No Reference \\
\hline$P$ & Wastren & $F$ & $1.26 E+10$ & $6.63 E+02$ & NS & $\bar{N}$ & No Reference \\
\hline $\mathbf{P}$ & Raw & $\mathrm{Fe}$ & \#VALUE! & \#VALUE! & AP102-3 & $Y$ & $\begin{array}{l}\text { Density reported for } 6470 \text { represents a composite sample. } \\
\text { Sample was kept heated to } 40^{\circ} \mathrm{C} \text {. }\end{array}$ \\
\hline$P$ & Raw & $\mathrm{Fe}$ & \#VALUE! & \#VALUE! & AP102-3 & $Y$ & $\begin{array}{l}\text { Density reported for G470 represents a composite sample. } \\
\text { Sample was kept heated to } 40^{\circ} \mathrm{C} \text {. }\end{array}$ \\
\hline $\mathbf{L}$ & HTCE InV. & $\mathrm{Fe}$ & $1,58 \mathrm{E}+11$ & $2.83 E+03$ & NA & $\bar{N}$ & No Reference \\
\hline$L$ & Raw & $\mathrm{Fe}$ & $1.90 E+09$ & $3.40 E+01$ & AP102-1 & $\mathbf{N}$ & Outdated Information \\
\hline$P$ & Wastren & $\mathrm{Fe}$ & $1.90 E+09$ & $3.40 E+01$ & NS & $N$ & No Reference \\
\hline$P$ & Braun & $\mathrm{Fe}$ & $1.90 E+09$ & $3.40 E+01$ & & $N$ & No Reference \\
\hline $\mathbf{P}$ & Raw & $\mathrm{Fe}$ & $1.05 E+04$ & $1.88 E-04$ & AP102-3 & $Y$ & $\begin{array}{l}\text { Density reported for } \mathrm{G} 358 \text { collected at } 334 \text { in from bottom } \\
\text { of tank at a } 150^{\circ} \text { angle. Sample was unheated, unheated } \\
\text { samples precipitated }\end{array}$ \\
\hline $\mathbf{P}$ & Raw & $\mathrm{Fe}$ & $7.16 E+03$ & $1.28 \mathrm{E}-04$ & AP102-3 & $Y$ & $\begin{array}{l}\text { Density not reported for G453. No assumptions could be } \\
\text { made }\end{array}$ \\
\hline$P$ & Raw & $\mathrm{Fe}$ & $6.44 E+03$ & $1.15 E-04$ & AP102-3 & Y & Density reported for $\mathrm{G} 443$ represents a composite sample \\
\hline $\mathbf{P}$ & Raw & $\mathrm{Fe}$ & $6.16 \mathrm{E}+03$ & $1.10 \mathrm{E}-04$ & AP102-3 & $Y$ & $\begin{array}{l}\text { Density reported for G348 collected at } 125 \text { in from bottom } \\
\text { of tank at a } 30^{\circ} \text { angle. Sample was unheated, unheated } \\
\text { samples precipitated }\end{array}$ \\
\hline
\end{tabular}




\begin{tabular}{|c|c|c|c|c|c|c|c|c|c|c|c|}
\hline & & & & & ank Waste & haracteriza & ion Sample Ir & ormation & & & \\
\hline Reported Chemical & $\begin{array}{c}\text { Memo } \\
\text { Date }\end{array}$ & $\begin{array}{c}\text { Start } \\
\text { Analysis } \\
\text { Date }\end{array}$ & $\begin{array}{c}\text { Finish } \\
\text { Analysis Date }\end{array}$ & $\begin{array}{c}\text { Start } \\
\text { Sample } \\
\text { Date } \\
\end{array}$ & $\begin{array}{c}\text { Finish } \\
\text { Sample } \\
\text { Date } \\
\end{array}$ & $\begin{array}{l}\text { Sample } \\
\text { Number }\end{array}$ & $\begin{array}{c}\text { Tank Farm } \\
\text { Number }\end{array}$ & $\begin{array}{c}\text { Lab Sample } \\
\text { Number }\end{array}$ & $\begin{array}{l}\text { Density } \\
(\mathrm{g} / \mathrm{mL})\end{array}$ & $\begin{array}{c}\text { Reported } \\
\text { Value } \\
\end{array}$ & $\begin{array}{r}\text { Repol } \\
\text { Un } \\
\end{array}$ \\
\hline Iron & $6 / 7 / 90$ & $4 / 30 / 89$ & $12 / 30 / 89$ & $4 / 27 / 89$ & $4 / 29 / 89$ & BOB2L8 & G303 & G348 & 1.21 & $6.01 E+03$ & ug $f$ \\
\hline Iron & $6 / 1 / 90$ & $4 / 30 / 89$ & $12 / 30 / 89$ & $4 / 27 / 89$ & $4 / 29 / 89$ & BOB2T9 & G327 & G443 & 1.20 & $4.78 E+03$ & ugh \\
\hline Iron & $6 / 1 / 90$ & $4 / 30 / 89$ & $12 / 30 / 89$ & $4 / 27 / 89$ & $4 / 29 / 89$ & BOB2NO & G307 & G453 & NA & $4.65 E+03$ & $u g n$ \\
\hline Iron & $6 / 1 / 90$ & $4 / 30 / 89$ & $12 / 30 / 89$ & $4 / 27 / 89$ & $4 / 29 / 89$ & BOB2M1 & G308 & G353 & 1.20 & $4.38 E+03$ & ug/t \\
\hline fron & $6 / 1 / 90$ & $4 / 30 / 89$ & $12 / 30 / 89$ & $4 / 27 / 89$ & $4 / 29 / 89$ & BOB2M7 & G319 & G460 & 1.20 & $4.16 E+03$ & ugh \\
\hline Iton & $6 / 1 / 90$ & $4 / 30 / 89$ & $12 / 30 / 89$ & $4 / 27 / 89$ & $4 / 29 / 89$ & $\mathrm{BOB2L7}$ & G299 & G342 & 1.20 & $4.14 E+03$ & $u g / h$ \\
\hline Iron & $6 / 1 / 90$ & $4 / 30 / 89$ & $12 / 30 / 89$ & $4 / 27 / 89$ & $4 / 29 / 89$ & BOB2M1 & G308 & G353. & 1.20 & $3.99 E+03$ & $u g / h$ \\
\hline Iron & $6 / 1 / 90$ & $4 / 30 / 89$ & $12 / 30 / 89$ & $4 / 27 / 89$ & $4 / 29 / 89$ & BOB2N3 & G322 & G338 & NA & $3.93 E+03$ & $u g / L$ \\
\hline Iron & $6 / 1 / 90$ & 4/30/89 & $12 / 30 / 89$ & $4 / 27 / 89$ & $4 / 29 / 89$ & BOB2M5 & G315 & G364 & 1.19 & $3.87 E+03$ & $u g / L$ \\
\hline Iron & $6 / 1 / 90$ & $4 / 30 / 89$ & $12 / 30 / 89$ & $4 / 27 / 89$ & $4 / 29 / 89$ & BOB2N4 & G323 & G341 & 1.20 & $3.87 E+03$ & ugh $/ \mathrm{h}$ \\
\hline Iron & $6 / 1 / 90$ & $4 / 30 / 89$ & $12 / 30 / 89$ & $4 / 27 / 89$ & $4 / 29 / 89$ & BOB2M7 & G319 & $\mathrm{G} 460$ & 1.20 & $3.78 E+03$ & ug $/$ \\
\hline Iron & $6 / 1 / 90$ & $4 / 30 / 89$ & $12 / 30 / 89$ & $4 / 27 / 89$ & $4 / 29 / 89$ & BOB2M8 & G301 & G449 & NA & $3.65 E+03$ & ugh. \\
\hline Iron & $6 / 1 / 90$ & $4 / 30 / 89$ & $12 / 30 / 89$ & $4 / 27 / 89$ & $4 / 29 / 89$ & BOB2NT & G310 & G454 & (1.19) & $3.49 E+03$ & ug $/ \mathrm{L}$ \\
\hline Iron & $6 / 1 / 90$ & $4 / 30 / 89$ & $12 / 30 / 89$ & $4 / 27 / 89$ & $4 / 29 / 89$ & BOB2N1 & G310 & G454 & $(1.19)$ & $3.45 E+03$ & $u g / h$ \\
\hline Iron & $6 / 1 / 90$ & 4/30/89 & 12/30/89 & $4 / 27 / 89$ & $4 / 29 / 89$ & $8082 L 7$ & G299 & G342 & 1.20 & $3.41 E+03$ & ug/L \\
\hline Iron & $6 / 1 / 90$ & $4 / 30 / 89$ & $12 / 30 / 89$ & $.4 / 27 / 89$ & $4 / 29 / 89$ & BOB2M4 & G313 & G363 & 1.20 & $3.37 E+03$ & ug/L \\
\hline Iron & $6 / 1 / 90$ & $4 / 30 / 89$ & $12 / 30 / 89$ & $4 / 27 / 89$ & $4 / 29 / 89$ & ВOB2M3 & G311 & G359 & 1.20 & $3.36 E+03$ & ug $/ \mathrm{L}$ \\
\hline Iron & $6 / 1 / 90$ & $4 / 30 / 89$ & $12 / 30 / 89$ & $4 / 27 / 89$ & $4 / 29 / 89$ & ВОВ2M5 & G315 & G364 & 1.19 & $3.36 \mathrm{E}+03$ & ug/L \\
\hline Iron & $6 / 1 / 90$ & $4 / 30 / 89$ & $12 / 30 / 89$ & $4 / 27 / 89$ & $4 / 29 / 89$ & вов2M6 & G318 & G459 & 1.20 & $3.31 E+03$ & ug $\Omega$ \\
\hline iron & $6 / 1 / 90$ & $4 / 30 / 89$ & $12 / 30 / 89$ & $4 / 27 / 89$ & $4 / 29 / 89$ & BOB2N2 & G317 & G333 & NA & $3.19 E+03$ & $u g h$ \\
\hline Iron & $6 / 1 / 90$ & $4 / 30 / 89$ & $12 / 30 / 89$ & $4 / 27 / 89$ & $4 / 29 / 89$ & BOB2M9 & G302 & G450 & $(1.20)$ & $3.14 E+03$ & ugh \\
\hline
\end{tabular}




\begin{tabular}{|c|c|c|c|c|c|c|c|}
\hline & & & Analyte Inforn & ation & & & \\
\hline Code & Source & Analyte & $\begin{array}{l}\text { Conc. } \\
\text { (ug/L) }\end{array}$ & Conc. (mol/L) & $\begin{array}{l}\text { Reference } \\
\text { Number }\end{array}$ & $\begin{array}{l}\text { Validated } \\
\text { (Y/N) }\end{array}$ & Notes \\
\hline$P$ & Raw & $\mathrm{Fe}$ & $6.01 E+03$ & $1.08 E-04$ & AP102-3 & $\gamma$ & $\begin{array}{l}\text { Density reported for } \mathbf{G 3 4 8} \text { collected at } 125 \text { in from bottom } \\
\text { of tank at a } 30^{\circ} \text { angle. Sample was unheated, unheated } \\
\text { samples precipitated }\end{array}$ \\
\hline $\mathbf{P}$ & Raw & $\mathrm{Fe}$ & $4.78 E+03$ & $8.56 E-05$ & AP102-3 & Y & Density reported for $\mathrm{G} 443$ represents a composite sample \\
\hline$P$ & Raw & $\mathrm{Fe}$ & $4.65 E+03$ & 8.33E-05 & AP102-3 & $\gamma$ & $\begin{array}{l}\text { Density not reported for G453. No assumptions could be } \\
\text { made }\end{array}$ \\
\hline $\mathbf{P}$ & Raw & $\mathrm{Fe}$ & $4.38 \mathrm{E}+03$ & $7.84 E-05$ & AP102-3 & $\mathbf{Y}$ & $\begin{array}{l}\text { Density reported for G353 collected at } 334 \text { in from bottom } \\
\text { of tank at a } 150^{\circ} \text { angle. Sample was unheated, unheated } \\
\text { samples precipitated }\end{array}$ \\
\hline $\mathbf{P}$ & Raw & $\mathrm{Fe}$ & $4.16 E+03$ & 7.45E-05 & AP102-3 & $\mathrm{Y}$ & $\begin{array}{l}\text { Density reported for } \mathrm{G} 460 \text { collected at } 226 \text { in from bottom } \\
\text { of tank at a } 270^{\circ} \text { angle. Sample was unheated, unheated } \\
\text { samples precipitated }\end{array}$ \\
\hline $\mathbf{P}$ & Raw & $\mathrm{Fe}$ & $4.14 E+03$ & 7.41E-05 & APr02-3 & Y & $\begin{array}{l}\text { Density reported for } 6342 \text { collected at } 306 \text { in from bottom } \\
\text { of tank at a } 30^{\circ} \text { angle. Sample was unheated, unheated } \\
\text { samples precipitated }\end{array}$ \\
\hline $\mathbf{P}$ & Raw & $\mathrm{Fe}$ & $3.99 E+03$ & 7.14E-05 & AP102-3 & $\gamma$ & $\begin{array}{l}\text { Density reported for } \mathrm{G} 353 \text { collected at } 334 \text { in from bottom } \\
\text { of tank at a } 150^{\circ} \text { angle. Sample was unheated, unheated } \\
\text { samples precipitated }\end{array}$ \\
\hline$P$ & Raw & $\mathrm{Fe}$ & $3.93 E+03$ & $7.04 E-05$ & AP102-3 & $\mathrm{Y}$ & $\begin{array}{l}\text { Density not reported for G338. No assumptions could be } \\
\text { made }\end{array}$ \\
\hline $\mathbf{P}$ & Raw & $\mathrm{Fe}$ & $3.87 E+03$ & 6.93E-05 & AP102-3 & $Y$ & $\begin{array}{l}\text { Density reported for } G 364 \text { collected at } 372 \text { in from bottom } \\
\text { of tank at a } 270^{\circ} \text { angle. Sample was unheated, unheated } \\
\text { samples precipitated }\end{array}$ \\
\hline$P$ & Raw & $\mathrm{Fe}$ & $3.87 E+03$ & $6.93 E-05$ & AP102-3 & $Y$ & $\begin{array}{l}\text { Density reported for } 6341 \text { collected at } 99 \text { in from bottom of } \\
\text { tank at a } 270^{\circ} \text { angle. }\end{array}$ \\
\hline $\mathbf{P}$ & Raw & $\mathrm{Fe}$ & $3.78 E+03$ & 6.77E-05 & AP102-3 & $\mathrm{Y}$ & $\begin{array}{l}\text { Density reported for G460 collected at } 226 \text { in from bottom } \\
\text { of tank at a } 270^{\circ} \text { angle. Sample was unheated, unheated } \\
\text { samples precipitated }\end{array}$ \\
\hline $\mathbf{P}$ & Raw & $\mathrm{Fe}$ & $3.65 E+03$ & $6.54 E-05$ & AP102-3 & $Y$ & $\begin{array}{l}\text { Density not reported for } 6449 \text {. No assumptions could be } \\
\text { made }\end{array}$ \\
\hline $\mathbf{P}$ & Raw & $\mathrm{Fe}$ & $3.49 E+03$ & $6.25 E-05$ & AP102-3 & Y & $\begin{array}{l}\text { Density not reported for G454. Density assumed same as } \\
G 471 \text { which was collected at } 208 \text { in from bottom of tank at } \\
\text { a } 150^{\circ} \text { angle and was keep heated to } 40^{\circ} \mathrm{C} \text {. }\end{array}$ \\
\hline $\mathbf{P}$ & Raw & $\mathrm{Fe}$ & $3.45 E \div 03$ & $6.18 E-05$ & AP102-3 & $Y$ & $\begin{array}{l}\text { Density not reported for G454. Density assumed same as } \\
\text { G471 which was collected at } 208 \text { in from bottom of tank at } \\
\text { a } 150^{\circ} \text { angle and was keep heated to } 40^{\circ} \mathrm{C} \text {. }\end{array}$ \\
\hline $\mathbf{P}$ & flaw & $\mathrm{Fe}$ & $3.41 E+03$ & $6.11 E-05$ & AP102.3 & $Y$ & $\begin{array}{l}\text { Density reported for } \mathbf{G} 342 \text { collected at } 306 \text { in from bottom } \\
\text { of tank at a } 30^{\circ} \text { angle. Sample was unheated, unheated } \\
\text { samples precipitated }\end{array}$ \\
\hline $\mathbf{P}$ & Raw & $\mathrm{Fe}$ & $3.37 E+03$ & $6.03 E-05$ & AP102-3 & $Y$ & $\begin{array}{l}\text { Density reported for G363 collected at } 18 \text { in from bottom of } \\
\text { tank at a } 150^{\circ} \text { angle. Sample was unheated, unheated } \\
\text { samples precipitated }\end{array}$ \\
\hline $\mathbf{P}$. & Raw & $\mathrm{Fe}$ & $3.36 E \div 03$ & $6.02 E-05$ & AP102-3 & $Y$ & $\begin{array}{l}\text { Density reported for } 6359 \text { collected at } 162 \text { in from bottom } \\
\text { of tank at a } 150^{\circ} \text { angle. Sample was unheated, Unheated } \\
\text { samples precipitated }\end{array}$ \\
\hline $\mathbf{P}$ & Raw & $\mathrm{Fe}$ & $3.36 \mathrm{E}+03$ & $6.02 E-05$ & AP102-3 & $Y$ & $\begin{array}{l}\text { Density reported for G364 collected at } 372 \text { in from bottom } \\
\text { of tank at a } 270^{\circ} \text { angle. Sample was unheated, unheated } \\
\text { samples precipitated }\end{array}$ \\
\hline $\mathbf{P}$ & Raw & $\mathrm{Fe}$ & $3.31 E+03$ & 5.93E-05 & AP102-3 & $Y$ & $\begin{array}{l}\text { Density reported for } \mathrm{G} 459 \text { collected at } 226 \text { in from bottom } \\
\text { of tank at a } 270^{\circ} \text { angle. Sample was unheated, unheated } \\
\text { samples precipitated }\end{array}$ \\
\hline$P$ & Raw & $\mathrm{Fe}$ & $3.19 E+03$ & 5.71E-05 & AP102-3 & $\mathbf{Y}$ & $\begin{array}{l}\text { Density not reported for } \mathbf{G 3 3 3} \text {. No assumptions could be } \\
\text { made }\end{array}$ \\
\hline $\mathbf{P}$ & Raw & $\mathbf{F e}$ & $3.14 E+03$ & $5.62 E-05$ & AP102-3 & Y & $\begin{array}{l}\text { Density not reported for G450. Density assumed same as } \\
6477 \text { which was collected at } 182 \text { in from bottom of tank at } \\
\text { a } 30^{\circ} \text { angle and was keep heated to } 40^{\circ} \mathrm{C} \text {. }\end{array}$ \\
\hline
\end{tabular}




\begin{tabular}{|c|c|c|c|c|c|c|c|c|c|c|c|}
\hline & & & & & ank Waste & haracteriz & ion Sample Ir & ormation & & & \\
\hline Reported Chemical & $\begin{array}{c}\text { Memo } \\
\text { Date }\end{array}$ & $\begin{array}{c}\text { Start } \\
\text { Analysis } \\
\text { Date } \\
\end{array}$ & $\begin{array}{c}\text { Finish } \\
\text { Analysis Date }\end{array}$ & $\begin{array}{c}\text { Start } \\
\text { Sample } \\
\text { Date } \\
\end{array}$ & $\begin{array}{c}\text { Finish } \\
\text { Sample } \\
\text { Date } \\
\end{array}$ & $\begin{array}{l}\text { Sample } \\
\text { Number }\end{array}$ & $\begin{array}{c}\text { Tank Farm } \\
\text { Number }\end{array}$ & $\begin{array}{c}\text { Lab Sample } \\
\text { Number }\end{array}$ & $\begin{array}{l}\text { Density } \\
\text { (g/mL) }\end{array}$ & $\begin{array}{c}\text { Reported } \\
\text { Value } \\
\end{array}$ & $\begin{array}{r}\text { Repor } \\
\text { Uni }\end{array}$ \\
\hline Iron & $6 / 1 / 90$ & $4 / 30 / 89$ & $12 / 30 / 89$ & $4 / 27 / 89$ & $4 / 29 / 89$ & BOB2MO & G306 & G352 & 1.20 & $3.11 E+03$ & ug $\pi$ \\
\hline Iron & $6 / 1 / 90$ & $4 / 30 / 89$ & $12 / 30 / 89$ & $4 / 27 / 89$ & $4 / 29 / 89$ & BOB2N4 & G323 & G341 & 1.20 & $3.09 E+03$ & ugh \\
\hline Iron & $6 / 1 / 90$ & $4 / 30 / 89$ & $12 / 30 / 89$ & $4 / 27 / 89$ & $4 / 29 / 89$ & BOB2M9 & G302 & G450 & $(1.20)$ & $3.06 E+03$ & ugh \\
\hline Iron & $6 / 1 / 90$ & $4 / 30 / 89$ & $12 / 30 / 89$ & $4 / 27 / 89$ & $4 / 29 / 89$ & BOB2N3 & G322 & G338 & NA & $3.04 E+03$ & $u g /$ \\
\hline Iron & $6 / 1 / 90$ & $4 / 30 / 89$ & $12 / 30 / 89$ & $4 / 27 / 89$ & $4 / 29 / 89$ & BOB2L9 & G305 & G349 & 1.20 & $2.98 E+03$ & $u g /$ \\
\hline leon & $6 / 1 / 90$ & $4 / 30 / 89$ & $12 / 30 / 89$ & $4 / 27 / 89$ & $4 / 29 / 89$ & BOB2L9 & G305 & G349 & 1.20 & $2.97 E+03$ & $u g / 2$ \\
\hline Iron & $6 / 1 / 90$ & $4 / 30 / 89$ & 12/30/89 & $4 / 27 / 89$ & $4 / 29 / 89$ & Вов2M3 & G311 & G359 & 1.20 & $2.92 E+03$ & $u g h$ \\
\hline Iron & $6 / 1 / 90$ & $4 / 30 / 89$ & $12 / 30 / 89$ & $4 / 27 / 89$ & $4 / 29 / 89$ & Вов2M6 & G318 & G459 & 1.20 & $2.90 E+03$ & $u g / h$ \\
\hline Iron & $6 / 1 / 90$ & $4 / 30 / 89$ & $12 / 30 / 89$ & $4 / 27 / 89$ & $4 / 29 / 89$ & BOB2M4 & G313 & G363 & 1.20 & $2.89 E+03$ & ug $\Omega$ \\
\hline Iron & $6 / 1 / 90$ & $4 / 30 / 89$ & $12 / 30 / 89$ & $4 / 27 / 89$ & $4 / 29 / 89$ & BOB2MO & G306 & G352 & 1.20 & $2.79 E+03$ & $u g / L$ \\
\hline Iron & $6 / 1 / 90$ & $4 / 30 / 89$ & $12 / 30 / 89$ & $4 / 27 / 89$ & $4 / 29 / 89$ & BOB2N2 & G317 & G333 & NA & $2.63 E+03$ & ugh \\
\hline Iron & $6 / 1 / 90$ & $4 / 30 / 89$ & $12 / 30 / 89$ & $4 / 27 / 89$ & $4 / 29 / 89$ & ВОВ2M8 & G301 & G449 & NA & $2.60 E+03$ & $u g / h$ \\
\hline Iron & $6 / 1 / 90$ & $4 / 30 / 89$ & $12 / 30 / 89$ & $4 / 27 / 89$ & $4 / 29 / 89$ & ВОВ2M2 & G309 & G358 & 1.20 & $2.51 E+03$ & ug/h \\
\hline Mercury & $6 / 1 / 90$ & $4 / 30 / 89$ & $12 / 30 / 89$ & $4 / 27 / 89$ & $4 / 29 / 89$ & BOB2M9 & $\mathrm{G} 302$ & G477* & 1.20 & $<1.00 E+01$ & ug/h \\
\hline Mercury & $6 / 1 / 90$ & $4 / 30 / 89$ & $12 / 30 / 89$ & $4 / 27 / 89$ & $4 / 29 / 89$ & ВОВ2M9 & G302 & G477* & 1.20 & $<1.00 E+01$ & ug/h \\
\hline Mercury & $6 / 1 / 90$ & $4 / 30 / 89$ & $12 / 30 / 89$ & $4 / 27 / 89$ & $4 / 29 / 89$ & BOB2N1 & G310 & $\mathrm{G} 471^{\circ}$ & 1.19 & $<1.00 E+01$ & ug $\Omega$ \\
\hline Mercury & $6 / 1 / 90$ & $4 / 30 / 89$ & $12 / 30 / 89$ & $4 / 27 / 89$ & $4 / 29 / 89$ & BOB2N1 & G310 & G471 & 1.19 & $<1.00 E+01$ & ug $/$. \\
\hline Mercury & $6 / 1 / 90$ & $4 / 30 / 89$ & $12 / 30 / 89$ & $4 / 27 / 89$ & $4 / 29 / 89$ & $\mathrm{BOB} 2 \mathrm{~N} 4$ & G323 & G476* & 1.21 & $<1.00 E+01$ & $4 g h$ \\
\hline Mercury & $6 / 1 / 90$ & $4 / 30 / 89$ & $12 / 30 / 89$ & $4 / 27 / 89$ & $4 / 29 / 89$ & BOB2N4 & G323 & G476* & 1.21 & $<1.00 E+01$ & ugn. \\
\hline Mercury & $6 / 1 / 90$ & $4 / 30 / 89$ & $12 / 30 / 89$ & $4 / 27 / 89$ & $4 / 29 / 89$ & ВOB2T9 & G327 & G443 & 1.20 & $<5.00 E+\infty$ & ugh. \\
\hline Mercury & $6 / 1 / 90$ & $4 / 30 / 89$ & $12 / 30 / 89$ & $4 / 27 / 89$ & $4 / 29 / 89$ & ВОВ2T9 & G327 & G443 & 1.20 & $<5.00 E+\infty$ & $u g / h$. \\
\hline
\end{tabular}




\begin{tabular}{|c|c|c|c|c|c|c|c|}
\hline \multirow[b]{2}{*}{ Code } & \multirow[b]{2}{*}{ Source } & \multicolumn{3}{|c|}{ Analyte Information } & \multirow[b]{2}{*}{$\begin{array}{c}\text { Reference } \\
\text { Number }\end{array}$} & \multirow[b]{2}{*}{$\begin{array}{l}\text { Validated } \\
(Y / N)\end{array}$} & \multirow[b]{2}{*}{ Notes } \\
\hline & & Analyte & $\begin{array}{l}\text { Conc. } \\
\text { (ug/L) }\end{array}$ & Conc. (mol/L) & & & \\
\hline $\mathbf{P}$ & Raw & $\mathrm{Fe}$ & $3.11 E+03$ & 5.57E-05 & AP102-3 & $\mathbf{Y}$ & $\begin{array}{l}\text { Density reported for } \mathrm{G} 352 \text { collected at } 34 \text { in from bottom of } \\
\text { tank at a } 30^{\circ} \text { angle. Sample was unheated, unheated } \\
\text { samples precipitated }\end{array}$ \\
\hline $\mathbf{P}$ & Raw & $\mathrm{Fe}$ & $3.09 E+03$ & 5.53E-05 & AP102-3 & $\mathbf{Y}$ & $\begin{array}{l}\text { Density reported for } 6341 \text { collected at } 99 \text { in from bottom of } \\
\text { tank at a } 270^{\circ} \text { angle. }\end{array}$ \\
\hline $\mathbf{P}$ & Raw & $\mathrm{Fe}$ & $3.06 E+03$ & $5.48 E-05$ & AP102-3 & $\mathbf{Y}$ & $\begin{array}{l}\text { Density not reported for G450. Density assumed same as } \\
\text { G477 which was collected at } 182 \text { in from bottom of tank at } \\
\text { a } 30^{\circ} \text { angle and was keep heated to } 40^{\circ} \mathrm{C} \text {. }\end{array}$ \\
\hline $\mathbf{P}$ & Raw & $\mathrm{Fe}$ & $3.04 E+03$ & 5.44E-05 & AP102-3 & $\mathbf{Y}$ & $\begin{array}{l}\text { Density not reported for G338. No assumptions could be } \\
\text { made }\end{array}$ \\
\hline $\mathbf{P}$ & Raw & $\mathrm{Fe}$ & $2.98 E+03$ & 5.34E-05 & AP102-3 & $Y$ & $\begin{array}{l}\text { Density reported for } \mathrm{G} 349 \text { collected at } 34 \text { in from bottom of } \\
\text { tank at a } 30^{\circ} \text { angle. Sample was unheated, unheated } \\
\text { samples precipitated }\end{array}$ \\
\hline $\mathbf{P}$ & Raw & $\mathrm{Fe}$ & $2.97 E+03$ & 5.32E-05 & AP102-3 & $Y$ & $\begin{array}{l}\text { Density reported for } \mathbf{G 3 4 9} \text { collected at } 34 \text { in from bottom of } \\
\text { tank at a } 30^{\circ} \text { angle. Sample was unheated, unheated } \\
\text { samples precipitated }\end{array}$ \\
\hline$P$ & Raw & $\mathrm{Fe}$ & $2.92 E+03$ & 5.23E-05 & AP102-3 & $Y$ & $\begin{array}{l}\text { Density reported for G359 collected at } 162 \text { in from bottom } \\
\text { of tank at a } 150^{\circ} \text { angle. Sample was unheated, unheated } \\
\text { samples precipitated }\end{array}$ \\
\hline $\mathbf{P}$ & Raw & $\mathrm{Fe}$ & $2.90 E+03$ & 5.19E-05 & AP102-3 & $Y$ & $\begin{array}{l}\text { Density reported for } 6459 \text { collected at } 226 \text { in from bottom } \\
\text { of tank at a } 270^{\circ} \text { angle. Sample was unheated, unheated } \\
\text { samples precipitated }\end{array}$ \\
\hline$P$ & Raw & $\mathrm{Fe}$ & $2.89 E+03$ & 5.17E-05 & AP102-3 & $Y$ & $\begin{array}{l}\text { Density reported for } 6363 \text { collected at } 18 \text { in from bottom of } \\
\text { tank at a } 150^{\circ} \text { angle. Sample was unheated, unheated } \\
\text { samples precipitated }\end{array}$ \\
\hline $\mathbf{P}$ & Raw & $\mathrm{Fe}$ & $2.79 E+03$ & 5.00E-05 & AP102-3 & $Y$ & $\begin{array}{l}\text { Density reported for } \mathrm{G} 352 \text { collected at } 34 \text { in from bottom of } \\
\text { tank at a } 30^{\circ} \text { angle. Sample was unheated, unheated } \\
\text { samples precipitated }\end{array}$ \\
\hline$P$ & Raw & $\mathrm{Fe}$ & $2.63 E+03$ & $4.71 E-05$ & AP102-3 & $Y$ & $\begin{array}{l}\text { Density not reported for G333. No assumptions could be } \\
\text { made }\end{array}$ \\
\hline$P$ & Raw & $\mathrm{Fe}$ & $2.60 E+03$ & $4.66 \mathrm{E}-05$ & AP102-3 & $\gamma$ & $\begin{array}{l}\text { Density not reported for G449. No assumptions could be } \\
\text { made }\end{array}$ \\
\hline $\mathbf{P}$ & Raw & $\mathrm{Fe}$ & $2.51 E+03$ & 4.49E-05 & AP102-3 & $Y$ & $\begin{array}{l}\text { Density reported for G358 collected at } 334 \text { in from bottom } \\
\text { of tank at a } 150^{\circ} \text { angle. Sample was unheated, unheated } \\
\text { samples precipitated }\end{array}$ \\
\hline $\mathbf{P}$ & Raw & $\mathrm{Hg}$ & \#VALUE! & \#VALUE! & AP102-3 & $Y$ & $\begin{array}{l}\text { Density reported for } G 477 \text { collected at } 182 \text { in from bottom } \\
\text { of tank at a } 30^{\circ} \text { angle and was keep heated to } 40^{\circ} \mathrm{C} \text {. }\end{array}$ \\
\hline $\mathbf{P}$ & Raw & $\mathrm{Hg}$ & FALUE! & \#VALUE! & AP102-3 & $Y$ & $\begin{array}{l}\text { Density reported for } \mathrm{G} 477 \text { collected at } 182 \text { in from bottom } \\
\text { of tank at a } 30^{\circ} \text { angle and was keep heated to } 40^{\circ} \mathrm{C} \text {. }\end{array}$ \\
\hline$P$ & Raw & $\mathrm{Hg}$ & \#VALUE! & \#VALUE! & AP102-3 & $Y$ & $\begin{array}{l}\text { Density reported for } \mathrm{G} 471 \text { collected at } 208 \text { in from bottom } \\
\text { of tank at a } 150^{\circ} \text { angle and was keep heated to } 40^{\circ} \mathrm{C} \text {. }\end{array}$ \\
\hline$P$ & Raw & $\mathrm{Hg}$ & \#VALUE! & \#VALUE! & AP102-3 & $Y$ & $\begin{array}{l}\text { Density reported for G471 collected at } 208 \text { in from bottom } \\
\text { of tank at a } 150^{\circ} \text { angle and was keep heated to } 40^{\circ} \mathrm{C} \text {. }\end{array}$ \\
\hline$P$ & Raw & $\mathrm{Hg}$ & *VALUE! & \#VALUE! & AP102-3 & $\gamma$ & $\begin{array}{l}\text { Density reported for G476 collected at } 99 \text { in from bottom of } \\
\text { tank at a } 270^{\circ} \text { angle and was keep heated to } 40^{\circ} \mathrm{C} \text {. }\end{array}$ \\
\hline$P$ & Raw & $\mathrm{Hg}$ & VALUEI & FALUEI & AP102-3 & $Y$ & $\begin{array}{l}\text { Density reported for G476 collected at } 99 \text { in from bottom of } \\
\text { tank at a } 270^{\circ} \text { angle and was keep heated to } 40^{\circ} \mathrm{C} \text {. }\end{array}$ \\
\hline$P$ & Raw & $\mathrm{Hg}$ & \#VALUEI & \#VALUE! & AP102-3 & $Y$ & Density reported for $\mathrm{G} 443$ represents a composite sample \\
\hline $\mathbf{P}$ & Raw & $\mathrm{Hg}$ & \#VALUe! & \#VALUE! & AP102-3 & $\mathbf{Y}$ & Density reported for $\mathrm{G} 443$ represents a composite sample \\
\hline
\end{tabular}




\begin{tabular}{|c|c|c|c|c|c|c|c|c|c|c|c|}
\hline$\tau / 6 n$ & $90+\exists 0 \varepsilon^{\prime \prime} b$ & 071 & $\varepsilon 989$ & ยเยง & †WZgOB & $68 / 6 \tau / t$ & $68 / \angle Z / t$ & 68/0ع/ZL & $68 / 0 \varepsilon / t$ & $06 / 4 / 9$ & wn!ssejod \\
\hline $7 / 60 n$ & $90+\exists เ \varepsilon *\llcorner$ & $O Z 1$ & $09+9$ & 6เEง & $\angle W Z 808$ & $68 / 6 z / b$ & $68 / \angle Z / b$ & 68/0ع/ZL & $68 / 0 \varepsilon / t$ & $06 / 1 / 9$ & unissejod \\
\hline $7^{\prime 6 n}$ & $90+\exists L \varepsilon^{*}\llcorner$ & $o z=1$ & てちદり & 6629 & $\angle 7 z 808$ & $68 / 62 / t$ & $68 / L Z / b$ & $68 / 0 \varepsilon / Z L$ & $68 / 0 \varepsilon / t$ & $06 / 1 / 9$ & wn!ssejod \\
\hline $7 / 6 n$ & $90+3 L \varepsilon^{\circ} L$ & $O Z^{\prime} L$ & ItES & દてદ૭ & †NZEOg & $68 / 62 / t$ & $68 / \angle Z / b$ & $68 / 0 \varepsilon / 2 L$ & $68 / 0 \varepsilon / t$ & $06 / 4 / 9$ & un!sseiod \\
\hline $7 / 8 \mathbf{n}$ & $90+\exists L \varepsilon^{\circ} l$ & $\forall N$ & $6 t+9$ & LOES & 8WZ808 & $68 / 62 / t$ & $68 / L Z / t$ & 68/0E/ZL & $68 / 0 \varepsilon / t$ & $06 / L / 9$ & wnilsselod \\
\hline $7 / 6 n$ & $90+\exists เ \varepsilon^{\prime} \downarrow$ & $0 z \cdot 1$ & Eד & LZED & 612808 & $68 / 62 / t$ & $68 / L Z / b$ & $68 / 0 \varepsilon / Z L$ & $68 / 0 \varepsilon / t$ & $06 / 1 / 9$ & wn!ssejod \\
\hline $7 / 8 n$ & $90+\exists Z \varepsilon^{\circ}\llcorner$ & $\forall N$ & ES† & LOES & ONZROB & $68 / 6 Z / \$$ & $68 / L Z / b$ & $68 / 0 \varepsilon / Z 1$ & $68 / 0 \varepsilon / t$ & $06 / 2 / 9$ & wn!ssetod \\
\hline $7^{6 n}$ & $90+\exists Z \varepsilon^{\prime} !$ & $\forall N$ & 8Е६९ & 乙ટЕ૭ & ENZ8Og & $68 / 62 / t$ & $68 / L Z / b$ & $68 / 0 \varepsilon / Z L$ & $68 / 0 \varepsilon / t$ & $06 / 1 / 9$ & un!sselod \\
\hline $7 / 8 n$ & $90+\exists Z \varepsilon\llcorner$ & $\left.10 Z^{*} L\right)$ & $0 s+5$ & Z૦\&S & 6W2808 & $68 / 62 / 5$ & $68 / L Z / b$ & $68 / 0 \varepsilon / Z L$ & $68 / 0 \varepsilon / t$ & $06 / 2 / 9$ & un!sseiod \\
\hline $7 / 8 n$ & $90+\exists \varepsilon \varepsilon^{\prime}\llcorner$ & $0 Z \cdot 1$ & 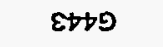 & $\angle Z E D$ & 612808 & $68 / 6 Z / t$ & $68 / L Z / t$ & $68 / 0 \varepsilon / 2 L$ & $68 / 0 \varepsilon / \hbar$ & $08 / 1 / 9$ & wn!ssejod \\
\hline $7 / 8 \pi$ & $90+\exists \varepsilon \varepsilon^{\circ}\llcorner$ & $(6 L \cdot L)$ & $\neg S t 9$ & OLES & INZgOB & $68 / 62 / t$ & $68 / L Z / t$ & $68 / 0 \varepsilon / 2 L$ & $68 / 0 \varepsilon / t$ & $06 / L / 9$ & wnı̣ssetod \\
\hline$\gamma^{8 \mathrm{~B}}$ & $90+\exists \varepsilon \varepsilon \cdot \downarrow$ & $\forall N$ & $\varepsilon s \neq 5$ & $\angle O E S$ & onzaog & $68 / 62 / 4$ & $68 / 2 Z / s$ & 68/0ع/LL & $68 / 0 \varepsilon / 7$ & $06 / 1 / 9$ & un!ssezod \\
\hline $7^{8 n}$ & $90+\exists \varepsilon \varepsilon^{\circ}\llcorner$ & $0 z \cdot\llcorner$ & เ†Eפ & ६२६९ & tNZ808 & $68 / 6 Z / t$ & $68 / L Z / b$ & $68 / 0 \varepsilon / Z L$ & $68 / 0 \varepsilon / t$ & $06 / 1 / 9$ & un!̣ssejod \\
\hline $7 / 6 n$ & $90+\exists \varepsilon \varepsilon^{\circ} \iota$ & $\forall N$ & 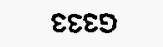 & $\angle I E D$ & ZNZROO & $68 / 62 / b$ & $68 / L Z / \hbar$ & $68 / 0 \varepsilon / Z L$ & $68 / 0 \varepsilon / t$ & $06 / 1 / 9$ & unı̣ssejod \\
\hline$\gamma^{B n}$ & $90+3 ৮ \varepsilon^{\prime} \downarrow$ & or:L & $09 \$ 5$ & GLEפ & $\angle W Z 808$ & $68 / 6 \tau / t$ & $68 / L Z / t$ & $68 / 0 \varepsilon / Z L$ & $68 / 0 \varepsilon / t$ & $06 / 1 / 9$ & wn!ssetod \\
\hline $7 / 6 n$ & $90+3 t \varepsilon^{\prime} L$ & 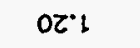 & $65 \rightarrow 9$ & 8เEง & 9 9wZgo8 & $68 / 6 \tau / t$ & $68 / L Z / b$ & $68 / 0 \varepsilon / 21$ & $68 / 0 \varepsilon / t$ & $06 / L / 9$ & wn!ssezod \\
\hline$\nu^{6 n}$ & $90+\exists S \varepsilon^{\prime} L$ & $6 L \div$ & †९อง & SIES & swzgog & $68 / 62 / b$ & $68 / \angle Z / b$ & $68 / 0 \varepsilon / Z \downarrow$ & $68 / 0 \varepsilon / t$ & $06 / 4 / 9$ & wn!ssejod \\
\hline $7 / 6 n$ & $90+39 \varepsilon^{\circ} L$ & $\forall N$ & $8 \varepsilon \varepsilon 9$ & 乙ZЕ૭ & ENZ808 & $68 / 6 z / t$ & $68 / L Z / \$$ & $68 / 0 \varepsilon / 2 L$ & $68 / 0 \varepsilon / t$ & $08 / 1 / 9$ & un!sseiod \\
\hline yon & $90+\exists \angle E^{\prime} L$ & $6 L L$ & $.0<t 5$ & $\angle Z \varepsilon \vartheta$ & 612808 & $68 / 62 / t$ & $68 / L Z / \$$ & $68 / 0 \varepsilon / Z 1$ & $68 / 0 \varepsilon / t$ & $06 / 4 / 9$ & unissetod \\
\hline$y 6 n$ & $90+30+1$ & $6 L^{\circ} \mathrm{L}$ & $.0<t 5$ & $\angle Z E S$ & 612808 & $68 / 62 / t$ & $68 / L Z / t$ & $68 / 0 \varepsilon / 2 L$ & $68 / 0 \varepsilon / t$ & $06 / L / 9$ & un!̣ssejod \\
\hline ग010 & $20-3 E+1$ & \pm 21 & SN & SN & $\mathrm{SN}$ & $\mathrm{SN}$ & $\mathrm{SN}$ & SN & $S N$ & SN & $+x$ \\
\hline i/gn & $90+\exists \angle \varepsilon^{\prime} l$ & SN & SN & $\angle Z \varepsilon Q$ & 612808 & SN & SN & SN & SN & $\varepsilon 6 / L / t$ & unisseiod \\
\hline 1/9n & $90+300^{\circ} L$ & SN & SN & $\angle Z E Q$ & 612808 & SN & SN & SN & SN & $\varepsilon 6 / L / t$ & wn!ssejod \\
\hline udd & $70 . \exists 6<^{\circ} \varepsilon$ & $\forall 9 \angle 996^{\circ} 0$ & SN & SN & SN & $\mathrm{SN}$ & SN & SN & SN & $68 / 01 / 2$ & $\mathrm{BH}$ \\
\hline uod & $t 0-300^{\circ} t$ & $10^{\circ} 1$ & SN & SN & $\mathbf{S N}$ & $\mathrm{SN}$ & SN & SN & SN & $68 / 01 / 2$ & BH \\
\hline $1 / 6 n$ & $100+300^{\circ} 9>$ & $6 L \cdot 2$ & $.0<+5$ & LZES & 612808 & $68 / 6 \tau / t$ & $68 / L Z / t$ & $68 / 0 \varepsilon / Z !$ & $68 / 0 \varepsilon / t$ & $06 / L / 9$ & Rnosaw \\
\hline i/6n & $100+300 \cdot 9>$ & $61 \cdot 1$ & $.0<+9$ & $\angle Z \varepsilon 9$ & 612808 & $68 / 6 \tau / t$ & $68 / L Z / 7$ & $68 / 0 \varepsilon / 21$ & $68 / 0 \varepsilon / t$ & $06 / L / 9$ & Nunasow \\
\hline $\begin{array}{l}\text { inn } \\
\text { sodoy }\end{array}$ & $\begin{array}{c}\text { enjen } \\
\text { peyoday }\end{array}$ & $\begin{array}{l}\text { (7w/6) } \\
\text { Ayssueg }\end{array}$ & $\begin{array}{c}\text { JogunN } \\
\text { opdwes qR7 }\end{array}$ & $\begin{array}{c}\text { JequnN } \\
\text { usef Xue } 1\end{array}$ & $\begin{array}{l}\text { JoqunN } \\
\text { opdwes }\end{array}$ & $\begin{array}{l}\text { ojea } \\
\text { गjdures } \\
\text { पs!lu! }\end{array}$ & $\begin{array}{l}\text { ojea } \\
\text { ajdures } \\
\text { jeis }\end{array}$ & $\begin{array}{c}\text { OIRO S!shjeut } \\
\text { 4s!u!s }\end{array}$ & 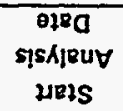 & $\begin{array}{l}\text { oped } \\
\text { owaw }\end{array}$ & 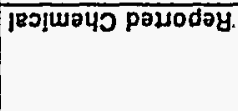 \\
\hline
\end{tabular}




\begin{tabular}{|c|c|c|c|c|c|c|c|}
\hline \multirow[b]{2}{*}{ Code } & \multirow[b]{2}{*}{ Source } & \multicolumn{3}{|c|}{ Analyte Information } & \multirow[b]{2}{*}{$\begin{array}{c}\text { Reference } \\
\text { Number }\end{array}$} & \multirow[b]{2}{*}{$\begin{array}{l}\text { Validated } \\
\text { (Y/N) }\end{array}$} & \multirow[b]{2}{*}{ Notes } \\
\hline & & Analyte & $\begin{array}{l}\text { Conc. } \\
\text { (ug/L) }\end{array}$ & Conc. (mol/L) & & & \\
\hline $\mathbf{P}$ & Raw & $\mathrm{Hg}$ & \#VALUE! & \#VALUE! & AP102-3 & $\mathbf{Y}$ & $\begin{array}{l}\text { Density reported for } G 470 \text { represents a composite sample. } \\
\text { Sample was kept heated to } 40^{\circ} \mathrm{C} \text {. }\end{array}$ \\
\hline $\mathbf{P}$ & Raw & $\mathrm{Hg}$ & VALUE! & \#ALUE! & AP102-3 & $\mathbf{Y}$ & $\begin{array}{l}\text { Density reported for } \mathrm{G} 470 \text { represents a composite sample. } \\
\text { Sample was kept heated to } 40^{\circ} \mathrm{C} \text {. }\end{array}$ \\
\hline $\mathbf{U}$ & RAW & $\mathrm{Hg}$ & $4.04 E+05$ & $2.01 E-03$ & AP102-2 & $\mathbf{N}$ & Projected value, not a real data point \\
\hline$\underline{U}$ & RAW & $\mathrm{Hg}$ & $3.63 E+05$ & $1.81 E-03$ & AP102-2 & $\mathbf{N}$ & Projected value, not a real data point \\
\hline $\mathbf{P}$ & TCD & $K$ & $1.40 E+12$ & $3.58 E+04$ & & $\mathbf{N}$ & THIS POINT NOT GIVEN IN REPORT WHC-SD-WM-TRP-169 \\
\hline$P$ & TCD & $\bar{K}$ & $1.37 E+12$ & $3.50 E+04$ & & $\mathbf{N}$ & THIS POINT NOT GIVEN IN REPORT WHC-SD-WM-TRP-169 \\
\hline L & HTCE Inv. & $\mathrm{K}$ & $5.60 E+11$ & $1.43 E+04$ & NA & $N$ & No Reference \\
\hline $\mathbf{P}$ & Raw & $\mathrm{k}$ & $1.40 E+06$ & $3.58 E-02$ & AP102-3 & $\mathbf{Y}$ & $\begin{array}{l}\text { Density reported for } \mathrm{G} 470 \text { represents a composite sample. } \\
\text { Sample was kept heated to } 40^{\circ} \mathrm{C} \text {. }\end{array}$ \\
\hline $\mathbf{P}$ & Raw & $\mathrm{k}$ & $1.37 E+06$ & $3.50 E-02$ & AP102-3 & $\mathbf{Y}$ & $\begin{array}{l}\text { Density reported for G470 represents a composite sample. } \\
\text { Sample was kept heated to } 40^{\circ} \mathrm{C} \text {. }\end{array}$ \\
\hline$?$ & Raw & K & $1.35 E+06$ & $3.45 E-02$ & AP102-3 & $\mathbf{Y}$ & $\begin{array}{l}\text { Density not reported for G338. No assumptions could be } \\
\text { made }\end{array}$ \\
\hline $\mathbf{P}$ & Raw & $k$ & $1.35 E+06$ & $3.45 E-02$ & AP102-3 & $\mathbf{Y}$ & $\begin{array}{l}\text { Density reported for G364 collected at } 372 \text { in from bottom } \\
\text { of tank at a } 270^{\circ} \text { angle. Sample was unheated, unheated } \\
\text { samples precipitated }\end{array}$ \\
\hline $\mathbf{P}$ & Raw & $\mathrm{k}$ & $1.34 \varepsilon+06$ & $3.43 E-02$ & AP102-3 & $\mathbf{Y}$ & $\begin{array}{l}\text { Density reported for } \mathbf{G} 459 \text { collected at } 226 \text { in from bottom } \\
\text { of tank at a } 270^{\circ} \text { angle. Sample was unheated, unheated } \\
\text { samples precipitated }\end{array}$ \\
\hline $\mathbf{P}$ & Raw & K & $1.34 E+06$ & $3.43 E-02$ & AP102-3 & $\mathbf{Y}$ & $\begin{array}{l}\text { Density reported for } G 460 \text { collected at } 226 \text { in from bottom } \\
\text { of tank at a } 270^{\circ} \text { angle. Sample was unheated, unheated } \\
\text { samples precipitated }\end{array}$ \\
\hline $\mathbf{P}$ & Raw & $\mathrm{K}$ & $1.33 E+06$ & $3.40 E-02$ & AP102-3 & $\mathbf{Y}$ & $\begin{array}{l}\text { Density not reported for G333. No assumptions could be } \\
\text { made }\end{array}$ \\
\hline $\mathbf{P}$ & Raw & K & $1.33 E+06$ & $3.40 E-02$ & AP102-3 & $\mathbf{Y}$ & $\begin{array}{l}\text { Density reported for } \mathrm{G} 341 \text { collected at } 99 \text { in from bottom of } \\
\text { tank at a } 270^{\circ} \text { angle. }\end{array}$ \\
\hline $\mathbf{P}$ & Raw & $\mathrm{K}$ & $1.33 E+06$ & $3.40 E-02$ & AP102-3 & $\mathbf{Y}$ & $\begin{array}{l}\text { Density not reported for } 6453 \text {. No assumptions could be } \\
\text { made }\end{array}$ \\
\hline $\mathbf{P}$ & Raw & $\mathrm{K}$ & $1.33 E+06$ & 3.40E-02 & AP102-3 & $\mathbf{Y}$ & $\begin{array}{l}\text { Density not reported for G454. Density assumed same as } \\
\text { G471 which was collected at } 208 \text { in from bottom of tank at } \\
\text { a } 150^{\circ} \text { angle and was keep heated to } 40^{\circ} \mathrm{C} \text {. }\end{array}$ \\
\hline $\mathbf{P}$ & Raw & K & $1.33 E+06$ & $3.40 E-02$ & AP102-3 & $\mathbf{Y}$ & Density reported for $\mathrm{G} 443$ represents a composite sample \\
\hline $\mathbf{P}$ & Raw & K & $1.32 E+06$ & 3.38E-02 & AP102-3 & $\mathbf{Y}$ & $\begin{array}{l}\text { Density not reported for G450. Density assumed same as } \\
\text { G477 which was collected at } 182 \text { in from bottom of tank at } \\
\text { a } 30^{\circ} \text { angle and was keep heated to } 40^{\circ} \mathrm{C} \text {. }\end{array}$ \\
\hline $\mathbf{P}$ & Raw & K & $1.32 E+06$ & $3.38 \mathrm{E}-02$ & AP102-3 & Y & $\begin{array}{l}\text { Density not reported for } \mathrm{G} 338 \text {. No assumptions could be } \\
\text { made }\end{array}$ \\
\hline $\mathbf{P}$ & Raw & K & $1.32 E+06$ & $3.38 \mathrm{E}-02$ & AP102-3 & $Y$ & $\begin{array}{l}\text { Density not reported for G453. No assumptions could be } \\
\text { made }\end{array}$ \\
\hline $\mathbf{P}$ & Raw & K & $1.31 E+06$ & $3.35 \mathrm{E}-02$ & AP102-3 & $\mathbf{Y}$ & Density reported for $\mathrm{G} 443$ represents a composite sample \\
\hline $\mathbf{P}$ & Raw & K & $1.31 E+06$ & 3.35E-02 & AP102-3 & $Y$ & $\begin{array}{l}\text { Density not reported for G449. No assumptions could be } \\
\text { made }\end{array}$ \\
\hline $\mathbf{P}$ & Raw & K & $1.31 E+06$ & 3.35E-02 & AP102-3 & Y & $\begin{array}{l}\text { Density reported for } G 341 \text { collected at } 99 \text { in from bottom of } \\
\text { tank at a } 270^{\circ} \text { angle. }\end{array}$ \\
\hline $\mathbf{P}$ & Raw & $k$ & $1.31 E+06$ & $3.35 E-02$ & AP102-3 & $Y$ & $\begin{array}{l}\text { Density reported for } \mathrm{G} 342 \text { collected at } 306 \text { in from bottom } \\
\text { of tank at a } 30^{\circ} \text { angle. Sample was unheated, unheated } \\
\text { samples precipitated }\end{array}$ \\
\hline$P$ & Raw & K & $1.31 E+06$ & $3.35 E-02$ & AP102-3 & $Y$ & $\begin{array}{l}\text { Density reported for G460 collected at } 226 \text { in from bottom } \\
\text { of tank at a } 270^{\circ} \text { angle. Sample was unheated, unheated } \\
\text { samples precipitated }\end{array}$ \\
\hline$P$ & Raw & $k$ & $1.30 E+06$ & 3.32E-02 & AP102-3 & $Y$ & $\begin{array}{l}\text { Density reported for } \mathrm{G} 363 \text { collected at } 18 \text { in from bottom of } \\
\text { tank at a } 150^{\circ} \text { angle. Sample was unheated, unheated } \\
\text { samples precipitated }\end{array}$ \\
\hline
\end{tabular}




\begin{tabular}{|c|c|c|c|c|c|c|c|c|c|c|c|}
\hline & & & & & ank Waste & haracteriz & ion Sample & ormation & & & \\
\hline Reported Chemical & $\begin{array}{l}\text { Memo } \\
\text { Date }\end{array}$ & $\begin{array}{c}\text { Start } \\
\text { Analysis } \\
\text { Date }\end{array}$ & $\begin{array}{c}\begin{array}{c}\text { Finish } \\
\text { Analysis Date }\end{array} \\
\end{array}$ & $\begin{array}{c}\text { Start } \\
\text { Sample } \\
\text { Date }\end{array}$ & $\begin{array}{c}\text { Finish } \\
\text { Sample } \\
\text { Date } \\
\end{array}$ & $\begin{array}{l}\text { Sample } \\
\text { Number }\end{array}$ & $\begin{array}{c}\text { Tank Farm } \\
\text { Number }\end{array}$ & $\begin{array}{c}\text { Lab Sample } \\
\text { Number }\end{array}$ & $\begin{array}{l}\text { Density } \\
(\mathrm{g} / \mathrm{mL})\end{array}$ & $\begin{array}{c}\text { Reported } \\
\text { Value }\end{array}$ & $\begin{array}{r}\text { Repo } \\
U_{n} \\
\end{array}$ \\
\hline Potassium & $6 / 7 / 90$ & $4 / 30 / 89$ & $12 / 30 / 89$ & $4 / 27 / 89$ & $4 / 29 / 89$ & 8OB2M5 & G315 & G364 & 1.19 & $1.30 E+06$ & ugl \\
\hline Potassium & $6 / 1 / 90$ & $4 / 30 / 89$ & $12 / 30 / 89$ & $4 / 27 / 89$ & $4 / 29 / 89$ & BOB2N2 & G317 & G333 & NA & $1.30 E+06$ & ugf \\
\hline Potassium & $6 / 1 / 90$ & 4/30/89 & $12 / 30 / 89$ & $4 / 27 / 89$ & $4 / 29 / 89$ & BOB2L7 & G299 & G342 & 1.20 & $1.29 E+06$ & $u g l$ \\
\hline Potassium & $6 / 1 / 90$ & $4 / 30 / 89$ & $12 / 30 / 89$ & $4 / 27 / 89$ & $4 / 29 / 89$ & BOB2L9 & G305 & G349 & 1.20 & $1.29 E+06$ & $u g n$ \\
\hline Potassium & $6 / 1 / 90$ & $4 / 30 / 89$ & $12 / 30 / 89$ & $4 / 27 / 89$ & $4 / 29 / 89$ & ВОВ2M4 & G313 & G363 & 1.20 & $1.29 E+06$ & $\operatorname{ug} h$ \\
\hline Potassium & $6 / 1 / 90$ & $4 / 30 / 89$ & $12 / 30 / 89$ & $4 / 27 / 89$ & $4 / 29 / 89$ & BOB2N1 & G310 & G454 & $(1.19)$ & $1.29 E+06$ & $u g / t$ \\
\hline Potassium & $6 / 1 / 90$ & $4 / 30 / 89$ & $12 / 30 / 89$ & $4 / 27 / 89$ & $4 / 29 / 89$ & BOB2L9 & G305 & G349 & 1.20 & $1.28 E+06$ & $u g / \Lambda$ \\
\hline Potassium & $6 / 1 / 90$ & $4 / 30 / 89$ & $12 / 30 / 89$ & $4 / 27 / 89$ & $4 / 29 / 89$ & $8082 \mathrm{M9}$ & G302 & G450 & $(1.20)$ & $1.28 E+06$ & $u g / h$ \\
\hline Potassium & $6 / 1 / 90$ & $4 / 30 / 89$ & $12 / 30 / 89$ & $4 / 27 / 89$ & $4 / 29 / 89$ & BOB2M1 & G308 & G353 & 1.20 & $1.27 E+06$ & $u g / L$ \\
\hline Potassium & $6 / 1 / 90$ & $4 / 30 / 89$ & $12 / 30 / 89$ & $4 / 27 / 89$ & $4 / 29 / 89$ & BOB2M 8 & G301 & G449 & NA & $1.27 E+06$ & $u g / L$ \\
\hline Potassium & $6 / 1 / 90$ & $4 / 30 / 89$ & $12 / 30 / 89$ & $4 / 27 / 89$ & 4/29/89 & BOB2L8 & G303 & G348 & 1.21 & $1.26 E+06$ & $u g / L$ \\
\hline Potassium & $6 / 1 / 90$ & $4 / 30 / 89$ & $12 / 30 / 89$ & $4 / 27 / 89$ & $4 / 29 / 89$ & BOB2MO & G306 & G352 & 1.20 & $1.26 E+06$ & ug/L. \\
\hline Potassium & $6 / 1 / 90$ & $4 / 30 / 89$ & $12 / 30 / 89$ & $4 / 27 / 89$ & $4 / 29 / 89$ & ВОВ2M6 & G318 & G459 & 1.20 & $1.26 E+06$ & ug/h \\
\hline Potassium & $6 / 1 / 90$ & $4 / 30 / 89$ & $12 / 30 / 89$ & $4 / 27 / 89$ & $4 / 29 / 89$ & BOB2L8 & G303 & G348 & 1.21 & $1.25 E+06$ & ug $\Omega$. \\
\hline Potassium & $6 / 1 / 90$ & $4 / 30 / 89$ & $12 / 30 / 89$ & $4 / 27 / 89$ & $4 / 29 / 89$ & BOB2M1 & G308 & G353 & 1.20 & $1.25 E+06$ & $u g / L$ \\
\hline Potassium & $6 / 1 / 90$ & $4 / 30 / 89$ & $12 / 30 / 89$ & $4 / 27 / 89$ & $4 / 29 / 89$ & BOB2MO & G306 & G352 & 1.20 & $1.23 E+06$ & $u g / h$ \\
\hline Potassium & $6 / 1 / 90$ & $4 / 30 / 89$ & $12 / 30 / 89$ & $4 / 27 / 89$ & 4/29/89 & BOB2M2 & 6309 & G358 & 1.20 & $1.22 E+06$ & $u g / h$ \\
\hline Potassium & $6 / 1 / 90$ & $4 / 30 / 89$ & $12 / 30 / 89$ & $4 / 27 / 89$ & $4 / 29 / 89$ & 8ОВ2M3 & G311 & G359 & 1.20 & $1.21 E+06$ & ug $/$ L \\
\hline Potassium & $6 / 1 / 90$ & $4 / 30 / 89$ & $12 / 30 / 89$ & $4 / 27 / 89$ & $4 / 29 / 89$ & ВOB2M2 & G309 & G358 & 1.20 & $1.20 E+06$ & $\operatorname{ug} \Omega$ \\
\hline Potassium & $6 / 1 / 90$ & $4 / 30 / 89$ & $12 / 30 / 89$ & $4 / 27 / 89$ & $4 / 29 / 89$ & ВОВ2M3 & G311 & G359 & 1.20 & $1.19 E+06$ & ug $/$. \\
\hline $\mathrm{Na}$ & $4 / 28 / 84$ & NS & NS & NS & NS & R8469 & NS & NS & NS & $1.25 E+04$ & $\overline{U G / M L}$ \\
\hline
\end{tabular}




\begin{tabular}{|c|c|c|c|c|c|c|c|}
\hline \multirow[b]{2}{*}{ Code } & \multirow[b]{2}{*}{ Source } & \multicolumn{3}{|c|}{ Analyte Information } & \multirow[b]{2}{*}{$\begin{array}{c}\text { Reference } \\
\text { Number }\end{array}$} & \multirow[b]{2}{*}{$\begin{array}{l}\text { Validated } \\
\text { (Y/N) }\end{array}$} & \multirow[b]{2}{*}{ Notes } \\
\hline & & Analyte & $\begin{array}{l}\text { Conc. } \\
\text { (ug/L) }\end{array}$ & Conc. (mol/L) & & & \\
\hline$P$ & Raw & K & $1.30 E+06$ & $3.32 E-02$ & AP102-3 & $Y$ & $\begin{array}{l}\text { Density reported for } 6364 \text { collected at } 372 \text { in from bottom } \\
\text { of tank at a } 270^{\circ} \text { angle. Sample was unheated, unheated } \\
\text { samples precipitated }\end{array}$ \\
\hline $\mathbf{P}$ & Raw & K & $1.30 E+06$ & $3.32 E-02$ & AP102-3 & $Y$ & $\begin{array}{l}\text { Density not reported for G333. No assumptions could be } \\
\text { made }\end{array}$ \\
\hline $\mathbf{P}$ & Raw & K & $1.29 E+06$ & $3.30 E-02$ & AP102-3 & $Y$ & $\begin{array}{l}\text { Density reported for } \mathrm{G} 342 \text { collected at } 306 \text { in from bottom } \\
\text { of tank at a } 30^{\circ} \text { angle. Sample was unheated, unheated } \\
\text { samples precipitated }\end{array}$ \\
\hline $\mathbf{P}$ & Raw & K & $1.29 E+06$ & $3.30 E-02$ & AP102-3 & $Y$ & $\begin{array}{l}\text { Density reported for } \mathrm{G} 349 \text { collected at } 34 \text { in from bottom of } \\
\text { tank at a } 30^{\circ} \text { angle. Sample was unheated, unheated } \\
\text { samples precipitated }\end{array}$ \\
\hline $\mathbf{P}$ & Raw & $x^{x}$ & $1.29 E+06$ & $3.30 E-02$ & AP102-3 & $Y$ & $\begin{array}{l}\text { Density reported for } \mathrm{G} 363 \text { collected at } 18 \text { in from bottom of } \\
\text { tank at a } 150^{\circ} \text { angle. Sample was unheated, unheated } \\
\text { samples precipitated }\end{array}$ \\
\hline $\mathbf{P}$ & Raw & $\kappa$ & $1.29 E+06$ & 3.30E-02 & AP102-3 & $Y$ & $\begin{array}{l}\text { Density not reported for G454. Density assumed same as } \\
\mathrm{G} 471 \text { which was collected at } 208 \text { in from bottom of tank at } \\
\text { a } 150^{\circ} \text { angle and was keep heated to } 40^{\circ} \mathrm{C} \text {. }\end{array}$ \\
\hline $\mathbf{P}$ & Raw & K & $1.28 E+06$ & 3.27E-02 & AP102-3 & $Y$ & $\begin{array}{l}\text { Density reported for } \mathrm{G} 349 \text { collected at } 34 \text { in from bottom of } \\
\text { tank at a } 30^{\circ} \text { angle. Sample was unheated, unheated } \\
\text { samples precipitated }\end{array}$ \\
\hline $\mathbf{P}$ & Raw & $k$ & $1.28 E+06$ & 3.27E-02 & AP102-3 & $\mathbf{Y}$ & $\begin{array}{l}\text { Density not reported for G450. Density assumed same as } \\
\mathrm{G} 477 \text { which was collected at } 182 \text { in from bottom of tank at } \\
\text { a } 30^{\circ} \text { angle and was keep heated to } 40^{\circ} \mathrm{C} \text {. }\end{array}$ \\
\hline $\mathbf{P}$ & Raw & $k$ & $1.27 E+06$ & $3.25 \mathrm{E}-02$ & AP102-3 & $Y$ & $\begin{array}{l}\text { Density reported for G353 collected at } 334 \text { in from bottom } \\
\text { of tank at a } 150^{\circ} \text { angle. Sample was unheated, unheated } \\
\text { samples precipitated }\end{array}$ \\
\hline $\mathbf{P}$ & Raw & $k$ & $1.27 E+06$ & $3.25 E-02$ & AP102-3 & $Y$ & $\begin{array}{l}\text { Density not reported for G449. No assumptions could be } \\
\text { made }\end{array}$ \\
\hline $\mathbf{P}$ & Raw & $k$ & $1.26 E+06$ & $3.22 \mathrm{E}-02$ & AP102-3 & $Y$ & $\begin{array}{l}\text { Density reported for } \mathrm{G348} \text { collected at } 125 \text { in from bottom } \\
\text { of tank at a } 30^{\circ} \text { angle. Sample was unheated, unheated } \\
\text { samples precipitated }\end{array}$ \\
\hline $\mathbf{P}$ & Raw & K & $1.26 E+06$ & $3.22 E-02$ & AP102-3 & $Y$ & $\begin{array}{l}\text { Density reported for } \mathrm{G} 352 \text { collected at } 34 \text { in from bottom of } \\
\text { tank at a } 30^{\circ} \text { angle. Sample was unheated, unheated } \\
\text { samples precipitated }\end{array}$ \\
\hline $\mathbf{P}$ & Raw & $\mathrm{K}$ & $1.26 \mathrm{E}+06$ & $3.22 E-02$ & AP102-3 & $Y$ & $\begin{array}{l}\text { Density reported for G459 coltected at } 226 \text { in from bottom } \\
\text { of tank at a } 270^{\circ} \text { angle. Sample was unheated, unheated } \\
\text { samples precipitated }\end{array}$ \\
\hline $\mathbf{P}$ & Raw & K & $1.25 \mathrm{E}+06$ & $3.20 E-02$ & AP102-3 & $Y$ & $\begin{array}{l}\text { Density reported for G348 collected at } 125 \text { in from bottom } \\
\text { of tank at a } 30^{\circ} \text { angle. Sample was unheated, unheated } \\
\text { samples precipitated }\end{array}$ \\
\hline$P$ & Raw & $k$ & $1.25 E+06$ & $3.20 E-02$ & AP102-3 & $Y$ & $\begin{array}{l}\text { Density reported for G353 collected at } 334 \text { in from bottom } \\
\text { of tank at a } 150^{\circ} \text { angle. Sample was unheated, unheated } \\
\text { samples precipitated }\end{array}$ \\
\hline $\mathbf{P}$ & Raw & k & $1.23 \mathrm{E}+06$ & 3.15E-02 & AP102-3 & $Y$ & $\begin{array}{l}\text { Density reported for } \mathrm{G} 352 \text { collected at } 34 \text { in from bottom of } \\
\text { tank at a } 30^{\circ} \text { angle. Sample was unheated, unheated } \\
\text { samples precipitated }\end{array}$ \\
\hline P & Raw & K & $1.22 E+06$ & $3.12 \mathrm{E}-02$ & AP102-3 & Y & $\begin{array}{l}\text { Density reported for G358 collected at } 334 \text { in from bottom } \\
\text { of tank at a } 150^{\circ} \text { angle. Sample was unheated, unheated } \\
\text { samples precipitated }\end{array}$ \\
\hline $\mathbf{P}$ & Raw & K & $1.21 E+06$ & 3.09E-02 & AP102-3 & $\mathbf{Y}$ & $\begin{array}{l}\text { Density reported for G359 collected at } 162 \text { in from bottom } \\
\text { of tank at a } 150^{\circ} \text { angle. Sample was unheated, unheated } \\
\text { samples precipitated }\end{array}$ \\
\hline $\mathbf{P}$ & Raw & K & $1.20 E+06$ & $3.07 E-02$ & AP102.3 & $\mathbf{Y}$ & $\begin{array}{l}\text { Density reported for } 6358 \text { collected at } 334 \text { in from bottom } \\
\text { of tank at a } 150^{\circ} \text { angle. Sample was unheated, unheated } \\
\text { samples precipitated }\end{array}$ \\
\hline$P$ & Raw & $k$ & $1.19 E+06$ & $3.04 E-02$ & AP102-3 & $\mathbf{Y}$ & $\begin{array}{l}\text { Density reported for G359 collected at } 162 \text { in from bottom } \\
\text { of tank at a } 150^{\circ} \text { angle. Sample was unheated, unheated } \\
\text { samples precipitated }\end{array}$ \\
\hline $\bar{P}$ & RAW & $\mathrm{Na}$ & $1.25 E+13$ & $5.43 E+05$ & AP102-1 & $\mathbf{N}$ & Outdated information \\
\hline
\end{tabular}




\begin{tabular}{|c|c|c|c|c|c|c|c|c|c|c|c|}
\hline & & & & & ank Waste & haracteriza & ion Sample I & ormation & & & \\
\hline Reported Chemical & $\begin{array}{c}\text { Memo } \\
\text { Date }\end{array}$ & $\begin{array}{c}\text { Start } \\
\text { Analysis } \\
\text { Date }\end{array}$ & $\begin{array}{c}\text { Finish } \\
\text { Analysis Date }\end{array}$ & $\begin{array}{c}\text { Start } \\
\text { Sample } \\
\text { Date }\end{array}$ & $\begin{array}{c}\text { Finish } \\
\text { Sample } \\
\text { Date }\end{array}$ & $\begin{array}{l}\text { Sample } \\
\text { Number }\end{array}$ & $\begin{array}{c}\text { Tank Farm } \\
\text { Number }\end{array}$ & $\begin{array}{c}\text { Lab Sample } \\
\text { Number }\end{array}$ & $\begin{array}{l}\text { Density } \\
\text { (g/mL) }\end{array}$ & $\begin{array}{l}\text { Reported } \\
\text { Value }\end{array}$ & $\begin{array}{r}\operatorname{Rep} \\
\mathbf{U}\end{array}$ \\
\hline $\mathrm{Na}$ & $4 / 28 / 84$ & NS & NS & NS & NS & NS & NS & NS & NS & $1.25 E+01$ & 9 \\
\hline $\mathrm{Na}$ & $4 / 28 / 84$ & NS & NS & NS & NS & $R-8469$ & NS & NS & NS & $1.25 E+04$ & ugn \\
\hline $\mathrm{Na}$ & $2 / 10 / 89$ & NS & NS & NS & NS & NS & NS & NS & 1.01 & $1.16 E+04$ & $\mathrm{Pr}$ \\
\hline $\mathrm{Na}$ & $2170 / 89$ & NS & NS & NS & NS & NS & NS & NS & 0.956764 & $1.14 E+04$ & $\overrightarrow{p r}$ \\
\hline Sodium & $6 / 1 / 90$ & $4 / 30 / 89$ & $12 / 30 / 89$ & $4 / 27 / 89$ & $4 / 29 / 89$ & BOB2L9 & G305 & G349 & 1.20 & $1.06 E+08$ & $u \varepsilon$ \\
\hline Sodium & $6 / 1 / 90$ & $4 / 30 / 89$ & $12 / 30 / 89$ & $4 / 27 / 89$ & $4 / 29 / 89$ & BOB2T9 & G327 & G470 & 1.19 & $1.06 E+0 B$ & ug \\
\hline Sodium & $6 / 1 / 90$ & $4 / 30 / 89$ & $12 / 30 / 89$ & $4 / 27 / 89$ & $4 / 29 / 89$ & BOB2NO & G307 & G453 & NA & $1.05 E+08$ & ug \\
\hline Sodium & $6 / 1 / 90$ & $4 / 30 / 89$ & $12 / 30 / 89$ & $4 / 27 / 89$ & $4 / 29 / 89$ & ВОВ2T9 & 6327 & G470* & 1.19 & $1.05 E+08$ & ug \\
\hline Sodium & $6 / 1 / 90$ & $4 / 30 / 89$ & $12 / 30 / 89$ & $4 / 27 / 89$ & $4 / 29 / 89$ & Вов2M9 & G302 & G450 & $(1.20)$ & $1.04 E+08$ & ug \\
\hline Sodium & $6 / 1 / 90$ & $4 / 30 / 89$ & $12 / 30 / 89$ & $4 / 27 / 89$ & $4 / 29 / 89$ & BOB2M9 & G302 & G450 & $(1.20)$ & $1.04 E+08$ & ug \\
\hline Sodium & $6 / 1 / 90$ & $4 / 30 / 89$ & $12 / 30 / 89$ & $4 / 27 / 89$ & $4 / 29 / 89$ & BOB2N3 & G322 & G338 & NA & $1.03 E+08$ & ugi \\
\hline Sodium & $6 / 1 / 90$ & 4/30/89 & $12 / 30 / 89$ & $4 / 27 / 89$ & $4 / 29 / 89$ & BOB2N1 & G310 & G454 & (1.19) & $1.03 E+08$ & ugi \\
\hline Sodium & $6 / 1 / 90$ & $4 / 30 / 89$ & $12 / 30 / 89$ & $4 / 27 / 89$ & $4 / 29 / 89$ & BOB2N3 & G322 & G338 & NA & $1.03 E+08$ & $\operatorname{ug} /$ \\
\hline Sodium & $6 / 1 / 90$ & $4 / 30 / 89$ & $12 / 30 / 89$ & $4 / 27 / 89$ & 4/29/89 & BOB2L8 & G303 & G348 & 1.21 & $1.03 E+08$ & ugl \\
\hline Sodium & $6 / 1 / 90$ & $4 / 30 / 89$ & $12 / 30 / 89$ & $4 / 27 / 89$ & $4 / 29 / 89$ & Вов2M2 & G309 & G358 & 1.20 & $1.03 E+08$ & ug/l \\
\hline Sodium & $6 / 1 / 90$ & $4 / 30 / 89$ & $12 / 30 / 89$ & $4 / 27 / 89$ & $4 / 29 / 89$ & вОВ2М5 & G315 & G364 & 1.19 & $1.03 E+08$ & ug/l \\
\hline Sodium & $6 / 1 / 90$ & $4 / 30 / 89$ & $12 / 30 / 89$ & $4 / 27 / 89$ & $4 / 29 / 89$ & BOB2N1 & G310 & G454 & (1.19) & $1.03 E+08$ & $u g / h$ \\
\hline Sodium & $6 / 1 / 90$ & $4 / 30 / 89$ & $12 / 30 / 89$ & $4 / 27 / 89$ & $4 / 29 / 89$ & BOB2L8 & G303 & G348 & 1.21 & $1.02 E+08$ & $u g h$ \\
\hline Sodium & $6 / 1 / 90$ & $4 / 30 / 89$ & $12 / 30 / 89$ & $4 / 27 / 89$ & $4 / 29 / 89$ & BOB2L9 & G305 & G349 & 1.20 & $1.02 E \div 08$ & ug $/ 4$ \\
\hline Sodium & $6 / 1 / 90$ & $4 / 30 / 89$ & $12 / 30 / 89$ & $4 / 27 / 89$ & $4 / 29 / 89$ & В082T9 & G327 & G443 & 1.20 & $1.02 E+08$ & $u g / h$ \\
\hline Sodium & $6 / 1 / 90$ & $4 / 30 / 89$ & $12 / 30 / 89$ & $4 / 27 / 89$ & $4 / 29 / 89$ & ВОВ2M6 & G318 & G459 & 1.20 & $1.02 \mathrm{E}+08$ & ug/L \\
\hline Sodium & $6 / 1 / 90$ & $4 / 30 / 89$ & $12 / 30 / 89$ & $4 / 27 / 89$ & $4 / 29 / 89$ & BOB2MO & G306 & G352 & 1.20 & $1.02 E+08$ & ugh \\
\hline Sodium & $6 / 1 / 90$ & $4 / 30 / 89$ & $12 / 30 / 89$ & $4 / 27 / 89$ & $4 / 29 / 89$ & вОВ2Т9 & G327 & G443 & 1.20 & $1.02 E+08$ & $u g /$ \\
\hline Sodium & $6 / 1 / 90$ & 4/30/89 & $12 / 30 / 89$ & $4 / 27 / 89$ & $4 / 29 / 89$ & BOB2M8 & G301 & G449 & NA & $1.02 E+08$ & ug $n$. \\
\hline Sodium & $6 / 1 / 90$ & $4 / 30 / 89$ & $12 / 30 / 89$ & $4 / 27 / 89$ & $4 / 29 / 89$ & BOB2NO & G307 & G453 & NA & $1.02 E+08$ & ugh \\
\hline
\end{tabular}




\begin{tabular}{|c|c|c|c|c|c|c|c|}
\hline & & & Inalyte Inforn & ation & & & \\
\hline Code & Source & Analyte & $\begin{array}{l}\text { Conc. } \\
\text { (ug/L) }\end{array}$ & Conc. (mol/L) & $\begin{array}{c}\text { Reference } \\
\text { Number }\end{array}$ & $\begin{array}{c}\text { Validated } \\
(Y / N)\end{array}$ & Notes \\
\hline 8 & Wastren & $\mathrm{Na}$ & $1.25 E+13$ & $5.43 E+05$ & NS & $N$ & No Reference \\
\hline$P$ & Braun & $\mathrm{Na}$ & $1.25 E+13$ & $5.43 E+05$ & & $\mathbf{N}$ & No Reference \\
\hline $\mathbf{U}$ & RAW & $\mathrm{Na}$ & $1.17 E+13$ & $5.09 E+05$ & AP102-2 & N & Projected value, not a real data point \\
\hline u & RAW & $\mathrm{Na}$ & $1.09 E+13$ & $4.74 \mathrm{E}+05$ & AP102-2 & $\mathbf{N}$ & Projected value, not a real data point \\
\hline$P$ & Raw & $\mathrm{Na}$ & $1.06 E+08$ & $4.61 E+\infty$ & AP102-3 & $Y$ & $\begin{array}{l}\text { Density reported for G349 collected at } 34 \text { in from bottom of } \\
\text { tank at a } 30^{\circ} \text { angle. Sample was unheated, unheated } \\
\text { samples precipitated }\end{array}$ \\
\hline$p$ & Raw & $\mathrm{Na}$ & $1.06 E+08$ & $4.61 E+\infty 0$ & AP102-3 & $Y$ & $\begin{array}{l}\text { Density reported for G470 represents a composite sample. } \\
\text { Sample was kept heated to } 40^{\circ} \mathrm{C} \text {. }\end{array}$ \\
\hline$p$ & Raw & $\mathrm{Na}$ & $1.05 E+08$ & $4.57 E+\infty 0$ & AP102-3 & $\mathrm{Y}$ & $\begin{array}{l}\text { Density not reported for G453. No assumptions could be } \\
\text { made }\end{array}$ \\
\hline $\mathbf{P}$ & Raw & $\mathrm{Na}$ & $1.05 E+08$ & $4.57 E+\infty 0$ & AP102-3 & $Y$ & $\begin{array}{l}\text { Density reported for } 6470 \text { represents a composite sample. } \\
\text { Sample was kept heated to } 40^{\circ} \mathrm{C} \text {. }\end{array}$ \\
\hline $\mathbf{P}$ & Raw & $\mathrm{Na}$ & $1.04 E+08$ & $4.52 E+\infty 0$ & AP102-3 & $Y$ & $\begin{array}{l}\text { Density not reported for G450. Density assumed same as } \\
\text { G477 which was collected at } 182 \text { in from bottom of tank at } \\
\text { a } 30^{\circ} \text { angle and was keep heated to } 40^{\circ} \mathrm{C} \text {. }\end{array}$ \\
\hline$P$ & Raw & $\mathrm{Na}$ & $1.04 E+08$ & $4.52 E+\infty$ & AP102-3 & $Y$ & $\begin{array}{l}\text { Density not reported for G450. Density assumed same as } \\
\text { G477 which was collected at } 182 \text { in from bottom of tank at } \\
\text { a } 30^{\circ} \text { angle and was keep heated to } 40^{\circ} \mathrm{C} \text {. }\end{array}$ \\
\hline$P$ & Raw & $\mathrm{Na}$ & $1.03 E+08$ & $4.48 E+\infty$ & AP102-3 & $Y$ & $\begin{array}{l}\text { Density not reported for G338. No assumptions could be } \\
\text { made }\end{array}$ \\
\hline$P$ & Raw & $\mathrm{Na}$ & $1.03 E+08$ & $4.48 E+\infty$ & AP102-3 & $Y$ & $\begin{array}{l}\text { Density not reported for G454. Density assumed same as } \\
\text { G471 which was collected at } 208 \text { in from bottom of tank at } \\
\text { a } 150^{\circ} \text { angle and was keep heated to } 40^{\circ} \mathrm{C} \text {. }\end{array}$ \\
\hline $\mathbf{P}$ & Raw & $\mathrm{Na}$ & $1.03 E+08$ & $4.48 E+\infty$ & AP102-3 & $Y$ & $\begin{array}{l}\text { Density not reported for G338. No assumptions could be } \\
\text { made }\end{array}$ \\
\hline$P$ & Raw & $\mathrm{Na}$ & $1.03 E+08$ & $4.48 E+\infty 0$ & AP102-3 & $Y$ & $\begin{array}{l}\text { Density reported for G348 collected at } 125 \text { in from bottom } \\
\text { of tank at a } 30^{\circ} \text { angle. Sample was unheated, unheated } \\
\text { samples precipitated }\end{array}$ \\
\hline$P$ & Raw & $\mathrm{Na}$ & $1.03 E+08$ & $4.48 E+\infty$ & AP102-3 & $Y$ & $\begin{array}{l}\text { Density reported for } G 358 \text { collected at } 334 \text { in from bottom } \\
\text { of tank at a } 150^{\circ} \text { angle. Sample was unheated, unheated } \\
\text { samples precipitated }\end{array}$ \\
\hline $\mathbf{P}$ & Raw & $\mathrm{Na}$ & $1.03 E+08$ & $4.48 E+\infty 0$ & AP102-3 & $\mathbf{Y}$ & $\begin{array}{l}\text { Density reported for G364 collected at } 372 \text { in from bottom } \\
\text { of tank at a } 270^{\circ} \text { angle. Sample was unheated, unheated } \\
\text { samples precipitated }\end{array}$ \\
\hline $\mathbf{P}$ & Raw & $\mathrm{Na}$ & $1.03 E+08$ & $4.48 E+\infty$ & AP102-3 & $\gamma$ & $\begin{array}{l}\text { Density not reported for G454. Density assumed same as } \\
\text { G471 which was collected at } 208 \text { in from bottom of tank at } \\
\text { a } 150^{\circ} \text { angle and was keep heated to } 40^{\circ} \mathrm{C} \text {. }\end{array}$ \\
\hline $\mathbf{P}$ & Raw & $\mathrm{Na}$ & $1.02 E+08$ & $4.43 E+\infty 0$ & AP102-3 & $Y$ & $\begin{array}{l}\text { Density reported for G348 collected at } 125 \text { in from bottom } \\
\text { of tank at a } 30^{\circ} \text { angle. Sample was unheated, unheated } \\
\text { samples precipitated }\end{array}$ \\
\hline $\mathbf{P}$ & Raw & $\mathrm{Na}$ & $1.02 E+08$ & $4.43 E+\infty$ & AP102-3 & $Y$ & $\begin{array}{l}\text { Density reported for } \mathrm{G} 349 \text { collected at } 34 \text { in from bottom of } \\
\text { tank at a } 30^{\circ} \text { angle. Sample was unheated, unheated } \\
\text { samples precipitated }\end{array}$ \\
\hline $\mathbf{P}$ & Raw & $\mathrm{Na}$ & $1.02 E+08$ & $4.43 E+\infty$ & AP102-3 & $\gamma$ & Density reported for $\mathrm{G} 443$ represents a composite sample \\
\hline $\mathbf{P}$ & Raw & $\mathrm{Na}$ & $1.02 E+08$ & $4.43 E \div 00$ & AP102-3 & $Y$ & $\begin{array}{l}\text { Density reported for } 6459 \text { collected at } 226 \text { in from bottom } \\
\text { of tank at a } 270^{\circ} \text { angle. Sample was unheated, unheated } \\
\text { samples precipitated }\end{array}$ \\
\hline$P$ & Raw & $\mathrm{Na}$ & $1.02 E+08$ & $4.43 E+\infty 0$ & AP102-3 & $Y$ & $\begin{array}{l}\text { Density reported for } \mathrm{G} 352 \text { collected at } 34 \text { in from bottom of } \\
\text { tank at a } 30^{\circ} \text { angle. Sample was unheated, unheated } \\
\text { samples precipitated }\end{array}$ \\
\hline P & Raw & $\mathrm{Na}$ & $1.02 E+08$ & $4.43 E+\infty 0$ & AP102-3 & $Y$ & Density reported for $\mathrm{G} 443$ represents a composite sample \\
\hline $\mathbf{P}$ & Raw & $\mathrm{Na}$ & $1.02 E+08$ & $4.43 E+\infty$ & AP102-3 & $Y$ & $\begin{array}{l}\text { Density not reported for G449. No assumptions could be } \\
\text { made }\end{array}$ \\
\hline$P$ & Raw & $\mathrm{Na}$ & $1.02 E+08$ & $4.43 E+00$ & AP102-3 & $Y$ & $\begin{array}{l}\text { Density not reported for } \mathrm{G} 453 \text {. No assumptions could be } \\
\text { made }\end{array}$ \\
\hline
\end{tabular}


TWRS Privatization Support Pr Chemical Liquic

\begin{tabular}{|c|c|c|c|c|c|c|c|c|c|c|c|}
\hline Reported Chemical & $\begin{array}{c}\text { Memo } \\
\text { Date }\end{array}$ & $\begin{array}{c}\text { Start } \\
\text { Analysis } \\
\text { Date }\end{array}$ & $\begin{array}{c}\text { Finish } \\
\text { Analysis Date }\end{array}$ & $\begin{array}{c}\text { Start } \\
\text { Sample } \\
\text { Date } \\
\end{array}$ & $\begin{array}{c}\text { Finish } \\
\text { Sample } \\
\text { Date }\end{array}$ & $\begin{array}{l}\text { Sample } \\
\text { Number }\end{array}$ & $\begin{array}{c}\text { Tank Farm } \\
\text { Number }\end{array}$ & $\begin{array}{c}\text { Lab Sample } \\
\text { Number }\end{array}$ & $\begin{array}{l}\text { Density } \\
(\mathrm{g} / \mathrm{mL})\end{array}$ & $\begin{array}{c}\text { Reported } \\
\text { Value } \\
\end{array}$ & $\begin{array}{r}\text { Repl } \\
\mathrm{U} \\
\end{array}$ \\
\hline Sodium & $6 / 1 / 90$ & $4 / 30 / 89$ & $12 / 30 / 89$ & $4 / 27 / 89$ & $4 / 29 / 89$ & 80B2M6 & G318 & G459 & 1.20 & $1.02 E+08$ & u! \\
\hline Sodium & $6 / 1 / 90$ & $4 / 30 / 89$ & $12 / 30 / 89$ & $4 / 27 / 89$ & $4 / 29 / 89$ & $\mathrm{BOB2M7}$ & G319 & G460 & 1.2 & $1.02 E+08$ & $u q$ \\
\hline Sodium & $6 / 1 / 90$ & $4 / 30 / 89$ & $12 / 30 / 89$ & $4 / 27 / 89$ & $4 / 29 / 89$ & BOB2N4 & G323 & G341 & 1.20 & $1.01 E+08$ & ug \\
\hline Sodium & $6 / 1 / 90$ & $4 / 30 / 89$ & $12 / 30 / 89$ & $4 / 27 / 89$ & $4 / 29 / 89$ & BOB2MO & G306 & G352 & 1.20 & $1.01 E+08$ & ug \\
\hline Sodium & $6 / 1 / 90$ & $4 / 30 / 89$ & $12 / 30 / 89$ & $4 / 27 / 89$ & $4 / 29 / 89$ & BOB2M1 & G308 & G353 & 1.20 & $1.01 E+08$ & ug \\
\hline Sodium & $6 / 1 / 90$ & $4 / 30 / 89$ & $12 / 30 / 89$ & $4 / 27 / 89$ & $4 / 29 / 89$ & BOB2M8 & G301 & G449 & NA & $1.01 E+08$ & ug \\
\hline Sodium & $6 / 1 / 90$ & $4 / 30 / 89$ & $12 / 30 / 89$ & $4 / 27 / 89$ & $4 / 29 / 89$ & BOB2N4 & G323 & G341 & 1.20 & $1.01 E+08$ & ug \\
\hline Sodium & $6 / 1 / 90$ & $4 / 30 / 89$ & $12 / 30 / 89$ & $4 / 27 / 89$ & $4 / 29 / 89$ & BOB2M4 & G313 & G363 & 1.20 & $1.01 E+08$ & ug \\
\hline Sodium & $6 / 1 / 90$ & $4 / 30 / 89$ & $12 / 30 / 89$ & $4 / 27 / 89$ & $4 / 29 / 89$ & BOB2N2 & G317 & G333 & NA & $1.00 E+08$ & ug. \\
\hline Sodium & $6 / 1 / 90$ & $4 / 30 / 89$ & $12 / 30 / 89$ & $4 / 27 / 89$ & $4 / 29 / 89$ & BOB2L7 & G299 & G342 & 1.20 & $1.00 E+08$ & ugl \\
\hline Sodium & $6 / 1 / 90$ & $4 / 30 / 89$ & $12 / 30 / 89$ & $4 / 27 / 89$ & $4 / 29 / 89$ & 80B2M5 & G315 & G364 & 1.19 & $1.00 E+08$ & ugi \\
\hline Sodium & $6 / 1 / 90$ & $4 / 30 / 89$ & $12 / 30 / 89$ & $4 / 27 / 89$ & $4 / 29 / 89$ & BOB2M7 & G319 & G460 & 1.2 & $1.00 E+08$ & ug $/$ \\
\hline Sodium & $6 / 1 / 90$ & $4 / 30 / 89$ & $12 / 30 / 89$ & $4 / 27 / 89$ & $4 / 29 / 89$ & BOB2L7 & G299 & G342 & 1.20 & $1.00 E+08$ & ugl \\
\hline Sodium & $6 / 1 / 90$ & $4 / 30 / 89$ & $12 / 30 / 89$ & $4 / 27 / 89$ & $4 / 29 / 89$ & BOB2M1 & G308 & G353 & 1.20 & $1.00 E+08$ & ug $/$ \\
\hline Sodium & $6 / 1 / 90$ & $4 / 30 / 89$ & $12 / 30 / 89$ & $4 / 27 / 89$ & $4 / 29 / 89$ & BOB2M4 & G313 & G363 & 1.20 & $9.99 E+07$ & ug/l \\
\hline Sodium & $6 / 1 / 90$ & $4 / 30 / 89$ & 12/30/89 & $4 / 27 / 89$ & $4 / 29 / 89$ & $8082 N 2$ & G317 & G333 & NA & $9.97 E+07$ & ugh \\
\hline Sodium & $6 / 1 / 90$ & $4 / 30 / 89$ & $12 / 30 / 89$ & $4 / 27 / 89$ & $4 / 29 / 89$ & ВОВ2M3 & G311 & G359 & 1.20 & $9.91 E+07$ & ug $/$ \\
\hline Sodium & $6 / 1 / 90$ & $4 / 30 / 89$ & $12 / 30 / 89$ & $4 / 27 / 89$ & $4 / 29 / 89$ & ВОВ2M2 & G309 & G358 & 1.20 & $9.88 E+07$ & $u g / h$ \\
\hline Sodium & $6 / 1 / 90$ & $4 / 30 / 89$ & $12 / 30 / 89$ & $4 / 27 / 89$ & $4 / 29 / 89$ & ВОВ2M3 & G311 & G359 & 1.20 & $9.80 E+07$ & $u g / h$ \\
\hline $\mathrm{OH}-$ & $2 / 10 / 89$ & NS & NS & NS & NS & NS & NS & NS & 0.956764 & $1.06 \mathrm{E}-01$ & $\bar{M}$ \\
\hline $\mathrm{OH}-$ & $2 / 10 / 89$ & NS & N5 & NS & NS & NS & NS & NS & 0.956764 & $9.06 \mathrm{E}-02$ & $\bar{M}$ \\
\hline $\mathrm{OH}-$ & $2 / 10 / 89$ & NS & NS & NS & NS & NS & NS & NS & 1.01 & $5.13 E-03$ & $\bar{M}$ \\
\hline Ammonia & $6 / 1 / 90$ & $4 / 30 / 89$ & $12 / 30 / 89$ & $4 / 27 / 89$ & $4 / 29 / 89$ & ВОВ2T9 & G327 & 6443 & 1.20 & $<1.60 E+02$ & ug/m \\
\hline Ammonia & $6 / 1 / 90$ & $4 / 30 / 89$ & $12 / 30 / 89$ & $4 / 27 / 89$ & $4 / 29 / 89$ & во82T9 & G327 & G443 & 1.20 & $<4.00 E+01$ & $u g / m$ \\
\hline
\end{tabular}




\begin{tabular}{|c|c|c|c|c|c|c|c|}
\hline \multirow[b]{2}{*}{ Code } & \multirow[b]{2}{*}{ Source } & \multicolumn{3}{|c|}{ Analyte Information } & \multirow[b]{2}{*}{$\begin{array}{c}\text { Reference } \\
\text { Number }\end{array}$} & \multirow[b]{2}{*}{$\begin{array}{c}\text { Yalidated } \\
\text { (Y/N) }\end{array}$} & \multirow[b]{2}{*}{ Notes } \\
\hline & & Analyte & $\begin{array}{l}\text { Conc. } \\
\text { (ug/L) }\end{array}$ & Conc. (mol/t) & & & \\
\hline $\mathbf{P}$ & Raw & $\mathrm{Na}$ & $1.02 E+08$ & $4.43 E+00$ & AP102-3 & $\mathbf{Y}$ & $\begin{array}{l}\text { Density reported for } \mathrm{G} 459 \text { collected at } 226 \text { in from bottom } \\
\text { of tank at a } 270^{\circ} \text { angle. Sample was unheated, unheated } \\
\text { samples precipitated }\end{array}$ \\
\hline $\mathbf{P}$ & Raw & $\mathrm{Na}$ & $1.02 E+08$ & $4.43 E+00$ & AP102-3 & $\mathbf{Y}$ & $\begin{array}{l}\text { Density reported for } \mathbf{G} 460 \text { collected at } 226 \text { in from bottom } \\
\text { of tank at a } 270^{\circ} \text { angle. Sample was unheated, unheated } \\
\text { samples precipitated }\end{array}$ \\
\hline $\mathbf{P}$ & Raw & $\mathrm{Na}$ & $1.01 E+08$ & $4.39 E+\infty$ & AP102-3 & $\mathbf{Y}$ & $\begin{array}{l}\text { Density reported for } \mathrm{G} 341 \text { collected at } 99 \text { in from bottom of } \\
\text { tank at a } 270^{\circ} \text { angle. }\end{array}$ \\
\hline $\mathbf{P}$ & Raw & $\mathrm{Na}$ & $1.01 E+08$ & $4.39 E+\infty 0$ & AP102-3 & $\mathbf{Y}$ & $\begin{array}{l}\text { Density reported for } \mathrm{G} 352 \text { collected at } 34 \text { in from bottom of } \\
\text { tank at a } 30^{\circ} \text { angle. Sample was unheated, unheated } \\
\text { samples precipitated }\end{array}$ \\
\hline $\mathbf{P}$ & Raw & $\mathrm{Na}$ & $1.01 E+08$ & $4.39 E+\infty 0$ & AP102-3 & $Y$ & $\begin{array}{l}\text { Density reported for } \mathrm{G} 353 \text { collected at } 334 \text { in from bottom } \\
\text { of tank at a } 150^{\circ} \text { angle. Sample was unheated, unheated } \\
\text { samples precipitated }\end{array}$ \\
\hline $\mathbf{P}$ & Raw & $\mathrm{Na}$ & $1.01 E+08$ & $4.39 E+00$ & AP102-3 & $Y$ & $\begin{array}{l}\text { Density not reported for G449. No assumptions could be } \\
\text { made }\end{array}$ \\
\hline $\mathbf{P}$ & Raw & $\mathrm{Na}$ & $1.01 E+08$ & $4.39 E+00$ & AP102-3 & $Y$ & $\begin{array}{l}\text { Density reported for } \mathrm{G} 341 \text { collected at } 99 \text { in from bottom of } \\
\text { tank at a } 270^{\circ} \text { angle. }\end{array}$ \\
\hline $\mathbf{P}$ & Raw & $\mathrm{Na}$ & $1.01 E+08$ & $4.39 E+00$ & AP102-3 & $Y$ & $\begin{array}{l}\text { Density reported for G363 collected at } 18 \text { in from bottom of } \\
\text { tank at a } 150^{\circ} \text { angle. Sample was unheated, unheated } \\
\text { samples precipitated }\end{array}$ \\
\hline $\mathbf{P}$ & Raw & $\mathrm{Na}$ & $1.00 E+08$ & $4.35 E+00$ & AP102-3 & $\mathbf{Y}$ & $\begin{array}{l}\text { Density not reported for G333. No assumptions could be } \\
\text { made }\end{array}$ \\
\hline $\mathbf{P}$ & Raw & $\mathrm{Na}$ & $1.00 E+08$ & $4.35 E+00$ & AP102-3 & $\mathbf{Y}$ & $\begin{array}{l}\text { Density reported for } \mathrm{G} 342 \text { collected at } 306 \text { in from bottom } \\
\text { of tank at a } 30^{\circ} \text { angle. Sample was unheated, unheated } \\
\text { samples precipitated }\end{array}$ \\
\hline $\mathbf{P}$ & Raw & $\mathrm{Na}$ & $1.00 E+08$ & $4.35 E+00$ & AP102-3 & $Y^{\prime}$ & $\begin{array}{l}\text { Density reported for G364 collected at } 372 \text { in from bottom } \\
\text { of tank at a } 270^{\circ} \text { angle. Sample was unheated, unheated } \\
\text { samples precipitated }\end{array}$ \\
\hline $\mathbf{P}$ & Raw & $\mathrm{Na}$ & $1.00 E+08$ & $4.35 E+\infty 0$ & AP102-3 & $\mathbf{Y}$ & $\begin{array}{l}\text { Density reported for G460 collected at } 226 \text { in from bottom } \\
\text { of tank at a } 270^{\circ} \text { angle. Sample was unheated, unheated } \\
\text { samples precipitated }\end{array}$ \\
\hline $\mathbf{P}$ & Raw & $\mathrm{Na}$ & $1.00 E+08$ & $4.35 E+\infty$ & AP102-3 & $Y$ & $\begin{array}{l}\text { Density reported for G342 collected at } 306 \text { in from bottom } \\
\text { of tank at a } 30^{\circ} \text { angle. Sample was unheated, unheated } \\
\text { samples precipitated }\end{array}$ \\
\hline$P$ & Raw & $\mathrm{Na}$ & $1.00 E+08$ & $4.35 E+00$ & AP102-3 & $\mathbf{Y}$ & $\begin{array}{l}\text { Density reported for } \mathrm{G} 353 \text { collected at } 334 \text { in from bottom } \\
\text { of tank at a } 150^{\circ} \text { angle. Sample was unheated, unheated } \\
\text { samples precipitated }\end{array}$ \\
\hline$P$ & Raw & $\mathrm{Na}$ & $9.99 E+07$ & $4.34 E+\infty$ & AP102-3 & Y & $\begin{array}{l}\text { Density reported for G363 collected at } 18 \text { in from bottom of } \\
\text { tank at a } 150^{\circ} \text { angle. Sample was unheated, unheated } \\
\text { samples precipitated }\end{array}$ \\
\hline $\mathbf{P}$ & Raw & $\mathrm{Na}$ & $9.97 E+07$ & $4.33 E+\infty 0$ & AP102-3 & $\mathbf{Y}$ & $\begin{array}{l}\text { Density not reported for G333. No assumptions could be } \\
\text { made }\end{array}$ \\
\hline $\mathbf{P}$ & Raw & $\mathrm{Na}$ & $9.91 E+07$ & $4.31 E+\infty 0$ & AP102-3 & $Y$ & $\begin{array}{l}\text { Density reported for } 6359 \text { collected at } 162 \text { in from bottom } \\
\text { of tank at a } 150^{\circ} \text { angle. Sample was unheated, unheated } \\
\text { samples precipitated }\end{array}$ \\
\hline $\mathbf{P}$ & Raw & $\mathrm{Na}$ & $9.88 E+07$ & $4.30 E+\infty$ & AP102-3 & $\mathbf{Y}$ & $\begin{array}{l}\text { Density reported for } \mathrm{G} 358 \text { collected at } 334 \text { in from bottom } \\
\text { of tank at a } 150^{\circ} \text { angle. Sample was unheated, unheated } \\
\text { samples precipitated }\end{array}$ \\
\hline $\mathbf{P}$ & Raw & $\mathrm{Na}$ & $9.80 E+07$ & $4.26 E+\infty$ & AP102-3 & $\mathbf{Y}$ & $\begin{array}{l}\text { Density reported for G359 collected at } 162 \text { in from bottom } \\
\text { of tank at a } 150^{\circ} \text { angle. Sample was unheated, unheated } \\
\text { samples precipitated }\end{array}$ \\
\hline $\mathbf{U}$ & RAW & $\mathrm{NaOH}$ & $4.22 E+12$ & $1.06 E+05$ & AP102-2 & $N$ & Projected value, not a real data point \\
\hline $\mathbf{U}$ & RAW & $\mathrm{NaOH}$ & $3.62 E+12$ & $9.05 E+04$ & AP102-2 & $N$ & Projected value, not a real data point \\
\hline $\mathbf{U}$ & RAW & $\mathrm{NaOH}$ & $2.05 E+11$ & $5.13 E+03$ & AP102-2 & $N$ & Projected value, not a real data point \\
\hline$P$ & Raw & NH3 & NALUE! & NALUE! & AP102-3 & $\mathbf{Y}$ & Density reported for $\mathrm{G} 443$ represents a composite sample \\
\hline $\mathbf{P}$ & Raw & NH3 & *VALUEI & \#VALUE! & AP102-3 & $\mathbf{Y}$ & Density reported for $\mathbf{G} 443$ represents a composite sample \\
\hline
\end{tabular}




\begin{tabular}{|c|c|c|c|c|c|c|c|c|c|c|c|}
\hline Reported Chemical & $\begin{array}{l}\text { Memo } \\
\text { Date }\end{array}$ & $\begin{array}{c}\text { Start } \\
\text { Analysis } \\
\text { Date }\end{array}$ & $\begin{array}{c}\text { Finish } \\
\text { Analysis Date }\end{array}$ & $\begin{array}{c}\text { Start } \\
\text { Sample } \\
\text { Date } \\
\end{array}$ & $\begin{array}{c}\text { Finish } \\
\text { Sample } \\
\text { Date }\end{array}$ & $\begin{array}{l}\text { Sample } \\
\text { Number }\end{array}$ & $\begin{array}{c}\text { Tank Farm } \\
\text { Number }\end{array}$ & $\begin{array}{c}\text { Lab Sample } \\
\text { Number }\end{array}$ & $\begin{array}{l}\text { Density } \\
(\mathrm{g} / \mathrm{mL})\end{array}$ & $\begin{array}{l}\text { Reported } \\
\text { Value }\end{array}$ & $\begin{array}{r}\text { Repc } \\
U_{i} \\
\end{array}$ \\
\hline Ammonia & $6 / 1 / 90$ & $4 / 30 / 89$ & $12 / 30 / 89$ & $4 / 27 / 89$ & $4 / 29 / 89$ & в0B2T9 & G327 & G443 & 1.20 & $<4.00 E+01$ & $u g f_{1}$ \\
\hline $\mathrm{NH3}$ & $2 / 10 / 89$ & NS & NS & NS & NS & NS & NS & NS & 1.01 & $3.77 E-01$ & $\mathbf{N}$ \\
\hline $\mathrm{NH3}$ & $2 / 10 / 89$ & NS & NS & NS & NS & NS & NS & NS & 0.956764 & 3.57E-01 & $\bar{N}$ \\
\hline Ammonia & $6 / 1 / 90$ & $4 / 30 / 89$ & $12 / 30 / 89$ & $4 / 27 / 89$ & $4 / 29 / 89$ & BOB2N1 & G310 & G471* & 1.19 & $4.13 E+02$ & ugh \\
\hline Ammonia & $6 / 1 / 90$ & $4 / 30 / 89$ & $12 / 30 / 89$ & $4 / 27 / 89$ & $4 / 29 / 89$ & BOB2N1 & 6310 & G471* & 1.19 & $4.10 E+02$ & $u g h$ \\
\hline Ammonia & $6 / 1 / 90$ & $4 / 30 / 89$ & $12 / 30 / 89$ & $4 / 27 / 89$ & $4 / 29 / 89$ & BOB2N1 & G310 & G471" & 1.19 & $3.08 E+02$ & ug/s \\
\hline Ammonia & $6 / 1 / 90$ & $4 / 30 / 89$ & $12 / 30 / 89$ & $4 / 27 / 89$ & $4 / 29 / 89$ & BOB2N1 & G310 & G471 * & 1.19 & $3.02 E+02$ & $u g / n$ \\
\hline Ammonia & $6 / 1 / 90$ & $4 / 30 / 89$ & $12 / 30 / 89$ & $4 / 27 / 89$ & $4 / 29 / 89$ & вов2М9 & G302 & G477* & 1.20 & $2.72 E+02$ & $u g / \pi$ \\
\hline Ammonia & $6 / 1 / 90$ & $4 / 30 / 89$ & $12 / 30 / 89$ & $4 / 27 / 89$ & $4 / 29 / 89$ & 8082M9 & G302 & G477" & 1.20 & $2.72 E+02$ & ug/n \\
\hline Ammonia & $6 / 1 / 90$ & $4 / 30 / 89$ & $12 / 30 / 89$ & $4 / 27 / 89$ & $4 / 29 / 89$ & BOB2T9 & G327 & G470* & 1.19 & $2.65 E+02$ & $u g / \kappa$ \\
\hline Ammonia & $6 / 1 / 90$ & $4 / 30 / 89$ & $12 / 30 / 89$ & $4 / 27 / 89$ & $4 / 29 / 89$ & ВOB2T9 & G327 & G470* & 1.19 & $2.58 E+02$ & ugin \\
\hline Ammonia & $6 / 1 / 90$ & $4 / 30 / 89$ & $12 / 30 / 89$ & $4 / 27 / 89$ & $4 / 29 / 89$ & BOB2N4 & G323 & G476* & 1.21 & $2.01 E+02$ & ug/T \\
\hline Ammonia & $6 / 1 / 90$ & $4 / 30 / 89$ & $12 / 30 / 89$ & $4 / 27 / 89$ & $4 / 29 / 89$ & BOB2N4 & G323 & G476* & 1.21 & $2.01 E+02$ & $u g / m$ \\
\hline Ammonia & $6 / 1 / 90$ & $4 / 30 / 89$ & $12 / 30 / 89$ & $4 / 27 / 89$ & $4 / 29 / 89$ & BOB2N4 & G323 & G476* & 1.21 & $1.95 E+02$ & $u g / m$ \\
\hline Ammonia & $6 / 1 / 90$ & $4 / 30 / 89$ & $12 / 30 / 89$ & $4 / 27 / 89$ & $4 / 29 / 89$ & BOB2N4 & G323 & G476* & 1.21 & $1.92 E+02$ & $\mathrm{ug} / \mathrm{m}$ \\
\hline Ammonia & $6 / 1 / 90$ & $4 / 30 / 89$ & $12 / 30 / 89$ & $4 / 27 / 89$ & $4 / 29 / 89$ & Вов2T9 & G327 & G443 & 1.20 & $1.64 E+02$ & $\mathrm{ug} / \mathrm{m}$ \\
\hline $\mathrm{Ni2}+$ & NS & NS & NS & NS & NS & NS & NS & NS & 1.24 & $2.47 E-03$ & Molel \\
\hline Nickel & $4 / 1 / 93$ & NS & NS & NS & NS & B082T9 & G327 & NS & NS & $3.45 E+04$ & UG $/ \mathrm{L}$ \\
\hline Nickel & $4 / 1 / 93$ & NS & NS & NS & NS & B0B2T9 & G327 & NS & NS & $2.78 E+04$ & $U \mathrm{UA}$ \\
\hline Nickel & $6 / 1 / 90$ & $4 / 30 / 89$ & $12 / 30 / 89$ & $4 / 27 / 89$ & $4 / 29 / 89$ & BOB2T9 & G327 & G470* & 1.19 & $3.45 E+04$ & ugh \\
\hline Nickel & $6 / 1 / 90$ & $4 / 30 / 89$ & $12 / 30 / 89$ & $4 / 27 / 89$ & $4 / 29 / 89$ & BOB2L9 & G305 & G349 & 1.20 & $2.82 E+04$ & ug $/$. \\
\hline Nickel & $6 / 1 / 90$ & $4 / 30 / 89$ & $12 / 30 / 89$ & $4 / 27 / 89$ & $4 / 29 / 89$ & BOB2T9 & G327 & G470* & 1.19 & $2.78 \mathrm{E}+04$ & $u g / L$ \\
\hline Nickel & $6 / 1 / 90$ & $4 / 30 / 89$ & $12 / 30 / 89$ & $4 / 27 / 89$ & $4 / 29 / 89$ & BOB2L9 & G305 & G349 & 1.20 & $2.77 E+04$ & ugh \\
\hline Nickel & $6 / 1 / 90$ & $4 / 30 / 89$ & $12 / 30 / 89$ & $4 / 27 / 89$ & $4 / 29 / 89$ & B082L8 & G303 & G348 & 1.21 & $2.75 E+04$ & ug/L. \\
\hline Nickel & $6 / 1 / 90$ & $4 / 30 / 89$ & $12 / 30 / 89$ & $4 / 27 / 89$ & $4 / 29 / 89$ & Вов2Т9 & G327 & 6443 & 1.20 & $2.75 E+04$ & $u g \Omega$ \\
\hline
\end{tabular}




\begin{tabular}{|c|c|c|c|c|c|c|c|}
\hline \multirow[b]{2}{*}{ Code } & \multirow[b]{2}{*}{ Source } & \multicolumn{3}{|c|}{ Analyte Information } & \multirow[b]{2}{*}{$\begin{array}{c}\text { Reference } \\
\text { Number }\end{array}$} & \multirow[b]{2}{*}{$\begin{array}{l}\text { Validated } \\
\text { [Y/N\} }\end{array}$} & \multirow[b]{2}{*}{ Notes } \\
\hline & & Analyte & $\begin{array}{l}\text { Conc. } \\
\text { (ug/L) }\end{array}$ & Conc. (mol/L) & & & \\
\hline $\mathbf{P}$ & Raw & NH3 & \#VALUE! & \#VALUE! & AP102-3 & $\mathbf{Y}$ & Density reported for $\mathrm{G} 443$ represents a composite sample \\
\hline $\mathbf{U}$ & RAW & $\mathrm{NH3}$ & $6.41 \mathrm{E}+12$ & $3.77 E+05$ & AP102-2 & $\mathbf{N}$ & Projected value, not a real data point \\
\hline U & RAW & NH3 & $6.07 E+12$ & $3.57 E+05$ & AP102-2 & $\mathbf{N}$ & Projected value, not a real data point \\
\hline $\mathbf{P}$ & Raw & NH3 & $4.13 E+05$ & $2.43 \mathrm{E}-02$ & AP102-3 & $\mathbf{Y}$ & $\begin{array}{l}\text { Density reported for G471 collected at } 208 \text { in from bottom } \\
\text { of tank at a } 150^{\circ} \text { angle and was keep heated to } 40^{\circ} \mathrm{C} \text {. }\end{array}$ \\
\hline $\mathbf{P}$ & Raw & NH3 & $4.10 E+05$ & $2.41 E-02$ & AP102-3 & $\mathbf{Y}$ & $\begin{array}{l}\text { Density reported for } \mathrm{G} 471 \text { collected at } 208 \text { in from bottom } \\
\text { of tank at a } 150^{\circ} \text { angle and was keep heated to } 40^{\circ} \mathrm{C} \text {. }\end{array}$ \\
\hline$P$ & Raw & NH3 & $3.08 \mathrm{E}+05$ & $1.81 E-02$ & AP102-3 & $\mathbf{Y}$ & $\begin{array}{l}\text { Density reported for G471 collected at } 208 \text { in from bottom } \\
\text { of tank at a } 150^{\circ} \text { angle and was keep heated to } 40^{\circ} \mathrm{C} \text {. }\end{array}$ \\
\hline$P$ & Raw & $\mathrm{NH3}$ & $3.02 E+05$ & $1.78 \mathrm{E}-02$ & AP102-3 & $Y$ & $\begin{array}{l}\text { Density reported for G471 collected at } 208 \text { in from bottom } \\
\text { of tank at a } 150^{\circ} \text { angle and was keep heated to } 40^{\circ} \mathrm{C} \text {. }\end{array}$ \\
\hline $\mathbf{P}$ & Raw & NH3 & $2.72 E+05$ & $1.60 \mathrm{E}-02$ & AP102-3 & $\mathbf{Y}$ & $\begin{array}{l}\text { Density reported for } \mathrm{G} 477 \text { collected at } 182 \text { in from bottom } \\
\text { of tank at a } 30^{\circ} \text { angle and was keep heated to } 40^{\circ} \mathrm{C} \text {. }\end{array}$ \\
\hline $\mathbf{P}$ & Raw & NH3 & $2.72 E+05$ & 1.60E-02 & AP102-3 & $Y$ & $\begin{array}{l}\text { Density reported for G477 collected at } 182 \text { in from bottom } \\
\text { of tank at a } 30^{\circ} \text { angle and was keep heated to } 40^{\circ} \mathrm{C} \text {. }\end{array}$ \\
\hline $\mathbf{P}$ & Raw & NH3 & $2.65 E+05$ & $1.56 \mathrm{E}-02$ & AP102-3 & $\mathbf{Y}$ & $\begin{array}{l}\text { Density reported for G470 represents a composite sample. } \\
\text { Sample was kept heated to } 40^{\circ} \mathrm{C} \text {. } \\
\end{array}$ \\
\hline$P$ & Raw & NH3 & $2.58 \varepsilon+05$ & 1.52E-02 & AP102-3 & $\mathbf{Y}$ & $\begin{array}{l}\text { Density reported for } \mathrm{G} 470 \text { represents a composite sample. } \\
\text { Sample was kept heated to } 40^{\circ} \mathrm{C} \text {. }\end{array}$ \\
\hline$P$ & Raw & NH3 & $2.01 E+05$ & $1.18 \mathrm{E}-02$ & AP102-3 & $Y$ & $\begin{array}{l}\text { Density reported for } \mathrm{G} 476 \text { collected at } 99 \text { in from bottom of } \\
\text { tank at a } 270^{\circ} \text { angle and was keep heated to } 40^{\circ} \mathrm{C} \text {. }\end{array}$ \\
\hline$P$ & Raw & NH3 & $2.01 E+05$ & $1.18 E-02$ & AP102-3 & $\gamma$ & $\begin{array}{l}\text { Density seported for G476 collected at } 99 \text { in from bottom of } \\
\text { tank at a } 270^{\circ} \text { angle and was keep heated to } 40^{\circ} \mathrm{C} \text {. }\end{array}$ \\
\hline $\mathbf{P}$ & Raw & NH3 & $1.95 E+05$ & $1.15 E-02$ & AP102-3 & $Y$ & $\begin{array}{l}\text { Density reported for G476 collected at } 99 \text { in from bottom of } \\
\text { tank at a } 270^{\circ} \text { angle and was keep heated to } 40^{\circ} \mathrm{C} \text {. }\end{array}$ \\
\hline $\mathbf{P}$ & Raw & $\mathrm{NH3}$ & $1.92 E+05$ & 1.13E-02 & AP102-3 & $\mathbf{Y}$ & $\begin{array}{l}\text { Density reported for G476 collected at } 99 \text { in from bottom of } \\
\text { tank at a } 270^{\circ} \text { angle and was keep heated to } 40^{\circ} \mathrm{C} \text {. }\end{array}$ \\
\hline $\mathbf{P}$ & Raw & $\mathrm{NH} 3$ & $1.64 E+05$ & $9.65 E-03$ & AP102-3 & $Y$ & Density reported for $\mathrm{G} 443$ represents a composite sample \\
\hline$L$ & HTCE Inv. & $\mathrm{Ni}$ & $1.45 E+11$ & $2.47 E+03$ & NA & $\bar{N}$ & No Reference \\
\hline $\mathbf{P}$ & TCD & $\mathrm{Ni}$ & $3.45 E+10$ & $5.88 E+02$ & & $\mathbf{N}$ & THIS POINT NOT GIVEN IN REPORT WHC-SD-WM-TRP-169 \\
\hline$P$ & TCD & $\mathrm{Ni}$ & $2.78 E+10$ & $4.74 E+02$ & & N & THIS POINT NOT GIVEN IN REPORT WHC-SD-WM-TRP-169 \\
\hline $\mathbf{P}$ & Raw & $\mathrm{Ni}$ & $3.45 E+04$ & $5.88 \mathrm{E}-04$ & AP102-3 & $\mathbf{Y}$ & $\begin{array}{l}\text { Density reported for G470 represents a composite sample. } \\
\text { Sample was kept heated to } 40^{\circ} \mathrm{C} \text {. }\end{array}$ \\
\hline $\mathbf{P}$ & Raw & $\mathrm{Ni}$ & $2.82 E+04$ & $4.80 E-04$ & AP102-3 & $\mathbf{Y}$ & $\begin{array}{l}\text { Density reported for G349 collected at } 34 \text { in from bottom of } \\
\text { tank at a } 30^{\circ} \text { angle. Sample was unheated, unheated } \\
\text { samples precipitated }\end{array}$ \\
\hline$P$ & Raw & $\mathrm{Ni}$ & $2.78 E+04$ & 4.74E-04 & AP102-3 & Y & $\begin{array}{l}\text { Density reported for } 6470 \text { represents a composite sample. } \\
\text { Sample was kept heated to } 40^{\circ} \mathrm{C} \text {. }\end{array}$ \\
\hline $\mathbf{P}$ & Raw & $\mathrm{Ni}$ & $2.77 E+04$ & 4.72E-04 & AP102-3 & $\mathrm{Y}$ & $\begin{array}{l}\text { Density reported for } \mathrm{G} 349 \text { collected at } 34 \text { in from bottom of } \\
\text { tank at a } 30^{\circ} \text { angle. Sample was unheated, unheated } \\
\text { samples precipitated }\end{array}$ \\
\hline$P$ & Raw & $\mathrm{Ni}$ & $2.75 E+04$ & 4.69E-04 & AP102-3 & $\gamma$ & $\begin{array}{l}\text { Density reported for G348 collected at } 125 \text { in from bottom } \\
\text { of tank at a } 30^{\circ} \text { angle. Sample was unheated, unheated } \\
\text { samples precipitated }\end{array}$ \\
\hline P & Raw & $\mathrm{Ni}$ & $2.75 E+04$ & 4.69E-04 & AP102-3 & $\mathbf{Y}$ & Density reported for $\mathrm{G} 443$ represents a composite sample \\
\hline
\end{tabular}




\begin{tabular}{|c|c|c|c|c|c|c|c|c|c|c|c|}
\hline & & & & & ank Waste & haracteriza & on Sample It & ormation & & & \\
\hline Reported Chemical & $\begin{array}{c}\text { Memo } \\
\text { Date }\end{array}$ & $\begin{array}{c}\text { Start } \\
\text { Analysis } \\
\text { Date } \\
\end{array}$ & $\begin{array}{c}\text { Finish } \\
\text { Analysis Date }\end{array}$ & $\begin{array}{c}\text { Start } \\
\text { Sample } \\
\text { Date } \\
\end{array}$ & $\begin{array}{c}\text { Finish } \\
\text { Sample } \\
\text { Date } \\
\end{array}$ & $\begin{array}{l}\text { Sample } \\
\text { Number }\end{array}$ & $\begin{array}{c}\text { Tank Farm } \\
\text { Number }\end{array}$ & $\begin{array}{c}\text { Lab Sample } \\
\text { Number }\end{array}$ & $\begin{array}{l}\text { Density } \\
(\mathrm{g} / \mathrm{mL})\end{array}$ & $\begin{array}{c}\text { Reported } \\
\text { Value } \\
\end{array}$ & $\begin{array}{r}\text { Rep } \\
\mathbf{U} \\
\end{array}$ \\
\hline Nicke! & $6 / 1 / 90$ & $4 / 30 / 89$ & $12 / 30 / 89$ & $4 / 27 / 89$ & $4 / 29 / 89$ & BOB2L8 & G303 & G348 & 1.21 & $2.74 E+04$ & 49 \\
\hline Nickel & $6 / 1 / 90$ & $4 / 30 / 89$ & $12 / 30 / 89$ & $4 / 27 / 89$ & $4 / 29 / 89$ & BOB2NO & G307 & G453 & NA & $2.74 E+04$ & $\mathrm{ug}$ \\
\hline Nickel & $6 / 1 / 90$ & $4 / 30 / 89$ & $12 / 30 / 89$ & $4 / 27 / 89$ & $4 / 29 / 89$ & В0В2M6 & G318 & G459 & 1.20 & $2.73 E+04$ & $u g$ \\
\hline Nickel & $6 / 1 / 90$ & $4 / 30 / 89$ & $12 / 30 / 89$ & $4 / 27 / 89$ & $4 / 29 / 89$ & BOB2M5 & G315 & G364 & 1.19 & $2.73 E+04$ & $u g$ \\
\hline Nickel & $6 / 1 / 90$ & $4 / 30 / 89$ & $12 / 30 / 89$ & $4 / 27 / 89$ & $4 / 29 / 89$ & BOB2N2 & G317 & G333 & NA & $2.71 E+04$ & ugr \\
\hline Nickel & $6 / 1 / 90$ & $4 / 30 / 89$ & $12 / 30 / 89$ & $4 / 27 / 89$ & $4 / 29 / 89$ & $B O B 2 N 1$ & G310 & G454 & $(1.19)$ & $2.71 E+04$ & ug \\
\hline Nickel & $6 / 1 / 90$ & $4 / 30 / 89$ & $12 / 30 / 89$ & $4 / 27 / 89$ & $4 / 29 / 89$ & BOB2N3 & G322 & G338 & NA & $2.70 E+04$ & ug $f$ \\
\hline Nickel & $6 / 1 / 90$ & $4 / 30 / 89$ & $12 / 30 / 89$ & $4 / 27 / 89$ & $4 / 29 / 89$ & ВОВ2M7 & G319 & G460 & 1.20 & $2.69 E+04$ & ugf \\
\hline Nickel & $6 / 1 / 90$ & $4 / 30 / 89$ & $12 / 30 / 89$ & $4 / 27 / 89$ & $4 / 29 / 89$ & BOB2N4 & G323 & G341 & 1.20 & $2.69 E+04$ & ugl \\
\hline Nickel & $6 / 1 / 90$ & $4 / 30 / 89$ & $12 / 30 / 89$ & $4 / 27 / 89$ & $4 / 29 / 89$ & BOB2N4 & G323 & G341 & 1.20 & $2.68 E+04$ & $u g h$ \\
\hline Nickel & $6 / 1 / 90$ & $4 / 30 / 89$ & $12 / 30 / 89$ & $4 / 27 / 89$ & $4 / 29 / 89$ & BOB2NO & G307 & G453 & NA & $2.68 E+04$ & ugh \\
\hline Nickel & $6 / 1 / 90$ & $4 / 30 / 89$ & $12 / 30 / 89$ & $4 / 27 / 89$ & $4 / 29 / 89$ & BOB2L7 & G299 & G342 & 1.20 & $2.68 \varepsilon+04$ & ug $/$ \\
\hline Nickel & $6 / 1 / 90$ & $4 / 30 / 89$ & $12 / 30 / 89$ & $4 / 27 / 89$ & $4 / 29 / 89$ & ВОВ2M9 & G302 & G450 & $(1.20)$ & $2.67 E+04$ & $u g / h$ \\
\hline Nickel & $6 / 1 / 90$ & $4 / 30 / 89$ & $12 / 30 / 89$ & $4 / 27 / 89$ & $4 / 29 / 89$ & BOB2N3 & G322 & G338 & NA & $2.67 E+04$ & $u g / h$ \\
\hline Nickel & $6 / 1 / 90$ & $4 / 30 / 89$ & $12 / 30 / 89$ & $4 / 27 / 89$ & $4 / 29 / 89$ & ВОВ2MO & G306 & G352 & 1.20 & $2.67 E+04$ & $u g R$ \\
\hline Nickel & $6 / 1 / 90$ & $4 / 30 / 89$ & $12 / 30 / 89$ & $4 / 27 / 89$ & $4 / 29 / 89$ & ВОВ2M1 & G308 & G353 & 1.20 & $2.66 E+04$ & ugh \\
\hline Nickel & $6 / 1 / 90$ & $4 / 30 / 89$ & $12 / 30 / 89$ & $4 / 27 / 89$ & $4 / 29 / 89$ & Во82M4 & G313 & G363 & 1.20 & $2.66 E+04$ & $u g / L$ \\
\hline Nickel & $6 / 1 / 90$ & $4 / 30 / 89$ & $12 / 30 / 89$ & $4 / 27 / 89$ & $4 / 29 / 89$ & BOB2N2 & G317 & G333 & NA & $2.66 \mathrm{E}+04$ & ugh \\
\hline Nickel & $6 / 1 / 90$ & $4 / 30 / 89$ & $12 / 30 / 89$ & $4 / 27 / 89$ & $4 / 29 / 89$ & BOB2M2 & G309 & G358 & 1.20 & $2.66 E+04$ & ugh \\
\hline Nickel & $6 / 1 / 90$ & $4 / 30 / 89$ & $12 / 30 / 89$ & $4 / 27 / 89$ & $4 / 29 / 89$ & BOB2T9 & G327 & G443 & 1.20 & $2.64 E+04$ & ug $h$ \\
\hline Nickel & $6 / 1 / 90$ & $4 / 30 / 89$ & $12 / 30 / 89$ & $4 / 27 / 89$ & $4 / 29 / 89$ & BOB2M7 & G319 & G460 & 1.20 & $2.64 E+04$ & ug $/$. \\
\hline Nickel & $6 / 1 / 90$ & $4 / 30 / 89$ & $12 / 30 / 89$ & $4 / 27 / 89$ & $4 / 29 / 89$ & BOB2MT & G308 & G353 & 1.20 & $2.63 E+04$ & ugh \\
\hline
\end{tabular}




\begin{tabular}{|c|c|c|c|c|c|c|c|}
\hline \multirow[b]{2}{*}{ Code } & \multirow[b]{2}{*}{ Source } & \multirow[b]{2}{*}{ Analivte } & \multicolumn{2}{|c|}{ Analyte Information } & \multirow[b]{2}{*}{$\begin{array}{c}\text { Reference } \\
\text { Number }\end{array}$} & \multirow[b]{2}{*}{$\begin{array}{c}\text { Validated } \\
(Y / N)\end{array}$} & \multirow[b]{2}{*}{ Notes } \\
\hline & & & $\begin{array}{l}\text { Conc. } \\
\text { (ug/L) }\end{array}$ & Conc. (mol/L) & & & \\
\hline$P$ & Raw & $\mathrm{Ni}$ & $2.74 E \div 04$ & $4.67 E-04$ & AP102-3 & $Y$ & $\begin{array}{l}\text { Density reported for } G 348 \text { collected at } 125 \text { in from bottom } \\
\text { of tank at a } 30^{\circ} \text { angle. Sample was unheated, unheated } \\
\text { samples precipitated }\end{array}$ \\
\hline$P$ & Raw & $\mathrm{Ni}$ & $2.74 E+04$ & $4.67 E-04$ & AP102-3 & $Y$ & $\begin{array}{l}\text { Density not reported for G453. No assumptions could be } \\
\text { made }\end{array}$ \\
\hline $\mathbf{P}$ & Raw & $\mathrm{Ni}$ & $2.73 E+04$ & $4.65 \mathrm{E}-0.4$ & AP102-3 & $\mathbf{Y}$ & $\begin{array}{l}\text { Density reported for G459 collected at } 226 \text { in from bottom } \\
\text { of tank at a } 270^{\circ} \text { angle. Sample was unheated, unheated } \\
\text { samples precipitated }\end{array}$ \\
\hline$P$ & Raw & $\mathbf{N i}$ & $2.73 E+04$ & $4.65 E-04$ & AP102-3 & $\mathbf{Y}$ & $\begin{array}{l}\text { Density reported for G364 collected at } 372 \text { in from bottom } \\
\text { of tank at a } 270^{\circ} \text { angle. Sample was unheated, unheated } \\
\text { samples precipitated }\end{array}$ \\
\hline$P$ & Raw & $\mathbf{N i}$ & $2.71 E+04$ & $4.62 \mathrm{E}-04$ & AP102-3 & $\gamma$ & $\begin{array}{l}\text { Density not reported for G333. No assumptions could be } \\
\text { made }\end{array}$ \\
\hline$P$ & Raw & $\mathrm{Ni}$ & $2.71 E+04$ & $4.62 E-04$ & AP102-3 & $Y$ & $\begin{array}{l}\text { Density not reported for G454. Density assumed same as } \\
\text { G471 which was collected at } 208 \text { in from bottom of tank at } \\
\text { a } 150^{\circ} \text { angle and was keep heated to } 40^{\circ} \mathrm{C} \text {. }\end{array}$ \\
\hline$P$ & Raw & $\mathrm{Ni}$ & $2.70 E+04$ & $4.60 \mathrm{E}-04$ & AP102-3 & $Y$ & $\begin{array}{l}\text { Density not reported for G338. No assumptions could be } \\
\text { made }\end{array}$ \\
\hline $\mathbf{P}$ & Raw & $\mathrm{Ni}$ & $2.69 E+04$ & $4.58 E-04$ & AP102-3 & Y & $\begin{array}{l}\text { Density reported for } \mathrm{G} 460 \text { collected at } 226 \text { in from bottom } \\
\text { of tank at a } 270^{\circ} \text { angle. Sample was unheated, unheated } \\
\text { samples precipitated }\end{array}$ \\
\hline $\mathbf{P}$ & Raw & $\mathrm{Ni}$ & $2.69 E+04$ & 4.58E-04 & AP102-3 & Y & $\begin{array}{l}\text { Density reported for } \mathrm{G} 341 \text { collected at } 99 \text { in from bottom of } \\
\text { tank at a } 270^{\circ} \text { angle. }\end{array}$ \\
\hline $\mathbf{P}$ & Raw & $\mathrm{Ni}$ & $2.68 E+04$ & 4.57E-04 & AP102-3 & $Y$ & $\begin{array}{l}\text { Density reported for G341 collected at } 99 \text { in from bottom of } \\
\text { tank at a } 270^{\circ} \text { angle. }\end{array}$ \\
\hline $\mathbf{P}$ & Raw & $\mathrm{Ni}$ & $2.68 \mathrm{E}+04$ & 4.57E-04 & AP102-3 & $Y$ & $\begin{array}{l}\text { Density not reported for G453. No assumptions could be } \\
\text { made }\end{array}$ \\
\hline $\mathbf{P}$ & Raw & $\mathrm{Ni}$ & $2.68 E+04$ & 4.57E-04 & AP102-3 & $Y$ & $\begin{array}{l}\text { Density reported for } \mathrm{G} 342 \text { collected at } 306 \text { in from bottom } \\
\text { of tank at a } 30^{\circ} \text { angle. Sample was unheated, unheated } \\
\text { samples precipitated }\end{array}$ \\
\hline $\mathbf{P}$ & Raw & $\mathrm{Ni}$ & $2.67 E+0.4$ & $4.55 E-04$ & AP102-3 & Y & $\begin{array}{l}\text { Density not reported for G450. Density assumed same as } \\
\text { G } 477 \text { which was collected at } 182 \text { in from bottom of tank at } \\
\text { a } 30^{\circ} \text { angle and was keep heated to } 40^{\circ} \mathrm{C} \text {. }\end{array}$ \\
\hline $\mathbf{P}$ & Raw & $\mathrm{Ni}$ & $2.67 E+04$ & $4.55 E-04$ & AP102-3 & $Y$ & $\begin{array}{l}\text { Density not reported for } G 338 \text {. No assumptions could be } \\
\text { made }\end{array}$ \\
\hline $\mathbf{P}$ & Raw & $\mathrm{Ni}$ & $2.67 E+04$ & $4.55 E-04$ & AP102-3 & $Y$ & $\begin{array}{l}\text { Density reported for } \mathrm{G} 352 \text { collected at } 34 \text { in from bottom of } \\
\text { tank at a } 30^{\circ} \text { angle. Sample was unheated, unheated } \\
\text { samples precipitated }\end{array}$ \\
\hline $\mathbf{P}$ & Raw & $\mathrm{Ni}$ & $2.66 \mathrm{E}+04$ & 4.53E-04 & AP102-3 & $\gamma$ & $\begin{array}{l}\text { Density reported for G353 collected at } 334 \text { in from bottom } \\
\text { of tank at a } 150^{\circ} \text { angle. Sample was unheated, unheated } \\
\text { samples precipitated }\end{array}$ \\
\hline $\mathbf{P}$ & Raw & $\mathbf{N i}$ & $2.66 E+04$ & 4.53E-04 & AP102-3 & Y & $\begin{array}{l}\text { Density reported for } \mathrm{G} 363 \text { collected at } 18 \mathrm{in} \text { from bottom of } \\
\text { tank at a } 150^{\circ} \text { angle. Sample was unheated, unheated } \\
\text { samples precipitated }\end{array}$ \\
\hline $\mathbf{P}$ & Raw & $\mathrm{Ni}$ & $2.66 \mathrm{E}+0.4$ & $4.53 E-04$ & AP102-3 & Y & $\begin{array}{l}\text { Density not reported for G333. No assumptions could be } \\
\text { made }\end{array}$ \\
\hline $\mathbf{P}$ & Raw & $\mathrm{Ni}$ & $2.66 E+04$ & $4.53 E-04$ & AP102-3 & $\mathbf{Y}$ & $\begin{array}{l}\text { Density reported for } \mathrm{G} 358 \text { collected at } 334 \text { in from bottom } \\
\text { of tank at a } 150^{\circ} \text { angle. Sample was unheated, unheated } \\
\text { samples precipitated }\end{array}$ \\
\hline $\mathbf{P}$ & Raw & $\mathrm{Ni}$ & $2.64 E+04$ & $4.50 E-0.4$ & AP102-3 & Y & Density reported for $\mathrm{G} 443$ represents a composite sample \\
\hline $\mathbf{P}$ & Raw & $\mathrm{Ni}$ & $2.64 E+04$ & $4.50 E-04$ & AP102-3 & $Y$ & $\begin{array}{l}\text { Density reported for } 6460 \text { collected at } 226 \text { in from bottom } \\
\text { of tank at a } 270^{\circ} \text { angle. Sample was unheated, unheated } \\
\text { samples precipitated }\end{array}$ \\
\hline $\mathbf{P}$ & Raw & $\mathrm{Ni}$ & $2.63 E+0.4$ & $4.48 E-04$ & AP102-3 & $Y$ & $\begin{array}{l}\text { Density reported for G353 collected at } 334 \text { in from bottom } \\
\text { of tank at a } 150^{\circ} \text { angle. Sample was unheated, unheated } \\
\text { samples precipitated }\end{array}$ \\
\hline
\end{tabular}


TWRS Privatization Support $F$

\begin{tabular}{|c|c|c|c|c|c|c|c|c|c|c|c|}
\hline & & & & & nk Waste & haracteriza & on Sample I & armation & & & \\
\hline Reported Chemical & $\begin{array}{c}\text { Memo } \\
\text { Date }\end{array}$ & $\begin{array}{c}\text { Start } \\
\text { Analysis } \\
\text { Date }\end{array}$ & $\begin{array}{c}\text { Finish } \\
\text { Analysis Date }\end{array}$ & $\begin{array}{c}\text { Start } \\
\text { Sample } \\
\text { Date } \\
\end{array}$ & $\begin{array}{c}\text { Finish } \\
\text { Sample } \\
\text { Date } \\
\end{array}$ & $\begin{array}{l}\text { Sample } \\
\text { Number }\end{array}$ & $\begin{array}{c}\text { Tank Farm } \\
\text { Number }\end{array}$ & $\begin{array}{c}\text { Lab Sample } \\
\text { Number }\end{array}$ & $\begin{array}{l}\text { Density } \\
\text { (g/mL) }\end{array}$ & $\begin{array}{c}\text { Reported } \\
\text { Value }\end{array}$ & $\begin{array}{r}R e p \\
4\end{array}$ \\
\hline Nickel & $6 / 1 / 90$ & $4 / 30 / 89$ & $12 / 30 / 89$ & $4 / 27 / 89$ & $4 / 29 / 89$ & В082M3 & G311 & G359 & 1.20 & $2.63 E+04$ & $u$ \\
\hline Nickel & $6 / 1 / 90$ & $4 / 30 / 89$ & $12 / 30 / 89$ & $4 / 27 / 89$ & $4 / 29 / 89$ & BOB2M4 & G313 & G363 & 1.20 & $2.63 E+04$ & $u_{i}$ \\
\hline Nickel & $6 / 1 / 90$ & $4 / 30 / 89$ & $12 / 30 / 89$ & $4 / 27 / 89$ & $4 / 29 / 89$ & BOB2N1 & G310 & G454 & $(1.19)$ & $2.62 E+04$ & us \\
\hline Nickel & $6 / 1 / 90$ & $4 / 30 / 89$ & $12 / 30 / 89$ & $4 / 27 / 89$ & $4 / 29 / 89$ & BOB2M8 & G301 & G449 & NA & $2.61 E+04$ & us \\
\hline Nickel & $6 / 1 / 90$ & $4 / 30 / 89$ & $12 / 30 / 89$ & $4 / 27 / 89$ & $4 / 29 / 89$ & BOB2M2 & G309 & G358 & 1.20 & $2.60 E+04$ & ug \\
\hline Nickel & $6 / 1 / 90$ & $4 / 30 / 89$ & $12 / 30 / 89$ & $4 / 27 / 89$ & $4 / 29 / 89$ & В082M6 & G318 & G459 & 1.20 & $2.60 E+04$ & ug \\
\hline Nickel & $6 / 1 / 90$ & $4 / 30 / 89$ & $12 / 30 / 89$ & $4 / 27 / 89$ & $4 / 29 / 89$ & BOB2L7 & G299 & G342 & 1.20 & $2.59 E+04$ & ug \\
\hline Nickel & $6 / 1 / 90$ & $4 / 30 / 89$ & $12 / 30 / 89$ & $4 / 27 / 89$ & $4 / 29 / 89$ & BO82MO & G306 & G352 & 1.20 & $2.58 E+04$ & ug \\
\hline Nickel & $6 / 1 / 90$ & $4 / 30 / 89$ & $12 / 30 / 89$ & $4 / 27 / 89$ & $4 / 29 / 89$ & BOB2M5 & G315 & G364 & 1.19 & $2.58 E+04$ & ug \\
\hline Nickel & $6 / 1 / 90$ & $4 / 30 / 89$ & $12 / 30 / 89$ & $4 / 27 / 89$ & $4 / 29 / 89$ & $8082 M 8$ & G301 & G449 & NA & $2.58 \mathrm{E}+04$ & $u g_{i}$ \\
\hline Nickel & $6 / 1 / 90$ & $4 / 30 / 89$ & $12 / 30 / 89$ & $4 / 27 / 89$ & $4 / 29 / 89$ & ВОВ2M3 & G311 & G359 & 1.20 & $2.56 E+04$ & ugr \\
\hline Nickel & $6 / 1 / 90$ & $4 / 30 / 89$ & $12 / 30 / 89$ & $4 / 27 / 89$ & $4 / 29 / 89$ & В082M9 & G302 & G450 & $(1.20)$ & $2.56 E+04$ & ugl \\
\hline No2- & NS & NS & NS & NS & NS & NS & NS & NS & 1.24 & $1.22 E+\infty$ & Mole \\
\hline Nitrite & $4 / 1 / 93$ & NS & NS & NS & NS & BOB2NO & G307 & NS & NS & $3.82 \mathrm{E}+04$ & $u g / n$ \\
\hline Nitrite & $4 / 1 / 93$ & NS & NS & NS & NS & BOB2NO & G307 & NS & NS & $3.74 E+04$ & $u g / \Omega$ \\
\hline $\mathrm{NO2-}$ & $4 / 28 / 84$ & NS & NS & NS & NS & NS & NS & NS & NS & $8.05 \mathrm{E}-04$ & $g \Omega$ \\
\hline NO2 & $4 / 28 / 84$ & NS & NS & NS & NS & R-8469 & NS & NS & NS & $1.75 \mathrm{E}-05$ & mole \\
\hline NO2 & $4 / 28 / 84$ & NS & NS & NS & NS & $R 8469$ & NS & NS & NS & $1.74 \mathrm{E}-05$ & $\bar{M}$ \\
\hline Nitrite & $6 / 1 / 90$ & $4 / 89$ & $12 / 89$ & $4 / 27 / 89$ & $4 / 29 / 89$ & BOB2T9 & G327 & G443 & 1.20 & $5.51 E+04$ & ug/r \\
\hline Nitrite & $6 / 1 / 90$ & $4 / 89$ & $12 / 89$ & $4 / 27 / 89$ & $4 / 29 / 89$ & ВОВ2T9 & G327 & G443 & 1.20 & $5.45 E+04$ & $u g / r$ \\
\hline Nitrite & $6 / 1 / 90$ & $4 / 89$ & $12 / 89$ & $4 / 27 / 89$ & $4 / 29 / 89$ & BOB2M7 & G319 & G460 & 1.2 & $4.75 E+04$ & $u g / m$ \\
\hline Nitrite & $6 / 1 / 90$ & $4 / 89$ & $12 / 89$ & $4 / 27 / 89$ & 4/29/89 & BOB2M7 & G319 & G460 & 1.2 & $4.70 E+04$ & $\mathrm{ug} / \mathrm{m}$ \\
\hline Nitrite & $6 / 1 / 90$ & $4 / 89$ & $12 / 89$ & $4 / 27 / 89$ & $4 / 29 / 89$ & ВОВ2Т9 & G327 & G470* & 1.19 & $4.18 E+04$ & ug/m \\
\hline Nitrite & $6 / 1 / 90$ & $4 / 89$ & $12 / 89$ & $4 / 27 / 89$ & $4 / 29 / 89$ & ВОВ2Т9 & G327 & G470* & 1.19 & $4.15 E+04$ & ug/m \\
\hline Nitrite & $6 / 1 / 90$ & $4 / 89$ & $12 / 89$ & $4 / 27 / 89$ & $4 / 29 / 89$ & BOB2M9 & G302 & G450 & $(1.20)$ & $4.10 E+04$ & $\mathrm{ug} / \mathrm{ml}$ \\
\hline
\end{tabular}


TWRS Privatization Support $P_{1}$ Chemical Liquic

\begin{tabular}{|c|c|c|c|c|c|c|c|c|c|c|c|}
\hline & & & & & ank Waste & haracteriza & ion Sample & ormation & & & \\
\hline Reported Chemical & $\begin{array}{c}\text { Memo } \\
\text { Date }\end{array}$ & $\begin{array}{c}\text { Start } \\
\text { Analysis } \\
\text { Date }\end{array}$ & $\begin{array}{c}\text { Finish } \\
\text { Analysis Date } \\
\end{array}$ & $\begin{array}{c}\text { Start } \\
\text { Sample } \\
\text { Date } \\
\end{array}$ & $\begin{array}{c}\text { Finish } \\
\text { Sample } \\
\text { Date } \\
\end{array}$ & $\begin{array}{l}\text { Sample } \\
\text { Number }\end{array}$ & $\begin{array}{c}\text { Tank Farm } \\
\text { Number }\end{array}$ & $\begin{array}{c}\text { Lab Sample } \\
\text { Number }\end{array}$ & $\begin{array}{l}\text { Density } \\
\text { (g/mL) }\end{array}$ & $\begin{array}{l}\text { Reported } \\
\text { Value }\end{array}$ & $\begin{array}{r}\text { Rep. } \\
\mathbf{U} \\
\end{array}$ \\
\hline Nitrite & $6 / 1 / 90$ & $4 / 89$ & $12 / 89$ & $4 / 27 / 89$ & $4 / 29 / 89$ & BOB2M9 & G302 & G477* & 1.20 & $4.10 E+04$ & uga \\
\hline Nitrite & $6 / 1 / 90$ & $4 / 89$ & $12 / 89$ & $4 / 27 / 89$ & $4 / 29 / 89$ & BOB2N4 & G323 & G476* & 1.21 & $4.08 E+04$ & ugl \\
\hline Nitrite & $6 / 1 / 90$ & $4 / 89$ & $12 / 89$ & $4 / 27 / 89$ & $4 / 29 / 89$ & BOB2N1 & G310 & G471* & 1.19 & $4.03 E+04$ & ug \\
\hline Nitrite & $6 / 1 / 90$ & $4 / 89$ & $12 / 89$ & $4 / 27 / 89$ & $4 / 29 / 89$ & BOB2NO & G307 & G453 & NA & $3.94 E+04$ & vgl \\
\hline Nitrite & $6 / 1 / 90$ & $4 / 89$ & $12 / 89$ & $4 / 27 / 89$ & $4 / 29 / 89$ & BOB2N2 & G317 & G333 & NA & $3.91 E+04$ & ugl \\
\hline Nitrite & $6 / 1 / 90$ & $4 / 89$ & $12 / 89$ & $4 / 27 / 89$ & $4 / 29 / 89$ & BOB2N2 & G317 & G333 & NA & $3.88 E+04$ & $\mathrm{ug} h$ \\
\hline Nitrite & $6 / 1 / 90$ & $4 / 89$ & $12 / 89$ & $4 / 27 / 89$ & $4 / 29 / 89$ & BOB2M8 & G301 & G449 & NA & $3.87 E+04$ & $u g / 1$ \\
\hline Nitrite & $6 / 1 / 90$ & $4 / 89$ & $12 / 89$ & $4 / 27 / 89$ & $4 / 29 / 89$ & BOB2N1 & G310 & G454 & (1.19) & $3.85 E+04$ & $\mathrm{ug} / \mathrm{t}$ \\
\hline Nitrite & $6 / 1 / 90$ & $4 / 89$ & $12 / 89$ & $4 / 27 / 89$ & $4 / 29 / 89$ & ВOB2M8 & G301 & G449 & NA & $3.84 E+04$ & $u g / t$ \\
\hline Nitrite & $6 / 1 / 90$ & $4 / 89$ & $12 / 89$ & $4 / 27 / 89$ & $4 / 29 / 89$ & BOB2N1 & G310 & G454 & $(1.19)$ & $3.84 E+04$ & $u g / r$ \\
\hline Nitrite & $6 / 1 / 90$ & $4 / 89$ & $12 / 89$ & $4 / 27 / 89$ & $4 / 29 / 89$ & BOB2N3 & G322 & G338 & $N A$ & $3.84 E+04$ & $u g / n$ \\
\hline Nitrite & $6 / 1 / 90$ & $4 / 89$ & $12 / 89$ & $4 / 27 / 89$ & $4 / 29 / 89$ & BOB2N4 & G323 & G341 & 1.20 & $3.84 E+04$ & $\mathrm{ug} / \mathrm{n}$ \\
\hline Nitrite & $6 / 1 / 90$ & $4 / 89$ & $12 / 89$ & $4 / 27 / 89$ & $4 / 29 / 89$ & ВOB2M9 & G302 & G450 & $(1.20)$ & $3.83 E+04$ & ug/n \\
\hline Nitrite & $6 / 1 / 90$ & $4 / 89$ & $12 / 89$ & $4 / 27 / 89$ & $4 / 29 / 89$ & BOB2N1 & G310 & G471* & 1.19 & $3.83 E+04$ & ug/ $/ \pi$ \\
\hline Nitrite & $6 / 1 / 90$ & $4 / 89$ & $12 / 89$ & $4 / 27 / 89$ & $4 / 29 / 89$ & ВОВ2M5 & G315 & G364 & 1.19 & $3.81 E \div 04$ & ug/rir \\
\hline Nitrite & $6 / 1 / 90$ & $4 / 89$ & $12 / 89$ & $4 / 27 / 89$ & $4 / 29 / 89$ & ВOВ2M9 & G302 & G450 & $(1.20)$ & $3.80 E+04$ & $\mathrm{ug} / \mathrm{m}$ \\
\hline Nitrite & $6 / 1 / 90$ & $4 / 89$ & $12 / 89$ & $4 / 27 / 89$ & $4 / 29 / 89$ & BOB2NO & G307 & G453 & NA & $3.80 E \div 04$ & ug/m \\
\hline Nitrite & $6 / 1 / 90$ & $4 / 89$ & $12 / 89$ & $4 / 27 / 89$ & $4 / 29 / 89$ & BOB2M5 & G315 & G364 & 1.19 & $3.80 E+04$ & $\mathrm{ug} / \mathrm{m}$ \\
\hline Nitrite & $6 / 1 / 90$ & $4 / 89$ & $12 / 89$ & $4 / 27 / 89$ & $4 / 29 / 89$ & BOB2M4 & G313 & G363 & 1.20 & $3.79 E+0.4$ & $u g / m^{\prime}$ \\
\hline Nitrite & $6 / 1 / 90$ & $4 / 89$ & $12 / 89$ & $4 / 27 / 89$ & $4 / 29 / 89$ & Вов2М6 & G318 & G459 & 1.20 & $3.79 E+04$ & ug/m! \\
\hline Nitrite & $6 / 1 / 90$ & $4 / 89$ & $12 / 89$ & $4 / 27 / 89$ & $4 / 29 / 89$ & BOB2N3 & G322 & G338 & NA & $3.78 E+04$ & $u g / m l$ \\
\hline Nitrite & $6 / 1 / 90$ & $4 / 89$ & $12 / 89$ & $4 / 27 / 89$ & $4 / 29 / 89$ & BOB2L9 & G305 & G349 & 1.20 & $3.76 E+04$ & ug/ml \\
\hline
\end{tabular}




\begin{tabular}{|c|c|c|c|c|c|c|c|}
\hline \multirow[b]{2}{*}{ Code } & \multirow[b]{2}{*}{ Source } & \multicolumn{3}{|c|}{ Analyte Information } & \multirow[b]{2}{*}{$\begin{array}{c}\text { Reference } \\
\text { Number }\end{array}$} & \multirow[b]{2}{*}{$\begin{array}{l}\text { Validated } \\
\text { (Y/N) }\end{array}$} & \multirow[b]{2}{*}{ Notes } \\
\hline & & Analyte & $\begin{array}{l}\text { Conc. } \\
\text { (ug/L) }\end{array}$ & Conc. (mol/L) & & & \\
\hline$P$ & AP102-3 & No2 & $4.10 E+07$ & 8.91E-01 & AP102-3 & $Y$ & $\begin{array}{l}\text { Density reported for } \mathrm{G} 477 \text { collected at } 182 \text { in from bottom } \\
\text { of tank at a } 30^{\circ} \text { angle and was keep heated to } 40^{\circ} \mathrm{C} \text {. }\end{array}$ \\
\hline $\mathbf{P}$ & TCD & No2 & $4.08 E+07$ & 8.87E-01 & AP102-3 & $Y$ & $\begin{array}{l}\text { Density reported for } \mathrm{G} 476 \text { collected at } 99 \text { in from bottom of } \\
\text { tank at a } 270^{\circ} \text { angle and was keep heated to } 40^{\circ} \mathrm{C} \text {. }\end{array}$ \\
\hline $\mathbf{P}$ & TCD & NO2 & $4.03 E+07$ & 8.76E-01 & AP102-3 & $Y$ & $\begin{array}{l}\text { Density reported for } \mathrm{G} 471 \text { collected at } 208 \text { in from bottom } \\
\text { of tank at a } 150^{\circ} \text { angle and was keep heated to } 40^{\circ} \mathrm{C} \text {. }\end{array}$ \\
\hline$P$ & TCD & NO2 & $3.94 E+07$ & 8.57E-01 & AP102-3 & $Y$ & $\begin{array}{l}\text { Density not reported for G453. No assumptions could be } \\
\text { made }\end{array}$ \\
\hline$P$ & TCD & NO2 & $3.91 E+07$ & $8.50 E-01$ & AP102-3 & $Y$ & $\begin{array}{l}\text { Density not reported for G333. No assumptions could be } \\
\text { made }\end{array}$ \\
\hline$P$ & TCD & NO2 & $3.88 E+07$ & 8.43E-01 & AP102-3 & $Y$ & $\begin{array}{l}\text { Density not reported for G333. No assumptions could be } \\
\text { made }\end{array}$ \\
\hline$P$ & TCD & NO2 & $3.87 E+07$ & 8.41E-01 & AP102-3 & $Y$ & $\begin{array}{l}\text { Density not reported for G449. No assumptions could be } \\
\text { made }\end{array}$ \\
\hline$P$ & TCD & $\mathrm{NO} 2$ & $3.85 E+07$ & 8.37E-01 & AP102-3 & $Y$ & $\begin{array}{l}\text { Density not reported for G454. Density assumed same as } \\
\text { G471 which was collected at } 208 \text { in from bottom of tank at } \\
\text { a } 150^{\circ} \text { angle and was keep heated to } 40^{\circ} \mathrm{C} \text {. }\end{array}$ \\
\hline$P$ & TCD & NO2 & $3.84 E+07$ & 8.35E-01 & AP102-3 & $\gamma$ & $\begin{array}{l}\text { Density not reported for } 6449 \text {. No assumptions could be } \\
\text { made }\end{array}$ \\
\hline$P$ & TCD & NO2 & $3.84 E+07$ & 8.35E-01 & AP102-3 & $Y$ & $\begin{array}{l}\text { Density not reported for G454. Density assumed same as } \\
\text { G471 which was collected at } 208 \text { in from bottom of tank at } \\
\text { a } 150^{\circ} \text { angle and was keep heated to } 40^{\circ} \mathrm{C} \text {. }\end{array}$ \\
\hline$P$ & TCD & NO2 & $3.84 E+07$ & 8.35E-01 & AP102-3 & $Y$ & $\begin{array}{l}\text { Density not reported for G338. Na assumptions could be } \\
\text { made }\end{array}$ \\
\hline$P$ & TCD & NO2 & $3.84 E+07$ & 8.35E-01 & AP102-3 & $Y$ & $\begin{array}{l}\text { Density reported for G341 collected at } 99 \text { in from bottom of } \\
\text { tank at a } 270^{\circ} \text { angle. }\end{array}$ \\
\hline$P$ & TCD & NO2 & $3.83 E+07$ & 8.33E-01 & AP102-3 & $Y$ & $\begin{array}{l}\text { Density not reported for G450. Density assumed same as } \\
\text { G477 which was collected at } 182 \text { in from bottom of tank at } \\
\text { a } 30^{\circ} \text { angle and was keep heated to } 40^{\circ} \mathrm{C} \text {. }\end{array}$ \\
\hline$P$ & TCD & NO2 & $3.83 E+07$ & 8.33E-01 & AP102-3 & $Y$ & $\begin{array}{l}\text { Density reported for } G 471 \text { collected at } 208 \text { in from bottom } \\
\text { of tank at a } 150^{\circ} \text { angle and was keep heated to } 40^{\circ} \mathrm{C} \text {. }\end{array}$ \\
\hline$P$ & TCD & NO2 & $3.81 E+07$ & 8.28E-01 & AP102-3 & $\gamma$ & $\begin{array}{l}\text { Density reported for G364 collected at } 372 \text { in from bottom } \\
\text { of tank at a } 270^{\circ} \text { angle. Sample was unheated, unheated } \\
\text { samples precipitated }\end{array}$ \\
\hline $\mathbf{P}$ & TCD & $\mathrm{NO} 2$ & $3.80 E+07$ & 8.26E-01 & AP102-3 & $Y$ & $\begin{array}{l}\text { Density not reported for G450. Density assumed same as } \\
\text { G } 477 \text { which was collected at } 182 \text { in from bottom of tank at } \\
\text { a } 30^{\circ} \text { angle and was keep heated to } 40^{\circ} \mathrm{C} \text {. }\end{array}$ \\
\hline$P$ & TCD & NO2 & $3.80 E+07$ & 8.26E-01 & AP102-3 & $Y$ & $\begin{array}{l}\text { Density not reported for } \mathrm{G} 453 \text {. No assumptions could be } \\
\text { made }\end{array}$ \\
\hline$P$ & TCD & NO2 & $3.80 E+07$ & $8.26 E-01$ & AP102-3 & $Y$ & $\begin{array}{l}\text { Density reported for } \mathrm{G} 364 \text { collected at } 372 \text { in from bottom } \\
\text { of tank at a } 270^{\circ} \text { angle. Sample was unheated, unheated } \\
\text { samples precipitated }\end{array}$ \\
\hline $\mathbf{P}$ & TCD & $\mathrm{NO} 2$ & $3.79 E+07$ & 8.24E-01 & AP102-3 & $Y$ & $\begin{array}{l}\text { Density reported for } \mathrm{G} 363 \text { collected at } 18 \text { in from bottom of } \\
\text { tank at a } 150^{\circ} \text { angle. Sample was unheated, unheated } \\
\text { samples precipitated }\end{array}$ \\
\hline$P$ & TCD & NO2 & $3.79 E+07$ & 8.24E-01 & AP102-3 & $Y$ & $\begin{array}{l}\text { Density reported for G459 collected at } 226 \text { in from bottom } \\
\text { of tank at a } 270^{\circ} \text { angle. Sample was unheated, unheated } \\
\text { samples precipitated }\end{array}$ \\
\hline $\mathbf{P}$ & TCD & NO2 & $3.78 E+07$ & $8.22 E-01$ & AP102-3 & $Y$ & $\begin{array}{l}\text { Density not reported for G338. No assumptions could be } \\
\text { made }\end{array}$ \\
\hline $\mathbf{P}$ & TCD & NO2 & $3.76 E+07$ & 8.17E-01 & AP102-3 & $Y$ & $\begin{array}{l}\text { Density reported for G349 collected at } 34 \text { in from bottom of } \\
\text { tank at a } 30^{\circ} \text { angle. Sample was unheated, unheated } \\
\text { samples precipitated }\end{array}$ \\
\hline
\end{tabular}




\begin{tabular}{|c|c|c|c|c|c|c|c|c|c|c|c|}
\hline & & & & & ank Waste & haracteriza & on Sample Ir & imation & & & \\
\hline Reported Chemical & $\begin{array}{c}\text { Memo } \\
\text { Date }\end{array}$ & $\begin{array}{c}\text { Start } \\
\text { Analysis } \\
\text { Date }\end{array}$ & $\begin{array}{c}\text { Finish } \\
\text { Analysis Date }\end{array}$ & $\begin{array}{c}\text { Start } \\
\text { Sample } \\
\text { Date } \\
\end{array}$ & $\begin{array}{c}\text { Finish } \\
\text { Sample } \\
\text { Date } \\
\end{array}$ & $\begin{array}{l}\text { Sample } \\
\text { Number }\end{array}$ & $\begin{array}{c}\text { Tank Farm } \\
\text { Number }\end{array}$ & $\begin{array}{c}\text { Lab Sample } \\
\text { Number }\end{array}$ & $\begin{array}{l}\text { Density } \\
(\mathrm{g} / \mathrm{mL})\end{array}$ & $\begin{array}{c}\text { Reported } \\
\text { Value } \\
\end{array}$ & $\begin{array}{r}\text { Repo } \\
U_{n} \\
\end{array}$ \\
\hline Nitrite & $6 / 1 / 90$ & $4 / 89$ & $12 / 89$ & $4 / 27 / 89$ & $4 / 29 / 89$ & BOB2M4 & G313 & G363 & 1.20 & $3.76 E+04$ & $\mathrm{ug} / \mathrm{s}$ \\
\hline Nitrite & $6 / 1 / 90$ & $4 / 89$ & $12 / 89$ & $4 / 27 / 89$ & $4 / 29 / 89$ & В0В2M3 & G311 & G359 & 1.20 & $3.74 E+04$ & $u g / n$ \\
\hline Nitrite & $6 / 1 / 90$ & $4 / 89$ & $12 / 89$ & $4 / 27 / 89$ & $4 / 29 / 89$ & $\mathrm{BOB} 2 \mathrm{M} 1$ & G308 & G353 & 1.20 & $3.72 E+04$ & $u g / \pi$ \\
\hline Nitrite & $6 / 1 / 90$ & $4 / 89$ & $12 / 89$ & $4 / 27 / 89$ & $4 / 29 / 89$ & BOB2L8 & G303 & G348 & 1.21 & $3.70 E+04$ & $u g / \pi$ \\
\hline Nitrite & $6 / 1 / 90$ & $4 / 89$ & $12 / 89$ & $4 / 27 / 89$ & $4 / 29 / 89$ & BOB2L8 & G303 & G510* & 1.21 & $3.70 E+04$ & $u g / \pi$ \\
\hline Nitrite & $6 / 1 / 90$ & $4 / 89$ & $12 / 89$ & $4 / 27 / 89$ & $4 / 29 / 89$ & В0В2MO & G306 & G352 & 1.20 & $3.70 E+04$ & $\mathrm{ug} / \mathrm{m}$ \\
\hline Nitrite & $6 / 1 / 90$ & $4 / 89$ & $12 / 89$ & $4 / 27 / 89$ & $4 / 29 / 89$ & ВОВ2M2 & G309 & G515* & $(1.20)$ & $3.70 E+04$ & $\mathrm{ug} / \mathrm{m}$ \\
\hline Nitrite & $6 / 1 / 90$ & $4 / 89$ & $12 / 89$ & $4 / 27 / 89$ & $4 / 29 / 89$ & Вов2M3 & G311 & G359 & 1.20 & $3.70 E+04$ & $u g / m$ \\
\hline Nitrite & $6 / 1 / 90$ & $4 / 89$ & $12 / 89$ & $4 / 27 / 89$ & $4 / 29 / 89$ & BOB2M6 & G318 & G517* & $(1.20)$ & $3.70 E+04$ & $\mathrm{ug} / \mathrm{m}^{\prime}$ \\
\hline Nitrite & $6 / 1 / 90$ & $4 / 89$ & $12 / 89$ & $4 / 27 / 89$ & $4 / 29 / 89$ & BOB2N4 & G323 & G341 & 1.20 & $3.70 E+04$ & $\mathrm{ug} / \mathrm{ml}$ \\
\hline Nitrite & $6 / 1 / 90$ & $4 / 89$ & $12 / 89$ & $4 / 27 / 89$ & $4 / 29 / 89$ & $\mathrm{BOB} 2 \mathrm{N4}$ & G323 & G476* & 1.21 & $3.70 E+04$ & $\mathrm{ug} / \mathrm{ml}$ \\
\hline Nitrite & $6 / 1 / 90$ & $4 / 89$ & $12 / 89$ & $4 / 27 / 89$ & $4 / 29 / 89$ & $\mathrm{BOB2M3}$ & G311 & G516* & $(1.20)$ & $3.69 E+04$ & $\mathrm{ug} / \mathrm{ml}$ \\
\hline Nitrite & $6 / 1 / 90$ & $4 / 89$ & $12 / 89$ & $4 / 27 / 89$ & $4 / 29 / 89$ & B082L7 & G299 & G342 & 1.20 & $3.69 E+04$ & $\mathrm{ug} / \mathrm{ml}$. \\
\hline Nitrite & $6 / 1 / 90$ & $4 / 89$ & $12 / 89$ & $4 / 27 / 89$ & $4 / 29 / 89$ & BOB2L.9 & G305 & G349 & 1.20 & $3.69 E+04$ & $\mathrm{ug} / \mathrm{mL}$ \\
\hline Nitrite & $6 / 1 / 90$ & $4 / 89$ & $12 / 89$ & $4 / 27 / 89$ & $4 / 29 / 89$ & BOB2M6 & G318 & G459 & 1.20 & $3.69 E+04$ & $\mathrm{ug} / \mathrm{mL}$ \\
\hline Nitrite & $6 / 1 / 90$ & $4 / 89$ & $12 / 89$ & $4 / 27 / 89$ & $4 / 29 / 89$ & 80B2M1 & G308 & G522* & $(1.20)$ & $3.67 E+04$ & $\mathrm{ug} / \mathrm{mL}$ \\
\hline Nitrite & $6 / 1 / 90$ & $4 / 89$ & $12 / 89$ & $4 / 27 / 89$ & $4 / 29 / 89$ & BOB2L7 & G299 & G509 * & $(1.20)$ & $3.65 E+04$ & $\mathrm{ug} / \mathrm{mL}$ \\
\hline Nitrite & $6 / 1 / 90$ & $4 / 89$ & $12 / 89$ & $4 / 27 / 89$ & $4 / 29 / 89$ & BOB2L8 & G303 & G348 & 1.21 & $3.65 E+04$ & ug $/ \mathrm{mL}$ \\
\hline Nitrite & $6 / 1 / 90$ & $4 / 89$ & $12 / 89$ & $4 / 27 / 89$ & $4 / 29 / 89$ & BOB2M2 & G309 & G358 & 1.20 & $3.65 E+04$ & $\mathrm{ug} / \mathrm{mL}$ \\
\hline Nitrite & $6 / 1 / 90$ & $4 / 89$ & $12 / 89$ & $4 / 27 / 89$ & $4 / 29 / 89$ & BOB2M2 & G309 & G358 & 1.20 & $3.65 E \div 04$ & $\mathrm{ug} / \mathrm{mL}$ \\
\hline
\end{tabular}




\begin{tabular}{|c|c|c|c|c|c|c|c|}
\hline \multirow[b]{2}{*}{ Code } & \multirow[b]{2}{*}{ Source } & \multicolumn{3}{|c|}{ Analyte Information } & \multirow[b]{2}{*}{$\begin{array}{c}\text { Reference } \\
\text { Number }\end{array}$} & \multirow[b]{2}{*}{$\begin{array}{l}\text { Validated } \\
\text { (Y/N) }\end{array}$} & \multirow[b]{2}{*}{ Notes } \\
\hline & & Analyte & $\begin{array}{l}\text { Conc. } \\
\text { (ug/L) }\end{array}$ & Conc. (mol/L) & & & \\
\hline $\mathbf{P}$ & TCD & NO2 & $3.76 E+07$ & 8.17E-01 & AP102-3 & $\mathbf{Y}$ & $\begin{array}{l}\text { Density reported for } \mathrm{G} 363 \text { collected at } 18 \text { in from bottom of } \\
\text { tank at a } 150^{\circ} \text { angle. Sample was unheated, unheated } \\
\text { samples precipitated }\end{array}$ \\
\hline $\mathbf{P}$ & TCD & NO2 & $3.74 E+07$ & 8.13E-01 & AP102-3 & $\mathbf{Y}$ & $\begin{array}{l}\text { Density reported for G359 collected at } 162 \text { in from bottom } \\
\text { of tank at a } 150^{\circ} \text { angle. Sample was unheated, unheated } \\
\text { samples precipitated }\end{array}$ \\
\hline $\mathbf{P}$ & $T C D$ & NO2 & $3.72 E+07$ & 8.09E-01 & AP102-3 & $\boldsymbol{Y}$ & $\begin{array}{l}\text { Density reported for G353 collected at } 334 \text { in from bottom } \\
\text { of tank at a } 150^{\circ} \text { angle. Sample was unheated, unheated } \\
\text { samples precipitated }\end{array}$ \\
\hline$P$ & $T C D$ & NO2 & $3.70 E+07$ & $8.04 E-01$ & AP102-3 & $\mathbf{Y}$ & $\begin{array}{l}\text { Density reported for } \mathrm{G} 348 \text { collected at } 125 \text { in from bottom } \\
\text { of tank at a } 30^{\circ} \text { angle. Sample was unheated, unheated } \\
\text { samples precipitated }\end{array}$ \\
\hline$P$ & TCD & No2 & $3.70 E+07$ & 8.04E-01 & AP102-3 & $\mathrm{Y}$ & $\begin{array}{l}\text { Density not reported for G510. Density assumed same as } \\
\text { G348 which was collected at } 125 \text { in from bottom of tank at } \\
\text { a } 30^{\circ} \text { angle. This sample was heated. }\end{array}$ \\
\hline $\mathbf{P}$ & $T C D$ & No2 & $3.70 E+07$ & 8.04E-01 & AP102-3 & $Y$ & $\begin{array}{l}\text { Density reported for } \mathrm{G} 352 \text { collected at } 34 \text { in from bottom of } \\
\text { tank at a } 30^{\circ} \text { angle. Sample was unheated, unheated } \\
\text { samples precipitated }\end{array}$ \\
\hline $\mathbf{P}$ & TCD & NO2 & $3.70 E+07$ & 8.04E-01 & AP102-3 & $\mathbf{Y}$ & $\begin{array}{l}\text { Density not reported for G515. Density assumed same as } \\
\text { G358 which was collected at } 334 \text { in from bottom of tank at } \\
\text { a } 150^{\circ} \text { angle. This sample was heated. }\end{array}$ \\
\hline $\mathbf{P}$ & TCD & NO2 & $3.70 E+07$ & 8.04E-01 & AP102-3 & $\mathbf{Y}$ & $\begin{array}{l}\text { Density reported for G359 collected at } 162 \text { in from bottom } \\
\text { of tank at a } 150^{\circ} \text { angle. Sample was unheated, unheated } \\
\text { samples precipitated }\end{array}$ \\
\hline$P$ & TCD & NO2 & $3.70 E+07$ & $8.04 E-01$ & AP102-3 & $\mathbf{Y}$ & $\begin{array}{l}\text { Density not reported for G517. Density assumed same as } \\
\text { G459 which was collected at } 226 \text { in from bottom of tank at } \\
\text { a } 270^{\circ} \text { angle. This sample was heated. }\end{array}$ \\
\hline$P$ & TCD & NO2 & $3.70 E+07$ & 8.04E-01 & AP102-3 & $Y$ & $\begin{array}{l}\text { Density reported for } \mathrm{G} 341 \text { collected at } 99 \text { in from bottom of } \\
\text { tank at a } 270^{\circ} \text { angle. }\end{array}$ \\
\hline$P$ & TCD & NO2 & $3.70 E+07$ & 8.04E-01 & AP102-3 & $\mathbf{Y}$ & $\begin{array}{l}\text { Density reported for G476 collected at } 99 \text { in from bottom of } \\
\text { tank at a } 270^{\circ} \text { angle and was keep heated to } 40^{\circ} \mathrm{C} \text {. }\end{array}$ \\
\hline$P$ & TCD & NO2 & $3.69 E+07$ & $8.02 E-01$ & AP102-3 & $\mathbf{Y}$ & $\begin{array}{l}\text { Density not reported for G516. Density assumed same as } \\
\text { G359 which was collected at } 162 \text { in from bottom of tank at } \\
\text { a } 150^{\circ} \text { angle. This sample was heated. }\end{array}$ \\
\hline $\mathbf{P}$ & TCD & NO2 & $3.69 E+07$ & $8.02 E-01$ & AP102-3 & $\gamma$ & $\begin{array}{l}\text { Density reported for G342 collected at } 306 \text { in from bottom } \\
\text { of tank at a } 30^{\circ} \text { angle. Sample was unheated, unheated } \\
\text { samples precipitated }\end{array}$ \\
\hline$P$ & TCD & NO2 & $3.69 E+07$ & $8.02 E-01$ & AP102-3 & $Y$ & $\begin{array}{l}\text { Density reported for G349 collected at } 34 \text { in from bottom of } \\
\text { tank at a } 30^{\circ} \text { angle. Sample was unheated, unheated } \\
\text { samples precipitated }\end{array}$ \\
\hline$P$ & TCD & NO2 & $3.69 E+07$ & $8.02 E-01$ & AP102-3 & $Y$ & $\begin{array}{l}\text { Density reported for G459 collected at } 226 \text { in from bottom } \\
\text { of tank at a } 270^{\circ} \text { angle. Sample was unheated, unheated } \\
\text { samples precipitated }\end{array}$ \\
\hline$P$ & TCD & NO2 & $3.67 E+07$ & $7.98 E-01$ & AP102-3 & $Y$ & $\begin{array}{l}\text { Density not reported for G522. Density assumed sams as } \\
\text { G353 which was collected at } 334 \text { in from bottom of tank at } \\
\text { a } 150^{\circ} \text { angle. This sample was heated. }\end{array}$ \\
\hline$P$ & TCD & NO2 & $3.65 E+07$ & 7.93E-01 & AP102-3 & $\mathbf{Y}$ & $\begin{array}{l}\text { Density not reported for G509. Density assumed same as } \\
\mathrm{G342} \text { which was collected at } 306 \text { in from bottom of tank at } \\
\text { a } 30^{\circ} \text { angle. This sample was heated. }\end{array}$ \\
\hline$P$ & TCD & $\mathrm{NO} 2$ & $3.65 E+07$ & 7.93E-01 & AP102-3 & $\mathbf{Y}$ & $\begin{array}{l}\text { Density reported for } G 348 \text { collected at } 125 \text { in from bottom } \\
\text { of tank at a } 30^{\circ} \text { angle. Sample was unheated, unheated } \\
\text { samples precipitated }\end{array}$ \\
\hline$P$ & TCD & $\mathrm{NO} 2$ & $3.65 E+07$ & 7.93E-01 & AP102-3 & $Y$ & $\begin{array}{l}\text { Density reported for } G 358 \text { collected at } 334 \text { in from bottom } \\
\text { of tank at a } 150^{\circ} \text { angle. Sample was unheated, unheated } \\
\text { samples precipitated }\end{array}$ \\
\hline $\mathbf{P}$ & TCD & No2 & $3.65 E+07$ & 7.93E-01 & AP102-3 & $\mathbf{Y}$ & $\begin{array}{l}\text { Density reported for G358 collected at } 334 \text { in from bottom } \\
\text { of tank at a } 150^{\circ} \text { angle. Sample was unheated, unheated } \\
\text { samples precipitated }\end{array}$ \\
\hline
\end{tabular}




\begin{tabular}{|c|c|c|c|c|c|c|c|c|c|c|c|}
\hline & & & & & ank Waste & haracteriz & Ion Sampla & ormation & & & \\
\hline Reported Chemical & $\begin{array}{c}\text { Memo } \\
\text { Date }\end{array}$ & $\begin{array}{c}\text { Start } \\
\text { Analysis } \\
\text { Date } \\
\end{array}$ & $\begin{array}{c}\text { Finish } \\
\text { Anzlysis Date }\end{array}$ & $\begin{array}{c}\text { Start } \\
\text { Sample } \\
\text { Date }\end{array}$ & $\begin{array}{c}\text { Finish } \\
\text { Sample } \\
\text { Date } \\
\end{array}$ & $\begin{array}{l}\text { Sample } \\
\text { Number }\end{array}$ & $\begin{array}{c}\text { Tank Farm } \\
\text { Number }\end{array}$ & $\begin{array}{c}\text { Lab Sample } \\
\text { Number }\end{array}$ & $\begin{array}{l}\text { Density } \\
\text { (g/mL) }\end{array}$ & $\begin{array}{c}\text { Reported } \\
\text { Value } \\
\end{array}$ & $\begin{array}{r}\text { Repa } \\
U_{n} \\
\end{array}$ \\
\hline Nitrite & $6 / 1 / 90$ & $4 / 89$ & $12 / 89$ & $4 / 27 / 89$ & $4 / 29 / 89$ & BOB2M6 & G318 & G517* & $(1.20)$ & $3.65 E+04$ & ught \\
\hline Nitrite & $6 / 1 / 90$ & $4 / 89$ & $12 / 89$ & $4 / 27 / 89$ & $4 / 29 / 89$ & BOB2MO & G306 & G352 & 1.20 & $3.64 E+04$ & ug/r \\
\hline Nitrite & $6 / 1 / 90$ & $4 / 89$ & $12 / 89$ & $4 / 27 / 89$ & $4 / 29 / 89$ & BOB2NO & G307 & G527* & NA & $3.64 E+04$ & ug/n \\
\hline Nitrite & $6 / 1 / 90$ & $4 / 89$ & $12 / 89$ & $4 / 27 / 89$ & $4 / 29 / 89$ & 8OB2MI & G308 & G522* & $(1.20)$ & $3.63 \varepsilon+04$ & $u g / n$ \\
\hline Nitrite & $6 / 1 / 90$ & $4 / 89$ & $12 / 89$ & $4 / 27 / 89$ & $4 / 29 / 89$ & Вов2M9 & G302 & G450 & $(1.20)$ & $3.62 E+0.4$ & $u g / \pi$ \\
\hline Nitrite & $6 / 1 / 90$ & $4 / 89$ & $12 / 89$ & $4 / 27 / 89$ & $4 / 29 / 89$ & BOB2M9 & G302 & G477* & 1.20 & $3.62 E+04$ & $u g / \pi$ \\
\hline Nitrite & $6 / 7 / 90$ & $4 / 89$ & $12 / 89$ & $4 / 27 / 89$ & $4 / 29 / 89$ & BOB2L7 & G299 & G342 & 1.20 & $3.60 E+04$ & $u g / m$ \\
\hline Nitrite & $6 / 1 / 90$ & $4 / 89$ & $12 / 89$ & $4 / 27 / 89$ & $4 / 29 / 89$ & $\mathrm{BOB2M4}$ & G313 & G533* & $(1.20)^{\circ}$ & $3.60 E+04$ & $u g / m$ \\
\hline Nitrite & $6 / 1 / 90$ & $4 / 89$ & $12 / 89$ & $4 / 27 / 89$ & $4 / 29 / 89$ & BOB2L9 & G305 & G511* & $(1.20)$ & $3.58 E+04$ & $\mathrm{ug} / \mathrm{m}$ \\
\hline Nitrite & $6 / 1 / 90$ & $4 / 89$ & $12 / 89$ & $4 / 27 / 89$ & $4 / 29 / 89$ & ВOB2MO & G306 & G521" & $(1.20)$ & $3.58 E+04$ & $\mathrm{ug} / \mathrm{ml}$ \\
\hline Nitrite & $6 / 1 / 90$ & $4 / 89$ & $12 / 89$ & $4 / 27 / 89$ & $4 / 29 / 89$ & BOB2M1 & G308 & G353 & 1.20 & $3.58 E+04$ & $u g / m l$ \\
\hline Nitrite & $6 / 1 / 90$ & $4 / 89$ & $12 / 89$ & $4 / 27 / 89$ & $4 / 29 / 89$ & BOB2L7 & G299 & G509* & $(1.20)$ & $3.57 E+04$ & $\mathrm{ug} / \mathrm{ml}$ \\
\hline Nitrite & $6 / 1 / 90$ & $4 / 89$ & $12 / 89$ & $4 / 27 / 89$ & $4 / 29 / 89$ & B082M9 & G302 & G477* & 1.20 & $3.56 \mathrm{E}+04$ & $\mathrm{ug} / \mathrm{ml}$ \\
\hline Nitrite & $6 / 1 / 90$ & $4 / 89$ & $12 / 89$ & $4 / 27 / 89$ & $4 / 29 / 89$ & BOB2M4 & G313 & G533* & $(1.20)$ & $3.55 E+04$ & $u g / m L$ \\
\hline Nitrite & $6 / 1 / 90$ & $4 / 89$ & $12 / 89$ & $4 / 27 / 89$ & $4 / 29 / 89$ & BOB2L9 & G305 & G511* & $(1.20)$ & $3.54 E+04$ & $\mathrm{ug} / \mathrm{mL}$ \\
\hline Nitrite & $6 / 1 / 90$ & $4 / 89$ & $12 / 89$ & $4 / 27 / 89$ & $4 / 29 / 89$ & BOB2M7 & G319 & G536* & $(1.20)$ & $3.53 E+04$ & $u g / m L$ \\
\hline Nitrite & 6/1/90 & $4 / 89$ & $12 / 89$ & $4 / 27 / 89$ & $4 / 29 / 89$ & BOB2M5 & G315 & G534" & (1.19) & $3.52 E+04$ & $\mathrm{ug} / \mathrm{mL}$ \\
\hline Nitrite & $6 / 1 / 90$ & $4 / 89$ & $12 / 89$ & $4 / 27 / 89$ & $4 / 29 / 89$ & вов2М9 & G302 & G477* & 1.20 & $3.50 E+04$ & $u g / \mathrm{mL}$ \\
\hline Nitrite & $6 / 1 / 90$ & $4 / 89$ & $12 / 89$ & $4 / 27 / 89$ & $4 / 29 / 89$ & BOB2M5 & G315 & G534* & (1.19) & $3.50 E+04$ & $u g / \mathrm{mL}$ \\
\hline Nitrite & $6 / 1 / 90$ & $4 / 89$ & $12 / 89$ & $4 / 27 / 89$ & $4 / 29 / 89$ & BOB2M2 & G309 & G515* & $(1.20)$ & $3.49 E+04$ & $u g / m L$ \\
\hline
\end{tabular}




\begin{tabular}{|c|c|c|c|c|c|c|c|}
\hline \multirow[b]{2}{*}{ Code } & \multirow[b]{2}{*}{ Source } & \multicolumn{3}{|c|}{ Analyte Information } & \multirow[b]{2}{*}{$\begin{array}{c}\text { Reference } \\
\text { Number }\end{array}$} & \multirow[b]{2}{*}{$\begin{array}{l}\text { Validated } \\
\text { [YNI }\end{array}$} & \multirow[b]{2}{*}{ Notes } \\
\hline & & Analyre & $\begin{array}{l}\text { Conc. } \\
\text { (ug/L) } \\
\end{array}$ & Conc. (mol/L) & & & \\
\hline $\mathbf{P}$ & TCD & $\mathrm{NO} 2$ & $3.65 E+07$ & 7.93E-01 & AP102-3 & $Y$ & $\begin{array}{l}\text { Density not reported for G517. Density assumed same as } \\
\text { G459 which was collected at } 226 \text { in from bottom of tank at } \\
\text { a } 270^{\circ} \text { angle. This sample was heated. }\end{array}$ \\
\hline$P$ & TCD & NO2 & $3.64 E+07$ & 7.91E-01 & AP102-3 & $Y$ & $\begin{array}{l}\text { Density reported for } \mathrm{G} 352 \text { collected at } 34 \text { in from bottom of } \\
\text { tank at a } 30^{\circ} \text { angle. Sample was unheated, unheated } \\
\text { samples precipitated }\end{array}$ \\
\hline $\mathbf{P}$ & TCD & NO2 & $3.64 E+07$ & 7.91E-01 & AP102-3 & Y & $\begin{array}{l}\text { Density not reported for G527. No assumptions could be } \\
\text { made }\end{array}$ \\
\hline $\mathbf{P}$ & TCD & No2 & $3.63 E+07$ & 7.89E-01 & AP102-3 & $Y$ & $\begin{array}{l}\text { Density not reported for G522. Density assumed same as } \\
\text { G353 which was collected at } 334 \text { in from bottom of tank at } \\
\text { a } 150^{\circ} \text { angle. This sample was heated. }\end{array}$ \\
\hline $\mathbf{P}$ & TCD & NO2 & $3.62 E+07$ & 7.87E-01 & AP102-3 & $Y$ & $\begin{array}{l}\text { Density not reported for G450. Density assumed same as } \\
\text { G477 which was collected at } 182 \text { in from bottom of tank at } \\
\text { a } 30^{\circ} \text { angle and was keep heated to } 40^{\circ} \mathrm{C} \text {. }\end{array}$ \\
\hline $\mathbf{P}$ & AP102-3 & NO2 & $3.62 E+07$ & 7.87E-01 & AP102-3 & $Y$ & $\begin{array}{l}\text { Density reported for } \mathrm{G} 477 \text { collected at } 182 \text { in from bottom } \\
\text { of tank at a } 30^{\circ} \text { angle and was keep heated to } 40^{\circ} \mathrm{C} \text {. }\end{array}$ \\
\hline$P$ & $T C D$ & NO2 & $3.60 E+07$ & $7.83 E-01$ & AP102-3 & $\mathbf{Y}$ & $\begin{array}{l}\text { Density reported for } \mathrm{G} 342 \text { collected at } 306 \text { in from bottom } \\
\text { of tank at a } 30^{\circ} \text { angle. Sample was unheated, unheated } \\
\text { samples precipitated }\end{array}$ \\
\hline $\mathbf{P}$ & TCD & NO2 & $3.60 E+07$ & $7.83 E-01$ & AP102-3 & $\gamma$ & $\begin{array}{l}\text { Density not reported for G533. Density assumed same as } \\
\text { G363 which was collected at } 18 \text { in from bottom of tank at a } \\
150^{\circ} \text { angle. This sample was heated. }\end{array}$ \\
\hline $\mathbf{P}$ & TCD & No2 & $3.58 E+07$ & $7.78 \mathrm{E}-01$ & AP102-3 & $Y$ & $\begin{array}{l}\text { Density not reported for G511. Density assumed same as } \\
\text { G349 which was callected at } 34 \text { in from bottom of tank at a } \\
30^{\circ} \text { angle. This sample was heated. }\end{array}$ \\
\hline $\mathbf{P}$ & $T C D$ & NO2 & $3.58 E+07$ & 7.78E-01 & AP102-3 & $\gamma$ & $\begin{array}{l}\text { Density not reported for G521. Density assumed same as } \\
G 352 \text { which was collected at } 34 \text { in from bottom of tank at a } \\
30^{\circ} \text { angle. This sample was heated. }\end{array}$ \\
\hline $\mathbf{P}$ & TCD & NO2 & $3.58 E+07$ & $7.78 \mathrm{E}-01$ & AP102-3 & $Y$ & $\begin{array}{l}\text { Density reported for G353 collected at } 334 \text { in from bottom } \\
\text { of tank at a } 150^{\circ} \text { angle. Sample was unheated, unheated } \\
\text { samples precipitated }\end{array}$ \\
\hline $\mathbf{P}$ & TCD & No2 & $3.57 E+07$ & $7.76 E-01$ & AP102-3 & $\mathrm{Y}$ & $\begin{array}{l}\text { Density not reported for G509. Density assumed same as } \\
\text { G342 which was collected at } 306 \text { in from bottom of tank at } \\
\text { a } 30^{\circ} \text { angle. This sample was heated. }\end{array}$ \\
\hline$P$ & AP102-3 & NO2 & $3.56 \mathrm{E}+07$ & $7.74 E-01$ & AP102-3 & $\mathbf{Y}$ & $\begin{array}{l}\text { Density reported for } \mathrm{G} 477 \text { collected at } 182 \text { in from bottom } \\
\text { of tank at a } 30^{\circ} \text { angle and was keep heated to } 40^{\circ} \mathrm{C} \text {. }\end{array}$ \\
\hline $\mathbf{P}$ & TCD & NO2 & $3.55 E+07$ & $7.72 E-01$ & AP102-3 & $Y$ & $\begin{array}{l}\text { Density not reported for G533. Density assumed same as } \\
\text { G363 which was collected at } 18 \text { in from bottom of tank at a } \\
150^{\circ} \text { angle. This sample was heated. }\end{array}$ \\
\hline $\mathbf{P}$ & TCD & NO2 & $3.54 \mathrm{E}+07$ & 7.70E-01 & AP102-3 & $Y$ & $\begin{array}{l}\text { Density nat reported for G511. Density assumed same as } \\
\text { G349 which was collected at } 34 \text { in from bottom of tank at a } \\
30^{\circ} \text { angle. This sample was heated. }\end{array}$ \\
\hline $\mathbf{P}$ & TCD & NO2 & $3.53 E+07$ & 7.67E-01 & AP102-3 & $Y$ & $\begin{array}{l}\text { Density not reported for G536. Density assumed same as } \\
\text { G460 which was collected at } 226 \text { in from bottom of tank at } \\
\text { a } 270^{\circ} \text { angle. This sample was heated. }\end{array}$ \\
\hline $\mathbf{P}$ & TCD & NO2 & $3.52 \mathrm{E}+07$ & $7.65 \mathrm{E}-01$ & AP102-3 & $Y$ & $\begin{array}{l}\text { Density not reported for G534. Density assumed same as } \\
\text { G364 which was collected at } 372 \text { in from bottom of tank at } \\
\text { a } 270^{\circ} \text { angle. This sample was heated. }\end{array}$ \\
\hline $\mathbf{P}$ & AP102-3 & No2 & $3.50 \mathrm{E}+07$ & 7.61E-01 & AP102-3 & $\gamma$ & $\begin{array}{l}\text { Density reported for } \mathrm{G} 477 \text { collected at } 182 \text { in from bottom } \\
\text { of tank at a } 30^{\circ} \text { angle and was keep heated to } 40^{\circ} \mathrm{C} \text {. }\end{array}$ \\
\hline$P$ & $T C D$ & NO2 & $3.50 E+07$ & 7.61E-01 & AP102-3 & $\mathbf{Y}$ & $\begin{array}{l}\text { Density not reported for G534. Density assumed same as } \\
\text { G364 which was collected at } 372 \text { in from bottom of tank at } \\
\text { a } 270^{\circ} \text { angle. This sample was heated. }\end{array}$ \\
\hline $\mathbf{P}$ & TCD & NO2 & $3.49 E+07$ & 7.59E-01 & AP102-3 & $Y$ & $\begin{array}{l}\text { Density not reported for G515. Density assumed same as } \\
\text { G358 which was collected at } 334 \text { in from bottom of tank at } \\
\text { a } 150^{\circ} \text { angle. This sample was heated. }\end{array}$ \\
\hline
\end{tabular}


TWRS Privatization Support $\mathrm{Pr}$ Chemical Liquic

\begin{tabular}{|c|c|c|c|c|c|c|c|c|c|c|c|}
\hline & & & & & ank Waste & haracteriza & ion Sample Is & ormation & & & \\
\hline Reported Chemical & $\begin{array}{c}\text { Memo } \\
\text { Date } \\
\end{array}$ & $\begin{array}{c}\text { Start } \\
\text { Analysis } \\
\text { Date } \\
\end{array}$ & $\begin{array}{c}\text { Finish } \\
\text { Analysis Date } \\
\end{array}$ & $\begin{array}{c}\text { Start } \\
\text { Sample } \\
\text { Date } \\
\end{array}$ & $\begin{array}{c}\text { Finish } \\
\text { Sample } \\
\text { Date } \\
\end{array}$ & $\begin{array}{l}\text { Sample } \\
\text { Number }\end{array}$ & $\begin{array}{c}\text { Tank Farm } \\
\text { Number }\end{array}$ & $\begin{array}{c}\text { Lab Sample } \\
\text { Number }\end{array}$ & $\begin{array}{l}\text { Density } \\
(\mathrm{g} / \mathrm{mL})\end{array}$ & $\begin{array}{c}\text { Reported } \\
\text { Value }\end{array}$ & $\begin{array}{r}\text { Repi } \\
\mathbf{U} \\
\end{array}$ \\
\hline Nitrite & $6 / 1 / 90$ & $4 / 89$ & $12 / 89$ & $4 / 27 / 89$ & $4 / 29 / 89$ & BOB2N3 & G322 & G529* & NA & $3.49 E+04$ & ugi \\
\hline Nitrite & $6 / 1 / 90$ & $4 / 89$ & $12 / 89$ & $4 / 27 / 89$ & $4 / 29 / 89$ & BOB2M7 & G319 & G536* & $(1.20)$ & $3.48 E+04$ & ug! \\
\hline Nitrite & $6 / 1 / 90$ & $4 / 89$ & $12 / 89$ & $4 / 27 / 89$ & $4 / 29 / 89$ & ВОВ2Мо & G306 & G521* & $(1.20)$ & $3.43 E+0.4$ & ugl \\
\hline Nitrite & $6 / 1 / 90$ & $4 / 89$ & $12 / 89$ & $4 / 27 / 89$ & $4 / 29 / 89$ & $\mathrm{BOB2M8}$ & G301 & G523* & NA & $3.42 E+04$ & 'ป̀g/ \\
\hline Nitrite & $6 / 1 / 90$ & $4 / 89$ & $12 / 89$ & $4 / 27 / 89$ & $4 / 29 / 89$ & BOB2N2 & G317 & G528* & NA & $3.41 E+04$ & $u g l$ \\
\hline Nitrite & $6 / 1 / 90$ & $4 / 89$ & $12 / 89$ & $4 / 27 / 89$ & $4 / 29 / 89$ & BOB2N3 & G322 & G529* & NA & $3.40 E+04$ & ugl \\
\hline Nitrite & $6 / 1 / 90$ & $4 / 89$ & $12 / 89$ & $4 / 27 / 89$ & $4 / 29 / 89$ & В0В2M8 & G301 & G523* & NA & $3.38 E+04$ & $u g \wedge$ \\
\hline Nitrite & $6 / 1 / 90$ & $4 / 89$ & $12 / 89$ & $4 / 27 / 89$ & $4 / 29 / 89$ & B0B2L8 & G303 & G510* & 1.21 & $3.26 E+04$ & ugh \\
\hline Nitrite & $6 / 1 / 90$ & $4 / 89$ & $12 / 89$ & $4 / 27 / 89$ & $4 / 29 / 89$ & $\mathrm{BOB} 2 \mathrm{~N} 2$ & G317 & G528* & NA & $3.15 E+04$ & $\mathrm{ug} / \mathrm{h}$ \\
\hline Nitrite & $6 / 1 / 90$ & $4 / 89$ & $12 / 89$ & $4 / 27 / 89$ & $4 / 29 / 89$ & BOB2M3 & G311 & G516* & $(1.20)$ & $3.13 E+04$ & $u g / 1$ \\
\hline Nitrite & $6 / 1 / 90$ & $4 / 89$ & $12 / 89$ & $4 / 27 / 89$ & $4 / 29 / 89$ & BOB2NO & G307 & G527* & NA & $3.09 E+04$ & ugh \\
\hline NO3 & $4 / 28 / 84$ & NS & NS & NS & NS & R8469 & NS & NS & NS & $<1.99 \mathrm{E}-03$ & $\bar{N}$ \\
\hline No3. & NS & NS & NS & NS & NS & NS & NS & NS & 1.24 & $1.81 \varepsilon+\infty$ & $\mathrm{Mol}$ \\
\hline NO3 & $4 / 28 / 84$ & NS & NS & NS & NS & R-8469 & NS & NS & NS & $1.99 \mathrm{E}-03$ & $\overline{\text { moll }}$ \\
\hline NO3- & $4 / 28 / 84$ & NS & NS & NS & NS & NS & NS & NS & NS & $1.23 \mathrm{E}-01$ & $g /$ \\
\hline Nitrate & $6 / 1 / 90$ & $4 / 30 / 89$ & $12 / 30 / 89$ & $4 / 27 / 89$ & $4 / 29 / 89$ & ВОВ2M7 & G319 & 6460 & 1.2 & $9.73 E+04$ & ught \\
\hline Nitrate & $6 / 1 / 90$ & $4 / 30 / 89$ & $12 / 30 / 89$ & $4 / 27 / 89$ & $4 / 29 / 89$ & BOB2M7 & G319 & G460 & 1.2 & $9.68 E+04$ & $u g / r$ \\
\hline Nitrate & $6 / 1 / 90$ & $4 / 30 / 89$ & $12 / 30 / 89$ & $4 / 27 / 89$ & $4 / 29 / 89$ & B082T9 & G327 & G470* & 1.19 & $8.52 E+04$ & ugh \\
\hline Nitrate & $6 / 1 / 90$ & $4 / 30 / 89$ & $12 / 30 / 89$ & $4 / 27 / 89$ & $4 / 29 / 89$ & BOB2NO & G307 & G527" & NA & $8.40 E+04$ & ug/t \\
\hline Nitrate & $6 / 1 / 90$ & $4 / 30 / 89$ & $12 / 30 / 89$ & $4 / 27 / 89$ & $4 / 29 / 89$ & ВOB2T9 & G327 & G470* & 1.19 & $8.34 E+04$ & $u g / r$ \\
\hline Nitrate & $6 / 1 / 90$ & $4 / 30 / 89$ & $12 / 30 / 89$ & $4 / 27 / 89$ & $4 / 29 / 89$ & BOB2M9 & G302 & $\mathrm{G} 477^{\circ}$ & 1.20 & $8.34 E+04$ & $u g / r$ \\
\hline Nitrate & $6 / 1 / 90$ & $4 / 30 / 89$ & $12 / 30 / 89$ & $4 / 27 / 89$ & $4 / 29 / 89$ & BOB2N4 & G323 & G476* & 1.21 & $8.25 E+04$ & $u g / r$. \\
\hline Nitrate & $6 / 1 / 90$ & $4 / 30 / 89$ & $12 / 30 / 89$ & $4 / 27 / 89$ & $4 / 29 / 89$ & BOB2N1 & G310 & G471" & 1.19 & $8.22 E+04$ & $u g / n$ \\
\hline Nittate & $6 / 1 / 90$ & $4 / 30 / 89$ & $12 / 30 / 89$ & $4 / 27 / 89$ & $4 / 29 / 89$ & BOB2NO & G307 & G453 & NA & $8.05 E+04$ & $u g / n$ \\
\hline Nitrate & $6 / 1 / 90$ & $4 / 30 / 89$ & $12 / 30 / 89$ & $4 / 27 / 89$ & $4 / 29 / 89$ & BOB2N2 & G317 & G333 & NA & $7.95 E+04$ & $u g / n$ \\
\hline Nitrate & $6 / 1 / 90$ & $4 / 30 / 89$ & $12 / 30 / 89$ & $4 / 27 / 89$ & $4 / 29 / 89$ & BOB2M9 & $\mathrm{G} 302$ & G450 & $(1.20)$ & $7.94 E+04$ & ug/n \\
\hline
\end{tabular}




\begin{tabular}{|c|c|c|c|c|c|c|c|}
\hline \multirow[b]{2}{*}{ Code } & \multirow[b]{2}{*}{ Source } & \multicolumn{3}{|c|}{ Analyte Information } & \multirow[b]{2}{*}{$\begin{array}{c}\text { Reference } \\
\text { Number }\end{array}$} & \multirow[b]{2}{*}{$\begin{array}{c}\text { Validated } \\
\text { (Y/N) }\end{array}$} & \multirow[b]{2}{*}{ Notes } \\
\hline & & Analyte & $\begin{array}{l}\text { Conc. } \\
\text { (ug/L) }\end{array}$ & Conc. (mol/L) & & & \\
\hline$P$ & TCD & NO2 & $3.49 E+07$ & 7.59E-01 & AP102-3 & $Y$ & $\begin{array}{l}\text { Density not reported for G529. No assumptions cọuld be } \\
\text { made }\end{array}$ \\
\hline $\mathbf{P}$ & TCD & NO2 & $3.48 E+07$ & 7.57E-01 & AP102-3 & $Y$ & $\begin{array}{l}\text { Density not reported for G536. Density assumed same as } \\
\text { G460 which was collected at } 226 \text { in from bottom of tank at } \\
\text { a } 270^{\circ} \text { angle. This sample was heated. }\end{array}$ \\
\hline$P$ & TCD & NO2 & $3.43 E+07$ & 7.46E-01 & AP102-3 & $Y$ & $\begin{array}{l}\text { Density not reported for G521. Density assumed same as } \\
\text { G352 which was collected at } 34 \text { in from bottom of tank at a } \\
30^{\circ} \text { angle. This sample was heated. }\end{array}$ \\
\hline$P$ & TCD & NO2 & $3.42 E+07$ & 7.43E-01 & AP102-3 & $\mathbf{Y}$ & $\begin{array}{l}\text { Density not reported for G523. No assumptions could be } \\
\text { made }\end{array}$ \\
\hline $\mathbf{P}$ & TCD & NO2 & $3.41 E+07$ & 7.41E-01 & AP102-3 & $Y$ & $\begin{array}{l}\text { Density not reported for G528. No assumptions could be } \\
\text { made }\end{array}$ \\
\hline$P$ & TCD & $\mathrm{NO} 2$ & $3.40 E+07$ & 7.39E-01 & AP102-3 & $\boldsymbol{Y}$ & $\begin{array}{l}\text { Density not reported for G529. No assumptions could be } \\
\text { made }\end{array}$ \\
\hline $\mathbf{P}$ & TCD & NO2 & $3.38 E+07$ & 7.35E-01 & AP102-3 & $Y$ & $\begin{array}{l}\text { Density not reported for G523. No assumptions could be } \\
\text { made }\end{array}$ \\
\hline$P$ & TCD & NO2 & $3.26 E+07$ & $\begin{array}{l}7.09 E-01 \\
-\end{array}$ & AP102-3 & $\mathbf{Y}$ & $\begin{array}{l}\text { Density not reported for G510. Density assumed same as } \\
\text { G348 which was collected at } 125 \text { in from bottom of tank at } \\
\text { a } 30^{\circ} \text { angle. This sample was heated. }\end{array}$ \\
\hline $\mathbf{P}$ & TCD & $\mathrm{NO2}$ & $3.15 E+07$ & $6.85 E-01$ & AP102-3 & $Y$ & $\begin{array}{l}\text { Density not reported for G528. No assumptions could be } \\
\text { made }\end{array}$ \\
\hline $\mathbf{P}$ & TCD & NO2 & $3.13 E+07$ & $6.80 E-01$ & AP102-3 & $Y$ & $\begin{array}{l}\text { Density not reported for G516. Density assumed same as } \\
\text { G359 which was collected at } 162 \text { in from bottom of tank at } \\
\text { a } 150^{\circ} \text { angle. This sample was heated. }\end{array}$ \\
\hline$P$ & TCD & NO2 & $3.09 E+07$ & $6.72 E-01$ & AP102-3 & $\mathbf{Y}$ & $\begin{array}{l}\text { Density not reported for G527. No assumptions could be } \\
\text { made }\end{array}$ \\
\hline$\underline{L}$ & Raw & NO3 & \#VALUE! & \#VALUE! & AP102-1 & N & Outdated Information \\
\hline$L$ & HTCE Inv. & NO3 & $1.12 E+14$ & $1.81 \mathrm{E}+06$ & NA & $\mathbf{N}$ & No Reference \\
\hline$P$ & Braun & NO3 & $1.23 E+11$ & $1.99 E+03$ & & $\bar{N}$ & No Reference \\
\hline $\mathbf{P}$ & Wastren & NO3 & $1.23 E+11$ & $1.98 E+03$ & NS & $\mathbf{N}$ & No Reference \\
\hline $\mathbf{P}$ & Raw & NO3 & $9.73 E+07$ & $1.57 E+\infty$ & AP102-3 & $Y$ & $\begin{array}{l}\text { Density reported for G460 collected at } 226 \text { in from bottom } \\
\text { of tank at a } 270^{\circ} \text { angle. Sample was unheated, unheated } \\
\text { samples precipitated }\end{array}$ \\
\hline $\mathbf{P}$ & Raw & NO3 & $9.68 \mathrm{E}+07$ & $1.56 E+\infty$ & AP102-3 & $Y$ & $\begin{array}{l}\text { Density reported for G460 collected at } 226 \text { in from bottom } \\
\text { of tank at a } 270^{\circ} \text { angle. Sample was unheated, unheated } \\
\text { samples precipitated }\end{array}$ \\
\hline$P$ & Raw & NO3 & $8.52 E+07$ & $1.37 E+\infty$ & AP102-3 & $Y$ & $\begin{array}{l}\text { Density reported for G470 represents a composite sample. } \\
\text { Sample was kept heated to } 40^{\circ} \mathrm{C} \text {. }\end{array}$ \\
\hline$P$ & Raw & NO3 & $8.40 E+07$ & $1.35 E+\infty 0$ & AP102-3 & $Y$ & $\begin{array}{l}\text { Density not reported for G527. No assumptions could be } \\
\text { made }\end{array}$ \\
\hline$P$ & Raw & NO3 & $8.34 E+07$ & $1.35 E+\infty$ & AP102-3 & $Y$ & $\begin{array}{l}\text { Density reported for G470 represents a composite sample. } \\
\text { Sample was kept heated to } 40^{\circ} \mathrm{C} \text {. }\end{array}$ \\
\hline $\mathbf{P}$ & Raw & NO3 & $8.34 E+07$ & $1.35 E+\infty 0$ & AP102-3 & $\mathbf{Y}$ & $\begin{array}{l}\text { Density reported for } \mathrm{G} 477 \text { collected at } 182 \text { in from bottom } \\
\text { of tank at a } 30^{\circ} \text { angle and was keep heated to } 40^{\circ} \mathrm{C} \text {. }\end{array}$ \\
\hline$P$ & Raw & NO3 & $8.25 E+07$ & $1.33 E+\infty$ & AP102-3 & $Y$ & $\begin{array}{l}\text { Density reported for G476 collected at } 99 \text { in from bottom of } \\
\text { tank at a } 270^{\circ} \text { angle and was keep heated to } 40^{\circ} \mathrm{C} \text {. }\end{array}$ \\
\hline$P$ & Raw & NO3 & $8.22 E+07$ & $1.33 E+\infty$ & AP102-3 & $Y$ & $\begin{array}{l}\text { Density reported for G471 collected at } 208 \text { in from bottom } \\
\text { of tank at a } 150^{\circ} \text { angle and was keep heated to } 40^{\circ} \mathrm{C} \text {. }\end{array}$ \\
\hline $\mathbf{P}$ & Raw & NO3 & $8.05 E+07$ & $1.30 E+\infty 0$ & AP102-3 & $Y$ & $\begin{array}{l}\text { Density not reported for } \mathbf{6 4 5 3} \text {. No assumptions could be } \\
\text { made }\end{array}$ \\
\hline$P$ & Raw & NO3 & $7.95 E+07$ & $1.28 E+\infty 0$ & AP102-3 & $\gamma$ & $\begin{array}{l}\text { Density not reported for G333. No assumptions could be } \\
\text { made }\end{array}$ \\
\hline $\mathbf{P}$ & Raw & NO3 & $7.94 E+07$ & $1.28 E+\infty$ & AP102-3 & $\mathbf{Y}$ & $\begin{array}{l}\text { Density not reported for G450. Density assumed same as } \\
\text { G477 which was collected at } 182 \text { in from bottom of tank at } \\
\text { a } 30^{\circ} \text { angle and was keep heated to } 40^{\circ} \mathrm{C} \text {. }\end{array}$ \\
\hline
\end{tabular}




\begin{tabular}{|c|c|c|c|c|c|c|c|c|c|c|c|}
\hline & & & & & nik Waste & haracteriza & on Sample I & Ormation & & & \\
\hline Reported Chemical & $\begin{array}{c}\text { Memo } \\
\text { Date } \\
\end{array}$ & $\begin{array}{c}\text { Start } \\
\text { Analysis } \\
\text { Date }\end{array}$ & $\begin{array}{c}\text { Finish } \\
\text { Analysis Date }\end{array}$ & $\begin{array}{c}\text { Start } \\
\text { Sample } \\
\text { Date } \\
\end{array}$ & $\begin{array}{c}\text { Finish } \\
\text { Sample } \\
\text { Date } \\
\end{array}$ & $\begin{array}{l}\text { Sample } \\
\text { Number }\end{array}$ & $\begin{array}{c}\text { Tank Farm } \\
\text { Number }\end{array}$ & $\begin{array}{c}\text { Lab Sample } \\
\text { Number }\end{array}$ & $\begin{array}{l}\text { Density } \\
\text { (g/mL) }\end{array}$ & $\begin{array}{c}\text { Reported } \\
\text { Value } \\
\end{array}$ & $\begin{array}{r}\text { Repo } \\
\text { Un } \\
\end{array}$ \\
\hline Nitrate & $6 / 1 / 90$ & $4 / 30 / 89$ & $12 / 30 / 89$ & $4 / 27 / 89$ & $4 / 29 / 89$ & BOB2N3 & G322 & G338 & NA & $7.93 E+04$ & ug/t \\
\hline Nitrate & $6 / 1 / 90$ & $4 / 30 / 89$ & $12 / 30 / 89$ & $4 / 27 / 89$ & $4 / 29 / 89$ & BOB2N2 & G317 & G333 & NA & $7.92 E+04$ & ug/r \\
\hline Nitrate & $6 / 1 / 90$ & $4 / 30 / 89$ & $12 / 30 / 89$ & $4 / 27 / 89$ & $4 / 29 / 89$ & BO82M1 & G308 & G522* & $(1.20)$ & $7.92 E+04$ & $u g / n$ \\
\hline Nitrate & $6 / 1 / 90$ & $4 / 30 / 89$ & $12 / 30 / 89$ & $4 / 27 / 89$ & $4 / 29 / 89$ & Вов2M2 & G309 & G515* & $(1.20)$ & $7.91 E+04$ & ug/n \\
\hline Nitrate & $6 / 1 / 90$ & $4 / 30 / 89$ & $12 / 30 / 89$ & $4 / 27 / 89$ & $4 / 29 / 89$ & $\mathrm{BOB} 2 \mathrm{~L} 7$ & G299 & G509* & $(7.20)$ & $7.90 E+04$ & $u g / \pi$ \\
\hline Nitrate & $6 / 1 / 90$ & $4 / 30 / 89$ & $12 / 30 / 89$ & $4 / 27 / 89$ & $4 / 29 / 89$ & ВОВ2M9 & G302 & G450 & $(1.20)$ & $7.90 E+04$ & $\mathrm{ug} / \mathrm{r}$ \\
\hline Nitrate & $6 / 1 / 90$ & $4 / 30 / 89$ & $12 / 30 / 89$ & $4 / 27 / 89$ & $4 / 29 / 89$ & $B O B 2 N 1$ & G310 & G454 & $(1.19)$ & $7.87 E+04$ & $u g / m$ \\
\hline Nitrate & $6 / 1 / 90$ & $4 / 30 / 89$ & $12 / 30 / 89$ & $4 / 27 / 89$ & $4 / 29 / 89$ & Вов2М8 & G301 & G449 & NA & $7.86 E+04$ & $\mathrm{ug} / \mathrm{m}$ \\
\hline Nitrate & $6 / 1 / 90$ & $4 / 30 / 89$ & $12 / 30 / 89$ & $4 / 27 / 89$ & $4 / 29 / 89$ & ВОВ2M5 & G315 & G364 & 1.19 & $7.85 E+04$ & $\mathrm{ug} / \mathrm{m}$ \\
\hline Nitrate & $6 / 1 / 90$ & $4 / 30 / 89$ & $12 / 30 / 89$ & $4 / 27 / 89$ & $4 / 29 / 89$ & В0В2M5 & G315 & G364 & 1.19 & $7.84 E+04$ & $\mathrm{ug} / \mathrm{m} !$ \\
\hline Nitrate & $6 / 1 / 90$ & $4 / 30 / 89$ & $12 / 30 / 89$ & $4 / 27 / 89$ & $4 / 29 / 89$ & $B O B 2 N 1$ & G310 & G454 & $(1.19)$ & $7.84 E+04$ & $\mathrm{ug} / \mathrm{ml}$ \\
\hline Nitrate & $6 / 1 / 90$ & $4 / 30 / 89$ & $12 / 30 / 89$ & $4 / 27 / 89$ & $4 / 29 / 89$ & BOB2NO & G307 & G453 & NA & $7.83 E+04$ & $\mathrm{ug} / \mathrm{ml}$ \\
\hline Nitrate & $6 / 1 / 90$ & $4 / 30 / 89$ & $12 / 30 / 89$ & $4 / 27 / 89$ & $4 / 29 / 89$ & в082M6 & G318 & G517* & $(1.20)$ & $7.83 E+04$ & $\mathrm{ug} / \mathrm{mL}$ \\
\hline Nitrate & $6 / 1 / 90$ & $4 / 30 / 89$ & $12 / 30 / 89$ & $4 / 27 / 89$ & $4 / 29 / 89$ & Вов2M1 & G308 & G522* & $(1.20)$ & $7.82 E+04$ & $\mathrm{ug} / \mathrm{ml}$ \\
\hline Nitrate & $6 / 1 / 90$ & $4 / 30 / 89$ & $12 / 30 / 89$ & $4 / 27 / 89$ & $4 / 29 / 89$ & BOB2N4 & G323 & G341 & 1.20 & $7.81 E+04$ & $u g / m L$ \\
\hline Nitrate & $6 / 1 / 90$ & $4 / 30 / 89$ & $12 / 30 / 89$ & $4 / 27 / 89$ & $4 / 29 / 89$ & Вов2М6 & G318 & G517* & $(1.20)$ & $7.81 E+04$ & $\mathrm{ug} / \mathrm{mL}^{\prime}$ \\
\hline Nitrate & $6 / 1 / 90$ & $4 / 30 / 89$ & $12 / 30 / 89$ & $4 / 27 / 89$ & $4 / 29 / 89$ & ВОВ2М3 & G311 & G516* & $(1.20)$ & $7.80 E+04$ & $\mathrm{ug} / \mathrm{mL}$ \\
\hline Nitrate & $6 / 1 / 90$ & $4 / 30 / 89$ & $12 / 30 / 89$ & $4 / 27 / 89$ & $4 / 29 / 89$ & BOB2N3 & G322 & G338 & NA & $7.80 E+04$ & $\mathrm{ug} / \mathrm{ml}$. \\
\hline Nitrate & $6 / 1 / 90$ & $4 / 30 / 89$ & $12 / 30 / 89$ & $4 / 27 / 89$ & $4 / 29 / 89$ & BOB2N1 & G310 & G471. & 1.19 & $7.79 E+04$ & $u g / m L$ \\
\hline Nitrate & $6 / 1 / 90$ & $4 / 30 / 89$ & $12 / 30 / 89$ & $4 / 27 / 89$ & $4 / 29 / 89$ & BOB2L8 & G303 & G510* & 1.21 & $7.77 E+04$ & $\mathrm{ug} / \mathrm{mL}$ \\
\hline Nitrate & $6 / 1 / 90$ & $4 / 30 / 89$ & $12 / 30 / 89$ & $4 / 27 / 89$ & $4 / 29 / 89$ & BOB2M4 & G313 & G533" & $(1.20)$ & $7.76 E+04$ & $\mathrm{ug} / \mathrm{mL}$ \\
\hline
\end{tabular}




\begin{tabular}{|c|c|c|c|c|c|c|c|}
\hline \multirow[b]{2}{*}{ Code } & \multirow[b]{2}{*}{ Source } & \multicolumn{3}{|c|}{ Analyte Information } & \multirow[b]{2}{*}{$\begin{array}{c}\text { Reference } \\
\text { Number }\end{array}$} & \multirow[b]{2}{*}{$\begin{array}{l}\text { Validated } \\
(Y / N)\end{array}$} & \multirow[b]{2}{*}{ Notes } \\
\hline & & Analyte & $\begin{array}{l}\text { Conc. } \\
\text { (ug/L) }\end{array}$ & Conc. (mol/L) & & & \\
\hline$P$ & Raw & $\mathrm{NO3}$ & $7.93 E+07$ & $1.28 \mathrm{E}+00$ & AP102-3 & $Y$ & $\begin{array}{l}\text { Density not reported for } \mathrm{G} 338 \text {. No assumptions could be } \\
\text { made }\end{array}$ \\
\hline$P$ & Raw & NO3 & $7.92 E+07$ & $1.28 \mathrm{E}+00$ & AP102-3 & $Y$ & $\begin{array}{l}\text { Density not reported for G333. No assumptions could be } \\
\text { made }\end{array}$ \\
\hline$P$ & Raw & NO3 & $7.92 E+07$ & $1.28 \mathrm{E}+00$ & AP102-3 & $Y$ & $\begin{array}{l}\text { Density not reported for G522. Density assumed same as } \\
\text { G353 which was collected at } 334 \text { in from bottom of tank at } \\
\text { a } 150^{\circ} \text { angle. This sample was heated. }\end{array}$ \\
\hline$P$ & Raw & NO3 & $7.91 E+07$ & $1.28 \varepsilon+\infty 0$ & AP102-3 & $\mathbf{Y}$ & $\begin{array}{l}\text { Density not reported for G515. Density assumed same as } \\
\text { G358 which was collected at } 334 \text { in from bottom of tank at } \\
\text { a } 150^{\circ} \text { angle. This sample was heated. }\end{array}$ \\
\hline$P$ & Raw & NO3 & $7.90 E+07$ & $1.27 E+\infty$ & AP102-3 & $\mathbf{Y}$ & $\begin{array}{l}\text { Density not reported for G509. Density assumed same as } \\
6342 \text { which was collected at } 306 \text { in from bottom of tank at } \\
\text { a } 30^{\circ} \text { angle. This sample was heated. }\end{array}$ \\
\hline$P$ & Raw & NO3 & $7.90 E+07$ & $1.27 E+\infty$ & AP102-3 & $Y$ & $\begin{array}{l}\text { Density not reported for G450. Density assumed same as } \\
\text { G477 which was collected at } 182 \text { in from bottom of tank at } \\
\text { a } 30^{\circ} \text { angle and was keep heated to } 40^{\circ} \mathrm{C} \text {. }\end{array}$ \\
\hline$P$ & Raw & NO3 & $7.87 E+07$ & $1.27 E+\infty$ & AP102-3 & $Y$ & $\begin{array}{l}\text { Density not reported for G } 454 \text {. Density assumed same as } \\
\text { G471 which was collected at } 208 \text { in from bottom of tank at } \\
\text { a } 150^{\circ} \text { angle and was keep heated to } 40^{\circ} \mathrm{C} \text {. }\end{array}$ \\
\hline$P$ & Raw & NO3 & $7.86 E+07$ & $1.27 E+\infty$ & AP102-3 & $Y$ & $\begin{array}{l}\text { Densiry not reported for G449. No assumptions could be } \\
\text { made }\end{array}$ \\
\hline$P$ & Raw & NO3 & $7.85 E+07$ & $1.27 E+\infty$ & AP102-3 & $\mathrm{Y}$ & $\begin{array}{l}\text { Density reported for G } 364 \text { collected at } 372 \text { in from bottom } \\
\text { of tank at a } 270^{\circ} \text { angle. Sample was unheated, unheated } \\
\text { samples precipitated }\end{array}$ \\
\hline$P$ & Raw & NO3 & $7.84 E+07$ & $1.26 E+\infty$ & AP102-3 & $\gamma$ & $\begin{array}{l}\text { Density reported for G364 collected at } 372 \text { in from bottom } \\
\text { of tank at a } 270^{\circ} \text { angle. Sample was unheated, unheated } \\
\text { samples precipitated }\end{array}$ \\
\hline$P$ & Raw & NO3 & $7.84 \mathrm{E}+07$ & $1.26 E+\infty$ & AP102-3 & $Y$ & $\begin{array}{l}\text { Density not reported for G454. Density assumed same as } \\
\text { G471 which was collected at } 208 \text { in from bottom of tank at } \\
\text { a } 150^{\circ} \text { angle and was keep heated to } 40^{\circ} \mathrm{C} \text {. }\end{array}$ \\
\hline $\mathbf{P}$ & Raw & NO3 & $7.83 E+07$ & $1.26 \mathrm{E}+\infty 0$ & AP102-3 & $Y$ & $\begin{array}{l}\text { Density not reported for } \mathrm{G} 453 \text {. No assumptions could be } \\
\text { made }\end{array}$ \\
\hline$P$ & Raw & NO3 & $7.83 E+07$ & $1.26 \mathrm{E}+\infty$ & AP102-3 & $Y$ & $\begin{array}{l}\text { Density not reported for G517. Density assumed same as } \\
\mathrm{G} 459 \text { which was collected at } 226 \text { in from bottom of tank at } \\
\text { a } 270^{\circ} \text { angle. This sample was heated. }\end{array}$ \\
\hline$P$ & Raw & NO3 & $7.82 E+07$ & $1.26 E+\infty$ & AP102-3 & Y & $\begin{array}{l}\text { Density not reported for G522. Density assumed same as } \\
\text { G353 which was collected at } 334 \text { in from bottom of tank at } \\
\text { a } 150^{\circ} \text { angle. This sample was heated. }\end{array}$ \\
\hline$P$ & Raw & No3 & $7.81 E+07$ & $1.26 E+\infty 0$ & AP102-3 & $Y$ & $\begin{array}{l}\text { Density reported for } \mathrm{G} 341 \text { collected at } 99 \text { in from bottom of } \\
\text { tank at a } 270^{\circ} \text { angle. }\end{array}$ \\
\hline $\mathbf{P}$ & Raw & NO3 & $7.81 E+07$ & $1.26 \mathrm{E}+\infty 0$ & AP102-3 & $\mathbf{Y}$ & $\begin{array}{l}\text { Density not reported for G517. Density assumed same as } \\
\text { G459 which was collected at } 226 \text { in from bottom of tank at } \\
\text { a } 270^{\circ} \text { angle. This sample was heated. }\end{array}$ \\
\hline$P$ & Raw & NO3 & $7.80 E+07$ & $1.26 E+\infty$ & AP102-3 & $Y$ & $\begin{array}{l}\text { Density not reported for G516. Density assumed same as } \\
\text { G359 which was collected at } 162 \text { in from bottom of tank at } \\
\text { a } 150^{\circ} \text { angle. This sample was heated. }\end{array}$ \\
\hline $\mathbf{P}$ & Raw & NO3 & $7.80 E+07$ & $1.26 E+\infty$ & AP102-3 & $\mathbf{Y}$ & $\begin{array}{l}\text { Density not reported for G338. No assumptions could be } \\
\text { made }\end{array}$ \\
\hline $\mathbf{P}$ & Raw & NO3 & $7.79 E+07$ & $1.26 E+\infty$ & AP102-3 & $\mathbf{Y}$ & $\begin{array}{l}\text { Density reported for G } 471 \text { collected at } 208 \text { in from bottom } \\
\text { of tank at a } 150^{\circ} \text { angle and was keep heated to } 40^{\circ} \mathrm{C} \text {. }\end{array}$ \\
\hline $\mathbf{P}$ & Raw & NO3 & $7.77 E+07$ & $1.25 E+00$ & AP102-3 & $Y$ & $\begin{array}{l}\text { Density not reported for G510. Density assumed same as } \\
\text { G348 which was collected at } 125 \text { in from bottom of tank at } \\
\text { a } 30^{\circ} \text { angle. This sample was heated. }\end{array}$ \\
\hline$P$ & Raw & No3 & $7.76 E+07$ & $1.25 E+\infty 0$ & AP102-3 & $Y$ & $\begin{array}{l}\text { Density not reported for G533. Density assumed same as } \\
6363 \text { which was collected at } 18 \text { in from bottom of tank at a } \\
150^{\circ} \text { angle. This sample was heated. }\end{array}$ \\
\hline
\end{tabular}




\begin{tabular}{|c|c|c|c|c|c|c|c|c|c|c|c|}
\hline Reported Chemical & $\begin{array}{c}\text { Memo } \\
\text { Date }\end{array}$ & $\begin{array}{c}\text { Start } \\
\text { Analysis } \\
\text { Date } \\
\end{array}$ & $\begin{array}{c}\text { Finish } \\
\text { Analysis Date }\end{array}$ & $\begin{array}{c}\text { Start } \\
\text { Sample } \\
\text { Date } \\
\end{array}$ & $\begin{array}{c}\text { Finish } \\
\text { Sample } \\
\text { Date } \\
\end{array}$ & $\begin{array}{l}\text { Sample } \\
\text { Number }\end{array}$ & $\begin{array}{c}\text { Tank Farm } \\
\text { Number }\end{array}$ & $\begin{array}{l}\text { Lab Sample } \\
\text { Number }\end{array}$ & $\begin{array}{l}\text { Density } \\
(\mathrm{g} / \mathrm{mL})\end{array}$ & $\begin{array}{c}\text { Reported } \\
\text { Value }\end{array}$ & $\begin{array}{r}\text { Repr } \\
U_{r} \\
\end{array}$ \\
\hline Nitrate & $6 / 1 / 90$ & $4 / 30 / 89$ & $12 / 30 / 89$ & $4 / 27 / 89$ & $4 / 29 / 89$ & $\mathrm{BOB2M4}$ & G313 & G363 & 1.20 & $7.76 E+04$ & ughl \\
\hline Nitrate & $6 / 1 / 90$ & $4 / 30 / 89$ & $12 / 30 / 89$ & $4 / 27 / 89$ & $4 / 29 / 89$ & BOB2M3 & G311 & G516* & $(1.20)$ & $7.76 E+04$ & $u g h$ \\
\hline Nitrate & $6 / 1 / 90$ & $4 / 30 / 89$ & $12 / 30 / 89$ & $4 / 27 / 89$ & $4 / 29 / 89$ & ВOB2MO & G306 & G352 & 1.20 & $7.75 E+04$ & $u g / t$ \\
\hline Nitrate & $6 / 1 / 90$ & $4 / 30 / 89$ & $12 / 30 / 89$ & $4 / 27 / 89$ & $4 / 29 / 89$ & BOB2M4 & G313 & G363 & 1.20 & $7.75 E+04$ & $u g / r_{1}$ \\
\hline Nitrate & $6 / 1 / 90$ & $4 / 30 / 89$ & $12 / 30 / 89$ & $4 / 27 / 89$ & $4 / 29 / 89$ & B082M4 & G313 & G533* & $(1.20)$ & $7.75 E+04$ & $u g / n$ \\
\hline Nitrate & $6 / 1 / 90$ & $4 / 30 / 89$ & $12 / 30 / 89$ & $4 / 27 / 89$ & $4 / 29 / 89$ & вов2M3 & G311 & G359 & 1.20 & $7.74 E+04$ & $u g / n$ \\
\hline Nitrate & $6 / 1 / 90$ & $4 / 30 / 89$ & $12 / 30 / 89$ & $4 / 27 / 89$ & $4 / 29 / 89$ & BOB2M3 & G311 & G516* & $(1.20)$ & $7.74 E+04$ & $u g / m$ \\
\hline Nitrate & $6 / 1 / 90$ & $4 / 30 / 89$ & $12 / 30 / 89$ & $4 / 27 / 89$ & $4 / 29 / 89$ & B082M8 & G301 & G449 & NA & $7.74 E+04$ & $u g / n$ \\
\hline Nitrate & $6 / 1 / 90$ & $4 / 30 / 89$ & $12 / 30 / 89$ & $4 / 27 / 89$ & $4 / 29 / 89$ & Вов2МO & G306 & G521* & $(1.20)$ & $7.73 E+04$ & $\mathrm{ug} / \mathrm{m}$ \\
\hline Nitrate & $6 / 1 / 90$ & $4 / 30 / 89$ & $12 / 30 / 89$ & $4 / 27 / 89$ & $4 / 29 / 89$ & BOB2M1 & G308 & G353 & 1.20 & $7.72 E+04$ & $\mathrm{ug} / \mathrm{m}$ \\
\hline Nitrate & $6 / 1 / 90$ & $4 / 30 / 89$ & $12 / 30 / 89$ & $4 / 27 / 89$ & $4 / 29 / 89$ & BOB2NO & G307 & G527* & NA & $7.72 E+04$ & $\mathrm{ug} / \mathrm{m}$ \\
\hline Nitrate & $6 / 1 / 90$ & $4 / 30 / 89$ & $12 / 30 / 89$ & $4 / 27 / 89$ & $4 / 29 / 89$ & ВОВ2M6 & G318 & G459 & 1.20 & $7.71 E+04$ & $u g / m$ \\
\hline Nitrate & $6 / 1 / 90$ & $4 / 30 / 89$ & $12 / 30 / 89$ & $4 / 27 / 89$ & $4 / 29 / 89$ & BOB2M3 & G311 & G359 & 1.20 & $7.69 E+04$ & $u g / m$ \\
\hline Nitrate & $6 / 1 / 90$ & $4 / 30 / 89$ & $12 / 30 / 89$ & $4 / 27 / 89$ & $4 / 29 / 89$ & BOB2NO & G307 & G527* & NA & $7.68 E+04$ & $\mathrm{ug} / \mathrm{ml}$ \\
\hline Nitrate & $6 / 1 / 90$ & $4 / 30 / 89$ & $12 / 30 / 89$ & $4 / 27 / 89$ & $4 / 29 / 89$ & BOB2L7 & G299 & G342 & 1.20 & $7.68 \mathrm{E}+04$ & $\mathrm{ug} / \mathrm{ml}$ \\
\hline Nitrate & $6 / 1 / 90$ & $4 / 30 / 89$ & $12 / 30 / 89$ & $4 / 27 / 89$ & $4 / 29 / 89$ & BOB2L9 & G305 & G349 & 1.20 & $7.64 E+04$ & $\mathrm{ug} / \mathrm{ml}$ \\
\hline Nitrate & $6 / 1 / 90$ & $4 / 30 / 89$ & $12 / 30 / 89$ & $4 / 27 / 89$ & $4 / 29 / 89$ & BOB2L8 & G303 & G348 & 1.21 & $7.63 E+04$ & $\mathrm{ug} / \mathrm{ml}$ \\
\hline Nitrate & $6 / 1 / 90$ & $4 / 30 / 89$ & $12 / 30 / 89$ & $4 / 27 / 89$ & $4 / 29 / 89$ & BOB2M9 & G302 & $\mathrm{G} 477^{*}$ & 1.20 & $7.63 E+04$ & $\mathrm{ug} / \mathrm{mL}$ \\
\hline Nitrate & $6 / 1 / 90$ & $4 / 30 / 89$ & $12 / 30 / 89$ & $4 / 27 / 89$ & $4 / 29 / 89$ & BOB2L7 & G299 & G509* & $(1.20)$ & $7.61 E+04$ & $\mathrm{ug} / \mathrm{mL}$ \\
\hline Nitrate & $6 / 1 / 90$ & $4 / 30 / 89$ & $12 / 30 / 89$ & $4 / 27 / 89$ & $4 / 29 / 89$ & BOB2L. & G305 & G349 & 1.20 & $7.60 \varepsilon+04$ & $\mathrm{ug} / \mathrm{mL}$ \\
\hline
\end{tabular}




\begin{tabular}{|c|c|c|c|c|c|c|c|}
\hline \multirow[b]{2}{*}{ Code } & \multirow[b]{2}{*}{ Source } & \multicolumn{3}{|c|}{ Analyte Information } & \multirow[b]{2}{*}{$\begin{array}{l}\text { Reference } \\
\text { Number }\end{array}$} & \multirow[b]{2}{*}{$\begin{array}{l}\text { Validated } \\
\text { (Y/N] }\end{array}$} & \multirow[b]{2}{*}{ Notes } \\
\hline & & Analyte & $\begin{array}{l}\text { Conc. } \\
\text { (ug/L) }\end{array}$ & Conc. (mol/L) & & & \\
\hline$P$ & Raw & NO3 & $7.76 \mathrm{E}+07$ & $1.25 E+00$ & AP102-3 & Y & $\begin{array}{l}\text { Density reported for } G 363 \text { collected at } 18 \text { in from bottom of } \\
\text { tank at a } 150^{\circ} \text { angle. Sample was unheated, unheated } \\
\text { samples precipitated }\end{array}$ \\
\hline$P$ & Raw & NO3 & $7.76 E+07$ & $1.25 E+00$ & AP102-3 & $Y$ & $\begin{array}{l}\text { Density not reported for G516. Density assumed same as } \\
\text { G359 which was collected at } 162 \text { in from bottom of tank at } \\
\text { a } 150^{\circ} \text { angle. This sample was heated. }\end{array}$ \\
\hline$P$ & Raw & NO3 & $7.75 E+07$ & $1.25 E+\infty 0$ & AP102-3 & $\mathbf{Y}$ & $\begin{array}{l}\text { Density reported for } 6352 \text { collected at } 34 \text { in from bottom of } \\
\text { tank at a } 30^{\circ} \text { angle. Sample was unheated, unheated } \\
\text { samples precipitated }\end{array}$ \\
\hline$P$ & Raw & $\mathrm{NO} 3$ & $7.75 E+07$ & $1.25 E+\infty 0$ & AP102-3 & $Y$ & $\begin{array}{l}\text { Density reported for G363 collected at } 18 \text { in from bottom of } \\
\text { tank at a } 150^{\circ} \text { angle. Sample was unheated, unheated } \\
\text { samples precipitated }\end{array}$ \\
\hline $\mathbf{P}$ & Raw & NO3 & $7.75 E+07$ & $1.25 E+00$ & AP102-3 & $\mathbf{Y}$ & $\begin{array}{l}\text { Density not reported for G533. Density assumed same as } \\
\text { G363 which was collected at } 18 \text { in from bottom of tank at a } \\
150^{\circ} \text { angle. This sample was heated. }\end{array}$ \\
\hline $\mathbf{P}$ & Raw & NO3 & $7.74 E+07$ & $1.25 E+\infty 0$ & AP102-3 & Y & $\begin{array}{l}\text { Density reported for G359 collected at } 162 \text { in from bottom } \\
\text { of tank at a } 150^{\circ} \text { angle. Sample was unheated, unheated } \\
\text { samples precipitated }\end{array}$ \\
\hline $\mathbf{P}$ & Raw & NO3 & $7.74 E+07$ & $1.25 E+\infty 0$ & AP102-3 & $Y$ & $\begin{array}{l}\text { Density not reported for G516. Density assumed same as } \\
\text { G359 which was collected at } 162 \text { in from bottom of tank at } \\
\text { a } 150^{\circ} \text { angle. This sample was heated. }\end{array}$ \\
\hline $\mathbf{P}$ & Raw & NO3 & $7.74 E+07$ & $1.25 E+\infty$ & AP102-3 & Y & $\begin{array}{l}\text { Density not reported for G449. No assumptions could be } \\
\text { made }\end{array}$ \\
\hline $\mathbf{P}$ & Raw & NO3 & $7.73 E+07$ & $1.25 E+\infty 0$ & AP102-3 & Y & $\begin{array}{l}\text { Density not reported for G521. Density assumed same as } \\
\text { G352 which was collected at } 34 \text { in from bottom of tank at a } \\
30^{\circ} \text { angle. This sample was heated. }\end{array}$ \\
\hline $\mathbf{P}$ & Raw & NO3 & $7.72 E+07$ & $1.25 E+\infty 0$ & AP102-3 & Y & $\begin{array}{l}\text { Density reported for } \mathrm{G} 353 \text { collected at } 334 \text { in from bottom } \\
\text { of tank at a } 150^{\circ} \text { angle. Sample was unheated, unheated } \\
\text { samples precipitated }\end{array}$ \\
\hline$P$ & Raw & NO3 & $7.72 E+07$ & $1.25 E+00$ & AP102-3 & Y & $\begin{array}{l}\text { Density not reported for G527. No assumptions could be } \\
\text { made }\end{array}$ \\
\hline$P$ & Raw & NO3 & $7.71 E+07$ & $1.24 E+00$ & AP102-3 & $\gamma$ & $\begin{array}{l}\text { Density reported for } \mathbf{G} 459 \text { collected at } 226 \text { in from bottom } \\
\text { of tank at a } 270^{\circ} \text { angle. Sample was unheated, unheated } \\
\text { samples precipitated }\end{array}$ \\
\hline$P$ & Raw & NO3 & $7.69 E+07$ & $1.24 E+\infty 0$ & AP102-3 & $\mathbf{Y}$ & $\begin{array}{l}\text { Density reported for G359 collected at } 162 \text { in from bottom } \\
\text { of tank at a } 150^{\circ} \text { angle. Sample was unheated, unheated } \\
\text { samples precipitated }\end{array}$ \\
\hline$P$ & Raw & NO3 & $7.68 E+07$ & $1.24 E+00$ & AP102-3 & $Y$ & $\begin{array}{l}\text { Density not reported for G527. No assumptions could be } \\
\text { made }\end{array}$ \\
\hline $\mathbf{P}$ & Raw & NO3 & $7.68 E+07$ & $1.24 E+00$ & AP102-3 & $Y$ & $\begin{array}{l}\text { Density seported for G342 collected at } 306 \text { in from bottom } \\
\text { of tank at a } 30^{\circ} \text { angle. Sample was unheated, unheated } \\
\text { samples precipitated }\end{array}$ \\
\hline$P$ & Raw & NO3 & $7.64 E+07$ & $1.23 E+\infty 0$ & AP102-3 & $Y$ & $\begin{array}{l}\text { Density reported for } G 349 \text { collected at } 34 \text { in from bottom of } \\
\text { tank at a } 30^{\circ} \text { angle. Sample was unheated, unheated } \\
\text { samples precipitated }\end{array}$ \\
\hline $\mathbf{P}$ & Raw & NO3 & $7.63 E+07$ & $1.23 E+00$ & AP102-3 & $Y$ & $\begin{array}{l}\text { Density reported for } \mathrm{G} 348 \text { collected at } 125 \text { in from bottom } \\
\text { of tank at a } 30^{\circ} \text { angle. Sample was unheated, unheated } \\
\text { samples precipitated }\end{array}$ \\
\hline$P$ & Raw & NO3 & $7.63 E+07$ & $1.23 E+\infty 0$ & AP102-3 & $Y$ & $\begin{array}{l}\text { Density reported for G477 collected at } 182 \text { in from bottom } \\
\text { of tank at a } 30^{\circ} \text { angle and was keep heated to } 40^{\circ} \mathrm{C} \text {. }\end{array}$ \\
\hline$P$ & Raw & NO3 & $7.61 E+07$ & $1.23 E+\infty$ & AP102-3 & $Y$ & $\begin{array}{l}\text { Density not reported for G509. Density assumed same as } \\
\text { G342 which was collected at } 306 \text { in from bottom of tank at } \\
\text { a } 30^{\circ} \text { angle. This sample was heated. }\end{array}$ \\
\hline$P$ & Raw & NO3 & $7.60 E+07$ & $1.23 E+\infty$ & AP102-3 & $Y$ & $\begin{array}{l}\text { Density reported for } \mathrm{G} 349 \text { collected at } 34 \text { in from bottom of } \\
\text { tank at a } 30^{\circ} \text { angle. Sample was unheated, unheated } \\
\text { samples precipitated }\end{array}$ \\
\hline
\end{tabular}


TWRS Privatization Support Proj Chemical Liquids

\begin{tabular}{|c|c|c|c|c|c|c|c|c|c|c|c|}
\hline & & & & & ank Wast & Sharacteriza & ion Sample & ormation & & & \\
\hline Reported Chemical & $\begin{array}{c}\text { Memo } \\
\text { Date }\end{array}$ & $\begin{array}{c}\text { Start } \\
\text { Analysis } \\
\text { Date } \\
\end{array}$ & $\begin{array}{c}\text { Finish } \\
\text { Analysis Date }\end{array}$ & $\begin{array}{c}\text { Start } \\
\text { Sample } \\
\text { Date } \\
\end{array}$ & $\begin{array}{c}\text { Finish } \\
\text { Sample } \\
\text { Date } \\
\end{array}$ & $\begin{array}{l}\text { Sample } \\
\text { Number }\end{array}$ & $\begin{array}{c}\text { Tank Farm } \\
\text { Number }\end{array}$ & $\begin{array}{c}\text { Lab Sample } \\
\text { Number }\end{array}$ & $\begin{array}{c}\text { Density } \\
\text { (g/mL) }\end{array}$ & $\begin{array}{c}\text { Reported } \\
\text { Value }\end{array}$ & $\begin{array}{r}\text { Repor } \\
\text { Unii } \\
\end{array}$ \\
\hline Nitrate & $6 / 1 / 90$ & $4 / 30 / 89$ & $12 / 30 / 89$ & $4 / 27 / 89$ & $4 / 29 / 89$ & BOB2M2 & G309 & G358 & 1.20 & $7.59 E+04$ & $\mathrm{ug} / \mathrm{m}$ \\
\hline Nitrate & $6 / 1 / 90$ & $4 / 30 / 89$ & $12 / 30 / 89$ & $4 / 27 / 89$ & $4 / 29 / 89$ & B082N4 & G323 & G476 * & 1.21 & $7.59 E+04$ & $\mathrm{ug} / \mathrm{m}$ \\
\hline Nitrate & $6 / 1 / 90$ & $4 / 30 / 89$ & $12 / 30 / 89$ & $4 / 27 / 89$ & $4 / 29 / 89$ & BOB2M5 & G315 & G534* & $(1.19)$ & $7.59 E+04$ & $\mathrm{ug} / \mathrm{m}$ \\
\hline Nitrate & $6 / 1 / 90$ & $4 / 30 / 89$ & $12 / 30 / 89$ & $4 / 27 / 89$ & $4 / 29 / 89$ & 8082M5 & G315 & G534* & $(1.19)$ & $7.59 E+04$ & $u g / m$ \\
\hline Nitrate & $6 / 1 / 90$ & $4 / 30 / 89$ & $12 / 30 / 89$ & $4 / 27 / 89$ & $4 / 29 / 89$ & BOB2M9 & G302 & G477* & 1.20 & $7.58 E+04$ & $u g / m$ \\
\hline Nitrate & $6 / 1 / 90$ & $4 / 30 / 89$ & $12 / 30 / 89$ & $4 / 27 / 89$ & $4 / 29 / 89$ & BOB2M7 & G319 & G536* & $(1.20)$ & $7.58 E+04$ & $u g / m$ \\
\hline Nitrate & $6 / 1 / 90$ & $4 / 30 / 89$ & $12 / 30 / 89$ & $4 / 27 / 89$ & $4 / 29 / 89$ & ВОВ2M2 & G309 & G358 & 1.20 & $7.58 E+04$ & ug/mi: \\
\hline Nitrate & $6 / 1 / 90$ & $4 / 30 / 89$ & $12 / 30 / 89$ & $4 / 27 / 89$ & $4 / 29 / 89$ & Bов2M6 & G318 & G459 & 1.20 & $7.58 E+04$ & $\mathrm{ug} / \mathrm{ml}$ \\
\hline Nitrate & $6 / 1 / 90$ & $4 / 30 / 89$ & $12 / 30 / 89$ & $4 / 27 / 89$ & $4 / 29 / 89$ & BOB2L9 & G305 & $6511^{\circ}$ & $(1.20)$ & $7.58 E+04$ & $u g / m l$ \\
\hline Nitrate & $6 / 1 / 90$ & $4 / 30 / 89$ & $12 / 30 / 89$ & $4 / 27 / 89$ & $4 / 29 / 89$ & ВОВ2M2 & G309 & G515* & $(1.20)$ & $7.57 E+04$ & $\mathrm{ug} / \mathrm{ml}$ \\
\hline Nitrate & $6 / 1 / 90$ & $4 / 30 / 89$ & $12 / 30 / 89$ & $4 / 27 / 89$ & $4 / 29 / 89$ & BOB2L8 & G303 & G348 & 1.21 & $7.55 E+04$ & $\mathrm{ug} / \mathrm{ml}$ \\
\hline Nitrate & $6 / 1 / 90$ & $4 / 30 / 89$ & $12 / 30 / 89$ & $4 / 27 / 89$ & $4 / 29 / 89$ & BOB2M9 & G302 & G477* & 1.20 & $7.55 E+04$ & $\mathrm{ug} / \mathrm{ml}$ \\
\hline Nitrate & $6 / 1 / 90$ & $4 / 30 / 89$ & $12 / 30 / 89$ & $4 / 27 / 89$ & $4 / 29 / 89$ & ВОВ2M7 & G319 & G536* & $(1.20)$ & $7.53 E+04$ & $u g / m !$ \\
\hline Nitrate & $6 / 1 / 90$ & $4 / 30 / 89$ & $12 / 30 / 89$ & $4 / 27 / 89$ & $4 / 29 / 89$ & BOB2L9 & G305 & G511* & $(1.20)$ & $7.52 E+0.4$ & ug/ml \\
\hline Nitrate & $6 / 1 / 90$ & $4 / 30 / 89$ & $12 / 30 / 89$ & $4 / 27 / 89$ & $4 / 29 / 89$ & B0B2L7 & G299 & G342 & 1.20 & $7.50 E+0.4$ & $u g / m l$ \\
\hline Nitrate & $6 / 1 / 90$ & $4 / 30 / 89$ & $12 / 30 / 89$ & $4 / 27 / 89$ & $4 / 29 / 89$ & в082M1 & G308 & G353 & 1.20 & $7.50 E+04$ & ug $/ \mathrm{mL}$ \\
\hline Nitrate & $6 / 1 / 90$ & $4 / 30 / 89$ & $12 / 30 / 89$ & $4 / 27 / 89$ & $4 / 29 / 89$ & BOB2N4 & G323 & 6341 & 1.20 & $7.49 E+0.4$ & $\mathrm{ug} / \mathrm{ml}$ \\
\hline Nitrate & $6 / 1 / 90$ & $4 / 30 / 89$ & $12 / 30 / 89$ & $4 / 27 / 89$ & $4 / 29 / 89$ & вов2MO & G306 & G521* & $(1.20)$ & $7.42 E+04$ & $u g / \mathrm{mL}$ \\
\hline Nitrate & $6 / 1 / 90$ & $4 / 30 / 89$ & $12 / 30 / 89$ & $4 / 27 / 89$ & $4 / 29 / 89$ & BOB2MO & G306 & G352 & 1.20 & $7.42 E+04$ & $\mathrm{ug} / \mathrm{mL}$ \\
\hline Nitrate & $6 / 1 / 90$ & $4 / 30 / 89$ & $12 / 30 / 89$ & $4 / 27 / 89$ & $4 / 29 / 89$ & BOB2N3 & G322 & G529* & NA & $7.36 E+04$ & $\mathrm{ug} / \mathrm{mL}$ \\
\hline
\end{tabular}




\begin{tabular}{|c|c|c|c|c|c|c|c|}
\hline \multirow[b]{2}{*}{ Code } & \multirow[b]{2}{*}{ Source } & \multicolumn{3}{|c|}{ Analyte Information } & \multirow[b]{2}{*}{$\begin{array}{l}\text { Reference } \\
\text { Number }\end{array}$} & \multirow[b]{2}{*}{$\begin{array}{l}\text { Validated } \\
\text { (Y/N) }\end{array}$} & \multirow[b]{2}{*}{ Notes } \\
\hline & & Analyte & $\begin{array}{l}\text { Conc. } \\
\text { (ug/L) }\end{array}$ & Conc. (mol/L) & & & \\
\hline $\mathbf{P}$ & Raw & No3 & $7.59 E+07$ & $1.22 E+\infty 0$ & AP102-3 & Y & $\begin{array}{l}\text { Density reported for G358 collected at } 334 \text { in from bottom } \\
\text { of tank at a } 150^{\circ} \text { angle. Sample was unheated, unheated } \\
\text { samples precipitated }\end{array}$ \\
\hline $\mathbf{P}$ & Raw & NO3 & $7.59 E+07$ & $1.22 E+\infty 0$ & $A P 102-3$ & $Y$ & $\begin{array}{l}\text { Density reported for } \mathrm{G} 476 \text { collected at } 99 \text { in from bottom of } \\
\text { tank at a } 270^{\circ} \text { angle and was keep heated to } 40^{\circ} \mathrm{C} \text {. }\end{array}$ \\
\hline $\mathbf{P}$ & Raw & NO3 & $7.59 E+07$ & $1.22 E+\infty 0$ & AP102-3 & $Y$ & $\begin{array}{l}\text { Density not reported for G534. Density assumed same as } \\
\text { G364 which was collected at } 372 \text { in from bottom of tank at } \\
\text { a } 270^{\circ} \text { angle. This sample was heated. }\end{array}$ \\
\hline $\mathbf{P}$ & Raw & NO3 & $7.59 E+07$ & $1.22 \mathrm{E}+\infty$ & AP102-3 & $Y$ & $\begin{array}{l}\text { Density not reported for G534. Density assumed same as } \\
\text { G364 which was collected at } 372 \text { in from bottom of tank at } \\
\text { a } 270^{\circ} \text { angle. This sample was heated. }\end{array}$ \\
\hline P. & Raw & NO3 & $7.58 E+07$ & $1.22 E+00$ & AP102-3 & $\gamma$ & $\begin{array}{l}\text { Density reported for } \mathrm{G} 477 \text { collected at } 182 \text { in from bottom } \\
\text { of tank at a } 30^{\circ} \text { angle and was keep heated to } 40^{\circ} \mathrm{C} \text {. }\end{array}$ \\
\hline $\mathbf{P}$ & Raw & NO3 & $7.58 E+07$ & $1.22 E+\infty 0$ & AP102-3 & $\gamma$ & $\begin{array}{l}\text { Density not reported for G536. Density assumed same as } \\
\text { G460 which was collected at } 226 \text { in from bottom of tank at } \\
\text { a } 270^{\circ} \text { angle. This sample was heated. }\end{array}$ \\
\hline $\mathbf{P}$ & Raw & $\mathrm{NO} 3$ & $7.58 E+07$ & $1.22 \mathrm{E}+\infty 0$ & AP102-3 & $Y$ & 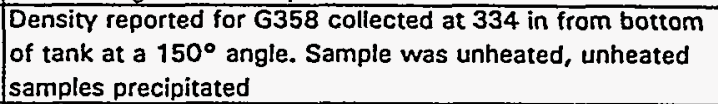 \\
\hline $\mathbf{P}$ & Raw & No3 & $7.58 E+07$ & $1.22 \mathrm{E}+00$ & AP102-3 & $Y$ & $\begin{array}{l}\text { Density reported for G459 collected at } 226 \text { in from bottom } \\
\text { of tank at a } 270^{\circ} \text { angle. Sample was unheated, unheated } \\
\text { samples precipitated }\end{array}$ \\
\hline $\mathbf{P}$ & Raw & NO3 & $7.58 E+07$ & $1.22 E+00$ & AP102-3 & $Y$ & $\begin{array}{l}\text { Density not reported for G511. Density assumed same as } \\
\text { G349 which was collected at } 34 \text { in from bottom of tank at a } \\
30^{\circ} \text { angle. This sample was heated. }\end{array}$ \\
\hline $\mathbf{P}$ & Raw & NO3 & $7.57 \mathrm{E}+07$ & $1.22 \mathrm{E}+00$ & AP102-3 & $Y$ & $\begin{array}{l}\text { Density not reported for G515. Density assumed same as } \\
\text { G358 which was collected at } 334 \text { in from bottom of tank at } \\
\text { a } 150^{\circ} \text { angle. This sample was heated. }\end{array}$ \\
\hline $\mathbf{P}$ & Raw & NO3 & $7.55 E+07$ & $1.22 E+\infty 0$ & AP102-3 & Y & $\begin{array}{l}\text { Density reported for } \mathrm{G} 348 \text { collected at } 125 \text { in from bottom } \\
\text { of tank at a } 30^{\circ} \text { angle. Sample was unheated, unheated } \\
\text { samples precipitated }\end{array}$ \\
\hline $\mathbf{P}$ & Raw & NO3 & $7.55 E+07$ & $1.22 E+\infty 0$ & AP102-3 & $Y$ & $\begin{array}{l}\text { Density reported for } \mathrm{G} 477 \text { collected at } 182 \text { in from bottom } \\
\text { of tank at a } 30^{\circ} \text { angle and was keep heated to } 40^{\circ} \mathrm{C} \text {. }\end{array}$ \\
\hline$P$ & Raw & NO3 & $7.53 E+07$ & $1.21 E+00$ & AP102-3 & $Y$ & $\begin{array}{l}\text { Density not reported for G536. Density assumed same as } \\
\text { G460 which was collected at } 226 \text { in from bottom of tank at } \\
\text { a } 270^{\circ} \text { angle. This sample was heated. }\end{array}$ \\
\hline $\mathbf{P}$ & Raw & NO3 & $7.52 E+07$ & $1.21 E+\infty$ & AP102-3 & $Y$ & $\begin{array}{l}\text { Density not reported for G511. Density assumed same as } \\
\text { G349 which was collected at } 34 \text { in from bottom of tank at a } \\
30^{\circ} \text { angle. This sample was heated. }\end{array}$ \\
\hline $\mathbf{P}$ & Raw & NO3 & $7.50 E+07$ & $1.21 E+\infty 0$ & AP102-3 & $Y$ & $\begin{array}{l}\text { Density reported for } \mathrm{G} 342 \text { collected at } 306 \text { in from bottom } \\
\text { of tank at a } 30^{\circ} \text { angle. Sample was unheated, unheated } \\
\text { samples precipitated }\end{array}$ \\
\hline$P$ & Raw & NO3 & $7.50 E+07$ & $1.21 E+\infty$ & AP102-3 & $Y$ & $\begin{array}{l}\text { Density reported for } G 353 \text { collected at } 334 \text { in from bottom } \\
\text { of tank at a } 150^{\circ} \text { angle. Sample was unheated, unheated } \\
\text { samples precipitated }\end{array}$ \\
\hline$P$ & Raw & NO3 & $7.49 E+07$ & $1.21 E+\infty$ & AP102-3 & $Y$ & $\begin{array}{l}\text { Density reported for G341 collected at } 99 \text { in from bottom of } \\
\text { tank at a } 270^{\circ} \text { angle. }\end{array}$ \\
\hline$P$ & Raw & NO3 & $7.42 E+07$ & $1.20 E+\infty$ & AP102-3 & $Y$ & $\begin{array}{l}\text { Density not reported for G521. Density assumed same as } \\
\text { G352 which was collected at } 34 \text { in from bottom of tank at a } \\
30^{\circ} \text { angle. This sample was heated. }\end{array}$ \\
\hline$P$ & Raw & NO3 & $7.42 E+07$ & $1.20 E+\infty$ & AP102-3 & $Y$ & $\begin{array}{l}\text { Density reported for } \mathrm{G} 352 \text { collected at } 34 \text { in from bottom of } \\
\text { tank at a } 30^{\circ} \text { angle. Sample was unheated, unheated } \\
\text { samples precipitated }\end{array}$ \\
\hline $\mathbf{P}$ & Raw & NO3 & $7.36 E+07$ & $1.19 E+\infty$ & AP102-3 & $\mathbf{Y}$ & $\begin{array}{l}\text { Density not reported for G529. No assumptions could be } \\
\text { made }\end{array}$ \\
\hline
\end{tabular}




\begin{tabular}{|c|c|c|c|c|c|c|c|c|c|c|c|}
\hline & & & & & nnk Waste & haracteriza & Oon Sample I & ormation & & & \\
\hline Reported Chemical & $\begin{array}{c}\text { Memo } \\
\text { Date }\end{array}$ & $\begin{array}{c}\text { Start } \\
\text { Analysis } \\
\text { Date }\end{array}$ & $\begin{array}{c}\text { Finish } \\
\text { Analysis Date } \\
\end{array}$ & $\begin{array}{c}\text { Start } \\
\text { Sample } \\
\text { Date } \\
\end{array}$ & $\begin{array}{c}\text { Finish } \\
\text { Sample } \\
\text { Date } \\
\end{array}$ & $\begin{array}{l}\text { Sample } \\
\text { Number }\end{array}$ & $\begin{array}{c}\text { Tank Farm } \\
\text { Number }\end{array}$ & $\begin{array}{c}\text { Lab Sample } \\
\text { Number }\end{array}$ & $\begin{array}{l}\text { Density } \\
(\mathrm{g} / \mathrm{mL})\end{array}$ & $\begin{array}{c}\text { Reported } \\
\text { Value } \\
\end{array}$ & $\begin{array}{r}\text { Repr } \\
\text { Ur } \\
\end{array}$ \\
\hline Nitrate & $6 / 1 / 90$ & $4 / 30 / 89$ & $12 / 30 / 89$ & $4 / 27 / 89$ & $4 / 29 / 89$ & BOB2N3 & G322 & G529* & NA & $7.30 E+04$ & ug $/$ \\
\hline Nitrate & $6 / 1 / 90$ & $4 / 30 / 89$ & $12 / 30 / 89$ & $4 / 27 / 89$ & $4 / 29 / 89$ & ВОВ2M8 & G301 & G523* & NA & $7.27 E+04$ & ug/t \\
\hline Nitrate & $6 / 1 / 90$ & $4 / 30 / 89$ & $12 / 30 / 89$ & $4 / 27 / 89$ & $4 / 29 / 89$ & вов2М8 & G301 & G523* & NA & $7.21 E+04$ & $u g / r$ \\
\hline Nitrate & $6 / 1 / 90$ & $4 / 30 / 89$ & $12 / 30 / 89$ & $4 / 27 / 89$ & $4 / 29 / 89$ & BOB2N2 & G317 & G528* & NA & $7.18 E+04$ & ug $/ \mathrm{t}$ \\
\hline Nitrate & $6 / 1 / 90$ & $4 / 30 / 89$ & $12 / 30 / 89$ & $4 / 27 / 89$ & $4 / 29 / 89$ & 808218 & G303 & G510* & 1.21 & $6.97 E+04$ & ug/r \\
\hline Nitrate & $6 / 1 / 90$ & $4 / 30 / 89$ & $12 / 30 / 89$ & $4 / 27 / 89$ & $4 / 29 / 89$ & BOB2N2 & G317 & G528* & NA & $6.68 E+04$ & ug/n \\
\hline Nitrate & $6 / 1 / 90$ & $4 / 30 / 89$ & $12 / 30 / 89$ & $4 / 27 / 89$ & $4 / 29 / 89$ & ВОВ2M3 & G311 & G516" & $(1.20)$ & $6.52 E+04$ & $u g / n$ \\
\hline Nitrate & $6 / 1 / 90$ & $4 / 30 / 89$ & $12 / 30 / 89$ & $4 / 27 / 89$ & $4 / 29 / 89$ & BOB2NO & G307 & G527" & NA & $6.45 E+04$ & $\mathrm{ug} / \mathrm{s}$ \\
\hline Nitrate & $6 / 1 / 90$ & $4 / 30 / 89$ & $12 / 30 / 89$ & $4 / 27 / 89$ & $4 / 29 / 89$ & BOB2T9 & G327 & G443 & 1.20 & $5.58 E+04$ & ug/r \\
\hline Nitrate & $6 / 1 / 90$ & $4 / 30 / 89$ & $12 / 30 / 89$ & $4 / 27 / 89$ & $4 / 29 / 89$ & BOB2T9 & G327 & G443 & 1.20 & $5.37 E+04$ & ug/r \\
\hline $\mathrm{OH}$ & $6 / 1 / 90$ & $4 / 30 / 89$ & $12 / 30 / 89$ & $4 / 27 / 89$ & $4 / 29 / 89$ & BOB2L9 & G305 & G349 & 1.20 & $9.52 E+03$ & $\mathrm{ug} / \mathrm{m}$ \\
\hline $\mathrm{OH}$ & $6 / 1 / 90$ & $4 / 30 / 89$ & $12 / 30 / 89$ & $4 / 27 / 89$ & $4 / 29 / 89$ & ВOB2MO & G306 & G352 & 1.20 & $9.47 E+03$ & $u g / m$ \\
\hline $\mathrm{OH}$ & $6 / 1 / 90$ & $4 / 30 / 89$ & $12 / 30 / 89$ & $4 / 27 / 89$ & $4 / 29 / 89$ & ВОВ2M2 & G309 & G358 & 1.20 & $9.40 E+03$ & $u g / m^{\prime}$ \\
\hline $\mathrm{OH}$ & 6/1/90 & $4 / 30 / 89$ & $12 / 30 / 89$ & $4 / 27 / 89$ & $4 / 29 / 89$ & BOB2N4 & G323 & G341 & 1.20 & $9.40 E+03$ & $\mathrm{ug} / \mathrm{ml}$ \\
\hline $\mathrm{OH}$ & $6 / 1 / 90$ & $4 / 30 / 89$ & $12 / 30 / 89$ & $4 / 27 / 89$ & $4 / 29 / 89$ & BOB2M5 & G315 & G364 & 1.19 & $9.33 E+03$ & $u g / m l$ \\
\hline $\mathrm{OH}$ & $6 / 1 / 90$ & $4 / 30 / 89$ & $12 / 30 / 89$ & $4 / 27 / 89$ & $4 / 29 / 89$ & вов2М9 & G302 & G477* & 1.20 & $9.33 E+03$ & $\mathrm{ug} / \mathrm{ml}$ \\
\hline $\mathrm{OH}$ & $6 / 1 / 90$ & $4 / 30 / 89$ & $12 / 30 / 89$ & $4 / 27 / 89$ & $4 / 29 / 89$ & Вов2М9 & G302 & G477* & 1.20 & $9.33 E+03$ & $u g / m L$ \\
\hline $\mathrm{OH}$ & $6 / 1 / 90$ & $4 / 30 / 89$ & $12 / 30 / 89$ & $4 / 27 / 89$ & $4 / 29 / 89$ & Вов2M6 & G318 & G459 & 1.20 & $9.27 E+03$ & $\mathrm{ug} / \mathrm{mL}$ \\
\hline OH & $6 / 1 / 90$ & $4 / 30 / 89$ & 12/30/89 & $4 / 27 / 89$ & $4 / 29 / 89$ & Bов2M4 & G313 & G363 & 1.20 & $9.20 E+03$ & $\mathrm{ug} / \mathrm{mL}$ \\
\hline $\mathrm{OH}$ & $6 / 1 / 90$ & $4 / 30 / 89$ & $12 / 30 / 89$ & $4 / 27 / 89$ & $4 / 29 / 89$ & Вов2Т9 & G327 & G443 & 1.20 & $9.20 E+03$ & $\mathrm{ug} / \mathrm{mL}$ \\
\hline $\mathrm{OH}$ & $6 / 1 / 90$ & $4 / 30 / 89$ & $12 / 30 / 89$ & $4 / 27 / 89$ & $4 / 29 / 89$ & BOB2T9 & G327 & G443 & 1.20 & $9.20 E+03$ & $\mathrm{ug} / \mathrm{mL}$ \\
\hline $\mathrm{OH}$ & $6 / 1 / 90$ & $4 / 30 / 89$ & $12 / 30 / 89$ & $4 / 27 / 89$ & $4 / 29 / 89$ & BOB2MO & G306 & G352 & 1.20 & $9.20 E+03$ & $\mathrm{ug} / \mathrm{mL}$ \\
\hline $\mathrm{OH}$ & $6 / 1 / 90$ & $4 / 30 / 89$ & $12 / 30 / 89$ & $4 / 27 / 89$ & $4 / 29 / 89$ & BOB2M1 & G308 & G353 & 1.20 & $9.20 E+03$ & $\mathrm{ug} / \mathrm{mL}$ \\
\hline
\end{tabular}




\begin{tabular}{|c|c|c|c|c|c|c|c|}
\hline \multirow[b]{2}{*}{ Code } & \multirow[b]{2}{*}{ Source } & \multicolumn{3}{|c|}{ Analyte Information } & \multirow[b]{2}{*}{$\begin{array}{l}\text { Reference } \\
\text { Numbet }\end{array}$} & \multirow[b]{2}{*}{$\begin{array}{l}\text { Validated } \\
\text { (Y/N) }\end{array}$} & \multirow[b]{2}{*}{ Notes } \\
\hline & & Analyto & $\begin{array}{l}\text { Conc. } \\
\text { (ug/L) }\end{array}$ & Conc. (mol/L) & & & \\
\hline $\mathbf{P}$ & Raw & NO3 & $7.30 E+07$ & $1.18 E+\infty 0$ & AP102-3 & $\gamma$ & $\begin{array}{l}\text { Density not reported for G529. No assumptions could be } \\
\text { made }\end{array}$ \\
\hline $\mathbf{P}$ & Raw & No3 & $7.27 E+07$ & $1.17 E+\infty 0$ & AP102-3 & $Y$ & $\begin{array}{l}\text { Density not reported for G523. No assumptions could be } \\
\text { made }\end{array}$ \\
\hline$P$ & Raw & NO3 & $7.21 E+07$ & $1.16 E+\infty$ & AP102-3 & $Y$ & $\begin{array}{l}\text { Density not reported for G523. No assumptions could be } \\
\text { made }\end{array}$ \\
\hline $\mathbf{P}$ & Raw & NO3 & $7.18 E+07$ & $1.16 E+\infty 0$ & AP102-3 & $Y$ & $\begin{array}{l}\text { Density not reported for G528. No assumptions could be } \\
\text { made }\end{array}$ \\
\hline $\mathbf{P}$ & Raw & NO3 & $6.97 E+07$ & $1.12 E+\infty 0$ & AP102-3 & $\mathbf{Y}$ & $\begin{array}{l}\text { Density not reported for G510. Density assumed same as } \\
\text { G348 which was collected at } 125 \text { in from bottom of tank at } \\
\text { a } 30^{\circ} \text { angle. This sample was heated. }\end{array}$ \\
\hline $\mathbf{P}$ & Raw & NO3 & $6.68 E+07$ & $1.08 E+\infty 0$ & AP102-3 & $\mathbf{Y}$ & $\begin{array}{l}\text { Density not reported for G528. No assumptions could be } \\
\text { made }\end{array}$ \\
\hline $\mathbf{P}$ & Raw & NO3 & $6.52 E+07$ & $1.05 E+00$ & AP102-3 & $Y$ & $\begin{array}{l}\text { Density not reported for G516. Density assumed same as } \\
\text { G359 which was collected at } 162 \text { in from bottom of tank at } \\
\text { a } 150^{\circ} \text { angle. This sample was heated. }\end{array}$ \\
\hline $\mathbf{P}$ & Raw & NO3 & $6.45 E+07$ & $1.04 E+00$ & AP102-3 & $Y$ & $\begin{array}{l}\text { Density not reported for G527. No assumptions could be } \\
\text { made }\end{array}$ \\
\hline $\mathbf{P}$ & Raw & NO3 & $5.58 E+07$ & $9.00 E-01$ & AP102-3 & $\gamma$ & Density reported for 6443 represents a composite sample \\
\hline $\mathbf{P}$ & Raw & NO3 & $5.37 E+07$ & 8.66E-01 & API02-3 & $Y$ & Density reported for 6443 represents a composite sample \\
\hline $\mathbf{P}$ & Raw & $\mathrm{OH}$ & $9.52 E+06$ & 5.6OE-01 & AP102-3 & $Y$ & $\begin{array}{l}\text { Density reported for } \mathrm{G} 349 \text { collected at } 34 \text { in from bottom of } \\
\text { tank at a } 30^{\circ} \text { angle. Sample was unheated, unheated } \\
\text { samples precipitated }\end{array}$ \\
\hline $\mathbf{P}$ & Raw & $\mathrm{OH}$ & $9.47 E+06$ & 5.57E-01 & AP102-3 & $Y$ & $\begin{array}{l}\text { Density reported for } \mathrm{G} 352 \text { collected at } 34 \text { in from bottom of } \\
\text { tank at a } 30^{\circ} \text { angle. Sample was unheated, unheated } \\
\text { samples precipitated }\end{array}$ \\
\hline $\mathbf{P}$ & Raw & $\mathrm{OH}$ & $9.40 E+06$ & $5.53 \mathrm{E}-01$ & AP102-3 & $\gamma$ & $\begin{array}{l}\text { Density reported for G358 collected at } 334 \text { in from bottom } \\
\text { of tank at a } 150^{\circ} \text { angle. Sample was unheated, unheated } \\
\text { samples precipitated }\end{array}$ \\
\hline $\mathbf{P}$ & Raw & $\mathrm{OH}$ & $9.40 E+06$ & $5.53 E-01$ & AP102-3 & $\mathbf{Y}$ & $\begin{array}{l}\text { Density reported for } \mathrm{G} 341 \text { collected at } 99 \text { in from bottom of } \\
\text { tank at a } 270^{\circ} \text { angle. }\end{array}$ \\
\hline $\mathbf{P}$ & Raw & $\mathrm{OH}$ & $9.33 E+06$ & 5.49E-01 & AP102-3 & Y & $\begin{array}{l}\text { Density reported for G364 collected at } 372 \text { in from bottom } \\
\text { of tank at a } 270^{\circ} \text { angle. Sample was unheated, unheated } \\
\text { samples precipitated }\end{array}$ \\
\hline $\mathbf{P}$ & Raw & $\mathrm{OH}$ & $9.33 E+06$ & 5.49E-01 & AP102-3 & $\mathbf{Y}$ & $\begin{array}{l}\text { Density reported for } G 477 \text { collected at } 182 \text { in from bottom. } \\
\text { of tank at a } 30^{\circ} \text { angle and was keep heated to } 40^{\circ} \mathrm{C} \text {. }\end{array}$ \\
\hline $\mathbf{P}$ & Raw & $\mathrm{OH}$ & $9.33 E+06$ & $5.49 E-01$ & AP102-3 & $\mathbf{Y}$ & $\begin{array}{l}\text { Density reported for G477 collected at } 182 \text { in from bottom } \\
\text { of tank at a } 30^{\circ} \text { angle and was keep heated to } 40^{\circ} \mathrm{C} \text {. }\end{array}$ \\
\hline $\mathbf{P}$ & Raw & $\mathrm{OH}$ & $9.27 E+06$ & $5.45 E-01$ & AP102-3 & $Y$ & $\begin{array}{l}\text { Density reported for } \mathrm{G} 459 \text { collected at } 226 \text { in trom bottom } \\
\text { of tank at a } 270^{\circ} \text { angle. Sample was unheated, unheated } \\
\text { samples precipitated }\end{array}$ \\
\hline $\mathbf{P}$ & Raw & $\mathrm{OH}$ & $9.20 E+06$ & 5.41E-01 & AP102-3 & $\mathbf{Y}$ & $\begin{array}{l}\text { Density reported for G363 collected at } 18 \text { in from bottom of } \\
\text { tank at a } 150^{\circ} \text { angle. Sample was unheated, unheated } \\
\text { samples precipitated }\end{array}$ \\
\hline $\mathbf{P}$ & Raw & $\mathrm{OH}$ & $9.20 E+06$ & 5.41E-01 & AP102-3 & $\mathbf{Y}$ & Density reported for 6443 represents a composite sample \\
\hline$P$ & Raw & $\mathrm{OH}$ & $9.20 E+06$ & $5.41 E-01$ & AP102-3 & $Y$ & Density reported for 6443 represents a composite sample \\
\hline $\mathbf{P}$ & Raw & $\mathrm{OH}$ & $9.20 E+06$ & 5.41E-01 & AP102-3 & $Y$ & $\begin{array}{l}\text { Density reported for } \mathrm{G} 352 \text { collected at } 34 \text { in from bottom of } \\
\text { tank at a } 30^{\circ} \text { angle. Sample was unheated, unheated } \\
\text { samples precipitated }\end{array}$ \\
\hline$P$ & Raw & $\mathrm{OH}$ & $9.20 E+06$ & $5.41 E-01$ & AP102-3 & $\gamma$ & $\begin{array}{l}\text { Density reported for } \mathrm{G} 353 \text { collected at } 334 \text { in from bottom } \\
\text { of tank at a } 150^{\circ} \text { angle. Sample was unheated, unheated } \\
\text { samples precipitated }\end{array}$ \\
\hline
\end{tabular}




\begin{tabular}{|c|c|c|c|c|c|c|c|c|c|c|c|}
\hline & & & & & ank Waste & haracteriz: & Ion Sample tr & ormation & & & \\
\hline Reported Chemical & $\begin{array}{c}\text { Memo } \\
\text { Date }\end{array}$ & $\begin{array}{c}\text { Start } \\
\text { Analysis } \\
\text { Date }\end{array}$ & $\begin{array}{c}\text { Finish } \\
\text { Analysis Date }\end{array}$ & $\begin{array}{c}\text { Start } \\
\text { Sample } \\
\text { Date }\end{array}$ & $\begin{array}{c}\text { Finish } \\
\text { Sample } \\
\text { Date } \\
\end{array}$ & $\begin{array}{l}\text { Sample } \\
\text { Number }\end{array}$ & $\begin{array}{c}\text { Tank Farm } \\
\text { Number }\end{array}$ & $\begin{array}{c}\text { Lab Sample } \\
\text { Number }\end{array}$ & $\begin{array}{l}\text { Density } \\
\text { (g/mL) }\end{array}$ & $\begin{array}{c}\text { Reported } \\
\text { Value }\end{array}$ & $\begin{array}{r}\text { Repa } \\
\text { Ur } \\
\end{array}$ \\
\hline $\mathrm{OH}$ & $6 / 1 / 90$ & $4 / 30 / 89$ & $12 / 30 / 89$ & $4 / 27 / 89$ & $4 / 29 / 89$ & BOB2M4 & G313 & G363 & 1.20 & $9.20 E+03$ & $u g / h$ \\
\hline $\mathrm{OH}$ & $6 / 1 / 90$ & $4 / 30 / 89$ & $12 / 30 / 89$ & $4 / 27 / 89$ & $4 / 29 / 89$ & ВОВ2M5 & G315 & G364 & 1.19 & $9.15 E+03$ & $u g / r$ \\
\hline $\mathrm{OH}$ & $6 / 1 / 90$ & $4 / 30 / 89$ & $12 / 30 / 89$ & $4 / 27 / 89$ & $4 / 29 / 89$ & $80 B 2 L 8$ & G303 & G348 & 1.21 & $9.13 E+03$ & $u g / r$ \\
\hline $\mathrm{OH}$ & $6 / 1790$ & $4 / 30 / 89$ & $12 / 30 / 89$ & $4 / 27 / 89$ & $4 / 29 / 89$ & BOB2M1 & G308 & G353 & 1.20 & $9.08 E+03$ & $u g / n$ \\
\hline $\mathrm{OH}$ & $6 / 1 / 90$ & $4 / 30 / 89$ & $12 / 30 / 89$ & $4 / 27 / 89$ & $4 / 29 / 89$ & ВOB2M3 & G311 & G359 & 1.20 & $9.08 E+03$ & $u g / n$ \\
\hline $\mathrm{OH}$ & $6 / 1 / 90$ & $4 / 30 / 89$ & $12 / 30 / 89$ & $4 / 27 / 89$ & $4 / 29 / 89$ & BOB2M6 & G318 & G459 & 1.20 & $9.08 E+03$ & $u g / n$ \\
\hline $\mathrm{OH}$ & $6 / 1 / 90$ & $4 / 30 / 89$ & $12 / 30 / 89$ & $4 / 27 / 89$ & $4 / 29 / 89$ & BOB2M7 & G319 & G460 & 1.20 & $9.08 E+03$ & $u g / n$ \\
\hline $\mathrm{OH}$ & $6 / 1 / 90$ & $4 / 30 / 89$ & $12 / 30 / 89$ & $4 / 27 / 89$ & $4 / 29 / 89$ & BOB2L7 & G299 & G342 & 1.20 & $9.08 E+03$ & ug/r \\
\hline $\mathrm{OH}$ & $6 / 1 / 90$ & $4 / 30 / 89$ & $12 / 30 / 89$ & $4 / 27 / 89$ & $4 / 29 / 89$ & ВOB2M2 & G309 & G358 & 1.20 & $9.08 E+03$ & $\mathrm{ug} / \mathrm{m}$ \\
\hline $\mathrm{OH}$ & $6 / 1 / 90$ & $4 / 30 / 89$ & $12 / 30 / 89$ & $4 / 27 / 89$ & $4 / 29 / 89$ & BOB2M3 & G311 & G359 & 1.20 & $9.08 E+03$ & $u g / m$ \\
\hline $\mathrm{OH}$ & $6 / 1 / 90$ & $4 / 30 / 89$ & $12 / 30 / 89$ & $4 / 27 / 89$ & $4 / 29 / 89$ & вов2М7 & G319 & G460 & 1.20 & $9.08 E+03$ & $\mathrm{ug} / \mathrm{m}$ \\
\hline $\mathrm{OH}$ & $6 / 1 / 90$ & $4 / 30 / 89$ & $12 / 30 / 89$ & $4 / 27 / 89$ & $4 / 29 / 89$ & BOB2N4 & G323 & G476 & 1.21 & $9.08 E+03$ & $\mathrm{ug} / \mathrm{m}^{\prime}$ \\
\hline $\mathrm{OH}$ & $6 / 1 / 90$ & $4 / 30 / 89$ & $12 / 30 / 89$ & $4 / 27 / 89$ & $4 / 29 / 89$ & BOB2LG & G305 & G349 & 1.20 & $9.01 E+03$ & $\mathrm{ug} / \mathrm{mi}$ \\
\hline $\mathrm{OH}$ & $6 / 1 / 90$ & $4 / 30 / 89$ & $12 / 30 / 89$ & $4 / 27 / 89$ & $4 / 29 / 89$ & BOB2T9 & G327 & $\mathrm{G} 443$ & 1.20 & $9.01 E+03$ & $\mathrm{ug} / \mathrm{m} !$ \\
\hline $\mathrm{OH}$ & $6 / 1 / 90$ & $4 / 30 / 89$ & $12 / 30 / 89$ & $4 / 27 / 89$ & $4 / 29 / 89$ & ВОВ2T9 & G327 & G443 & 1.20 & $9.01 E+03$ & $\mathrm{ug} / \mathrm{ml}$ \\
\hline $\mathrm{OH}$ & $6 / 1 / 90$ & $4 / 30 / 89$ & $12 / 30 / 89$ & $4 / 27 / 89$ & $4 / 29 / 89$ & BOB2N4 & G323 & G341 & 1.20 & $8.94 E+03$ & $\mathrm{ug} / \mathrm{ml}$ \\
\hline $\mathrm{OH}$ & $6 / 1 / 90$ & $4 / 30 / 89$ & $12 / 30 / 89$ & $4 / 27 / 89$ & 4/29/89 & BOB2L7 & G299 & G342 & 1.20 & $8.94 E+03$ & $\mathrm{ug} / \mathrm{ml}$ \\
\hline $\mathrm{OH}$ & $6 / 1 / 90$ & $4 / 30 / 89$ & $12 / 30 / 89$ & $4 / 27 / 89$ & $4 / 29 / 89$ & BOB2L8 & G303 & G348 & 1.21 & $8.94 E+03$ & $\mathrm{ug} / \mathrm{ml}$ \\
\hline $\mathrm{OH}$ & $6 / 1 / 90$ & $4 / 30 / 89$ & $12 / 30 / 89$ & $4 / 27 / 89$ & $4 / 29 / 89$ & BOB2N1 & G310 & $\mathrm{G} 471^{\circ}$ & 1.19 & $8.94 E+03$ & $\mathrm{ug} / \mathrm{ml}$ \\
\hline $\mathrm{OH}$ & $6 / 1 / 90$ & $4 / 30 / 89$ & $12 / 30 / 89$ & $4 / 27 / 89$ & $4 / 29 / 89$ & ВОВ2Т9 & G327 & G470* & 1.19 & $8.81 E+03$ & $u g / m L$ \\
\hline OH & $6 / 1 / 90$ & $4 / 30 / 89$ & $12 / 30 / 89$ & $4 / 27 / 89$ & $4 / 29 / 89$ & BOB2N4 & G323 & G476* & 1.21 & $8.81 E+03$ & $\mathrm{ug} / \mathrm{mL}$ \\
\hline
\end{tabular}




\begin{tabular}{|c|c|c|c|c|c|c|c|}
\hline \multirow[b]{2}{*}{ Code } & \multirow[b]{2}{*}{ Source } & \multicolumn{3}{|c|}{ Analyte Information } & \multirow[b]{2}{*}{$\begin{array}{l}\text { Reference } \\
\text { Number }\end{array}$} & \multirow[b]{2}{*}{$\begin{array}{l}\text { Validated } \\
\text { (Y/N) }\end{array}$} & \multirow[b]{2}{*}{ Notes } \\
\hline & & Analyte & $\begin{array}{l}\text { Conc. } \\
\text { lug/L) }\end{array}$ & Conc. (mol/L) & & & \\
\hline $\mathbf{P}$ & Raw & $\mathrm{OH}$ & $9.20 E+06$ & $5.41 E-01$ & AP102-3 & $Y$ & $\begin{array}{l}\text { Density reported for G363 collected at } 18 \text { in from bottom of } \\
\text { tank at a } 150^{\circ} \text { angle. Sample was unheated, unheated } \\
\text { samples precipitated }\end{array}$ \\
\hline $\mathbf{P}$ & Raw & $\mathrm{OH}$ & $9.15 E+06$ & $5.38 \mathrm{E}-01$ & AP102-3 & $Y$ & $\begin{array}{l}\text { Density reported for G364 collected at } 372 \text { in from bottom } \\
\text { of tank at a } 270^{\circ} \text { angle. Sample was unheated, unheated } \\
\text { samples precipitated }\end{array}$ \\
\hline $\mathbf{P}$ & Raw & $\mathrm{OH}$ & $9.13 E+06$ & 5.37E-01 & AP102-3 & $Y$ & $\begin{array}{l}\text { Density reported for } 6348 \text { collected at } 125 \text { in from bottom } \\
\text { of tank at a } 30^{\circ} \text { angle. Sample was unheated, unheated } \\
\text { samples precipitated }\end{array}$ \\
\hline $\mathbf{P}$ & Raw & $\mathrm{OH}$ & $9.08 E+06$ & 5.34E-01 & AP102-3 & $\mathbf{Y}$ & $\begin{array}{l}\text { Density reported for } \mathrm{G} 353 \text { collected at } 334 \text { in from bottom } \\
\text { of tank at a } 150^{\circ} \text { angle. Sample was unheated, unheated } \\
\text { samples precipitated }\end{array}$ \\
\hline $\mathbf{P}$ & Raw & $\mathrm{OH}$ & $9.08 E+06$ & $5.34 E-01$ & AP102-3 & $Y$ & $\begin{array}{l}\text { Density reported for G359 collected at } 162 \text { in from bottom } \\
\text { of tank at a } 150^{\circ} \text { angle. Sample was unheated, unheated } \\
\text { samples precipitated }\end{array}$ \\
\hline $\mathbf{P}$ & Raw & $\mathrm{OH}$ & $9.08 E+06$ & 5.34E-01 & AP102-3 & $\gamma$ & $\begin{array}{l}\text { Density reported for } 6459 \text { collected at } 226 \text { in from bottom } \\
\text { of tank at a } 270^{\circ} \text { angle. Sample was unheated, unheated } \\
\text { samples precipitated }\end{array}$ \\
\hline $\mathbf{P}$ & Raw & $\mathrm{OH}$ & $9.08 E+06$ & 5.34E-01 & AP102-3 & $Y$ & $\begin{array}{l}\text { Density reported for G460 collected at } 226 \text { in from bottom } \\
\text { of tank at a } 270^{\circ} \text { angle. Sample was unheated, unheated } \\
\text { samples precipitated }\end{array}$ \\
\hline$P$ & Raw & $\mathrm{OH}$ & $9.08 E+06$ & 5.34E-01 & AP102-3 & $\gamma$ & $\begin{array}{l}\text { Density reported for G342 collected at } 306 \text { in from bottom } \\
\text { of tank at a } 30^{\circ} \text { angle. Sample was unheated, unheated } \\
\text { samples precipitated }\end{array}$ \\
\hline $\mathbf{P}$ & Raw & $\mathrm{OH}$ & $9.08 E+06$ & $5.34 E-01$ & AP102-3 & Y & $\begin{array}{l}\text { Density reported for G358 collected at } 334 \text { in from bottom } \\
\text { of tank at a } 150^{\circ} \text { angle. Sample was unheated, unheated } \\
\text { samples precipitated }\end{array}$ \\
\hline$P$ & Raw & $\mathrm{OH}$ & $9.08 E+06$ & $5.34 \mathrm{E}-01$ & AP102-3 & $Y$ & $\begin{array}{l}\text { Density reported for G359 collected at } 162 \text { in from bottom } \\
\text { of tank at a } 150^{\circ} \text { angle. Sample was unheated, unheated } \\
\text { samples precipitated }\end{array}$ \\
\hline$P$ & Raw & $\mathrm{OH}$ & $9.08 E+06$ & $5.34 E-01$ & AP102-3 & $Y$ & $\begin{array}{l}\text { Density reported for } \mathrm{G} 460 \text { collected at } 226 \text { in from bottom } \\
\text { of tank at a } 270^{\circ} \text { angle. Sample was unheated, unheated } \\
\text { samples precipitated }\end{array}$ \\
\hline $\mathbf{P}$ & Raw & $\mathrm{OH}$ & $9.08 E+06$ & 5.34E-01 & AP102-3 & $\gamma$ & $\begin{array}{l}\text { Density reported for } G 476 \text { collected at } 99 \text { in from bottom of } \\
\text { tank at a } 270^{\circ} \text { angle and was keep heated to } 40^{\circ} \mathrm{C} \text {. }\end{array}$ \\
\hline $\mathbf{P}$ & Raw & $\mathrm{OH}$ & $9.01 E+06$ & $5.30 \mathrm{E}-01$ & AP102-3 & $Y$ & $\begin{array}{l}\text { Density reported for G349 collected at } 34 \text { in from bottom of } \\
\text { tank at a } 30^{\circ} \text { angle. Sample was unheated, unheated } \\
\text { samples precipitated }\end{array}$ \\
\hline$P$ & Raw & $\mathrm{OH}$ & $9.01 E+06$ & 5.30E-01 & AP102-3 & $Y$ & Density reported for $\mathrm{G} 443$ represents a composite sample \\
\hline $\mathbf{P}$ & Raw & $\mathrm{OH}$ & $9.01 E+06$ & 5.30E-01 & AP102-3 & Y & Density reported for $G 443$ represents a composite sample \\
\hline $\mathbf{P}$ & Raw & $\mathrm{OH}$ & $8.94 E+06$ & $5.26 \mathrm{E}-01$ & AP102-3 & Y & $\begin{array}{l}\text { Density reported for G341 collected at } 99 \text { in from bottom of } \\
\text { tank at a } 270^{\circ} \text { angle. }\end{array}$ \\
\hline$P$ & Raw & $\mathrm{OH}$ & $8.94 E+06$ & $5.26 \mathrm{E}-01$ & AP102-3 & $\gamma$ & $\begin{array}{l}\text { Density reported for } 6342 \text { collected at } 306 \text { in from bottom } \\
\text { of tank at a } 30^{\circ} \text { angle. Sample was unheated, unheated } \\
\text { samples precipitated }\end{array}$ \\
\hline$P$ & Raw & $\mathrm{OH}$ & $8.94 E+06$ & $5.26 E-01$ & AP102-3 & Y & $\begin{array}{l}\text { Density reported for } \mathrm{G} 348 \text { collected at } 125 \text { in from bottom } \\
\text { of tank at a } 30^{\circ} \text { angle. Sample was unheated, unheated } \\
\text { samples precipitated }\end{array}$ \\
\hline 8 & Raw & $\mathrm{OH}$ & $8.94 E+06$ & 5.26E-01 & AP102-3 & Y & $\begin{array}{l}\text { Density reported for } G 471 \text { collected at } 208 \text { in from bottom } \\
\text { of tank at a } 150^{\circ} \text { angle and was keep heated to } 40^{\circ} \mathrm{C} \text {. }\end{array}$ \\
\hline $\mathbf{P}$ & Raw & $\mathrm{OH}$ & $8.81 E+06$ & $5.18 E-01$ & AP102-3 & $Y$ & $\begin{array}{l}\text { Density reported for G470 represents a composite sample. } \\
\text { Sample was kept heated to } 40^{\circ} \mathrm{C} \text {. }\end{array}$ \\
\hline $\mathbf{P}$ & Raw & $\mathrm{OH}$ & $8.81 E+06$ & 5.18E-01 & AP102-3 & $Y$ & $\begin{array}{l}\text { Density reported for G476 collected at } 99 \text { in from bottom of } \\
\text { tank at a } 270^{\circ} \text { angle and was keep heated to } 40^{\circ} \mathrm{C} \text {. }\end{array}$ \\
\hline
\end{tabular}




\begin{tabular}{|c|c|c|c|c|c|c|c|c|c|c|c|}
\hline & & & & & ank Waste & haracteriza & ion Sample I & ormation & & & \\
\hline Reported Chemical & $\begin{array}{c}\text { Memo } \\
\text { Date }\end{array}$ & $\begin{array}{c}\text { Start } \\
\text { Analysis } \\
\text { Date }\end{array}$ & $\begin{array}{c}\text { Finish } \\
\text { Analysis Date }\end{array}$ & $\begin{array}{c}\text { Start } \\
\text { Sample } \\
\text { Date } \\
\end{array}$ & $\begin{array}{c}\text { Finish } \\
\text { Sample } \\
\text { Date }\end{array}$ & $\begin{array}{l}\text { Sample } \\
\text { Number }\end{array}$ & $\begin{array}{c}\text { Tank Farm } \\
\text { Number }\end{array}$ & $\begin{array}{c}\text { Lab Sampla } \\
\text { Number }\end{array}$ & $\begin{array}{l}\text { Density } \\
(\mathrm{g} / \mathrm{mL})\end{array}$ & $\begin{array}{c}\text { Reported } \\
\text { Value }\end{array}$ & $\begin{array}{r}\text { Repori } \\
\text { Unii }\end{array}$ \\
\hline $\mathrm{OH}$ & $6 / 1 / 90$ & $4 / 30 / 89$ & $12 / 30 / 89$ & $4 / 27 / 89$ & $4 / 29 / 89$ & ВОВ2M9 & G302 & G477* & 1.20 & $8.81 E+03$ & $\mathrm{ug} / \mathrm{m}$ \\
\hline $\mathrm{OH}$ & $6 / 1 / 90$ & $4 / 30 / 89$ & $12 / 30 / 89$ & $4 / 27 / 89$ & $4 / 29 / 89$ & BOB2N1 & G310 & G471* & 1.19 & $8.69 E+03$ & $u g / m$ \\
\hline $\mathrm{OH}$ & $6 / 1 / 90$ & $4 / 30 / 89$ & $12 / 30 / 89$ & $4 / 27 / 89$ & $4 / 29 / 89$ & BOB2T9 & G327 & G470 & 1.19 & $8.62 E+03$ & $\mathrm{ug} / \mathrm{m}$ \\
\hline $\mathrm{OH}$ & $6 / 1 / 90$ & $4 / 30 / 89$ & $12 / 30 / 89$ & $4 / 27 / 89$ & $4 / 29 / 89$ & BOB2M9 & -6302 & G477* & 1.20 & $\cdot 7.91 E+03$ & $u g / m$ \\
\hline Phosphorus & $6 / 1 / 90$ & $4 / 30 / 89$ & $12 / 30 / 89$ & $4 / 27 / 89$ & $4 / 29 / 89$ & ВОВ2Т9 & G327 & G470. & 1.19 & $3.88 E+06$ & ug/L \\
\hline Phosphorus & $6 / 1 / 90$ & $4 / 30 / 89$ & $12 / 30 / 89$ & $4 / 27 / 89$ & $4 / 29 / 89$ & BOB2T9 & G327 & G470* & 1.19 & $3.55 E+06$ & ug/t. \\
\hline Phosphorus & $6 / 1 / 90$ & $4 / 30 / 89$ & $12 / 30 / 89$ & $4 / 27 / 89$ & $4 / 29 / 89$ & BOB2NO & G307 & G453 & $N A ̈$ & $3.53 E+06$ & $u g / L$ \\
\hline Phosphorus & $6 / 1 / 90$ & $4 / 30 / 89$ & $12 / 30 / 89$ & $4 / 27 / 89$ & $4 / 29 / 89$ & $\mathrm{BOB} 2 \mathrm{~N} 3$ & G322 & G338 & NA & $3.44 E+06$ & $u g / L$. \\
\hline Phosphorus & $6 / 1 / 90$ & $4 / 30 / 89$ & $12 / 30 / 89$ & $4 / 27 / 89$ & $4 / 29 / 89$ & BOB2N3 & G322 & G338 & NA & $3.44 E+06$ & ug/L \\
\hline Phosphorus & $6 / 1 / 90$ & $4 / 30 / 89$ & $12 / 30 / 89$ & $4 / 27 / 89$ & $4 / 29 / 89$ & BOB2N1 & G310 & G454 & $(1.19)$ & $3.42 E+06$ & ug/L \\
\hline Phosphorus & $6 / 1 / 90$ & $4 / 30 / 89$ & $12 / 30 / 89$ & $4 / 27 / 89$ & $4 / 29 / 89$ & BOB2N1 & G310 & G454 & (17.19) & $3.38 E+06$ & ug/L. \\
\hline Phosphorus & $6 / 1 / 90$ & $4 / 30 / 89$ & $12 / 30 / 89$ & $4 / 27 / 89$ & $4 / 29 / 89$ & BOB2L9 & G305 & G349 & 1.20 & $3.30 E+06$ & ug/L \\
\hline Phosphorus & $6 / 1 / 90$ & $4 / 30 / 89$ & $12 / 30 / 89$ & $4 / 27 / 89$ & $4 / 29 / 89$ & BOB2NO & 6307 & G453 & NA & $3.30 E+06$ & ug/L \\
\hline Phosphorus & $6 / 1 / 90$ & $4 / 30 / 89$ & $12 / 30 / 89$ & $4 / 27 / 89$ & $4 / 29 / 89$ & BOB2N2 & G317 & G333 & NA & $3.29 E+06$ & ug/L \\
\hline Phosphorus & $6 / 1 / 90$ & $4 / 30 / 89$ & $32 / 30 / 89$ & $4 / 27 / 89$ & $4 / 29 / 89$ & BOB2N2 & G317 & G333 & NA & $3.25 E+06$ & ug/L \\
\hline Phosphorus & $6 / 1 / 90$ & $4 / 30 / 89$ & $12 / 30 / 89$ & $4 / 27 / 89$ & $4 / 29 / 89$ & BOB2M6 & G318 & G459 & 1.20 & $3.25 E+06$ & $u g / L$ \\
\hline Phosphorus & $6 / 1 / 90$ & $4 / 30 / 89$ & $12 / 30 / 89$ & $4 / 27 / 89$ & $4 / 29 / 89$ & BOB2M6 & G318 & G459 & 1.20 & $3.19 E+06$ & ug/L \\
\hline Phosphorus & $6 / 1 / 90$ & $4 / 30 / 89$ & $12 / 30 / 89$ & $4 / 27 / 89$ & $4 / 29 / 89$ & BO82M7 & G319 & G460 & 1.20 & $3.13 E+06$ & $u g / L$ \\
\hline Phosphorus & $6 / 1 / 90$ & $4 / 30 / 89$ & $12 / 30 / 89$ & $4 / 27 / 89$ & $4 / 29 / 89$ & ВОВ2M7 & G319 & G460 & 1.20 & $3.12 E+06$ & $u g / L$ \\
\hline Phosphorus & $6 / 1 / 90$ & $4 / 30 / 89$ & $12 / 30 / 89$ & $4 / 27 / 89$ & $4 / 29 / 89$ & $8082 \mathrm{M9}$ & G302 & G450 & $(1.20)$ & $3.08 E+06$ & $u g R$ \\
\hline Phosphorus & $6 / 1 / 90$ & $4 / 30 / 89$ & $12 / 30 / 89$ & $4 / 27 / 89$ & $4 / 29 / 89$ & $80 B 2 N 4$ & G323 & G341 & 1.20 & $3.08 E+06$ & $u g / L$ \\
\hline Phosphorus & $6 / 1 / 90$ & $4 / 30 / 89$ & $12 / 30 / 89$ & $4 / 27 / 89$ & $4 / 29 / 89$ & $B 082 L 7$ & G299 & G342 & 1.20 & $3.07 E+06$ & ug/L \\
\hline
\end{tabular}




\begin{tabular}{|c|c|c|c|c|c|c|c|}
\hline \multirow[b]{2}{*}{ Code } & \multirow[b]{2}{*}{ Source } & \multicolumn{3}{|c|}{ Analyte Information } & \multirow[b]{2}{*}{$\begin{array}{c}\text { Reference } \\
\text { Number }\end{array}$} & \multirow[b]{2}{*}{$\begin{array}{l}\text { Validated } \\
\text { (Y/N) }\end{array}$} & \multirow[b]{2}{*}{ Notes } \\
\hline & & Analyte & $\begin{array}{l}\text { Conc. } \\
\text { (ug/L) }\end{array}$ & Conc. (mol/L) & & & \\
\hline $\mathbf{P}$ & Raw & $\mathrm{OH}$ & $8.81 E+06$ & $5.18 \mathrm{E}-01$ & AP102-3 & $Y$ & $\begin{array}{l}\text { Density reported for G477 collected at } 182 \text { in from bottom } \\
\text { of tank at a } 30^{\circ} \text { angle and was keep heated to } 40^{\circ} \mathrm{C} \text {. }\end{array}$ \\
\hline $\mathbf{P}$ & Raw & $\mathrm{OH}$ & $8.69 E+06$ & 5.11E-01 & AP102-3 & $Y$ & $\begin{array}{l}\text { Density reported for } G 471 \text { collected at } 208 \text { in from bottom } \\
\text { of tank at a } 150^{\circ} \text { angle and was keep heated to } 40^{\circ} \mathrm{C} \text {. }\end{array}$ \\
\hline $\mathbf{P}$ & Raw & $\mathrm{OH}$ & $8.62 E+06$ & 5.07E-01 & AP102-3 & $Y$ & $\begin{array}{l}\text { Density reported for G470 represents a composite sample. } \\
\text { Sample was kept heated to } 40^{\circ} \mathrm{C} \text {. }\end{array}$ \\
\hline $\mathbf{P}$ & Raw & $\mathrm{OH}$ & $7.91 E+06$ & $4.65 E-01$ & AP102-3 & $Y$ & $\begin{array}{l}\text { Density reported for } G 477 \text { collected at } 182 \text { in from bottom } \\
\text { of tank at a } 30^{\circ} \text { angle and was keep heated to } 40^{\circ} \mathrm{C} \text {. }\end{array}$ \\
\hline $\mathbf{P}$ & Raw & $\mathbf{P}$ & $3.88 E+06$ & $\# N / A$ & AP102-3 & $Y$ & $\begin{array}{l}\text { Density reported for } \mathrm{G} 470 \text { represents a composite sample. } \\
\text { Sample was kept heated to } 40^{\circ} \mathrm{C} \text {. }\end{array}$ \\
\hline $\mathbf{P}$ & Raw & $\mathbf{P}$ & $3.55 E+06$ & $\# N / A$ & AP102-3 & $Y$ & $\begin{array}{l}\text { Density reported for G470 represents a composite sample. } \\
\text { Sample was kept heated to } 40^{\circ} \mathrm{C} \text {. }\end{array}$ \\
\hline$P$ & Raw & $\mathbf{P}$ & $3.53 E+06$ & $\# N / A$ & AP102-3 & $Y$ & $\begin{array}{l}\text { Density not reported for G453. No assumptions could be } \\
\text { made }\end{array}$ \\
\hline$P$ & Raw & $\mathbf{P}$ & $3.44 \mathrm{E}+06$ & $\# N / A$ & AP102-3 & $Y$ & $\begin{array}{l}\text { Density not reported for G338. No assumptions could be } \\
\text { made }\end{array}$ \\
\hline$P$ & Raw & $\mathbf{P}$ & $3.44 E+06$ & $\#$ N/A & AP102-3 & $\mathbf{Y}$ & $\begin{array}{l}\text { Density not reported for G338. No assumptions could be } \\
\text { made }\end{array}$ \\
\hline$P$ & Raw & $P$ & $3.42 E+06$ & $\# N / A$ & AP102-3 & $Y$ & $\begin{array}{l}\text { Density not reported for G454. Density assumed same as } \\
\text { G471 which was collected at } 208 \text { in from bottom of tank at } \\
\text { a } 150^{\circ} \text { angle and was keep heated to } 40^{\circ} \mathrm{C} \text {. }\end{array}$ \\
\hline$P$ & Raw & $P$ & $3.38 E+06$ & $\# N / A$ & AP102-3 & $\gamma$ & $\begin{array}{l}\text { Density not reported for } \mathrm{G} 454 \text {. Density assumed same as } \\
\text { G471 which was collected at } 208 \text { in from bottom of tank at } \\
\text { a } 150^{\circ} \text { angle and was keep heated to } 40^{\circ} \mathrm{C} \text {. }\end{array}$ \\
\hline$P$ & Raw & $\mathbf{P}$ & $3.30 E+06$ & $\# N / A$ & AP102-3 & $\mathbf{Y}$ & $\begin{array}{l}\text { Density reported for } \mathrm{G} 349 \text { collected at } 34 \text { in from bottom of } \\
\text { tank at a } 30^{\circ} \text { angle. Sample was unheated, unheated } \\
\text { samples precipitated }\end{array}$ \\
\hline$P$ & Raw & $P$ & $3.30 E+06$ & $\# N / A$ & AP102-3 & $Y$ & $\begin{array}{l}\text { Density not reported for } \mathrm{G} 453 \text {. No assumptions could be } \\
\text { made }\end{array}$ \\
\hline $\mathbf{P}$ & Raw & $P$ & $3.29 E+06$ & $\#$ N/A & AP102-3 & $Y$ & $\begin{array}{l}\text { Density not reported for } \mathrm{G} 333 \text {. No assumptions could be } \\
\text { made }\end{array}$ \\
\hline $\mathbf{P}$ & Raw & $\mathbf{P}$ & $3.25 E+06$ & $\# N / A$ & AP102-3 & $Y$ & $\begin{array}{l}\text { Density not reported for G333. No assumptions could be } \\
\text { made }\end{array}$ \\
\hline$P$ & Raw & $P$ & $3.25 E+06$ & $\# N / A$ & AP102-3 & $Y$ & $\begin{array}{l}\text { Density reported for G } 459 \text { collected at } 226 \text { in from bottom } \\
\text { of tank at a } 270^{\circ} \text { angle. Sample was unheated, unheated } \\
\text { samples precipitated }\end{array}$ \\
\hline$P$ & Raw & $\mathbf{P}$ & $3.19 E+06$ & $\# N / A$ & AP102-3 & $Y$ & $\begin{array}{l}\text { Density reported for } \mathrm{G} 459 \text { collected at } 226 \text { in from bottom } \\
\text { of tank at a } 270^{\circ} \text { angle. Sample was unheated, unheated } \\
\text { samples precipitated }\end{array}$ \\
\hline$P$ & Raw & $\mathbf{P}$ & $3.13 E+06$ & $\# N / A$ & AP102-3 & $Y$ & $\begin{array}{l}\text { Density reported for G460 collected at } 226 \text { in from bottom } \\
\text { of tank at a } 270^{\circ} \text { angle. Sample was unheated, unheated } \\
\text { samples precipitated }\end{array}$ \\
\hline$P$ & Raw & $\mathbf{P}$ & $3.12 E+06$ & $\#$ N/A & AP102-3 & $Y$ & $\begin{array}{l}\text { Density reported for G } 460 \text { collected at } 226 \text { in from bottom } \\
\text { of tank at a } 270^{\circ} \text { angle. Sample was unheated, unheated } \\
\text { samples precipitated }\end{array}$ \\
\hline$P$ & Raw & $\mathbf{P}$ & $3.08 E+06$ & $* N / A$ & AP102-3 & $Y$ & $\begin{array}{l}\text { Density not reported for G450. Density assumed same as } \\
\mathrm{G} 477 \text { which was collected at } 182 \text { in from bottom of tank at } \\
\text { a } 30^{\circ} \text { angle and was keep heated to } 40^{\circ} \mathrm{C} \text {. }\end{array}$ \\
\hline$P$ & Raw & $P$ & $3.08 E+06$ & $\#$ N/A & AP102-3 & $Y$ & $\begin{array}{l}\text { Density reported for G341 collected at } 99 \text { in from bottom of } \\
\text { tank at a } 270^{\circ} \text { angle. }\end{array}$ \\
\hline $\mathbf{P}$ & Raw & $P$ & $3.07 E+06$ & $\# N / A$ & AP102-3 & $Y$ & $\begin{array}{l}\text { Density reported for G342 collected at } 306 \text { in from bottom } \\
\text { of tank at a } 30^{\circ} \text { angle. Sample was unheated, unheated } \\
\text { samples precipitated }\end{array}$ \\
\hline
\end{tabular}




\begin{tabular}{|c|c|c|c|c|c|c|c|c|c|c|c|}
\hline & & & & & ank Wast & haracteriz & On Sample I & ormation & & & \\
\hline Reported Chemical & $\begin{array}{c}\text { Memo } \\
\text { Date }\end{array}$ & $\begin{array}{c}\text { Start } \\
\text { Analysis } \\
\text { Date }\end{array}$ & $\begin{array}{c}\text { Finish } \\
\text { Analysis Date }\end{array}$ & $\begin{array}{c}\text { Start } \\
\text { Sample } \\
\text { Date } \\
\end{array}$ & $\begin{array}{c}\text { Finish } \\
\text { Sample } \\
\text { Date } \\
\end{array}$ & $\begin{array}{l}\text { Sample } \\
\text { Number }\end{array}$ & $\begin{array}{c}\text { Tank Farm } \\
\text { Number }\end{array}$ & $\begin{array}{c}\text { Lab Sample } \\
\text { Number }\end{array}$ & $\begin{array}{l}\text { Density } \\
\text { (g/mL) }\end{array}$ & $\begin{array}{l}\text { Reported } \\
\text { Value }\end{array}$ & $\begin{array}{r}\text { Repor } \\
\text { Uni }\end{array}$ \\
\hline Phosphorus & $6 / 1 / 90$ & $4 / 30 / 89$ & $12 / 30 / 89$ & $4 / 27 / 89$ & $4 / 29 / 89$ & BOB2L9 & G305 & G349 & 1.20 & $3.07 E+06$ & $u g / h$ \\
\hline Phosphorus & $6 / 1 / 90$ & $4 / 30 / 89$ & $12 / 30 / 89$ & $4 / 27 / 89$ & $4 / 29 / 89$ & BOB2M8 & G301 & G449 & NA & $3.06 E+06$ & $u g h$ \\
\hline Phosphorus & $6 / 1 / 90$ & $4 / 30 / 89$ & $12 / 30 / 89$ & $4 / 27 / 89$ & $4 / 29 / 89$ & ВOB2M3 & G311 & G359 & 1.20 & $3.06 E+06$ & ugh \\
\hline Phosphorus & $6 / 1 / 90$ & $4 / 30 / 89$ & $12 / 30 / 89$ & $4 / 27 / 89$ & $4 / 29 / 89$ & BOB2N4 & G323 & G341 & 1.20 & $3.05 E+06$ & ug/l \\
\hline Phosphorus & $6 / 1 / 90$ & $4 / 30 / 89$ & $12 / 30 / 89$ & $4 / 27 / 89$ & $4 / 29 / 89$ & BOB2L8 & G303 & G348 & 1.21 & $3.03 E+06$ & $u g / h$ \\
\hline Phosphorus & $6 / 1 / 90$ & $4 / 30 / 89$ & $12 / 30 / 89$ & $4 / 27 / 89$ & $4 / 29 / 89$ & BOB2M3 & G311 & G359 & 1.20 & $3.01 E+06$ & $u g h$ \\
\hline Phosphorus & $6 / 1 / 90$ & $4 / 30 / 89$ & $12 / 30 / 89$ & $4 / 27 / 89$ & $4 / 29 / 89$ & BOB2L7 & G299 & G342 & 1.20 & $2.99 E+06$ & $u g h$ \\
\hline Phosphorus & $6 / 1 / 90$ & $4 / 30 / 89$ & $12 / 30 / 89$ & $4 / 27 / 89$ & $4 / 29 / 89$ & Вов2М9 & G302 & G450 & $(1.20)$ & $2.98 E+06$ & ug/h \\
\hline Phosphorus & $6 / 1 / 90$ & $4 / 30 / 89$ & $12 / 30 / 89$ & $4 / 27 / 89$ & $4 / 29 / 89$ & BOB2L8 & G303 & G348 & 1.21 & $2.96 E+06$ & ugh. \\
\hline Phosphorus & $6 / 1 / 90$ & $4 / 30 / 89$ & $12 / 30 / 89$ & $4 / 27 / 89$ & $4 / 29 / 89$ & BOB2M2 & G309 & G358 & 1.20 & $2.95 E+06$ & ug/L \\
\hline Phosphorus & $6 / 1 / 90$ & $4 / 30 / 89$ & $12 / 30 / 89$ & $4 / 27 / 89$ & $4 / 29 / 89$ & ВОВ2MO & G306 & G352 & 1.20 & $2.94 E+06$ & $u g / L$ \\
\hline Phasphorus & $6 / 1 / 90$ & $4 / 30 / 89$ & $12 / 30 / 89$ & $4 / 27 / 89$ & $4 / 29 / 89$ & ВОВ2M2 & G309 & G358 & 1.20 & $2.90 E+06$ & $u g / h$ \\
\hline Phosphorus & $6 / 1 / 90$ & $4 / 30 / 89$ & $12 / 30 / 89$ & $4 / 27 / 89$ & $4 / 29 / 89$ & BOB2M1 & G30B & G353 & 1.20 & $2.89 E+06$ & ug/L \\
\hline Phosphorus & $6 / 1 / 90$ & $4 / 30 / 89$ & $12 / 30 / 89$ & $4 / 27 / 89$ & $4 / 29 / 89$ & BOB2TS & G327 & G443 & 1.20 & $2.86 E+06$ & $u g / L$ \\
\hline Phosphorus & $6 / 1 / 90$ & $4 / 30 / 89$ & $12 / 30 / 89$ & $4 / 27 / 89$ & $4 / 29 / 89$ & BOB2T9 & G327 & G443 & 1.20 & $2.86 E+06$ & ug/L \\
\hline Phosphorus & $6 / 1 / 90$ & $4 / 30 / 89$ & $12 / 30 / 89$ & $4 / 27 / 89$ & $4 / 29 / 89$ & ВOB2M8 & G301 & G449 & NA & $2.81 E+06$ & $u g / L$ \\
\hline Phosphorus & $6 / 1 / 90$ & $4 / 30 / 89$ & $12 / 30 / 89$ & $4 / 27 / 89$ & $4 / 29 / 89$ & BOB2MO & G306 & G352 & 1.20 & $2.81 E+06$ & ug/h \\
\hline Phosphorus & $6 / 1 / 90$ & $4 / 30 / 89$ & $12 / 30 / 89$ & $4 / 27 / 89$ & $4 / 29 / 89$ & ВOB2M1 & G308 & G353 & 1.20 & $2.79 E+06$ & ug $\Omega$ \\
\hline Phosphorus & $6 / 1 / 90$ & $4 / 30 / 89$ & $12 / 30 / 89$ & $4 / 27 / 89$ & $4 / 29 / 89$ & BOB2M4 & G313 & G363 & 1.20 & $2.68 E+06$ & ug/L \\
\hline Phosphorus & $6 / 1 / 90$ & $4 / 30 / 89$ & $12 / 30 / 89$ & $4 / 27 / 89$ & $4 / 29 / 89$ & BOB2M5 & G315 & G364 & 1.19 & $2.68 E+06$ & ug/h \\
\hline Phosphorus & $6 / 1 / 90$ & $4 / 30 / 89$ & $12 / 30 / 89$ & $4 / 27 / 89$ & $4 / 29 / 89$ & $80 B 2 M 4$ & G313 & G363 & 1.20 & $2.59 E+06$ & $u g / 2$ \\
\hline
\end{tabular}




\begin{tabular}{|c|c|c|c|c|c|c|c|}
\hline \multirow[b]{2}{*}{ Code } & \multirow[b]{2}{*}{ Source } & \multicolumn{3}{|c|}{ Analyte Information } & \multirow[b]{2}{*}{$\begin{array}{c}\text { Reference } \\
\text { Number }\end{array}$} & \multirow[b]{2}{*}{$\begin{array}{c}\text { Validated } \\
(\mathrm{Y} / \mathrm{N})\end{array}$} & \multirow[b]{2}{*}{ Notes } \\
\hline & & Analyte & $\begin{array}{l}\text { Conc. } \\
\text { (ug/L) }\end{array}$ & Conc. (mol/L) & & & \\
\hline$P$ & Raw & $\mathbf{P}$ & $3.07 E+06$ & $\# N / A$ & AP102-3 & $Y$ & $\begin{array}{l}\text { Density reported for } \mathrm{G} 349 \text { collected at } 34 \text { in from bottom of } \\
\text { tank at a } 30^{\circ} \text { angle. Sample was unheated, unheated } \\
\text { samples precipitated }\end{array}$ \\
\hline$P$ & Raw & $\mathbf{P}$ & $3.06 \mathrm{E}+06$ & $\# N / A$ & AP102-3 & $\mathbf{Y}$ & $\begin{array}{l}\text { Density not reported for G449. No assumptions could be } \\
\text { made }\end{array}$ \\
\hline$P$ & Raw & $P$ & $3.06 E \div 06$ & $\# N / A$ & AP102-3 & $\mathbf{Y}$ & $\begin{array}{l}\text { Density reported for G359 collected at } 162 \text { in from bottom } \\
\text { of tank at a } 150^{\circ} \text { angle. Sample was unheated, unheated } \\
\text { samples precipitated }\end{array}$ \\
\hline$P$ & Raw & $\mathbf{P}$ & $3.05 E+06$ & $\# N / A$ & AP102-3 & $\mathbf{Y}$ & $\begin{array}{l}\text { Density reported for } G 341 \text { collected at } 99 \text { in from bottom of } \\
\text { tank at a } 270^{\circ} \text { angle. }\end{array}$ \\
\hline $\mathbf{P}$ & Raw & $P$ & $3.03 E+06$ & $\# N / A$ & AP102-3 & $Y$ & $\begin{array}{l}\text { Density reported for G348 collected at } 125 \text { in from bottom } \\
\text { of tank at a } 30^{\circ} \text { angle. Sample was unheated, unheated } \\
\text { samples precipitated }\end{array}$ \\
\hline $\mathbf{P}$ & Raw & $\mathbf{P}$ & $3.01 E+06$ & $\# N / A$ & AP102-3 & $Y$ & $\begin{array}{l}\text { Density reported for G359 collected at } 162 \text { in from bottom } \\
\text { of tank at a } 150^{\circ} \text { angle. Sample was unheated, unheated } \\
\text { samples precipitated }\end{array}$ \\
\hline $\mathbf{P}$ & Raw & $P$ & $2.99 E+06$ & $\# N / A$ & AP102-3 & $\gamma$ & $\begin{array}{l}\text { Density reported for } G 342 \text { collected at } 306 \text { in from bottom } \\
\text { of tank at a } 30^{\circ} \text { angle. Sample was unheated, unheated } \\
\text { samples precipitated }\end{array}$ \\
\hline $\mathbf{P}$ & Raw & $P$ & $2.98 E+06$ & $\# N / A$ & AP102-3 & $\mathbf{Y}$ & $\begin{array}{l}\text { Density not reported for } \mathrm{G} 450 \text {. Density assumed same as } \\
\mathrm{G} 477 \text { which was collected at } 182 \text { in from bottom of tank at } \\
\text { a } 30^{\circ} \text { angle and was keep heated to } 40^{\circ} \mathrm{C} \text {. }\end{array}$ \\
\hline $\mathbf{P}$ & Raw & $P$ & $2.96 E+06$ & $\# N / A$ & AP102-3 & $\mathbf{Y}$ & $\begin{array}{l}\text { Density reported for } G 348 \text { collected at } 125 \text { in from bottom } \\
\text { of tank at a } 30^{\circ} \text { angle. Sample was unheated, unheated } \\
\text { samples precipitated }\end{array}$ \\
\hline $\mathbf{P}$ & Raw & $P$ & $2.95 E+06$ & $\# N / A$ & AP102-3 & $\mathbf{Y}$ & $\begin{array}{l}\text { Density reported for G358 collected at } 334 \text { in from bottom } \\
\text { of tank at a } 150^{\circ} \text { angle. Sample was unheated, unheated } \\
\text { samples precipitated }\end{array}$ \\
\hline $\mathbf{P}$ & Raw & $P$ & $2.94 E+06$ & $\# N / A$ & AP102-3 & $\mathbf{Y}$ & $\begin{array}{l}\text { Density reported for } \mathrm{G} 352 \text { collected at } 34 \text { in from bottom of } \\
\text { tank at a } 30^{\circ} \text { angle. Sample was unheated, unheated } \\
\text { samples precipitated }\end{array}$ \\
\hline $\mathbf{P}$ & Raw & $\mathbf{P}$ & $2.90 E+06$ & $\# N / A$ & AP102-3 & $Y$ & $\begin{array}{l}\text { Density reported for } \mathrm{G} 358 \text { collected at } 334 \text { in from bottom } \\
\text { of tank at a } 150^{\circ} \text { angle. Sample was unheated, unheated } \\
\text { samples precipitated }\end{array}$ \\
\hline $\mathbf{P}$ & Raw & $P$ & $2.89 E+06$ & $\# N / A$ & AP102-3 & $\mathbf{Y}$ & $\begin{array}{l}\text { Density reported for G353 collected at } 334 \text { in from bottom } \\
\text { of tank at a } 150^{\circ} \text { angle. Sample was unheated, unheated } \\
\text { samples precipitated }\end{array}$ \\
\hline $\mathbf{P}$ & Raw & $\mathbf{P}$ & $2.86 E+06$ & $\# N / A$ & AP102-3 & $\gamma$ & Density reported for $\mathrm{G} 443$ represents a composite sample \\
\hline $\mathbf{P}$ & Raw & $P$ & $2.86 \mathrm{E}+06$ & $\# N / A$ & AP102-3 & Y. & Density reported for $\mathbf{G} 443$ represents a composite sample \\
\hline $\mathbf{P}$ & Raw & $P$ & $2.81 E+06$ & $\# N / A$ & AP102-3 & $Y$ & $\begin{array}{l}\text { Density not reported for G449. No assumptions could be } \\
\text { made }\end{array}$ \\
\hline $\mathbf{P}$ & Raw & $\mathbf{P}$ & $2.81 E+06$ & $\because N / A$ & AP102-3 & $Y$ & $\begin{array}{l}\text { Density reported for G352 collected at } 34 \text { in from bottom of } \\
\text { tank at a } 30^{\circ} \text { angle. Sample was unheated, unheated } \\
\text { samples precipitated }\end{array}$ \\
\hline$P$ & Raw & $\mathbf{P}$ & $2.79 E+06$ & $\#$ N/A & AP102-3 & $\mathbf{Y}$ & $\begin{array}{l}\text { Density reported for G353 collected at } 334 \text { in from bottom } \\
\text { of tank at a } 150^{\circ} \text { angle. Sample was unheated, unheated } \\
\text { samples precipitated }\end{array}$ \\
\hline $\mathbf{P}$ & Raw & $\mathbf{P}$ & $2.68 E+06$ & $\#$ \#/A & AP102-3 & $\gamma$ & $\begin{array}{l}\text { Density reported for G363 collected at } 18 \text { in from bottom of } \\
\text { tank at a } 150^{\circ} \text { angle. Sample was unheated, unheated } \\
\text { samples precipitated }\end{array}$ \\
\hline $\mathbf{P}$ & Raw & $\mathbf{P}$ & $2.68 E+06$ & \#N/A & AP102-3 & $Y$ & $\begin{array}{l}\text { Density reported for G364 collected at } 372 \text { in from bottom } \\
\text { of tank at a } 270^{\circ} \text { angle. Sample was unheated, unheated } \\
\text { samples precipitated }\end{array}$ \\
\hline P & Raw & $\mathbf{P}$ & $2.59 E+06$ & $\# N / A$ & AP102-3 & $\mathbf{Y}$ & $\begin{array}{l}\text { Density reported for G363 collected at } 18 \text { in from bottom of } \\
\text { tank at a } 150^{\circ} \text { angle. Sample was unheated, unheated } \\
\text { samples precipitated }\end{array}$ \\
\hline
\end{tabular}




\begin{tabular}{|c|c|c|c|c|c|c|c|c|c|c|c|}
\hline \multicolumn{12}{|c|}{ Tank Waste Characterization Sample Information } \\
\hline Reported Chemical & $\begin{array}{c}\text { Memo } \\
\text { Date }\end{array}$ & $\begin{array}{c}\text { Start } \\
\text { Analysis } \\
\text { Date } \\
\end{array}$ & $\begin{array}{c}\text { Finish } \\
\text { Analysis Date } \\
\end{array}$ & $\begin{array}{c}\text { Start } \\
\text { Sample } \\
\text { Date } \\
\end{array}$ & $\begin{array}{c}\text { Finish } \\
\text { Sample } \\
\text { Date } \\
\end{array}$ & $\begin{array}{l}\text { Sample } \\
\text { Number }\end{array}$ & $\begin{array}{c}\text { Tank Farm } \\
\text { Number }\end{array}$ & $\begin{array}{c}\text { Lab Sample } \\
\text { Number }\end{array}$ & $\begin{array}{l}\text { Density } \\
(\mathrm{g} / \mathrm{mL})\end{array}$ & $\begin{array}{c}\text { Reported } \\
\text { Value } \\
\end{array}$ & $\begin{array}{r}\text { Repoi } \\
\text { Unil }\end{array}$ \\
\hline Phosphorus & $6 / 1 / 90$ & $4 / 30 / 89$ & $12 / 30 / 89$ & $4 / 27 / 89$ & $4 / 29 / 89$ & BOB2M5 & G315 & G364 & 1.19 & $2.53 E+06$ & ugl \\
\hline Lead & $6 / 1 / 90$ & $4 / 30 / 89$ & $12 / 30 / 89$ & $4 / 27 / 89$ & $4 / 29 / 89$ & ВОВ2Т9 & G327 & G470* & 1.19 & $<6.20 E+0.4$ & ugh \\
\hline Lead & $6 / 1 / 90$ & $4 / 30 / 89$ & $12 / 30 / 89$ & $4 / 27 / 89$ & $4 / 29 / 89$ & ВОВ2Т9 & G327 & G470* & 1.19 & $<6.20 E+0$. & $u g n$ \\
\hline Lead & $6 / 1 / 90$ & $4 / 30 / 89$ & $12 / 30 / 89$ & $4 / 27 / 89$ & $4 / 29 / 89$ & BOB2N2 & G317 & G333 & NA & $<1.55 E+03$ & $u g h$ \\
\hline Lead & $6 / 1 / 90$ & $4 / 30 / 89$ & $12 / 30 / 89$ & $4 / 27 / 89$ & $4 / 29 / 89$ & $8082 N 3$ & G322 & G338 & NA & $<1.55 E+03$ & ugf \\
\hline Lead & $6 / 1 / 90$ & $4 / 30 / 89$ & $12 / 30 / 89$ & $4 / 27 / 89$ & $4 / 29 / 89$ & BOB2N4 & G323 & G341 & 1.20 & $<1.55 E+03$ & ug/ \\
\hline Lead & $6 / 1 / 90$ & $4 / 30 / 89$ & $12 / 30 / 89$ & $4 / 27 / 89$ & $4 / 29 / 89$ & BOB2L7 & G299 & G342 & 1.20 & $<1.55 E+03$ & ugh \\
\hline Lead & $6 / 1 / 90$ & $4 / 30 / 89$ & $12 / 30 / 89$ & $4 / 27 / 89$ & $4 / 29 / 89$ & BOB2L8 & G303 & 6348 & 1.21 & $<1.55 E+03$ & $u g / h$ \\
\hline Lead & $6 / 1 / 90$ & $4 / 30 / 89$ & $12 / 30 / 89$ & $4 / 27 / 89$ & $4 / 29 / 89$ & BOB2MO & G306 & G352 & 1.20 & $<1.55 E+03$ & ug/h \\
\hline Lead & $6,1 / 90$ & $4 / 30 / 89$ & $12 / 30 / 89$ & $4 / 27 / 89$ & $4 / 29 / 89$ & BOB2M1 & G308 & G353 & 1.20 & $<1.55 E+03$ & $u g / L$ \\
\hline Lead & $6 / 1 / 90$ & $4 / 30 / 89$ & $12 / 30 / 89$ & $4 / 27 / 89$ & $4 / 29 / 89$ & BOB2M2 & G309 & G358 & 1.20 & $<1.55 E+03$ & ught. \\
\hline Lead & $6 / 1 / 90$ & $4 / 30 / 89$ & $12 / 30 / 89$ & $4 / 27 / 89$ & $4 / 29 / 89$ & BOB2M3 & G311 & G359 & 1.20 & $<1.55 E+03$ & $u g / h$ \\
\hline Lead & $6 / 1 / 90$ & $4 / 30 / 89$ & $12 / 30 / 89$ & $4 / 27 / 89$ & $4 / 29 / 89$ & BOB2M4 & G313 & G363 & 1.20 & $<1.55 E+03$ & $u g h$ \\
\hline Lead & $6 / 1 / 90$ & $4 / 30 / 89$ & $12 / 30 / 89$ & $4 / 27 / 89$ & $4 / 29 / 89$ & BOB2M5 & 6315 & G364 & 1.19 & $<1.55 E+03$ & ug $\Omega$ \\
\hline Lead & $6 / 1 / 90$ & $4 / 30 / 89$ & $12 / 30 / 89$ & $4 / 27 / 89$ & $4 / 29 / 89$ & BOB2T9 & G327 & G443 & 1.20 & $<1.55 E+03$ & $u g / L$ \\
\hline Lead & $6 / 1 / 90$ & $4 / 30 / 89$ & $12 / 30 / 89$ & $4 / 27 / 89$ & $4 / 29 / 89$ & ВОВ2M8 & G301 & G449 & NA & $<1.55 E+03$ & $u g /$ \\
\hline Lead & $6 / 1 / 90$ & $4 / 30 / 89$ & $12 / 30 / 89$ & $4 / 27 / 89$ & $4 / 29 / 89$ & Вов2M9 & G302 & G450 & $(1.20)$ & $<1.55 E+03$ & ug/h \\
\hline Lead & $6 / 1 / 90$ & $4 / 30 / 89$ & $12 / 30 / 89$ & $4 / 27 / 89$ & $4 / 29 / 89$ & BOB2NO & G307 & G453 & NA & $<1.55 E+03$ & $u g h$ \\
\hline Lead & $6 / 1 / 90$ & $4 / 30 / 89$ & $12 / 30 / 89$ & $4 / 27 / 89$ & $4 / 29 / 89$ & BOB2M6 & G318 & G459 & 1.20 & $<1.55 E+03$ & $u g / h$ \\
\hline Lead & $6 / 1 / 90$ & $4 / 30 / 89$ & $12 / 30 / 89$ & $4 / 27 / 89$ & $4 / 29 / 89$ & BOB2M7 & G319 & G460 & 1.20 & $<1.55 E+03$ & $\omega g / L$ \\
\hline Lead & $6 / 1 / 90$ & $4 / 30 / 89$ & $12 / 30 / 89$ & $4 / 27 / 89$ & $4 / 29 / 89$ & $\mathrm{BOB} 2 \mathrm{~N} 2$ & G317 & G333 & NA & $<1.55 E+03$ & ug/h. \\
\hline Lead & $6 / 1 / 90$ & $4 / 30 / 89$ & $12 / 30 / 89$ & $4 / 27 / 89$ & $4 / 29 / 89$ & BOB2N3 & G322 & G338 & NA & $<1.55 E+03$ & $u g \Omega$ \\
\hline Lead & $6 / 1 / 90$ & $4 / 30 / 89$ & $12 / 30 / 89$ & $4 / 27 / 89$ & $4 / 29 / 89$ & BOB2N4 & G323 & G341 & 1.20 & $<1.55 E+03$ & ugh \\
\hline
\end{tabular}




\begin{tabular}{|c|c|c|c|c|c|c|c|}
\hline \multirow[b]{2}{*}{ Code } & \multirow[b]{2}{*}{ Source } & \multicolumn{3}{|c|}{ Analyte Information } & \multirow[b]{2}{*}{$\begin{array}{c}\text { Reference } \\
\text { Number }\end{array}$} & \multirow[b]{2}{*}{$\begin{array}{l}\text { Validated } \\
\text { (Y/N) }\end{array}$} & \multirow[b]{2}{*}{ Notes } \\
\hline & & Analyte & $\begin{array}{l}\text { Conc. } \\
\text { (ug/L) }\end{array}$ & Conc. (mol/L) & & & \\
\hline $\mathbf{P}$ & Raw & $\mathbf{P}$ & $2.53 E+06$ & $\#$ \#/A & AP102-3 & $\mathbf{Y}$ & $\begin{array}{l}\text { Density reported for } G 364 \text { collected at } 372 \text { in from bottom } \\
\text { of tank at a } 270^{\circ} \text { angle. Sample was unheated, unheated } \\
\text { samples precipitated }\end{array}$ \\
\hline $\mathbf{P}$ & Raw & $\mathrm{Pb}$ & \#ALUE! & \#ALUEI & AP102-3 & $\gamma$ & $\begin{array}{l}\text { Density reported for G470 represents a composite sample. } \\
\text { Sample was kept heated to } 40^{\circ} \mathrm{C} \text {. }\end{array}$ \\
\hline $\mathbf{P}$ & Raw & $\mathrm{Pb}$ & \#VALUE! & ¿VALUE! & AP102-3 & $Y$ & $\begin{array}{l}\text { Density reported for } \mathrm{G} 470 \text { represents a composite sample. } \\
\text { Sample was kept heated to } 40^{\circ} \mathrm{C} \text {. }\end{array}$ \\
\hline $\mathbf{P}$ & Raw & $\mathrm{Pb}$ & \#VALUE! & \#ALUE! & AP102-3 & $\gamma$ & $\begin{array}{l}\text { Density not reported for G333. No assumptions could be } \\
\text { made }\end{array}$ \\
\hline $\mathbf{P}$ & Raw & $\mathrm{Pb}$ & *VALUEI & \#ALUE! & AP102-3 & $Y$ & $\begin{array}{l}\text { Density not reported for G338. No assumptions could be } \\
\text { made }\end{array}$ \\
\hline $\mathbf{P}$ & Raw & $\mathrm{Pb}$ & \#ALUEI & \#VALUE! & AP102-3 & $\gamma$ & $\begin{array}{l}\text { Density reported for G341 collected at } 99 \text { in from bottom of } \\
\text { tank at a } 270^{\circ} \text { angle. }\end{array}$ \\
\hline $\mathbf{P}$ & Raw & $\mathrm{Pb}$ & \#VALUE! & \#VALUE! & AP102-3 & $\mathrm{Y}$ & $\begin{array}{l}\text { Density reported for } \mathrm{G} 342 \text { collected at } 306 \text { in from bottom } \\
\text { of tank at a } 30^{\circ} \text { angle. Sample was unheated, unheated } \\
\text { samples precipitated }\end{array}$ \\
\hline $\mathbf{P}$ & Raw & $\mathrm{Pb}$ & \#VALUE! & \#VALUE! & AP102-3 & $Y$ & $\begin{array}{l}\text { Density reported for } \mathrm{G} 348 \text { collected at } 125 \text { in from bottom } \\
\text { of tank at a } 30^{\circ} \text { angle. Sample was unheated, unheated } \\
\text { samples precipitated }\end{array}$ \\
\hline $\mathbf{P}$ & Raw & $\mathrm{Pb}$ & \#ALUE! & \#VALUE! & AP102-3 & $Y$ & $\begin{array}{l}\text { Density reported for } \mathrm{G} 352 \text { collected at } 34 \text { in from bottom of } \\
\text { tank at a } 30^{\circ} \text { angle. Sample was unheated, unheated } \\
\text { samples precipitated }\end{array}$ \\
\hline $\mathbf{P}$ & Raw & $\mathrm{Pb}$ & \#VALUE! & \#VALUE! & AP102-3 & $Y$ & $\begin{array}{l}\text { Density reported for } \mathrm{G} 353 \text { collected at } 334 \text { in from bottom } \\
\text { of tank at a } 150^{\circ} \text { angle. Sample was unheated, unheated } \\
\text { samples precipitated }\end{array}$ \\
\hline$P$ & Raw & $\mathrm{Pb}$ & \#VALUE! & \#VALUE! & AP102-3 & Y & $\begin{array}{l}\text { Density reported for G358 collected at } 334 \text { in from bottom } \\
\text { of tank at a } 150^{\circ} \text { angle. Sample was unheated, unheated } \\
\text { samples precipitated }\end{array}$ \\
\hline$P$ & Raw & $\mathrm{Pb}$ & \#VALUE! & \#VALUE! & AP102-3 & $Y$ & $\begin{array}{l}\text { Density reported for } \mathrm{G359} \text { collected at } 162 \text { in from bottom } \\
\text { of tank at a } 150^{\circ} \text { angle. Sample was unheated, unheated } \\
\text { samples precipitated }\end{array}$ \\
\hline$P$ & Raw & $\mathrm{Pb}$ & \$VALUE! & \#VALUE! & AP102-3 & $Y$ & $\begin{array}{l}\text { Density reported for G363 collected at } 18 \text { in from bottom of } \\
\text { tank at a } 150^{\circ} \text { angle. Sample was unheated, unheated } \\
\text { samples precipitated }\end{array}$ \\
\hline $\mathbf{P}$ & Raw & $\mathrm{Pb}$ & \#VALUE! & \#VALUE! & AP102-3 & $Y$ & $\begin{array}{l}\text { Density reported for G364 collected at } 372 \text { in from bottom } \\
\text { of tank at a } 270^{\circ} \text { angle. Sample was unheated, unheated } \\
\text { samples precipitated }\end{array}$ \\
\hline $\mathbf{P}$ & Raw & $\mathrm{Pb}$ & \#VALUE! & \#VALUE! & AP102-3 & $Y$ & Density reported for $\mathrm{G} 443$ represents a composite sample \\
\hline $\mathbf{P}$ & Raw & $\mathrm{Pb}$ & \#VALUE! & *VALUEl & AP102-3 & $Y$ & $\begin{array}{l}\text { Density not reported for G449. No assumptions could be } \\
\text { made }\end{array}$ \\
\hline $\mathbf{P}$ & Raw & $\mathrm{Pb}$ & \#VALUE! & \#ALUE! & AP102-3 & Y & $\begin{array}{l}\text { Density not reported for G450. Density assumed same as } \\
\text { G477 which was collected at } 182 \text { in from bottom of tank at } \\
\text { a } 30^{\circ} \text { angle and was keep heated to } 40^{\circ} \mathrm{C} \text {. }\end{array}$ \\
\hline $\mathbf{P}$ & Raw & $\mathrm{Pb}$ & \#VALUE! & *VALUEI & AP102-3 & $\gamma$ & $\begin{array}{l}\text { Density not reported for G453. No assumptions could be } \\
\text { made }\end{array}$ \\
\hline $\mathbf{P}$ & Raw & $\mathrm{Pb}$ & \#VALUE! & \#VALUE! & AP102-3 & $Y$ & $\begin{array}{l}\text { Density reported for G } 459 \text { collected at } 226 \text { in from bottom } \\
\text { of tank at a } 270^{\circ} \text { angle. Sample was unheated, unheated } \\
\text { samples precipitated }\end{array}$ \\
\hline $\mathbf{P}$ & Raw & $\mathrm{Pb}$ & \#ALUE! & FaLUE! & AP102-3 & Y & $\begin{array}{l}\text { Density reported for G460 collected at } 226 \text { in from bottom } \\
\text { of tank at a } 270^{\circ} \text { angle. Sample was unheated, unheated } \\
\text { samples precipitated }\end{array}$ \\
\hline $\mathbf{P}$ & Raw & $\mathrm{Pb}$ & \#VALUEI & \#VALUEI & AP102-3 & $\gamma$ & $\begin{array}{l}\text { Density not reported for G333. No assumptions could be } \\
\text { made }\end{array}$ \\
\hline $\mathbf{P}$ & Raw & $\mathrm{Pb}$ & \#VALUE! & \#ALUE! & AP102-3 & $\gamma$ & $\begin{array}{l}\text { Density not reported for G338. No assumptions could be } \\
\text { made }\end{array}$ \\
\hline $\mathbf{P}$ & Raw & $\mathrm{Pb}$ & ALUE! & \#VALUEI & AP102-3 & $Y$ & $\begin{array}{l}\text { Density reported for } G 341 \text { collected at } 99 \text { in from bottom of } \\
\text { tank at a } 270^{\circ} \text { angle. }\end{array}$ \\
\hline
\end{tabular}


TWRS Privatization Support Pro Chemical Liquid.

\begin{tabular}{|c|c|c|c|c|c|c|c|c|c|c|c|}
\hline & & & & & ank Waste & haracteriza & ion Sample I & ormation & & & \\
\hline Reported Chemical & $\begin{array}{c}\text { Memo } \\
\text { Date }\end{array}$ & $\begin{array}{c}\text { Start } \\
\text { Analysis } \\
\text { Date }\end{array}$ & $\begin{array}{c}\text { Finish } \\
\text { Analysis Date }\end{array}$ & $\begin{array}{c}\text { Start } \\
\text { Sample } \\
\text { Date }\end{array}$ & $\begin{array}{c}\text { Finish } \\
\text { Sample } \\
\text { Date } \\
\end{array}$ & $\begin{array}{l}\text { Sample } \\
\text { Number }\end{array}$ & $\begin{array}{c}\text { Tank Farm } \\
\text { Number }\end{array}$ & $\begin{array}{c}\text { Lab Sample } \\
\text { Number }\end{array}$ & $\begin{array}{l}\text { Density } \\
(\mathrm{g} / \mathrm{mL})\end{array}$ & $\begin{array}{c}\text { Reported } \\
\text { Value }\end{array}$ & $\begin{array}{r}\text { Repo } \\
\text { Un }\end{array}$ \\
\hline Lead & $6 / 1 / 90$ & $4 / 30 / 89$ & $12 / 30 / 89$ & $4 / 27 / 89$ & $4 / 29 / 89$ & $\mathrm{BOB} 2 \mathrm{L7}$ & G299 & G342 & 1.20 & $<1.55 E+03$ & ug. \\
\hline Lead & $6 / 1 / 90$ & $4 / 30 / 89$ & $12 / 30 / 89$ & $4 / 27 / 89$ & $4 / 29 / 89$ & B082L8 & G303 & G348 & 1.21 & $<1.55 E+03$ & ugt \\
\hline Lead & $6 / 1 / 90$ & $4 / 30 / 89$ & $12 / 30 / 89$ & $4 / 27 / 89$ & $4 / 29 / 89$ & BOB2L9 & G305 & G349 & 1.20 & $<1.55 E+03$ & ugi \\
\hline Lead & $6 / 1 / 90$ & $4 / 30 / 89$ & $12 / 30 / 89$ & $4 / 27 / 89$ & $4 / 29 / 89$ & ВОВ2МO & G306 & G352 & 1.20 & $<7.55 \varepsilon+03$ & $\mathbf{u g l}$ \\
\hline Lead & $6 / 1 / 90$ & $4 / 30 / 89$ & $12 / 30 / 89$ & $4 / 27 / 89$ & $4 / 29 / 89$ & BOB2M1 & G308 & G353 & 1.20 & $<1.55 E+03$ & $u g /$. \\
\hline Lead & $6 / 1 / 90$ & $4 / 30 / 89$ & $12 / 30 / 89$ & $4 / 27 / 89$ & $4 / 29 / 89$ & ВОВ2M2 & G309 & G358 & 1.20 & $<1.55 E+03$ & ug $/ 1$ \\
\hline Lead & $6 / 1 / 90$ & $4 / 30 / 89$ & $12 / 30 / 89$ & $4 / 27 / 89$ & $4 / 29 / 89$ & В082M4 & G313 & G363 & 1.20 & $<1.55 E+03$ & $u g h$ \\
\hline Lead & $6 / 1 / 90$ & $4 / 30 / 89$ & $12 / 30 / 89$ & $4 / 27 / 89$ & $4 / 29 / 89$ & Вов2T9 & G327 & G443 & 1.20 & $<1.55 E+03$ & $\operatorname{ug} n$ \\
\hline Lead & $6 / 1 / 90$ & $4 / 30 / 89$ & $12 / 30 / 89$ & $4 / 27 / 89$ & $4 / 29 / 89$ & вОВ2M8 & G301 & G449 & NA & $<1.55 E+03$ & $\mathrm{ug} \lambda$ \\
\hline Lead & $6 / 1 / 90$ & $4 / 30 / 89$ & $12 / 30 / 89$ & $4 / 27 / 89$ & $4 / 29 / 89$ & вов2М9 & G302 & G450 & $(1.20)$ & $<1.55 E+03$ & ug/h \\
\hline Lead & $6 / 1 / 90$ & $4 / 30 / 89$ & $12 / 30 / 89$ & $4 / 27 / 89$ & $4 / 29 / 89$ & BOB2NO & G307 & G453 & NA & $<1.55 E+03$ & ugh \\
\hline Lead & $6 / 1 / 90$ & $4 / 30 / 89$ & $12 / 30 / 89$ & $4 / 27 / 89$ & $4 / 29 / 89$ & BOB2N1 & G310 & G454 & (1.19) & $<1.55 E+03$ & $u g / h$ \\
\hline Lead & $6 / 1 / 90$ & $4 / 30 / 89$ & $12 / 30 / 89$ & $4 / 27 / 89$ & $4 / 29 / 89$ & В0В2M6 & G318 & G459 & 1.20 & $<1.55 E+03$ & $u g / L$ \\
\hline Lead & $6 / 1 / 90$ & $4 / 30 / 89$ & $12 / 30 / 89$ & $4 / 27 / 89$ & $4 / 29 / 89$ & BOB2M7 & G319 & G460 & 1.20 & $<1.55 E+03$ & $u g h$ \\
\hline $\mathrm{Pb} 2+$ & NS & NS & NS & NS & NS & NS & NS & NS & 1.24 & $4.00 \mathrm{E}-05$ & $\overline{\text { Mole/ }}$ \\
\hline Lead & $4 / 1 / 93$ & NS & NS & NS & NS & BOB2T9 & G327 & NS & NS & $1.55 E+03$ & $U G /$ \\
\hline Lead & $4 / 1 / 93$ & NS & NS & NS & NS & BOB2T9 & G327 & NS & NS & $1.55 E+03$ & $U G M$ \\
\hline Lead & $6 / 1 / 90$ & $4 / 30 / 89$ & $12 / 30 / 89$ & $4 / 27 / 89$ & $4 / 29 / 89$ & 80B2M5 & G315 & G364 & 1.19 & $5.33 E+03$ & ug/L \\
\hline Lead & $6 / 1 / 90$ & $4 / 30 / 89$ & $12 / 30 / 89$ & $4 / 27 / 89$ & $4 / 29 / 89$ & BOB2M3 & G311 & G359 & 1.20 & $3.22 E+03$ & ug $/ \mathrm{h}$ \\
\hline Lead & $6 / 1 / 90$ & $4 / 30 / 89$ & $12 / 30 / 89$ & $4 / 27 / 89$ & $4 / 29 / 89$ & BOB2L9 & G305 & G349 & 1.20 & $2.64 E+03$ & ugh \\
\hline Lead & $6 / 1 / 90$ & $4 / 30 / 89$ & $12 / 30 / 89$ & $4 / 27 / 89$ & $4 / 29 / 89$ & BOB2N1 & G310 & G454 & (1.19) & $1.95 E+03$ & ug $/ \mathrm{L}$ \\
\hline PO4 & $4 / 28 / 84$ & NS & NS & NS & NS & R8469 & NS & NS & NS & $1.56 \mathrm{E}-01$ & $M$ \\
\hline $\mathrm{PO4}$ & $4 / 28 / 84$ & NS & NS & NS & NS & R-8469 & NS & NS & NS & $1.56 \mathrm{E}-01$ & moles, \\
\hline P04- & $4 / 28 / 84$ & NS & NS & NS & NS & NS & NS & NS & NS & $1.48 E+01$ & g/L \\
\hline P043. & NS & NS & NS & NS & NS & NS & NS & NS & 1.24 & $1.09 \mathrm{E}-01$ & Mole/ \\
\hline
\end{tabular}




\begin{tabular}{|c|c|c|c|c|c|c|c|}
\hline \multirow[b]{2}{*}{ Code } & \multirow[b]{2}{*}{ Source } & \multicolumn{3}{|c|}{ Analyte Information } & \multirow[b]{2}{*}{$\begin{array}{l}\text { Reference } \\
\text { Number }\end{array}$} & \multirow[b]{2}{*}{$\begin{array}{c}\text { Validated } \\
(\mathrm{Y} / \mathrm{N})\end{array}$} & \multirow[b]{2}{*}{ Notes } \\
\hline & & Analyte & $\begin{array}{l}\text { Conc. } \\
\text { (ug/L) }\end{array}$ & Conc. (mol/L) & & & \\
\hline $\mathbf{P}$ & Raw & $\mathrm{Pb}$ & \#VALUE! & \#VALUE! & AP102-3 & $\mathbf{Y}$ & $\begin{array}{l}\text { Density reported for } \mathrm{G} 342 \text { collected at } 306 \text { in from bottom } \\
\text { of tank at a } 30^{\circ} \text { angle. Sample was unheated, unheated } \\
\text { samples precipitated }\end{array}$ \\
\hline $\mathbf{P}$ & Raw & $\mathrm{Pb}$ & \#VALUE! & \#VALUE! & AP102-3 & $Y$ & $\begin{array}{l}\text { Density reported for G348 collected at } 125 \text { in from bottom } \\
\text { of tank at a } 30^{\circ} \text { angle. Sample was unheated, unheated } \\
\text { samples precipitated }\end{array}$ \\
\hline $\mathbf{P}$ & Raw & $\mathrm{Pb}$ & \#VALUE! & \#VALUE! & AP102-3 & $\mathbf{Y}$ & $\begin{array}{l}\text { Density reported for } \mathbf{G} 349 \text { collected at } 34 \text { in from bottom of } \\
\text { tank at a } 30^{\circ} \text { angle. Sample was unheated, unheated } \\
\text { samples precipitated }\end{array}$ \\
\hline $\mathbf{P}$ & Raw & $\mathrm{Pb}$ & \#VALUEI & \#VALUEI & AP102-3 & $\mathbf{Y}$ & $\begin{array}{l}\text { Density reported for } \mathrm{G} 352 \text { collected at } 34 \text { in from bottom of } \\
\text { tank at a } 30^{\circ} \text { angle. Sample was unheated, unheated } \\
\text { samples precipitated }\end{array}$ \\
\hline $\mathbf{P}$ & Raw & $\mathrm{Pb}$ & \#ALUE! & \#VALUE! & AP102-3 & $\mathbf{Y}$ & $\begin{array}{l}\text { Density reported for G353 collected at } 334 \text { in from bottom } \\
\text { of tank at a } 150^{\circ} \text { angle. Sample was unheated, unheated } \\
\text { samples precipitated }\end{array}$ \\
\hline $\mathbf{P}$ & Raw & $\mathrm{Pb}$ & \#VALUE! & \#VALUE! & AP102-3 & $\mathbf{Y}$ & $\begin{array}{l}\text { Density reported for G358 collected at } 334 \text { in from bottom } \\
\text { of tank at a } 150^{\circ} \text { angle. Sample was unheated, unheated } \\
\text { samples precipitated }\end{array}$ \\
\hline $\mathbf{P}$ & Raw & $\mathrm{Pb}$ & \#ALUE! & \#VAlue! & AP102-3 & $\mathbf{Y}$ & $\begin{array}{l}\text { Density reported for G363 collected at } 18 \text { in from bottom of } \\
\text { tank at a } 150^{\circ} \text { angle. Sample was unheated, unheated } \\
\text { samples precipitated }\end{array}$ \\
\hline $\mathbf{P}$ & Raw & $\mathrm{Pb}$ & \#VALUE! & \#VALUE! & AP102-3 & $\gamma$ & Density reported for $\mathrm{G} 443$ represents a composite sample \\
\hline $\mathbf{P}$ & Raw & $\mathrm{Pb}$ & \#VALUE! & \#VALUE! & AP102-3 & $\mathbf{Y}$ & $\begin{array}{l}\text { Density not reported for G449. No assumptions could be } \\
\text { made }\end{array}$ \\
\hline $\mathbf{P}$ & Raw & $\mathrm{Pb}$ & \#VALUE! & \#VALUEI & AP102-3 & $\mathbf{Y}$ & $\begin{array}{l}\text { Density not reported for G450. Density assumed same as } \\
\mathrm{G} 477 \text { which was collected at } 182 \text { in from bottom of tank at } \\
\text { a } 30^{\circ} \text { angle and was keep heated to } 40^{\circ} \mathrm{C} \text {. }\end{array}$ \\
\hline$P$ & Raw & $\mathrm{Pb}$ & \#VALUE! & \#VALUE! & AP102-3 & $\mathrm{Y}$ & $\begin{array}{l}\text { Density not reported for G453. No assumptions could be } \\
\text { made }\end{array}$ \\
\hline $\mathbf{P}$ & Raw & $\mathrm{Pb}$ & \#VALUE! & \#VALUE! & AP102-3 & $\mathbf{Y}$ & $\begin{array}{l}\text { Density not reported for G454. Density assumed same as } \\
\text { G471 which was collected at } 208 \text { in from bottom of tank at } \\
\text { a } 150^{\circ} \text { angle and was keep heated to } 40^{\circ} \mathrm{C} \text {. }\end{array}$ \\
\hline $\mathbf{p}$ & Raw & $\mathrm{Pb}$ & \#VALUE! & \#ALUE! & AP102-3 & $\mathbf{Y}$ & $\begin{array}{l}\text { Density reported for G } 459 \text { collected at } 226 \text { in from bottom } \\
\text { of tank at a } 270^{\circ} \text { angle. Sample was unheated, unheated } \\
\text { samples precipitated }\end{array}$ \\
\hline$P$ & Raw & $\mathrm{Pb}$ & *VALUE! & *VALUEI & AP102-3 & $\gamma$ & $\begin{array}{l}\text { Density reported for G } 460 \text { collected at } 226 \text { in from bottom } \\
\text { of tank at a } 270^{\circ} \text { angle. Sample was unheated, unheated } \\
\text { samples precipitated }\end{array}$ \\
\hline$\underline{L}$ & HTCE Inv. & $\mathrm{Pb}$ & $8.29 E+09$ & $4.00 E+01$ & NA & $\mathbf{N}$ & No Reference \\
\hline $\mathbf{P}$ & TCD & $\mathbf{P b}$ & $1.55 E+09$ & $7.48 E+\infty$ & & $\mathbf{N}$ & THIS POINT NOT GIVEN IN REPORT WHC-SD-WM-TRP-169 \\
\hline $\bar{P}$ & TCD & $\mathrm{Pb}$ & $1.55 E+09$ & $7.48 E+00$ & & $\bar{N}$ & THIS POINT NOT GIVEN IN REPORT WHC-SD-WM-TRP-169 \\
\hline $\mathbf{P}$ & Raw & $\mathbf{P b}$ & $5.33 E+03$ & 2.57E-05 & AP102-3 & $\mathbf{Y}$ & $\begin{array}{l}\text { Density reported for G364 collected at } 372 \text { in from bottom } \\
\text { of tank at a } 270^{\circ} \text { angle. Sample was unheated, unheated } \\
\text { samples precipitated }\end{array}$ \\
\hline $\mathbf{P}$ & Raw & $\mathrm{Pb}$ & $3.22 E+03$ & 1.55E-05 & AP102-3 & $\mathbf{Y}$ & $\begin{array}{l}\text { Density reported for G359 collected at } 162 \text { in from bottom } \\
\text { of tank at a } 150^{\circ} \text { angle. Sample was unheated, unheated } \\
\text { samples precipitated }\end{array}$ \\
\hline $\mathbf{P}$ & Raw & Pb & $2.64 E+03$ & $1.27 \mathrm{E}-05$ & AP102-3 & $\mathbf{Y}$ & $\begin{array}{l}\text { Density reported for G349 collected at } 34 \text { in from bottom of } \\
\text { tank at a } 30^{\circ} \text { angle. Sample was unheated, unheated } \\
\text { samples precipitated }\end{array}$ \\
\hline $\mathbf{P}$ & Raw & $\mathrm{Pb}$ & $1.95 E+03$ & $9.41 E-06$ & AP102-3 & $Y$ & $\begin{array}{l}\text { Density not reported for G454. Density assumed same as } \\
\text { G471 which was collected at } 208 \text { in from bottom of tank at } \\
\text { a } 150^{\circ} \text { angle and was keep heated to } 40^{\circ} \mathrm{C} \text {. }\end{array}$ \\
\hline$L$ & Raw & P04 & $1.48 E+13$ & $1.56 E+05$ & AP102-1 & $\mathbf{N}$ & Outdated Information \\
\hline$P$ & Braun & P04 & $1.48 E+13$ & $1.56 \mathrm{E}+05$ & & $\mathbf{N}$ & No Reference \\
\hline $\mathbf{P}$ & Wastren & PO4 & $1.48 \mathrm{E}+13$ & $1.56 \mathrm{E}+05$ & NS & $N$ & No Reference \\
\hline L & HTCE Inv. & PO4 & $1.04 E+13$ & $1.09 E+05$ & $\overline{N A}$ & $\mathrm{~N}$ & No Reference \\
\hline
\end{tabular}


TWRS Privatization Support Pro] Chemical Liquids

\begin{tabular}{|c|c|c|c|c|c|c|c|c|c|c|c|}
\hline & & & & & ank Waste & haracteriza & Oon Sample I & ormation & & & \\
\hline Reported Chemical & $\begin{array}{c}\text { Memo } \\
\text { Date }\end{array}$ & $\begin{array}{c}\text { Start } \\
\text { Analysis } \\
\text { Date }\end{array}$ & $\begin{array}{c}\begin{array}{c}\text { Finish } \\
\text { Analysis Date }\end{array} \\
\end{array}$ & $\begin{array}{c}\text { Start } \\
\text { Sample } \\
\text { Date } \\
\end{array}$ & $\begin{array}{c}\text { Finish } \\
\text { Sample } \\
\text { Date } \\
\end{array}$ & $\begin{array}{l}\text { Sample } \\
\text { Number }\end{array}$ & $\begin{array}{c}\text { Tank Farm } \\
\text { Number }\end{array}$ & $\begin{array}{c}\text { Lab Sample } \\
\text { Number }\end{array}$ & $\begin{array}{l}\text { Density } \\
(\mathrm{g} / \mathrm{mL})\end{array}$ & $\begin{array}{c}\text { Reported } \\
\text { Value } \\
\end{array}$ & $\begin{array}{r}\text { Repor } \\
\text { Uni } \\
\end{array}$ \\
\hline Phosphate & $6 / 1 / 90$ & $4 / 30 / 89$ & $12 / 30 / 89$ & $4 / 27 / 89$ & $4 / 29 / 89$ & ВОВ2T9 & G327 & $6470^{*}$ & 1.19 & $1.37 E+04$ & $u g / \pi$ \\
\hline Phosphate & $6 / 1 / 90$ & $4 / 30 / 89$ & $12 / 30 / 89$ & $4 / 27 / 89$ & $4 / 29 / 89$ & BOB2T9 & G327 & G443 & 1.20 & $1.33 E+04$ & $u g / m$ \\
\hline Phosphate & $6 / 1 / 90$ & $4 / 30 / 89$ & $12 / 30 / 89$ & $4 / 27 / 89$ & $4 / 29 / 89$ & ВОВ2T9 & G327 & G443 & 1.20 & $1.32 E+0.4$ & $\mathrm{ug} / \mathrm{m}$ \\
\hline Phosphate & $6 / 1 / 90$ & $4 / 30 / 89$ & $12 / 30 / 89$ & $4 / 27 / 89$ & $4 / 29 / 89$ & BOB2M9 & G302 & G477* & 1.20 & $1.29 E+04$ & $\mathrm{ug} / \mathrm{m}$ \\
\hline Phosphate & $6 / 1 / 90$ & $4 / 30 / 89$ & $12 / 30 / 89$ & $4 / 27 / 89$ & $4 / 29 / 89$ & 80B2T9 & G327 & G470* & 1.19 & $1.29 E+04$ & $\mathrm{ug} / \mathrm{m}$ \\
\hline Phosphate & $6 / 1 / 90$ & $4 / 30 / 89$ & $12 / 30 / 89$ & $4 / 27 / 89$ & $4 / 29 / 89$ & BOB2N1 & G310 & G471* & 1.19 & $1.28 E+04$ & $u g / m$ \\
\hline Phosphate & $6 / 1 / 90$ & $4 / 30 / 89$ & $12 / 30 / 89$ & $4 / 27 / 89$ & $4 / 29 / 89$ & BOB2N4 & G323 & G476* & 1.21 & $1.28 E+04$ & $\mathrm{ug} / \mathrm{m}$ \\
\hline Phosphate & $6 / 1 / 90$ & $4 / 30 / 89$ & $12 / 30 / 89$ & $4 / 27 / 89$ & $4 / 29 / 89$ & BOB2M1 & G308 & G522* & $(1.20)$ & $1.24 E+04$ & $\mathrm{ug} / \mathrm{m}$ \\
\hline Phosphate & $6 / 1 / 90$ & $4 / 30 / 89$ & $12 / 30 / 89$ & $4 / 27 / 89$ & $4 / 29 / 89$ & BOB2M1 & G308 & G522* & $(1.20)$ & $1.24 E+04$ & $\mathrm{ug} / \mathrm{m}$ \\
\hline Phosphate & $6 / 1 / 90$ & $4 / 30 / 89$ & $12 / 30 / 89$ & $4 / 27 / 89$ & $4 / 29 / 89$ & BOB2NO & G307 & G527* & NA & $1.24 E+04$ & $\mathrm{ug} / \mathrm{m}^{\prime}$ \\
\hline Phosphate & $6 / 1 / 90$ & $4 / 30 / 89$ & $12 / 30 / 89$ & $4 / 27 / 89$ & $4 / 29 / 89$ & ВОВ2M2 & 6309 & G515* & $(1.20)$ & $1.23 E+04$ & $\mathrm{ug} / \mathrm{m}$ \\
\hline Phosphate & $6 / 1 / 90$ & $4 / 30 / 89$ & $12 / 30 / 89$ & $4 / 27 / 89$ & $4 / 29 / 89$ & вов2мо & G306 & G521* & $(1.20)$ & $1.23 E+04$ & $\mathrm{ug} / \mathrm{ml}$ \\
\hline Phosphate & $6 / 1 / 90$ & $4 / 30 / 89$ & $12 / 30 / 89$ & $4 / 27 / 89$ & $4 / 29 / 89$ & BOB2L7 & G299 & G509* & $(1.20)$ & $1.22 E+04$ & $u_{g} / \mathrm{ml}$ \\
\hline Phosphate & $6 / 1 / 90$ & $4 / 30 / 89$ & $12 / 30 / 89$ & $4 / 27 / 89$ & $4 / 29 / 89$ & Вов2M4 & G313 & G533* & $(1.20)$ & $1.22 E+04$ & $\mathrm{ug} / \mathrm{ml}$ \\
\hline Phosphate & $6 / 1 / 90$ & $4 / 30 / 89$ & $12 / 30 / 89$ & $4 / 27 / 89$ & $4 / 29 / 89$ & вов2M4 & G313 & G533* & $(1.20)$ & $1.22 E+04$ & $\mathrm{ug} / \mathrm{ml}$ \\
\hline Phosphate & $6 / 1 / 90$ & $4 / 30 / 89$ & $12 / 30 / 89$ & $4 / 27 / 89$ & $4 / 29 / 89$ & ВОВ2M3 & G311 & G516* & $(1.20)$ & $1.21 E+04$ & $\mathrm{ug} / \mathrm{ml}$ \\
\hline Phosphate & $6 / 1 / 90$ & $4 / 30 / 89$ & $12 / 30 / 89$ & $4 / 27 / 89$ & $4 / 29 / 89$ & ВОВ2M3 & G311 & G516* & $(1.20)$ & $1.20 \varepsilon+04$ & ugiml \\
\hline Phosphate & $6 / 1 / 90$ & $4 / 30 / 89$ & $12 / 30 / 89$ & $4 / 27 / 89$ & $4 / 29 / 89$ & $8082 \mathrm{MB}$ & G311 & G516" & $(1.20)$ & $1.20 E+04$ & $\mathrm{ug} / \mathrm{mL}$ \\
\hline Phosphate & $6 / 1 / 90$ & $4 / 30 / 89$ & $12 / 30 / 89$ & $4 / 27 / 89$ & $4 / 29 / 89$ & 8OB2MO & G306 & G521* & $(1.20)$ & $1.20 E+0.4$ & $\mathrm{ug} / \mathrm{mL}$ \\
\hline Phosphate & $6 / 1 / 90$ & $4 / 30 / 89$ & $12 / 30 / 89$ & $4 / 27 / 89$ & $4 / 29 / 89$ & BOB2M5 & G315 & G534* & (1.19) & $1.20 E+04$ & $\mathrm{ug} / \mathrm{mL}$ \\
\hline Phosphate & $6 / 1 / 90$ & $4 / 30 / 89$ & $12 / 30 / 89$ & $4 / 27 / 89$ & $4 / 29 / 89$ & BOB2N1 & G310 & $\mathrm{G} 471^{\circ}$ & 1.19 & $1.19 E+04$ & $\mathrm{ug} / \mathrm{mL}$ \\
\hline
\end{tabular}




\begin{tabular}{|c|c|c|c|c|c|c|c|}
\hline \multirow[b]{2}{*}{ Code } & \multirow[b]{2}{*}{ Source } & \multicolumn{3}{|c|}{ Analyte Information } & \multirow[b]{2}{*}{$\begin{array}{l}\text { Reference } \\
\text { Number }\end{array}$} & \multirow[b]{2}{*}{$\begin{array}{l}\text { Validated } \\
\text { (Y/N) }\end{array}$} & \multirow[b]{2}{*}{ Notes } \\
\hline & & Analyte & $\begin{array}{l}\text { Conc. } \\
\text { (ug/L) }\end{array}$ & Conc. (mol/L) & & & \\
\hline $\mathbf{P}$ & Raw & PO4 & $1.37 E+07$ & $1.44 E-01$ & AP102-3 & $\mathbf{Y}$ & $\begin{array}{l}\text { Density reported for } \mathrm{G} 470 \text { represents a composite sample. } \\
\text { Sample was kept heated to } 40^{\circ} \mathrm{C} \text {. }\end{array}$ \\
\hline $\mathbf{P}$ & Raw & PO4 & $1.33 E+07$ & $1.40 E-01$ & AP102-3 & $\mathbf{Y}$ & Density reported for $\mathrm{G} 443$ represents a composite sample \\
\hline $\mathbf{P}$ & Raw & PO4 & $1.32 \mathrm{E}+07$ & $1.39 E-01$ & AP102-3 & $\mathbf{Y}$ & Density reported for $\mathrm{G} 443$ represents a composite sample \\
\hline $\boldsymbol{P}$ & Raw & $\mathrm{PO} 4$ & $1.29 E+07$ & $1.36 E-01$ & AP102-3 & $\mathbf{Y}$ & $\begin{array}{l}\text { Density reported for } \mathrm{G} 477 \text { collected at } 182 \text { in from bottom } \\
\text { of tank at a } 30^{\circ} \text { angle and was keep heated to } 40^{\circ} \mathrm{C} \text {. }\end{array}$ \\
\hline $\mathbf{P}$ & Raw & PO4 & $1.29 E+07$ & $1.36 E-01$ & AP102-3 & $\mathbf{Y}$ & $\begin{array}{l}\text { Density reported for } \mathrm{G} 470 \text { represents a composite sample. } \\
\text { Sample was kept heated to } 40^{\circ} \mathrm{C} \text {. }\end{array}$ \\
\hline $\mathbf{P}$ & Raw & PO4 & $1.28 E+07$ & $1.35 E-01$ & AP102-3 & $\mathbf{Y}$ & $\begin{array}{l}\text { Density reported for } \mathrm{G} 471 \text { collected at } 208 \text { in from bottom } \\
\text { of tank at a } 150^{\circ} \text { angle and was keep heated to } 40^{\circ} \mathrm{C} \text {. }\end{array}$ \\
\hline $\mathbf{P}$ & Raw & PO4 & $1.28 E+07$ & 1.35E-01 & AP102-3 & $\mathbf{Y}$ & $\begin{array}{l}\text { Density reported for } \mathrm{G} 476 \text { collected at } 99 \text { in from bottom of } \\
\text { tank at a } 270^{\circ} \text { angle and was keep heated to } 40^{\circ} \mathrm{C} \text {. }\end{array}$ \\
\hline $\mathbf{P}$ & Raw & PO4 & $1.24 E+07$ & $1.31 E-01$ & AP102-3 & $\mathbf{Y}$ & $\begin{array}{l}\text { Density not reported for G522. Density assumed same as } \\
\text { G353 which was collected at } 334 \text { in from bottom of tank at } \\
\text { a } 150^{\circ} \text { angle. This sample was heated. }\end{array}$ \\
\hline $\mathbf{P}$ & Raw & PO4 & $1.24 E+07$ & 1.31E-01 & AP102-3 & $\mathbf{Y}$ & $\begin{array}{l}\text { Density not reported for G522. Density assumed same as } \\
\text { G353 which was collected at } 334 \text { in from bottom of tank at } \\
\text { a } 150^{\circ} \text { angle. This sample was heated. }\end{array}$ \\
\hline $\mathbf{P}$ & Raw & PO4 & $1.24 E+07$ & $1.31 E-01$ & AP102-3 & $\mathbf{Y}$ & $\begin{array}{l}\text { Density not reported for } \mathbf{6 5 2 7} \text {. No assumptions could be } \\
\text { made }\end{array}$ \\
\hline $\mathbf{P}$ & Raw & PO4 & $1.23 E+07$ & $1.30 E-01$ & AP102-3 & $\mathbf{Y}$ & $\begin{array}{l}\text { Density not reported for G515. Density assumed same as } \\
\text { G358 which was collected at } 334 \text { in from bottom of tank at } \\
\text { a } 150^{\circ} \text { angle. This sample was heated. }\end{array}$ \\
\hline$P$ & Raw & 804 & $1.23 E+07$ & $1.30 E-01$ & AP102-3 & $Y$ & $\begin{array}{l}\text { Density not reported for G521. Density assumed same as } \\
\text { G352 which was collected at } 34 \text { in from bottom of tank at a } \\
30^{\circ} \text { angle. This sample was heated. }\end{array}$ \\
\hline$P$ & Raw & PO4 & $1.22 E+07$ & $1.28 \mathrm{E}-01$ & AP102-3 & $\mathbf{Y}$ & $\begin{array}{l}\text { Density not reported for } \mathrm{G509} \text {. Density assumed same as } \\
\mathrm{G} 342 \text { which was collected at } 306 \text { in from bottom of tank at } \\
\text { a } 30^{\circ} \text { angle. This sample was heated. }\end{array}$ \\
\hline $\mathbf{P}$ & Raw & PO4 & $1.22 E+07$ & $1.28 E-01$ & AP102-3 & $Y$ & $\begin{array}{l}\text { Density not reported for G533. Density assumed same as } \\
\text { G363 which was collected at } 18 \text { in from bottom of tank at a } \\
150^{\circ} \text { angle. This sample was heated. }\end{array}$ \\
\hline $\mathbf{P}$ & Raw & $\mathrm{PO4}$ & $1.22 E+07$ & $1.28 E-01$ & AP102-3 & $Y$ & $\begin{array}{l}\text { Density not reported for G533. Density assumed same as } \\
\text { G363 which was collected at } 18 \text { in from bottom of tank at a } \\
150^{\circ} \text { angle. This sample was heated. }\end{array}$ \\
\hline $\mathbf{P}$ & Raw & PO4 & $1.21 E+07$ & 1.27E-01 & AP102-3 & $\mathbf{Y}$ & $\begin{array}{l}\text { Density not reported for G516. Density assumed same as } \\
\text { G359 which was collected at } 162 \text { in from bottom of tank at } \\
\text { a } 150^{\circ} \text { angle. This sample was heated. }\end{array}$ \\
\hline $\mathbf{P}$ & Raw & PO4 & $1.20 E+07$ & $1.26 E-01$ & AP102-3 & $\mathbf{Y}$ & $\begin{array}{l}\text { Density not reported for G516. Density assumed same as } \\
\text { G359 which was collected at } 162 \text { in trom bottom of tank at } \\
\text { a } 150^{\circ} \text { angle. This sample was heated. }\end{array}$ \\
\hline$P$ & Raw & PO4 & $1.20 E+07$ & $1.26 \mathrm{E}-01$ & AP102-3 & $\mathbf{Y}$ & $\begin{array}{l}\text { Density not reported for G516. Density assumed same as } \\
\text { G359 which was collected at } 162 \text { in from bottom of tank at } \\
\text { a } 150^{\circ} \text { angle. This sample was heated. }\end{array}$ \\
\hline$P$ & Raw & PO4 & $1.20 E+07$ & $1.26 \mathrm{E}-01$ & AP102-3 & $Y$ & $\begin{array}{l}\text { Density not reported for G521. Density assumed same as } \\
\text { G352 which was collected at } 34 \text { in from bottom of tank at a } \\
30^{\circ} \text { angle. This sample was heated. }\end{array}$ \\
\hline $\mathbf{P}$ & Raw & PO4 & $1.20 E+07$ & $1.26 \mathrm{E}-01$ & AP102-3 & $Y$ & $\begin{array}{l}\text { Density not reported for G534. Density assumed same as } \\
\text { G364 which was collected at } 372 \text { in from bottom of tank at } \\
\text { a } 270^{\circ} \text { angle. This sample was heated. }\end{array}$ \\
\hline $\mathbf{P}$ & Raw & PO4 & $1.19 E+07$ & $1.25 E-01$ & AP102-3 & $\mathbf{Y}$ & $\begin{array}{l}\text { Density reported for G } 471 \text { collected at } 208 \text { in from bottom } \\
\text { of tank at a } 150^{\circ} \text { angle and was keep heated to } 40^{\circ} \mathrm{C} \text {. }\end{array}$ \\
\hline
\end{tabular}




\begin{tabular}{|c|c|c|c|c|c|c|c|c|c|c|c|}
\hline & & & & & ank Waste & haracteriz: & on Sample 1 & ormation & & & \\
\hline Reported Chemical & $\begin{array}{c}\text { Memo } \\
\text { Date }\end{array}$ & $\begin{array}{c}\text { Start } \\
\text { Analysis } \\
\text { Date }\end{array}$ & $\begin{array}{c}\text { Finish } \\
\text { Analysis Date }\end{array}$ & $\begin{array}{c}\text { Start } \\
\text { Sample } \\
\text { Date }\end{array}$ & $\begin{array}{c}\text { Finish } \\
\text { Sample } \\
\text { Date } \\
\end{array}$ & $\begin{array}{l}\text { Sample } \\
\text { Number }\end{array}$ & $\begin{array}{c}\text { Tank Farm } \\
\text { Number }\end{array}$ & $\begin{array}{c}\text { Lab Sample } \\
\text { Number }\end{array}$ & $\begin{array}{l}\text { Density } \\
(\mathrm{g} / \mathrm{mL})\end{array}$ & $\begin{array}{c}\text { Reported } \\
\text { Value }\end{array}$ & $\begin{array}{r}\text { Repor } \\
\text { Unit }\end{array}$ \\
\hline Phosphate & $6 / 1 / 90$ & $4 / 30 / 89$ & $12 / 30 / 89$ & $4 / 27 / 89$ & $4 / 29 / 89$ & BOB2N4 & G323 & G476* & 1.21 & $1.19 E+04$ & $u g / \pi$ \\
\hline Phosphate & $6 / 1 / 90$ & $4 / 30 / 89$ & $12 / 30 / 89$ & $4 / 27 / 89$ & $4 / 29 / 89$ & ВОВ2M7 & G319 & G536* & $(1.20)$ & $1.19 E+04$ & $u g / n$ \\
\hline Phosphate & $6 / 1 / 90$ & $4 / 30 / 89$ & $12 / 30 / 89$ & $4 / 27 / 89$ & $4 / 29 / 89$ & ВОВ2M7 & G319 & G460 & 1.2 & $1.19 E+04$ & $u g / \pi$ \\
\hline Phosphate & $6 / 1 / 90$ & $4 / 30 / 89$ & $12 / 30 / 89$ & $4 / 27 / 89$ & $4 / 29 / 89$ & BOB2L7 & G299 & G509* & $(1.20)$ & $1.19 E+04$ & $\mathrm{ug} / \mathrm{m}$ \\
\hline Phosphate & $6 / 1 / 90$ & $4 / 30 / 89$ & $12 / 30 / 89$ & $4 / 27 / 89$ & $4 / 29 / 89$ & во82M7 & G319 & G460 & 1.2 & $1.18 E+04$ & ug/m \\
\hline Phosphate & $6 / 1 / 90$ & $4 / 30 / 89$ & $12 / 30 / 89$ & $4 / 27 / 89$ & $4 / 29 / 89$ & BOB2L8 & G303 & G510* & 1.21 & $1.18 E+04$ & $\mathrm{ug} / \mathrm{m}$ \\
\hline Phosphate & $6 / 1 / 90$ & $4 / 30 / 89$ & $12 / 30 / 89$ & $4 / 27 / 89$ & $4 / 29 / 89$ & В082M5 & G315 & G534* & $(1.19)$ & $1.18 E+04$ & $\mathrm{ug} / \mathrm{m}$ \\
\hline Phosphate & $6 / 1 / 90$ & $4 / 30 / 89$ & $12 / 30 / 89$ & $4 / 27 / 89$ & $4 / 29 / 89$ & Вов2М7 & G319 & G536* & $(1.20)$ & $1.18 E+04$ & $\mathrm{ug} / \mathrm{mi}$ \\
\hline Phosphate & $6 / 1 / 90$ & $4 / 30 / 89$ & $12 / 30 / 89$ & $4 / 27 / 89$ & $4 / 29 / 89$ & Вов2М6 & G318 & G517* & $(1.20)$ & $1.16 E+04$ & $\mathrm{ug} / \mathrm{ml}$ \\
\hline Phosphate & $6 / 1 / 90$ & $4 / 30 / 89$ & $12 / 30 / 89$ & $4 / 27 / 89$ & $4 / 29 / 89$ & ВОВ2M8 & G301 & G523" & NA & $1.16 E+04$ & $\mathrm{ug} / \mathrm{mt}$ \\
\hline Phosphate & $6 / 1 / 90$ & $4 / 30 / 89$ & $12 / 30 / 89$ & $4 / 27 / 89$ & $4 / 29 / 89$ & 80B2M8 & G301 & G523* & NA & $1.15 E+04$ & ug/ml \\
\hline Phosphate & $6 / 1 / 90$ & $4 / 30 / 89$ & $12 / 30 / 89$ & $4 / 27 / 89$ & $4 / 29 / 89$ & BOB2M2 & G309 & G515* & $(1.20)$ & $1.15 E+04$ & $\mathrm{ug} / \mathrm{mL}$ \\
\hline Phosphate & $6 / 1 / 90$ & $4 / 30 / 89$ & $12 / 30 / 89$ & $4 / 27 / 89$ & $4 / 29 / 89$ & Вов2M9 & $\mathrm{G} 302$ & G477* & 1.20 & $1.14 E+04$ & $u g / m L$ \\
\hline Phosphate & $6 / 1 / 90$ & $4 / 30 / 89$ & $12 / 30 / 89$ & $4 / 27 / 89$ & $4 / 29 / 89$ & BOB2M6 & G318 & G517* & $(1.20)$ & $1.14 E+04$ & $\mathrm{ug} / \mathrm{mL}$ \\
\hline Phosphate & $6 / 1 / 90$ & $4 / 30 / 89$ & $12 / 30 / 89$ & $4 / 27 / 89$ & $4 / 29 / 89$ & BOB2N3 & G322 & G529: & NA & $1.13 E+04$ & $\mathrm{ug} / \mathrm{mL}$ \\
\hline Phosphate & $6 / 1 / 90$ & $4 / 30 / 89$ & $12 / 30 / 89$ & $4 / 27 / 89$ & $4 / 29 / 89$ & BOB2L9 & G305 & G511* & $(1.20)$ & $1.13 E+04$ & $u g / \mathrm{mL}$ \\
\hline Phosphate & $6 / 1 / 90$ & $4 / 30 / 89$ & $12 / 30 / 89$ & $4 / 27 / 89$ & $4 / 29 / 89$ & BOB2N3 & G322 & G529. & NA & $1.13 E+04$ & $\mathrm{ug} / \mathrm{mL}$ \\
\hline Phosphate & $6 / 1 / 90$ & $4 / 30 / 89$ & $12 / 30 / 89$ & $4 / 27 / 89$ & $4 / 29 / 89$ & BOB2L9 & G305 & G511* & $(1.20)$ & $1.11 E+04$ & $u g / m L$ \\
\hline Phosphate & $6 / 1 / 90$ & $4 / 30 / 89$ & $12 / 30 / 89$ & $4 / 27 / 89$ & $4 / 29 / 89$ & BOB2N2 & G317 & G528* & NA & $1.10 E+04$ & $\mathrm{ug} / \mathrm{mL}$ \\
\hline Phosphate & $6 / 1 / 90$ & $4 / 30 / 89$ & $12 / 30 / 89$ & $4 / 27 / 89$ & $4 / 29 / 89$ & $80 B 2 M 9$ & G302 & G477* & 1.20 & $1.07 E+04$ & ug $/ \mathrm{mL}$ \\
\hline Phosphate & $6 / 1 / 90$ & $4 / 30 / 89$ & $12 / 30 / 89$ & $4 / 27 / 89$ & $4 / 29 / 89$ & BOB2L.8 & G303 & G510* & 1.21 & $1.07 E+04$ & $\mathrm{ug} / \mathrm{mL}$ \\
\hline
\end{tabular}




\begin{tabular}{|c|c|c|c|c|c|c|c|}
\hline \multirow[b]{2}{*}{ Code } & \multirow[b]{2}{*}{ Source } & \multicolumn{3}{|c|}{ Analyte Information } & \multirow[b]{2}{*}{$\begin{array}{c}\text { Reference } \\
\text { Number }\end{array}$} & \multirow[b]{2}{*}{$\begin{array}{l}\text { Validated } \\
\text { (Y/N) }\end{array}$} & \multirow[b]{2}{*}{ Notes } \\
\hline & & Analyte & $\begin{array}{l}\text { Cone. } \\
\text { (ug/L) }\end{array}$ & Conc. (mol/L) & & & \\
\hline $\mathbf{P}$ & Raw & PO4 & $1.19 E+07$ & $1.25 \mathrm{E}-01$ & AP102-3 & $\mathbf{Y}$ & $\begin{array}{l}\text { Density reported for } G 476 \text { collected at } 99 \text { in from bottom of } \\
\text { tank at a } 270^{\circ} \text { angle and was keep heated to } 40^{\circ} \mathrm{C} \text {. }\end{array}$ \\
\hline $\mathbf{P}$ & Raw & PO4 & $1.19 E+07$ & $1.25 \mathrm{E}-01$ & AP102-3 & $Y$ & $\begin{array}{l}\text { Density not reported for G536. Density assumed same as } \\
G 460 \text { which was collected at } 226 \text { in from bottom of tank at } \\
\text { a } 270^{\circ} \text { angle. This sample was heated. }\end{array}$ \\
\hline $\mathbf{P}$ & Raw & PO4 & $1.19 E+07$ & $1.25 E-01$ & AP102-3 & $\mathbf{Y}$ & $\begin{array}{l}\text { Density reported for } 6460 \text { collected at } 226 \text { in from bottom } \\
\text { of tank at a } 270^{\circ} \text { angle. Sample was unheated, unheated } \\
\text { samples precipitated }\end{array}$ \\
\hline $\mathbf{P}$ & Raw & PO4 & $1.19 E+07$ & $1.25 \mathrm{E}-01$ & AP102-3 & $Y$ & $\begin{array}{l}\text { Density not reported for G509. Density assumed same as } \\
G 342 \text { which was collected at } 306 \text { in from bottom of tank at } \\
\text { a } 30^{\circ} \text { angle. This sample was heated. }\end{array}$ \\
\hline $\mathbf{P}$ & Raw & PO4 & $1.18 E+07$ & $1.24 E-01$ & AP102-3 & $Y$ & $\begin{array}{l}\text { Density reported for G460 collected at } 226 \text { in from bottom } \\
\text { of tank at a } 270^{\circ} \text { angle. Sample was unheated, unheated } \\
\text { samples precipitated }\end{array}$ \\
\hline $\mathbf{P}$ & Raw & PO4 & $1.18 \mathrm{E}+07$ & $1.24 E-01$ & AP102-3 & $\mathrm{Y}$ & $\begin{array}{l}\text { Density not reported for G510. Density assumed same as } \\
G 348 \text { which was collected at } 125 \text { in from bottom of tank at } \\
\text { a } 30^{\circ} \text { angle. This sample was heated. }\end{array}$ \\
\hline $\mathbf{P}$ & Raw & PO4 & $1.18 \mathrm{E}+07$ & $1.24 \mathrm{E}-01$ & AP102-3 & $\gamma$ & $\begin{array}{l}\text { Density not reported for } G 534 \text {. Density assumed same as } \\
6364 \text { which was collected at } 372 \text { in from bottom of tank at } \\
\text { a } 270^{\circ} \text { angle. This sample was heated. }\end{array}$ \\
\hline $\mathbf{P}$ & Raw & PO4 & $1.18 E+07$ & $1.24 E-01$ & AP102-3 & $Y$ & $\begin{array}{l}\text { Density not reported for G536. Density assumed same as } \\
\text { G460 which was collected at } 226 \text { in from bortom of tank at } \\
\text { a } 270^{\circ} \text { angle. This sample was heated. }\end{array}$ \\
\hline $\mathbf{P}$ & Raw & PO4 & $1.16 E+07$ & $1.22 E-01$ & AP102-3 & $Y$ & $\begin{array}{l}\text { Density not reported for G517. Density assumed same as } \\
G 459 \text { which was collected at } 226 \text { in from bottom of tank at } \\
\text { a } 270^{\circ} \text { angle. This sample was heated. }\end{array}$ \\
\hline $\mathbf{P}$ & Raw & PO4 & $1.16 \mathrm{E}+07$ & $1.22 E-01$ & AP102-3 & $Y$ & $\begin{array}{l}\text { Density not reported for G523. No assumptions could be } \\
\text { made }\end{array}$ \\
\hline $\mathbf{P}$ & Raw & PO4 & $1.15 E+07$ & $1.21 E-01$ & AP102-3 & $Y$ & $\begin{array}{l}\text { Density not reported for G523. No assumptions could be } \\
\text { made }\end{array}$ \\
\hline $\mathbf{P}$ & Raw & PO4 & $1.15 \mathrm{E}+07$ & $1.21 E-01$ & AP102-3 & $Y$ & $\begin{array}{l}\text { Density not reported for G515. Density assumed same as } \\
\text { G358 which was collected at } 334 \text { in from bottom of tank at } \\
\text { a } 150^{\circ} \text { angle. This sample was heated. }\end{array}$ \\
\hline $\mathbf{P}$ & Raw & $\mathrm{PO} 4$ & $1.14 E+07$ & 1.20E-01 & AP102-3 & $Y$ & $\begin{array}{l}\text { Density reported for } \mathrm{G} 477 \text { collected at } 182 \text { in from bottom } \\
\text { of tank at a } 30^{\circ} \text { angle and was keep heated to } 40^{\circ} \mathrm{C} \text {. }\end{array}$ \\
\hline $\mathbf{P}$ & Raw & PO4 & $1.14 E+07$ & $1.20 \mathrm{E}-01$ & AP102-3 & $Y$ & $\begin{array}{l}\text { Density not reported for G517. Density assumed same as } \\
\text { G459 which was collected at } 226 \text { in from bottom of tank at } \\
\text { a } 270^{\circ} \text { angle. This sample was heated. }\end{array}$ \\
\hline $\mathbf{P}$ & Raw & PO4 & $1.13 E+07$ & $1.19 E-01$ & AP102-3 & $\gamma$ & $\begin{array}{l}\text { Density not reported for G529. No assumptions could be } \\
\text { made }\end{array}$ \\
\hline $\mathbf{P}$ & Raw & PO4 & $1.13 E+07$ & 1.19E-01 & AP102-3 & $Y$ & $\begin{array}{l}\text { Density not reported for G511. Density assumed same as } \\
\text { G349 which was collected at } 34 \text { in from bottom of tank at a } \\
30^{\circ} \text { angle. This sample was heated. }\end{array}$ \\
\hline $\mathbf{P}$ & Raw & PO4 & $1.13 E+07$ & 1.19E-0t & AP102-3 & $Y$ & $\begin{array}{l}\text { Density not reported for G529. No assumptions could be } \\
\text { made }\end{array}$ \\
\hline $\mathbf{P}$ & Raw & PO4 & $1.11 E+07$ & $1.17 \mathrm{E}-01$ & AP102-3 & $Y$ & $\begin{array}{l}\text { Density not reported for G511. Density assumed same as } \\
\text { G349 which was collected at } 34 \text { in from bottom of tank at a } \\
30^{\circ} \text { angle. This sample was heated. }\end{array}$ \\
\hline$P$ & Raw & PO4 & $1.10 E+07$ & 1.16E-01 & AP102-3 & $Y$ & $\begin{array}{l}\text { Density not reported for G528. No assumptions could be } \\
\text { made }\end{array}$ \\
\hline $\mathbf{P}$ & Raw & PO4 & $1.07 E \div 07$ & 1.13E-01 & AP102-3 & $Y$ & $\begin{array}{l}\text { Density reported for G477 collected at } 182 \text { in from bottom } \\
\text { of tank at a } 30^{\circ} \text { angle and was keep heated to } 40^{\circ} \mathrm{C} \text {. }\end{array}$ \\
\hline$P$ & Raw & PO4 & $1.07 E+07$ & $1.13 \mathrm{E}-01$ & AP102-3 & $Y$ & $\begin{array}{l}\text { Density not reported for G510. Density assumed same as } \\
\text { G348 which was collected at } 125 \text { in from bottom of tank at } \\
\text { a } 30^{\circ} \text { angle. This sample was heated. }\end{array}$ \\
\hline
\end{tabular}




\begin{tabular}{|c|c|c|c|c|c|c|c|c|c|c|c|}
\hline & & & & & ank Waste & haracteriza & Ion Sample In & ormation & & & \\
\hline Reported Chemicat & $\begin{array}{l}\text { Memo } \\
\text { Date }\end{array}$ & $\begin{array}{c}\text { Start } \\
\text { Analysis } \\
\text { Date }\end{array}$ & $\begin{array}{c}\text { Finish } \\
\text { Analysis Date }\end{array}$ & $\begin{array}{c}\text { Start } \\
\text { Sample } \\
\text { Date } \\
\end{array}$ & $\begin{array}{c}\text { Finish } \\
\text { Sample } \\
\text { Date } \\
\end{array}$ & $\begin{array}{l}\text { Sample } \\
\text { Number }\end{array}$ & $\begin{array}{c}\text { Tank Farm } \\
\text { Number }\end{array}$ & $\begin{array}{c}\text { Lab Sample } \\
\text { Number }\end{array}$ & $\begin{array}{l}\text { Density } \\
\text { (g/mL) }\end{array}$ & $\begin{array}{c}\text { Reported } \\
\text { Value } \\
\end{array}$ & $\begin{array}{r}\text { Rep } \\
\text { U } \\
\end{array}$ \\
\hline Phosphate & $6 / 1 / 90$ & $4 / 30 / 89$ & $12 / 30 / 89$ & $4 / 27 / 89$ & $4 / 29 / 89$ & вов2М9 & G302 & G477* & 1.20 & $1.06 E+04$ & ug. \\
\hline Phosphate & $6 / 1 / 90$ & $4 / 30 / 89$ & $12 / 30 / 89$ & $4 / 27 / 89$ & $4 / 29 / 89$ & BOB2N2 & G317 & G528* & NA & $1.05 E+04$ & ugI \\
\hline Phosphate & $6 / 1 / 90$ & $4 / 30 / 89$ & $12 / 30 / 89$ & $4 / 27 / 89$ & $4 / 29 / 89$ & BOB2L.9 & G305 & G349 & 1.20 & $1.04 E+04$ & ugl \\
\hline Phosphate & $6 / 1 / 90$ & $4 / 30 / 89$ & $12 / 30 / 89$ & $4 / 27 / 89$ & $4 / 29 / 89$ & BOB2L9 & G305 & G349 & 1.20 & $1.04 E+04$ & ug $\prime$ \\
\hline Phosphate & $6 / 1 / 90$ & $4 / 30 / 89$ & $12 / 30 / 89$ & $4 / 27 / 89$ & $4 / 29 / 89$ & $80 B 2 M 3$ & G311 & G516* & $(1.20)$ & $1.03 E+04$ & ugt \\
\hline Phosphate & $6 / 1 / 90$ & $4 / 30 / 89$ & $12 / 30 / 89$ & $4 / 27 / 89$ & $4 / 29 / 89$ & BOB2NO & G307 & G527* & NA & $1.02 E+04$ & ugls \\
\hline Phosphate & $6 / 1 / 90$ & $4 / 30 / 89$ & $12 / 30 / 89$ & $4 / 27 / 89$ & $4 / 29 / 89$ & BOB2N2 & G317 & G333 & NA & $9.95 E+03$ & $u g h$ \\
\hline Phosphate & $6 / 1 / 90$ & $4 / 30 / 89$ & $12 / 30 / 89$ & $4 / 27 / 89$ & $4 / 29 / 89$ & BOB2N1 & G310 & G454 & $(1.19)$ & $9.90 E+03$ & $u g / r$ \\
\hline Phosphate & $6 / 1 / 90$ & $4 / 30 / 89$ & $12 / 30 / 89$ & $4 / 27 / 89$ & $4 / 29 / 89$ & BOB2N1 & G310 & G454 & (1.19) & $9.86 E+03$ & $u g / k$ \\
\hline Phosphate & $6 / 1 / 90$ & $4 / 30 / 89$ & $12 / 30 / 89$ & $4 / 27 / 89$ & $4 / 29 / 89$ & BOB2N2 & G317 & G333 & NA & $9.69 E+03$ & $u g / r$ \\
\hline Phosphate & $6 / 1 / 90$ & $4 / 30 / 89$ & $12 / 30 / 89$ & $4 / 27 / 89$ & $4 / 29 / 89$ & BOB2N3 & G322 & G338 & NA & $9.65 E+03$ & $u g / r$ \\
\hline Phosphate & $6 / 1 / 90$ & $4 / 30 / 89$ & $12 / 30 / 89$ & $4 / 27 / 89$ & $4 / 29 / 89$ & BOB2NO & G307 & G453 & NA & $9.60 E+03$ & $u g / r$ \\
\hline Phosphate & $6 / 1 / 90$ & $4 / 30 / 89$ & $12 / 30 / 89$ & $4 / 27 / 89$ & $4 / 29 / 89$ & BOB2N3 & G322 & G338 & NA & $9.51 E+03$ & ug/n \\
\hline Phosphate & $6 / 1 / 90$ & $4 / 30 / 89$ & $12 / 30 / 89$ & $4 / 27 / 89$ & $4 / 29 / 89$ & BOB2NO & G307 & G453 & NA & $9.39 E+03$ & $u g / n$ \\
\hline Phosphate & $6 / 1 / 90$ & $4 / 30 / 89$ & $12 / 30 / 89$ & $4 / 27 / 89$ & $4 / 29 / 89$ & BOB2M6 & G318 & G459 & 1.20 & $9.34 E+03$ & $u g / \pi$ \\
\hline Phosphate & $6 / 1 / 90$ & $4 / 30 / 89$ & $12 / 30 / 89$ & $4 / 27 / 89$ & 4/29/89 & вов2M8 & G301 & G449 & NA & $9.30 E+03$ & $u g / r$ \\
\hline Phosphate & $6 / 1 / 90$ & $4 / 30 / 89$ & $12 / 30 / 89$ & $4 / 27 / 89$ & $4 / 29 / 89$ & ВоB2M6 & G318 & G459 & 1.20 & $9.26 \mathrm{E}+03$ & $u g / \pi$ \\
\hline Phosphate & $6 / 1 / 90$ & $4 / 30 / 89$ & $12 / 30 / 89$ & $4 / 27 / 89$ & $4 / 29 / 89$ & Вов2М9 & G302 & G450 & $(1.20)$ & $9.22 E+03$ & $u g / \pi$ \\
\hline Phosphate & $6 / 1 / 90$ & $4 / 30 / 89$ & $12 / 30 / 89$ & $4 / 27 / 89$ & $4 / 29 / 89$ & ВОВ2M8 & G301 & G449 & NA & $9.20 E+03$ & $\mathrm{ug} / \mathrm{m}$ \\
\hline Phosphate & $6 / 1 / 90$ & $4 / 30 / 89$ & $12 / 30 / 89$ & $4 / 27 / 89$ & $4 / 29 / 89$ & ВОВ2M9 & G302 & G450 & $(1.20)$ & $9.19 E+03$ & $\mathrm{ug} / \mathrm{m}$ \\
\hline Phosphate & $6 / 1 / 90$ & $4 / 30 / 89$ & $12 / 30 / 89$ & $4 / 27 / 89$ & $4 / 29 / 89$ & BOB2M5 & G315 & G364 & 1.19 & $9.19 E+03$ & $u g / m$ \\
\hline Phosphate & $6 / 1 / 90$ & $4 / 30 / 89$ & $12 / 30 / 89$ & $4 / 27 / 89$ & $4 / 29 / 89$ & ВОВ2M2 & G309 & G358 & 1.20 & $9.15 E+03$ & $\mathrm{ug} / \mathrm{m}$ \\
\hline
\end{tabular}




\begin{tabular}{|c|c|c|c|c|c|c|c|}
\hline \multirow[b]{2}{*}{ Code } & \multirow[b]{2}{*}{ Source } & \multicolumn{3}{|c|}{ Analyte Information } & \multirow[b]{2}{*}{$\begin{array}{l}\text { Reference } \\
\text { Number }\end{array}$} & \multirow[b]{2}{*}{$\begin{array}{l}\text { Validated } \\
\text { (Y/N) }\end{array}$} & \multirow[b]{2}{*}{ Notes } \\
\hline & & Analyte & $\begin{array}{l}\text { Conc. } \\
\text { (ug/L) }\end{array}$ & Conc. (mol/L) & & & \\
\hline $\mathbf{P}$ & Raw & 804 & $1.06 E+07$ & $1.12 E-01$ & AP102-3 & $\mathbf{Y}$ & $\begin{array}{l}\text { Density reported for } \mathrm{G} 477 \text { collected at } 182 \text { in from bottom } \\
\text { of tank at a } 30^{\circ} \text { angle and was keep heated to } 40^{\circ} \mathrm{C} \text {. }\end{array}$ \\
\hline $\mathbf{P}$ & Raw & $\mathrm{PO} 4$ & $1.05 E+07$ & $1.11 E-01$ & AP102-3 & $Y$ & $\begin{array}{l}\text { Density not reported for G528. No assumptions could be } \\
\text { made }\end{array}$ \\
\hline $\mathbf{P}$ & Raw & PO4 & $1.04 E+07$ & 1.10E-01 & AP102-3 & $Y$ & $\begin{array}{l}\text { Density reported for } \mathrm{G} 349 \text { collected at } 34 \text { in from bottom of } \\
\text { tank at a } 30^{\circ} \text { angle. Sample was unheated, unheated } \\
\text { samples precipitated }\end{array}$ \\
\hline $\mathbf{P}$ & Raw & PO4 & $1.04 E+07$ & $1.10 E-01$ & AP102-3 & $\mathbf{Y}$ & $\begin{array}{l}\text { Density reported for G349 collected at } 34 \text { in from bottom of } \\
\text { tank at a } 30^{\circ} \text { angle. Sample was unheated, unheated } \\
\text { samples precipitated }\end{array}$ \\
\hline $\mathbf{P}$ & Raw & PO4 & $1.03 E+07$ & $1.08 E-01$ & AP102-3 & $\mathbf{Y}$ & $\begin{array}{l}\text { Density not reported for G516. Density assumed same as } \\
\text { G359 which was collected at } 162 \text { in from bottom of tank at } \\
\text { a } 150^{\circ} \text { angle. This sample was heated. }\end{array}$ \\
\hline $\mathbf{P}$ & Raw & $\mathrm{PO} 4$ & $1.02 E+07$ & $1.07 E-01$ & AP102-3 & $\mathrm{Y}$ & $\begin{array}{l}\text { Density not reported for } \mathrm{G} 527 \text {. No assumptions could be } \\
\text { made }\end{array}$ \\
\hline $\mathbf{P}$ & Raw & PO4 & $9.95 E+06$ & $1.05 E-01$ & AP102-3 & $\mathbf{Y}$ & $\begin{array}{l}\text { Density not reported for G333. No assumptions could be } \\
\text { made }\end{array}$ \\
\hline $\mathbf{P}$ & Raw & PO4 & $9.90 \varepsilon+06$ & $1.04 E-01$ & AP102-3 & $Y$ & $\begin{array}{l}\text { Density not reported for G454. Density assumed same as } \\
\text { G471 which was collected at } 208 \text { in from bottom of tank at } \\
\text { a } 150^{\circ} \text { angle and was keep heated to } 40^{\circ} \mathrm{C} \text {. }\end{array}$ \\
\hline $\mathbf{P}$ & Raw & $\mathrm{PO4}$ & $9.86 E+06$ & $1.04 E-01$ & AP102-3 & $\mathbf{Y}$ & $\begin{array}{l}\text { Density not reported for G } 454 \text {. Density assumed same as } \\
G 471 \text { which was collected at } 208 \text { in from bottom of tank at } \\
\text { a } 150^{\circ} \text { angle and was keep heated to } 40^{\circ} \mathrm{C} \text {. }\end{array}$ \\
\hline $\mathbf{P}$ & Raw & PO4 & $9.69 E+06$ & $1.02 E-01$ & AP102-3 & $Y$ & $\begin{array}{l}\text { Density not reported for G333. No assumptions could be } \\
\text { made }\end{array}$ \\
\hline $\mathbf{P}$ & Raw & PO4 & $9.65 E+06$ & $1.02 E-01$ & AP102-3 & $\mathbf{Y}$ & $\begin{array}{l}\text { Density not reported for G338. No assumptions could be } \\
\text { made }\end{array}$ \\
\hline $\mathbf{P}$ & Raw & PO4 & $9.60 E+06$ & $1.01 E-01$ & AP102-3 & $Y$ & $\begin{array}{l}\text { Density not reported for G453. No assumptions could be } \\
\text { made }\end{array}$ \\
\hline$P$ & Raw & PO4 & $9.51 E+06$ & $1.00 \mathrm{E}-01$ & AP102-3 & $Y$ & $\begin{array}{l}\text { Density not reported for G338. No assumptions could be } \\
\text { made }\end{array}$ \\
\hline $\mathbf{P}$ & Raw & $\mathrm{PO} 4$ & $9.39 E+06$ & $9.89 E-02$ & AP102-3 & $\mathbf{Y}$ & $\begin{array}{l}\text { Density not reported for G453. No assumptions could be } \\
\text { made }\end{array}$ \\
\hline$P$ & Raw & PO4 & $9.34 E+06$ & $9.83 E-02$ & AP102-3 & $Y$ & $\begin{array}{l}\text { Density reported for } 6459 \text { collected at } 226 \text { in from bottom } \\
\text { of tank at a } 270^{\circ} \text { angle. Sample was unheated, unheated } \\
\text { samples precipitated }\end{array}$ \\
\hline$P$ & Raw & PO4 & $9.30 E+06$ & $9.79 E-02$ & AP102-3 & Y & $\begin{array}{l}\text { Density not reported for G449. No assumptions could be } \\
\text { made }\end{array}$ \\
\hline$P$ & Raw & $\mathrm{PO} 4$ & $9.26 E+06$ & $9.75 \mathrm{E}-02$ & AP102-3 & $Y$ & $\begin{array}{l}\text { Density reported for G459 collected at } 226 \text { in from bottom } \\
\text { of tank at a } 270^{\circ} \text { angle. Sample was unheated, unheated } \\
\text { samples precipitated }\end{array}$ \\
\hline$P$ & Raw & PO4 & $9.22 E+06$ & $9.71 E-02$ & AP102-3 & $Y$ & $\begin{array}{l}\text { Density not reported for } \mathrm{G} 450 \text {. Density assumed same as } \\
\mathrm{G} 477 \text { which was collected at } 182 \text { in from bottom of tank at } \\
\text { a } 30^{\circ} \text { angle and was keep heated to } 40^{\circ} \mathrm{C} \text {. }\end{array}$ \\
\hline $\mathbf{P}$ & Raw & PO4 & $9.20 E+06$ & $9.69 E-02$ & AP102-3 & $Y$ & $\begin{array}{l}\text { Density not reported for G449. No assumptions could be } \\
\text { made }\end{array}$ \\
\hline$P$ & Raw & PO4 & $9.19 E+06$ & $9.68 \mathrm{E}-02$ & AP102-3 & $\mathbf{Y}$ & $\begin{array}{l}\text { Density not reported for G450. Density assumed same as } \\
G 477 \text { which was collected at } 182 \text { in from bottom of tank at } \\
\text { a } 30^{\circ} \text { angle and was keep heated to } 40^{\circ} \mathrm{C} \text {. }\end{array}$ \\
\hline$P$ & Raw & PO4 & $9.19 E+06$ & $9.68 \mathrm{E}-02$ & AP102-3 & $Y$ & $\begin{array}{l}\text { Density reported for G364 collected at } 372 \text { in from bottom } \\
\text { of tank at a } 270^{\circ} \text { angle. Sample was unheated, unheated } \\
\text { samples precipitated }\end{array}$ \\
\hline $\mathbf{P}$ & Raw & PO4 & $9.15 E+06$ & $9.63 E-02$ & AP102-3 & Y & $\begin{array}{l}\text { Density reported for G358 collected at } 334 \text { in from bottom } \\
\text { of tank at a } 150^{\circ} \text { angle. Sample was unheated, unheated } \\
\text { samples precipitated }\end{array}$ \\
\hline
\end{tabular}




\begin{tabular}{|c|c|c|c|c|c|c|c|c|c|c|c|}
\hline & & & & & ank Waste & hiaracteriza & ion Sample & ormation & & & \\
\hline Reported Chemical & $\begin{array}{c}\text { Memo } \\
\text { Date }\end{array}$ & $\begin{array}{c}\text { Start } \\
\text { Analysis } \\
\text { Date }\end{array}$ & $\begin{array}{c}\text { Finish } \\
\text { Analysis Date } \\
\end{array}$ & $\begin{array}{c}\text { Start } \\
\text { Sample } \\
\text { Date } \\
\end{array}$ & $\begin{array}{c}\text { Finish } \\
\text { Sample } \\
\text { Date } \\
\end{array}$ & $\begin{array}{l}\text { Sample } \\
\text { Number }\end{array}$ & $\begin{array}{c}\text { Tank Farm } \\
\text { Number }\end{array}$ & $\begin{array}{c}\text { Lab Sample } \\
\text { Number }\end{array}$ & $\begin{array}{l}\text { Density } \\
\text { (g/mL) }\end{array}$ & $\begin{array}{c}\text { Reported } \\
\text { Value } \\
\end{array}$ & $\begin{array}{r}\text { Repor } \\
\text { Uni } \\
\end{array}$ \\
\hline Phosphate & $6 / 1 / 90$ & $4 / 30 / 89$ & $12 / 30 / 89$ & $4 / 27 / 89$ & $4 / 29 / 89$ & ВОВ2M2 & G309 & G358 & 1.20 & $9.05 E+03$ & $\mathrm{ug} / \mathrm{n}$ \\
\hline Phosphate & $6 / 1 / 90$ & $4 / 30 / 89$ & $12 / 30 / 89$ & $4 / 27 / 89$ & $4 / 29 / 89$ & B082M5 & G315 & G364 & 1.19 & $9.05 E+03$ & $u g / m$ \\
\hline Phosphate & $6 / 1 / 90$ & $4 / 30 / 89$ & $12 / 30 / 89$ & $4 / 27 / 89$ & $4 / 29 / 89$ & вов2МО & G306 & G352 & 1.20 & $8.87 E+03$ & $\mathrm{ug} / \mathrm{m}$ \\
\hline Phosphate & $6 / 1 / 90$ & $4 / 30 / 89$ & $12 / 30 / 89$ & $4 / 27 / 89$ & $4 / 29 / 89$ & ВОВ2M3 & G311 & G359 & 1.20 & $8.87 E+03$ & $\mathrm{ug} / \mathrm{m}$ \\
\hline Phosphate & $6 / 1 / 90$ & $4 / 30 / 89$ & $12 / 30 / 89$ & $4 / 27 / 89$ & $4 / 29 / 89$ & В0В2M4 & G313 & G363 & 1.20 & $8.85 E+03$ & $\mathrm{ug} / \mathrm{m}$ \\
\hline Phosphate & $6 / 1 / 90$ & $4 / 30 / 89$ & $12 / 30 / 89$ & $4 / 27 / 89$ & $4 / 29 / 89$ & вов2М3 & G311 & G359 & 1.20 & $8.85 E+03$ & $u g / m$ \\
\hline Phosphate & $6 / 1 / 90$ & $4 / 30 / 89$ & $12 / 30 / 89$ & $4 / 27 / 89$ & $4 / 29 / 89$ & BOB2M1 & G308 & G353 & 1.20 & $8.84 E+03$ & ug/mi \\
\hline Phosphate & $6 / 1 / 90$ & $4 / 30 / 89$ & $12 / 30 / 89$ & $4 / 27 / 89$ & $4 / 29 / 89$ & BOB2L8 & G303 & G348 & 1.21 & $8.79 E+03$ & $\mathrm{ug} / \mathrm{ml}$ \\
\hline Phosphate & 6/1/90 & $4 / 30 / 89$ & $12 / 30 / 89$ & $4 / 27 / 89$ & $4 / 29 / 89$ & BOB2M4 & G313 & G363 & 1.20 & $8.77 E+03$ & $\mathrm{ug} / \mathrm{ml}$ \\
\hline Phosphate & $6 / 1 / 90$ & $4 / 30 / 89$ & $12 / 30 / 89$ & $4 / 27 / 89$ & $4 / 29 / 89$ & BÓB2MO & G306 & G352 & 1.20 & $8.76 E+03$ & $\mathrm{ug} / \mathrm{ml}$ \\
\hline Phosphate & $6 / 1 / 90$ & $4 / 30 / 89$ & $12 / 30 / 89$ & $4 / 27 / 89$ & $4 / 29 / 89$ & BOB2N4 & G323 & G341 & 1.20 & $8.75 E+03$ & ug/mi \\
\hline Phosphate & $6 / 1 / 90$ & $4 / 30 / 89$ & $12 / 30 / 89$ & $4 / 27 / 89$ & $4 / 29 / 89$ & BOB2L7 & G299 & G342 & 1.20 & $8.73 E+03$ & $\mathrm{ug} / \mathrm{mL}$ \\
\hline Phosphate & $6 / 1 / 90$ & $4 / 30 / 89$ & $12 / 30 / 89$ & $4 / 27 / 89$ & $4 / 29 / 89$ & BO82M1 & G308 & G353 & 1.20 & $8.69 E+03$ & $u g / \mathrm{mL}$ \\
\hline Phosphate & $6 / 1 / 90$ & $4 / 30 / 89$ & $12 / 30 / 89$ & $4 / 27 / 89$ & $4 / 29 / 89$ & BOB2L7 & G299 & G342 & 1.20 & $8.59 E+03$ & $\mathrm{ug} / \mathrm{mL}$ \\
\hline Phosphate & $6 / 1 / 90$ & $4 / 30 / 89$ & $12 / 30 / 89$ & $4 / 27 / 89$ & $4 / 29 / 89$ & BOB2L8 & G303 & G348 & 1.21 & $8.55 E+03$ & $\mathrm{ug} / \mathrm{mL}$ \\
\hline Phosphate & $6 / 1 / 90$ & $4 / 30 / 89$ & $12 / 30 / 89$ & $4 / 27 / 89$ & $4 / 29 / 89$ & BOB2N4 & G323 & G341 & 1.20 & $8.51 E+03$ & $\mathrm{ug} / \mathrm{mL}$ \\
\hline Phosphate & $6 / 1 / 90$ & 4/30/89 & $12 / 30 / 89$ & $4 / 27 / 89$ & $4 / 29 / 89$ & BOB2NO & G307 & G527* & NA & $8.27 E+03$ & $u g / m L$ \\
\hline Phosphate & $6 / 1 / 90$ & $4 / 30 / 89$ & $12 / 30 / 89$ & $4 / 27 / 89$ & $4 / 29 / 89$ & BOB2NO & G307 & G527* & NA & $8.09 E+03$ & $u g / \mathrm{mL}$ \\
\hline Antimony & $6 / 1 / 90$ & $4 / 30 / 89$ & $12 / 30 / 89$ & $4 / 27 / 89$ & $4 / 29 / 89$ & ВОВ2T9 & G327 & G470* & 1.19 & $<2.10 E+05$ & $u g / L$ \\
\hline Antimony & $6 / 1 / 90$ & $4 / 30 / 89$ & $12 / 30 / 89$ & $4 / 27 / 89$ & $4 / 29 / 89$ & BOB2T9 & G327 & G470* & 1.19 & $<2.10 E+05$ & ug $n$ \\
\hline Antimony & $6 / 1 / 90$ & $4 / 30 / 89$ & $12 / 30 / 89$ & $4 / 27 / 89$ & $4 / 29 / 89$ & BOB2N2 & G317 & G333 & NA & $<5.25 E+03$ & ugh \\
\hline Antimony & $6 / 1 / 90$ & $4 / 30 / 89$ & $12 / 30 / 89$ & $4 / 27 / 89$ & $4 / 29 / 89$ & BOB2N3 & G322 & G338 & NA & $<5.25 E+03$ & ugh : \\
\hline
\end{tabular}




\begin{tabular}{|c|c|c|c|c|c|c|c|}
\hline \multirow[b]{2}{*}{ Code } & \multirow[b]{2}{*}{ Source } & \multicolumn{3}{|c|}{ Analyte Information } & \multirow[b]{2}{*}{$\begin{array}{l}\text { Reference } \\
\text { Number }\end{array}$} & \multirow[b]{2}{*}{$\begin{array}{l}\text { Validated } \\
\text { (Y/N) }\end{array}$} & \multirow[b]{2}{*}{ Notes } \\
\hline & & Analyte & $\begin{array}{l}\text { Conc. } \\
\text { (ug/L) }\end{array}$ & Conc. $(\mathrm{mol} / \mathrm{L})$ & & & \\
\hline$P$ & Raw & $\mathrm{PO4}$ & $9.05 E+06$ & 9.53E-02 & AP102-3 & $Y$ & $\begin{array}{l}\text { Density reported for } \mathrm{G} 358 \text { collected at } 334 \text { in from bottom } \\
\text { of tank at a } 150^{\circ} \text { angle. Sample was unheated, unheated } \\
\text { samples precipitated }\end{array}$ \\
\hline$P$ & Raw & $\mathrm{PO} 4$ & $9.05 E \div 06$ & $9.53 \mathrm{E}-02$ & AP102-3 & $Y$ & $\begin{array}{l}\text { Density reported for G364 collected at } 372 \text { in from bottom } \\
\text { of tank at a } 270^{\circ} \text { angle. Sample was unheated, unheated } \\
\text { samples precipitated }\end{array}$ \\
\hline$P$ & Raw & PO4 & $8.87 E+06$ & 9.34E-02 & AP102-3 & $\mathrm{Y}$ & $\begin{array}{l}\text { Density reported for } \mathrm{G} 352 \text { collected at } 34 \text { in from bottom of } \\
\text { tank at a } 30^{\circ} \text { angle. Sample was unheated, unheated } \\
\text { samples precipitated }\end{array}$ \\
\hline$P$ & Raw & PO4 & $8.87 E+06$ & $9.34 E-02$ & AP102-3 & $Y$ & $\begin{array}{l}\text { Density reported for G359 collected at } 162 \text { in from bottom } \\
\text { of tank at a } 150^{\circ} \text { angle. Sample was unheated, unheated } \\
\text { samples precipitated }\end{array}$ \\
\hline $\mathbf{P}$ & Raw & PO4 & $8.85 E+06$ & $9.32 \mathrm{E}-02$ & AP102-3 & $Y$ & $\begin{array}{l}\text { Density reported for G363 collected at } 18 \text { in from bottom of } \\
\text { tank at a } 150^{\circ} \text { angle. Sample was unheated, unheated } \\
\text { samples precipitated }\end{array}$ \\
\hline$P$ & Raw & $\mathrm{PO4}$ & $8.85 E+06$ & $9.32 E-02$ & AP102-3 & Y & $\begin{array}{l}\text { Density reported for G359 collected at } 162 \text { in from bottom } \\
\text { of tank at a } 150^{\circ} \text { angle. Sample was unheated, unheated } \\
\text { samples precipitated }\end{array}$ \\
\hline $\mathbf{P}$ & Raw & 804 & $8.84 E+06$ & $9.31 E-02$ & AP102-3 & $Y$ & $\begin{array}{l}\text { Density reported for } \mathrm{G} 353 \text { collected at } 334 \text { in from bottom } \\
\text { of tank at a } 150^{\circ} \text { angle. Sample was unheated, unheated } \\
\text { samples precipitated }\end{array}$ \\
\hline$P$ & Raw & PO4 & $8.79 E \div 06$ & $9.26 \mathrm{E}-02$ & AP102-3 & $\gamma$ & $\begin{array}{l}\text { Density reported for } \mathrm{G} 348 \text { collected at } 125 \text { in from bottom } \\
\text { of tank at a } 30^{\circ} \text { angle. Sample was unheated, unheated } \\
\text { samples precipitated }\end{array}$ \\
\hline$P$ & Raw & 804 & $8.77 E \div 06$ & $9.23 E-02$ & AP102-3 & $Y$ & $\begin{array}{l}\text { Density reported for } G 363 \text { collected at } 18 \text { in from bottom of } \\
\text { tank at a } 150^{\circ} \text { angle. Sample was unheated, unheated } \\
\text { samples precipitated }\end{array}$ \\
\hline$P$ & Raw & PO4 & $8.76 E+06$ & $9.22 \mathrm{E}-02$ & AP102-3 & $\gamma$ & $\begin{array}{l}\text { Density reported for } \mathrm{G} 352 \text { collected at } 34 \text { in from bottom of } \\
\text { tank at a } 30^{\circ} \text { angle. Sample was unheated, unheated } \\
\text { samples precipitated }\end{array}$ \\
\hline $\mathbf{P}$ & Raw & PO4 & $8.75 E+06$ & 9.21E-02 & AP102-3 & $\mathbf{Y}$ & $\begin{array}{l}\text { Density reported for } 6341 \text { collected at } 99 \text { in from bottom of } \\
\text { tank at a } 270^{\circ} \text { angle. }\end{array}$ \\
\hline $\mathbf{P}$ & Raw & PO4 & $8.73 E+06$ & $9.19 E-02$ & AP102-3 & $Y$ & $\begin{array}{l}\text { Density reported for } 6342 \text { collected at } 306 \text { in from bottom } \\
\text { of tank at a } 30^{\circ} \text { angle. Sample was unheated, unheated } \\
\text { samples precipitated }\end{array}$ \\
\hline$P$ & Raw & PO4 & $8.69 E+06$ & $9.15 E-02$ & AP102-3 & $Y$ & $\begin{array}{l}\text { Density reported for G353 collected at } 334 \text { in from bottom } \\
\text { of tank at a } 150^{\circ} \text { angle. Sample was unheated, unheated } \\
\text { samples precipitated }\end{array}$ \\
\hline $\mathbf{P}$ & Raw & PO4 & $8.59 E+06$ & 9.04E-02 & AP102-3 & $Y$ & $\begin{array}{l}\text { Density reported for G342 collected at } 306 \text { in from bottom } \\
\text { of tank at a } 30^{\circ} \text { angle. Sample was unheated, unheated } \\
\text { samples precipitated }\end{array}$ \\
\hline$P$ & Raw & PO4 & $8.55 E \div 06$ & $9.00 \mathrm{E}-02$ & AP102-3 & $Y$ & $\begin{array}{l}\text { Density reported for G348 collected at } 125 \text { in from bottom } \\
\text { of tank at a } 30^{\circ} \text { angle. Sample was unheated, unheated } \\
\text { samples precipitated }\end{array}$ \\
\hline ? & Raw & PO4 & $8.51 E+06$ & 8.96E-02 & AP102-3 & $Y$ & $\begin{array}{l}\text { Density reported for G341 collected at } 99 \text { in from bottom of } \\
\text { tank at a } 270^{\circ} \text { angle. }\end{array}$ \\
\hline$P$ & Raw & PO4 & $8.27 E+06$ & 8.71E-O2 & AP102-3 & $Y$ & $\begin{array}{l}\text { Density not reported for G527. No assumptions could be } \\
\text { made }\end{array}$ \\
\hline$P$ & Raw & PO4 & $8.09 E+06$ & 8.52E-02 & AP102-3 & $Y$ & $\begin{array}{l}\text { Density not reported for G527. No assumptions could be } \\
\text { made }\end{array}$ \\
\hline$P$ & Raw & Sb & \#VALUEI & *VALUe! & AP102-3 & $Y$ & $\begin{array}{l}\text { Density reported for G470 represents a composite sample. } \\
\text { Sample was kept heated to } 40^{\circ} \mathrm{C} \text {. }\end{array}$ \\
\hline$P$ & Raw & Sb & \#VALUE! & \#VALUE! & AP102-3 & $Y$ & $\begin{array}{l}\text { Density reported for G470 represents a composite sample. } \\
\text { Sample was kept heated to } 40^{\circ} \mathrm{C} \text {. }\end{array}$ \\
\hline $\mathbf{P}$ & Raw & $\mathrm{Sb}$ & "VALUE! & \#VALUE! & AP102-3 & $\gamma$ & $\begin{array}{l}\text { Density not reported for G333. No assumptions could be } \\
\text { made }\end{array}$ \\
\hline $\mathbf{P}$ & Raw & Sb & \#VALUE! & \#VALUE! & AP102-3 & $Y$ & $\begin{array}{l}\text { Density not reported for G338. No assumptions could be } \\
\text { made }\end{array}$ \\
\hline
\end{tabular}




\begin{tabular}{|c|c|c|c|c|c|c|c|c|c|c|c|}
\hline & & & & & ank Waste & haracteriza & ion Sample & ormation & & & \\
\hline Reported Chemical & $\begin{array}{c}\text { Memo } \\
\text { Date }\end{array}$ & $\begin{array}{c}\text { Start } \\
\text { Analysis } \\
\text { Date }\end{array}$ & $\begin{array}{c}\text { Finish } \\
\text { Analysis Date } \\
\end{array}$ & $\begin{array}{c}\text { Start } \\
\text { Sample } \\
\text { Date }\end{array}$ & $\begin{array}{c}\text { Finish } \\
\text { Sample } \\
\text { Date } \\
\end{array}$ & $\begin{array}{l}\text { Sample } \\
\text { Number }\end{array}$ & $\begin{array}{c}\text { Tank Farm } \\
\text { Number }\end{array}$ & $\begin{array}{c}\text { Lab Sample } \\
\text { Number }\end{array}$ & $\begin{array}{l}\text { Density } \\
\text { (g/mL) }\end{array}$ & $\begin{array}{l}\text { Reported } \\
\text { Value }\end{array}$ & $\begin{array}{r}\text { Rep } \\
\mathbf{C}\end{array}$ \\
\hline Antimony & $6 / 1 / 90$ & $4 / 30 / 89$ & $12 / 30 / 89$ & $4 / 27 / 89$ & $4 / 29 / 89$ & BOB2L8 & G303 & G348 & 1.21 & $<5.25 E+03$ & $\mathbf{u}$ \\
\hline Antimony & $6 / 1 / 90$ & $4 / 30 / 89$ & $12 / 30 / 89$ & $4 / 27 / 89$ & $4 / 29 / 89$ & BOB2L.9 & G305 & G349 & 1.20 & $<5.25 E+03$ & $u_{4}$ \\
\hline Antimany & $6 / 1 / 90$ & $4 / 30 / 89$ & $12 / 30 / 89$ & $4 / 27 / 89$ & $4 / 29 / 89$ & ВОВ2MO & G306 & G352 & 1.20 & $<5.25 E+03$ & ugf \\
\hline Antimony & $6 / 1 / 90$ & $4 / 30 / 89$ & $12 / 30 / 89$ & $4 / 27 / 89$ & $4 / 29 / 89$ & ВOB2M1 & G308 & G353 & 1.20 & $<5.25 E+03$ & us \\
\hline Antimony & $6 / 1 / 90$ & $4 / 30 / 89$ & $12 / 30 / 89$ & $4 / 27 / 89$ & $4 / 29 / 89$ & ВОВ2M2 & G309 & G358 & 1.20 & $<5.25 E+03$ & uद̨ \\
\hline Antimony & $6 / 1 / 90$ & $4 / 30 / 89$ & $12 / 30 / 89$ & $4 / 27 / 89$ & $4 / 29 / 89$ & 8082M5 & G315 & G364 & 1.19 & $<5.25 E+03$ & us \\
\hline Antimony & $6 / 1 / 90$ & $4 / 30 / 89$ & $12 / 30 / 89$ & $4 / 23 / 89$ & $4 / 29 / 89$ & BOB2T9 & G327 & G443 & 1.20 & $<5.25 E+03$ & ug] \\
\hline Antimony & $6 / 1 / 90$ & $4 / 30 / 89$ & $12 / 30 / 89$ & $4 / 27 / 89$ & $4 / 29 / 89$ & BOB2M8 & G301 & G449 & NA & $<5.25 E+03$ & ug \\
\hline Antimony & $6 / 1 / 90$ & $4 / 30 / 89$ & $12 / 30 / 89$ & $4 / 27 / 89$ & $4 / 29 / 89$ & BOB2NO & G307 & G453 & NA & $<5.25 E+03$ & $u g$ \\
\hline Antimony & $6 / 1 / 90$ & $4 / 30 / 89$ & $12 / 30 / 89$ & $4 / 27 / 89$ & $4 / 29 / 89$ & ВОВ2M6 & G318 & G459 & 1.20 & $<5.25 E+03$ & ug \\
\hline Antimony & $6 / 1 / 90$ & $4 / 30 / 89$ & $12 / 30 / 89$ & $4 / 27 / 89$ & $4 / 29 / 89$ & ВОВ2M7 & G319 & G460 & 1.20 & $<5.25 E+03$ & ug. \\
\hline Antimony & $6 / 1 / 90$ & $4 / 30 / 89$ & $12 / 30 / 89$ & $4 / 27 / 89$ & $4 / 29 / 89$ & BOB2L8 & G303 & G348 & 1.21 & $<5.25 E+03$ & $u g$ \\
\hline Antimony & $6 / 1 / 90$ & $4 / 30 / 89$ & $12 / 30 / 89$ & $4 / 27 / 89$ & $4 / 29 / 89$ & BOB2L9 & G305 & G349 & 1.20 & $<5.25 E+03$ & ug! \\
\hline Antimony & $6 / 1 / 90$ & $4 / 30 / 89$ & $12 / 30 / 89$ & $4 / 27 / 89$ & $4 / 29 / 89$ & BOB2MO & G306 & G352 & 1.20 & $<5.25 E+03$ & ugf \\
\hline Antimony & $6 / 1 / 90$ & $4 / 30 / 89$ & $12 / 30 / 89$ & $4 / 27 / 89$ & $4 / 29 / 89$ & BOB2M1 & G308 & G353 & 1.20 & $<5.25 E+03$ & ugl \\
\hline Antimony & $6 / 1 / 90$ & $4 / 30 / 89$ & $12 / 30 / 89$ & $4 / 27 / 89$ & $4 / 29 / 89$ & BOB2M2 & G309 & G358 & 1.20 & $<5.25 E+03$ & ug/. \\
\hline Antimony & $6 / 1 / 90$ & $4 / 30 / 89$ & $12 / 30 / 89$ & $4 / 27 / 89$ & $4 / 29 / 89$ & BOB2M3 & G311 & G359 & 1.20 & $<5.25 E+03$ & ug $n$ \\
\hline Antimony & $6 / 1 / 90$ & $4 / 30 / 89$ & $12 / 30 / 89$ & $4 / 27 / 89$ & $4 / 29 / 89$ & BOB2M5 & G315 & G364 & 1.19 & $<5.25 E+03$ & ugh \\
\hline Antimony & $6 / 1 / 90$ & $4 / 30 / 89$ & $12 / 30 / 89$ & $4 / 27 / 89$ & $4 / 29 / 89$ & ВОВ2Т9 & G327 & G443 & 1.20 & $<5.25 E+03$ & $u g h$ \\
\hline Antimony & $6 / 1 / 90$ & $4 / 30 / 89$ & $12 / 30 / 89$ & $4 / 27 / 89$ & $4 / 29 / 89$ & BOB2M8 & G301 & G449 & NA & $<5.25 E+03$ & ug $/$ \\
\hline Antimony & $6 / 1 / 90$ & $4 / 30 / 89$ & $12 / 30 / 89$ & $4 / 27 / 89$ & $4 / 29 / 89$ & 8082M9 & G302 & G450 & $(1.20)$ & $<5.25 E+03$ & ugn \\
\hline
\end{tabular}




\begin{tabular}{|c|c|c|c|c|c|c|c|}
\hline & & & nalyte Info & ration & & & \\
\hline Code & Source & Analyte & $\begin{array}{l}\text { Conc. } \\
\text { (ug/L) }\end{array}$ & Conc. (mol/L) & $\begin{array}{c}\text { Reference } \\
\text { Number }\end{array}$ & $\begin{array}{c}\text { Validated } \\
(Y / N)\end{array}$ & Notes \\
\hline $\mathbf{P}$ & Raw & Sb & \#VALUE! & *VALUE! & AP102-3 & $Y$ & $\begin{array}{l}\text { Density reported for } 6348 \text { collected at } 125 \text { in from bottom } \\
\text { of tank at a } 30^{\circ} \text { angle. Sample was unheated, unheated } \\
\text { samples precipitated }\end{array}$ \\
\hline$P$ & Raw & $\mathrm{Sb}$ & \#VAL.UE! & FALUE! & AP102-3 & $Y$ & $\begin{array}{l}\text { Density reported for } 6349 \text { collected at } 34 \text { in from bottom of } \\
\text { tank at a } 30^{\circ} \text { angle. Sample was unheated, unheated } \\
\text { samples precipitated }\end{array}$ \\
\hline$P$ & Raw & Sb & \#ALUE! & Nalue! & AP102-3 & $Y$ & $\begin{array}{l}\text { Density reported for } \mathrm{G} 352 \text { collected at } 34 \text { in from bottom of } \\
\text { tank at a } 30^{\circ} \text { angle. Sample was unheated, unheated } \\
\text { samples precipitated }\end{array}$ \\
\hline $\mathbf{P}$ & Raw & Sb & \#VALUE! & *VALUEI & AP102-3 & $Y$ & $\begin{array}{l}\text { Density reported for G353 collected at } 334 \text { in from bottom } \\
\text { of tank at a } 150^{\circ} \text { angle. Sample was unheated, unheated } \\
\text { samples precipitated }\end{array}$ \\
\hline $\mathbf{P}$ & Raw & $\mathrm{Sb}$ & \#VALUE! & NALUE! & AP102-3 & $\mathbf{Y}$ & $\begin{array}{l}\text { Density reported for G358 collected at } 334 \text { in from bottom } \\
\text { of tank at a } 150^{\circ} \text { angle. Sample was unheated, unheated } \\
\text { samples precipitated }\end{array}$ \\
\hline$P$ & Raw & $\mathrm{Sb}$ & \#VALUE! & \#VALUE! & AP102-3 & $\mathbf{Y}$ & $\begin{array}{l}\text { Density reported for G364 collected at } 372 \text { in from botiom } \\
\text { of tank at a } 270^{\circ} \text { angle. Sample was unheated, unheated } \\
\text { samples precipitated }\end{array}$ \\
\hline $\mathbf{P}$ & Raw & Sb & \#VALUE! & *VALUE! & AP102-3 & $Y$ & Density reported for $\mathrm{G} 443$ represents a composite sample \\
\hline $\mathbf{P}$ & Raw & Sb & \#VALUE! & NALUE! & AP102-3 & Y & $\begin{array}{l}\text { Density not reported for G449. No assumptions could be } \\
\text { made }\end{array}$ \\
\hline $\mathbf{P}$ & Raw & Sb & \#VALUE! & \#VALUE! & AP102-3 & $Y$ & $\begin{array}{l}\text { Density not reported for G453. No assumptions could be } \\
\text { made }\end{array}$ \\
\hline $\mathbf{P}$ & Raw & Sb & \#VAL.UE! & NALUE! & AP102-3 & $Y$ & $\begin{array}{l}\text { Density reported for } \mathrm{G} 459 \text { collected at } 226 \text { in from bottom } \\
\text { of tank at a } 270^{\circ} \text { angle. Sample was unheated, unheated } \\
\text { samples precipitated }\end{array}$ \\
\hline $\mathbf{P}$ & Raw & Sb & \#ALUE! & $\#$ \#ALUEI & AP102-3 & $Y$ & $\begin{array}{l}\text { Density reported for } \mathrm{G} 460 \text { collected at } 226 \text { in from bottom } \\
\text { of tank at a } 270^{\circ} \text { angle. Sample was unheated, unheated } \\
\text { samples precipitated }\end{array}$ \\
\hline$\cdot P$ & Raw & Sb & \#VALUE! & \#VALUE! & AP102-3 & Y & $\begin{array}{l}\text { Density reported for G348 collected at } 125 \text { in from bottom } \\
\text { of tank at a } 30^{\circ} \text { angle. Sample was unheated, unheated } \\
\text { samples precipitated }\end{array}$ \\
\hline $\mathbf{P}$ & Raw & Sb & \#VALUE! & \#VALUE! & AP102-3 & Y & $\begin{array}{l}\text { Density reported for } \mathrm{G} 349 \text { collected at } 34 \text { in from bottom of } \\
\text { tank at a } 30^{\circ} \text { angle. Sample was unheated, unheated } \\
\text { samples precipitated }\end{array}$ \\
\hline$P$ & Raw & Sb & \#VALUE! & \#VALUE! & AP102-3 & $Y$ & 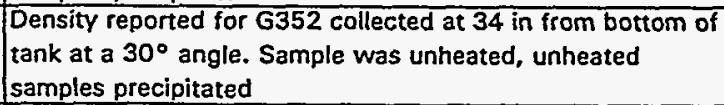 \\
\hline $\mathbf{P}$ & Raw & Sb & \#VALUE! & WALUE! & AP102-3 & $\mathbf{Y}$ & $\begin{array}{l}\text { Density reported for G353 collected at } 334 \text { in from bottom } \\
\text { of tank at a } 150^{\circ} \text { angle. Sample was unheated, unheated } \\
\text { samples precipitated }\end{array}$ \\
\hline $\mathbf{P}$ & Raw & Sb & \#VALUE! & *VALUEI & AP102-3 & Y & $\begin{array}{l}\text { Density reported for } 6358 \text { collected at } 334 \text { in from bottom } \\
\text { of tank at a } 150^{\circ} \text { angle. Sample was unheated, unheated } \\
\text { samples precipitated }\end{array}$ \\
\hline $\mathbf{P}$ & Raw & $\mathrm{Sb}$ & \#VALUE! & FALUE! & AP102-3 & $\gamma$ & $\begin{array}{l}\text { Density reported for G359 collected at } 162 \text { in from bottom } \\
\text { of tank at a } 150^{\circ} \text { angle. Sample was unheated, unheated } \\
\text { samples precipitated }\end{array}$ \\
\hline $\mathbf{P}$ & Raw & $\mathrm{Sb}$ & \#VALUE! & NVALUE! & AP102-3 & Y & $\begin{array}{l}\text { Density reported for G364 collected at } 372 \text { in from bottom } \\
\text { of tank at a } 270^{\circ} \text { angle. Sample was unheated, unheated } \\
\text { samples precipitated }\end{array}$ \\
\hline P & Raw & Sb & \#VALUE! & *VALUE! & AP102-3 & Y & Density reported for 6443 represents a composite sample \\
\hline $\mathbf{P}$ & Raw & Sb & \#VALUE! & \#VALUE! & AP102-3 & $Y$ & $\begin{array}{l}\text { Density not reported for G449. No assumptions could be } \\
\text { made }\end{array}$ \\
\hline $\mathbf{P}$ & Raw & $\mathrm{Sb}$ & \#VALUE! & \#ALUE! & AP102-3 & $Y$ & $\begin{array}{l}\text { Density not reported for G450. Density assumed same as } \\
\text { G477 which was collected at } 182 \text { in from bottom of tank at } \\
\text { a } 30^{\circ} \text { angle and was keep heated to } 40^{\circ} \mathrm{C} \text {. }\end{array}$ \\
\hline
\end{tabular}


TWRS Privatization Support Pr Chemical Liquid

\begin{tabular}{|c|c|c|c|c|c|c|c|c|c|c|c|}
\hline & & & & & ank Wast & haracteriza & ion Sample & ormation & & & \\
\hline Reported Chemical & $\begin{array}{l}\text { Memo } \\
\text { Date }\end{array}$ & $\begin{array}{c}\text { Start } \\
\text { Analysis } \\
\text { Date }\end{array}$ & $\begin{array}{c}\text { Finish } \\
\text { Analysis Date } \\
\end{array}$ & $\begin{array}{c}\text { Start } \\
\text { Sample } \\
\text { Date } \\
\end{array}$ & $\begin{array}{c}\text { Finish } \\
\text { Sample } \\
\text { Date }\end{array}$ & $\begin{array}{l}\text { Sample } \\
\text { Number }\end{array}$ & $\begin{array}{c}\text { Tank Farm } \\
\text { Number }\end{array}$ & $\begin{array}{c}\text { Lab Sample } \\
\text { Number }\end{array}$ & $\begin{array}{l}\text { Density } \\
(\mathrm{g} / \mathrm{mL})\end{array}$ & $\begin{array}{c}\text { Reported } \\
\text { Value }\end{array}$ & $\begin{array}{r}\text { Repl } \\
U_{4} \\
\end{array}$ \\
\hline Antimony & $6 / 1 / 90$ & $4 / 30 / 89$ & $12 / 30 / 89$ & $4 / 27 / 89$ & $4 / 29 / 89$ & вов2М6 & G318 & G459 & 1.20 & $<5.25 E+03$ & ug \\
\hline Antimony & $6 / 1 / 90$ & $4 / 30 / 89$ & $12 / 30 / 89$ & $4 / 27 / 89$ & $4 / 29 / 89$ & вов2M7 & G319 & G460 & 1.20 & $<5.25 E+03$ & ug \\
\hline Antimony & $6 / 1 / 90$ & $4 / 30 / 89$ & $12 / 30 / 89$ & $4 / 27 / 89$ & $4 / 29 / 89$ & BOB2N4 & G323 & G341 & 1.20 & $<5.25 E+03$ & $u g /$ \\
\hline$S b$ & $2 / 10 / 89$ & NS & NS & NS & NS & NS & NS & NS & 0.956764 & $7.58 E+00$ & $\overrightarrow{p p}$ \\
\hline Antimony & $6 / 1 / 90$ & $4 / 30 / 89$ & $12 / 30 / 89$ & $4 / 27 / 89$ & $4 / 29 / 89$ & Вов2M4 & G313 & G363 & 1.20 & $9.47 E+03$ & ug \\
\hline Antimony & $6 / 1 / 90$ & $4 / 30 / 89$ & $12 / 30 / 89$ & $4 / 27 / 89$ & $4 / 29 / 89$ & B082L7 & G299 & G342 & 1.20 & $7.57 E+03$ & ug \\
\hline Antimony & $6 / 1 / 90$ & $4 / 30 / 89$ & $12 / 30 / 89$ & $4 / 27 / 89$ & $4 / 29 / 89$ & вов2М3 & G311 & G359 & 1.20 & $6.58 E+03$ & ug \\
\hline Antimony & $6 / 1 / 90$ & $4 / 30 / 89$ & $12 / 30 / 89$ & $4 / 27 / 89$ & $4 / 29 / 89$ & BOB2N3 & G322 & G338 & NA & $6.56 E+03$ & ug. \\
\hline Antimony & $6 / 1 / 90$ & $4 / 30 / 89$ & $12 / 30 / 89$ & $4 / 27 / 89$ & $4 / 29 / 89$ & вов2L7 & G299 & G342 & 1.20 & $6.34 E+03$ & $u g_{1}$ \\
\hline Antimony & $6 / 1 / 90$ & $4 / 30 / 89$ & $12 / 30 / 89$ & $4 / 27 / 89$ & $4 / 29 / 89$ & В082M9 & G302 & G450 & $(1.20)$ & $6.00 E+03$ & ugi \\
\hline Antimony & $6 / 1 / 90$ & $4 / 30 / 89$ & $12 / 30 / 89$ & $4 / 27 / 89$ & $4 / 29 / 89$ & BOB2N4 & G323 & G341 & 1.20 & $6.00 E+03$ & ugl \\
\hline Antimony & $6 / 1 / 90$ & $4 / 30 / 89$ & $12 / 30 / 89$ & $4 / 27 / 89$ & $4 / 29 / 89$ & BOB2NO & - 6307 & G453 & NA & $5.98 \mathrm{E}+03$ & ugl \\
\hline Antimony & $6 / 1 / 90$ & $4 / 30 / 89$ & $12 / 30 / 89$ & $4 / 27 / 89$ & $4 / 29 / 89$ & BOB2N2 & G317 & G333 & NA & $5.82 E+03$ & ugl \\
\hline Antimony & $6 / 1 / 90$ & $4 / 30 / 89$ & $12 / 30 / 89$ & $4 / 27 / 89$ & $4 / 29 / 89$ & вов2M4 & G313 & G363 & 1.20 & $5.53 E+03$ & ugi \\
\hline Antimony & $6 / 1 / 90$ & $4 / 30 / 89$ & $12 / 30 / 89$ & $4 / 27 / 89$ & $4 / 29 / 89$ & BOB2N1 & G310 & G454 & $(1.19)$ & $5.39 E+03$ & ug $/$ \\
\hline Antimony & $6 / 1 / 90$ & $4 / 30 / 89$ & $12 / 30 / 89$ & $4 / 27 / 89$ & $4 / 29 / 89$ & BOB2N1 & G310 & G454 & $(1.19)$ & $5.37 E+03$ & $u g /$. \\
\hline $\mathrm{Se}$ & $2 / 10 / 89$ & NS & NS & NS & NS & NS & NS & NS & 0.956764 & $4.74 \mathrm{E}-02$ & $\overline{p p n}$ \\
\hline Selenium & $6 / 1 / 90$ & $4 / 30 / 89$ & $12 / 30 / 89$ & $4 / 27 / 89$ & $4 / 29 / 89$ & BOB2N1 & G310 & G471 • & 1.19 & 6.79E-01 & $u g / \pi$ \\
\hline Selenium & $6 / 1 / 90$ & $4 / 30 / 89$ & $12 / 30 / 89$ & $4 / 27 / 89$ & $4 / 29 / 89$ & ВОВ2M9 & G302 & G477* & 1.20 & $6.15 E-01$ & $u g / \pi$ \\
\hline Selenium & $6 / 1 / 90$ & $4 / 30 / 89$ & $12 / 30 / 89$ & $4 / 27 / 89$ & $4 / 29 / 89$ & вов2Т9 & G327 & G470* & 1.19 & $6.02 E-01$ & $u g / \pi$ \\
\hline Selenium & $6 / 1 / 90$ & $4 / 30 / 89$ & $12 / 30 / 89$ & $4 / 27 / 89$ & $4 / 29 / 89$ & вов2Т9 & G327 & G470 & 1.19 & 5.95E-01 & $u g / \pi$ \\
\hline Selenium & $6 / 1 / 90$ & $4 / 30 / 89$ & $12 / 30 / 89$ & $4 / 27 / 89$ & $4 / 29 / 89$ & BOB2N4 & G323 & G476" & 1.21 & 5.93E-01 & $u g / m$ \\
\hline Selenium & $6 / 1 / 90$ & $4 / 30 / 89$ & $12 / 30 / 89$ & $4 / 27 / 89$ & $4 / 29 / 89$ & BOB2N1 & G310 & G471* & 1.19 & 5.88E-01 & $\mathrm{ug} / \mathrm{m}$ \\
\hline
\end{tabular}




\begin{tabular}{|c|c|c|c|c|c|c|c|}
\hline \multirow[b]{2}{*}{ Code } & \multirow[b]{2}{*}{ Source } & \multicolumn{3}{|c|}{ Analyte Information } & \multirow[b]{2}{*}{$\begin{array}{l}\text { Reference } \\
\text { Number }\end{array}$} & \multirow[b]{2}{*}{$\begin{array}{l}\text { Validated } \\
\text { (Y/N) }\end{array}$} & \multirow[b]{2}{*}{ Notes } \\
\hline & & Analyte & $\begin{array}{l}\text { Conc. } \\
\text { (ug/L) }\end{array}$ & Conc. $(\mathrm{mol} / \mathrm{L})$ & & & \\
\hline$P$ & Raw & Sb & \#VALUE! & \#VALUE! & AP102-3 & Y & $\begin{array}{l}\text { Density reported for G459 collected at } 226 \text { in from bottom } \\
\text { of tank at a } 270^{\circ} \text { angle. Sample was unheated, unheated } \\
\text { samples precipitated }\end{array}$ \\
\hline $\mathbf{P}$ & Raw & Sb & \#VALUE! & \#VALUE! & AP102-3 & Y & $\begin{array}{l}\text { Density reported for } \mathrm{G} 460 \text { collected at } 226 \text { in from bottom } \\
\text { of tank at a } 270^{\circ} \text { angle. Sample was unheated, unheated } \\
\text { samples precipitated }\end{array}$ \\
\hline $\mathbf{P}$ & Raw & Sb & VALUE! & *Value! & AP102-3 & $\mathbf{Y}$ & $\begin{array}{l}\text { Density reported for G341 collected at } 99 \text { in from bottom of } \\
\text { tank at a } 270^{\circ} \text { angle. }\end{array}$ \\
\hline $\bar{U}$ & RAW & Sb & $7.25 \mathrm{E}+09$ & $5.95 E+01$ & AP102-2 & $\mathbf{N}$ & Projected value, not a real data point \\
\hline $\mathbf{P}$ & Raw & Sb & $9.47 E+03$ & 7.78E-05 & AP102-3 & $Y$ & $\begin{array}{l}\text { Density reported for } G 363 \text { collected at } 18 \text { in from bottom of } \\
\text { tank at a } 150^{\circ} \text { angle. Sample was unheated, unheated } \\
\text { samples precipitated }\end{array}$ \\
\hline$P$ & Raw & Sb & $7.57 E+03$ & $6.22 E-05$ & AP102-3 & $Y$ & $\begin{array}{l}\text { Density reported for } \mathrm{G} 342 \text { collected at } 306 \text { in from bottom } \\
\text { of tank at a } 30^{\circ} \text { angle. Sample was unheated, unheated } \\
\text { samples precipitated }\end{array}$ \\
\hline $\mathbf{P}$ & Raw & Sb & $6.58 E+03$ & $5.40 E-05$ & AP102-3 & $Y$ & $\begin{array}{l}\text { Density reported for G359 collected at } 162 \text { in from bottom } \\
\text { of tank at a } 150^{\circ} \text { angle. Sample was unheated, unheated } \\
\text { samples precipitated }\end{array}$ \\
\hline $\mathbf{P}$ & Raw & Sb & $6.56 E+03$ & 5.39E-05 & AP102-3 & $Y$ & $\begin{array}{l}\text { Density not reported for G338. No assumptions could be } \\
\text { made }\end{array}$ \\
\hline$P$ & Raw & Sb & $6.34 E+03$ & 5.21E-05 & $A P 102-3$ & $\mathbf{Y}$ & $\begin{array}{l}\text { Density reported for } G 342 \text { collected at } 306 \text { in from bottom } \\
\text { of tank at a } 30^{\circ} \text { angle. Sample was unheated, unheated } \\
\text { samples precipitated }\end{array}$ \\
\hline$P$ & Raw & Sb & $6.00 E+03$ & 4.93E-05 & AP102-3 & $Y$ & $\begin{array}{l}\text { Density not reported for G450. Density assumed same as } \\
\text { G } 477 \text { which was collected at } 182 \text { in from bottom of tank at } \\
\text { a } 30^{\circ} \text { angle and was keep heated to } 40^{\circ} \mathrm{C} \text {. }\end{array}$ \\
\hline$P$ & Raw & Sb & $6.00 E+03$ & 4.93E-05 & AP102-3 & $\gamma$ & $\begin{array}{l}\text { Density reported for } \mathrm{G} 341 \text { collected at } 99 \text { in from bottom of } \\
\text { tank at a } 270^{\circ} \text { angle. }\end{array}$ \\
\hline$P$ & Raw & Sb & $5.98 E+03$ & 4.91E-05 & AP102-3 & $\gamma$ & $\begin{array}{l}\text { Density not reported for } \mathrm{G} 453 \text {. No assumptions could be } \\
\text { made }\end{array}$ \\
\hline$P$ & Raw & Sb & $5.82 E+03$ & 4.78E-05 & AP102-3 & $Y$ & $\begin{array}{l}\text { Density not reported for G333. No assumptions could be } \\
\text { made }\end{array}$ \\
\hline$P$ & Raw & $\mathbf{S b}$ & $5.53 E+03$ & 4.54E-05 & AP102-3 & $\gamma$ & $\begin{array}{l}\text { Density reported for G363 collected at } 18 \text { in from bottom of } \\
\text { tank at a } 150^{\circ} \text { angle. Sample was unheated, unheated } \\
\text { samples precipitated }\end{array}$ \\
\hline$P$ & Raw & $\mathrm{Sb}$ & $5.39 E+03$ & 4.43E-05 & AP102-3 & $Y$ & $\begin{array}{l}\text { Density not reported for G454. Density assumed same as } \\
G 471 \text { which was collected at } 208 \text { in from bottom of tank at } \\
\text { a } 150^{\circ} \text { angle and was keep heated to } 40^{\circ} \mathrm{C} \text {. }\end{array}$ \\
\hline$P$ & Raw & So & $5.37 E+03$ & 4.41E-05 & AP102-3 & $\gamma$ & $\begin{array}{l}\text { Density not reported for G454. Density assumed same as } \\
\mathrm{G} 471 \text { which was collected at } 208 \text { in from bottom of tank at } \\
\text { a } 150^{\circ} \text { angle and was keep heated to } 40^{\circ} \mathrm{C} \text {. }\end{array}$ \\
\hline $\mathrm{U}$ & RAW & $\mathrm{Se}$ & $4.53 E+07$ & $5.74 \mathrm{E}-01$ & AP102-2 & $N$ & Projected value, not a real data point \\
\hline $\mathbf{P}$ & Raw & Se & $6.79 E+02$ & 8.60E-06 & AP102-3 & $Y$ & $\begin{array}{l}\text { Density reported for } 6471 \text { collected at } 208 \text { in from bottom } \\
\text { of tank at a } 150^{\circ} \text { angle and was keep heated to } 40^{\circ} \mathrm{C} \text {. }\end{array}$ \\
\hline $\mathbf{P}$ & Raw & Se & $6.15 E+02$ & 7.79E-06 & AP102-3 & $Y$ & $\begin{array}{l}\text { Density reported for } \mathrm{G} 477 \text { collected at } 182 \text { in from bottom } \\
\text { of tank at a } 30^{\circ} \text { angle and was keep heated to } 40^{\circ} \mathrm{C} \text {. }\end{array}$ \\
\hline $\mathbf{P}$ & Raw & Se & $6.02 \mathrm{E}+02$ & $7.62 E-06$ & AP102-3 & Y & $\begin{array}{l}\text { Density reported for G470 represents a composite sample. } \\
\text { Sample was kept heated to } 40^{\circ} \mathrm{C} \text {. }\end{array}$ \\
\hline $\mathbf{P}$ & Raw & Se & $5.95 E+02$ & 7.54E-06 & AP102-3 & $Y$ & $\begin{array}{l}\text { Density reported for G470 represents a composite sample. } \\
\text { Sample was kept heated to } 40^{\circ} \mathrm{C} \text {. }\end{array}$ \\
\hline $\mathbf{P}$ & Raw & Se & $5.93 E+02$ & 7.51E-06 & AP102-3 & $\mathbf{Y}$ & $\begin{array}{l}\text { Density reported for G476 collected at } 99 \text { in from bottom of } \\
\text { tank at a } 270^{\circ} \text { angle and was keep heated to } 40^{\circ} \mathrm{C} \text {. }\end{array}$ \\
\hline $\mathbf{P}$ & Raw & Se & $5.88 E+02$ & $7.45 E-06$ & AP102-3 & $\mathbf{Y}$ & $\begin{array}{l}\text { Density reported for } \mathrm{G} 471 \text { collected at } 208 \text { in from bottom } \\
\text { of tank at a } 150^{\circ} \text { angle and was keep heated to } 40^{\circ} \mathrm{C} \text {. }\end{array}$ \\
\hline
\end{tabular}




\begin{tabular}{|c|c|c|c|c|c|c|c|c|c|c|c|}
\hline & & & & & nk Waste & haracteriza & ion Sample it & ormation & & & \\
\hline Reported Chemical & $\begin{array}{c}\text { Memo } \\
\text { Date }\end{array}$ & $\begin{array}{c}\text { Start } \\
\text { Analysis } \\
\text { Date }\end{array}$ & $\begin{array}{c}\text { Finish } \\
\text { Analysis Date }\end{array}$ & $\begin{array}{c}\text { Start } \\
\text { Sample } \\
\text { Date }\end{array}$ & $\begin{array}{c}\text { Finish } \\
\text { Sample } \\
\text { Date }\end{array}$ & $\begin{array}{l}\text { Sample } \\
\text { Number }\end{array}$ & $\begin{array}{c}\text { Tank Farm } \\
\text { Number }\end{array}$ & $\begin{array}{c}\text { Lab Sample } \\
\text { Number }\end{array}$ & $\begin{array}{l}\text { Density } \\
\text { (g/mL) }\end{array}$ & $\begin{array}{c}\text { Reported } \\
\text { Value }\end{array}$ & $\begin{array}{r}\text { Reps } \\
U_{1}\end{array}$ \\
\hline Selenium & $6 / 1 / 90$ & $4 / 30 / 89$ & $12 / 30 / 89$ & $4 / 27 / 89$ & $4 / 29 / 89$ & BOB2N4 & G323 & G476* & 1.21 & $5.63 E-01$ & ugl \\
\hline Selenium & $6 / 1 / 90$ & $4 / 30 / 89$ & $12 / 30 / 89$ & $4 / 27 / 89$ & $4 / 29 / 89$ & Вов2M9 & G302 & G477" & 1.20 & $5.63 E-01$ & uge! \\
\hline Selenium & $6 / 1 / 90$ & $4 / 30 / 89$ & $12 / 30 / 89$ & $4 / 27 / 89$ & $4 / 29 / 89$ & ВОВ2MO & G306 & G352 & 1.20 & 4.69E-01 & ugl \\
\hline Selenium & $6 / 1 / 90$ & $4 / 30 / 89$ & $12 / 30 / 89$ & $4 / 27 / 89$ & $4 / 29 / 89$ & BOB2M4 & G313 & G363 & 1.20 & $4.68 \mathrm{E}-01$ & $u g /$ \\
\hline Selenium & $6 / 1 / 90$ & $4 / 30 / 89$ & $12 / 30 / 89$ & $4 / 27 / 89$ & $4 / 29 / 89$ & BOB2M1 & G308 & G353 & 1.20 & 4.64E-01 & $u g / h$ \\
\hline Selenium & $6 / 1 / 90$ & $4 / 30 / 89$ & $12 / 30 / 89$ & $4 / 27 / 89$ & $4 / 29 / 89$ & Вов2MO & G306 & G352 & 1.20 & $4.56 \mathrm{E}-01$ & $u g / i$ \\
\hline Selenium & $6 / 1 / 90$ & $4 / 30 / 89$ & $12 / 30 / 89$ & $4 / 27 / 89$ & $4 / 29 / 89$ & BOB2M1 & G308 & G353 & 1.20 & 4.56E-01 & ug/i \\
\hline Selenium & $6 / 1 / 90$ & $4 / 30 / 89$ & $12 / 30 / 89$ & $4 / 27 / 89$ & $4 / 29 / 89$ & ВОВ2Т9 & G327 & G443 & 1.20 & $4.24 E-01$ & $\mathrm{ug} / \mathrm{r}$ \\
\hline Selenium & $6 / 1 / 90$ & $4 / 30 / 89$ & $12 / 30 / 89$ & $4 / 27 / 89$ & $4 / 29 / 89$ & $8082 M 4$ & G313 & G363 & 1.20 & $4.22 E-01$ & $u g / t$ \\
\hline Selenium & $6 / 1 / 90$ & $4 / 30 / 89$ & $12 / 30 / 89$ & $4 / 27 / 89$ & $4 / 29 / 89$ & Вов2Т9 & G327 & G443 & 1.20 & $4.00 E-01$ & $u g / r$ \\
\hline Selenium & $6 / 1 / 90$ & $4 / 30 / 89$ & $12 / 30 / 89$ & $4 / 27 / 89$ & $4 / 29 / 89$ & $80 B 2 M 2$ & G309 & G358 & 1.20 & 3.98E-01 & $u g / r$ \\
\hline Selenium & $6 / 1 / 90$ & $4 / 30 / 89$ & $12 / 30 / 89$ & $4 / 27 / 89$ & $4 / 29 / 89$ & BOB2M2 & G309 & G358 & 1.20 & 3.95E-01 & $u g / r$ \\
\hline Selenium & $6 / 1 / 90$ & $4 / 30 / 89$ & $12 / 30 / 89$ & $4 / 27 / 89$ & $4 / 29 / 89$ & $80 B 2 L 7$ & G299 & G342 & 1.20 & $3.88 E-01$ & $u g / n$ \\
\hline Selenium & $6 / 1 / 90$ & $4 / 30 / 89$ & $12 / 30 / 89$ & $4 / 27 / 89$ & $4 / 29 / 89$ & $\mathrm{BOB} 2 \mathrm{MT}$ & 6319 & G460 & .1 .2 & $3.84 E-01$ & $u g / n$ \\
\hline Selenium & $6 / 1 / 90$ & $4 / 30 / 89$ & $12 / 30 / 89$ & $4 / 27 / 89$ & $4 / 29 / 89$ & BOB2M6 & G318 & G459 & 1.20 & 3.83E-01 & $u g / \pi$ \\
\hline Selenium & $6 / 1 / 90$ & $4 / 30 / 89$ & $12 / 30 / 89$ & $4 / 27 / 89$ & $4 / 29 / 89$ & BOB2M3 & G311 & G359 & 1.20 & $3.74 E-01$ & $u g / \pi$ \\
\hline Selenium & $6 / 1 / 90$ & $4 / 30 / 89$ & $12 / 30 / 89$ & $4 / 27 / 89$ & $4 / 29 / 89$ & ВОВ2M7 & G319 & G460 & 1.2 & $3.72 E-01$ & $u g / \pi$ \\
\hline Selenium & $6 / 1 / 90$ & $4 / 30 / 89$ & $12 / 30 / 89$ & $4 / 27 / 89$ & $4 / 29 / 89$ & ВOB2M3 & G311 & G359 & 1.20 & $3.69 E-01$ & ug/ $/ \pi$ \\
\hline Selenium & $6 / 1 / 90$ & $4 / 30 / 89$ & $12 / 30 / 89$ & $4 / 27 / 89$ & $4 / 29 / 89$ & В082M6 & G318 & G459 & 1.20 & 3.57E-01 & ug/m \\
\hline Selenium & $6 / 1 / 90$ & $4 / 30 / 89$ & $12 / 30 / 89$ & $4 / 27 / 89$ & $4 / 29 / 89$ & BOB2N4 & G323 & G341 & 1.20 & $3.51 E-01$ & $\mathrm{ug} / \mathrm{rr}$ \\
\hline Selenium & $6 / 1 / 90$ & $4 / 30 / 89$ & $12 / 30 / 89$ & $4 / 27 / 89$ & $4 / 29 / 89$ & BOB2N4 & G323 & G341 & 1.20 & $3.41 E-01$ & $u g /$ r \\
\hline
\end{tabular}




\begin{tabular}{|c|c|c|c|c|c|c|c|}
\hline \multirow[b]{2}{*}{ Code } & \multirow[b]{2}{*}{ Source } & \multicolumn{3}{|c|}{ Analyte Information } & \multirow[b]{2}{*}{$\begin{array}{c}\text { Reference } \\
\text { Number }\end{array}$} & \multirow[b]{2}{*}{$\begin{array}{l}\text { Validated } \\
\text { (Y/N) }\end{array}$} & \multirow[b]{2}{*}{ Notes } \\
\hline & & Analyte & $\begin{array}{l}\text { Conc. } \\
\text { (ugg/L) }\end{array}$ & Conc. (mol/L) & & & \\
\hline$P$ & Raw & Se & $5.63 E+02$ & 7.13E-06 & AP102-3 & $Y$ & $\begin{array}{l}\text { Density reported for } \mathrm{G} 476 \text { collected at } 99 \text { in from bottom of } \\
\text { tank at a } 270^{\circ} \text { angle and was keep heated to } 40^{\circ} \mathrm{C} \text {. }\end{array}$ \\
\hline $\mathbf{P}$ & Raw & $\mathrm{Se}$ & $5.63 E+02$ & $7.13 E-06$ & AP102-3 & $Y$ & $\begin{array}{l}\text { Density reported for } \mathrm{G} 477 \text { collected at } 182 \text { in from bottom } \\
\text { of tank at a } 30^{\circ} \text { angle and was keep heated to } 40^{\circ} \mathrm{C} \text {. }\end{array}$ \\
\hline $\mathbf{P}$ & Raw & $\mathrm{Se}$ & $4.69 E+02$ & $5.94 \mathrm{E}-06$ & AP102-3 & $\mathbf{Y}$ & $\begin{array}{l}\text { Density reported for G352 collected at } 34 \text { in from bottom of } \\
\text { tank at a } 30^{\circ} \text { angle. Sample was unheated, unheated } \\
\text { samples precipitated }\end{array}$ \\
\hline$P$ & Raw & Se & $4.68 E+02$ & 5.93E-06 & AP102-3 & $Y$ & $\begin{array}{l}\text { Density reported for } \mathrm{G} 363 \text { collected at } 18 \text { in from bottom of } \\
\text { tank at a } 150^{\circ} \text { angle. Sample was unheated, unheated } \\
\text { samples precipitated }\end{array}$ \\
\hline$P$ & Raw & $\mathrm{Se}$ & $4.64 E+02$ & 5.88E-06 & AP102-3 & $Y$ & $\begin{array}{l}\text { Density reported for } \mathrm{G} 352 \text { collected at } 34 \text { in from bottom of } \\
\text { tank at a } 30^{\circ} \text { angle. Sample was unheated, unheated } \\
\text { samples precipitated }\end{array}$ \\
\hline$P$ & Raw & $\mathrm{Se}$ & $4.56 E \div 02$ & 5.78E-06 & AP102-3 & $Y$ & $\begin{array}{l}\text { Density reported for } \mathrm{G} 352 \text { collected at } 34 \text { in from bottom of } \\
\text { tank at a } 30^{\circ} \text { angle. Sample was unheated, unheated } \\
\text { samples precipitated }\end{array}$ \\
\hline$P$ & Raw & $\mathrm{Se}$ & $4.56 E+02$ & 5.78E-06 & AP102-3 & $Y$ & $\begin{array}{l}\text { Density reported for } \mathrm{G} 352 \text { collected at } 34 \text { in from bottom of } \\
\text { tank at a } 30^{\circ} \text { angle. Sample was unheated, unheated } \\
\text { samples precipitated }\end{array}$ \\
\hline$P$ & Raw & $\mathrm{Se}$ & $4.24 E+02$ & 5.37E-06 & AP102-3 & $Y$ & Density reported for $\mathrm{G} 443$ represents a composite sample \\
\hline$P$ & Raw & Se & $4.22 E+02$ & 5.34E-06 & AP102-3 & $Y$ & $\begin{array}{l}\text { Density reported for G363 collected at } 18 \text { in from bottom of } \\
\text { tank at a } 150^{\circ} \text { angle. Sample was unheated, unheated } \\
\text { samples precipitated }\end{array}$ \\
\hline $\mathbf{P}$ & Raw & Se & $4.00 E+02$ & 5.07E-06 & AP102-3 & Y & Density reported for $\mathrm{G} 443$ represents a composite sample \\
\hline $\mathbf{P}$ & Raw & $\mathrm{Se}$ & $3.98 E+02$ & $5.04 E-06$ & AP102-3 & $Y$ & $\begin{array}{l}\text { Density reported for G358 collected at } 334 \text { in from bottom } \\
\text { of tank at a } 150^{\circ} \text { angle. Sample was unheated, unheated } \\
\text { samples precipitated }\end{array}$ \\
\hline$P$ & Raw & Se & $3.95 E+02$ & $5.00 E-06$ & AP102-3 & Y & $\begin{array}{l}\text { Density reported for } \mathrm{G} 358 \text { collected at } 334 \text { in from bottom } \\
\text { of tank at a } 150^{\circ} \text { angle. Sample was unheated, unheated } \\
\text { samples precipitated }\end{array}$ \\
\hline$P$ & Raw & Se & $3.88 E+02$ & 4.91E-06 & AP102-3 & $Y$ & $\begin{array}{l}\text { Density reported for } \mathrm{G} 342 \text { collected at } 306 \text { in from bottom } \\
\text { of tank at a } 30^{\circ} \text { angle. Sample was unheated, unheated } \\
\text { samples precipitated }\end{array}$ \\
\hline$P$ & Raw & Se & $3.84 E+02$ & $4.86 E-06$ & AP102-3 & $Y$ & $\begin{array}{l}\text { Density reported for } 6460 \text { collected at } 226 \text { in from bottom } \\
\text { of tank at a } 270^{\circ} \text { angle. Sample was unheated, unheated } \\
\text { samples precipitated }\end{array}$ \\
\hline$P$ & Raw & Se & $3.83 E+02$ & 4.85E-06 & AP102-3 & $\mathbf{Y}$ & $\begin{array}{l}\text { Density reported for G } 459 \text { collected at } 226 \text { in from bottom } \\
\text { of tank at a } 270^{\circ} \text { angle. Sample was unheated, unheated } \\
\text { samples precipitated }\end{array}$ \\
\hline$P$ & Raw & $\mathrm{Se}$ & $3.74 E+02$ & 4.74E-06 & AP102-3 & $Y$ & $\begin{array}{l}\text { Density reported for G359 collected at } 162 \text { in from bottom } \\
\text { of tank at a } 150^{\circ} \text { angle. Sample was unheated, unheated } \\
\text { samples precipitated }\end{array}$ \\
\hline$P$ & Raw & $\mathrm{Se}$ & $3.72 E+02$ & 4.71E-O6 & AP102-3 & $Y$ & $\begin{array}{l}\text { Density reported for } \mathrm{G} 460 \text { collected at } 226 \text { in from bottom } \\
\text { of tank at a } 270^{\circ} \text { angle. Sample was unheated, unheated } \\
\text { samples precipitated }\end{array}$ \\
\hline$P$ & Raw & Se & $3.69 E+02$ & $4.67 E-06$ & AP102-3 & $\gamma$ & $\begin{array}{l}\text { Density reported for } 6359 \text { collected at } 162 \text { in from bottom } \\
\text { of tank at a } 150^{\circ} \text { angle. Sample was unheated, unheated } \\
\text { samples precipitated }\end{array}$ \\
\hline$P$ & Raw & $\mathrm{Se}$ & $3.57 E+02$ & $4.52 E-06$ & AP102-3 & $Y$ & $\begin{array}{l}\text { Density reported for G459 collected at } 226 \text { in from bottom } \\
\text { of tank at a } 270^{\circ} \text { angle. Sample was unheated, unheated } \\
\text { samples precipitated }\end{array}$ \\
\hline$P$ & Raw & Se & $3.51 E+02$ & $4.45 E-06$ & AP102-3 & $Y$ & $\begin{array}{l}\text { Density reported for } \mathrm{G} 341 \text { collected at } 99 \text { in from bottom of } \\
\text { tank at a } 270^{\circ} \text { angle. }\end{array}$ \\
\hline $\mathbf{P}$ & Raw & $\mathrm{Se}$ & $3.41 E+02$ & 4.32E-06 & AP102-3 & $Y$ & $\begin{array}{l}\text { Density reported for } \mathrm{G} 341 \text { collected at } 99 \text { in from bottom o } \\
\text { tank at a } 270^{\circ} \text { angle. }\end{array}$ \\
\hline
\end{tabular}




\begin{tabular}{|c|c|c|c|c|c|c|c|c|c|c|c|}
\hline & & & & & ank Waste & haracteriza & ion Sample & ormation & & & \\
\hline Reported Chemical & $\begin{array}{c}\text { Memo } \\
\text { Date } \\
\end{array}$ & $\begin{array}{c}\text { Start } \\
\text { Analysis } \\
\text { Date } \\
\end{array}$ & $\begin{array}{c}\text { Finish } \\
\text { Analysis Date }\end{array}$ & $\begin{array}{c}\text { Start } \\
\text { Sample } \\
\text { Date } \\
\end{array}$ & $\begin{array}{c}\text { Finish } \\
\text { Sample } \\
\text { Date } \\
\end{array}$ & $\begin{array}{l}\text { Sample } \\
\text { Number }\end{array}$ & $\begin{array}{c}\text { Tank Farm } \\
\text { Number }\end{array}$ & $\begin{array}{c}\text { Lab Sample } \\
\text { Number }\end{array}$ & $\begin{array}{l}\text { Density } \\
(\mathrm{g} / \mathrm{mL})\end{array}$ & $\begin{array}{c}\text { Reported } \\
\text { Value } \\
\end{array}$ & $\begin{array}{r}\text { Rep } \\
\mathbf{U}\end{array}$ \\
\hline Selenium & $6 / 1 / 90$ & $4 / 30 / 89$ & $12 / 30 / 89$ & $4 / 27 / 89$ & $4 / 29 / 89$ & BOB2L7 & G299 & G342 & 1.20 & 3.40E-01 & ug \\
\hline Selenium & $6 / 1 / 90$ & $4 / 30 / 89$ & $12 / 30 / 89$ & $4 / 27 / 89$ & $4 / 29 / 89$ & BOB2L8 & G303 & G348 & 1.21 & $3.40 E-01$ & ugil \\
\hline Selenium & $6 / 1 / 90$ & $4 / 30 / 89$ & $12 / 30 / 89$ & $4 / 27 / 89$ & $4 / 29 / 89$ & BOB2L8 & G303 & G348 & 1.21 & 3.17E-01 & ugt \\
\hline Selenium & $6 / 1 / 90$ & $4 / 30 / 89$ & $12 / 30 / 89$ & $4 / 27 / 89$ & $4 / 29 / 89$ & BOB2L9 & G305 & G349 & 1.20 & $3.12 E-01$ & ugl \\
\hline Selenium & $6 / 1 / 90$ & $4 / 30 / 89$ & $12 / 30 / 89$ & $4 / 27 / 89$ & $4 / 29 / 89$ & BOB2L9 & G305 & G349 & 1.20 & 2.91E-01 & ugl \\
\hline Selenium & $6 / 1 / 90$ & $4 / 30 / 89$ & $12 / 30 / 89$ & $4 / 27 / 89$ & $4 / 29 / 89$ & BOB2M5 & G315 & G364 & 1.19 & $2.64 \mathrm{E}-01$ & $\mathrm{ug} /$ \\
\hline Selenium & $6 / 1 / 90$ & $4 / 30 / 89$ & $12 / 30 / 89$ & $4 / 27 / 89$ & $4 / 29 / 89$ & BOB2M5 & G315 & G364 & 1.19 & $2.56 E-01$ & $u g_{i}$ \\
\hline S042- & NS & NS & NS & NS & NS & NS & NS & NS & 1.24 & 1.42E-01 & MOI \\
\hline 504 & $4 / 28 / 84$ & NS & NS & NS & NS & $R 8469$ & NS & NS & NS & $1.48 \mathrm{E}-02$ & $\mathrm{M}$ \\
\hline 504 & $4 / 28 / 84$ & NS & NS & NS & NS & $\mathrm{R}-8469$ & NS & NS & NS & $1.48 \mathrm{E}-02$ & $\mathrm{~mol}$ \\
\hline 504 & $4 / 28 / 84$ & NS & NS & NS & NS & NS & NS & NS & NS & $1.42 \mathrm{E}+\infty 0$ & $\overline{g l}$ \\
\hline Sulfate & $6 / 1 / 90$ & $4 / 30 / 89$ & $12 / 30 / 89$ & $4 / 27 / 89$ & $4 / 29 / 89$ & BOB2NO & G307 & G527* & NA & $5.17 E+03$ & ugh \\
\hline Sulfate & $6 / 1 / 90$ & $4 / 30 / 89$ & $12 / 30 / 89$ & $4 / 27 / 89$ & $4 / 29 / 89$ & В0В2T9 & G327 & G443 & 1.20 & $5.03 E+03$ & ug/i \\
\hline Sulfate & $6 / 1 / 90$ & $4 / 30 / 89$ & $12 / 30 / 89$ & $4 / 27 / 89$ & $4 / 29 / 89$ & BOB2T9 & G327 & G443 & 1.20 & $4.99 E+03$ & ug/ \\
\hline Sulfate & $6 / 1 / 90$ & $4 / 30 / 89$ & $12 / 30 / 89$ & $4 / 27 / 89$ & $4 / 29 / 89$ & $80 \mathrm{~B} 2 \mathrm{M} 2$ & G309 & G515* & $(1.20)$ & $4.86 E+03$ & $\mathrm{ug} / \mathrm{t}$ \\
\hline Sulfate & $6 / 1 / 90$ & $4 / 30 / 89$ & $12 / 30 / 89$ & $4 / 27 / 89$ & $4 / 29 / 89$ & BOB2M6 & G318 & G517* & $(1.20)$ & $4.86 E+03$ & $\mathrm{ug} / \mathrm{t}$ \\
\hline Sulfate & $6 / 1 / 90$ & $4 / 30 / 89$ & $12 / 30 / 89$ & $4 / 27 / 89$ & $4 / 29 / 89$ & BOB2L7 & G299 & G509* & $(1.20)$ & $4.85 E+03$ & $u g / t$ \\
\hline Sulfate & $6 / 1 / 90$ & $4 / 30 / 89$ & $12 / 30 / 89$ & $4 / 27 / 89$ & $4 / 29 / 89$ & ВOB2M3 & G311 & G516* & $(7.20)$ & $4.85 E+03$ & ug/s \\
\hline Sulfate & $6 / 1 / 90$ & $4 / 30 / 89$ & $12 / 30 / 89$ & $4 / 27 / 89$ & $4 / 29 / 89$ & BOB2L8 & G303 & G510* & 1.21 & $4.84 E+03$ & $u g / r$ \\
\hline Sulfate & $6 / 1 / 90$ & $4 / 30 / 89$ & $12 / 30 / 89$ & $4 / 27 / 89$ & $4 / 29 / 89$ & BOB2M6 & G318 & G517* & $(1.20)$ & $4.83 E+03$ & ug/n \\
\hline Sulfate & $6 / 1 / 90$ & 4/30/89 & $12 / 30 / 89$ & $4 / 27 / 89$ & $4 / 29 / 89$ & $80 B 2 M 2$ & G309 & G515* & $(1.20)$ & $4.79 E+03$ & $u g / n$ \\
\hline Sulfate & $6 / 7 / 90$ & $4 / 30 / 89$ & $12 / 30 / 89$ & $4 / 27 / 89$ & $4 / 29 / 89$ & BOB2M9 & G302 & G477* & 1.20 & $4.78 E+03$ & ug/n \\
\hline Sulfate & $6 / 1 / 90$ & $4 / 30 / 89$ & $12 / 30 / 89$ & $4 / 27 / 89$ & $4 / 29 / 89$ & ВOВ2M9 & G302 & G477* & 1.20 & $4.77 E+03$ & ug/n \\
\hline
\end{tabular}




\begin{tabular}{|c|c|c|c|c|c|c|c|}
\hline \multirow[b]{2}{*}{ Code } & \multirow[b]{2}{*}{ Source } & \multicolumn{3}{|c|}{ Analyte Information } & \multirow[b]{2}{*}{$\begin{array}{c}\text { Reference } \\
\text { Number }\end{array}$} & \multirow[b]{2}{*}{$\begin{array}{c}\text { Validated } \\
\text { (Y/N) }\end{array}$} & \multirow[b]{2}{*}{ Notes } \\
\hline & & Anaiyte & $\begin{array}{l}\text { Conc. } \\
\text { (ug/L) }\end{array}$ & Conc. (mol/h) & & & \\
\hline $\mathbf{P}$ & Raw & Se & $3.40 E+02$ & 4.31E-06 & AP102-3 & $Y$ & $\begin{array}{l}\text { Density reported for G342 collected at } 306 \text { in from bottom } \\
\text { of tank at a } 30^{\circ} \text { angle. Sample was unheated, unheated } \\
\text { samples precipitated }\end{array}$ \\
\hline $\mathbf{P}$ & Raw & $\mathrm{Se}$ & $3.40 E+02$ & $4.31 E-06$ & AP102-3 & Y & $\begin{array}{l}\text { Density reported for G348 collected at } 125 \text { in from bottom } \\
\text { of tank at a } 30^{\circ} \text { angle. Sample was unheated, unheated } \\
\text { samples precipitated }\end{array}$ \\
\hline $\mathbf{P}$ & Raw & Se & $3.17 E+02$ & 4.01E-06 & AP102-3 & $Y$ & $\begin{array}{l}\text { Density reported for } \mathrm{G} 348 \text { collected at } 125 \text { in from bottom } \\
\text { of tank at a } 30^{\circ} \text { angle. Sample was unheared, unheated } \\
\text { samples precipitated }\end{array}$ \\
\hline$P$ & Raw & Se & $3.12 E+02$ & $3.95 E-06$ & AP102-3 & $Y$ & $\begin{array}{l}\text { Density reported for G349 collected at } 34 \text { in from bottom of } \\
\text { tank at a } 30^{\circ} \text { angle. Sample was unheated, unheated } \\
\text { samples precipitated }\end{array}$ \\
\hline$P$ & Raw & Se & $2.91 E+02$ & 3.69E-06 & AP102-3 & $Y$ & $\begin{array}{l}\text { Density reported for } \mathrm{G} 349 \text { collected at } 34 \text { in from bottom of } \\
\text { tank at a } 30^{\circ} \text { angle. Sample was unheated, unheated } \\
\text { samples precipitated }\end{array}$ \\
\hline $\mathbf{P}$ & Raw & $\mathrm{Se}$ & $2.64 E+02$ & $3.34 E-06$ & AP102-3 & $Y$ & $\begin{array}{l}\text { Density reported for G364 collected at } 372 \text { in from bottom } \\
\text { of tank at a } 270^{\circ} \text { angle. Sample was unheated, unheated } \\
\text { samples precipitated }\end{array}$ \\
\hline$P$ & Raw & Se & $2.56 \mathrm{E}+02$ & $3.24 E-06$ & AP102-3 & $Y$ & $\begin{array}{l}\text { Density reported for G364 collected at } 372 \text { in from bottom } \\
\text { of tank at a } 270^{\circ} \text { angle. Sample was unheated, unheated } \\
\text { samples precipitated }\end{array}$ \\
\hline L & HTCE Inv. & SO4 & $1.37 E+13$ & $1.43 E+05$ & NA & $\mathbf{N}$ & No Reference \\
\hline $\mathrm{L}$ & Raw & SO4 & $1.42 E+12$ & $1.48 E+04$ & AP102-1 & $N$ & Outdated information \\
\hline$P$ & Braun & 504 & $1.42 E+12$ & $1.48 E+04$ & & $\bar{N}$ & No Reference \\
\hline$P$ & Wastren & 504 & $1.42 \mathrm{E}+12$ & $1.48 \mathrm{E}+04$ & NS & $N$ & No Reference \\
\hline$P$ & Raw & so4 & $5.17 E+06$ & $5.39 E-02$ & AP102-3 & $\mathbf{Y}$ & $\begin{array}{l}\text { Density not reported for G527. No assumptions could be } \\
\text { made }\end{array}$ \\
\hline $\mathbf{P}$ & Raw & SO4 & $5.03 E \div 06$ & $5.24 E-02$ & AP102-3 & $Y$ & Density reported for $\mathbf{G 4 4 3}$ represents a composite sample \\
\hline $\mathbf{P}$ & Raw & so4 & $4.99 E+06$ & $5.20 \mathrm{E}-02$ & AP102-3 & $\mathbf{Y}$ & Density reported for $\mathrm{G} 443$ represents a composite sample \\
\hline $\mathbf{P}$ & Raw & SO4 & $4.86 \mathrm{E}+06$ & $5.06 \mathrm{E}-02$ & AP102-3 & $\mathbf{Y}$ & $\begin{array}{l}\text { Density not reported for G515. Density assumed same as } \\
6358 \text { which was collected at } 334 \text { in from bottom of tank at } \\
\text { a } 150^{\circ} \text { angle. This sample was heated. }\end{array}$ \\
\hline $\mathbf{P}$ & Raw & SO4 & $4.86 E+06$ & 5.06E-02 & AP102-3 & Y & $\begin{array}{l}\text { Density not reported for } G 517 \text {. Density assumed same as } \\
\text { G459 which was collected at } 226 \text { in from bottom of tank at } \\
\text { a } 270^{\circ} \text { angle. This sample was heated. }\end{array}$ \\
\hline $\mathbf{P}$ & Raw & SO4 & $4.85 E+06$ & $5.05 E-02$ & AP102-3 & Y & $\begin{array}{l}\text { Density not reported for G509. Density assumed same as } \\
\text { G342 which was collected at } 306 \text { in from bottom of tank at } \\
\text { a } 30^{\circ} \text { angle. This sample was heated. }\end{array}$ \\
\hline $\mathbf{P}$ & Raw & SO4 & $4.85 E+06$ & $5.05 \mathrm{E}-02$ & AP102-3 & $\mathbf{Y}$ & $\begin{array}{l}\text { Density not reported for G516. Density assumed same as } \\
\text { G359 which was collected at } 162 \text { in from bottom of tank at } \\
\text { a } 150^{\circ} \text { angle. This sample was heated. }\end{array}$ \\
\hline $\mathbf{P}$ & Raw & SO4 & $4.84 E+06$ & 5.04E-02 & AP102-3 & $\gamma$ & $\begin{array}{l}\text { Density not reported for G510. Density assumed same as } \\
\text { G348 which was collected at } 125 \text { in from bottom of tank at } \\
\text { a } 30^{\circ} \text { angle. This sample was heated. }\end{array}$ \\
\hline $\mathbf{P}$ & Raw & SO4 & $4.83 E+06$ & $5.03 \mathrm{E}-02$ & AP102-3 & $Y$ & $\begin{array}{l}\text { Density not reported for G517. Density assumed same as } \\
\text { G459 which was collected at } 226 \text { in from bottom of tank at } \\
\text { a } 270^{\circ} \text { angle. This sample was heated. }\end{array}$ \\
\hline$P$ & Raw & so4 & $4.79 E+06$ & 4.99E-02 & AP102-3 & $Y$ & $\begin{array}{l}\text { Density not reported for G515. Density assumed same as } \\
\text { G358 which was collected at } 334 \text { in from bottom of tank at } \\
\text { a } 150^{\circ} \text { angle. This sample was heated. }\end{array}$ \\
\hline$P$ & Raw & so4 & $4.78 E+06$ & $4.98 E-02$ & AP102-3 & $\mathbf{Y}$ & $\begin{array}{l}\text { Density reported for G477 collected at } 182 \text { in from bottom } \\
\text { of tank at a } 30^{\circ} \text { angle and was keep heated to } 40^{\circ} \mathrm{C} \text {. }\end{array}$ \\
\hline$P$ & Raw & SO4 & $4.77 E+06$ & 4.97E-02 & AP102-3 & $Y$ & $\begin{array}{l}\text { Density reported for } \mathrm{G} 477 \text { collected at } 182 \text { in from bottom } \\
\text { of tank at a } 30^{\circ} \text { angle and was keep heated to } 40^{\circ} \mathrm{C} \text {. }\end{array}$ \\
\hline
\end{tabular}


TWRS Privatization Support Proj. Chemical Liquids

\begin{tabular}{|c|c|c|c|c|c|c|c|c|c|}
\hline \multirow[b]{2}{*}{ Reported Chemical } & \multicolumn{9}{|c|}{ Tank Waste Characterization Sample Information } \\
\hline & $\begin{array}{c}\text { Memo } \\
\text { Date } \\
\end{array}$ & $\begin{array}{c}\text { Start Analysis } \\
\text { Date }\end{array}$ & $\begin{array}{c}\text { Sample } \\
\text { Date }\end{array}$ & $\begin{array}{l}\text { Sample } \\
\text { Number }\end{array}$ & $\begin{array}{c}\text { Tank Farm } \\
\text { Number }\end{array}$ & $\begin{array}{c}\text { Lab Sample } \\
\text { Number }\end{array}$ & $\begin{array}{c}\text { Density } \\
(\mathrm{g} / \mathrm{mL})\end{array}$ & $\begin{array}{c}\text { Reported } \\
\text { Value } \\
\end{array}$ & Reported Unit \\
\hline $\mathrm{Al3}+$ & NA & NA & NA & NA & NA & NA & 1.33 & $8.05 \mathrm{E}-01$ & Mole/L \\
\hline Aluminum & $3 / 1 / 93$ & NA & $3 / 10 / 93$ & $808 V \times 4$ & G238 & G401 & 1.389 & $1.40 \mathrm{E}+07$ & $\mathrm{UG} / \mathrm{L}$ \\
\hline Aluminum & $3 / 1 / 93$ & NA & $3 / 10 / 93$ & $\mathrm{~B} 08 \mathrm{~V} \times 8$ & G244 & G412 & 1.377 & $1.37 E+07$ & UG/L \\
\hline Aluminum & $3 / 1 / 93$ & NA & $3 / 10 / 93$ & $808 V \times 5$ & G240 & G402 & 1.338 & $1.24 E+07$ & UG/L \\
\hline Aluminum & $3 / 1 / 93$ & NA & $3 / 10 / 93$ & $808 \mathrm{~V} \times 9$ & G245 & G413 & 1.335 & $1.23 E+07$ & UG/L \\
\hline Aluminum & $3 / 1 / 93$ & NA & $3 / 10 / 93$ & B08VX6 & G241 & G407 & 1.338 & $1.19 E+07$ & UG/L \\
\hline Aluminum & $3 / 1 / 93$ & NA & $3 / 10 / 93$ & $\mathrm{NA}$ & G250 & G422 & 1.348 & $1.19 \varepsilon+07$ & UG/L \\
\hline Al & $3 / 1 / 93$ & NA & $3 / 10 / 93$ & NA & NA & NA & 1.336 & 11700 & $u g / m L$ \\
\hline Aluminum & $3 / 1 / 93$ & $\mathrm{NA}$ & $3 / 10 / 93$ & BOB3D1 & NA & G378 & 1.334 & $1.16 \mathrm{E}+07$ & UG/L \\
\hline Aluminum & $3 / 1 / 93$ & NA & $3 / 10 / 93$ & $\mathrm{BOB} 3 \mathrm{C} 8$ & G248 & G417 & 1.327 & $1.16 E+07$ & UG \\
\hline Aluminum & $3 / 1 / 93$ & NA & $3 / 10 / 93$ & BOB3D2 & NA & G379 & 1.329 & $1.15 E \div 07$ & $U G / L$ \\
\hline Aluminum & $3 / 1 / 93$ & NA & $3 / 10 / 93$ & B08VX1 & G253 & G392 & 1.341 & $1.13 E+07$ & UGL \\
\hline Aluminum & $3 / 1 / 93$ & NA & $3 / 10 / 93$ & $808 \vee \times 0$ & G251 & G391 & 1.344 & $1.11 E+07$ & UG/L \\
\hline Aluminum & $3 / 1 / 93$ & NA & $3 / 10 / 93$ & $808 \vee \times 7$ & G243 & G409 & 7.298 & $1.05 E+07$ & $U G / L$ \\
\hline Aluminum & $3 / 1 / 93$ & NA & $3 / 10 / 93$ & $\mathrm{~B} 08 \mathrm{~V} \times 3$ & G271 & G397 & 1.3 & $9.93 E+06$ & UG/L \\
\hline Aluminum & $3 / 1 / 93$ & NA & $3 / 10 / 93$ & B08VX2 & G254 & G396 & 1.298 & $9.76 E+06$ & $\mathrm{UG} / \mathrm{L}$ \\
\hline Aluminum & $3 / 1 / 93$ & NA & $3 / 10 / 93$ & B0B3C9 & G249 & G418 & 1.3 & $9.72 E+06$ & UG/L \\
\hline Arsenic & $3 / 1 / 93$ & NA & $3 / 10 / 93$ & B0B3D1 & $\mathrm{NA}$ & G378 & 1.334 & 1.03 & $\mathrm{ug} / \mathrm{mL}$ \\
\hline Arsenic & $3 / 1 / 93$ & NA & $3 / 10 / 93$ & BOB3D2 & NA & G379 & 1.329 & 0.787 & $\mathrm{ug} / \mathrm{mL}$ \\
\hline Barium & $3 / 1 / 93$ & NA & $3 / 10 / 93$ & $\mathrm{BOB} 3 \mathrm{C} 8$ & G248 & G417 & 1.327 & $<3.75 \mathrm{E}+02$ & UG/L \\
\hline Barium & $3 / 1 / 93$ & NA & $3 / 10 / 93$ & $B 08 \vee \times 7$ & G243 & G409 & 1.298 & $1.45 \mathrm{E}+03$ & UG/L \\
\hline Barium & $3 / 1 / 93$ & NA & $3 / 10 / 93$ & B08VX6 & G241 & G407 & 1.338 & $7.47 E+02$ & UG/L \\
\hline Barium & $3 / 1 / 93$ & NA & $3 / 10 / 93$ & B08VX3 & G271 & G397 & 1.3 & $5.97 E+02$ & UG/L \\
\hline Barium & $3 / 1 / 93$ & NA & $3 / 10 / 93$ & $\mathrm{BOB} 3 \mathrm{D} 2$ & NS & G379 & 1.329 & $5.47 E+02$ & $\mathrm{UG} / \mathrm{L}$ \\
\hline Barium & $3 / 1 / 93$ & NA & $3 / 10 / 93$ & B08VX8 & G244 & G412 & 1.377 & $5.44 \mathrm{E}+02$ & UG/L \\
\hline Barium & $3 / 1 / 93$ & NA & $3 / 10 / 93$ & B08VX1 & G253 & G392 & 1.341 & $5.40 E+02$ & $\mathbf{U G} / \mathrm{L}$ \\
\hline Barium & $3 / 1 / 93$ & NA & $3 / 10 / 93$ & B08V $\times 9$ & G245 & G413 & 1.335 & $5.35 E+02$ & UG/L \\
\hline Barium & $3 / 7 / 93$ & NA & $3 / 10 / 93$ & BOB3C9 & G249 & G418 & 1.3 & $5.32 \mathrm{E}+02$ & UG/L \\
\hline Barium & $3 / 1 / 93$ & NA & $3 / 10 / 93$ & $808 \mathrm{~V} \times 0$ & G251 & G391 & 1.344 & $5.23 E+02$ & UG/L \\
\hline Barium & $3 / 1 / 93$ & NA & $3 / 10 / 93$ & BOB3D1 & NS & G378 & 1.334 & $5.16 E+02$ & UG $/ L$ \\
\hline Barium & $3 / 1 / 93$ & $\mathrm{NA}$ & $3 / 10 / 93$ & $808 \vee \times 5$ & G240 & G402 & 1.338 & $5.08 E+02$ & $U G / L$ \\
\hline Barium & $3 / 1 / 93$ & NA & $3 / 10 / 93$ & B08VX2 & G254 & G396 & 1.298 & $4.82 E+02$ & UG/L \\
\hline Barium & $3 / 1 / 93$ & NA & $3 / 10 / 93$ & $B 08 \mathrm{~V} \times 4$ & G238 & G401 & 1.389 & $4.65 E+02$ & UG/L \\
\hline Beryllium & $3 / 1 / 93$ & NA & $3 / 10 / 93$ & $\mathrm{BOB3C8}$ & G248 & G417 & 1.327 & $<3.75 E+03$ & UG/L \\
\hline Beryllium & $3 / 1 / 93$ & NA & $3 / 10 / 93$ & B08VX4 & G238 & G401 & 1.389 & $1.68 E+03$ & UG/L \\
\hline Berylium & $3 / 1 / 93$ & $N A$ & $3 / 10 / 93$ & B08VX6 & G241 & G407 & 1.338 & $1.62 \mathrm{E}+03$ & UG/L \\
\hline Beryllium & $3 / 1 / 93$ & NA & $3 / 10 / 93$ & BOB3D2 & NS & G379 & 1.329 & $1.53 E+03$ & UG/L \\
\hline Bervillium & $3 / 1 / 93$ & NA & $3 / 10 / 93$ & $808 \mathrm{~V} \times 5$ & G240 & G402 & 1.338 & $1.51 \mathrm{E}+03$ & UG \\
\hline Bervilium & $3 / 1 / 93$ & $\mathrm{NA}$ & $3 / 10 / 93$ & B08VX1 & G253 & G392 & 1.341 & $1.47 E+03$ & UG/L \\
\hline Beryllium & $3 / 1 / 93$ & NA & $3 / 10 / 93$ & B0B3D1 & NS & G378 & 1.334 & $1.47 E+03$ & UGIL \\
\hline Beryllium & $3 / 1 / 93$ & NA & $3 / 10 / 93$ & B08VX0 & G251 & G391 & 1.344 & $1.46 E+03$ & UG $/ L$ \\
\hline Beryllium & $3 / 1 / 93$ & $N A$ & $3 / 10 / 93$ & $808 \vee \times 9$ & G245 & G413 & 1.335 & $1.44 E+03$ & UG/L \\
\hline Beryilium & $3 / 1 / 93$ & NA & $3 / 10 / 93$ & $\mathrm{BOB} 3 \mathrm{C} 9$ & G249 & G418 & 1.3 & $1.24 E+03$ & UG $\Omega$ \\
\hline Beryilium & $3 / 1 / 93$ & NA & $3 / 10 / 93$ & $\mathrm{~B} 08 \mathrm{~V} \times 3$ & G271 & G397 & 1.3 & $1.22 E+03$ & UG $/ \mathrm{L}$ \\
\hline Beryllium & $3 / 1 / 93$ & NA & $3 / 10 / 93$ & $808 V \times 2$ & G254 & G396 & 1.298 & 1.17E+03 & $\mathrm{UG} / \mathrm{L}$ \\
\hline Beryllium & $3 / 1 / 93$ & NA & $3 / 10 / 93$ & B08VX8 & G244 & G412 & 1.377 & $1.62 E+02$ & UG/L \\
\hline Bervilium & $3 / 1 / 93$ & NA & $3 / 10 / 93$ & B08VX7 & G243 & G409 & 1.298 & $1.40 E+02$ & UG/L \\
\hline Cadmium & $3 / 1 / 93$ & NA & $3 / 10 / 93$ & $\mathrm{BOB} 3 \mathrm{C} 8$ & G248 & G417 & 1.327 & $<7.50 \mathrm{E}+03$ & UG $/ L$ \\
\hline Cadmium & $3 / 1 / 93$ & NA & $3 / 10 / 93$ & BOB3D2 & NA & G379 & 1.329 & $2.05 E+03$ & $U G / L$ \\
\hline Cadmium & $3 / 1 / 93$ & NA & $3 / 10 / 93$ & B08VX3 & G271 & G397 & 1.3 & $1.97 E+03$ & UG/L \\
\hline
\end{tabular}




\begin{tabular}{|c|c|c|c|c|c|c|c|}
\hline \multirow[b]{2}{*}{ Zode } & \multirow[b]{2}{*}{ Source } & \multicolumn{3}{|c|}{ Analyte information } & \multirow[b]{2}{*}{$\begin{array}{l}\text { Reference } \\
\text { Number }\end{array}$} & \multirow[b]{2}{*}{$\begin{array}{c}\text { Validated } \\
(Y / N)\end{array}$} & \multirow[b]{2}{*}{ Notes } \\
\hline & & Analyte & Conc. (ug/L) & Conc. (mol/L) & & & \\
\hline $\mathbf{L}$ & HTCE Inv. & Al & $2.17 E+07$ & $8.05 \mathrm{E}-01$ & NA & $\bar{N}$ & HTCE \\
\hline$\overline{P-3}$ & Raw & Al & $1.40 E+07$ & $5.17 \mathrm{E}-01$ & AP105-2 & $\bar{Y}$ & Average of Primary and Duplicate results \\
\hline P-3 & Raw & Al & $1.37 E+07$ & $5.08 \mathrm{E}-01$ & AP105-2 & $\mathbf{Y}$ & Average of Primary and Duplicate results \\
\hline P-3 & Raw & Al & $1.24 \mathrm{E}+07$ & $4.60 \mathrm{E}-01$ & AP105-2 & $\bar{Y}$ & Average of Primary and Duplicate results \\
\hline P-3 & Raw & Al & $1.23 E+07$ & $4.56 \mathrm{E}-01$ & AP105-2 & $\bar{Y}$ & Average of Primary and Duplicate results \\
\hline P-3 & Raw & Al & $1.19 E+07$ & $4.41 \mathrm{E}-01$ & AP105-2 & $\mathbf{Y}$ & Average of Primary and Duplicate results \\
\hline P-3 & Raw & Al & $1.19 E+07$ & 4.41E-01 & AP105-2 & $\bar{Y}$ & Average of Primary and Duplicate results \\
\hline $\mathbf{P}$ & Raw & Al & $1.17 E+07$ & 4.34E-01 & AP105-2 & $\mathbf{Y}$ & $\begin{array}{l}\text { Statistical average of } 13 \text { indivisual tank samples and } \\
2 \text { composite samples, and representsthe "nominal } \\
\text { value" for this anaiyte }\end{array}$ \\
\hline P-3 & Raw & Al & $1.16 E+07$ & 4.30E-01 & AP105-2 & $\mathbf{Y}$ & Average of Primary and Duplicate results \\
\hline P.3 & Raw & Al & $1.16 \mathrm{E}+07$ & 4.28E-01 & AP105-2 & $\mathbf{Y}$ & Average of Primary and Duplicate results \\
\hline P-3 & Raw & Al & $1.15 E+07$ & $4.26 \mathrm{E}-01$ & AP105-2 & $\mathbf{Y}$ & Average of Primary and Duplicate results \\
\hline P-3 & Raw & Al & $1.13 E+07$ & 4.17E-01 & AP105-2 & $\mathbf{Y}$ & Average of Primary and Duplicate results \\
\hline P-3 & Raw & Al & $1.11 E+07$ & $4.11 \mathrm{E}-01$ & AP105-2 & $Y$ & Average of Primary and Duplicate results \\
\hline P-3 & Raw & Al & $1.05 E+07$ & $3.89 \mathrm{E}-01$ & AP105-2 & $\mathbf{Y}$ & Average of Primary and Duplicate results \\
\hline$\overline{P-3}$ & Raw & Al & $9.93 \mathrm{E}+06$ & $3.68 \mathrm{E}-01$ & AP105-2 & $\mathbf{Y}$ & Average of Primary and Duplicate results \\
\hline P.3 & Raw & Al & $9.76 E+06$ & $3.62 \mathrm{E}-01$ & AP105.2 & $Y$ & Average of Primary and Duplicate results \\
\hline$\underline{p-3}$ & Raw & $\mathrm{Al}$ & $9.72 E+06$ & 3.60E-01 & AP105-2 & $\bar{Y}$ & Average of Primary and Duplicate results \\
\hline$\overline{\mathbf{P}-3}$ & Raw & As & $1.03 E+03$ & $1.37 \mathrm{E}-05$ & AP105-2 & $Y$ & Average of Primary and Duplicate results \\
\hline p-3 & Raw & As & $7.87 E+02$ & $1.05 E-05$ & AP105-2 & $\mathbf{Y}$ & Average of Primary and Duplicate results \\
\hline $\mathbf{P}-3$ & Raw & $\mathrm{Ba}$ & $<3.75 E+02$ & \#VALUE! & AP105-2 & NR & Average of Primary and Duplicate results \\
\hline P.3 & Raw & $\mathbf{B a}$ & $1.45 E+03$ & $1.05 \mathrm{E}-05$ & AP105-2 & $\bar{Y}$ & Average of Primary and Duplicate results \\
\hline P-3 & Raw & $\mathrm{Ba}$ & $7.47 \mathrm{E}+02$ & 5.44E-06 & AP105-2 & NR & Average of Primary and Duplicate results \\
\hline $\mathrm{P}-3$ & Raw & $\mathrm{Ba}$ & $5.97 \mathrm{E}+02$ & 4.35E-06 & AP105-2 & NR & Average of Primary and Duplicate results \\
\hline$\overline{P-3}$ & Raw & $\mathrm{Ba}$ & $5.47 E+02$ & $3.98 \mathrm{E}-06$ & AP105-2 & NR & Average of Primary and Duplicate results \\
\hline P-3 & Raw & $\overline{\mathrm{Ba}}$ & $5.44 \mathrm{E}+02$ & 3.96E-06 & AP105-2 & NR & Average of Primary and Duplicate results \\
\hline$\overline{P-3}$ & Raw & $\mathrm{Ba}$ & $5.40 E+02$ & 3.93E-06 & AP105-2 & NR & Average of Primary and Duplicate results \\
\hline$\overline{P-3}$ & Raw & $\mathrm{Ba}$ & $5.35 E+02$ & 3.90E-06 & AP105-2 & NR & Average of Primary and Duplicate results \\
\hline$\overline{p-3}$ & Raw & $\mathrm{Ba}$ & $5.32 E+02$ & 3.87E-06 & AP105-2 & NR & Average of Primary and Duplicate results \\
\hline$\overline{p-3}$ & Raw & $\mathbf{B a}$ & $5.23 E+02$ & 3.81E-06 & AP105-2 & NR & Average of Primary and Duplicate results \\
\hline P-3 & Raw & $\mathrm{Ba}$ & $5.16 E+02$ & $3.76 \mathrm{E}-06$ & AP105-2 & NR & Average of Primary and Duplicate results \\
\hline$\overline{p-3}$ & Raw & $\mathrm{Ba}$ & $5.08 \mathrm{E}+02$ & $3.70 \mathrm{E}-06$ & AP105-2 & NR & Average of Primary and Duplicate results \\
\hline$\overline{p-3}$ & Raw & $8 \mathrm{Ba}$ & $4.82 E+02$ & 3.51E-06 & AP105-2 & NR & Average of Primary and Duplicate results \\
\hline$\overline{-3}$ & Raw & $\overline{\mathrm{Ba}}$ & $4.65 E+02$ & $3.39 E-06$ & AP105-2 & $\bar{Y}$ & Average of Primary and Duplicate results \\
\hline $3-3$ & Raw & $\mathrm{Be}$ & $<3.75 E+03$ & \#VALUE! & AP105-2 & NR & Average of Primary and Duplicate results \\
\hline $2-3$ & Raw & $\mathrm{Be}$ & $1.68 \mathrm{E}+03$ & $1.86 \mathrm{E}-04$ & AP105-2 & $\bar{Y}$ & Average of Primary and Duplicate results \\
\hline $2-3$ & Raw & $\mathrm{Be}$ & $1.62 \mathrm{E}+03$ & $1.79 E-04$ & AP105-2 & NR & Average of Primary and Duplicate results \\
\hline 3.3 & Raw & $\overline{\mathrm{Be}}$ & $1.53 E+03$ & 1.70E-04 & AP105-2 & NR & Average of Primary and Duplicate results \\
\hline$\overline{3-3}$ & Raw & $\overline{B e}$ & $1.51 \mathrm{E}+03$ & 1.67E-04 & AP105-2 & NR & Average of Primary and Duplicate results \\
\hline 3 & Raw & $\mathrm{Be}$ & $1.47 \mathrm{E}+03$ & $1.63 \mathrm{E}-04$ & AP105-2 & NR & Average of Primary and Duplicate results \\
\hline 5.3 & Raw & $\overline{\mathrm{Be}}$ & $1.47 E+03$ & $1.63 E-04$ & AP105-2 & NR & Average of Primary and Duplicate results \\
\hline $2-3$ & Raw & $\mathrm{Be}$ & $1.46 \mathrm{E}+03$ & $1.62 \mathrm{E}-04$ & AP105-2 & NR & Average of Primary and Duplicate results \\
\hline 3.3 & Raw & $\mathrm{Be}$ & $1.44 E+03$ & $1.60 \mathrm{E}-04$ & AP105-2 & NR & Average of Primary and Duplicate results \\
\hline 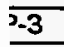 & Raw & $\mathrm{Be}$ & $1.24 \mathrm{E}+03$ & $1.37 E-04$ & AP105-2 & NR & Average of Primary and Duplicate results \\
\hline 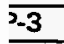 & Raw & $\overline{\mathrm{Be}}$ & $1.22 \mathrm{E}+03$ & $1.35 \mathrm{E}-04$ & AP105-2 & NR & Average of Primary and Duplicate results \\
\hline$\sqrt{-3}$ & Raw & $\mathrm{Be}$ & $1.17 \mathrm{E}+03$ & $1.30 \mathrm{E}-04$ & AP105-2 & NR & Average of Primary and Duplicate results \\
\hline$\overline{3}$ & Raw & $8 \mathrm{Be}$ & $1.62 E+02$ & $1.80 E-05$ & AP105-2 & NR & Average of Primary and Duplicate results \\
\hline$\overline{3-3}$ & Raw & $\overrightarrow{\mathrm{Be}}$ & $1.40 E+02$ & $1.55 \mathrm{E}-05$ & AP105-2 & NR & Average of Primary and Duplicate results \\
\hline 3 & Raw & $\mathrm{Cd}$ & $<7.50 E+03$ & \#VALUE! & AP105-2 & NR & Average of Primary and Duplicate results \\
\hline$\sqrt[3]{-3}$ & Raw & $\mathrm{Cd}$ & $2.05 E+03$ & 1.82E-05 & AP105-2 & $\mathrm{Y}$ & Average of Primary and Duplicate results \\
\hline$\overline{2-3}$ & Raw & $\overline{C d}$ & $1.97 E+03$ & $1.75 \mathrm{E}-05$ & AP105-2 & NR & Average of Primary and Duplicate results \\
\hline
\end{tabular}




\begin{tabular}{|c|c|c|c|c|c|c|c|c|c|}
\hline \multirow[b]{2}{*}{ Reported Chemical } & \multicolumn{9}{|c|}{ Tank Waste Characterization Sample Information } \\
\hline & $\begin{array}{c}\text { Memo } \\
\text { Date }\end{array}$ & $\begin{array}{c}\text { Start Analysis } \\
\text { Date }\end{array}$ & $\begin{array}{c}\text { Start } \\
\text { Sample } \\
\text { Date } \\
\end{array}$ & $\begin{array}{l}\text { Sample } \\
\text { Number }\end{array}$ & $\begin{array}{c}\text { Tank Farm } \\
\text { Number }\end{array}$ & \begin{tabular}{|c|} 
Lab Sample \\
Number
\end{tabular} & $\begin{array}{l}\text { Density } \\
\text { (g/mL) }\end{array}$ & $\begin{array}{l}\text { Reported } \\
\text { Value }\end{array}$ & Reported Unit \\
\hline Cadmium & $3 / 1 / 93$ & NA & $3 / 10 / 93$ & $808 \mathrm{~V} \times 7$ & G243 & G409 & 1.298 & $1.96 E+03$ & UG/L \\
\hline Cadmium & $3 / 1 / 93$ & NA & $3 / 10 / 93$ & $\mathrm{BOB3C9}$ & $\mathbf{G} 249$ & G418 & 1.3 & $1.87 \varepsilon+03$ & UG/L \\
\hline Cadmium & $3 / 1 / 93$ & NA & $3 / 10 / 93$ & $\mathrm{~B} 08 \mathrm{~V} \times 2$ & G254 & G396 & 1.298 & $1.78 \mathrm{E}+03$ & UG/L \\
\hline Cadmium & $3 / 1 / 93$ & NA & $3 / 10 / 93$ & $808 \mathrm{VX} 4$ & G238 & G401 & 1.389 & $1.73 E+03$ & UG/L \\
\hline Cadmium & $3 / 1 / 93$ & NA & $3 / 10 / 93$ & $B 08 \vee \times 8$ & G244 & G412 & 1.377 & $1.70 \mathrm{E}+03$ & UG/L \\
\hline Cadmium & $3 / 1 / 93$ & NA & $3 / 10 / 93$ & $808 \mathrm{X} \times 0$ & G251 & G391 & 1.344 & $1.64 E+03$ & UG/L \\
\hline Cadmium & $3 / 1 / 93$ & NA & $3 / 10 / 93$ & B08VX1 & G253 & G392 & 1.341 & $1.62 \mathrm{E}+03$ & UG $\Omega$ \\
\hline Cadmium & $3 / 1 / 93$ & NA & $3 / 10 / 93$ & B0B3D1 & NS & G378 & 1.334 & $1.99 E+02$ & UG/L \\
\hline Cadmium & $3 / 1 / 93$ & NA & $3 / 10 / 93$ & B08VX6 & G241 & G407 & 1.338 & $1.81 E+02$ & UG/L \\
\hline Cadmium & $3 / 1 / 93$ & NA & $3 / 10 / 93$ & $808 \mathrm{~V} \times 5$ & G240 & $\mathrm{G} 402$ & 1.338 & $1.75 E+02$ & UG/L \\
\hline Cadmium & $3 / 1 / 93$ & NA & $3 / 10 / 93$ & B08VX9 & G245 & G413 & 1.335 & $1.75 E+02$ & $U G / L$ \\
\hline Chloride & $3 / 1 / 93$ & NA & $3 / 10 / 93$ & B08VX6 & G241 & G407 & 1.338 & $6.96 E+03$ & $\mathrm{ug} / \mathrm{mL}$ \\
\hline $\mathrm{Cl}-$ & NA & NA & NA & NA & NA & $\mathrm{NA}$ & 1.33 & 1.22E-01 & Mole/L \\
\hline Chloride & $3 / 1 / 93$ & NA & $3 / 10 / 93$ & B08VX5 & G240 & G402 & 1.338 & $4.07 E+03$ & $\mathrm{ug} / \mathrm{mL}$ \\
\hline Chloride & $3 / 1 / 93$ & NA & $3 / 10 / 93$ & $808 \mathrm{~V} \times 4$ & G238 & G401 & 1.389 & $3.36 E+03$ & $\mathrm{ug} / \mathrm{mL}$ \\
\hline Chloride & $3 / 1 / 93$ & NA & $3 / 10 / 93$ & $808 \mathrm{VX7}$ & G243 & G409 & 1.298 & $3.23 E+03$ & $\mathrm{ug} / \mathrm{mL}$ \\
\hline Chloride & $3 / 1 / 93$ & NA & $3 / 10 / 93$ & BOB3D2 & NA & G379 & 1.329 & $2.69 E+03$ & ug/mL \\
\hline Chloride & $3 / 1 / 93$ & NA & $3 / 10 / 93$ & Bo8VX2 & G254 & G396 & 1.298 & $2.68 E+03$ & $\mathrm{ug} / \mathrm{mL}$ \\
\hline Chloride & $3 / 1 / 93$ & NA & $3 / 10 / 93$ & B08VX4 & G238 & G401 & 1.389 & $2.68 \mathrm{E}+03$ & $\mathrm{ug} / \mathrm{mL}$ \\
\hline Chloride & $3 / 1 / 93$ & NA & $3 / 10 / 93$ & BOB3D1 & NA & G378 & 1.334 & $2.65 E+03$ & $\mathrm{ug} / \mathrm{mL}$ \\
\hline Chloride & $3 / 1 / 93$ & NA & $3 / 10 / 93$ & $B 08 V \times 1$ & G253 & G392 & 1.341 & $2.64 E+03$ & $\mathrm{ug} / \mathrm{mL}$ \\
\hline Chloride & $3 / 1 / 93$ & NA & $3 / 10 / 93$ & B08VX6 & G241 & G407 & 1.338 & $2.62 E+03$ & $\mathrm{ug} / \mathrm{mL}$ \\
\hline Chloride & $3 / 1 / 93$ & NA & $3 / 10 / 93$ & $80 B 3 \mathrm{C} 9$ & G249 & G418 & 1.3 & $2.58 E+03$ & $4 \mathrm{~g} / \mathrm{mL}$ \\
\hline $\mathrm{Cl}^{-}$ & $3 / 1 / 93$ & NA & $3 / 10 / 93$ & NA & NA & NA & 1.336 & $2.49 E+03$ & $u g / m L$ \\
\hline Chloride & $3 / 1 / 93$ & NA & $3 / 10 / 93$ & $808 \mathrm{~V} \times 8$ & G244 & $\mathrm{G} 412$ & 1.377 & $2.40 E+03$ & $\mathrm{ug} / \mathrm{mL}$ \\
\hline $\mathrm{Cl}^{\circ}$. & $3 / 1 / 93$ & NA & $3 / 10 / 93$ & NA & NA & NA & 1.336 & $2.36 E+03$ & $\mathrm{ug} / \mathrm{mL}$ \\
\hline Chloride & $3 / 1 / 93$ & NA & $3 / 10 / 93$ & B08VX5 & G240 & G402 & 1.338 & $2.28 E+03$ & $\mathrm{ug} / \mathrm{mL}$ \\
\hline Chloride & $3 / 1 / 93$ & NA & $3 / 10 / 93$ & Bo8VX9 & G245 & G413 & 1.335 & $2.26 \mathrm{E}+03$ & $\mathrm{ug} / \mathrm{mL}$ \\
\hline Chloride & $3 / 1 / 93$ & NA & $3 / 10 / 93$ & $\mathrm{~B} 08 \mathrm{~V} \times 6$ & G241 & G407 & 1.338 & $2.19 E+03$ & $\mathrm{ug} / \mathrm{mL}$ \\
\hline Chloride & $3 / 1 / 93$ & NA & $3 / 10 / 93$ & B08VX0 & G251 & G391 & 1.344 & $2.18 \mathrm{E}+03$ & $\mathrm{ug} / \mathrm{mL}$ \\
\hline Chloride & $3 / 1 / 93$ & NA & $3 / 10 / 93$ & BOBVX3 & G271 & G397 & 1.3 & $1.99 E+03$ & $\mathrm{ug} / \mathrm{mL}$ \\
\hline Chloride & $3 / 1 / 93$ & NA & $3 / 10 / 93$ & $\mathrm{BOB3C8}$ & G248 & G417 & 1.327 & $1.97 \varepsilon+03$ & $\mathrm{ug} / \mathrm{mL}$ \\
\hline Chloride & $3 / 1 / 93$ & NA & $3 / 10 / 93$ & B08VX7 & G243 & G409 & 1.298 & $1.87 E+03$ & $\mathrm{ug} / \mathrm{mL}$ \\
\hline Chloride & $3 / 1 / 93$ & NA & $3 / 10 / 93$ & B08VX2 & G254 & G396 & 1.298 & $1.76 E+03$ & $\mathrm{ug} / \mathrm{mL}$ \\
\hline Chloride & $3 / 1 / 93$ & NA & $3 / 10 / 93$ & B08VX7 & G243 & G409 & 1.298 & $1.74 E+03$ & $u g / m L$ \\
\hline Chloride & $3 / 1 / 93$ & NA & $3 / 10 / 93$ & $\mathrm{~B} 08 \mathrm{~V} \times 3$ & G271 & G397 & 1.3 & $1.73 E+03$ & $\mathrm{ug} / \mathrm{mL}$ \\
\hline Chloride & $3 / 1 / 93$ & NA & $3 / 10 / 93$ & BOB3C9 & G249 & G418 & 1.3 & $1.68 \mathrm{E}+03$ & $\mathrm{ug} / \mathrm{mL}$ \\
\hline Chloride & $3 / 1 / 93$ & NA & $3 / 10 / 93$ & $\mathrm{~B} 08 \mathrm{~V} \times 2$ & G254 & G396 & 1.298 & $1.46 \mathrm{E}+03$ & $\mathrm{ug} / \mathrm{mL}$ \\
\hline Chloride & $3 / 1 / 93$ & NA & $3 / 10 / 93$ & BOB3C9 & G249 & G418 & 1.3 & $1.14 \mathrm{E}+03$ & $\mathrm{ug} / \mathrm{mL}$ \\
\hline Cyanide & $3 / 1 / 93$ & NA & $3 / 10 / 93$ & BOB3D1 & NA & G378 & 1.334 & $1.89 \mathrm{E}+01$ & $\mathrm{ug} / \mathrm{mL}$ \\
\hline Cyanide & $3 / 1 / 93$ & NA & $3 / 10 / 93$ & 808302 & NA & G379 & 1.329 & $1.77 E+01$ & $u \mathrm{~g} / \mathrm{mL}$ \\
\hline Chromium & $3 / 1 / 93$ & $\mathrm{NA}$ & $3 / 10 / 93$ & B08VX4 & G238 & G401 & 1.389 & $2.19 E+05$ & $\mathrm{UG} / \mathrm{L}$ \\
\hline Chromium & $3 / 1 / 93$ & $\mathrm{NA}$ & $3 / 10 / 93$ & B08VX8 & G244 & $\mathrm{G} 412$ & 1.377 & $2.07 E+05$ & $U G \Omega$ \\
\hline Chromium & $3 / 1 / 93$ & $\mathrm{NA}$ & $3 / 10 / 93$ & $\mathrm{BOB3C8}$ & G248 & G417 & 1.327 & $1.99 E+05$ & UG $\Omega$ \\
\hline Chromium & $3 / 1 / 93$ & NA & $3 / 10 / 93$ & B08VX6 & G241 & G407 & 1.338 & $1.97 E+05$ & $U G \Omega$ \\
\hline Chromium & $3 / 1 / 93$ & NA & $3 / 10 / 93$ & NA & 6250 & G422 & 1.348 & $1.97 E+05$ & UG/L \\
\hline
\end{tabular}




\begin{tabular}{|c|c|c|c|c|c|c|c|}
\hline \multirow[b]{2}{*}{ Code } & \multirow[b]{2}{*}{ Source } & \multicolumn{3}{|c|}{ Analyte Information } & \multirow[b]{2}{*}{$\begin{array}{l}\text { Reference } \\
\text { Number }\end{array}$} & \multirow[b]{2}{*}{$\begin{array}{l}\text { Validated } \\
\qquad(Y / N)\end{array}$} & \multirow[b]{2}{*}{ Notes } \\
\hline & & Analyte & Conc. (ug/L) & Conc. (mol/L) & & & \\
\hline P-3 & Raw & $\mathrm{Cd}$ & $1.87 E+03$ & $1.66 \mathrm{E}-05$ & AP105-2 & NR & Average of Primary and Duplicate results \\
\hline $\mathrm{P}-3$ & Raw & Cd & $1.78 \mathrm{E}+03$ & $1.58 \mathrm{E}-05$ & AP105-2 & NR & Average of Primary and Duplicate results \\
\hline $\mathbf{P - 3}$ & Raw & $\mathrm{Cd}$ & $1.73 E+03$ & $1.53 \mathrm{E}-05$ & AP105-2 & NR & Average of Primary and Duplicate results \\
\hline$P-3$ & Raw & Cd & $1.62 \mathrm{E}+03$ & 1.44E-05 & AP105-2 & NR & Average of Primary and Duplicate results \\
\hline P-3 & Raw & $\mathrm{Cd}$ & $1.99 E+02$ & 1.77E-06 & AP105-2 & NR & Average of Primary and Duplicate results \\
\hline $\mathrm{P}-3$ & Raw & $\mathrm{Cd}$ & $1.81 E+02$ & $1.61 E-06$ & AP105-2 & NR & Average of Primary and Duplicate results \\
\hline $\mathrm{P}-\mathbf{3}$ & Raw & $\mathrm{Cd}$ & $1.75 E+02$ & 1.56E-06 & AP105-2 & NR & Average of Primary and Duplicate results \\
\hline$P-3$ & Raw & $\mathrm{Cd}$ & $1.75 \mathrm{E} \div 02$ & 1.56E-06 & AP105-2 & NR & Average of Primary and Duplicate results \\
\hline P-3 & Raw & $\mathrm{Cl}$ & $6.96 E+06$ & $1.96 \mathrm{E}-01$ & AP105-2 & Y & $\begin{array}{l}\text { Average of Primary and Duplicate results; Possible } \\
\text { outlier }\end{array}$ \\
\hline P-3 & Raw & $\mathrm{Cl}$ & $2.69 E+06$ & 7.59E-02 & AP105-2 & $\bar{Y}$ & Average of Primary and Duplicate results \\
\hline P-3 & Raw & $\mathrm{Cl}$ & $2.68 E \div 06$ & 7.56E-02 & AP105-2 & $Y$ & Added / Average of Primary and Duplicate results \\
\hline $\mathrm{P}-3$ & Raw & $\overline{\mathrm{Cl}}$ & $2.68 E+06$ & $7.56 \mathrm{E}-02$ & AP105-2 & $\bar{Y}$ & Average of Primary and Duplicate results \\
\hline$P-3$ & Raw & $\mathrm{Cl}$ & $2.65 E \div 06$ & 7.46E-02 & AP105-2 & $\bar{Y}$ & Average of Primary and Duplicate results \\
\hline$P-3$ & Raw & $\mathrm{Cl}$ & $2.64 E+06$ & $7.43 E-02$ & AP105-2 & $\bar{Y}$ & Average of Primary and Duplicate results \\
\hline $\mathrm{P}-3$ & Raw & $\mathrm{Cl}$ & $2.62 \mathrm{E}+06$ & $7.39 E-02$ & AP105-2 & $\bar{Y}$ & Average of Primary and Duplicate results \\
\hline $\mathrm{P}-\mathbf{3}$ & Raw & $\mathrm{Cl}$ & $2.58 E+06$ & $7.28 E-02$ & AP105-2 & $\bar{Y}$ & Average of Primary and Duplicate results \\
\hline $\mathbf{P}$ & Raw & $\mathrm{Cl}$ & $2.49 E+06$ & 7.02E-02 & AP105-2 & $Y$ & $\begin{array}{l}\text { Average of all } 30 \text { or more sample results, including } \\
\text { outliers }\end{array}$ \\
\hline P-3 & Raw & $\mathrm{Cl}$ & $2.40 E+06$ & 6.76E-02 & AP105-2 & $\bar{Y}$ & Average of Primary and Duplicate results \\
\hline $\mathbf{P}$ & Raw & $\mathrm{Cl}$ & 2.3 & 6.66E-02 & AP105-2 & $Y$ & $\begin{array}{l}\text { Average of } 30 \text { or fewer sample results with outliers } \\
\text { removed; represents "nominal value" }\end{array}$ \\
\hline P-3 & Raw & $\mathrm{Cl}$ & $1.76 E+06$ & 4.96E-02 & AP105-2 & Y & Added / Average of Primary and Duplicate results \\
\hline P-3 & Raw & $\mathrm{Cl}$ & $1.74 E+06$ & 4.91E-02 & AP105-2 & Y & Added / Average of Primary and Duplicate results \\
\hline P.3 & Raw & $\mathrm{Cl}$ & $1.73 E+06$ & 4.87E-02 & AP105-2 & $\bar{Y}$ & Average of Primary and Duplicate results \\
\hline P-3 & Raw & $\mathrm{Cl}$ & $1.68 \mathrm{E}+06$ & $4.72 \mathrm{E}-02$ & AP105-2 & $\bar{Y}$ & Average of Primary and Duplicate results \\
\hline P.3 & Raw & $\mathrm{Cl}$ & $1.46 E+06$ & $4.12 \mathrm{E}-02$ & AP105-2 & $\bar{Y}$ & Average of Primary and Duplicate results \\
\hline$\overline{\mathbf{P}-3}$ & Raw & $\mathrm{Cl}$ & $1.14 \mathrm{E}+06$ & $3.20 \mathrm{E}-02$ & AP105-2 & $\bar{Y}$ & Average of Primary and Duplicate results \\
\hline P-3 & Raw & $\overline{\mathrm{CN}}$ & $1.89 E+04$ & 7.27E-04 & AP105-2 & $\bar{Y}$ & Average of Primary and Duplicate results \\
\hline$\overline{P-3}$ & Raw & $\mathrm{CN}$ & $1.77 E+04$ & $6.81 E-04$ & AP105-2 & $\bar{Y}$ & Average of Primary and Duplicate results \\
\hline P-3 & Raw & $\mathrm{Cr}$ & $2.19 E+05$ & $4.21 \mathrm{E}-03$ & AP105-2 & $\bar{Y}$ & Average of Primary and Duplicate results \\
\hline$\overline{\mathrm{P}-3}$ & Raw & $\mathrm{Cr}$ & $2.07 E+05$ & $3.97 E-03$ & AP105-2 & $\bar{Y}$ & Average of Primary and Duplicate results \\
\hline$\overline{\mathrm{P}-3}$ & Raw & $\mathrm{Cr}$ & $1.99 E+05$ & $3.83 E-03$ & AP105-2 & $\bar{Y}$ & Average of Primary and Duplicate results \\
\hline$\overline{P-3}$ & Raw & $\mathrm{Cr}$ & $1.97 E+05$ & $3.79 E-03$ & AP105-2 & $\bar{Y}$ & Average of Primary and Duplicate results \\
\hline$\overline{P-3}$ & Raw & $\mathrm{Cr}$ & $1.97 E+05$ & $3.79 E-03$ & AP105-2 & $\bar{Y}$ & \\
\hline
\end{tabular}




\begin{tabular}{|c|c|c|c|c|c|c|c|c|c|}
\hline \multirow[b]{2}{*}{ Reported Chemical } & \multicolumn{9}{|c|}{ Tank Waste Characterization Sample information } \\
\hline & $\begin{array}{c}\text { Memo } \\
\text { Date }\end{array}$ & $\begin{array}{c}\text { Start Analysis } \\
\text { Date }\end{array}$ & $\begin{array}{c}\text { Start } \\
\text { Sample } \\
\text { Date }\end{array}$ & $\begin{array}{l}\text { Sample } \\
\text { Number }\end{array}$ & $\begin{array}{c}\text { Tank Farm } \\
\text { Number }\end{array}$ & $\begin{array}{c}\text { Lab Sample } \\
\text { Number }\end{array}$ & $\begin{array}{l}\text { Density } \\
\text { (g/mL) }\end{array}$ & $\begin{array}{l}\text { Reported } \\
\text { Value }\end{array}$ & Reported Unit \\
\hline Chromium & $3 / 1 / 93$ & NA & $3 / 10 / 93$ & BOB3D2 & NA & G379 & 1.329 & $1.96 \mathrm{E}+05$ & UG/L \\
\hline Chromium & $3 / 1 / 93$ & NA & $3 / 10 / 93$ & $808 V \times 5$ & G240 & G402 & 1.338 & $1.94 E+05$ & UG/L \\
\hline Chromium & $3 / 1 / 93$ & NA & $3 / 10 / 93$ & B0B3D1 & $\mathrm{NA}$ & G378 & 1.334 & $1.94 \mathrm{E}+05$ & $U G / L$ \\
\hline $\mathrm{Cr}$ & $3 / 1 / 93$ & NA & $3 / 10 / 93$ & NA & NA & NA & 1.336 & $1.87 E+02$ & $\mathrm{ug} / \mathrm{mL}$ \\
\hline Chromium & $3 / 1 / 93$ & NA & $3 / 10 / 93$ & $808 \mathrm{~V} \times 9$ & G245 & G413 & 1.335 & $1.87 E+05$ & UG/L \\
\hline Chromium & $3 / 1 / 93$ & NA & $3 / 10 / 93$ & $\mathrm{~B} 08 \mathrm{~V} \times 1$ & G253 & G392 & 1.341 & $1.80 E+05$ & UG/L \\
\hline Chromium & $3 / 1 / 93$ & $\overline{N A}$ & $3 / 10 / 93$ & B08VX0 & G251 & G391 & 1.344 & $1.79 E+05$ & UG/L \\
\hline Chromium & $3 / 1 / 93$ & NA & $3 / 10 / 93$ & $808 \mathrm{~V} \times 7$ & G243 & G409 & 1.298 & $1.76 E+05$ & UG $/ \mathrm{L}$ \\
\hline Chromium & $3 / 1 / 93$ & NA & $3 / 10 / 93$ & BOB3C9 & G249 & G418 & 1.3 & $1.60 E+05$ & UG $/ L$ \\
\hline Chromium & $3 / 1 / 93$ & NA & $3 / 10 / 93$ & B08VX3 & G271 & G397 & 1.3 & $1.54 E+05$ & UG/L \\
\hline Chromium & $3 / 1 / 93$ & NA & $3 / 10 / 93$ & B08VX2 & G254 & G396 & 1.298 & $1.45 E+05$ & UG/L \\
\hline Fluoride & $3 / 1 / 93$ & NA & $3 / 10 / 93$ & B08VX0 & G251 & G391 & 1.344 & $<4.01 E+01$ & $\mathrm{ug} / \mathrm{mL}$ \\
\hline Fluoride & $3 / 1 / 93$ & NA & $3 / 10 / 93$ & BO8VX1 & G253 & G392 & 1.341 & $<4.01 E+01$ & $\mathrm{ug} / \mathrm{mL}$ \\
\hline Fluoride & $3 / 1 / 93$ & NA & $3 / 10 / 93$ & Bo8VX2 & G254 & G396 & 1.298 & $<2.01 E+01$ & $\mathrm{ug} / \mathrm{mL}$ \\
\hline Fluoride & $3 / 1 / 93$ & NA & $3 / 10 / 93$ & $808 V \times 3$ & G271 & G397 & 1.3 & $<4.01 E+01$ & $\mathrm{ug} / \mathrm{mL}$ \\
\hline Fluoride & $3 / 1 / 93$ & NA & $3 / 10 / 93$ & $B 08 V \times 3$ & G271 & G397 & 1.3 & $<2.01 E+01$ & $\mathrm{ug} / \mathrm{mL}$ \\
\hline Fluoride & $3 / 1 / 93$ & NA & $3 / 10 / 93$ & B08VX4 & G238 & G401 & 1.389 & $<1.01 E+01$ & $\mathrm{ug} / \mathrm{mL}$ \\
\hline Fluoride & $3 / 1 / 93$ & NA & $3 / 10 / 93$ & $808 V \times 5$ & G240 & G402 & 1.338 & $<5.10 E+00$ & $\mathrm{ug} / \mathrm{mL}$ \\
\hline Fluoride & $3 / 1 / 93$ & NA & $3 / 10 / 93$ & Bo8VX6 & G241 & G407 & 1.338 & $<2.09 E+02$ & $\mathrm{ug} / \mathrm{mL}$ \\
\hline Fluoride & $3 / 1 / 93$ & NA & $3 / 10 / 93$ & вов3С & G249 & G418 & 1.3 & $<1.68 \mathrm{E}+02$ & $u g / m L$ \\
\hline Fluoride & $3 / 1 / 93$ & NA & $3 / 10 / 93$ & BOB3C9 & G249 & G418 & 1.3 & $<2.01 E+01$ & $u g / m L$ \\
\hline Fluoride & $3 / 1 / 93$ & NA & $3 / 10 / 93$ & BOB3D1 & NS & G378 & 1.334 & $<1.01 E+01$ & $u g / m L$ \\
\hline Fluoride & $3 / 1 / 93$ & NA & $3 / 10 / 93$ & $\mathrm{BOB} 302$ & NS & G379 & 1.329 & $<1.01 E+01$ & $u g / m L$ \\
\hline$F$ & $3 / 1 / 93$ & NA & $3 / 10 / 93$ & NA & NA & NA & 1.336 & $<8.86 E+03$ & $\mathrm{ug} / \mathrm{mL}$ \\
\hline Fluoride & $3 / 1 / 93$ & NA & $3 / 10 / 93$ & $808 V \times 2$ & G254 & G396 & 1.298 & $8.78 E+03$ & $\mathrm{ug} / \mathrm{mL}$ \\
\hline F. & $\mathrm{NA}$ & NA & $\mathrm{NA}$ & NA & NA & $\mathrm{NA}$ & 1.33 & $2.18 E-01$ & Mole/L \\
\hline Fluoride & $3 / 1 / 93$ & NA & $3 / 10 / 93$ & NA & NA & P1530 & 1.332 & $1.54 E+03$ & $\mathrm{ug} / \mathrm{mL}$ \\
\hline Fluoride & $3 / 1 / 93$ & NA & $3 / 10 / 93$ & вовзС8 & G248 & G417 & 1.327 & $1.40 E+03$ & $u g / m L$ \\
\hline Fluoride & $3 / 1 / 93$ & NA & $3 / 10 / 93$ & B08VX8 & G244 & G412 & 1.377 & $6.57 E \div 02$ & $\mathrm{ug} / \mathrm{mL}$ \\
\hline Iron & $3 / 1 / 93$ & NA & $3 / 10 / 93$ & $\mathrm{~B} 0 \mathrm{B3C} 8$ & G248 & G417 & 1.327 & $<1.92 \mathrm{E}+04$ & UG/L \\
\hline $\mathrm{Fe} 3+($ total Fe) & NA & $\mathrm{NA}$ & NA & NA & NA & NA & 1.33 & $9.24 E-03$ & Mole/L \\
\hline Iron & $3 / 1 / 93$ & NA & $3 / 10 / 93$ & $808 \mathrm{~V} \times 3$ & G271 & G397 & 1.3 & $9.67 E+03$ & UG/L \\
\hline
\end{tabular}




\begin{tabular}{|c|c|c|c|c|c|c|c|}
\hline & & & nalyte informat & & & & \\
\hline Sode & Source & Analyte & Conc. (ug/L) & $\mid$ Conc. (mol/h) & $\begin{array}{l}\text { Reference } \\
\text { Number }\end{array}$ & $\begin{array}{l}\text { Validated } \\
(Y / N)\end{array}$ & Notes \\
\hline P-3 & Raw & $\mathrm{Cr}$ & $1.96 \mathrm{E}+05$ & $3.76 \mathrm{E}-03$ & AP105-2 & $Y$ & Average of Primary and Duplicate results \\
\hline $\mathrm{P}-3$ & Raw & $\mathrm{Cr}$ & $1.94 E+05$ & $3.73 \mathrm{E}-03$ & AP105-2 & $\bar{Y}$ & Average of Primary and Duplicate results \\
\hline$\overline{P-3}$ & Raw & $\mathrm{Cr}$ & $1.94 E+05$ & $3.73 E-03$ & AP105-2 & $\bar{Y}$ & Average of Primary and Duplicate results \\
\hline $\mathbf{P}$ & Raw & $\mathrm{Cr}$ & $1.87 E+05$ & $3.60 \mathrm{E}-03$ & AP105-2 & Y & $\begin{array}{l}\text { Statistical average of } 13 \text { individual tank samples and } \\
2 \text { composite samples, and represents the "nomonal } \\
\text { value" for this analyte. }\end{array}$ \\
\hline$\overline{P-3}$ & Raw & $\mathrm{Cr}$ & $1.87 E+05$ & $3.60 \mathrm{E}-03$ & AP105-2 & $\bar{Y}$ & Average of Primary and Duplicate results \\
\hline$\overline{\mathrm{P}-3}$ & Raw & $\mathrm{Cr}$ & $1.80 E+05$ & $3.46 \mathrm{E}-03$ & AP105-2 & $Y$ & Average of Primary and Duplicate results \\
\hline$\overline{\mathrm{P}-3}$ & Raw & $\mathrm{Cr}$ & $1.79 E+05$ & $3.44 \mathrm{E}-03$ & AP105-2 & $\mathbf{Y}$ & Average of Primary and Duplicate results \\
\hline$\overline{P-3}$ & Raw & $\mathrm{Cr}$ & $1.76 \mathrm{E}+05$ & $3.38 \mathrm{E}-03$ & AP105-2 & $\mathbf{Y}$ & Average of Primary and Duplicate results \\
\hline $\mathrm{p}-3$ & Raw & $\mathrm{Cr}$ & $1.60 E+05$ & $3.08 \mathrm{E}-03$ & AP105-2 & $\bar{Y}$ & Average of Primary and Duplicate results \\
\hline $\mathrm{P}-3$ & Raw & $\mathrm{Cr}$ & $1.54 \mathrm{E}+05$ & $2.96 \mathrm{E}-03$ & AP105-2 & $\bar{Y}$ & Average of Primary and Duplicate results \\
\hline$\overline{\mathrm{p-3}}$ & Raw & $\mathrm{Cr}$ & $1.45 E+05$ & $2.79 E-03$ & AP105-2 & $\bar{Y}$ & Average of Primary and Duplicate results \\
\hline P-3 & Raw & F & \#VALUE! & \#VALUE! & AP105-2 & N & $\begin{array}{l}\text { Average of Primary and Duplicate results; Matrix } \\
\text { interference }\end{array}$ \\
\hline P-3 & Raw & $F$ & \#VALUE! & \#VALUE! & AP105-2 & N & $\begin{array}{l}\text { Average of Primary and Duplicate results; Matrix } \\
\text { interference }\end{array}$ \\
\hline P-3 & Raw & $F$ & \#VALUE! & \#VALUE! & AP105-2 & $N$ & $\begin{array}{l}\text { Average of Primary and Duplicate results; Matrix } \\
\text { interference }\end{array}$ \\
\hline P-3 & Raw & $F$ & \#VALUE! & \#VALUE! & AP105-2 & $\mathrm{N}$ & $\begin{array}{l}\text { Average of Primary and Duplicate results; Matrix } \\
\text { interference }\end{array}$ \\
\hline P-3 & Raw & $F$ & \#VALUE! & \#VALUE! & AP105-2 & $\mathrm{N}$ & $\begin{array}{l}\text { Average of Primary and Duplicate results; Matrix } \\
\text { interference }\end{array}$ \\
\hline P-3 & Raw & $F$ & \#VALUE! & \#VALUEl & AP105-2 & $\mathbf{N}$ & $\begin{array}{l}\text { Average of Primary and Duplicate results; Matrix } \\
\text { interference }\end{array}$ \\
\hline$P-3$ & Raw & $F$ & \#VALUE! & \#VALUE! & AP105-2 & $\mathbf{N}$ & $\begin{array}{l}\text { Average of Primary and Duplicate results; Matrix } \\
\text { interference }\end{array}$ \\
\hline$P-3$ & Raw & $F$ & \#VALUE! & \#VALUE! & AP105-2 & N & $\begin{array}{l}\text { Average of Primary and Duplicate results; Matrix } \\
\text { interference }\end{array}$ \\
\hline$P-3$ & Raw & $\mathbf{F}$ & \#VALUE! & \#VALUE! & AP105-2 & $N$ & $\begin{array}{l}\text { Average of Primary and Duplicate results; Matrix } \\
\text { interference }\end{array}$ \\
\hline$P-3$ & Raw & F & \#VALUE! & \#VALUE! & AP105-2 & $\mathrm{N}$ & $\begin{array}{l}\text { Average of Primary and Duplicate results; Matrix } \\
\text { interference }\end{array}$ \\
\hline$?-3$ & Raw & $F$ & \#VALUE! & \#VALUE! & AP105-2 & $N$ & $\begin{array}{l}\text { Average of Primary and Duplicate results; Matrix } \\
\text { interference }\end{array}$ \\
\hline$?-3$ & Raw & $F$ & \#VALUE! & \#VALUE! & AP105-2 & $\mathbf{N}$ & $\begin{array}{l}\text { Average of Primary and Duplicate results; Matrix } \\
\text { interference }\end{array}$ \\
\hline$P$ & Raw & $\mathbf{F}$ & \#VALUE! & \#VALUE! & AP105-2 & $Y$ & $\begin{array}{l}\text { Retain "<" synbol, highest of all } 30 \text { or more samples } \\
\text { results, including ouliers, possible matrix interference }\end{array}$ \\
\hline $3-3$ & Raw & $\mathbf{F}$ & $8.78 E+06$ & 4.62E-01 & AP105-2 & N & $\begin{array}{l}\text { Average of Primary and Duplicate results; Matrix } \\
\text { interference }\end{array}$ \\
\hline $\bar{L}$ & HTCE InV. & $\bar{F}$ & $4.15 E+06$ & $2.18 \mathrm{E}-07$ & NA & $\mathbf{N}$ & HTCE \\
\hline 3.3 & Raw & $F$ & $1.54 \mathrm{E}+06$ & $8.11 E-02$ & AP105-2 & $Y$ & $\begin{array}{l}\text { Average of Primary and Duplicate results; Specific ion } \\
\text { electrode method }\end{array}$ \\
\hline $2-3$ & Raw & $F$ & $1.40 E+06$ & 7.37E-02 & AP105-2 & $N$ & $\begin{array}{l}\text { Average of Primary and Duplicate results; Matrix } \\
\text { interference }\end{array}$ \\
\hline $3-3$ & Raw & $F$ & $6.57 E+05$ & $3.46 \mathrm{E}-02$ & AP105-2 & $N$ & $\begin{array}{l}\text { Average of Primary and Duplicate results; Matrix } \\
\text { interference }\end{array}$ \\
\hline 3 & Raw & $\mathrm{Fe}$ & $<1.92 \mathrm{E}+04$ & \#VALUE! & AP105-2 & NR & Average of Primary and Duplicate results \\
\hline $\bar{L}$ & HTCE Inv. & $\mathrm{Fe}$ & $5.16 E+05$ & $9.24 \mathrm{E}-03$ & NA & $N$ & HTCE \\
\hline$\sqrt{-3}$ & Raw & $\mathrm{Fe}$ & $9.67 \mathrm{E}+03$ & $1.73 \mathrm{E}-04$ & AP105-2 & $\bar{Y}$ & Average of Primary and Duplicate results \\
\hline
\end{tabular}


TWRS Privatization Support Pro

\begin{tabular}{|c|c|c|c|c|c|c|c|c|c|}
\hline \multirow[b]{2}{*}{ Reported Chemical } & \multicolumn{9}{|c|}{ Tank Waste Characterization Sample Information } \\
\hline & $\begin{array}{l}\text { Memo } \\
\text { Date }\end{array}$ & $\begin{array}{c}\text { Start Analysis } \\
\text { Date }\end{array}$ & $\begin{array}{c}\text { Sample } \\
\text { Date }\end{array}$ & $\begin{array}{l}\text { Sample } \\
\text { Number }\end{array}$ & $\begin{array}{c}\text { Tank Farm } \\
\text { Number }\end{array}$ & $\begin{array}{c}\text { Lab Sample } \\
\text { Number }\end{array}$ & $\begin{array}{l}\text { Density } \\
\text { (g/mL) }\end{array}$ & $\begin{array}{l}\text { Reported } \\
\text { Value }\end{array}$ & Reported Unit \\
\hline Iron & $3 / 1 / 93$ & NA & $3 / 10 / 93$ & B08VX8 & G244 & G412 & 1.377 & $8.98 E+03$ & UG/L \\
\hline Iron & $3 / 1 / 93$ & NA & $3 / 10 / 93$ & $808 V \times 7$ & G243 & G409 & 1.298 & $7.23 E+03$ & UG/L \\
\hline $\mathrm{Fe}$ & $3 / 1 / 93$ & NA & $3 / 10 / 93$ & NS & NS & NS & 1.336 & 6.6 & $\mathrm{ug} / \mathrm{mL}$ \\
\hline Iron & $3 / 1 / 93$ & NA & $3 / 10 / 93$ & $808 V \times 2$ & G254 & G396 & 1.298 & $6.60 E+03$ & UG/L \\
\hline Tron & $3 / 1 / 93$ & NA & $3 / 10 / 93$ & $808 V \times 0$ & G251 & G391 & 1.344 & $6.57 E+03$ & UG/L \\
\hline Tron & $3 / 1 / 93$ & NA & $3 / 10 / 93$ & B08VX4 & G238 & G401 & 1.389 & $6.48 E+03$ & UG/L \\
\hline Iron & $3 / 1 / 93$ & NA & $3 / 10 / 93$ & $\mathrm{BOB} 302$ & NS & G379 & 1.329 & $6.23 E+03$ & UG/L \\
\hline fron & $3 / 1 / 93$ & NA & $3 / 10 / 93$ & BOB3C9 & G249 & G418 & 1.3 & $6.23 E+03$ & UG/L \\
\hline Iron & $3 / 1 / 93$ & NA & $3 / 10 / 93$ & B08VX1 & G253 & G392 & 1.341 & $6.10 E+03$ & $\mathrm{UG} / \mathrm{L}$ \\
\hline Iron & $3 / 1 / 93$ & NA & $3 / 10 / 93$ & B0B3D1 & NS & G378 & 1.334 & $6.04 \mathrm{E}+03$ & UG/L \\
\hline Iron & $3 / 1 / 93$ & NA & $3 / 10 / 93$ & B08VX6 & G241 & G407 & 1.338 & $5.79 E+03$ & UG $/ L$ \\
\hline Iron & $3 / 1 / 93$ & NA & $3 / 10 / 93$ & B08VX9 & G245 & G413 & 1.335 & $5.49 E+03$ & UG/L \\
\hline Iron & $3 / 1 / 93$ & NA & $3 / 10 / 93$ & $808 \vee \times 5$ & G240 & G402 & 1.338 & $4.98 \mathrm{E}+03$ & UG/L \\
\hline Potassium & $3 / 1 / 93$ & NA & $3 / 10 / 93$ & $808 V \times 8$ & G244 & G412 & 1.377 & $3.70 E+07$ & UG/L \\
\hline Potassium & $3 / 1 / 93$ & NA & $3 / 70 / 93$ & 80B3D1 & NA & G378 & 1.334 & $3.31 E+07$ & UG/L \\
\hline Potassium & $3 / 1 / 93$ & NA & $3 / 10 / 93$ & NA & G250 & G422 & 1.348 & $3.30 E+07$ & UGIL \\
\hline Potassium & $3 / 1 / 93$ & NA & $3 / 10 / 93$ & 808302 & NA & G379 & 1.329 & $3.29 \mathrm{E}+07$ & UG/L \\
\hline Potassium & $3 / 1 / 93$ & NA & $3 / 10 / 93$ & $808 \vee \times 5$ & G240 & G402 & 1.338 & $3.16 E+07$ & UG/L \\
\hline Potassium & $3 / 1 / 93$ & NA & $3 / 10 / 93$ & B08VX9 & G245 & G413 & 1.335 & $3.14 E+07$ & UG/L \\
\hline K & $3 / 1 / 93$ & NA & $3 / 10 / 93$ & NA & NA & NA & 1.336 & 31000 & $\mathrm{ug} / \mathrm{mL}$ \\
\hline Potassium & $3 / 1 / 93$ & NA & $3 / 10 / 93$ & $\mathrm{BOB3C8}$ & G248 & G417 & 1.327 & $3.08 \mathrm{E}+07$ & UG/L \\
\hline Potassium & $3 / 1 / 93$ & NA & $3 / 10 / 93$ & B08VX6 & G241 & G407 & 1.338 & $3.05 E+07$ & UG/L \\
\hline Potassium & $3 / 1 / 93$ & NA & $3 / 10 / 93$ & B08VX4 & G238 & G401 & 1.389 & $3.02 E+07$ & $\mathrm{UG} / \mathrm{L}$ \\
\hline Potassium & $3 / 1 / 93$ & NA & $3 / 10 / 93$ & B08VX7 & G243 & G409 & 1.298 & $3.00 E+07$ & UG/L \\
\hline Potassium & $3 / 1 / 93$ & NA & $3 / 10 / 93$ & $\mathrm{BOB3C9}$ & G249 & G418 & 1.3 & $2.99 E+07$ & UG/L \\
\hline Potassium & $3 / 1 / 93$ & NA & $3 / 10 / 93$ & B08VX1 & G253 & G392 & 1.341 & $2.95 E+07$ & UG/L \\
\hline Potassium & $3 / 1 / 93$ & NA & $3 / 10 / 93$ & $808 V \times 0$ & G251 & G391 & 1.344 & $2.91 E+07$ & UG/L \\
\hline Potassium & $3 / 1 / 93$ & NA & $3 / 10 / 93$ & B08VX3 & G271 & G397 & 1.3 & $2.90 E+07$ & UG/L \\
\hline Potassium & $3 / 1 / 93$ & NA & $3 / 10 / 93$ & B08VX2 & G254 & G396 & 1.298 & $2.83 E+07$ & $U G / L$ \\
\hline$K+$ & NA & NA & $\mathrm{NA}$ & NA & NA & NA & 1.33 & $1.94 E-01$ & Mole/L \\
\hline Sodium & $3 / 1 / 93$ & NA & $3 / 10 / 93$ & $808 \mathrm{VX} 4$ & G238 & G401 & 1.389 & $1.93 E+08$ & UG/L \\
\hline Sodium & $3 / 1 / 93$ & NA & $3 / 10 / 93$ & $808 V \times 8$ & G244 & $\mathrm{G} 412$ & 1.377 & $1.90 \mathrm{E}+08$ & UG/L \\
\hline Sodium & $3 / 1 / 93$ & NA & $3 / 10 / 93$ & $\mathrm{NA}$ & G250 & G422 & 1.348 & $1.74 E+08$ & UG/L \\
\hline Sodium & $3 / 1 / 93$ & NA & $3 / 10 / 93$ & BOB301 & NA & G378 & 1.334 & $1.72 E+08$ & UG/L \\
\hline Sodium & $3 / 1 / 93$ & $\mathrm{NA}$ & $3 / 10 / 93$ & $808 \vee \times 5$ & G240 & G402 & 1.338 & $1.71 E+08$ & UG/L \\
\hline Sodium & $3 / 1 / 93$ & NA & $3 / 10 / 93$ & 808302 & NA & G379 & 1.329 & $1.71 \mathrm{E}+08$ & UG/L \\
\hline Sodium & $3 / 1 / 93$ & NA & $3 / 10 / 93$ & B08VX9 & G245 & G413 & 1.335 & $1.69 E+08$ & UG/L \\
\hline $\mathrm{Na}$ & $3 / 1 / 93$ & NA & $3 / 10 / 93$ & NA & NA & NA & 1.336 & $1.67 E+05$ & $\mathrm{ug} / \mathrm{mL}$ \\
\hline Sodium & $3 / 1 / 93$ & NA & $3 / 10 / 93$ & $808 \mathrm{~V} \times 6$ & G241 & G407 & 1.338 & $1.67 E+08$ & UG/L \\
\hline Sodium & $3 / 1 / 93$ & NA & $3 / 10 / 93$ & $808 \mathrm{~V} \times 1$ & G253 & G392 & 1.341 & $1.65 E+08$ & UGIL \\
\hline Sodium & $3 / 1 / 93$ & NA & $3 / 10 / 93$ & BOBVXO & G251 & G391 & 1.344 & $1.62 E+08$ & $\mathrm{UG} / \mathrm{L}$ \\
\hline Sodium & $3 / 1 / 93$ & NA & $3 / 10 / 93$ & $\mathrm{BOB} 3 \mathrm{C} 8$ & G248 & G417 & 1.327 & $1.62 \mathrm{E}+08$ & UG/L \\
\hline Sodium & $3 / 1 / 93$ & NA & $3 / 10 / 93$ & $808 \vee \times 7$ & G243 & G409 & 1.298 & $1.50 E+08$ & UG/L \\
\hline Sodium & $3 / 1 / 93$ & NA & $3 / 10 / 93$ & $\mathrm{BOB} 3 \mathrm{C9}$ & G249 & G418 & 1.3 & $1.49 E+08$ & UG/L \\
\hline Sodium & $3 / 1 / 93$ & NA & $3 / 10 / 93$ & B08VX3 & G271 & G397 & 1.3 & $1.49 E+08$ & $\mathrm{UG} / \mathrm{L}$ \\
\hline Sodium & $3 / 1 / 93$ & NA & $3 / 10 / 93$ & $808 \mathrm{~V} \times 2$ & G254 & G396 & 1.298 & $1.46 E+08$ & UG/L \\
\hline Ammonia & $3 / 1 / 93$ & NA & $3 / 10 / 93$ & BOB3D1 & NS & G378 & 1.334 & $<40$ & $\mathrm{ug} / \mathrm{mL}$ \\
\hline
\end{tabular}




\begin{tabular}{|c|c|c|c|c|c|c|c|}
\hline & & & ralyte Informa & tion & & & \\
\hline Code & Source & Analyte & Conc. (ug/L) & Conc. (mollu) & $\begin{array}{c}\text { Reference } \\
\text { Number }\end{array}$ & $\begin{array}{l}\text { Validated } \\
\text { (Y/N) }\end{array}$ & Notes \\
\hline P-3 & Raw & $\mathrm{Fe}$ & $8.98 E+03$ & $1.61 \mathrm{E}-04$ & AP105-2 & NR & Average of Primary and Duplicate results \\
\hline p-3 & Raw & $\mathrm{Fe}$ & $7.23 \mathrm{E}+03$ & $1.29 E-04$ & AP105-2 & NR & Average of Primary and Duplicate results \\
\hline $\mathbf{P}$ & Raw & $\mathrm{Fe}$ & $6.60 E+03$ & $1.18 \mathrm{E}-04$ & AP105-2 & NR & \\
\hline$\overline{p-3}$ & Raw & $\mathrm{Fe}$ & $6.60 E+03$ & $1.18 \mathrm{E}-04$ & AP105-2 & NR & Average of Primary and Duplicate results \\
\hline P-3 & Raw & $\mathrm{Fe}$ & $6.57 E+03$ & $1.18 \mathrm{E}-04$ & AP105-2 & NR & Average of Primary and Duplicate results \\
\hline$\overline{\mathrm{P}-3}$ & Raw & $\mathrm{Fe}$ & $6.48 E+03$ & $1.16 \mathrm{E}-04$ & AP105-2 & NR & Average of Primary and Duplicate results \\
\hline$\overline{P-3}$ & Raw & $\mathrm{Fe}$ & $6.23 E+03$ & $1.12 \mathrm{E}-04$ & AP105-2 & NR & Average of Primary and Duplicate results \\
\hline P-3 & Raw & $\mathrm{Fe}$ & $6.23 E+03$ & $1.11 E-04$ & AP105-2 & NR & Average of Primary and Duplicate results \\
\hline$\overline{P-3}$ & Raw & $\mathrm{Fe}$ & $6.10 E+03$ & $1.09 \mathrm{E}-04$ & AP105-2 & NR & Average of Primary and Duplicate results \\
\hline P-3 & Raw & $\mathrm{Fe}$ & $6.04 E+03$ & $1.08 \mathrm{E}-04$ & AP105-2 & NR & Average of Primary and Duplicate results \\
\hline P-3 & Raw & Fe & $5.79 E+03$ & $1.04 \mathrm{E}-04$ & AP105-2 & NR & Average of Primary and Duplicate results \\
\hline$\overline{\mathrm{P}-3}$ & Raw & $\mathrm{Fe}$ & $5.49 E+03$ & $9.83 E-05$ & AP105-2 & NR & Average of Primary and Duplicate results \\
\hline$\overline{P-3}$ & Raw & $\mathrm{Fe}$ & $4.98 E+03$ & $8.91 E-05$ & AP105-2 & NA & Average of Primary and Duplicate results \\
\hline$\overline{\mathrm{P}-3}$ & Raw & $K$ & $3.70 E+07$ & $9.46 E-01$ & AP105-2 & $\bar{Y}$ & Average of Primary and Duplicate results \\
\hline P-3 & Raw & $\bar{K}$ & $3.31 E+07$ & $8.47 E-01$ & AP105-2 & $\bar{Y}$ & Average of Primary and Duplicate results \\
\hline $\mathrm{P}-3$ & Raw & $\bar{K}$ & $3.30 E+07$ & $8.44 E-01$ & AP105-2 & $\bar{Y}$ & Average of Primary and Duplicate results \\
\hline$\overline{\mathbf{P}-3}$ & Raw & $\bar{K}$ & $3.29 E+07$ & 8.41E-01 & AP105-2 & $\bar{Y}$ & Average of Primary and Duplicate results \\
\hline $\mathbf{P}-3$ & Raw & $\bar{K}$ & $3.16 E+07$ & $8.08 \mathrm{E}-01$ - & AP105-2 & $\bar{Y}$ & Average of Primary and Duplicate results \\
\hline$\overline{P-3}$ & Raw & $\bar{K}$ & $3.14 E+07$ & $8.03 E-01$ & AP105-2 & $\bar{Y}$ & Average of Primary and Duplicate results \\
\hline $\mathbf{P}$ & Raw & K & $3.10 E+07$ & 7.93E-01 & AP105-2 & $\mathbf{Y}$ & $\begin{array}{l}\text { Statistical average of } 13 \text { individual tank samples and } \\
2 \text { composite samples, and represents the "nomonal } \\
\text { value" for this analyte. }\end{array}$ \\
\hline$\overline{\mathbf{P}-3}$ & Raw & $\bar{K}$ & $3.08 E+07$ & $7.86 \mathrm{E}-01$ & AP105-2 & $\bar{Y}$ & Average of Primary and Duplicate results \\
\hline$\overline{\mathbf{P}-3}$ & Raw & $\bar{K}$ & $3.05 E+07$ & $7.79 E-01$ & AP105-2 & $\bar{Y}$ & Average of Primary and Duplicate results \\
\hline$\overline{\mathbf{P}-3}$ & Raw & $\mathrm{K}$ & $3.02 E+07$ & $7.72 \mathrm{E}-01$ & AP105-2 & $\bar{Y}$ & Average of Primary and Duplicate results \\
\hline$\overline{\mathrm{P}-3}$ & Raw & $\bar{K}$ & $3.00 E+07$ & 7.67E-01 & AP105-2 & $\bar{Y}$ & Average of Primary and Duplicate results \\
\hline$\overline{P-3}$ & Raw & $\mathrm{K}$ & $2.99 E+07$ & $7.65 E-01$ & AP105-2 & $\bar{Y}$ & Average of Primary and Duplicate results \\
\hline$\overline{P-3}$ & Raw & $\bar{K}$ & $2.95 E+07$ & $7.53 E-01$ & AP105-2 & $\bar{Y}$ & Average of Primary and Duplicate results \\
\hline$\overline{\mathrm{P}-3}$ & Raw & $\bar{K}$ & $2.91 E+07$ & 7.44E-01 & AP105-2 & $\bar{Y}$ & Average of Primary and Duplicate results \\
\hline$\overline{P-3}$ & Raw & $\bar{K}$ & $2.90 E+07$ & $7.42 \mathrm{E}-01$ & AP105-2 & $\bar{Y}$ & Average of Primary and Duplicate results \\
\hline$\overline{P-3}$ & Raw & $\mathrm{K}$ & $2.83 E+07$ & $7.24 E-01$ & AP105-2 & $\bar{Y}$ & Average of Primary and Duplicate results \\
\hline $\bar{L}$ & HTCE InV. & $\bar{K}$ & $7.58 \mathrm{E}+06$ & $1.94 E-01$ & NA & $\bar{N}$ & HTCE \\
\hline$\overline{P-3}$ & Raw & $\overline{\mathrm{Na}}$ & $1.93 E+08$ & $8.39 E+00$ & AP105-2 & $\bar{Y}$ & Average of Primary and Duplicate results \\
\hline$\overline{P-3}$ & Raw & $\mathrm{Na}$ & $1.90 E+08$ & $8.26 E+00$ & AP105-2 & $\bar{Y}$ & Average of Primary and Duplicate results \\
\hline$P-3$ & Raw & $\mathrm{Na}$ & $1.74 E+08$ & $7.57 E+00$ & AP105-2 & $\bar{Y}$ & Average of Primary and Duplicate results \\
\hline$\overline{p-3}$ & Raw & $\mathrm{Na}$ & $1.72 E+08$ & $7.48 \mathrm{E}+00$ & AP105-2 & $\bar{Y}$ & Average of Primary and Duplicate results \\
\hline $2-3$ & Raw & $\mathrm{Na}$ & $1.71 E+08$ & $7.43 E+00$ & AP105-2 & $\bar{Y}$ & Average of Primary and Duplicate results \\
\hline $2-3$ & Raw & $\mathrm{Na}$ & $1.71 E+08$ & $7.43 E+00$ & AP105-2 & $\bar{Y}$ & Average of Primary and Duplicate results \\
\hline$\overline{3-3}$ & Raw & $\mathrm{Na}$ & $1.69 E+08$ & $7.35 \mathrm{E}+00$ & AP105-2 & $\bar{Y}$ & Average of Primary and Duplicate results \\
\hline $\mathbf{P}$ & Raw & $\mathrm{Na}$ & $1.67 E+08$ & $7.26 \mathrm{E}+00$ & AP105-2 & $\mathrm{Y}$ & $\begin{array}{l}\text { Added, Statistical average of } 13 \text { individual tank } \\
\text { samples and } 2 \text { composite samples, and represents the } \\
\text { "nomonal value" for this analyte. }\end{array}$ \\
\hline$\overline{2-3}$ & Raw & $\overline{\mathrm{Na}}$ & $1.67 E+08$ & $7.26 \mathrm{E}+00$ & AP105-2 & $\bar{Y}$ & Average of Primary and Duplicate results \\
\hline 5 & Raw & $\mathrm{Na}$ & $1.65 E+08$ & $7.15 E+00$ & AP105-2 & $\bar{Y}$ & Average of Primary and Duplicate results \\
\hline 3 & Raw & $\mathrm{Na}$ & $1.62 E+08$ & $7.04 E+00$ & AP105-2 & $\bar{Y}$ & Average of Primary and Duplicate results \\
\hline 3.3 & Raw & $\mathrm{Na}$ & $1.62 E+08$ & $7.04 \mathrm{E}+00$ & AP105-2 & $\bar{Y}$ & Average of Primary and Duplicate results \\
\hline$\overline{-3}$ & Raw & $\mathrm{Na}$ & $1.50 E+08$ & $6.52 \mathrm{E}+00$ & AP105-2 & $\bar{Y}$ & Average of Primary and Duplicate results \\
\hline 3 & Raw & $\mathrm{Na}$ & $1.49 E+08$ & $6.48 E+00$ & AP105-2 & $\bar{Y}$ & Average of Primary and Duplicate results \\
\hline 3 & Raw & $\mathrm{Na}$ & $1.49 E+08$ & $6.46 E+00$ & AP105-2 & $\dot{Y}$ & Average of Primary and Duplicate results \\
\hline 3 & Raw & $\mathrm{Na}$ & $1.46 E+08$ & $6.35 E+00$ & AP105-2 & $\bar{Y}$ & Average of Primary and Duplicate results \\
\hline $2-3$ & Raw & $\mathrm{NH} 3$ & \#VALUE! & \#VALUE! & AP105-2 & $N$ & $\begin{array}{l}\text { Average of Primary and Duplicate results; Less than } \\
\text { value }\end{array}$ \\
\hline
\end{tabular}


TWRS Privatization Support Pro Chemical Liquids

\begin{tabular}{|c|c|c|c|c|c|c|c|c|c|}
\hline \multirow[b]{2}{*}{ Reported Chemical } & \multicolumn{9}{|c|}{ Tank Waste Characterization Sample Information } \\
\hline & $\begin{array}{c}\text { Memo } \\
\text { Date } \\
\end{array}$ & $\begin{array}{c}\text { Start Analysis } \\
\text { Date }\end{array}$ & $\begin{array}{c}\text { Start } \\
\text { Sample } \\
\text { Date } \\
\end{array}$ & $\begin{array}{l}\text { Sample } \\
\text { Number }\end{array}$ & $\begin{array}{c}\text { Tank Farm } \\
\text { Number }\end{array}$ & $\begin{array}{c}\text { Lab Sample } \\
\text { Number }\end{array}$ & $\begin{array}{l}\text { Density } \\
(\mathrm{g} / \mathrm{mL})\end{array}$ & $\begin{array}{l}\text { Reported } \\
\text { Value } \\
\end{array}$ & Reported Unit \\
\hline Nickel & $3 / 1 / 93$ & NA & $3 / 10 / 93$ & $\mathrm{BOB} 3 \mathrm{C} 8$ & G248 & \begin{tabular}{|l|} 
G417 \\
\end{tabular} & 1.327 & $<1.63 E+04$ & UG/L \\
\hline $\mathrm{Ni2+}$ & NA & NA & NA & NA & NA & $\mathrm{NA}$ & 1.33 & $5.63 \mathrm{E}-03$ & Mole/L \\
\hline Nickel & $3 / 1 / 93$ & NA & $3 / 10 / 93$ & B08VX4 & G238 & G401 & 1.389 & $1.23 E+04$ & UG/L \\
\hline Nickel & $3 / 1 / 93$ & NA & $3 / 10 / 93$ & B08VX6 & G241 & G407 & 1.338 & $1.20 E+04$ & UG/L \\
\hline Nickel & $3 / 1 / 93$ & NA & $3 / 10 / 93$ & B08VX8 & G244 & G412 & 1.377 & $1.19 E+04$ & UG/L \\
\hline Nickel & $3 / 1 / 93$ & NA & $3 / 10 / 93$ & B0B3D2 & NS & G379 & 1.329 & $1.14 E+04$ & UG/L \\
\hline Nickel & $3 / 1 / 93$ & NA & $3 / 10 / 93$ & B08VX5 & G240 & $\mathrm{G} 402$ & 1.338 & $1.12 \mathrm{E}+04$ & UG/L \\
\hline $\mathrm{Ni}$ & $3 / 1 / 93$ & NA & $3 / 10 / 93$ & NS & NS & NS & $1.3 \overline{36}$ & 11 & $\mathrm{ug} / \mathrm{mL}$ \\
\hline Nickel & $3 / 1 / 93$ & NA & $3 / 10 / 93$ & BO8VX0 & G251 & G391 & 1.344 & $1.10 E+04$ & UG/L \\
\hline Nickel & $3 / 1 / 93$ & NA & $3 / 10 / 93$ & B0B3D1 & NS & G378 & 1.334 & $1.10 E+04$ & $\mathrm{UG} / \mathrm{L}$ \\
\hline Nickel & $3 / 1 / 93$ & NA & $3 / 10 / 93$ & B08VX9 & G245 & G413 & 7.335 & $1.09 E+04$ & UG/L \\
\hline Nickel & $3 / 1 / 93$ & $\mathrm{NA}$ & $3 / 10 / 93$ & B08VX1 & G253 & G392 & 1.341 & $1.08 \mathrm{E}+04$ & UG/L \\
\hline Nickel & $3 / 1 / 93$ & NA & $3 / 10 / 93$ & B08VX7 & 6243 & G409 & 1.298 & $1.05 E+04$ & UG/L \\
\hline Nickel & $3 / 1 / 93$ & NA & $3 / 10 / 93$ & B08VX3 & G271 & G397 & 1.3 & $9.70 E+03$ & UG/L \\
\hline Nickel & $3 / 1 / 93$ & NA & $3 / 10 / 93$ & B083C9 & G249 & G418 & 1.3 & $9.61 E+03$ & UG/L \\
\hline Nickel & $3 / 1 / 93$ & NA & $3 / 10 / 93$ & $B 08 \vee \times 2$ & G254 & G396 & 1.298 & $8.99 E+03$ & UG/L \\
\hline Nitrite & $3 / 1 / 93$ & NA & $3 / 10 / 93$ & BO8VX2 & G254 & G396 & 1.298 & $9.86 E+04$ & $\mathrm{ug} / \mathrm{mL}$ \\
\hline Nitrite & $3 / 1 / 93$ & NA & $3 / 10 / 93$ & $808 \mathrm{~V} \times 4$ & G238 & G401 & 1.389 & $6.23 E+04$ & $\mathrm{ug} / \mathrm{mL}$ \\
\hline Nitrite & $3 / 1 / 93$ & NA & $3 / 10 / 93$ & $808 \vee X 7$ & G243 & G409 & 1.298 & $6.21 E+04$ & $\mathrm{ug} / \mathrm{mL}$ \\
\hline Nitrite & $3 / 1 / 93$ & NA & $3 / 10 / 93$ & $808 V \times 5$ & G240 & G402 & 1.338 & $5.77 E+04$ & $\mathrm{ug} / \mathrm{mL}$ \\
\hline Nitrite & $3 / 1 / 93$ & NA & $3 / 10 / 93$ & $\mathrm{~B} 08 \mathrm{~V} \times 4$ & G238 & G401 & 1.389 & $5.48 E+04$ & $u g / \mathrm{mL}$ \\
\hline Nitrite & $3 / 1 / 93$ & $\mathrm{NA}$ & $3 / 10 / 93$ & B08VX6 & G241 & G407 & 1.338 & $5.35 E+04$ & $\mathrm{ug} / \mathrm{mL}$ \\
\hline Nitrite & $3 / 1 / 93$ & NA & $3 / 10 / 93$ & B08VX8 & G244 & G412 & 1.377 & $5.05 E+04$ & $\mathrm{ug} / \mathrm{mL}$ \\
\hline Nitrite & $3 / 7 / 93$ & NA & $3 / 10 / 93$ & $\mathrm{~B} 08 \mathrm{8} \times 0$ & G251 & G391 & 1.344 & $5.00 \mathrm{E}+04$ & $\mathrm{ug} / \mathrm{mL}$ \\
\hline Nitrite & $3 / 1 / 93$ & NA & $3 / 10 / 93$ & $\mathrm{BOB302}$ & NA & G379 & 1.329 & $4.90 E+04$ & $\mathrm{ug} / \mathrm{mL}$ \\
\hline $\mathrm{NO}_{2}^{-}$ & $3 / 1 / 93$ & NA & $3 / 10 / 93$ & NA & NA & NA & 1.336 & 48800 & $u g / m L$ \\
\hline Nitrite & $3 / 1 / 93$ & NA & $3 / 10 / 93$ & $808 V \times 9$ & G245 & G413 & 1.335 & $4.88 E+04$ & $\mathrm{ug} / \mathrm{mL}$ \\
\hline Nitrite & $3 / 1 / 93$ & NA & $3 / 10 / 93$ & BOB3D1 & NA & G378 & 1.334 & $4.88 E+04$ & $\mathrm{ug} / \mathrm{mL}$ \\
\hline Nitrite & $3 / 1 / 93$ & NA & $3 / 10 / 93$ & $\mathrm{~B} 08 \mathrm{~V} \times 1$ & G253 & G392 & 1.341 & $4.84 E+04$ & $\mathrm{ug} / \mathrm{mL}$ \\
\hline $\mathrm{NO}_{2}=$ & $3 / 1 / 93$ & NA & $3 / 10 / 93$ & NA & NA & NA & 1.336 & 48300 & $\mathrm{ug} / \mathrm{mL}$ \\
\hline Nitrite & $3 / 1 / 93$ & NA & $3 / 10 / 93$ & $\mathrm{~B} 08 \mathrm{~V} \times 3$ & G271 & G397 & 1.3 & $4.66 \mathrm{E}+04$ & $\mathrm{ug} / \mathrm{mL}$ \\
\hline Nitrite & $3 / 1 / 93$ & NA & $3 / 10 / 93$ & B083C8 & G248 & G417 & 1.327 & $4.62 \mathrm{E}+04$ & $\mathrm{ug} / \mathrm{mL}$ \\
\hline Nitrite & $3 / 1 / 93$ & NA & $3 / 10 / 93$ & NA & G250 & G422 & 1.348 & $4.58 \mathrm{E}+04$ & $\mathrm{ug} / \mathrm{mL}$ \\
\hline Nitrite & $3 / 1 / 93$ & NA & $3 / 10 / 93$ & $\mathrm{NA}$ & G250 & G422 & 1.348 & $4.56 \mathrm{E}+04$ & $\mathrm{ug} / \mathrm{mL}$ \\
\hline Nitrite & $3 / 1 / 93$ & NA & $3 / 10 / 93$ & $B 08 V \times 2$ & G254 & G396 & 1.298 & $4.21 E+04$ & $\mathrm{ug} / \mathrm{mL}$ \\
\hline Nitrite & $3 / 1 / 93$ & NA & $3 / 10 / 93$ & $808 V \times 5$ & G240 & G402 & 1.338 & $4.21 E+04$ & $\mathrm{ug} / \mathrm{mL}$ \\
\hline Nitrite & $3 / 1 / 93$ & NA & $3 / 10 / 93$ & $808 V \times 3$ & G271 & G397 & 1.3 & $4.18 \mathrm{E}+04$ & $\mathrm{ug} / \mathrm{mL}$ \\
\hline Nitrite & $3 / 1 / 93$ & $\mathrm{NA}$ & $3 / 10 / 93$ & $80 \mathrm{~B} 3 \mathrm{C} 9$ & G249 & G418 & 1.3 & $4.01 E+04$ & $\mathrm{ug} / \mathrm{mL}$ \\
\hline NO2. & NA & NA & NA & NA & NA & NA & 1.33 & 8.04E-01 & Mole/L \\
\hline Nitrite & $3 / 1 / 93$ & NA & $3 / 10 / 93$ & B08VX7 & G243 & G409 & 1.298 & $3.67 E+04$ & $\mathrm{ug} / \mathrm{mL}$ \\
\hline Nitrite & $3 / 1 / 93$ & $\mathrm{NA}$ & $3 / 10 / 93$ & $B 08 V \times 7$ & G243 & G409 & 1.298 & $3.60 E+04$ & $\mathrm{ug} / \mathrm{mL}$ \\
\hline Nitrite & $3 / 1 / 93$ & NA & $3 / 10 / 93$ & во83С9 & G249 & G418 & 1.3 & $2.33 E+04$ & $\mathrm{ug} / \mathrm{mL}$ \\
\hline Nitrate & $3 / 1 / 93$ & NA & $3 / 10 / 93$ & $808 V \times 2$ & G254 & G396 & 1.298 & $3.39 E+05$ & $\mathrm{ug} / \mathrm{mL}$ \\
\hline Nitrate & $3 / 1 / 93$ & NA & $3 / 10 / 93$ & B08VX4 & G238 & G401 & 1.389 & $2.23 E+05$ & $\mathrm{ug} / \mathrm{mL}$ \\
\hline
\end{tabular}




\begin{tabular}{|c|c|c|c|c|c|c|c|}
\hline \multirow[b]{2}{*}{ Code } & \multirow[b]{2}{*}{ Source } & \multicolumn{3}{|c|}{ Analyte information } & \multirow[b]{2}{*}{$\begin{array}{l}\text { Reference } \\
\text { Number }\end{array}$} & \multirow[b]{2}{*}{$\begin{array}{l}\text { Validated } \\
\text { (Y/N) }\end{array}$} & \multirow[b]{2}{*}{ Notes } \\
\hline & & Analyte & Conc. (ug/L) & Conc. $(\mathrm{mol} / \mathrm{L})$ & & & \\
\hline$P-3$ & Raw & $\mathrm{Ni}$ & $<1.63 \mathrm{E}+04$ & \#VALUE! & AP105-2 & NR & Average of Primary and Duplicate results \\
\hline $\mathrm{L}$ & HTCE lnv. & $\overline{\mathrm{Ni}}$ & $3.30 E+05$ & $5.62 \mathrm{E}-03$ & NA & $\bar{N}$ & HTCE \\
\hline$\overline{P-3}$ & Raw & $\overline{\mathrm{Ni}}$ & $1.23 E+04$ & $2.10 E-04$ & AP105-2 & $\bar{Y}$ & Average of Primary and Duplicate results \\
\hline$P-3$ & Raw & $\mathrm{Ni}$ & $1.20 E+04$ & 2.04E-04 & AP105-2 & NR & Average of Primary and Duplicate results \\
\hline P-3 & Raw & $\mathrm{Ni}$ & $1.19 E+04$ & $2.03 \mathrm{E}-04$ & AP105-2 & NR & Average of Primary and Duplicate results \\
\hline P-3 & Raw & $\mathrm{Ni}$ & $1.14 E+04$ & $1.93 \mathrm{E}-04$ & AP105-2 & NR & Average of Primary and Duplicate results \\
\hline P-3 & Raw & $\mathrm{Ni}$ & $1.12 E+04$ & $1.91 \mathrm{E}-04$ & AP105-2 & NR & Average of Primary and Duplicate results \\
\hline$P$ & Raw & $\mathrm{Ni}$ & $1.10 E+04$ & $1.87 \mathrm{E}-04$ & AP105-2 & NR & \\
\hline$\overline{\mathrm{P}-3}$ & Raw & $\mathrm{Ni}$ & $1.10 E+04$ & $1.87 E-04$ & AP105-2 & NR & Average of Primary and Duplicate results \\
\hline $\mathrm{P}-3$ & Raw & $\mathrm{Ni}$ & $1.10 E+04$ & $1.87 E-04$ & AP105-2 & NR & Average of Primary and Duplicate results \\
\hline$\overline{P-3}$ & Raw & $\mathrm{Ni}$ & $1.09 E+04$ & $1.86 \mathrm{E}-04$ & AP105-2 & NR & Average of Primary and Duplicate results \\
\hline P-3 & Raw & $\mathrm{Ni}$ & $1.08 E+04$ & $1.84 \mathrm{E}-04$ & AP105-2 & NR & Average of Primary and Duplicate results \\
\hline P-3 & Raw & $\mathrm{Ni}$ & $1.05 E \div 04$ & $1.78 \mathrm{E}-04$ & AP105-2 & NR & Average of Primary and Duplicate results \\
\hline $\mathrm{P}-3$ & Raw & $\mathrm{Ni}$ & $9.70 E+03$ & $1.65 \mathrm{E}-04$ & AP105-2 & NR & Average of Primary and Duplicate results \\
\hline P-3 & Raw & $\mathrm{Ni}$ & $9.61 E+03$ & $1.64 \mathrm{E}-04$ & AP105-2 & NR & Average of Primary and Duplicate results \\
\hline$\overline{P-3}$ & Raw & $\mathrm{Ni}$ & $8.99 E+03$ & $1.53 \mathrm{E}-04$ & AP105-2 & NR & Average of Primary and Duplicate results \\
\hline P-3 & Raw & $\mathrm{NO}_{2}$ & $9.86 E+07$ & $2.14 E+00$ & AP105-2 & Y & $\begin{array}{l}\text { Average of Primary and Duplicate resuits; Possible } \\
\text { outlier }\end{array}$ \\
\hline P-3 & Raw & $\mathrm{NO}_{2}$ & $6.23 \varepsilon+07$ & $1.35 E+00$ & AP105-2 & $\mathrm{Y}$ & Average of Primary and Duplicate results \\
\hline$\overline{P-3}$ & Raw & $\mathrm{NO}_{2}$ & $6.21 E+07$ & $1.35 \mathrm{E}+00$ & AP105-2 & $Y$ & Average of Primary and Duplicate results \\
\hline$\overline{P-3}$ & Raw & $\mathrm{NO}_{2}$ & $5.77 E+07$ & $1.25 E+00$ & AP105-2 & $\bar{Y}$ & Average of Primary and Duplicate results \\
\hline P-3 & Raw & $\mathrm{NO}_{2}$ & $5.48 E+07$ & $1.19 E+00$ & AP105-2 & $\bar{Y}$ & Average of Primary and Duplicate results \\
\hline P-3 & Raw & $\mathrm{NO}_{2}$ & $5.35 E+07$ & $1.16 E+00$ & AP105-2 & $\bar{\gamma}$ & Average of Primary and Duplicate results \\
\hline$\overline{P-3}$ & Raw & $\mathrm{NO}_{2}$ & $5.05 E+07$ & $1.10 \mathrm{E}+00$ & AP105-2 & $\bar{Y}$ & Average of Primary and Duplicate results \\
\hline $\mathrm{P}-3$ & Raw & $\mathrm{NO}_{2}$ & $5.00 E+07$ & $1.09 E+00$ & AP105-2 & $\bar{Y}$ & Average of Primary and Duplicate results \\
\hline$\overline{P-3}$ & Raw & $\mathrm{NO}_{2}$ & $4.90 E+07$ & $1.06 \mathrm{E}+00$ & AP105-2 & $\bar{Y}$ & Average of Primary and Duplicate results \\
\hline $\mathbf{P}$ & Raw & $\mathrm{NO}_{2}$ & $4.88 E+07$ & $1.06 E+00$ & AP105-2 & $Y$ & $\begin{array}{l}\text { Average of all } 30 \text { or more sample results, including } \\
\text { outliers }\end{array}$ \\
\hline$\overline{P-3}$ & Raw & $\mathrm{NO}_{2}$ & $4.88 E+07$ & $1.06 E+00$ & AP105-2 & $Y$ & Average of Primary and Duplicate results \\
\hline$\overline{P-3}$ & Raw & $\mathrm{NO}_{2}$ & $4.88 \mathrm{E}+07$ & $1.06 E+00$ & AP105-2 & $\bar{Y}$ & Average of Primary and Duplicate results \\
\hline P-3 & Raw & $\mathrm{NO}_{2}$ & $4.84 E+07$ & $1.05 E+00$ & AP105-2 & $Y$ & Average of Primary and Duplicate results \\
\hline$P$ & Raw & $\mathrm{NO}_{2}$ & $4.83 E+07$ & $1.05 E+00$ & AP105-2 & $Y$ & $\begin{array}{l}\text { Average of } 30 \text { or fewer sample results with outliers } \\
\text { removed; represents "nominal value" }\end{array}$ \\
\hline$P-3$ & Raw & $\mathrm{NO}_{2}$ & $4.66 E+07$ & $1.01 E+00$ & AP105-2 & $\bar{Y}$ & Average of Primary and Duplicate results \\
\hline$\overline{\mathrm{P}-3}$ & Raw & $\mathrm{NO}_{2}$ & $4.62 \mathrm{E}+07$ & $1.00 E+00$ & AP105-2 & $\bar{Y}$ & Average of Primary and Duplicate results \\
\hline P-3 & Raw & $\mathrm{NO}_{2}$ & $4.58 E+07$ & 9.96E-01 & AP105-2 & $\bar{Y}$ & Average of Primary and Duplicate results \\
\hline$\overline{\mathrm{P}-3}$ & Raw & $\mathrm{NO}_{2}$ & $4.56 E+07$ & 9.91E-01 & AP105-2 & $\bar{Y}$ & Average of Primary and Duplicate results \\
\hline P-3 & Raw & $\mathrm{NO}_{2}$ & $4.21 E+07$ & $9.15 \mathrm{E}-01$ & AP105-2 & $\mathrm{Y}$ & Average of Primary and Duplicate results \\
\hline$\overline{P-3}$ & Raw & $\mathrm{NO}_{2}$ & $4.21 E+07$ & $9.14 \mathrm{E}-01$ & AP105-2 & $\bar{Y}$ & Average of Primary and Duplicate results \\
\hline$\overline{P-3}$ & Raw & $\mathrm{NO}_{2}$ & $4.18 \mathrm{E}+07$ & $9.08 \mathrm{E}-01$ & AP105-2 & $Y$ & Average of Primary and Duplicate results \\
\hline$\overline{p-3}$ & Raw & $\mathrm{NO}_{2}$ & $4.01 E+07$ & 8.71E-01 & AP105-2 & $\bar{Y}$ & Average of Primary and Duplicate results \\
\hline $\mathbf{L}$ & HTCE Inv. & $\mathrm{NO}_{2}$ & $3.70 \mathrm{E}+07$ & $8.04 \mathrm{E}-01$ & NA & $N$ & HTCE \\
\hline $\mathrm{P-3}$ & Raw & $\mathrm{NO}_{2}$ & $3.67 E+07$ & 7.97E-01 & AP105-2 & $Y$ & Average of Primary and Duplicate results \\
\hline P-3 & Raw & $\mathrm{NO}_{2}$ & $3.60 \mathrm{E}+07$ & $7.83 \mathrm{E}-01$ & AP105-2 & $\bar{Y}$ & Average of Primary and Duplicate results \\
\hline P-3 & Raw & $\mathrm{NO}_{2}$ & $2.33 E+07$ & 5.07E-01 & AP105-2 & $Y$ & $\begin{array}{l}\text { Average of Primary and Duplicate results; Possible } \\
\text { outlier }\end{array}$ \\
\hline P-3 & Raw & $\mathrm{NO}_{3}$ & $3.39 E+08$ & $5.47 E+00$ & AP105-2 & $\mathrm{Y}$ & $\begin{array}{l}\text { Average of Primary and Duplicate results; Possible } \\
\text { outlier }\end{array}$ \\
\hline P-3 & Raw & $\mathrm{NO}_{3}$ & $2.23 E+08$ & $3.60 E+00$ & AP105-2 & $\bar{Y}$ & Average of Primary and Duplicate results \\
\hline
\end{tabular}


TWRS Privatization Support Proje Chemical Liquids

\begin{tabular}{|c|c|c|c|c|c|c|c|c|c|}
\hline \multicolumn{10}{|c|}{ Tank Waste Characterization Sample Information } \\
\hline Reported Chemical & $\begin{array}{c}\text { Memo } \\
\text { Date }\end{array}$ & $\begin{array}{c}\text { Start Analysis } \\
\text { Date }\end{array}$ & $\begin{array}{c}\text { Start } \\
\text { Sample } \\
\text { Date } \\
\end{array}$ & $\begin{array}{l}\text { Sample } \\
\text { Number } \\
\end{array}$ & $\begin{array}{c}\text { Tank Farm } \\
\text { Number }\end{array}$ & $\begin{array}{c}\text { Lab Sample } \\
\text { Number }\end{array}$ & $\begin{array}{l}\text { Density } \\
(\mathrm{g} / \mathrm{mL})\end{array}$ & $\begin{array}{c}\text { Reported } \\
\text { Value } \\
\end{array}$ & Reported Unit \\
\hline Nitrate & $3 / 1 / 93$ & NA & $3 / 10 / 93$ & B08VX4 & G238 & G401 & 1.389 & $2.19 E+05$ & $\mathrm{ug} / \mathrm{mL}$ \\
\hline Nitrate & $3 / 1 / 93$ & NA. & $3 / 10 / 93$ & B08VX7 & G243 & G409 & 1.298 & $2.17 E+05$ & $\mathrm{ug} / \mathrm{mL}$ \\
\hline No3- & NA & NA & NA & NA & NA & NA & 1.33 & $3.31 E+00$ & Mole/L \\
\hline Nitrate & $3 / 1 / 93$ & NA & $3 / 10 / 93$ & $808 \mathrm{~V} \times 5$ & G240 & G402 & 1.338 & $1.98 \mathrm{E}+05$ & $\mathrm{ug} / \mathrm{mL}$ \\
\hline Nitrate & $3 / 1 / 93$ & NA & $3 / 10 / 93$ & $\mathrm{~B} 08 \mathrm{~V} \times 8$ & G244 & G412 & 1.377 & $1.94 \mathrm{E}+05$ & $\mathrm{ug} / \mathrm{mL}$ \\
\hline Nitrate & $3 / 1 / 93$ & NA & $3 / 10 / 93$ & BO83D2 & NA & G379 & 1.329 & $1.70 \mathrm{E}+05$ & $\mathrm{ug} / \mathrm{mL}$ \\
\hline Nitrate & $3 / 1 / 93$ & $\overline{N A}$ & $3 / 10 / 93$ & BOB3D1 & NA & G378 & 1.334 & $1.70 E+05$ & $\mathrm{ug} / \mathrm{mL}$ \\
\hline $\mathrm{NO}_{3}^{-}$ & $3 / 1 / 93$ & NA & $3 / 10 / 93$ & NA & NA & NA & 1.336 & 168000 & $u g / m L$ \\
\hline Nitrate & $3 / 1 / 93$ & NA & $3 / 10 / 93$ & $808 \mathrm{~V} \times 6$ & G241 & G407 & 1.338 & $1.68 \mathrm{E}+05$ & $\mathrm{ug} / \mathrm{mL}$ \\
\hline Nitrate & $3 / 1 / 93$ & NA & $3 / 10 / 93$ & B08VX9 & G245 & G413 & 1.335 & $1.67 \mathrm{E}+05$ & $\mathrm{ug} / \mathrm{mL}$ \\
\hline $\mathrm{NO}_{3} 0^{-}$ & $3 / 1 / 93$ & NA & $3 / 10 / 93$ & NA & NA & NA & 1.336 & 165000 & ug/mL \\
\hline Nitrate & $3 / 1 / 93$ & NA & $3 / 10 / 93$ & NA & G250 & G422 & 1.348 & $1.61 E+05$ & $\mathrm{ug} / \mathrm{mL}$ \\
\hline Nitrate & $3 / 1 / 93$ & NA & $3 / 10 / 93$ & $\mathrm{BOB3C8}$ & G248 & G417 & 1.327 & $1.61 E+05$ & $\mathrm{ug} / \mathrm{mL}$ \\
\hline Nitrate & $3 / 1 / 93$ & NA & $3 / 10 / 93$ & NA & G250 & G422 & 1.348 & $1.60 E+05$ & $\mathrm{ug} / \mathrm{mL}$ \\
\hline Nitrate & $3 / 1 / 93$ & NA & $3 / 10 / 93$ & $808 \mathrm{~V} \times 0$ & G251 & G391 & 1.344 & $1.58 \mathrm{E}+05$ & $\mathrm{ug} / \mathrm{mL}$ \\
\hline Nitrate & $3 / 1 / 93$ & $\mathrm{NA}$ & $3 / 10 / 93$ & $808 \mathrm{VX1}$ & G253 & G392 & 1.341 & $1.52 E+05$ & $\mathrm{ug} / \mathrm{mL}$ \\
\hline Nitrate & $3 / 1 / 93$ & $\mathrm{NA}$ & $3 / 10 / 93$ & $808 \mathrm{~V} \times 3$ & G271 & G397 & 1.3 & $1.45 E+05$ & $\mathrm{ug} / \mathrm{mL}$ \\
\hline Nitrate & $3 / 1 / 93$ & $\mathrm{NA}$ & $3 / 10 / 93$ & $808 \mathrm{~V} \times 5$ & G240 & G402 & 1.338 & $1.45 E \div 05$ & $\mathrm{ug} / \mathrm{mL}$ \\
\hline Nitrate & $3 / 1 / 93$ & NA & $3 / 10 / 93$ & $808 \mathrm{~V} \times 2$ & G254 & G396 & 1.298 & $1.32 \mathrm{E}+05$ & $\mathrm{ug} / \mathrm{mL}$ \\
\hline Nitrate & $3 / 1 / 93$ & $\mathrm{NA}$ & $3 / 10 / 93$ & $\mathrm{~B} 08 \mathrm{~V} \times 3$ & G271 & G397 & 1.3 & $1.32 E+05$ & $\mathrm{ug} / \mathrm{mL}$ \\
\hline Nitrate & $3 / 1 / 93$ & NA & $3 / 10 / 93$ & $808 \mathrm{~V} \times 7$ & G243 & G409 & 1.298 & $1.28 E+05$ & $\mathrm{ug} / \mathrm{mL}$ \\
\hline Nitrate & $3 / 1 / 93$ & NA & $3 / 10 / 93$ & BOB3C9 & G249 & G418 & 1.3 & $1.25 E+05$ & $\mathrm{ug} / \mathrm{mL}$ \\
\hline Nitrate & $3 / 1 / 93$ & NA & $3 / 10 / 93$ & вовзС & G249 & G418 & 1.3 & $8.18 E+04$ & $\mathrm{ug} / \mathrm{mL}$ \\
\hline $\mathrm{OH}$ & $3 / 1 / 93$ & NA & $3 / 10 / 93$ & NA & NA & NA & 1.336 & $5.48 E+04$ & $\mathrm{ug} / \mathrm{mL}$ \\
\hline $\mathrm{OH}$ & $3 / 1 / 93$ & NA & $3 / 10 / 93$ & NA & NA & NA & 1.336 & $5.31 E+04$ & $\mathrm{ug} / \mathrm{mL}$ \\
\hline Lead & $3 / 1 / 93$ & NA & $3 / 10 / 93$ & $\mathrm{BOB} 3 \mathrm{C} 8$ & G248 & G417 & 1.327 & $<7.75 \mathrm{E}+04$ & $U G / L$ \\
\hline Lead & $3 / 1 / 93$ & NA & $3 / 10 / 93$ & $808 V \times 6$ & G241 & G407 & 1.338 & $<5.21 E+03$ & UG/L \\
\hline $\mathrm{Pb2}+$ & NA & NA & NA & NA & NA & $\mathrm{NA}$ & 1.33 & $9.02 \mathrm{E}-05$ & Mole/L \\
\hline Lead & $3 / 1 / 93$ & NA & $3 / 10 / 93$ & B08VX7 & G243 & G409 & 1.298 & $7.62 \mathrm{E}+03$ & $\mathrm{UG} / \mathrm{L}$ \\
\hline Lead & $3 / 1 / 93$ & NA & $3 / 10 / 93$ & $\mathrm{~B} 08 \mathrm{~V} \times 3$ & G271 & G397 & 1.3 & $5.66 E+03$ & $\mathrm{UG} / \mathrm{L}$ \\
\hline Lead & $3 / 1 / 93$ & NA & $3 / 10 / 93$ & B08VX8 & G244 & G412 & 1.377 & $5.58 E+03$ & $U G / L$ \\
\hline Lead & $3 / 1 / 93$ & $\mathrm{NA}$ & $3 / 10 / 93$ & B08VX5 & G240 & G402 & 1.338 & $5.50 E+03$ & UG/L \\
\hline $\mathrm{Pb}$ & $3 / 1 / 93$ & NA & $3 / 10 / 93$ & NS & NS & NS & 1.336 & 5.39 & ug/mL \\
\hline Lead & $3 / 1 / 93$ & NA & $3 / 10 / 93$ & $808 V \times 4$ & G238 & G401 & 1.389 & $5.34 E+03$ & UG/L \\
\hline Lead & $3 / 1 / 93$ & NA & $3 / 10 / 93$ & Bo8VX0 & G251 & G391 & 1.344 & $5.29 E+03$ & $\mathrm{UG} / \mathrm{L}$ \\
\hline Lead & $3 / 1 / 93$ & NA & $3 / 10 / 93$ & B08VX2 & G254 & G396 & 1.298 & $5.26 E+03$ & UGIL \\
\hline Lead & $3 / 1 / 93$ & NA & $3 / 10 / 93$ & $80 \overline{B 302}$ & NS & G379 & 1.329 & $5.02 E+03$ & $\mathrm{UG} / \mathrm{L}$ \\
\hline Lead & $3 / 1 / 93$ & NA & $3 / 10 / 93$ & $808 \mathrm{~V} \times 9$ & G245 & G413 & 1.335 & $4.78 E+03$ & UG/L \\
\hline Lead & $3 / 1 / 93$ & NA & $3 / 10 / 93$ & B08VX1 & 6253 & G392 & 1.341 & $4.76 E+03$ & $\mathrm{UG} / \mathrm{L}$ \\
\hline Lead & $3 / 1 / 93$ & NA & $3 / 10 / 93$ & B0B301 & NS & G378 & 1.334 & $4.51 E+03$ & UG/L \\
\hline Lead & $3 / 1 / 93$ & NA & $3 / 10 / 93$ & BOB3C9 & G249 & G418 & 1.3 & $4.19 E+03$ & UG/L \\
\hline Phosphate & $3 / 1 / 93$ & NA & $3 / 10 / 93$ & B08VX0 & G251 & G391 & 1.344 & $<4.01 E+02$ & $\mathrm{ug} / \mathrm{mL}$ \\
\hline Phosphate & $3 / 1 / 93$ & NA & $3 / 10 / 93$ & B08VX0 & G251 & G391 & 1.344 & $<1.00 E+02$ & $\mathrm{ug} / \mathrm{mL}$ \\
\hline
\end{tabular}




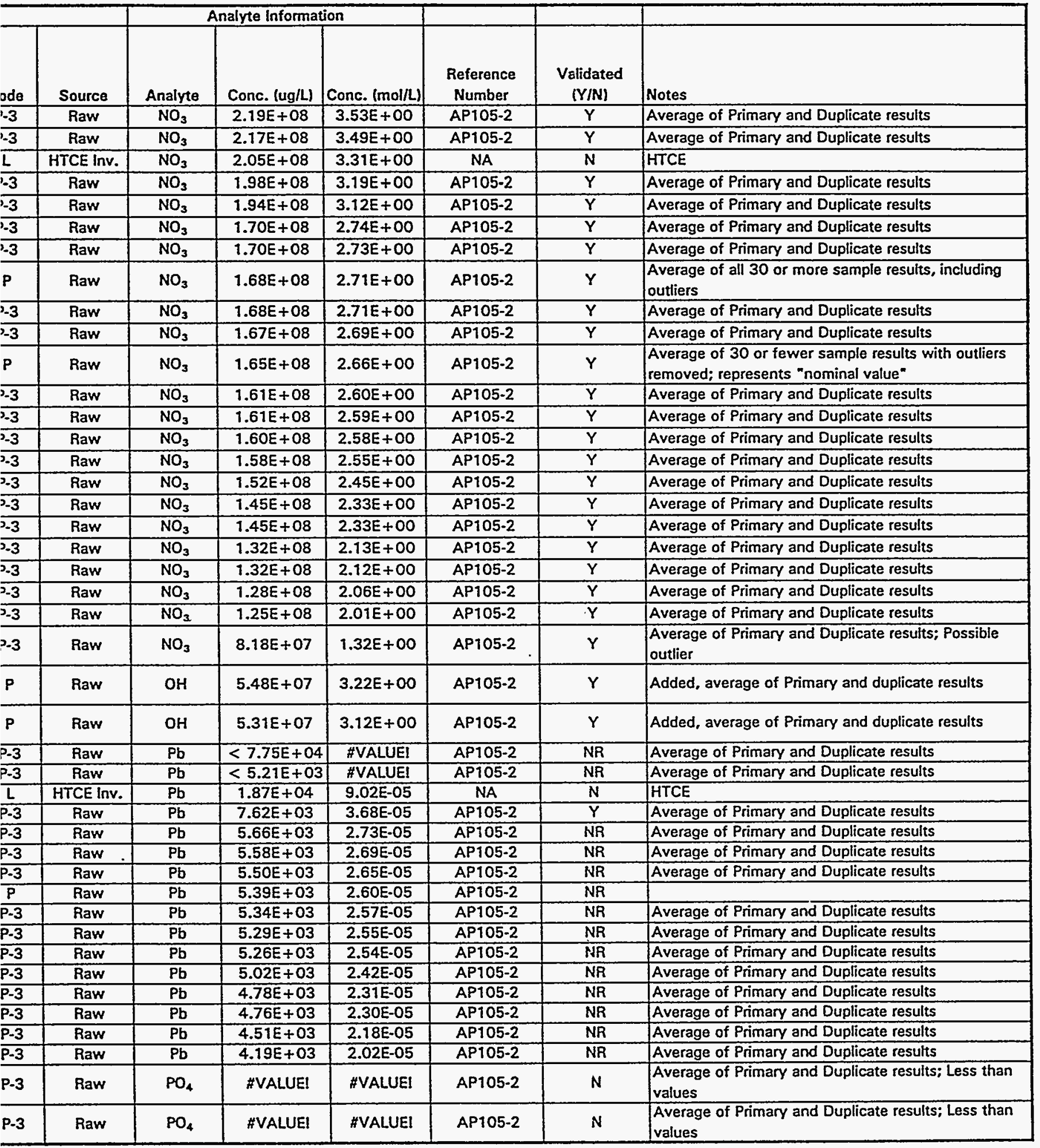




\begin{tabular}{|c|c|c|c|c|c|c|c|c|c|}
\hline \multirow[b]{2}{*}{ Reported Chemical } & \multicolumn{9}{|c|}{ Tank Waste Characterization Sample Information } \\
\hline & $\begin{array}{c}\text { Memo } \\
\text { Date }\end{array}$ & $\begin{array}{c}\text { Start Analysis } \\
\text { Date }\end{array}$ & $\begin{array}{c}\text { Sample } \\
\text { Date }\end{array}$ & $\begin{array}{l}\text { Sample } \\
\text { Number }\end{array}$ & $\begin{array}{c}\text { Tank Farm } \\
\text { Number }\end{array}$ & $\begin{array}{c}\text { Lab Sample } \\
\text { Number }\end{array}$ & $\begin{array}{c}\text { Density } \\
\text { (g/mL) }\end{array}$ & $\begin{array}{c}\text { Reported } \\
\text { Value }\end{array}$ & Reported Unit \\
\hline Phosphate & $3 / 1 / 93$ & NA & $3 / 10 / 93$ & $808 \mathrm{VX1}$ & G253 & G392 & 1.341 & $<4.01 E+02$ & $\mathrm{ug} / \mathrm{mL}$ \\
\hline Phosphate & $3 / 1 / 93$ & NA & $3 / 10 / 93$ & BO8VX1 & G253 & G392 & 1.341 & $<5.10 E+01$ & $\mathrm{ug} / \mathrm{mL}$ \\
\hline Phosphate & $3 / 1 / 93$ & NA & $3 / 10 / 93$ & $808 \mathrm{~V} \times 2$ & G254 & G396 & 1.298 & $<4.01 E+02$ & $\mathrm{ug} / \mathrm{mL}$ \\
\hline Phosphate & $3 / 1 / 93$ & NA & $3 / 10 / 93$ & $\mathrm{BOB3C8}$ & G248 & G417 & 1.327 & $<8.61 E+02$ & $\mathrm{ug} / \mathrm{mL}$ \\
\hline Phosphate & $3 / 1 / 93$ & $\overline{N A}$ & $3 / 10 / 93$ & $80 \mathrm{B3C8}$ & G248 & G417 & 1.327 & $<1.00 E+02$ & $u g / m L$ \\
\hline P043. & $\mathrm{NA}$ & NA & NA & NA & NA & NA & 1.33 & $1.59 E-01$ & Mole/L \\
\hline Phosphate & $3 / 1 / 93$ & NA & $3 / 10 / 93$ & BOB3C9 & G249 & G418 & 1.3 & $2.36 E+03$ & $\mathrm{ug} / \mathrm{mL}$ \\
\hline Phosphate & $3 / 1 / 93$ & NA & $3 / 10 / 93$ & $808 \mathrm{~V} \times 3$ & G271 & G397 & 1.3 & $6.76 E+02$ & $\mathrm{ug} / \mathrm{mL}$ \\
\hline Phosphate & $3 / 1 / 93$ & NA & $3 / 10 / 93$ & BOB3C9 & G249 & G418 & 1.3 & $5.98 E+02$ & $u g / m L$ \\
\hline Phosphate & $3 / 1 / 93$ & NA & $3 / 10 / 93$ & $\mathrm{~B} 08 \mathrm{~V} \times 4$ & G238 & G401 & 1.389 & $5.81 E+02$ & $\mathrm{ug} / \mathrm{mL}$ \\
\hline Phosphate & $3 / 1 / 93$ & NA & $3 / 10 / 93$ & $808 \mathrm{~V} \times 3$ & G271 & G397 & 1.3 & $5.68 E+02$ & $\mathrm{ug} / \mathrm{mL}$ \\
\hline Phosphate & $3 / 1 / 93$ & NA & $3 / 10 / 93$ & $808 \vee \times 7$ & G243 & G409 & 1.298 & $5.51 E+02$ & $\mathrm{ug} / \mathrm{mL}$ \\
\hline Phosphate & $3 / 1 / 93$ & NA & $3 / 10 / 93$ & $808 \mathrm{~V} \times 2$ & G254 & G396 & 1.298 & $5.48 E+02$ & $\mathrm{ug} / \mathrm{mL}$ \\
\hline $\mathrm{PO}_{4}^{3-}$ & $3 / 1 / 93$ & NA & $3 / 10 / 93$ & NA & NA & NA & 1.336 & 538 & $\mathrm{ug} / \mathrm{mL}$ \\
\hline Phosphate & $3 / 1 / 93$ & NA & $3 / 10 / 93$ & NA & G250 & $\mathrm{G} 422$ & 1.348 & $4.90 E+02$ & $\mathrm{ug} / \mathrm{mL}$ \\
\hline Phosphate & $3 / 1 / 93$ & NA & $3 / 10 / 93$ & $808 \mathrm{~V} \times 5$ & G240 & G402 & 1.338 & $4.78 E+02$ & $\mathrm{ug} / \mathrm{mL}$ \\
\hline $\mathrm{PO}_{4}^{3-}$ & $3 / 1 / 93$ & NA & $3 / 10 / 93$ & NA & NA & NA & 1.336 & 440 & $\mathrm{ug} / \mathrm{mL}$ \\
\hline Phosphate & $3 / 1 / 93$ & NA & $3 / 10 / 93$ & NA & G250 & G422 & 1.348 & $4.26 E+02$ & $\mathrm{ug} / \mathrm{mL}$ \\
\hline Phosphate & $3 / 1 / 93$ & NA & $3 / 10 / 93$ & Bo8VX9 & G245 & G413 & 1.335 & $4.23 E+02$ & $u g / m L$ \\
\hline Phosphate & $3 / 1 / 93$ & $\mathrm{NA}$ & $3 / 10 / 93$ & B08VX7 & G243 & G409 & 1.298 & $4.02 E+02$ & $\mathrm{ug} / \mathrm{mL}$ \\
\hline Phosphate & $3 / 1 / 93$ & NA & $3 / 10 / 93$ & $808 \mathrm{~V} \times 8$ & G244 & G412 & 1.377 & $3.75 E+02$ & $\mathrm{ug} / \mathrm{mL}$ \\
\hline Phosphate & $3 / 1 / 93$ & NA & $3 / 10 / 93$ & B08VX6 & G241 & G407 & 1.338 & $3.68 E+02$ & $\mathrm{ug} / \mathrm{mL}$ \\
\hline Phosphate & $3 / 1 / 93$ & NA & $3 / 10 / 93$ & $808 \mathrm{~V} \times 8$ & G244 & G412 & 1.377 & $3.25 E+02$ & $\mathrm{ug} / \mathrm{mL}$ \\
\hline Phosphate & $3 / 1 / 93$ & NA & $3 / 10 / 93$ & $8083 \mathrm{D1}$ & NA & G378 & 1.334 & $3.01 E+02$ & $\mathrm{ug} / \mathrm{mL}$ \\
\hline Phosphate & $3 / 1 / 93$ & $\mathrm{NA}$ & $3 / 10 / 93$ & BOB3D2 & $\mathrm{NA}$ & G379 & 1.329 & $3.00 \mathrm{E}+02$ & $\mathrm{ug} / \mathrm{mL}$ \\
\hline Phosphate & $3 / 1 / 93$ & NA & $3 / 10 / 93$ & $808 \mathrm{~V} \times 6$ & G241 & G407 & 1.338 & $2.80 E+02$ & $\mathrm{ug} / \mathrm{mL}$ \\
\hline Phosphate & $3 / 1 / 93$ & NA & $3 / 10 / 93$ & $B 08 V \times 4$ & G238 & G401 & 1.389 & $2.47 \mathrm{E}+02$ & $4 \mathrm{~g} / \mathrm{mL}$ \\
\hline Phosphate & $3 / 1 / 93$ & NA & $3 / 10 / 93$ & $808 V \times 5$ & G240 & $\mathrm{G} 402$ & 1.338 & $2.16 E+02$ & ug $/ \mathrm{mL}$ \\
\hline Antimony & $3 / 1 / 93$ & $\overline{N A}$ & $3 / 10 / 93$ & B08VX4 & G238 & G401 & 1.389 & $<5.61 E+03$ & UG/L \\
\hline Antimony & $3 / 1 / 93$ & $\mathrm{NA}$ & $3 / 10 / 93$ & $808 \mathrm{~V} \times 0$ & G251 & G391 & 1.344 & $<5.25 E+03$ & UG/L \\
\hline Antimony & $3 / 1 / 93$ & NA & $3 / 10 / 93$ & $808 \mathrm{~V} \times 1$ & G253 & G392 & 1.341 & $<5.25 E+03$ & UG/L \\
\hline Antimony & $3 / 1 / 93$ & $\mathrm{NA}$ & $3 / 10 / 93$ & $B 08 V \times 2$ & G254 & G396 & 1.298 & $<5.25 E+03$ & UG/L \\
\hline Antimony & $3 / 1 / 93$ & NA & $3 / 10 / 93$ & $808 \mathrm{~V} \times 3$ & G271 & G397 & 1.3 & $<5.25 E+03$ & UG/L \\
\hline Antimony & $3 / 1 / 93$ & NA & $3 / 10 / 93$ & $808 V \times 5$ & G240 & G402 & 7.338 & $<5.25 E+03$ & UG \\
\hline Antimony & $3 / 1 / 93$ & NA & $3 / 10 / 93$ & $B 08 \vee \times 9$ & G245 & G413 & 1.335 & $<5.25 E+03$ & UG/L \\
\hline Antimony & $3 / 1 / 93$ & NA & $3 / 10 / 93$ & $\mathrm{BOB} 3 \mathrm{C9}$ & G249 & G418 & 1.3 & $<5.25 E+03$ & UGL \\
\hline Antimony & $3 / 1 / 93$ & NA & $3 / 10 / 93$ & 80B301 & NS & G378 & 1.334 & $<5.25 E+03$ & UG/L \\
\hline Antimony & $3 / 1 / 93$ & NA & $3 / 10 / 93$ & B083D2 & NS & G379 & 1.329 & $<5.25 E+03$ & UG/L \\
\hline Antimony & $3 / 1 / 93$ & NA & $3 / 10 / 93$ & $\mathrm{BOB3C8}$ & G248 & G417 & 1.327 & $<2.63 E+03$ & UG/L \\
\hline Antimony & $3 / 1 / 93$ & $\mathrm{NA}$ & $3 / 10 / 93$ & B08VX6 & G241 & G407 & 1.338 & $<1.29 E+04$ & UG/L \\
\hline Antimony & $3 / 1 / 93$ & NA & $3 / 10 / 93$ & $808 V \times 7$ & G243 & G409 & 1.298 & $<1.29 E+04$ & UG/L \\
\hline Antimony & $3 / 1 / 93$ & $\mathrm{NA}$ & $3 / 10 / 93$ & $808 \mathrm{~V} \times 8$ & G244 & G412 & 1.377 & $6.76 \mathrm{E}+03$ & UG/L \\
\hline Selenium & $3 / 1 / 93$ & NA & $3 / 10 / 93$ & BOB3D1 & NS & G378 & 1.334 & $2.50 \mathrm{E}-01$ & $\mathrm{ug} / \mathrm{mL}$ \\
\hline
\end{tabular}




\begin{tabular}{|c|c|c|c|c|c|c|c|}
\hline \multirow[b]{2}{*}{ Sode } & \multirow[b]{2}{*}{ Source } & \multicolumn{3}{|c|}{ Analyte information } & \multirow[b]{2}{*}{$\begin{array}{l}\text { Reference } \\
\text { Number }\end{array}$} & \multirow[b]{2}{*}{$\begin{array}{l}\text { Validated } \\
(Y / N)\end{array}$} & \multirow[b]{2}{*}{ Notes } \\
\hline & & Analyte & Conc. (ug/L) & Conc. (mol/L) & & & \\
\hline P-3 & Raw & $\mathrm{PO}_{4}$ & \#VALUE! & \#VALUE! & AP105-2 & $N$ & $\begin{array}{l}\text { Average of Primary and Duplicate results; Less than } \\
\text { values }\end{array}$ \\
\hline P-3 & Raw & $\mathrm{PO}_{4}$ & \#VALUE! & \#VALUE! & AP105-2 & $N$ & $\begin{array}{l}\text { Average of Primary and Duplicate results; Less than } \\
\text { values }\end{array}$ \\
\hline P-3 & Raw & $\mathrm{PO}_{4}$ & \#VALUE! & \#VALUE! & AP105-2 & $\mathbf{N}$ & $\begin{array}{l}\text { Average of Primary and Duplicate results; Less than } \\
\text { values }\end{array}$ \\
\hline$\overline{\mathrm{P}-3}$ & Raw & $\mathrm{PO}_{4}$ & \#VALUE! & \#VALLUE! & AP105-2 & $N$ & Average of Primary and Duplicate results \\
\hline L & HTCE InV. & $\mathrm{PO}_{4}$ & $1.51 \mathrm{E}+07$ & $1.58 \mathrm{E}-01$ & NA & $N$ & HTCE \\
\hline P-3 & Raw & $\mathrm{PO}_{4}$ & $2.36 E+06$ & $2.48 E-02$ & AP105-2 & $Y$ & $\begin{array}{l}\text { Average of Primary and Duplicate results; Possible } \\
\text { outlier }\end{array}$ \\
\hline P-3 & Raw & $\mathrm{PO}_{4}$ & $5.51 E+05$ & $5.80 \mathrm{E}-03$ & AP105-2 & $Y$ & Average of Primary and Duplicate results \\
\hline $\mathrm{P}-3$ & Raw & $\mathrm{PO}_{4}$ & $5.48 \mathrm{E}+05$ & 5.77E-03 & AP105-2 & $\bar{Y}$ & Average of Primary and Duplicate results \\
\hline $\mathbf{P}$ & Raw & $\mathrm{PO}_{4}$ & 538000 & $5.66 \mathrm{E}-03$ & AP105-2 & $Y$ & $\begin{array}{l}\text { Average of all } 30 \text { or more sample results, including } \\
\text { outliers }\end{array}$ \\
\hline$\overline{\mathrm{P}-3}$ & Raw & $\mathrm{PO}_{4}$ & $4.90 E+05$ & 5.16E-03 & AP105-2 & $\bar{Y}$ & Average of Primary and Duplicate results \\
\hline$\overline{\mathrm{P}-3}$ & Raw & $\mathrm{PO}_{4}$ & $4.78 E+05$ & $5.03 E-03$ & AP105-2 & $\bar{Y}$ & Average of Primary and Duplicate results \\
\hline$P$ & Raw & $\mathrm{PO}_{4}$ & 440000 & $4.63 \mathrm{E}-03$ & AP105-2 & $Y$ & $\begin{array}{l}\text { Average of } 30 \text { or fewer sample results with outliers } \\
\text { removed; represents "nominal value" }\end{array}$ \\
\hline$\overline{P-3}$ & Raw & $\mathrm{PO}_{4}$ & $4.26 E+05$ & 4.48E-03 & AP105-2 & $\bar{Y}$ & Average of Primary and Duplicate results \\
\hline$P-3$ & Raw & $\mathrm{PO}_{4}$ & $4.23 E+05$ & $4.45 \mathrm{E}-03$ & AP105-2 & $\bar{Y}$ & Average of Primary and Duplicate results \\
\hline$\overline{\mathrm{P}-3}$ & Raw & $\mathrm{PO}_{4}$ & $4.02 E+05$ & $4.23 \mathrm{E}-03$ & AP105-2 & $\bar{Y}$ & Average of Primary and Duplicate results \\
\hline$\overline{\mathrm{P}-3}$ & Raw & Sb & $<5.61 E+03$ & \#VALUE! & AP105-2 & NR & Average of Primary and Duplicate results \\
\hline$\overline{P-3}$ & Raw & $\mathrm{Sb}$ & $<5.25 E+03$ & \#VALUE! & AP105-2 & NR & Average of Primary and Duplicate results \\
\hline$\overline{\mathrm{P}-3}$ & Raw & $\mathrm{Sb}$ & $<5.25 E+03$ & \#VALUE! & AP105-2 & NR & Average of Primary and Duplicate results \\
\hline$\overline{\mathrm{P}-3}$ & Raw & Sb & $<5.25 E+03$ & \#VALUE! & AP105-2 & NR & Average of Primary and Duplicate results \\
\hline P-3 & Raw & Sb & $<5.25 E+03$ & \#VALUE! & AP105-2 & NR & Average of Primary and Duplicate results \\
\hline $\mathrm{P}-3$ & Raw & Sb & $<5.25 \mathrm{E}+03$ & \#VALUE! & AP105-2 & NR & Average of Primary and Duplicate results \\
\hline$\overline{P-3}$ & Raw & Sb & $<5.25 E+03$ & \#VALUE! & AP105-2 & NR & Average of Primary and Duplicate results \\
\hline$\overline{\mathrm{P}-3}$ & Raw & $\overline{\mathrm{Sb}}$ & $<5.25 E+03$ & \#VALUE! & AP105-2 & NR & Average of Primary and Duplicate results \\
\hline$\overline{\mathrm{P}-3}$ & Raw & $\mathrm{Sb}$ & $<5.25 E+03$ & \#VALUE! & AP105-2 & NR & Average of Primary and Duplicate results \\
\hline$\overline{P-3}$ & Raw & $\overline{S b}$ & $<5.25 E+03$ & \#VALUE! & AP105-2 & NR & Average of Primary and Duplicate results \\
\hline$\overline{\mathrm{P}-3}$ & Raw & Sb & $<2.63 E+03$ & \#VALUE! & AP105-2 & NR & Average of Primary and Duplicate results \\
\hline$\overline{\mathrm{P}-3}$ & Raw & Sb & $<1.29 E+04$ & \#VALUE! & AP105-2 & NR & Average of Primary and Duplicate results \\
\hline$\overline{\mathrm{P}-3}$ & Raw & $\mathrm{Sb}$ & $<1.29 E+04$ & \#VALUE! & AP105-2 & NR & Average of Primary and Duplicate results \\
\hline$\overline{p-3}$ & Raw & $\mathrm{Sb}$ & $6.76 E+03$ & 5.55E-05 & AP105-2 & $\mathbf{Y}$ & Average of Primary and Duplicate results \\
\hline $\mathrm{P}-3$ & Raw & $\mathrm{Se}$ & $2.50 E+02$ & $3.17 E-06$ & AP105-2 & NR & Average of Primary and Duplicate results \\
\hline
\end{tabular}


TWRS Privatization Support Pre Chemical Liquid:

\begin{tabular}{|c|c|c|c|c|c|c|c|c|c|}
\hline \multirow[b]{2}{*}{ Reported Chemical } & \multicolumn{9}{|c|}{ Tank Waste Characterization Sample Information } \\
\hline & $\begin{array}{c}\text { Memo } \\
\text { Date }\end{array}$ & $\begin{array}{c}\text { Start Analysis } \\
\text { Date }\end{array}$ & $\begin{array}{c}\text { Start } \\
\text { Sample } \\
\text { Date } \\
\end{array}$ & $\begin{array}{l}\text { Sample } \\
\text { Number }\end{array}$ & $\begin{array}{c}\text { Tank Farm } \\
\text { Number }\end{array}$ & $\begin{array}{c}\text { Lab Sample } \\
\text { Number }\end{array}$ & $\begin{array}{l}\text { Density } \\
(\mathrm{g} / \mathrm{mL})\end{array}$ & $\begin{array}{c}\text { Reported } \\
\text { Value }\end{array}$ & Reported Unit \\
\hline Selenium & $3 / 1 / 93$ & NA & $3 / 10 / 93$ & BOB3D2 & NS & G379 & 1.329 & 2.50E-01 & $\mathrm{ug} / \mathrm{mL}$ \\
\hline Selenium & $3 / 1 / 93$ & NA & $3 / 10 / 93$ & B08VX8 & G244 & G412 & 1.377 & $1.95 E-01$ & $\mathrm{ug} / \mathrm{mL}$ \\
\hline Selenium & $3 / 1 / 93$ & NA & $3 / 10 / 93$ & $808 \vee \times 9$ & G245 & G413 & 1.335 & $1.61 \mathrm{E}-01$ & $\mathrm{ug} / \mathrm{mL}$ \\
\hline Selenium & $3 / 1 / 93$ & NA & $3 / 10 / 93$ & B08VX2 & G254 & G396 & 1.298 & 1.61E-01 & ug/mL \\
\hline Selenium & $3 / 1 / 93$ & NA & $3 / 10 / 93$ & $808 \mathrm{~V} \times 3$ & $\mathrm{G} 271$ & G397 & 1.3 & 1.60 E-01 & $\mathrm{ug} / \mathrm{mL}$ \\
\hline Selenium & $3 / 1 / 93$ & NA & $3 / 10 / 93$ & $808 \mathrm{~V} \times 4$ & G238 & G401 & 1.389 & $1.59 \mathrm{E}-01$ & $\mathrm{ug} / \mathrm{mL}$ \\
\hline Selenium & $3 / 1 / 93$ & NA & $3 / 10 / 93$ & $808 V \times 4$ & G238 & G401 & 1.389 & $1.59 \mathrm{E}-01$ & $\mathrm{ug} / \mathrm{mL}$ \\
\hline Selenium & $3 / 1 / 93$ & NA & $3 / 10 / 93$ & $808 \mathrm{~V} \times 7$ & G243 & G409 & 1.298 & $1.43 \mathrm{E}-01$ & $\mathrm{ug} / \mathrm{mL}$ \\
\hline Selenium & $3 / 1 / 93$ & NA & $3 / 10 / 93$ & $80 \mathrm{B3C9}$ & G249 & G418 & 1.3 & 1.36E-01 & $\mathrm{ug} / \mathrm{mL}$ \\
\hline Selenium & $3 / 1 / 93$ & NA & $3 / 10 / 93$ & BOB3C8 & G248 & G417 & 1.327 & $1.32 \mathrm{E}-01$ & $\mathrm{ug} / \mathrm{mL}$ \\
\hline Selenium & $3 / 1 / 93$ & NA & $3 / 10 / 93$ & B08VX0 & G251 & G391 & 1.344 & $1.28 \mathrm{E}-01$ & $\mathrm{ug} / \mathrm{mL}$ \\
\hline Selenium & $3 / 1 / 93$ & NA & $3 / 10 / 93$ & $808 V \times 5$ & G240 & G402 & 1.338 & $1.26 \mathrm{E}-01$ & $\mathrm{ug} / \mathrm{mL}$ \\
\hline Selenium & $3 / 1 / 93$ & NA & $3 / 10 / 93$ & B08VX6 & G241 & G407 & 1.338 & $1.25 \mathrm{E}-01$ & $\mathrm{ug} / \mathrm{mL}$ \\
\hline Selenium & $3 / 1 / 93$ & NA & $3 / 10 / 93$ & B08VX6 & G241 & G407 & 1.338 & $1.19 \mathrm{E}-01$ & $\mathrm{ug} / \mathrm{mL}$ \\
\hline Selenium & $3 / 1 / 93$ & NA & $3 / 10 / 93$ & B08VX1 & G253 & G392 & 1.341 & 1.17E-01 & $\mathrm{ug} / \mathrm{mL}$ \\
\hline Sulfate & $3 / 1 / 93$ & NA & $3 / 10 / 93$ & B08VX4 & G238 & G401 & 1.389 & $<1.01 E+02$ & $\mathrm{ug} / \mathrm{mL}$ \\
\hline Sulfate & $3 / 1 / 93$ & NA & $3 / 10 / 93$ & BOB3D1 & NS & G378 & 1.334 & $<1.01 E+02$ & $\mathrm{ug} / \mathrm{mL}$ \\
\hline Sulfate & $3 / 1 / 93$ & NA & $3 / 10 / 93$ & 808302 & NS & G379 & 1.329 & $<1.01 E+02$ & $\mathrm{ug} / \mathrm{mL}$ \\
\hline S042- & NA & NA & NA & NA & NA & NA & 7.33 & $1.75 \mathrm{E}-01$ & Mole/L \\
\hline Sulfate & $3 / 1 / 93$ & NA & $3 / 10 / 93$ & ВовзС 9 & G249 & G418 & 1.3 & $1.20 E+04$ & $\mathrm{ug} / \mathrm{mL}$ \\
\hline Sulfate & $3 / 1 / 93$ & NA & $3 / 10 / 93$ & B08VX7 & G243 & G409 & 1.298 & $4.88 E+03$ & $\mathrm{ug} / \mathrm{mL}$ \\
\hline Sulfate & $3 / 1 / 93$ & NA & $3 / 10 / 93$ & B08VX7 & G243 & G409 & 1.298 & $4.28 E+03$ & $\mathrm{ug} / \mathrm{mL}$ \\
\hline Sulfate & $3 / 1 / 93$ & NA & $3 / 10 / 93$ & B08VX6 & G241 & G407 & 1.338 & $4.04 E+03$ & $\mathrm{ug} / \mathrm{mL}$ \\
\hline Sulfate & $3 / 1 / 93$ & NA & $3 / 10 / 93$ & B0B3C9 & G249 & G418 & 1.3 & $3.39 E+03$ & $\mathrm{ug} / \mathrm{mL}$ \\
\hline Sulfate & $3 / 1 / 93$ & NA & $3 / 10 / 93$ & $808 \mathrm{~V} \times 3$ & G271 & G397 & 1.3 & $3.33 E+03$ & $u g / m L$ \\
\hline Sulfate & $3 / 1 / 93$ & NA & $3 / 10 / 93$ & B0B3D1 & NA & G378 & 1.334 & $3.24 E+03$ & $\mathrm{ug} / \mathrm{mL}$ \\
\hline Sulfate & $3 / 1 / 93$ & NA & $3 / 10 / 93$ & B08VX2 & G254 & G396 & 1.298 & $3.20 E+03$ & ug/mL \\
\hline Sulfate & $3 / 1 / 93$ & NA & $3 / 10 / 93$ & B08VX7 & G243 & G409 & 1.298 & $3.00 \mathrm{E}+03$ & $\mathrm{ug} / \mathrm{mL}$ \\
\hline Sulfate & $3 / 1 / 93$ & NA & $3 / 10 / 93$ & $\mathrm{~B} 08 \mathrm{~V} \times 3$ & G271 & G397 & 1.3 & $2.94 E+03$ & $\mathrm{ug} / \mathrm{mL}$ \\
\hline $\mathrm{SO}_{4}{ }^{2-}$ & $3 / 1 / 93$ & NA & $3 / 10 / 93$ & NA & NA & NA & 1.336 & 2610 & $\mathrm{ug} / \mathrm{mL}$ \\
\hline $\mathrm{SO}_{4}^{2-}$ & $3 / 1 / 93$ & NA & $3 / 10 / 93$ & NA & NA & NA & 1.336 & 2420 & $\mathrm{ug} / \mathrm{mL}$ \\
\hline Sulfate & $3 / 1 / 93$ & NA & $3 / 10 / 93$ & B08VX2 & G254 & G396 & 1.298 & $2.31 E+03$ & $\mathrm{ug} / \mathrm{mL}$ \\
\hline Sulfate & $3 / 1 / 93$ & NA & $3 / 10 / 93$ & $\mathrm{BOB} 3 \mathrm{C} 8$ & G248 & G417 & 1.327 & $2.20 \mathrm{E}+03$ & $\mathrm{ug} / \mathrm{mL}$ \\
\hline Sulfate & $3 / 1 / 93$ & NA & $3 / 10 / 93$ & NA & G250 & G422 & 1.348 & $2.07 E+03$ & $\mathrm{ug} / \mathrm{mL}$ \\
\hline Sulfate & $3 / 1 / 93$ & $\mathrm{NA}$ & $3 / 10 / 93$ & B08VX9 & G245 & G413 & 1.335 & $1.97 E+03$ & $\mathrm{ug} / \mathrm{mL}$ \\
\hline Sulfate & $3 / 1 / 93$ & NA & $3 / 10 / 93$ & BOB3D2 & NA & G379 & 1.329 & $1.97 \mathrm{E}+03$ & $u g / m L$ \\
\hline Sulfate & $3 / 1 / 93$ & NA & $3 / 10 / 93$ & $808 \mathrm{~V} \times 1$ & G253 & G392 & 1.341 & $1.77 E+03$ & $\mathrm{ug} / \mathrm{mL}$ \\
\hline Sulfate & $3 / 1 / 93$ & NA & $3 / 10 / 93$ & B08VX0 & G251 & G391 & 1.344 & $1.68 E+03$ & $\mathrm{ug} / \mathrm{mL}$ \\
\hline Sulfate & $3 / 1 / 93$ & NA & $3 / 10 / 93$ & $808 V \times 5$ & G240 & G402 & 1.338 & $1.52 \mathrm{E}+03$ & $\mathrm{ug} / \mathrm{mL}$ \\
\hline Sulfate & $3 / 1 / 93$ & NA & $3 / 10 / 93$ & NA & G250 & G422 & 1.348 & $1.37 \mathrm{E}+03$ & $\mathrm{tg} / \mathrm{mL}$ \\
\hline Sulfate & $3 / 1 / 93$ & NA & $3 / 10 / 93$ & NA & G250 & G422 & 1.348 & $1.34 \mathrm{E}+03$ & $u g / m L$ \\
\hline Sulfate & $3 / 1 / 93$ & $\mathrm{NA}$ & $3 / 10 / 93$ & B08VX8 & G244 & $\mathrm{G} 412$ & 1.377 & $1.27 E+03$ & $\mathrm{ug} / \mathrm{mL}$ \\
\hline Sulfate & $3 / 1 / 93$ & NA & $3 / 10 / 93$ & $808 \mathrm{~V} \times 4$ & G238 & G401 & 1.389 & $1.13 E+03$ & $\mathrm{ug} / \mathrm{mL}$ \\
\hline
\end{tabular}




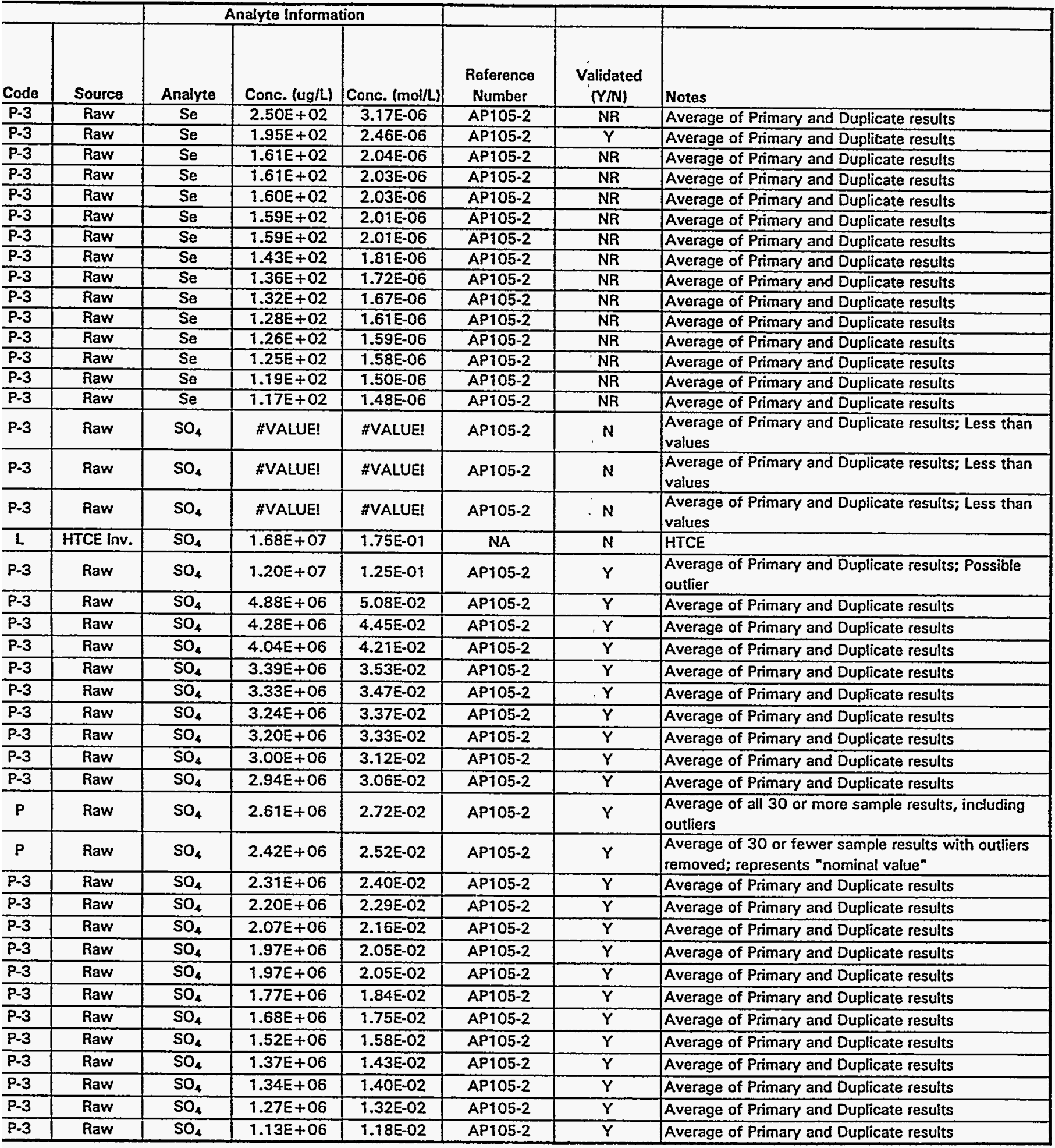




\begin{tabular}{|c|c|c|c|c|c|c|c|c|c|}
\hline & \multicolumn{9}{|c|}{ Tank Waste Characterization Sample Information } \\
\hline Reported Chemical & $\begin{array}{c}\text { Memo } \\
\text { Date }\end{array}$ & $\begin{array}{c}\text { Start Analysis } \\
\text { Date }\end{array}$ & $\begin{array}{c}\text { Start } \\
\text { Sample } \\
\text { Date } \\
\end{array}$ & $\begin{array}{c}\text { Sample } \\
\text { Number } \\
\end{array}$ & $\begin{array}{c}\text { Tank Farm } \\
\text { Number }\end{array}$ & $\begin{array}{c}\text { Lab Sample } \\
\text { Number } \\
\end{array}$ & $\begin{array}{c}\text { Density } \\
(\mathrm{g} / \mathrm{mL})\end{array}$ & $\begin{array}{c}\text { Reported } \\
\text { Value } \\
\end{array}$ & Reported Unit \\
\hline Sulfate & $3 / 1 / 93$ & NA & $3 / 10 / 93$ & $808 V \times 5$ & G240 & G402 & 1.338 & $4.42 E+02$ & $\mathrm{ug} / \mathrm{mL}$ \\
\hline TOC wt\% C (wet) & NA & NA & NA & NA & NA & $\mathrm{NA}$ & 1.33 & 8.01E-01 & wt\% \\
\hline TOC & $11 / 11 / 92$ & NS & NS & T3635 & NS & NS & 1.44 & 3.75 & $g / L$ \\
\hline TOC & $11 / 11 / 92$ & NS & NS & T3789 & NS & NS & 1.426 & 3.61 & $g / L$ \\
\hline Total organic carbon & $3 / 1 / 93$ & NA & $3 / 10 / 93$ & B08VX4 & G238 & G401 & 1.389 & $3.24 E+03$ & $\mathrm{ug} / \mathrm{mL}$ \\
\hline Total organic carbon & $3 / 1 / 93$ & NA & $3 / 10 / 93$ & B0B3D2 & NA & G379 & 7.329 & $3.03 E+03$ & $\mathrm{ug} / \mathrm{mL}$ \\
\hline Total organic carbon & $3 / 1 / 93$ & NA & $3 / 10 / 93$ & $808 \mathrm{~V} \times 5$ & G240 & G402 & 1.338 & $3.01 E+03$ & $\mathrm{ug} / \mathrm{mL}$ \\
\hline Total organic carbon & $3 / 1 / 93$ & NA & $3 / 10 / 93$ & $808 \mathrm{~V} \times 3$ & G271 & G397 & 1.3 & $3.00 E+03$ & $\mathrm{ug} / \mathrm{mL}$ \\
\hline Total organic carbon & $3 / 1 / 93$ & NA & $3 / 10 / 93$ & B083C9 & G249 & G418 & 1.3 & $2.99 E+03$ & $\mathrm{ug} / \mathrm{mL}$ \\
\hline Total organic carbon & $3 / 1 / 93$ & NA & $3 / 10 / 93$ & B08VX6 & G241 & G407 & 1.338 & $2.85 E+03$ & $\mathrm{ug} / \mathrm{mL}$ \\
\hline Total organic carbon & $3 / 1 / 93$ & NA & $3 / 10 / 93$ & $\mathrm{~B} 08 \mathrm{~V} \times 0$ & G251 & G391 & 1.344 & $2.83 E+03$ & $\mathrm{ug} / \mathrm{mL}$ \\
\hline Total organic carbon & $3 / 1 / 93$ & NA & $3 / 10 / 93$ & NA & G250 & G422 & 1.348 & $2.80 E+03$ & $\mathrm{ug} / \mathrm{mL}$ \\
\hline Total organic carbon & $3 / 1 / 93$ & NA & $3 / 10 / 93$ & B08VX8 & G244 & G412 & 1.377 & $2.80 E+03$ & $\mathrm{ug} / \mathrm{mL}$ \\
\hline Total organic carbon & $3 / 1 / 93$ & NA & $3 / 10 / 93$ & $808 \mathrm{X} \times 7$ & G243 & G409 & 1.298 & $2.77 E+03$ & $\mathrm{ug} / \mathrm{mL}$ \\
\hline Total organic carbon & $3 / 1 / 93$ & NA & $3 / 10 / 93$ & 808301 & NA & G378 & 1.334 & $2.77 E+03$ & $\mathrm{ug} / \mathrm{mL}$ \\
\hline TOC & $3 / 1 / 93$ & NA & $3 / 10 / 93$ & NA & NA & NA & 1.336 & 2750 & $u g / m L$ \\
\hline Total organic carbon & $3 / 1 / 93$ & NA & $3 / 10 / 93$ & B08VX3 & G271 & G397 & 1.3 & $2.75 E+03$ & $\mathrm{ug} / \mathrm{mL}$ \\
\hline Total organic carbon & $3 / 1 / 93$ & NA & $3 / 10 / 93$ & B08VX2 & G254 & G396 & 1.298 & $2.72 E+03$ & $\mathrm{ug} / \mathrm{mL}$ \\
\hline Total organic carbon & $3 / 1 / 93$ & NA & $3 / 10 / 93$ & $\mathrm{BOB} 3 \mathrm{C} 8$ & G248 & G417 & 1.327 & $2.62 E+03$ & $\mathrm{ug} / \mathrm{mL}$ \\
\hline Total organic carbon & $3 / 1 / 93$ & NA & $3 / 10 / 93$ & B08VX9 & G245 & G413 & 1.335 & $2.53 E+03$ & $\mathrm{ug} / \mathrm{mL}$ \\
\hline TOC & $11 / 11 / 92$ & NS & NS & T3954 & NS & NS & 1.1963 & 2.32 & g/L \\
\hline Total organic carbon & $3 / 1 / 93$ & NA & $3 / 10 / 93$ & B08VX1 & G253 & G392 & 1.341 & $2.26 E+03$ & $\mathrm{ug} / \mathrm{mL}$ \\
\hline Total organic carbon & $3 / 1 / 93$ & NA & $3 / 10 / 93$ & $\mathrm{~B} 08 \mathrm{~V} \times 8$ & G244 & G412 & 1.377 & $1.85 E+03$ & $\mathrm{ug} / \mathrm{mL}$ \\
\hline$U$ & $3 / 1 / 93$ & NA & $3 / 10 / 93$ & NA & NA & G379 & 1.329 & $4.86 E+01$ & $\mathrm{ug} / \mathrm{mL}$ \\
\hline $\mathrm{u}$ & $3 / 1 / 93$ & NA & $3 / 10 / 93$ & NA & NA & G378 & 1.334 & $3.58 E+01$ & $\mathrm{ug} / \mathrm{mL}$ \\
\hline
\end{tabular}




\begin{tabular}{|c|c|c|c|c|c|c|c|}
\hline \multirow[b]{2}{*}{ ode } & \multirow[b]{2}{*}{ Source } & \multicolumn{3}{|c|}{ Analyte Information } & \multirow[b]{2}{*}{$\begin{array}{c}\text { Reference } \\
\text { Number }\end{array}$} & \multirow[b]{2}{*}{$\begin{array}{l}\text { Validated } \\
\text { (YN) }\end{array}$} & \multirow[b]{2}{*}{ Notes } \\
\hline & & Analyte & Conc. (ug/L) & Conc. (mol/L) & & & \\
\hline 2.3 & Raw & $\mathrm{SO}_{4}$ & $4.42 E+05$ & $4.60 E-03$ & AP105-2 & $Y$ & $\begin{array}{l}\text { Average of Primary and Duplicate results; Possible } \\
\text { outlier }\end{array}$ \\
\hline$\underline{L}$ & HTCE InV. & TOC & $1.07 E+07$ & NS & NA & $\mathbf{N}$ & HTCE \\
\hline L & Raw & TOC & $3.75 E+06$ & NS & AP105-1 & N & $\begin{array}{l}\text { Analysis of evaporator slurry samples. The } \\
\text { evaporator slurry analysis represents only a } \\
\text { "sanpshot", and does not represent the final } \\
\text { composition of the waste in } 105-A P\end{array}$ \\
\hline L & Raw & TOC & $3.61 E+06$ & NS & AP105-1 & N & $\begin{array}{l}\text { Analysis of evaporator slurry samples. The } \\
\text { evaporator slurry analysis represents only a } \\
\text { "sanpshot", and does not represent the final } \\
\text { composition of the waste in 105-AP }\end{array}$ \\
\hline$\overline{-3}$ & Raw & TOC & $3.24 E+06$ & $2.70 E-01$ & AP105-2 & $Y$ & Average of Primary and Duplicate results \\
\hline 3 & Raw & TOC & $3.03 E+06$ & 2.52E-01 & AP105-2 & $\bar{Y}$ & Average of Primary and Duplicate results \\
\hline$\overline{-3}$ & Raw & TOC & $3.01 E+06$ & 2.50E-01 & AP105-2 & $\bar{Y}$ & Average of Primary and Duplicate results \\
\hline-3 & Raw & TOC & $3.00 E+06$ & 2.50E-01 & AP105-2 & $\bar{Y}$ & Average of Primary and Duplicate results \\
\hline-3 & Raw & TOC & $2.99 \mathrm{E}+06$ & $2.49 E-01$ & AP105-2 & $\bar{Y}$ & Average of Primary and Duplicate results \\
\hline$\overline{-3}$ & Raw & TOC & $2.85 E+06$ & 2.37 E-01 & AP105-2 & $\bar{Y}$ & Average of Primary and Duplicate results \\
\hline-3 & Raw & TOC & $2.83 E+06$ & $2.35 E-01$ & AP105-2 & $\bar{Y}$ & Average of Primary and Duplicate results \\
\hline$\overline{-3}$ & Raw & TOC & $2.80 E+06$ & $2.33 E-01$ & AP105-2 & $\bar{Y}$ & Average of Primary and Duplicate results \\
\hline-3 & Raw & TOC & $2.80 E+06$ & $2.33 \mathrm{E}-01$ & AP105-2 & $\bar{Y}$ & Average of Primary and Duplicate results \\
\hline-3 & Raw & TOC & $2.77 E+06$ & $2.31 E-01$ & AP105-2 & $\bar{Y}$ & Average of Primary and Duplicate results \\
\hline-3 & Raw & TOC & $2.77 E+06$ & $2.30 \mathrm{E}-01$ & AP105-2 & $\bar{Y}$ & Average of Primary and Duplicate results \\
\hline $\mathbf{P}$ & Raw & TOC & $2.75 E+06$ & NA & AP105-2 & $\mathrm{Y}$ & $\begin{array}{l}\text { Statistical average of } 13 \text { indivisual tank samples and } \\
2 \text { composite samples, and represents the "nominal } \\
\text { value" for this analyte }\end{array}$ \\
\hline-3 & Raw & TOC & $2.75 E+06$ & 2.29E-01 & AP105-2 & $\bar{Y}$ & Average of Primary and Duplicate results \\
\hline-3 & Raw & TOC & $2.72 E+06$ & $2.27 E-01$ & AP105-2 & $\bar{Y}$ & Average of Primary and Duplicate results \\
\hline$-\overline{3}$ & Raw & TOC & $2.62 E+06$ & 2.18E-01 & AP105-2 & $Y$ & Average of Primary and Duplicate results \\
\hline-3 & Raw & TOC & $2.53 E+06$ & $2.10 \mathrm{E}-01$ & AP105-2 & $\bar{Y}$ & Average of Primary and Duplicate results \\
\hline $\mathbf{L}$ & Raw & TOC & $2.32 E+06$ & NS & AP105-1 & N & $\begin{array}{l}\text { Analysis of evaporator slurry samples. The } \\
\text { evaporator slurry analysis represents only a } \\
\text { "sanpshot", and does not represent the final } \\
\text { composition of the waste in } 105-\text { AP }\end{array}$ \\
\hline-3 & Raw & TOC & $2.26 E+06$ & $1.88 \mathrm{E}-01$ & AP105-2 & $\bar{Y}$ & Average of Primary and Duplicate results \\
\hline-3 & Raw & TOC & $1.85 E+06$ & $1.54 \mathrm{E}-01$ & AP105-2 & $\bar{Y}$ & Average of Primary and Duplicate results \\
\hline-3 & Raw & $u$ & $4.86 E+04$ & $2.04 \mathrm{E}-04$ & AP105-2 & $Y$ & $\begin{array}{l}\text { Average of Primary and Duplicate results; Composite } \\
\text { sample }\end{array}$ \\
\hline-3 & Raw & $u$ & $3.58 E+04$ & $1.50 \mathrm{E}-04$ & AP105-2 & $Y$ & $\begin{array}{l}\text { Average of Primary and Duplicate results; Composite } \\
\text { sample }\end{array}$ \\
\hline
\end{tabular}


TWRS Privatization Support Projı Chemical Liquids 1

\begin{tabular}{|c|c|c|c|c|c|c|c|c|c|}
\hline \multicolumn{10}{|c|}{ Tank Waste Characterization Sample Information } \\
\hline Reported Chemical & $\begin{array}{c}\text { Memo } \\
\text { Date }\end{array}$ & $\begin{array}{c}\text { Start Analysis } \\
\text { Date }\end{array}$ & $\begin{array}{c}\text { Start } \\
\text { Sample } \\
\text { Date } \\
\end{array}$ & $\begin{array}{l}\text { Sample } \\
\text { Number }\end{array}$ & $\begin{array}{c}\text { Tank Farm } \\
\text { Number }\end{array}$ & $\begin{array}{c}\text { Lab Sample } \\
\text { Number }\end{array}$ & $\begin{array}{l}\text { Density } \\
\text { (g/mL) }\end{array}$ & Reported Value & Reported Ur \\
\hline $\mathrm{Al}$ & $4 / 29 / 85$ & $4 / 21 / 85$ & NA & T5350 & NA & NA & 1.028 & $<8.09 \mathrm{E}-03$ & Moles/L \\
\hline Aluminum & $12 / 19 / 91$ & $7 / 17 / 90$ & $6 / 28 / 90$ & NA & NA & R8795 & 1.540 & $1.300 E+00$ & $M$ \\
\hline Aluminum & $12 / 19 / 91$ & $7 / 17 / 90$ & $6 / 28 / 90$ & NA & NA & $\mathbf{R} 8796$ & 1.540 & $1.280 E+00$ & $M$ \\
\hline Al & $9 / 18 / 86$ & NA & $6 / 23 / 86$ & T621 & NS & NS & 1.440 & 1.14 & Moles/L \\
\hline Aluminum & $12 / 19 / 91$ & $7 / 17 / 90$ & $6 / 28 / 90$ & NA & R4798 & R7897 & 1.560 & $1.105 E+00$ & $M$ \\
\hline Al & $9 / 18 / 86$ & NA & $7 / 2 / 86$ & R9896 & NA & NA & 1.500 & 1.06 & Moles/L \\
\hline Aluminum & $12 / 19 / 91$ & $7 / 17 / 90$ & $6 / 28 / 90$ & NA & R4794 & R7891 & 1.570 & $1.050 E+00$ & $M$ \\
\hline $\mathrm{Al}$ & $9 / 18 / 86$ & NA & $6 / 26 / 86$ & R9894 & $\mathrm{NA}$ & $\mathrm{NA}$ & 1.490 & 1.05 & Moles/L \\
\hline Aluminum & $12 / 19 / 91$ & $7 / 17 / 90$ & $6 / 28 / 90$ & NA & R4793 & R7890 & 1.535 & $1.045 \mathrm{E}+00$ & $M$ \\
\hline Aluminum & $12 / 19 / 91$ & $7 / 17 / 90$ & $6 / 28 / 90$ & NA & $\mathrm{R} 4802$ & R7887/R7903 & 1.550 & $1.045 E+00$ & $M$ \\
\hline Aluminum & $12 / 19 / 91$ & $7 / 17 / 90$ & $6 / 28 / 90$ & NA & $R 4801$ & $R 7902$ & 1.560 & $1.040 \mathrm{E}+00$ & $M$ \\
\hline Aluminum & $12 / 19 / 91$ & $7 / 17 / 90$ & $6 / 28 / 90$ & NA & $\mathrm{R} 4800$ & R7901 & 1.560 & $1.040 \mathrm{E}+00$ & $\bar{M}$ \\
\hline Aluminum & $12 / 19 / 91$ & $7 / 17 / 90$ & $6 / 28 / 90$ & NA & $R 4796$ & R7895 & 1.625 & $1.035 E+00$ & $\bar{M}$ \\
\hline $\mathrm{Al}$ & $6 / 28 / 90$ & NA & $\mathrm{NA}$ & $n / a$ & $\mathrm{NA}$ & NA & 1.560 & 1.033 & Moles/L \\
\hline Aluminum & $12 / 19 / 91$ & $7 / 17 / 90$ & $6 / 28 / 90$ & NA & R4799 & R7898 & 1.555 & $1.030 E+00$ & $M$ \\
\hline Aluminum & $12 / 19 / 91$ & $7 / 17 / 90$ & $6 / 28 / 90$ & NA & $\mathrm{R} 4792$ & R7889 & 1.530 & 1.030 & $M$ \\
\hline Aluminum & $12 / 19 / 91$ & $7 / 17 / 90$ & $6 / 28 / 90$ & NA & R4797 & R7896 & 1.560 & $1.015 E+00$ & $M$ \\
\hline Aluminum & $12 / 19 / 91$ & $7 / 17 / 90$ & $6 / 28 / 90$ & NA & R4803 & R7888/R7904 & 1.575 & $1.008 E+00$ & $\bar{M}$ \\
\hline $\mathrm{Al} 3+$ & NA & NA & NA & NA & NA & NA & 1.39 & $1.00 E+00$ & Mole/L \\
\hline$\overline{A l}$ & $9 / 18 / 86$ & NA & $6 / 30 / 86$ & R9895 & NA & NA & 1.540 & 0.96 & Moles/L \\
\hline Aluminum & $12 / 19 / 91$ & $7 / 17 / 90$ & $6 / 28 / 90$ & $\mathrm{NA}$ & R4795 & $\overline{R 7894}$ & 1.540 & 9.510E-01 & $M$ \\
\hline $\mathrm{Al}$ & $12 / 30 / 81$ & NA & NA & $\mathrm{T} 1048$ & NA & NA & 1.324 & 0.824 & Moles \\
\hline $\mathrm{Al}$ & $12 / 30 / 81$ & NA & NA & T1049 & NA & NA & 1.329 & 0.799 & Moles \\
\hline $\mathrm{Al}$ & $9 / 18 / 86$ & NA & $6 / 23 / 86$ & R9893 & $\mathrm{NA}$ & NA & 1.370 & 0.79 & Moles/L \\
\hline $\mathrm{Al}$ & $9 / 18 / 86$ & NA & $7 / 6 / 86$ & R9897 & NA & NA & 1.460 & 0.76 & Moles $/ \mathrm{L}$ \\
\hline Al & $9 / 18 / 86$ & NA & $6 / 26 / 86$ & T686 & NS & NS & 1.310 & 0.75 & Moles/L \\
\hline $\mathrm{Al}^{+3}$ & $4 / 3 / 84$ & NA & NA & 89414 & NA & NA & 1.330 & 0.33 & Moles/L \\
\hline $\mathrm{Al}^{+3}$ & $4 / 3 / 84$ & $\mathrm{NA}$ & NA & R9408 & NA & NA & 1.330 & 0.33 & Moles $/ \mathrm{L}$ \\
\hline Al & $9 / 18 / 86$ & NA & $6 / 30 / 86$ & T812 & NS & NS & 1.130 & 0.3 & Moles/L \\
\hline Al & $9 / 18 / 86$ & NA & $7 / 2 / 86$ & T882 & NS & NS & 1.320 & 0.28 & Moles/L \\
\hline $\mathrm{Al}^{+3}$ & $11 / 20 / 84$ & NA & NA & R2644 & NS & NS & NS & 0.074 & Moles/L \\
\hline $\mathrm{Al}$ & $2 / 20 / 86$ & $11 / 14 / 85$ & NA & T2641 & NS & NS & 1.010 & 0.057 & Moles $/ \mathrm{L}$ \\
\hline$A$ & $2 / 20 / 86$ & $11 / 14 / 85$ & NA & $T 2601$ & NS & NS & 1.010 & 0.045 & Moles/L \\
\hline $\mathrm{Al}$ & $8 / 18 / 85$ & $8 / 18 / 85$ & NA & T9368 & NA & NA & 1.040 & 0.0441 & Moles/L \\
\hline $\mathrm{Al}^{+3}$ & $11 / 20 / 84$ & NA & NA & $\mathrm{R} 2645$ & NS & NS & NS & 0.0104 & Moles/L \\
\hline$\overline{A l}$ & $4 / 29 / 86$ & $3 / 30 / 86$ & NA & $T 7697$ & NS & NS & 0.993 & 0.002 & Moles/L \\
\hline $\mathrm{Al}$ & $4 / 29 / 86$ & $3 / 30 / 86$ & NA & $T 7698$ & NS & NS & 0.993 & 0.002 & Moles/L \\
\hline Arsenic & $12 / 19 / 91$ & $7 / 17 / 90$ & $6 / 28 / 90$ & NA & NA & R8798 & 1.540 & $<1.33 \mathrm{E}-07$ & $\bar{M}$ \\
\hline Barium & $12 / 19 / 91$ & $7 / 17 / 90$ & $6 / 28 / 90$ & NA & NA & R8796 & 1.540 & $<2.60$ E-06 & $M$ \\
\hline Barium & $12 / 19 / 91$ & $7 / 17 / 90$ & $6 / 28 / 90$ & NA & R4801 & R7902 & 1.560 & $<6.62 E-05$ & $M$ \\
\hline Barium & $12 / 19 / 91$ & $7 / 17 / 90$ & $6 / 28 / 90$ & NA & R4800 & R7901 & 1.560 & $<6.62 \mathrm{E}-05$ & $M$ \\
\hline Barium & $12 / 19 / 91$ & $7 / 17 / 90$ & $6 / 28 / 90$ & NA & R4799 & R7898 & 1.555 & $<6.62 E .05$ & $\vec{M}$ \\
\hline Barium & $12 / 19 / 91$ & $7 / 17 / 90$ & $6 / 28 / 90$ & NA & R4798 & R7897 & 1.560 & $<6.62 E-05$ & $M$ \\
\hline Barium & $12 / 19 / 91$ & $7 / 17 / 90$ & $6 / 28 / 90$ & NA & R4797 & R7896 & 1.560 & $<6.62 E-05$ & $M$ \\
\hline Barium & $12 / 19 / 91$ & $7 / 17 / 90$ & $6 / 28 / 90$ & NA & R4796 & R7895 & 1.625 & $<6.62 \mathrm{E}-05$ & $M$ \\
\hline Barium & $12 / 19 / 91$ & $7 / 17 / 90$ & $6 / 28 / 90$ & NA & R4795 & R7894 & 1.540 & $<8.81 E-05$ & $M$ \\
\hline Barium & $12 / 19 / 91$ & $7 / 17 / 90$ & $6 / 28 / 90$ & $\mathrm{NA}$ & R4794 & R7891 & 1.570 & $<6.62 E-05$ & $M$ \\
\hline Barium & $12 / 19 / 91$ & $7 / 17 / 90$ & $6 / 28 / 90$ & NA & R4793 & R7890 & 1.535 & $<6.62 \mathrm{E}-05$ & $M$ \\
\hline
\end{tabular}




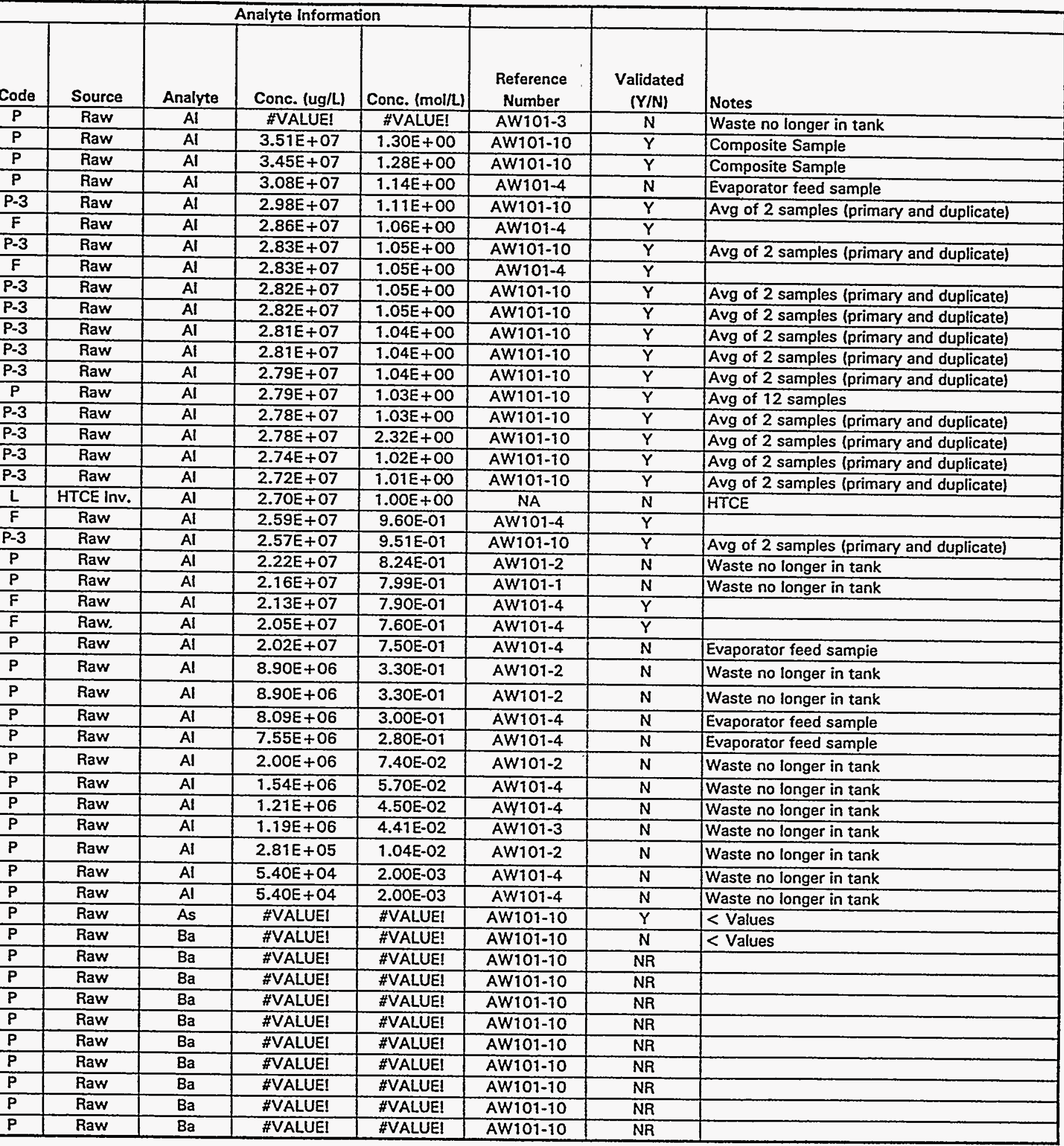


TWRS Privatization Support Proj Chemical Liquids

\begin{tabular}{|c|c|c|c|c|c|c|c|c|c|}
\hline & & & & Tank W & Characteriza & tion Sample Inform & ion & & \\
\hline Reported Chemical & $\begin{array}{c}\text { Memo } \\
\text { Date } \\
\end{array}$ & $\begin{array}{c}\text { Start Analysis } \\
\text { Date }\end{array}$ & $\begin{array}{c}\text { Start } \\
\text { Sample } \\
\text { Date }\end{array}$ & $\begin{array}{l}\text { Sample } \\
\text { Number }\end{array}$ & $\begin{array}{c}\text { Tank Farm } \\
\text { Number }\end{array}$ & $\begin{array}{c}\text { Lab Sample } \\
\text { Number } \\
\end{array}$ & $\begin{array}{c}\text { Density } \\
(\mathrm{g} / \mathrm{mL})\end{array}$ & Reported Valua & Reported U \\
\hline Barium & $12 / 19 / 91$ & $7 / 17 / 90$ & $6 / 28 / 90$ & $\mathrm{NA}$ & $\mathrm{R} 4792$ & R7889 & 1.530 & $<6.62 \mathrm{E}-05$ & $M$ \\
\hline Barium & $12 / 19 / 91$ & $7 / 17 / 90$ & $6 / 28 / 90$ & NA & R4803 & R7888/R7904 & 1.575 & $<6.62 \mathrm{E}-05$ & $M$ \\
\hline Barium & $12 / 19 / 91$ & $7 / 17 / 90$ & $6 / 28 / 90$ & NA & R4802 & R7887/R7903 & 1.550 & $<6.62 \mathrm{E}-05$ & $M$ \\
\hline Barium & $12 / 19 / 91$ & $7 / 17 / 90$ & $6 / 28 / 90$ & $\overline{N A}$ & NA & R8795 & 1.540 & $6.250 \mathrm{E}-06$ & $M$ \\
\hline Calcium & $12 / 19 / 91$ & $7 / 17 / 90$ & $6 / 28 / 90$ & NA & R4796 & R7895 & 1.625 & $2.565 \mathrm{E}-03$ & $M$ \\
\hline Calcium & $12 / 19 / 91$ & $7 / 17 / 90$ & $6 / 28 / 90$ & $\mathrm{NA}$ & 84798 & R7897 & 1.560 & $1.735 E-03$ & $M$ \\
\hline Calcium & $12 / 19 / 91$ & $7 / 17 / 90$ & $6 / 28 / 90$ & NA & R4795 & R7894 & 1.540 & $1.460 \mathrm{E}-03$ & $\bar{M}$ \\
\hline Calcium & $12 / 19 / 91$ & $7 / 17 / 90$ & $6 / 28 / 90$ & NA & R4797 & R7896 & 1.560 & $5.575 \mathrm{E}-04$ & $M$ \\
\hline $\mathrm{Ca}$ & $6 / 23 / 86$ & NS & NS & NS & NS & NS & 1.37 & 0.00158 & Wt\% \\
\hline $\mathrm{Ca}$ & $9 / 18 / 86$ & NS & NS & NS & NS & NS & 1.53 & 0.00136 & Wt\% \\
\hline $\mathrm{Ca}$ & $6 / 26 / 86$ & NS & NS & NS & NS & NS & 1.49 & 0.00139 & Wt\% \\
\hline Calcium & $12 / 19 / 91$ & $7 / 17 / 90$ & $6 / 28 / 90$ & NA & $R 4800$ & R7901 & 1.560 & 5.065E-04 & $M$ \\
\hline Calcium & $12 / 19 / 91$ & $7 / 17 / 90$ & $6 / 28 / 90$ & NA & R4799 & R7898 & 1.555 & 4.955E-04 & $M$ \\
\hline Calcium & $12 / 19 / 91$ & $7 / 17 / 90$ & $6 / 28 / 90$ & NA & 84802 & R7887/R7903 & 1.550 & $4.680 \mathrm{E}-04$ & $M$ \\
\hline $\mathrm{Ca}$ & $7 / 2 / 86$ & NS & NS & NS & NS & NS & 7.5 & 0.00124 & Wt\% \\
\hline Calcium & $12 / 19 / 91$ & $7 / 17 / 90$ & $6 / 28 / 90$ & NA & $\mathrm{R} 4793$ & R7890 & 1.535 & $4.480 \mathrm{E}-04$ & $M$ \\
\hline Calcium & $12 / 19 / 91$ & $7 / 17 / 90$ & $6 / 28 / 90$ & NA & $\mathrm{R} 4803$ & R7888 / R7904 & 1.575 & $4.265 \mathrm{E}-04$ & $M$ \\
\hline Calcium & $12 / 19 / 91$ & $7 / 17 / 90$ & $6 / 28 / 90$ & $\mathrm{NA}$ & R4794 & R7891 & 1.570 & $4.180 \mathrm{E}-04$ & $\bar{M}$ \\
\hline Calcium & $12 / 19 / 91$ & $7 / 17 / 90$ & $6 / 28 / 90$ & NA & R4792 & R7889 & 1.530 & 4.150 E-04 & $M$ \\
\hline Calcium & $12 / 19 / 91$ & $7 / 17 / 90$ & $6 / 28 / 90$ & NA & R4801 & R7902 & 1.560 & $4.140 \mathrm{E}-04$ & $M$ \\
\hline Cadmium & $12 / 19 / 91$ & $7 / 17 / 90$ & $6 / 28 / 90$ & NA & R4801 & R7902 & 1.560 & $<1.08 \mathrm{E}-04$ & $M$ \\
\hline Cadmium & $12 / 19 / 91$ & $7 / 17 / 90$ & $6 / 28 / 90$ & NA & R4800 & $\overline{R 7901}$ & 1.560 & $<1.08 E-04$ & $M$ \\
\hline Cadmium & $12 / 19 / 91$ & $7 / 17 / 90$ & $6 / 28 / 90$ & NA & R4799 & $R 7898$ & 1.555 & $<1.08 \mathrm{E}-04$ & $M$ \\
\hline Cadmium & $12 / 19 / 91$ & $7 / 17 / 90$ & $6 / 28 / 90$ & NA & R4798 & R7897 & 1.560 & $<1.08 \mathrm{E}-04$ & $M$ \\
\hline Cadmium & $12 / 19 / 91$ & $7 / 17 / 90$ & $6 / 28 / 90$ & NA & R4797 & R7896 & 1.560 & $<1.08 \mathrm{E}-04$ & $\bar{M}$ \\
\hline Cadmium & $12 / 19 / 91$ & $7 / 17 / 90$ & $6 / 28 / 90$ & NA & R4796 & R7895 & 1.625 & $<1.08 \mathrm{E}-04$ & $M$ \\
\hline Cadmium & $12 / 19 / 91$ & $7 / 17 / 90$ & $6 / 28 / 90$ & NA & R4795 & 87894 & 1.540 & $<1.08 \mathrm{E}-04$ & $M$ \\
\hline Cadmium & $12 / 19 / 91$ & $7 / 17 / 90$ & $6 / 28 / 90$ & NA & R4794 & R7891 & 1.570 & $<1.08 \mathrm{E}-04$ & $M$ \\
\hline Cadmium & $12 / 19 / 91$ & $7 / 17 / 90$ & $6 / 28 / 90$ & NA & R4793 & R7890 & 1.535 & $<1.08 \mathrm{E}-04$ & $M$ \\
\hline Cadmium & $12 / 19 / 91$ & $7 / 17 / 90$ & $6 / 28 / 90$ & NA & R4792 & R7889 & 1.530 & $<1.08 E-04$ & $M$ \\
\hline Cadmium & $12 / 19 / 91$ & $7 / 17 / 90$ & $6 / 28 / 90$ & NA & 84803 & R7888/R7904 & 1.575 & $<1.08 \mathrm{E}-04$ & $M$ \\
\hline Cadmium & $12 / 19 / 91$ & $7 / 17 / 90$ & $6 / 28 / 90$ & $\mathrm{NA}$ & $\mathrm{R} 4802$ & R7887 / R7503 & 1.550 & $<1.08 \mathrm{E}-04$ & $M$ \\
\hline Cadmium & $12 / 19 / 91$ & $7 / 17 / 90$ & $6 / 28 / 90$ & NA & NA & R8795 & 1.540 & $6.280 \mathrm{E}-05$ & $M$ \\
\hline Cadmium & $12 / 19 / 91$ & $7 / 17 / 90$ & $6 / 28 / 90$ & NA & NA & R8796 & 1.540 & 5.470E-05 & $\bar{M}$ \\
\hline $\mathrm{Cl}$ & $9 / 18 / 86$ & NA & $6 / 26 / 86$ & R9894 & NA & NA & 1.490 & 0.23 & Moles/L \\
\hline $\mathrm{Cl}$ & $9 / 18 / 86$ & NA & $6 / 23 / 86$ & $T 621$ & NS & NS & 1.440 & 0.18 & Moles/L \\
\hline $\mathrm{Cl}$ & $9 / 18 / 86$ & NA & $6 / 30 / 86$ & R9895 & NA & NA & 1.540 & 0.18 & Moles/L \\
\hline $\mathrm{Cl}$ & $9 / 18 / 86$ & NA & $7 / 2 / 86$ & R9896 & NA & NA & 1.500 & 0.17 & Moles/L \\
\hline Chloride & $12 / 19 / 91$ & $7 / 17 / 90$ & $6 / 28 / 90$ & NA & NA & R8801 & 1.540 & $1.690 \mathrm{E}-01$ & $\bar{M}$ \\
\hline Chloride & $12 / 19 / 91$ & $7 / 17 / 90$ & $6 / 28 / 90$ & NA & $R 4801$ & R7923/R7942 & 1.560 & $1.600 \mathrm{E}-01$ & $M$ \\
\hline Chloride & $12 / 19 / 91$ & $7 / 17 / 90$ & $6 / 28 / 90$ & NA & $\mathrm{R} 4803$ & R7927/R7944 & 1.575 & $1.595 E-01$ & $M$ \\
\hline Chloride & $12 / 19 / 91$ & $7 / 17 / 90$ & $6 / 28 / 90$ & NA & $\mathrm{R} 4800$ & R7922/R7941 & 1.560 & $1.595 \mathrm{E}-01$ & $M$ \\
\hline Chloride & $12 / 19 / 91$ & $7 / 17 / 90$ & $6 / 28 / 90$ & $\mathrm{NA}$ & R4799 & R7921/R7940 & 1.555 & $1.595 \mathrm{E}-01$ & $M$ \\
\hline Chloride & $12 / 19 / 91$ & $7 / 17 / 90$ & $6 / 28 / 90$ & NA & NA & R8802 & 1.540 & $1.540 \mathrm{E}-01$ & $\vec{M}$ \\
\hline Chloride & $12 / 19 / 91$ & $7 / 17 / 90$ & $6 / 28 / 90$ & NA & $\mathrm{R} 4802$ & R7926/R7943 & 1.550 & $1.535 \mathrm{E}-01$ & $M$ \\
\hline $\mathrm{Cl}-$ & $\mathrm{NA}$ & NA & NA & $\overline{N A}$ & NA & NA & 1.39 & $1.49 \mathrm{E}-01$ & Mole/L \\
\hline Chloride & $6 / 28 / 90$ & NA & NA & NA & NA & NA & 1.560 & 0.146 & Moles/L \\
\hline Chloride & $12 / 19 / 91$ & $7 / 17 / 90$ & $6 / 28 / 90$ & NA & R4798 & $R 7920 / R 7937$ & 1.560 & $1.400 \mathrm{E}-01$ & $M$ \\
\hline Chloride & $12 / 19 / 91$ & $7 / 17 / 90$ & $6 / 28 / 90$ & NA & R4797 & R7919/R7936 & 1.560 & $1.400 E-01$ & $M$ \\
\hline
\end{tabular}




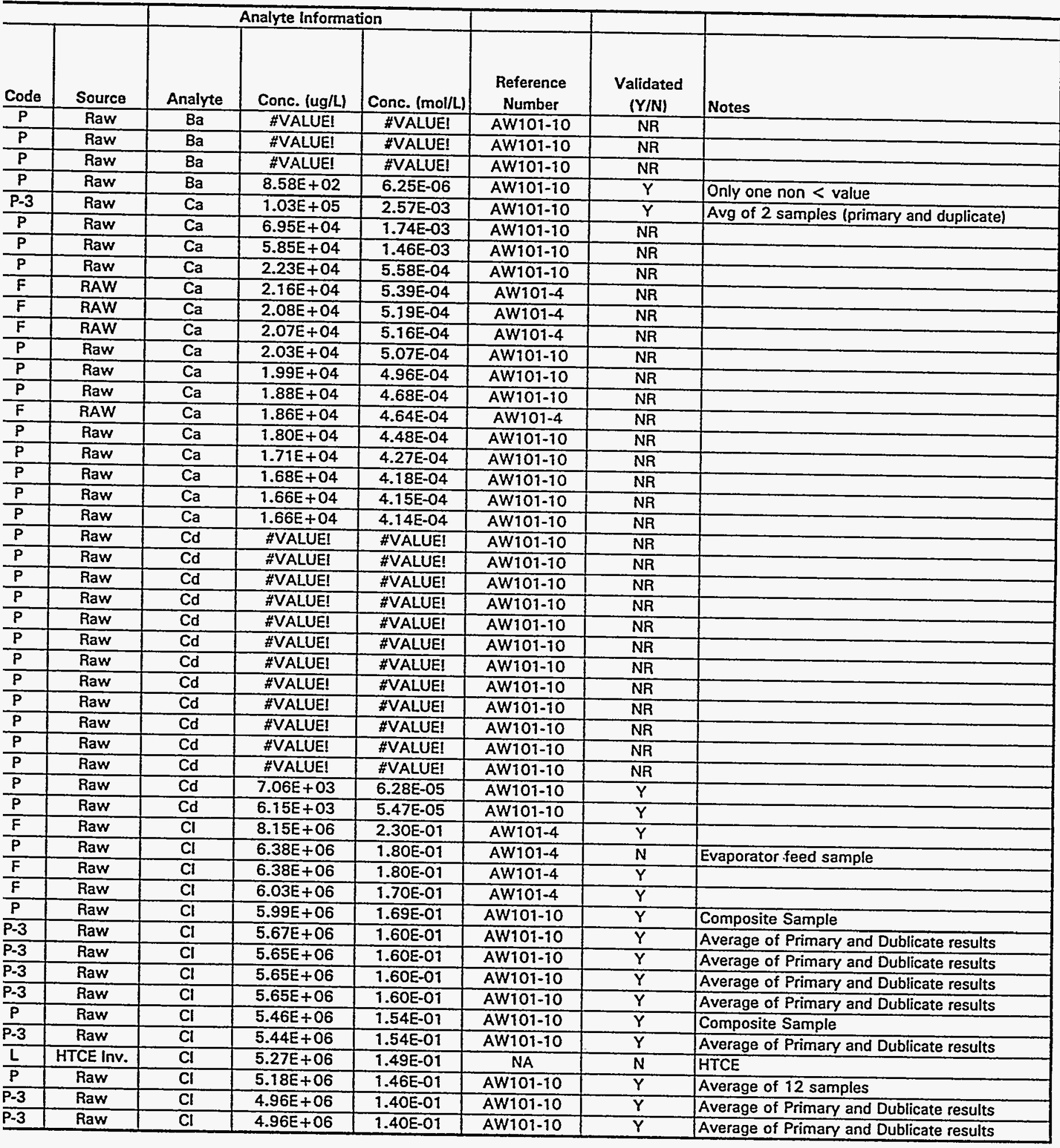




\begin{tabular}{|c|c|c|c|c|c|c|c|c|c|}
\hline & & & & Tank Was & Characteriza & in Sample Inform & Son & & \\
\hline Reported Chemical & $\begin{array}{c}\text { Memo } \\
\text { Date }\end{array}$ & $\begin{array}{c}\text { Start Analysis } \\
\text { Date }\end{array}$ & $\begin{array}{c}\text { Start } \\
\text { Sample } \\
\text { Date } \\
\end{array}$ & $\begin{array}{l}\text { Sample } \\
\text { Number }\end{array}$ & $\begin{array}{c}\text { Tank Farm } \\
\text { Number }\end{array}$ & $\begin{array}{c}\text { Lab Sample } \\
\text { Number }\end{array}$ & $\begin{array}{c}\text { Density } \\
(\mathrm{g} / \mathrm{mL})\end{array}$ & Reported Value & Reported $U_{1}$ \\
\hline Chloride & $12 / 19 / 91$ & $7 / 17 / 90$ & $6 / 28 / 90$ & NA & R4793 & R7913/R7930 & 1.535 & $1.390 \mathrm{E}-01$ & $M$ \\
\hline Chloride & $12 / 19 / 91$ & $7117 / 90$ & $6 / 28 / 90$ & NA & R4792 & R7912/R7929 & 1.530 & $1.390 \mathrm{E}-01$ & $\bar{M}$ \\
\hline Chloride & $12 / 19 / 91$ & $7 / 17 / 90$ & $6 / 28 / 90$ & NA & R4794 & R7914/R7933 & 1.570 & $1.375 \mathrm{E}-01$ & $M$ \\
\hline Chloride & $12 / 19 / 91$ & $7 / 17 / 90$ & $6 / 28 / 90$ & NA & R4796 & R7916/R7935 & 1.625 & $1.355 \mathrm{E}-01$ & $M$ \\
\hline $\mathrm{Cl}$ & $9 / 18 / 86$ & NA & $6 / 26 / 86$ & T686 & NS & NS & 1.310 & 0.13 & Moles/L \\
\hline $\mathrm{Cl}$ & $9 / 18 / 86$ & NA & $6 / 23 / 86$ & R9893 & NA & NA & 1.370 & 0.13 & Moles/L \\
\hline Chloride & $12 / 19 / 91$ & $7 / 17 / 90$ & $6 / 28 / 90$ & NA & R4795 & R7915/R7934 & 1.540 & $1.290 \mathrm{E}-01$ & $M$ \\
\hline $\mathrm{Cl}$ & $9 / 18 / 86$ & NA & $7 / 6 / 86$ & R9897 & NA & NA & 1.460 & 0.12 & Moles/L \\
\hline CI & $9 / 18 / 86$ & NA & $7 / 2 / 86$ & $T 882$ & NS & NS & 1.320 & 0.11 & Moles/L \\
\hline $\mathrm{Cl}$ & $9 / 18 / 86$ & NA & $6 / 30 / 86$ & $T 812$ & NS & NS & 1.130 & 0.04 & Moles/L \\
\hline $\mathrm{Cl}$ & $8 / 18 / 85$ & $8 / 18 / 85$ & NA & T9368 & NA & NA & 1.040 & 0.00754 & Moles/L \\
\hline $\mathrm{Cl}$ & $2 / 20 / 86$ & $11 / 14 / 85$ & NA & $T 2641$ & NS & NS & 1.010 & 0.005 & Moles/L \\
\hline Cl & $4 / 29 / 86$ & $3 / 30 / 86$ & NA & T7697 & NS & NS & 0.993 & 0.002 & Moles/L \\
\hline $\mathrm{Cl}$ & $4 / 29 / 86$ & $3 / 30 / 86$ & NA & T7698 & NS & NS & 0.993 & 0.002 & Moles/L \\
\hline $\mathrm{Cl}$ & $4 / 29 / 85$ & $4 / 21 / 85$ & NA & $T 5350$ & NA & NA & 1.028 & 0.00116 & Moles/L \\
\hline Chromium & $12 / 19 / 91$ & $7 / 17 / 90$ & $6 / 28 / 90$ & NA & R4795 & R7894 & 1.540 & $7.170 \mathrm{E}-03$ & $M$ \\
\hline $\mathrm{Cr}$ & $9 / 18 / 86$ & NA & $7 / 2 / 86$ & R9896 & NA & NA & 1.5 & 0.00513 & $M$ \\
\hline Chromium & $12 / 19 / 91$ & $7 / 17 / 90$ & $6 / 28 / 90$ & $\mathrm{NA}$ & $\mathrm{NA}$ & R8795 & 1.540 & $4.690 \mathrm{E}-03$ & $M$ \\
\hline Chromium & $12 / 19 / 91$ & $7 / 17 / 90$ & $6 / 28 / 90$ & NA & NA & R8796 & 1.540 & $4.460 \mathrm{E}-03$ & $M$ \\
\hline $\mathrm{Cr}$ & $9 / 18 / 86$ & NA & $6 / 26 / 86$ & R9894 & NA & NA & 1.49 & 0.00433 & $M$ \\
\hline Chromium & $12 / 19 / 91$ & $7 / 17 / 90$ & $6 / 28 / 90$ & NA & $R 4796$ & R7895 & 1.625 & $4.260 \mathrm{E}-03$ & $M$ \\
\hline Chromium & $12 / 19 / 91$ & $7 / 17 / 90$ & $6 / 28 / 90$ & NA & $R 4798$ & R7897 & 1.560 & $3.720 E-03$ & $M$ \\
\hline $\mathrm{Cr}$ & $9 / 18 / 86$ & NA & $6 / 23 / 86$ & R9893 & NA & NA & 1.37 & 0.00275 & $M$ \\
\hline Chromium & $12 / 19 / 91$ & $7 / 17 / 90$ & $6 / 28 / 90$ & NA & R4803 & R7888/R7904 & 1.575 & $2.665 \mathrm{E}-03$ & $M$ \\
\hline Chromium & $12 / 19 / 91$ & $7 / 17 / 90$ & $6 / 28 / 90$ & NA & R4799 & $R 7898$ & 1.555 & $2.625 \mathrm{E}-03$ & $M$ \\
\hline Chromium & $12 / 19 / 91$ & $7 / 17 / 90$ & 6128190 & NA & R4792 & R7889 & 1.530 & $2.460 \mathrm{E}-03$ & $\bar{M}$ \\
\hline Chromium & $12 / 19 / 91$ & $7 / 17 / 90$ & $6 / 28 / 90$ & NA & R4797 & R7896 & 1.560 & $2.450 \mathrm{E}-03$ & $M$ \\
\hline Chromium & $12 / 19 / 91$ & $7 / 17 / 90$ & $6 / 28 / 90$ & NA & $\mathrm{R} 4800$ & R7901 & 1.560 & $2.425 E-03$ & $M$ \\
\hline Chromium & $12 / 19 / 91$ & $7 / 17 / 90$ & $6 / 28 / 90$ & NA & R4794 & R7891 & 1.570 & $2.415 \mathrm{E}-03$ & $\bar{M}$ \\
\hline Chromium & $12 / 19 / 91$ & $7 / 17 / 90$ & $6 / 28 / 90$ & NA & R4793 & R7890 & 1.535 & $2.385 \mathrm{E}-03$ & $M$ \\
\hline Chromium & $12 / 19 / 91$ & $7 / 17 / 90$ & $6 / 28 / 90$ & NA & R4801 & R7902 & 1.560 & $2.270 \mathrm{E}-03$ & $\bar{M}$ \\
\hline Chromium & $12 / 19 / 91$ & $7 / 17 / 90$ & $6128 / 90$ & NA & $\mathrm{R} 4802$ & R7887/R7903 & 1.550 & $2.210 \mathrm{E}-03$ & $M$ \\
\hline Fluoride & $6 / 28 / 90$ & NA & NA & NA & $\mathrm{NA}$ & NA & 1.560 & $<4.015 \mathrm{E}-03$ & Moles/L \\
\hline Fluoride & $12 / 19 / 91$ & $7 / 17 / 90$ & $6 / 28 / 90$ & NA & R4803 & R7927 / R7944 & 1.575 & $<1.795 \mathrm{E}-03$ & M \\
\hline Fluoride & $12 / 19 / 91$ & $7 / 17 / 90$ & $6 / 28 / 90$ & NA & $\mathrm{R} 4802$ & $R 7926$ / R7943 & 1.550 & $<1.795 \mathrm{E}-03$ & $M$ \\
\hline Fluoride & $12 / 19 / 91$ & $7 / 17 / 90$ & $6 / 28 / 90$ & NA & R4801 & R7923 / R7942 & 1.560 & $<3.535 \mathrm{E}-03$ & $M$ \\
\hline Fluoride & $12 / 19 / 91$ & $7 / 17 / 90$ & $6 / 28 / 90$ & NA & $R 4800$ & $R 7922$ / R7941 & 1.560 & $<3.535 \mathrm{E}-03$ & $M$ \\
\hline Fluoride & $12 / 19 / 91$ & $7 / 17 / 90$ & $6 / 28 / 90$ & NA & R4799 & R7921 / R7940 & 1.555 & $<3.535 \mathrm{E}-03$ & $M$ \\
\hline Fluoride & $12 / 19 / 91$ & $7 / 17 / 90$ & $6 / 28 / 90$ & NA & R4798 & R7920 / R7937 & 1.560 & $<5.85 \mathrm{E}-03$ & $M$ \\
\hline Fluoride & $12 / 19 / 91$ & $7 / 17 / 90$ & $6 / 28 / 90$ & NA & $\mathrm{R} 4797$ & R7919 / R7936 & 1.560 & $<4.11 \mathrm{E}-03$ & $M$ \\
\hline Fluoride & $12 / 19 / 91$ & $7 / 17 / 90$ & $6 / 28 / 90$ & NA & R4796 & R7916 / R7935 & 1.625 & $<5.85 \mathrm{E}-03$ & $M$ \\
\hline
\end{tabular}




\begin{tabular}{|c|c|c|c|c|c|c|c|}
\hline & & & nalyte Informa & & & & \\
\hline Sode & Source & Analyte & Conc. (ug/L) & Conc. $(\mathrm{mol} / \mathrm{L})$ & $\begin{array}{c}\text { Reference } \\
\text { Number }\end{array}$ & $\begin{array}{c}\text { Validated } \\
(Y / N)\end{array}$ & Notes \\
\hline$\overline{\mathrm{P}-3}$ & Raw & $\mathrm{Cl}$ & $4.93 E+06$ & $1.39 \mathrm{E}-01$ & AW101-10 & $Y$ & Average of Primary and Dublicate results \\
\hline$\overline{P-3}$ & Raw & $\mathrm{Cl}$ & $4.93 E+06$ & $1.39 \mathrm{E}-01$ & AW101-10 & $\bar{Y}$ & Average of Primary and Dublicate results \\
\hline P-3 & Raw & $\mathrm{Cl}$ & $4.87 E+06$ & $1.38 \mathrm{E}-01$ & AW101-10 & $\bar{Y}$ & Average of Primary and Dublicate results \\
\hline$F$ & Raw & $\mathrm{Cl}$ & $4.61 E+06$ & $1.30 \mathrm{E}-01$ & AW101-4 & $\bar{Y}$ & \\
\hline$\overline{P-3}$ & Raw & $\mathrm{Cl}$ & $4.57 E+06$ & $1.29 \mathrm{E}-01$ & AW101-10 & $\bar{Y}$ & Average of Primary and Dublicate results \\
\hline $\mathbf{F}$ & Raw & $\mathrm{Cl}$ & $4.25 E+06$ & $1.20 \mathrm{E}-01$ & AW101-4 & $\bar{Y}$ & \\
\hline$P$ & Raw & $\mathrm{Cl}$ & $3.90 E+06$ & $1.10 \mathrm{E}-01$ & AW101-4 & $N$ & Evaporator feed sample \\
\hline$P$ & Raw & $\mathrm{Cl}$ & $1.42 E+06$ & $4.00 \mathrm{E}-02$ & AW101-4 & $N$ & Evaporator feed sample \\
\hline $\mathbf{P}$ & Raw & $\mathrm{Cl}$ & $2.67 E+05$ & $7.54 E-03$ & AW101-3 & $N$ & Waste no longer in tank \\
\hline $\mathbf{P}$ & Raw & $\mathrm{Cr}$ & $3.73 E+05$ & 7.17E-03 & AW $101-10$ & $Y$ & \\
\hline$F$ & RAW & $\mathrm{Cr}$ & $2.67 \mathrm{E}+05$ & $5.13 E-03$ & AW101-4 & $\bar{Y}$ & \\
\hline $\mathbf{P}$ & Raw & $\mathrm{Cr}$ & $2.44 E+05$ & 4.69E-03 & AW101-10 & $\bar{Y}$ & Composite Sample \\
\hline $\mathbf{P}$ & Raw & $\mathrm{Cr}$ & $2.32 \mathrm{E}+05$ & $4.46 \mathrm{E}-03$ & AW101-10 & $Y$ & Composite Sample \\
\hline $\mathbf{F}$ & RAW & $\mathrm{Cr}$ & $2.25 E+05$ & $4.33 \mathrm{E}-03$ & AW101-4 & $Y$ & \\
\hline$P$ & Raw & $\mathrm{Cr}$ & $2.22 E+05$ & $4.26 \mathrm{E}-03$ & AW101-10 & $\bar{Y}$ & \\
\hline $\mathbf{P}$ & Raw & $\mathrm{Cr}$ & $1.93 E+05$ & $3.72 \mathrm{E}-03$ & AW101-10 & $\bar{Y}$ & \\
\hline$F$ & RAW & $\mathrm{Cr}$ & $1.43 E+05$ & $2.75 \mathrm{E}-03$ & AW101-4 & $\bar{Y}$ & \\
\hline $\mathbf{P}$ & Raw & $\overline{\mathrm{Cr}}$ & $1.39 \mathrm{E} \div 05$ & $2.67 E-03$ & AW101-10 & $\bar{Y}$ & \\
\hline $\mathbf{P}$ & Raw. & $\mathrm{Cr}$ & $1.36 E+05$ & $2.63 \mathrm{E}-03$ & AW101-10 & $Y$ & \\
\hline$P$ & Raw & $\mathrm{Cr}$ & $1.28 \mathrm{E}+05$ & $2.46 \mathrm{E}-03$ & AW101-10 & $\bar{Y}$ & \\
\hline$P$ & Raw & $\mathrm{Cr}$ & $1.27 \mathrm{E}+05$ & $2.45 \mathrm{E}-03$ & AW101-10 & $Y$ & \\
\hline$P$ & Raw & $\mathrm{Cr}$ & $1.26 E+05$ & $2.43 E-03$ & AW101-10 & $\bar{Y}$ & \\
\hline$P-3$ & Raw & $F$ & \#VALUE! & \#VALUE! & AW101-10 & $\mathbf{N}$ & $\begin{array}{l}\text { Average of Primary and Dublicate results; }< \\
\text { Values }\end{array}$ \\
\hline$P-3$ & Raw & $F$ & \#VALUEI & \#VALUE! & AW101-10 & $N$ & $\begin{array}{l}\text { Average of Primary and Dublicate results; }< \\
\text { Values }\end{array}$ \\
\hline$P-3$ & Raw & $F$ & \#VALUE! & \#VALUE! & AW101-10 & $N$ & $\begin{array}{l}\text { Average of Primary and Dublicate results; }< \\
\text { Values }\end{array}$ \\
\hline$?-3$ & Raw & $F$ & \#VALUE! & \#VALUE! & AW101-10 & $N$ & $\begin{array}{l}\text { Average of Primary and Dublicate results; }< \\
\text { Values }\end{array}$ \\
\hline$P-3$ & Raw & $F$ & \#VALUE! & \#VALUUE! & AW101-10 & $N$ & $\begin{array}{l}\text { Average of Primary and Dublicate results; < } \\
\text { Values }\end{array}$ \\
\hline$P-3$ & Raw & $\mathbf{F}$ & \#VALUEI & \#VALUE! & AW101-10 & $N$ & $\begin{array}{l}\text { Average of Primary and Dublicate results; < } \\
\text { Values }\end{array}$ \\
\hline
\end{tabular}


TWRS Privatization Support Prc Chemical Liquids

\begin{tabular}{|c|c|c|c|c|c|c|c|c|c|}
\hline & & & & Tank Was & Characteriza & on Sample Inform & ion & & \\
\hline Reported Chemical & $\begin{array}{c}\text { Memo } \\
\text { Date }\end{array}$ & $\begin{array}{c}\text { Start Analysis } \\
\text { Date }\end{array}$ & $\begin{array}{c}\text { Start } \\
\text { Sample } \\
\text { Date }\end{array}$ & $\begin{array}{l}\text { Sample } \\
\text { Number }\end{array}$ & $\begin{array}{c}\text { Tank Farm } \\
\text { Number }\end{array}$ & $\begin{array}{c}\text { Lab Sample } \\
\text { Number }\end{array}$ & $\begin{array}{l}\text { Density } \\
(\mathrm{g} / \mathrm{mL}) \\
\end{array}$ & Reported Value & Reported \\
\hline Fluoride & $12 / 19 / 91$ & $7 / 17 / 90$ & $6 / 28 / 90$ & NA & R4795 & R7915 / R7934 & 1.540 & $<5.85 \mathrm{E}-03$ & $M$ \\
\hline Fluoride & $12 / 19 / 91$ & $7 / 17 / 90$ & $6 / 28 / 90$ & NA & R4794 & R7914 / R7933 & 1.570 & $<4.11 \mathrm{E}-03$ & $M$ \\
\hline Fluoride & $12 / 19 / 91$ & $7 / 17 / 90$ & $6 / 28 / 90$ & NA & R4793 & R7913 / R7930 & 1.535 & $<4.11 \mathrm{E}-03$ & $M$ \\
\hline Fluoride & $12 / 19 / 91$ & $7 / 17 / 90$ & $6 / 28 / 90$ & NA & NA & R8801 & 1.540 & $<1.06 \mathrm{E}-03$ & $M$ \\
\hline Fluoride & $12 / 19 / 91$ & $7 / 17 / 90$ & $6 / 28 / 90$ & NA & NA & $\mathrm{R} 8802$ & 1.540 & $<1.06 \mathrm{E}-03$ & $M$ \\
\hline Fluoride & $12 / 19 / 91$ & $7 / 17 / 90$ & $6 / 28 / 90$ & NA & R4792 & R7912/R7929 & 1.530 & $<4.11 \mathrm{E}-03$ & $M$ \\
\hline F- & NA & NA & NA & NA & NA & NA & 1.39 & $1.82 \mathrm{E}-01$ & Mole/L \\
\hline F & $7 / 2 / 86$ & NS & NS & NS & NS & NS & 1.500 & 0.14 & Moles/L \\
\hline$F$ & $9 / 18 / 86$ & NA & $6 / 23 / 86$ & T621 & NS & NS & 1.440 & 0.09 & Moles $/ L$ \\
\hline$F$ & $6 / 23 / 86$ & NS & NS & NS & NS & NS & 1.370 & 0.09 & Moles/L \\
\hline$F$ & $9 / 18 / 86$ & NA & $6 / 26 / 86$ & T686 & NS & NS & 1.310 & 0.08 & Moles/L \\
\hline$F$ & $9 / 18 / 86$ & NA & $7 / 2 / 86$ & $T 882$ & NS & NS & 1.320 & 0.08 & Moles $/ \mathrm{L}$ \\
\hline$F$ & $6 / 30 / 86$ & NS & NS & NS & NS & NS & 1.540 & 0.08 & Moles/L \\
\hline$F$ & $6 / 26 / 86$ & NS & NS & NS & NS & NS & 1.490 & 0.08 & Moles/L \\
\hline$F$ & $9 / 18 / 86$ & NA & $7 / 6 / 86$ & R9897 & NS & NS & 1.460 & 0.07 & Moles/L \\
\hline$F$ & $9 / 18 / 86$ & NA & $6 / 30 / 86$ & T812 & NS & NS & 1.130 & 0.04 & Moles/L \\
\hline$F$ & $8 / 18 / 85$ & $8 / 18 / 85$ & NA & T9368 & $\mathrm{NA}$ & NA & 1.040 & 0.0148 & Moles/L \\
\hline$F$ & $2 / 20 / 86$ & $11 / 14 / 85$ & NA & T2601 & NS & NS & 1.010 & 0.01 & Moles $/ \mathrm{L}$ \\
\hline$F$ & $2 / 20 / 86$ & $11 / 14 / 85$ & NA & T2641 & NS & NS & 1.010 & 0.01 & Moles/L \\
\hline$F$ & $4 / 29 / 86$ & $3 / 30 / 86$ & NA & T7697 & NS & NS & 0.993 & 0.007 & Moles/L \\
\hline$F$ & $4 / 29 / 86$ & $3 / 30 / 86$ & NA & T7698 & NS & NS & 0.993 & 0.007 & Moles/L \\
\hline$F$ & $11 / 9 / 84$ & NS & NS & R-3185 & NS & NS & 1.024 & 0.005 & moles $n$ \\
\hline$F$ & $11 / 9 / 84$ & NA & NA & R3185 & NS & NS & 1.024 & 0.005 & Moles/L \\
\hline$F$ & $4 / 29 / 85$ & $4 / 21 / 85$ & NA & $T 5350$ & NA & $\mathrm{NA}$ & 1.028 & 0.00484 & Moles/L \\
\hline Iron & $6 / 28 / 90$ & $\mathrm{NA}$ & NA & NA & NA & NA & 1.560 & $<7.864 \mathrm{E}-04$ & Moles/L \\
\hline Iron & $12 / 19 / 91$ & $7 / 17 / 90$ & $6 / 28 / 90$ & NA & R4801 & R7902 & 1.560 & $<4.33 \mathrm{E}-04$ & $M$ \\
\hline
\end{tabular}

A- 


\begin{tabular}{|c|c|c|c|c|c|c|c|}
\hline & \multicolumn{3}{|c|}{ Analyte information } & \multirow[b]{2}{*}{$\begin{array}{l}\text { Reference } \\
\text { Number }\end{array}$} & \multirow[b]{2}{*}{$\begin{array}{l}\text { Validated } \\
\text { (YIN) }\end{array}$} & \multirow[b]{2}{*}{ Notes } \\
\hline Code & Source & Analyte & Conc. (ug/L) & Conc. $(\mathrm{mol} / \mathrm{L})$ & & & \\
\hline P-3 & Raw & $\mathbf{F}$ & \#VALUE! & \#VALUE! & AW101-10 & $N$ & $\begin{array}{l}\text { Average of Primary and Dublicate results; }< \\
\text { Values }\end{array}$ \\
\hline P-3 & Raw & $\mathbf{F}$ & \#VALUE! & \#VALUE! & AW101-10 & $N$ & $\begin{array}{l}\text { Average of Primary and Dublicate results; < } \\
\text { Values }\end{array}$ \\
\hline$P$ & Raw & $F$ & \#VALUE! & \#VALUEI & AW101-10 & $N$ & Composite Sample; < Values \\
\hline $\mathbf{P}$ & Raw & $F$ & \#VALUE! & \#VALUE! & AW101-10 & $N$ & Composite Sample; < values \\
\hline $\mathbf{P}$ & Raw & $F$ & \#VALUE! & \#VALUE! & AW101-10 & $Y$ & highest less than \\
\hline$L$ & HTCE Inv. & $F$ & $3.45 E+06$ & $1.82 E-01$ & NA & $N$ & HTCE \\
\hline$F$ & Raw & $F$ & $2.66 \mathrm{E}+06$ & $1.40 \mathrm{E}-01$ & AW101-4 & $N$ & $\begin{array}{l}\text { Suspicious because feed and slurry samples } \\
\text { (i.e. the liquid from the slurry samples) are all } \\
\text { about the same. You can't see any evidence of } \\
\text { concentrating in the evaporator }\end{array}$ \\
\hline $\mathbf{P}$ & Raw & $F$ & $1.71 E+06$ & $9.00 \mathrm{E}-02$ & AW101-4 & $\bar{N}$ & Evaporator feed sample \\
\hline$F$ & Raw & F & $1.71 E+06$ & $9.00 \mathrm{E}-02$ & AW101-4 & $N$ & $\begin{array}{l}\text { Suspicious because feed and slurry samples } \\
\text { (i.e. the liquid from the slurry samples) are all } \\
\text { about the same. You can't see any evidence of } \\
\text { concentrating in the evaporator }\end{array}$ \\
\hline $\mathbf{P}$ & Raw & $F$ & $1.52 E+06$ & $8.00 \mathrm{E}-02$ & AW101-4 & $N$ & Evaporator feed sample \\
\hline $\mathbf{P}$ & Raw & $F$ & $1.52 E+06$ & $8.00 \mathrm{E}-02$ & AW101-4 & $N$ & Evaporator feed sample \\
\hline $\mathbf{F}$ & Raw & $F$ & $1.52 E+06$ & 8.00E-02 & AW101-4 & $N$ & $\begin{array}{l}\text { Suspicious because feed and slurry samples } \\
\text { (i.e. the liquid from the slurry samples) are all } \\
\text { about the same. You can't see any evidence of } \\
\text { concentrating in the evaporator }\end{array}$ \\
\hline$P$ & Raw & $\mathrm{F}$ & $7.60 E+05$ & $4.00 E-02$ & AW101-4 & $\mathbb{N}$ & Evaporator feed sample \\
\hline $\mathbf{P}$ & Raw & $F$ & $2.81 E+05$ & $1.48 \mathrm{E}-02$ & AW101-3 & $\mathbf{N}$ & Waste no longer in tank \\
\hline$P$ & Raw & $F$ & $1.90 E+05$ & $1.00 \mathrm{E}-02$ & AW101-4 & $\mathbf{N}$ & Waste no longer in tank \\
\hline$P$ & Raw & $F$ & $1.90 E+05$ & $1.00 \mathrm{E}-02$ & AW101-4 & $N$ & Waste no longer in tank \\
\hline$P$ & Raw & $F$ & $1.33 E+05$ & $7.00 \mathrm{E}-03$ & AW101-4 & $\mathbf{N}$ & Waste no longer in tank \\
\hline$P$ & Raw & $\bar{F}$ & $1.33 E+05$ & $7.00 \mathrm{E}-03$ & AW101-4 & $N$ & Waste no longer in tank \\
\hline$P$ & Braun & $\mathbf{F}$ & $9.50 E+04$ & $5.00 \mathrm{E}-03$ & NA & $N$ & Waste no longer in tank \\
\hline$P$ & Raw & $F$ & $9.50 E+04$ & $5.00 \mathrm{E}-03$ & AW101-2 & $N$ & Waste no longer in tank \\
\hline$P$ & Raw & $F$ & $9.20 E+04$ & $4.84 E-03$ & AW101-3 & $N$ & Waste no longer in tank \\
\hline$P$ & Raw & $\mathrm{Fe}$ & \#VALUE! & \#VALUE! & AW101-10 & NR & \\
\hline $\mathrm{P}$ & Raw & $\mathrm{Fe}$ & \#VALUE! & \#VALUE! & AW101-10 & NR & \\
\hline
\end{tabular}


TWRS Privatization Support Proj Chemical Liquids

\begin{tabular}{|c|c|c|c|c|c|c|c|c|c|}
\hline & & & & Tank Was & Characteriza & ion Sample Inform & ion & & \\
\hline Reported Chemical & $\begin{array}{c}\text { Memo } \\
\text { Date }\end{array}$ & $\begin{array}{c}\text { Start Analysis } \\
\text { Date }\end{array}$ & $\begin{array}{c}\text { Start } \\
\text { Sample } \\
\text { Date } \\
\end{array}$ & $\begin{array}{l}\text { Sample } \\
\text { Number }\end{array}$ & $\begin{array}{c}\text { Tank Farm } \\
\text { Number }\end{array}$ & $\begin{array}{c}\text { Lab Sample } \\
\text { Number }\end{array}$ & $\begin{array}{l}\text { Density } \\
\text { (g/mL) }\end{array}$ & Reported Value & Reported U \\
\hline Iron & $12 / 19 / 91$ & $7 / 17 / 90$ & $6 / 28 / 90$ & NA & $R 4800$ & R7901 & 1.560 & $<4.33 \mathrm{E}-04$ & $M$ \\
\hline Iron & $12 / 19 / 91$ & $7 / 17 / 90$ & $6 / 28 / 90$ & NA & R4799 & R7898 & 1.555 & $<4.33 \mathrm{E}-04$ & $M$ \\
\hline Iron & $12 / 19 / 91$ & $7 / 17 / 90$ & $6 / 28 / 90$ & NA & R4797 & R7896 & 1.560 & $<4.33 E-04$ & $M$ \\
\hline Iron & $12 / 19 / 91$ & $7 / 17 / 90$ & $6 / 28 / 90$ & $\overline{N A}$ & $\mathrm{R} 4794$ & R7891 & 1.570 & $<4.33 E-04$ & $M$ \\
\hline Iron & $12 / 19 / 91$ & $7 / 17 / 90$ & $6 / 28 / 90$ & NA & R4793 & R7890 & 1.535 & $<4.33 \mathrm{E}-04$ & $M$ \\
\hline Iron & $12 / 19 / 91$ & $7 / 17 / 90$ & $6 / 28 / 90$ & NA & R4792 & R7889 & 1.530 & $<4.33 E-04$ & $M$ \\
\hline Iron & $12 / 19 / 91$ & $7 / 17 / 90$ & $6 / 28 / 90$ & NA & $\mathrm{R} 4803$ & R7888 / R7904 & 1.575 & $<4.33 E-04$ & $M$ \\
\hline Iron & $12 / 19 / 91$ & $7 / 17 / 90$ & $6 / 28 / 90$ & NA & $\mathrm{R} 4802$ & R7887/R7903 & 1.550 & $<4.33 \mathrm{E}-04$ & $M$ \\
\hline $\mathrm{Fe}^{+3}$ & $4 / 3 / 84$ & NA & NA & R9414 & NA & NA & 1.370 & 0.024 & Moles/L \\
\hline $\mathrm{Fe}^{+3}$ & $4 / 3 / 84$ & NA & NA & R9408 & NA & NA & 1.330 & 0.023 & Moles/L \\
\hline $\mathrm{Fe} 3+($ total Fe) & NA & NA & NA & NA & NA & NA & 1.39 & $6.83 \mathrm{E}-03$ & Mole/L \\
\hline Iron & $12 / 19 / 91$ & $7 / 17 / 90$ & $6 / 28 / 90$ & NA & R4796 & R7895 & 1.625 & $2.120 \mathrm{E}-03$ & $M$ \\
\hline Iron & $12 / 19 / 91$ & $7 / 17 / 90$ & $6 / 28 / 90$ & NA & R4795 & R7894 & 1.540 & $1.830 \mathrm{E}-03$ & $M$ \\
\hline Iron & $12 / 19 / 91$ & $7 / 17 / 90$ & $6 / 28 / 90$ & NA & R4798 & R7897 & 1.560 & $1.590 \mathrm{E}-03$ & $M$ \\
\hline$\% \mathrm{H} 2 \mathrm{O}$ & $12 / 19 / 91$ & $7 / 17 / 90$ & $6 / 28 / 90$ & NA & NA & R8799 & NA & $4.50 E+01$ & $w t \%$ \\
\hline$\% \mathrm{H} 2 \mathrm{O}$ & $12 / 19 / 91$ & $7 / 17 / 90$ & $6 / 28 / 90$ & NA & NA & R8798 & NA & $4.22 E+01$ & $w t \%$ \\
\hline Mercury & $12 / 19 / 91$ & $7 / 17 / 90$ & $6 / 28 / 90$ & NA & $\mathrm{R} 4803$ & R7927/R7944 & 1.575 & $<1.10 \mathrm{E}-01$ & $\mathrm{mg} / \mathrm{L}$ \\
\hline Mercury & $12 / 19 / 91$ & $7 / 17190$ & $6 / 28 / 90$ & NA & $\mathrm{R} 4802$ & R7926 / R7943 & 1.550 & $<1.10 E-01$ & $\mathrm{mg} / \mathrm{L}$ \\
\hline Mercury & $12 / 19 / 91$ & $7 / 17 / 90$ & $6 / 28 / 90$ & NA & R4801 & R7923 / R7942 & 1.560 & $<1.65 \mathrm{E}-01$ & $\mathrm{mg} / \mathrm{L}$ \\
\hline Mercury & $12 / 19 / 91$ & $7 / 17 / 90$ & $6 / 28 / 90$ & NA & R4800 & R7922 / R7941 & 1.560 & $<1.65 \mathrm{E}-01$ & $\mathrm{mg} / \mathrm{L}$ \\
\hline Mercury & $12 / 19 / 91$ & $7 / 17 / 90$ & $6 / 28 / 90$ & NA & R4799 & R7921 / R7940 & 1.555 & $<1.65 \mathrm{E}-01$ & mgih \\
\hline Mercury & $12 / 19 / 91$ & $7 / 17 / 90$ & $6 / 28 / 90$ & NA & R4798 & R7920/R7937 & 1.560 & $<1.65 E-01$ & $\mathrm{mg} / \mathrm{L}$ \\
\hline Mercury & $12 / 19 / 91$ & $7 / 17 / 90$ & $6 / 28 / 90$ & NA & R4797 & R7919/R7936 & 1.560 & $<1.65 E-01$ & $\mathrm{mg} / \mathrm{L}$ \\
\hline Mercury & $12 / 19 / 91$ & $7 / 17 / 90$ & $6 / 28190$ & NA & R4796 & R7916/R7935 & 1.625 & $<1.65 \mathrm{E}-01$ & $\mathrm{mg} / \mathrm{l}$ \\
\hline Mercury & $12 / 19 / 91$ & $7 / 17 / 90$ & $6 / 28 / 90$ & NA & $\mathrm{R} 4795$ & R7915/R7934 & 1.540 & $<1.65 E-01$ & $\mathrm{mg} / \mathrm{L}$ \\
\hline Mercury & $12 / 19 / 91$ & $7 / 17 / 90$ & $6 / 28 / 90$ & NA & R4794 & R7914/R7933 & 1.570 & $<1.65 \mathrm{E}-01$ & $\mathrm{mg} / \mathrm{L}$ \\
\hline Mercury & $12 / 19 / 91$ & $7 / 17 / 90$ & $6 / 28 / 90$ & NA & R4793 & R7913/R7930 & 1.535 & $<1.65 \mathrm{E}-01$ & $\mathrm{mg} / \mathrm{h}$ \\
\hline Mercury & $12 / 19 / 91$ & $7 / 17 / 90$ & $6 / 28 / 90$ & NA & R4792 & R7912/R7929 & 1.530 & $<1.77 \mathrm{E}-01$ & $m g / h$ \\
\hline Mercury & $12 / 19 / 91$ & $7 / 17 / 90$ & $6 / 28 / 90$ & NA & NA & R8798 & 1.540 & $3.340 \mathrm{E}-02$ & $\mathrm{mg} / \mathrm{L}$ \\
\hline Potassium & $12 / 19 / 91$ & $7 / 17 / 90$ & $6 / 28 / 90$ & NA & R4792 & R7889 & 1.530 & $1.435 E+00$ & $M$ \\
\hline Potassium & $12 / 19 / 91$ & $7 / 17 / 90$ & $6 / 28 / 90$ & NA & R4793 & R7890 & 1.535 & $1.395 \mathrm{E}+00$ & $M$ \\
\hline Potassium & $12 / 19 / 91$ & $7 / 17 / 90$ & $6 / 28 / 90$ & NA & R4794 & R7891 & 1.570 & $1.320 \mathrm{E}+00$ & $M$ \\
\hline $\bar{k}$ & $9 / 18 / 86$ & NA & $7 / 6 / 86$ & R9897 & NA & NA & 1.460 & 1.27 & Moles/L \\
\hline Potassium & $12 / 19 / 91$ & $7 / 17 / 90$ & $6 / 28 / 90$ & NA & NA & R8795 & 1.540 & $1.260 E+00$ & $M$ \\
\hline Potassium & $12 / 19 / 91$ & $7 / 17 / 90$ & $6 / 28 / 90$ & NA & NA & R8796 & 1.540 & $1.260 E+00$ & $M$ \\
\hline Potassium & $12 / 19 / 91$ & $7 / 17 / 90$ & $6 / 28 / 90$ & NA & R4798 & R7897 & 1.560 & $1.095 E+00$ & $M$ \\
\hline Potassium & $12 / 19 / 91$ & $7 / 17 / 90$ & $6 / 28 / 90$ & NA & R4798 & R7897 & 1.560 & $1.080 E+00$ & $M$ \\
\hline Potassium & $6 / 28 / 90$ & NA & NA & NA & NA & NA & 1.560 & 1.068 & Moles/L \\
\hline$k$ & $9 / 18 / 86$ & NA & $7 / 2 / 86$ & ค9896 & NA & NA & 1.500 & 1.06 & Moles/L \\
\hline Potassium & $12 / 19 / 91$ & $7 / 17 / 90$ & $6 / 28 / 90$ & $\mathrm{NA}$ & R4802 & $\mathrm{R} 7887 / \mathrm{R7903}$ & 1.550 & $9.960 \mathrm{E}-01$ & $\bar{M}$ \\
\hline Potassium & $12 / 19 / 91$ & $7 / 17 / 90$ & $6 / 28 / 90$ & NA & R4794 & R7891 & 1.570 & 9.910E-01 & $M$ \\
\hline Potassium & $12 / 19 / 91$ & $7 / 17 / 90$ & $6 / 28 / 90$ & NA & R4796 & R7895 & 1.625 & 9.910E-01 & $M$ \\
\hline Potassium & $12 / 19 / 91$ & $7 / 17 / 90$ & $6 / 28 / 90$ & NA & $\mathbf{R 4 7 9 3}$ & R7890 & 1.535 & $9.885 \mathrm{E}-01$ & $M$ \\
\hline Potassium & $12 / 19 / 91$ & $7117 / 90$ & $6 / 28 / 90$ & NA & R4800 & R7901 & 1.560 & $9.880 \mathrm{E}-01$ & $M$ \\
\hline Potassium & $12 / 19 / 91$ & $7 / 17 / 90$ & $6 / 28 / 90$ & NA & R4796 & R7895 & 1.625 & $9.870 \mathrm{E}-01$ & $M$ \\
\hline Potassium & $12 / 19 / 91$ & $7 / 17 / 90$ & $6 / 28 / 90$ & NA & $\mathrm{R} 4801$ & R7902 & 1.560 & 9.715E-01 & $M$ \\
\hline Potassium & $12 / 19 / 91$ & $7 / 17 / 90$ & $6 / 28 / 90$ & NA & R4792 & R7889 & 1.530 & 9.595E-01 & $M$ \\
\hline Potassium & $12 / 19 / 91$ & $7 / 17 / 90$ & $6 / 28 / 90$ & NA & $\mathrm{R} 4801$ & R7902 & 1.560 & $9.555 \mathrm{E}-01$ & $M$ \\
\hline Potassium & $12 / 19 / 91$ & $7 / 17 / 90$ & $6 / 28 / 90$ & NA & R4799 & R7898 & 1.555 & $9.490 \mathrm{E}-01$ & $M$ \\
\hline
\end{tabular}




\begin{tabular}{|c|c|c|c|c|c|c|c|}
\hline \multirow[b]{2}{*}{ Code } & \multirow[b]{2}{*}{ Source } & \multicolumn{3}{|c|}{ Analyte Information } & \multirow[b]{2}{*}{$\begin{array}{c}\text { Reference } \\
\text { Number }\end{array}$} & \multirow[b]{2}{*}{$\begin{array}{c}\text { Validated } \\
\text { (Y/N) }\end{array}$} & \multirow[b]{2}{*}{ Notes } \\
\hline & & Analyte & Conc. (ug/L) & Conc. (mol/L) & & & \\
\hline$P$ & Raw & $\mathrm{Fe}$ & \#VALUE! & \#VALUE! & AW101-10 & NR & \\
\hline $\mathrm{P}$ & Raw & $\mathrm{Fe}$ & \#VALUE! & \#VALUE! & AW101-10 & NR & \\
\hline$P$ & Raw & $\mathrm{Fe}$ & \#VALUE! & \#VALUE! & AW101-10 & NR & \\
\hline$P$ & Raw & $\mathrm{Fe}$ & \#VALUE! & \#VALUE! & AW101-10 & NR & \\
\hline$P$ & Raw & $\mathrm{Fe}$ & \#VALUE! & \#VALUE! & AW101-10 & NR & \\
\hline$P$ & Raw & $\mathrm{Fe}$ & \#VALUE! & \#VALUEI & AW101-10 & NR & \\
\hline$P$ & Raw & $\mathrm{Fe}$ & \#VALUE! & \#VALUE! & AW101-10 & NR & \\
\hline$P$ & Raw & $\mathrm{Fe}$ & \#VALUE! & \#VALUE! & AW101-10 & NR & \\
\hline$P$ & Raw & $\mathrm{Fe}$ & $1.34 E+06$ & $2.40 \mathrm{E}-02$ & AW101-2 & NR & \\
\hline$P$ & Raw & $\mathrm{Fe}$ & $1.28 E+06$ & $2.30 \mathrm{E}-02$ & AW101-2 & NR & \\
\hline$L$ & HTCE Inv. & $\mathrm{Fe}$ & $3.81 E+05$ & $6.83 \mathrm{E}-03$ & NA & NR & \\
\hline$P$ & Raw & $\mathrm{Fe}$ & $1.18 \mathrm{E}+05$ & $2.12 \mathrm{E}-03$ & AW101-10 & $Y$ & highest valid data point for $\mathrm{Fe}$ \\
\hline$P$ & Raw & $\mathrm{Fe}$ & $1.02 E+05$ & $1.83 \mathrm{E}-03$ & AW101-10 & NR & \\
\hline $\mathrm{P}$ & Raw & $\mathrm{Fe}$ & $8.88 E+04$ & $1.59 \mathrm{E}-03$ & AW101-10 & NR & \\
\hline NA & Raw & $\mathrm{H} 2 \mathrm{O}$ & $\mathrm{NA}$ & NA & AW101-10 & $\bar{Y}$ & \\
\hline NA & Raw & $\mathrm{H} 2 \mathrm{O}$ & NA & NA & AW101-10 & $Y$ & \\
\hline P-3 & Raw & $\mathrm{Hg}$ & \#VALUE! & \#VALUE! & AW101-10 & NR & Average of Primary and Dublicate results \\
\hline P-3 & Raw & $\mathrm{Hg}$ & \#VALUE! & \#VALUE! & AW101-10 & NR & Average of Primary and Dublicate results \\
\hline P-3 & Raw & $\mathrm{Hg}$ & \#VALUE! & \#VALUE! & AW101-10 & NR & Average of Primary and Dublicate results \\
\hline P-3 & Raw & $\mathrm{Hg}$ & \#VALUE! & $\ddot{\# V A L U E ! ~}$ & AW101-10 & NR & Average of Primary and Dublicate results \\
\hline $\mathrm{P}-3$ & Raw & $\mathrm{Hg}$ & \#VALUE! & \#VALUE! & AW101-10 & NR & Average of Primary and Dublicate results \\
\hline $\mathrm{P}-3$ & Raw & $\mathrm{Hg}$ & \#VALUEI & \#VALUE! & AW101-10 & NR & Average of Primary and Dublicate results \\
\hline $\mathrm{P}-3$ & Raw & $\mathrm{Hg}$ & \#VALUE! & \#VALUE! & AW $101-10$ & NR & Average of Primary and Dublicate results \\
\hline $\mathrm{P}-3$ & Raw & $\mathrm{Hg}$ & \#VALUE! & \#VALUE! & AW101-10 & NR & Average of Primary and Dublicate results \\
\hline $\mathrm{P}-3$ & Raw & $\mathrm{Hg}$ & \#VALUE! & \#VALUE! & AW101-10 & NR & Average of Primary and Dublicate results \\
\hline P-3 & Raw & $\mathrm{Hg}$ & \#VALUE! & \#VALUE! & AW101-10 & NR & Average of Primary and Dublicate results \\
\hline P-3 & Raw & $\mathrm{Hg}$ & \#VALUE! & \#VALUE! & AW101-10 & NR & Average of Primary and Dublicate results \\
\hline$P$ & Raw & $\mathrm{Hg}$ & \#VALUE! & \#VALUE! & AW101-10 & NR & \\
\hline$P$ & Raw & $\mathrm{Hg}$ & $3.34 \mathrm{E}+01$ & $1.67 E-07$ & AW101-10 & $\bar{Y}$ & Only non $<$ value for $\mathrm{Hg}$ \\
\hline$P$ & Raw & $\mathrm{k}$ & $5.61 E+07$ & $1.43 E+00$ & AW $101-10$ & $\bar{Y}$ & \\
\hline$P$ & Raw & $K$ & $5.45 E+07$ & $1.39 E+00$ & AW101-10 & $Y$ & \\
\hline$P$ & Raw & $K$ & $5.16 E+07$ & $1.32 \mathrm{E}+00$ & AW101-10 & $\bar{Y}$ & \\
\hline$F$ & Raw & $K$ & $4.97 E+07$ & $1.27 E+00$ & AW101-4 & $\bar{Y}$ & \\
\hline$P$ & Raw & $K$ & $4.93 E+07$ & $1.26 \mathrm{E}+00$ & AW101-10 & $\mathrm{Y}$ & Composite Sample \\
\hline$P$ & Raw & $K$ & $4.93 E+07$ & $1.26 \mathrm{E}+00$ & AW101-10 & $\bar{Y}$ & Composite Sample \\
\hline$P$ & Raw & $\bar{K}$ & $4.28 E+07$ & $1.09 \mathrm{E}+00$ & AW101-10 & $\bar{Y}$ & \\
\hline$P$ & Raw & $K$ & $4.22 E+07$ & $1.08 E+00$ & AW101-10 & $\bar{Y}$ & Rerun \\
\hline$P$ & Raw & $K$ & $4.18 E+07$ & $1.07 E+00$ & AW101-10 & $\bar{Y}$ & Avg of all 10 samples \\
\hline$F$ & Raw & $K$ & $4.14 E+07$ & $1.06 E+00$ & AW101-4 & $\bar{Y}$ & \\
\hline$P$ & Raw & $K$ & $3.89 E+07$ & 9.96E-01 & AW101-10 & $\bar{Y}$ & \\
\hline$P$ & Raw & $K$ & $3.87 \mathrm{E}+07$ & 9.91E-01 & AW101-10 & $\bar{Y}$ & Rerun \\
\hline$P$ & Raw & $K$ & $3.87 E+07$ & 9.91E-01 & AW101-10 & $Y$ & \\
\hline$P$ & Raw & $k$ & $3.86 \mathrm{E}+07$ & $9.88 \mathrm{E}-01$ & AW101-10 & $\bar{Y}$ & Rerun \\
\hline$P$ & Raw & $K$ & $3.86 E+07$ & $9.88 E-01$ & AW101-10 & $Y$ & \\
\hline$P$ & Raw & $K$ & $3.86 E+07$ & 9.87E-01 & AW101-10 & $Y$ & Rerun \\
\hline$P$ & Raw & $K$ & $3.80 E \div 07$ & $9.71 \mathrm{E}-01$ & AW101-10 & $\bar{Y}$ & \\
\hline$P$ & Raw & $K$ & $3.75 E+07$ & $9.59 \mathrm{E}-01$ & AW101-10 & $\bar{Y}$ & Rerun \\
\hline$P$ & Raw & $K$ & $3.74 E+07$ & $9.55 \mathrm{E}-01$ & AW101-10 & $\bar{Y}$ & Rerun \\
\hline$P$ & Raw & $K$ & $3.71 E+07$ & $9.49 \mathrm{E}-01$ & AW $101-10$ & $Y$ & Rerun \\
\hline
\end{tabular}




\begin{tabular}{|c|c|c|c|c|c|c|c|c|c|}
\hline \multicolumn{10}{|c|}{ Tank Waste Characterization Sample Information } \\
\hline Reported Chemical & $\begin{array}{c}\text { Memo } \\
\text { Date }\end{array}$ & $\begin{array}{c}\text { Start Analysis } \\
\text { Date }\end{array}$ & $\begin{array}{c}\text { Start } \\
\text { Sample } \\
\text { Date }\end{array}$ & $\begin{array}{l}\text { Sample } \\
\text { Number }\end{array}$ & $\begin{array}{c}\text { Tank Farm } \\
\text { Number }\end{array}$ & $\begin{array}{c}\text { Lab Sample } \\
\text { Number }\end{array}$ & $\begin{array}{l}\text { Density } \\
\text { (g/ml) }\end{array}$ & Reported Value & Reported U \\
\hline Potassium & $12 / 19 / 91$ & $7 / 17 / 90$ & $6 / 28 / 90$ & NA & $\mathrm{R} 4803$ & R7888/R7904 & 1.575 & $9.415 \mathrm{E}-01$ & $\bar{M}$ \\
\hline $\bar{K}$ & $9 / 18 / 86$ & NA & $6 / 30 / 86$ & R9895 & NA & NA & 1.540 & 0.94 & Moles/L \\
\hline Potassium & $12 / 19 / 91$ & $7 / 17 / 90$ & $6 / 28 / 90$ & NA & R4800 & A7901 & 1.560 & $9.375 \mathrm{E}-01$ & $\bar{M}$ \\
\hline Potassium & $12 / 19 / 91$ & $7 / 17 / 90$ & $6 / 28 / 90$ & NA & R4795 & R7894 & 1.540 & $9.375 \mathrm{E}-01$ & $M$ \\
\hline Potassium & $12 / 19 / 91$ & $7 / 17 / 90$ & $6 / 28 / 90$ & NA & $\mathrm{R} 4802$ & R7887/R7903 & 1.550 & $9.155 \mathrm{E}-01$ & $\bar{M}$ \\
\hline Potassium & $12 / 19 / 91$ & $7 / 17 / 90$ & $6 / 28 / 90$ & NA & R4799 & R7898 & 1.555 & $8.990 E-01$ & $M$ \\
\hline Potassium & $12 / 19 / 91$ & $7 / 17 / 90$ & $6 / 28 / 90$ & NA & R4803 & R7888/R7904 & 1.575 & $8.955 \mathrm{E}-01$ & $M$ \\
\hline Potassium & $12 / 19 / 91$ & $7 / 17 / 90$ & $6 / 28 / 90$ & $\overline{N A}$ & R4795 & R7894 & 1.540 & $8.825 \mathrm{E}-01$ & $\bar{M}$ \\
\hline Potassium & $12 / 19 / 91$ & $7 / 17 / 90$ & $6 / 28 / 90$ & NA & R4797 & R7896 & 1.560 & $8.710 \mathrm{E}-01$ & $M$ \\
\hline$K$ & $9 / 18 / 86$ & NA & $6 / 26 / 86$ & R9894 & NA & NA & 1.490 & 0.87 & Moles/L \\
\hline Potassium & $12 / 19 / 91$ & $7 / 17 / 90$ & $6 / 28 / 90$ & NA & R4797 & R7896 & 1.560 & $8.430 E-01$ & $M$ \\
\hline $\mathrm{K}$ & $9 / 18 / 86$ & NA & $7 / 2 / 86$ & T882 & NS & NS & 1.320 & 0.73 & Moles/L \\
\hline$K$ & $9 / 18 / 86$ & NA & $6 / 26 / 86$ & T686 & NS & NS & 1.310 & 0.62 & Moles/L \\
\hline $\mathrm{K}$ & $9 / 18 / 86$ & NA & $6 / 23 / 86$ & R9893 & NA & NA & 1.370 & 0.59 & Moles/L \\
\hline $\bar{K}$ & $9 / 18 / 86$ & NA & $6 / 23 / 86$ & T621 & NS & NS & 1.440 & 0.58 & Moles/L \\
\hline$k$ & $9 / 18 / 86$ & NA & $6 / 30 / 86$ & $T 812$ & NS & NS & 1.130 & 0.33 & Moles/L \\
\hline$K+$ & NA & $\overline{\mathrm{NA}}$ & NA & NA & $\overline{N A}$ & NA & 1.39 & $1.38 \mathrm{E}-01$ & Mole/L \\
\hline $\mathrm{K}$ & $4 / 29 / 86$ & $3 / 30 / 86$ & $\overline{N A}$ & T7697 & NS & NS & 0.993 & 0.015 & Moles/L \\
\hline$\ddot{K}$ & $4 / 29 / 86$ & $3 / 30 / 86$ & NA & T7698 & NS & NS & 0.993 & 0.011 & Moles/L \\
\hline$K$ & $11 / 9 / 84$ & NS & NS & $R-3185$ & NS & $\widehat{N S}$ & 1.024 & 0.006 & moles $n$ \\
\hline $\mathrm{K}$ & $11 / 9 / 84$ & NA & NA & R3185 & NS & NS & 1.024 & 0.006 & Moles $/ \mathrm{L}$ \\
\hline $\bar{K}$ & $4 / 29 / 85$ & $4 / 21 / 85$ & NA & T5350 & NA & NA & 1.028 & 0.00279 & Moles/L \\
\hline$K$ & $8 / 18 / 85$ & $8 / 18 / 85$ & NA & T9368 & NA & NA & 1.040 & 0.00274 & Moles/L \\
\hline $\bar{k}$ & $2 / 20 / 86$ & $11 / 14 / 85$ & NA & $T 2601$ & NS & NS & 1.010 & 0.002 & Moles \\
\hline$K$ & $2 / 20 / 86$ & $11 / 14 / 85$ & NA & T2641 & NS & NS & 1.010 & 0.002 & Moles \\
\hline Sodium & $12 / 19 / 91$ & $7 / 17 / 90$ & $6 / 28 / 90$ & NA & NA & R8795 & 1.540 & $1.340 E+01$ & $M$ \\
\hline Sodium & $12 / 19 / 91$ & $7 / 17 / 90$ & $6 / 28 / 90$ & $\overline{N A}$ & $\overline{\mathrm{NA}}$ & R8796 & 1.540 & $1.340 E+01$ & $M$ \\
\hline $\mathrm{Na}$ & $9 / 18 / 86$ & NA & $7 / 2 / 86$ & R9896 & NA & NA & 1.5 & 11.2 & $M$ \\
\hline $\mathrm{Na}$ & $9 / 18 / 86$ & $\overline{N A}$ & $6 / 26 / 86$ & 89894 & NA & NA & 1.49 & 11.1 & $\bar{M}$ \\
\hline$\overline{\mathrm{Na}}$ & $9 / 18 / 86$ & $\overline{\mathrm{NA}}$ & $6 / 30 / 86$ & R9895 & NA & $\widehat{N A}$ & 1.54 & 10.9 & $\vec{M}$ \\
\hline Sodium & $12 / 19 / 91$ & $7 / 17 / 90$ & $6 / 28 / 90$ & $\overline{\mathrm{NA}}$ & $\mathrm{R} 4793$ & R7890 & 1.535 & $1.040 E+01$ & $\bar{M}$ \\
\hline Sodium & $12 / 19 / 91$ & $7 / 17 / 90$ & $6 / 28 / 90$ & $\overline{N A}$ & $\mathrm{R} 4792$ & R7889 & 1.530 & $1.035 E+01$ & $M$ \\
\hline Sodium & $12 / 19 / 91$ & $7 / 17 / 90$ & $6 / 28 / 90$ & NA & $R 4798$ & R7897 & 1.560 & $1.025 E+01$ & $M$ \\
\hline Sodium & $12 / 19 / 91$ & $7 / 17 / 90$ & $6 / 28 / 90$ & NA & R4796 & R7895 & 1.625 & $1.025 E+01$ & $M$ \\
\hline Sodium & $12 / 19 / 91$ & $7 / 17 / 90$ & $6 / 28 / 90$ & NA & 84800 & R7901 & 1.560 & $1.020 E+01$ & $M$ \\
\hline Sodium & $12 / 19 / 91$ & $7 / 17 / 90$ & $6 / 28 / 90$ & $\overline{\mathrm{NA}}$ & $\mathrm{R} 4802$ & R7887/R7903 & 1.550 & $1.015 E+01$ & $M$ \\
\hline Sodium & $12 / 19 / 91$ & $7 / 17 / 90$ & $6 / 28 / 90$ & $\overline{N A}$ & R4794 & R7891 & 1.570 & $1.007 E+01$ & $\bar{M}$ \\
\hline Sodium & $12 / 19 / 91$ & $7 / 17 / 90$ & $6 / 28 / 90$ & NA & R4801 & R7902 & 1.560 & $9.875 E+00$ & $\bar{M}$ \\
\hline Sodium & $12 / 19 / 91$ & $7 / 17 / 90$ & $6 / 28 / 90$ & $\widehat{\mathrm{NA}}$ & R4799 & R7898 & 1.555 & $9.870 E+00$ & $M$ \\
\hline Sodium & $12 / 19 / 91$ & $7 / 77 / 90$ & $6 / 28 / 90$ & NA & R4803 & R7888/R7904 & 1.575 & $9.655 E+00$ & $M$ \\
\hline Sodium & $12 / 19 / 91$ & $7 / 17 / 90$ & $6 / 28 / 90$ & NA & R4797 & R7896 & 1.560 & $9.635 E+00$ & $M$ \\
\hline Sodium & $12 / 19 / 91$ & $7 / 17 / 90$ & $6 / 28 / 90$ & NA & R4795 & R7894 & 1.540 & $9.460 E+00$ & $M$ \\
\hline $\mathrm{Na}$ & $9 / 18 / 86$ & NA & $7 / 2 / 86$ & T882 & NS & NS & 1.32 & 8.31 & $M$ \\
\hline$\overline{\mathrm{Na}}$ & $9 / 18 / 86$ & NA & $6 / 26 / 86$ & T686 & NS & NS & 1.31 & 7.33 & $M$ \\
\hline $\mathrm{Na}$ & $9 / 18 / 86$ & $\overline{\mathrm{NA}}$ & $6 / 23 / 86$ & R9893 & NA & $\overline{N A}$ & 1.37 & 6.9 & $M$ \\
\hline $\mathrm{Na}$ & $9 / 18 / 86$ & NA & $6 / 23 / 86$ & $T 621$ & NS & NS & 1.44 & 6.65 & $M$ \\
\hline $\mathrm{Na}$ & $9 / 18 / 86$ & $\overline{N A}$ & $7 / 6 / 86$ & T959 & NS & NS & 1.23 & 5.77 & $M$ \\
\hline $\mathrm{Na}$ & $9 / 18 / 86$ & NA & $6 / 30 / 86$ & T812 & NS & NS & 1.13 & 3.12 & $M$ \\
\hline $\mathrm{Na}$ & $11 / 9 / 84$ & NS & NS & $\mathrm{R}-3185$ & NS & NS & 1.024 & 1.11 & moles $n$ \\
\hline
\end{tabular}




\begin{tabular}{|c|c|c|c|c|c|c|c|}
\hline \multirow[b]{2}{*}{ Code } & \multirow[b]{2}{*}{ Source } & \multicolumn{3}{|c|}{ Analyte Information } & \multirow[b]{2}{*}{$\begin{array}{c}\text { Reference } \\
\text { Number }\end{array}$} & \multirow[b]{2}{*}{$\begin{array}{c}\text { Validated } \\
\text { (Y/N) }\end{array}$} & \multirow[b]{2}{*}{ Notes } \\
\hline & & Analyte & Conc. (ug/L) & Conc. (mol/L) & & & \\
\hline$P$ & Raw & $K$ & $3.68 E+07$ & $9.41 \mathrm{E}-01$ & AW101-10 & $\bar{Y}$ & \\
\hline$F$ & Raw & $K$ & $3.68 E+07$ & $9.40 \mathrm{E}-01$ & AW101-4 & $\bar{Y}$ & \\
\hline $\bar{P}$ & Raw & $k$ & $3.67 E+07$ & 9.37E-01 & AW101-10 & $\bar{Y}$ & Rerun \\
\hline $\mathbf{P}$ & Raw & $K$ & $3.51 E+07$ & $8.99 E-01$ & AW101-10 & $\bar{Y}$ & \\
\hline $\mathbf{P}$ & Raw & $\mathrm{K}$ & $3.50 E+07$ & $8.95 E-01$ & AW $101-10$ & $\bar{Y}$ & Rerun \\
\hline $\mathbf{P}$ & Raw & $\bar{K}$ & $3.45 E+07$ & $8.82 E-01$ & AW101-10 & $Y$ & Rerun \\
\hline $\mathbf{P}$ & Raw & $K$ & $3.41 E+07$ & $8.71 E-01$ & AW101-10 & $\bar{Y}$ & Rerun \\
\hline $\mathbf{F}$ & Raw & $K$ & $3.40 E+07$ & $8.70 E-01$ & AW101-4 & $\bar{Y}$ & \\
\hline$\overline{\mathbf{P}}$ & Raw & $\mathrm{K}$ & $3.30 E+07$ & $8.43 E-01$ & AW101-10 & $\bar{Y}$ & \\
\hline $\bar{P}$ & Raw & $\mathrm{K}$ & $1.29 E+07$ & $3.30 \mathrm{E}-01$ & AW101-4 & $N$ & Evaporator feed sample \\
\hline$L$ & HTCE Inv. & $K$ & $5.40 E+06$ & $1.38 \mathrm{E}-01$ & NA & $N$ & HTCE \\
\hline$P$ & Raw & $K$ & $5.87 E+05$ & $1.50 \mathrm{E}-02$ & AW101-4 & $\mathbf{N}$ & Waste no longer in tank \\
\hline $\mathrm{P}$ & Raw & $K$ & $4.30 E+05$ & 1.10E-02 & AW101-4 & $\mathbf{N}$ & Waste no longer in tank \\
\hline $\mathbf{P}$ & Braun & $\mathrm{K}$ & $2.35 E+05$ & $6.00 \mathrm{E}-03$ & NA & $\bar{N}$ & Waste no longer in tank \\
\hline $\mathrm{P}$ & Raw & $K$ & $2.35 E+05$ & 6.00E-03 & AW101-2 & $\mathbf{N}$ & Waste no longer in tank \\
\hline$P$ & Raw & $\bar{K}$ & $1.09 E+05$ & $2.79 E-03$ & AW101-3 & $\mathbf{N}$ & Waste no longer in tank \\
\hline $\mathbf{P}$ & Raw & $K$ & $1.07 E+05$ & 2.74E-03 & AW101-3 & $N$ & Waste no longer in tank \\
\hline$P$ & Raw & $K$ & $7.82 E+04$ & $2.00 E-03$ & AW101-4 & $N$ & Waste no longer in tank \\
\hline$P$ & Raw & $K$ & $7.82 E+04$ & $2.00 E-03$ & AW101-4 & $\bar{N}$ & Waste no longer in tank \\
\hline $\mathbf{P}$ & Raw & $\mathrm{Na}$ & $3.08 E+08$ & $1.34 E+01$ & AW101-10 & $Y$ & Composite Sample \\
\hline $\mathbf{P}$ & Raw & $\mathrm{Na}$ & $3.08 \mathrm{E}+08$ & $1.34 E+01$ & AW101-10 & $\bar{Y}$ & Composite Sample \\
\hline $\mathbf{F}$ & RAW & $\mathrm{Na}$ & $2.58 E+08$ & $1.12 \mathrm{E}+01$ & AW101-4 & $\bar{Y}$ & \\
\hline 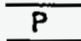 & Raw & $\mathrm{Na}$ & $2.31 E+08$ & $1.01 E+01$ & AW101-10 & $\bar{Y}$ & \\
\hline $\bar{P}$ & Raw & $\mathrm{Na}$ & $2.27 E+08$ & $9.88 E+00$ & AW101-10 & $\bar{Y}$ & \\
\hline $\mathrm{P}$ & Raw & $\mathrm{Na}$ & $2.27 E+08$ & $9.87 E+00$ & AW101-10 & $\mathbf{Y}$ & \\
\hline$P$ & Raw & $\mathrm{Na}$ & $2.22 E+08$ & $9.66 E+00$ & AW101-10 & $\mathbf{Y}$ & \\
\hline $\mathbf{P}$ & Raw & $\mathrm{Na}$ & $2.22 E+08$ & $9.64 E+00$ & AW101-10 & $Y$ & \\
\hline $\mathbf{P}$ & Raw & $\mathrm{Na}$ & $2.18 E+08$ & $9.46 E+\infty$ & AW101-10 & $\mathbf{Y}$ & \\
\hline $\mathrm{U}$ & RAW & $\mathrm{Na}$ & $1.91 E+08$ & $8.31 E+\infty 0$ & AW101-4 & $N$ & Evaporator feed sample \\
\hline $\mathbf{U}$ & RAW & $\mathrm{Na}$ & $1.69 \mathrm{E}+08$ & $7.33 E+00$ & AW101-4 & $N$ & Evaporator feed sample \\
\hline$F$ & RAW & $\mathrm{Na}$ & $1.59 E+08$ & $6.90 E+\infty 0$ & AW101-4 & $\mathrm{Y}$ & \\
\hline $\mathrm{U}$ & RAW & $\mathrm{Na}$ & $1.53 E+08$ & $6.65 E+\infty$ & AW101-4 & $\mathrm{N}$ & Evaporator feed sample \\
\hline $\mathrm{U}$ & RAW & $\mathrm{Na}$ & $1.33 E+08$ & $5.77 E+\infty$ & AW101-4 & $\mathbf{N}$ & Evaporator feed sample \\
\hline$U$ & RAW & $\mathrm{Na}$ & $7.18 E+07$ & $3.12 E+00$ & AW101-4 & $N$ & Evaporator feed sample \\
\hline $\mathbf{P}$ & Braun & $\mathrm{Na}$ & $2.55 E+07$ & $1.11 E+00$ & NA & $N$ & Waste no longer in tank \\
\hline
\end{tabular}


TWRS Privatization Support Pró Chemical Liquids

\begin{tabular}{|c|c|c|c|c|c|c|c|c|c|}
\hline & & & & Tank Was & Characteriz & Son Sample Infom & ion & & \\
\hline Reported Chemical & $\begin{array}{c}\text { Memo } \\
\text { Date }\end{array}$ & $\begin{array}{c}\text { Start Analysis } \\
\text { Date }\end{array}$ & $\begin{array}{c}\text { Start } \\
\text { Sample } \\
\text { Date } \\
\end{array}$ & $\begin{array}{l}\text { Sample } \\
\text { Number }\end{array}$ & $\begin{array}{c}\text { Tank Farm } \\
\text { Number }\end{array}$ & $\begin{array}{c}\text { Lab Sample } \\
\text { Number }\end{array}$ & $\begin{array}{l}\text { Density } \\
\text { (g/mL) }\end{array}$ & Reported Value & Reported U \\
\hline $\mathrm{Na}$ & $4 / 29 / 85$ & $4 / 21 / 85$ & NA & T5351 & NA & NA & 1.0411 & 1.11 & $M$ \\
\hline $\mathrm{Na}$ & $11 / 9 / 84$ & NA & NA & R3185 & NS & NS & 1.024 & 1.11 & $\bar{M}$ \\
\hline $\mathrm{Na}$ & $8 / 21 / 85$ & $8 / 18 / 85$ & NA & T9368 & NA & NA & 1.0392 & 1.02 & $M$ \\
\hline $\mathrm{Na}$ & $8 / 18 / 85$ & $8 / 18 / 85$ & NA & T9368 & NA & NA & 1.0392 & 1.02 & $M$ \\
\hline $\mathrm{Na}$ & $4 / 29 / 85$ & $4 / 21 / 85$ & NA & T5350 & NA & NA & 1.0279 & 0.873 & $M$ \\
\hline $\mathrm{Na}$ & $11 / 6 / 85$ & $11 / 14 / 85$ & $\overline{N A}$ & T2601 & NS & NS & 1.01 & 0.648 & $M$ \\
\hline $\mathrm{Na}$ & $11 / 6 / 85$ & $11 / 14 / 85$ & $\mathrm{NA}$ & T2641 & NS & NS & 1.01 & 0.516 & $M$ \\
\hline $\mathrm{Na}$ & $3 / 30 / 86$ & $3 / 30 / 86$ & NA & T7698 & $\ldots N S$ & NS & 0.993 & 0.375 & $M$ \\
\hline $\mathrm{Na}$ & $3 / 30 / 86$ & $3 / 30 / 86$ & NA & $T 7697$ & NS & NS & 0.997 & 0.349 & $M$ \\
\hline $\mathrm{NH3}$ & $9 / 18 / 86$ & NA & $6 / 26 / 86$ & T686 & NS & NS & 1.31 & 0.04 & $M$ \\
\hline NH3 & $9 / 18 / 86$ & NA & $6 / 26 / 86$ & R9894 & NA & NA & 1.49 & 0.03 & $\bar{M}$ \\
\hline NH3 & $6 / 23 / 86$ & NS & NS & NS & NS & NS & 1.37 & 0.02 & $M$ \\
\hline NH3 & $11 / 6 / 85$ & $11 / 14 / 85$ & NA & T2641 & NS & NS & 1.01 & 0.014 & $M$ \\
\hline NH3 & $3 / 30 / 86$ & $3 / 30 / 86$ & NA & T7698 & NS & NS & 0.993 & 0.013 & $M$ \\
\hline NH3 & $8 / 21 / 85$ & $8 / 18 / 85$ & NA & T9368 & NA & NA & 1.0392 & 0.0119 & $M$ \\
\hline $\mathrm{NH4}$ & $8 / 18 / 85$ & $8 / 18 / 85$ & NA & T9368 & NA & NA & 1.0392 & 0.0119 & $\bar{M}$ \\
\hline $\mathrm{NH3}$ & $4 / 29 / 85$ & $4 / 21 / 85$ & NA & $T 5350$ & NA & NA & 1.0279 & 0.0115 & $M$ \\
\hline $\mathrm{NH3}$ & $7 / 2 / 86$ & NS & NS & NS & NS & NS & 1.5 & 0.01 & $M$ \\
\hline $\mathrm{NH3}$ & $11 / 9 / 84$ & NS & NS & $8-3185$ & NS & NS & 1.024 & 0.007 & moles I \\
\hline $\mathrm{NH3}$ & $11 / 9 / 84$ & NA & NA & R3185 & NS & NS & 7.024 & 0.007 & $M$ \\
\hline $\mathrm{NH3}$ & $11 / 6 / 85$ & $11 / 14 / 85$ & NA & T2601 & NS & NS & 1.01 & 0.006 & $\bar{M}$ \\
\hline NH3 & $9 / 18 / 86$ & NA & $7 / 2 / 86$ & T882 & NS & NS & 1.32 & 0.00477 & $M$ \\
\hline $\mathrm{NH3}$ & $9 / 18 / 86$ & NA & $6 / 23 / 86$ & T621 & NS & NS & 1.44 & 0.00159 & $M$ \\
\hline $\mathrm{Ni2}+$ & NA & NA & NA & NA & NA & NA & 1.39 & $5.14 E-03$ & Mole/L \\
\hline Nitrite & $12 / 19 / 91$ & $7 / 17 / 90$ & $6 / 28 / 90$ & NA & R4796 & R7916/R7935 & 1.625 & $2.370 E+00$ & $M$ \\
\hline Nitrite & $12 / 19 / 91$ & $7 / 17 / 90$ & $6 / 28 / 90$ & NA & R4792 & R7912/R7929 & 1.530 & $2.370 E+00$ & $M$ \\
\hline Nitrite & $12 / 19 / 91$ & $7 / 17 / 90$ & $6 / 28 / 90$ & NA & R4794 & R7914/R7933 & 1.570 & $2.340 E+00$ & $M$ \\
\hline Nitrite-D & $12 / 19 / 91$ & $7 / 17 / 90$ & $6 / 28 / 90$ & NA & NA & 88801 & 1.540 & $2.290 E+00$ & $M$ \\
\hline Nitrite & $12 / 19 / 91$ & $7 / 17 / 90$ & $6 / 28 / 90$ & NA & $\mathrm{R} 4793$ & $R 7913 / R 7930$ & 1.535 & $2.280 \mathrm{E}+00$ & $\vec{M}$ \\
\hline Nitrite-D & $12 / 19 / 91$ & $7 / 17 / 90$ & $6 / 28 / 90$ & NA & NA & R8802 & 1.540 & $2.260 E+00$ & $M$ \\
\hline Nitrite & $12 / 19 / 91$ & $7 / 17 / 90$ & $6 / 28 / 90$ & NA & $\mathrm{R} 4800$ & R7922/R7941 & 1.560 & $2.215 E+00$ & $M$ \\
\hline Nitrite & $12 / 19 / 91$ & $7 / 17 / 90$ & $6 / 28 / 90$ & NA & R4795 & R7915/R7934 & 1.540 & $2.215 E+00$ & $M$ \\
\hline Nitrite-D & $6 / 28 / 90$ & NA & NA & NA & NA & NA & 1.560 & 2.212 & Moles/L \\
\hline Nitrite-S & $12 / 19 / 91$ & $7 / 17 / 90$ & $6 / 28 / 90$ & NA & NA & $R 8801$ & 1.540 & $2.170 E+00$ & $M$ \\
\hline Nitrite-S & $6 / 28 / 90$ & NA & NA & NA & NA & NA & 1.560 & 2.163 & Moles/L \\
\hline Nitrite-S & $12 / 19 / 91$ & $7 / 17 / 90$ & $6 / 28 / 90$ & NA & NA & R8802 & 1.540 & $2.160 E+00$ & $\bar{M}$ \\
\hline Nitrite & $12 / 19 / 91$ & $7 / 17 / 90$ & $6 / 28 / 90$ & NA & $R 4803$ & R7927/R7944 & 1.575 & $2.140 E+00$ & $\bar{M}$ \\
\hline Nitrite & $12 / 19 / 91$ & $7 / 17 / 90$ & $6 / 28 / 90$ & $\mathrm{NA}$ & R4799 & R7921/R7940 & 1.555 & $2.140 E+00$ & $M$ \\
\hline Nitrite & $12 / 19 / 91$ & $7 / 17 / 90$ & $6 / 28 / 90$ & NA & $R 4802$ & R7926/R7943 & 1.550 & $2.135 E+00$ & $M$ \\
\hline Nitrite & $12 / 19 / 91$ & $7 / 17 / 90$ & $6 / 28 / 90$ & NA & R4801 & R7923/R7942 & 1.560 & $2.130 E+00$ & $M$ \\
\hline Nitrite & $12 / 19 / 91$ & $7 / 17 / 90$ & $6 / 28 / 90$ & $\mathrm{NA}$ & R4797 & R7919/R7936 & 1.560 & $2.105 E+00$ & $\bar{M}$ \\
\hline Nitrite & $12 / 19 / 91$ & $7 / 17 / 90$ & $6 / 28 / 90$ & NA & $R 4798$ & R7920/R7937 & 1.560 & $2.100 E+00$ & $M$ \\
\hline $\mathrm{NO}_{2}$ & $9 / 18 / 86$ & $\mathrm{NA}$ & $7 / 2 / 86$ & R9896 & NA & NA & 1.500 & 2.04 & Moles/L \\
\hline $\mathrm{NO}_{2}$ & $9 / 18 / 86$ & NA & $6 / 30 / 86$ & $\mathrm{R} 9895$ & $\mathrm{NA}$ & NA & 1.540 & 1.89 & Moles/L \\
\hline $\mathrm{NO}_{2}$ & $9 / 18 / 86$ & NA & $6 / 26 / 86$ & 89894 & NA & NA & 1.490 & 1.85 & Moles/L \\
\hline $\mathrm{NO}_{2}$ & $12 / 30 / 81$ & NA & NA & $\mathrm{T} 1049$ & NA & NA & 1.329 & 1.59 & Moles \\
\hline $\mathrm{NO}_{2}$ & $12 / 30 / 81$ & NA & NA & $T 1048$ & $\mathrm{NA}$ & NA & 1.324 & 1.52 & Moles \\
\hline NO2- & NA & NA & NA & NA & NA & NA & 1.39 & $1.51 E+00$ & Mole/L \\
\hline
\end{tabular}




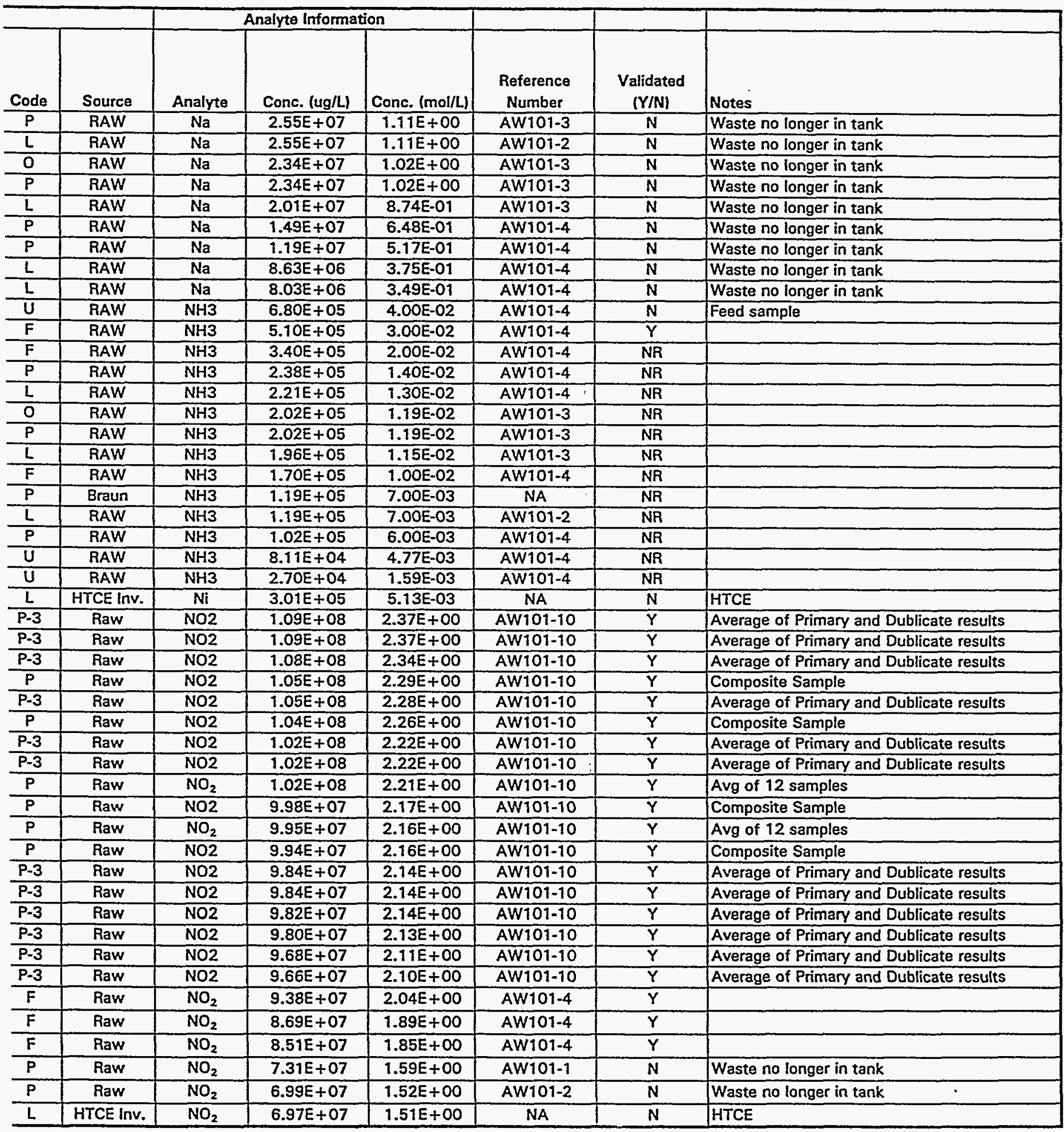




\begin{tabular}{|c|c|c|c|c|c|c|c|c|c|}
\hline & & & & Tank Wast & Characteriza & ion Sample Inform & ion & & \\
\hline Reported Chemical & $\begin{array}{c}\text { Memo } \\
\text { Date } \\
\end{array}$ & $\begin{array}{c}\text { Start Analysis } \\
\text { Date }\end{array}$ & $\begin{array}{c}\text { Start } \\
\text { Sample } \\
\text { Date }\end{array}$ & $\begin{array}{l}\text { Sample } \\
\text { Number }\end{array}$ & $\begin{array}{c}\text { Tank Farm } \\
\text { Number }\end{array}$ & $\begin{array}{c}\text { Lab Sample } \\
\text { Number }\end{array}$ & $\begin{array}{l}\text { Density } \\
(\mathrm{g} / \mathrm{mL})\end{array}$ & Reported Value & Reported U \\
\hline $\mathrm{NO}_{2}$ & $9 / 18 / 86$ & NA & $6 / 23 / 86$ & R9893 & NA & NA & 1.370 & 1.42 & Moles $/ L$ \\
\hline $\mathrm{NO}_{2}$ & $9 / 18 / 86$ & NA & $7 / 6 / 86$ & $\mathrm{R} 9897$ & NA & NA & 1.460 & 1.37 & Moles/L \\
\hline $\mathrm{NO}_{2}$ & $9 / 18 / 86$ & NA & $6 / 23 / 86$ & T621 & NS & NS & 1.440 & 1.36 & Moles/L \\
\hline $\mathrm{NO}_{2}$ & $9 / 18 / 86$ & NA & $6 / 26 / 86$ & T686 & NS & NS & 1.310 & 1.26 & Moles $/ L$ \\
\hline $\mathrm{NO}_{2}$ & $9 / 18 / 86$ & NA & $7 / 2 / 86$ & $\mathrm{~T} 882$ & NS & NS & 1.320 & 1.22 & Moles $/ \mathrm{L}$ \\
\hline $\mathrm{NO}_{-2}^{-}$ & $4 / 3 / 84$ & NA & NA & $\mathrm{R} 9408$ & NA & NA & 1.330 & 0.85 & Moles/L \\
\hline $\mathrm{NO}_{2}^{-}$ & $4 / 3 / 84$ & NA & NA & $\mathrm{R} 9414$ & NA & NA & 1.330 & 0.84 & Moles/L \\
\hline $\mathrm{NO}_{2}$ & $9 / 18 / 86$ & NA & $6 / 30 / 86$ & $\mathrm{~T} 812$ & NS & NS & 1.130 & 0.51 & Moles/L \\
\hline $\mathrm{NO}_{-2}^{-}$ & $11 / 20 / 84$ & NA & NA & $\mathrm{R} 2644$ & NA & NA & NA & 0.122 & Moles/L \\
\hline $\mathrm{NO}_{2}^{-}$ & $11 / 20 / 84$ & NA & NA & $\mathrm{R} 2645$ & NA & NA & NA & 0.069 & Moles/L \\
\hline $\mathrm{NO2}$ & $8 / 18 / 85$ & $8 / 18 / 85$ & NA & T9368 & NA & NA & 1.040 & 0.0446 & Moles/L \\
\hline $\mathrm{NO2}$ & $4 / 29 / 85$ & $4 / 21 / 85$ & NA & T5350 & NA & NA & 1.028 & 0.0437 & Moles/L \\
\hline NO2 & $11 / 9 / 84$ & NS & NS & $\mathrm{R}-3185$ & NS & NS & 1.024 & 0.043 & moles/1 \\
\hline $\mathrm{NO}-2$ & $11 / 9 / 84$ & NA & NA & R3185 & NS & NS & 1.024 & 0.043 & Moles \\
\hline $\mathrm{NO}_{2}$ & $2 / 20 / 86$ & $11 / 14 / 85$ & NA & T2601 & NS & NS & 1.010 & 0.042 & Moles/L \\
\hline $\mathrm{NO}_{2}$ & $4 / 29 / 86$ & $3 / 30 / 86$ & NA & $T 7698$ & NS & NS & 0.993 & 0.036 & Moles/L \\
\hline $\mathrm{NO}_{2}$ & $4 / 29 / 86$ & $3 / 30 / 86$ & NA & 77697 & NS & NS & 0.993 & 0.035 & Moles/L \\
\hline $\mathrm{NO}_{2}$ & $2 / 20 / 86$ & $11 / 14 / 85$ & NA & $T 2641$ & NS & NS & 1.010 & 0.032 & Moles/L \\
\hline $\mathrm{NO}_{3}$ & $9 / 18 / 86$ & NA & $7 / 6 / 86$ & R9897 & NS & NS & 1.460 & 4.7 & Moles/L \\
\hline Nitrate & $12 / 19 / 91$ & $7 / 17 / 90$ & $6 / 28 / 90$ & NA & NA & R8801 & 1.540 & $3.810 E+00$ & $M$ \\
\hline $\mathrm{NO}_{3}$ & $9 / 18 / 86$ & $\mathrm{NA}$ & $7 / 2 / 86$ & R9896 & NA & NA & 1.500 & 3.73 & Moles/L \\
\hline Nitrate & $12 / 19 / 91$ & $7 / 17 / 90$ & $6 / 28 / 90$ & NA & R4796 & R7916 / R7935 & 1.625 & $3.665 E+00$ & $\bar{M}$ \\
\hline Nitrate & $12 / 19 / 91$ & $7 / 17 / 90$ & $6 / 28 / 90$ & NA & R4794 & R7914/R7933 & 1.570 & $3.615 \mathrm{E}+00$ & $M$ \\
\hline Nitrate & $12 / 19 / 91$ & $7 / 17 / 90$ & $6 / 28 / 90$ & $\mathrm{NA}$ & R4793 & R7913/R7930 & 1.535 & $3.520 E+00$ & $M$ \\
\hline Nitrate & $12 / 19 / 91$ & $7 / 17 / 90$ & $6 / 28 / 90$ & $\mathrm{NA}$ & $\mathrm{R} 4803$ & R7927/R7944 & 1.575 & $3.475 E+00$ & $M$ \\
\hline Nitrate & $12 / 19 / 91$ & $7 / 17 / 90$ & $6 / 28 / 90$ & NA & $R 4800$ & R7922 / R7941 & 1.560 & $3.470 E+00$ & $\bar{M}$ \\
\hline Nitrate & $12 / 19 / 91$ & $7 / 17 / 90$ & $6 / 28 / 90$ & NA & R4799 & R7921/R7940 & 1.555 & $3.460 \mathrm{E}+00$ & $\bar{M}$ \\
\hline Nitrate & $6 / 28 / 90$ & NA & NA & NA & NA & NA & 1.560 & 3.456 & Moles/L \\
\hline Nitrate & $12 / 19 / 91$ & $7 / 17 / 90$ & $6 / 28 / 90$ & NA & $\mathrm{R} 4802$ & R7926/R7943 & 1.550 & $3.455 \mathrm{E}+00$ & $M$ \\
\hline Nitrate & $12 / 19 / 91$ & $7 / 17 / 90$ & $6 / 28 / 90$ & NA & R4801 & R7923/R7942 & 1.560 & $3.450 E+00$ & $M$ \\
\hline NO3- & NA & $\mathrm{NA}$ & NA & NA & NA & NA & 1.39 & $3.44 \mathrm{E}+00$ & Mole/L \\
\hline Nitrate & $12 / 19 / 91$ & $7 / 17 / 90$ & $6 / 28 / 90$ & NA & $\mathrm{NA}$ & $\mathrm{R} 8802$ & 1.540 & $3.430 \mathrm{E}+00$ & $M$ \\
\hline Nitrate & $12 / 19 / 91$ & $7 / 17 / 90$ & $6 / 28 / 90$ & NA & R4792 & R7912/R7929 & 1.530 & $3.425 \mathrm{E}+00$ & $M$ \\
\hline Nitrate & $12 / 19 / 91$ & $7 / 17 / 90$ & $6 / 28 / 90$ & NA & R4798 & R7920/R7937 & 1.560 & $3.400 E+00$ & $M$ \\
\hline Nitrate & $12 / 19 / 91$ & $7 / 17 / 90$ & $6 / 28 / 90$ & NA & R4795 & R7915/R7934 & 1.540 & $3.335 E+00$ & $M$ \\
\hline Nitrate & $12 / 19 / 91$ & $7 / 17 / 90$ & $6 / 28 / 90$ & NA & R4797 & R7919/R7936 & 1.560 & $3.205 E+00$ & $M$ \\
\hline $\mathrm{NO}_{3}$ & $9 / 18 / 86$ & $\mathrm{NA}$ & $6 / 30 / 86$ & R9895 & $\mathrm{NA}$ & $\mathrm{NA}$ & 1.540 & 3.2 & Moles/L \\
\hline $\mathrm{NO}_{3}$ & $9 / 18 / 86$ & $\mathrm{NA}$ & $6 / 23 / 86$ & $T 621$ & NS & NS & 1.440 & 3.16 & Moles/L \\
\hline $\mathrm{NO}_{3}$ & $12 / 30 / 81$ & $\mathrm{NA}$ & $\mathrm{NA}$ & T1049 & NA & NA & 1.329 & 3.11 & Moles \\
\hline $\mathrm{NO}_{3}$ & $12 / 30 / 81$ & NA & NA & T1048 & NA & NA & 1.324 & 2.98 & Moles \\
\hline
\end{tabular}




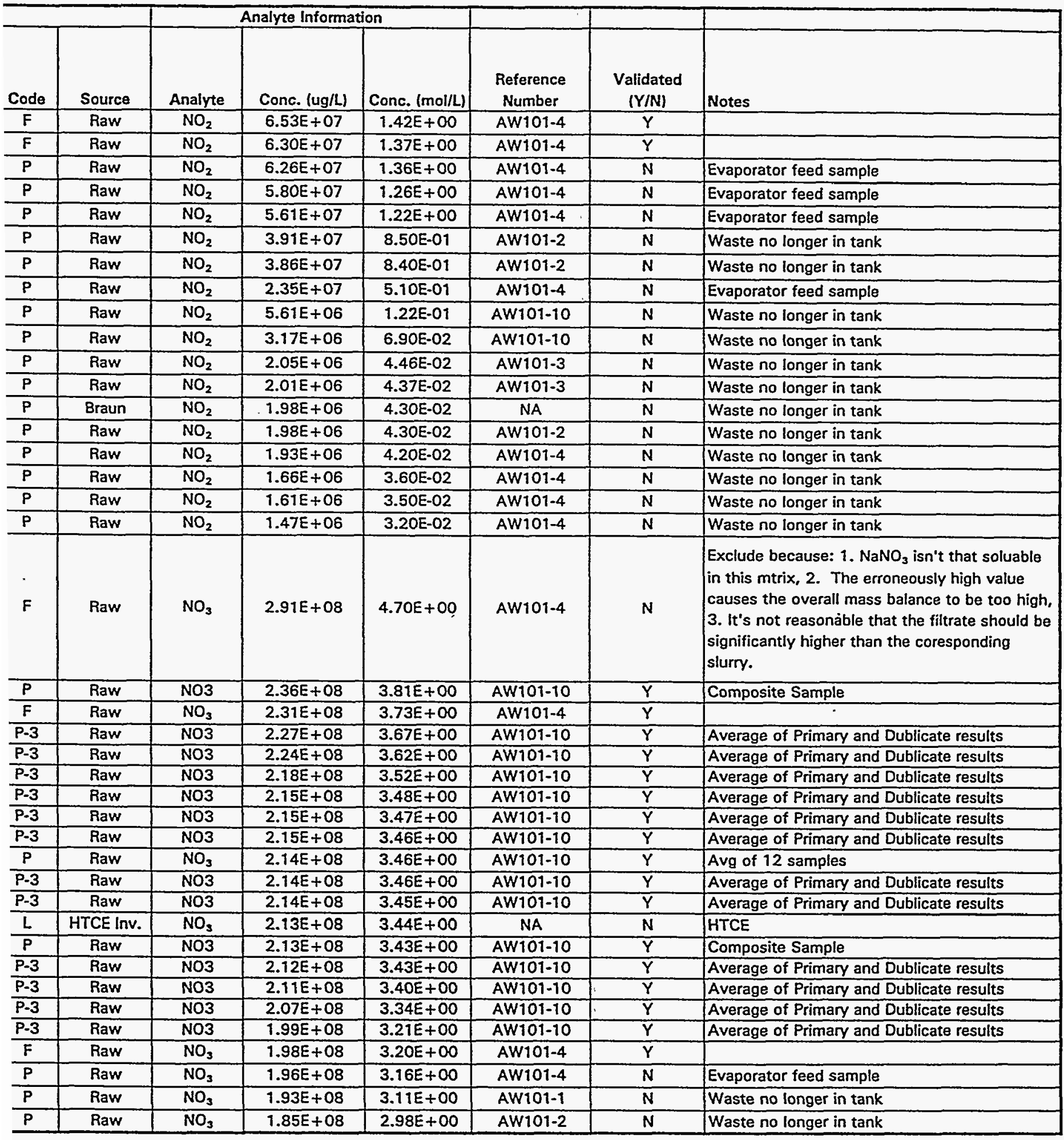


TWRS Privatization Support Pro, Chemical Liquids

\begin{tabular}{|c|c|c|c|c|c|c|c|c|c|}
\hline \multicolumn{10}{|c|}{ Tank Waste Characterization Sample Information } \\
\hline Reported Chemical & $\begin{array}{c}\text { Memo } \\
\text { Date }\end{array}$ & $\begin{array}{c}\text { Start Analysis } \\
\text { Date }\end{array}$ & $\begin{array}{c}\text { Start } \\
\text { Sample } \\
\text { Date } \\
\end{array}$ & $\begin{array}{l}\text { Sample } \\
\text { Number }\end{array}$ & $\begin{array}{c}\text { Tank Farm } \\
\text { Number }\end{array}$ & $\begin{array}{c}\text { Lab Sample } \\
\text { Number }\end{array}$ & $\begin{array}{c}\text { Density } \\
(\mathrm{g} / \mathrm{mL})\end{array}$ & Reported Value & Reported U \\
\hline $\mathrm{NO}_{3}$ & $9 / 18 / 86$ & NA & $6 / 26 / 86$ & R9894 & $\overrightarrow{N A}$ & NA & 1.490 & 2.88 & Moles/L \\
\hline $\mathrm{NO}_{3}^{-}$ & $4 / 3 / 84$ & NA. & NA & R9414 & NA & NA & 1.330 & 2.85 & Moles/L \\
\hline $\mathrm{NO}_{3}{ }^{-}$ & $4 / 3 / 84$ & NA & NA & R9408 & NA & NA & 1.330 & 2.79 & Moles/L \\
\hline $\mathrm{NO}_{3}$ & $9 / 18 / 86$ & NA & $6 / 23 / 86$ & R9893 & NA & NA & 1.370 & 2.52 & Moles/L \\
\hline $\mathrm{NO}_{3}$ & $9 / 18 / 86$ & NA & $7 / 2 / 86$ & T882 & NS & NS & 1.320 & 2.37 & Moles/L \\
\hline $\mathrm{NO}_{3}$ & $9 / 18 / 86$ & NA & $6 / 26 / 86$ & T686 & NS & NS & 1.310 & 2 & Moles $/ L$ \\
\hline $\mathrm{NO}_{3}$ & $9 / 18 / 86$ & NA & $6 / 30 / 86$ & T812 & NS & NS & 1.130 & 0.99 & Moles/L \\
\hline $\mathrm{NO}_{3}{ }^{\circ}$ & $11 / 20 / 84$ & NA & NA & $\mathrm{R} 2644$ & NA & $\mathrm{NA}$ & $\mathrm{NA}$ & 0.368 & Moles/L \\
\hline $\mathrm{NO}_{3}^{-}$ & $11 / 20 / 84$ & NA & NA & $R 2645$ & NA & NA & NA & 0.237 & Moles $\mathrm{L}$ \\
\hline NO3 & $11 / 9 / 84$ & NS & NS & $R-3185$ & NS & NS & 1.024 & 0.19 & moles $\|$ \\
\hline $\mathrm{NO}_{3}$ & $11 / 9 / 84$ & NA & $\mathrm{NA}$ & R3185 & NS & NS & 1.024 & 0.19 & Moles/L \\
\hline $\mathrm{NO}_{3}$ & $2 / 20 / 86$ & $11 / 14 / 85$ & NA & $T 2641$ & NS & NS & 1.010 & 0.184 & Moles/L \\
\hline $\mathrm{NO}_{3}$ & $4 / 29 / 86$ & $3 / 30 / 86$ & $\mathrm{NA}$ & $T 7697$ & NS & NS & 0.993 & 0.182 & Moles/L \\
\hline $\mathrm{NO}_{3}$ & $4 / 29 / 86$ & $3 / 30 / 86$ & $\mathrm{NA}$ & T7698 & NS & NS & 0.993 & 0.171 & Moles/L \\
\hline $\mathrm{NO3}$ & $4 / 29 / 85$ & $4 / 21 / 85$ & NA & $T 5350$ & NA & $\mathrm{NA}$ & 1.028 & 0.163 & Moles/L \\
\hline $\mathrm{NO}_{3}$ & $2 / 20 / 86$ & $11 / 14 / 85$ & NA & $T 2601$ & NS & NS & 1.010 & 0.157 & Moles/L \\
\hline NO3 & $8 / 18 / 85$ & $8 / 18 / 85$ & NA & $T 9368$ & $\mathrm{NA}$ & NA & 1.040 & 0.0121 & Moles/L \\
\hline $\mathrm{OH}$ & $9 / 18 / 86$ & NA & $6 / 30 / 86$ & R9895 & NA & NA & 1.54 & 5.58 & $M$ \\
\hline $\mathrm{OH}$ & $9 / 18 / 86$ & NA & $7 / 2 / 86$ & R9896 & NA & NA & 1.5 & 5.35 & $\vec{M}$ \\
\hline Hydroxide & $12 / 19 / 91$ & $7 / 17 / 90$ & $6 / 28 / 90$ & NA & $R 4800$ & R7958 / R7976 & 1.560 & $5.250 E+00$ & $M$ \\
\hline $\mathrm{OH}$ & $9 / 18 / 86$ & NA & $7 / 6 / 86$ & R9897 & NA & NA & 1.53 & 5.25 & $M$ \\
\hline Hydroxide & $12 / 19 / 91$ & $7 / 17 / 90$ & $6 / 28 / 90$ & NA & $\mathrm{R} 4802$ & R7962/R7978 & 1.550 & $5.155 E+00$ & $M$ \\
\hline Hydroxide & $12 / 19 / 91$ & $7 / 17 / 90$ & $6 / 28 / 30$ & NA & $\mathrm{R} 4793$ & R7949/R7965 & 1.535 & $5.155 E+00$ & $M$ \\
\hline Hydroxide & $12 / 19 / 91$ & $7 / 17 / 90$ & $6 / 28 / 90$ & NA & R4799 & R7957/R7973 & 1.555 & $5.125 \mathrm{E}+00$ & $\bar{M}$ \\
\hline Hydroxide & $12 / 19 / 91$ & $7 / 17 / 90$ & $6 / 28 / 90$ & NA & R4798 & R7956/R7972 & 1.560 & $5.110 E+00$ & $\bar{M}$ \\
\hline Hydroxide & $12 / 19 / 91$ & $7 / 17 / 90$ & $6 / 28 / 90$ & NA & R4792 & R7948/R7964 & 1.530 & $5.095 E+00$ & $M$ \\
\hline Hydroxide & $12 / 19 / 91$ & $7 / 17 / 90$ & $6 / 28 / 90$ & $\mathrm{NA}$ & R4801 & R7959/R7977 & 1.560 & $5.085 E+00$ & $M$ \\
\hline Hydroxide & $12 / 19 / 91$ & $7 / 17 / 90$ & $6 / 28 / 90$ & NA & 84797 & R7955 / R7971 & 1.560 & $5.075 E+00$ & $M$ \\
\hline Hydroxide & $12 / 19 / 91$ & $7 / 17 / 90$ & $6 / 28 / 90$ & NA & R4796 & R795 / R7970 & 1.625 & $5.050 E+00$ & $M$ \\
\hline Hydroxide & $12 / 19 / 91$ & $7 / 17 / 90$ & $6 / 28 / 90$ & NA & R4794 & R7950 / R7966 & 1.570 & $5.045 \mathrm{E}+00$ & $M$ \\
\hline Hydroxide & $12 / 19 / 91$ & $7 / 17 / 90$ & $6 / 28 / 90$ & NA & NA & R8799 & 1.540 & $4.950 E+00$ & $M$ \\
\hline Hydroxide & $12 / 19 / 91$ & $7 / 17 / 90$ & $6 / 28 / 90$ & NA & R4795 & R7951 / R7969 & 1.540 & $4.900 \mathrm{E}+00$ & $M$ \\
\hline Hydroxide & $12 / 19 / 91$ & $7 / 17 / 90$ & $6 / 28 / 90$ & NA & R4803 & R7963/R7979 & 1.575 & $4.820 E+00$ & $M$ \\
\hline $\mathrm{OH}$ & $9 / 18 / 86$ & $\mathrm{NA}$ & $6 / 26 / 86$ & R9894 & NA & NA & 1.49 & 4.78 & $M$ \\
\hline Hydroxide & $12 / 19 / 91$ & $7 / 17 / 90$ & $6 / 28 / 90$ & NA & NA & R8798 & 1.540 & $4.510 \mathrm{E}+00$ & $M$ \\
\hline $\mathrm{OH}$ & $9 / 18 / 86$ & NA & $6 / 23 / 86$ & T621 & NS & NS & 1.44 & 4.61 & $M$ \\
\hline $\mathrm{OH}$ & $9 / 18 / 86$ & NA & $7 / 2 / 86$ & T882 & NS & NS & 1.32 & 3.87 & $M$ \\
\hline $\mathrm{OH}$ & $12 / 30 / 81$ & NA & NA & $T 1049$ & NA & $\overline{N A}$ & 1.329 & 1.56 & $M$ \\
\hline $\mathrm{OH}$ & $12 / 30 / 81$ & NA & NA & T1048 & NA & NA & 1.324 & 1.56 & $M$ \\
\hline $\mathrm{OH}$ & $9 / 18 / 86$ & NA & $6 / 23 / 86$ & R9893 & $\overline{N A}$ & NA & 1.37 & 3.33 & $M$ \\
\hline $\mathrm{OH}$ & $9 / 18 / 86$ & NA & $6 / 26 / 86$ & T686 & NS & NS & 1.31 & 3.18 & $M$ \\
\hline $\mathrm{OH}$ & $9 / 18 / 86$ & NA & $6 / 30 / 86$ & $\mathrm{~T} 812$ & NS & NS & 1.13 & 1.34 & $M$ \\
\hline $\mathrm{OH}$ & $9 / 18 / 86$ & NA & $7 / 6 / 86$ & T959 & NS & NS & 1.23 & 2.9 & $\bar{M}$ \\
\hline $\mathrm{OH}-$ & $2 / 15 / 84$ & NA & NA & R9414 & NA & NA & 1.37 & 1.2 & $M$ \\
\hline $\mathrm{OH}-$ & $2 / 15 / 84$ & NA & NA & R9408 & NA & NA & 1.33 & 1.2 & $M$ \\
\hline $\mathrm{OH}$ & $11 / 9 / 84$ & NS & NS & $\mathrm{R}-3185$ & NS & NS & 1.024 & 0.745 & moles $/$ \\
\hline $\mathrm{OH}$ & $11 / 9 / 84$ & NA & NA & R3185 & NS & NS & 1.024 & 0.745 & $M$ \\
\hline
\end{tabular}




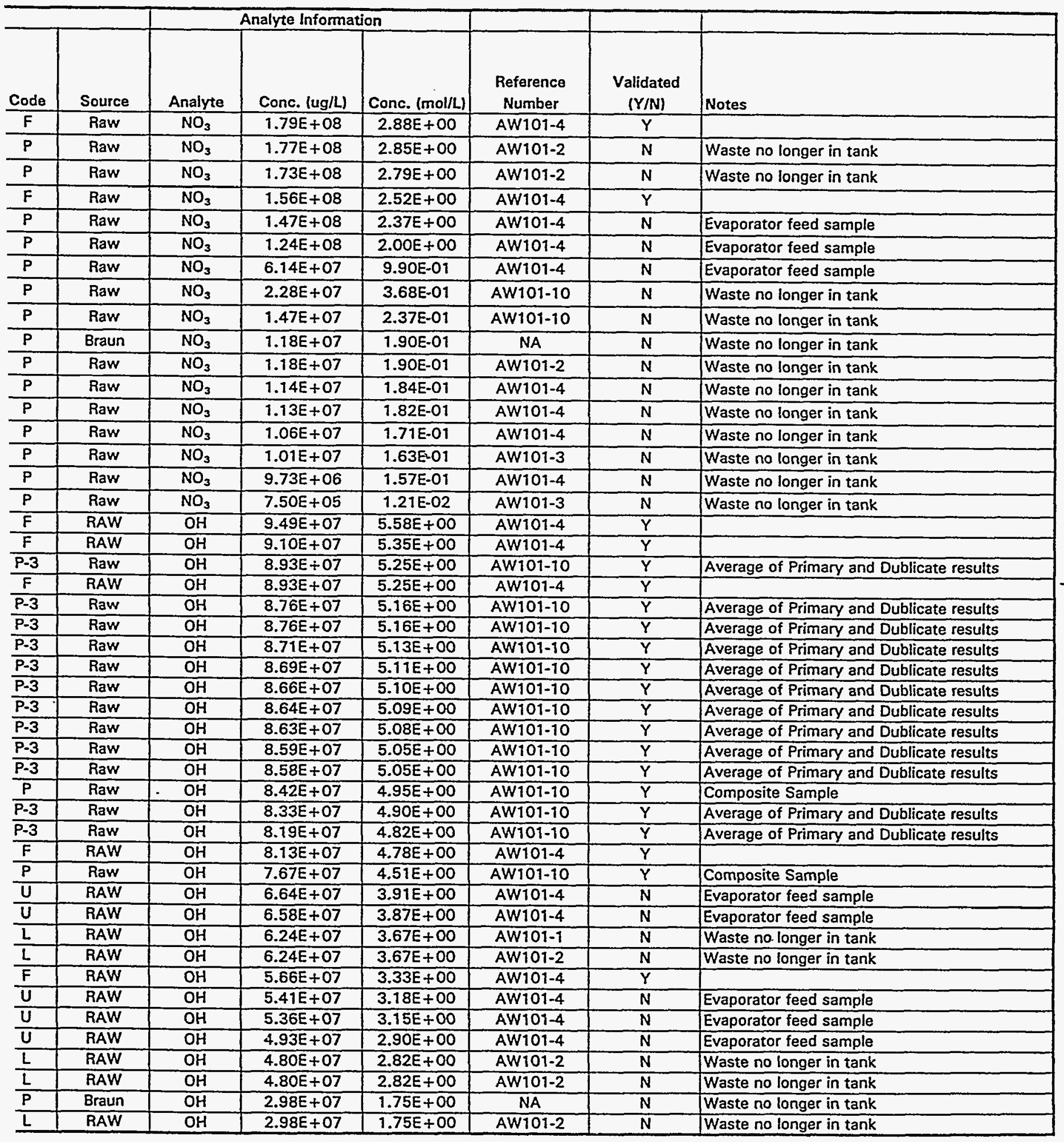




\begin{tabular}{|c|c|c|c|c|c|c|c|c|c|}
\hline & & & & Tank Was & Characteriza & ion Sample Inform & ion & & \\
\hline Reported Chemical & $\begin{array}{c}\text { Memo } \\
\text { Date }\end{array}$ & $\begin{array}{c}\text { Start Analysis } \\
\text { Date }\end{array}$ & $\begin{array}{c}\text { Start } \\
\text { Sample } \\
\text { Date }\end{array}$ & $\begin{array}{l}\text { Sample } \\
\text { Number }\end{array}$ & $\begin{array}{c}\text { Tank Farm } \\
\text { Number }\end{array}$ & $\begin{array}{c}\text { Lab Sample } \\
\text { Number }\end{array}$ & $\begin{array}{l}\text { Density } \\
(\mathrm{g} / \mathrm{mL})\end{array}$ & Reported Value & Reported I \\
\hline $\mathrm{OH}-$ & $11 / 20 / 84$ & NA & NA & R2644 & NS & NS & NS & 0.691 & Mole/L \\
\hline $\mathrm{OH}-$ & $11 / 20 / 84$ & NA & NA & R2645 & NS & NS & NS & 0.669 & Mole/L \\
\hline $\mathrm{OH}-$ & $8 / 21 / 85$ & $8 / 18 / 85$ & NA & T9368 & NA & NA & 1.0392 & 0.6177 & $M$ \\
\hline$\overline{\mathrm{OH}}$ & $8 / 18 / 85$ & $8 / 18 / 85$ & NA & T9368 & NA & NA & 1.0392 & 0.6177 & $M$ \\
\hline $\mathrm{OH}-$ & $11 / 6 / 85$ & $11 / 14 / 85$ & NA & T2601 & NS & NS & 1.01 & 0.607 & $\bar{M}$ \\
\hline $\mathrm{OH}-$ & $4 / 29 / 85$ & $4 / 21 / 85$ & NA & T5351 & NA & NA & 1.0411 & 0.601 & $M$ \\
\hline $\mathrm{OH}$ - & $4 / 29 / 85$ & $4 / 21 / 85$ & NA & $T 5350$ & NA & NA & 1.0279 & 0.585 & $M$ \\
\hline $\mathrm{OH}-$ & $11 / 6 / 85$ & $11 / 14 / 85$ & NA & T2641 & NS. & NS & 1.01 & 0.505 & $M$ \\
\hline $\mathrm{OH}$ & $3 / 30 / 86$ & $3 / 30 / 86$ & NA & T7697 & NS & NS & 0.997 & 0.208 & $\bar{M}$ \\
\hline $\mathrm{OH}$ & $3 / 30 / 86$ & $3 / 30 / 86$ & NA & T7698 & NS & NS & 0.993 & 0.203 & $\vec{M}$ \\
\hline Lead & $12 / 19 / 91$ & $7 / 17 / 90$ & $6 / 28 / 90$ & NA & R4801 & R7902 & 1.560 & $<1.46$ E-03 & $\bar{M}$ \\
\hline Lead & $12 / 19 / 91$ & $7 / 17 / 90$ & $6 / 28 / 90$ & NA & R4800 & R7901 & 1.560 & $<1.46 E-03$ & $M$ \\
\hline Lead & $12 / 19 / 91$ & $7 / 17 / 90$ & $6 / 28 / 90$ & $\mathrm{NA}$ & R4799 & R7898 & 1.555 & $<1.46 \mathrm{E}-03$ & $M$ \\
\hline Lead & $12 / 19 / 91$ & $7 / 17 / 90$ & $6 / 28 / 90$ & NA & $R 4798$ & R7897 & 1.560 & $<1.46 \mathrm{E}-03$ & $M$ \\
\hline Lead & $12 / 19 / 91$ & $7 / 17 / 90$ & $6 / 28 / 90$ & NA & R4797 & R7896 & 1.560 & $<1.46 \mathrm{E}-03$ & $M$ \\
\hline Lead & $12 / 19 / 91$ & $7 / 17 / 90$ & $6 / 28 / 90$ & NA & $R 4796$ & R7895 & 1.625 & $<1.46 E-03$ & $M$ \\
\hline Lead & $12 / 19 / 91$ & $7 / 17 / 90$ & $6 / 28 / 90$ & NA & R4795 & 87894 & 1.540 & $<1.46 \mathrm{E}-03$ & $M$ \\
\hline Lead & $12 / 19 / 91$ & $7 / 17 / 90$ & $6 / 28 / 90$ & NA & R4794 & R7891 & 1.570 & $<1.46 \mathrm{E}-03$ & $M$ \\
\hline Lead & $12 / 19 / 91$ & $7 / 17 / 90$ & $6 / 28 / 90$ & NA & R4793 & R7890 & 1.535 & $<1.46 \mathrm{E}-03$ & $M$ \\
\hline Lead & $12 / 19 / 91$ & $7 / 17 / 90$ & $6 / 28 / 90$ & NA & R4792 & R7889 & 1.530 & $<1.46 \mathrm{E}-03$ & $M$ \\
\hline Lead & $12 / 19 / 91$ & $7 / 17 / 90$ & $6 / 28 / 90$ & $\mathrm{NA}$ & R4803 & R7888/R7904 & 1.575 & $<1.46 \mathrm{E}-03$ & $M$ \\
\hline Lead & $12 / 19 / 91$ & $7 / 17 / 90$ & $6 / 28 / 90$ & NA & $\mathrm{R} 4802$ & R7887 / R7903 & 1.550 & $<1.46 \mathrm{E}-03$ & $\bar{M}$ \\
\hline Lead & $6 / 28 / 90$ & NA & NA & NA & $\mathrm{NA}$ & $\mathrm{NA}$ & 1.560 & $<1.460 \mathrm{E}-03$ & Moles/L \\
\hline $\mathrm{Pb} 2+$ & NA & $\mathrm{NA}$ & NA & NA & $\mathrm{NA}$ & $\mathrm{NA}$ & 1.39 & $9.56 \mathrm{E}-05$ & Mole/L \\
\hline P043- & NA & NA & NA & NA & NA & NA & 1.39 & $1.68 \mathrm{E}-01$ & Mole/L \\
\hline $\mathrm{PO}_{4}$ & $9 / 18 / 86$ & NA & $6 / 23 / 86$ & R9893 & NA & NA & 1.370 & 0.11 & Moles/L \\
\hline $\mathrm{PO}_{4}$ & $12 / 30 / 81$ & $\mathrm{NA}$ & NA & T1049 & NA & NA & 1.329 & 0.089 & Moles \\
\hline $\mathrm{PO}_{4}$ & $12 / 30 / 81$ & NA & NA & $T 1048$ & NA & NA & 1.324 & 0.084 & Moles \\
\hline Phosphate & $12 / 19 / 91$ & $7 / 17 / 90$ & $6 / 28 / 90$ & NA & R4795 & R7915/R7934 & 1.540 & $6.510 \mathrm{E}-02$ & $M$ \\
\hline $\mathrm{PO}_{4}$ & $9 / 18 / 86$ & $\mathrm{NA}$ & $7 / 2 / 86$ & R9896 & NA & $\mathrm{NA}$ & 1.500 & 0.04 & Moles/L \\
\hline $\mathrm{PO}_{4}$ & $9 / 18 / 86$ & NA & $6 / 26 / 86$ & R9894 & NA & NA & 1.490 & 0.04 & Moles/L \\
\hline $\mathrm{PO}_{4}$ & $9 / 18 / 86$ & NA & $7 / 6 / 86$ & R9897 & NA & NA & 1.460 & 0.04 & Moles/L \\
\hline $\mathrm{PO}_{4}^{-3}$ & $4 / 3 / 84$ & NA & NA & $\mathrm{R} 9414$ & NA & $\mathrm{NA}$ & 1.370 & 0.036 & Moles/L \\
\hline $\mathrm{PO}_{4}^{-3}$ & $4 / 3 / 84$ & NA & NA & R9408 & NA & NA & 1.330 & 0.035 & Moles/L \\
\hline Phosphate & $12 / 19 / 91$ & $7 / 17 / 90$ & $6 / 28 / 90$ & $\mathrm{NA}$ & R4796 & R7916 / R7935 & 1.625 & $3.300 \mathrm{E}-02$ & $M$ \\
\hline $\mathrm{PO}_{4}$ & $9 / 18 / 86$ & NA & $6 / 26 / 86$ & T686 & NS & NS & 1.310 & 0.03 & Moles/L \\
\hline $\mathrm{PO}_{4}$ & $9 / 18 / 86$ & $\mathrm{NA}$ & $6 / 30 / 86$ & R9895 & NA & NA & 1.540 & 0.03 & Moles/L \\
\hline Phosphate & $12 / 19 / 91$ & $7 / 17 / 90$ & $6 / 28 / 90$ & NA & R4803 & R7927/R7944 & 1.575 & $2.960 \mathrm{E}-02$ & $M$ \\
\hline Phosphate & $12 / 19 / 91$ & $7 / 17 / 90$ & $6 / 28 / 90$ & NA & R4798 & R7920/R7937 & 1.560 & $2.430 \mathrm{E}-02$ & $M$ \\
\hline Phosphate & $6 / 28 / 90$ & NA & $\mathrm{NA}$ & NA & NA & NA & 1.560 & 0.02218 & Moles/L \\
\hline $\mathrm{PO}_{4}$ & $9 / 18 / 86$ & $\mathrm{NA}$ & $7 / 2 / 86$ & T882 & NS & NS & 1.320 & 0.02 & Moles/L \\
\hline Phosphate & $12 / 19 / 91$ & $7 / 17 / 90$ & $6 / 28 / 90$ & $\mathrm{NA}$ & R4800 & R7922/R7941 & 1.560 & $1.815 \mathrm{E}-02$ & $M$ \\
\hline Phosphate & $12 / 19 / 91$ & $7 / 17 / 90$ & $6 / 28 / 90$ & NA & R4797 & R7919/R7936 & 1.560 & $1.530 \mathrm{E}-02$ & $M$ \\
\hline $\mathrm{PO}_{4}^{-3}$ & $11 / 20 / 84$ & NS & NS & R2644 & NS & NS & NS & 0.015 & Moles/L \\
\hline Phosphate & $12 / 19 / 91$ & $7 / 17 / 90$ & $6 / 28 / 90$ & NA & R4792 & $R 7912 / R 7929$ & 1.530 & $1.395 \mathrm{E}-02$ & $M$ \\
\hline Phosphate & $12 / 19 / 91$ & $7 / 17 / 90$ & $6 / 28 / 90$ & NA & R4793 & R7913/R7930 & 1.535 & $1.380 \mathrm{E}-02$ & $M$ \\
\hline Phosphate & $12 / 19 / 91$ & $7 / 17 / 90$ & $6 / 28 / 90$ & $\mathrm{NA}$ & 84801 & R7923/R7942 & 1.560 & $1.375 \mathrm{E}-02$ & $M$ \\
\hline
\end{tabular}




\begin{tabular}{|c|c|c|c|c|c|c|c|}
\hline \multirow[b]{2}{*}{ Code } & \multirow[b]{2}{*}{ Source } & \multicolumn{3}{|c|}{ Analyte Information } & \multirow[b]{2}{*}{$\begin{array}{c}\text { Reference } \\
\text { Number }\end{array}$} & \multirow[b]{2}{*}{$\begin{array}{c}\text { Validated } \\
(\mathrm{Y} / \mathrm{N})\end{array}$} & \multirow[b]{2}{*}{ Notes } \\
\hline & & Analyte & Conc. (ug/L) & Conc. (mol/L) & & & \\
\hline 9 & RAW & $\mathrm{OH}$ & $2.76 E+07$ & $1.62 E+00$ & AW101-2 & $N$ & Waste no longer in tank \\
\hline$P$ & RAW & $\mathrm{OH}$ & $2.68 E+07$ & $1.58 \mathrm{E}+00$ & AW101-2 & $N$ & Waste no longer in tank \\
\hline 0 & RAW & $\mathrm{OH}$ & $2.47 E+07$ & $1.45 E+00$ & AW101-3 & $N$ & Waste no longer in tank \\
\hline$P$ & RAW & $\mathrm{OH}$ & $2.47 E+07$ & $1.45 E+00$ & AW101-3 & $N$ & Waste no longer in tank \\
\hline $\mathbf{P}$ & RAW & $\mathrm{OH}$ & $2.43 E+07$ & $1.43 E+00$ & AW101-4 & $N$ & Waste no longer in tank \\
\hline $\mathbf{P}$ & RAW & $\mathrm{OH}$ & $2.40 E+07$ & $1.41 E+00$ & AW101-3 & $\mathrm{N}$ & Waste no longer in tank \\
\hline$L$ & RAW & $\overline{\mathrm{OH}}$ & $2.34 E+07$ & $1.38 E+00$ & AW101-3 & $\mathbf{N}$ & Waste no longer in tank \\
\hline$P$ & RAW & $\mathrm{OH}$ & $2.02 E+07$ & $1.19 E+00$ & AW101-4 & $\mathbf{N}$ & Waste no longer in tank \\
\hline$L$ & RAW & $\mathrm{OH}$ & $8.32 \mathrm{E}+06$ & $4.89 \mathrm{E}-01$ & AW101-4 & $N$ & Waste no longer in tank \\
\hline$L$ & RAW & $\mathrm{OH}$ & $8.12 E+06$ & $4.78 \mathrm{E}-01$ & AW101-4 & $N$ & Waste no longer in tank \\
\hline$P$ & Raw & $\mathrm{Pb}$ & \#VALUE! & \#VALUE! & AW101-10 & $N$ & $<$ Values \\
\hline$P$ & Raw & $\mathrm{Pb}$ & \#VALUE! & \#VALUE! & AW101-10 & $N$ & $<$ Values \\
\hline $\mathbf{P}$ & Raw & $\mathrm{Pb}$ & \#VALUE! & \#VALUE! & AW101-10 & $N$ & $<$ Values \\
\hline $\mathbf{P}$ & Raw & $\mathrm{Pb}$ & \#VALUE! & \#VALUE! & AW101-10 & $\bar{N}$ & $<$ Values \\
\hline$P$ & Raw & $\mathrm{Pb}$ & \#VALUE! & \#VALUE! & AW101-10 & $N$ & $<$ Values \\
\hline$P$ & Raw & $\mathrm{Pb}$ & \#VALUE! & \#VALUE! & AW101-10 & $N$ & $<$ Values \\
\hline$P$ & Raw & $\mathrm{Pb}$ & \#VALUE! & \#VALUE! & AW101-10 & $N$ & $<$ Values \\
\hline $\mathrm{P}$ & Raw & 80 & \#VALUE! & \#VALUE! & AW101-10 & $\mathrm{N}$ & $<$ Values \\
\hline$P$ & Raw & $\mathrm{Pb}$ & \#VALUE! & \#VALUE! & AW101-10 & $N$ & $<$ Values \\
\hline$P$ & Raw & $\mathrm{Pb}$ & \#VALUE! & \#VALUEI & AW101-10 & $N$ & $<$ Values \\
\hline$P$ & Raw & $\mathrm{Pb}$ & \#VALUE! & \#VALUE! & $A W 101-10$ & $N$ & $\leq$ Values \\
\hline$P$ & Raw & $\mathrm{Pb}$ & \#VALUE! & \#VALUE! & AW101-10 & $N$ & $<$ Values \\
\hline$P$ & Raw & $\mathrm{Pb}$ & \#VALUE! & \#VALUE! & AW101-10 & $\bar{Y}$ & Detection limit \\
\hline$L$ & HTCE Inv. & $\mathrm{Pb}$ & \#VALUE! & \#VALUEI & $\mathrm{NA}$ & $N$ & HTCE \\
\hline$\underline{L}$ & HTCE Inv. & $\mathrm{PO}_{4}$ & $1.59 E+07$ & $1.68 \mathrm{E}-01$ & NA & $N$ & HTCE \\
\hline$F$ & Raw & $\mathrm{PO}_{4}$ & $1.04 E+07$ & $1.10 \mathrm{E}-01$ & AW101-4 & $Y$ & \\
\hline$P$ & Raw & $\mathrm{PO}_{4}$ & $8.45 E+06$ & $8.90 \mathrm{E}-02$ & AW101-1 & $N$ & Waste no longer in tank \\
\hline$P$ & Raw & $\mathrm{PO}_{4}$ & $7.98 \mathrm{E}+06$ & $8.40 \mathrm{E}-02$ & AW101-2 & $N$ & Waste no longer in tank \\
\hline $\mathrm{P}-3$ & Raw & PO4 & $6.18 E+06$ & $6.51 E-02$ & AW101-10 & $\bar{Y}$ & Average of Primary and Dublicate results \\
\hline $\mathbf{F}$ & Raw & $\mathrm{PO}_{4}$ & $3.80 E+06$ & $4.00 \mathrm{E}-02$ & AW101-4 & $\bar{Y}$ & \\
\hline $\mathbf{F}$ & Raw & $\mathrm{PO}_{4}$ & $3.80 E+06$ & $4.00 \mathrm{E}-02$ & AW101-4 & $\bar{Y}$ & \\
\hline$F$ & Raw & $\mathrm{PO}_{4}$ & $3.80 E+06$ & $4.00 \mathrm{E}-02$ & AW101-4 & $Y$ & \\
\hline$P$ & Raw & $\mathrm{PO}_{4}$ & $3.42 \mathrm{E}+06$ & $3.60 E-02$ & AW101-2 & N & Waste no longer in tank \\
\hline$P$ & Raw & $\mathrm{PO}_{4}$ & $3.32 E+06$ & $3.50 E-02$ & AW101-2 & $N$ & Waste no longer in tank \\
\hline P-3 & Raw & PO4 & $3.14 \mathrm{E}+06$ & $3.30 \mathrm{E}-02$ & AW101-10 & $\bar{Y}$ & Average of Primary and Dublicate results \\
\hline $\mathbf{P}$ & Raw & $\mathrm{PO}_{4}$ & $2.85 E+06$ & $3.00 E-02$ & AW101-4 & $\mathrm{N}$ & Evaporator feed sample \\
\hline$F$ & Raw & $\mathrm{PO}_{4}$ & $2.85 E+06$ & $3.00 \mathrm{E}-02$ & AW101-4 & $Y$ & \\
\hline$P-3$ & Raw & PO4 & $2.81 E+06$ & $2.96 \mathrm{E}-02$ & AW101-10 & $\bar{Y}$ & Average of Primary and Dublicate results \\
\hline$P-3$ & Raw & P04 & $2.31 E+06$ & $2.43 \mathrm{E}-02$ & AW101-10 & $\mathbf{Y}$ & Average of Primary and Dublicate results \\
\hline$P$ & Raw & $\mathrm{PO}_{4}$ & $2.11 E+06$ & $2.22 \mathrm{E}-02$ & AW101-10 & $Y$ & (best value) /Avg of 12 samples \\
\hline $\mathbf{P}$ & Raw & $\mathrm{PO}_{4}$ & $1.90 E+06$ & 2.00E-02 & AW101-4 & $N$ & Evaporator feed sample \\
\hline P-3 & Raw & PO4 & $1.72 \mathrm{E}+06$ & $1.82 \mathrm{E}-02$ & AW101-10 & $Y$ & Average of Primary and Dublicate results \\
\hline$P-3$ & Raw & 804 & $1.45 E+06$ & $1.53 \mathrm{E}-02$ & AW101-10 & $\bar{Y}$ & Average of Primary and Dublicate results \\
\hline$P$ & Raw & $\mathrm{PO}_{4}$ & $1.42 E+06$ & $1.50 E-02$ & AW101-2 & $N$ & Waste no longer in tank \\
\hline$\underline{P-3}$ & Raw & PO4 & $1.33 \mathrm{E}+06$ & $1.40 E-02$ & AW101-10 & $Y$ & Average of Primary and Dublicate results \\
\hline $\mathrm{P}-3$ & Raw & PO4 & $1.31 E+06$ & $1.38 \mathrm{E}-02$ & AW101-10 & $Y$ & Average of Primary and Dublicate results \\
\hline P-3 & Raw & P04 & $1.31 E+06$ & $1.38 \mathrm{E}-02$ & AW101-10 & $\bar{Y}$ & Average of Primary and Dublicate results \\
\hline
\end{tabular}


TWRS Privatization Support Pro Chemical Liquids

\begin{tabular}{|c|c|c|c|c|c|c|c|c|c|}
\hline & & & & Tank Was & Characteriza & ion Sample Inform & ion & & \\
\hline Reported Chemical & $\begin{array}{c}\text { Memo } \\
\text { Date }\end{array}$ & $\begin{array}{c}\text { Start Analysis } \\
\text { Date }\end{array}$ & $\begin{array}{c}\text { Sample } \\
\text { Date }\end{array}$ & $\begin{array}{l}\text { Sample } \\
\text { Number }\end{array}$ & $\begin{array}{c}\text { Tank Farm } \\
\text { Number }\end{array}$ & $\begin{array}{c}\text { Lab Sample } \\
\text { Number }\end{array}$ & $\begin{array}{l}\text { Density } \\
\text { (g/mL) }\end{array}$ & Reported Value & Reported $\mathrm{V}$ \\
\hline Phosphate & $12 / 19 / 91$ & $7 / 17 / 90$ & $6 / 28 / 90$ & NA & R4799 & R7921 / R7940 & 1.555 & $1.335 \mathrm{E}-02$ & $M$ \\
\hline Phosphate & $12 / 19 / 91$ & $7 / 17 / 90$ & $6 / 28 / 90$ & NA & $\mathrm{R} 4794$ & R7914/R7933 & 1.570 & 1.310E-02 & $M$ \\
\hline Phosphate & $12 / 19 / 91$ & $7 / 17 / 90$ & $6 / 28 / 90$ & NA & R4802 & R7926/R7943 & 1.550 & $1.275 \mathrm{E}-02$ & $M$ \\
\hline $\mathrm{PO}_{4}$ & $9 / 18 / 86$ & NA & $6 / 23 / 86$ & T621 & NS & NS & 1.440 & 0.01 & Moles $/ \mathrm{L}$ \\
\hline $\mathrm{PO}_{4}$ & $9 / 18 / 86$ & NA & $6 / 30 / 86$ & T812 & NS & NS & 1.130 & 0.01 & Moles $/ L$ \\
\hline $\mathrm{PO}_{4}$ & $4 / 29 / 86$ & $3 / 30 / 86$ & NA & $T 7697$ & NS & NS & 0.993 & 0.007 & Moles/L \\
\hline $\mathrm{PO}_{4}^{-3}$ & $11 / 20 / 84$ & NA & NA. & $\mathrm{R} 2644$ & NA & NA & NA & 0.004 & Moles/L \\
\hline $\mathrm{PO}_{4}$ & $2 / 20 / 86$ & $11 / 14 / 85$ & NA & T2601 & NS & NS & 1.010 & 0.003 & Moles/L \\
\hline $\mathrm{PO}_{4}^{-3}$ & $11 / 20 / 84$ & NA & NA & R2645 & NA & NA & NA & 0.0015 & Moles $/ L$ \\
\hline PO4 & $4 / 29 / 85$ & $4 / 21 / 85$ & NA & T5350 & NA & NA & 1.028 & 0.0011 & Moles/L \\
\hline $\mathrm{PO4}$ & $11 / 9 / 84$ & NS & NS & R-3185 & NS & NS & 1.024 & 0.001 & moles $n$ \\
\hline $\mathrm{PO}_{4}$ & $11 / 9 / 84$ & NA & NA & $R 3185$ & NS & NS & 1.024 & 0.001 & Moles \\
\hline $\mathrm{PO}_{4}$ & $2 / 20 / 86$ & $11 / 14 / 85$ & NA & T2641 & NS & NS & 1.010 & 0.001 & Moles /L \\
\hline PO4 & $8 / 18 / 85$ & $8 / 18 / 85$ & NA & T9368 & $\mathrm{NA}$ & NA & 1.040 & 0.00029 & Moles $/ L$ \\
\hline $\mathrm{PO}_{4}$ & $4 / 29 / 86$ & $3 / 30 / 86$ & NA & $T 7698$ & NS & NS & 0.993 & 0.0002 & Moles/L \\
\hline. $\mathrm{SO}_{4}$ & $9 / 18 / 86$ & $\mathrm{NA}$ & $6 / 23 / 86$ & T621 & NS & NS & 1.440 & $<1.00 \mathrm{E}-02$ & Moles $/ \mathrm{L}$ \\
\hline $\mathrm{SO}_{4}$ & $9 / 18 / 86$ & NA & $6 / 26 / 86$ & T686 & NS & NS & 1.310 & $<1.0 \mathrm{E}-02$ & Moles/L \\
\hline $\mathrm{SO}_{4}$ & $6 / 30 / 86$ & NS & NS & NS & NS & NS & 1.540 & $<0.01 E-02$ & Moles/L \\
\hline $\mathrm{SO}_{4}$ & $6 / 26 / 86$ & NS & NS & NS & NS & NS & 1.490 & $<1.40 E-01$ & Moles/L \\
\hline $\mathrm{SO}_{4}$ & $4 / 29 / 86$ & $3 / 30 / 86$ & NA & $T 7697$ & NS & NS & 0.993 & $<5.00 \mathrm{E}-03$ & Moles/L \\
\hline $\mathrm{SO}_{4}$ & $4 / 29 / 86$ & $3 / 30 / 86$ & NA & $T 7698$ & NS & NS & 0.993 & $<5.00 \mathrm{E}-03$ & Moles/L \\
\hline $\mathrm{SO}_{4}$ & $9 / 18 / 86$ & NA & $7 / 6 / 86$ & R9897 & NS & NS & 1.460 & $<0.01$ & Moles/L \\
\hline S042- & NA & NA & NA & NA & $\mathrm{NA}$ & NA & 1.39 & $2.17 E-01$ & Mole/L \\
\hline $\mathrm{SO}_{4}{ }^{-2}$ & $4 / 3 / 84$ & NA & NA & R9408 & NA & NA & 1.330 & 0.064 & Moles/L \\
\hline $\mathrm{SO}_{4}{ }^{-2}$ & $4 / 3 / 84$ & NA & NA & R9414 & NA & NA & 1.370 & 0.062 & Moles/L \\
\hline $\mathrm{SO}_{4}$ & $9 / 18 / 86$ & $\mathrm{NA}$ & $6 / 30 / 86$ & T812 & NS & NS & 1.130 & 0.05 & Moles/L \\
\hline Sulfate & $12 / 19 / 91$ & $7 / 17 / 90$ & $6 / 28 / 90$ & NA & NA & R8801 & 1.540 & $2.350 \mathrm{E}-02$ & $M$ \\
\hline Sulfate & $12 / 19 / 91$ & $7 / 17 / 90$ & $6 / 28 / 90$ & $\mathrm{NA}$ & NA & $R 8802$ & 1.540 & $2.170 \mathrm{E}-02$ & $M$ \\
\hline Sulfate & $12 / 19 / 91$ & $7 / 17 / 90$ & $6 / 28 / 90$ & NA & R4796 & R7916/R7935 & 9.625 & $2.145 \mathrm{E}-02$ & $M$ \\
\hline Sulfate & $12 / 19 / 91$ & $7 / 17 / 90$ & $6 / 28 / 90$ & NA & R4795 & R7915 / R7934 & 1.540 & $1.570 \mathrm{E}-02$ & $M$ \\
\hline Sulfate & $12 / 19 / 91$ & $7 / 17 / 90$ & $6 / 28 / 90$ & NA & R4798 & R7920/R7937 & 1.560 & $1.535 \mathrm{E}-02$ & $M$ \\
\hline Sulfate & $12 / 19 / 91$ & $7 / 17 / 90$ & $6 / 28 / 90$ & NA & R4800 & R7922/R7941 & 1.560 & $1.109 \mathrm{E}-02$ & $M$ \\
\hline Sulfate & $12 / 19 / 91$ & $7 / 17 / 90$ & $6 / 28 / 90$ & $\mathrm{NA}$ & R4797 & R7919/R7936 & 1.560 & $1.048 \mathrm{E}-02$ & $M$ \\
\hline $\mathrm{SO}_{4}$ & $9 / 18 / 86$ & NA & $7 / 2 / 86$ & T882 & NS & NS & 1.320 & 0.01 & Moles/L \\
\hline $\mathrm{SO}_{4}$ & $9 / 18 / 86$ & $\mathrm{NA}$ & $7 / 2 / 86$ & R9896 & NA & $\mathrm{NA}$ & 1.500 & 0.01 & Moles/L \\
\hline $\mathrm{SO}_{4}$ & $9 / 18 / 86$ & NA & $6 / 23 / 86$ & R9893 & NA & NA & 1.370 & 0.01 & Moles/L \\
\hline Sulfate & $12 / 19 / 91$ & $7 / 17 / 90$ & $6 / 28 / 90$ & NA & $\mathrm{R} 4793$ & R7913/R7930 & 1.535 & 8.290 E-03 & $M$ \\
\hline Sulfate & $12 / 19 / 91$ & $7 / 17 / 90$ & $6 / 28 / 90$ & $\mathrm{NA}$ & R4792 & R7912 / R7929 & 1.530 & $8.195 \mathrm{E}-03$ & $M$ \\
\hline Sulfate & $12 / 19 / 91$ & $7 / 17 / 90$ & $6 / 28 / 90$ & NA & R4794 & R7914 / R7933 & 1.570 & $7.910 \mathrm{E}-03$ & $M$ \\
\hline Sulfate & $12 / 19 / 91$ & $7 / 17 / 90$ & $6 / 28 / 90$ & $\mathrm{NA}$ & R4799 & $R 7921 / R 7940$ & 1.555 & $7.790 \mathrm{E}-03$ & $M$ \\
\hline Sulfate & $12 / 19 / 91$ & $7 / 17 / 90$ & $6 / 28 / 90$ & NA & $\mathrm{R} 4801$ & R7923/R7942 & 1.560 & $7.735 E-03$ & $M$ \\
\hline Sulfate & $12 / 19 / 91$ & $7 / 17 / 90$ & $6 / 28 / 90$ & NA & R4802 & R7926/R7943 & 1.550 & $7.440 \mathrm{E}-03$ & $M$ \\
\hline Sulfate & $12 / 19 / 91$ & $7 / 17 / 90$ & $6 / 28 / 90$ & NA & R4803 & R7927/R7944 & 1.575 & 7.395E-03 & $M$ \\
\hline 504 & $4 / 29 / 85$ & $4 / 21 / 85$ & NA & $T 5350$ & NA & NA & 1.028 & 0.00324 & Moles/L \\
\hline 504 & $8 / 18 / 85$ & $8 / 18 / 85$ & NA & T9368 & NA & NA & 1.040 & 0.0014 & Moles/L \\
\hline $\mathrm{SO}_{4}$ & $2 / 20 / 86$ & $11 / 14 / 85$ & NA & T2601 & NS & NS & 1.010 & 0.001 & Moles/L \\
\hline
\end{tabular}




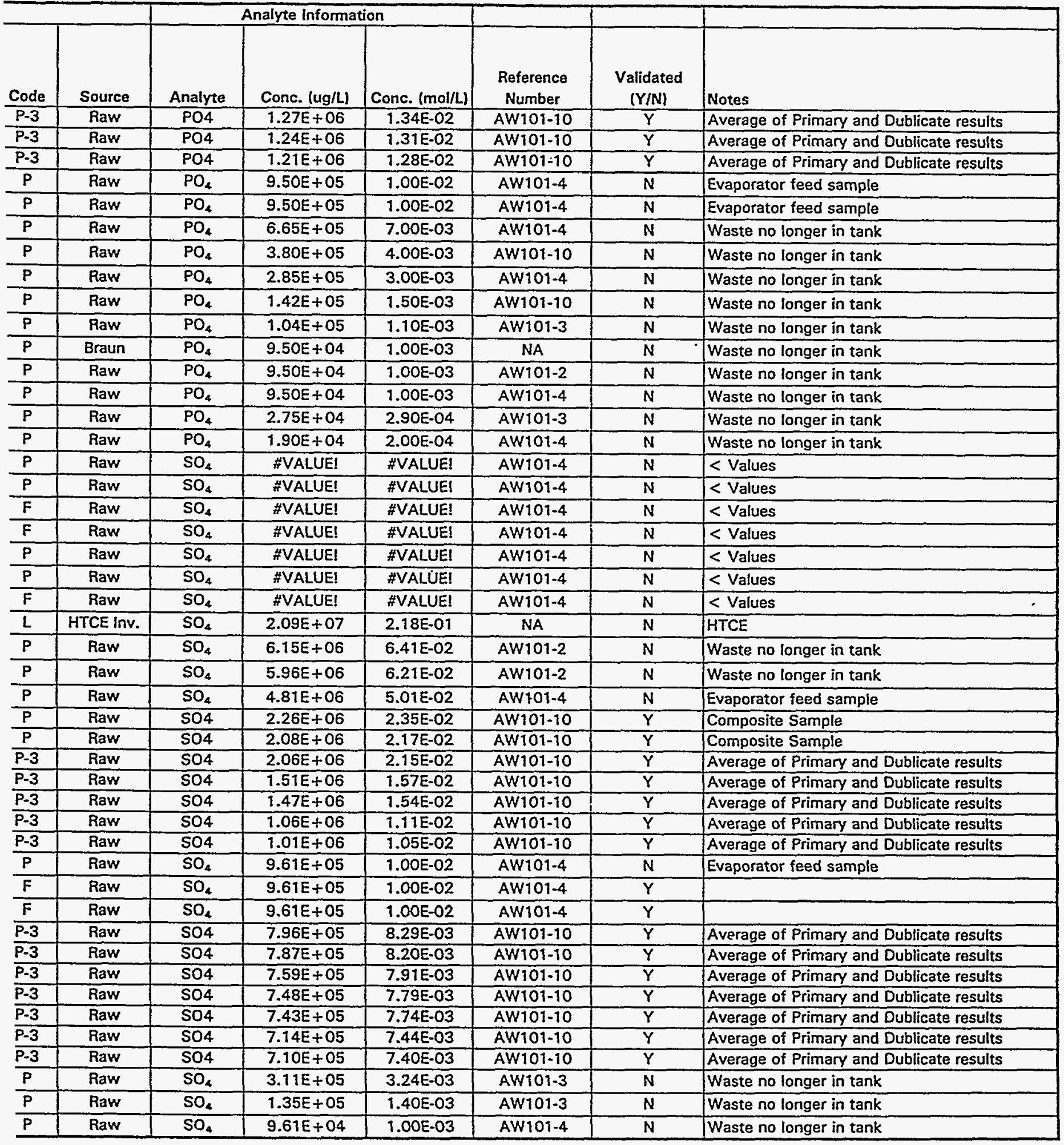




\begin{tabular}{|c|c|c|c|c|c|c|c|c|}
\hline \multirow{3}{*}{ nit } & \multirow[b]{2}{*}{ Code } & \multirow[b]{2}{*}{ Source } & \multicolumn{3}{|c|}{ Analyte Information } & \multirow[b]{2}{*}{$\begin{array}{c}\text { Reference } \\
\text { Number }\end{array}$} & \multirow[b]{2}{*}{$\begin{array}{c}\text { Validated } \\
\text { (Y/N) }\end{array}$} & \multirow[b]{2}{*}{ Notes } \\
\hline & & & Analyte & Conc. (ug/L) & Conc. (mol/L) & & & \\
\hline & $\mathbf{P}$ & Raw & $\mathrm{SO}_{4}$ & $9.61 E+04$ & $1.00 \mathrm{E}-03$ & AW101-4 & $N$ & Waste no longer in tank \\
\hline & $P$ & Raw & TOC & $3.58 \mathrm{E}+07$ & $2.98 \mathrm{E}+00$ & AW101-10 & $N$ & Waste no longer in tank \\
\hline & $P$ & Raw & TOC & $2.80 E+07$ & $2.34 E+00$ & AW101-2 & $N$ & Waste no longer in tank \\
\hline & $P$ & Raw & TOC & $2.80 \mathrm{E}+07$ & $2.34 \mathrm{E}+00$ & AW101-2 & $N$ & Waste no longer in tank \\
\hline & $L$ & HTCE Inv. & TOC & $1.33 E+07$ & $1.11 E+00$ & NA & $N$ & HTCE \\
\hline & $P$ & Raw & TOC & $9.09 E+06$ & $7.58 E-01$ & AW101-2 & $\mathbf{N}$ & Waste no longer in tank \\
\hline & $P$ & Raw & TOC & $6.12 \mathrm{E}+06$ & $5.10 \mathrm{E}-01$ & AW101-4 & $N$ & Evaporator feed sample \\
\hline & $F$ & Raw & TOC & $5.48 E+06$ & 4.57E-01 & AW101-4 & $\bar{Y}$ & \\
\hline & $F$ & Raw & TOC & $5.20 E+06$ & $4.33 \mathrm{E}-01$ & AW101-4 & $\bar{Y}$ & \\
\hline & $F$ & Raw & TOC & $4.95 E+06$ & 4.13E-01 & AW101-4 & $\bar{Y}$ & \\
\hline & $F$ & Raw & TOC & $3.53 E+06$ & & AW101-4 & $\bar{Y}$ & \\
\hline & $F$ & Raw & TOC & $3.48 E+06$ & $2.90 \mathrm{E}-01$ & AW101-4 & $\bar{Y}$ & \\
\hline & $P$ & Raw & TOC & $3.45 E+06$ & 2.88E-01 & AW101-4 & $N$ & Evaporator feed sample \\
\hline & $P$ & Raw & TOC & $2.83 E+06$ & $2.36 \mathrm{E}-01$ & AW 101-10 & $\mathbf{N}$ & Waste no longer in tank \\
\hline & $P$ & Raw & TOC & $2.53 E+06$ & 2.11E-01 & AW101-10 & $\bar{Y}$ & Composite Sample \\
\hline & P.3 & Raw & TOC & $2.46 E+06$ & $2.05 E-01$ & AW101-10 & $\bar{Y}$ & Avg of 2 values \\
\hline & $P$ & Raw & TOC & $2.39 \mathrm{E}+06$ & $1.99 \mathrm{E}-01$ & AW101-10 & $\bar{Y}$ & Composite Sample \\
\hline & $P$ & Raw & TOC & $1.42 E+06$ & $1.18 \mathrm{E}-01$ & AW101-4 & $N$ & Evaporator feed sample \\
\hline & $P$ & Raw & TOC & $1.11 E+06$ & $9.25 \mathrm{E}-02$ & AW101-10 & $N$ & Waste no longer in tank \\
\hline & $\mathbf{P}$ & Braun & TOC & $7.57 E+05$ & $6.31 E-02$ & NA & $N$ & Waste no longer in tank \\
\hline & $P$ & Raw & TOC & $6.57 \mathrm{E}+05$ & $5.48 \mathrm{E}-02$ & AW101-4 & $N$ & Waste no longer in tank \\
\hline & $\mathbf{P}$ & Raw & TOC & $6.19 E+05$ & $5.16 \mathrm{E}-02$ & AW101-4 & $N$ & Waste no longer in tank \\
\hline & $P$ & Raw & TOC & $6.00 E+05$ & $5.00 \mathrm{E}-02$ & AW101-3 & $N$ & Waste no longer in tank \\
\hline & $\bar{P}$ & Raw & TOC & $4.34 \mathrm{E}+05$ & $3.62 \mathrm{E}-02$ & AW101-4 & $N$ & Waste no longer in tank \\
\hline & $P$ & Raw & TOC & $4.24 E+05$ & $3.53 E-02$ & AW101-4 & $\bar{N}$ & Waste no longer in tank \\
\hline & $P$ & Raw & TOC & $3.68 E+05$ & $3.07 E-02$ & AW101-3 & $\mathbf{N}$ & Waste no longer in tank \\
\hline & $P$ & Raw & TOC & $3.10 E+05$ & $2.58 E-02$ & AW101-4 & $N$ & Waste no longer in tank \\
\hline
\end{tabular}


TWRS Privatization Support Proj Chemical Liquids

\begin{tabular}{|c|c|c|c|c|c|c|c|c|c|}
\hline \multirow[b]{2}{*}{ Reported Chemical } & \multicolumn{9}{|c|}{ Tank Waste Characterization Sample Information } \\
\hline & $\begin{array}{c}\text { Memo } \\
\text { Date } \\
\end{array}$ & $\begin{array}{c}\text { Start Analysis } \\
\text { Date }\end{array}$ & $\begin{array}{c}\text { Sample } \\
\text { Date }\end{array}$ & $\begin{array}{l}\text { Sample } \\
\text { Number }\end{array}$ & $\begin{array}{c}\text { Tank Farm } \\
\text { Number }\end{array}$ & $\begin{array}{c}\text { Lab Sample } \\
\text { Number }\end{array}$ & $\begin{array}{c}\text { Density } \\
(\mathrm{g} / \mathrm{mL})\end{array}$ & Reported Value & Reported U \\
\hline $\mathrm{SO}_{4}$ & $2 / 20 / 86$ & $11 / 14 / 85$ & NA & T2641 & NS & NS & 1.010 & 0.001 & Moles/L. \\
\hline TOC & $11 / 20 / 84$ & NA & NA & R2644 & NA & NA & NA & 35.8 & g/L \\
\hline TOC & $4 / 3 / 84$ & NA & NA & R9414 & NA & NA & 1.330 & 28.04 & $\mathrm{~g} / \mathrm{L}$ \\
\hline TOC & $4 / 3 / 84$ & NA & NA & R9408 & NA & NA & 1.330 & 28.04 & $\mathrm{~g} / \mathrm{L}$ \\
\hline TOC wt\% C (wet) & NA & NA & NA & NA & NA & $\mathrm{NA}$ & 1.39 & 9.56E-01 & $w t \%$ \\
\hline TOC & $11 / 9 / 84$ & NA & NA & R3185 & NS & NS & 1.024 & 0.757 & Moles/L \\
\hline TOC & $9 / 18 / 86$ & NA & $6 / 23 / 86$ & T621 & NS & NS & 1.440 & 6.12 & $g / L$ \\
\hline TOC & $9 / 18 / 86$ & NA & $7 / 2 / 86$ & R9896 & NA & NA & 1.500 & 5.48 & $g / L$ \\
\hline TOC & $9 / 18 / 86$ & NA & $6 / 30 / 86$ & R9895 & NA & NA & 1.540 & 5.2 & $g / L$ \\
\hline TOC & $9 / 18 / 86$ & NA & $6 / 26 / 86$ & R9894 & NA & NA & 1.490 & 4.95 & $g / L$ \\
\hline TOC & $9 / 18 / 86$ & NA & $7 / 6 / 86$ & R9897 & NA & NA & 1.460 & 3.53 & $g / L$ \\
\hline TOC & $9 / 18 / 86$ & NA & $6 / 23 / 86$ & R9893 & NA & NA & 1.370 & 3.48 & $g / L$ \\
\hline TOC & $9 / 18 / 86$ & NA & $6 / 26 / 86$ & T686 & NS & NS & 1.310 & 3.45 & $g / L$ \\
\hline TOC & $11 / 20 / 84$ & NA & NA & 82644 & NA & NA & NA & 2.83 & $g / L$ \\
\hline TOC & $12 / 19 / 91$ & $7 / 17 / 90$ & $6 / 28 / 90$ & NA & NA & $\mathrm{R} 8802$ & 1.540 & 2.53 & $g / L$ \\
\hline TOC & $6 / 28 / 90$ & $\mathrm{NA}$ & NA & NA & NA & NA & 1.560 & 2.46 & $g / h$ \\
\hline TOC & $12 / 19 / 91$ & $7 / 17 / 90$ & $6 / 28 / 90$ & NA & NA & $R 8801$ & 1.540 & 2.39 & $g / L$ \\
\hline TOC & $9 / 18 / 86$ & NA & $6 / 30 / 86$ & $T 812$ & NS & NS & 1.130 & 1.42 & $g / L$ \\
\hline TOC & $11 / 20 / 84$ & NA & $\mathrm{NA}$ & R2645 & NA & NA & NA & 1.11 & $g / L$ \\
\hline TOC & $11 / 9 / 84$ & NS & NS & R-3185 & NS & NS & 1.024 & 0.757 & $\mathrm{gm} / \mathrm{l}$ \\
\hline TOC & $2 / 20 / 86$ & $11 / 14 / 85$ & NA & T2641 & NS & NS & 1.010 & 0.657 & $g / L$ \\
\hline TOC & $2 / 20 / 86$ & $11 / 14 / 85$ & NA & T2601 & NS & NS & 1.010 & 0.619 & $g / L$ \\
\hline TOC & $4 / 29 / 85$ & $4 / 21 / 85$ & NA & T5350 & NA & NA & 1.028 & 0.6 & GM/ C \\
\hline TOC & $4 / 29 / 86$ & $3 / 30 / 86$ & NA & T7697 & NS & NS & 0.993 & 0.434 & $g / L$ \\
\hline TOC & $4 / 29 / 86$ & $3 / 30 / 86$ & NA & T7698 & NS & NS & 0.993 & 0.424 & $g / L$ \\
\hline TOC & $8 / 18 / 85$ & $8 / 18 / 85$ & $\mathrm{NA}$ & T9368 & NA & NA & 1.040 & 0.368 & GM/I-C \\
\hline TOC & $9 / 18 / 86$ & NA & $7 / 2 / 86$ & T882 & NS & NS & 1.320 & 0.31 & $g / L$ \\
\hline
\end{tabular}


TWRS Privatization Support Pre Chemical Liquids

\begin{tabular}{|c|c|c|c|c|c|c|c|c|c|}
\hline \multicolumn{10}{|c|}{ Tank Waste Characterization Sample Information } \\
\hline Reported Chemical & $\begin{array}{c}\text { Memo } \\
\text { Date }\end{array}$ & $\begin{array}{c}\text { Start Analysis } \\
\text { Date }\end{array}$ & $\begin{array}{c}\text { Start } \\
\text { Sample } \\
\text { Date } \\
\end{array}$ & Sample Number & $\begin{array}{c}\text { Tank Farm } \\
\text { Number }\end{array}$ & $\begin{array}{c}\text { Lab Sample } \\
\text { Number }\end{array}$ & $\begin{array}{l}\text { Density } \\
\text { (g/mL) }\end{array}$ & Reported Value & Rep \\
\hline $\mathrm{Al}$ & $12 / 3 / 79$ & NS & NS & 1466 & NS & NS & 1.414 & $1.44 E+00$ & \\
\hline Al & $12 / 3 / 79$ & NS & NS & 1465 & NS & NS & 1.41 & $1.43 E+00$ & \\
\hline Al & $12 / 3 / 79$ & NS & NS & $1494 B$ & NS & NS & 1.42 & $1.42 E+00$ & \\
\hline Al & $12 / 3 / 79$ & NS & NS & 1495B & NS & NS & 1.448 & $1.42 E+00$ & \\
\hline Al & $12 / 3 / 79$ & NS & NS & $1495 \mathrm{~A}$ & NS & NS & 1.419 & $1.38 E+00$ & \\
\hline Al & $12 / 3 / 79$ & NS & NS & $1494 \mathrm{~A}$ & NS & NS & 1.0414 & $1.36 E+00$ & \\
\hline Al & $12 / 3 / 79$ & NS & NS & 1463 & NS & NS & 1.384 & $1.09 E+00$ & \\
\hline Al & $12 / 3 / 79$ & NS & NS & 1464 & NS & NS & 1.409 & $1.03 E+00$ & \\
\hline Al & $11 / 18 / 85$ & NS & NS & RAT-AZ101-1 & NS & NS & 1.17 & 0.481 & $n$ \\
\hline Al & $1 / 10 / 86$ & NA & $11 / 1 / 85$ & NA & $\mathrm{NA}$ & NA & 1.17 & 4.81E-01 & \\
\hline Al & $4 / 8 / 86$ & NA & $11 / 1 / 85$ & NA & NA & NA & NA & 4.80E-01 & \\
\hline Al & $8 / 7 / 86$ & NS & NS & R9964/R0247 & NS & NS & 1.2 & 0.44 & $n$ \\
\hline Al & $10 / 1 / 87$ & NS & NS & RAT-AZ101-6A & NS & NS & NS & 0.44 & $\bar{n}$ \\
\hline Al & $3 / 11 / 88$ & NA & NA & $T 3493$ & NA & NA & NA & 4.40E-01 & \\
\hline Al & $3 / 11 / 88$ & NA & NA & T3493 & NA & NA & NA & 4.40E-01 & \\
\hline Al & $2 / 8 / 89$ & NA & $10 / 1 / 87$ & T3493 & NA & NA & NA & $4.40 E-01$ & \\
\hline Al & $9 / 1 / 89$ & NS & NS & RAT-AZ101-4A & NS & NS & 1.2 & 0.332 & $m$ \\
\hline $\mathrm{Al3}+$ & NA & NA & NA & NA & NA & NA & 1.12 & 3.68E-01 & $\underline{\Lambda}$ \\
\hline Aluminum & $7 / 26 / 95$ & NA & $3 / 3 / 95$ & BOD9P5 & NA & NA & NA & 9475 & 1 \\
\hline Aluminum & $7 / 26 / 95$ & NA & $3 / 3 / 95$ & BOD9N9 & NA & NA & NA & 9275 & 2 \\
\hline Aluminum & $7 / 26 / 95$ & NA & $3 / 3 / 95$ & BOD9P2 & NA & NA & NA & 9220 & $\underline{\mathbf{t}}$ \\
\hline $\mathrm{NaAlO} 2$ & $8 / 27 / 81$ & NS & NS & NS & NS & NS & 1.143 & $3.14 \mathrm{E}-01$ & \\
\hline $\mathrm{Al}$ & $9 / 13 / 85$ & NS & NS & RAT-AZ101-3A & NS & NS & 1.17 & 0.302 & $\bar{\pi}$ \\
\hline $\mathrm{Al}$ & $9 / 13 / 85$ & NA & NA & NA & NA & NA & 1.17 & $3.02 E-01$ & \\
\hline $\mathrm{Al}$ & May 89 & NS & NS & NS & NS & NS & NS & $2.69 E+00$ & \\
\hline $\mathrm{Al}$ & $9 / 7 / 90$ & NS & NS & RAT-AZ101-5A & NS & NS & 1.22 & 81.6 & $\mathrm{un}$ \\
\hline Al & $6 / 1 / 84$ & NS & NS & RAT-AZ101-2B & NS & NS & NS & 0.099 & $\bar{m}$ \\
\hline $\mathrm{Al}$ & $6 / 1 / 84$ & NS & NS & RAT-AZ101-2A & NS & NS & NS & 0.097 & $\bar{m}$ \\
\hline Al & $9 / 10 / 84$ & NA & $6 / 1 / 84$ & NA & NA & NA & NA & $9.70 E-02$ & \\
\hline $\mathrm{NaAlO2}$ & $4 / 26 / 78$ & NS & NS & NS & NS & NS & 1.133 & $7.70 \mathrm{E}-02$ & \\
\hline Al & $12 / 28 / 83$ & NS & NS & NS & NS & NS & NS & $7.70 E-02$ & \\
\hline Al & $1 / 1 / 81$ & NS & NS & NS & NS & NS & NS & NS & \\
\hline Al & $1 / 10 / 86$ & NA & $11 / 1 / 85$ & NA & NA & NA & NA & 4.49E-01 & 1 \\
\hline As & $9 / 1 / 93$ & NA & $4 / 1 / 89$ & Core 2 & $\overline{\mathrm{NA}}$ & NA & 1.24 & 0.077 & $\bar{u}$ \\
\hline
\end{tabular}




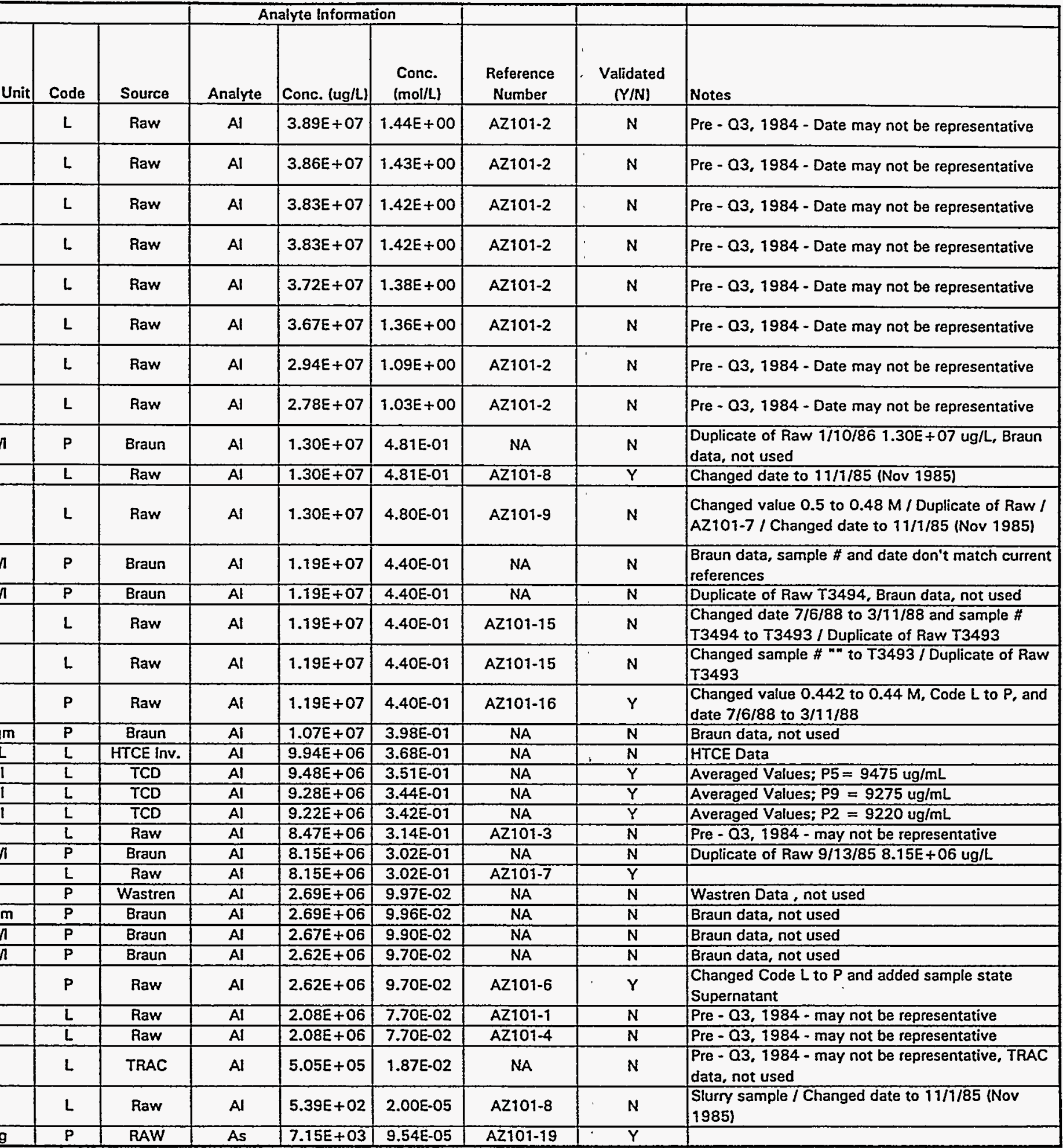




\begin{tabular}{|c|c|c|c|c|c|c|c|c|c|}
\hline \multirow[b]{2}{*}{ Reported Chemical } & \multicolumn{9}{|c|}{ Tank Waste Characterization Sample Information } \\
\hline & $\begin{array}{c}\text { Memo } \\
\text { Date }\end{array}$ & $\begin{array}{c}\text { Start Analysis } \\
\text { Date } \\
\end{array}$ & $\begin{array}{c}\text { Start, } \\
\text { Sample } \\
\text { Date } \\
\end{array}$ & Sample Number & $\begin{array}{c}\text { Tank Farm } \\
\text { Number }\end{array}$ & $\begin{array}{c}\text { Lab Sample } \\
\text { Number }\end{array}$ & $\begin{array}{l}\text { Density } \\
\text { (g/mL) }\end{array}$ & Reported Value & Repct \\
\hline As & $9 / 1 / 90$ & NS & NS & RAT-AZ101-5A & NS & NS & 1.22 & 0.077 & $\mathrm{um}$ \\
\hline As & NS & NS & NS & NS & NS & NS & NS & 0.00704 & \\
\hline Ba & $9 / 1 / 93$ & NA & $4 / 1 / 89$ & Core 2 & NA & NA & 1.24 & 0.015 & un \\
\hline $\mathrm{Ba}$. & $9 / 1 / 90$ & NS & NS & RAT-AZ101-5A & NS & NS & 1.22 & 0.015 & un \\
\hline $\mathrm{Ba}$ & NS & NS & NS & NS & NS & NS & NS & 0.00215 & \\
\hline $\mathbf{B a}$ & $9 / 1 / 89$ & NS & NS & RAT-AZ101-4A & NS & NS & 1.2 & 0.00001 & $\mathrm{~mm}$ \\
\hline $\mathrm{Ba}$ & $9 / 29 / 89$ & NA & $4 / 1 / 89$ & Core 1 & NA & NA & 1.2 & 0.00001 & $\overrightarrow{m r}$ \\
\hline $\mathrm{Be}$ & $9 / 1 / 93$ & NA & $4 / 1 / 89$ & Core 2 & NA & $\mathrm{NA}$ & 1.24 & 0.01 & UiI \\
\hline $\mathrm{Be}$ & $9 / 1 / 90$ & NS & NS & RAT-AZ101-5A & NS & NS & 1.22 & 0.01 & $\mathbf{u m}$ \\
\hline $\mathrm{Be}$ & & NS & NS & NS & NS & NS & NS & 0.00011 & \\
\hline $\mathrm{Ca}$ & $4 / 8 / 86$ & NA & $11 / 1 / 85$ & NA & NA & NA & NA & 0.016 & \\
\hline $\mathrm{Ca}$ & $11 / 18 / 85$ & NS & NS & RAT-AZ101-1 & NS & NS & 1.17 & 0.00026 & mi \\
\hline $\mathrm{Ca}$ & $4 / 8 / 86$ & NA & $11 / 1 / 85$ & NA & NA & NA & NA & 0.00026 & \\
\hline $\mathrm{Ca}$ & $1 / 10 / 86$ & $\mathrm{NA}$ & $11 / 1 / 85$ & NA & NA & NA & 1.17 & 0.00026 & \\
\hline $\mathrm{Ca}$ & $9 / 1 / 93$ & NA & $4 / 1 / 89$ & Core 2 & NA & NA & 1.24 & 0.196 & $\overrightarrow{u r}$ \\
\hline $\mathrm{Ca}$ & $9 / 1 / 90$ & NS & NS & RAT-AZ101-5A & NS & NS & 1.22 & 0.196 & umı \\
\hline $\mathrm{Ca}$ & NS & NS & NS & NS & NS & NS & NS & 0.00958 & \\
\hline $\mathrm{Ca}$ & $9 / 13 / 85$ & NS & NS & RAT-AZ101-3A & NS & NS & 1.17 & 0.000152 & $m \mathrm{~m}$ \\
\hline $\mathrm{Ca}$ & $9 / 13 / 85$ & NA & NA & NA & NA & NA & 1.17 & 0.000152 & \\
\hline $\mathrm{Ca}$ & $9 / 1 / 89$ & NS & NS & RAT-AZ101-4A & NS & NS & 1.2 & 0.00009 & $\overrightarrow{\mathrm{mm}}$ \\
\hline $\mathrm{Ca}$ & $9 / 29 / 89$ & NA & $4 / 1 / 89$ & Core 1 & $\mathrm{NA}$ & NA & 1.2 & 0.00009 & $\overline{m n}$ \\
\hline $\mathrm{Ca}$ & $9 / 29 / 89$ & $\mathrm{NA}$ & $4 / 1 / 89$ & Core 1 & $\mathrm{NA}$ & NA & 1.2 & 0.0000146 & $\mathrm{mrl}$ \\
\hline $\mathrm{Cd}$ & $9 / 1 / 93$ & NA & $4 / 1 / 89$ & Core 2 & $\mathrm{NA}$ & NA & 1.24 & 0.032 & $\mathrm{un}$ \\
\hline $\mathrm{Cd}$ & $9 / 1 / 90$ & NS & NS & RAT-AZ101-5A & NS & NS & 1.22 & 0.032 & um: \\
\hline $\mathrm{Cd}$ & NS & NS & NS & NS & NS & NS & NS & 0.00439 & $\bar{c}$ \\
\hline $\mathrm{Ce}$ & $9 / 29 / 89$ & NA & $4 / 1 / 89$ & Core 1 & NA & NA & 1.2 & 0.00000476 & $\overrightarrow{\mathrm{mrt}}$ \\
\hline Chloride & $7 / 26 / 95$ & NA & $3 / 3 / 95$ & BOD9P7 & NA & NA & NA & $<158$ & $U_{i}^{i}$ \\
\hline Chloride & $7 / 26 / 95$ & NA & $3 / 3 / 95$ & 8009P9 & NA & NA & NA & $<158$ & $\overline{U C}$ \\
\hline $\mathrm{Cl}-$ & $\mathrm{NA}$ & NA & $\mathrm{NA}$ & NA & NA & NA & 1.12 & $5.38 \mathrm{E}-02$ & $\overrightarrow{M l}$ \\
\hline $\mathrm{Cl}$ & $10 / 1 / 87$ & NS & NS & RAT-AZ101-6A & NS & NS & NS & 0.019 & $m$ \\
\hline $\mathrm{Cl}$ & $3 / 11 / 88$ & NA & NA & T3493 & NA & NA & NA & $1.90 E-02$ & 1 \\
\hline $\mathrm{Cl}$ & $3 / 11 / 88$ & NA & NA & NA & $\mathrm{NA}$ & NA & $\overline{N A}$ & $1.90 \mathrm{E}-02$ & 1 \\
\hline $\mathrm{Cl}$ & $2 / 8 / 89$ & NA & $10 / 1 / 87$ & T3493 & NA & NA & $\mathrm{NA}$ & $1.90 \mathrm{E}-02$ & I \\
\hline $\mathrm{Cl}$ & $11 / 18 / 85$ & NS & NS & RAT-AZ101-1 & NS & NS & 1.17 & 0.00907 & mo \\
\hline $\mathrm{Cl}$ & $1 / 10 / 86$ & NA & $11 / 1 / 85$ & NA & $\mathrm{NA}$ & NA & 1.17 & $9.07 E-03$ & I \\
\hline $\mathrm{Cl}-$ & May 89 & NS & NS & NS & NS & NS & NS & 3.11E-01 & a \\
\hline $\mathrm{Cl}$ & $9 / 1 / 90$ & NS & NS & RAT-AZ101-5A & NS & NS & 1.22 & 7.19 & ume \\
\hline $\mathrm{Cl}$ & $4 / 8 / 86$ & NA & $11 / 1 / 85$ & $\mathrm{NA}$ & NA & NA & NA & $8.00 \mathrm{E}-03$ & $T$ \\
\hline Chloride & $7 / 26 / 95$ & NA & $3 / 3 / 95$ & BOD9P6 & NA & NA & $\overline{N A}$ & 187 & $\overline{U C}$ \\
\hline Chloride & $7 / 26 / 95$ & NA & $3 / 3 / 95$ & BOD9P9 & NA & NA & NA & 183 & $\overline{U E}$ \\
\hline Chloride & $7 / 26 / 95$ & $\mathrm{NA}$ & $3 / 3 / 95$ & BOD9P7 & NA & NA & NA & 173 & $\overline{U E}$ \\
\hline $\mathrm{Cl}$ & $9 / 1 / 89$ & NS & NS & RAT-AZ101-4A & NS & NS & 1.2 & 0.00002 & $\overline{m m}$ \\
\hline $\mathrm{Cl}$ & $1 / 1 / 81$ & NS & NS & NS & NS & NS & NS & NS & h \\
\hline $\mathrm{Cr}$ & $9 / 29 / 89$ & NA & $4 / 1 / 89$ & Core 1 & NA & NA & 1.2 & 0.013 & $\overline{\mathrm{mm}}$ \\
\hline
\end{tabular}




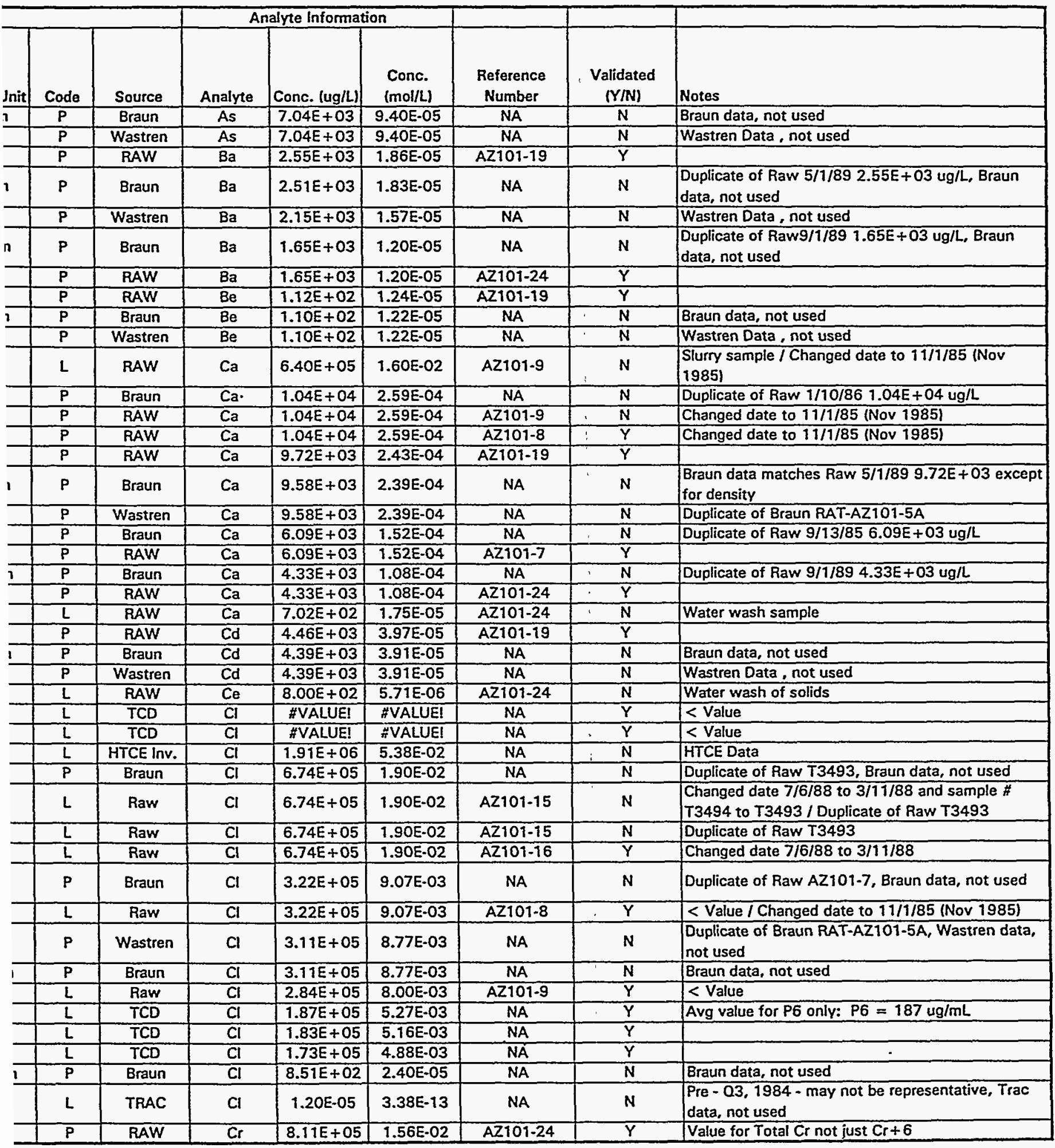




\begin{tabular}{|c|c|c|c|c|c|c|c|c|c|}
\hline \multicolumn{10}{|c|}{ Tank Waste Characterization Sample Information } \\
\hline Reported Chemical & $\begin{array}{l}\text { Memo } \\
\text { Date }\end{array}$ & $\begin{array}{l}\text { Start Analysis } \\
\text { Date }\end{array}$ & $\begin{array}{c}\text { Start } \\
\text { Sample } \\
\text { Date } \\
\end{array}$ & Sample Number & $\begin{array}{c}\text { Tank Farm } \\
\text { Number }\end{array}$ & $\begin{array}{c}\text { Lab Sample } \\
\text { Number }\end{array}$ & $\begin{array}{l}\text { Density } \\
\text { (g/mL) }\end{array}$ & Reported Value & $\operatorname{Rep}$ \\
\hline $\mathrm{Cr}$ & $9 / 1 / 93$ & $\mathrm{NA}$ & $4 / 1 / 89$ & Core 2 & NA & NA & 1.24 & 8.29 & 1 \\
\hline $\mathrm{Cr}$ & $9 / 1 / 90$ & NS & NS & RAT-A.Z101-5A & NS & NS & 1.22 & 8.29 & 4 \\
\hline $\mathrm{Cr}$ & $5 / 1 / 89$ & NS & NS & NS & NS & NS & NS & 0.526 & \\
\hline $\mathrm{Cr}$ & $9 / 1 / 89$ & NS & NS & RAT-AZ101-4A & NS & NS & 1.2 & 0.013 & $m$ \\
\hline $\mathrm{Cr}$ & $3 / 11 / 88$ & NA & NA & T3494 & NA & NA & $\overline{N A}$ & 0.013 & \\
\hline $\mathrm{Cr}$ & $9 / 29 / 89$ & NA & $4 / 1 / 89$ & Core 1 & NA & NA & 1.2 & 0.0103 & $\bar{n}$ \\
\hline $\mathrm{Cr}$ & $9 / 29 / 89$ & $\mathrm{NA}$ & $4 / 1 / 89$ & Core 1 & NA & NA & 1.2 & 0.0098 & - \\
\hline $\mathrm{CrO} 4$ & $9 / 1 / 89$ & NS & NS & RAT-AZ101-4A & NS & NS & 1.2 & 0.0098 & $\mathbf{m}$ \\
\hline $\mathrm{Cr}$ & $11 / 18 / 85$ & NS & NS & RAT-AZ101-1 & NS & NS & 1.17 & 0.0112 & $r$ \\
\hline $\mathrm{Cr}$ & $1 / 10 / 86$ & NA & $11 / 1 / 85$ & NA & NA & NA & 1.17 & 0.0112 & \\
\hline $\mathrm{Cr}$ & $4 / 8 / 86$ & NA & $11 / 1 / 85$ & NA & NA & NA & NA & 0.011 & \\
\hline $\mathrm{Cr}_{\mathrm{r}}$ & $8 / 7 / 86$ & NS & NS & R9964/R0247 & NS & NS & 1.2 & 0.01 & 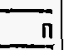 \\
\hline $\mathrm{Cr}$ & $9 / 13 / 85$ & NS & NS & RAT-AZ101-3A & NS & NS & 1.17 & 0.00771 & 11 \\
\hline $\mathrm{Cr}$ & $9 / 13 / 85$ & NA & NA & NA & NA & NA & 1.17 & 0.00771 & \\
\hline $\mathrm{Cr}$ & $12 / 28 / 83$ & NS & NS & NS & NS & NS & NS & 0.006 & \\
\hline $\mathrm{CrO4}$ & $5 / 1 / 89$ & NS & NS & NS & NS & NS & NS & 0.187 & \\
\hline $\mathrm{Cr}+6$ & $9 / 1 / 93$ & NA & $4 / 1 / 89$ & Core 2 & NA & NA & 1.24 & 1.32 & $\underline{u}$ \\
\hline $\mathrm{CrO} 4$ & $9 / 1 / 90$ & NS & NS & RAT-AZ101-5A & NS & NS & 1.22 & 1.32 & ur \\
\hline$F$ & $10 / 1 / 87$ & NS & NS & RAT-AZ101-6A & NS & NS & NS & 0.097 & $\pi$ \\
\hline$F$ & $3 / 11 / 88$ & MA & NA & $T 3493$ & NA & NA & NA & $9.70 \mathrm{E}-02$ & $i$ \\
\hline$F$ & $3 / 11 / 88$ & NA & NA & NA & NA & NA & NA & $9.70 \mathrm{E}-02$ & \\
\hline$F$ & $2 / 8 / 89$ & $\mathrm{NA}$ & $10 / 1 / 87$ & T3493 & NA & NA & NA & $9.70 \mathrm{E}-02$ & \\
\hline$F$ & $11 / 18 / 85$ & NS & NS & RAT-AZ101-1 & NS & NS & 1.17 & 0.089 & n \\
\hline$F$ & $1 / 10 / 86$ & NA & $11 / 1 / 85$ & NA & NA & NA & 1.17 & $8.90 E-02$ & \\
\hline$F$ & $4 / 8 / 86$ & NA & $11 / 1 / 85$ & NA & NA & NA & NA & $8.70 E-02$ & \\
\hline Fluoride & $7 / 26 / 95$ & NA & $3 / 3 / 95$ & B009P6 & NA & NA & NA & 1620 & $i j$ \\
\hline Fluoride & $7 / 26 / 95$ & NA & $3 / 3 / 95$ & B0D997 & NA & NA & NA & 1590 & u \\
\hline$F$ & $9 / 1 / 89$ & NS & NS & RAT-A2101-4A & NS & NS & 1.2 & 0.068 & mil \\
\hline Fluoride & $7 / 26 / 95$ & NA & $3 / 3 / 95$ & BOD9P9 & NA & NA & NA & 1540 & i) \\
\hline$F$ & $9 / 1 / 90$ & NS & NS & RAT-AZ101-5A & NS & NS & 1.22 & 30.6 & $u \pi$ \\
\hline F- & May 89 & NS & NS & NS & NS & NS & NS & 7.09E-01 & . \\
\hline $\mathrm{F}-$ & NA & NA & NA & NA & NA & NA & 1.12 & $2.47 E-02$ & $\vec{M}$ \\
\hline$F$ & $9 / 13 / 85$ & NS & NS & RAT-AZ101-3A & NS & NS & 1.17 & 0.0198 & m \\
\hline$F$ & $9 / 13 / 85$ & NA & NA & NA & NA & NA & 1.17 & $1.98 \mathrm{E}-02$ & \\
\hline$F$ & 877186 & NS & NS & R9964/R0247 & NS & NS & 1.2 & 0.00461 & $\bar{m}$ \\
\hline$F$ & $1 / 1 / 81$ & NS & NS & NS & NS & NS & NS & NS & \\
\hline Iron & $7 / 26 / 95$ & NA & $3 / 3 / 95$ & BOD9N9 & NA & NA & NA & $<10.1$ & U \\
\hline Iron & $7 / 26 / 95$ & $\mathrm{NA}$ & $3 / 3 / 95$ & BOD9P2 & NA & NA & NA & $<10.1$ & $\mathrm{U}$ \\
\hline Iron & $7 / 26 / 95$ & NA & $3 / 3 / 95$ & B0D9P5 & NA & $\mathrm{NA}$ & NA & $<10.1$ & $\overline{\mathbf{U}}$ \\
\hline
\end{tabular}




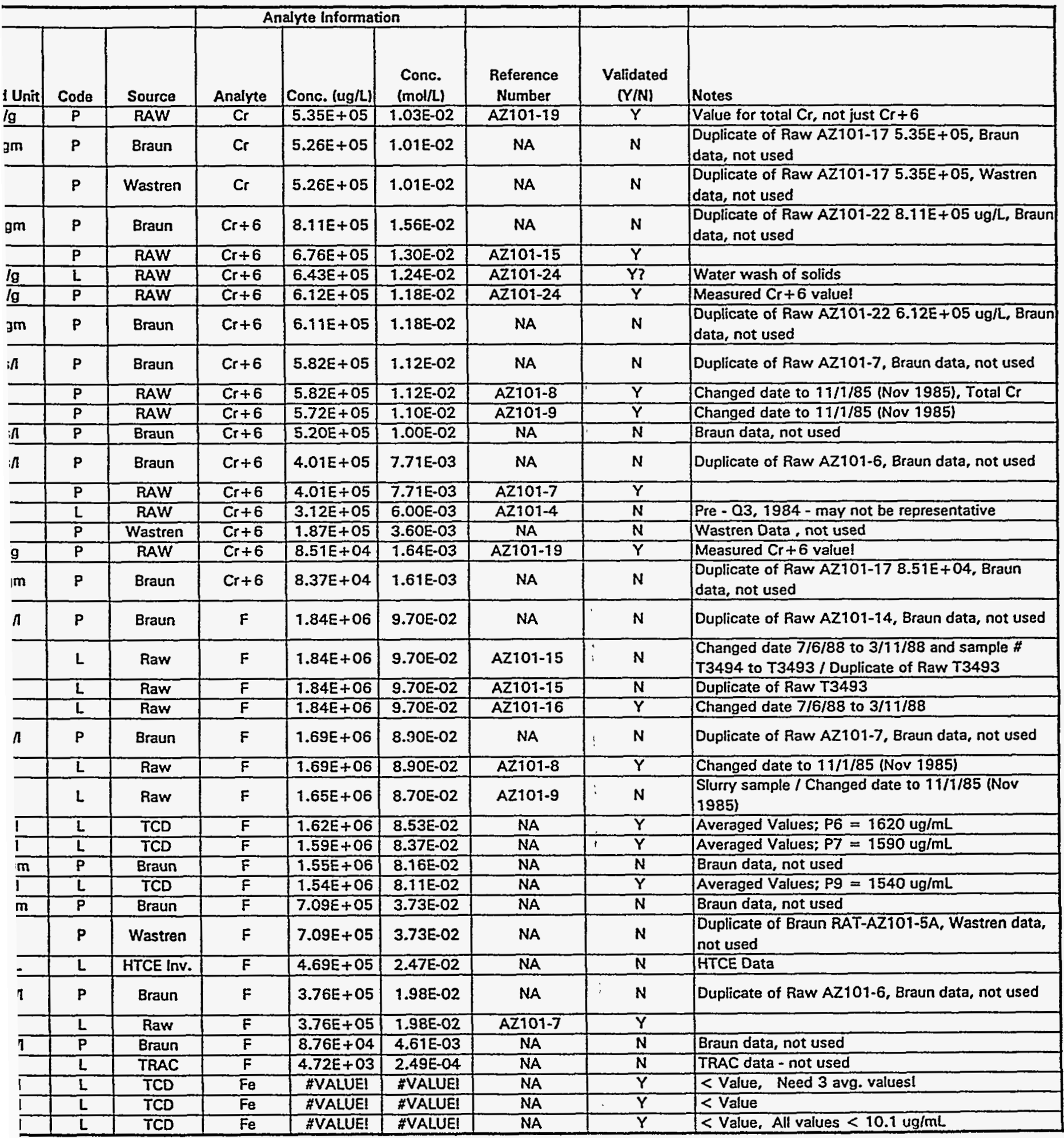




\begin{tabular}{|c|c|c|c|c|c|c|c|c|c|}
\hline \multicolumn{10}{|c|}{ Tank Waste Characterization Sample Information } \\
\hline Reported Chemical & $\begin{array}{c}\text { Memo } \\
\text { Date }\end{array}$ & $\begin{array}{c}\text { Start Analysis } \\
\text { Date }\end{array}$ & $\begin{array}{c}\text { Start } \\
\text { Sample } \\
\text { Date } \\
\end{array}$ & Sample Number & $\begin{array}{c}\text { Tank Fam } \\
\text { Number }\end{array}$ & $\begin{array}{c}\text { Lab Sample } \\
\text { Number }\end{array}$ & $\begin{array}{l}\text { Density } \\
\text { (g/mL) }\end{array}$ & Reported Value & Rer \\
\hline Iron & $7 / 26 / 95$ & NA & $3 / 3 / 95$ & BOD9N9 & NA & $\mathrm{NA}$ & NA & $<10$ & \\
\hline Iron & $7 / 26 / 95$ & NA & $3 / 3 / 95$ & B0D9P2 & NA & NA & NA & $<10$ & \\
\hline Iron & $7 / 26 / 95$ & NA & $3 / 3 / 95$ & BOD9P5 & NA & NA & NA & $<10$ & \\
\hline Fe & $4 / 8 / 86$ & NS & NS & NS & NS & NS & NS & $6.70 E-02$ & \\
\hline $\mathrm{Fe}$ & $12 / 28 / 83$ & NS & NS & NS & NS & NS & NS & $2.00 \mathrm{E}-03$ & \\
\hline $\mathrm{Fe} 3+($ total $\mathrm{Fe})$ & $\mathrm{NA}$ & NA & NA & NA & NA & NA & 1.12 & $1.94 \mathrm{E}-03$ & \\
\hline $\mathrm{Fe}$ & $1 / 1 / 81$ & NS & NS & & NS & NS & NS & NS & \\
\hline $\mathrm{Fe}$ & $9 / 1 / 90$ & NS & NS & RAT-A2101-5A & NS & NS & 1.22 & 0.351 & $\underline{\mathbf{u}}$ \\
\hline $\mathrm{Fe}$ & May 89 & NS & NS & NS & NS & NS & NS & $2.39 E-02$ & \\
\hline $\mathrm{Fe}$ & $9 / 1 / 89$ & NS & NS & RAT-AZ101-4A & NS & NS & 1.2 & 0.0002 & $\bar{\pi}$ \\
\hline $\mathrm{Fe}$ & $1 / 10 / 86$ & NA & $11 / 1 / 85$ & NA & NA & NA & NA & $1.16 \mathrm{E}-02$ & \\
\hline $\mathrm{K}$ & $4 / 8 / 86$ & NA & $11 / 1 / 85$ & NA & NA & $\mathrm{NA}$ & NA & 1.20E-01 & \\
\hline $\bar{k}$ & $11 / 18 / 85$ & NS & NS & RAT-AZ101-1 & NS & NS & 1.17 & 0.116 & $r$ \\
\hline $\mathrm{K}$ & $1 / 10 / 86$ & NA & $11 / 1 / 85$ & NA & NA & NA & 1.17 & $1.16 E-01$ & \\
\hline$K$ & $8 / 7 / 86$ & NS & NS & R9964/R0247 & NS & NS & 1.2 & 0.11 & $\underline{r}$ \\
\hline$K$ & $9 / 1 / 89$ & NS & NS & RAT-AZ101-4A & NS & NS & 1.2 & 0.088 & $\underline{m}$ \\
\hline K & $9 / 13 / 85$ & NS & NS & RAT-AZ101-3A & NS & NS & 1.17 & 0.0764 & $n$ \\
\hline $\bar{K}$ & $9 / 13 / 85$ & NA & NA & NA & NA & NA & 1.17 & $7.64 \mathrm{E}-02$ & \\
\hline$k$ & May 89 & NS & NS & NS & NS & NS & NS & $2.49 E+\infty 0$ & \\
\hline$K$ & $9 / 1 / 90$ & NS & NS & RAT-AZ101-5A & NS & NS & 1.22 & 52.1 & ur \\
\hline $\bar{k}+$ & NA & NA & NA & NA & NA & NA & 1.12 & $2.03 E-03$ & $A$ \\
\hline$k$ & $1 / 1 / 81$ & NS & NS & & NS & NS & NS & NS & \\
\hline$k$ & $1 / 10 / 86$ & NA & $11 / 1 / 85$ & NA & NA & NA & NA & $1.25 \mathrm{E}-01$ & \\
\hline $\mathrm{La}$ & $9 / 1 / 93$ & NA & $4 / 1 / 89$ & Core 2 & NA & NA & 1.24 & 0.023 & u \\
\hline La & $9 / 1 / 90$ & NS & NS & RAT-AZ101-5A & NS & NS & 1.22 & 0.023 & un \\
\hline La & $5 / 1 / 89$ & NS & NS & NS & NS & NS & NS & 0.0039 & \\
\hline $\mathrm{Na}$ & $4 / 8 / 86$ & NA & $11 / 1 / 85$ & NA & NA & NA & NA & 5 & \\
\hline $\mathrm{Na}$ & $4 / 8 / 86$ & NA & $11 / 1 / 85$ & NA & NA & NA & NA & 4.9 & \\
\hline $\mathrm{Na}$ & $11 / 18 / 85$ & NS & NS & RAT-AZ101-1 & NS & NS & 1.17 & 4.89 & m \\
\hline $\mathrm{Na}$ & $1 / 10 / 86$ & NA & $11 / 1 / 85$ & NA & NA & NA & 1.17 & 4.89 & \\
\hline $\mathrm{Na}$ & $10 / 29 / 90$ & NA & NA & R101283 & $\mathrm{NA}$ & NA & NA & 4.89 & \\
\hline $\mathrm{Na}$ & $8 / 7 / 86$ & NS & NS & R9964/R0247 & NS & NS & 1.2 & 4.82 & $\mathrm{~m}$ \\
\hline $\mathrm{Na}$ & $10 / 29 / 90$ & $5 / 2 / 90$ & NA & R280187 & NA & $\mathrm{NA}$ & NA & 4.82 & \\
\hline $\mathrm{Na}$ & $9 / 1 / 89$ & NS & NS & RAT-AZ101-4A & NS & NS & 1.2 & 3.76 & $\mathrm{mr}$ \\
\hline $\mathrm{Na}$ & $9 / 29 / 89$ & NA & $4 / 1 / 89$ & Core 1 & NA & NA & 1.2 & 3.76 & $\mathrm{mi}$ \\
\hline $\mathrm{Na}$ & $10 / 1 / 87$ & NS & NS & RAT-AZ101-6A & NS & NS & NS & 4.42 & $\mathrm{me}$ \\
\hline $\mathrm{Na}$ & $3 / 11 / 88$ & NA & NA & T3493 & NA & NA & NA & 4.42 & \\
\hline $\mathrm{Na}$ & $3 / 11 / 88$ & NA & NA & NA & NA & NA & NA & 4.42 & \\
\hline $\mathrm{Na}$ & $2 / 8 / 89$ & NA & $10 / 1 / 89$ & $T 3493$ & NA & $\mathrm{NA}$ & NA & 4.42 & \\
\hline
\end{tabular}




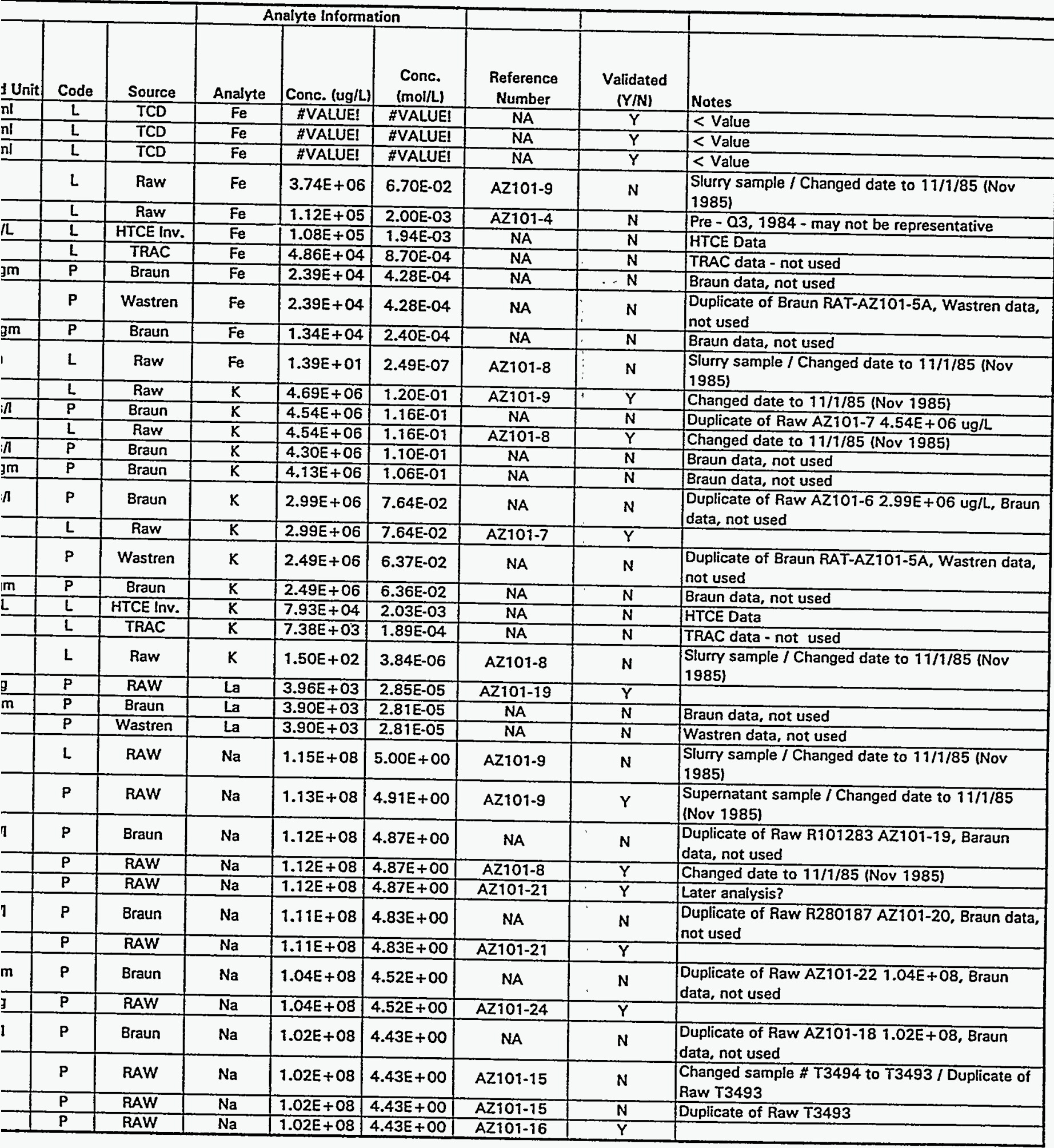




\begin{tabular}{|c|c|c|c|c|c|c|c|c|c|}
\hline Reported Chemical & $\begin{array}{c}\text { Memo } \\
\text { Date }\end{array}$ & $\begin{array}{c}\text { Start Analysis } \\
\text { Date }\end{array}$ & $\begin{array}{c}\text { Start } \\
\text { Sample } \\
\text { Date } \\
\end{array}$ & Sample Number & $\begin{array}{c}\text { Tank Farm } \\
\text { Number }\end{array}$ & $\begin{array}{c}\text { Lab Sample } \\
\text { Number }\end{array}$ & $\begin{array}{l}\text { Density } \\
(\mathrm{g} / \mathrm{mL})\end{array}$ & Reported Value & Rep. \\
\hline $\mathrm{Na}$ & $10 / 30 / 90$ & $5 / 2 / 90$ & NA & R110388 & NA & NA & NA & 4.42 & \\
\hline Sodium & $7 / 26 / 95$ & $\overline{N A}$ & $3 / 3 / 95$ & $8009 P 5$ & NA & NA & NA & 97300 & \\
\hline Sodium & $7 / 26 / 95$ & NA & $3 / 3 / 95$ & BOD9N9 & NA & NA & $\mathrm{NA}$ & 94800 & \\
\hline Sodium & $7 / 26 / 95$ & NA & $3 / 3 / 95$ & BOD9P2 & NA & NA & NA & 93600 & \\
\hline $\mathrm{Na}$ & $9 / 13 / 85$ & NS & NS & RAT-AZ101-3A & NS & NS & 1.17 & 3.13 & $r$ \\
\hline $\mathrm{Na}$ & $9 / 13 / 85$ & NA & NA & R130985 & NA & $\widehat{N A}$ & NA & 3.13 & \\
\hline $\mathrm{Na}$ & $9 / 13 / 85$ & NA & NA & R130985 & NA & NA & 1.17 & 3.13 & \\
\hline Na TOT & 8127181 & NS & NS & NS & NS & NS & 1.143 & NS & \\
\hline $\mathrm{Na}$ & $9 / 1 / 93$ & NA & $4 / 1 / 89$ & Core 2 & NA & NA & 7.24 & 2020 & 4 \\
\hline $\mathrm{Na}$ & $9 / 1 / 90$ & NS & NS & RAT-AZ101-5A & NS & NS & 1.22 & 2020 & uㅡ \\
\hline $\mathrm{Na}$ & NS & NS & NS & NS & NS & NS & NS & 56.7 & \\
\hline NaTOT & $4 / 26 / 78$ & NS & NS & NS & NS & NS & 1.133 & NS & \\
\hline $\mathrm{Na}$ & $6 / 1 / 84$ & NS & NS & RAT-AZ101-2B & NS & NS & NS & 1.07 & II \\
\hline $\mathrm{Na}$ & $9 / 10 / 84$ & NA & $6 / 1 / 84$ & NA & NA & NA & NA & 1.07 & \\
\hline $\mathrm{Na}$ & $6 / 1 / 84$ & NS & NS & RAT-AZ101-2A & NS & NS & NS & 1.06 & ir \\
\hline $\mathrm{Na}$ & $9 / 10 / 84$ & $\mathrm{NA}$ & $6 / 1 / 84$ & NA & NA & NA & NA & 1.06 & \\
\hline $\mathrm{Na}$ & $2 / 5190$ & NA & NA & R100984 & NA & NA & NA & 1.06 & \\
\hline Nd & $9 / 1 / 93$ & NA & $4 / 1 / 89$ & Core 2 & NA & NA & 1.24 & 0.008 & u \\
\hline $\mathrm{Nd}$ & $9 / 1 / 90$ & NS & NS & RAT-AZ101-5A & NS & NS & 1.22 & 0.008 & un \\
\hline Nd & $5 / 1 / 89$ & NS & NS & NS & NS & NS & $\mathrm{NA}$ & 0.00141 & \\
\hline $\mathrm{Ni}$ & $4 / 8 / 86$ & NA & $11 / 1 / 85$ & NA & NA & NA & NA & $8.20 E-03$ & \\
\hline $\mathrm{Ni2t}$ & NA & NA & $\mathrm{NA}$ & $\mathrm{NA}$ & $\mathrm{NA}$ & NA & 1.12 & $6.14 \mathrm{E}-04$ & $\bar{M}$ \\
\hline $\mathrm{Ni}$ & $9 / 1 / 89$ & NS & NS & RAT-AZ101-4A & NS & NS & 1.2 & 0.0002 & $\mathbf{m i r}$ \\
\hline $\mathrm{Ni}$ & May 89 & NS & NS & NS & NS & NS & NS & $3.08 \mathrm{E}-03$ & \\
\hline $\mathrm{Ni}$ & 9/1/90 & NS & NS & RAT-AZ101-5A & NS & NS & 1.22 & 0.043 & ur \\
\hline $\mathrm{Ni}$ & $1 / 1 / 81$ & NS & NS & NS & NS & NS & NS & NS & \\
\hline $\mathrm{Ni}$ & $1 / 10 / 86$ & NA & $11 / 1 / 85$ & NA & NA & NA & NA & $1.03 E-03$ & $f$ \\
\hline NO2 & $9 / 1 / 90$ & NS & NS & RAT-AZ101-5A & NS & NS & 1.22 & 2370 & um \\
\hline No2- & May 89 & NS & NS & NS & NS & NS & NS & $1.33 E+02$ & 1 \\
\hline NO2 & $12 / 3 / 79$ & NS & N5 & $1494 \mathrm{~A}$ & NS & NS & 1.0414 & $2.68 E+00$ & \\
\hline NO2 & $12 / 3 / 79$ & NS & NS & 1495B & NS & NS & 1.448 & $2.68 E+00$ & \\
\hline No2 & $12 / 3 / 79$ & NS & NS & 1465 & $\mathrm{NS}$ & NS & 1.41 & $2.65 E+00$ & \\
\hline NO2 & $12 / 3 / 79$ & NS & NS & 1494B & NS & NS & 1.42 & $2.65 E+00$ & \\
\hline No2 & $12 / 3779$ & NS & NS & $1495 \mathrm{~A}$ & NS & NS & 1.419 & $2.64 E+00$ & \\
\hline No2 & $12 / 3 / 79$ & NS & NS & 1464 & NS & NS & 1.409 & $2.49 E+00$ & \\
\hline NO2 & $12 / 3 / 79$ & NS & NS & 1463 & NS & NS & 1.384 & $2.43 E+00$ & \\
\hline No2 & $12 / 3 / 79$ & NS & NS & 1466 & NS & NS & 1.414 & $2.37 E+00$ & \\
\hline Nitrite & $3 / 3 / 95$ & $\mathrm{NA}$ & $3 / 3 / 95$ & B009P7 & NA & NA & NA & 59000 & $\overline{U C}$ \\
\hline Nitrite & $3 / 3 / 95$ & NA & $3 / 3 / 95$ & BOD9P6 & NA & NA & NA & 56600 & $U$ \\
\hline $\mathrm{NO} 2$ & $9 / 1 / 89$ & NS & NS & RAT-AZ101-4A & NS & NS & 1.2 & 1 & $\mathrm{~mm}$ \\
\hline Nitrite & $3 / 3 / 95$ & NA & $3 / 3 / 95$ & BODSP9 & NA & NA & NA & 55050 & UC \\
\hline $\mathrm{NO2}$ & $10 / 1 / 87$ & NS & NS & RAT-AZ101-6A & NS & NS & NS & 0.79 & mo \\
\hline
\end{tabular}




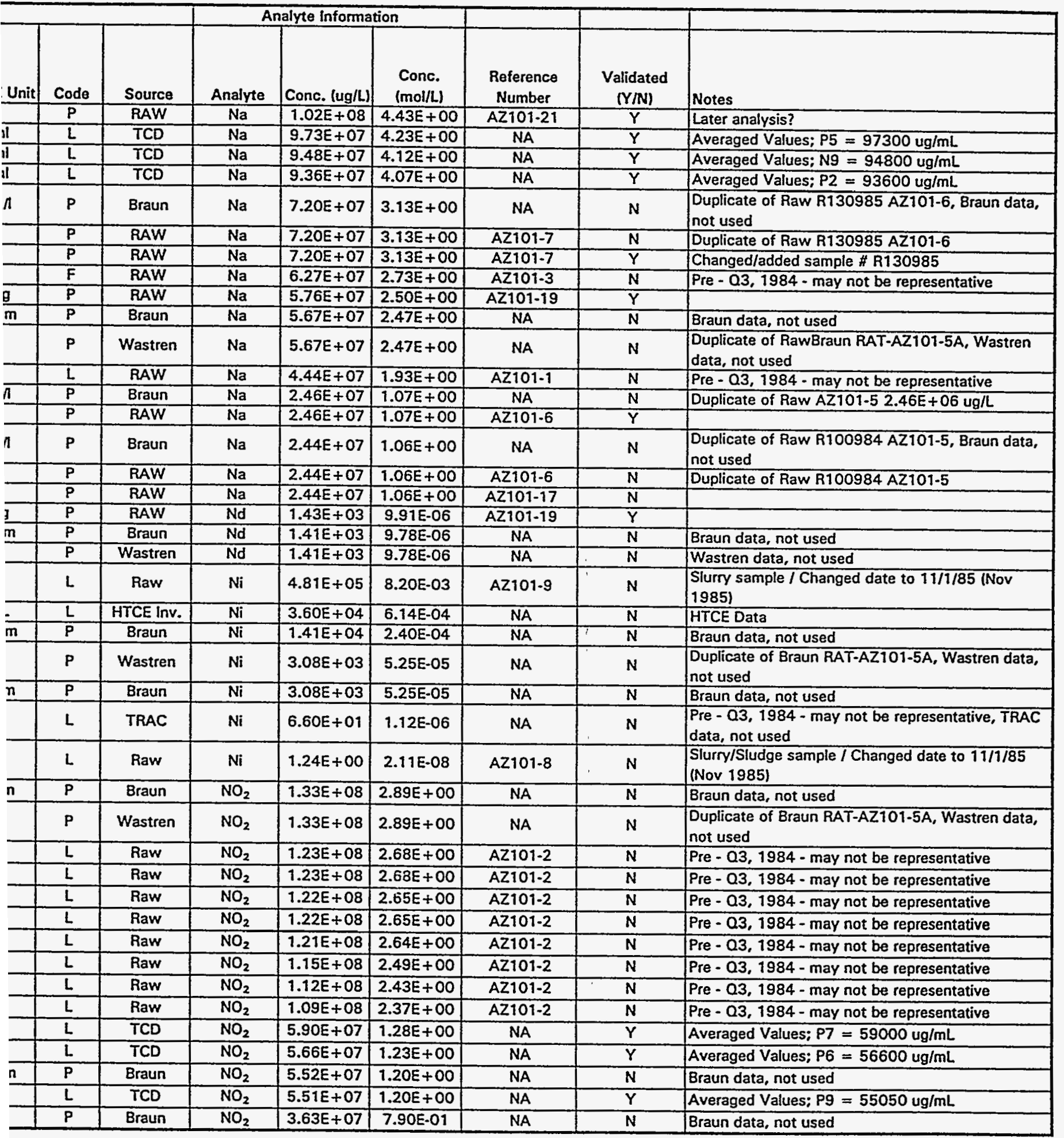




\begin{tabular}{|c|c|c|c|c|c|c|c|c|c|}
\hline \multicolumn{10}{|c|}{ Tank Waste Characterization Sample Information } \\
\hline Reported Chemical & $\begin{array}{c}\text { Memo } \\
\text { Date }\end{array}$ & $\begin{array}{c}\text { Start Analysis } \\
\text { Date } \\
\end{array}$ & $\begin{array}{c}\text { Start } \\
\text { Sample } \\
\text { Date } \\
\end{array}$ & Sample Number & $\begin{array}{c}\text { Tank Farm } \\
\text { Number }\end{array}$ & $\begin{array}{c}\text { Lab Sample } \\
\text { Number }\end{array}$ & $\begin{array}{l}\text { Density } \\
(\mathrm{g} / \mathrm{mL})\end{array}$ & Reported Value & Rep \\
\hline NO2 & $3 / 11 / 88$ & NA & NA & T3493 & NA & NA & NA & $7.90 \mathrm{E}-01$ & \\
\hline NO2 & $2 / 8 / 89$ & NA & NA & T3493 & NA & $\overline{\mathrm{NA}}$ & NA & 7.90E-01 & \\
\hline NO2 & $8 / 7 / 86$ & NS & NS & R9964/R0247 & NS & NS & 1.2 & 0.61 & $\underline{n}$ \\
\hline NO2 & $9 / 13 / 85$ & NS & NS & RAT-AZ101-3A & NS & NS & 1.17 & 0.589 & $n$ \\
\hline $\mathrm{NO2}$ & $9 / 13 / 85$ & NA & NA & NA & NA & $\overrightarrow{\mathrm{NA}}$ & 1.17 & $5.89 E-01$ & \\
\hline NaNO2 & $8 / 27 / 81$ & NS & NS & NS & NS & NS & 1.143 & $5.38 E-01$ & \\
\hline NO2- & NA & NA & NA & NA & NA & NA & 1.12 & 4.74E-01 & $A$ \\
\hline NO2 & $11 / 18 / 85$ & NS & NS & RAT-AZ101-1 & NS & NS & 1.17 & 0.441 & $\pi$ \\
\hline NO2 & $1 / 10 / 86$ & NA & $11 / 1 / 85$ & $\mathrm{NA}$ & NA & $\overline{N A}$ & 1.17 & $4.41 \mathrm{E}-01$ & \\
\hline NO2 & $4 / 8 / 86$ & NA & $11 / 1 / 85$ & NA & NA & NA & NA & 4.30E-01 & \\
\hline NO2 & $12 / 28 / 83$ & NS & NS & NS & NS & NS & NS & $2.70 E-01$ & \\
\hline NO2 & $6 / 1 / 84$ & NS & NS & RAT-AZ101-2B & NS & NS & NS & 0.028 & $m$ \\
\hline NO2 & $6 / 1 / 84$ & NS & NS & RAT-AZ101-2A & NS & NS & NS & 0.027 & $\mathbf{m}$ \\
\hline NO2 & $9 / 10 / 84$ & NA & $6 / 1 / 84$ & NA & $\overline{N A}$ & $\mathrm{NA}$ & NA & $2.70 E-02$ & \\
\hline NaNO2 & $4 / 26 / 78$ & NS & NS & NS & NS & NS & 1.133 & $2.40 \mathrm{E}-02$ & \\
\hline NO2 & $1 / 1 / 81$ & NS & NS & NS & NS & NS & NS & NS & \\
\hline $\mathrm{NO3}$ & $12 / 3 / 79$ & NS & NS & $1494 B$ & NS & NS & 1.42 & $3.01 E+00$ & \\
\hline NO3 & $12 / 3 / 79$ & NS & NS & $1495 \mathrm{~A}$ & NS & NS & 1.419 & $2.98 E+00$ & \\
\hline $\mathrm{NO3}$ & $12 / 3 / 79$ & NS & NS & 1465 & NS & NS & 1.41 & $2.95 E+00$ & \\
\hline $\mathrm{NO3}$ & $12 / 3 / 79$ & NS & NS & 1466 & NS & NS & 1.414 & $2.94 E+00$ & \\
\hline NO3 & $12 / 3 / 79$ & NS & NS & $1494 \mathrm{~A}$ & NS & NS & 1.0414 & $2.91 E+00$ & \\
\hline $\mathrm{NO3}$ & $12 / 3 / 79$ & NS & NS & $1495 \mathrm{~B}$ & NS & NS & 1.448 & $2.90 E+00$ & \\
\hline $\mathrm{NO3}$ & $12 / 3 / 79$ & NS & NS & 1464 & NS & NS & 1.409 & $2.80 E+00$ & \\
\hline $\mathrm{NO3}$ & $12 / 3 / 79$ & NS & NS & 1463 & NS & NS & 1.384 & $2.67 E+00$ & \\
\hline $\mathrm{NO3}$ & $11 / 18 / 85$ & NS & NS & RAT-AZ101-1 & NS & NS & 1.17 & 1.75 & $m$ \\
\hline No3 & $1 / 10 / 86$ & NA & $11 / 1 / 85$ & NA & NA & NA & 1.17 & $1.75 E+00$ & \\
\hline NO3 & $4 / 8 / 86$ & NA & $91 / 1 / 85$ & NA & NA & NA & NA & $1.70 E+00$ & \\
\hline NO3 & $9 / 1 / 90$ & NS & NS & RAT-AZ101-5A & NS & NS & 1.22 & 1380 & um \\
\hline NO3- & May 89 & NS & NS & NS & NS & NS & NS & $1.04 E+02$ & \\
\hline $\mathrm{NaNO3}$ & $4 / 26 / 78$ & NS & NS & NS & NS & NS & 1.133 & $1.55 E+00$ & \\
\hline NO3 & $9 / 13 / 85$ & NS & NS & RAT-AZ101-3A & NS & NS & 1.17 & 1.45 & $m$ \\
\hline NO3 & $9 / 13 / 85$ & NA & NA & $\mathrm{NA}$ & NA & NA & 1.17 & $1.45 E+00$ & \\
\hline NO3 & $10 / 1 / 87$ & NS & NS & RAT-AZ101-6A & NS & NS & NS & 1.39 & mi \\
\hline NO3 & $3 / 11 / 88$ & NA & NA & T3493 & NA & NA & NA & $1.39 E+00$ & \\
\hline NO3 & $2 / 8 / 89$ & $\mathrm{NA}$ & $10 / 1 / 87$ & T3493 & NA & NA & NA & $1.39 E+00$ & \\
\hline NO3 & $9 / 1 / 89$ & NS & NS & RAT-AZ101-4A & NS & NS & 1.2 & 1.04 & $\mathrm{~mm}$ \\
\hline Nitrate & $7 / 26 / 95$ & NA & $3 / 3 / 95$ & BOD9P7 & NA & $\mathrm{NA}$ & NA & 68250 & U \\
\hline Nitrate & $7 / 26 / 95$ & NA & $3 / 3 / 95$ & BOD9P6 & NA & NA & NA & 65100 & 4 \\
\hline
\end{tabular}




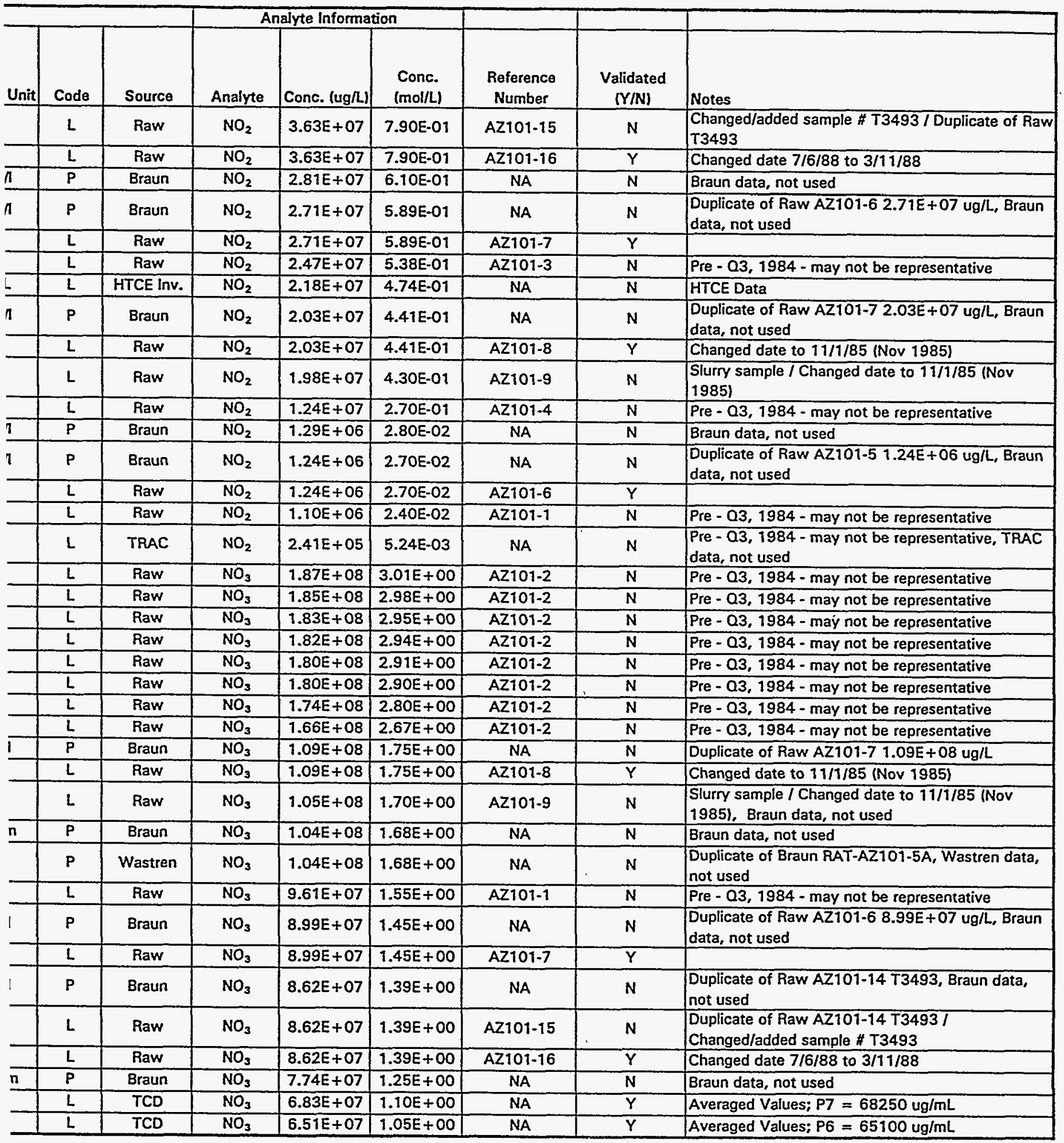




\begin{tabular}{|c|c|c|c|c|c|c|c|c|c|}
\hline \multirow[b]{2}{*}{ Reported Chemical } & \multicolumn{9}{|c|}{ Tank Waste Characterization Sample Information } \\
\hline & $\begin{array}{c}\text { Memo } \\
\text { Date }\end{array}$ & $\begin{array}{c}\text { Start Analysis } \\
\text { Date }\end{array}$ & $\begin{array}{c}\text { Start } \\
\text { Sample } \\
\text { Date } \\
\end{array}$ & Sample Number & $\begin{array}{c}\text { Tank Farm } \\
\text { Number }\end{array}$ & $\begin{array}{c}\text { Lab Sample } \\
\text { Number }\end{array}$ & $\begin{array}{c}\text { Density } \\
\text { (g/mL) }\end{array}$ & Reported Value & Repo: \\
\hline Nitrate & $7 / 26 / 95$ & NA & $3 / 3 / 95$ & B009P9 & NA & NA & NA & 64700 & u \\
\hline $\mathrm{NaNO3}$ & $8 / 27 / 81$ & NS & NS & NS & NS & NS & 1.143 & 9.31E-01 & \\
\hline No3 & $12 / 28 / 83$ & NS & NS & NS & NS & NS & NS & $7.70 \mathrm{E}-01$ & \\
\hline No3- & NA & NA & NA & NA & NA & $\overline{N A}$ & 1.12 & $5.76 E-01$ & $M$ \\
\hline NO3 & $6 / 1 / 84$ & NS & NS & RAT-AZ101-2B & NS & NS & NS & 0.4 & $m$ \\
\hline NO3 & $6 / 1 / 84$ & NS & NS & RAT-AZ101-2A & NS & NS & NS & 0.39 & $m_{\prime}$ \\
\hline No3 & $9 / 10 / 84$ & $\mathrm{NA}$ & $6 / 1 / 84$ & NA & NA & NA & NA & $3.90 \mathrm{E}-01$ & \\
\hline NO3 & $1 / 1 / 81$ & NS & NS & NS & NS & NS & NS & NS & 1 \\
\hline NO3 & $8 \longdiv { 7 1 8 6 }$ & NS & NS & R9964/R0247 & NS & NS & 1.2 & 0.08 & $\mathrm{mi}$ \\
\hline $\mathrm{OH}$ & $12 / 3 / 79$ & NS & NS & 1464 & NS & NS & 1.409 & 3.04 & \\
\hline $\mathrm{OH}$ & $12 / 3 / 79$ & NS & NS & 1463 & NS & NS & 1.384 & 2.94 & \\
\hline $\mathrm{OH}$ & $12 / 3 / 79$ & NS & NS & 1495B & NS & NS & 1.448 & 2.7 & \\
\hline $\mathrm{OH}$ & $12 / 3 / 79$ & NS & NS & 1465 & NS & NS & 1.41 & 2.68 & \\
\hline $\mathrm{OH}$ & $12 / 3 / 79$ & NS & NS & $1494 A$ & NS & NS & 1.0414 & 2.66 & \\
\hline $\mathrm{OH}$ & $12 / 3 / 79$ & NS & NS & 1495A & NS & NS & 1.419 & 2.66 & \\
\hline $\mathrm{OH}$ & $12 / 3 / 79$ & NS & NS & $1494 B$ & NS & NS & 1.42 & 2.65 & \\
\hline $\mathrm{OH}$ & $12 / 3 / 79$ & NS & NS & 1466 & NS & NS & 1.414 & 2.64 & \\
\hline $\mathrm{OH}$ & $4 / 8 / 86$ & NA & $11 / 1 / 85$ & NA & NA & NA & NA & 1.1 & \\
\hline $\mathrm{OH}$ & $11 / 18 / 85$ & NS & NS & RAT-AZ101-1 & NS & NS & 1.17 & 1.09 & $\mathrm{mc}$ \\
\hline $\mathrm{OH}$ & $1 / 10 / 86$ & $\mathrm{NA}$ & $11 / 1 / 85$ & NA & $\mathrm{NA}$ & $\mathrm{NA}$ & 1.17 & 1.09 & \\
\hline $\mathrm{OH}$ & $10 / 29 / 90$ & $5 / 2 / 90$ & NA & R101283 & NA & NA & NA & 1.09 & \\
\hline $\mathrm{OH}$ & $8 / 7 / 86$ & NS & NS & R9964/R0247 & NS & NS & 1.2 & 0.95 & $\mathrm{mo}$ \\
\hline $\mathrm{OH}$ & $10 / 29 / 90$ & $5 / 2 / 90$ & NA & $R 280187$ & NA & NA & NA & 0.95 & \\
\hline $\mathrm{OH}$ & $10 / 1 / 87$ & NS & NS & RAT-AZ101-6A & NS & NS & NS & 0.9 & $\mathrm{mo}$ \\
\hline $\mathrm{OH}$ & $3 / 11 / 88$ & NA & NA & NA & NA & NA & NA & 0.9 & 7 \\
\hline $\mathrm{OH}$ & $2 / 8 / 89$ & NA & $10 / 1 / 87$ & T3493 & NA & NA & NA & 0.9 & 7 \\
\hline $\mathrm{OH}$ & $10 / 30 / 90$ & $5 / 2 / 90$ & $\mathrm{NA}$ & $R 110388$ & NA & NA & NA & 0.9 & 7 \\
\hline $\mathrm{OH}$ & $9 / 13 / 85$ & NS & NS & RAT-AZ101-3A & NS & NS & 1.17 & 0.688 & $\mathrm{mo}$ \\
\hline $\mathrm{OH}$ & $9 / 13 / 85$ & NA & NA & R130985 & NA & NA & NA & 0.688 & 7 \\
\hline $\mathrm{OH}$ & $9 / 13 / 85$ & NA & NA & NA & NA & NA & 1.17 & 0.688 & $T$ \\
\hline Hydroxide & $7 / 26 / 95$ & NA & $3 / 3 / 95$ & B0D9P4 & NA & NA & 1.2 & 12500 & UE \\
\hline Hydroxide & $7 / 26 / 95$ & NA & $3 / 3 / 95$ & BOD9P4 & NA & NA & 1.2 & 11400 & UE \\
\hline Hydraxide & $7 / 26 / 95$ & NA & $3 / 3 / 95$ & BOD9P1 & NA & NA & 1.18 & 11300 & $U_{1:}$ \\
\hline Hydroxide & $7 / 26 / 95$ & NA & $3 / 3 / 95$ & $\mathrm{~B} 009 \mathrm{P4}$ & NA & NA & 1.2 & 11300 & US \\
\hline Hydroxide & $7 / 26 / 95$ & NA & $3 / 3 / 95$ & B009P1 & NA & NA & 1.18 & 11250 & UE \\
\hline Hydroxide & $7 / 26 / 95$ & NA & $3 / 3 / 95$ & BOD9P1 & NA & NA & 1.18 & 11200 & UC \\
\hline Hydroxide & $7 / 26 / 95$ & NA & $3 / 3 / 95$ & BOD9N8 & NA & NA & 1.2 & 11200 & UG \\
\hline Hydroxide & $7 / 26 / 95$ & NA & $3 / 3 / 95$ & BOD9N8 & NA & NA & 1.2 & 11150 & UC \\
\hline Hydroxide & $7 / 26 / 95$ & NA & $3 / 3 / 95$ & BOD9N8 & NA & NA & 1.2 & 11100 & $\mathrm{UC}$ \\
\hline $\mathrm{NaOH}$ & $8 / 27 / 81$ & NS & NS & NS & NS & NS & 1.143 & 0.578 & 7 \\
\hline $\mathrm{OH}$ & $6 / 1 / 84$ & NS & NS & RAT-AZ101-2A & NS & NS & NS & 0.212 & mo \\
\hline $\mathrm{OH}$ & $9 / 10 / 84$ & NA & $6 / 1 / 84$ & NA & NA & NA & NA & 0.212 & 1 \\
\hline $\mathrm{OH}$ & $6 / 1 / 84$ & NS & NS & RAT-AZ101-2B & NS & NS & NS & 0.204 & $\mathrm{mo}$ \\
\hline $\mathrm{OH}$ & $9 / 10 / 84$ & NA & $6 / 1 / 84$ & NA & NA & NA & NA & 0.204 & 7 \\
\hline $\mathrm{OH}$ & $12 / 28 / 83$ & NS & NS & R281283 & NS & NS & NS & 0.19 & 7 \\
\hline $\mathrm{OH}$ & $12 / 28 / 83$ & NS & NS & NS & NS & NS & NS & 0.19 & 1 \\
\hline $\mathrm{NaOH}$ & $4 / 26 / 78$ & NS & NS & NS & NS & NS & 1.133 & 0.278 & $i$ \\
\hline $\mathrm{Pb}$ & $4 / 8 / 86$ & NA & $11 / 1 / 85$ & NA & NA & NA & NA & 6.30E-04 & 1 \\
\hline
\end{tabular}




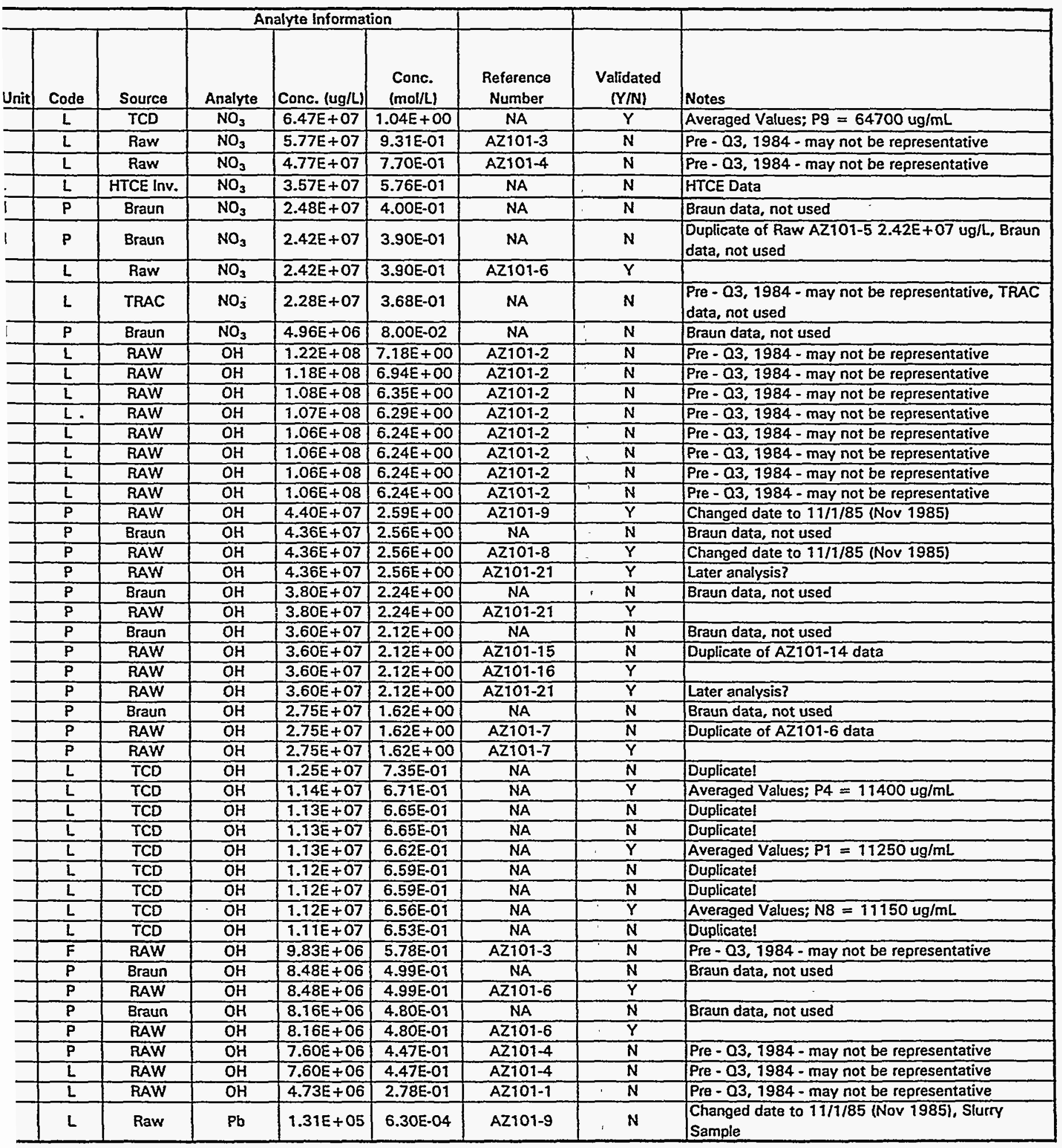




\begin{tabular}{|c|c|c|c|c|c|c|c|c|c|}
\hline & & & & k Waste Characteri & ation Sample & Information & & & \\
\hline Reported Chemical & $\begin{array}{c}\text { Memo } \\
\text { Date }\end{array}$ & $\begin{array}{c}\text { Start Analysis } \\
\text { Date } \\
\end{array}$ & $\begin{array}{c}\text { Start } \\
\text { Sample } \\
\text { Date }\end{array}$ & Sample Number & $\begin{array}{c}\text { Tank Farm } \\
\text { Number }\end{array}$ & $\begin{array}{c}\text { Lab Sample } \\
\text { Number }\end{array}$ & $\begin{array}{l}\text { Density } \\
\text { (g/mL) }\end{array}$ & Reported Value & $R \in$ \\
\hline $\mathrm{Pb}$ & $11 / 18 / 85$ & NS & NS & RAT-AZ101-1 & NS & NS & 1.17 & 0.0004 & \\
\hline $\mathrm{Pb}$ & $1 / 10 / 86$ & $\mathrm{NA}$ & $11 / 1 / 85$ & NA & NA & $\mathrm{NA}$ & 1.17 & 4.00E-04 & \\
\hline $\mathrm{Pb}$ & $9 / 1 / 90$ & NS & NS & RAT-AZ101-5A & NS & NS & 1.22 & 0.019 & $u$ \\
\hline $\mathrm{Pb}$ & May 89 & NS & NS & NS & NS & NS & NS & $4.80 E-03$ & \\
\hline $\mathrm{Pb} 2+$ & NA & NA & NA & NA & NA & NA & 1.12 & $7.89 \mathrm{E}-06$ & \\
\hline $\mathrm{Pb}$ & $1 / 1 / 81$ & NS & NS & NS & NS & NS & NS & NS & \\
\hline $\mathrm{PO4}$ & $4 / 8 / 86$ & NA & $11 / 1 / 85$ & NA & NA & NA & NA & $<0.025$ & \\
\hline PO4 & $12 / 28 / 83$ & NS & NS & NS & NS & NS & NS & 7.70E-02 & \\
\hline $\mathrm{Na3PO4}$ & $8 / 27 / 81$ & NS & NS & NS & NS & NS & 1.143 & $3.84 \mathrm{E}-02$ & \\
\hline $\mathrm{PO4}$ & $12 / 3 / 79$ & NS & NS & 1495B & NS & NS & 1.448 & $2.94 E-02$ & \\
\hline PO4 & $12 / 3 / 79$ & NS & NS & $1495 \mathrm{~A}$ & NS & NS & 1.419 & $2.90 E-02$ & \\
\hline $\mathrm{PO4}$ & $12 / 3 / 79$ & NS & NS & $1494 \mathrm{~A}$ & NS & NS & 1.0414 & 2.83E-02 & \\
\hline PO4 & $11 / 18 / 85$ & NS & NS & RAT-AZ101-1 & NS & NS & 1.17 & 0.028 & 7 \\
\hline PO4 & $12 / 3 / 79$ & NS & NS & 1465 & NS & NS & 1.41 & $2.80 E-02$ & \\
\hline P04 & $1 / 10 / 86$ & NA & $11 / 1 / 85$ & $\mathrm{NA}$ & NA & NA & 1.17 & $2.80 \mathrm{E}-02$ & \\
\hline$P 04$ & $9 / 1 / 90$ & NS & NS & RAT-AZ101-5A & NS & NS & 1.22 & 22.8 & u \\
\hline PO4 & $12 / 3 / 79$ & NS & NS & $1494 \mathrm{~B}$ & NS & NS & 1.42 & 2.73E-02 & \\
\hline $\mathrm{PO4}$ & $12 / 3 / 79$ & NS & NS & 1464 & NS & NS & 1.409 & 2.60E-02 & \\
\hline $\mathrm{PO4}$ & $12 / 3 / 79$ & NS & NS & 1466 & NS & NS & 1.414 & $2.40 \mathrm{E}-02$ & \\
\hline PO4 & $12 / 3 / 79$ & NS & NS & 1463 & NS & NS & 1.384 & $2.20 \mathrm{E}-02$ & \\
\hline P04 & $3 / 1 / 89$ & NS & NS & RAT-AZ101-4A & NS & NS & 1.2 & 0.017 & $m$ \\
\hline $\mathrm{PO} 4$ & $10 / 1 / 87$ & NS & NS & RAT-AZ101-6A & NS & NS & NS & 0.017 & n \\
\hline PO4 & $3 / 11 / 88$ & NA & NA & T3493 & NA & NA & NA & 1.70E-02 & \\
\hline PO4 & $3 / 11 / 88$ & NA & NA & T3493 & NA & NA & NA & $1.70 E-02$ & \\
\hline $\mathrm{PO4}$ & $2 / 8 / 89$ & NA & $10 / 1 / 87$ & T3493 & NA & NA & NA & $1.70 \mathrm{E}-02$ & \\
\hline Phosphate & $7 / 26 / 95$ & NA & $3 / 3 / 95$ & B0D9P9 & NA & NA & NA & 1365 & 1 \\
\hline Phosphate & $7 / 26 / 95$ & NA & $3 / 3 / 95$ & BOD9P7 & NA & NA & NA & 1335 & i \\
\hline PO43- & NA & NA & $\mathrm{NA}$ & NA & NA & NA & 1.12 & $1.39 \mathrm{E}-02$ & 1 \\
\hline Phosphate & $7 / 26 / 95$ & NA & $3 / 3 / 95$ & 80D9P6 & NA & NA & NA & 1220 & $\mathrm{~T}$ \\
\hline PO4- & May 89 & NS & NS & NS & NS & NS & NS & $3.24 E-01$ & \\
\hline Se & $9 / 1 / 89$ & NS & NS & RAT-AZ101-4A & NS & NS & 1.2 & 0.003 & $m r$ \\
\hline Se & $9 / 29 / 89$ & NA & $4 / 1 / 89$ & Core 1 & NA & NA & 1.2 & 0.003 & $m$ \\
\hline $\mathrm{Se}$ & $9 / 29 / 89$ & $\mathrm{NA}$ & $4 / 1 / 89$ & Core 1 & NS & NS & 1.2 & 0.0000633 & $m$ \\
\hline 504 & $12 / 3 / 79$ & NS & NS & $1495 \mathrm{~A}$ & NS & NS & 1.419 & $2.31 E-01$ & \\
\hline SO4- & May 89 & NS & NS & NS & NS & NS & NS & $1.88 \mathrm{E}+01$ & \\
\hline SO4 & $9 / 1 / 90$ & NS & NS & RAT-AZ101-5A & NS & NS & 1.22 & 160 & un \\
\hline Sulfate & $7 / 26 / 95$ & NA & $3 / 3 / 95$ & B0D9P7 & NA & NA & NA & 16000 & $\bar{U}$ \\
\hline 504 & $3 / 11 / 88$ & NA & NA & $\mathrm{T3494}$ & $\mathrm{NA}$ & $\mathrm{NA}$ & NA & $1.62 \mathrm{E}-01$ & \\
\hline $\mathrm{SO} 4$ & $10 / 1 / 87$ & NS & NS & RAT-AZ101-6A & NS & NS & NS & 0.16 & $\mathbf{m}$ \\
\hline 504 & $3 / 11 / 88$ & NA & NA & T3493 & NA & NA & NA & $1.60 \mathrm{E}-01$ & \\
\hline 504 & $2 / 8 / 89$ & NA & $10 / 1 / 87$ & T3493 & NA & NA & NA & $1.60 \mathrm{E}-01$ & \\
\hline Sulfate & $7 / 26 / 95$ & NA & $3 / 3 / 95$ & B0D9P6 & $\mathrm{NA}$ & NA & NA & 15200 & $\mathrm{u}$ \\
\hline
\end{tabular}




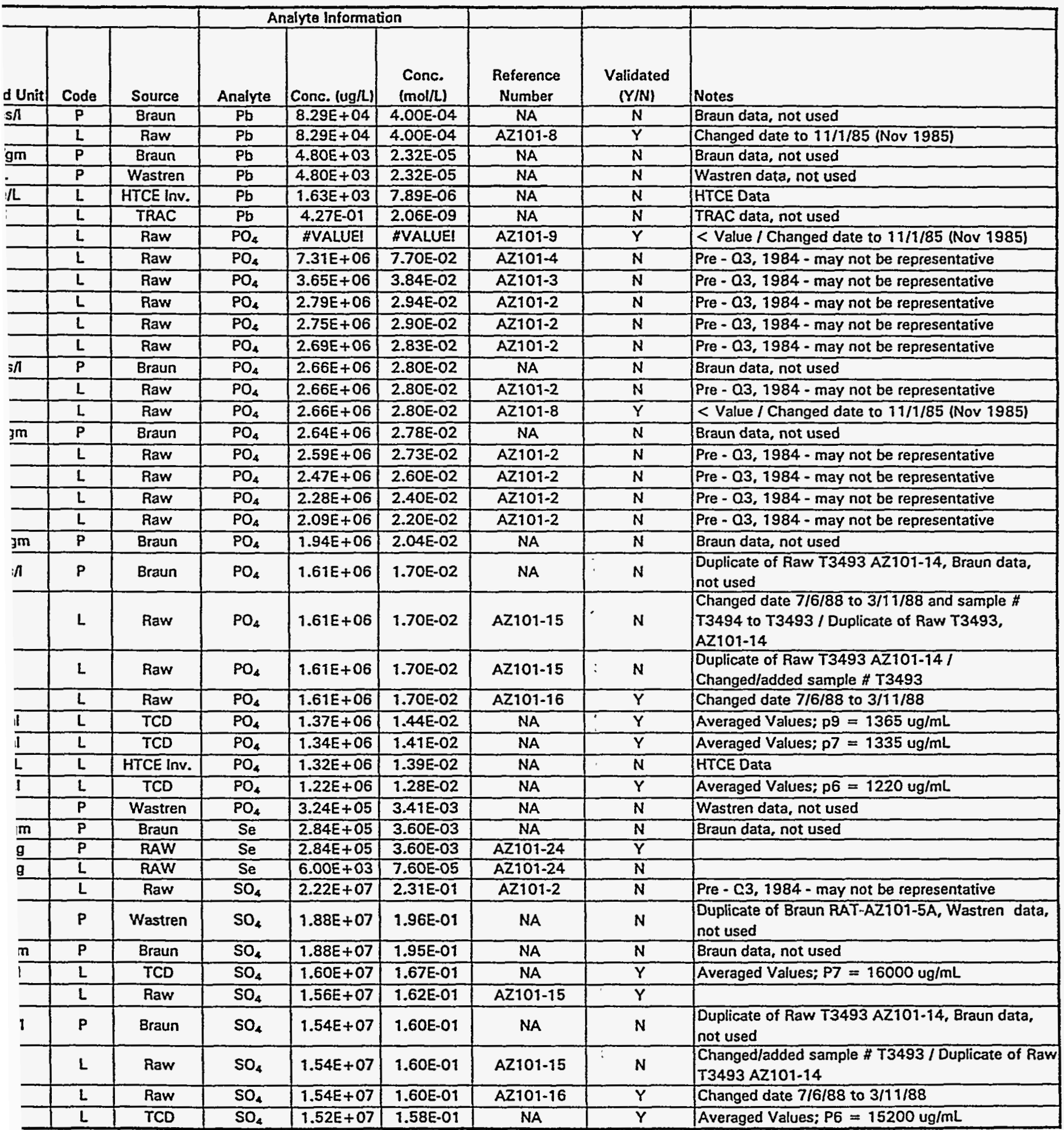




\begin{tabular}{|c|c|c|c|c|c|c|c|c|c|}
\hline \multicolumn{10}{|c|}{ Tank Waste Characterization Sample Information } \\
\hline Reported Chemical & $\begin{array}{c}\text { Memo } \\
\text { Date }\end{array}$ & $\begin{array}{c}\text { Start Analysis } \\
\text { Date }\end{array}$ & $\begin{array}{c}\text { Start } \\
\text { Sample } \\
\text { Date }\end{array}$ & Sample Number & $\begin{array}{l}\text { Tank Farm } \\
\text { Number }\end{array}$ & $\begin{array}{c}\text { Lab Sample } \\
\text { Number }\end{array}$ & $\begin{array}{l}\text { Density } \\
\text { (g/mL) }\end{array}$ & Reported Value & $\operatorname{Re} \mid$ \\
\hline Sulfate & $7 / 26 / 95$ & NA & $3 / 3 / 95$ & BOD9P9 & NA & NA & NA & 15100 & \\
\hline SO4 & $9 / 1 / 89$ & NS & NS & RAT-AZ101-4A & NS & NS & 1.2 & 0.13 & $n$ \\
\hline so4 & $11 / 18 / 85$ & NS & NS & RAT-AZ101-1 & NS & NS & 1.17 & 0.151 & \\
\hline 504 & $1 / 10 / 86$ & NA & $11 / 1 / 85$ & NA & $\mathrm{NA}$ & $\mathrm{NA}$ & 1.17 & $1.51 \mathrm{E}-01$ & \\
\hline so4 & $4 / 8 / 86$ & NA & $11 / 1 / 85$ & NA & NA & NA & NA & $1.50 \mathrm{E}-01$ & \\
\hline S042- & $\overline{N A}$ & NA & NA & $\overline{N A}$ & NA & NA & 1.12 & $1.21 \mathrm{E} \cdot 01$ & \\
\hline SO4 & $9 / 13 / 85$ & NS & NS & RAT-AZ101-3A & NS & NS & 1.17 & 0.105 & \\
\hline 504 & $9 / 13 / 85$ & NA & NA & NA & NA & $\overline{\mathrm{NA}}$ & 1.17 & $1.05 \mathrm{E}-01$ & \\
\hline 504 & $12 / 3 / 79$ & NS & NS & $1494 \mathrm{~A}$ & NS & NS & 1.0414 & $5.30 \mathrm{E}-02$ & \\
\hline SO4 & $6 / 1 / 84$ & NS & NS & RAT-AZ101-2B & NS & NS & NS & 0.025 & \\
\hline SO4 & $6 / 1 / 84$ & NS & NS & RAT-AZ101-2A & NS & NS & NS & 0.023 & , \\
\hline 504 & $9 / 10 / 84$ & NA & $6 / 1 / 84$ & $\overline{N A}$ & NA & $\overline{N A}$ & $\overline{N A}$ & $2.30 \mathrm{E}-02$ & \\
\hline SO4 & $1 / 1 / 81$ & NS & NS & NS & NS & NS & NS & NS & \\
\hline 504 & $12 / 28 / 83$ & NS & NS & NS & NS & NS & NS & $1.00 \mathrm{E}-02$ & \\
\hline SO4 & $8 / 7 / 86$ & NS & NS & R9964/R0247 & NS & NS & 1.2 & 0.00688 & 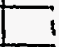 \\
\hline $\mathrm{Sr}$ & $9 / 1 / 89$ & NS & NS & RAT-AZ101 & NS & NS & 1.2 & 0.00001 & $n$ \\
\hline $\mathrm{Sr}$ & $9 / 29 / 89$ & NA & $4 / 1 / 89$ & Core 1 & NA & NA & 1.2 & 0.00001 & $i$ \\
\hline $\mathrm{Sr}$ & $9 / 1 / 93$ & NA & $4 / 1 / 89$ & Core 2 & NA & NA & 1.24 & 0.002 & 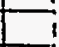 \\
\hline $\mathrm{Sr}$ & $9 / 1 / 90$ & NS & NS & RAT-AZ101 & NS & NS & 1.22 & 0.002 & 4 \\
\hline $\mathrm{Sr}$ & $5 / 1 / 89$ & NS & NS & NS & NS & NS & NS & 0.000214 & \\
\hline $\mathrm{Sr}$ & $1 / 10 / 86$ & NA & $11 / 1 / 85$ & NA & NA & NA & NA & 0.0000609 & \\
\hline $\mathrm{Te}$ & $9 / 1 / 93$ & NA & $4 / 1 / 89$ & Core 2 & NA & NA & 1.24 & 0.017 & $i$ \\
\hline $\mathrm{Te}$ & $9 / 1 / 90$ & NS & NS & RAT-AZ101-5A & NS & NS & 1.22 & 0.017 & u \\
\hline $\mathrm{Te}$ & $5 / 1 / 89$ & NS & NS & NS & NS & NS & NS & 0.00265 & \\
\hline TOC & $4 / 26 / 78$ & NS & NS & NS & NS & NS & 1.133 & $2.80 E+01$ & \\
\hline TOC & $12 / 3779$ & NS & NS & 1495B & NS & NS & 1.448 & $1.70 E+01$ & \\
\hline TOC & $12 / 3 / 79$ & NS & NS & 1495A & NS & NS & 1.419 & $1.61 \mathrm{E}+01$ & \\
\hline TOC & $12 / 3 / 79$ & NS & NS & $1494 \mathrm{~A}$ & NS & NS & 1.0414 & $1.58 E+01$ & \\
\hline TOC & $12 / 3 / 79$ & NS & NS & 1465 & NS & NS & 1.41 & $1.36 \mathrm{E}+01$ & \\
\hline TOC & $12 / 3 / 79$ & NS & NS & 1466 & NS & NS & 1.414 & $1.36 E+01$ & \\
\hline TOC & $12 / 3 / 79$ & NS & NS & $1494 \mathrm{~B}$ & NS & NS & 1.42 & $1.31 \mathrm{E}+01$ & \\
\hline TOC & $12 / 3 / 79$ & NS & NS & 1463 & NS & NS & 1.384 & $1.16 E+01$ & \\
\hline TOC & $12 / 3 / 79$ & NS & NS & 1464 & NS & NS & 1.409 & $1.16 \mathrm{E}+01$ & \\
\hline TOC & $12 / 3 / 79$ & NS & NS & 1494A & NS & NS & 1.0414 & $9.84 E+00$ & \\
\hline TOC & $12 / 3 / 79$ & NS & NS & 1494B & NS & NS & 1.42 & $9.08 E+00$ & \\
\hline TOC & $12 / 28 / 83$ & NS & NS & NS & NS & NS & NS & $7.31 \mathrm{E}-01$ & \\
\hline TOC & $8 / 27 / 81$ & NS & NS & NS & NS & NS & 1.143 & $3.06 \mathrm{E}+00$ & \\
\hline TOC & $1 / 10 / 86$ & NA & $11 / 1 / 85$ & NA & NA & NA & NA & $2.29 E+\infty 0$ & \\
\hline TOC $w$ t $C$ (wet) & NA & NA & NA & NA & $\overline{\mathrm{NA}}$ & NA & 1.12 & 1.67E-01 & \\
\hline TOC & $4 / 8 / 86$ & NA & $11 / 1 / 85$ & NA & NA & NA & NS & $1.70 E+00$ & \\
\hline TOC & $9 / 13 / 85$ & NS & NS & RAT-AZ101-3A & NS & NS & 1.17 & 1.09 & \\
\hline
\end{tabular}




\begin{tabular}{|c|c|c|c|c|c|c|c|c|}
\hline \multirow[b]{2}{*}{ dUnit } & \multirow[b]{2}{*}{ Code } & \multirow[b]{2}{*}{ Source } & \multicolumn{3}{|c|}{ Analyte-Information } & \multirow[b]{2}{*}{$\begin{array}{l}\text { Reference } \\
\text { Number }\end{array}$} & \multirow[b]{2}{*}{$\begin{array}{l}\text { Validated } \\
\text { (Y/N) }\end{array}$} & \multirow[b]{2}{*}{ Notes } \\
\hline & & & Analyte & Conc. (ug/L) & $\begin{array}{l}\text { Conc. } \\
\text { (mol/L) }\end{array}$ & & & \\
\hline$\overline{m l}$ & L & TCD & $\mathrm{SO}_{4}$ & $1.51 E+07$ & $1.57 \mathrm{E}-01$ & NA & $\bar{\gamma}$ & Averaged Values; P9 $=15100 \mathrm{ug} / \mathrm{mL}$ \\
\hline $\operatorname{lgm}$ & $\mathbf{P}$ & Braun & $\mathrm{SO}_{4}$ & $1.50 E+07$ & $1.56 \mathrm{E}-01$ & NA & $N$ & Braun data, not used \\
\hline \multirow[t]{3}{*}{ sn } & $\mathbf{P}$ & Braun & $\mathrm{SO}_{4}$ & $1.45 E+07$ & $1.51 E-01$ & NA & $\mathbf{N}$ & $\begin{array}{l}\text { Duplicate of Raw AZ101-7 1.45E +07 ug/L, Braun } \\
\text { data, not used }\end{array}$ \\
\hline & $\mathbf{L}$ & Raw & $\mathrm{SO}_{4}$ & $1.45 E+07$ & 1.51E-01 & A2101.8 & $\bar{Y}$ & Changed date to $11 / 1 / 85$ (Nov 1985 ) \\
\hline & $\mathbf{P}$ & Raw & $\mathrm{SO}_{4}$ & $1.44 E+07$ & $1.50 E-01$ & AZ101-9 & $\mathbf{Y}$ & $\begin{array}{l}\text { Changed code } L \text { to } P \text { added sample state } \\
\text { Supernatant / Changed date to } 11 / 1 / 85 \text { (Nov 1985) }\end{array}$ \\
\hline$\overline{1 / L}$ & $L$ & HTCE Inv. & $\mathrm{SO}_{4}$ & $1.16 E+07$ & $1.21 E-01$ & NA & $N$ & HTCE Data \\
\hline \multirow[t]{3}{*}{$s \AA$} & $\mathbf{P}$ & Braun & $\mathrm{SO}_{4}$ & $1.01 E+07$ & $1.05 E-01$ & NA & $\mathbf{N}$ & $\begin{array}{l}\text { Duplicate of Raw AZ101-6 1.01E7 ug/L, Braun } \\
\text { data, not used }\end{array}$ \\
\hline & L & Raw & $\mathrm{SO}_{4}$ & $\mid 1.01 E+07$ & $1.05 \mathrm{E}-01$ & AZ101-7 & $Y$ & \\
\hline & L & Raw & $\mathrm{SO}_{4}$ & $5.09 E+06$ & $5.31 E-02$ & AZ101-2 & $\mathrm{N}$ & Pre - 03, 1984 - may not be representative \\
\hline$\overline{s \pi}$ & $P$ & Braun & $\mathrm{SO}_{4}$ & $2.40 E+06$ & $2.50 E-02$ & NA & $N$ & Braun data, not used \\
\hline \multirow[t]{2}{*}{$s / 1$} & $\mathbf{P}$ & Braun & $\mathrm{SO}_{4}$ & $2.21 E+06$ & $2.30 \mathrm{E}-02$ & NA & $\mathbf{N}$ & $\begin{array}{l}\text { Duplicate of Raw AZ101-5 2.21E + 06 ug/L, Braun } \\
\text { data, not used }\end{array}$ \\
\hline & $L$ & Raw & $\mathrm{SO}_{4}$ & $2.21 E+06$ & $2.30 \mathrm{E}-02$ & $A Z 101-6$ & Y & \\
\hline \multirow[t]{2}{*}{ i } & $\mathbf{L}$ & TRAC & $\mathrm{SO}_{4}$ & $1.21 \mathrm{E}+06$ & $1.27 \mathrm{E}-02$ & NA & $\mathbf{N}$ & TRAC data - not used \\
\hline & $L$ & Raw & $\mathrm{SO}_{4}$ & $9.61 E+05$ & $1.00 \mathrm{E}-02$ & $A Z 101-4$ & $N$ & Pre - 03, 1984 - may not be representative \\
\hline$s \pi$ & $\mathbf{P}$ & Braun & $\mathrm{SO}_{4}$ & $6.61 E+05$ & $6.89 \mathrm{E}-03$ & NA & $\mathbf{N}$ & Braun data, not used \\
\hline $\operatorname{gm}$ & $\mathbf{P}$ & Braun & Sr & $1.05 E+03$ & $1.20 E-05$ & NA & N & $\begin{array}{l}\text { Duplicate of Raw AZ101-22 1.05E+03 ug/L, Braun } \\
\text { data, not used }\end{array}$ \\
\hline$\overline{l g}$ & $\mathrm{P}$ & RAW & $\mathrm{Sr}$ & $1.05 E+03$ & $1.20 \mathrm{E}-05$ & AZ101-24 & $\bar{Y}$ & Total Sr! \\
\hline $\lg$ & $\mathbf{P}$ & RAW & Sr & $2.17 E+02$ & $2.48 \mathrm{E}-06$ & AZ101-19 & $\bar{Y}$ & Total Sr! \\
\hline \multirow[t]{2}{*}{ gm } & $P$ & Braun & Sr & $2.14 E+02$ & $2.44 \mathrm{E}-06$ & NA & $\mathrm{N}$ & Braun data, not used \\
\hline & $\mathbf{P}$ & Wastren & Sr & $2.14 E+02$ & 2.44E-06 & NA & $\mathbf{N}$ & $\begin{array}{l}\text { Duplicate of Braun RAT-AZ101 2.14E+02 ug/h, } \\
\text { Wastren data, not used }\end{array}$ \\
\hline$?$ & $\mathbf{P}$ & RAW & $\mathrm{Sr}$ & $6.70 \mathrm{E}-02$ & $7.65 \mathrm{E}-10$ & AZ101-8 & $\mathbf{N}$ & $\begin{array}{l}\text { Siurry sample / Changed date to } 11 / 1 / 85 \text { (Nov } \\
1985 \text { ) }\end{array}$ \\
\hline$\underline{\lg }$ & $P$ & RAW & $\mathrm{Te}$ & $2.69 E+03$ & $2.11 E-05$ & AZ101-19 & $\bar{Y}$ & \\
\hline \multirow[t]{16}{*}{$g m$} & $P$ & Braun & Te & $2.65 E+03$ & $2.08 \mathrm{E}-05$ & NA & $\mathbf{N}$ & Braun data, not used \\
\hline & $P$ & Wastren & Te & $2.65 E+03$ & $2.08 \mathrm{E}-05$ & NA & $\mathbf{N}$ & Wastren data, not used \\
\hline & $L$ & Raw & TOC & $2.80 E+07$ & $2.33 E+00$ & AZ101-1 & $\mathbf{N}$ & Pre - 03, 1984 - may not be representative \\
\hline & L & Raw & TOC & $1.70 E+07$ & $1.42 E+00$ & AZ101-2 & $\mathbf{N}$ & Pre - 03, 1984-may not be representative \\
\hline & $\bar{L}$ & Raw & TOC & $1.61 E+07$ & $1.34 E+00$ & AZ101-2 & $\mathbf{N}$ & Pre - 03, 1984-may not be representative \\
\hline & $\mathrm{L}$ & Raw & TOC & $1.58 E+07$ & $1.32 E+00$ & AZ101-2 & $N$ & Pre $-03,1984-$ may not be representative \\
\hline & $\mathrm{L}$ & Raw & TOC & $1.36 E+07$ & $1.13 E+00$ & $A Z 101-2$ & $N$ & Pre - $03,1984-$ may not be representative \\
\hline & $\bar{L}$ & Raw & TOC & $1.36 \mathrm{E}+07$ & $1.13 E+00$ & $A Z 101-2$ & $\mathbf{N}$ & Pre $-03,1984-$ may not be representative \\
\hline & $L$ & Raw & TOC & $1.31 E+07$ & $1.09 E+00$ & $A Z 101-2$ & $N$ & Pre - 03, $1984-$ may not be representative \\
\hline & $\mathrm{L}$ & $\overline{\text { Raw }}$ & TOC & $1.16 E+07$ & $9.67 E-01$ & AZ101-2 & $N$ & Pre $-03,1984-$ may not be representative \\
\hline & $\mathrm{L}$ & Raw & TOC & $1.16 \mathrm{E}+07$ & $9.67 E-01$ & AZ101-2 & $\mathbf{N}$ & Pre - 03, 1984-may not be representative \\
\hline & $\mathbf{L}$ & Raw & TOC & $9.84 E+06$ & $8.20 E-01$ & AZ101-2 & $\bar{N}$ & Pre - 03,1984 - may not be representative \\
\hline & $L$ & Raw & TOC & $9.08 \mathrm{E}+06$ & $7.57 E-01$ & $A Z 101-2$ & $N$ & Pre - 03,1984-may not be representative \\
\hline & $\mathbf{L}$ & Raw & TOC & $8.78 E+06$ & $7.32 \mathrm{E}-01$ & $A Z 101-4$ & $\mathrm{~N}$ & Pre - 03, 1984 - may not be representative \\
\hline & $\mathbf{L}$ & Raw & TOC & $3.06 \mathrm{E}+06$ & $2.55 \mathrm{E}-01$ & AZ101.3 & $\mathbf{N}$ & Pre - 03, 1984 - may not be representative \\
\hline & $\mathbf{L}$ & Raw & TOC & $2.29 E+06$ & $1.91 E-01$ & $A Z 101-8$ & N & $\begin{array}{l}\text { Slurry Sample / Changed date to } 11 / 1 / 85 \text { (Nov } \\
\text { 1985) }\end{array}$ \\
\hline \multirow[t]{2}{*}{5} & $L$ & HTCE Inv. & TOC & $1.87 E+06$ & $1.56 E-01$ & NA & $N$ & HTCE Data \\
\hline & $L$ & Raw & TOC & $1.70 E+06$ & $1.42 \mathrm{E}-01$ & $A Z 101-9$ & $\mathbf{N}$ & $\begin{array}{l}\text { Slurry Sample / Changed date to } 11 / 1 / 85 \text { (Nov } \\
1985 \text { ) }\end{array}$ \\
\hline$i$ & $\bar{P}$ & Braun & TOC & $1.09 E+06$ & $9.08 E-02$ & NA & $\mathbf{N}$ & Braun data, not used \\
\hline
\end{tabular}




\begin{tabular}{|c|c|c|c|c|c|c|c|c|c|}
\hline & \multicolumn{9}{|c|}{ Tank Waste Characterization Sample Information } \\
\hline Reported Chemical & $\begin{array}{c}\text { Memo } \\
\text { Date }\end{array}$ & $\begin{array}{c}\text { Start Analysis } \\
\text { Date } \\
\end{array}$ & $\begin{array}{c}\text { Start } \\
\text { Sample } \\
\text { Date } \\
\end{array}$ & Sample Number & $\begin{array}{c}\text { Tank Famm } \\
\text { Number }\end{array}$ & $\begin{array}{c}\text { Lab Sample } \\
\text { Number }\end{array}$ & $\begin{array}{l}\text { Density } \\
\text { (g/mL) }\end{array}$ & Reported Value & Repe \\
\hline TOC & $9 / 13 / 85$ & NA & NA & $\mathrm{NA}$ & NA & NA & 1.17 & $1.09 \mathrm{E}+00$ & \\
\hline Total organic carbon & $7 / 26 / 95$ & NA & $3 / 3 / 95$ & BOD9P9 & NA & NA & $\mathrm{NA}$ & 1090 & $T$ \\
\hline Total organic carbon & $7 / 26 / 95$ & NA & $3 / 3 / 95$ & BOD9P7 & NA & NA & NA & 1075 & 7 \\
\hline Total organic carbon & $7 / 26 / 95$ & NA & $3 / 3 / 95$ & BOD9P6 & NA & NA & NA & 938 & $\mathrm{i}$ \\
\hline TOC & $11 / 18 / 85$ & NS & NS & RAT-AZ101-1 & NS & NS & 1.17 & 0.562 & \\
\hline TOC & $1 / 10 / 86$ & NA & $11 / 1 / 85$ & NA & NA & NA & 1.17 & $5.62 E-01$ & \\
\hline TOC & $10 / 1 / 87$ & NS & NS & RAT-AZ101-6A & NS & NS & NS & 0.43 & ! \\
\hline TOC & $3 / 11 / 88$ & NA & NA & NA & NA & NA & NA & $4.30 E-01$ & \\
\hline TOC & $3 / 11 / 88$ & NA & NA & T3493 & NA & NA & NA & 4.30E-01 & \\
\hline TOC & $2 / 8 / 89$ & NA & $10 / 1 / 87$ & T3493 & NA & NA & $\mathrm{NA}$ & $4.30 \mathrm{E}-01$ & \\
\hline TOC & May 89 & NS & NS & NS & NS & NS & NS & $1.24 \mathrm{E}-01$ & \\
\hline $\mathbf{U}$ & $1 / 10 / 86$ & NA & $11 / 1 / 85$ & NA & NA & NA & NA & 0.311 & \\
\hline U & $9 / 1 / 89$ & NS & NS & RAT-AZ101-4A & NS & NS & 1.2 & 0.000025 & $\mathrm{mi}$ \\
\hline U & $9 / 29 / 89$ & NA & $4 / 1 / 89$ & Core 1 & NA & NA & 1.2 & 0.000025 & $m$ \\
\hline U & $9 / 13 / 85$ & NS & NS & RAT-AZ101-3A & NS & NS & 1.17 & 0.0059 & \\
\hline U & $9 / 13 / 85$ & NA & NA & NA & NA & NA & 1.17 & 0.0059 & \\
\hline U & $9 / 29 / 89$ & NA & $4 / 1 / 89$ & Core 1 & NA & NA & 1.2 & 0.0000175 & $\pi$ \\
\hline $\mathbf{U}$ & $11 / 18 / 85$ & NS & NS & RAT-AZ101-1A & NS & NS & NS & 0.0049 & \\
\hline$U$ & $9 / 13 / 85$ & NA & NA & NA & NA & NA & 1.17 & 0.000106 & \\
\hline $\mathrm{V}$ & 9/1/93 & NA & $4 / 1 / 89$ & Core 2 & NA & NA & 1.24 & 0.014 & ur \\
\hline $\mathrm{v}$ & $9 / 1 / 90$ & NS & NS & RAT-AZ101-5A & NS & NS & 1.22 & 0.014 & uni \\
\hline$v$ & $5 / 1 / 89$ & NS & NS & NS & NS & NS & NS & 0.00087 & \\
\hline
\end{tabular}




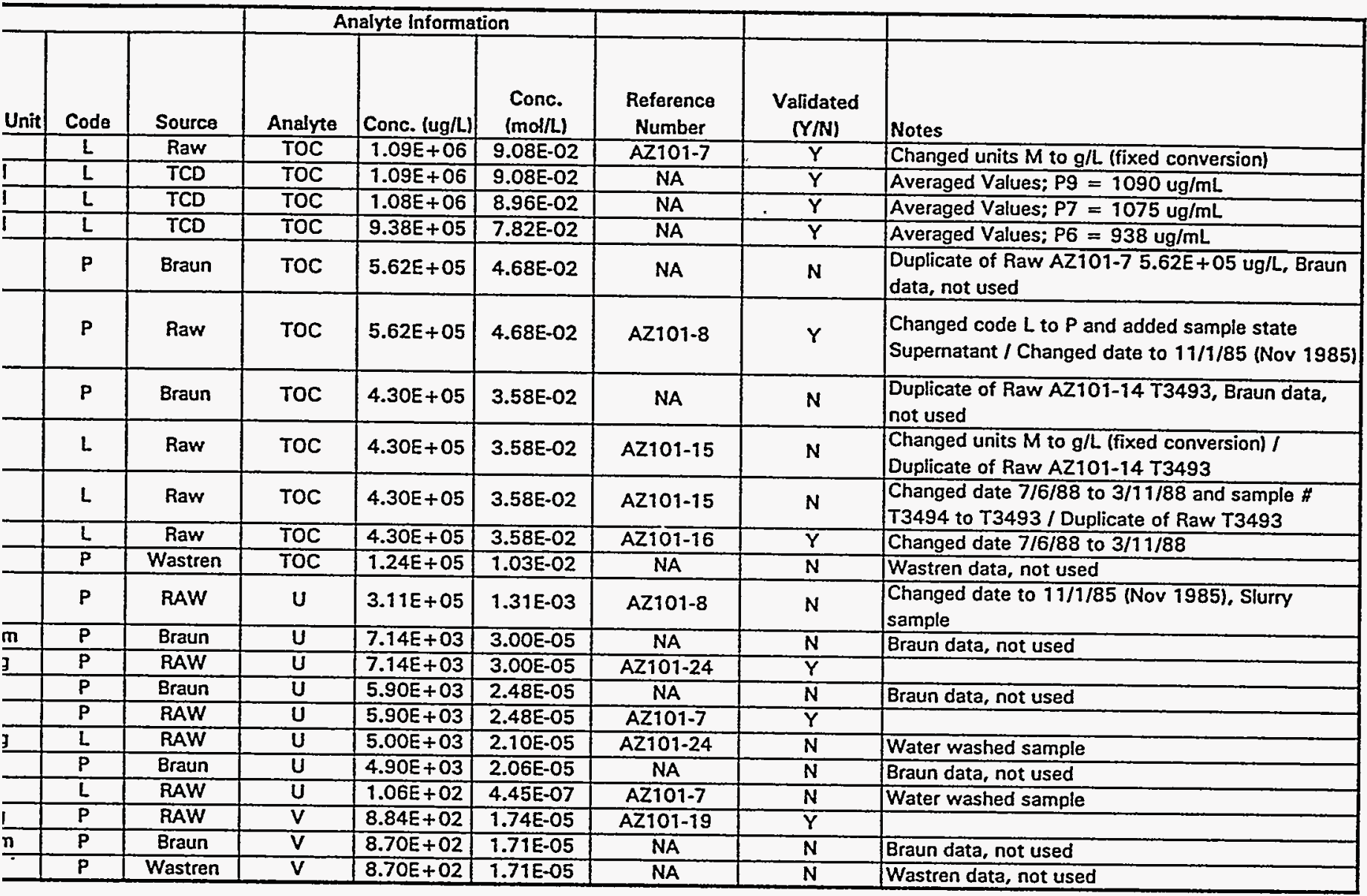




\begin{tabular}{|c|c|c|c|c|c|c|c|c|c|}
\hline & & & & Tank Waste & racterization & ample Informati & & & \\
\hline $\begin{array}{l}\text { Reported } \\
\text { Chemical }\end{array}$ & Memo Date & $\begin{array}{c}\text { Start } \\
\text { Analysis } \\
\text { Date } \\
\end{array}$ & $\begin{array}{c}\text { Start Sample } \\
\text { Date }\end{array}$ & Sample Number & $\begin{array}{c}\text { Tank Farm } \\
\text { Number }\end{array}$ & $\begin{array}{c}\text { Lab Sample } \\
\text { Number }\end{array}$ & $\begin{array}{c}\text { Density } \\
\text { (g/mL) }\end{array}$ & $\begin{array}{c}\text { Reported } \\
\text { Value }\end{array}$ & Reported U \\
\hline $\mathrm{Al}$ & $1 / 1 / 77$ & NS & NS & NS & NS & NS & NS & NS & NS \\
\hline $\mathrm{Al}$ & $10 / 31 / 86$ & NS & $6 / 30 / 85$ & NS & NS & NS & 1.73 & $1.99 \mathrm{E}+03$ & umol/g \\
\hline Al & Aug 89 & NS & NS & NS & NS & NS & NS & $8.00 E+01$ & $g / L$ \\
\hline Al & $10 / 31 / 86$ & NS & $6 / 30 / 85$ & NS & NS & NS & 1.11 & $4.82 \mathrm{E}+02$ & umol/g \\
\hline Cd & $8 / 31 / 89$ & NS & $6 / 30 / 85$ & Composite Core & NS & NS & 1.11 & $4.82 E+02$ & umol/g \\
\hline $\mathrm{Al3}+$ & NS & NS & NS & NS & NS & NS & 1.39 & $5.18 \mathrm{E}-01$ & Mole/L \\
\hline Al & $11 / 4 / 81$ & NS & NS & RAT-AZ102-2B & NS & NS & 1.4 & 4.70E-01 & moles $n$ \\
\hline $\mathrm{Al}$ & $5 / 5 / 82$ & NS & $11 / 4 / 81$ & NS & NS & NS & 1.4 & 4.70E-01 & $M$ \\
\hline AI & $2 / 3 / 81$ & NS & NS & R-3699 & NS & NS & 1.11 & $2.38 \mathrm{E}-01$ & moles $/ 1$ \\
\hline $\mathrm{Al}$ & $2 / 4 / 81$ & NS & NS & R3699 & NS & NS & 1.11 & $2.38 \mathrm{E}-01$ & $M$ \\
\hline Al & $2 / 3 / 81$ & NS & NS & $R-3700$ & NS & NS & 1.11 & 2.22E-01 & moles $/$ \\
\hline $\mathrm{Al}$ & $2 / 4 / 81$ & NS & NS & R3700 & NS & NS & 1.11 & $2.22 \mathrm{E}-01$ & $M$ \\
\hline$\overline{\mathrm{Al}}$ & $6 / 25 / 75$ & NS & NS & RAT-AZ102-4a & NS & NS & 1.39 & $1.80 E-01$ & moles $/$ \\
\hline $\mathrm{Al}$ & $6 / 26 / 75$ & NS & NS & NS & NS & NS & 1.392 & $1.80 \mathrm{E}-01$ & $M$ \\
\hline $\mathrm{Al}$ & $12 / 3 / 75$ & NS & NS & 1456 & NS & NS & 1.393 & $1.36 \mathrm{E}-01$ & $M$ \\
\hline $\mathrm{Al}$ & $7 / 27 / 79$ & NS & NS & RAT-AZ102-4D & NS & NS & 1.41 & $1.35 \mathrm{E}-01$ & moles/l \\
\hline $\mathrm{NaAlO2}$ & $7 / 28 / 79$ & NS & NS & NS & NS & NS & 1.41 & $1.35 \mathrm{E}-01$ & $M$ \\
\hline $\mathrm{AlO2}$ & $7 / 28 / 79$ & NS & NS & RAT-AZ102-6 & NS & NS & 1.41 & $1.35 \mathrm{E}-01$ & moles/ \\
\hline $\mathrm{Al}$ & $2 / 19 / 82$ & NS & NS & T3251 & NS & NS & 1.03 & 1.30E-01 & $M$ \\
\hline $\mathrm{Al}$ & $11 / 5 / 81$ & NS & NS & RAT-AZ102-2A & NS & NS & 1.09 & $1.20 \mathrm{E}-01$ & moles/l \\
\hline Al & $5 / 5 / 82$ & NS & NS & NS & NS & NS & 1.09 & $1.20 \mathrm{E}-01$ & $M$ \\
\hline Al & $10 / 30 / 86$ & NS & NS & $\mathrm{R} 280783$ & NS & NS & NS & $1.20 \mathrm{E}-01$ & $\bar{M}$ \\
\hline$A 13+$ & NS & NS & NS & NS & NS & NS & 1.04 & $1.09 E-01$ & Mole/L \\
\hline $\mathrm{Al}$ & $10 / 1 / 83$ & NS & NS & RAT-AZ102-3 & NS & NS & NS & 1.00E-01 & moles $/$ \\
\hline Al & $3 / 10 / 84$ & NS & NS & NS & NS & NS & NS & 1.00E-01 & $M$ \\
\hline $\mathrm{Al}$ & $7 / 5 / 84$ & NS & NS & T3495 & NS & NS & NS & $1.00 \mathrm{E}-01$ & $M$ \\
\hline $\mathrm{Al}$ & $10 / 31 / 86$ & NS & NS & R081287 & NS & NS & NS & $1.00 \mathrm{E}-01$ & $M$ \\
\hline $\mathrm{Al}$ & $10 / 21 / 83$ & NS & NS & NS & NS & NS & NS & $9.70 \mathrm{E}-02$ & $M$ \\
\hline $\mathrm{Al}$ & $3 / 10 / 84$ & NS & NS & NS & NS & NS & NS & $9.70 \mathrm{E}-02$ & $M$ \\
\hline $\mathrm{Al}$ & $12 / 13 / 83$ & NS & $7 / 31 / 83$ & $\mathrm{~T} 1521$ & NS & NS & NS & $9.30 \mathrm{E}-02$ & $\bar{M}$ \\
\hline $\mathrm{Al}$ & $12 / 7 / 83$ & NS & NS & $T 1520$ & NS & NS & NS & $8.50 \mathrm{E}-02$ & $M$ \\
\hline $\mathrm{Al}$ & $10 / 30 / 86$ & NS & NS & R060386 & NS & NS & NS & $8.50 \mathrm{E}-02$ & $M$ \\
\hline Aluminum & $7 / 26 / 95$ & N/A & $3 / 2 / 91$ & B00908 & $A Z-102-1$ & S95T000266 & 1.1 & $1.56 E+03$ & UG/ml \\
\hline Aluminum & $7 / 26 / 95$ & N/A & $3 / 2 / 91$ & BOD9R2 & $A Z-102-3$ & S95T000268 & 1.1 & $1.55 E+03$ & UG/ml \\
\hline Aluminum & $7 / 26 / 95$ & N/A & $3 / 2 / 91$ & BOD9RO & $A Z-102-2$ & S95T000267 & 1.1 & $1.55 E+03$ & $\mathrm{UG} / \mathrm{ml}$ \\
\hline Aluminum & $7 / 26 / 95$ & N/A & $3 / 2 / 91$ & B00908 & $A Z-102-1$ & S95T000266 & 1.1 & $1.54 E+03$ & $\mathrm{UG} / \mathrm{ml}$ \\
\hline Aluminum & $7 / 26 / 95$ & N/A & $3 / 2 / 91$ & BOD9R0 & $A Z-102-2$ & S95T000267 & 1.1 & $1.54 \mathrm{E}+03$ & $\mathrm{UG} / \mathrm{ml}$ \\
\hline Aluminum & $7 / 26 / 95$ & N/A & $3 / 2 / 91$ & BOD9R2 & $A Z-102-3$ & S95T000268 & 1.1 & $1.54 E+03$ & $\mathrm{UG} / \mathrm{ml}$ \\
\hline $\mathrm{Al}$ & $8 / 26 / 77$ & NS & NS & RAT-AZ102-4B & NS & NS & 1.39 & $3.10 \mathrm{E}-02$ & moles/I \\
\hline $\mathrm{NaAlO} 2$ & $8 / 27 / 77$ & NS & NS & NS & NS & NS & 1.391 & $3.10 \mathrm{E}-02$ & $M$ \\
\hline $\mathrm{Al}$ & $9 / 19 / 78$ & NS & NS & RAT-AZ102-4C & NS & NS & 1.39 & $2.40 \mathrm{E}-02$ & moles $\Lambda$ \\
\hline $\mathrm{Al}$ & $9 / 20 / 78$ & NS & NS & NS & NS & NS & 1.39 & $2.40 \mathrm{E}-02$ & $M$ \\
\hline $\mathrm{NaAlO} 2$ & $4 / 26 / 74$ & NS & NS & NS & NS & NS & 1.299 & $1.90 \mathrm{E}-02$ & $M$ \\
\hline Al & Aug 89 & NS & NS & NS & NS & NS & NS & $1.05 \mathrm{E}-01$ & g/L \\
\hline
\end{tabular}




\begin{tabular}{|c|c|c|c|c|c|c|c|}
\hline & & & Analyte Inform & & & & \\
\hline Code & Source & Analyte & Conc. (ug/L) & Conc. (mol/L) & $\begin{array}{c}\text { Reference } \\
\text { Number }\end{array}$ & $\begin{array}{c}\text { Validated } \\
\text { (Y/N) }\end{array}$ & Notes \\
\hline $\mathbf{L}$ & TRAC & Al & NS & NS & NS & $\bar{N}$ & Analysis Prior to $9 / 86$ \\
\hline $\mathrm{BI}$ & Raw & Al & $9.29 E+07$ & $3.44 E+00$ & AZ102-18 & D & NS \\
\hline G & Wastren & Al & $8.00 E+07$ & $2.97 E+\infty 0$ & NS & N & Won't use Braun, Wastren, or HTCE Inv. Data \\
\hline El & Raw & $\mathrm{Al}$ & $1.44 E+07$ & $5.35 E-01$ & $A Z 102-18$ & D & NS \\
\hline El & Raw & Al & $1.44 E+07$ & 5.35E-01 & AZ102-23 & $\mathbf{N}$ & $\begin{array}{l}\text { Solid sample after has been washed with } \\
\text { fresh water }\end{array}$ \\
\hline s & HTCE Inv. & Al & $1.40 E+07$ & $5.18 E-01$ & NS & N & Won't use Braun, Wastren, or HTCE Inv. Data \\
\hline$s$ & Braun & Al & $1.27 E+07$ & 4.70E-01 & NS & N & Won't use Braun, Wastren, or HTCE Inv. Data \\
\hline S & Raw & $\mathrm{Al}$ & $1.27 E+07$ & 4.70E-01 & AZ102-11 & $\bar{N}$ & Analysis Prior to $9 / 86$ \\
\hline $\bar{P}$ & Braun & Al & $6.42 E+06$ & $2.38 \mathrm{E}-01$ & NS & $\mathbf{N}$ & Analysis Prior to $9 / 86$ \\
\hline$L$ & Raw & Al & $6.42 E+06$ & $2.38 \mathrm{E}-01$ & $\mathrm{AZ102-8}$ & $N$ & Analysis Prior to $9 / 86$ \\
\hline$P$ & Braun & Al & $5.99 E+06$ & $2.22 \mathrm{E}-01$ & NS & $\mathbf{N}$ & Analysis Prior to $9 / 86$ \\
\hline L & Raw & $\mathrm{Al}$ & $5.99 E+06$ & $2.22 \mathrm{E}-01$ & $A Z 102-8$ & $N$ & Analysis Prior to $9 / 86$ \\
\hline$P$ & Braun & $\mathrm{Al}$ & $4.86 E+06$ & 1.80E-01 & NS & $\mathbf{N}$ & Analysis Prior to $9 / 86$ \\
\hline$L$ & Raw & Al & $4.86 E+06$ & $1.80 \mathrm{E}-01$ & AZ102-6 & $\mathbf{N}$ & Analysis Prior to $9 / 86$ \\
\hline$L$ & Raw & $\mathrm{Al}$ & $3.67 E+06$ & $1.36 \mathrm{E}-01$ & AZ102-3 & $\mathrm{N}$ & Analysis Prior to $9 / 86$ \\
\hline$P$ & Braun & $\mathrm{Al}$ & $3.64 E+06$ & $1.35 \mathrm{E}-01$ & NS & $N$ & Analysis Prior to $9 / 86$ \\
\hline L & Raw & $\mathrm{Al}$ & $3.64 E+06$ & $1.35 E-01$ & $A Z 102-5$ & $N$ & Analysis Prior to $9 / 86$ \\
\hline $\mathbf{R}$ & Braun & Al & $3.64 E+06$ & $1.35 E-01$ & NS & $N$ & Won't use Braun, Wastren, or HTCE Inv. Data \\
\hline$L$ & Raw & $\overrightarrow{A l}$ & $3.51 E+06$ & $1.30 \mathrm{E}-01$ & A2102-10 & $N$ & Analysis Prior to $9 / 86$ \\
\hline$P$ & Braun & $\overline{A l}$ & $3.24 E+06$ & $1.20 \mathrm{E}-01$ & NS & $N$ & Analysis Prior to $9 / 86$ \\
\hline $\mathrm{L}$ & Raw & Al & $3.24 E+06$ & $1.20 \mathrm{E}-01$ & AZ102-11 & $N$ & Analysis Prior to $9 / 86$ \\
\hline$\underline{L}$ & Raw & $\overline{A l}$ & $3.24 E+06$ & $1.20 \mathrm{E}-01$ & AZ102-20 & $\mathbf{N}$ & Analysis Prior to $9 / 86$ \\
\hline L & HTCE Inv. & $\mathrm{Al}$ & $2.94 E+06$ & $1.09 E-01$ & NS & N & Won't use Braun, Wastren, or HTCE Inv. Data \\
\hline $\mathbf{P}$ & Braun & $\mathrm{Al}$ & $2.70 E+06$ & $1.00 \mathrm{E}-01$ & NS & $N$ & Analysis Prior to $9 / 86$ \\
\hline$L$ & Raw & $\mathrm{Al}$ & $2.70 E+06$ & $1.00 \mathrm{E}-01$ & AZ102-16 & $N$ & Analysis Prior to $9 / 86$ \\
\hline$L$ & Raw & Al & $2.70 \mathrm{E}+06$ & $1.00 \mathrm{E}-01$ & $A Z 102-16$ & $\mathbf{N}$ & Analysis Prior to $9 / 86$ \\
\hline$L$ & Raw & $\mathrm{AI}$ & $2.70 E+06$ & $1.00 \mathrm{E}-01$ & AZ102-22 & $N$ & Not a traceable reference \\
\hline $\mathbf{L}$ & Raw & $\mathrm{Al}$ & $2.62 E+06$ & $9.70 \mathrm{E}-02$ & $A Z 102-13$ & $\mathbf{N}$ & Analysis Prior to $9 / 86$ \\
\hline $\mathbf{L}$ & Raw & $\mathrm{Al}$ & $2.62 E+06$ & $9.70 \mathrm{E}-02$ & AZ102-16 & $N$ & Analysis Prior to $9 / 86$ \\
\hline $\mathbf{s}$ & Raw & $\mathrm{Al}$ & $2.51 E+06$ & $9.30 \mathrm{E}-02$ & AZ102-14 & $\bar{N}$ & No units for concentration given in report \\
\hline $\mathbf{L}$ & Raw & $\mathrm{Al}$ & $2.29 E+06$ & $8.50 E-02$ & AZ102-14 & $\bar{N}$ & Analysis Prior to $9 / 86$ \\
\hline$L$ & Raw & $\mathrm{Al}$ & $2.29 E+06$ & 8.50E-02 & AZ102-21 & $\bar{N}$ & Not a traceable reference \\
\hline$\overline{\mathrm{P}-2}$ & Raw & $\mathrm{Al}$ & $1.56 \mathrm{E}+06$ & $5.78 E-02$ & $A 2102-24$ & $Y$ & Updated per memo 8/17/95 \\
\hline$\underline{p-1}$ & Raw & Al & $1.55 \mathrm{E}+06$ & $5.74 \mathrm{E}-02$ & AZ102-24 & $\bar{Y}$ & Updated per memo $8 / 17 / 95$ \\
\hline P-2 & Raw & $\mathrm{Al}$ & $1.55 E+06$ & $5.74 \mathrm{E}-02$ & AZ102-24 & $\bar{Y}$ & Updated per memo 8/17/95 \\
\hline$\overline{p-1}$ & Raw & Al & $1.54 E+06$ & $5.71 E-02$ & AZ102-24 & $\bar{Y}$ & Updated per memo 8/17/95 \\
\hline$\overline{P-1}$ & Raw & Al & $1.54 \mathrm{E}+06$ & 5.71E-02 & $A Z 102-24$ & $\bar{Y}$ & Updated per memo 8/17/95 \\
\hline P-2 & Raw & $\overline{A l}$ & $1.54 \mathrm{E}+06$ & $5.71 E-02$ & $A Z 102-24$ & $\bar{Y}$ & Updated per memo $8 / 17 / 95$ \\
\hline$P$ & Braun & $\mathrm{Al}$ & $8.36 \mathrm{E}+05$ & $3.10 \mathrm{E}-02$ & NS & $\mathbf{N}$ & Analysis Prior to $9 / 86$ \\
\hline$F$ & Raw & $\mathrm{Al}$ & $8.36 E+05$ & $3.10 E-02$ & AZ102-4 & $\mathrm{N}$ & Analysis Prior to 9/86 \\
\hline$P$ & Braun & $\mathrm{Al}$ & $6.48 E+05$ & $2.40 E-02$ & NS & $N$ & Analysis Prior to $9 / 86$ \\
\hline$L$ & Raw & $\overline{\mathrm{Al}}$ & $6.48 E \div 05$ & $2.40 E-02$ & $A Z 102-6$ & $\bar{N}$ & Analysis Prior to $9 / 86$ \\
\hline$L$ & Raw & $\mathrm{Al}$ & $5.13 E+05$ & 1.90E-02 & $A Z 102-1$ & $\mathbf{N}$ & Analysis Prior to $9 / 86$ \\
\hline $\mathbf{P}$ & Wastren & Al & $1.05 E+05$ & 3.89E-03 & NS & $\mathbf{N}$ & Won't use Braun, Wastren, or HTCE Inv. Data \\
\hline
\end{tabular}




\begin{tabular}{|c|c|c|c|c|c|c|c|c|c|}
\hline & & & & Tank Waste C & racterization & mple Informa & & & \\
\hline $\begin{array}{l}\text { Reported } \\
\text { Chemical }\end{array}$ & Memo Date & $\begin{array}{c}\text { Start } \\
\text { Analysis } \\
\text { Date } \\
\end{array}$ & $\begin{array}{c}\text { Start Sample } \\
\text { Date }\end{array}$ & Sample Number & $\begin{array}{c}\text { Tank Farm } \\
\text { Number }\end{array}$ & $\begin{array}{c}\text { Lab Sample } \\
\text { Number }\end{array}$ & $\begin{array}{l}\text { Density } \\
\text { (g/mL) }\end{array}$ & $\begin{array}{l}\text { Reported } \\
\text { Value }\end{array}$ & Reported Uni \\
\hline $\mathrm{Al}$ & $9 / 1 / 93$ & N/A & $6 / 30 / 85$ & N/A & N/A & N/A & 1.14 & $3.30 E+00$ & umol/g \\
\hline $\mathrm{Al}$ & $10 / 31 / 86$ & NS & $6 / 30 / 85$ & NS & NS & NS & 1.14 & $3.30 E+00$ & umol/g \\
\hline Al & $11 / 4 / 81$ & NS & NS & RAT-AZ102-2C & NS & NS & 1.26 & 2.00E-03 & moles/l \\
\hline Al & $8 / 31 / 89$ & NS & $6 / 30 / 85$ & Composite Core & NS & NS & $(1.0)$ & $1.51 E+00$ & umol/g \\
\hline$\overline{A l}$ & $1 / 30 / 82$ & NS & NS & $T-5387$ & NS & NS & 1.008 & $1.19 \mathrm{E}-03$ & moles $/$ \\
\hline $\mathrm{Al}$ & $2 / 19 / 82$ & NS & NS & T5387 & NS & NS & 1.008 & $1.19 \mathrm{E}-03$ & $M$ \\
\hline $\mathrm{Al}$ & $10 / 31 / 86$ & NS & $6 / 30 / 85$ & NS & NS & NS & NS & $1.51 E+00$ & umol/g \\
\hline$A l$ & $5 / 3 / 86$ & NS & NS & L1 & NS & NS & NS & $5.62 E+02$ & umol/g \\
\hline Al & $5 / 3 / 86$ & NS & NS & L3 & NS & NS & NS & $1.61 E+03$ & umol/g \\
\hline Al & $5 / 3 / 86$ & NS & NS & L4 & NS & NS & NS & $1.70 E+03$ & umol/g \\
\hline $\mathrm{Al}$ & $12 / 3 / 75$ & NS & NS & 1458 & NS & NS & 1.391 & $1.36 \mathrm{E}-01$ & $M$ \\
\hline $\mathrm{Al}$ & $12 / 3 / 75$ & NS & NS & 1457 & NS & NS & 1.398 & 1.27E-01 & $M$ \\
\hline $\mathrm{Al}$ & $12 / 3 / 75$ & NS & NS & 1459 & NS & NS & 1.386 & $1.23 \mathrm{E}-01$ & $M$ \\
\hline$\overline{A l}$ & $12 / 3 / 75$ & NS & NS & $1496 \mathrm{D}$ & NS & NS & 1.36 & $1.13 \mathrm{E}-01$ & $M$ \\
\hline $\mathrm{Al}$ & $12 / 3 / 75$ & NS & NS & $1496 \mathrm{C}$ & NS & NS & 1.379 & 1.11E-01 & $M$ \\
\hline $\mathrm{Al}$ & $12 / 3 / 75$ & NS & NS & $1496 \mathrm{~B}$ & NS & NS & 1.38 & $1.09 \mathrm{E}-01$ & $\bar{M}$ \\
\hline $\mathrm{Al}$ & $12 / 3 / 75$ & NS & NS & $1496 \mathrm{~A}$ & NS & NS & 1.378 & $1.06 \mathrm{E}-01$ & $M$ \\
\hline As & $10 / 31 / 86$ & NS & $6 / 30 / 85$ & Composite Core & NS & NS & (1.0) & $<1.20 \mathrm{e}-02$ & umol/g \\
\hline $\mathrm{Ce}$ & $8 / 31 / 89$ & NS & $6 / 30 / 85$ & Composite Core & NS & NS & 1.11 & $<1.90 e+00$ & umol/g \\
\hline As & $8 / 1 / 85$ & NS & NS & NS & NS & $\cdot \mathrm{NS}$ & NS & $4.42 E-03$ & $g / L$ \\
\hline As & $9 / 1 / 93$ & N/A & $6 / 30 / 85$ & N/A & N/A & N/A & 1.14 & $5.00 \mathrm{E}-02$ & umol/g \\
\hline As & $10 / 31 / 86$ & NS & $6 / 30 / 85$ & NS & NS & NS & 1.14 & $5.00 \mathrm{E}-02$ & umol/g \\
\hline As & $10 / 31 / 86$ & NS & $6 / 30 / 85$ & NS & NS & NS & NS & $5.00 \mathrm{E}-02$ & umol/g \\
\hline As & $12 / 13 / 83$ & NS & $7 / 31 / 83$ & T1520 & NS & NS & NS & $2.80 E+00$ & $\mathrm{ppm}$ \\
\hline Ba & $10 / 31 / 86$ & NS & $6 / 30 / 85$ & NS & NS & NS & 1.73 & $4.03 E+00$ & umol/g \\
\hline $\mathrm{Ba}$ & NS & NS & NS & NS & NS & NS & NS & $8.25 E-01$ & g/L \\
\hline $\mathrm{Ba}$ & $11 / 5 / 81$ & NS & NS & RAT-AZ102-2B & NS & NS & 1.4 & 2.76E-03 & moles $/$ \\
\hline $\mathrm{Ba}$ & $10 / 31 / 86$ & NS & $6 / 30 / 85$ & NS & NS & NS & 1.11 & $1.02 E+00$ & umol/g \\
\hline $\mathrm{Ce}$ & $8 / 31 / 89$ & NS & $6 / 30 / 85$ & Composite Core & NS & NS & 1.11 & $1.02 E+\infty 0$ & umol/g \\
\hline $\mathrm{Ba}$ & NS & NS & NS & NS & NS & NS & NS & $8.10 E-04$ & g/L \\
\hline $\mathrm{Ba}$ & $9 / 1 / 93$ & N/A & $6 / 30 / 85$ & N/A & N/A & N/A & 1.14 & $5.00 E-03$ & umol/g \\
\hline $\mathrm{Ba}$ & $10 / 31 / 86$ & NS & $6 / 30 / 85$ & Composite Core & NS & NS & $(1.0)$ & $2.00 E-03$ & umol/g \\
\hline $\mathrm{Be}$ & $10 / 31 / 86$ & NS & $6 / 30 / 85$ & NS & NS & NS & 1.73 & $1.50 E+00$ & umol/g \\
\hline $\mathrm{Be}$ & $8 / 1 / 85$ & NS & NS & NS & NS & NS & NS & 2.01E-02 & $g / L$ \\
\hline $\mathrm{Be}$ & $10 / 31 / 86$ & NS & $6 / 30 / 85$ & NS & NS & NS & 1.11 & 4.70E-01 & umol/g \\
\hline $\mathrm{Ce}$ & $10 / 31 / 86$ & NS & $6 / 30 / 85$ & Composite Core & NS & NS & 1.11 & $4.70 E-01$ & umol/g \\
\hline
\end{tabular}




\begin{tabular}{|c|c|c|c|c|c|c|c|}
\hline & & & Analyte inform & & & & \\
\hline jode & Source & Analyte & Conc. (ug/L) & Conc. (mol/L) & $\begin{array}{l}\text { Reference } \\
\text { Number }\end{array}$ & $\begin{array}{c}\text { Validated } \\
(\mathrm{Y} / \mathrm{N})\end{array}$ & Notes \\
\hline $\mathrm{Cl}$ & Raw & $\mathrm{Al}$ & $1.01 E+05$ & $3.76 \mathrm{E}-03$ & AZ102-23 & $Y$ & \\
\hline $\mathrm{Cl}$ & Raw & $\mathrm{Al}$ & $1.01 E+05$ & $3.76 \mathrm{E}-03$ & AZ102-18 & D & NS \\
\hline $\mathbf{R}$ & Braun & Al & $5.40 E+04$ & 2.00E-03 & NS & $N$ & Won't use Braun, Wastren, or HTCE Inv. Data \\
\hline $\mathrm{Fl}$ & Raw & Al & $4.07 E+04$ & $1.51 \mathrm{E}-03$ & $A Z 102-23$ & $\mathbf{N}$ & Water wash of solid sample not supernatent \\
\hline$P$ & Braun & Al & $3.21 E+04$ & $1.19 \mathrm{E}-03$ & NS & $N$ & Analysis Prior to $9 / 86$ \\
\hline$L$ & Raw & Al & $3.21 E+04$ & $1.19 \mathrm{E}-03$ & AZ102-9 & $\mathbf{N}$ & Analysis Prior to $9 / 86$ \\
\hline FI & Raw & Al & $0.00 \mathrm{E}+00$ & $0.00 E+00$ & $A Z 102-18$ & $\mathrm{D}$ & NS \\
\hline s & Raw & Al & $0.00 E+00$ & $0.00 E+00$ & AZ102-17 & D & Points already in $D B$ as part of $A Z_{-} 102-23$ \\
\hline $\mathbf{S}$ & Raw & Al & $0.00 E+00$ & $0.00 E+00$ & $A Z 102-17$ & D & Points already in DB as part of $A Z_{-}{ }_{1} 102-23$ \\
\hline $\mathbf{S}$ & Raw & Al & $0.00 E+00$ & $0.00 E+00$ & $A Z 102-17$ & $D$ & Points already in DB as part of $A Z_{-} 102-23$ \\
\hline$L$ & Raw & $\mathrm{Al}$ & $3.67 E+06$ & $1.36 \mathrm{E}-01$ & $A Z 102-3$ & $N$ & Analysis Prior to $9 / 86$ \\
\hline$L$ & Raw & Al & $3.43 E+06$ & $1.27 \mathrm{E}-01$ & $A Z 102-3$ & $\mathbf{N}$ & Analysis Prior to $9 / 86$ \\
\hline $\mathbf{L}$ & Raw & $\mathrm{Al}$ & $3.32 E+06$ & 1.23E-01 & $A Z 102-3$ & $N$ & Analysis Prior to $9 / 86$ \\
\hline$\underline{L}$ & Raw & Al & $3.05 E+06$ & $1.13 E-01$ & $A Z 102-3$ & $N$ & Analysis Prior to $9 / 86$ \\
\hline$L$ & Raw & $\mathrm{Al}$ & $2.99 E+06$ & $1.11 \mathrm{E}-01$ & $A Z 102-3$ & $N$ & Analysis Prior to $9 / 86$ \\
\hline$L$ & Raw & $\mathrm{Al}$ & $2.94 E+06$ & $1.09 \mathrm{E}-01$ & $A Z 102-3$ & $N$ & Analysis Prior to $9 / 86$ \\
\hline$L$ & Raw & $\mathrm{Al}$ & $2.86 E+06$ & $1.06 \mathrm{E}-01$ & AZ102-3 & $N$ & Analysis Prior to $9 / 86$ \\
\hline FI & Raw & As & \#VALUEI & \#VALUE! & $A Z 102-18$ & $N$ & Water wash of solid sample not supernatent \\
\hline El & Raw & As & \#VALUEI & \#VALUE! & $A Z 102-18$ & $\mathbf{N}$ & $\begin{array}{l}\text { Solid sample after has been washed with } \\
\text { fresh water }\end{array}$ \\
\hline$P$ & Wastren & As & $4.42 \mathrm{E}+03$ & $5.90 \mathrm{E}-05$ & NS & $N$ & Analysis Prior to $9 / 86$ \\
\hline$\overline{\mathrm{Cl}}$ & Raw & As & $4.27 E+03$ & $5.70 E-05$ & AZ102-23 & $Y$ & \\
\hline $\mathrm{Cl}$ & Raw & As & $4.27 E+03$ & $5.70 \mathrm{E}-05$ & $A Z 102-18$ & $\bar{D}$ & NS \\
\hline$\underline{P}$ & Raw & As & $0.00 E+00$ & $0.00 E+00$ & $A Z 102-18$ & $N$ & Point in DB already_cc $8 / 15 / 95$ \\
\hline $\mathrm{L}$ & Raw & As & $0.00 E+00$ & $0.00 E+00$ & $A Z 102-14$ & N & $\begin{array}{l}\text { Wrong Reference no As measured in } \\
\text { referenced report }\end{array}$ \\
\hline B! & Raw & $\mathrm{Ba}$ & $9.57 E+05$ & 6.97E-03 & AZ102-18 & D & NS \\
\hline G & Wastren & Ba & $8.25 E+05$ & $6.01 E-03$ & NS & N & Won't use Braun, Wastren, or HTCE Inv. Data \\
\hline $\mathbf{S}$ & Braun & $\mathrm{Ba}$ & $3.79 E+05$ & $2.76 \mathrm{E}-03$ & NS & N & Won't use Braun, Wastren, or HTCE inv. Data \\
\hline$\underline{E}$ & Raw & $\mathrm{Ba}$ & $1.55 E+05$ & $1.13 \mathrm{E}-03$ & $A Z 102-18$ & $\mathrm{D}$ & NS \\
\hline El & Raw & $\mathrm{Ba}$ & $1.55 E+05$ & $1.13 \mathrm{E}-03$ & $A Z 102-23$ & $\mathbf{N}$ & $\begin{array}{l}\text { Solid sample after has been washed with } \\
\text { fresh water }\end{array}$ \\
\hline $\mathbf{P}$ & Wastren & $\mathrm{Ba}$ & $8.10 E+02$ & $5.90 E-06$ & NS & N & Won't use Braun, Wastren, or HTCE Inv. Data \\
\hline$\underline{a}$ & Raw & $\mathrm{Ba}$ & $7.83 E+02$ & $5.70 \mathrm{E}-06$ & AZ102-23 & $\bar{Y}$ & \\
\hline FI & Raw & $\mathrm{Ba}$ & $2.75 E+02$ & $2.00 E-06$ & $A Z 102-18$ & $N$ & Water wash of solid sample not supernatent \\
\hline$\underline{3 !}$ & Raw & $\mathrm{Be}$ & $2.34 E+04$ & $2.60 \mathrm{E}-03$ & $A Z 102-18$ & D & NS \\
\hline $\mathbf{G}$ & Wastren & $\mathrm{Be}$ & $2.01 E \div 04$ & $2.23 E-03$ & NS & $\mathbf{N}$ & Won't use Braun, Wastren, or HTCE Inv. Data \\
\hline EI & Raw & $8 \mathrm{e}$ & $4.70 E+03$ & $5.22 \mathrm{E}-04$ & AZ102-18 & D & NS \\
\hline EI & Raw & $8 e$ & $4.70 E+03$ & $5.22 \mathrm{E}-04$ & $A Z 102-23$ & N & $\begin{array}{l}\text { Solid sample after has been washed with } \\
\text { fresh water }\end{array}$ \\
\hline
\end{tabular}




\begin{tabular}{|c|c|c|c|c|c|c|c|c|c|}
\hline & & & & Tank Waste C & racterization & mple Informati & & & \\
\hline $\begin{array}{l}\text { Reported } \\
\text { Chemical }\end{array}$ & Memo Date & $\begin{array}{c}\text { Start } \\
\text { Analysis } \\
\text { Date }\end{array}$ & $\mid \begin{array}{c}\text { Start Sample } \\
\text { Date }\end{array}$ & Sample Number & $\begin{array}{c}\text { Tank Farm } \\
\text { Number }\end{array}$ & $\begin{array}{c}\text { Lab Sample } \\
\text { Number }\end{array}$ & $\begin{array}{l}\text { Density } \\
(\mathrm{g} / \mathrm{mL})\end{array}$ & $\begin{array}{c}\text { Reported } \\
\text { Value }\end{array}$ & Reported Uni \\
\hline $\mathrm{Be}$ & NS & NS & NS & NS & NS & NS & NS & $1.17 E-04$ & $g / L$ \\
\hline $\mathrm{Be}$ & $9 / 1 / 93$ & N/A & $6 / 30 / 85$ & N/A & N/A & N/A & 1.14 & 1.10E-02 & umol $/ \mathrm{g}$ \\
\hline $\mathrm{Be}$ & $10 / 31 / 86$ & NS & $6 / 30 / 85$ & NS & NS & NS & 1.14 & $1.10 E-02$ & umol/g \\
\hline $\mathrm{Be}$ & $10 / 31 / 86$ & NS & $6 / 30 / 85$ & Composite Core & NS & NS & $(1.0)$ & 2.00E-03 & umol/g \\
\hline $\mathrm{Ca}$ & $10 / 31 / 86$ & NS & $6 / 30 / 85$ & NS & NS & NS & 1.73 & $7.54 E+01$ & umol/g \\
\hline $\mathrm{Ca}$ & NS & NS & NS & NS & NS & NS & NS & $4.50 E+00$ & g/L \\
\hline $\mathrm{Ca}$ & $11 / 5 / 81$ & NS & NS & RAT-AZ102-2B & NS & NS & 1.4 & $3.40 \mathrm{E}-02$ & moles/I \\
\hline $\mathrm{Ca}$ & $10 / 31 / 86$ & NS & $6 / 30 / 85$ & NS & NS & NS & 1.11 & $2.01 E+01$ & umol/g \\
\hline $\mathrm{Cl}$ & $8 / 31 / 89$ & NS & $6 / 30 / 85$ & Composite Core & NS & NS & 1.11 & $2.01 E+01$ & umol/g \\
\hline $\mathrm{Ca}$ & $11 / 5 / 81$ & NS & NS & RAT-AZ102-2A & NS & NS & 1.09 & $1.60 \mathrm{E}-03$ & moles/l \\
\hline $\mathrm{Ca}$ & $11 / 5 / 81$ & NS & NS & RAT-AZ102-2C & NS & NS & 1.26 & 8.63E-04 & moles/l \\
\hline $\mathrm{Ca}$ & $2 / 4 / 81$ & NS & NS & $\mathrm{R}-3700$ & NS & NS & 1.11 & $5.36 \mathrm{E}-04$ & moles/l \\
\hline $\mathrm{Ca}$ & $2 / 4 / 81$ & NS & NS & $R-3699$ & NS & NS & 1.11 & 4.76E-04 & moles $/ 1$ \\
\hline $\mathrm{Ca}$ & NS & NS & NS & NS & NS & NS & NS & $6.24 E-03$ & $g / L$ \\
\hline $\mathrm{Ca}$ & $9 / 1 / 93$ & N/A & $6 / 30 / 85$ & N/A & N/A & N/A & 1.14 & 1.32E-01 & umol/g \\
\hline $\mathrm{Ca}$ & $10 / 31 / 86$ & NS & $6 / 30 / 85$ & NS & NS & NS & 1.14 & $1.32 \mathrm{E}-01$ & umol/g \\
\hline $\mathrm{Ca}$ & $10 / 31 / 86$ & NS & $6 / 30 / 85$ & Composite Core & NS & NS & $(1.0)$ & $8.20 E-02$ & umol/g \\
\hline Cd & $10 / 31 / 86$ & NS & $6 / 30 / 85$ & NS & NS & NS & 1.73 & $1.33 E+02$ & umol/g \\
\hline Cd & $8 / 1 / 85$ & NS & NS & NS & NS & NS & NS & $2.23 E+01$ & $g / L$ \\
\hline $\mathrm{Cd}$ & $10 / 31 / 86$ & NS & $6 / 30 / 85$ & NS & NS & NS & 1.11 & $3.28 \mathrm{E}+01$ & umol/g \\
\hline $\mathrm{Cl}$ & $8 / 31 / 89$ & NS & $6 / 30 / 85$ & Composite Core & NS & NS & 1.11 & $3.28 \mathrm{E}+01$ & umol/g \\
\hline$C d$ & $11 / 5 / 81$ & NS & NS & RAT-AZ102-2B & NS & NS & 1.4 & $5.69 E-04$ & moles $/$ \\
\hline Cd & NS & NS & NS & NS & NS & NS & NS & $1.99 E-03$ & $g / L$ \\
\hline Cd & $9 / 1 / 93$ & N/A & $6 / 30 / 85$ & N/A & N/A & N/A & 1.14 & $1.50 \mathrm{E}-02$ & umol/g \\
\hline Cd & $10 / 31 / 86$ & NS & $6 / 30 / 85$ & NS & NS & NS & 1.14 & $1.50 E-02$ & umol/g \\
\hline Cd & $10 / 31 / 86$ & NS & $6 / 30 / 85$ & Composite Core & NS & NS & $\{1.0\}$ & $5.00 E-03$ & umol/g \\
\hline $\mathrm{Ce}$ & $10 / 31 / 86$ & NS & $6 / 30 / 85$ & NS & NS & NS & 1.11 & $1.83 E+00$ & umol/g \\
\hline $\mathrm{Cl}$ & $8 / 31 / 89$ & NS & $6 / 30 / 85$ & Composite Core & NS & NS & 1.11 & $1.83 E+00$ & umol/g \\
\hline $\mathrm{Ce}$ & $11 / 5 / 81$ & NS & NS & RAT-AZ102-2B & NS & NS & 1.4 & $5.03 E-04$ & moles $\Lambda$ \\
\hline $\mathrm{Ce}$ & $8 / 1 / 85$ & NS & NS & NS & NS & NS & NS & $1.22 \mathrm{E}-02$ & $g / L$ \\
\hline $\mathrm{Ce}$ & $9 / 1 / 93$ & N/A & $6 / 30 / 85$ & N/A & N/A & $N / A$ & 1.14 & $7.40 \mathrm{E}-02$ & umol/g \\
\hline $\mathrm{Ce}$ & $10 / 31 / 86$ & NS & $6 / 30 / 85$ & NS & NS & NS & 1.14 & $7.40 E-02$ & umol/g \\
\hline
\end{tabular}




\begin{tabular}{|c|c|c|c|c|c|c|c|}
\hline & & & Analyte Inform & & & & \\
\hline :ode & Source & Analyte & Conc. (ug/L) & Conc. $(\mathrm{mol} / \mathrm{L})$ & $\begin{array}{c}\text { Reference } \\
\text { Number }\end{array}$ & $\begin{array}{l}\text { Validated } \\
\text { (Y/N) }\end{array}$ & Notes \\
\hline $\mathbf{P}$ & Wastren & $\mathrm{Be}$ & $1.17 E+02$ & $1.30 E-05$ & NS & $\mathbf{N}$ & Won't use Braun, Wastren, or HTCE Inv. Data \\
\hline $\mathrm{Cl}$ & Raw & $\mathrm{Be}$ & $1.13 E+02$ & $1.25 \mathrm{E}-05$ & $A Z 102-23$ & $Y$ & \\
\hline Cl & Raw & $\mathrm{Be}$ & $1.13 E+02$ & $1.25 \mathrm{E}-05$ & $A Z 102-18$ & $D$ & NS \\
\hline FI & Raw & $\mathrm{Be}$ & $1.80 E+01$ & $2.00 E-06$ & $A Z 102-18$ & $N$ & Water wash of solid sample not supernatent \\
\hline $\mathrm{BI}$ & Raw & $\mathrm{Ca}$ & $5.23 E+06$ & $1.30 \mathrm{E}-01$ & $A Z 102-18$ & D & NS \\
\hline $\mathbf{G}$ & Wastren & $\mathrm{Ca}$ & $4.50 E+06$ & $1.12 \mathrm{E}-\mathrm{O} 1$ & NS & N & Won't use Braun, Wastren, or HTCE Inv. Data \\
\hline s & Braun & $\mathrm{Ca}$ & $1.36 E+06$ & $3.40 E-02$ & NS & $N$ & Won't use Braun, Wastren, or HTCE Inv. Data \\
\hline EI & Raw & $\mathrm{Ca}$ & $8.94 E+05$ & $2.23 \mathrm{E}-02$ & AZ102-18 & D & NS \\
\hline El & Raw & $\mathrm{Ca}$ & $8.94 E+05$ & 2.23E-02 & $A Z 102-23$ & $\mathbf{N}$ & $\begin{array}{l}\text { Solid sample after has been washed with } \\
\text { fresh water }\end{array}$ \\
\hline$P$ & Braun & $\mathrm{Ca}$ & $6.41 E+04$ & $1.60 E-03$ & NS & $N$ & Won't use Braun, Wastren, or HTCE Inv. Data \\
\hline$Y$ & Braun & $\mathrm{Ca}$ & $3.46 \mathrm{E}+04$ & $8.63 E-04$ & NS & $N$ & Won't use Braun, Wastren, or HTCE Inv. Data \\
\hline $\mathbf{P}$ & Braun & $\mathrm{Ca}$ & $2.15 E+04$ & $5.36 \mathrm{E}-04$ & NS & N & Won't use Braun, Wastren, or HTCE Inv. Data \\
\hline$P$ & Braun & $\mathrm{Ca}$ & $1.91 E+04$ & $4.76 \mathrm{E}-04$ & NS & N & Won't use Braun, Wastren, or HTCE Inv. Data \\
\hline $\mathbf{P}$ & Wastren & $\mathrm{Ca}$ & $6.24 E+03$ & $1.56 \mathrm{E}-04$ & NS & $N$ & Won't use Braun, Wastren, or HTCE Inv. Data \\
\hline $\mathrm{Cl}$ & Raw & $\mathrm{Ca}$ & $6.03 E+03$ & $1.50 \mathrm{E}-04$ & $A Z 102-23$ & $Y$ & \\
\hline Cl & Raw & $\mathrm{Ca}$ & $6.03 E+03$ & $1.50 \mathrm{E}-04$ & $A Z 102-18$ & D & NS \\
\hline $\mathrm{FI}$ & Raw & $\mathrm{Ca}$ & $3.29 E+03$ & $8.20 E-05$ & $A Z 102-18$ & $\mathbf{N}$ & Water wash of solid sample not supematent \\
\hline BI & Raw & $\mathrm{Cd}$ & $2.59 E+07$ & $2.30 \mathrm{E}-01$ & AZ102-18 & D & NS \\
\hline $\mathbf{G}$ & Wastren & Cd & $2.23 E+07$ & $1.98 \mathrm{E}-01$ & NS & $\mathbf{N}$ & Won't use Braun, Wastren, or HTCE Inv. Data \\
\hline$\overline{E I}$ & Raw & $\mathrm{Cd}$ & $4.09 E+06$ & $3.64 \mathrm{E}-02$ & $A \geq 102-18$ & $D$ & NS \\
\hline El & Raw & Cd & $4.09 E+06$ & $3.64 E-02$ & $A Z 102-23$ & N & $\begin{array}{l}\text { Solid sample after has been washed with } \\
\text { fresh water }\end{array}$ \\
\hline $\mathbf{S}$ & Braun & Cd & $6.40 E+04$ & $5.69 E-04$ & NS & $\mathbf{N}$ & Won't use Braun, Wastren, or HTCE Inv. Data \\
\hline $\mathbf{P}$ & Wastren & Cd & $1.99 E+03$ & $1.77 \mathrm{E}-05$ & NS & $N$ & Won't use Braun, Wastren, or HTCE Inv. Data \\
\hline $\mathrm{Cl}$ & Raw & $\mathrm{Cd}$ & $1.92 E+03$ & $1.71 \mathrm{E}-05$ & $A Z 102-23$ & $\bar{Y}$ & \\
\hline Cl & Raw & Cd & $1.92 E+03$ & $1.71 \mathrm{E}-05$ & $A Z 102-18$ & $\mathbf{D}$ & NS \\
\hline FI & Raw & Cd & $5.62 E+02$ & $5.00 E-06$ & $A Z 102-18$ & $\mathbf{N}$ & Water wash of solid sample not supernatent \\
\hline El & Raw & $\mathrm{Ce}$ & $2.85 E+05$ & $2.03 E-03$ & $A Z 102-18$ & D & NS \\
\hline El & Raw & $\mathrm{Ce}$ & $2.85 E+05$ & 2.03E-03 & $A Z 102-23$ & N & $\begin{array}{l}\text { Solid sample after has been washed with } \\
\text { fresh water }\end{array}$ \\
\hline $\mathbf{S}$ & Braun & $\mathrm{Ce}$ & $7.05 E+04$ & $5.03 E-04$ & NS & $N$ & Won't use Braun, Wastren, or HTCE Inv. Data \\
\hline $\mathbf{P}$ & Wastren & $\mathrm{Ce}$ & $1.22 E+04$ & 8.71E-05 & NS & $\mathbf{N}$ & Won't use Braun, Wastren, or HTCE Inv. Data \\
\hline $\mathrm{Cl}$ & Raw & $\mathrm{Ce}$ & $1.18 E+04$ & $8.44 E-05$ & $A Z 102-23$ & $Y$ & \\
\hline $\mathrm{Cl}$ & Raw & $\mathrm{Ce}$ & $1.18 E+04$ & 8.44E-05 & AZ102-18 & D & NS \\
\hline
\end{tabular}




\begin{tabular}{|c|c|c|c|c|c|c|c|c|c|}
\hline & & & & Tank Waste C & racterization & imple Informati & & & \\
\hline $\begin{array}{l}\text { Reported } \\
\text { Chemical } \\
\end{array}$ & Memo Date & $\begin{array}{c}\text { Start } \\
\text { Analysis } \\
\text { Date }\end{array}$ & $\begin{array}{c}\text { Start Sample } \\
\text { Date }\end{array}$ & Sample Number & $\begin{array}{c}\text { Tank Farm } \\
\text { Number }\end{array}$ & $\begin{array}{c}\text { Lab Sample } \\
\text { Number }\end{array}$ & $\begin{array}{c}\text { Density } \\
\text { (g/mL) }\end{array}$ & $\begin{array}{c}\text { Reported } \\
\text { Value }\end{array}$ & Reported U \\
\hline $\mathrm{Ce}$ & $10 / 31 / 86$ & NS & $6 / 30 / 85$ & Composite Core & NS & NS & $(1.0)$ & $1.10 E-02$ & umol/g \\
\hline Chloride & $7 / 26 / 95$ & N/A & $3 / 2 / 91$ & BOD9R3 & $A Z-102-1$ & S95T000263 & 1.1 & $<1.21 e+02$ & $\mathrm{UG} / \mathrm{ml}$ \\
\hline Chloride & $7 / 26 / 95$ & N/A & $3 / 2 / 91$ & BOD9R3 & $A Z-102-1$ & S95T000263 & 1.1 & $<1.21 e+02$ & $\mathrm{UG} / \mathrm{ml}$ \\
\hline $\mathrm{Cl}$ & $12 / 13 / 83$ & NS & $7 / 31 / 83$ & T1521 & NS & NS & NS & $<6.00 \mathrm{e}-01$ & $M$ \\
\hline Chloride & $7 / 26 / 95$ & N/A & $3 / 2 / 91$ & BOD9R4 & $A Z-102-2$ & S95T000264 & 1.1 & $<6.60 e+01$ & $\mathrm{UG} / \mathrm{ml}$ \\
\hline Chloride & $7 / 26 / 95$ & N/A & $3 / 2 / 91$ & BOD9R5 & $A Z-102-3$ & S95T000265 & 1.1 & $<6.60 e+01$ & $\mathrm{UG} / \mathrm{ml}$ \\
\hline Chloride & $7 / 26 / 95$ & N/A & $3 / 2 / 91$ & BOD9R4 & $A Z-102-2$ & 595T000264 & 1.1 & $<6.60 e+01$ & $\mathrm{UG} / \mathrm{ml}$ \\
\hline Chloride & $7 / 26 / 95$ & N/A & $3 / 2 / 91$ & BOD9R5 & $A Z-102-3$ & 5957000265 & 1.1 & $<6.60 e+01$ & UG/ml \\
\hline $\mathrm{Cl}$ & $1 / 1 / 77$ & NS & NS & NS & NS & NS & NS & NS & NS \\
\hline $\mathrm{Cl}$ & $12 \sqrt{7 / 83}$ & NS & NS & $T 1520$ & NS & NS & NS & $6.10 \mathrm{E}-02$ & $M$ \\
\hline Cl- & NS & NS & NS & NS & NS & NS & 1.39 & 4.90E-02 & Mole/L \\
\hline $\mathrm{Cl}-$ & NS & NS & NS & NS & NS & NS & 1.04 & $1.74 \mathrm{E}-02$ & Mole/L \\
\hline $\mathrm{Cl}$ & $10 / 31 / 86$ & NS & $6 / 30 / 85$ & NS & NS & NS & 1.73 & $4.15 E+00$ & umol/g \\
\hline $\mathrm{Cl}$ & $10 / 1 / 83$ & NS & NS & RAT-AZ102-3 & NS & NS & NS & $7.00 \mathrm{E}-03$ & moles/l \\
\hline $\mathrm{Cl}$ & $3 / 10 / 84$ & NS & $7 / 31 / 83$ & T3495 & NS & NS & NS & 7.00E-03 & $M$ \\
\hline $\mathrm{Cl}$ & $3 / 10 / 84$ & NS & $7 / 31 / 83$ & NS & NS & NS & NS & $7.00 \mathrm{E}-03$ & $\bar{M}$ \\
\hline $\mathrm{Cl}$ & $7 / 5 / 84$ & NS & $7 / 31 / 83$ & T3495 & NS & NS & NS & $7.00 \mathrm{E}-03$ & $M$ \\
\hline $\mathrm{Cl}-$ & Aug 89 & NS & NS & NS & NS & NS & NS & $2.19 \mathrm{E}-01$ & $g / L$ \\
\hline $\mathrm{Cl}$ & $6 / 26 / 75$ & NS & NS & RAT-AZ102-4a & NS & NS & 1.39 & $5.00 \mathrm{E}-03$ & moles/1 \\
\hline $\mathrm{Cl}$ & $6 / 26 / 75$ & NS & NS & NS & NS & NS & 1.392 & $5.00 \mathrm{E}-03$ & $M$ \\
\hline $\mathrm{Cl}-$ & Aug 89 & NS & NS & NS & NS & NS & NS & 7.07E-02 & $g / L$ \\
\hline $\mathrm{Cl}$ & $9 / 1 / 93$ & N/A & $6 / 30 / 85$ & N/A & N/A & N/A & 1.14 & $1.69 \mathrm{E}+00$ & umol/g \\
\hline $\mathrm{Cl}$ & $10 / 31 / 86$ & NS & $6 / 30 / 85$ & NS & NS & NS & 1.14 & $1.69 \mathrm{E}+00$ & umol/g \\
\hline $\mathrm{Cl}$ & $8 / 31 / 89$ & NS & $6 / 30 / 85$ & Composite Core & NS & NS & 1.11 & $6.10 E-01$ & umol/g \\
\hline $\mathrm{Cl}$ & $10 / 31 / 86$ & NS & $6 / 30 / 85$ & NS & NS & NS & 1.11 & $5.64 \mathrm{E}-01$ & umol/g \\
\hline $\mathrm{Cl}$ & $8 / 31 / 89$ & NS & $6 / 30 / 85$ & Composite Core & NS & NS & $(1.0)$ & $5.64 E-01$ & umol/g \\
\hline $\mathrm{Cl}$ & $10 / 31 / 86$ & NS & $6 / 30 / 85$ & NS & NS & NS & NS & $6.10 \mathrm{E}-01$ & umol/g \\
\hline Co & $8 / 1 / 85$ & NS & NS & NS & NS & NS & NS & $8.83 E-03$ & $g / L$ \\
\hline Co & $9 / 1 / 93$ & N/A & $6 / 30 / 85$ & N/A & N/A & N/A & 1.14 & $1.27 \mathrm{E}-01$ & umol/g \\
\hline $\mathrm{Cl}$ & $8 / 31 / 89$ & NS & $6 / 30 / 85$ & Composite Core & NS & NS & 1.11 & $2.54 E+02$ & umol/g \\
\hline TIC & $8 / 31 / 89$ & NS & $6 / 30 / 85$ & Composite Core & NS & NS & $(1.0)$ & $1.33 E+02$ & umol/g \\
\hline $\mathrm{Cr}$ & $10 / 31 / 86$ & NS & $6 / 30 / 85$ & NS & NS & NS & 1.73 & $3.70 E+01$ & umol/g \\
\hline $\mathrm{Cr}$ & NS & NS & NS & NS & NS & NS & NS & $2.87 E+\infty 0$ & $g / L$ \\
\hline $\mathrm{Cr}$ & $9 / 1 / 93$ & N/A & $6 / 30 / 85$ & N/A & N/A & N/A & 1.14 & $1.69 E+01$ & umol/g \\
\hline $\mathrm{Cr}$ & $10 / 31 / 86$ & NS & $6 / 30 / 85$ & NS & NS & NS & 1.14 & $1.69 \mathrm{E}+01$ & umol/g \\
\hline $\mathrm{Cr}$ & $10 / 31 / 86$ & NS & $6 / 30 / 85$ & NS & NS & NS & 1.11 & $7.16 E+\infty$ & umol/g \\
\hline $\mathrm{Cl}$ & $8 / 31 / 89$ & NS & $6 / 30 / 85$ & Composite Core & NS & NS & 1.11 & $7.16 E+\infty 0$ & umol/g \\
\hline
\end{tabular}




\begin{tabular}{|c|c|c|c|c|c|c|c|}
\hline \multirow[b]{2}{*}{ Code } & \multirow[b]{2}{*}{ Source } & \multicolumn{3}{|c|}{ Analyte Information } & \multirow[b]{2}{*}{$\begin{array}{c}\text { Reference } \\
\text { Number }\end{array}$} & \multirow[b]{2}{*}{$\begin{array}{c}\text { Validated } \\
\text { (Y/N) }\end{array}$} & \multirow[b]{2}{*}{ Notes } \\
\hline & & Analyte & Conc. (ug/L) & Conc. $(\mathrm{mol} / \mathrm{L})$ & & & \\
\hline $\mathrm{FI}$ & Raw & $\mathrm{Ce}$ & $1.54 E+03$ & $1.10 \mathrm{E}-05$ & $A Z 102-18$ & $N$ & Water wash of solid sample not supernatent \\
\hline$P-1$ & Raw & $\mathrm{Cl}$ & \#VALUE! & \#VALUE! & $A Z 102-24$ & $Y$ & Updated per memo 8/17/95 \\
\hline P-2 & Raw & $\mathrm{Cl}$ & \#VALUE! & \#VALUE! & $\mathrm{AZ102-24}$ & $Y$ & Updated per memo 8/17/95 \\
\hline $\mathbf{S}$ & Raw & $\mathrm{Cl}$ & \#VALUE! & \#VALUE! & AZ102-14 & $N$ & No units for concentration given in report \\
\hline P-1 & Raw & $\mathrm{Cl}$ & \#VALUEI & \#VALUE! & $A Z 102-24$ & $Y$ & Updated per memo 8/17/95 \\
\hline $\mathrm{P}-1$ & Raw & $\mathrm{Cl}$ & \#VALUEI & \#VALUE! & $A Z 102-24$ & $\bar{Y}$ & Updated per memo $8 / 17 / 95$ \\
\hline P-2 & Raw & $\mathrm{Cl}$ & \#VALUEI & \#VALUE! & AZ102-24 & $Y$ & Updated per memo $8 / 17 / 95$ \\
\hline$P-2$ & Raw & $\mathrm{Cl}$ & \#VALUE! & \#VALUEI. . & $\mathrm{A} 2102-24$ & $Y$ & Updated per memo 8/17/95 \\
\hline$L$ & TRAC & $\mathrm{Cl}$ & NS & NS & NS & $\mathrm{N}$ & Analysis Prior to $9 / 86$ \\
\hline$\underline{L}$ & Raw & $\mathrm{Cl}$ & $2.16 E+06$ & $6.10 \mathrm{E}-02$ & $A Z 102-14$ & $N$ & GFV \\
\hline $\mathbf{s}$ & HTCE Inv. & $\mathrm{Cl}$ & $1.74 E+06$ & $4.90 E-02$ & NS & N & Won't use Braun, Wastren, or HTCE Inv. Data \\
\hline $\mathbf{L}$ & HTCE Inv. & $\mathrm{Cl}$ & $6.16 E+05$ & $1.74 \mathrm{E}-02$ & NS & N & Won't use Braun, Wastren, or HTCE Inv. Data \\
\hline $\mathrm{BI}$ & Raw & $\mathrm{Cl}$ & $2.55 \mathrm{E}+05$ & $7.18 \mathrm{E}-03$ & AZ102-18 & D & NS \\
\hline$P$ & Braun & $\mathrm{Cl}$ & $2.48 \mathrm{E}+05$ & $7.00 \mathrm{E}-03$ & NS & $\mathrm{N}$ & GFV \\
\hline $\mathbf{L}$ & Raw & $\mathrm{Cl}$ & $2.48 E+05$ & 7.00E-03 & $A Z 102-16$ & N & $\begin{array}{l}\text { These data are so messed up it will be easier } \\
\text { to reenter them. }\end{array}$ \\
\hline L & Raw & $\mathrm{Cl}$ & $2.48 \mathrm{E}+05$ & $7.00 E-03$ & AZ102-16 & $N$ & Point in DB already cc $8 / 15 / 95$ \\
\hline $\mathbf{L}$ & Raw & $\mathrm{Cl}$ & $2.48 E+05$ & $7.00 E-03$ & AZ102-16 & $\mathbf{N}$ & Point in DB already_cc_8/15/95 \\
\hline G & Wastren & $\mathrm{Cl}$ & $2.19 E+05$ & $6.18 E-03$ & NS & $N$ & Won't use Braun, Wastren, or HTCE Inv. Data \\
\hline $\mathbf{P}$ & Braun & $\mathrm{Cl}$ & $1.77 E+05$ & $5.00 \mathrm{E}-03$ & NS & $\mathbf{N}$ & Analysis Prior to $9 / 86$ \\
\hline$\underline{L}$ & Raw & $\mathrm{Cl}$ & $1.77 E+05$ & $5.00 \mathrm{E}-03$ & AZ102-6 & $\mathbf{N}$ & Analysis Prior to $9 / 86$ \\
\hline $\mathbf{P}$ & Wastren & $\mathrm{Cl}$ & $7.07 E+04$ & $1.99 E-03$ & NS & $N$ & Won't use Braun, Wastren, or HTCE Inv. Data \\
\hline $\mathrm{Cl}$ & Raw & $\mathrm{Cl}$ & $6.83 E+04$ & $1.93 \mathrm{E}-03$ & A2102-23 & $\bar{Y}$ & \\
\hline $\mathrm{Cl}$ & Raw & $\mathrm{Cl}$ & $6.83 E+04$ & $1.93 \mathrm{E}-03$ & $A Z 102-18$ & $\mathbf{D}$ & NS \\
\hline El & Raw & $\mathrm{Cl}$ & $2.40 E+04$ & $6.77 E-04$ & $A Z 102-23$ & $\mathbf{N}$ & $\begin{array}{l}\text { Solid sample after has been washed with } \\
\text { fresh water }\end{array}$ \\
\hline $\mathrm{El}$ & Raw & Cl & $2.22 E+04$ & $6.26 \mathrm{E}-04$ & $A Z 102-18$ & D & NS \\
\hline FI & Raw & $\mathrm{Cl}$ & $2.00 E+04$ & $5.64 \mathrm{E}-04$ & $A Z 102-23$ & N & Water wash of solid sample not supernatent \\
\hline $\mathrm{FI}$ & Raw & $\mathbf{C I}$ & $0.00 E+00$ & $0.00 E+00$ & $A Z 102-18$ & D & NS \\
\hline $\mathbf{P}$ & Wastren & Co & $8.83 E+03$ & $1.50 E-04$ & NS & $N$ & Won't use Braun, Wastren, or HTCE Inv. Data \\
\hline $\mathrm{Cl}$ & Raw & Co & $8.53 E+03$ & $1.45 \mathrm{E}-04$ & AZ102-23 & $\bar{Y}$ & \\
\hline El & Raw & $\cos$ & $1.69 E+07$ & $2.82 \mathrm{E}-01$ & $A Z 102-23$ & $\mathbf{N}$ & $\begin{array}{l}\text { Solid sample after has been washed with } \\
\text { fresh water }\end{array}$ \\
\hline$F I$ & Raw & $\cos$ & $7.98 E+06$ & $1.33 E-01$ & $A Z 102-23$ & $\mathbf{N}$ & Water wash of solid sample not supernatent \\
\hline BI & Raw & $\mathrm{Cr}$ & $3.33 \mathrm{E}+06$ & $6.40 \mathrm{E}-02$ & $A Z 102-18$ & D & NS \\
\hline G & Wastren & $\mathrm{Cr}$ & $2.87 E+06$ & $5.52 \mathrm{E}-02$ & NS & N & Won't use Braun, Wastren, or HTCE Inv. Data \\
\hline$\overline{\mathrm{Cl}}$ & Raw & $\mathrm{Cr}$ & $1.00 E+06$ & $1.93 \mathrm{E}-02$ & $\mathrm{AZ102-23}$ & $\bar{Y}$ & \\
\hline $\mathrm{Cl}$ & Raw & $\mathrm{Cr}$ & $1.00 E+06$ & $1.93 \mathrm{E}-02$ & AZ102-18 & D & NS \\
\hline$\underline{E l}$ & Raw & $\mathrm{Cr}$ & $4.13 E+05$ & 7.95E-03 & $\mathrm{AZ102-18}$ & $D$ & NS \\
\hline El & Raw & $\mathrm{Cr}$ & $4.13 E+05$ & $7.95 E-03$ & $A Z 102-23$ & $N$ & $\begin{array}{l}\text { Solid sample after has been washed with } \\
\text { fresh water }\end{array}$ \\
\hline
\end{tabular}


TWRS Privatization Support Pr Chemical Liquid:

\begin{tabular}{|c|c|c|c|c|c|c|c|c|c|}
\hline & & & & Tank Waste C & racterization & ample informati & & & \\
\hline $\begin{array}{l}\text { Reported } \\
\text { Chemical }\end{array}$ & Memo Date & $\begin{array}{c}\text { Start } \\
\text { Analysis } \\
\text { Date } \\
\end{array}$ & $\begin{array}{c}\text { Start Sample } \\
\text { Date }\end{array}$ & Sample Number & $\begin{array}{c}\text { Tank Farm } \\
\text { Number }\end{array}$ & $\begin{array}{c}\text { Lab Sample } \\
\text { Number }\end{array}$ & $\begin{array}{l}\text { Density } \\
(\mathrm{g} / \mathrm{mL})\end{array}$ & $\begin{array}{c}\text { Reported } \\
\text { Value }\end{array}$ & Reported C \\
\hline $\mathrm{Cr}$ & $11 / 5 / 81$ & NS & NS & RAT-AZ102-2B & NS & NS & 1.4 & $5.29 E-03$ & moles $/ 1$ \\
\hline $\mathrm{Cr}$ & $8 / 31 / 89$ & NS & $6 / 30 / 85$ & Composite Core & NS & NS & $(1.0)$ & $2.88 \mathrm{E}+00$ & umol/g \\
\hline $\mathrm{Cr}$ & $11 / 5 / 81$ & NS & NS & RAT-AZ102-2C & NS & NS & 1.26 & $2.27 E-03$ & moles n \\
\hline $\mathrm{Cr}$ & $10 / 31 / 86$ & NS & $6 / 30 / 85$ & NS & NS & NS & NS & $2.88 E+00$ & umol/g \\
\hline $\mathrm{Cr}$ & $8 / 1 / 85$ & NS & NS & NS & NS & NS & NS & $1.04 E+\infty 0$ & $g / L$ \\
\hline $\mathrm{CrO} 4$ & $8 / 1 / 85$ & NS & NS & NS & NS & NS & NS & 2.77E-01 & $g / L$ \\
\hline $\mathrm{Cr}$ & $2 / 4 / 81$ & NS & NS & $R-3700$ & NS & NS & 1.11 & $4.18 \mathrm{E}-03$ & moles $/$ \\
\hline $\mathrm{Cr}$ & $2 / 4 / 81$ & NS & NS & $\mathrm{R}-3699$ & NS & NS & 1.11 & $4.11 E-03$ & moles 1 \\
\hline $\mathrm{Cr}+6$ & $10 / 31 / 86$ & NS & $6 / 30 / 85$ & Composite Core & NS & NS & $(1.0)$ & $2.50 E+00$ & umol/g \\
\hline $\mathrm{Cr}+6$ & $8 / 31 / 89$ & NS & $6 / 30 / 85$ & Composite Core & NS & NS & $(1.0)$ & $2.50 E+00$ & umol/g \\
\hline $\mathrm{Cr}+6$ & $9 / 1 / 93$ & N/A & $6 / 30 / 85$ & N/A & N/A & N/A & 1.14 & $2.02 E+00$ & umol/g \\
\hline$C_{r+6}$ & $10 / 31 / 86$ & NS & $6 / 30 / 85$ & NS & NS & NS & 1.14 & $2.02 E+00$ & umol/g \\
\hline $\mathrm{Cr}$ & $11 / 5 / 81$ & NS & NS & RAT-AZ102-2A & NS & NS & 1.09 & $2.11 E-03$ & moles/1 \\
\hline$F$ & $1 / 1 / 77$ & NS & NS & NS & NS & NS & NS & NS & NS \\
\hline Fluoride & $7 / 26 / 95$ & N/A & $3 / 2 / 91$ & BOD9R3 & $A Z-102-1$ & 5957000263 & 1.1 & $1.08 E+03$ & $\mathrm{UG} / \mathrm{ml}$ \\
\hline Fluoride & $7 / 26 / 95$ & N/A & $3 / 2 / 91$ & B009R3 & $A Z-102-1$ & S95T000263 & 1.1 & $1.07 E \div 03$ & $\mathrm{UG} / \mathrm{ml}$ \\
\hline Fluoride & $7 / 26 / 95$ & N/A & $3 / 2 / 91$ & BOD9R4 & $A Z-102-2$ & S95T000264 & 1.1 & $1.03 E+03$ & UG/mI \\
\hline Fluoride & $7 / 26 / 95$ & N/A & $3 / 2 / 91$ & BOD9R5 & $A Z-102-3$ & 595T000265 & 1.1 & $1.03 E+03$ & $\mathrm{UG} / \mathrm{ml}$ \\
\hline Fluoride & $7 / 26 / 95$ & N/A & $3 / 2 / 91$ & BOD9R5 & $A Z-102-3$ & S95T000265 & 1.1 & $1.00 E+03$ & $\mathrm{UG} / \mathrm{ml}$ \\
\hline $\mathrm{F}=$ & Aug 89 & NS & NS & NS & NS & NS & NS & $8.72 E-01$ & $g / L$ \\
\hline$F$ & $9 / 1 / 93$ & N/A & $6 / 30 / 85$ & N/A & N/A & N/A & 1.14 & $3.89 E+01$ & umol/g \\
\hline$F$ & $10 / 31 / 86$ & NS & $6 / 30 / 85$ & NS & NS & NS & 1.14 & $3.89 E+01$ & umol/g \\
\hline Fluoride & $7 / 26 / 95$ & N/A & $3 / 2 / 91$ & BOD9R4 & $A Z-102-2$ & S95T000264 & 1.1 & $7.98 E+02$ & $\mathrm{UG} / \mathrm{ml}$ \\
\hline$F$ & $10 / 31 / 86$ & NS & $6 / 30 / 85$ & NS & NS & NS & 1.73 & $2.42 \mathrm{E}+01$ & umol/g \\
\hline$F$ & $12 / 7 / 83$ & NS & NS & $T 1520$ & NS & NS & NS & $3.80 E-02$ & $M$ \\
\hline$F$ & $10 / 1 / 83$ & NS & NS & RAT-AZ102-3 & NS & NS & NS & $3.70 E-02$ & moles \\
\hline$F$ & $3 / 10 / 84$ & NS & NS & NS & NS & NS & NS & $3.70 E-02$ & $M$ \\
\hline$F$ & $3 / 10 / 84$ & NS & NS & NS & NS & NS & NS & $3.70 E-02$ & $M$ \\
\hline$F$ & $7 / 5 / 84$ & NS & NS & $T 3495$ & NS & NS & NS & 3.70E-02 & $M$ \\
\hline F- & Aug 89 & NS & NS & NS & NS & NS & NS & 6.85E-01 & $g / L$ \\
\hline$F$ & $9 / 20 / 78$ & NS & NS & RAT-AZ102-4C & NS & NS & 1.39 & 1.65E-02 & moles $n$ \\
\hline$F$ & $9 / 20 / 78$ & NS & NS & NS & NS & NS & 1.39 & $1.65 \mathrm{E}-02$ & $M$ \\
\hline$F$ & $2 / 4 / 81$ & NS & NS & $R-3699$ & NS & NS & 1.11 & 1.41E-02 & moles $n$ \\
\hline$F$ & $2 / 4 / 81$ & NS & NS & R-3700 & NS & NS & 1.11 & $1.41 \mathrm{E}-02$ & moles $n$ \\
\hline$F$ & $2 / 4 / 81$ & NS & NS & R3699 & NS & NS & 1.11 & $1.41 \mathrm{E}-02$ & $M$ \\
\hline$F$ & $2 / 4 / 81$ & NS & NS & R3700 & NS & NS & 1.11 & 1.41E-02 & $M$ \\
\hline F- & NS & NS & NS & NS & NS & NS & 1.04 & $1.02 E-02$ & Molen \\
\hline$F$ & $6 / 26 / 75$ & NS & NS & RAT-AZ102-4a & NS & NS & 1.39 & $9.00 \mathrm{E}-03$ & moles $\Lambda$ \\
\hline$F$ & $6 / 26 / 75$ & NS & NS & NS & NS & NS & 1.392 & $9.00 \mathrm{E}-03$ & $M$ \\
\hline
\end{tabular}




\begin{tabular}{|c|c|c|c|c|c|c|c|}
\hline & & \multicolumn{3}{|c|}{ Analyte Information } & \multirow[b]{2}{*}{$\begin{array}{c}\text { Reference } \\
\text { Number }\end{array}$} & \multirow[b]{2}{*}{$\begin{array}{c}\text { Validated } \\
(Y / N)\end{array}$} & \multirow[b]{2}{*}{ Notes } \\
\hline Code & Source & Analyte & Conc. (ug/L) & Conc. (mol/L) & & & \\
\hline $\mathbf{S}$ & Braun & $\mathrm{Cr}$ & $2.75 E+05$ & $5.29 E-03$ & NS & $N$ & Won't use Braun, Wastren, or HTCE Inv. Data \\
\hline FI & Raw & $\mathrm{Cr}$ & $1.50 E+05$ & 2.88E-03 & $A Z 102-23$ & $N$ & Water wash of solid sample not supernatent \\
\hline $\mathbf{Y}$ & Braun & $\mathrm{Cr}$ & $1.18 E+05$ & 2.27E-03 & NS & $\mathbf{N}$ & Won't use Braun, Wastren, or HTCE Inv. Data \\
\hline $\mathbf{F I}$ & Raw & $\mathrm{Cr}$ & $0.00 E+00$ & $0.00 E+00$ & $A 2102-18$ & D & NS \\
\hline P & Wastren & $\operatorname{Cr}(\mathrm{V})$ & $1.04 E+06$ & $2.00 E-02$ & NS & $\mathbf{N}$ & Won't use Braun, Wastren, or HTCE Inv. Data \\
\hline $\mathbf{P}$ & Wastren & Crivi) & 2.77E+05 & 5.33E-03 & NS & $\mathbf{N}$ & Won't use Braun, Wastren, or HTCE Inv. Data \\
\hline $\mathbf{P}$ & Braun & Cr(VI) & $2.17 E+05$ & $4.18 \mathrm{E}-03$ & NS & $\mathbf{N}$ & Won't use Braun, Wastren, or HTCE Inv. Data \\
\hline $\mathbf{P}$ & Braun & $\mathrm{Cr}(\mathrm{VI})$ & $2.14 E+05$ & 4.11E-03 & NS & $\mathbf{N}$ & Won't use Braun, Wastren, or HTCE Inv. Data \\
\hline FI & Raw & $\mathrm{Cr}(\mathrm{V})$ & $1.30 E+05$ & 2.50E-03 & $A Z 102-18$ & $\mathbf{N}$ & Water wash of solid sample not supernatent \\
\hline $\mathrm{FI}$ & Raw & $\mathrm{Cr}(\mathrm{V} I)$ & $1.30 E+05$ & $2.50 E-03$ & AZ102-23 & $\mathbf{N}$ & Water wash of solid sample not supernatent \\
\hline $\mathrm{Cl}$ & Raw & CrivI) & $1.20 E+05$ & $2.30 \mathrm{E}-03$ & AZ102-23 & $\bar{Y}$ & \\
\hline $\mathrm{Cl}$ & Raw & $\mathrm{Cr}(\mathrm{V})$ & $1.20 E+05$ & $2.30 E-03$ & $A 2102-18$ & D & NS \\
\hline $\mathbf{P}$ & Braun & $\mathrm{Cr}(\mathrm{V} I)$ & $1.10 E+05$ & 2.11E-03 & NS & $N$ & Won't use Braun, Wastren, or HTCE Inv. Data \\
\hline L & TRAC & $F$ & NS & NS & NS & $N$ & Analysis Prior to $9 / 86$ \\
\hline$P-1$ & Raw & $F$ & $1.08 E+06$ & $5.68 \mathrm{E}-02$ & $A Z 102-24$ & $\mathbf{Y}$ & Updated per memo 8/17/95 \\
\hline$P-2$ & Raw & $F$ & $1.07 E+06$ & $5.63 \mathrm{E}-02$ & AZ102-24 & $Y$ & Updated per memo $8 / 17 / 95$ \\
\hline$P-2$ & Raw & $F$ & $1.03 E+06$ & $5.42 \mathrm{E}-02$ & AZ102-24 & $\bar{Y}$ & Updated per memo $8 / 17 / 95$ \\
\hline$P-2$ & Raw & $F$ & $1.03 E+06$ & $5.42 \mathrm{E}-02$ & $A Z 102-24$ & $\mathbf{Y}$ & Updated per memo $8 / 17 / 95$ \\
\hline$P-1$ & Raw & $F$ & $1.00 E+06$ & $5.26 \mathrm{E}-02$ & AZ102-24 & $\bar{Y}$ & Updated per memo 8/17/95 \\
\hline$P$ & Wastren & $F$ & $8.72 \mathrm{E}+05$ & $4.59 \mathrm{E}-02$ & NS & $N$ & Analysis Prior to $9 / 86$ \\
\hline $\mathrm{Cl}$ & Raw & $F$ & $8.43 E+05$ & $4.43 \mathrm{E}-02$ & $A Z 102-23$ & $\bar{Y}$ & \\
\hline $\mathrm{Cl}$ & Raw & $F$ & $8.43 E+05$ & $4.43 E-02$ & AZ102-18 & D & NS \\
\hline$P-1$ & Raw & $\mathbf{F}$ & $7.98 E+05$ & $4.20 \mathrm{E}-02$ & AZ102-24 & $\bar{Y}$ & Updated per memo 8/17/95 \\
\hline $\mathrm{BI}$ & Raw & $F$ & $7.95 E+05$ & $4.19 \mathrm{E}-02$ & AZ102-18 & D & NS \\
\hline$L$ & Raw & $F$ & $7.22 \mathrm{E}+05$ & $3.80 E-02$ & AZ102-14 & $\bar{N}$ & Analysis Prior to $9 / 86$ \\
\hline $\mathrm{P}$ & Braun & $\mathbf{F}$ & $7.03 E+05$ & $3.70 \mathrm{E}-02$ & NS & $\mathrm{N}$ & Analysis Prior to $9 / 86$ \\
\hline$L$ & Raw & $\mathbf{F}$ & $7.03 \mathrm{E}+05$ & $3.70 \mathrm{E}-02$ & $A Z 102-16$ & $N$ & Analysis Prior to $9 / 86$ \\
\hline $\mathbf{L}$ & Raw & $\bar{F}$ & $7.03 E+05$ & $3.70 \mathrm{E}-02$ & $A 2102-16$ & $\mathrm{~N}$ & Analysis Prior to $9 / 86$ \\
\hline$L$ & Raw & $F$ & $7.03 E+05$ & $3.70 \mathrm{E}-02$ & $A Z 102-16$ & $\mathbf{N}$ & Analysis Prior to $9 / 86$ \\
\hline G & Wastren & $F$ & $6.85 E+05$ & 3.61E-02 & NS & $\mathbf{N}$ & Won't use Braun, Wastren, or HTCE Inv. Data \\
\hline$P$ & Braun & $F$ & $3.14 E+05$ & $1.65 \mathrm{E}-02$ & NS & $N$ & Analysis Prior to $9 / 86$ \\
\hline$L$ & Raw & $F$ & $3.14 \mathrm{E}+05$ & $1.65 E-02$ & AZ102-6 & $\mathbf{N}$ & Analysis Prior to $9 / 86$ \\
\hline$P$ & Braun & $F$ & $2.68 E+05$ & $1.41 \mathrm{E}-02$ & NS & $N$ & Analysis Prior to $9 / 86$ \\
\hline$P$ & Braun & $F$ & $2.68 \mathrm{E}+05$ & $1.41 \mathrm{E}-02$ & NS & $\mathbf{N}$ & Analysis Prior to $9 / 86$ \\
\hline$L$ & Raw & $F$ & $2.68 E+05$ & $1.41 \mathrm{E}-02$ & $A Z 102-8$ & $N$ & Analysis Prior to $9 / 86$ \\
\hline$L$ & Raw & $F$ & $2.68 \mathrm{E}+05$ & $1.41 E-02$ & $A Z 102-8$ & $N$ & Analysis Prior to $9 / 86$ \\
\hline $\mathbf{L}$ & HTCE Inv. & $\mathbf{F}$ & $1.94 E+05$ & $1.02 \mathrm{E}-02$ & NS & N & Won't use Braun, Wastren, or HTCE Inv. Data \\
\hline$P$ & Braun & $F$ & $1.71 E+05$ & $9.00 \mathrm{E}-03$ & NS & $\bar{N}$ & Analysis Prior to $9 / 86$ \\
\hline$L$ & Raw & $F$ & $1.71 E+05$ & $9.00 E-03$ & $A Z 102-6$ & $N$ & Analysis Prior to $9 / 86$ \\
\hline
\end{tabular}




\begin{tabular}{|c|c|c|c|c|c|c|c|c|c|}
\hline & & & & Tank Waste C & racterization & ample Informatic & & & \\
\hline $\begin{array}{l}\text { Reported } \\
\text { Chemical }\end{array}$ & Memo Date & $\begin{array}{c}\text { Start } \\
\text { Analysis } \\
\text { Date }\end{array}$ & $\begin{array}{c}\text { Start Sample } \\
\text { Date }\end{array}$ & Sample Number & $\begin{array}{c}\text { Tank Farm } \\
\text { Number }\end{array}$ & $\begin{array}{c}\text { Lab Sample } \\
\text { Number }\end{array}$ & $\begin{array}{l}\text { Density } \\
\text { (g/mL) }\end{array}$ & $\begin{array}{c}\text { Reported } \\
\text { Value }\end{array}$ & Reported Ur \\
\hline$F-$ & NS & NS & NS & NS & NS & NS & 1.39 & 7.35E-03 & Mole/L \\
\hline$F$ & $8 / 31 / 89$ & NS & $6 / 30 / 85$ & Cómposite Core & NS & NS & 11.01 & $5.21 E+00$ & umol/g \\
\hline$F$ & $10 / 31 / 86$ & NS & $6 / 30 / 85$ & NS & NS & NS & 1.11 & $4.29 E+00$ & umol/g \\
\hline TIC & $8 / 31 / 89$ & NS & $6 / 30 / 85$ & Composite Core & NS & NS & 1.11 & $4.29 E+00$ & umal/g \\
\hline$F$ & $10 / 31 / 86$ & NS & $6 / 30 / 85$ & NS & NS & NS & NS & $5.21 E+00$ & umol/g \\
\hline Iron & $7 / 26 / 95$ & N/A & $3 / 2 / 91$ & 800908 & $A Z-102-1$ & S95T000266 & 1.1 & $<1.00 e+01$ & UG/mi \\
\hline Iron & $7 / 26 / 95$ & N/A & $3 / 2 / 91$ & 800980 & $A Z-102-2$ & S95T000267 & 1.1 & $<1.008+01$ & $\mathrm{UG} / \mathrm{ml}$ \\
\hline Iron & $7 / 26 / 95$ & N/A & $3 / 2 / 91$ & BOD9R2 & $A Z-102-3$ & \$95T000268 & 1.1 & $<1.000+01$ & $\mathrm{UG} / \mathrm{ml}$ \\
\hline Iron & $7 / 26 / 95$ & N/A & $3 / 2 / 91$ & B00908 & $A Z-102-1$ & S95T000266 & 1.1 & $<1.00 e+01$ & $\mathrm{UG} / \mathrm{ml}$ \\
\hline Iron & $7 / 26 / 95$ & N/A & $3 / 2 / 91$ & BOD9RO & $A Z-102-2$ & S95T000267 & 1.1 & $<1.00 e+01$ & $\mathrm{UG} / \mathrm{ml}$ \\
\hline Iron & $7 / 26 / 95$ & N/A & $3 / 2 / 91$ & BOD9R2 & $A Z-102-3$ & 595T000268 & 1.1 & $<1.00 e+01$ & $\mathrm{UG} / \mathrm{ml}$ \\
\hline $\mathrm{Fe}$ & $1 / 1 / 77$ & NS & NS & NS & NS & NS & NS & NS & NS \\
\hline $\mathrm{Fe}$ & Aug 89 & NS & NS & NS & NS & NS & NS & $1.96 E+02$ & $g / L$ \\
\hline $\mathrm{Fe} 3+($ total Fe $)$ & NS & NS & NS & NS & NS & NS & 1.39 & $1.54 \mathrm{E}+00$ & Mole/L \\
\hline $\mathrm{Fe}$ & $12 / 13 / 83$ & NS & $7 / 31 / 83$ & $T 1521$ & NS & NS & NS & $9.40 \mathrm{E}-01$ & $M$ \\
\hline TIC & $8 / 31 / 89$ & NS & $6 / 30 / 85$ & Composite Core & NS & NS & 1.11 & $5.70 E+02$ & $\mathrm{umal} / \mathrm{g}$ \\
\hline $\mathrm{Fe}$ & $11 / 5 / 81$ & NS & NS & RAT-AZ102-2B & NS & NS & 1.4 & $3.40 \mathrm{E}-01$ & moles/l \\
\hline $\mathrm{Fe}$ & $5 / 5 / 82$ & NS & $11 / 4 / 81$ & NS & NS & NS & 1.4 & $3.40 \mathrm{E}-01$ & $M$ \\
\hline $\mathrm{Fe} 3+($ total Fe) & NS & NS & NS & NS & NS & NS & 1.04 & $6.96 \mathrm{E}-04$ & Mole/L \\
\hline Fo & $2 / 4 / 81$ & NS & NS & $\mathrm{R}-3699$ & NS & NS & 1.11 & 2.17E-04 & moles/l \\
\hline $\mathrm{Fe}$ & $2 / 4 / 81$ & NS & NS & $R-3700$ & NS & NS & 1.11 & 2.17E-04 & moles/I \\
\hline $\mathrm{Fe}$ & $2 / 4 / 81$ & NS & NS & R3699 & NS & NS & 1.11 & $2.17 \mathrm{E}-04$ & $\bar{M}$ \\
\hline $\mathrm{Fe}$ & $2 / 4 / 81$ & NS & NS & 83700 & NS & NS & 1.11 & $2.17 E-04$ & $\bar{M}$ \\
\hline $\mathrm{Fo}$ & Aug 89 & NS & NS & NS & NS & NS & NS & $1.12 \mathrm{E}-02$ & $g / L$ \\
\hline $\mathrm{Fe}$ & $9 / 1 / 93$ & N/A & $6 / 30 / 85$ & N/A & N/A & N/A & 1.14 & $1.70 \mathrm{E}-01$ & umol/g \\
\hline $\mathrm{Fe}$ & $10 / 31 / 86$ & NS & $6 / 30 / 85$ & Composite Core & NS & NS & $(1.0)$ & $9.30 \mathrm{E}-02$ & umol/g \\
\hline $\mathrm{Fe}$ & $5 / 3 / 86$ & NS & NS & L3 & NS & NS & NS & $7.78 E+02$ & umol/g \\
\hline $\mathrm{Fe}$ & $5 / 3 / 86$ & NS & NS & L4 & NS & NS & NS & $1.10 E+03$ & umol/g \\
\hline $\mathrm{Fe}$ & $5 / 3 / 86$ & NS & NS & LI & NS & NS & NS & $1.99 E+03$ & umol/s \\
\hline $\bar{K}$ & $10 / 31 / 86$ & NS & $6 / 30 / 85$ & NS & NS & NS & 1.11 & $<2.20 e+01$ & umol/g \\
\hline TIC & $8 / 31 / 89$ & NS & $6 / 30 / 85$ & Composite Core & NS & NS & 1.11 & $<2.20 e+01$ & umol/g \\
\hline K & $5 / 3 / 86$ & NS & NS & L4 & NS & NS & NS & $<6.12 \mathrm{e}+01$ & umol/g \\
\hline K & $5 / 3 / 86$ & NS & NS & L1 & NS & NS & NS & $<6.15 e+01$ & umol/g \\
\hline
\end{tabular}




\begin{tabular}{|c|c|c|c|c|c|c|c|}
\hline \multirow[b]{2}{*}{ Code } & \multirow[b]{2}{*}{ Source } & \multicolumn{3}{|c|}{ Analyte information } & \multirow[b]{2}{*}{$\begin{array}{c}\text { Reference } \\
\text { Number } \\
\end{array}$} & \multirow[b]{2}{*}{$\begin{array}{c}\text { Validated } \\
\text { (Y/N) }\end{array}$} & \multirow[b]{2}{*}{ Notes } \\
\hline & & Analyte & Conc. (ug/L) & Conc. (mol/L) & & & \\
\hline $\mathbf{S}$ & HTCE Inv. & $F$ & $1.40 E+05$ & 7.35E-03 & NS & $\mathbf{N}$ & Won't use Braun, Wastren, or HTCE Inv. Data \\
\hline $\mathrm{FI}$ & Raw & $\mathbf{F}$ & $9.90 E+04$ & $5.21 \mathrm{E}-03$ & $A Z 102-23$ & $N$ & Water wash of solid sample not supernatent \\
\hline $\mathrm{EI}$ & Raw & $F$ & $9.05 E+04$ & $4.76 \mathrm{E}-03$ & AZ102-18 & $\mathrm{D}$ & NS \\
\hline EI & Raw & $\mathbf{F}$ & $9.05 E+04$ & $4.76 \mathrm{E}-03$ & $A Z 102-23$ & $\mathbf{N}$ & $\begin{array}{l}\text { Solid sample after has been washed with } \\
\text { fresh water }\end{array}$ \\
\hline $\mathbf{F I}$ & Raw & $\mathbf{F}$ & $0.00 E+00$ & $0.00 E+00$ & $A Z 102-18$ & D & NS \\
\hline P-1 & Raw & $\mathrm{Fe}$ & \#VALUE! & \#VALUE! & AZ102-24 & $Y$ & Updated per memo $8 / 17 / 95$ \\
\hline P-1 & Raw & $\mathrm{Fe}$ & \#VALUE! & \#VALUE! & $A Z 102-24$ & $\mathrm{Y}$ & Updated per memo $8 / 17 / 95$ \\
\hline$P-1$ & Raw & $\mathrm{Fe}$ & \#VALUE! & \#VALUE! & $A Z 102-24$ & $Y$ & Updated per memo 8/17/95 \\
\hline P-2 & Raw & $\mathrm{Fe}$ & \#VALUE! & \#VALUE! & AZ102-24 & $Y$ & Updated per memo $8 / 17 / 95$ \\
\hline $\mathrm{P}-2$ & Raw & $\mathrm{Fe}$ & \#VALUE! & \#VALUE! & $A Z 102-24$ & $\bar{Y}$ & Updated per memo $8 / 17 / 95$ \\
\hline P-2 & Raw & $\mathrm{Fe}$ & \#VALUE! & \#VALUE! & $A Z 102-24$ & $Y$ & Updated per memo 8/17/95 \\
\hline L & TRAC & $\mathrm{Fe}$ & NS & NS & NS & $\mathrm{N}$ & Analysis Prior to $9 / 86$ \\
\hline G & Wastren & $\mathrm{Fe}$ & $1.96 E+08$ & $3.51 E+00$ & NS & N & Won't use Braun, Wastren, or HTCE Inv. Data \\
\hline s & HTCE Inv. & $\mathrm{Fe}$ & $8.60 E+07$ & $1.54 E+00$ & NS & N & Won't use Braun, Wastren, or HTCE Inv. Data \\
\hline $\mathbf{s}$ & Raw & $\mathrm{Fe}$ & $5.25 E+07$ & $9.40 \mathrm{E}-01$ & $A Z 102-14$ & $\mathbf{N}$ & No units for concentration given in report \\
\hline El & Raw & $\mathrm{Fe}$ & $3.53 E+07$ & $6.33 \mathrm{E}-01$ & $A Z 102-18$ & N & $\begin{array}{l}\text { Solid sample after has been washed with } \\
\text { fresh water }\end{array}$ \\
\hline $\mathbf{s}$ & Braun & $\mathrm{Fe}$ & $1.90 E+07$ & $3.40 E-01$ & NS & $\mathbf{N}$ & Won't use Braun, Wastren, or HTCE Inv. Data \\
\hline$\underline{s}$ & Raw & $\mathrm{Fe}$ & $1.90 E+07$ & $3.40 \mathrm{E}-01$ & $A Z 102-11$ & $\mathrm{~N}$ & Analysis Prior to $9 / 86$ \\
\hline $\mathbf{L}$ & HTCE Inv. & $\mathrm{Fe}$ & $3.88 E+04$ & $6.96 \mathrm{E}-04$ & NS & $\mathbf{N}$ & Won't use Braun, Wastren, or HTCE Inv. Data \\
\hline $\mathbf{P}$ & Braun & Fe & $1.21 E+04$ & $2.17 E-04$ & NS & $\mathbf{N}$ & Won't use Braun, Wastren, or HTCE Inv. Data \\
\hline $\mathbf{P}$ & Braun & $\mathrm{Fe}$ & $1.21 E+04$ & 2.17E-04 & NS & $N$ & Won't use Braun, Wastren, or HTCE Inv. Data \\
\hline$\underline{L}$ & Raw & $\mathrm{Fe}$ & $1.21 \mathrm{E}+04$ & $2.17 E-04$ & $A Z 102-8$ & $N$ & Analysis Prior to $9 / 86$ \\
\hline$\underline{L}$ & Raw & $\mathrm{Fe}$ & $1.21 E+04$ & $2.17 E-04$ & AZ102-8 & $N$ & Analysis Prior to $9 / 86$ \\
\hline $\mathbf{P}$ & Wastren & $\mathrm{Fe}$ & $1.12 E+04$ & $2.01 E-04$ & NS & $\mathbf{N}$ & Won't use Braun, Wastren, or HTCE Inv. Data \\
\hline $\mathrm{Cl}$ & Raw & $\mathrm{Fe}$ & $1.08 E+04$ & $1.94 E-04$ & $A Z 102-23$ & $\bar{Y}$ & \\
\hline FI & Raw & $\mathrm{Fe}$ & $5.19 E+03$ & $9.30 E-05$ & $A Z 102-18$ & $\mathbf{N}$ & Water wash of solid sample not supernatent \\
\hline $\mathbf{s}$ & Raw & $\mathrm{Fe}$ & $0.00 \mathrm{E}+00$ & $0.00 E+00$ & $A Z 102-17$ & D & Points already in DB as part of $A Z_{-} 102-23$ \\
\hline $\mathbf{S}$ & Raw & $\mathrm{Fe}$ & $0.00 E+00$ & $0.00 E+00$ & $A 2102-17$ & D & Points already in DB as part of $A Z_{-} 102-23$ \\
\hline s & Raw & $\mathrm{Fe}$ & $0.00 E+00$ & $0.00 E+00$ & $A Z 102-17$ & D & Points already in DB as part of AZ_102-23 \\
\hline EI & Raw & $\mathrm{K}$ & \#VALUE! & \#VALUEI & $A Z 102-18$ & $D$ & NS \\
\hline El & Raw & K & \#VALUEI & \#ALUE! & $A Z 102-23$ & $N$ & $\begin{array}{l}\text { Solid sample after has been washed with } \\
\text { fresh water }\end{array}$ \\
\hline $\mathbf{S}$ & Raw & K & \#VALUE! & \#VALUE! & $A Z 102-17$ & D & Points already in DB as part of AZ_102-23 \\
\hline s & Raw & $k$ & \#VALUE! & \#VALUE! & $A Z 102-17$ & D & Points already in DB as part of AZ_102-23 \\
\hline
\end{tabular}




\begin{tabular}{|c|c|c|c|c|c|c|c|c|c|}
\hline \multicolumn{10}{|c|}{ Tank Waste Characterization Sample Information } \\
\hline $\begin{array}{l}\text { Reported } \\
\text { Chemical } \\
\end{array}$ & Memo Date & $\begin{array}{c}\text { Start } \\
\text { Analysis } \\
\text { Date }\end{array}$ & $\begin{array}{c}\text { Start Sample } \\
\text { Date }\end{array}$ & Sample Number & $\begin{array}{c}\text { Tank Farm } \\
\text { Number }\end{array}$ & $\begin{array}{c}\text { Lab Sample } \\
\text { Number }\end{array}$ & $\begin{array}{c}\text { Density } \\
\text { (g/mL) }\end{array}$ & $\begin{array}{c}\text { Reported } \\
\text { Value } \\
\end{array}$ & Reported Un \\
\hline$k$ & $5 / 3 / 86$ & NS & NS & L3 & NS & NS & NS & $<6.34 \theta+01$ & umol/g \\
\hline$K$ & $1 / 1 / 77$ & NS & NS & NS & NS & NS & NS & NS & NS \\
\hline$k$ & Aug 89 & NS & NS & NS & NS & NS & NS & $3.09 E+00$ & $\mathbf{g} / \mathbf{L}$ \\
\hline$k$ & Aug 89 & NS & NS & NS & NS & NS & NS & $1.49 E+00$ & $g / L$ \\
\hline $\mathrm{K}$ & $9 / 1 / 93$ & N/A & $6 / 30 / 85$ & N/A & N/A & N/A & 1.14 & $3.23 E+01$ & $\mathrm{umol} / \mathrm{g}$ \\
\hline $\mathrm{K}$ & $10 / 31 / 86$ & NS & $6 / 30 / 85$ & NS & NS & NS & 1.14 & $3.23 E+01$ & umol/g \\
\hline$K$ & $11 / 5 / 81$ & NS & NS & RAT-AZ102-2A & NS & NS & 1.09 & $1.00 E-02$ & moles $\Lambda$ \\
\hline $\mathrm{K}$ & $5 / 5 / 82$ & NS & NS & NS & NS & NS & 1.09 & $1.00 E-02$ & $M$ \\
\hline$k$ & $11 / 5 / 81$ & NS & NS & RAT-AZ102-2C & NS & NS & 1.26 & 8.66E-03 & moles/l \\
\hline$K$ & $8 / 31 / 89$ & NS & $6 / 30 / 85$ & Composite Core & NS & NS & $(1.0)$ & $5.72 E+00$ & umol/g \\
\hline$\cdot \mathrm{K}$ & $1 / 30 / 82$ & NS & NS & T-5387 & NS & NS & 1.008 & $5.80 E-04$ & moles $n$ \\
\hline$K$ & $2 / 19 / 82$ & NS & NS & T5387 & NS & NS & 1.008 & $5.80 E-04$ & $M$ \\
\hline$k+$ & NS & NS & NS & NS & NS & NS & 1.39 & $3.85 E-04$ & Mole/L \\
\hline$K+$ & NS & NS & NS & NS & NS & NS & 1.04 & 7.78E-05 & Mole/L \\
\hline$K$ & $10 / 31 / 86$ & NS & $6 / 30 / 85$ & NS & NS & NS & NS & $5.72 E+00$ & umol/g \\
\hline La & $10 / 31 / 86$ & NS & $6 / 30 / 85$ & NS & NS & NS & 1.73 & $3.24 E+01$ & umol/g \\
\hline La & $8 / 1 / 85$ & NS & NS & NS & NS & NS & NS & $6.71 E+00$ & $g / L$ \\
\hline La & $11 / 5 / 81$ & NS & NS & RAT-AZ102-2B & NS & NS & 1.4 & $1.42 \mathrm{E}-02$ & moles $/ 1$ \\
\hline La & $10 / 31 / 86$ & NS & $6 / 30 / 85$ & NS & NS & NS & 1.11 & $8.02 E+00$ & umol/g \\
\hline TIC & 8/31/89 & NS & $6 / 30 / 85$ & Composite Core & NS & NS & 1.11 & $8.02 E+\infty 0$ & umol/g \\
\hline La & $8 / 1 / 85$ & NS & NS & NS & NS & NS & NS & $1.80 E-03$ & $g / L$ \\
\hline La & $9 / 1 / 93$ & N/A & $6 / 30 / 85$ & N/A & N/A & N/A & 1.14 & $1.10 E-02$ & umol/g \\
\hline La & $10 / 31 / 86$ & NS & $6 / 30 / 85$ & NS & NS & NS & 1.14 & $1.10 \mathrm{E}-02$ & umol/g \\
\hline La & $10 / 31 / 86$ & NS & $6 / 30 / 85$ & Composite Core & NS & NS & $(1.0)$ & 2.00E-03 & umol/g \\
\hline $\mathrm{Na}$ & $7 / 28 / 79$ & NS & NS & RAT-AZ102-6 & NS & NS & 1.41 & $1.09 E+01$ & moles $/ 1$ \\
\hline $\mathrm{Na}$ & $7 / 28 / 79$ & NS & NS & RAT-AZ102-4D & NS & NS & 1.41 & $8.51 E+00$ & moles $/ 1$ \\
\hline $\mathrm{Na}$ & $5 / 2 / 86$ & NS & NS & R280783 & NS & NS & NS & $8.51 E+00$ & $M$ \\
\hline $\mathrm{Na}$ & $9 / 20 / 78$ & NS & NS & RAT-AZ102-4C & NS & NS & 1.39 & $8.14 E+00$ & moles II \\
\hline $\mathrm{Na}$ & $11 / 5 / 81$ & NS & NS & RAT-AZ102-2B & NS & NS & 1.4 & $7.08 E+00$ & moles $n$ \\
\hline $\mathrm{Na}$ & $11 / 5 / 81$ & NS & NS & RAT-AZ102-2C & NS & NS & 1.26 & $4.49 E+00$ & moles $/$ \\
\hline $\mathrm{Na}$ & $10 / 31 / 86$ & NS & $6 / 30 / 85$ & NS & NS & NS & 1.73 & $2.57 E+03$ & umol/g \\
\hline $\mathrm{Na}$ & NS & NS & NS & NS & NS & NS & NS & $8.80 E+01$ & $g / L$ \\
\hline $\mathrm{Na}$ & $6 / 26 / 75$ & NS & NS & RAT-AZ102-4a & NS & NS & 1.39 & $3.00 E+00$ & moles 1 \\
\hline
\end{tabular}




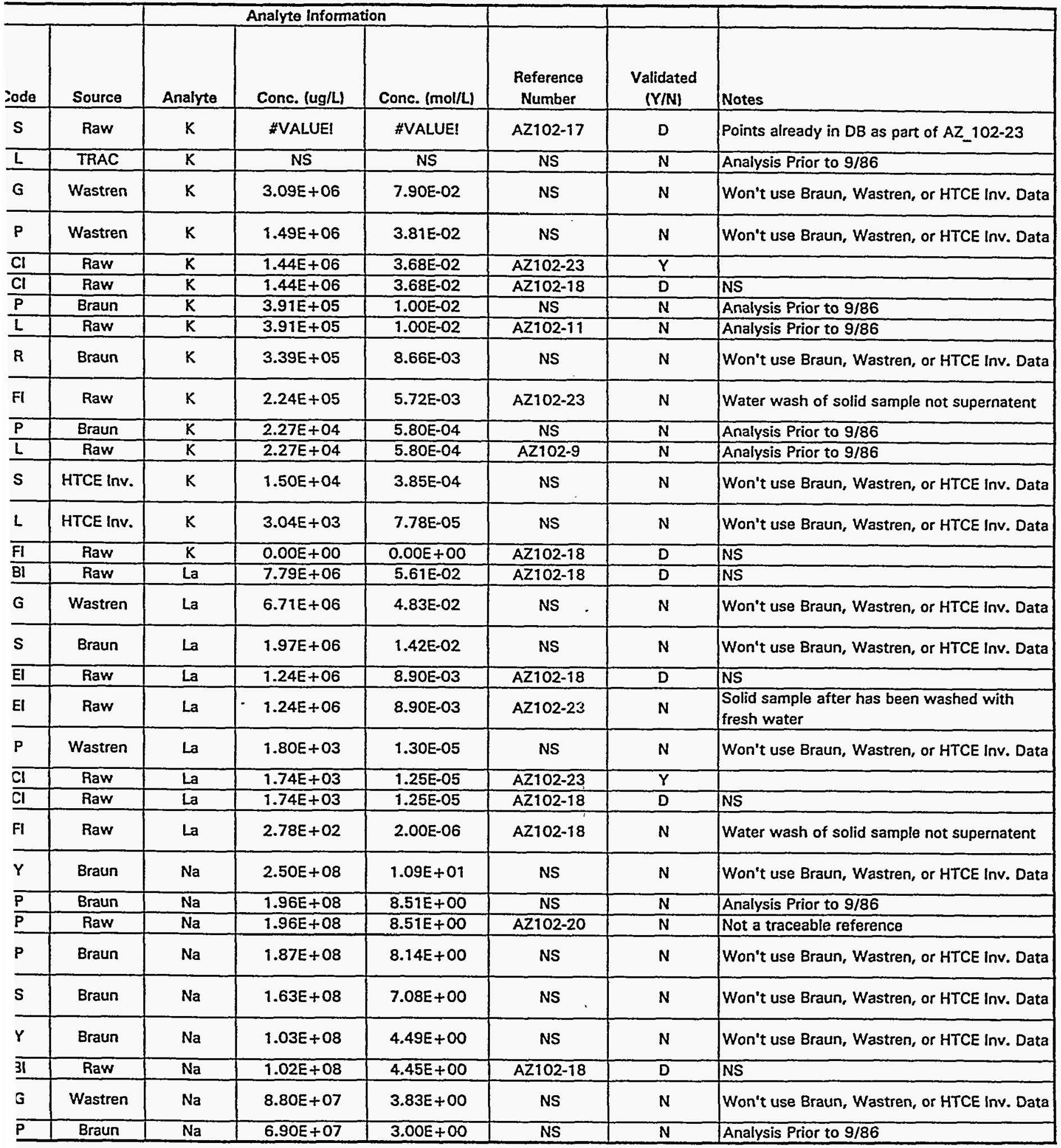




\begin{tabular}{|c|c|c|c|c|c|c|c|c|c|}
\hline & & & & Tank Waste C & racterization & ample Informati & & & \\
\hline $\begin{array}{l}\text { Reported } \\
\text { Chemical }\end{array}$ & Memo Date & $\begin{array}{c}\text { Start } \\
\text { Analysis } \\
\text { Date }\end{array}$ & $\begin{array}{c}\text { Start Sample } \\
\text { Date }\end{array}$ & Sample Number & $\begin{array}{c}\text { Tank Farm } \\
\text { Number }\end{array}$ & $\begin{array}{c}\text { Lab Sample } \\
\text { Number }\end{array}$ & $\begin{array}{l}\text { Density } \\
(\mathrm{g} / \mathrm{mL})\end{array}$ & $\begin{array}{c}\text { Reported } \\
\text { Value } \\
\end{array}$ & Reported $U_{i}$ \\
\hline $\mathrm{Na}$ & $11 / 5 / 81$ & NS & NS & RAT-AZ102-2A & NS & NS & 1.09 & $2.77 E+00$ & moles/I \\
\hline $\mathrm{Na}$ & $5 / 2 / 86$ & NS & NS & R60386 & NS & NS & NS & $2.77 E+00$ & $M$ \\
\hline $\mathrm{Na}$ & $2 / 4 / 81$ & NS & NS & $R-3699$ & NS & NS & 1.11 & $2.72 E+00$ & moles/l \\
\hline $\mathrm{Na}$ & $2 / 4 / 81$ & NS & NS & R-3700 & NS & NS & 1.11 & $2.65 E+00$ & molesn \\
\hline Sodium & $7 / 26 / 95$ & N/A & $3 / 2 / 91$ & 800908 & $A Z-102-1$ & S95T000266 & 1.1 & $5.37 E+04$ & $\mathrm{UG} / \mathrm{ml}$ \\
\hline Sodium & $7 / 26 / 95$ & N/A & $3 / 2 / 91$ & BOD9RO & $A Z-102-2$ & S95T000267 & 1.1 & $5.34 E+04$ & $\mathrm{UG} / \mathrm{ml}$ \\
\hline Sodium & $7 / 26 / 95$ & N/A & $3 / 2 / 91$ & BOD9R2 & $A Z-102-3$ & S95T000268 & 1.1 & $5.31 E+04$ & $\mathrm{UG} / \mathrm{ml}$ \\
\hline Sodium & $7 / 26 / 95$ & N/A & $3 / 2 / 91$ & B00908 & $A Z-102-1$ & S95T000266 & 1.1 & $5.30 E+04$ & $\mathrm{UG} / \mathrm{ml}$ \\
\hline Sodium & $7 / 26 / 95$ & N/A & $3 / 2 / 91$ & BOD9RO & $A Z-102-2$ & S95T000267 & 1.1 & $5.30 E+04$ & $\mathrm{UG} / \mathrm{ml}$ \\
\hline Sodium & $7 / 26 / 95$ & N/A & $3 / 2 / 91$ & BOD9R2 & $A Z-102-3$ & S95T000268 & 1.1 & $5.29 E+04$ & $\mathrm{UG} / \mathrm{ml}$ \\
\hline $\mathrm{Na}$ & NS & NS & NS & NS & NS & NS & NS & $4.96 E+01$ & $g / L$ \\
\hline $\mathrm{Na}$ & $9 / 1 / 93$ & N/A & $6 / 30 / 85$ & N/A & N/A & N/A & 1.14 & $1.83 E+03$ & umol/g \\
\hline $\mathrm{Na}$ & $10 / 31 / 86$ & NS & $6 / 30 / 85$ & NS & NS & NS & 1.14 & $1.83 E+03$ & umol/g \\
\hline $\mathrm{Na}$ & $10 / 1 / 83$ & NS & NS & RAT-AZ102-3 & NS & NS & NS & $1.61 E+00$ & moles $/ 1$ \\
\hline $\mathrm{Na}$ & $5 / 2 / 86$ & NS & NS & $\mathrm{R} 880311$ & NS & NS & NS & $1.61 E+00$ & $M$ \\
\hline $\mathrm{Na}$ & $5 / 2 / 86$ & NS & NS & R81287 & NS & NS & NS & $1.57 E+00$ & $M$ \\
\hline $\mathrm{NaOH}$ & $7 / 28 / 79$ & NS & NS & RAT-AZ102-6 & NS & NS & 1.41 & $8.20 E-01$ & moles $n$ \\
\hline $\mathrm{Na}$ & $10 / 31 / 86$ & NS & $6 / 30 / 85$ & NS & NS & NS & 1.11 & $4.49 \mathrm{E}+02$ & umol/g \\
\hline TIC & $8 / 31 / 89$ & NS & $6 / 30 / 85$ & Composite Core & NS & NS & 1.11 & $4.49 E+02$ & umol/g \\
\hline $\mathrm{Na}$ & $8 / 31 / 89$ & NS & $6 / 30 / 85$ & Composite Core & NS & NS & $(1.0)$ & $3.31 E+02$ & umol/g \\
\hline $\mathrm{Na}$ & $1 / 30 / 82$ & NS & NS & $T-5387$ & NS & NS & 1.008 & $5.02 \mathrm{E}-02$ & moles $/ 1$ \\
\hline $\mathrm{Na}$ & $10 / 31 / 86$ & NS & $6 / 30 / 85$ & NS & NS & NS & NS & $3.31 E+02$ & umol/g \\
\hline Nd & $10 / 31 / 86$ & NS & $6 / 30 / 85$ & NS & NS & NS & 1.73 & $2.02 \mathrm{E}+01$ & umol/g \\
\hline Nd & $8 / 1 / 85$ & NS & NS & NS & NS & NS & NS & $4.34 E+00$ & g/L \\
\hline Nd & $11 / 5 / 81$ & NS & NS & RAT-AZ102-2B & NS & NS & 1.4 & $1.12 E-02$ & moles/1 \\
\hline Nd & $10 / 31 / 86$ & NS & $6 / 30 / 85$ & NS & NS & NS & 1.11 & $5.90 E+00$ & umol/g \\
\hline $\mathrm{Cr}$ & $8 / 31 / 89$ & NS & $6 / 30 / 85$ & Composite Core & NS & NS & 1.11 & $5.90 E+00$ & umol/g \\
\hline Nd & $8 / 1 / 85$ & NS & NS & NS & NS & NS & NS & $5.62 E-03$ & $g / L$ \\
\hline Nd & $10 / 31 / 86$ & NS & $6 / 30 / 85$ & NS & NS & NS & 1.14 & $3.30 E-02$ & umol/g \\
\hline Nd & $10 / 31 / 86$ & NS & $6 / 30 / 85$ & Composite Core & NS & NS & (1.0) & $5.00 E-03$ & $\mathrm{umol} / \mathrm{g}$ \\
\hline $\mathrm{Ni}$ & $1 / 1 / 77$ & NS & NS & NS & NS & NS & NS & NS & NS \\
\hline $\mathrm{Ni}$ & Aug 89 & NS & NS & NS & NS & NS & NS & $1.32 E+01$ & g/L \\
\hline $\mathrm{Ni}$ & $12 / 13 / 83$ & NS & $7 / 31 / 83$ & T1521 & NS & NS & NS & $6.20 \mathrm{E}-02$ & $\bar{M}$ \\
\hline $\mathrm{Cr}$ & $8 / 31 / 89$ & NS & $6 / 30 / 85$ & Composite Core & NS & NS & 1.11 & $3.62 E+01$ & umol/g \\
\hline $\mathrm{Ni} 2+$ & NS & NS & NS & NS & NS & NS & 1.39 & $1.01 E-02$ & Mole/L \\
\hline
\end{tabular}




\begin{tabular}{|c|c|c|c|c|c|c|c|}
\hline \multirow[b]{2}{*}{ Code } & \multirow[b]{2}{*}{ Source } & \multicolumn{3}{|c|}{ Analyte Information } & \multirow[b]{2}{*}{$\begin{array}{c}\text { Reference } \\
\text { Number }\end{array}$} & \multirow[b]{2}{*}{$\begin{array}{c}\text { Validated } \\
(Y / N)\end{array}$} & \multirow[b]{2}{*}{ Notes } \\
\hline & & Analyte & Conc. (ug/L) & Conc. (mol/L) & & & \\
\hline $\mathbf{P}$ & Braun & $\mathrm{Na}$ & $6.37 E+07$ & $2.77 E+00$ & NS & $N$ & Won't use Braun, Wastren, or HTCE Inv. Data \\
\hline$P$ & Raw & $\mathrm{Na}$ & $6.37 E+07$ & $2.77 \mathrm{E}+00$ & $A Z 102-21$ & $N$ & Not a traceable reference \\
\hline$P$ & Braun & $\mathrm{Na}$ & $6.25 E+07$ & $2.72 E+\infty 0$ & NS & $N$ & Won't use Braun, Wastren, or HTCE Inv. Data \\
\hline $\mathbf{P}$ & Braun & $\mathrm{Na}$ & $6.09 E+07$ & $2.65 E+\infty 0$ & NS & $N$ & Won't use Braun, Wastren, or HTCE Inv. Data \\
\hline $\mathrm{P}-2$ & Raw & $\mathrm{Na}$ & $5.37 E+07$ & $2.34 E+00$ & AZ102-24 & $\mathrm{Y}$ & Updated per merno 8/17/95 \\
\hline $\mathrm{P}-2$ & Raw & $\mathrm{Na}$ & $5.34 \mathrm{E}+07$ & $2.32 E+00$ & $A Z 102-24$ & $Y$ & Updated per memo 8/17/95 \\
\hline $\mathrm{P}-1$ & Raw & $\mathrm{Na}$ & $5.31 E+07$ & $2.31 E+00$ & AZ102-24 & $\bar{Y}$ & Updated per memo 8/17/95 \\
\hline $\mathrm{P}-1$ & Raw & $\mathrm{Na}$ & $5.30 E+07$ & $2.31 E+00$ & $A Z 102-24$ & $\bar{Y}$ & Updated per memo 8/17/95 \\
\hline$P-1$ & Raw & $\mathrm{Na}$ & $5.30 E+07$ & $2.31 E+00$ & $A Z 102-24$ & $Y$ & Updated per memo 8/17/95 \\
\hline P-2 & Raw & $\mathrm{Na}$ & $5.29 E+07$ & $2.30 E+00$ & AZ102-24 & $Y$ & Updated per memo $8 / 17 / 95$ \\
\hline $\mathbf{P}$ & Wastren & $\mathrm{Na}$ & $4.96 \mathrm{E}+07$ & $2.16 E+00$ & NS : & $\mathbf{N}$ & Won't use Braun, Wastren, or HTCE Inv. Data \\
\hline $\mathrm{Cl}$ & Raw & $\mathrm{Na}$ & $4.80 E+07$ & $2.09 E+00$ & AZ102-23 & $Y$ & \\
\hline $\mathrm{Cl}$ & Raw & $\mathrm{Na}$ & $4.80 E+07$ & $2.09 E+00$ & $A Z 102-18$ & $D$ & NS \\
\hline$P$ & Braun & $\mathrm{Na}$ & $3.70 E+07$ & $1.61 \mathrm{E}+00$ & NS & $N$ & Analysis Prior to $9 / 86$ \\
\hline$P$ & Raw & $\mathrm{Na}$ & $3.70 E+07$ & $1.61 E+00$ & $A 7102-19$ & $\mathbf{N}$ & Not a traceable reference \\
\hline$P$ & Raw & $\mathrm{Na}$ & $3.61 E+07$ & $1.57 E+00$ & $A Z 102-22$ & $N$ & Not a traceable reference \\
\hline$Y$ & Braun & $\mathrm{Na}$ & $1.89 E+07$ & $8.20 E-01$ & NS & N & Won't use Braun, Wastren, or HTCE Inv. Data \\
\hline EI & Raw & $\mathrm{Na}$ & $1.15 E+07$ & $4.98 E-01$ & $A Z 102-18$ & $\mathrm{D}$ & NS \\
\hline EI & Raw & $\mathrm{Na}$ & $1.15 E+07$ & $4.98 \mathrm{E}-01$ & $A Z 102-23$ & $N$ & $\begin{array}{l}\text { Solid sample after has been washed with } \\
\text { fresh water }\end{array}$ \\
\hline $\mathrm{FI}$ & Raw & $\mathrm{Na}$ & $7.61 E+06$ & $3.31 \mathrm{E}-01$ & $A \geq 102-23$ & $\mathrm{~N}$ & Water wash of solid sample not supernatent \\
\hline $\mathbf{P}$ & Braun & $\mathrm{Na}$ & $1.15 E+06$ & 5.02E-02 & NS & $N$ & Analysis Prior to $9 / 86$ \\
\hline $\mathrm{FI}$ & Raw & $\mathrm{Na}$ & $0.00 E+00$ & $0.00 E+00$ & $A 2102-18$ & D & NS \\
\hline BI & Raw & $\mathrm{Nd}$ & $5.04 E+06$ & $3.49 \mathrm{E}-02$ & $A Z 102-18$ & D & NS \\
\hline G & Wastren & Nd & $4.34 E+06$ & 3.01E-02 & NS & $\mathrm{N}$ & Won't use Braun, Wastren, or HTCE Inv. Data \\
\hline S & Braun & Nd & $1.62 E+06$ & $1.12 \mathrm{E}-02$ & NS & $N$ & Won't use Braun, Wastren, or HTCE Inv. Data \\
\hline EI & Raw & $\mathrm{Nd}$ & $9.44 E+05$ & $6.55 \mathrm{E}-03$ & $A Z 102-18$ & $\bar{D}$ & NS \\
\hline El & Raw & Nd & $9.44 E+05$ & $6.55 \mathrm{E}-03$ & AZ102-23 & $\mathbf{N}$ & $\begin{array}{l}\text { Solid sample after has been washed with } \\
\text { fresh water }\end{array}$ \\
\hline $\mathbf{P}$ & Wastren & Nd & $5.62 E+03$ & $3.90 E-05$ & NS & $N$ & Won't use Braun, Wastren, or HTCE Inv. Data \\
\hline $\mathrm{Cl}$ & Raw & Nd & $5.42 \mathrm{E}+03$ & $3.76 \mathrm{E}-05$ & $A Z 102-18$ & D & NS \\
\hline FI & Raw & Nd & $7.21 E+02$ & $5.00 E-06$ & $A Z 102-18$ & $\mathbf{N}$ & Water wash of solid sample not supernatent \\
\hline L & TRAC & $\mathrm{Ni}$ & NS & NS & NS & $N$ & Analysis Prior to $9 / 86$ \\
\hline G & Wastren & $\mathrm{Ni}$ & $1.32 E+07$ & 2.25E-01 & NS & $N$ & Won't use Braun, Wastren, or HTCE Inv. Data \\
\hline $\mathbf{s}$ & Raw & $\mathrm{Ni}$ & $3.64 E+06$ & $6.20 \mathrm{E}-02$ & AZ102-14 & $N$ & No units for concentration given in report \\
\hline $\mathrm{EI}$ & Raw & $\mathrm{Ni}$ & $2.36 E+06$ & $4.02 E-02$ & $A Z 102-18$ & $N$ & $\begin{array}{l}\text { Solid sample after has been washed with } \\
\text { fresh water }\end{array}$ \\
\hline $\mathbf{s}$ & HTCE Inv. & $\mathrm{Ni}$ & $5.91 E+05$ & $1.01 E-02$ & NS & $\mathbf{N}$ & Won't use Braun, Wastren, or HTCE lnv. Data \\
\hline
\end{tabular}




\begin{tabular}{|c|c|c|c|c|c|c|c|c|c|}
\hline \multicolumn{10}{|c|}{ Tank Waste Characterization Sample Information } \\
\hline $\begin{array}{l}\text { Reported } \\
\text { Chemical }\end{array}$ & Memo Date & $\begin{array}{c}\text { Start } \\
\text { Analysis } \\
\text { Date } \\
\end{array}$ & $\begin{array}{c}\text { Start Sample } \\
\text { Date }\end{array}$ & Sample Number & $\begin{array}{c}\text { Tank Farm } \\
\text { Number }\end{array}$ & $\begin{array}{c}\text { Lab Sample } \\
\text { Number }\end{array}$ & $\begin{array}{l}\text { Density } \\
\text { (g/mL) }\end{array}$ & $\begin{array}{c}\text { Reported } \\
\text { Value }\end{array}$ & Reported $U_{r}$ \\
\hline $\mathrm{Ni}$ & $11 / 5 / 81$ & NS & NS & RAT-AZ102-2B & NS & NS & 1.4 & 2.64E-03 & moles/ \\
\hline $\mathrm{Ni}$ & $5 / 5 / 82$ & NS & $11 / 4 / 81$ & NS & NS & NS & 1.4 & $2.64 \mathrm{E}-03$ & $M$ \\
\hline $\mathrm{Ni}$ & $11 / 5 / 81$ & NS & NS & RAT-AZ102-2C & NS & NS & 1.26 & $6.24 E-04$ & moles/l \\
\hline $\mathrm{Ni}$ & $11 / 5 / 81$ & NS & NS & RAT-AZ102-2A & NS & NS & 1.09 & $4.90 E-04$ & moles $\Pi$ \\
\hline $\mathrm{Ni}$ & $5 / 5 / 82$ & NS & $11 / 4 / 81$ & NS & NS & NS & 1.09 & $4.90 E-04$ & $M$ \\
\hline $\mathrm{Ni}$ & Aug 89 & NS & NS & NS & NS & NS & NS & $2.22 E-03$ & $g /$ \\
\hline $\mathrm{Ni}$ & $9 / 1 / 93$ & N/A & $6 / 30 / 85$ & N/A & N/A & N/A & 1.14 & $3.20 E-02$ & umol/g \\
\hline $\mathrm{Ni} 2+$ & NS & NS & NS & NS & NS & NS & 1.04 & $2.21 E-05$ & Mole/L \\
\hline $\mathrm{Ni}$ & $10 / 31 / 86$ & NS & $6 / 30 / 85$ & Composite Core & NS & NS & $(1.0)$ & $1.00 \mathrm{E}-02$ & umol/g \\
\hline $\mathrm{Ni}$ & $5 / 3 / 86$ & NS & NS & L3 & NS & NS & NS & $2.54 E+01$ & umol/g \\
\hline $\mathrm{Ni}$ & $5 / 3 / 86^{\circ}$ & NS & NS & L4 & NS & NS & NS & $7.06 E+01$ & umol/g \\
\hline $\mathrm{Ni}$ & $5 / 3 / 86$ & NS & NS & L1 & NS & NS & NS & $1.50 E+02$ & umol/g \\
\hline $\mathrm{NO2}$ & $1 / 1 / 77$ & NS & NS & NS & NS & NS & NS & NS & NS \\
\hline Nitrite & $7 / 26 / 95$ & N/A & $3 / 2 / 91$ & BOD9R3 & $A Z-102-1$ & S95T000263 & 1.1 & $2.96 E+04$ & UG/ml \\
\hline Nitrite & $7 / 26 / 95$ & N/A & $3 / 2 / 91$ & BOD9R3 & $A Z-102-1$ & S95T000263 & 1.1 & $2.95 E+04$ & $\mathrm{UG} / \mathrm{ml}$ \\
\hline Nitrite & $7 / 26 / 95$ & N/A & $3 / 2 / 91$ & BOD9R5 & $A Z-102-3$ & S95T000265 & 1.1 & $2.83 E+04$ & UG/ml \\
\hline Nitrite & $7 / 26 / 95$ & N/A & $3 / 2 / 91$ & BOD9R5 & $A Z-102-3$ & S95T000265 & 1.1 & $2.81 E+04$ & UG/ml \\
\hline Nitrite & $7 / 26 / 95$ & N/A & $3 / 2 / 91$ & BOD9R4 & $A Z-102-2$ & S95T000264 & 1.1 & $2.80 E+04$ & $\mathrm{UG} / \mathrm{ml}$ \\
\hline $\mathrm{NO2}$ & $10 / 31 / 86$ & NS & $6 / 30 / 85$ & NS & NS & NS & 1.73 & $3.35 \mathrm{E}+02$ & umol/g \\
\hline NaNO2 & $7 / 28 / 79$ & NS & NS & NS & NS & NS & 1.41 & $5.36 \mathrm{E}-01$ & $M$ \\
\hline NO2 & $7 / 28 / 79$ & NS & NS & RAT-AZ102-6 & NS & NS & 1.41 & $5.36 \mathrm{E}-01$ & moles $n$ \\
\hline NO2 & $7 / 28 / 79$ & NS & NS & RAT-AZ102-4D & NS & NS & 1.41 & 5.30E-01 & moles $\|$ \\
\hline NO2- & Aug 89 & NS & NS & NS & NS & NS & NS & $2.30 E+01$ & $g / L$ \\
\hline Nitrite & $7 / 26 / 95$ & N/A & $3 / 2 / 91$ & BOD9R4 & $A Z-102-2$ & S95T000264 & 1.1 & $2.22 E+04$ & $\mathrm{UG} / \mathrm{ml}$ \\
\hline NO2 & $12 / 3 / 75$ & NS & NS & $1496 \mathrm{~A}$ & NS & NS & 1.378 & $4.18 \mathrm{E}-01$ & $M$ \\
\hline NO2 & $12 / 3 / 75$ & NS & NS & $1496 \mathrm{~B}$ & NS & NS & 1.38 & $4.18 E-01$ & $M$ \\
\hline $\mathrm{NO2}$ & $12 / 3 / 75$ & NS & NS & $1496 C$ & NS & NS & 1.379 & $4.18 \mathrm{E}-01$ & $M$ \\
\hline NO2 & $12 / 3 / 75$ & NS & NS & 14960 & NS & NS & 1.36 & $4.18 \mathrm{E}-01$ & $M$ \\
\hline $\mathrm{NO2}$ & $12 / 3 / 75$ & NS & NS & 1458 & NS & NS & 1.391 & 2.97E-01 & $M$ \\
\hline $\mathrm{NO2}$ & $12 / 3 / 75$ & NS & NS & 1459 & NS & NS & 1.386 & $2.83 \mathrm{E}-01$ & $M$ \\
\hline NO2 & $12 / 3 / 75$ & NS & NS & 1457 & NS & NS & 1.398 & $2.74 E-01$ & $M$ \\
\hline NO2 & $10 / 1 / 83$ & NS & NS & RAT-AZ102-3 & NS & NS & NS & 2.70E-01 & moles $n$ \\
\hline NO2 & $3 / 10 / 84$ & NS & $7 / 31 / 83$ & NS & NS & NS & NS & $2.70 E-01$ & $M$ \\
\hline NO2 & $3 / 10 / 84$ & NS & $7 / 31 / 83$ & NS & NS & NS & NS & $2.70 \mathrm{E}-01$ & $M$ \\
\hline $\mathrm{NO2}$ & $7 / 5 / 84$ & NS & $7 / 31 / 83$ & T3495 & NS & NS & NS & $2.70 \mathrm{E}-01$ & $M$ \\
\hline No2 & $12 / 13 / 83$ & NS & $7 / 31 / 83$ & $T 1520$ & NS & NS & NS & $2.70 \mathrm{E}-01$ & $M$ \\
\hline
\end{tabular}




\begin{tabular}{|c|c|c|c|c|c|c|c|}
\hline & & & Analyte Inform & & & & \\
\hline Code & Source & Analyte & Conc. (ug/L) & Conc. (mol/L) & $\begin{array}{c}\text { Reference } \\
\text { Number }\end{array}$ & $\begin{array}{c}\text { Validated } \\
(\mathrm{Y} / \mathrm{N}) \\
\end{array}$ & Notes \\
\hline $\mathbf{s}$ & Braun & $\mathrm{Ni}$ & $1.55 E+05$ & 2.64E-03 & -NS & $\mathbf{N}$ & Won't use Braun, Wastren, or HTCE Inv. Data \\
\hline s & Raw & $\mathrm{Ni}$ & $1.55 E+05$ & $2.64 E-03$ & AZ102-11 & $N$ & Analysis Prior to $9 / 86$ \\
\hline ฉ & Braun & $\mathrm{Ni}$ & $3.66 E+04$ & $6.24 \mathrm{E}-04$ & NS & N & Won't use Braun, Wastren, or HTCE inv. Data \\
\hline $\mathbf{P}$ & Braun & $\mathrm{Ni}$ & $2.88 E+04$ & $4.90 E-04$ & NS & $\mathbf{N}$ & Won't use Braun, Wastren, or HTCE Inv. Data \\
\hline $\mathbf{L}$ & Raw & $\mathrm{Ni}$ & $2.88 E+04$ & $4.90 \mathrm{E}-04$ & $A Z 102-11$ & $\mathbf{N}$ & Analyșis Prior to 9/86 \\
\hline $\mathbf{P}$ & Wastren & $\mathrm{Ni}$ & $2.22 E+03$ & $3.78 \mathrm{E}-05$ & NS & $\mathbf{N}$ & Won't use Braun, Wastren, or HTCE Inv. Data \\
\hline $\mathrm{Cl}$ & Raw & $\mathrm{Ni}$ & $2.14 E+03$ & 3.65E-05 & AZ102-23 & $\bar{Y}$ & \\
\hline $\mathbf{L}$ & HTCE Inv. & $\mathrm{Ni}$ & $1.30 E+03$ & $2.21 E-05$ & NS & N & Won't use Braun, Wastren, or HTCE Inv. Data \\
\hline FI & Raw & $\mathrm{Ni}$ & $5.87 E \div 02$ & $1.00 \mathrm{E}-05$ & $A Z 102-18$ & N & Water wash of solid sample not supernatent \\
\hline $\mathbf{S}$ & Raw & $\mathrm{Ni}$ & $0.00 E+00$ & $0.00 E+\infty 0$ & $A Z 102-17$ & D & Points already in DB as part of $A Z_{-} 102-23$ \\
\hline $\mathbf{s}$ & Raw & $\mathrm{Ni}$ & $0.00 E+00$ & $0.00 E+00$ & $A Z 102-17$ & D & Points already in DB as part of $A Z_{-} 102-23$ \\
\hline s & Raw & $\mathrm{Ni}$ & $0.00 E+00$ & $0.00 E+\infty$ & AZ102-17 & $D$ & Points already in DB as part of $A Z_{-} 102-23$ \\
\hline$L$ & TRAC & NO2 & NS & NS & NS & $N$ & Analysis Prior to $9 / 86$ \\
\hline$P-1$ & Raw & NO2 & $2.96 E+07$ & $6.43 \mathrm{E}-01$ & AZ102-24 & $\bar{Y}$ & Updated per memo 8/17/95 \\
\hline $\mathrm{P}-2$ & Raw & NO2 & $2.95 E+07$ & $6.41 \mathrm{E}-01$ & AZ102-24 & $\bar{Y}$ & Updated per memo $8 / 17 / 95$ \\
\hline$\underline{P-1}$ & Raw & NO2 & $2.83 E+07$ & $6.15 E-01$ & AZ102-24 & $\bar{Y}$ & Updated per memo $8 / 17 / 95$ \\
\hline P-2 & Raw & NO2 & $2.81 E+07$ & $6.11 E-01$ & AZ102-24 & $\mathbf{Y}$ & Updated per memo $8 / 17 / 95$ \\
\hline P-2 & Raw & NO2 & $2.80 E+07$ & $6.09 \mathrm{E}-01$ & AZ102-24 & $Y$ & Updated per memo $8 / 17 / 95$ \\
\hline $\mathrm{Bl}$ & Raw & NO2 & $2.67 \mathrm{E}+07$ & $5.80 \mathrm{E}-01$ & AZ102-18 & D & NS \\
\hline $\mathbf{L}$ & Raw & NO2 & $2.47 E+07$ & $5.36 \mathrm{E}-01$ & $A Z 102-5$ & $N$ & Analysis Prior to $9 / 86$ \\
\hline R & Braun & NO2 & $2.47 E+07$ & $5.36 \mathrm{E}-01$ & NS & $N$ & Won't use Braun, Wastren, or HTCE Inv. Data \\
\hline $\mathbf{P}$ & Braun & No2 & $2.44 E+07$ & $5.30 E-01$ & NS & $N$ & Won't use Braun, Wastren, or HTCE Inv. Data \\
\hline G & Wastren & NO2 & $2.30 E+07$ & $5.00 E-01$ & NS & $N$ & Won't use Braun, Wastren, or HTCE Inv. Data \\
\hline $\mathrm{P}-1$ & Raw & NO2 & $2.22 E+07$ & $4.83 \mathrm{E}-01$ & $A Z 102-24$ & $Y$ & Updated per memo 8/17/95 \\
\hline$L$ & Raw & NO2 & $1.92 E+07$ & 4.18E-01 & AZ102-3 & $N$ & Analysis Prior to $9 / 86$ \\
\hline$L$ & Raw & NO2 & $1.92 E+07$ & $4.18 \mathrm{E}-01$ & AZ102-3 & $N$ & Analysis Prior to $9 / 86$ \\
\hline$L$ & Raw & NO2 & $1.92 E+07$ & $4.18 \mathrm{E}-01$ & $A Z 102-3$ & $N$ & Analysis Prior to $9 / 86$ \\
\hline L & Raw & $\mathrm{NO} 2$ & $1.92 E+07$ & $4.18 \mathrm{E}-01$ & AZ102-3 & $\mathbf{N}$ & Analysis Prior to $9 / 86$ \\
\hline$L$ & Raw & NO2 & $1.37 E+07$ & 2.97E-01 & AZ102-3 & $N$ & Analysis Prior to $9 / 86$ \\
\hline$\underline{L}$ & Raw & $\mathrm{NO2}$ & $1.30 E+07$ & $2.83 \mathrm{E}-01$ & $A Z 102.3$ & $N$ & Analysis Prior to $9 / 86$ \\
\hline$\underline{L}$ & Raw & $\mathrm{NO2}$ & $1.26 E+07$ & $2.74 E-01$ & $A Z 102-3$ & $N$ & Analysis Prior to $9 / 86$ \\
\hline $\mathbf{P}$ & Braun & NO2 & $1.24 E+07$ & $2.70 E-01$ & NS & $\mathbf{N}$ & Won't use Braun, Wastren, or HTCE Inv. Data \\
\hline L & Raw & NO2 & $1.24 E+07$ & $2.70 E-01$ & AZ102-16 & $\mathbf{N}$ & $\begin{array}{l}\text { These data are so messed up it will be easier } \\
\text { to reenter them. }\end{array}$ \\
\hline L & Raw & NO2 & $1.24 E+07$ & $2.70 E-01$ & AZ102-16 & $\mathbf{N}$ & Point in DB already_cc $8 / 15 / 95$ \\
\hline$\underline{L}$ & Raw & NO2 & $1.24 \mathrm{E}+07$ & 2.70E-01 & AZ102-16 & $N$ & Point in DB already_cc $8 / 15 / 95$ \\
\hline$L$ & Raw & NO2 & $1.24 E+07$ & $2.70 \mathrm{E}-01$ & AZ102-14 & $\bar{N}$ & No units for concentration given in report \\
\hline
\end{tabular}


TWRS Privatization Support Prn Chemical Liquids

\begin{tabular}{|c|c|c|c|c|c|c|c|c|c|}
\hline \multirow[b]{2}{*}{$\begin{array}{l}\text { Reported } \\
\text { Chemical }\end{array}$} & \multirow[b]{2}{*}{ Memo Date } & \multicolumn{7}{|c|}{ Tank Waste Characterization Sample Information } & \multirow[b]{2}{*}{ Reported U } \\
\hline & & $\begin{array}{c}\text { Start } \\
\text { Analysis } \\
\text { Date }\end{array}$ & $\begin{array}{c}\text { Start Sample } \\
\text { Date }\end{array}$ & Sample Number & $\begin{array}{c}\text { Tank Farm } \\
\text { Number }\end{array}$ & $\begin{array}{c}\text { Lab Sample } \\
\text { Number }\end{array}$ & $\begin{array}{c}\text { Density } \\
\text { (g/mL) }\end{array}$ & $\begin{array}{c}\text { Reported } \\
\text { Value }\end{array}$ & \\
\hline NO2 & $2 / 4 / 81$ & NS & NS & R-3700 & NS & NS & 1.11 & 2.65E-01 & moles $n$ \\
\hline NO2- & $2 / 4 / 81$ & NS & NS & R3700 & NS & NS & 1.11 & $2.65 \mathrm{E}-01$ & $M$ \\
\hline NO2 & $12 / 3 / 75$ & NS & NS & 1456 & NS & NS & 1.393 & $2.63 \mathrm{E}-01$ & $\underline{M}$ \\
\hline NO2 & $2 / 4 / 81$ & NS & NS & R-3699 & NS & NS & 1.11 & $2.63 E-01$ & moles $\Lambda$ \\
\hline NO2- & $2 / 4 / 81$ & NS & NS & R3699 & NS & NS & 1.11 & $2.63 \mathrm{E}-01$ & $\vec{M}$ \\
\hline NO2 & $6 / 26 / 75$ & NS & NS & RAT-AZ102-4a & NS & NS & 1.39 & $2.00 E-01$ & moles $/ 1$ \\
\hline NO2- & $6 / 26 / 75$ & NS & NS & NS & NS & NS & 1.392 & $2.00 \mathrm{E}-01$ & $M$ \\
\hline NO2 & $11 / 5 / 81$ & NS & NS & RAT-AZ102-2A & NS & NS & 1.09 & $1.90 \mathrm{E}-01$ & moles $n$ \\
\hline NO2 & $5 / 5 / 82$ & NS & $11 / 4 / 81$ & NS & NS & NS & 1.09 & $1.90 \mathrm{E}-01$ & $\bar{M}$ \\
\hline $\mathrm{NaNO} 2$ & $4 / 26 / 74$ & NS & NS & NS & NS & NS & 1.299 & $1.84 \mathrm{E}-01$ & $M$ \\
\hline No2- & NS & NS & NS & NS & NS & NS & 1.39 & $1.71 E-01$ & Mole/L \\
\hline NO2- & NS & NS & NS & NS & NS & NS & 1.04 & 1.70E-01 & Mole/L \\
\hline NO2 & $8 / 31 / 89$ & NS & $6 / 30 / 85$ & Composite Core & NS & NS & $(1.0)$ & $9.39 E+01$ & umol/g \\
\hline NO2. & Aug 89 & NS & NS & NS & NS & NS & NS & $3.33 E+00$ & $g / L$ \\
\hline NO2 & $9 / 1 / 93$ & N/A & $6 / 30 / 85$ & N/A & N/A & N/A & 1.14 & $6.13 E+01$ & umol/g \\
\hline NO2 & $10 / 31 / 86$ & NS & $6 / 30 / 85$ & Composite Core & NS & NS & 1.14 & $6.13 E+01$ & umol/g \\
\hline NO2 & $2 / 19 / 82$ & NS & $12 / 1 / 81$ & T3251 & NS & NS & 1.03 & $6.00 E-02$ & $M$ \\
\hline $\mathrm{NaNO} 2$ & $8 / 27 / 77$ & NS & NS & NS & NS & NS & 1.391 & 5.70E-02 & $\bar{M}$ \\
\hline $\mathrm{NO} 2$ & $8 / 27 / 77$ & NS & NS & RAT-AZ102-4B & NS & NS & 1.39 & $5.70 E-02$ & moles $\Lambda$ \\
\hline NO2 & $10 / 31 / 86$ & NS & $6 / 30 / 85$ & NS & NS & NS & 1.11 & $4.54 E+01$ & umol/g \\
\hline $\mathrm{Cr}$ & $8 / 31 / 89$ & NS & $6 / 30 / 85$ & Composite Core & NS & NS & 1.11 & $4.54 E+01$ & umol/g \\
\hline NO2 & $1 / 30 / 82$ & NS & NS & $T-5387$ & NS & NS & 1.008 & $3.46 \mathrm{E}-03$ & moles $/$ \\
\hline NO2 & $2 / 19 / 82$ & NS & NS & $T 5387$ & NS & NS & 1.008 & $3.46 E-03$ & $M$ \\
\hline NO2 & $10 / 31 / 86$ & NS & $6 / 30 / 85$ & NS & NS & NS & NS & $9.39 E+01$ & umol/g \\
\hline NO3 & $1 / 1 / 77$ & NS & NS & NS & NS & NS & NS & NS & NS \\
\hline NO3 & $12 / 3 / 75$ & NS & NS & $1496 \mathrm{~A}$ & NS & NS & 1.378 & $4.33 E+00$ & $M$ \\
\hline NO3 & $12 / 3 / 75$ & NS & NS & 14968 & NS & NS & 1.38 & $4.32 \mathrm{E}+00$ & $M$ \\
\hline NO3 & $12 / 3 / 75$ & NS & NS & $1496 \mathrm{C}$ & NS & NS & 1.379 & $4.32 E+00$ & $M$ \\
\hline NO3 & $6 / 26 / 75$ & NS & NS & RAT-AZ102-4a & NS & NS & 1.39 & $4.26 E+00$ & moles $\Lambda$ \\
\hline NO3 & $6 / 26 / 75$ & NS & NS & NS & NS & NS & 1.392 & $4.26 \mathrm{E}+00$ & $M$ \\
\hline $\mathrm{NO3}$ & $12 / 3 / 75$ & NS & NS & 14960 & NS & NS & 1.36 & $4.20 E+00$ & $M$ \\
\hline NaNO3 & $8 / 27 / 77$ & NS & NS & NS & NS & NS & 1.391 & $4.08 E+00$ & $\bar{M}$ \\
\hline $\mathrm{NO3}$ & $8 / 27 / 77$ & NS & NS & RAT-AZ102-4B & NS & NS & 1.39 & $4.08 E+00$ & moles $/$ \\
\hline NO3 & $12 / 3 / 75$ & NS & NS & 1458 & NS & NS & 1.391 & $3.74 E+00$ & $M$ \\
\hline NO3 & $7 / 28 / 79$ & NS & NS & RAT-AZ102-4D & NS & NS & 1.41 & $3.72 E+\infty 0$ & moles $n$ \\
\hline NO3 & $12 / 3 / 75$ & NS & NS & 1459 & NS & NS & 1.386 & $3.59 E+00$ & $M$ \\
\hline $\mathrm{NO3}$ & $12 / 3 / 75$ & NS & NS & 1457 & NS & NS & 1.398 & $2.92 \mathrm{E}+00$ & $M$ \\
\hline NaNO3 & $4 / 26 / 74$ & N5 & NS & NS & NS & NS & 1.299 & $2.89 E+00$ & $M$ \\
\hline NO3 & $12 / 3 / 75$ & NS & NS & 1456 & NS & NS & 1.393 & $2.29 E+00$ & $M$ \\
\hline
\end{tabular}




\begin{tabular}{|c|c|c|c|c|c|c|c|}
\hline & & & Analyte Inform & & & & \\
\hline Code & Source & Analyte & Conc. (ug/L) & Conc. (mol/L) & $\begin{array}{c}\text { Reference } \\
\text { Number }\end{array}$ & $\begin{array}{c}\text { Validated } \\
(Y / N)\end{array}$ & Notes \\
\hline $\mathbf{P}$ & Braun & NO2 & $1.22 \mathrm{E}+07$ & $2.65 E-01$ & NS & $\mathbf{N}$ & Won't use Braun, Wastren, or HTCE Inv. Data \\
\hline L & Raw & NO2 & $1.22 E+07$ & $2.65 E-01$ & AZ102-8 & $\mathbf{N}$ & Analysis Prior to $9 / 86$ \\
\hline $\mathbf{L}$ & Raw & NO2 & $1.21 E+07$ & $2.63 \mathrm{E}-01$ & AZ102-3 & $\mathbf{N}$ & Analysis Prior to $9 / 86$ \\
\hline $\mathbf{P}$ & Braun & NO2 & $1.21 \mathrm{E}+07$ & 2.63E-01 & NS & $\mathbf{N}$ & Won't use Braun, Wastren, or HTCE Inv. Data \\
\hline L & Raw & NO2 & $1.21 E+07$ & $2.63 E-01$ & $A 2102-8$ & $\mathbf{N}$ & Analysis Prior to $9 / 86$ \\
\hline $\mathbf{P}$ & Braun & No2 & $9.20 E+06$ & $2.00 E-01$ & NS & $\mathbf{N}$ & Analysis Prior to $9 / 86$ \\
\hline$L$ & Raw & $\mathrm{NO2}$ & $9.20 E+06$ & 2.00E-01 & $A Z 102-6$ & $\mathrm{~N}$ & Analysis Prior to $9 / 86$ \\
\hline$P$ & Braun & NO2 & $8.74 E+06$ & $1.90 E-01$ & NS & $N$ & Won't use Braun, Wastren, or HTCE inv. Data \\
\hline$L$ & Raw & NO2 & $8.74 E+06$ & $1.90 \mathrm{E}-01$ & AZ102-11 & $N$ & Analysis Prior to $9 / 86$ \\
\hline$L$ & Raw & NO2 & $8.47 E+06$ & $1.84 \mathrm{E}-01$ & $A Z 102-1$ & $N$ & Analysis Prior to $9 / 86$ \\
\hline $\mathbf{s}$ & HTCE Inv. & NO2 & $7.85 E+06$ & $1.71 \mathrm{E}-01$ & NS & N & Won't use Braun, Wastren, or HTCE Inv. Data \\
\hline $\mathbf{L}$ & HTCE Inv. & NO2 & $7.84 E+06$ & $1.70 E-01$ & NS & $\mathbf{N}$ & Won't use Braun, Wastren, or HTCE Inv. Data \\
\hline FI & Raw & NO2 & $4.32 E+06$ & $9.39 \mathrm{E}-02$ & AZ102-23 & $\mathbf{N}$ & Water wash of solid sample not supernatent \\
\hline$P$ & Wastren & NO2 & $3.33 E+06$ & 7.24E-02 & NS & $\mathbf{N}$ & Won't use Braun, Wastren, or HTCE Inv. Data \\
\hline $\mathrm{Cl}$ & Raw & NO2 & $3.22 E+06$ & $6.99 \mathrm{E}-02$ & AZ102-23 & $\bar{Y}$ & \\
\hline $\mathrm{Cl}$ & Raw & NO2 & $3.22 \mathrm{E}+06$ & $6.99 \mathrm{E}-02$ & AZ102-18 & $\bar{D}$ & NS \\
\hline$L$ & Raw & $\mathrm{NO2}$ & $2.76 \mathrm{E}+06$ & $6.00 \mathrm{E}-02$ & $A Z 102-10$ & $\mathbf{N}$ & Analysis Prior to $9 / 86$ \\
\hline$F$ & Raw & NO2 & $2.62 E+06$ & 5.70E-02 & $A Z 102-4$ & $\mathbf{N}$ & Analysis Prior to $9 / 86$ \\
\hline$P$ & Braun & NO2 & $2.62 E+06$ & $5.70 \mathrm{E}-02$ & NS & $\mathrm{N}$ & Analysis Prior to $9 / 86$ \\
\hline El & Raw & NO2 & $2.32 E+06$ & $5.04 E-02$ & $A Z 102-18$ & $D$ & NS \\
\hline EI & Raw & No2 & $2.32 E+06$ & $5.04 \mathrm{E}-02$ & AZ102-23 & $\mathbf{N}$ & $\begin{array}{l}\text { Solid sample after has been washed with } \\
\text { fresh water }\end{array}$ \\
\hline$P$ & Braun & NO2 & $1.59 E+05$ & $3.46 \mathrm{E}-03$ & NS & $N$ & Won't use Braun, Wastren, or HTCE Inv. Data \\
\hline $\mathbf{L}$ & Raw & NO2 & $1.59 E+05$ & $3.46 \mathrm{E}-03$ & $A Z 102-9$ & $\mathbf{N}$ & $\begin{array}{l}\text { Not a traceable reference, analysis Prior to } \\
9 / 86\end{array}$ \\
\hline FI & Raw & NO2 & $0.00 E+00$ & $0.00 E+00$ & $A Z 102-18$ & D & NS \\
\hline$L$ & TRAC & NO3 & NS & NS & NS & $\mathbf{N}$ & Analysis Prior to $9 / 86$ \\
\hline L & Raw & NO3 & $2.69 E+08$ & $4.33 E+00$ & $A Z 102-3$ & $\mathbf{N}$ & Analysis Prior to $9 / 86$ \\
\hline$L$ & Raw & NO3 & $2.68 E+08$ & $4.32 E+00$ & $A Z 102-3$ & $\mathbf{N}$ & Analysis Prior to $9 / 86$ \\
\hline$L$ & Raw & NO3 & $2.68 E+08$ & $4.32 E+\infty 0$ & A2102-3 & $\mathbf{N}$ & Analysis Prior to $9 / 86$ \\
\hline $\mathbf{P}$ & Braun & NO3 & $2.64 E+08$ & $4.26 E+\infty 0$ & NS & $N$ & Analysis Prior to $9 / 86$ \\
\hline$L$ & Raw & .NO3 & $2.64 E+08$ & $4.26 E+00$ & $A Z 102-6$ & $N$ & Analysis Prior to $9 / 86$ \\
\hline L & Raw & NO3 & $2.60 E+08$ & $4.20 E+00$ & $A Z 102-3$ & $\mathbf{N}$ & Analysis Prior to $9 / 86$ \\
\hline $\mathbf{F}$ & Raw & NO3 & $2.53 E+08$ & $4.08 \mathrm{E}+00$ & AZ102-4 & $N$ & Analysis Prior to $9 / 86$ \\
\hline$P$ & Braun & NO3 & $2.53 E+08$ & $4.08 E+00$ & NS & $N$ & Analysis Prior to $9 / 86$ \\
\hline$L$ & Raw & NO3 & $2.32 E+08$ & $3.74 \mathrm{E}+00$ & AZ102-3 & $\mathrm{N}$ & Analysis Prior to $9 / 86$ \\
\hline $\mathbf{P}$ & Braun & NO3 & $2.31 E+08$ & $3.72 E+\infty$ & NS & $N$ & Won't use Braun, Wastren, or HTCE Inv. Data \\
\hline$L$ & Raw & NO3 & $2.23 E+08$ & $3.59 E+00$ & AZ102-3 & $\mathbf{N}$ & Analysis Prior to $9 / 86$ \\
\hline$L$ & Raw & NO3 & $1.81 E+08$ & $2.92 E+00$ & $A Z 102-3$ & $\mathbf{N}$ & Analysis Prior to $9 / 86$ \\
\hline$L$ & Raw & NO3 & $1.79 E+08$ & $2.89 E+00$ & $A Z 102-1$ & $\mathbf{N}$ & Analysis Prior to $9 / 86$ \\
\hline $\mathbf{L}$ & Raw & NO3 & $1.42 E+08$ & $2.29 E+00$ & AZ102-3 & $\mathbf{N}$ & Analysis Prior to $9 / 86$ \\
\hline
\end{tabular}




\begin{tabular}{|c|c|c|c|c|c|c|c|c|c|}
\hline & & & & Tank Waste C & racterization & ample Informati & & & \\
\hline $\begin{array}{l}\text { Reported } \\
\text { Chemical }\end{array}$ & Memo Date & $\begin{array}{c}\text { Start } \\
\text { Analysis } \\
\text { Date } \\
\end{array}$ & $\begin{array}{c}\text { Start Sample } \\
\text { Date }\end{array}$ & Sample Number & $\begin{array}{c}\text { Tank Farm } \\
\text { Number }\end{array}$ & $\begin{array}{c}\text { Lab Sample } \\
\text { Number }\end{array}$ & $\begin{array}{l}\text { Density } \\
\text { (g/mL) }\end{array}$ & $\begin{array}{c}\text { Reported } \\
\text { Value } \\
\end{array}$ & Reported Un \\
\hline NO3 & $2 / 4 / 81$ & NS & NS & $R-3700$ & NS & NS & 1.11 & $8.41 E-01$ & moles $n$ \\
\hline $\mathrm{NO3}$ & $2 / 4 / 81$ & NS & NS & 83700 & NS & NS & 1.11 & $8.41 \mathrm{E}-01$ & $M$ \\
\hline NO3 & $2 / 4 / 81$ & NS & NS & $R-3699$ & NS & NS & 1.11 & $8.29 E-01$ & moles/l \\
\hline No3 & $2 / 4 / 81$ & NS & NS & $\mathrm{R} 3699$ & NS & NS & 1.11 & $8.29 \mathrm{E}-01$ & $M$ \\
\hline NO3 & $11 / 5 / 81$ & NS & NS & RAT-AZ102-2A & NS & NS & 1.09 & $7.80 \mathrm{E}-01$ & moles n \\
\hline NO3 & $5 / 5 / 82$ & NS & $11 / 4 / 81$ & NS & NS & NS & 1.09 & $7.80 \mathrm{E}-01$ & $M$ \\
\hline NO3 & $11 / 5 / 81$ & NS & NS & RAT-AZ102-2B & NS & NS & 1.4 & 6.30E-01 & moles $\Lambda$ \\
\hline NO3 & $5 / 5 / 82$ & NS & $11 / 4 / 81$ & NS & NS & NS & 1.4 & $6.30 \mathrm{E}-01$ & $M$ \\
\hline Nitrate & $7 / 26 / 95$ & N/A & $3 / 2 / 91$ & BOD9R3 & $A Z-102-1$ & S95T000263 & 1.1 & $2.59 \mathrm{E}+04$ & $\mathrm{UG} / \mathrm{ml}$ \\
\hline Nitrate & $7 / 26 / 95$ & N/A & $3 / 2 / 91$ & BOD9R3 & $A Z-102-1$ & S95T000263 & 1.1 & $2.52 E+04$ & UG/m! \\
\hline NO3 & $2 / 19 / 82$ & NS & $12 / 1 / 81$ & T3251 & NS & NS & 1.03 & $4.00 \mathrm{E}-01$ & $\bar{M}$ \\
\hline NO3- & NS & NS & NS & NS & NS & NS & 1.39 & $3.94 E-01$ & Mole/L \\
\hline Nitrate & $7 / 26 / 95$ & N/A & $3 / 2 / 91$ & BOD9R5 & $A Z-102-3$ & S95T000265 & 1.1 & $2.43 E+04$ & $\mathrm{UG} / \mathrm{ml}$ \\
\hline Nitrate & $7 / 26 / 95$ & N/A & $3 / 2 / 91$ & BOD9R5 & $A Z-102-3$ & S95T000265 & 1.1 & $2.43 E+04$ & UG/ml \\
\hline Nitrate & $7 / 26 / 95$ & N/A & $3 / 2 / 91$ & BOD9R4 & $A Z-102-2$ & 595T000264 & 1.1 & $2.38 \mathrm{E}+04$ & UG/ml \\
\hline NO3 & $10 / 1 / 83$ & NS & NS & RAT-AZ102-3 & NS & NS & NS & 3.50E-01 & moles/l \\
\hline NO3 & $3 / 10 / 84$ & NS & $7 / 31 / 83$ & NS & NS & NS & NS & 3.50E-01 & $M$ \\
\hline NO3 & $3 / 10 / 84$ & NS & $7 / 31 / 83$ & NS & NS & NS & NS & $3.50 \mathrm{E}-01$ & $M$ \\
\hline $\mathrm{NO} 3$ & $7 / 5 / 84$ & NS & $7 / 31 / 83$ & T3495 & NS & NS & NS & $3.50 \mathrm{E}-01$ & $\bar{M}$ \\
\hline $\mathrm{NO3}$ & $12 / 13 / 83$ & NS & $7 / 31 / 83$ & $T 1520$ & NS & NS & NS & $3.50 \mathrm{E}-01$ & $M$ \\
\hline Nitrate & $7 / 26 / 95$ & N/A & $3 / 2 / 91$ & BOD9R4 & $A Z-102-2$ & S95T000264 & 1.1 & $1.91 E+04$ & $\mathrm{UG} / \mathrm{ml}$ \\
\hline NO3- & Aug 89 & NS & NS & NS & NS & NS & NS & $6.78 E+00$ & $g / L$ \\
\hline NO3 & $9 / 1 / 93$ & N/A & $6 / 30 / 85$ & $N / A$ & N/A & N/A & 1.14 & $9.27 E+01$ & umol/g \\
\hline NO3 & $10 / 31 / 86$ & NS & $6 / 30 / 85$ & NS & NS & NS & 1.14 & $9.27 \mathrm{E}+01$ & umol/g \\
\hline NO3 & $10 / 31 / 86$ & NS & $6 / 30 / 85$ & NS & NS & NS & 1.73 & $4.06 E+01$ & umol/g \\
\hline NO3- & Aug 89 & NS & NS & NS & NS & NS & NS & $3.75 E+00$ & $g / L$ \\
\hline NO3. & NS & NS & NS & NS & NS & NS & 1.04 & 4.47E-02 & Mole/L \\
\hline NO3 & $1 / 30 / 82$ & NS & NS & $T-5387$ & NS & NS & 1.008 & $2.45 \mathrm{E}-02$ & moles $n$ \\
\hline NO3 & $2 / 19 / 82$ & NS & NS & $T 5387$ & NS & NS & 1.008 & $2.45 E-02$ & $M$ \\
\hline NO3 & $10 / 31 / 86$ & NS & $6 / 30 / 85$ & NS & NS & NS & 1.11 & $1.22 \mathrm{E}+01$ & umol/g \\
\hline $\mathrm{Cr}$ & $8 / 31 / 89$ & NS & $6 / 30 / 85$ & Composite Core & NS & NS & 1.11 & $1.22 E+01$ & umol/g \\
\hline NO3 & $8 / 31 / 89$ & NS & $6 / 30 / 85$ & Composite Core & NS & NS & $(1.0)$ & $1.26 E+01$ & umol/g \\
\hline NO3 & $10 / 31 / 86$ & NS & $6 / 30 / 85$ & NS & NS & NS & NS & $1.26 \mathrm{E}+01$ & umol/g \\
\hline $\mathrm{OH}$ & $8 / 27 / 77$ & NS & NS & RAT-AZ102-4B & NS & NS & 1.39 & $1.10 E+00$ & moles $n$ \\
\hline $\mathrm{OH}$ & $6 / 26 / 75$ & NS & NS & RAT-AZ102-4a & NS & NS & 1.39 & $1.03 E+00$ & molesn \\
\hline $\mathrm{OH}$ & $9 / 20 / 78$ & NS & NS & RAT-AZ102-4C & NS & NS & 1.39 & $9.98 \mathrm{E}-01$ & moles/l \\
\hline
\end{tabular}




\begin{tabular}{|c|c|c|c|c|c|c|c|}
\hline \multirow[b]{2}{*}{ Code } & \multirow[b]{2}{*}{ Source } & \multicolumn{3}{|c|}{ Analyte Information } & \multirow[b]{2}{*}{$\begin{array}{c}\text { Reference } \\
\text { Number }\end{array}$} & \multirow[b]{2}{*}{$\begin{array}{c}\text { Validated } \\
\text { (Y/N) }\end{array}$} & \multirow[b]{2}{*}{ Notes } \\
\hline & & Analyte & Conc. (ug/L) & Conc. (mol/L) & & & \\
\hline $\mathbf{P}$ & Braun & NO3 & $5.22 E+07$ & $8.41 E-01$ & NS & $N$ & Won't use Braun, Wastren, or HTCE Inv. Data \\
\hline$L$ & Raw & NO3 & $5.22 E+07$ & $8.41 \mathrm{E}-01$ & AZ102-8 & $N$ & Analysis Prior to $9 / 86$ \\
\hline $\mathbf{P}$ & Braun & NO3 & $5.14 \mathrm{E}+07$ & $8.29 E-01$ & NS & $N$ & Won't use Braun, Wastren, or HTCE Inv. Data \\
\hline$L$ & Raw & NO3 & $5.14 E+07$ & $8.29 E-01$ & $A Z 102-8$ & $N$ & Analysis Prior to $9 / 86$ \\
\hline $\mathbf{P}$ & Braun & NO3 & $4.84 E+07$ & $7.80 E-01$ & NS & $N$ & Won't use Braun, Wastren, or HTCE Inv. Data \\
\hline $\mathbf{L}$ & Raw & NO3 & $4.84 E+07$ & 7.80E-01 & A2102-11 & $N$ & Analysis Prior to $9 / 86$ \\
\hline $\mathbf{s}$ & Braun & NO3 & $3.91 E+07$ & $6.30 E-01$ & NS & $\mathbf{N}$ & Won't use Braun, Wastren, or HTCE Inv. Data \\
\hline S & Raw & NO3 & $3.91 E+07$ & $6.30 E-01$ & AZ102-11 & $N$ & Analysis Prior to $9 / 86$ \\
\hline$P-1$ & Raw & NO3 & $2.59 E+07$ & $4.18 \mathrm{E}-01$ & $A Z 102-24$ & $\bar{Y}$ & Updated per memo $8 / 17 / 95$ \\
\hline$P-2$ & Raw & NO3 & $2.52 E+07$ & 4.06E-01 & $A Z 102-24$ & $Y$ & Updated per memo 8/17/95 \\
\hline L & Raw & NO3 & $2.48 E+07$ & $4.00 E-01$ & AZ102-10 & $\mathbf{N}$ & Analysis Prior to $9 / 86$ \\
\hline $\mathbf{s}$ & HTCE Inv. & NO3 & $2.44 E+07$ & $3.94 E-01$ & NS & $\mathbf{N}$ & Won't use Braun, Wastren, or HTCE Inv. Data \\
\hline$P-1$ & Raw & NO3 & $2.43 E+07$ & $3.92 \mathrm{E}-01$ & A2102-24 & $\bar{Y}$ & Updated per memo $8 / 17 / 95$ \\
\hline$\overline{P-2}$ & Raw & NO3 & $2.43 E+07$ & $3.92 \mathrm{E}-01$ & AZ102-24 & $Y$ & Updated per memo $8 / 17 / 95$ \\
\hline$\underline{\mathrm{P}-2}$ & Raw & NO3 & $2.38 E+07$ & $3.84 \mathrm{E}-01$ & AZ102-24 & $\bar{Y}$ & Updated per memo 8/17/95 \\
\hline $\mathbf{P}$ & Braun & NO3 & 2.17E+07 & 3.50E-01 & NS & $N$ & Won't use Braun, Wastren, or HTCE Inv. Data \\
\hline$L$ & Raw & NO3 & $2.17 E+07$ & $3.50 E-01$ & $A Z 102-16$ & $\mathbf{N}$ & $\begin{array}{l}\text { These data are so messed up it will be easier } \\
\text { to reenter them. }\end{array}$ \\
\hline $\mathbf{L}$ & Raw & No3 & $2.17 E+07$ & $3.50 \mathrm{E}-01$ & $A Z 102-16$ & $\mathbf{N}$ & Point in DB already_cc_8/15/95 \\
\hline$L$ & Raw & NO3 & $2.17 E+07$ & $3.50 E-01$ & AZ102-15 & $N$ & Point in DB already cc $8 / 15 / 95$ \\
\hline$\underline{L}$ & Raw & NO3 & $2.17 E+07$ & $3.50 E-01$ & AZ102-14 & $\mathbf{N}$ & No units for concentration given in report \\
\hline $\mathrm{P}-1$ & Raw & NO3 & $1.91 E+07$ & $3.08 \mathrm{E}-01$ & AZ102-24 & $Y$ & Updated per memo 8/17/95 \\
\hline $\mathbf{P}$ & Wastren & NO3 & $6.78 E+06$ & $1.09 E-01$ & NS & $N$ & Won't use Braun, Wastren, or HTCE Inv. Data \\
\hline $\mathrm{Cl}$ & Raw & NO3 & $6.55 E+06$ & $1.06 \mathrm{E}-01$ & AZ102-23 & $\bar{Y}$ & \\
\hline$\overline{\mathrm{Cl}}$ & Raw & NO3 & $6.55 \mathrm{E}+06$ & $1.06 \mathrm{E}-01$ & $A Z 102-18$ & D & NS \\
\hline$\underline{B l}$ & Raw & NO3 & $4.36 \mathrm{E}+06$ & $7.02 \mathrm{E}-02$ & $A 2102-18$ & D & NS \\
\hline G & Wastren & NO3 & $3.75 E+06$ & $6.05 E-02$ & NS & $\mathbf{N}$ & Won't use Braun, Wastren, or HTCE Inv. Data \\
\hline $\mathbf{L}$ & HTCE Inv. & NO3 & $2.77 E+06$ & 4.47E-02 & NS & $\mathbf{N}$ & Won't use Braun, Wastren, or HTCE Inv. Data \\
\hline $\mathbf{P}$ & Braun & NO3 & $1.52 E+06$ & $2.45 \mathrm{E}-02$ & NS & N & Won't use Braun, Wastren, or HTCE Inv. Data \\
\hline L & Raw & NO3 & $1.52 E+06$ & $2.45 E-02$ & $A Z 102-9$ & N & $\begin{array}{l}\text { Not a traceable reference, analysis Prior to } \\
9 / 86\end{array}$ \\
\hline El & Raw & NO3 & $8.40 E+05$ & $1.35 \mathrm{E}-02$ & $A Z 102-18$ & D & NS \\
\hline$\exists I$ & Raw & NO3 & $8.40 E+05$ & $1.35 E-02$ & $A Z 102-23$ & $\mathbf{N}$ & $\begin{array}{l}\text { Solid sample after has been washed with } \\
\text { fresh water }\end{array}$ \\
\hline$=$ & Raw & NO3 & $7.81 E+05$ & $1.26 \mathrm{E}-02$ & $A Z 102-23$ & $\mathbf{N}$ & Water wash of solid sample not supernatent \\
\hline 1 & Raw & NO3 & $0.00 E+00$ & $0.00 E+00$ & $A Z 102-18$ & $\mathrm{D}$ & NS \\
\hline P & Braun & $\mathrm{OH}$ & $1.87 E+07$ & $1.10 E+00$ & NS & $\mathrm{N}$ & Analysis Prior to $9 / 86$ \\
\hline P & Braun & $\mathrm{OH}$ & $1.75 E+07$ & $1.03 E+00$ & NS & $\mathrm{N}$ & Analysis Prior to $9 / 86$ \\
\hline$?$ & Braun & $\mathrm{OH}$ & $1.70 E+07$ & $9.98 \mathrm{E}-01$ & NS & $N$ & Won't use Braun, Wastren, or HTCE Inv. Data \\
\hline
\end{tabular}


TWRS Privatization Support Proje Chemical Liquids $f$

\begin{tabular}{|c|c|c|c|c|c|c|c|c|c|}
\hline \multicolumn{10}{|c|}{ Tank Waste Characterization Sample Information } \\
\hline $\begin{array}{l}\text { Reported } \\
\text { Chemical } \\
\end{array}$ & Memo Date & $\begin{array}{c}\text { Analysis } \\
\text { Date }\end{array}$ & $\begin{array}{c}\text { Start Sample } \\
\text { Date }\end{array}$ & Sample Number & $\begin{array}{c}\text { Tank Farm } \\
\text { Number }\end{array}$ & $\begin{array}{c}\text { Lab Samplo } \\
\text { Number }\end{array}$ & $\begin{array}{c}\text { Density } \\
(\mathrm{g} / \mathrm{mL})\end{array}$ & $\begin{array}{c}\text { Reported } \\
\text { Value } \\
\end{array}$ & Reported Uni \\
\hline $\mathrm{OH}$ & $7 / 28 / 79$ & NS & NS & RAT-AZ102-4D & NS & NS & 1.41 & $8.20 E-01$ & moles $n$ \\
\hline $\mathrm{NaOH}$ & $7 / 28 / 79$ & NS & NS & RAT-AZ102-6 & NS & NS & 1.41 & 8.20E-01 & moles/I \\
\hline $\mathrm{OH}$ & $2 / 4 / 81$ & NS & NS & R-3699 & NS & NS & 1.11 & $6.92 E-01$ & moles $\AA$ \\
\hline $\mathrm{OH}$ & $2 / 4 / 81$ & NS & NS & R-3700 & NS & NS & 1.11 & $6.68 \mathrm{E}-01$ & moles $n$ \\
\hline $\mathrm{OH}$ & $11 / 5 / 81$ & NS & NS & RAT-AZ102-2A & NS & NS & 1.09 & 3.50E-01 & moles $/$ \\
\hline $\mathrm{OH}$ & $5 / 2 / 86$ & NS & NS & $\mathrm{R} 60386$ & NS & NS & NS & 3.50E-01 & $M$ \\
\hline $\mathrm{OH}$ & $10 / 1 / 83$ & NS & NS & RAT-AZ102-3 & NS & NS & NS & $2.00 E-01$ & moles/l \\
\hline$\overline{\mathrm{OH}}$ & $5 / 2 / 86$ & NS & NS & $R 880311$ & NS & NS & NS & $2.00 \mathrm{E}-01$ & $\bar{M}$ \\
\hline $\mathrm{OH}$ & $5 / 2 / 86$ & NS & NS & $R 81287$ & NS & NS & NS & 1.80 E-01 & $\bar{M}$ \\
\hline Hydroxide & $7 / 26 / 95$ & N/A & $3 / 2 / 91$ & B0D909 & $A Z-102-2$ & S95T000246 & 1.1 & $1.99 \mathrm{E}+03$ & $\mathrm{UG} / \mathrm{ml}$ \\
\hline Hydroxide & $7 / 26 / 95$ & N/A & $3 / 2 / 91$ & B00909 & $A Z-102-2$ & S95T000246 & 1.1 & $1.86 E+03$ & $\mathrm{UG} / \mathrm{ml}$ \\
\hline Hydroxide & $7 / 26 / 95$ & N/A & $3 / 2 / 91$ & BOD9R1 & $A Z-102-3$ & S95T000247 & 1.1 & $1.84 \mathrm{E}+03$ & $\mathrm{UG} / \mathrm{ml}$ \\
\hline Hydroxide & $7 / 26 / 95$ & N/A & $3 / 2 / 91$ & BOD9R1 & $A Z-102-3$ & S95T000247 & 1.1 & $1.83 E+03$ & $\mathrm{UG} / \mathrm{ml}$ \\
\hline $\mathrm{OH}-$ & $8 / 31 / 89$ & NS & $6 / 30 / 85$ & Composite Core & NS & NS & $(1.0)$ & $1.08 E+01$ & umol/g \\
\hline $\mathrm{OH}$ & $1 / 30 / 82$ & NS & NS & $T-5387$ & NS & NS & 1.008 & $7.14 E-03$ & moles $/$ \\
\hline $\mathrm{Pb}$ & $1 / 1 / 77$ & NS & NS & NS & NS & NS & NS & NS & NS \\
\hline $\mathrm{Pb}$ & Aug 89 & NS & NS & NS & NS & NS & NS & $1.63 E+00$ & $g / L$ \\
\hline $\mathrm{Pb}$ & $11 / 5 / 81$ & NS & NS & RAT-AZ102-2B & NS & NS & 1.4 & 7.00E-03 & moles $A$ \\
\hline $\mathrm{Pb}$ & $5 / 5 / 82$ & NS & $11 / 4 / 81$ & NS & NS & NS & 1.4 & $7.00 \mathrm{E}-03$ & $\bar{M}$ \\
\hline $\mathrm{Cr}$ & $8 / 31 / 89$ & NS & $6 / 30 / 85$ & Composite Core & NS & NS & 1.11 & $1.78 E+00$ & umol/g \\
\hline $\mathrm{Pb}$ & Aug 89 & NS & NS & NS & NS & NS & NS & $6.11 E-03$ & $g / L$ \\
\hline $\mathrm{Pb}$ & $9 / 1 / 93$ & N/A & $6 / 30 / 85$ & N/A & N/A & N/A & 1.14 & $2.50 E-02$ & umol/g \\
\hline $\mathrm{Pb}$ & $10 / 31 / 86$ & NS & $6 / 30 / 85$ & Composite Core & NS & NS & $(1.0)$ & $5.00 E-03$ & umol/g \\
\hline $\mathrm{Pb} 2+$ & NS & NS & NS & NS & NS & NS & 1.39 & $2.79 E-06$ & Mole/L \\
\hline $\mathrm{Pb} 2+$ & NS & NS & NS & NS & NS & NS & 1.04 & $4.39 E-07$ & Mole/L \\
\hline $\mathrm{Pb}$ & $5 / 3 / 86$ & NS & NS & L4 & NS & NS & NS & $2.60 E+\infty 0$ & umol/g \\
\hline $\mathrm{Pb}$ & $5 / 3 / 86$ & NS & NS & L1 & NS & NS & NS & $3.60 E+00$ & umol/g \\
\hline $\mathrm{Pb}$ & $5 / 3 / 86$ & NS & NS & L3 & NS & NS & NS & $4.80 E+\infty 0$ & umol/g \\
\hline Phosphate & $7 / 26 / 95$ & N/A & $3 / 2 / 91$ & BOD9R4 & $A Z-102-2$ & S95T000264 & 1.1 & $<3.96 e+02$ & UG/ml \\
\hline Phosphate & $7 / 26 / 95$ & N/A & $3 / 2 / 91$ & B0D9R5 & $A Z-102-3$ & S95T000265 & 1.1 & $<3.96 \mathrm{e}+02$ & $\mathrm{UG} / \mathrm{ml}$ \\
\hline Phosphate & $7 / 26 / 95$ & N/A & $3 / 2 / 91$ & BOD9R4 & $A Z-102-2$ & S95T000264 & 1.1 & $<3.96 e+02$ & $\mathrm{UG} / \mathrm{ml}$ \\
\hline Phosphate & $7 / 26 / 95$ & N/A & $3 / 2 / 91$ & B0D9R5 & $A Z-102-3$ & S95T000265 & 1.1 & $<3.96 \mathrm{e}+02$ & $\mathrm{UG} / \mathrm{ml}$ \\
\hline Phosphate & $7 / 26 / 95$ & N/A & $3 / 2 / 91$ & BOD9R3 & $A Z-102-1$ & S95T000263 & 1.1 & $<7.26 \mathrm{e}+02$ & $\mathrm{UG} / \mathrm{ml}$ \\
\hline
\end{tabular}




\begin{tabular}{|c|c|c|c|c|c|c|c|}
\hline & & & Analyte Inform & & & & \\
\hline :ode & Source & Analyte & Conc. (ug/L) & Conc. (mol/L) & $\begin{array}{c}\text { Reference } \\
\text { Number }\end{array}$ & $\begin{array}{c}\text { Validated } \\
\text { (Y/N) }\end{array}$ & Notes \\
\hline$P$ & Braun & $\mathrm{OH}$ & $1.39 E+07$ & $8.20 E-01$ & NS & $N$ & Won't use Braun, Wastren, or HTCE Inv. Data \\
\hline$\gamma$ & Braun & $\mathrm{OH}$ & $1.39 E+07$ & $8.20 E-01$ & NS & $N$ & Won't use Braun, Wastren, or HTCE Inv. Data \\
\hline$P$ & Braun & $\mathrm{OH}$ & $1.18 E+07$ & $6.92 \mathrm{E}-01$ & NS & $\mathbf{N}$ & Won't use Braun, Wastren, or HTCE Inv. Data \\
\hline $\mathbf{P}$ & Braun & $\mathrm{OH}$ & $1.14 \mathrm{E}+07$ & $6.68 \mathrm{E}-01$ & NS & $N$ & Won't use Braun, Wastren, or HTCE inv. Data \\
\hline P & Braun & $\mathrm{OH}$ & $5.95 E+06$ & 3.5OE-01 & NS & $\mathbf{N}$ & Won't use Braun, Wastren, or HTCE Inv. Data \\
\hline$P$ & Raw & $\mathrm{OH}$ & $5.95 E+06$ & $3.50 E-01$ & AZ102-21 & $\mathbf{N}$ & Not a traceable reference \\
\hline$P$ & Braun & $\mathrm{OH}$ & $3.40 E+06$ & 2.00E-01 & NS & $N$ & Won't use Braun, Wastren, or HTCE Inv. Data \\
\hline$P$ & Raw & $\mathrm{OH}$ & $3.40 E+06$ & $2.00 E-01$ & $A Z 102-19$ & $\mathbf{N}$ & Not a traceable reference \\
\hline$P$ & Raw & $\mathrm{OH}$ & $3.06 E+06$ & $1.80 \mathrm{E}-01$ & $A Z 102-22$ & $\mathbf{N}$ & Not a traceable reference \\
\hline$\overline{p-1}$ & Raw & $\mathrm{OH}$ & $1.99 E+06$ & $1.17 \mathrm{E}-01$ & $A Z 102-24$ & $\bar{Y}$ & \\
\hline$\overline{p-2}$ & Raw & $\mathrm{OH}$ & $1.86 \mathrm{E}+06$ & $1.09 E-01$ & $A Z 102-24$ & $\bar{Y}$ & \\
\hline$p-1$ & Raw & $\mathrm{OH}$ & $1.84 E+06$ & $1.08 E-01$ & AZ102-24 & $\bar{Y}$ & \\
\hline$?-2$ & Raw & $\mathrm{OH}$ & $1.83 E+06$ & $1.08 \mathrm{E}-01$ & AZ102-24 & $\bar{Y}$ & \\
\hline FI & Raw & $\mathrm{OH}$ & $1.84 E+05$ & $1.08 \mathrm{E}-02$ & $A Z 102-23$ & $\mathbf{N}$ & Water wash of solid sample not supernatent \\
\hline $\mathbf{P}$ & Braun & $\mathrm{OH}$ & $1.21 E+05$ & 7.14E-03 & NS & $\mathbf{N}$ & Won't use Braun, Wastren, or HTCE Inv. Data \\
\hline$\underline{L}$ & TRAC & $\mathrm{Pb}$ & NS & NS & NS & $N$ & Analysis Prior to $9 / 86$ \\
\hline G & Wastren & $\mathrm{Pb}$ & $1.63 E+06$ & $7.87 E-03$ & NS & $\mathbf{N}$ & Won't use Braun, Wastren, or HTCE Inv. Data \\
\hline $\mathbf{S}$ & Braun & $\mathrm{Pb}$ & $1.45 E+06$ & 7.00E-03 & NS & $\mathbf{N}$ & Won't use Braun, Wastren, or HTCE Inv. Data \\
\hline$\underline{s}$ & Raw & $\mathrm{Pb}$ & $1.45 E+06$ & 7.00E-03 & AZ102-11 & $N$ & Analysis Prior to $9 / 86$ \\
\hline EI & Raw & $\mathrm{Pb}$ & $4.09 E+05$ & $1.98 E-03$ & AZ102-18 & $\mathbf{N}$ & $\begin{array}{l}\text { Solid sample after has been washed with } \\
\text { fresh water }\end{array}$ \\
\hline$P$ & Wastren & $\mathrm{Pb}$ & $6.11 E+03$ & 2.95E-05 & NS & $N$ & Won't use Braun, Wastren, or HTCE Inv. Data \\
\hline$\overline{\mathrm{Cl}}$ & Raw & $\mathrm{Pb}$ & $5.91 E+03$ & 2.85E-05 & AZ102-23 & $\bar{Y}$ & \\
\hline $\mathbf{F l}$ & Raw & $\mathrm{Pb}$ & $1.04 E+03$ & 5.00E-06 & $A Z 102-18$ & $N$ & Water wash of solid sample not supernatent \\
\hline S & HTCE Inv. & $\mathrm{Pb}$ & $5.77 E+02$ & 2.79E-06 & NS & N & Won't use Braun, Wastren, or HTCE Inv. Data \\
\hline $\mathbf{L}$ & HTCE Inv. & $\mathrm{Pb}$ & $9.09 E+01$ & 4.39E-07 & NS & $\mathbf{N}$ & Won't use Braun, Wastren, or HTCE Inv. Data \\
\hline $\mathbf{S}$ & Raw & $\mathrm{Pb}$ & $0.00 E+00$ & $0.00 E+\infty$ & $A Z 102-17$ & $\mathbf{D}$ & Points already in DB as part of AZ_102-23 \\
\hline $\mathbf{S}$ & Raw & $\mathrm{Pb}$ & $0.00 E+00$ & $0.00 E+00$ & $A Z 102-17$ & $D$ & Points already in DB as part of AZ_102-23 \\
\hline $\mathbf{S}$ & Raw & $\mathrm{Pb}$ & $0.00 E+00$ & $0.00 E+\infty$ & $A Z 102-17$ & D & Points already in DB as part of AZ_102-23 \\
\hline 3 & Raw & PO4 & \#VALUE! & \#VALUE! & $A Z 102-24$ & $Y$ & Updated per memo 8/17/95 \\
\hline $3-1$ & Raw & PO4 & \#VALUE! & \#VALUE! & $A Z 102-24$ & $\mathbf{Y}$ & Updated per memo 8/17/95 \\
\hline $2-2$ & Raw & P04 & \#VALUE! & \#VALUE! & AZ102-24 & $\bar{Y}$ & Updated per memo $8 / 17 / 95$ \\
\hline 3 & Raw & PO4 & \#VALUE! & \#VALUE! & AZ102-24 & $Y$ & Updated per memo 8/17/95 \\
\hline $3-1$ & Raw & P04 & \#VALUE! & \#VALUE! & AZ102-24 & $\mathbf{Y}$ & Updated per memo $8 / 17 / 95$ \\
\hline
\end{tabular}




\begin{tabular}{|c|c|c|c|c|c|c|c|c|c|}
\hline & & & & Tank Waste C & racterization & ample Informatic & & & \\
\hline $\begin{array}{l}\text { Reported } \\
\text { Chemical }\end{array}$ & Memo Date & $\begin{array}{c}\text { Start } \\
\text { Analysis } \\
\text { Date }\end{array}$ & $\begin{array}{c}\text { Start Sample } \\
\text { Date }\end{array}$ & Sample Number & $\begin{array}{l}\text { Tank Farm } \\
\text { Number }\end{array}$ & $\begin{array}{c}\text { Lab Sample } \\
\text { Number }\end{array}$ & $\begin{array}{l}\text { Density } \\
\text { (g/mL) }\end{array}$ & $\begin{array}{c}\text { Reported } \\
\text { Value }\end{array}$ & Reported U \\
\hline Phosphate & $7 / 26 / 95$ & N/A & $3 / 2 / 91$ & BOD9R3 & $A Z-102-1$ & S95T000263 & 1.1 & $<7.26 e+02$ & UG/ml \\
\hline PO4 & $11 / 5 / 81$ & NS & NS & RAT-AZ102-2B & NS & NS & 1.4 & 8.70E-01 & moles/l \\
\hline PO4 & $5 / 5 / 82$ & NS & $11 / 4 / 81$ & NS & NS & NS & 1.4 & $8.70 \mathrm{E}-01$ & $M$ \\
\hline PO4 & $11 / 5 / 81$ & NS & NS & RAT-AZ102-2C & NS & NS & 1.26 & $3.90 E-01$ & moles $/ 1$ \\
\hline PO4 & $11 / 5 / 81$ & NS & NS & RAT-AZ102-2A & NS & NS & 1.09 & $1.00 E-01$ & males $n$ \\
\hline $\mathrm{PO4}$ & $5 / 5 / 82$ & NS & $11 / 4 / 81$ & $\because N S$ & NS & NS & 1.09 & $1.00 \mathrm{E}-01$ & $\bar{M}$ \\
\hline $\mathrm{PO4}$ & $12 / 13 / 83$ & NS & $7 / 31 / 83$ & $T 1520$ & NS & NS & NS & 5.50E-02 & $\bar{M}$ \\
\hline $\mathrm{PO4}$ & $12 / 13 / 83$ & NS & $7 / 31 / 83$ & T1521 & NS & NS & NS & $2.00 \mathrm{E}-02$ & $M$ \\
\hline $\mathrm{PO4}$ & $6 / 26 / 75$ & NS & NS & RAT-AZ102-4a & NS & NS & 1.39 & $1.10 E-02$ & moles $/ 1$ \\
\hline PO4 & $6 / 26 / 75$ & NS & NS & NS & NS & NS & 1.392 & $1.10 \mathrm{E}-02$ & $M$ \\
\hline $\mathrm{Na3PO4}$ & $7 / 28 / 79$ & NS & NS & NS & NS & NS & 1.41 & $1.00 \mathrm{E}-02$ & $M$ \\
\hline PO4 & $7 / 28 / 79$ & NS & NS & RAT-AZ102-4D & NS & NS & 1.41 & $1.00 E-02$ & moles $/$ \\
\hline PO4 & $7 / 28 / 79$ & NS & NS & RAT-AZ102-6 & NS & NS & 1.41 & $1.00 \mathrm{E}-02$ & moles/1 \\
\hline PO4 & $2 / 4 / 81$ & NS & NS & $R-3699$ & NS & NS & 1.11 & 9.61E-03 & moles $n$ \\
\hline $\mathrm{PO4}$ & $2 / 4 / 81$ & NS & NS & R3699 & NS & NS & 1.11 & $9.61 \mathrm{E}-03$ & $M$ \\
\hline PO4 & $2 / 4 / 81$ & NS & NS & R-3700 & NS & NS & 1.11 & $9.38 \mathrm{E}-03$ & moles/ \\
\hline P04 & $2 / 4 / 81$ & NS & NS & R3700 & NS & NS & 1.11 & $9.38 E-03$ & $M$ \\
\hline $\mathrm{PO4}$ & $12 / 3 / 75$ & NS & NS & $1496 B$ & NS & NS & 1.38 & $8.42 \mathrm{E}-03$ & $M$ \\
\hline PO4 & $12 / 3 / 75$ & NS & NS & $1496 C$ & NS & NS & 1.379 & 8.31E-03 & $\bar{M}$ \\
\hline PO4 & $12 / 3 / 75$ & NS & NS & $1496 \mathrm{~A}$ & NS & NS & 1.378 & $8.17 E-03$ & $M$ \\
\hline $\mathrm{PO4}$ & $12 / 3 / 75$ & NS & NS & 1496D & NS & NS & 1.36 & 8.09E-03 & $M$ \\
\hline $\mathrm{PO4}$ & $12 / 3 / 75$ & NS & NS & 1459 & NS & NS & 1.386 & $7.61 \mathrm{E}-03$ & $M$ \\
\hline $\mathrm{PO4}$ & $10 / 31 / 86$ & NS & $6 / 30 / 85$ & NS & NS & NS & 1.73 & $4.38 E+00$ & umol/g \\
\hline PO4 & $12 / 3 / 75$ & NS & NS & 1457 & NS & NS & 1.398 & $7.12 E-03$ & $M$ \\
\hline PO4- & Aug 89 & NS & NS & NS & NS & NS & NS & $6.20 \mathrm{E}-01$ & $g / L$ \\
\hline $\mathrm{Na3PO4}$ & $8 / 27 / 77$ & NS & NS & NS & NS & NS & 1.391 & $6.00 \mathrm{E}-03$ & $M$ \\
\hline $\mathrm{PO4}$ & $8 / 27 / 77$ & NS & NS & RAT-AZ102-4B & NS & NS & 1.39 & $6.00 E-03$ & moles/l \\
\hline PO4 & $2 / 19 / 82$ & NS & $12 / 1 / 81$ & $T 3251$ & NS & NS & 1.03 & $6.00 E-03$ & $M$ \\
\hline$P 04$ & $12 / 3 / 75$ & NS & NS & 1458 & NS & NS & 1.391 & $5.53 E-03$ & $M$ \\
\hline PO4 & $10 / 1 / 83$ & NS & NS & RAT-AZ102-3 & NS & NS & NS & $5.00 E-03$ & moles $\|$ \\
\hline PO4 & $3 / 10 / 84$ & NS & $7 / 31 / 83$ & NS & NS & NS & NS & $5.00 E-03$ & $M$ \\
\hline PO4 & $3 / 10 / 84$ & NS & $7 / 31 / 83$ & NS & NS & NS & NS & $5.00 E-03$ & $M$ \\
\hline PO4 & $7 / 5 / 84$ & NS & $7 / 31 / 83$ & T3495 & NS & NS & NS & $5.00 E-03$ & $\bar{M}$ \\
\hline PO43- & NS & NS & NS & NS & NS & NS & 1.39 & $4.46 E-03$ & Mole/L \\
\hline PO4 & $12 / 3 / 75$ & NS & NS & 1456 & NS & NS & 1.393 & $3.28 \mathrm{E}-03$ & $M$ \\
\hline PO4 & $10 / 31 / 86$ & NS & $6 / 30 / 85$ & NS & NS & NS & 1.11 & $2.26 E+00$ & umol/g \\
\hline $\mathrm{Cr}$ & $8 / 31 / 89$ & NS & $6 / 30 / 85$ & Composite Core & NS & NS & 1.11 & $2.26 E+00$ & umol/g \\
\hline PO4- & Aug 89 & NS & NS & NS & NS & NS & NS & $1.52 E-01$ & $g / L$ \\
\hline
\end{tabular}




\begin{tabular}{|c|c|c|c|c|c|c|c|}
\hline & & & Analyte Inform & & & & \\
\hline Code & Source & Analyte & Conc. (ug/L) & Conc. $\{\mathrm{mol} / \mathrm{L}\}$ & $\begin{array}{c}\text { Reference } \\
\text { Number }\end{array}$ & $\begin{array}{c}\text { Validated } \\
\text { (Y/N) }\end{array}$ & Notes \\
\hline $\mathrm{P}-2$ & Raw & $\mathrm{PO4}$ & \#VALUEI & \#VALUE! & AZ102-24 & $Y$ & Updated per memo $8 / 17 / 95$ \\
\hline s & Braun & PO4 & $8.26 E+07$ & 8.70E-01 & NS & $N$ & Won't use Braun, Wastren, or HTCE Inv. Data \\
\hline $\mathbf{S}$ & Raw & PO4 & $8.26 \mathrm{E}+07$ & $8.70 E-01$ & $A Z 102-11$ & $N$ & Analysis Prior to $9 / 86$ \\
\hline $\mathbf{R}$ & Braun & PO4 & $3.70 E+07$ & 3.90E-01 & NS & $N$ & Won't use Braun, Wastren, or HTCE Inv. Data \\
\hline $\mathbf{P}$ & Braun & PO4 & $9.50 E+06$ & $1.00 E-01$ & NS & $N$ & Won't use Braun, Wastren, or HTCE Inv. Data \\
\hline L & Raw & PO4 & $9.50 \mathrm{E}+06$ & $1.00 \mathrm{E}-01$ & AZ102-11 & $\mathbf{N}$ & Analysis Prior to $9 / 86$ \\
\hline$L$ & Raw & P04 & $5.22 E+06$ & $5.50 E-02$ & AZ102-14 & $\mathrm{N}$ & No units for concentration given in report \\
\hline $\mathbf{S}$ & Raw & PO4 & $1.90 E+06$ & $2.00 \mathrm{E}-02$ & AZ102-14 & $N$ & No units for concentration given in report \\
\hline $\mathbf{P}$ & Braun & PO4 & $1.04 \mathrm{E}+06$ & $1.10 \mathrm{E}-02$ & NS & $\mathbf{N}$ & Analysis Prior to $9 / 86$ \\
\hline $\mathrm{L}$ & Raw & $\mathrm{PO4}$ & $1.04 E+06$ & $1.10 \mathrm{E}-02$ & AZ102-6 & $\mathbf{N}$ & Analysis Prior to $9 / 86$ \\
\hline$L$ & Raw & PO4 & $9.50 E+05$ & $1.00 \mathrm{E}-02$ & AZ102-5 & $\mathbf{N}$ & Analysis Prior to $9 / 86$ \\
\hline $\mathbf{P}$ & Braun & PO4 & $9.50 E+05$ & $1.00 E-02$ & NS & $N$ & Won't use Braun, Wastren, or HTCE Inv. Data \\
\hline $\mathbf{R}$ & Braun & PO4 & $9.50 E+05$ & $1.00 E-02$ & NS & $\mathbf{N}$ & Won't use Braun, Wastren, or HTCE Inv. Data \\
\hline $\mathbf{P}$ & Braun & PO4 & $9.13 E+05$ & $9.61 E-03$ & NS & $\mathbf{N}$ & Won't use Braun, Wastren, or HTCE Inv. Data \\
\hline$L$ & Raw & PO4 & $9.13 E+05$ & $9.61 \mathrm{E}-03$ & AZ102-8 & $\mathbf{N}$ & Analysis Prior to $9 / 86$ \\
\hline $\mathbf{P}$ & Braun & PO4 & $8.91 E+05$ & $9.38 E-03$ & NS & $N$ & Won't use Braun, Wastren, or HTCE Inv. Data \\
\hline $\mathrm{L}$ & Raw & PO4 & $8.91 E+05$ & $9.38 \mathrm{E}-03$ & $A Z 102-8$ & $N$ & Analysis Prior to $9 / 86$ \\
\hline$L$ & Raw & P04 & $8.00 E+05$ & $8.42 E-03$ & $A Z 102-3$ & $N$ & Analysis Prior to $9 / 86$ \\
\hline$L$ & Raw & $\mathrm{PO4}$ & $7.89 E+05$ & $8.31 E-03$ & $A Z 102-3$ & $\mathbf{N}$ & Analysis Prior to $9 / 86$ \\
\hline$L$ & Raw & P04 & $7.76 \mathrm{E}+05$ & 8.17E-03 & $A Z 102-3$ & $N$ & Analysis Prior to $9 / 86$ \\
\hline 1 & Raw & $\mathrm{PO4}$ & $7.68 \mathrm{E}+05$ & $8.09 \mathrm{E}-03$ & $A Z 102-3$ & $\mathbf{N}$ & Analysis Prior to $9 / 86$ \\
\hline $\mathrm{L}$ & Raw & PO4 & $7.23 E+05$ & $7.61 \mathrm{E}-03$ & $A Z 102-3$ & $\mathbf{N}$ & Analysis Prior to $9 / 86$ \\
\hline $\mathrm{BI}$ & Raw & $\mathrm{PO4}$ & $7.20 E+05$ & $7.58 \mathrm{E}-03$ & $A Z 102-18$ & $\mathrm{D}$ & NS \\
\hline $\mathrm{L}$ & Raw & $\mathrm{PO4}$ & $6.76 E+05$ & $7.12 \mathrm{E}-03$ & $A Z 102-3$ & $N$ & Analysis Prior to $9 / 86$ \\
\hline G & Wastren & PO4 & $6.20 E+05$ & $6.53 E-03$ & NS & $\mathbf{N}$ & Won't use Braun, Wastren, or HTCE Inv. Data \\
\hline$F$ & Raw & PO4 & $5.70 E+05$ & $6.00 \mathrm{E}-03$ & $A Z 102-4$ & $\mathbf{N}$ & Analysis Prior to $9 / 86$ \\
\hline$P$ & Braun & PO4 & $5.70 E+05$ & $6.00 \mathrm{E}-03$ & NS & $\therefore \mathrm{N}$ & Analysis Prior to 9/86 \\
\hline$L$ & Raw & PO4 & $5.70 E+05$ & $6.00 \mathrm{E}-03$ & AZ102-10 & $\mathrm{N}$ & Analysis Prior to $9 / 86$ \\
\hline $\mathbf{L}$ & Raw & PO4 & $5.25 E+05$ & $5.53 E-03$ & AZ102-3 & $N$ & Analysis Prior to $9 / 86$ \\
\hline $\mathbf{P}$ & Braun & PO4 & $4.75 E+05$ & 5.00E-03 & NS & N & Won't use Braun, Wastren, or HTCE Inv. Data \\
\hline L & Raw & PO4 & $4.75 E+05$ & $5.00 E-03$ & $A Z 102-16$ & $\mathbf{N}$ & $\begin{array}{l}\text { These data are so messed up it will be easier } \\
\text { to reenter them. }\end{array}$ \\
\hline$L$ & Raw & PO4 & $4.75 E+05$ & $5.00 E-03$ & AZ102-16 & $N$ & Point in DB already ce $8 / 15 / 95$ \\
\hline$L$ & Raw & $\mathrm{PO4}$ & $4.75 \mathrm{E}+05$ & $5.00 \mathrm{E}-03$ & $A 2102-16$ & $\mathbf{N}$ & Point in DB already_cc_8/15/95 \\
\hline $\mathbf{s}$ & HTCE Inv. & PO4 & $4.24 E+05$ & $4.46 \mathrm{E}-03$ & NS & N & Won't use Braun, Wastren, or HTCE Inv. Data \\
\hline $\mathrm{L}$ & Raw & $\mathrm{PO4}$ & $3.12 E+05$ & $3.28 E-03$ & $A Z 102-3$ & $N$ & Analysis Prior to $9 / 86$ \\
\hline El & Raw & P04 & $2.38 \varepsilon+05$ & $2.51 E-03$ & AZ102-1,8 & D & NS \\
\hline El & Raw & PO4 & $2.38 E+05$ & 2.51E-03 & $A Z 102-23$ & $\mathbf{N}$ & $\begin{array}{l}\text { Solid sample after has been washed with } \\
\text { fresh water }\end{array}$ \\
\hline$P$ & Wastren & PO4 & $1.52 E+05$ & $1.60 \mathrm{E}-03$ & NS & $\mathbf{N}$ & Won't use Braun, Wastren, or HTCE Inv. Data \\
\hline
\end{tabular}




\begin{tabular}{|c|c|c|c|c|c|c|c|c|c|}
\hline & & & & Tank Waste C & racterization & ample Informati & & & \\
\hline $\begin{array}{l}\text { Reported } \\
\text { Chemical }\end{array}$ & Memo Date & $\begin{array}{c}\text { Analysis } \\
\text { Date }\end{array}$ & $\begin{array}{c}\text { Start Sample } \\
\text { Date }\end{array}$ & Sample Number & $\begin{array}{c}\text { Tank Farm } \\
\text { Number }\end{array}$ & $\begin{array}{c}\text { Lab Sample } \\
\text { Number }\end{array}$ & $\begin{array}{l}\text { Density } \\
\text { ( } \mathrm{g} / \mathrm{mL})\end{array}$ & $\begin{array}{l}\text { Reported } \\
\text { Value }\end{array}$ & Reported Un \\
\hline $\mathrm{PO4}$ & $9 / 1 / 93$ & N/A & $6 / 30 / 85$ & N/A & N/A & N/A & 1.14 & $1.36 E+00$ & umol/g \\
\hline PO4 & $10 / 31 / 86$ & NS & $6 / 30 / 85$ & NS & NS & NS & 1.14 & $1.36 \mathrm{E}+00$ & umol/g \\
\hline PO43- & NS & NS & NS & NS & NS & NS & 1.04 & $7.25 E-04$ & Mole/L \\
\hline $\mathrm{PO} 4$ & $8 / 31 / 89$ & NS & $6 / 30 / 85$ & Composite Core & NS & NS & $(1.0)$ & 4.21E-01 & umol/g \\
\hline PO4 & $1 / 30 / 82$ & NS & NS & $T-5387$ & NS & NS & 1.008 & $3.10 \mathrm{E}-04$ & moles $/ 1$ \\
\hline PO4 & $2 / 19 / 82$ & NS & NS & $T 5387$ & NS & NS & 1.008 & 3.10E-04 & $M$ \\
\hline $\mathrm{PO4}$ & $10 / 31 / 86$ & NS & $6 / 30 / 85$ & NS & NS & NS & NS & $4.21 E-01$ & umol/g \\
\hline $\mathrm{Sb}$ & $9 / 1 / 93$ & N/A & $6 / 30 / 85$ & N/A & N/A & N/A & 1.14 & $<7.40 \mathrm{e}-02$ & umol/g \\
\hline$F$ & $8 / 31 / 89$ & NS & $6 / 30 / 85$ & Composite Core & NS & NS & 1.11 & $<2.50 e+00$ & umol/g \\
\hline $\mathrm{Se}$ & NS & NS & NS & NS & NS & NS & NS & $1.12 \mathrm{E}-02$ & $g / L$ \\
\hline Se & $9 / 1 / 93$ & N/A & $6 / 30 / 85$ & N/A & $\overline{N / A}$ & N/A & 1.14 & $1.20 \mathrm{E}-01$ & umol/g \\
\hline Se & $10 / 31 / 86$ & NS & $6 / 30 / 85$ & NS & NS & NS & 1.14 & $1.20 \mathrm{E}-01$ & umol/g \\
\hline Se & $10 / 31 / 86$ & NS & $6 / 30 / 85$ & Composite Core & NS & NS & $(1.0)$ & $2.80 \mathrm{E}-02$ & umol/g \\
\hline SO4 & $5 / 5 / 82$ & NS & $11 / 4 / 81$ & NS & NS & NS & 1.09 & $<2.00 \mathrm{e}-02$ & $M$ \\
\hline 504 & $1 / 1 / 77$ & NS & NS & NS & NS & NS & NS & NS & NS \\
\hline S04 & $12 / 13 / 83$ & NS & $7 / 31 / 83$ & $T 1521$ & NS & NS & NS & $1.90 E+00$ & $M$ \\
\hline SO4 & $6 / 26 / 75$ & NS & NS & RAT-AZ102-4a & NS & NS & 1.39 & $9.04 \mathrm{E}-01$ & moles $/ 1$ \\
\hline SO4 & $6 / 26 / 75$ & NS & NS & NS & NS & NS & 1.392 & $9.04 E-01$ & $M$ \\
\hline Sulfate & $7 / 26 / 95$ & N/A & $3 / 2 / 91$ & BOD9R3 & $A Z-102-1$ & S95T000263 & 1.1 & $1.88 \mathrm{E}+04$ & $\mathrm{UG} / \mathrm{ml}$ \\
\hline Sulfate & $7 / 26 / 95$ & N/A & $3 / 2 / 91$ & BOD9R3 & $A Z-102-1$ & S95T000263 & 1.1 & $1.86 \mathrm{E}+04$ & $\mathrm{UG} / \mathrm{ml}$ \\
\hline Sulfate & $7 / 26 / 95$ & N/A & $3 / 2 / 91$ & 80D9R5 & $A Z-102-3$ & S95T000265 & 1.1 & $1.77 E+04$ & $\mathrm{UG} / \mathrm{ml}$ \\
\hline Sulfate & $7 / 26 / 95$ & N/A & $3 / 2 / 91$ & BOD9R4 & $A Z-102-2$ & $595 T 000264$ & 1.1 & $1.76 E+04$ & $\mathrm{UG} / \mathrm{ml}$ \\
\hline Sulfate & $7 / 26 / 95$ & N/A & $3 / 2 / 91$ & BOD9R5 & $A Z-102-3$ & $595 T 000265$ & 1.1 & $1.73 E+04$ & $\mathrm{UG} / \mathrm{ml}$ \\
\hline so4- & Aug 89 & NS & NS & NS & NS & NS & NS & $1.41 E+01$ & $g / L$ \\
\hline Sulfate & $7 / 26 / 95$ & N/A & $3 / 2 / 91$ & 8009R4 & $A Z-102-2$ & S95T000264 & 1.1 & $1.38 E+04$ & $\mathrm{UG} / \mathrm{ml}$ \\
\hline 504 & $9 / 1 / 93$ & N/A & $6 / 30 / 85$ & N/A & N/A & N/A & 1.14 & $1.24 E+02$ & $\mathrm{umol} / \mathrm{g}$ \\
\hline S04 & $10 / 31 / 86$ & NS & $6 / 30 / 85$ & NS & NS & NS & 1.14 & $1.24 E+02$ & $\mathrm{umol} / \mathrm{g}$ \\
\hline S04 & $10 / 31 / 86$ & NS & $6 / 30 / 85$ & NS & NS & NS & 1.73 & $6.81 E+01$ & umol/g \\
\hline so4 & $3 / 10 / 84$ & NS & $7 / 31 / 83$ & NS & NS & NS & NS & $1.13 E-01$ & $M$ \\
\hline so4 & $10 / 1 / 83$ & NS & NS & RAT-AZ102-3 & NS & NS & NS & $1.10 E-01$ & moles/l \\
\hline SO4 & $3 / 10 / 84$ & NS & $7 / 31 / 83$ & NS & NS & NS & NS & $1.10 \mathrm{E}-01$ & $M$ \\
\hline SO4 & $7 / 5 / 84$ & NS & $7 / 31 / 83$ & T3495 & NS & NS & NS & $1.10 \mathrm{E}-01$ & $M$ \\
\hline S04 & $12 / 13 / 83$ & NS & $7 / 31 / 83$ & T1520 & NS & NS & NS & $1.10 \mathrm{E}-01$ & $M$ \\
\hline SO4 & $10 / 31 / 86$ & NS & NS & $\mathrm{R} 880311$ & NS & NS & NS & $1.10 \mathrm{E}-01$ & $M$ \\
\hline 504 & $10 / 31 / 86$ & NS & NS & R081287 & NS & NS & NS & $1.10 \mathrm{E}-01$ & $M$ \\
\hline so4- & Aug 89 & NS & NS & NS & NS & NS & NS & $9.75 E+00$ & $g / L$ \\
\hline SO4 & $11 / 5 / 81$ & NS & NS & RAT-AZ102-2B & NS & NS & 1.4 & $1.00 \mathrm{E}-01$ & moles $/$ \\
\hline 504 & $5 / 5 / 82$ & NS & $11 / 4 / 81$ & NS & NS & NS & 1.4 & $1.00 \mathrm{E}-01$ & $M$ \\
\hline
\end{tabular}




\begin{tabular}{|c|c|c|c|c|c|c|c|}
\hline & & & Analyte Inform & & & & \\
\hline Yode & Source & Analyte & Conc. (ug/L) & Conc. (mol/L) & $\begin{array}{c}\text { Reference } \\
\text { Number }\end{array}$ & $\begin{array}{c}\text { Validated } \\
(\mathrm{Y} / \mathrm{N})\end{array}$ & Notes \\
\hline $\mathrm{Cl}$ & Raw & P04 & $1.47 \mathrm{E}+05$ & $1.55 \mathrm{E}-03$ & AZ102-23 & $Y$ & \\
\hline $\mathrm{Cl}$ & Raw & $\mathrm{PO4}$ & $1.47 E+05$ & $1.55 \mathrm{E}-03$ & $A 2102-18$ & $\bar{D}$ & NS \\
\hline L & HTCE Inv. & PO4 & $6.89 E+04$ & $7.25 E-04$ & NS & $N$ & Won't use Braun, Wastren, or HTCE Inv. Data \\
\hline $\mathrm{FI}$ & Raw & PO4 & $4.00 E+04$ & $4.21 \varepsilon-04$ & $A Z 102-23$ & $N$ & Water wash of solid sample not supernatent \\
\hline$P$ & Braun & PO4 & $2.94 E+04$ & $3.10 E-04$ & NS & N & Won't use Braun, Wastren, or HTCE Inv. Data \\
\hline L & Raw & P04 & $2.94 E+04$ & $3.10 E-04$ & $A Z 102-9$ & $\mathbf{N}$ & $\begin{array}{l}\text { Not a traceable reference, analysis Prior to } \\
9 / 86\end{array}$ \\
\hline $\mathrm{FI}$ & Raw & PO4 & $0.00 E+00$ & $0.00 E+00$ & AZ102-18 & D & NS \\
\hline $\mathrm{Cl}$ & Raw & $\mathrm{Sb}$ & \#VALUE! & \#VALUE! & $A Z 102-23$ & $Y$ & \\
\hline El & Raw & Se & \#VALUE! & \#VALUE! & $A Z 102-18$ & $N$ & $\begin{array}{l}\text { Solid sample after has been washed with } \\
\text { fresh water }\end{array}$ \\
\hline $\mathbf{P}$ & Wastren & Se & $1.12 E+04$ & $1.42 \mathrm{E}-04$ & NS & $N$ & Won't use Braun, Wastren, or HTCE Inv. Data \\
\hline $\mathrm{Cl}$ & Raw & Se & $1.08 E+04$ & $1.37 E-04$ & AZ102-23 & $\bar{Y}$ & \\
\hline $\mathrm{Cl}$ & Raw & Se & $1.08 E+04$ & $1.37 E-04$ & AZ102-18 & $\bar{D}$ & NS \\
\hline $\mathbf{F l}$ & Raw & $\mathrm{Se}$ & $2.21 E+03$ & $2.80 E-05$ & $A Z 102-18$ & $N$ & Water wash of solid sample not supernatent \\
\hline $\mathbf{L}$ & Raw & SO4 & \#VALUE! & \#VALUE! & AZ102-11 & $\mathrm{N}$ & Analysis Prior to $9 / 86$ \\
\hline $\mathbf{L}$ & TRAC & SO4 & NS & NS & NS & $\mathbf{N}$ & Analysis Prior to $9 / 86$ \\
\hline $\mathbf{s}$ & Raw & SO4 & $1.83 E+08$ & $1.90 E+00$ & AZ102-14 & $\mathbf{N}$ & No units for concentration given in report \\
\hline $\mathbf{P}$ & Braun & SO4 & $8.68 E+07$ & 9.04E-01 & NS & $\mathrm{N}$ & Analysis Prior to $9 / 86$ \\
\hline $\mathbf{L}$ & Raw & SO4 & $8.68 E+07$ & 9.04E-01 & $A Z 102-6$ & $N$ & Analysis Prior to $9 / 86$ \\
\hline$P-1$ & Raw & SO4 & $1.88 E+07$ & $1.96 \mathrm{E}-01$ & AZ102-24 & $\bar{Y}$ & Updated per memo $8 / 17 / 95$ \\
\hline$P-2$ & Raw & SO4 & $1.86 E+07$ & $1.94 \mathrm{E}-01$ & $\mathrm{AZ102-24}$ & $Y$ & Updated per memo 8/17/95 \\
\hline P-2 & Raw & SO4 & $1.77 E+07$ & $1.84 \mathrm{E}-01$ & AZ102-24 & $\mathrm{Y}$ & Updated per memo 8/17/95 \\
\hline P-2 & Raw & SO4 & $1.76 E+07$ & $1.83 \mathrm{E}-01$ & AZ102-24 & $Y$ & Updated per memo 8/17/95 \\
\hline P-1 & Raw & SO4 & $1.73 E+07$ & $1.80 \mathrm{E}-01$ & AZ102-24 & $\bar{Y}$ & Updated per memo 8/17/95 \\
\hline $\mathbf{P}$ & Wastren & SO4 & $1.41 E+07$ & $1.47 E-01$ & NS & $\mathbf{N}$ & Won't use Braun, Wastren, or HTCE Inv. Data \\
\hline$P-1$ & Raw & SO4 & $1.38 E+07$ & $1.44 \mathrm{E}-01$ & AZ102-24 & $Y$ & Updated per memo 8/17/95 \\
\hline $\mathrm{Cl}$ & Raw & SO4 & $1.36 E+07$ & $1.41 \mathrm{E}-01$ & $A Z 102-23$ & $Y$ & \\
\hline $\mathrm{Cl}$ & Raw & SO4 & $1.36 E+07$ & $1.41 \mathrm{E}-01$ & AZ102-18 & $\mathrm{D}$ & NS \\
\hline$\underline{B 1}$ & Raw & SO4 & $1.13 E+07$ & $1.18 \mathrm{E}-01$ & AZ102-18 & D & NS \\
\hline L & Raw & SO4 & $1.09 E+07$ & $1.13 \mathrm{E}-01$ & $A Z 102-16$ & $\mathbf{N}$ & $\begin{array}{l}\text { These data are so messed up it will be easier } \\
\text { to reenter them. }\end{array}$ \\
\hline $\mathbf{P}$ & Braun & SO4 & $1.06 E+07$ & $1.10 E-01$ & NS & N & Won't use Braun, Wastren, or HTCE Inv. Data \\
\hline L & Raw & SO4 & $1.06 E+07$ & $1.10 \mathrm{E}-01$ & AZ102-16 & $N$ & Point in DB already_cc_8/15/95 \\
\hline $\mathbf{L}$ & Raw & SO4 & $1.06 E+07$ & $1.10 \mathrm{E}-01$ & AZ102-16 & $N$ & Point in DB already_cc $8 / 15 / 95$ \\
\hline $\mathbf{L}$ & Raw & SO4 & $1.06 \mathrm{E}+07$ & $1.10 E-01$ & AZ102-14 & $N$ & No units for concentration given in report \\
\hline $\mathbf{L}$ & Raw & SO4 & $1.06 E+07$ & $1.10 \mathrm{E}-01$ & AZ102-19 & $N$ & Not a traceable reference \\
\hline $\mathbf{L}$ & Raw & SO4 & $1.06 E+07$ & $1.10 \mathrm{E}-01$ & AZ102-22 & $N$ & Not a traceable reference \\
\hline $\mathbf{G}$ & Wastren & so4 & $9.75 E+06$ & $1.01 E-01$ & NS & $N$ & Won't use Braun, Wastren, or HTCE Inv. Data \\
\hline $\mathbf{S}$ & Braun & SO4 & $9.61 E+06$ & $1.00 E-01$ & NS & $N$ & Won't use Braun, Wastren, or HTCE Inv. Data \\
\hline $\mathbf{S}$ & Raw & SO4 & $9.61 E+06$ & $1.00 E-01$ & $A 2102-11$ & $\mathbf{N}$ & Analysis Prior to $9 / 86$ \\
\hline
\end{tabular}




\begin{tabular}{|c|c|c|c|c|c|c|c|c|c|}
\hline & & & & Tank Waste C & Iracterization & mple Informat & & & \\
\hline $\begin{array}{l}\text { Reported } \\
\text { Chemical }\end{array}$ & Memo Date & $\begin{array}{c}\text { Start } \\
\text { Analysis } \\
\text { Date }\end{array}$ & $\begin{array}{c}\text { Start Sample } \\
\text { Date }\end{array}$ & Sample Number & $\begin{array}{c}\text { Tank Farm } \\
\text { Number }\end{array}$ & $\begin{array}{c}\text { Lab Sample } \\
\text { Number }\end{array}$ & $\begin{array}{l}\text { Density } \\
\text { (g/mL) }\end{array}$ & $\begin{array}{c}\text { Reported } \\
\text { Value } \\
\end{array}$ & Reported Uni \\
\hline so42- & NS & NS & NS & NS & NS & NS & 1.39 & $9.03 E-02$ & Mole/L \\
\hline SO4 & $11 / 5 / 81$ & NS & NS & RAT-AZ102-2C & NS & NS & 1.26 & $5.00 E-02$ & moles/l \\
\hline so42. & NS & NS & NS & NS & NS & NS & 1.04 & 4.6OE-02 & Mole/L \\
\hline so4 & $2 / 4 / 81$ & NS & NS & R-3699 & NS & NS & 1.11 & $2.75 E-02$ & moles $n$ \\
\hline SO4 & $2 / 4 / 81$ & NS & NS & R-3700 & NS & NS & 1.11 & $2.75 \mathrm{E}-02$ & moles/1 \\
\hline 504 & $2 / 4 / 81$ & NS & NS & R3699 & NS & NS & 1.11 & $2.75 E-02$ & $\bar{M}$ \\
\hline 504 & $2 / 4 / 81$ & NS & NS & 83700 & NS & NS & 1.11 & $2.75 E-02$ & $M$ \\
\hline SO4 & $11 / 5 / 81$ & NS & NS & RAT-AZ102-2A & NS & NS & 1.09 & $2.00 \mathrm{E}-02$ & moles $n$ \\
\hline SO4 & $10 / 30 / 86$ & NS & NS & R060386 & NS & NS & NS & $2.00 \mathrm{E}-02$ & $M$ \\
\hline SO4 & $8 / 31 / 89$ & NS & $6 / 30 / 85$ & Composite Core & NS & NS & $(1.0)$ & $1.95 E+01$ & umol/g \\
\hline SO4 & $10 / 31 / 86$ & NS & $6 / 30 / 85$ & NS & NS & NS & 1.11 & $7.30 E+00$ & umol/g \\
\hline$F$ & $8 / 31 / 89$ & NS & $6 / 30 / 85$ & Composite Core & NS & NS & 1.11 & $7.30 E+00$ & umol/g \\
\hline SO4 & $10 / 31 / 86$ & NS & $6 / 30 / 85$ & NS & NS & NS & NS & $1.95 E+01$ & umol/g \\
\hline $\mathrm{Sr}$ & $10 / 31 / 86$ & NS & $6 / 30 / 85$ & NS & NS & NS & 1.73 & $3.73 E+00$ & umol/g \\
\hline Sr & $8 / 1 / 85$ & NS & NS & NS & NS & NS & NS & 4.87E-01 & $g /$ \\
\hline $\mathrm{Sr}$ & $11 / 5 / 81$ & NS & NS & RAT-AZ102-28 & NS & NS & 1.4 & $1.38 E-03$ & moles $/$ \\
\hline Sr & $10 / 31 / 86$ & NS & $6 / 30 / 85$ & NS & NS & NS & 1.11 & $9.00 \mathrm{E}-01$ & umol/g \\
\hline$F$ & $8 / 31 / 89$ & NS & $6 / 30 / 85$ & Composite Core & NS & NS & 1.11 & $9.00 E-01$ & umol/g \\
\hline Sr & $8 / 1 / 85$ & NS & NS & NS & NS & NS & NS & $2.07 E-04$ & $g / L$ \\
\hline $\mathrm{Sr}$ & $9 / 1 / 93$ & N/A & $6 / 30 / 85$ & N/A & N/A & N/A & 1.14 & $2.00 \mathrm{E}-03$ & umol/g \\
\hline $\mathrm{Sr}$ & $10 / 31 / 86$ & NS & $6 / 30 / 85$ & NS & NS & NS & 1.14 & $2.00 E-03$ & umol/g \\
\hline $\mathrm{Sr}$ & $10 / 31 / 86$ & NS & $6 / 30 / 85$ & NS & NS & NS & NS & $0.00 E+00$ & umol/g \\
\hline $\mathrm{Sr}$ & $8 / 31 / 89$ & NS & $6 / 30 / 85$ & Composite Core & NS & NS & NS & $0.00 E+00$ & umol/g \\
\hline $\mathrm{Sr}$ & $8 / 31 / 89$ & NS & $6 / 30 / 85$ & $\mathrm{L1}$ & NS & NS & NS & $3.00 E+00$ & umol/g \\
\hline TC & $9 / 1 / 93$ & N/A & $6 / 30 / 85$ & N/A & N/A & N/A & 1.14 & $6.98 E+02$ & umol/g \\
\hline $\mathrm{Te}$ & $10 / 31 / 86$ & NS & $6 / 30 / 85$ & Composite Core & NS & NS & $(1.0)$ & $<4.00 \mathrm{e}-03$ & umol/g \\
\hline $\mathrm{Te}$ & $10 / 31 / 86$ & NS & $6 / 30 / 85$ & NS & NS & NS & 1.11 & $1.36 E+00$ & umol/g \\
\hline$F$ & $8 / 31 / 89$ & NS & $6 / 30 / 85$ & Composite Core & NS & NS & 1.11 & $1.36 E+00$ & umol/g \\
\hline$T e$ & $9 / 1 / 93$ & N/A & $6 / 30 / 85$ & N/A & N/A & N/A & 1.14 & $1.80 E-02$ & umol/g \\
\hline $\mathrm{Te}$ & $10 / 31 / 86$ & NS & $6 / 30 / 85$ & NS & NS & NS & 1.14 & $1.80 \mathrm{E}-02$ & umol/g \\
\hline Te & $8 / 1 / 85$ & NS & NS & NS & NS & NS & NS & 2.17E-03 & $g / L$ \\
\hline $\mathbf{F}$ & $8 / 31 / 89$ & NS & $6 / 30 / 85$ & Composite Core & NS & NS & 1.11 & $<1.00 e+01$ & umol/g \\
\hline$\pi$ & $10 / 31 / 86$ & NS & $6 / 30 / 85$ & Composite Core & NS & NS & (1.0) & $<6.10 \mathrm{e}-02$ & $\mathrm{umol} / \mathrm{g}$ \\
\hline
\end{tabular}




\begin{tabular}{|c|c|c|c|c|c|c|c|}
\hline \multirow[b]{2}{*}{ :ode } & \multirow[b]{2}{*}{ Source } & \multicolumn{3}{|c|}{ Analyte Information } & \multirow[b]{2}{*}{$\begin{array}{c}\text { Reference } \\
\text { Number }\end{array}$} & \multirow[b]{2}{*}{$\begin{array}{c}\text { Validated } \\
\text { (Y/N) }\end{array}$} & \multirow[b]{2}{*}{ Notes } \\
\hline & & Analyte & Conc. (ug/L) & Conc. (mol/L) & & & \\
\hline $\mathbf{S}$ & HTCE Inv. & SO4 & $8.68 E+06$ & $9.03 E-02$ & NS & $N$ & Won't use Braun, Wastren, or HTCE inv. Data \\
\hline $\mathbf{R}$ & Braun & so4 & $4.80 E+06$ & $5.00 \mathrm{E}-02$ & NS & $N$ & Won't use Braun, Wastren, or HTCE Inv. Data \\
\hline L & HTCE InV. & so4 & $4.42 E+06$ & $4.60 E-02$ & NS & $N$ & Won't use Braun, Wastren, or HTCE Inv. Data \\
\hline $\mathbf{P}$ & Braun & SO4 & $2.64 E+06$ & $2.75 \mathrm{E}-02$ & NS & $N$ & Won't use Braun, Wastren, or HTCE Inv. Data \\
\hline $\mathbf{P}$ & Braun & SO4 & $2.64 \mathrm{E}+06$ & $2.75 E-02$ & NS & $\mathbf{N}$ & Won't use Braun, Wastren, or HTCE Inv. Data \\
\hline$L$ & Raw & SO4 & $2.64 \mathrm{E}+06$ & $2.75 E-02$ & AZ102-8 & $N$ & Analysis Prior to $9 / 86$ \\
\hline$\underline{L}$ & Raw & SO4 & $2.64 E+06$ & $2.75 \mathrm{E}-02$ & $\mathrm{AZ102-8}$ & $N$ & Analysis Prior to $9 / 86$ \\
\hline $\mathbf{P}$ & Braun & SO4 & $1.92 E+06$ & $2.00 \mathrm{E}-02$ & NS & $N$ & Won't use Braun, Wastren, or HTCE Inv. Data \\
\hline LL & Raw & 504 & $1.92 E+06$ & $2.00 E-02$ & $A Z 102-21$ & $\mathrm{~N}$ & Not a traceable reference \\
\hline $\mathrm{Fl}$ & Raw & SO4 & $1.87 E \div 06$ & 1.95E-02 & $A Z 102-23$ & $N$ & Water wash of solid sample not supernatent \\
\hline$\underline{E l}$ & Raw & 504 & $7.78 \mathrm{E}+05$ & $8.10 E-03$ & AZ102-18 & $\mathrm{D}$ & NS \\
\hline EI & Raw & so4 & $7.78 E+05$ & $8.10 E-03$ & $A Z 102-23$ & $\mathbf{N}$ & $\begin{array}{l}\text { Solid sample after has been washed with } \\
\text { fresh water }\end{array}$ \\
\hline$\overline{F I}$ & Raw & SO4 & $0.00 E+00$ & $0.00 E+00$ & $A Z 102-18$ & $\mathrm{D}$ & NS \\
\hline$\underline{B 1}$ & Raw & Sr & $5.65 E+05$ & $6.45 \mathrm{E}-03$ & $A 2102-18$ & D & NS \\
\hline G & Wastren & Sr & $4.87 E+05$ & $5.56 \mathrm{E}-03$ & NS & $\mathbf{N}$ & Won't use Braun, Wastren, or HTCE Inv. Data \\
\hline $\mathbf{S}$ & Braun & $\mathrm{Sr}$ & $1.21 E+05$ & $1.38 E-03$ & NS & $N$ & Won't use Braun, Wastren, or HTCE Inv. Data \\
\hline EI & Raw & $\mathrm{Sr}$ & $8.75 E+04$ & 9.99E-04 & AZ102-18 & D & NS \\
\hline EI & Raw & Sr & $8.75 E+04$ & $9.99 E-04$ & $A Z 102-23$ & $\mathbf{N}$ & $\begin{array}{l}\text { Solid sample after has been washed with } \\
\text { fresh water }\end{array}$ \\
\hline $\mathbf{P}$ & Wastren & Sr & $2.07 E+02$ & 2.36E-06 & NS & $\mathbf{N}$ & Won't use Braun, Wastren, or HTCE Inv. Data \\
\hline Cl & Raw & $\mathrm{Sr}$ & $2.00 E+02$ & $2.28 \mathrm{E}-06$ & AZ102-23 & $\mathrm{Y}$ & \\
\hline$\overline{\mathrm{Cl}}$ & Raw & $S r$ & $2.00 E+02$ & $2.28 \mathrm{E}-06$ & $A Z 102-18$ & $\mathrm{D}$ & NS \\
\hline$\underline{F I}$ & Raw & Sr & $0.00 E+00$ & $0.00 E+00$ & $A Z 102-18$ & $\mathbf{N}$ & value reported as 0.000 \\
\hline FI & Raw & Sr & $0.00 E+00$ & $0.00 E+00$ & $A Z 102-23$ & $N$ & value reported as 0.000 \\
\hline$\underline{Y}$ & Raw & Sr & $0.00 E+00$ & $0.00 E+00$ & $A Z 102-23$ & D & NS \\
\hline$\underline{\mathrm{Cl}}$ & Raw & TC & $9.55 E+06$ & $7.96 \mathrm{E}-01$ & $A Z 102-23$ & $\mathbf{Y}$ & \\
\hline $\mathbf{F I}$ & Raw & Te & \#VALUE! & \#VALUE! & AZ102-18 & $\mathbf{N}$ & Water wash of solid sample not supernatent \\
\hline$\underline{E}$ & Raw & Te & $1.93 E+05$ & $1.51 \mathrm{E}-03$ & AZ102-18 & $\mathrm{D}$ & NS \\
\hline $\mathrm{EI}$ & Raw & Te & $1.93 E+05$ & $1.51 E-03$ & $A Z 102-23$ & $\mathbf{N}$ & $\begin{array}{l}\text { Solid sample after has been washed with } \\
\text { fresh water }\end{array}$ \\
\hline$\overline{\mathrm{Cl}}$ & Raw & $\mathrm{Te}$ & $2.62 E+03$ & $2.05 E-05$ & AZ102-23 & $Y$ & \\
\hline$\underline{\underline{\mathrm{Cl}}}$ & Raw & $\mathrm{Te}$ & $2.62 E+03$ & 2.05E-05 & $A Z 102-18$ & D & NS \\
\hline $\mathbf{P}$ & Wastren & $\mathrm{Te}$ & $2.17 E+03$ & $1.70 E-05$ & NS & $\mathbf{N}$ & Won't use Braun, Wastren, or HTCE Inv. Data \\
\hline El & Raw & $\Pi$ & \#VALUE! & \#VALUEl & AZ102-18 & $\mathbf{N}$ & $\begin{array}{l}\text { Solid sample after has been washed with } \\
\text { fresh water }\end{array}$ \\
\hline FI & Raw & $\mathrm{TI}$ & \#VALUE! & \#VALUE! & $A Z 102-18$ & $\mathbf{N}$ & Water wash of solid sample not supernatent \\
\hline
\end{tabular}


TWRS Privatization Support Pro Chemical Liquids

\begin{tabular}{|c|c|c|c|c|c|c|c|c|c|}
\hline $\begin{array}{l}\text { Reported } \\
\text { Chemical }\end{array}$ & Memo Date & $\begin{array}{c}\text { Start } \\
\text { Analysis } \\
\text { Date }\end{array}$ & $\begin{array}{c}\text { Start Sample } \\
\text { Date }\end{array}$ & Sample Number & $\begin{array}{c}\text { Tank Farm } \\
\text { Number }\end{array}$ & $\begin{array}{c}\text { Lab Sample } \\
\text { Number }\end{array}$ & $\begin{array}{l}\text { Density } \\
(\mathrm{g} / \mathrm{mL})\end{array}$ & $\begin{array}{c}\text { Reported } \\
\text { Value } \\
\end{array}$ & Reported U \\
\hline$\pi$ & $8 / 1 / 85$ & NS & NS & NS & NS & NS & NS & $3.93 E-02$ & $g / L$ \\
\hline$\pi$ & $10 / 31 / 86$ & NS & $6 / 30 / 85$ & NS & NS & NS & 1.14 & $1.63 \mathrm{E}-01$ & umol/g \\
\hline $\mathrm{TI}$ & $9 / 1 / 93$ & N/A & $6 / 30 / 85$ & N/A & N/A & N/A & 1.14 & $1.63 E-01$ & umol/g \\
\hline $\begin{array}{c}\text { TOC wt \% C } \\
\text { (wet) }\end{array}$ & NS & NS & NS & NS & NS & NS & 1.39 & $2.77 E+00$ & $w t \%$ \\
\hline TOC & $10 / 31 / 86$ & NS & $6 / 30 / 85$ & NS & NS & NS & NS & $2.63 E+02$ & umol/g \\
\hline$F$ & $8 / 31 / 89$ & NS & $6 / 30 / 85$ & Composite Core & NS & NS & 1.11 & $9.00 E+01$ & umol/g \\
\hline TOC & $10 / 31 / 86$ & NS & $6 / 30 / 85$ & NS & NS & NS & NS & $1.12 E+02$ & umol/g \\
\hline $\begin{array}{c}\text { TOC wt\% C } \\
\text { (wet) }\end{array}$ & NS & NS & NS & NS & NS & NS & 1.04 & $2.03 E-03$ & $w t \%$ \\
\hline TOC & $12 / 3 / 75$ & NS & NS & 1459 & NS & NS & 1.386 & $1.23 E+02$ & $g / L$ \\
\hline TOC & $12 / 3 / 75$ & NS & NS & $1496 \mathrm{C}$ & NS & NS & 1.379 & $1.22 \mathrm{E}+02$ & $g / L$ \\
\hline TOC & $6 / 26 / 75$ & NS & NS & RAT-AZ102-4a & NS & NS & 1.39 & $1.22 \mathrm{E}+02$ & $\mathrm{gm} / \mathrm{s}$ \\
\hline TOC & $6 / 26 / 75$ & NS & NS & NS & NS & NS & 1.392 & $1.22 E+02$ & $g / L$ \\
\hline TOC & $12 / 3 / 75$ & NS & NS & 1456 & NS & NS & 1.393 & $1.12 E+02$ & $g / h$ \\
\hline TOC & $12 / 3 / 75$ & NS & NS & 1457 & NS & NS & 1.398 & $1.12 \mathrm{E}+02$ & $g / L$ \\
\hline TOC & $12 / 3 / 75$ & NS & NS & 1458 & NS & NS & 1.391 & $1.12 E+02$ & $g / L$ \\
\hline TOC & $12 / 3 / 75$ & NS & NS & 14960 & NS & NS & 1.36 & $8.82 E+01$ & $\mathrm{~g} / \mathrm{L}$ \\
\hline TOC & $12 / 3 / 75$ & NS & NS & $1496 \mathrm{~A}$ & NS & NS & 1.378 & $8.80 E+01$ & $g / L$ \\
\hline TOC & $12 / 3 / 75$ & NS & NS & $1496 \mathrm{~B}$ & NS & NS & 1.38 & $8.78 \mathrm{E}+01$ & $g / L$ \\
\hline TOC & $12 / 3 / 75$ & NS & NS & $1496 \mathrm{C}$ & NS & NS & 1.379 & $8.72 E+01$ & $g / L$ \\
\hline TOC & $12 / 3 / 75$ & NS & NS & $1496 \mathrm{~A}$ & NS & NS & 1.378 & $8.70 E+01$ & $g / L$ \\
\hline TOC & $12 / 3 / 75$ & NS & NS & 14968 & NS & NS & 1.38 & $8.68 \mathrm{E}+01$ & $g / L$ \\
\hline TOC & $12 / 3 / 75$ & NS & NS & $1496 \mathrm{D}$ & NS & NS & 1.36 & $8.52 E+01$ & $g / L$ \\
\hline TOC & $8 / 27 / 77$ & NS & NS & NS & NS & NS & 1.391 & $7.74 \mathrm{E}+01$ & $g / L$ \\
\hline TOC & $9 / 20 / 78$ & NS & NS & RAT-AZ102-4C & NS & NS & 1.39 & $7.60 E+01$ & $\mathrm{gm} / \mathrm{I}$ \\
\hline TOC & $9 / 20 / 78$ & NS & NS & NS & NS & NS & 1.39 & $7.60 E+01$ & $g / L$ \\
\hline TOC & $7 / 28 / 79$ & NS & NS & RAT-AZ102-4D & NS & NS & 1.41 & $7.00 E+01$ & $\mathrm{gm} / \mathrm{I}$ \\
\hline TOC & $7 / 28 / 79$ & NS & NS & NS & NS & NS & 1.41 & $7.00 E+01$ & $g / 1$ \\
\hline TOC & $7 / 28 / 79$ & NS & NS & RAT-AZ102-6 & NS & NS & 1.41 & $7.00 E+01$ & $\mathrm{gm} n$ \\
\hline TOC & $10 / 30 / 86$ & NS & NS & $\mathrm{R} 280783$ & NS & NS & NS & $7.00 \mathrm{E}+01$ & $g / L$ \\
\hline $\mathrm{Cd}$ & $8 / 31 / 89$ & NS & $6 / 30 / 85$ & Composite Core & NS & NS & 1.73 & $1.06 \mathrm{E}+01$ & $g / L$ \\
\hline TOC & $12 / 13 / 83$ & NS & $7 / 31 / 83$ & NS & NS & NS & NS & $1.05 \mathrm{E}+01$ & $g / L$ \\
\hline TOC & $11 / 5 / 81$ & NS & NS & RAT-AZ102-2B & NS & NS & 1.4 & $8.02 E+00$ & $g \mathrm{~m} / 1$ \\
\hline TOC & $5 / 5 / 82$ & NS & $11 / 4 / 81$ & NS & NS & NS & 1.4 & $8.02 E+00$ & $g / L$ \\
\hline Cd & $8 / 31 / 89$ & NS & $6 / 30 / 85$ & Composite Core & NS & NS & 1.73 & $8.00 E+00$ & $g / L$ \\
\hline TOC & $11 / 5 / 81$ & NS & NS & RAT-AZ102-2C & NS & NS & 1.26 & $6.14 E+00$ & $\mathrm{gm} / \mathrm{l}$ \\
\hline TOC & Aug 89 & NS & NS & NS & NS & NS & NS & $4.70 E+00$ & $g / L$ \\
\hline
\end{tabular}




\begin{tabular}{|c|c|c|c|c|c|c|c|}
\hline \multirow[b]{2}{*}{ Code } & \multirow[b]{2}{*}{ Source } & \multicolumn{3}{|c|}{ Analyte Information } & \multirow[b]{2}{*}{$\begin{array}{c}\text { Reference } \\
\text { Number }\end{array}$} & \multirow[b]{2}{*}{$\begin{array}{c}\text { Validated } \\
\text { (Y/N) }\end{array}$} & \multirow[b]{2}{*}{ Notes } \\
\hline & & Analyte & Conc. (ug/L) & Conc. (mol/L) & & & \\
\hline$P$ & Wastren & $\mathrm{TI}$ & $3.93 E+04$ & $1.92 E-04$ & NS & $\mathbf{N}$ & Won't use Braun, Wastren, or HTCE Inv. Data \\
\hline $\mathrm{Cl}$ & Raw & $\pi$ & $3.80 E+04$ & $1.86 \mathrm{E}-04$ & $A Z 102-18$ & $D$ & NS \\
\hline $\mathrm{Cl}$ & Raw & $\pi$ & $3.80 E+04$ & $1.86 E-04$ & A2102-23 & $\bar{Y}$ & \\
\hline s & HTCE Inv. & TOC & $3.85 E+19$ & $3.21 E+12$ & NS & $N$ & Won't use Braun, Wastren, or HTCE Inv. Data \\
\hline $\mathbf{S}$ & Raw & TOC & $5.05 E+18$ & $4.21 E+11$ & $A Z 102-18$ & $\mathbf{N}$ & $\begin{array}{l}\text { No TOC values given in the partial report } \\
\text { available_cc_8/15/95; may be in AZ-102-23 }\end{array}$ \\
\hline El & Raw & TOC & $1.73 E+18$ & $1.44 E+11$ & $A Z 102-23$ & $N$ & $\begin{array}{l}\text { Solid sample after has been washed with } \\
\text { fresh water }\end{array}$ \\
\hline $\mathbf{L}$ & Raw & TOC & $1.61 E+18$ & $1.34 E+11$ & $A Z 102-18$ & $\mathbf{N}$ & $\begin{array}{l}\text { No TOC values given in the partial report } \\
\text { available_cc_8/15/95; may be in AZ-102-23 }\end{array}$ \\
\hline $\mathbf{L}$ & HTCE Inv. & TOC & $2.11 E+16$ & $1.76 E+09$ & NS & $\mathbf{N}$ & Won't use Braun, Wastren, or HTCE Inv. Data \\
\hline $\mathrm{L}$ & Raw & TOC & $1.23 E+08$ & $1.03 E+01$ & $A Z 102-3$ & $N$ & Analysis Prior to $9 / 86$ \\
\hline$L$ & Raw & TOC & $1.22 \mathrm{E}+08$ & $1.02 E+01$ & $A Z 102-3$ & $\mathbf{N}$ & Analysis Prior to $9 / 86$ \\
\hline$P$ & Braun & TOC & $1.22 E+08$ & $1.02 \mathrm{E}+01$ & NS & $N$ & Analysis Prior to $9 / 86$ \\
\hline$L$ & Raw & TOC & $1.22 \mathrm{E}+08$ & $1.02 E+01$ & $A Z 102-6$ & $N$ & Analysis Prior to $9 / 86$ \\
\hline$L$ & Raw & TOC & $1.12 E+08$ & $9.33 E+00$ & $A Z 102-3$ & $N$ & Analysis Prior to $9 / 86$ \\
\hline$\underline{L}$ & Raw & TOC & $1.12 E+08$ & $9.33 E+00$ & AZ102-3 & $N$ & Analysis Prior to $9 / 86$ \\
\hline$L$ & Raw & TOC & $1.12 E+08$ & $9.33 E+00$ & $A Z 102-3$ & $N$ & Analysis Prior to $9 / 86$ \\
\hline L & Raw & TOC & $8.82 E+07$ & $7.35 E+00$ & $A Z 102-3$ & $N$ & Analysis Prior to $9 / 86$ \\
\hline $\mathrm{L}$ & Raw & TOC & $-8.80 E+07$ & $7.33 E+00$ & $A Z 102-3$ & $N$ & Analysis Prior to $9 / 86$ \\
\hline L & Raw & TOC & $8.78 E+07$ & $7.32 E+00$ & AZ102-3 & $N$ & Analysis Prior to $9 / 86$ \\
\hline $\mathrm{L}$ & Raw & TOC & $8.72 E+07$ & $7.27 E+00$ & $A Z 102-3$ & $N$ & Analysis Prior to 9/86 \\
\hline $\mathbf{L}$ & Raw & TOC & $8.70 E+07$ & $7.25 E+00$ & $A Z 102-3$ & $\mathbf{N}$ & Analysis Prior to $9 / 86$ \\
\hline $\mathrm{L}$ & Raw & TOC & $8.68 \mathrm{E}+07$ & $7.23 E+00$ & $A Z 102-3$ & $\mathbf{N}$ & Analysis Prior to $9 / 86$ \\
\hline $\mathrm{L}$ & Raw & TOC & $8.52 \mathrm{E}+07$ & $7.10 \mathrm{E}+00$ & AZ102-3 & $\mathbf{N}$ & Analysis Prior to $9 / 86$ \\
\hline$F$ & Raw & TOC & $7.74 E+07$ & $6.45 E+00$ & $A Z 102-4$ & $\bar{N}$ & Analysis Prior to $9 / 86$ \\
\hline$P$ & Braun & TOC & $7.60 E+07$ & $6.33 E+00$ & NS & $\mathbf{N}$ & Won't use Braun, Wastren, or HTCE Inv. Data \\
\hline $\mathbf{L}$ & Raw & TOC & $7.60 E+07$ & $6.33 E+00$ & $\mathrm{AZ102-6}$ & $\mathrm{N}$ & Analysis Prior to $9 / 86$ \\
\hline $\mathbf{P}$ & Braun & TOC & $7.00 E+07$ & $5.83 E+00$ & NS & N & Won't use Braun, Wastren, or HTCE Inv. Data \\
\hline $\mathbf{L}$ & Raw & TOC & $7.00 E+07$ & $5.83 E+00$ & $A Z 102-5$ & $\mathrm{~N}$ & Analysis Prior to $9 / 86$ \\
\hline $\mathbf{R}$ & Braun & TOC & $7.00 E+07$ & $5.83 E+00$ & NS & $\mathbf{N}$ & Won't use Braun, Wastren, or HTCE Inv. Data \\
\hline $\mathrm{L}$ & Raw & TOC & $7.00 E+07$ & $5.83 E+00$ & $A Z 102-20$ & $N$ & Not a traceable reference \\
\hline $\mathrm{BI}$ & Raw & TOC & $1.06 E+07$ & $8.83 \mathrm{E}-01$ & $A Z 102-14$ & $\mathrm{D}$ & Duplicate of Data in Kaizer AZ-102-23 \\
\hline S & Raw & TOC & $1.05 E+07$ & $8.75 \mathrm{E}-01$ & AZ102-14 & $\mathbf{N}$ & Calculated not measured \\
\hline $\mathbf{S}$ & Braun & TOC & $8.02 E+06$ & $6.68 \mathrm{E}-01$ & NS & $N$ & Won't use Braun, Wastren, or HTCE Inv. Data \\
\hline $\mathbf{S}$ & Rlaw & TOC & $8.02 E+06$ & $6.68 \mathrm{E}-01$ & AZ102-11 & $N$ & Analysis Prior to $9 / 86$ \\
\hline BI & Raw & TOC & $8.00 E+06$ & 6.67E-01 & AZ102-14 & D & Duplicate of Data in Kaizer AZ-102-23 \\
\hline R & Braun & TOC & $6.14 \mathrm{E}+06$ & $5.12 \mathrm{E}-01$ & NS & $N$ & Won't use Braun, Wastren, or HTCE Inv. Data \\
\hline $\mathbf{G}$ & Wastren & TOC & $4.70 E+06$ & $3.92 \mathrm{E}-01$ & NS & $N$ & Won't use Braun, Wastren, or HTCE Inv. Data \\
\hline
\end{tabular}




\begin{tabular}{|c|c|c|c|c|c|c|c|c|c|}
\hline & & & & Tank Waste $C$ & racterization & ample informati & & & \\
\hline $\begin{array}{l}\text { Reported } \\
\text { Chemical }\end{array}$ & Memo Date & $\begin{array}{c}\text { Start } \\
\text { Analysis } \\
\text { Date } \\
\end{array}$ & $\begin{array}{c}\text { Start Sample } \\
\text { Date }\end{array}$ & Sample Number & $\begin{array}{c}\text { Tank Farm } \\
\text { Number }\end{array}$ & $\begin{array}{c}\text { Lab Sample } \\
\text { Number }\end{array}$ & $\begin{array}{l}\text { Density } \\
(\mathrm{g} / \mathrm{mL})\end{array}$ & $\begin{array}{c}\text { Reported } \\
\text { Value } \\
\end{array}$ & Reported UnI \\
\hline TOC & $11 / 5 / 81$ & NS & NS & RAT-AZ102-2A & NS & NS & 1.09 & $3.81 E+00$ & $\mathrm{gm} / 1$ \\
\hline TOC & $5 / 5 / 82$ & NS & $11 / 4 / 81$ & NS & NS & NS & 1.09 & $3.81 E+00$ & $g / L$ \\
\hline TOC & $10 / 30 / 86$ & NS & NS & R060386 & NS & NS & NS & $3.81 E+00$ & $g / L$ \\
\hline TOC & Aug 89 & NS & NS & NS & NS & NS & NS & $1.59 E+00$ & $g / L$ \\
\hline $\begin{array}{c}\text { Tot. Organic } \\
\text { Carbon }\end{array}$ & $7 / 26 / 95$ & N/A & $3 / 2 / 91$ & BOD9R3 & $A Z-102-1$ & S95T000263 & 1.1 & $1.58 E+03$ & UG/ml, C \\
\hline $\begin{array}{c}\text { Tot. Organic } \\
\text { Carbon }\end{array}$ & $7 / 26 / 95$ & N/A & $3 / 2 / 91$ & BOD9R4 & $A Z-102-2$ & S95T000264 & 1.1 & $1.56 \mathrm{E}+03$ & UG/ml, C \\
\hline $\begin{array}{c}\text { Tot. Organic } \\
\text { Carbon }\end{array}$ & $7 / 26 / 95$ & N/A & $3 / 2 / 91$ & BOD9R3 & $A Z-102-1$ & S95T000263 & 1.1 & $1.55 E+03$ & UG/ml, C \\
\hline TOC & $9 / 1 / 93$ & N/A & $6 / 30 / 85$ & N/A & N/A & N/A & 1.14 & $1.12 \mathrm{E}+02$ & umol/g, C \\
\hline TOC & $10 / 1 / 83$ & NS & NS & RAT-AZ102-3 & NS & NS & NS & $1.52 E+00$ & $\mathrm{gm} / \mathrm{l}$ \\
\hline Toc & $3 / 10 / 84$ & NS & $7 / 31 / 83$ & NS & NS & NS & NS & $1.52 E+00$ & $g / L$ \\
\hline TOC & $3 / 10 / 84$ & NS & $7 / 31 / 83$ & NS & NS & NS & NS & $1.52 E+00$ & $g / l$ \\
\hline TOC & $7 / 5 / 84$ & NS & $7 / 31 / 83$ & T3495 & NS & NS & NS & $1.52 \mathrm{E}+00$ & $g / L$ \\
\hline TOC & $10 / 31 / 86$ & NS & NS & R880311 & NS & NS & NS & $1.52 \mathrm{E}+00$ & $g / L$ \\
\hline $\begin{array}{c}\text { Tot. Organic } \\
\text { Carbon } \\
\end{array}$ & $7 / 26 / 95$ & N/A & $3 / 2 / 91$ & BOD9R4 & $A Z-102-2$ & S95T000264 & 1.1 & $1.51 E+03$ & UG/ml, C \\
\hline $\begin{array}{c}\text { Tot. Organic } \\
\text { Carbon }\end{array}$ & $7 / 26 / 95$ & N/A & $3 / 2 / 91$ & BOD9R5 & $A Z-102-3$ & S95T000265 & 1.1 & $1.42 E+03$ & UG/ml, C \\
\hline $\begin{array}{c}\text { Tot. Organic } \\
\text { Carbon }\end{array}$ & $7 / 26 / 95$ & N/A & $3 / 2 / 91$ & BOD9R5 & $A Z-102-3$ & S95T000265 & 1.1 & $1.40 E+03$ & UG/ml, C \\
\hline TOC & $12 / 13 / 83$ & NS & $7 / 31 / 83$ & NS & NS & NS & NS & $7.20 E-01$ & $g / L$ \\
\hline TOC & $10 / 31 / 86$ & NS & NS & R081287 & NS & NS & NS & 7.20E-01 & g/L \\
\hline TOC & $1 / 30 / 82$ & NS & NS & $T-5387$ & NS & NS & 1.008 & 5.22E-02 & $\mathrm{gm} / \mathrm{I}$ \\
\hline TOC & $2 / 19 / 82$ & NS & NS & T5387 & NS & NS & 1.008 & $5.22 E-02$ & $g / l$ \\
\hline TOC & $8 / 31 / 89$ & NS & $6 / 30 / 85$ & Composite Core & NS & NS & NS & $0.00 E+00$ & umol/g \\
\hline$u$ & $11 / 5 / 81$ & NS & NS & RAT-AZ102-2B & NS & NS & 1.4 & $5.99 E+00$ & $\mathrm{gm} / \mathrm{I}$ \\
\hline$u$ & $11 / 5 / 81$ & NS & NS & RAT-AZ102-2C & NS & NS & 1.26 & $1.64 E+00$ & $\mathrm{gm} / \mathrm{l}$ \\
\hline $\mathrm{U}$ & $11 / 5 / 81$ & NS & NS & RAT-AZ102-2A & NS & NS & 1.09 & $1.56 E-01$ & $\mathrm{gm} / \mathrm{l}$ \\
\hline$U$ & $10 / 1 / 83$ & NS & NS & RAT-AZ102-3 & NS & NS & NS & $5.00 E-02$ & $\mathrm{gm} / \mathrm{h}$ \\
\hline$u$ & $2 / 4 / 81$ & NS & NS & $R-3700$ & NS & NS & 1.11 & $2.95 E-02$ & $\mathrm{gm} / \mathrm{I}$ \\
\hline$u$ & $2 / 4 / 81$ & NS & NS & $R-3699$ & NS & NS & 1.11 & $2.78 E-02$ & $\mathrm{gm} / \mathrm{I}$ \\
\hline $\mathrm{v}$ & $10 / 31 / 86$ & NS & $6 / 30 / 85$ & NS & NS & NS & 1.11 & $3.70 \mathrm{E}-01$ & umol/g \\
\hline $\mathrm{Fe}$ & $10 / 31 / 86$ & NS & $6 / 30 / 85$ & Composite Core & NS & NS & 1.11 & $3.70 \mathrm{E}-01$ & umol/g \\
\hline v & $8 / 1 / 85$ & NS & NS & NS & NS & NS & NS & $2.10 E-03$ & $g / L$ \\
\hline $\mathrm{V}$ & $9 / 1 / 93$ & N/A & $6 / 30 / 85$ & $\mathrm{~N} / \mathrm{A}$ & N/A & N/A & 1.14 & $3.50 \mathrm{E}-02$ & umol/g \\
\hline
\end{tabular}




\begin{tabular}{|c|c|c|c|c|c|c|c|}
\hline & & & Analyte Inform & & & & \\
\hline Sode & Source & Analyte & Conc. (ug/L) & Conc. (mol/L) & $\begin{array}{c}\text { Referenice } \\
\text { Number }\end{array}$ & $\begin{array}{c}\text { Validated } \\
\text { (Y/N) }\end{array}$ & Notes \\
\hline $\mathbf{P}$ & Braun & TOC & $3.81 E+06$ & $3.18 E-01$ & NS & $\mathbf{N}$ & Won't use Braun, Wastren, or HTCE Inv. Data \\
\hline $\mathrm{L}$ & Raw & TOC & $3.81 E+06$ & $3.18 \mathrm{E}-01$ & $A 2102-11$ & $\mathbf{N}$ & Analysis Prior to $9 / 86$ \\
\hline $\bar{L}$ & Raw & TOC & $3.81 E+06$ & $3.18 \mathrm{E}-01$ & AZ102-21 & $\mathrm{N}$ & Not a traceable reference \\
\hline$P$ & Wastren & TOC & $1.59 E+06$ & 1.33E-01 & NS & $N$ & Won't use Braun, Wastren, or HTCE Inv. Data \\
\hline P-1 & Raw & TOC & $1.58 E+06$ & $1.32 \mathrm{E}-01$ & $A Z 102-24$ & Y & $\begin{array}{l}\text { Updated per memo } 8 / 17 / 95 \text {, determined by } \\
\text { coulometry }\end{array}$ \\
\hline P-1 & Raw & TOC & $1.56 E+06$ & $1.30 E-01$ & $A Z 102-24$ & $Y$ & $\begin{array}{l}\text { Updated per memo 8/17/95, determined by } \\
\text { coulometry }\end{array}$ \\
\hline P-2 & Raw & TOC & $1.55 E+06$ & $1.29 E-01$ & $A Z 102-24$ & Y & $\begin{array}{l}\text { Updated per memo } 8 / 17 / 95 \text {, determined by } \\
\text { coulometry }\end{array}$ \\
\hline $\mathrm{Cl}$ & Raw & TOC & $1.53 E+06$ & $1.28 \mathrm{E}-01$ & $\mathrm{AZ102-23}$ & $\bar{Y}$ & \\
\hline$P$ & Braun & TOC & $1.52 E+06$ & $1.27 E-01$ & NS & N & Won't use Braun, Wastren, or HTCE Inv. Data \\
\hline L & Raw & TOC & $1.52 E+06$ & $1.27 \mathrm{E}-01$ & $A Z 102-16$ & $N$ & $\begin{array}{l}\text { These data are so messed up it will be easier } \\
\text { to reenter them. }\end{array}$ \\
\hline$L$ & Raw & TOC & $1.52 E+06$ & 1.27E-01 & AZ102-16 & $\mathbf{N}$ & Point in DB already_cc 8/15/95 \\
\hline$L$ & Raw & TOC & $1.52 E+06$ & $1.27 \mathrm{E}-01$ & $A Z 102-16$ & $\mathbf{N}$ & Point in DB already cc_8/15/95 \\
\hline$L$ & Raw & TOC & $1.52 E+06$ & $1.27 E-01$ & AZ102-19 & $\mathrm{N}$ & Not a traceable reference \\
\hline P-2 & Raw & TOC & $1.51 E+06$ & $1.26 \mathrm{E}-01$ & $A Z 102-24$ & Y & $\begin{array}{l}\text { Updated per memo } 8 / 17 / 95 \text {, determined by } \\
\text { coulometry }\end{array}$ \\
\hline P-1 & Raw & TOC & $1.42 E+06$ & $1.18 \mathrm{E}-01$ & $A Z 102-24$ & $\gamma$ & $\begin{array}{l}\text { Updated per memo } 8 / 17 / 95 \text {, determined by } \\
\text { coulometry }\end{array}$ \\
\hline P-2 & Raw & TOC & $1.40 E+06$ & $1.17 \mathrm{E}-01$ & $A Z 102-24$ & $Y$ & $\begin{array}{l}\text { Updated per memo } 8 / 17 / 95 \text {, determined by } \\
\text { coulometry }\end{array}$ \\
\hline$L$ & Raw & TOC & $7.20 E+05$ & $6.00 \mathrm{E}-02$ & $A Z 102-14$ & $\mathbf{N}$ & Calculated not measured \\
\hline$L$ & Raw & TOC & $7.20 E+05$ & $6.00 \mathrm{E}-02$ & $A Z 102-22$ & $\mathbf{N}$ & Not a traceable reference \\
\hline$P$ & Braun & TOC & $5.22 E+04$ & 4.35E-03 & NS & $N$ & Won't use Braun, Wastren, or HTCE inv. Data \\
\hline $\mathbf{L}$ & Raw & TOC & $5.22 E+04$ & 4.35E-03 & $A Z 102-9$ & $\mathbf{N}$ & $\begin{array}{l}\text { Not a traceable reference, analysis Prior to } \\
9 / 86\end{array}$ \\
\hline $\mathrm{FI}$ & Raw & TOC & $0.00 E+00$ & $0.00 E+00$ & AZ102-23 & $\mathbf{N}$ & value reported as 0.000 \\
\hline $\mathbf{s}$ & Braun & $U$ & $5.99 E+06$ & $2.52 E-02$ & NS & $\mathbf{N}$ & Won't use Braun, Wastren, or HTCE Inv. Data \\
\hline Y & Braun & $u$ & $1.64 E+06$ & $6.89 E-03$ & NS & $N$ & Won't use Braun, Wastren, or HTCE Inv. Data \\
\hline $\mathbf{P}$ & Braun & $u$ & $1.56 E+05$ & $6.55 \mathrm{E}-04$ & NS & $\mathbf{N}$ & Won't use Braun, Wastren, or HTCE Inv. Data \\
\hline$P$ & Braun & $u$ & $5.00 E+04$ & $2.10 E-04$ & NS & N & Won't use Braun, Wastren, or HTCE Inv. Data \\
\hline$P$ & Braun & $u$ & $2.95 E+04$ & $1.24 E-04$ & NS & N & Won't use Braun, Wastren, or HTCE Inv. Data \\
\hline$P$ & Braun & $u$ & $2.78 E+04$ & $1.17 \mathrm{E}-04$ & NS & N & Won't use Braun, Wastren, or HTCE Inv. Data \\
\hline El & Raw & $\bar{v}$ & $2.09 E+04$ & 4.11E-04 & $A Z 102-18$ & $\mathrm{D}$ & NS \\
\hline El & Raw & V & $2.09 E+04$ & $4.11 E-04$ & AZ102-23 & $N$ & $\begin{array}{l}\text { Solid sample after has been washed with } \\
\text { fresh water }\end{array}$ \\
\hline $\mathbf{P}$ & Wastren & V & $2.10 E+03$ & $4.12 \mathrm{E}-05$ & NS & $N$ & Won't use Braun, Wastren, or HTCE Inv. Data \\
\hline $\mathrm{Cl}$ & Raw & $\mathrm{V}$ & $2.03 E+03$ & $3.99 E-05$ & $\mathrm{AZ102-23}$ & $\bar{Y}$ & \\
\hline
\end{tabular}




\begin{tabular}{|c|c|c|c|c|c|c|c|c|c|}
\hline \multicolumn{10}{|c|}{ Tank Waste Characterization Sample Information } \\
\hline $\begin{array}{c}\text { Reported } \\
\text { Chemical } \\
\mathrm{V} \\
\end{array}$ & $\begin{array}{c}\text { Memo Date } \\
10 / 31 / 86 \\
\end{array}$ & $\begin{array}{c}\begin{array}{c}\text { Start } \\
\text { Analysis } \\
\text { Date }\end{array} \\
\text { NS }\end{array}$ & 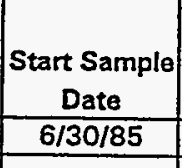 & $\frac{\text { Sample Number }}{\text { NS }}$ & $\begin{array}{c}\begin{array}{c}\text { Tank Farm } \\
\text { Number }\end{array} \\
\text { NS } \\
\end{array}$ & $\begin{array}{c}\text { Lab Sample } \\
\text { Number }\end{array}$ & $\begin{array}{c}\begin{array}{c}\text { Density } \\
(\mathrm{g} / \mathrm{m} L)\end{array} \\
1.14\end{array}$ & $\begin{array}{c}\begin{array}{c}\text { Reported } \\
\text { Value }\end{array} \\
3.50 \mathrm{E}-02 \\
\end{array}$ & $\frac{\text { Reported Un }}{\text { umol/g }}$ \\
\hline v & $8 / 31 / 89$ & NS & $6 / 30 / 85$ & Composite Core & NS & NS & $(1.0)$ & $7.00 E-03$ & umol/g \\
\hline$V$ & $10 / 31 / 86$ & NS & $6 / 30 / 85$ & NS & NS & NS & NS & $7.00 \mathrm{E}-03$ & umol/g \\
\hline
\end{tabular}

A- 


\begin{tabular}{|c|c|c|c|c|c|c|c|}
\hline & & \multicolumn{3}{|c|}{ Analyte Information } & \multirow[b]{2}{*}{$\begin{array}{c}\text { Reference } \\
\text { Number }\end{array}$} & \multirow[b]{2}{*}{$\begin{array}{c}\text { Validated } \\
\text { (Y/N) }\end{array}$} & \multirow[b]{2}{*}{ Notes } \\
\hline Code & Source & Analyte & Conc. (ug/L) & Conc. (mol/L) & & & \\
\hline $\mathrm{Cl}$ & Raw & $\mathrm{V}$ & $2.03 E+03$ & $3.99 E-05$ & $A Z 102-18$ & $\mathrm{D}$ & NS \\
\hline FI & Raw & v & $3.57 E+02$ & 7.00E-06 & $A Z 102-23$ & N & Water wash of solid sample not supernatent \\
\hline $\mathrm{FI}$ & Raw & $\mathrm{V}$ & $0.00 E+00$ & $0.00 E+00$ & $A Z 102-18$ & $\bar{D}$ & NS \\
\hline
\end{tabular}

